WILLIAM DUNCAN MCKIM

GRADUATE OF

COLUMBIA UNIVERSITY

A. B., 1875; A. M., 1878; M. D., 1878

QP34

L232

v.1

Culumbia ofnimersity in the Citipuf

College of 枹ysticians and Surgeons

Zibrary

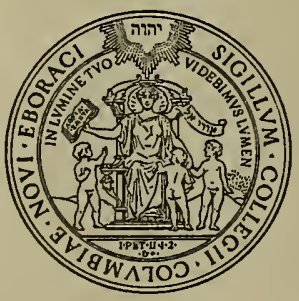








$$
\begin{gathered}
\text { A MANUAL } \\
\text { HUMAN }- \text { PHYSIOLOGY. }
\end{gathered}
$$





\section{A MANUAL OF}

\section{HUMAN PHYSIOLOGY,}

INCLUDING

\section{HISTOLOGY AND MICROSCOPICAL ANATOMY;}

WITH SPECIAL REFERENCE TO THE REQUIREMENTS OF

\section{PRACTICAL MEDICINE.}

D R. L. L A N D O I S,

PROFESSOR OF PHYSIOLOGY AND DIRECTOR OF THE PHYSIOLOGICAL INSTITUTE, UNIVERSITY OF GREIFSWALD.

TRANSLATED FROM THE FOURTH GERMAN EDITION.

\section{WITH ADDITIONS BY}

IV I L L I A M S T I R L I N G, M.D., Sc. D., REGIUS PROFESSOR OF THE INSTITUTES OF MEDICINE OR PHYSIOLOGY IN THE UNIVERSITY OF ABERDEEN.

WITII 176 IIIUSTRATIONS.

V O L. I.

PHILADELPHIA:

P. BLAKISTON, SON, AND COMPANY, IOI 2 WALNUT STEET.

1885 .

[All Rights Reserved.] 


$$
\begin{aligned}
& \mathrm{Q}_{34} \\
& \begin{array}{l}
\mathrm{L}_{232} \\
\mathrm{~V} .1
\end{array}
\end{aligned}
$$




\section{SIR JOSEPH LISTER, BARONET,}

M.D., D.C.L., LL.D., F.R.SS. (LOXD, AXD EDIX.),

PROFESSOR OF CLINICAL SURGERY IN EING'S COLLEGE, LONDON, SURGEOY-EXTRAORDLYARY TO TEE QCEEX;

FORMERLY REGIOS PROFESSOR OF CLIYICAL SURGERY IX THE UXIVERSITY OF EDINBCRGH.

iN ADMIRATION OF

Tlye attir of seienter,

WHOSE BRILLLANT DISCOVERIES HAVE REVOLUTIONISED

MEDICAL PRACTICE, AND CONTRIBUTED INCALCULABLY TO THE

WELL-BEING OF MANKIND;

IND IN GRATITUDE TO

Tye שeardex,

WHOSE NOBLE EARNESTNESS IN INCULCATIYY

THE SACREDNESS OF HUMAN LIFE

STIRRED THE HEARTS OF ALL WHO HEARD HIM :

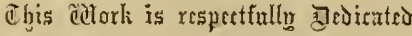

BY HIS FORIIER PUPIL,

THE TRANSLATOR. 



\section{PREFACE.}

THE fact that Professor LandoIs' "Lehrbuch der Physiologie des MIenschen" has already passed through Four large Editions since its first appearance in 1880 , shows that in some special way it has met the wants of Students and Practitioners in Germany. The characteristic which has thus commended the work will be found mainly to lie in its eminent practicality; and it is this consideration which has induced me to undertake the task of putting it into an English dress for English readers.

Landois' work, in fact, forms a Bridge between Physiology and the Practice of Medicine. It never loses sight of the fact that the Student of to-day is the practising Physician of to-morrow. Thus, to every Section is appended-after a full description of the normal processesa short résumé of the pathological variations, the object of this being to direct the attention of the Student, from the outset, to the field of his future practice, and to show him to what extent pathological processes are a disturbance of the normal activities.

In the same way, the work offers to the busy physician in practice a ready means of refreshing his memory on the theoretical aspects of Medicine. He can pass backwards from the examination of pathological phenomena to the normal processes, and, in the study of these, find new indications and new lights for the appreciation and treatment of the cases under consideration.

With this object in view, all the methods of investigation which may with advantage be used by the Practitioner, are carefully and fully described; and Histology, also, occupies a larger place than is usually assigned to it in Text-books of Physiology.

A word as to my own share in the present version :-

(1.) In the task of translating, I have endeavoured throughout to convey the author's meaning accurately, without a too rigid adherence to the original. Those who from experience know something of the difficulties of such an undertaking will be most ready to pardon any shortcomings they may detect. 
(2.) Very considerable additions have been made to the Histological, and also (where it has seemed necessary) to the Physiological sections. All such additions are enclosed within square brackets [ ]. I have to acknowledge my indebtedness to many valuable Papers in the various Medical Journals-British and Foreign-and also to the Histological Treatises of Cadiat, Ranvier, and Klein; Quain's Anatomy, vol. II., ninth edition; Hermann's Handbuch der Physiologie; and the Textbooks on Physiology, by Rutherford, Foster, and Kirkes; Gamgee's Physiological Chemistry; Ewald's Digestion; and Roberts's Digestive Ferments.

(3.) The Illustrations have been increased in number from 106 in the Fourth German Edition to 176 in the English version. These additional Diagrams, with the sources whence derived, are distinguished in the List of Woodcuts by an asterisk.

There only remains for me now to express my thanks to all who have kindly helped in the progress of the work, either by furnishing Illustrations or otherwise - especially to Drs. Byrom Bramwell, Dudgeon, Lauder Brunton, and Knott; Mr. Hawksley; Professors Hamilton and M'Kendrick; to my esteemed teacher and friend, Professor Ludwig, of Leipzic; and, finally, to my friend, Mr. A. W. Pobertson, MI.A., formerly Assistant Librarian in the University, and now Librarian of the Aberdeen Public Library, for much valuable assistance while the work was passing through the press.

The Second Part will, it is hoper, be issued early in 1885 .

In conclusion-and forgetting for the moment my own connection with it-I heartily commend the work per se to the attention of Medical Men, and can wish for it no better fate than that it may speedily become as popular in this country as it is in its Fatherland.

WILLIAM STIRLING.

ABERDEEN UNIVERSITY,

November, 1884. 


\section{GENERAL CONTENTS.}

\section{INTRODUCTION.}

The Scope of Physiology, and its Relation to the other Branches of Natural

Science,

Matter,

Forces, .

ation of Energy,

Vital Energy and Life,

\section{PHYSIOLOGY OF THE BLOOD.}

SECTION

1. Physical Properties of the Blood, . . . . . . . . 1

2. Microscopic Examination of the Blood, . . . . . . . 3

3. Histology of the Human Red Blood-Corpuscles, . $\quad$. $\quad$. $\quad$. $\quad$. 7

4. Effects of Reagents on the Blood-Corpuscles, . $\quad$. $\quad . \quad$. $\quad$. 7

5. Preparation of the Stroma-Making Blood "Lake-Coloured," . . 10

6. Form and Size of the Blood-Corpuscles of Different Animals, . . 11

7. Origin of the Red Blood-Corpuscles, . . . . . . . 12

8. Decay of the Red Blood-Corpuscles, . . . . . . . 16

9. The Colourless Corpuscles--Leucocytes, . . . . . $\quad$. 17

10. Abnormal Changes of the Blood-Corpuscles, . . . . . $\quad 22$

11. Chemical Constituents of the Red Blood-Corpuscles, . . . . 23

12. Preparation of Hæmoglobin Crystals, . . . . . . . 24

13. Quantitative Estimation of Hæmoglobin, . . . . . . 25

14. Use of the Spectroscope, . . . . . . . . . 27

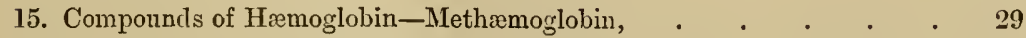

16. Carbonic Oxide-Hæmoglobin, . . . . . . . . 31

17. Poisoning by Carbonic Oxide, . . . . . . . . . 32

18. Decomposition of Hæmoglobin, . . . . . . . . . 33

19. Hæmin and Blood Tests, . . . . . . . . . 34

20. Hæmatoidin, . . . . . . . . . . . 35

21. The Colourless Proteid of Hæmoglobin, . . . . . . . 36

22. Proteids of the Stroma, . . . . . . . . . . . $\quad$. 36

23. The other Constituents of Red Blood-Corpuscles, $\quad$. . . . $\quad 36$

24. Chemical Composition of the Colourless Corpuscles, . . . $\quad$. 37

25. Blood-Plasma, and its Relation to Serum, . . . . . . . 37

26. Preparation of Plasma, . . . . . . . . . . 38

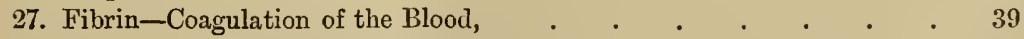

28. General Phenomena of Coagulation, . . . . . . . 40

29. Cause of the Coagulation of the Blood, . . . . . . . 43

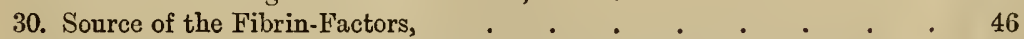

31. Relation of the Red Blood-Corpuscles to the Formation of Fibrin, . $\quad 47$ 
32. Chemical Composition of the Plasma and Serum, . . . . 49

33. The Gases of the Blood, . . . . . . . . . 51

34. Extraction of the Blood Gases, . . . . . . . . 53

35. Quantitative Estimation of the Blood Gases, . . . . . . 55

36. The Blood Gases, . . . . . . . . . . . 55

37. Is ozone $\left(\mathrm{O}_{3}\right)$ present in Blood? . . . . . . . . $\quad$. 57

38. Carbonic Acid and Nitrogen in Blood, . . . . . . . 58

39. Arterial and Venous Blood, . . . . . . . . . 59

40. Quantity of Blood, . . . . . . . . . . 60

41. Variations from the Normal Conditions of the Blood, . . . . 61

\section{PHYSIOLOGY OF THE CIRCULATION.}

42. General View of the Circulation, . . . . . . . . 65

43. The Heart, . . . . . . . . . . . . 66

44. Arrangement of the Cardiac Muscular Fibres, . . . . . . 67

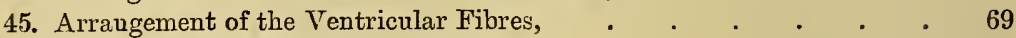

46. Pericardium, Endocardium, Valves, . . . . . . . 71

47. Self-Steering Action of the Heart, . . . . . . . . 73

48. The Movements of the Heart, . . . . . . . . 76

49. Pathological Disturbances of Cardiac Action, _ . . . . $\quad$. 79

50. The Apex-Beat-the Cardiogram, . . . . . . . 80

51. The Time occupied by the Cardiac Movements, . . . . . 85

52. Pathological Disturbance of the Cardiac Impulse, $\quad$. $\quad$. $\quad$. $\quad$. $\quad 89$

53. The Heart-Sounds, . . . . . . . . . . 91

54. Variations of the Heart-Sounds, . . . . . . . . 95

55. The Duration of the Movements of the Heart, . . . . $\quad$ - 96

56. Innervation of the Heart, . . . . . . . . . . 97

57. The Cardiac Nerves, . . . . . . . . . . . 97

58. The Automatic Motor-Centres of the Heart, . . . . . . . $\quad$. 98

59. The Cardio-Pneumatic Movements, . . . . . . 109

60. Influence of the Respiratory Pressure on the Heart, . . . . 111

\section{THE CIRCULATION.}

61. The Flow of Fluids through Tubes, . . . . . . . 115

62. Propelling Force, Velocity of Current, Lateral Pressure, . $\quad$. 115

63. Currents through Capillary Tubes, . . . . . . . 118

64. Movements of Fluids and Wave-Motion in Elastic Tubes, . . $\quad 118$

65. Structure and Properties of the Blood-Vessels, . . . . . 119

66. The Pulse-Historical, . . . . . . . . . 127

67. Instruments for Investigating the Pulse, . . . . . . 128

68. The Pulse-Curve or Sphygmogram, . . . . . . . 136

69. Dicrotic Pulse, . . . . . . . . . . . 140

70. Characters of the Pulse, . . . . . . . . . 141

71. Variations in the Strength, Tension, and Volume of the Pulse, . . 143

72. The Pulse-Curves of various Arteries, . . . . . . . 144

73. Anacrotism, . . . . . . . . . . 146

74. Influence of the Respiratory Movements on the Pulse-Curve, . . 148

75. Influence of Pressure upon the Form of the Pulse-Wave, . . . 151

76. Rapidity of Transmission of Pulse-Waves, . . . . . . 152

77. Propagation of the Pulse-Wave in Elastic Tubes, . . . . $\quad 152$

78. Velocity of the Pulse-Wave in Man, . . . . . . . 154 
79. Further Pulsatile Phenomena, . . . . . . . , 156

80. Vibrations Communicated to the Body by the Action of the Heart, , 157

81. The Blood-Current, . . . . . . . . . . 159

S2. Schemata of the Circulation, . . . . . . . . . 161

83. Capacity of the Ventricles, . . . . . . . . . 161

84. Estimation of the Blood-Pressure, . . . . . . . 162

S5. Blood-Pressure in the Arteries, . . . . . . . . 166

86. Blood-Pressure in the Capillaries, . . . . . . . 173

87. Blood-Pressure in the Veins, . . . . . . . . $\quad$. 175

S8. Blood-Pressure in the Pulmonary Artery, . . . . . . 177

89. Measurement of the Velocity of the Blood-Stream, . . . . 179

90. Velocity of the Blood in Arteries, Capillaries, and Veins, . . $\quad 182$

91. Estimation of the Capacity of the Ventricles, . . . . . 184

92. The Duration of the Circulation, . . . . . . . . 184

93. Work of the Heart, . . . . . . . . . . 185

94. Blood-Current in the Smallest Vessels, . . . . . . 186

95. Passage of the Blood-Corpuscles out of the Vessels-[Diapedesis], . 189

96. Movement of the Blood in the Veins, . . . . . . . 190

97. Sounds or Bruits within Arteries, . . . . . . . 192

98. Venous Murmurs, . . . . . . . . . . 193

99. The Venous Pulse-Phlebogram, . . . . . . . . . 194

100. Distribution of the Blood, . . . . . . . . . 196

101. Plethysmugraphy, . . . . . . . . . . 197

102. Transfusion of Blood, . . . . . . . . . . . . 199

\section{THE BLOOD-GLANDS.}

103. The Spleen-Thymus-Thyroid-Supra-Renal Capsules-Hypophysis

Cerebri-Coccygeal and Carotid Glands, . . . . 203

104. Comparative, . . . . . . . . . . . 215

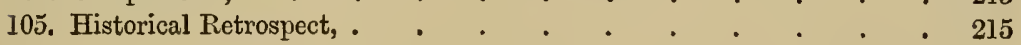

\section{PHYSIOLOGY OF RESPIRATION.}

106. Structure of the Air-Passages and Lungs, . . . . . 217

107. Mechanism of Respiration, . . . . . . . . . 226

108. Quantity of Gases Respired, . . . . . . . . . $\quad$. 227

109. Number of Respirations, . . . . . . . . . . . . $\quad$ • 229

110. Time occupied by the Respiratory Movements, . . . . . . 229

111. Pathological Variations of the Respiratory Movements, . . $\quad 233$

112. General View of the Respiratory Muscles, . . . . . 234

113. Action of the Individual Respiratory Muscles, . . . . . 235

114. Relative Size of the Chest, . . . . . . . . 240

115. Pathological Variations of the Percussion Sounds, . . . . 244

116. The Normal Respiratory Sounds, . . . . . . . 245

117. Pathological Respiratory Sounds, . . . . . . . 245

118. Pressure in the Air-Passages during Respiration, . . . . 247

119. Appendix to Respiration, . . . . . . . . . 248

120. Peculiarly Modified Respiratory Sounds, . . . . . . . 248

121. Quantitative Estimation of $\mathrm{CO}_{2}, \mathrm{O}$, and Watery Vapour, . . . 250

122. Methods of Investigation, . . . . . . . . . 250

123. Composition and Properties of Atmospheric Air, . . . . . 254 
SECTION

124. Composition of Expired Air,

125. Daily Quantity of Gases Exchanged, . . . . . . . 256

126. Review of the Daily Gaseous Income and Expenditure, _ . . . 256

127. Conditions Influencing the Gaseous Exchanges, . . . . . . 256

128. Diffusion of Gases within the Lungs, . . . . . . $\quad 259$

129. Exchange of Gases between the Blood and the Air, . . . . 260

130. Dissociation of Gases, . . . . . . . . . 263

131. Cutaneous Respiration, . . . . . . . . . 264

132. Internal Respiration, . . . . . . . . . . 265

133. Respiration in a Closed Space, . . . . . . . . 267

134. Dyspnœa and Asphyxia, . . . . . . . . . 268

135. Respiration of Foreign Gases, . . . . . . . . 271

136. Accidental Impurities of the Air, . . . . . . . . . 272

137. Ventilation of Rooms, . . . . . . . . . . 272

138. Formation of Mucus, . . . . . . . . . 273

139. Antiou of the Atmospheric Pressure, . . . . . . . 275

140. Comparative and Historical, . . . . . . . , 277

\section{PHYSIOLOGY OF DIGESTION}

141. The Mouth and its Glands, . . . . . . . . . 279

142. The Salivary Glands, . . . . . . . . . 280

143. Histological Changes in the Salivary Glands, . . . . . 283

144. The Nerves of the Salivary Glands, . . . . . . . 285

145. Action of Nerves on the Salivary Secretion, . . . . . 286

146. The Saliva of the Individual Glands, . . . . . . . 291

147. The Mixed Saliva in the Mouth, . . . . . . . . 292

148. Physiological Action of Saliva, . . . . . . . . 294

149. Tests for Sugar, . . . . . . . . . . . . . . 297

150. Quantitative Estimation of Sugar, . . . . . . . . . 298

151. Mechanism of the Digestive Apparatus, . . . . . . 298

152. Introduction of the Food, . . . . . . . . . . 298

153. The Movements of Mastication, . . . . . . . . 299

154. Structure and Development of the Teeth, . . . . . . 300

155. Movements of the Tongue, . . . . . . . . . 304

156. Deglutition, . • . . • • • . . . . . 305

157. Movements of the Stomach, . . . . . . . . 309

158. Vomiting, . . . . . . . . . . . . 310

159. Movements of the Intestine, . . . . . . . . 312

160. Excretion of Fæcal Matter, . . . . . . . . . 313

161. Influence of Nerves on the Intestine, . . . . . . . . . 316

162. Structure of the Stomach, . . . . . . . . . 321

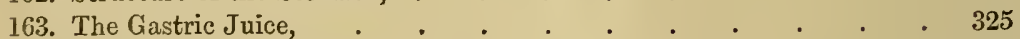

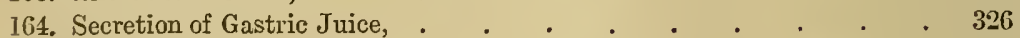

165. Methods of obtaining Gastric Juice, . . . . . . 330

166. Process of Gastric Digestion, . . . . . . . . 331

167. Gases in the Stomach, . . . . . . . . . . 336

168. Structure of the Pancreas, . . . . . . . . . 337

169. The Pancreatic Juice, . . . . . . . . . . . 339

170. Digestive Action of the Pancreatic Juice, . . . . . . 340

171. The Secretion of the Pancreatic Juice, . $\quad$. . . . . . . 340

172. Preparation of Peptonised Food, . . . . . . . . 345 
173. Structure of the Liver, . . . . . . . . . . 346

174. Chemical Composition of the Liver-Cells, . . . . . 350

175. Diabetes Mellitus, or Glycosuria, . . . . . . 352

176. The Functions of the Liver, . . . . . . . . . 35

177. Constituents of the Bile, . . . . . . . . . . 354

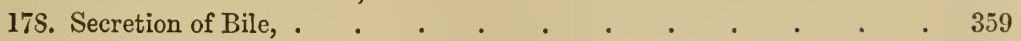

179. Excretion of Bile, . . . . . . . . . . . $\quad$. 361

1S0. Reabsorption of Bile, . . . . . . . . . . 362

181. Functions of the Bile, . . . . . . . . . . 365

1S2. Fate of the Bile in the Intestine, . . . . . . . . 367

183. The Intestinal Juice, . . . . . . . . . . 368

184. Fermentation Processes in the Intestine, . . . . . . 371

185. Processes in the Large Intestine, . . . . . . . . 377

186. Pathological Variations, . . . . . . . . . 350

1S7. Comparative Physiology, . $\quad$. $\quad . \quad$. $\quad . \quad$. . . . 353

1S8. Historical Retrospect, . . . . . . . . . . 354

\section{PHYSIOLOGY OF ABSORPTION.}

189. The Organs of Absorption, . . . . . . . . . 386

190. Structure of the Small and Large Intestines, . . . . . . . 356

191. Absorption of the Digested Food, . . . . . . . 392

192. Absorptive Activity of the Wall of the Intestine, . . . . 395

193. Influence of the Nervous System, . . . . . . . 400

194. Feeding with "Nutrient Enemata," . . . . . . . 400

195. Chyle-Vessels and Lymphatics, . . . . . . . . 401

196. Origin of the Lymphatics, . . . . . . . . . 402

197. The Lymph-Glands, . . . . . . . . . . 406

198. Properties of Chyle and Lympli, . . . . . . . . . 409

199. Quantity of Lymph and Chyle, . . . . . . . . . 412

200. Origin of Lymph, . . . . . . . . . . . 413

201. Movement of Clyyle and Lymph, . . . . . . . . . 415

202. Absorption of Parenchymatous Effusions, . . . . . . 4IS

203. Congestion of Lymph, Serous Effusions and Eilema, . . . . . 4 I9

201. Comparative Physiology, . . , . . . . . . 420

205. Historical Retrospect, . . . . . . . . . . 421

VI. PHYSIOLOGY OF ANIMAL HEAT.

206. Sources of Heat, • . . . . . . . . . . 422

207. Homoiothermal and Poikilothermal Animals, . . . . . 426

208. Methods of Estimating Temperature-Thermometry, . . . . 427

209. Temperature-Topography, . . . . . . . . 430

210. Conditions Influencing the Temperature of Organs, . . . . . 432

211. Estimation of the Amount of Heat-Calorimetry, . . . . 433

212. Thermal Conductivity of Animal Tissues, . . . . . . 436

213. Variations of the Mean Temperature, . . . . . . . . $\quad$. 437

214. Regulation of the Temperature, . . . . . . . . 441

215. Income and Expenditure of Heat, . . . . . . . . 445

216. Variations in Heat Production, . . . . . . . . 447

217. Relation of Heat Production to Bodily Work, . . . . . 447

218. Accommodation for Different Temperatures, . . . . . 448 
219. Storage of Heat in the Body,

220. Fever,

221. Artificial Increase of the Temperature,

222. Employment of Heat, .

223. Increase of Temperature post mortem, . . . . . . . . . 453

224. Action of Cold on the Body, . . . . . . . . . . 454

225. Artificial Lowering of Temperature, . . . . . . . . . . 455

226. Employment of Cold, . . . . . . . . . . . 456

227. Heat of Inflamed Parts, . . . . . . . . . 457

228. Historical and Comparative, . . . . . . . . 457

\section{PHYSIOLOGY OF THE IMETABOLIC PHENOMENA OF THE BODY.}

229. General View of Food-Stuffs, . . . . . . . . 458

230. Structure and Secretion of the Mammary Glands, . . . . . 461

231. Milk and its Preparations, . . . . . . . . . . 464

232. Eggs, • . . . • . . . • .. . . . 468

233. Flesh and its Preparations, . . . . . . . . . . . 469

234. Vegetable Foods, . . . . . . . . . . . 471

235. Condiments-Tea and Alcohol, . . . . . . . . 473

\section{PHENOMENA AND LAIVS OF METABOLISM.}

236. Equilibrium of the Metabolism, . . . . . . . 476

237. Metabolism during Hunger and Starvation, . . . . . . 482

238. Metabolism during a purely Flesh Diet, . . . . . . 485

239. A Diet of Fat or of Carbohydrates, . . . . . . . . . . 486

240. Mixture of Flesh and Fat, . . . . . . . . . . 486

241. Origin of Fat in the Body, . . . . . . . . . 487

242. Corpulence, . . . . . . . . . . . . 488

243. The Metabolism of the Tissues, . . . . . . . 490

244. Regeneration of the Tissues, . . . . . . . . . 493

245. Transplantation of the Tissues, . . . . . . . . 497

246. Increase in Size and Weight during Growth, . . . . . 497

\section{GENERAL VIEW OF THE CHEMICAL CONSTITUENTS OF THE ORGANISM.}

247. Inorganic Constituents,

248. Organic Constituents-Proteids, . . . . . . . 500

249. The Animal and Vegetable Proteids and their Properties, . . 502

250. The Albuminoids, . . . . . . . . . . 504

251. The Fats, . . . . . . . . . . 508

252. The Carbohydrates, . . . . . . . . 511

253. Historical Retrospect, . . . . . . . . . . 514 


\section{LIST OF ILLUSTRATIONS.}

FIGERE

1. Human coloured blood-corpuscles, - . - . . 3

2. Malassez's apparatus for estimating the number of blood-corpuscles, . 4

*3. Gower's hæmacytometer (Hawksley), . . . . . 6

4. Red blood-corpuscles showing various changes of shaje, . . 9

5. Vaso-formative cells, . . . . . . . 14

6. White blood-corpuscles, . . . . . . 18

7. Blood-plates and their derivatives, . . . . . 21

8. Hæmoglobin crystals, . . . . . , . 24

*9. Gower's hæmoglobinometer (Hawksley), . . . . . 26

10. Scheme of a spectroscope, . . . . . . . 28

11. Various spectra of hæmoglobin, . . . . . $\quad$. 29

12. Hæmin crystals, . $\quad$. $\quad$. $\quad$. $\quad$. 34

13. Hæmin crystals prepared from traces of blood, . . . . 34

14. Hæmatoidin crystals, . . . . . . . 35

15. Scheme of Pfluger's gas-pump, . $\quad$. $\quad . \quad$. $\quad . \quad$. 53

16. Scheme of the circulation, . . . . . . . $\quad$. 65

17. Muscular fibres from the heart, . . . . . 66

18. Muscular fibres in the left auricle, . . . . . 68

19. Muscular fibres in the ventricles, . . . . . 70

*20. Lymphatic from the pericardium (Cadiat), . . . . 71

*21. Section of the endocardium (Cadiat), . . . . . 71

*22. Purkinje's fibres (Ranvier), . . . . . . . 73

23. Cast of the ventricles of the human heart, . . . . $\quad$. 77

24. The closed semilunar valves, . . . . . . 78

*25. Various cardiographs (Hermann), . . . . . . . . $\quad$ S1

25a. Curves of the apex-beat, . . . . . . . $\quad$. 82

26. Changes of the heart during systole, . . . . . . $\quad$. 83

27. Curves from a rabbit's ventricle, . . . . . . 86

*28. Marey's registering tambour (Hermann), . . . . . 87

29. Curves obtained with a cardiac sound, . . . . . SS

30. Curves from the cardiac impulse, . . . . $\quad 90$

31. Position of the heart in the chest (Luschka and Gairdner), . $\quad$. 93

31a. Bipolar nerve-cells from a frog's heart, . . . . 98

*32. Scheme of a frog-manometer (Stirling), . . . . . . $\quad$. 102

*32a. Perfusion cannula (Kronecker and Stirling), . . . . 102

*33. Roy's tonometer (Stirling), . . . . . . 103

*34. Luciani's groups of cardiac pulsations (Hermann), . . . 104

"35. Curves of a frog's heart at different temperatures (Hermanu), , , 105

36. Cardio-pneumograph of Landois, . . . . . 110 
FIGURE

37. Apparatus for showing the effect of respiration,

38. Cylindrical vessel,

39. Cylindrical vessel with manometers,

40. Small artery with its various coats,

41. Capillaries injected with silver nitrate,

*42. Longitudinal section of a vein at a valve (Cadiat)

43. Poiseuille's pulse-measurer,

44. Sphygmometer of Herisson,

45. Scheme of Marey's sphygmograph, . . . . . . 129

*46. Marey's improved sphygmograph (B. Bramwell), . . . . 130

*47. Scheme of Marey's sphygmograph in working order (B. Bramwell), . 130

*48. Scheme of Marey's sphygmograph after increase of the pressure (B. Bramwell),

*49. Dudgeon's sphygmograph (Dudgeon), .

*50. Mode of applying Dudgeon's sphygmograph (Dudgeon),

*51. Sphygmogram (Dudgeon),

52. Scheme of Brondgeest's pansphygmograph

53. Scheme of Landois' angiograph,

54. Pulse-curves of the carotid, radial, and posterior tibial arteries,

55. Landois' gas-sphygmoscope, .

56. Hæmautographic curve, .

*57. Sphygmogram of radial artery (Dudgeon),

58. Sphygmograms of various arteries, : . . . . . . $\quad$. 138

59. Pulsus dicrotus, P. caprizans, P. monocrotus, . . . . . 140

60. Pulsus alternans,

6I. Curves of the posterior tibial and pedal arteries, . . . 145

62. Anacrotic pulse-curves, . $\quad . \quad$. $\quad . \quad$. $\quad$. 147

63. Influence of the respiration on the sphygmogram, . . . 148

64. Curves of the radial and carotid arteries during Müller's and Valsalva's experiments,

65. Pulsus paradoxus, . : . . . . . 150

66. Varions radial curves altered by pressure, . . . . $\quad$. 151

67. Apparatus for measuring the velocity of the pulse-wave in an elastic tube,

67a. Tracing obtained from $6 \bar{T}$,

68. Pulse tracings of the radial and carotid arteries, . . . 155

69. Tracings from the posterior, tibial, and carotid arteries, . . 156

70. Apparatus for registering the molar motions of the body, . . $\quad$. 157

71. Vibration and heart curves, . . . . . . 158

72. Ludwig and Fick's kymographs, . . . . . . 163

*73. Ludwig's improved revolving cylinder (Hermann), . . . 164

*74. Blood-pressure tracing of the carotid of a dog (Hermann), . . 165

*75. Fick's spring kymograph by Hering (Hermann), . . . . 166

*76. Depressor curve (Stirling), . . . . . . . 168

77. Blood-pressure and respiration tracings takeu simultaneously, $\quad 169$

*78. Blood-pressure tracing during stimulation of the vagus (Stirling), . 173

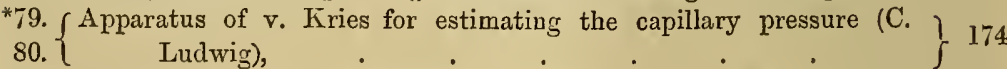

81. Volkmann's hæmadromometer, . . . . . 180

82. Ludwig and Dogiel's rheometer, . $\quad$. $\quad . \quad$. 180

83. Vierordt's læmatachometer, . . . . . . . 181

84. Dromograph, . . . . . . . . . 182 
85. Diapedesis,

86. Various forms of venous pulse,

87. Mosso's plethysmograph,

*88. Trabeculæ of the spleen (Cadiat),

*89. Adenoid tissue of spleen (Cadiat), . . . . . . 203

*90. Malpighian corpuscle of the spleen (Cadiat), . $\quad$. $\quad . \quad 205$

*91. Tracing of a splenic curve (Roy), . . . . . $\quad$. 209

*92. Thymus gland (Carliat), . . . . . . . . 212

*93. Elements of the thymus gland (Cadiat), . . . . . 212

*94. Thyroid gland (Cadiat),

213

*95. Supra-renal capsule (Cadiat), . . . . . . . 214

*97. Human bronchus (Hamilton), . $\quad$. $\quad$. . . 219

*98. Air-vesicles injected witl silver nitrate (Hamilton), . . . 221

99. Scheme of the air-vesicles of lung, . . . . . 222

*100. Interlobular septa of lung (Hamilton), . . . . . 223

101. Scheme of Hutchinson's spirometer,' . . . . . 228

102. Marey's stethograph (M'Kendrick), . . . . . 230

103. Brondgeest's tambour and curve, . . . . . 230

104. Pneumatogram, . $\quad$. . . . . 231

105. Section through diaphragm (Hermann), . . . . 236

106. Action of intercostal muscles, . . . . . . . 237

107. Cyrtometer curve, . . . . . . . 241

108. Sibson's thoracometer, . . . . . . . . 242

109. Topography of the lungs and heart, . . . . . 243

110. Andiral and Gavarret's respiration apparatus, . . . . 251

111. Scharling's apparatus, . . . . . . 251

112. Regnault and Reiset's apparatus, . . . . . . . 252

113. v. Pettenkofer's apparatus, . . . . . . . 253

114. Valentin and Brunner's apparatus, . $\quad$. $\quad . \quad$. $\quad$. 255

115. Objects found in sputum, . . . . . . . 274

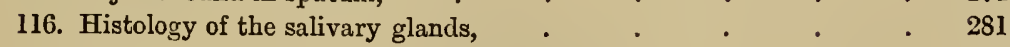

*117. Human sub-maxillary gland (Heidenhain), . $\quad$. $\quad$. $\quad$. 282

*118. $\{$ Sections of a serous gland (Heidenhain), . . . . 284

*120. Diagram of a salivary gland (L. Brunton), . . . . . . 289

121. Apparatus for estimation of sugar, . . . . . 298

122. Vertical section of a tooth, $\quad . \quad+\quad . \quad . \quad . \quad . \quad 300$

123. Dentine, . . . . . . . . 300

124. Dentine and enamel, . $\quad$. $\quad$. $\quad$. $\quad$. $\quad . \quad$. 301

125. Dentine and crusta petrosa, . $\quad$. . . 302

126.

127. Development of a tooth,

128.

302 and 303

129. Perinæum and its muscles, $\quad$. $\quad$. $\quad . \quad$. 314

130. Levator ani externus and internus, . $\quad . \quad$. $\quad . \quad$. 315

*131. Auerbach's plexus (Cadiat), . . . . . . . $\quad$. 317

*132. Meissner's plexus (Cadiat), . . . . . . . 317

133. Surface section of gastric mucous membrane, . . . . . 321

134. Fundus gland of the stomach, . . . . . . . 322

135. Pyloric gland and goblet-cells,. . . . . . $\quad$. 323

136. Scheme of the gastric mucous membrane, . . . . 324

*137. Pyloric mucous membrane (Hermann), . . . . 326 
${ }^{*}$ 138. Pyloric glands during digestion (Hermann),

139. Section of the tubes of the pancreas (Hermann), ․ $\quad . \quad 337$

140. Changes of the pancreatic cells during activity, . . . . 338

141. Scheme of a liver lobule, . $\quad . \quad$. $\quad . \quad . \quad . \quad 347$

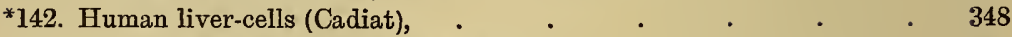

${ }^{*}$ 143. Liver-cells during fasting (Hermann), . . . . . . . 348

144. Various appearances of the liver-cells, $\quad$ • $\quad . \quad 3 \quad$. $\quad$. 349

${ }^{*}$ 145. Cholesterin (Aitken), . . . . . . . . 358

*146. Lieberkïhn's gland (Hermann), . . . . . . . 369

147. Bacterium aceti and B. butyricus, . $\quad$. $\quad . \quad$. 373

148. Bacillus subtilis, . . . . . . . . 375

*149. Villi of small intestine injected (Cadiat), . . . . . . . 387

150. Scheme of an intestinal villus, $\quad . \quad$. $\quad . \quad$. $\quad .388$

*151. Villi and Lieberkühn's follicles (Cadiat), . . . . . . 389

*152. Section of a solitary follicle (Cadiat), . $\quad . \quad$. $\quad$. $\quad$. $\quad$. 390

*153. Section of a Peyer's patch (Cadiat), . . . . . . 391

*154. Section of Auerbach's plexus (Cadiat), . . . . . 391

*155. Lieberkühn's gland (Hermann), . . . . . . . 392

156. Endosmometer, . . . . . . . 393

157. Origin of lymphatics in the tendon of diaphragm, . . . . 403

*158. Lymphatics of diaphragm silvered (Ranvier), . . . . 403

159. Perivascular lymphatics, . . . . . . . 405

160. Stomata from lymph-sac of frog, . . . . . . 405

161. Section of two lymph-follicles, . . . . . . . 406

*162. Scheme of a lymphatic gland (Knott), . . . . 407

163. Part of a lymphatic gland , . . . . . 408

${ }^{*}$ 164. Section of central tendon of diaphragm (Brunton), . . . . 416

*165. Section of fascia lata of a dog (Brunton), . . . . 416

166. Water calorimeter of Favre and Silbermann, . . . . 422

167. Walferdin's metastatic thermometer, . . . . . . 427

168. Scheme of thermo-electric arrangements, . . . . . 428

169. Kopp's apparatus for specific heat, . . . . . . 435

170. Daily variations of temperature, . . . . . . 439

*171. Acini of the mammary gland of a sheep (Cadiat), . . . 462

172. Milk-glands during inaction and secretion, . . . . . 462

*173. Section of a grain of wheat (Blyth), . . . . . 471

174. Yeast-cells growing, . . . . . . . . . . 474

175. Composition of animal and vegetable foods, . . . . . $\quad . \quad 479$

176. Starch grains (Blyth), . . . . . . . 512

[The illustrations indicated by the word Hermann, are from Hermann's Handbuch der Physiologie; by Cadiat, from Cadiat's Traité d'Anatomie Générale; by Ranvier, from Ranvier's Traité Technique d'Histologie; by Brunton, from The Practitioner; and by Hamilton, from Hamilton's Pathology of Bronchitis.] 


\section{Introduction.}

The Scope of Physiology and its Relations to other Branches of Natural Science.

Physiology is the science of the vital phenomena of organisms, or broadly, it is the Doctrine of Life. Correspondingly to the divisions of organisms, we distinguish-(1) Animal Physiology; (2) Vegetable Physiology; and (3) the Physiology of the Lowest Living Organisms, which stand on the border line of animals and plants-i.e., the so-called Protistce of Hæckel, micro-organisms, and those elementary organisms or cells which exist on the same level.

The object of Physiology is to establish these phenomena, to determine their regularity and causes, and to refer them to the general fundamental laws of Natural Science, viz., the Laws of Physics and of Chemistry.

The following Scheme shows the relation of Physiology to the allied branches of Natural Science :-

\section{Biology.}

The science of organised beings or organisms (animals, plants, protistæ, and elementary organisms).

\section{Morphology.}

The doctrine of the form of organisms.

\section{General}

Morphology. Morphology.

The doctrine of the The doctrine of formed elemen- the parts and taryconstituents organs of organof organisms.

(Histology) - (Organology
(a) Histology of Plants,
(b) Histology of Animals.

\section{Physiology.}

The doctrine of the vital phenomena of organisms.

$\begin{array}{cc}\text { General } & \text { Special } \\ \text { Physiology. } & \text { Physiology. }\end{array}$

The doctrine of The doctrine of vital phenomena the activities of in general- the individual (a) Of Plants, organs(b) Of Animals. (a) Of Plants, (b) Of Animals. 


\section{Embryology.}

The doctrine of the generation and development of organisms.

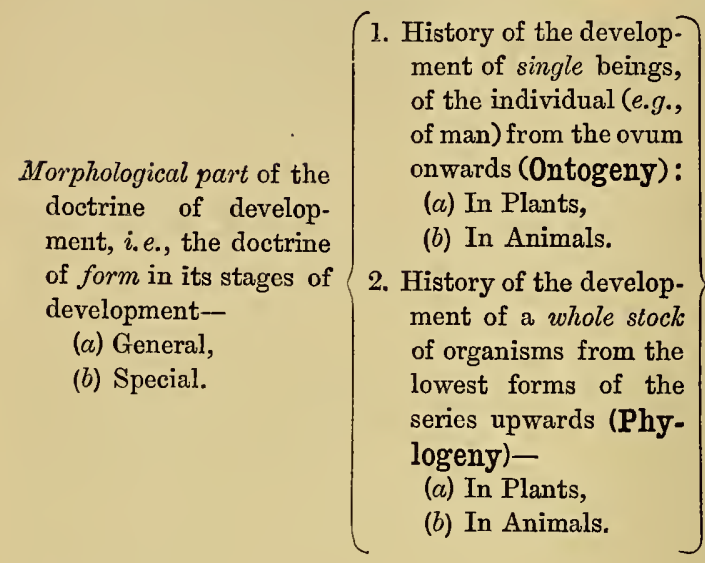

Physiological part of the doctrine of development, i.e., the doctrine of the activity during development-

(a) General,

(b) Special.

Morphology and Physiology are of equal rank in biological science, and a previous acquaintance with Morphology is assumed as a basis for the comprehension of Physiology, since the work of an organ can only be properly understood when its external form and its internal arrangements are known. Development occupies a middle place between Morphology and Physiology; it is a morphological discipline in so far as it is concerned with the description of the parts of the developing organism; it is a physiological doctrine in so far as it studies the activities and vital phenomena during the course of development.

\section{Matter.}

The entire visible world, including all organisms, consists of matter, i.e., of substance which occupies space.

We distinguish ponderable matter which has weight, and imponderable matter which cannot be weighed in a balance. The latter is generally termed ether.

In ponderable materials, again, we distinguish their form, i.e., the nature of their limiting surfaces; further, their volume, i.e., the amount of space which they occupy; and lastly, their aggregate condition, i.e., whether they are solid, fluid, or gaseous bodies.

Ether.-The ether fills the space of the universe, certainly as far as the most distant visible stars. This ether, notwithstanding its imponderability, possesses distinct mechanical properties; it is infinitely more attenuated than any known kind of gas, and behaves more like 
a solid body than a gas, resembling a gelatinous mass rather than the air. It participates in the luminous phenomena due to the vibrations of the atoms of the fixed stars, and hence it is the transmitter of light, which is conducted by means of its vibrations, with inconceivable rapidity $(42,220$ geographical miles per sec.) to our visual organs (Tyndall).

Imponderable matter (ether) and ponderable matter are not separated sharply from each other; rather does the ether penetrate into all the spaces existing between the smallest particles of ponderable matter.

Particles.-Supposing that ponderable matter were to be subdivided continuously into smaller and smaller portions, until we reached the last stage of division in which it is possible to recognise the aggregate condition of the matter operated upon, we should call the finely-divided portions of matter in this state particles. Particles of iron would still be recognised as solid, particles of water as fluid, particles of oxygen as gaseous.

Molecules._Supposing, however, the process of division of the particles to be carried further still, we should at last reach a limit beyond which, neither by mechanical nor by physical means, could any further division be effected. We should have arrived at the molecules. A molecule, therefore, is the smallest amount of matter which can still exist in a free condition, and which as a unit no longer exhibits the aggregate condition.

Atoms.-But even molecules are not the final units of matter, since every molecule consists of a group of smaller units, called atoms. An atom cannot exist by itself in a free condition, but the atoms unite with other similar or dissimilar atoms to form groups, which are called molecules. Atoms are incapable of further sub-division, hence their name. We assume that the atoms are invariably of the same size, and that they are solid. From a chemical point of view, the atom of an elementary body (element) is the smallest amount of the element which can enter into a chemical combination. Just as ponderable matter consists in its ultinate parts of ponderable atoms, so does the ether consist of analogous small ether-atoms.

Ponderable and Imponderable Atoms.-The ponderable atoms within ponderable matter are arranged in a definite relation to the etheratoms. The ponderable atoms mutually attract each other, and similarly, they attract the imponderable ether-atoms; but the etheratoms repel each other. Hence, in ponderable masses, ether-atoms surround every ponderable atom. These masses, in virtue of the attraction of the ponderable atoms, tend to come together, but only 
to the extent permitted by the surrounding ether-atoms. Thus, the ponderable atoms can never come so close as not to leave interspaces. All matter must, therefore, be regarded as more or less loose and open in texture, a condition due to the interpenetrating ether-atoms, which resist the direct contact of the ponderable atoms.

Aggregate Condition of Atoms.-The relative arrangement of the molecules, i.e., the smallest particles of matter, which can be isolated in a free condition, determines the aggregate condition of the body.

Within a solid body, characterised by the permanence of its volume, as well as by the independence of its form, the molecules are so arranged that they cannot readily be displaced from their relative positions.

Fluid bodies, although their volume is permanent, readily change their shape, and their molecules are in a condition of continual movement.

When this movement of the molecules takes so wide a range that the individual molecules fly apart, the body becomes gaseous, and as such, is characterised by the instability of its form as well as by the changeableness of its volume.

Physics is the study of these molecules and their motions.

\section{Forces.}

\section{Gravitation-Work done.}

All phenomena appertain to matter. These phenomena are the appreciable expression of the forces inherent in matter. The forces themselves are not appreciable, they are the causes of the phenomena.

1. Gravitation.-The law of gravitation postulates that every particle of ponderable matter in the universe, attracts every other particle with a certain force. This force is inversely as the square of the distance. Further, the attractive force is directly proportional to the amount of the attracting matter, without any reference to the quality of the body. We may estimate the intensity of gravitation, by the extent of the movement which it communicates to a body allowed to fall, for one second, through a given distance, in a space free from air. Such a body will fall in vacuo 9.809 metres per second. This fact has been arrived at experimentally.

Let us represent $g=9.809$ metres, the final velocity of the freely falling body at the end of one second. The velocity, $\mathrm{V}$, of the freely falling body is proportional to the time, $t$, so that

$$
\mathrm{V}=g t . . . . . . .(1) ;
$$


i.e., at the end of the lst sec., $\mathrm{V}=g, \mathrm{I}=g=9.809 \mathrm{M}$-the distance traversed-

$$
s=\frac{g}{2} t^{2}
$$

i.e., the distances are as the square of the times. Hence, from (1) and (2) it follows (by eliminating $t$ ) that-

$$
\mathrm{V}=\sqrt{2 g s} \cdot \text {. . . . . . . (3). }
$$

The velocities are as the square roots of the distances traversed-

Therefore,

$$
\frac{\mathrm{V}^{2}}{2 g}=s
$$

The freely falling body, and in fact every freely moving body, possesses kinetic energy, and is in a certain sense a magazine of energy. The kinetic energy of any moving body is always equal to the product of its weight (estimated by the balance), and the height to which it would rise from the earth, if it were thrown from the earth with its own velocity.

Let $\mathrm{W}$ represent the kinetic energy of the moving body, and $\mathrm{P}$ its weight, then $\mathrm{W}=\mathrm{P} . \mathrm{s}$, so that from (4) it follows that-

$$
\mathrm{W}=\mathrm{P} \frac{\mathrm{V}}{2 g}
$$

Hence, the kinetic energy of a body is proportional to the square of its velocity.

Work.-If a force (pressure, strain, tension) be so applied to a body as to move it, a certain amount of work is performed. The amount of work is equal to the product of the amount of the pressure or strain which moves the body, and of the distance through which it is moved.

Let $\mathrm{K}$ represent the force acting on the body, and $\mathrm{S}$ the distance, then the work $\mathrm{W}=\mathrm{KS}$. The attraction between the earth and any body raised above it is a source of work.

It is usual to express the value of $\mathrm{K}$ in kilogrammes, and $\mathrm{S}$ in metres, so that the "unit of work" is the kilogramme-metre, i.e., the force which is required to raise 1 kilo. to the height of 1 metre.

2. Potential Energy.-The transformation of Potential into Kinetic energy, and conversely: Besides kinetic energy, there is also "potential energy," or energy of position. By this term are meant various forms of energy, which are suspended in their action, and which, although they may cause motion, are not in themselves motion. A coiled watchspring kept in this position, a stone resting upon a tower, are instances of bodies possessing potential energy, or the energy of position. It requires merely a push to develop kinetic from the potential energy, or to transform potential into kinetic energy. 
Work, $w$, was performed in raising the stone to rest upon the tower.

$w=p, s$, where $p=$ the weight and $s=$ the height,

$p=m . g$, is = the product of the mass $(m)$, and the force of gravity $(g)$, so that $w=m g s$.

This is at the same time the expression for the potential energy of the stone. This potential energy may readily be transformed into kinetic energy by merely pushing the stone so that it falls from the tower. The kinetic energy of the stone is equal to the final velocity with which it impinges upon the earth.

$$
\begin{aligned}
\mathrm{V} & =\sqrt{2 g s} \text { (see above (3). } \\
\mathrm{V}^{2} & =2 g s . \\
m \mathrm{~V}^{2} & =2 m g s . \\
m & =m g s .
\end{aligned}
$$

$m g s$ was the expression for the potential energy of the stone while it was still resting on the height; $\frac{m}{2} \mathrm{~V}_{2}$ is the kinetic energy corresponding to this potential energy (Brïcke).

Potential energy may be transformed into mechanical energy under the most varied conditions; it may also be transferred from one body to another.

The movement of a pendulum is a striking example of the former. When the pendulum is at the highest point of its excursion, it must be regarded as absolutely at rest for an instant, and as endowed with potential energy, thus corresponding with the raised stone in the previous instance. During the swing of the pendulum, this potential energy is changed into kinetic energy, which is greatest when the pendulum is moving most rapidly towards the vertical. As it rises again from the vertical position, it moves more slowly, and the kinetic energy is changed into potential energy, which once more reaches its maximum, when the pendulum comes to rest at the utmost limit of its excursion. Were it not for the resistances continually opposed to its movements, such as the resistance of the air, and friction, the movement of the pendulum, due to the alternating change of kinetic into potential energy and vice versâ, would continue uninterruptedly, as with a mathematical pendulum. Suppose the swinging ball of the pendulum, when exactly in a vertical position, impinged upou a resting but movable sphere, the potential energy of the ball of the pendulum would be transferred directly to the sphere, provided that the elasticity of the ball of the pendulum and the sphere were complete; the pendulum would come to rest, while the sphere would move onward with an equal amount of kinetic energy, provided there were no resistance to its movement. This is an example of the transference of kinetic energy from one body to another. Lastly, suppose that a stretched watch-spring on uncoiling causes another spring to become coiled; and we have another example of the transference of kinetic energy from one body to another.

The following general statement is deducible from the foregoing examples:-If, in a system, the individual moving masses approach the final position of equilibrium, then in this system the sum of the kinetic 
energies increases; if, on the other hand, the particles move away from the final position of equilibrium, then the sum of the potential energies is increased at the expense of the kinetic energies, i.e., the kinetic energies diminish (Brücke).

The pendulum, which, after swinging from the highest point of its excursion, approaches the vertical position, i.e., the position of equilibrium of a passive pendulum, has in this position the largest amount of potential energy; as it again ascends to the highest point of its excursion on the other side, it again gradually receives the maximum of potential energy at the expense of the gradually diminish. ing movement, and therefore of the kinetic energy.

3. Heat-Its Relation to Potential and Kinetic Energy.-If a lead weight be thrown from a high tower to the earth, and if it strike an unyielding substance, the movement of the mass of lead is not only arrested, but the kinetic energy (which to the eye appears to be lost), is transformed into a lively vibratory movement of the atoms. When the lead meets the earth, heat is produced. The amount of heat produced is proportional to the kinetic energy, which is transformed through the concussion. At the moment when the lead weight reaches the earth, the atoms are thrown into vibrations; they impinge upon each other; then rebound again from each other in consequence of their elasticity, which opposes their direct juxtaposition; they fly asunder to the maximum extent permitted by the attractive force of the ponderable atoms, and thus oscillate to and fro. All the atoms vibrate like a pendulum, until their movement is communicated to the ethereal atoms surrounding them on every side, i.e., until the heat of the heated mass is "radiatel." Heat is thus a vibratory movement of the atoms.

As the amount of heat produced is proportional to the kinetic energy, which is transformed through the concussion, we must find an adequate measure for both forces.

Heat-Unit.-As a standard of measure of heat, we have the "heatunit" or calorie. The "heat-unit" or calorie is the amount of energy required to raise the temperature of 1 gramme of water $1^{\circ}$ centigrade. The "heat-unit" corresponds to 425.5 gramme-metres, i.e., the same energy required to heat 1 gramme of water $1^{\circ} \mathrm{C}$. would raise a weight of 425.5 grammes to the height of 1 metre; or, a weight of 425.5 grammes, if allowed to fall from the height of 1 metre, would by its concussion, produce as much heat as would raise the temperature of 1 gramme of water $1^{\circ} \mathrm{C}$. The "mechanical equivalent" of the heat-unit is, therefore, $425 \cdot 5$ gramme-metres.

It is evident, that from the collision of moving masses, an immeasurable amount of heat can be produced. Let us apply what has already beeu said to the earth. Suppose the earth to be disturbed in its orbit, and suppose further that, owing to 
the attraction of the sun, it were to impinge on the latter (whereby, according to J. R. Mayer, its final velocity would be 85 geographical miles per second), the amount of heat produced by the collision would be equal to that produced by the combustion of a mass of pure charcoal more than 5000 times as heavy (Julius Robert Mayer, Helmholtz).

Thus, the heat of the sun itself can be produced by the collision of masses of cold matter. If the cold matter of the universe were thrown into space, and there left to the attraction of its particles, the collision of these particles would ultimately produce the light of the stars. At the present time, numerous cosmic bodies collide in space, while innumerable small meteors $(94,000-188,000$ billions of kilos. per minute) fall into the sun. The force of gravity is perhaps, in fact, the only source of all heat (J. R. Mayer, Tyndall).

We have a homely example of the transformation of kinetic energy into heat in the fact, that a blacksmith may make a piece of iron red-hot by hammering it. Of the conversion of heat into kinetic energy, we have an example in the hot watery vapour (steam) of the steam-engine raising the piston. An example of the conversion of potential energy into heat occurs, in a metallic spring, when it uncoils and is so placed as to rub against a rough surface, producing heat by friction.

4. Chemical Affinity: Relation to Heat.-Whilst gravity acts upon the particles of matter without reference to the composition of the body, there is another atomic force which acts between atoms of a chemically different nature; this is chemical affinity. This is the force, in virtue of which the atoms of chemically-different bodies unite to form a chemical compound. The force itself varies greatly between the atoms of different chemical bodies; thus, we speak of strong chemical affinities and weak affinities. Just as we were able to estimate the potential energy of a body in motion, from the amount of heat which was produced when it collided with an unyielding body, so we can measure the amount of the chemical affinity by the amount of heat which is formed, when the atoms of chemically-different bodies unite to form a chemical compound. As a rule, heat is formed when separate, chemically-different atoms, form a compound body. When in virtue of chemical affinity, the atoms of 1 kilo. of hydrogen and 8 kilos. of oxygen unite to form the chemical compound water, an amount of heat is thereby evolved which is equal to that produced by a weight of 47,000 kilos. falling and colliding with the earth from a height of 1000 feet above the surface of the earth. If 1 gram. of $H$ be burned along with the requisite amount of $\mathrm{O}$ to form water, it yields 34,460 heat-units or calories; and 1 gram. carbon burned to carbonic acid (carbon dioxide) yields 8,080 heat-units. Wherever, in chemical processes, strong chemical affinities are satisfied, heat is set free-i.e., chemical affinity is changed into heat. Chemical affinity is a form of potential energy obtaining between the most different atoms, which during chemical processes is changed into heat. Conversely, in those chemical processes where strong affinities are dissolved, and chemically-united atoms thereby pulled asunder, there must be a diminution of temperature, or, 
as it is said, heat becomes latent-that is, the energy of the heat which has become latent is changed into chemical energy, and this, after decomposition of the compound chemical body, is again represented by the chemical affinity between its isolated different atoms.

\section{Law of the Conservation of Energy.}

Julius Robert Mayer and Helmholtz have established the important law, that in a system which does not receive any influence and impression from without, the sum of all the forces acting within it is always the same. The various forms of energy can be transformed one into the other, so that kinetic energy may be transformed into potential energy and vice versâ, but there is never any part of the energy lost. The transformation takes place in such measure that, from a certain definite amount of one form of energy, a definite amount of another can be obtained.

The various forms of energy acting in organisms occur in the following modifications :-

1. Molar motion (ordinary movements), as in the movements of the whole body, of the limbs, or of the intestines, and even those observable microscopically in connection with cells.

2. Movements of Atoms as Heat.-We know, in connection with the vibration of atoms, that the number of vibrations in the unit of time determines whether the oscillations appear as heat, light, or chemically-active vibrations. Heat-vibrations have the smallest number, while chemically-active vibrations have the largest number, light-vibrations standing between the two. In the human body, we only observe heat-vibrations, but some of the lower animals are capable of exhibiting the phenomena of light.

In the human organism, the molar movements in the individual organs are constantly being transformed into heat, e.g., the kinetic energy in the organs of the circulation is transformed by friction into heat. The measure of this is the "unit of work" =1 gramme-metre, and the "unit of heat" $=425.5$ gramme-metres.

3. Potential Energy.-The organism contains many chemical compounds which are characterised by the great complexity of their constitution, by the imperfect saturation of their affinities, and hence, by their great tendency to split up into simpler bodies.

The body can transform the potential energy into heat as well as into kinetic energy, the latter always in conjunction with the former, but the former always by itself alone. The simplest measure of the potential energy is the amount of heat, which can be obtained by complete combustion of the chemical compounds representing the potential 
energy. The number of work-units can then be calculated from the amount of heat produced.

4. The phenomena of electricity, magnetism, and diamagnetism may be recognised in two directions, as movements of the smallest particles, which are recognised in the glowing of a thin wire when it is traversed by strong electrical currents (against considerable resistance), and also as molar movement, as in the attraction or repulsion of the magnetic needle. Electrical phenomena are manifested in our bodies by muscle, nerve, and glands, but these phenomena are relatively small in amount when compared with the other forms of energy. It is not improbable that the electrical phenomena of our bodies become almost completely transformed into heat. As yet experiment has not determined with accuracy a "unit of electricity," directly comparable with the "heat-unit" and the "work-unit."

It is quite certain that within the organism, one form of energy can be transformed into another form, and that a certain amount of one form will yield a definite amount of another form; further, that new energy never arises spontaneously, nor is energy, already present, ever destroyed, so that in the organism the law of the conservation of energy is continually in action.

\section{Animals and Plants.}

The animal body contains a quantity of chemically-potential energy stored up in its constituents. The total amount of the energy present in the human body might be measured, by burning completely an entire human body in a calorimeter, and thereby determining how many heatunits are produced when it is reduced to ashes (see Animal Heat, p. 422).

The chemical compounds containing the potential energy are characterised by the complicated relative position of their atoms, by a comparatively imperfect saturation of the affinities of their atoms, by the relatively small amount of oxygen which they contain, by their great tendency to decomposition, and the facility with which they undergo it.

If a man were not supplied with food, he would lose 50 grammes of his body-weight every hour; the material part of his body, which contains the potential energy, is used up, oxygen is absorbed, and a continual process of combustion takes place; by the process of combustion, simpler substances are formed from the more complex compounds, whereby potential is converted into kinetic energy. It is immaterial whether the combustion is rapid or slow; the same amount 
of the same chemical substances always produces the same amount of kinetic energy, i.e., of heat.

A person when fasting, experiences after a certain time, the disagreeable feeling of exhaustion of his reserve of potential energy, hunger sets in, and he takes food. All food for the animal kingdom is obtained, either directly or indirectly, from the vegetable hingdom. Even carnivora, which eat the flesh of other animals, only eat organised matter which has been formed from vegetable food. The existence of the animal kingdom presupposes the existence of the vegetable kingdom.

All substances, therefore, necessary for the food of animals occur in vegetables. Besides water and the inorganic constituents, plants contain, amongst other organic compounds, the following three chief representatives of food-stuffs-fats, carbohydrates, and proteids.

All these contain stores of potential energy, in virtue of their complex chemical constitution.

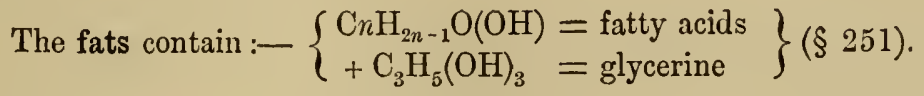

The carbohydrates contain :- $-\mathrm{C}_{6} \mathrm{H}_{10} \mathrm{O}_{5}$. . . (\$252).

The proteids contain per cent.:- $\left\{\begin{array}{c}\text { C. } 51 \cdot 5-54 \cdot 5 \\ \text { H. } 6 \cdot 9-7 \cdot 3 \\ \text { N. } 15 \cdot 2-17 \cdot 0 \\ \text { O. } 20 \cdot 9-23 \cdot 5 \\ \text { S. } 0 \cdot 3-2 \cdot 0\end{array}\right\}$ (\$s 248 and

A man, who takes a certain amount of this food adds thereto oxygen from the air in the process of respiration. Combustion or oxidation then takes place, whereby chemically potential energy is transformed into heat.

It is evident, that the products of this combustion must be bodies of simpler constitution-bodies with less complex arrangement of their atoms, with the greatest possible saturation of the affinities of their atoms, of greater stability, partly rich, in $\mathrm{O}$, and possessing either no potential energy, or only very little. These bodies are carbonic acid (carbon dioxide), $\mathrm{CO}_{2}$; water, $\mathrm{H}_{2} \mathrm{O}$; and as the chief representative of the nitrogenous excreta, urea $\left(\mathrm{CO}\left(\mathrm{NH}_{2}\right)_{2}\right)$, which has still a small amount of potential energy, but which outside the body readily splits into $\mathrm{CO}_{2}$ and ammonia $\left(\mathrm{NH}_{3}\right)$.

The human body is an organism in which, by the phenomena of oxidation, the complex nutritive materials of the vegetable kingdom, which are highly charged with potential energy, are transformed into simple chemical bodies, whereby the potential energy is transformed 
into the equivalent amount of kinetic energy (heat, work, electrical phenomena).

But how do plants form these complex food-stuffs so rich in potential energy? It is plain, that the potential energy of plants must be obtained from some other form of energy. This potential energy is supplied to plants by the rays of the sun, whose chemical light-rays are absorbed by plants. Without the rays of the sun there could be no plants. Plants absorb from the air and the soil, $\mathrm{CO}_{2}, \mathrm{H}_{2} \mathrm{O}, \mathrm{NH}_{3}$, and $\mathrm{N}$, of which carbonic acid, water, and ammonia (from urea), are also produced by the excreta of animals. Plants absorb the kinetic energy of light from the sun's rays and transform it into potential energy, which is accumulated during the growth of the plant in its tissues, and in the. food-stuffs produced in them during their growth. This formation of complex chemical compounds is accompanied by the simultaneous excretion of 0 .

Occasionally, kinetic energy, such as we universally meet with in animals, is liberated in plants. Many plants develop considerable quantities of heat in their flowers-e.g., the arum tribe. We must also remember that, during the formation of the solid parts of plants, when fluid juices are changed into solid masses, heat is set free. In plants, under certain circumstances, $\mathrm{O}$ is absorbed, and $\mathrm{CO}_{2}$ is excreted, but these processes are so trivial as compared with the typical condition in the vegetable kingdom, that they may be regarded as of small moment.

Plants, therefore, are organisms which, by a reduction process, transform simple stable combinations into complex compounds, whereby potential solar energy is transformed into the chemically-potential energy of vegetable tissues. Animals are living beings, which, by oxidation, decompose or break up the complex grouping of atoms manufactured by plants, whereby potential is transformed into kinetic energy. Thus, there is a constant circulation of matter and a constant exchange of energy between plants and animals. All the energy of animals is derived from plants. All the energy of plants arises from the sun. Thus the sun is the cause, the original source of all energy in the organism, i.e., of the whole of life.

As the formation of solar heat and solar light is explicable by the gravitation of masses, gravity is perhaps the original form of energy of all life.

We may thus represent the formation of kinetic energy in the animal body from the potential energy of plants. Let us suppose the atoms of the substances formed in organisms, as simple small bodies, balls, or blocks. As long as these lie in a single layer, or in a few layers, upon the surface, there is a stable arrangement, and they continue to remain at rest. If, however, an artificial tower be built of these blocks, so that an unstable erection is produced, and the same tower be afterwards knocked down, then for this purpose we require-(1) 
the motor power of the workman who lifts and carries the blocks; (2) a blow or other impulse from without applied to the unstable structurewhen the atoms will fall together, and as they fall collide with each other and produce heat. Thus, the energy employed by the workman is again transformed into the last-named form of energy.

In plants, the complex unstable building of the groups of atoms is carried on, the constructor being the sun. In animals, which eat plants, the complex groups of the atoms are tumbled down, with the liberation of kinetic energy.

\section{Vital Energy and Life.}

The forces which act in organisms, in plants and animals are exactly the same as are recognisable as acting in dead matter. A so-called "vital force," as a special force of a peculiar kind, causing and governing the vital phenomena of living beings, does not exist. The forces of all matter, of organised as well as unorganised, exist in connection with their smallest particles or atoms. As, however, the smallest particles of organised matter are, for the most part, arranged in a very complicated way, compared with the much simpler composition of inorganic bodies, so the forces of the organism, connected with the smallest particles, yield more complicated phenomena and combinations, whereby it is excessively difficult to ascribe the vital phenomena in organisms to the simple fundamental laws of physics and chemistry.

The Exchange of Material, or Metabolism (Stoffwechsel) as a Sign of Life.-Nevertheless, there appears to be a special exchange of matter and energy peculiar to living beings. This consists in the capacity of organisms to assimilate the matter of their surroundings, and to work it up into their own constitution, so that it forms for a time an integral part of the living being, to be given off again. The whole series of phenomena is called Metabolism or Stoffwechsel, which consists in the introduction, assimilation, integration, and excretion of matter.

We have already shown, that the metabolism of plants and that of animals are quite different. The processes, as already described, are actually what occur in the typical higher plants and animals.

But there is a large group of organisms which, throughout their entire organisation, exhibit so low a degree of development, that by some observers they are considered as undifferentiated "ground-forms." They are regarded as neither plants nor animals, and are the most simple forms of animated matter. Hæckel has called these organisms Protistoe, as being the original and primitive forms.

We must assume that, corresponding with their simpler vital conditions, their metabolism is also simpler, but on this point we still require further observations. 



\section{Physiology of the Blood.}

[THE blood is aptly described by Claude Bernard as an internal medium, which acts as a "go-between" for the outer world and the tissues. Into it are poured those substances which have been subjected to the action of the digestive fluids, and in the lungs or other respiratory organs it receives oxygen. It thus contains new substances, but in its passage through the tissues it gives up some of these new substances, and receives in exchange certain effete and more or less useless substances which have to be got rid of. Its composition is thus highly complex, containing, as it does, things both new and old. It is at once a great pabulum-supplying medium, and a channel for getting rid of useless materials. As the composition of the organs through which the blood flows varies, it is evident that its composition must vary in different parts of the circulatory system; and it also varies in the same individual under different conditions. Still, with slight variations, there are certain general physical, histological, and chemical properties which characterise blood as a whole.]

\section{Physical Properties of the Blood.}

(1.) Colour.-The colour of blood varies from a bright scarlet-red in the arteries to a deep, dark, bluish-red in the veins. Oxygen (and, therefore, the air) makes the blood bright-red; want of oxygen makes it dark. Blood free from oxygen (and also venous blood) is dichroic -i.e., by reflected light it appears dark-red, while by transmitted light it is green (Briicke).

In thin layers blood is opaque, as is easily shown by shaking blood so as to form bubbles, or by allowing blood to fall upon a plate with a pattern on it, and pouring it off again. Blood behaves, therefore, like an "opaque colour" (Rollett), as its colouring-matter is suspended in the form of fine particles-the blood-corpuscles.

Hence, it is possible to separate the colouring-matter from the fluid part of the blood by filtration. This is accomplished by mixing the blood with fluids which render the blood-corpuscles sticky or rough. If mammalian blood be treated with one-seventh of its volume of solution of sodic sulphate, or if frog's blood be mixed with a two per cent. solution of sugar and filtered, the shrivelled corpuscles, now robbed of part of their water, remain upon the filter. 
(2.) Reaction.-The reaction is alkaline, owing to the presence of disodic phosphate, $\mathrm{Na}_{2}, \mathrm{H}, \mathrm{PO}_{4}$ (Maly). After blood is shed, its alkalinity rapidly diminishes, and this occurs more rapidly the greater the alkalinity of the blood. This is due to the formation of an acid, in which, perhaps, the coloured corpuscles take part, owing to the decomposition of their colouring-matter. A high temperature and the addition of an alkali favour the formation of the acid (N. Zuntz).

The alkalinity is less in persons suffering from anæmia, cachectic conditions, and chronic rheumatism (Lépine). After the prolonged use of soda, the alkali in the ash of blood is increased (Dubelir).

Methods.-Owing to the colour of the blood we cannot employ ordinary litmus paper to test its reaction. One or other of the following methods may be used :(1.) Moisten a strip of glazed red litmus paper with solution of common salt, and dip it quickly into the blood, or allow a drop of blood to fall on the paper, and rapidly wipe it off before its colouring-matter has time to penetrate and tinge the paper (Zuntz). (2.) Kühne made a small cup of parchment paper which was placed in water in a watch-glass. The colourless diffusate was afterwards tested with litmus paper. (3.) Liebreich used thin plates of plaster-of-Paris of a perfectly nentral reaction. These are dried, and afterwards moistened with a neutral solution of litmus. When a drop of blood is placed upon the porous plate, the fluid part of the blood passes into it, while the corpuscles remain at the surface. The corpuscles are washed off with water, and the altered colour of the litmusstained slab is apparent. [(4.) Schäfer uses dry faintly-reddlened glazed litmus paper, and on it is placed a drop of blood, which is wiped off after a few seconds. The place where the blood rested is indicated by a well-defined blue patch upon a red or violet gromd.]

The alkaline reaction of blood is diminished : $(\alpha)$ By great muscular exertion, owing to the formation of a large amount of acid in the muscles; $(\beta)$ during coagulation; $(\gamma)$ in old blood, or blood dissolved by water from old blood-stains, such blood being usually acid. Fresh cruor has a stronger alkaline reaction than serum.

(3.) Odour.-Blood emits a peculiar odour (Halitus sanguinis), which differs in animals and man.

It depends upon the presence of volatile fatty acids. If concentrated sulphuric acid be added to blood, whereby the volatile fatty acids are set free from their combinations with alkalies, the characteristic odour becomes much more perceptible (Barruel).

(4.) Taste.-Blood has a saline taste, depending upon the salts dissolved in the fluid of the blood.

(5.) Specific Gravity.-The specific gravity is 1,055 (extreme limits $1,045-1,075)$; in women and young persons it is somewhat less. The specific gravity of the blood-corpuscles is 1,105 , that of the plasma 1,027. Hence, the corpuscles tend to sink.

The specific gravity of the red blood-corpuscles is estimated by allowing the corpuscles to subside to the bottom (which occurs most readily in the blood of the horse); but it is more correctly estimated by placing the blood in a tall cylindrical vessel, and setting the latter in the radius of the revolving disc of a centrifugal apparatus, the base of the cylinder being directed outwards. The drinking of water and hunger diminish the specific gravity tem- 
porarily, while thirst and the digestion of dry food raise it. If blood be passed through an organ artificially, its specific gravity rises in consequence of the absorption of dissolved matters and the giving off of water. It falls after hæmorrhage, and is less in badly-nourished individuals.

\section{Microscopic Examination of the Blood.}

[Blood, when examined by the microscope, is seen to consist of an enormous number of corpuscles-coloured and colourless-floating in a transparent fluid, the plasma, or liquor sanguinis.]

The RED blood-corpuscles were discovered in frog's blood by Swammerdam in 1658, and in human blood by Leenwenhoek in 1673 .

Characters of Human Blood-( $a_{0}$ ) Form.-The human red bloodcorpuscles are circular, coin-shaped, homogeneous discs, with saucer-like depressions on both surfaces, and with rounded margins; in other words, they are bi-concave, circular discs.

(b.) Size.-According to Welcker the diameter $\left(\begin{array}{ll}a & b\end{array}\right)$ is $7 \cdot 7 \mu, *$ the greatest thickness $(c d) 1.9 \mu$ (Fig. 1, C) [i.e., it is $\frac{7}{3500}$ to $\frac{1}{3200}$ of an inch in diameter, and about one-fourth of that in thickness].

The corpuscles are slightly diminished in size by septic fever, inanition, after the subcutaneous injection of morphia, increased bodily temperature, and $\mathrm{CO}_{2}$; while they are increased by $\mathrm{O}$, watery condition of the blood, cold, consumption of alcohol, quinine, hydrocyanic acid, andl acute anæmia (Manasseïn).

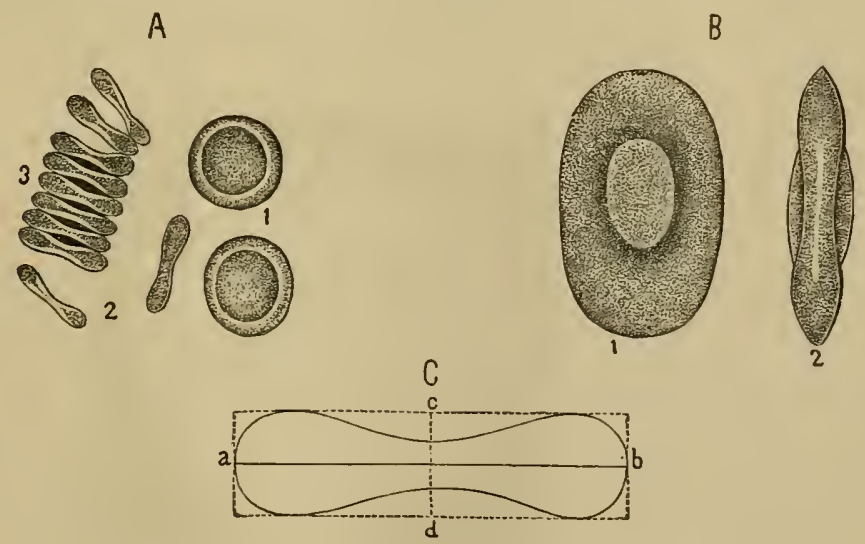

Fig. 1.

A, Human coloured blood-corpuscles-1, seen on the flat; 2 , on edge; 3 , roulean of coloured corpuscles slightly separated. B, Coloured amphibian blood-corpuscles-1, seen on the flat, and 2, on edge. C, Ideal transverse section of a human coloured blood-corpuscle magnified 5,000 times linear; $a b$, diameter ; $c d$, thickness.

* The Greek letter $\mu$ represents one-thousandth of a millimetre $(\mu=0.001 \mathrm{~mm}$.), and is the sign of a micro-millimetre, or a micron. 
If the total amount of blood in a man be taken at 4,400 cubic centimetres, the corpuscles therein contained have a surface of 2,816 square metres, which is equal to a square surface with a side of 80 paces; 176 cubic centimetres of blood, pass through the lungs in a second, and the blood-corpuscles in this amount of blood have a superficies of 81 square metres, equal to a square surface with a side of 13 paces (IVelcker).

(c.) Weight.-The weight of a blood-corpuscle, according to Welcker, is 0.00008 milligrammes.

(d.) Number.-According to Vierordt, the number exceeds 5,000,000 per cubic millimetre in the male, and 4,500,000 in the female; so that, in $10 \mathrm{lbs}$. of blood, there are 25 billions of corpuscles.

The venous blood of the small cutaneous veins contains more red corpuscles than arterial blood. As a general rule, the number is in inverse ratio to the amount of plasma; hence, the number must vary with the state of contraction of the blood-vessels, the pressure-diffusion currents, and other conditions. The use of solid food increases their

A

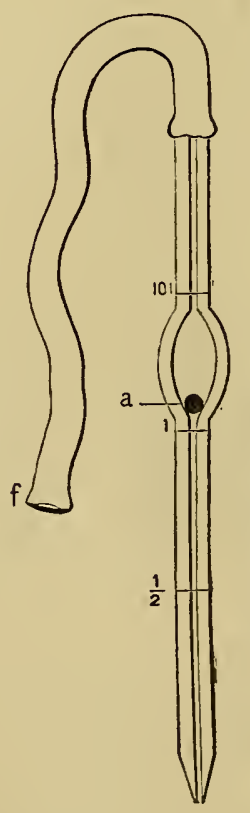

B
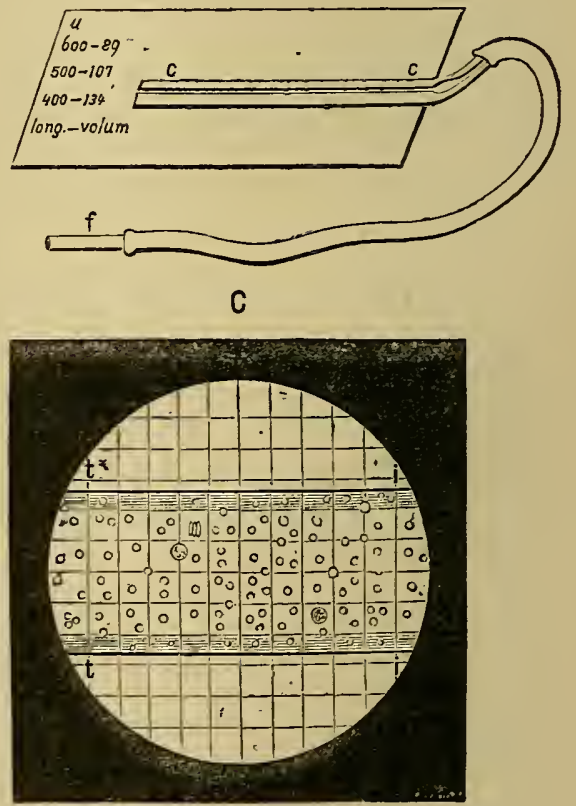

Fig. 2.

Apparatus of Malassez for estimating the number of blood-corpuscles. A, the melangeur, or pipette, for mixing the blood with the artificial serum. $f$, tube for sucking up these fluids. $B$, the artificial capillary tube, with an elastic tube, $f$, attached for filling it. $\mathrm{C}$, appearance of $\mathrm{B}$ under the microscope when it is filled with blood. The squares are due to a piece of glass divided into squares, which is put in the ocular of the microscope. 
number, copious draughts of water reduce it; during inanition the number is relatively increased, because the blood plasma undergoes decomposition sooner than the blood-corpuscles themselves (Buntzen). The blood of the newly-born child contains a considerably larger number of red corpuscles than the blood of the mother (Panum), while Hayem found that the number diminished after the fourth day. In persons of robust constitution the number is larger than in the weakly, and those who live in the country have more than those who live in town.

(The pathological conditions which affect the number of corpuscles are given at p. 22).

a. Malassez's Method of Estimating the number of Blool-Corpuscles. - The pointed end of a glass pipette (Fig. 2, A), the mixer, is dipped into the blood, and by sncking the elastic tube, $f$, blood is drawn into the tube until it reaches the mark, $\frac{1}{8}$, on the stem of the pipette, or until the mark, 1 , is reached. The carefully-cleaned point of the pipette is dipped into the artificial serum, and this is sucked into the pipette until it reaches the mark, 101. The artificial serum consists of 1 vol. of solution of gum arabic (S. G. 1,020) and 3 vols. of a solution of equal parts of sodic sulphate and sodic chloride (S. G. 1,020). The process of mixing the two fluids is aided by the presence of a little glass ball $(a)$ in the bulb of the pipette. If blood is sucked up to the mark, $\frac{1}{2}$, the strength of the mixture is $1: 200$; if to the mark, 1 , it is $1: 100$. A small drop of the mixture is allowed to rnn into the artificial capillary tube $(c c)$ (the first portions are not used in order to obtain a uniform sample from the bulb of the pipette). The mixture passes by capillarity into the capillary tube, which, when full, is emptied by blowing through the thin caoutchouc tube, $f$, and then again filled to $\frac{2}{3}$, and the mixture sucked into the middle of the capillary tube. The capillary tube is firmly fixed to a glass slide (B) with Canada balsam, and on it is inscribed the following numbers :-

$\begin{array}{lllllc}\text { Length. } & & & & & \text { Volume. } \\ 600 \mu & . & . & \text {. } & \text {. } & 89 \\ 500 \mu & . & . & . & . & 107 \\ 400 \mu & . & . & . & . & 134\end{array}$

i.e., a length of the capillary tube of 600,500 , and $400 \mu$ contains $\frac{1}{89}, \frac{1}{10}, \frac{1}{134}$, cubic millimetre.

In order to count the corpuscles, the same combination of lenses must always be used. Select Hartnack's objective, No. 5 (Nachet, No. 2); the ocular contains a piece of glass divided into 100 squares. The tube of the microscope must be so made that it can be pulled out and in. A micrometer, divided into $\frac{1}{100}$ millimetre, is placed upon the stage of the microscope: 1 division, therefore, $=10 \mu$ ( $\mu=\frac{1}{1000}$ millimetre). The tube is now pulled ont until the outer lines of the divided ocular $(t t, i i)$ exactly cover 600,500 , or $400 \mu\left(500 \mu=\frac{1}{2} \mathrm{~mm}\right.$. is most convenient). A mark is made on the tube of the microscope to indicate how far it must be drawn ont to accomplish this object, and, having been made, it indicates, once for all, how far the tube must be drawn out to indicate exactly $500 \mu$. The capillary tube is then filled and placed on the stage, instead of the micrometer, when a picture like $\mathrm{C}$ is obtained. The length of the capillary tube, from $t t$ to $i$, is $500 \mu$. All the corpuscles observable between $t t$ aud $i i$ are now counted. Suppose 315 corpnscles to be counted between $t t$ and $i i$, the number, 315 , is then multiplied by 107 (which stands opposite 500 on $\mathrm{B}$ ) aud also by 100 (when the mixture of blood and serum was $1: 100$ ), or by 200 as the case may be-i.e., 
$315 \times 107 \times 100=3,370,000$ blood-corpuscles in 1 cubic millimetre. (After the experiment the instruments must be carefully washed with distilled water.)

To estimate the colourless corpuscles only, mix the blood with 10 parts of 0.5 per cent. solution of acetic acid, which destroys all the red corpuscles (Thoma).

Varions forms of apparatus for the same purpose have been devised by Thoma, Zeiss, Abbé, and Gowers.

[The following is a description of Gowers' instrument (Fig. 3):- "The Homacytometer consists of-(l.) A small pipette, which, when filled to the mark on its stem, holds exactly 995 cubic millimetres. It is furnished with an India-rubber tube and mouthpiece to facilitate filling and emptying. (2.) A capillary tube marked to contain exactly 5 cubic millimetres, with India-rubber tube for filling, \&c. (3.) A small glass jar in which the dilution is made. (4.) A glass stimer for mixing the blood and solution in the glass jar. (5.) A brass stage plate, carrying a glass slip, on which is a cell, $\frac{1}{5}$ of a millimetre deep. The bottom of this is divided into $\frac{1}{10}$ millimetre squares. Upon the top of the cell rests the cover glass, which is kept in its place by the pressure of two springs proceeding from the ends of the stage plate."

The diluting solution used is a solution of sodic sulphate in distilled water, S. G. 1,025, or the following-sodic sulphate, 104 grains; acetic acid, 1 drachm; distilled water, 4 ozs.

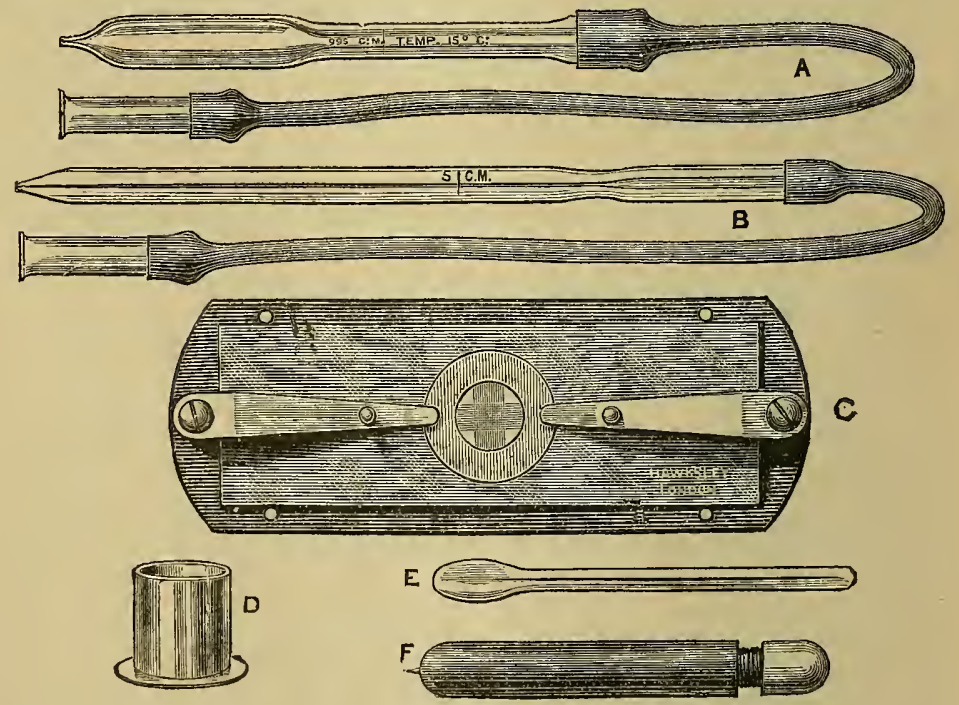

Fig. 3.

Gowers' apparatus, made by Hawksley, London. A, Pipette for measuring the diluting solution. B, Capillary tube for measuring the blood. C, Cell with divisions on the floor, mounted on a slide, to which springs are fixed to secure the cover glass. D, Vessel in which the solution is made. E, Spud for mixing the blood and solution. F, Guarded spear-pointed needle.

" 995 cubic millimetres of the solution are placed in the mixing jar; 5 cubic millimetres of blood are drawn iuto the capillary tube from a puncture in the 
finger, and then blown into the solution. The two fluids are well mixed by rotating the stirrer between the thumb and finger, and a small drop of this dilution is placed in the centre of the cell, the covering glass gently put upon the cell, and secured by the two springs, and the plate placed upon the stage of the microscope. The lens is then focussed for the squares. In a few minutes the corpuscles have sunk to the bottom of the cell, and are seen at rest on the squares. The number in ten squares is then counted, and this, multiplied by 10,000 , gives the number in a cubic millimetre of blood."

Welcker attempted to ascertain the number of corpuscles by estimating the colouring-power of the blood. His method was not exact, but other observers have constructed apparatus for determining the amount of hamoglobin.

(c.) Red blood-corpuscles are characterised by their great ELASTICITY, FLEXIBILITY, and SOFTNLSS. [The elastic property is shown by the great extent to which red corpuscles still within the circulation may be distorted, and yet resume their original form as soon as the pressure is removed.]

\section{Histology of the Human Red Blood-Corpuscles.}

When observed singly, blood-corpuscles have a yellow colour with a slight tinge of green; they seem to be devoid of an envelope, are certainly non-nucleated, and appear to be homogeneous throughout. Each corpuscle consists (1.) of a framework, an exceedingly pale, transparent, soft protoplasm-the stromu (Rollett); and (2.) of the red pigment, or hæmoglobin, which impregnates the stroma, much as fluid passes into and is retained in the interstices of a bath-sponge. Some observers (Böttcher, Eberhardt, Stricker), maintain that the corpuscles contain a nucleus, but this is certainly a mistake.

\section{Effects of Reagents.}

(A.) Vital Phenomena.-Blood-corpuscles contained in shed bloodor even in defibrinated blood, when it is reintroduced into the circulation-retain their vitality and functions undiminished. Heat acts powerfully on their vitality, for if blood be heated to $52^{\circ} \mathrm{C}$, the vitality of the red corpuscles is extinguished. Mammalian blood may be kept for four or five days in a vessel under iced water, and still retain its functions; but if it be kept longer, and reintroduced into the circulation, the corpuscles rapidly break up-a proof that they have lost their vitality (Landois). Blood freshly shed from an artery, frequently shows a transformation of the corpuscles into a peculiar mulberry-shape. [This is the so-called crenation of the coloured corpuscles. It is produced by poisoning with Calabar bean (T. R. Fraser), and also by the addition of a 2 per cent. solution of common salt]. The 
blood of many persons crenates spontaneously-a condition ascribed to an active contraction of the stroma (Klebs), but it is doubtful if this is the carse. Max Schultze observed that the red corpuscles of the embryo-chick undergo active contraction.

(B.) External Characters.--Many agents affect the external characters of the corpuscles.

(a.) The Colour is changed by many gases. O makes blood scarlet, want of $\mathrm{O}$ renders it dark bluish-red, $\mathrm{CO}$ makes it cherry-red, NO violet-red. There is no difference between the shape of corpuscles in arterial and venous blood, as was supposed by Harless. All reagents (e.g., a concentrated solution of sodic sulphate), which cause great shrinking of the coloured corpuscles, produce a very bright scarlet or brick-red colour (Bartholinus, 1661). The red colour so produced is quite different from the scarlet-red of arterial blood. Reagents which render blood-corpuscles globular darken the blood, e.g., water. [The contrast is very striking, if we compare blood to which a 10 per cent. solution of common salt has been added with blood to which water has been added. With reflected light the one is bright-red, and the other a very dark deep crimson, almost black.]

(b.) Change of Position and Form.-A very common phenomenon in shed blood is the tendency of the corpuscles to run into rouleaux (Fig. 1, A, 3).

Conditions that increase the coagulability of the blood favour this phenomenon, which is ascribed by Dogiel to the attraction of the discs and the formation of a sticky substance. [The cause of the arrangement of the red corpuscles into rouleaux is by no means clear. They may be detached from each other by gently touching the cover-glass, but the rouleaux may reform. Lister suggested that the surfaces of the corpuscles were so altered that they became adhesive, and thus cohered. Norris has made some ingenious experiments with corks weighted with tacks or pins, so as to produce partial submersion of the cork discs. These discs rapidly cohere, owing to capillarity, and form rouleaux. If the dises be completely submerged they remain apart, as occurs with unaltered blood-corpuscles within the blood-vessels. If, however, the corpuscles be dipped in petroleum, and then placed in water, rouleaux are formed]. If reagents which cause the corpuscles to swell up be added to the blood, the corpuscles become globular and the rouleaux break up. According to E. Weber and Suchard, the uniting medium is not fibrin (although it may sometimes assume a fibrous form), but belongs to the peripheral layer of the corpuscles.

(c.) The Changes of Form which, after blood is shed, the red corpuscles undergo until they are gradually dissolved, are important. Some reagents rapidly produce this series of events-e.g., the discharge of a Leyden jar causes the corpuscles to crenate, so that their surfaces are beset with large or small projections (Fig. $4, c, d, e, g, h)$; it also causes the corpuscles to assume a spherical form $(i, i)$, when they are smaller than normal. The corpuscles so altered are sticky, and run together like drops of oil, 
forming larger spheres. The prolonged action of the electrical spark causes the hæmoglobin to separate from the stroma $(k)$, whereby the fluid part of the blood is reddened, while the stroma is recognisable only as a faint shadow (l). Similar forms are to be found in decomposing blood, as well as after the action of many other reagents.

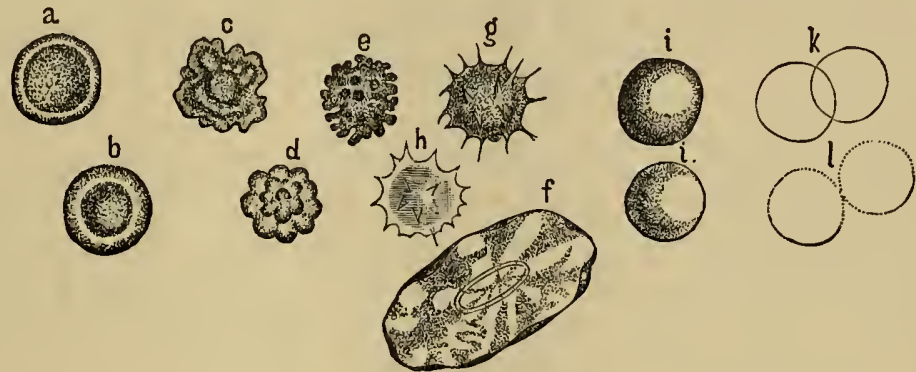

Fig. 4.

Red blood-corpuscles, showing various changes of shape- $a, b$, normal human red corpuscles, with the central depression more or less in focus; $c, d, e$, mulberry forms; $g, h$, crenated corpuscles; $k$, pale decolourised corpuscles; $l$, stroma; $f$, a frog's blood-corpuscle, partly shrivelled, owing to the action of a strong saline solution.

Action of Heat.-When blood is heated, on a warm stage, to $52^{\circ} \mathrm{C}$. the corpuscles begin to undergo remarkable changes. Some of them become spherical, others biscuit-shaped; some are perforated, while in others small portions become detached and swim about in the surrounding fluid, a proof that heat destroys the histological individuality of the corpuscles (Max Schultze). If the heat be continued, the corpuscles are ultimately dissolved.

Cytozoon or Würmchen-Gaule's Experiment.-The following remarkable observation made by Gaule deserves mention here:-A few drops of freshlyshed frog's blood are mixed with 5 cc. of 0.6 per cent. solution of common salt, and the mixture defibrinated by shaking it along with a few cc. of mercury. A drop of the defibrinated blood is examined on a hot stage $\left(30^{\circ}-32^{\circ} \mathrm{C}\right.$.) under a microscope, when a protoplasmic mass, the so-called "wiirmchen," escapes with a lively movement from many corpuscles, and nltimately dissolves. Similar "cytozoa" were discovered by Gaule in the epithelium of the cornea, of the stomach and intestine, in connective tissue, in most of the large glands, and in the retina (frog, triton). In mammals also he found similar but smaller structures. Most probably these structures are parasitic in their nature, as suggested by Ray Lankester, who called the parasite Drepanidium ranarum.

If a finger moistened with blood be rapidly drawn across a warm slip of glass, so that the fluid dries rapidly, very remarkable corpuscleshapes, showing their great ductility and softness, are observed under the microscope. 
If blood be mixed with concentrated gum, and if concentrated salt solution be added to it under the microscope, the corpuscles assume elongated forms (Lindwurm). Similar forms are obtained by mixing blood with an equal volume of gelatine at $36^{\circ} \mathrm{C}$., allowing it to cool, and then making sections of the coagulated mass (Rollett). The corpuscles may be broken up by pressing firmly on the cover-glass. In all these experiments no trace of an envelope is observed.

\section{Preparation of the Stroma-Making Blood "Lake-Coloured."}

There are many reagents which separate the hæmoglobin from the stroma. The hæmoglobin dissolves in the serum; the blood then becomes transparent, as it contains its colouring matter in solution, and hence it is called "lake-coloured" by Rollett. Lake-coloured blood is dark-red. The aggregate condition of the hæmoglobin is not altered, when the corpuscles are dissolved-it only changes its place, leaving the stroma and passing into the serum. Hence, the temperature of the blood is not lowered thereby (Landois). To obtain a large quantity of the stroma, add ten volumes of a solution of common salt ( 1 vol. concentrated solution, and 15 to 20 vols. of water) to one volume of defibrinated blood, when the stromata are thrown down as a whitish precipitate.

The following reagents cause a separation of the stroma from the hæmoglobin :-

(a.) Physical Agents.-1. Heating the blood to $60^{\circ} \mathrm{C}$. (Schultze); the temperature, however, varies for the blood of different animals. 2. Repeated freezing and thawing of the blood (Rollett). 3. Sparks from an electrical machine (but not after the addition of salts to the blood) (Rollett); the constant and induced currents (Neumann).

(b.) Chemically active Substances produced within the Body.-4. Bile (Hünefeld), or bile salts (Plattner, v. Dusch). 5. Serum of other species of animals (Laudois); thus dog's serum and frog's serum dissolve the blood-corpuscles of the rabbit in a few minutes. 6. The addition of lake-coloured blood of many species of animals (Landois).

(c.) Other Chemical Reagents.-7. Water. S. Conduction of vapour of chloroform (Böttcher); ether (v. Wittich); amyls, small quantities of alcohol (Rollett); thymol (Marchand); nitrobenzol, ethylic ether, aceton, petroleum ether, etc. (L. Lewin). 9. Antimonuretted hydrogen, arseniuretted hydrogen; carbon disulphide (Hiunefeld, Hermann); boracic acid (2 per cent.), added to amphibian blood, causes the red mass (which also encloses the nucleus when such is present), the so-called zooid, to separate from the ocoid. The zooid may shrink from the periphery of the corpuscle, or it may even pass out of the corpuscle altogether (Briicke); Bricke regards the stroma in a certain sense as a house, in which the remainder of the substance of the corpuscle, the chief part endowed with vital phenomena, lives. 11. Strong solutions of acids dissolve the corpuscles; more dilute solutions cause precipitates in the hæmoglobin. This is easily seen with carbolic acid (Hüls and Landois; Stirling and Rannie). 12. Alkalies of moderate strength cause sudden solution. A 10 per cent. solution of potash, placed at the margin of a cover-glass, shows the process of solution going on under the micro- 
scope. At first the corpuscles become globular, and so appear smaller, but afterwards they burst like soap-bubbles.

[Tannic Acid.-A freshly prepared solution of tannic acid has a remarkable effect on the coloured blood-corpuscles of man and animals - causing a separation of the hæmoglobin and the stroma. The usual effect is to produce one or more granular buds of hæmoglobin on the side of the corpuscles; more rarely the hæmoglobin collects around the nucleus, if such be present (IV. Roberts).]

[Ammonium or Potassium Sulpho-cyanide remores the hæmoglobin, and reveals a reticular structure-intra-nuclear plexus of fibrils (Stirling and Rannie).]

The quantity of gases contained in the blood-corpuscles exercises an important influence on their solubility. The corpuscles of venous blood, which contains much $\mathrm{CO}_{2}$, are more easily dissolved than those of arterial blood; while between both stands blood containing CO (Landois, Litterski). When the gases are completely removed from the blood, it becomes lake-coloured.

\section{Form and Size of the Blood-Corpuscles of Different Animals.}

All mammals (with the exception of the camel, llama, alpaca, and their allies), and the cyclostomata amongst fishes-e.g., Petromyzon, possess circular disc-shaped corpuscles.

Elliptical corpuscles without a nucleus are found in the above-named mammals, while all birds, reptiles, amphibians (Fig. 1, B, 1, 2), and fishes (except cyclostomata) have nucleated elliptical bi-convex corpuscles.

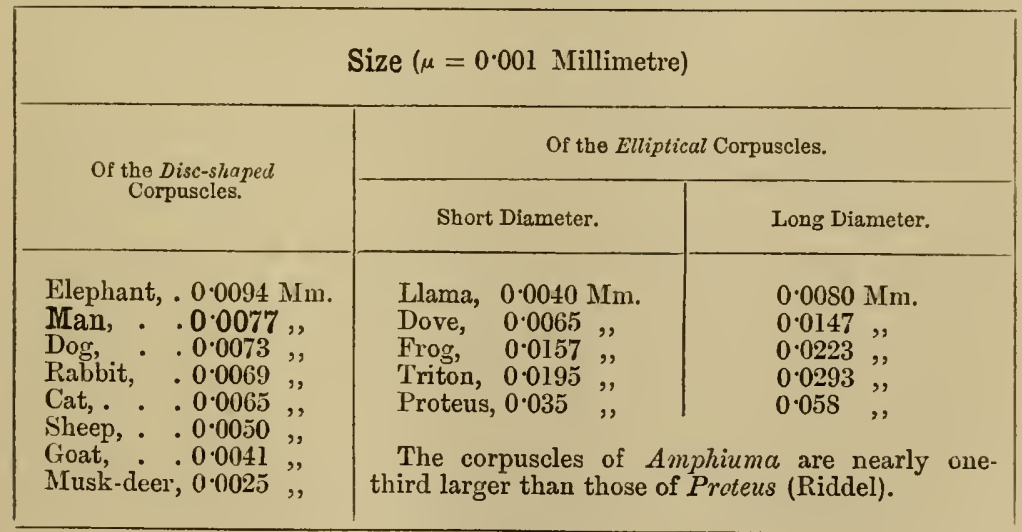

Amongst vertebrates, amphioxus has colourless blood-invertebrates generally have colourless blood, with colourless corpuscles; but the earth-worm, and the larva of the large gnats, \&c., have red blood whose plasma contains hæmoglobin, while the blood-corpuscles themselves are colourless.

[Elaborate measurements of the blood-corpuscles have been made in 
this country by Gulliver, but the relative size may be best appreciated by comparing the corpuscles from various vertebrates.]

Many invertebrates possess red, violet, brown, or green opalescent blood with colourless corpuscles (amœboid cells). In cephalopods, and some crabs, the blood is blue, owing to the presence of a colouring-matter (Hamo-cyanin) which contains copper, and combines with $\mathrm{O}$ (Bert, Rabuteau \& Papillon, Frédéricq, and Krukenberg). The large blood-corpuscles of many amphibia, e.g., amphiuma, are visible to the naked eye. The blood-corpuscles of the frog contain, in addition to a nucleus, a nucleolus (Auerbach, Ranvier), [and the same is true of the coloured corpuscles of the newt (Stirling). The nucleolus is revealed by acting on the corpuscles with dilute alcohol (1, alcohol; 2, water; Ranvier's "alcool au tiers").] It is evident that the larger the blood-corpuscles are, the smaller must be the number and total superficies of corpuscles in a given volume of blood. In birds, how. ever, the number is relatively larger than in other classes of vertebrates, notwithstanding the larger size of their corpuscles; this, doubtless, has a relation to the very energetic metabolism that takes place in birds (Malassez).

Amongst mammals, carnivora have more blood-corpuscles than herbivora. Welcker has ascertained that goat's blood contains $9,720,000$ corpuscles per cubic millimetre; the llama's, 13,000,000; the bullfinch's, 3,600,000; the lizard's, $1,420,000$; the frog's, 404,000 ; the proteus', 36,000. In hybernating animals, Vierordt found that the number of corpuscles diminished from $7,000,000$ to $2,000,000$ per cubic millimetre during hybernation.

\section{Origin of the Red Blood-Corpuscles.}

\section{(A.) Origin of the Nucleated Red Corpuscles during Embryonic} Life.-Blood-corpuscles are developed in the fowl during the first days of embryonic life. [They appear in groups within the large branched cells of the mesoblast, in the vascular area of the blastoderm outside the developing body of the chick or embyro, where they form the "blood-islands" of Pander. The mother-cells form an irregular network by the union of the processes of adjoining cells, and meantime the central masses split up, and the nuclei multiply. The small nucleated masses of protoplasm, which represent the blood-corpuscles, acquire a reddish hue, while the surrounding protoplasm, and also that of the processes, becomes vacuolated or hollowed out, constituting a branching system of canals; the outer part of the cells remaining with their nuclei to form the walls of the future blood-vessels. A fluid appears within this system of branched canals in which the corpuscles lie, and gradually a communication is established with the bloodvessels developed in connection with the heart.]

[According to Klein, the nuclei of the protoplasmic wall may also proliferate, and give rise to new corpuscles, which are washed away to form blood-corpuscles.] At first the corpuscles are devoid of pigment, nucleated, globular, larger and more irregular than the permanent corpuscles, and they also exhibit amœboid movements. They become 
coloured, retain their nucleus, and are capable of undergoing multiplication by division; and, in fact, Remak observed all the stages of the process of division. The process of division is best seen from the 3rd -5th day of incubation. Increase by division also takes place in the larvæ of the salamander, triton, and toad (Flemming, Peremeschko).

After the liver is developed, blood-corpuscles seem to be formed in it (E. H. Weber, Kölliker). Protoplasmic, nucleated, colourless cells are carried by the vena porta from the spleen into the liver, where they take up pigment. Neumann found in the liver of the embryo protoplasmic cells containing red blood-corpuscles. The spleen is also regarded as a centre of their formation, but this seems to be the case only during embryonic life (Neumann). Here the red corpuscles are said to arise from yellow, round, nucleated cells, which represent transition forms. Foa and Salvioli found red corpuscles forming endogenously within large protoplasmic cells in lymphatic glands. In the later period of embryonic life, the characteristic non-nucleated corpuscles seem to be developed from the nucleated corpuscles. The nucleus becomes smaller and smaller, breaks up, and gradually disappears. In the human embryo at the fourth week only nucleated corpuscles are found; at the third month their number is still $\frac{1}{4}-\frac{1}{8}$ of the total corpuscles, while at the end of foetal life nucleated bloodcorpuscles are very rarely found. Of course, in animals with nucleated blood-corpuscles, the nucleus of the embryonic blood-corpuscles remains.

(B.) Development of Blood-Vessels, Formation of Blood-Vessels and Blood-Corpuscles during Post-embryonic Life. - Kölliker assumed that, in the tail of the tadpole, capillaries are formed by the anastomoses of the processes of branched and radiating connective tissuecorpuscles. These corpuscles lose their nuclei and protoplasm, become hollowed out, join with neighbouring capillaries, and thus form new blood-channels. Von Golubew, on the other hand, opposes this view. He assumes that the blood capillaries in the tail of the tadpole give off solid buds at different places, which grow more and more into the surrounding tissues, and anastomose with each other; their protoplasm and contents disappearing, they become hollow and a branched system of capillaries is formed in the tissues. Ranvier, be it remarked, noticed the same mode of growth in the omentum of newly-born kittens.

The latter observer has recently studied the development of bloodvessels and blood-corpuscles in the omentum of young rabbits. These animals, when a week old, have, in their omentum, little white or milk spots ("taches laiteuses," Ranvier), in which lie "vaso-formative" cells, i.e., highly refractive cells of variable shape, with long cylindrical protoplasmic processes (Fig. 5). In its refractive power the protoplasm 
of these cells resembles that of lymph-corpuscles. Long rod-like

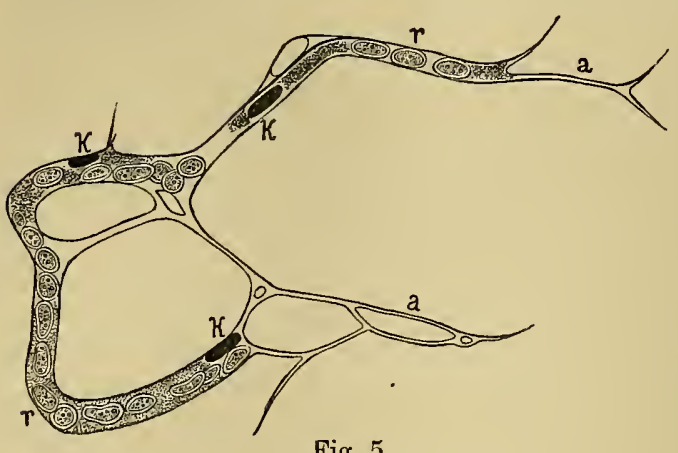

Fig. 5.

Formation of red blood-corpuscles within "vasoformative cells," from the omentum of a rabbit seven days old. $r, r$, the formed corpuscles. $\mathrm{K}, \mathrm{K}$, nuclei of the vaso-formative cell. $a, a$, processes which ultimately unite to form capillaries. nuclei lie within these cells $(\mathrm{K}, \mathrm{K})$, and also red blood-corpuscles $(r, r)$, and both are surrounded with protoplasm. These vasoformative cells give off protoplasmic points and processes $(a, a)$ some of which end free, while others form a network. Here and there elongated connective tissue-corpuscles lie on the branches, and ultimately form

the adventitia of the blood-vessel.

The vaso-formative cells have many forms : they may be elongated cylinders ending in points, or more round and oval, resembling lymph cells, or they may be modified connective tissue-corpuscles, as observed by Schäfer in the subcutaneous tissue of young rats. These cells are always the seat of origin of non-nucleated red blood-corpuscles, which arise in the protoplasm of vaso-formative cells, as chlorophyll grains or starch grannles arise within the cells of plants. The corpuscles escape and are washed into the circulation, when the cells form connections with the circulatory system by means of their processes. It is probable that the vessels so formed in the omentum are only temporary. May it not be that there are many other situations in the body where blood is regenerated?

[The observations of Schäfer also prove the intra-cellular origin of red blood-corpuscles, and although this mode usually ceases before birth, still it is found in the rat at birth. The protoplasm of the subcutaneous connective tissue-corpuscles, which are derived from the mesoblast, has in it small coloured globules about the size of a coloured corpuscle. The mother-cells elongate, become pointed at their ends, and unite with processes from adjoining cells. The cells become vacuolated; fluid or plasma, in which the liberated corpuscles float, appears in their interior, and ultimately a communication is established with the general circulation.]

Similar observations have been made by Neumann in the embryonic liver; by Wissotzky in the rabbit's amnion; by Klein in the embryo chick; and by Leboucq and Hayem in various animals; all of which go to show that at a certain early 
period of development blood-corpuscles are formed within other large cells of the mesoblast, and that part of the protoplasm of these blood-forming cells remains to form the wall of the future blood-vessel.

(C.) Later Formation of Red Blood-Corpuscles.-There is much diversity of opinion as to how coloured blood-corpuscles are formed in mammals at a later period. [They have been described as derived from colourless corpuscles, one set of observers (including Kölliker) maintaining that the nucleus of these corpuscles disappears, while the peri-nuclear portion remains, becomes flattened and coloured, and assumes the characters of the mammalian blood-corpuscles. On the other hand, other observers (including Wharton Jones, Gulliver, Busk, Huxley, and Balfour) are of opinion that the nucleus becomes pigmented, and forms the future blood-corpuscle. It is still donbtful, however, whether coloured corpuscles are developed in either of these ways.] Neumann and Bizzozero described peculiar corpuscles occurring in the red marrow of bone, which they maintain become developed into coloured blood-corpuscles, undergoing a series of changes, and forming a series of intermediate forms, which may be detected in the red marrow. Bizzozero holds that it is the nucleus of the marrow-cell which is coloured, while Neumann thinks it is the perinuclear part which becomes coloured, and forms the blood-corpuscle. Schäfer's observations on the red marrow of the guinea-pig rather tend to confirm Neumann's view.

These transition cells are said by Erb to be more numerous after severe hæmorrhage, the number of them occurring in the blood corresponding with the energy of the formative process. In dogs and guinea-pigs which he had rendered anæmic, Bizzozero found in the marrow and spleen nucleated red blood-corpuscles, which increased by division.

According to Neumann, the bone-marrou" of adults contains all transition forms, from nucleated coloured corpuscles to true red bloodcorpuscles. After copious hæmorrhage, these transition forms appear in numbers in the blood-stream.

Red or blood-forming marrow occurs in the bones of the skull, and in most of the bones of the trunk, while the bones of the extremities either contain yellow marrow (which is essentially fatty in its nature), or, at most, it is only the heads of the long bones that contain red marrow. Where the blood regeneration process is very active, however, the yellow marrow may be changed into red, even throughout all the bones of the extremities (Neumann).

Rindfleisch also regards the connective substance of the red marrow and the spleen as the mother-tissue of the red blood-corpuscles, the connective substance or the hæmatogenous connective tissue either temporarily or permanently forming red blood-corpuscles. Once the red corpuscles are formed, they easily enter the blood-stream, as the capillaries and veins of the red marrow have either no walls 
(Hoyer, Kollmann), or exceedingly thin perforated walls. Similar conditions obtain in the spleen.

Bizzozero and Torre found that after severe hæmorrhage in birds, the marrow of the bones contained globular, granular, nucleated cells, whose protoplasm was coloured with hæmoglobin, while between these and the oval biconvex nucleated corpuscles of the bird, there were numerous transition stages. The spleen of the bird seems to be of much less importance in the formation of blood-corpuscles (Korn). All these observations prove that the red marrow of the bones is a great manufactory for coloured blood-corpuscles.

v. Recklinghausen observed the direct transformation of these intermediate forms into blood-corpuscles in frog's blood, which was kept for several days in a moist chamber. A. Schmidt and Semmer found large lymph cells in the blood, filled with granules of hæmogoblin, and they regard these as intermediate forms between colourless and coloured corpuscles.

[Malassez, from an investigation of the red marrow of young kids, finds that the cells of the red marrow and certain cells in the spleen form rounded coloured projections or buds on their surface. These get detached and form young blood-corpuscles, which soon become disc-shaped; while the mother-cell itself continues to produce other coloured corpuscles. Thus gemmation of the splenic and medullary cells constitutes one great process in the manufacture of blood-corpuscles. Hence it is apparent why diseases of bone in children lead to anæmia, and soon bring about a cachectic condition.]

\section{Decay of the Red Blood-Corpuscles.}

The blood-corpuscles must positively undergo decay within a limited time, and the liver is regarded as one of the chief places in which their disintegration occurs, because bile-pigments are formed from hæmoglobin, and the blood of the hepatic vein contains fewer red corpuscles than the blood of the portal vein.

The splenic pulp contains cells which seem to indicate that coloured corpuscles are broken up within it. These are the so-called "bloodcorpuscle-containing cells." Quincke's observations go to show that the red corpuscles-which may live from three to four weeks-when about to disintegrate, are taken up by white blood-corpuscles, and by the cells of the spleen and the bone-marrow, and are stored up chiefly in the spleen and marrow of bone. They are transformed, partly into coloured, and partly into colourless proteids which contain iron, and are either deposited in a granular form, or are dissolved. Part of the products of decomposition is used for the formation of new bloodcorpuscles in the marrow and in the spleen, and also perhaps in the liver, while a portion of the iron is excreted by the liver in the bile.

That the normal red blood-corpuscles and other particles suspended in the bloodstream are not taken up in this way, may be due to their being smooth and polished. 
As the corpuscles grow older and become more rigid, they, as it were, are caught by the amœboid cells. As cells containing blood-corpuscles are very rarely found in the general circulation, one may assume that the occurrence of these cells within the spleen, liver, and marrow of bone is favoured by the slowness of the circulation in these organs (Quincke).

Pathological. - In certain pathological conditions, ferruginous substances derived from the red blood-corpuscles are found in the spleen, in the marrow of bone, and in the capillaries of the liver :-(1.) When the disintegration of bloodcorpuscles is increased, as in anæmia (Stahel). (2.) When the formation of red blood-corpuscles from the old material is diminished. If the excretion from the liver cells be prevented, iron accumulates within them ; it is also more abundant in the blood-serum, and it may even accumulate in the secretory cells of the cortex of the kidney and pancreas, in gland cells, and in the tissue elements of other organs (Quincke). When the amount of blood is greatly increased (in dogs), after four weeks an enormous number of granules containing iron occur in the leucocytes of the liver capillaries, the cells of the spleen, bone-marrow, lymph-glands, the liver cells, and the epithelium of the cortex of the kidney (Quincke). The iron reaction in the two last situations occurs after the introduction of hæmoglobin, or of salts of iron into the blood (Glacveck and v. Stark).

When we reflect how rapidly (relatively) large quantities of blood are replaced after hæmorrhage and after menstruation, it is evident that there must be a brisk manufactory somewhere. As to the number of corpuscles which daily decay, we have in some measure an index in the amount of bile-pigment and urine-pigment resulting from the transformation of the liberated hæmoglobin.

\section{The Colourless Corpuscles (Leucocytes).}

Blood, like many other tissues, contains a number of cells or corpuscles which reach it from without; the corpuscles vary somewhat in form, and are called colourless or white blood-corpuscles, or "leucocytes" (Hewson, 1770). Similar corpuscles are found in lympl, adenoid tissue, marrow of bone, as wandering cells or leucocytes, in connective tissue, and also between glandular and epithelial cells. They all consist of more or less spherical masses of protoplasm, which is sticky, highly refractile, soft, capable of movement, and devoid of an envelope (Fig. 6). When they are quite fresh (A) it is difficult to detect the nucleus, but after they have been shed for some time, or after the addition of water (B), or acetic acid, the nucleus (which is usually a compound one) appears ; acetic acid clears up the perinuclear protoplasm, and reveals the presence of the nuclei, of which the number varies from one to four, although generally three are found. The subsequent addition of magenta solution stains the nuclei deeply. Water makes the contents more turbid, and causes the corpuscles to swell up. One or more nucleoli may be present in the nucleus. The corpuscles contain proteids, but they also contain fats, lecithin, and salts (p. 37). The size of the corpuscles varies from four 
to thirteen $\mu$, and as a rule they are about $\frac{1}{2500}$ of an inch in diameter,
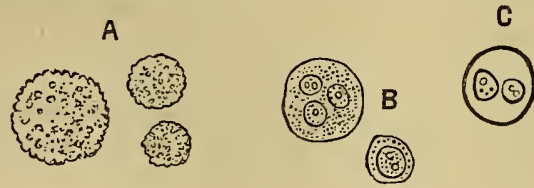

D

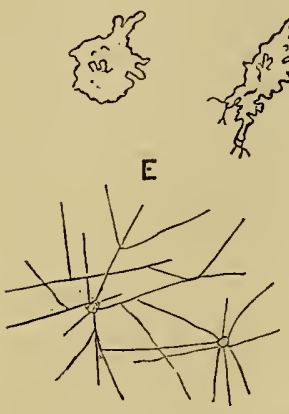

Fig. 6.

White blood-corpuscles-A, Human, without the addition of any reagent. B, after the addition of water, nuclei visible. $\mathrm{C}$, after the action of acetic acid. D, Frog's corpuscles showing changes of shape due to amøoboid movement. E, Fibrils of fibrin from coagulated blood. F, Elementary granules.

the same size as the coloured blood-corpuscles; and in the smallest the layer of the protoplasm is extremely thin. They all have the property of exhibiting amoboid movements which are very apparent in the larger corpuscles. These movements were discovered by Wharton Jones in the skate, and by Davine in the corpuscles of man. Max Schultze describes three different forms in human blood :-

(1.) The smallest, round forms, less than the red corpuscles, with one to two nuclei, and a very small amount of protoplasm;

(2.) Round forms,

(3.) The large amœboid corpuscles, with much protoplasm and distinctly evident movements.

[When a drop of human blood is examined under the microscope, more especially after the coloured blood-corpuscles have run into rouleaux, the colourless corpuscles may readily be detected, there being usually three or four of them visible in the field at once. They adhere to the glass slide, for if the cover-glass be moved, the coloured corpuscles readily glide over each other, while the colourless can be seen still adhering to the slide.

White Corpuscles of Newt's Blood.-The characters of the colourless corpuscles are best studied in a drop of newt's blood. Cut off the tip of the tail and express a drop of blood on to a slide, cover it with a thin glass, and examine.

Neglecting the coloured corpuscles, search for the colourless, of which there are three varieties:-

(1.) The Large Finely Granular Corpuscle, which is about $\frac{1}{100}$ of an 
inch in diameter, irregular in outline, with fine processes or pseudopodia, projecting from its surface. It rapidly changes its shape at the ordinary temperature, and in its interior a bi- or tri-partite nucleus may be seen, surrounded with fine granular protoplasm, whose outline is continually changing. Sometimes vacuoles are seen in the protoplasm.

(2.) The Coarsely Granular Variety is less common than the firstmentioned, but when detected its characters are distinct. The protoplasm contains, besides a nucleus, a large number of highly refractive granules, and the corpuscle usually exhibits active amœboid movements; suddenly the grantules may be seen to rush from one side of the corpuscle to the other. The processes are usually more blunt than those emitted by (1). The relation between these two kinds of corpuscles has not been ascertained.

(3.) The Small Colourless Corpuscles are more like the ordinary human colourless corpuscle, and they, too, exhibit amoboid movements.

Two kinds of colourless corpuscles like (1.) and (2.) exist in frog's blood. In the coarsely granular corpuscles the glancing granules may be of a fatty nature, since they dissolve in alcohol and ether, but other granules exist which are insoluble in these fluids, and the nature of which is unknown. Very large colourless corpuscles exist in the axolotl's blood (Ranvier).

Action of Reagents.-(a.) Water, when added slowly, causes the colourless corpuscles to become globular, and the granules within them to exhibit Brownian movements (Richardson, Stricker). (b.) Pigments, such as magenta or carmine, stain the nuclei very deeply, and the protoplasm to a less extent. (c.) Dilute Acetic Acid clears up the surrounding protoplasm and brings clearly into view the composite nucleus, which may be stained thereafter with magenta. (d.) Iodine gives a faint port-wine colour (horse's blood indicating the presence of glycogen best). (e.) Dilute Alcohol causes the formation of clear blebs on the surface of the corpuscles, and brings the nuclei clearly into view (Ranvier, Stirling).]

[A delicate plexus of fibrils-intra-nuclear plexus-exists within the nucleus just as in other cells. It is very probable that the protoplasm itself is pervaded by a similar plexus of fibrils, and that it is continuous with the intra-nuclear plexus.]

The colourless corpuscles divide, and in this way reproduce themselves (Klein).

The Number of Colourless Blood-Corpuscles is very much less than that of the red corpuscles, and is subject to considerable variations.

It is certain that the colourless corpuscles are very much fewer in 
shed blood than in blood still within the circulation. Immediately after blood is shed, an enormous number of white corpuscles disappear (see Formation of Fibrin, p. 47).

Al. Schmidt estimates the number that remain at $\frac{1}{10}$ of the whole originally present in the circulating blood. The proportion is greater in children than in adults (Bouchut and Dubrisay).

The following table gives the number in shed blood:-

Number of White Corpuscles in Proportion to Red Corpuscles-

\begin{tabular}{|c|c|c|}
\hline In Normal Conditions. & \multicolumn{1}{|c|}{ In Different Places. } & \multicolumn{1}{|c|}{ In Different Conditions. } \\
\hline $1: 335$ (Welcker). & $\begin{array}{c}\text { Splenic Vein, 1:60 } \\
\text { Splenic Artery, 1:2,260 } \\
\text { Hepatic Vein, 1:170 } \\
\text { Portal Vein, 1:740 } \\
\text { Generally more numerous } \\
\text { in Veins than Arteries. }\end{array}$ & $\begin{array}{c}\text { Increased by } \\
\text { Prolonged Suppuration, } \\
\text { Parturition, Leukæmia, } \\
\text { Quinine, Bitters. } \\
\text { Diminished by } \\
\text { Hunger, Bad Nourishment. }\end{array}$ \\
\hline
\end{tabular}

The old method of Welcker for estimating the number of colourless corpuscles is unsatisfactory. The blood was defibrinated, placed in a tall vessel, and allowed to subside, when a layer of colourless corpuscles was obtained immediately under a laycr of serum. [It is better to use the hæmocytometer (p. 6) as improved by Gowers.]

The Amœboid Movements of the white corpuscles (so-called because they resemble the movements of amoba) consist in an alternate contraction and relaxation of the protoplasm surrounding the nucleus. Processes are given off from the surface, and are retracted again (like the pseudopodia of amoba).

There is an internal current in the protoplasm, and the nucleus has also been observed to change its form (Lavdowsky). Two series of phenomena result from these movements:-(1.) The "wandering" or locomotion of the corpuscles due to the extension and retraction of their processes; (2.) the absorption of small particles into their interior (fat, pigment, foreign bodies). The particles adhere to the sticky external surface, are carried into the interior by the internal currents (Preyer), and may eventually be excreted, just as particles are taken up by amoba and the effete particles excreted. [Max Schultze observed that coloured particles were readily taken up by these corpuscles.]

On a hot stage $\left(35^{\circ}-40^{\circ} \mathrm{C}\right.$.) the colourless corpuscles of mammals retain their movements for a long time; at $40^{\circ} \mathrm{C}$. for two to three hours; at $50^{\circ} \mathrm{C}$. the proteids are coagulated and cause "heat-rigor" and death. In cold-blooded animals (frogs) colourless corpuscles may be seen to crawl 
out of small coagula, in a moist chamber, and move about in the serum. Induction shocks cause them to withdraw their processes and become spherical, and, if the shocks be not too severe, their movements recommence. Strong shocks liill them. $O$ is necessary for their movements. These amœboid movements are of special interest on account of the "wandering out" (diapedesis) of colonrless bloodcorpuscles through the walls of the blood-vessels (Waller, Cohnheim).

The chyle contains leucocytes, which are more resistant than those of the blood, but less so than those of the coagulable transudations (Heyl). The leucocytes of the lymphatic glands may also be dissolved (Rauschenbach).

Relation to Aniline Pigments.-Ehrlich has observed a remarkable relation of the white corpuscles to acid (eosin, picric acid, aurantia), basic (dahlia, acetate of rosanilin), or neutral (picrate of rosanilin) reactions. The smallest protoplasmic granules of the cells have different chemical affinities for these pigments. Thus Ehrlich distinguishes "eosinophile," "basophile," and "neutrophile" granules within the cells. Eosinophile granules occur in the leucocytes of amphibia, and in the marrow of their bones. Human leucocytes exhibit a neutrophile reaction, except in the case of those corpuscles that have large ovoid nuclei: the former are said to be the early stage of the latter. The eosinophile corpuscles are greatly increased in leukæmia. The basophile granules occur chiefly in connective tissuecorpuscles and in the neighbourhood of epithelium-they are always greatly increased where chronic inflammation occurs.

III. Special attention has recently been directed to a third element
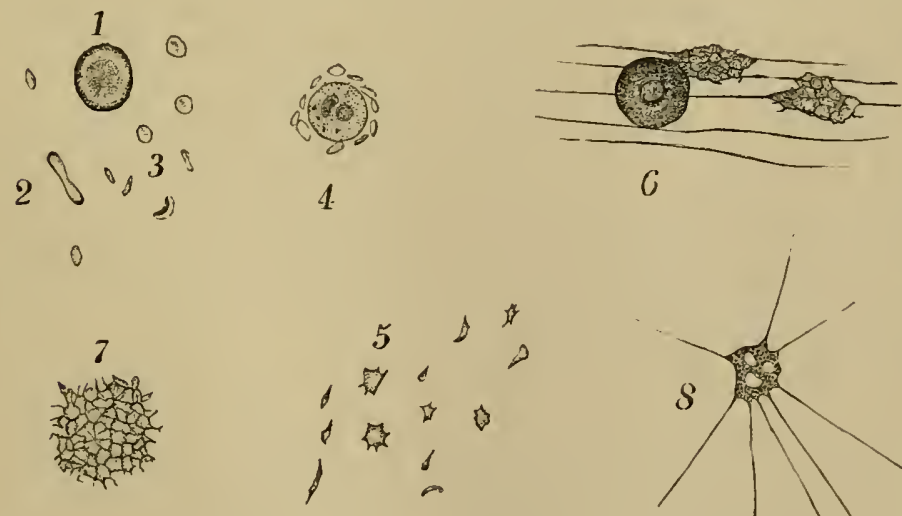

Fig. 7 .

"Blood-plates" and their derivatives, partly after Bizzozero and Laker. 1, Red blood-corpuscles on the flat. 2, From the side. 3, Unchanged blood-plates. 4, A lymph-corpuscle, surrounded with blood-plates. 5, Blood-plates variously altered, 6, A lymph-corpuscle with two heaps of fused bloodplates and threads of fibrin. 7, Group of blood-plates fused or run together. 8, A similar small heap of partially dissolved blood-plates with fibrils of fibrin. 
of the blood, the "blood-plates" of Bizzozero; pale, colourless, biconcave discs of variable size (mean, $3 \mu$ ). According to Hayem (who called these structures HÆMATOBLASTS, supposing that they were an early stage in the development of the red blood-corpuscles), they are forty times as numerous as the leucocytes. These blood-plates may be recognised in circulating blood, as in the mesentery of the guinea-pig. They are precipitated in enormous numbers upon threads suspended in fresh-shed blood (Bizzozero). They may be obtained from blood flowing directly from a blood-vessel, on mixing it with 1 per cent. solution of osmic acid or Hayem's fluid (mercury bichloride 0.5 , sodium carbonate 5, sodium chlorate 1 , distilled water 200-Laker). They undergo a rapid change in shed blood (Fig. 7, 5), disintegrating, forming small particles, and ultimately dissolving. When several occur together they rapidly unite, form small groups (7), and collect into masses resembling "stroma-fibrin" (p. 48). These masses may be associated in coagulated blood with fibrils of fibrin.

Bizzozero believes that they yield the material for the formation of fibrin during coagulation of the blood. It is not yet determined whether they are derived from partially disintegrated leucocytes, or whether they are independent formations. Along with the leucocytes they are concerned in the formation of fibrin (Hlava). These structures were known to earlier observers (Max Schultze, Riess, and others); but their significance has been variously interpreted.

IV. Blood, especially after a microscopic preparation has been made for a short time, is seen to contain Elementary GrandLes (Fig. 6, F), [i.e., the elementary particles of Zimmermann and Beale. They are irregular bodies, much smaller than the ordinary corpuscles, and appear to consist of masses of protoplasm detached from the surface of leucocytes, or derived from the disintegration of these corpuscles, or of the blood-plates. Others, again, are completely spherical granules, either consisting of some proteid substance or fatty in their nature. The protoplasmic and the proteid granules disappear on the addition of acetic acid, while the fatty granules (which are most numerous after a diet rich in fats) dissolve in ether].

V. In COAGULATED blood, delicate fibrils or threads of FIBRIN (Fig. $6, \mathrm{E}$ and $6,8,6)$ are seen, more especially after the corpuscles have run into rouleaux. At the nodes of these fibres are found granules which closely resemble those described under III.

[These granules and fibres are stained by magenta and iodine, but not by carmine or picro-carmine (Ranvier).]

\section{Abnormal Changes of the Red and White Blood-Corpuscles.}

(1.) All hæmorrhages diminish the number of red corpuscles (at most onehalf), and so does menstruation. The loss is partly covered hy the absorption of 
fluid from the tissues. Menstruation shows us that a moderate loss of red corpuscles is replaced within twenty-eight days. When a large amount of blood is lost, so that all the vital processes are lowered, the time may be extended to five weeks. In acute fevers, as the temperature increases, the number of red corpuscles diminishes, while the white corpuscles increase in number (Riegel \& Boeckmann).

(2.) Diminished production of new red corpuscles causes a decrease, since blood-corpuscles are continually being used up. In chlorotic girls there seems to be a congenital weakness in the blood-forming and bloor-propelling apparatus, the cause of which is to be songht for in some faulty condition of the meso-blast. In them the heart and the blood-vessels are small, and the absolute number of corpuscles may be diminished one-half, although the relative number may be retained, while in the corpuscles themselves the hæmoglobin is diminished almost one-third (Duncan, Quincke); but it rises again after the administration of iron (Hayem). The adninistration of iron increases the amount of hrmoglobin in the blood (Scherpf). The amonnt of iron in the blood may be diminished one-half. [The action of iron in anæmic persons has been known since the time of Sydenham. Hayem also finds that in certain forms of anæmia there is considerable variation in the size of the red corpuscles, and that in chronic anæmia the mean diameter of the corpuscles is always less than normal $(7 \mu$ to $6 \mu)$. There is, moreover, a persistent alteration in the volume, colouring power, and consistence of the corpuscles, consequently a want of accord between the number of the corpuscles and their colouring power-i.e., the amount of hæmoglobin which they contain, as was pointed out by Johann Duncan.] In so-called pernicious ancemia, in which the continued decrease in the red corpuscles may ultimately produce death, there is undoubtedly a serere affection of the blood-forning apparatus. The corpuscles assume many abnormal and bizarre forms (microcytes), often being oral or tailed, irregularly shaped, and sometimes very pale; while numerous cells containing blood-corpuscles are found in the marrow of bone (Riess). Curiously enough in this disease, although the red blood-corpuscles are diminished in number, some may be larger and contain more hæmoglobin than do normal corpuscles (Laache). The number of coloured corpuscles is also diminished in chronic poisoning by lead or miasmata, and also by the poison of syphilis.

(3.) Abnormal forms of the red corpuscles have been observed affer severe burns (Lesser); the corpuscles are much smaller, and under the influence of the heat, particles seem to be detached from them just as can be seen happening under the microscope as the effect of heat (Wertheim). Disintegration of the corpuscles into fine droplets has been observed in various diseases, as in severe malarial fevers. The dark granules of a pigment closely related to hæmatin are derived from the granules arising from the disintegration of the blood-corpuscles, and these particles float in the blood (Melancemia). They are partly absorbed by the colourless corpuscles, but they are also deposited in the spleeu, liver, brain, and bcnemarrow (Arnstein). Sometimes the red corpuscles are abnormally soft, and readily yield to pressure.

The white corpuscles are enormously increased in number in Leukamia (J. H. Bennett and Virchow); sometimes even to the extent of the red corpuscles. In some cases the blood looks as if it were mixed with milk. The colourless corpuscles seem to be formed chiefly in bone-marrow (Neumann), but also in the spleen aud lymphatic glands.

\section{Chemical Constituents of the Red Blood-Corpuscles.}

(1.) The colouring-matter or hæmoglobin (Hb) (Hæmato-globulin, Hæmato-crystallin) is the cause of the red colour of blood; it also occurs 
in muscle, and in traces in the fluid part of blood, but in this last case only as the result of the solution of some red corpuscles. Its percentage composition is :-C $53 \cdot 85, \mathrm{H} 7 \cdot 32, \mathrm{~N} 16 \cdot 17, \mathrm{Fe} \quad 0.42, \mathrm{~S} \quad 0.39$, O 21.84 (dog). Its rational formula is unknown, but Preyer gives the empirical formula $\mathrm{C}_{600}, \mathrm{H}_{960}, \mathrm{~N}_{154}, \mathrm{Fe}, \mathrm{S}_{3}, \mathrm{O}_{179}$. Although it is a colloid substance it crystallises (Hünefeld 1840, Reichert) in all classes of vertebrates, according to the rhombic system, and chiefly in rhombic plates or prisms; in the guinea-pig in rhombic tetrahedra (v. Lang); in the squirrel, however, it yields hexagonal plates. The varying forms, perhaps, correspond to slight differences in the chemical composition in different cases.

Crystals separate from the blood of all classes of vertebrata during the slow evaporation of lake-coloured blood, but with varying facility.

The colouring-matter crystallises very readily from the blood of man, dog, mouse, guinea-pig, rat, cat, hedgehog, horse, rabbit, birds, fishes ; with difficulty from that of the sheep, ox, and pig. Coloured crystals are not obtained from the blood of the frog. More rarely a crystal is formed from a single corpuscle enclosing the stroma. Crystals have been found near the nucleus of the large corpuscles of fishes, and in this class of vertebrates colourless crystals have been observed.
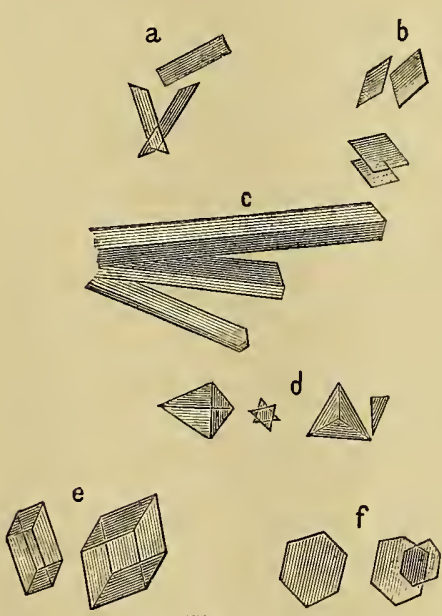

Fig. 8.

Hæmoglobin crystals - a, b, from human blood; c, from the cat; $d$, from the guinea-pig; e, hamster; f, squirrel.
Hæmoglobin crystals are doubly refractive and pleo-chromatic; they are bluish-red with transmitted light, scarlet-red by reflected light. They contain from 3 to 9 per cent. water of crystallisation, and are soluble in water, but more so in dilute alkalies. They are insoluble in alcohol, ether, chloroform, and fats. The solutions are dichroic; red in reflected light, and green in transmitted light.

In the act of crystallisation the hæmoglobin seems to undergo some internal change. Before it crystallises it does not diffuse like a true colloid, and it also rapidly decomposes hydric peroxide. If it be redissolved after crystallisation it diffuses, although only to a small extent, but it no longer decomposes hydric peroxide, and is decolourised by it. A body like an acid is deposited from hæmoglobin at the positive pole of a battery.

\section{Preparation of Hæmoglobin Crystals.}

Method of Rollett.-Place defibrinated blood in a platinum capsule, allow the capsule and the blood to freeze by setting them in a freezing-mixture, and 
then gradually to thaw; pour the lake-coloured blood into a plate, until it forms a stratum not more than $1 \frac{1}{2} \mathrm{~m} . \mathrm{m}$. in thickness, and allow it to evaporate slowly in a cool place, when crystals will separate.

Method of Hoppe-Seyler.-Mix defibrinated blood with ten volumes of a 20 per cent. salt solution, and allow it to stand for two days. Remove the clear upper fluid with a pipette, wash the thick deposit of blood-corpuscles with water, and afterwards shake it for a long time with an equal volume of ether, which dissolves the blood-corpuscles. Remove the ether, filter the lake-coloured blood, add to it of its rolume of cold $\left(0^{\circ}\right)$ alcohol, and allow the mixture to stand in the cold for several days. The numerous crystals can be collected in a filter and pressed between folds of blotting-paper.

Method of Gscheidlen.-Crystals several centimetres in length were obtained by taking defibrinated blood which harl been exposed for twenty-four hours to the air, and keeping it in a closed tube of narrow calibre for several days at $37^{\circ} \mathrm{C}$. When the blood is spread on glass, the crystals form rapidly. [Vaccine tubes answer very well.]

[Method of Stirling and Brito.-It is in many cases sufficient to mix a drol' of blood with a few drops of water on a microscopic slide, and to seal up the preparation. After a few days beantiful crystals are developed. The acdition of water to the blood of some animals, such as the rat and guinea-pig, is rapidly followed by the formation of crystals of hrmoglobin. Very large crystals may be obtained from the stomach of the leech several days after it has sucked blood.]

\section{Quantitative Estimation of Hæmoglobin.}

(a.) From the Amount of Iron.-As dry ( $100^{\circ} \mathrm{C}$.) hremoglobin contains 0.42 per cent. of iron, the amount of iron may be calculated from the amount of hæmoglobin. If $m$ represents the percentage amount of metallic iron, then the percentage of hamoglobin in blool is

$$
=\frac{100 m}{0.42}
$$

The procedure is the following:-Calcine a weighed quantity of blood, and exhaust the ash with $\mathrm{HCl}$ to obtain ferric chloride, which is transformed into ferrous chloride. The solution is then titrated with potassic permanganate.

(b.) Colorimetric Method.-Prepare a dilute watery solution of hæmoglobin crystals of a known strength. With this compare an aqueons dilution of the blood to be investigated, by adding water to it until the colour of the test solution is obtained. Of conrse, the solutions must be compared in vessels with parallel sides and of exactly the same width, so as to give the same thickness of fluid (Hoppe-Seyler). [In the vessel with parallel sicles, or, hamatinometer, the sides are exactly one centimetre apart. Instead of using a standard solntion of oxyhæmoglobin, a solution of picro-carminate of ammonia may be used (Rajewsky, Malassez.) ]

(c.) By the Spectroscope.-Preyer found that a 0.S per cent. watery solution (l c.m. thick), allowed the red, the yellow, and the first strip of green to be seen (Fig. 11, 1). Take the blood to be investigated (about $0.5 \mathrm{c.m}$.), and dilute it with water until it shows exactly the same optical effects in the spectroscope. If $k$ is the percentage of $\mathrm{Hb}$, which allows green to pass through $(0 . \mathrm{S}$ per cent.), $b$, the volume of blood investigated (about $0.5 \mathrm{c.m}$.), $w$, the necessary amount of water added to dilute it, then $x=$ the percentage of $\mathrm{Hb}$ in the blood to be investigated-

$$
x=\frac{k(2 c+b)}{b}
$$


[ (d.) The Hæmoglobinometer of Gowers is used for the clinical estimation of læmoglobin.]

"The tint of the dilution of a given volume of blood with distilled water is taken as the index of the amount of hæmoglobin. The distilled water rapidly dissolves out all the hæmoglobin, as is shown by the fact that the tint of the dilution undergoes no change on standing. The colour of a dilntion of average normal blood one hundred times is taken as the standard. The quantity of hæmoglobin is indicated by the amount of distilled water needed to obtain the tint with the same volume of blood under examination as was taken of the standard. On account of the instability of a standard dilution of blood, tinted glycerine-jelly is employed instead. This is perfectly stable, and by means of carmine and picrocarmine the exact tint of diluted blood can be obtained.

The apparatus consists of two glass tubes of exactly the same size. One contains (D) a standard of the tint of a dilution of 20 cubic m.m. of blood, in 2 cubic centimetres of water (1 in 100).

The second tube (C) is graduated, 100 degrees $=$ two centimetres $(100$ times twenty cubic millimetres).

The twenty cubic millimetres of blood are measured by a capillary pipette (B) (similar to, but larger than that used for the hæmacytometer). This quantity of the blood to be tested is ejected into the bottom of the tube, a few drops of distilled water being first placed in the latter. The mixture is rapidly agitated to prevent the coagulation of the blood. The distilled water is then added drop by drop (from the pipette stopper of a bottle [A] supplied for that purpose) until the tint of the dilution is the same as that of the standard, and the amount of water which has been added (i.e., the degree of dilution) indicates the amount of hæmoglobin.

Since average normal blood yields the tint of the standard at 100 degrees of dilution, the number of degrees of dilution necessary to obtain the same tint with

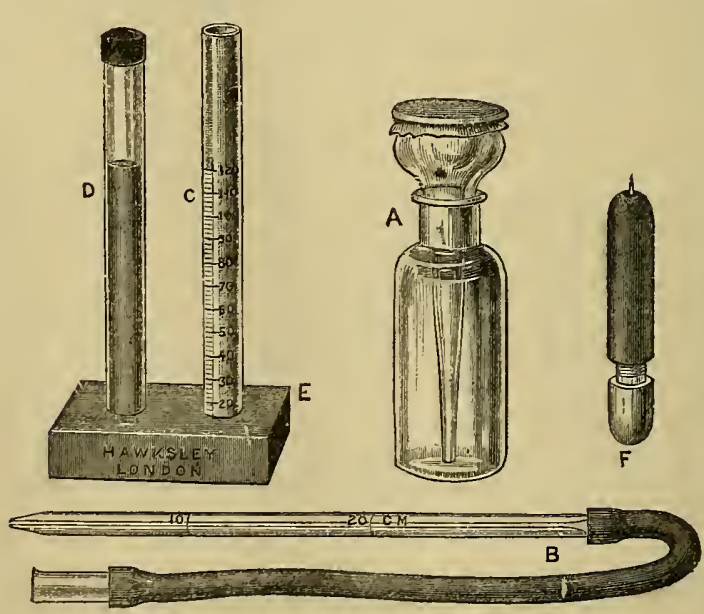

Fig. 9. a given specimen of blood is the percentage proportion of the hæmoglobin contained in it, compared to the normal.

For instance, the 20 cubic millimetres of blood from a patient with anæmia gave the standard tint at 30 degrees of dilution. Hence it contained only 30 per cent. of the normal quantity of hæmoglobin. By ascertaining with the hæmacytometer the corpuscular richness of the blood, we are able to compare the two. A fraction, of which the numerator is the per-

A, pipette bottle for distilled water; $\mathrm{B}$, capillary pipette; C, graduated tube; $\mathrm{D}$, tube with standard dilution; $\mathrm{F}$, lancet for pricking the finger. centage of hæmoglobin, and the denominator the percentage of corpuscles, gives at once

the average value per corpuscle. Thus the blood mentioned above containing 30 per cent. of hæmoglobill, contained 60 per cent. of corpuscles; hence the average 
value of each corpuscle was $\frac{30}{6} \frac{0}{0}$ or $\frac{1}{2}$ of the normal. Variations in the amount of hremoglobin may be recorded on the same chart as that employed for the corpuscles.

In using the instrument, the tint may be estimated by holding the tubes between the eye and the window, or by placing a piece of white paper behind the tubes; the former is perhaps the best. Care must be taken that the tubes are always held in the line of light, not below it. In the latter case some light is reflected from the suspended corpuscles from which the hæmoglobin has been dissolved. If the value of the corpuscles is small, then a perceptibly paler tint is seen when the tubes are held below the line of illumination. If all the light is transmitted directly through the tubes, the corpuscles do not interfere with the tint.

In using the instrument it will be found that, during 6 or $S$ degrees of dilution, it is difficult to distinguish a difference between the tint of the tubes. It is therefore neccssary to note the degree at which the colour of the dilution ceases to be deeper than the standard, and also that at which it is distinctly paler. The degree midway between these two will represent the hæmoglobin percentage.

The instrument is only expected to yield approximate results, ascurate within 2 or 3 per cent. It has, however, been found of much utility in clinical observation."]

The amount of hæmoglobin in man is 12 to 15 per cent., in the woman 12 to 14 per cent., during preguancy 9 to 12 per cent. (Preyer). According to Leichtenstern, $\mathrm{Hb}$ is in greatest amount in the blood of the newly-born infant, but after ten weeks the excess disappears. Between six months and five years, it becomes least in amount, reaches its second highest maximum between twenty-one and forty-five, and then sinks again. From the tenth year onwards the blood of the female is poorer in $\mathrm{Hb}$. The taking of fool causes a temporary decrease of the $\mathrm{Hb}$, owing to the dilution of the blool.

Pathological.-A decrease is observable during recovery from febrile conditions, and also during phthisis, cancer, ulcer of the stomach, cardiac disease, chronic diseases, chlorosis, lenkæmia, pernicious anæmia, and during the rapid mercurial treatment of syphilitic persous.

\section{Use of the Spectroscope.}

As the spectroscope is frequently used in the investigation of blood and other substances of the body, it will be convenient to give a short description of the instrument here (Fig. 10). It consists of-(1.) a tube, A, which has at its peripheral end a slit, S (that can be narrowed or widened). At the other end a collecting lens, $\mathrm{C}$ (called a collimator) is placed, so that its focus is in exact line with the slit. Light (from the sun or a lamp) passes through the slit, and thus goes parallel through $\mathrm{C}$ to-(2.) the prism, $\mathrm{P}$, which decomposes the parallel rays into a coloured spectrum, $r-v$.-(3.) An astronomical telescope is clirected to the spectrum, $r-v$, and the observer, $\mathrm{B}$, with the aid of the telescope, sees the spectrum magnified from six to eight times; - 4 .) a third tube, D, contains a delicate scale, M, on glass, whose image, when illuminated, is reflected from the prism to the eye of the observer, so that he sces the spectrum, and over or above it the scale. To keep out other rays of light the inner ends of the three tubes are covered by metal or by a dark cloth (see also Blood in urine).

[The micro-spectroscope, e.g., that known as the "Sorby-Browning" microspectroscope is very useful when small quantities of a solution are to be examined.] 
[Every spectroscope ought to give two spectra, so that the position of any absorp. tion band may be definitely ascertained. The spectroscope is fitted into the ocular end of the tube of a microscope instead of the eye-piece. Small cells for containing the fluid to be examined are made from short pieces of barometer-tubes cemented to a plate of glass.]

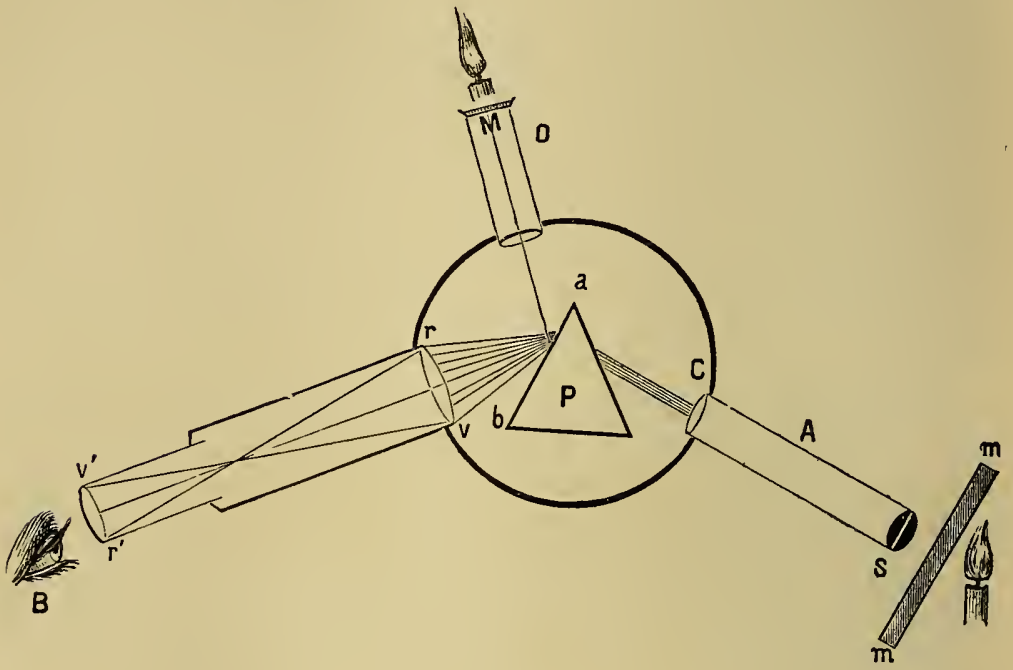

Fig. 10.

Scheme of a spectroscope for observing the spectrum of blood-A, tube; S, slit; $m m$, layer of blood with flame in front of it; $\mathbf{P}$, prism ; $\mathbf{M}$, scale ; $\mathrm{B}$, eye of observer looking through a telescope; $r v$, spectrum.

Absorption Spectra.-If a coloured medium (e.g., a solution of blood) be placed between the slit and a source of light, all the rays of coloured light do not pass through it_-some are absorbed ; many yellow rays are absorbed by blood, hence that part of the spectrum appears dark to the observer. On account of this absorption, such a spectrum is called an "absorption spectrum."

Flame Spectra.-If mineral substances be burned on a platinumwire in a non-luminous flame (Bunsen's burner) in front of the slit, the elements present in the mineral or ash give special coloured band or bands, which have a definite position. Sodium gives a yellow, potassium a red and a violet line. These substances are found in burning the ashes of almost all organs.

If sunlight be allowed to fall upon the slit, the spectrum shows a large number of lines (Fraunhofer's lines) which occupy definite positions in the coloured spectrum. These lines are indicated by the letters A, B, C, D, etc., a, b, c, etc. (Fig. 11). 


\section{Compounds of Hæmoglobin with 0; 0xyhæmoglobin, and Methæmoglobin.}

(1.) Oxyhæmoglobin $\left(\mathrm{O}_{2} \mathrm{Hb}\right)$ behaves as a weak acid, and occurs to the extent of 86.78 to 94.30 per cent. in dry red human corpuscles (Jüdell). It is formed very readily whenever $\mathrm{Hb}$ comes into contact with $\mathrm{O}$ or atmospherie air. 1 gramme $\mathrm{Hb}$ unites with 1.6 to 1.8 cubic centimetres of $\mathrm{O}$ at $0^{\circ}$ and $760 \mathrm{~mm}$. $\mathrm{Hg}$ pressure. Oxyhæmoglobin is a very loose chemical compound, and is slightly less soluble than $\mathrm{Hb}$; its spectrum shows in the yellow and the green, two dark absorption-bands (Hoppe-Seyler) whose length and breadth in a 0.18 per cent. solution are given in Fig. $11(2)$.

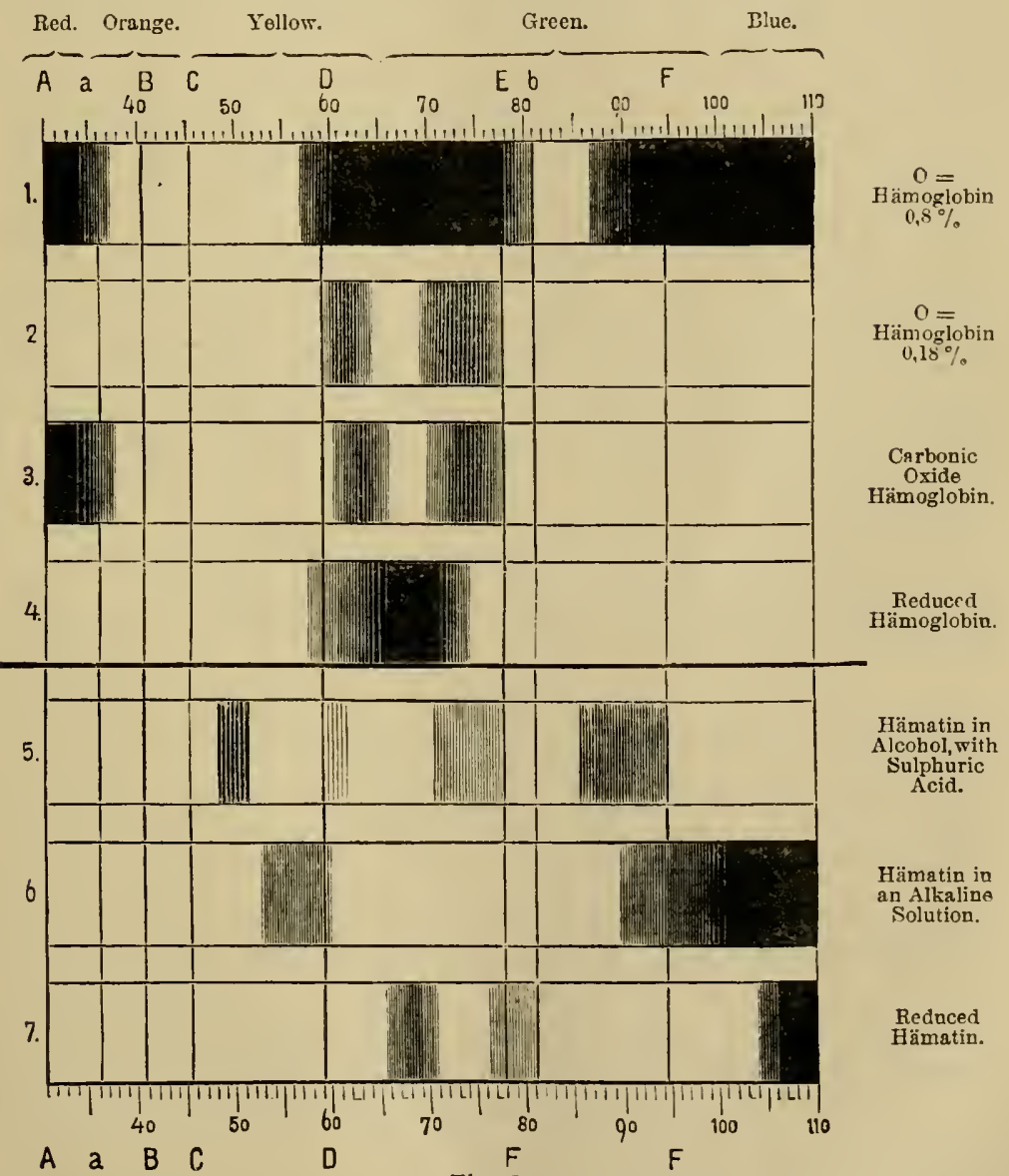

Fig. 11.

Various spectra of hæmoglobin and its compounds. 
[The two absorption-bands lie between the lines $\mathrm{D}$ and $\mathrm{E}$, the band nearer $\mathrm{D}$ being more sharply defined and narrower than the second band, which is wider and less clearly marked-off, and lies nearer E.]

It occurs in the blood-corpuscles, circulating in arteries and capillaries, as was shown by the spectroscopic examination of the ear of a rabbit, of the prepuce and the web of the fingers (Vierordt).

Reduction of 0xyhæmoglobin.-It gives up its $\mathrm{O}$ very readily, however, even when means which set free absorbed gases are used. It is reduced by the removal of the gases by the air-pump, by the conduction through its solution of other gases (CO \& NO), and by heating to the boiling point. In the circulating blood its $O$ is very rapidly given up to the tissues, so that in suffocated animals only reduced hoemoglobin is found in the arteries. Some constituents of the serum and sugar use up $\mathrm{O}$. By adding to a solution of oxyhæmoglobin reducing substances-e.g., ammonium sulphide, ammoniated tartarate of zinc oxide solution, iron filings, or Stokes's fluid [tartaric acid, iron proto-sulphate, and excess of ammonia] - the two absorption bands of the spectrum disappear, and reduced hcemoglobin (gas-free) (Fig. 11, 4), with one absorption band is formed (Stokes, 1864). [The single band which is obtained from reduced hæmoglobin lies between $\mathrm{D}$ and $\mathrm{E}$, and its most deeply shaded portion is opposite the interval between the two bands of oxyhæmoglobin. Its edges are less sharply defined. The colour of the blood changes from a bright red to a brownish tint. Hoppe-Seyler applies the term Hcemoglobin to the reduced substance to distinguish it from oxyhæmoglobin.]

The two bands are reproduced by shaking the reduced hæmoglobin with air, whereby $\mathrm{O}_{2} \mathrm{Hb}$ is again formed. Solutions of oxyhæmoglobin are readily distinguished by their scarlet colour from the purplish tint of reduced hæmoglobin.

If a string be tied round the base of two fingers so as to interrupt the circulation, the spectroscopic examination shows that the oxyhæmoglobin rapidly passes into reduced $\mathrm{Hb}$ (Vierordt). Cold delays this reduction (Filehne).

The spectroscopic examination of small blood-stains is often of the utmost forensic importance. A minimal drop is sufficient. Dissolve in a few drops of distilled water, and place in a thin glass tube in front of the slit of the spectroscope.

(2.) Methæmoglobin (Hoppe-Seyler) contains more O than oxyhæmoglobin (Fig. 11,5). Chemically it is fairly stable, contains $O$, and crystallises (Hüfner and $J$. Ott). It is obtained by acting upon a solution of reduced or oxyhæmoglobin with oxidising reagents; best, however, by adding crystals of potassic ferridcyanide. It shows four absorption bands like an acid solution of hæmatin, that between $\mathbf{C}$ and $\mathrm{D}$ being the only one sharply defined.

If a trace of ammonia be added to such a solution, it gives an alkaline solution of methæmoglobin, which shows two bands like oxyhæmoglobin, of which the first 
one is the broader, and extends more into the red. If ammonium sulphide be added to the methæmoglobin solution, reduced $\mathrm{Hb}$ is formed (Jäderholm). Methæmoglobin is produced in old brown blood-stains, in the crusts of bloody wounds, in blood cysts-farther by the addition of minute traces of acid to blood, or by heating blood with a trace of alkali. Sorby and Jäderholm regard it as a peroxidised hæmoglobin, but this view is opposed by Hoppe-Seyler. It may also be prepared by acting upon blood with potassic chlorate and nitrate, or nitrate of amyl, which gives to blood a chocolate-brown colour (Saarbach, Gamgee).

\section{Carbonic 0xide-Hæmoglobin.}

(3.) CO-Hæmoglobin is a more stable chemical compound than the foregoing, and is produced at once when carbonic oxide is brought into contact with pure $\mathrm{Hb}$ or $\mathrm{O}_{2} \mathrm{Hb}$ (Cl. Bernard, 185i). It has an intensely florid or cherry-red colour, and gives two absorption-bands, very like those of $\mathrm{O}_{2} \mathrm{Hb}$, but they are slightly closer together and lie more towards the violet (Fig. 11,3). Reducing substances (which act upon $\mathrm{HbO}_{2}$ ) do not affect these bands, i.e., they cannot convert the $\mathrm{CO}$ compound into reduced Hb. Another good test to distinguish it from $\mathrm{HbO}_{2}$ is the soda test. If a 10 per cent. solution of caustic soda be added to a solution of $\mathrm{CO}-\mathrm{Hb}$, and heated, it gives a cinnabar-red colour; while, with an $\mathrm{HbO}_{2}$ solution, it gives a dark-brown, greenish, greasy mass (Hoppe-Seyler). Oxidising substances [solutions of potassic permanganate $(0.025$ per cent.), potassic chlorate ( 5 per cent.), and dilute chlorine solution] make solutions of $\mathrm{CO}-\mathrm{Hb}$, cherry-red in colour, while they turn solutions of $\mathrm{HbO}_{2}$ pale yellow. After this treatment both solutions show the absorption-bands of methæmoglobin. If ammonium sulphide be added, $\mathrm{HbO}_{2}$ and $\mathrm{CO}-\mathrm{Hb}$ are re-formed.

On account of its stability $\mathrm{CO}-\mathrm{Hb}$ resists external influences and even putrefaction for a long time (Hoppe-Seyler), and the two bands of the spectrum may be visible after many months. Landois obtained the soda test and spectroscopic bands in the blood of a woman poisoned 18 months previously by $\mathrm{CO}$, and after great putrefaction of the body had taken place.

If $\mathrm{CO}$ is breathed by man, or if air containing it be inspired, it gradually displaces the $\mathrm{O}$, volume for volume, out of the $\mathrm{Hb}$ (L. Meyer), and death soon occurs; $1,000 \mathrm{ccm}$ inspired at once will kill a man. A very small quantity in the air $\left(\frac{1}{400-\frac{1}{1000}}\right)$ suffices, in a relatively short time, to form a large quantity of $\mathrm{CO}-\mathrm{Hb}$ (Gréhant). As continued contact with other gases (such as the passing of $\mathrm{O}$ through it for a very long time) gradually separates the $\mathrm{CO}$ from the $\mathrm{Hb}$ (with the formation of $\mathrm{O}_{2} \mathrm{Hb}$-Donders), it happens that, in very partial poisoning with $\mathrm{CO}$, the blood gradually gets rid of the latter. A high degree of poisoning necessitates the transfusion of blood (p. 61).

[Gamgee and Zuntz also find that although the $\mathrm{CO}-\mathrm{Hb}$ compound is very stable, yet it may be reduced by passing air or neutral gases through it for a lengthened period; it is also reduced when blood is boiled in the mercurial pump.] 


\section{Phenomena of Poisoning by Carbonic 0xide. Other Compounds of Hæmoglobin.}

Carbonic oxide is formed during incomplete combustion of coal or coke, and passes into the air of the room, provided there is not a free outlet for the products of combustion. It occurs to the extent of 12-28 per cent. in ordinary gas, which largely owes its poisonous jroperties to the presence of $\mathrm{CO}$. If the $\mathrm{O}$ be gradually displaced from the blood by the respiration of air containing $\mathrm{CO}$, life can ouly be maintained as Iong as sufficient $\mathrm{O}$ can be obtained from the blood to support the oxidations necessary for life. Death occurs before all the 0 is displaced from the blood. $\mathrm{CO}$ has no effect when directly applied to muscle and nerve. When it is inhaled, there is first stimulation and afterwards paralysis of the nervous system, as shown by the symptoms induced, e.g., violent headache, great restlessness, excitement, increased activity of the heart and respiration, salivation, tremors, and spasms. Later, unconsciousness, weakness, and paralysis occur, laboured respiration, diminished heart-beat, and lastly, complete loss of sensibility, cessation of the respiration and heart-beat, and death. At first the temperature rises several teuths of a degree, but it soon falls $1^{\circ}$ or more. The pulse is also increased at first, but afterwards it becomes very small and frequent.

In poisoning with pure $\mathrm{CO}$ there is no dyspncea, but sometimes muscular spasms occur, the coma not being very marked. There is also temporary but pronounced paralysis of the limbs, followed by violent spasms. After death the heart and brain are congested with intensely florid blood. In poisoning with the vapour of charcoal, where $\mathrm{CO}$ and $\mathrm{CO}_{2}$ both occur, there is a varying degree of coma ; pro. nounced dyspnca, muscular spasms which may last several minutes, gradual paralysis and asphyxia, moniliform contractions and subsequent dilatation of the blood-vessels, with congestion of various organs, occur, accompanied by a fall of the blood-pressure (Klebs), indicating initial stimulation and subsequent paralysis of the vaso-motor centre. This also explains the variations in the temperature and the occasional occurrence of sugar in the urine after poisoning with $\mathrm{CO}$. After death, the blood-vessels are found to be filled with fluid blood of an exquisitely bright cherry-red colour, while all the muscles and viscera and exposed parts of the body (such as the lips) have the same colour. The brain is soft and friable, there are catarrh of the respiratory organs and degeneration of the muscles, and great congestion and degeneration of the liver, kidneys, and spleen. The spots of lividity, post-mortem, are bright red. After recovery from poisoning with $\mathrm{CO}$, there may be paraplegia and (although more rarely) disturbances of the cerebral activity. The poisonous action of the vapours of combustion was known to Aristotle.

(4.) Nitric 0xide-Hæmoglobin (NO-Hb) -is formed when $\mathrm{NO}$ is brought into contact with $\mathrm{Hb}$ (L. Hermann).

As $\mathrm{NO}$ has a great affinity for $\mathrm{O}$, red fumes of nitrogen peroxide $\left(\mathrm{NO}_{2}\right)$ being formed whenever the two gases meet, it is clear that, in order to prepare $\mathrm{NO}-\mathrm{Hb}$, the $\mathrm{O}$ must first be removed. This may be done by passing $\mathrm{H}$ through it, [or ammonia may be added to the blood, and a stream of NO passed through it; the ammonia combines with all the acid formed by the union of the NO with the $\mathrm{O}$ of the blood]. $\mathrm{NO}-\mathrm{Hb}$ is a more stable chemical compound than $\mathrm{CO}-\mathrm{Hb}$, which, as we have seen, is again more stable than $\mathrm{O}_{2} \mathrm{Hb}$. It has a bluish-violet tint, and also gives two absorption-bands in the spectrum similar to those of the other two compounds, but not so intense. These bands are not abolished by the action of reducing agents.

The three compounds of $\mathrm{Hb}$, with $\mathrm{O}, \mathrm{CO}$, and $\mathrm{NO}$, are crystalline; like $\mathrm{Hb}$, they are isomorphous, and their solutions are not dichroic. One 
gramme $\mathrm{Hb}$ unites with $1.33-1.35$ c.c.m. of each of the gases at $0^{\circ}$ and 1 metre pressure (Preyer, L. Hermann).

(5.) Cyanogen, $\mathrm{CNH}$ (Hoppe-Seyler), and acetylene, $\mathrm{C}_{2} \mathrm{H}_{2}$ (Bistrow and Liebreich), form easily decomposable compounds with $\mathrm{Hb}$. The former occurs in poisoning with hydrocyanic acid, and has a spectrum identical with that of $\mathrm{O}_{2} \mathrm{Hb}$, and, like $\mathrm{O}_{2} \mathrm{Hb}$, it is reduced by spccial agents. [The existence of these com. pounds is, however, highly doubtful (Gamgee).]

(6.) If $\mathrm{CO}_{3}$ be passed through a solution of oxylhmoglobin for a considerable time, reduced $\mathrm{Hb}$ is first formed; but if the process be prolonged the $\mathrm{Hb}$ is decomposed, a precipitate of globulin is thrown down, and an absorption-band, similar to that obtained when $\mathrm{Hb}$ is decomposed with acids, is observed (see p. 33).

\section{Decomposition of Hæmoglobin.}

In solution and in the dry state $\mathrm{Hb}$ gradually becomes decomposed, whereby the iron-containing pigment hæmatin, along with certain bye-products, formic, lactic, and butyric acids arc formed.

Hæmoglobin, however, may be decomposed at once into-(1) a body containing iron hamatin, and-(2) a colourless proteid closely related to globulin;-by (a.) the addition of all acids, even by $\mathrm{CO}_{2}$ in the presence of plenty of water; $(b$.$) strong alkalies; (c$.$) all reagents which coagulate$ albumin, and by heat at $70^{\circ}-80^{\circ} \mathrm{C}$. ; $(d$. $)$ by ozone.

(A.) Hæuratin $\left(\mathrm{C}_{6 \mathrm{~S}}, \mathrm{H}_{70}, \mathrm{~N}_{8}, \mathrm{Fe}_{2}, \mathrm{O}_{10}\right)$ forms about 4 per cent. of hæmoglobin (dog). It is insoluble in water, alcohol, and ether; soluble in dilute alkalies and acids, and in acidulated ether and alcohol.

When $H b$ containing $O$ is decomposed, hæmatin is formed at once; while $H b$ free from $O$ on being decomposed forms first a purplish-red body, H.EMOCHROMOGEN $\left(\mathrm{C}_{34}, \mathrm{H}_{36}, \mathrm{~N}_{4}, \mathrm{Fe} \mathrm{O}_{5}\right)$, which contains less $\mathrm{O}$, and is a precursor of hæmatin. In the presence of $\mathrm{O}$ it becomes oxidised, and passes into hæmatin. In solution it gives the spectrum shown in Fig. II, 7 (Hoppe-Seyler). Dilute acids in an alkaline solution deprive hæmochromogen of its iron, and HEMATO-PORPIIYRIN, a substance which remains stable in contact with air, is produced. It may also be produced from hæmatin by the action of acids, so that hæmatin is an oxidation stage of hæmochromogen.

(a) Hcematin in acid solution.-Lecanu extracted it from dry blood-corpuscles by using alcohol containing sulphuric and tartaric acids. If acetic acid be added to a solution of $\mathrm{Hb}$, a mahogany-brown fluid is obtained, containing homatin in acid solution, which gives a spectrum with four absorption-bands in the yellow and green (Fig. 11, 5).

( $\beta$ ) If this solution be treated with excess of ammonia, hamatin in alkatine solution is formed, which gives one absorption-band on the boundary line between red and yellow (Fig. 11, 6).

$(\gamma)$ Reducing agents cause this band to disappear, and produce in the yellow two broad bands, which are due to the presence of "reduced hamatin" (Fig. 11, 7).

(o) When hæmoglobin is extravasated into the subcutaneous tissue, it becomes so altered that ultimately hydrated oxide of iron appears in its place. 


\section{Hæmin and Blood Tests.}

In 1853 Teichmann prepared crystals from blood, which HoppeSeyler showed to be chloride of hcematin or hydrochlorate of hæmatin. The presence of these crystals is used as a test for blood-stains or blood in solution. These crystals of hæmin (Fig. 12) are prepared by adding a small crystal of common salt to dry blood on a glass slide, and then an excess of glacial acetic acid; the whole is gently heated until bubbles of gas are given off. On allowing the preparation to cool, the characteristic hæmin crystals are obtained (Hæmatin, $+2 \mathrm{HCl}$ ).

Characters.-When well-formed, the crystals are small microscopic rhombic plates, or rhombic rods; sometimes they are single - at other times they are aggregated in groups, often crossing each other. Some kinds of blood (ox and pig) yield very irregular, scarcely crystalline, masses. The crystalline forms of hæmin are identical in all the different kinds of blood that have been examined (Jahnke, Högyes). They are doubly refractive and pleo-chromatic; by transmitted light they are mahoganybrown, and by reflected light bluish-black, glancing like steel. They give a brown streak on porcelain.

(1.) Preparation from Dry Blood-Stains.-Place a few particles of the blood-stain on a glass slide, add 2 to 3 drops of glacial acetic acid and a small crystal of common salt; cover with a cover-glass, and heat gently over the flame of a spirit-lamp until bubbles of gas are given off. On cooling; the crystals appear in the preparation (Fig. 13).

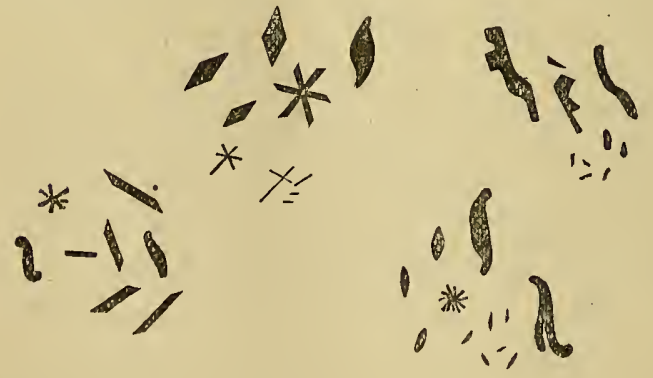

Fig. 12.

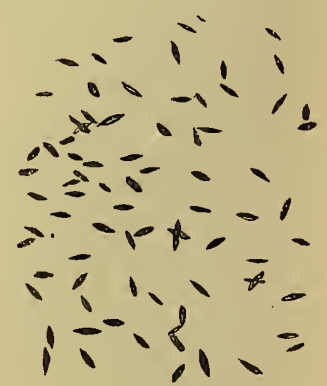

Fig. 13.

Hæmin Crystals of various forms.

Hæmin Crystals prepared from traces of blood.

(2.) From Stains on Porous Bodies.-The stained object (cloth, wood, blotting-paper, earth) is extracted with a small quantity of dilute caustic potash, and afterwards with water in a watch-glass. Both solutions are carefully filtered, and tannic acid and glacial acetic acid are added until an acid reaction is obtained. The dark precipitate which is formed is collected on a filter and washed. A small part of 
it is placed on a microscope slide, a granule of common salt is added, and the whole dried; the dry stain is treated as in (1.) (Struwe).

(3.) From Fluid Blood.-Dry the blood slowly at a low temperature, and proceerl as in (1.)

(4.) From very Dilute Solutions of Hæmoglobin.-(a.) Struwe's Method-Add to the fluid, ammonia, tannic acid, and afterwards glacial acetic acid, until it is acid; soon a black precipitate of tannate of hæmatin is thrown down. This is isolated, washed, dried, and treated as in (1.), but instead of $\mathrm{NaCl}$ a granule of ammonium chloride is added.

(b.) Guning and van Geuns recommend the addition of zinc acetate, which gives a reddish precipitate; this precipitate is to be treated as in (1.)

Hæmin crystals may sometimes be prepared from putrefying or lake-coloured blood, but they are very small, and here the test often fails. When mixed with iron-rust, as on iron-weapons, the bloodcrystals are generally not formed. In such cases, scrape off the stains and boil them with dilute caustic potash. If blood be present, the dissolved hæmativ forms a fluid, which in a thin layer is green; in a thick layer red (H. Rose).

Chemical Characters. - Hæmin crystals have been prepared from all classes of vertebrates and from the blood of the earth-worm.

They are insoluble in water, alcohol, ether, chloroform; but $\mathrm{H}_{2} \mathrm{SO}_{4}$ dissolves them, expelling the $\mathrm{HCl}$, and giving a violet-red colour. Ammonia also dissolves them, and if the resulting solution be evaporated, heated to $130^{\circ} \mathrm{C}$, and treated with boiling water (which extracts the ammonium chloride), pure hoematin is obtained (Hoppe-Seyler) as a bluish-black substance, which on being pounded forms a brown and amorphous powder. Its solutions in caustic alkalies are dichroic; in reflected light, brownish-red; in transmitted light, in a thick stratum, red-in a thin one, olive-green. The acid solutions are monochromatic and brown.

An alcoholic solution of hæmatin, when reduced by tin and hydrochloric acid, yields urobilin (Hoppe-Seyler), (compare Bile).

\section{Hæmatoidin.}

Virchow discovered this important derivative from hæmoglobin. It occurs in the body wherever blood stagnates outside the circulation, and becomes decomposed-as when blood is extravasated into the tissues -e.g., the brain-in solidified hlood-plugs (thrombus); invariably in the Graafian follicles. It contains no iron $\left(\mathrm{C}_{32}, \mathrm{H}_{36}, \mathrm{~N}_{4}, \mathrm{O}_{6}\right)$, and crystallises in clinorhombic prisms (Fig. 14) of a yellowish-brown colour. It is soluble in warm alkalies, carbon disulphide, benzol, and chloroform. Very probably it is identical with one of the bile pigments-bili-rubin (Valenteiner). [When acted upon by impure nitric acid (Gmelin's reaction), it gives the same play of colours as bile.]

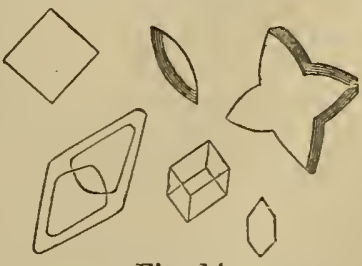

Fig. 14.

Hæmatoidin Crystals. 
In cases where a large amount of blood has undergone solution within the blood-vessels (as by injecting foreign blood), hæmatoidin crystals have been found in the urine (v. Recklinghausen, Landois).

\section{1. (B.) The Colourless Proteid of Hæmoglobin.}

It is closely related to globulin; but, while the latter is precipitated by all acids, even by $\mathrm{CO}_{2}$, and re-dissolved on passing $\mathrm{O}$ through it, the proteid of hæmoglobin, on the other hand, is not dissolved after precipitation on passing through it a stream of $\mathrm{O}$.

As crystals of hæmoglobin can be decolourised under special circumstances, it is probable that these owe their crystalline form to the proteid which they contain. Landois placed crystals of hæmoglobin along with alcohol in a dialyser, putting ether acidulated with sulphuric acid outside, and thereby obtained colourless crystals. [If frog's blood be sealed up on a microscopic slide, along with a few drops of water for several days, long colourless acicular crystals are developed in it (Stirling and Brito).]

\section{II. Proteids of the Stroma.}

Dry red human blood-corpuscles contain from $5 \cdot 10-12 \cdot 24$ per cent. of these proteids, but little is known about them (Jüdell). One of them is globulin, which is combined with a body resembling nuclein (Wooldridge), and traces of a diastatic ferment (v. Wittich). The stroma tends to form masses which resemble fibrin (Landois).

L. Brunton found a body resembling mucin in the nuclei of red blood-corpuscles, and Miescher detected nuclein.

\section{The Other Constituents of Red Blood-Corpuscles.}

III. Lecithin (0.35-0.72 per cent.) in dry blood-corpuscles (Jüdell), and also in brain, yelk, and seminal fluid.

It is regarcled as a glycero-phosphate of neurin, in which, in the radical of glycero-phosphoric acid, two atoms of $\mathrm{H}$ are replaced by two of the radical of stearic acid. By gentle heat glycero-phosphoric acid is split up into glycerine and phosphoric acid.

\section{Cholesterin (0.25 per cent.);-no Fats.}

These substances are obtained by extracting stromata or blood itself with ether. When the ether evaporates, the characteristic globular forms ("myelin-forms") of lecithin and crystals of cholesterin are recognised. The amount of lecithin may be determined from the amount of phosphorus in the ethereal extract.

IV. Water (681.63 per 1,000-C. Schmidt).

V. SALTS $(7 \cdot 28$ per $1,000,-$ C. Schmidt $)$, chiefly compounds of 
potash and phosphoric acid; the phosphoric acid is derived only from the burned lecithin; while the greater part of the sulphuric acid in the analysis is derived from the burning of the hrmoglobin.

Analysis of Blood.-1,000 parts, by weight, of HORSE's BLOoD contain : -

$344 \cdot 18$ blood-corpuscles (containing about 128 per cent. of solids).

655.52 plasma (containing about 10 per cent. of solids).

1,000 parts, by weight, of yOIST BLOOD-CORPUSCLES contain :-

Solids, $367 \cdot 9$ (pig); $400 \cdot 1$ (ox).

Water, 632.1 ,, $599 \cdot 9$,

The solids are :-

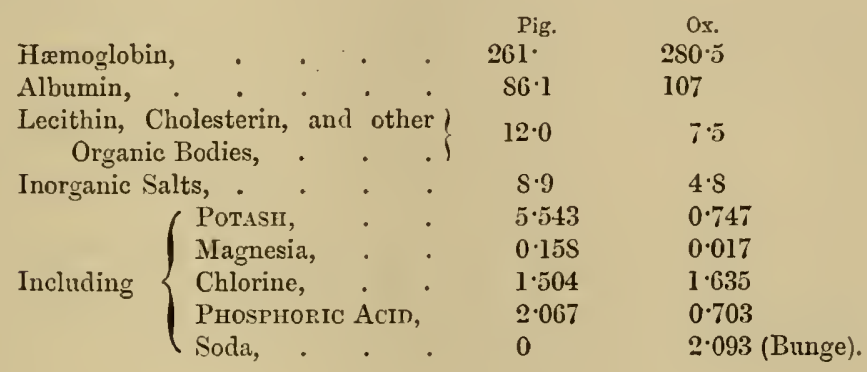

\section{Chemical Composition of the Colourless Corpuscles.}

Investigations have been made on pus cells, which closely resemble colourless blood-corpuscles. They contain scveral proteids; alkali albuminate, a proteid which coagulates at $48^{\circ} \mathrm{C}$., and another resembling myosin, fibrino-plastin, and a coagulating ferment; nuclein in the nuclei (Miescher); perhaps also glycogen (Salomon), lecithin, and extractives.

100 parts, by weight, of dry PEs contain :-

Earthy Phosphates, . . $0.416 \mid$ Potash, . . . . . 0.201 Sodic Phosphate, . . $0.606 \mid$ Sodic Chloride, . . . 0.143

\section{Blood-Plasma and its Relation to Serum.}

The unaltered fluid in which the blood-corpuscles float is called plasma, or liquor sanguinis. This fluid, however, after blood is withdrawn from the vessels, rapidly undergoes a change, owing to the formation of a solid fibrous substance, FIBRIN, which seems to be produced by the coming together of three special substances, the so-called fibrin-factors. After this occurs, the new fluid which remains no longer coagulates spontaneously (it is plasna, minus the fibrin-factors), and is called serum. Apart from 
the presence of the fibrin-factors, the chemical composition of plasma and serum is the same.

[When blood coagulates, the following rearrangement of its elements takes place :-

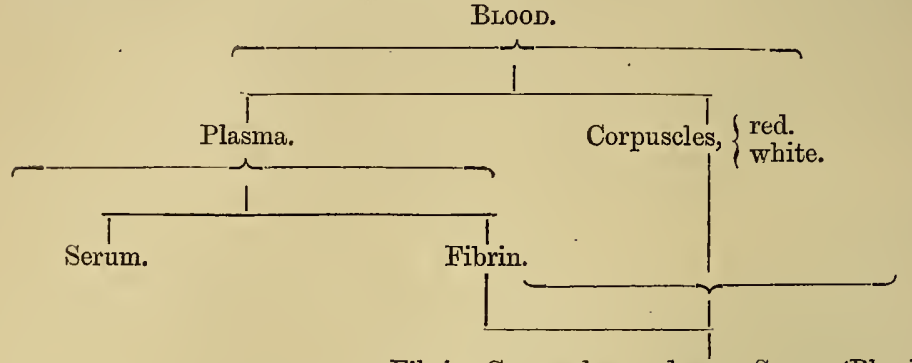

Fibrin, Corpuscles, and some Serum (Blood Clot).]

The serum, however, still contains a portion of the fibrin-ferment, and also some of the fibrino-plastin or fibrino-plastic substance. Plasma is a clear, transparent, slightly thickish fluid, which, in most animals (rabbit, ox, cat, dog), is almost colourless; in man it is yellow, and in the horse citron-yellow.

\section{Preparation of Plasma.}

(A.) Without Admixture.-Taking advantage of the fact that plasma, when cooled to $0^{\circ}$ outside the body, does not coagulate for a considerable time, Brücke prepares the plasma thus:- Selecting the blood of the horse (because it coagulates slowly, and its corpuscles sink rapidly to the bottom), he receives it, as it flows from an artery, in a tall narrow glass, placed in a freezing-mixture, and cooled to $0^{\circ}$. The blood remains fluid, and, the coloured corpuscles subsiding in a few hours, the plasma remains above as a clear layer, which can be removed with a cooled pipette. If this plasma be then passed through a cooled filter, it is robbed of all its colourless corpuscles.

[Burdon-Sanderson uses a vessel consisting of three compartments - the outer and inner contain ice, while the blood of the horse is caught in the central compartment, which does not exceed half-an-inch in diameter.]

The quantity of plasma may be roughly (but only roughly) estimated by using a tall, graduated measuring-glass. If the plasma be warmed, it soon coagulates (owing to the formation of fibrin), and passes into a trembling jelly. If, however, it be beaten with a glass-rod, the fibrin is obtained as a white stringy mass, adhering to the rod. The quantity of fibrin in a given volume of plasma is about $0.7-1$ per cent., although it varies much in different cases. 
(B.) With Admixture.-Blood flowing from an artery is caught in a tall graduated measure containing $\frac{1}{7}$ th of its volume of a concentrated solution of sodic sulphate (Hewson) - or in a 25 per cent. solution of magnesic sulphate (1 vol. to 4 vols. blood: Semmer) -or 1 vol. blood with 2 vols. of a 4 per cent. solution of monophosphate of potash (Masia). When the blood is mixed with these fluids and put in a cool place, the corpuscles subside, and the clear stratum of plasma mixed with the salts may be removed with a pipette. If the salts be removed by dialysis, coagulation occurs; or it may be caused by the addition of water (Joh. Müller). Blood which is mixed with a 4 per cent. solution of common salt does not coagulate, so that it also may be used for the preparation of plasma. [For frog's blood Johannes Müller used a $\frac{1}{2}$ per cent: solution of cane sugar, which permits the corpuscles to be separated from the plasma by filtration. The plasma mixed with the sugar coagulates in a short time.]

\section{Fibrin-Coagulation of the Blood.}

General Characters.-Fibrin is that substance which, becoming solid in shed blood, in plasma and in lymph causes coagulation. In these fluids, when left to themselves, fibrin is formed, consisting of innumerable, excessively delicate, closely-packed, microscopic, doubly refractive (Hermann) fibrils (Fig. 6, E). These fibrils entangle the blood-corpuscles as in a spider's web, and form with them a jelly-like, solid mass called the BLOOD-CLOT (placenta sanguinis). At first the clot is very soft, and after the first 2 to 15 minutes a few fibres may be found on its surface; these may be removed with a needle, while the interior of the clot is still fluid. The fibres ultimately extend throughout the entire mass, which, in this stage, has been called cruor. After from 12 to 15 hours the fibrin contracts, or, at least, shrinks more and more closely around the corpuscles, and a fairly solid, trembling, jelly-like clot, which can be cut with a knife, is formed. During this time the clot has expressed from its substance a fluid-the BLOOD-SERUM. The clot takes the shape of the vessel in which the blood coagulates. Fibrin may be obtained by washing away the corpuscles from the clot with a stream of water.

Crusta Phlogistica.-If the corpuscles subside very rapidly, and if the blood coagulates slowly, the upper stratum of the clot is not red, but only yellowish, on account of the absence of coloured corpuscles. This is regularly the case in horse's blood, and in human blood it is observed especially in inflammations; hence this layer has been called crusta phlogistica. Such blood contains more fibrin, and so coagulates more slowly. 
The crusta is formed under other circumstances, but the cause of its formation is not always clear-e.g., with increased S.G. of the corpuscles, or diminished S.G. of the plasma (as in hydræmia and chlorosis), whereby the corpuscles sink more rapidly, and also during pregnancy. The taller and narrower the glass, the thicker is the crusta (compare $\S 41$ ). The upper end of the clot, where there are few corpuscles, shrinks more, and is therefore smaller than the rest of the clot. This upper, lighter-coloured layer is called the "buffy" coat; this, however, gradually passes both as to size and colour into the normal dark-coloured clot. [Sometimes the upper surface of the clot is concave or cupped. The older physicians used to attribute great importance to this condition, and also to the occurrence of the crusta phlogistica, or buffy coat.]

Defibrinated Blood.-If freshly-shed blood be beaten or whipped with a glass-rod or with a bundle of twigs, fibrin is deposited on the rod or twigs in the form of a solid, tibrous, yellowish-white, elastic mass, and the blood which remains is called "defibrinated blood." [The twigs and fibrin must be washed in a stream of water to remove adhering corpuscles.]

Coagulation of Plasma.-Plasma shows phenomena exactly analogous, save that there is no well-defined clot, owing to the absence of the resisting corpuscles; there is, however, always a soft, trembling jelly formed, when plasma coagulates.

Properties of Fibrin.-Although the fibrin appears voluminous, it only occurs to the extent of 0.2 per cent. $(0.1$ to 0.3 per cent.) in the blood. The amount varies considerably in two samples of the same blood (Sig. Mayer). It is insoluble in water and ether; alcohol shrivels it by extracting water; dilute hydrochloric acid $(0 \cdot 1$ per cent.) causes it to swell up and become clear, and changes it into syntonin or acidalbumin. When fresh, it has a grayish-yellow fibrous appearance, and is elastic ; when dried, it is horny, transparent, brittle, and friable.

When fresh it dissolves in 6 to 8 per cent. solutions of sodium nitrate or sulphate, in dilute alkalies, and in ammonia-thus forming alkali-albuminate. Heat does not coagulate these solutions. If, however, to a solution of fibrin in 0.05 per cent. soda solution, there be added acids, or (the faintly alkaline) lactate, formate, butyrate, acetate, or valerianate of ammonia or soda, coagulation occurs (Deutschmann). Hydric peroxide is rapidly decomposed by fibrin (Thénard).

According to H. Nasse, the first appearance of a coagulum occurs in man's blood after $3 \mathrm{~min} .45 \mathrm{sec}$, in womau's blood after $2 \mathrm{~min} .20 \mathrm{sec}$. Age has no effect; withdrawal of food accelerates coagulation (H. Vierordt).

\section{General Phenomena of Coagulation.}

\section{Blood which is in direct contact with the living and unaltered} blood-vessels does not coagulate (Brïcke, 1857). This important fact was proved by Brïcke, who filled the heart of a tortoise with blood which had stood 15 minutes exposed to the air at $0^{\circ}$, and kept it in a moist chamber. The blood was still fluid at the end of $5 \frac{1}{2}$ hours, while the 
heart itself still continued to beat. He observed that at $0^{\circ}$ the blood was uncoagulated in the contracting heart of a tortoise after eight days. Blood inside a contracting frog's heart preserved under mercury does not coagulate. If the wall of the vessel be altered by pathological processes (e.g., if the intima becomes rough and uneven, or undergoes inflammatory change) coagulation is apt to occur at these places. Blood rapidly coagulates in a dead heart, or in blood-vessels (but not in capillaries) or other canals (e.g., the ureter) (Virchow).

If blood stagnates in a living vessel, coagulation begins in the central axis, because here there is no contact with the wall of the living bloodvessel. This influence of the wall of blood-vesscls was, to some extent, known to Thackrah (1819) and to Sir Astley Cooper.

II. Conditions which Hinder or Delay Coagulation.-(a.) The addition of small quantities of alkalies and ammonia, or of concentrated solutions of neutral salts of the alkalies and earths (alkaline chlorides, sulphates, phosphates, nitrates, carbonates). Magnesic sulphate acts most favourably in delaying coagulation ( 1 vol. solution of 28 per cent. to $3 \frac{1}{2}$ vol. blood of the horse).

(b.) The precipitation of the fibrinoplastin by arding weak acids, or by $\mathrm{CO}_{2}$.

By the addition of acelic acid until the reaction is acid, the coagulation is completely arrested. A large amount of $\mathrm{CO}_{2}$ delays it, and hence venous blood coagulates more slowly than arterial. Hence, also, the blood of suffocated persons remains fluid.

(c.) The addition of egg-albumin, syrup, glycerine, and much water. If uncoagulated blood be brought into contact with a layer of alreadyformed fibrin, coagulation occurs later.

(d.) By cold at $0^{\circ}$ coagulation may be delayed for one hour (J. Davy.) If blood is frozen at once, after thawing, it is still fluid, and then coagulates (Hewson). When shed blood is under high pressure it coagulates slowly (Landois).

(e.) Blood of embryo-fowls does not coagulate before the 12th or 14th day of incubation (Boll); that of the hepatic vein very slightly; menstrual blood shows little tendency to coagulate when alkaline mucus from the vagina is mixed with it. If it be rapidly discharged, it coagulates in masses.

$(f$.$) Blood rich in fibrin from inflamed parts coagulates slowly. In "bleeders"$ (hæmophilia), coagulation seems not to take place, owing to a want of the substances producing fibrin; hence, in these cases, wounds of vessels are not plugged with fibrin. Albertoni observed that if tryptic pancreas ferment (dissolved in glycerine), be injected into the blood of an animal, blood does not coagulate. Schmidt-Mülheim found that after the injection of pure peptone into the blood $(0 \cdot 3$ to 0.6 grammes per kilo.) of a dog, the blood lost its power of coagulating. A substance is formed in the plasma, which prevents coagulation, but which is 
precipitated by $\mathrm{CO}_{2}$. Lymph behaves similarly (Fano). After peptones are injected, there is a great solution of leucocytes in the blood (v. Samson-Himmelstjerna).

\section{Coagulation is Accelerated-(a.) By Contact with Foreign} Substances of all kinds; hence, threads or needles introduced into arteries are rapidly covered with fibrin. Even the introduction of air-bubbles into the circulation accelerates it, and the pathologically altered wall of a vessel acts like a foreign body. Blood shed from an artery rapidly coagulates on the walls of vessels, on the surfaces exposed freely to air, and on the rods or twigs by which it is beat. The passage through it of indifferent gases, such as N. and $\mathrm{H}_{\text {., and }}$ the addition of $\mathrm{H}_{2} \mathrm{O}$ have the same effect.

(b.) Heating from $39^{\circ}$ to $55^{\circ} \mathrm{C}$, rapidly facilitates coagulation (Hewson).

(c.) Agitation of the blood, as shown by Hewson and Hunter.

IV. Rapidity of Coagulation.-Amongst vertebrates, the blood of birds (especially of the pigeon), coagulates almost momentarily; in cold-blooded animals, coagulation occurs much more slowly, while mammals stand midway between the two. [The blood of a fowl begins to coagulate in a-half to one and a-half minute; that of a pig, sheep, rabbit, in a-half to one and a-half minute; of a $\operatorname{dog}$, one to three minutes; of a horse and ox, five to thirteen minutes; of man, three to four minutes; solidification is completed in nine to eleven minutes, but rather sooner in the case of women (Nasse)]. The blood of invertebrates, which is usually colourless, forms a soft whitish clot of fibrin. Even in lymph and chyle, a small soft clot is formed.

V. When coagulation occurs, the aggregate condition of the fibrinfactors is altered, so that heat must be set free (Valentin, 1884, Schiffer, Lépine). The rise in the temperature may be ascertained with a very delicate thermometer.

VI. In blood shed from an artery, the degree of alkalinity diminishes from the time of its being shed until coagulation is completed (Pflïger and Zuntz). This is probably due to a decomposition in the blood, whereby an acid is developed, which diminishes the alkalinity (p.2).

VII. Whether or not electricity is developed, is not positively proved. Hermann supposes that the parts already coagulated are negative, while non-coagulated parts are positive; but this has not been clearly shown.

VIII. During coagulation there is a diminution of the $\mathrm{O}$ in the blood, although a similar decrease also occurs in non-coagulated blood. Traces of ammonia are also given off, which Richardson erroneously supposed to be the cause of the coagulation of the blood. [This is refuted-(1.) by the fact that blood, when collected under mercury (whereby no escape of ammonia is possible), also coagulates; and (2.) by the follow- 
ing experiment of Lister:- $\mathrm{He}$ placed two ligatures on a vein containing blood, moistening one-half of the outer surface of the vein with ammonia, and leaving the other half intact. The blood coagulated in the first half, and not in the other, owing to the properties of the wall of the vein of the former being altered. Lister also proved that blood will remain fluid for hours in a vein after it has been freely exposed to the air, and even after it has been poured in a thin stream from one vein to another.] Neither the decrease of $\mathrm{O}$ nor the evolution of ammonia seems to have any causal connection with the formation of fibrin.

\section{Cause of the Coagulation of the Blood.}

Alexander Schmidt stated that fibrin is formed by the coming together of two proteid substances which occur dissolved in the plasma or liquor sanguinis, viz.:-(1.) Fibrinogen, i.e., the substance which yields the chief mass of the fibrin, and (2.) Fibrinoplastic substance or fibrinoplastin. In order to determine the coagulation a ferment seems to be necessary, and this is supplied by (3.) the fibrin-ferment.

[The serous sacs of the body contain a fluid which in some respects closely resembles lymph. The pericardium contains pericardial fluid, which in some animals coagulates spontancously (e.g., in the rabbit, ox, horse, and sheep), if the fluid be removed immediately after death. If this be not done till several hours after death, the fluid does not coagulate spontaneously. The fluid of the tunica vaginalis of the testis, again, sometimes accunulates to a great extent, and constitutes hydrocele, but this fluid shows no tendency to coagulate spontaneously. Andrew Buchanan found, however, that if to the fluid of ascites, to pleuritic fluid, or to hydrocele fluid, there be added clear blood-serum, then coagulation takes place, i.e., two fluids-neither of which shows any tendency by itself to coagulate-form a clot when they are mixed. He also found that if "washed blood clot" (which consists of a mixture of fibrin and colourless corpuscles) be added to hydrocele fluid, coagulation occurred. Denis mixed uncoagulated blood with a saturated solution of sodic sulphate, allowed the corpuscles to subside, and decanted the clear fluid which was mixed with sodic chloride, until a large amount of precipitate had been obtained. The precipitate, when washed with a saturated solution of sodic chloride, he called plasmine. If plasmine be mixed with water, it coagulates spontaneously, resulting in the formation of fibrin, while another proteid remains in solution. According to the view of Denis, fibrin is produced by the splitting up of plasmine into two bodics-fibrin and an insoluble proteid.]

[Researches of A. Schmidt. - This observer rediscovered the chief facts already known to Buchanan, viz., that some fluids which do not coagulate spontaneously, clot when mixed with other fluids, which also show no tendency to coagulate spontaneously, e.g., hydrocele fluid and blood-serum. He proceeded to isolate from these fluids the bodies which are described as fibrinogen and fibrinoplastin. The bodies so obtained were not pure, but Schmidt supposed that the formation of fibrin was due to the interaction of these two proteids. The reason why hydrocele fluid did not coagulate, he said, was that it contained fibrinogen and no fibrinoplastin, while blood-serum contained the latter, but not 
the former. Schmidt afterwards discovered that these two substances may be present in a fluid, and yet that coagulation may not occur (e.g., occasionally in hydrocele fluid). He supposed, therefore, that blood or blood-serum contained some other constituent necessary for coagulation. This he afterwards isolated in an impure condition and called fibrin-ferment (Gamgee). ]

Properties of these Substances.-Fibrinogen and fibrinoplastin are not distinguished from each other by well-marked chemical characters. Still they differ as follows:-

(a.) Fibrinoplastin is more easily precipitated from its solutions than fibrinogen.

(b.) It is more readily redissolved when once it is precipitated.

(c.) It forms when precipitated a very light granular powder.

(d.) Fibrinogen adlieres as a sticky deposit to the side of the vessel. It coagulates at $56^{\circ} \mathrm{C}$.

Both substances closely resemble globulin in their chemical composition (Kühne called fibrinoplastin paraglobulin), and in their reactions they are not unlike myosin. Like all globulins, they require a trace of common salt for their solution.

On account of their great similarity, both substances are not usually prepared from blood-plasma. Fibrinogen is prepared from serous transudations (pericardial, abdominal, or pleuritic fluid, or the fluid of hydrocele), which contain no fibrinoplastin. Fibrinoplastin is most readily prepared from serum, in which there is still plenty of fibrinoplastin, but no fibrinogen.

Preparation of Fibrinoplastin.-(a.) Dilute blood-serum with twelve times its volume of ice-cold water, and almost neutralise it with acetic acid, [add 4 drops of a 25 per cent. solution of acetic acid to every 120 c.c. of diluted serum]; or (b.) pass a stream of carbonic acid through the diluted serum, which soon becomes turbid; and after a time a fine white powder, copious and granular, is precipitated (Schmidt, 1862).

[(c.) The serum may be dialysed for a day; at the end of this time the conterits of the dialyser have become turbid, and when a current of $\mathrm{CO}_{2}$ is passed through them, a precipitate of fibrinoplastin is obtained. Schmidt's fibrinoplastin has also been called SERUM-GIOBULIN (Hammarsten) or PARAGLOBULIN (Kühne).]

Schmidt found that 100 c.c. of the serum of ox blood yielded 0.7 to 0.8 grms.; horse serum, 0.3 to 0.56 grms. of dry fibrinoplastin. Fibrinoplastin occurs not only in serum, but also in red blood-corpuscles, in the fluids of connective tissue, and in the juices of the cornea.

$[(d$.$) Method of Hammarsten.-All the fibrinoplastin in serum is$ not precipitated either by adding acetic acid or by $\mathrm{CO}_{2}$. Hammarsten found, however, that if crystals of magnesium sulphate be added to complete saturation, it precipitates the whole of the serum-globulin, but does not precipitate serum-albumin (Gamgee); it seems that in 
the ox and horse serum-globulin is more abundant than scrum-albunin, while in the dog and rabbit the reverse obtains.]

Preparation of Fibrinogen.-This is best prepared from hydrocele fluid, although it may also be obtained from the fluids of serous cavities-e.g., the pleura, pericardium, or peritoneum. It does not exist in blood-serum, althongh it does exist in blood-plasma, lymph, and chyle, from which it may be obtained by a stream of $\mathrm{CO}_{2}$, after the paraglobulin is precipitated. (a.) Dilute hydrocele fluid with ten to fifteen times its volume of water, and pass a stream of $\mathrm{CO}_{2}$ through it; or $(b$.) carefnlly neutralise it by adding acetic acid. (c.) Add powdered common salt to saturation to a serous transudation, when a sticky glutinous (not very abundant) precipitate of fibrinogen is obtained.

[Hammarsten and Eichwald find that, although paraglobulin and fibrinogen are soluble in solutions of common salt (containing 5 to 8 per cent. of the salt), a saline solution of 12 to 16 per cent. is required to precipitate the fibrinogen, leaving still in solution paraglobulin, which is not precipitated until the amount of salt exceeds 20 per cent. (Gamgee).]

Hammarsten found that it may be prepared from blood (of the horse) by first precipitating all the serum-globulin or fibrinoplastin with crystals of magnesium sulphate, and subsequent filtration, which removes the corpuscles; a clear salted plasma is thus obtained. If to the filtrate a saturated solution of common salt be added, a turbid, flaky, inpure precipitate of fibrinogen is obtained. This may be dissolved in dilute common salt, and again precipitated by a saturated solution of $\mathrm{NaCl}$.

Properties of the Fibrin-Factors. -They are insoluble in pure water, but dissolve in water containing $O$ in solution. Both are soluble in very dilute alkalies-e.g., caustic soda, and are precipitated from this solution by $\mathrm{CO}_{2}$. They are soluble in dilute common salt-like all globulins-but if a certain amount of comımon salt be added in excess they are precipitated. Very dilute hydrochloric acid dissolves them, but after several hours they become changed into a body resembling syntonin or acid-albumin.

Fibrinogen dissolved in a weak solution of common salt ( 1 to 5 per cent.) is re-precipitated on adding water, so that it resembles fibrin. Its solution in common salt coagulates at $52^{\circ}$ to $55^{\circ} \mathrm{C}$. (Hammarsten, Frédéricq).

[Frédéricq finds that fibrinogen exists as such in the plasma, it coagulates at $56^{\circ} \mathrm{C}$, and the plasma thereafter is uncoagulable (Gamgee).]

Preparation of the Fibrin-Ferment.-Mix blood-serum (ox) with twenty times its volume of strong alcohol, and filter off the deposit thereby produced after one month. The deposit on the filter consists of albumin and the ferment; dry it carefully over sulphuric acid, and reduce to a powder. Triturate 1 gramme of the powder with 65 c.c.m. of water for ten minutes, and filter. The ferment is dissolved by the 
water, and passes through the filter, while the coagulated albumin remains behind (Schmidt).

In the preparation of fibrinoplastin, the ferment is carried down with it mechanically. The ferment seems to be formed first in fluids outside the body, very probably by the solution of the colourless corpuscles. More ferment is formed in the blood the longer the interval between its being shed and its coagulation. It is destroyed at $80^{\circ} \mathrm{C}$.

[Gamgee's Method.-Buchanan's "washed blood-clot" (p. 43) is digested in an 8 per cent. solntion of common salt. The solution so obtained possesses in an intense degree the properties of Schmidt's fibrin-ferment.]

Coagulation Experiments.-According to A. Schmidt, if the pure solutions of (1) fibrinogen, (2) fibrinoplastin, and (3) fibrin-ferment be mixed, fibrin is formed. The process goes on best at the temperature of the body; it is delayed at $0^{\circ}$; and the ferment is destroyed at the boiling point. The presence of $\mathrm{O}$ seems necessary for coagulation. The amount of ferment appears to be immaterial; large quantities produce more rapid coagulation, but the amount of fibrin formed is not greater.

The amount of salts present has a remarkable relation to coagulation. Solutions of the fibrin-factors deprived of salts, and redissolved in very dilute caustic soda, when mixed, do not coagulate until sufficient $\mathrm{NaCl}$ be added to make a 1 per cent. solution of this salt (Schmidt).

When blood or blood-plasma coagulates, all the fibrinogen is used up, so that the serum contains only fibrinoplastin and fibrin-ferment; hence, the addition of hydrocele fluid (which contains fibrinogen) to serum causes coagulation.

According to Hammarsten, fibrin is formed when the ferment is added to a solution of fibrinogen.

[Hammarsten's Theory of Coagulation.-Hammarsten's researches lead him to believe that fibrinoplastin is quite unnecessary for coagulation. According to him, fibrin is formed from one body, viz., fibrinogen, which is present in plasma when it is acted upon by the fibrin-ferment; the latter, however, has not been obtained in a pure state. Neither he nor Schmidt asserts that this body is of the nature of a ferment, although they use the term for convenience. It is quite certain that fibrin may be formed when no fibrinoplastin is present, coagulation being caused by the addition of calcic chloride or casein prepared in a special way. But, whether one or two proteids be required, in all cases it is clear that a certain quantity of salts, especially of $\mathrm{NaCl}$, is necessary.]

\section{Source of the Fibrin-Factors.}

Al. Schmidt maintains that all the three substances out of which fibrin is said to be formed, arise from the breaking up of colourless blood-corpuscles. In the blood of man and mammals fibrinogen exists, dissolved in the circulating blood as a dissolution product of the 
retrogressive changes of the white corpuscles. Plasma contains dissolved fibrinogen and serum-albumin. The circulating blood is very rich in lymph or white cells, much richer, indeed, than was formerly supposed (Schmidt, Landois). As soon as blood is shed from an artery, enormous numbers of the colourless corpuscles are dissolved (Mantegazza)-according to Alex. Schmidt $71 \cdot 7$ per cent. (horse). First, the body of the cell disappears, and then the nucleus (Hlava). The products of their dissolution are dissolved in the plasma, and one of these products is fibrinoplastin. At the same time the fibrin-ferment is also produced, so that it would seem not to exist in the intact bloodcorpuscles. Fibrinoplastin and fibrin-ferment are also produced by the "transition forms" of blood-corpuscles, i.e., those forms which are intermediate between the red and the white corpuscles. They seem to break up immediately after blood is shed. The blood-plates (p. 21), are also probably sources of these substances.

In amphibians and birds, the red nucleated corpuscles rapidly break up after blood is shed, and yield the substance or substances which form fibrin. Al. Schmidt convinced himself that in these animals fibrinogen is also a constituent of the blood-corpuscles.

It is clear, therefore, according to Schmidt's view, that as soon as the blood-corpuscles, white or red, are dissolved, the fibrin-factors pass into solution, and the formation of fibrin by the union of the three substances will ensue.

[It is worthy of remark to recall the conclusion arrived at by And. Buchanan, viz., that the potential element of his "washed blood-clot" resided in the colourless corpuscles, "primary cells or vesicles." He, like Schmidt, found that the buffy-coat of horse's blood, which is very rich in white corpuscles, produced coagulation rapidly. Buchanan compared the action of his washed clot to that of rennet in coagulating milk.]

Pathological.-Al. Schmidt and his pupils, Jakowicki and Birk, have shown that some ferment, probably derived from the dissolntion of colourless corpuscles, is found in circulating blood, and that it is more abundant in venous than in arterial blood, while it is most abundant in shed blood. It is specially remarkable that in septic fever the amount of ferment in blood may increase to such an extent as to permit the occurrence of spontaneous coagulation (thrombosis), which may even produce death (Arn. Köhler). In febrile cases generally, the amount of ferment is somewhat more abundant (Edelberg and Birk). After the injection of ichor into the blood an enormous number of colourless corpuscles are dissolved (F. Hoffmann).

\section{Relation of the Red Blood-Corpuscles to the Formation of Fibrin.}

That the red blood-corpuscles may participate in the production of fibrin is proved by many experiments. 
Hoppe-Seyler showed that the nucleated blood-corpuscles of birds, when treated with water, give a copious precipitate which resembles fibrin. Heynsius observed a similar result after the blood of fowls had been acted upon by water and dilute solution of common salt, and he also states that nearly 90 per cent. of the total fibrin may bo obtained from the washed blood-corpuscles of the horse, when the corpuscles are gradually dissolved. Semmer discovered that he could cause defibrinated frog's blood to coagulate by mixing it with 4 to 6 times its volume of water. On adding 10 to 12 drops of a 0.2 per cent. solution of soda to 1 c.c.m. of defibrinated frog's blood, Semmer and A. Schmidt found that it became converted into a structureless glutinous mass, in which neutralisation with acetic acid produced fibres of fibrin. No fibrin was formed from serum. The same observers diluted 4 c.c.m. of defibrinated frog's blood with 20 c.c.m. of water containing $\mathrm{CO}_{2}$. The hæmoglobin was thereby dissolved in the water, while the colourless stromata fell to the bottom. When this deposit was mixed with a solution of sodium hydrate, a similar glutinous mass was obtained, which yielded fibrin on being neutralised with acetic acid. No such result was obtained from hæmoglobin.

In 1874, Landois observed under the microscope that the stromata of the red blood-corpuscles of mammals passed into fibrin. If a drop of defibrinated rabbit's blood be placed in serum of frog's blood, without mixing them, the red corpuscles can be seen collecting together; their surfaces are sticky, and they can only be separated by a certain pressure on the cover-glass, whereby some of the new spherical corpuscles are drawn out into threads. The corpuscles soon become spherical, and those at the margin allow the hæmoglobin to escape, when the decolourisation progresses, from the margin inwards, until at last there remains a mass of stroma adhering together. The stromasubstance is very sticky, but soon the cell-contours disappear, and the stromata adhere and form fine fibres. Thus (according to Landois) the formation of fibrin from red blood-corpuscles can be traced step by step. The red corpuscles of man and animals, when dissolved in the serum of other animals, show much the same phenomena.

Stroma-Fibrin and Plasma-Fibrin.-Landois calls fibrin formed direct from stroma, stroma-fibrin. Fibrin which is formed in the usual way by the fibrin-factors he calls plasma-fibrin. The stroma-fibrin is closely related chemically to stroma itself; and as yet the two kinds of fibrin have not been sharply distinguished chemically. Substances which rapidly dissolve red corpuscles cause extensive coagulation, e.g., injection of bile or bile salts, or lake-coloured blood, into arteries (Naunyn and Francken). After the injection of foreign blood the newly-injected blood often breaks up in the blood-vessels of the recipient, while 
the finer ressels are frequently found plugged with small thrombi (see Transfusion, p. 61).

Coagulable Fluids. - With regard to coagulability, fluids containing proteids may be classified thus :-

(1.) Those that coagulate spontaneously, i.e., blood, lymph, chyle.

(2.) Those capable of coagulating, e.g., fluids secreted pathologically in scrous cavities; for example, hydrocele fluid, which, as usually containing fibrinogen only, does not coagulate spontaneonsly, coagulates ou the addition of fibrinoplastin and ferment (or of blood-serum in which both occur).

(3.) Those which do not coagulate, e.g., milk or seminal fluid, which do not seem to contain fibrinogen.

\section{Chemical Composition of the Plasma and Serum.}

I. Proteids occur to the amount of 8 to 10 per cent. in the plasma. Only $0 \cdot 2$ per cent. of these go to form fibrin. When coagulation has taken place, and after the separation of the fibrin, the plasma becomes converted into serum. The S. G. of human serum is 1,027 to 1,029 . It contains several proteids. [According to Hammarsten, human serum contains $9 \cdot 2075$ per cent. of solids, - of these, $3 \cdot 103=$ serum-globulin, and $4.516=$ serum-albumin, i.e., in the ratio of $1: 1.511$.]

(a.) Serum-Globulin (Th. Weyl) or Para-Globulin $2-4$ p.c., was formerly believed to occur in much smaller amount than it actually does. Hammarsten found that if serum be diluted with two volumes of water, and crystals of magnesium sulphate be added to saturation, serum-globulin is precipitated, but not serum-albumin. In the serum of the horse and ox serum-globulin is more abundant than serumalbumin, while in the serum of the rabbit and $\log$ the reverse is the case. It is soluble in 10 per cent. solution of common salt, and coagulates at $75^{\circ} \mathrm{C}$.

[Serum-globulin was carefully described by Pannm under the name of "Seruncasein;" by Al. Schmidt, as "Fibrino-plastic substance;" and by Kühne, as "Para-globulin."]

As already mentioned, it may also be precipitated, in part, by diluting serum with 10 to 15 vols. of water, and passing a stream of $\mathrm{CO}_{2}$ through it (p. 44). If a trace of acetic acid be added to serum after the separation of the serum-globulin. Kiihne finds that a fine precipitate of what he calls soda-albuminate occurs. [It is, however, highly doubtful if an alkali-albuminate does occur in the blood. Hammarsten found that $\mathrm{CO}_{2}$ does not precipitate all the serum-globulin, so that it is improbable that Kithne's soda-albuminate exists as a distinct substance in serum.]

According to A. E. Burckhard, magnesium sulphate not only precipitates serum-globulin, but also another proteid substance more closely resembling albumin. During hunger the globulin increases and the albumin diminishes.

(b.) Serum-Albumin.-Its solutions begin to be turbid at $60^{\circ} \mathrm{C}$, and coagulation occurs at $73^{\circ} \mathrm{C}$., the fluid becoming slightly more 
alkaline at the same time. The amount is about $3-4$ p. c. (Frédéricq). If sodium chloride be cautiously added to serum, the coagulating temperature may be lowered to $50^{\circ} \mathrm{C}$. It has a rotatory power of $-56^{\circ}$. It is changed into syntonin or acid-albumin by the action of dilute $\mathrm{HCl}$, and by dilute alkalies into alkali-albuminate.

[Although serum-albumin is closely related to egg-albumin they differ: (a.) as regards their action upon polarised light; (b.) the precipitate produced by adding $\mathrm{HCl}$ or $\mathrm{HNO}_{3}$ is readily soluble in 4 c.c.m. of the reagent in the case of serum-albumin, while the precipitate in egg-albumin is dissolved with very great difficulty ; $\left(c_{.}\right)$egg-albumin, injected into the veins, is excreted in the urine as a foreign body, while serum-albumin is not (Stockvis).

Serum-albumin has never been obtained free from salts, even when it is dialysed for a very long time, as was maintained by Aronstein, whose results have not been confirmed by Heynsius, Haas, Huizinga, Salkowski, and others.]

After all the para-globulin (serum-globulin) in serum is precipitated by magnesinm sulphate, serum-albumin still remains in solution. If this solution be heated to 40 or $50^{\circ} \mathrm{C}$. a copious precipitate of non-coagulated serum-albumin is obtained, which is soluble in water. If the serum-albumin be filtered from the fluid, and if the clear fluid be heated to over $60^{\circ} \mathrm{C}$., Frédéricq found that it becomes turbid from the precipitation of other proteids; the amount of these other bodies, however, is small.

II. Fats $(0.1$ to 0.2 per cent.).-Neutral fats (tristearin, tripalmitin, triolein) occur in the blood in the form of small microscopic granules, which, after a meal rich in fat (or milk), render the serum quite milky.

The amount of fat in the serum of fasting animals is about 0.2 per cent.; during digestion 0.4 to 0.6 per cent.; and in dogs fed on a diet rich in fat it may be 1.25 per cent. There are also minute traces of fatty acids (succinic). Röhrig showed that soluble soaps-i.e., alkaline salts of the fatty acids-cannot exist in the blood. [Cholesterin may be considered along with the fats. It occurs in considerable amount in nerve-tissues, and, like fats, is extracted by ether from the dry residue of blood-serum. Hoppe-Seyler found 0.019 to 0.314 per cent. in the serum of the blood of fattened geese. There is no fat in the red blood-corpuscles (Hoppe-Seyler). Lecithin (and protagon) occur in serum and also in the blood-corpuscles.]

III. Traces of Grape Sugar (0.05 per cent.) occur normally in blood and serum, and also a trace of glycogen.

The amount of grape sugar in the blood increases with the absorption of sugar from the intestine, and this increase is most obvious in the blood of the portal and hepatic reins; there is also a slight increase in the arterial blood, but there it is rapidly changed. The presence of sugar is ascertained by coagulating blood by boiling it with sodium sulphate, pressing out the fluid and testing it for sugar with Fehling's solution (Cl. Bernard). Pavy coagulates the blood with alcohol.

IV. Extractives.-Kreatin, urea $(0.01$ to 0.085 per cent. in the: 
dog), hippuric acid, succinic acid, and uric acid (more abundant in gouty conditions), hypoxanthin, all occur in very small amounts.

The plasma and serum contain a yellow pigment, or perhaps several pigments. One of these is called cholepyrrhin (horse, calf), and is identical with the bile pigment of the same name (Hammarsten). [Rabbit's serum is colourless.] Thudichum regards the yellow pigment as hutein ; Maly, as hydrobilirubin; and MacMunn as choletelin.

$V$. Sarcolactic Acid and Indican, also in small amount.

VI. Salts.-The amount of inorganic salts (.085 to 09 per cent.) contained in the serum is slightly less than in the plasma, as a small amount of lime and magnesic phosphate is removed by the fibrin (Briicke). The most abundant salt is sodium chloride ( 0.5 per cent.), and next to it sodium carbonate [which exists in the plasma, most probably in the condition of sodium hydric carbonate $\left(\mathrm{NaHCO}_{3}\right)$. There is a small amount of potassic chloricle, and also sulphuric and phosphoric acids, lime and magnesia. It is most important to note that the soda salts are far more abundant in the serum than the potassium salts. The ratio may be as high as $10: 1$.]

Salts in human blood-sernm (Hoppe-Seyler).

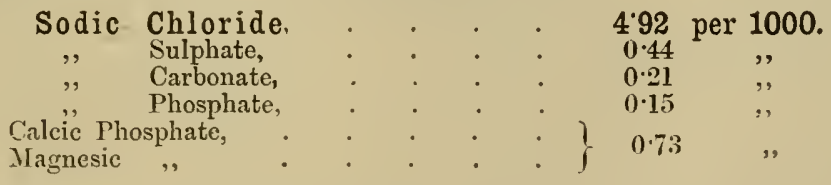

VII. Water abont 90 per cent.

\section{The Gases of the Blood.}

\section{Absorption of Gases by Solid Bodies and by Fluids.}

Absorption by Solid Bodies.-A considerable attraction exists between the particles of solid porous bodies and gaseous substances, so that gases are attracted and condensed within the pores of solid bodies-i.e., the gases are absorbed. Thus, one rolume of boxwood charcoal (at $12^{\circ} \mathrm{C}$. and ordinary barometric pressure) absorbs 35 volumes $\mathrm{CO}_{2}-9 \cdot 4 \mathrm{rol}$. $\mathrm{O}-7.5 \mathrm{vol} . \mathrm{N}-1.75$ vol. $\mathrm{H}$. Heat is always formed when gases are absorbed, and the amount of heat evolved bears a relation to the energy with which the absorption takes place. Non-porous bodies are similarly inrested by a layer of condensed gases on their surface.

By Fluids.-Fluids can also absorb gases. A known quantity of fluid at different pressures always absorbs the same volume of gas. Whether the pressure be great or small, the volume of the gas absorbed is equally great (IV. Henry). But according to Boyle and Mariotte's law (1679), when the pressure within the sane volume of gas is increased, the volume varies inversely as the pressure.

Hence it follows that, with varying pressure, the volume of gas absorbed remains 
the same, but the quantity of gas (veight, density) is directly proportional to the pressure. If the pressure $=0$, the weight of the gas absorbed must also $=0$. As a necessary result of this, we see that (1.) fuids can be freed of their absorbed gases in a vacuum under an air-pump.

Coefficient of absorption means the volume of a gas (at $0^{\circ} \mathrm{C}$ ) which is absorbed by a unit of volume of a liquid (at $760 \mathrm{~mm}$. $\mathrm{Hg}$ ) at a given temperature. The volume of a gas absorbed, and therefore the coefficient of absorption, is quite independent of the pressure, while the weight of the gas is proportional to it. Temperature has an important influence on the coefficient of absorption. With a low temperature, it is greatest; it diminishes as the temperature increases; and at the boiling point it $=0$. Hence, it follows that-(2.) Absorbed gases may be expelled from fuids simply by causing the fluids to boil. The coefficient of absorption diminishes for different fluids and gases, with increasing temperature, in a special, and by no means uniform, manner, which must be determined empirically for each liquid and gas. Thus the coefficient of absorption for $\mathrm{CO}_{2}$ in water diminishes with an increasing temperature, while that for $\mathrm{H}$ in water remains unchanged between 0 and $20^{\circ} \mathrm{C}$.

\section{Diffusion and Absorption of Gases.}

Diffusion of Gases.-Gases which do not enter into chemical combinations with each other, mix with each other in quite a regular proportion. If, e.g., the necks of two flasks be placed in communication by means of a glass or other tube, and if the lower flask contain $\mathrm{CO}_{2}$, and the upper one $\mathrm{H}$, the gases mix quite independently. of their different specific gravities, both gases forming in each flask a perfectly uniform mixture. This phenomenon is called the diffusion of gases. If a porous membrane be previously inserted between the gases, the exchange of gases still goes on through the membrane. But (as with endosmosis in fluids) the gases pass with unequal rapidity through the pores, so that at the beginning of the experiment a larger amount of gas is found on one side of the membrane than on the other. According to Graham, the rapidity of the diffusion of the gases through the pores is inversely proportional to the square root of their specific gravities. (According to Bunsen, however, this is not quite correct.)

Different Gases forming a Gaseous Mixture do not Exert Pressure upon one another.-Gases, therefore, pass into a space filled with another gas, as they would pass into a vacuum. If the surface of a fluid containing absorbed gases be placed in contact with a very large quantity of another gas, the absorbed gases diffuse into the latter. Hence, absorbed gases can be removed by (3.) passing a stream of another gas through the fluid, or by merely shaking up the fuid with another gas.

If two or more gases are mixed in a closed space over a fluid, as the different gases existing in a gaseous mixture exert no pressure upon each other, the several gases are absorbed. The weight of each absorbed is proportional to the pressure under which each gas would be, were it the only gas in the space. This pressure is called the partial pressure of a gas (Bunsen). The absorption of gases from their mixtures, therefore, is proportional to the partial pressure. The partial pressure of a gas in a space is at the same time the expression for the tension of the gas absorbed by a fluid.

The air contains 0.2096 volume of $O$, and 0.7904 volume $N$. If 1 volume of the air be placed under a pressure, $\mathrm{P}$, over water, the partial pressure under which $\mathrm{O}$ is absorbed $=0.2096 . \mathrm{P}$; that for $\mathrm{N},=0.7904 . \mathrm{P}$. At $0^{\circ} \mathrm{C}$, and $760 \mathrm{~m} . \mathrm{m}$. pressure, 1 volume of water absorbs 0.02477 volume of air, consisting of 0.00862 volume 0 , and 0.01615 volume $N$. It contains, therefore, 34 per cent. $O$, and 66 per cent. N. Therefore, water absorbs from the air a mixture of gases containing a larger percentage of $O$ than the air itself. 


\section{Extraction of the Blood Gases.}

The extraction of the gases from the blood, and their collection for chemical analysis, are carried out by means of the mercurial pump (C. Ludwig). Fig. 15 shows in a diagrammatic form the arrangement of Pflüger's gas-pump.

It consists of a RECEPTACLE FOR THE BLOOD, or "BLOOD-BULB" (A), a glass. globe capable of containing 250 to 300 c.c.m., connected above and below with tubes, each of which is provided with a stop-cock, $a$ and $b ; b$ is an ordinary stopcock, while $a$ has through its long axis a perforation which opens at $x$, and is so arranged that, according to the position of the bandle, it leads up into the

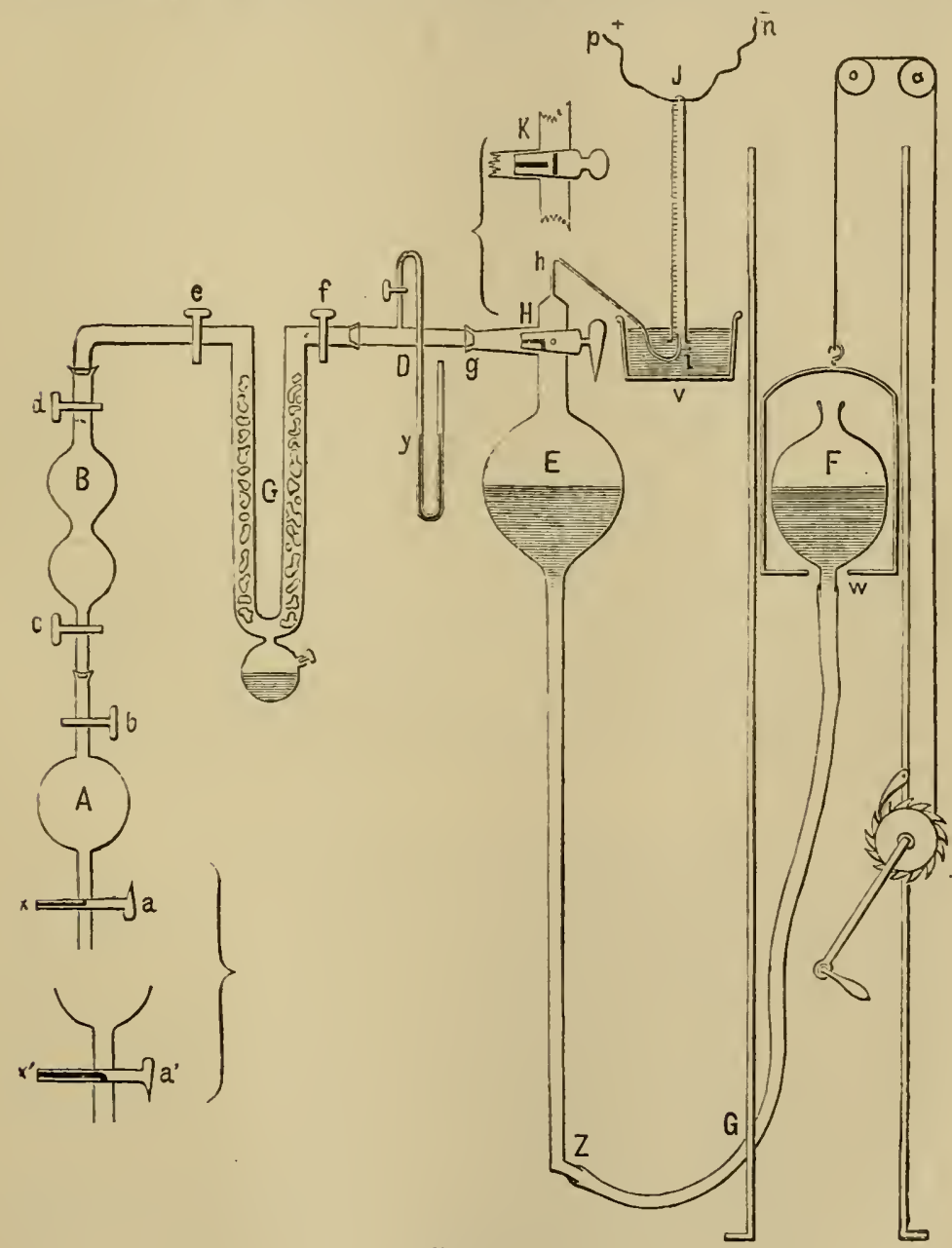

Fig. 15.

Scheme of PGïger's gas-pump-A, blood-bulb; $a$, stop-cock, with a longitudinal perforation opening upwards; $a^{\prime}$, the same opening downwards; $b$ and $c$, stop-cocks; B, froth-chamber; $d, e, f$, stop-cocks; G, drying-chambers, containing sulphuric acid and pumice-sţone; D, tube, witl manometer, $y$. 
blood bulb (position $x, a$ ), or downwards through the lower tube (position $x^{\prime}, a^{\prime}$ ). This blood-bulb is first completely emptied of air (by means of a mercurial air-pump), and then carefully weighed. One end $\left(x^{\prime}\right)$ of it is tied into an artery or a vein of an animal, and when the lower stop-cock is placed in the position $(x a)$ blood flows into the receptacle. When the necessary amount of blood is collected, the lower stop-cock is put into the position, $x^{\prime}, a^{\prime}$, and the bloodbulb, after being cleaned most carefully, is weighed to ascertain the weight of the amount of blood collected. The second part of the apparatus consists of the frothchamber, B, leading upwards and downwards into tubes, each of which is provided with an ordinary stop-cock, $c$ and $d$. The froth-chamber, as its name denotes, is to catch the froth which is formed during the energetic evolution of the gases from the blood. The lower aperture of the froth-chamber is connected by means of a well-ground tube with the blood-bulb, while above it communicates with the third part of the apparatus, the drying-chamber, G. This consists of a $\mathrm{U}$-shaped tube, provided below with a small glass-bulb, which is half filled with sulphuric acid, while in its limbs are placed pieces of pumice-stone also moistened with sulphuric acid. As the blood-gases pass through this apparatus (which may be shut off by the stop-cocks, $e$ and $f$ ) they are freed from their watery vapour by the sulphuric acid, so that they pass quite dry through the stop-cock, $f$. The short well-ground tube, $\mathrm{D}$, is fixed to $f$, and to the former is attached the small barometric tube or manometer, $y$, which indicates the extent of the vacuum. From $\mathrm{D}$ we pass to the pump proper. This consists of two large glass-bulbs which are continued above and below into open tubes; the lower tubes, $Z$ and $w$, being united by a cacutchouc tribe, G. Both the bulbs and the caoutchouc tube contain mercury - the bulbs being about half-full, and $\mathrm{F}$ being larger than $\mathrm{E}$. The bulb, $\mathrm{E}$, is fixed; but $\mathrm{F}$ can be raised or lowered by means of a pulley with a rack and pinion motion. If $\mathrm{F}$ be raised, $\mathrm{E}$ is filled; if $\mathrm{F}$ be lowered, $\mathrm{E}$ is emptied. The upper end of $\mathbf{E}$ divides into two tubes, $g$ and $h$, of which $g$ is united to $\mathrm{D}$. The ascending tube, $h$-gas-delivery tube-is very narrow, and is bent so that its free end clips into a vessel containing mercury $(v)-$ a pneumatic trough-and the opening is placed exactly under the tube for collecting the gases, the eudiometer, $J$, which is also filled with mercury. Where $g$ and $H$ unite, there is a two-way stop-cock, which in one position, $\mathrm{H}$, places $\mathrm{E}$ in communication with $\mathrm{A}, \mathrm{B}, \mathrm{G}, \mathrm{D}$ the chambers to be exhausted, and in the position, $K$, shuts off $A, B, G, D$, and places the bulb, $\mathrm{E}$, in communication with the gas-delivery tube, $h$, and the eudiometer, $\mathrm{J}$.

B, G, D are completely emptied of air, thus :-The stop-cock is placed in the position, $\mathrm{K}$; raise $\mathrm{F}$ until drops of mercury issue from the fine tube, $i$ (not yet placed under J); place the stop.cock in the position $H$, lower $F$; stop-cock in position, $K$, and so on until the barometer, $y$, indicates a complete vacuum. $J$ is now placed over $i$. Open the cocks, $c$ and $b$, so that the blood-bulb, A, communicates with the rest of the apparatus, and the blood-gases froth up in $B$, and after being dried in $\mathrm{G}$ pass towards $\mathrm{E}$. Lower $\mathrm{F}$, and they pass into $\mathrm{E}$; stop-cock in position, $K$, raise $F$, and the gases are collected in $J$ under mercury. The repeated lowering and raising of $F$ with the corresponding position of the stopcocks ultimately drives all the gases into $\mathrm{J}$. The removal of the gases is greatly facilitated by placing the blood-bulb, $\mathrm{A}$, in a vessel containing water at $60^{\circ} \mathrm{C}$.

It is well to remove the gases from the blood immediately after it is collected. from a blood-vessel, because the $O$ undergoes a diminution if the blood be kept. Of course, in making several analyses it is difficult to do this, and the best plan to pursue in that case is to keep the receptacles containing the blood on ice.

Mayow (1670) observed that gases were given off from blood in vacuo. Magnus (1837) investigated the percentage composition of the blood-gases. The more important recent investigations have been made by Lothar Meyer (1857), and by the pupils of C. Ludwig and E. Pflüger. 


\section{Quantitative Estimation of the Blood-Gases.}

The gases obtained from blood consist of $\mathrm{O}, \mathrm{CO}_{2}$ and $\mathrm{N}$. Pflüger obtained (at $0^{\circ} \mathrm{C}$. and 1 metre $\mathrm{Hg}$ pressure), $4.7 \cdot 3$ volumes per cent. consisting of

O 16.9 per cent. $-\mathrm{CO}_{2} 29$ per cent. - N. $1 \cdot 4$ per cent. As is shown in Fig. 15, the gases are obtained in an eudiometer, i.e., in a narrow tube, $J$, closed at one end, and with a very exact scale marked on it, and having two fine platinum wires melted into its upper end, with their free-ends projecting into the tube ( $p$ and $n)$.

(1.) Estimation of the $\mathrm{CO}_{2} .-\mathrm{A}$ small ball of fused caustic potash, fixed on a platinum wire, is introduced into the mixture of gases through the lower end of the eudiometer nuder cover of the mercury. The surface of the potash ball it moistenerl before it is introduced. The $\mathrm{CO}_{2}$ unites with the potash to form potassium carbonate. After it has been in for a considerable time ( 24 hours), it is withdrawn in a similar manncr. The climinution in volume inclicates the amont of $\mathrm{CO}_{2}$ absorvel.

(2.) Estimation of the 0.- (u.) Just as in estimating the $\mathrm{CO}_{2}$, a ball of phosphorus on a platinum wire is introduced into the endiometer (Bertholet); it absorbs the $\mathrm{O}$ and forms phosphoric acid. Another plan is to employ a small papier-maché ball saturated with pyrogallic acid in caustic potash, which rapidly absorbs $O$ (Liebig). After the ball is removed, the diminution in volume indicates the quantity of $O$.

(b.) The $O$ is most easily and accurately estimated by exploding it in the eudiometer (Volta and Bunsen). Introduce a sufficient quantity of $\mathbf{H}$ into the eudiometer, and accurately ascertain its volume; an electrical spark is now passed between the wires, $p$ and $n$, through the mixture of gases; the $O$ and $H$ unite to form water, which causes a dimiuution in the volume of the gases in the eudiometer, of which $\frac{1}{3}$ is due to the $\mathrm{O}$ nsed to form water $\left(\mathrm{H}_{2} \mathrm{O}\right)$.

(c.) Estimation of the N.--When the $\mathrm{CO}_{2}$ and $\mathrm{O}$ are estimated by the above method, the remainder is pure $\mathrm{N}$.

\section{The Blood Gases.}

I. Oxygen exists in arterial blood $(\operatorname{dog})$ on an average to the extent of 17 volumes per cent. (at $0^{\circ} \mathrm{C}$. and 1 metre $\mathrm{Hg}$ pressure) (Pfliiger). According to Pfliiger, arterial blood (dog) is saturated to $\frac{9}{10}$ with $\mathrm{O}$, while, according to Hüfner, it is saturated to the extent of $\frac{14}{15}$. In venous blood the quantity varies very greatly; in the blood of a passive muscle 6 volumes per cent. have been found; while in the blood after asphyxia it is absent, or occurs only in traces. It is certainly more abundant in the comparatively red blood of active glands (salivary glands, kidney), than in ordinary dark venous blood.

The $\mathbf{0}$ in Blood occurs-(a.) simply absorbed in the plasma. This is only a minimal amount, and does not exceed what distilled water at the temperature of the body would take up at the partial pressure of the $\mathrm{O}$ in the air of the lungs (Lothar Meyer). According to 
Fernet, serum takes up slightly more $O$ than corresponds to the pressure, and this is, perhaps, due to the trace of hæmoglobin contained in the plasma or the serum, and which is derived from the solution of red corpuscles.

(b.) Almost the total 0 of the blood is chemically united, and, therefore, not subject to the law of absorption. It is loosely united to the hæmoglobin of the red corpuscles, with which it forms oxylcemoglobin (p. 29).

The absorption of this quantity of $O$ is completely independent of pressure; hence, animals confined in a closed space until they are nearly asphyxiated, can use up almost all the 0 from the surrounding atmosphere. The fact of the union being independent of pressure is proved by the following:-The blood only gives off copiously its chemically united 0 , when the atmospheric pressure is lowered to 20 millimetres, $\mathrm{Hg}$. (Worm Müller); and, conversely, blood only takes up a little more 0 when the pressure is increased to 6 atmospheres (Bert).

Physical Methods of obtaining 0 from Blood.-Notwithstanding this chemical union between the $\mathrm{Hb}$ and $\mathrm{O}$, however, the total $\mathrm{O}$ of the blood can be expelled from its state of combination by those means which set free absorbed gases- $(a$.$) by introducing blood into a torri-$ cellian vacuum; $(b$.$) by boiling; (c.) by the conduction of other gases$ $[\mathrm{H}, \mathrm{N}, \mathrm{CO}$ or $\mathrm{NO}]$ through the blood, because the chemical union of the oxyhæmoglobin is so loose that it is decomposed even by these physical means.

Chemical Reagents.-Amongst chemical reagents the following reducing substances-ammonium sulphide, sulphuretted hyärogen, alkaline solutions of sub-salts, iron filings, \&c., rob blood of its O (p. 30).

With regard to the taking up of $\mathrm{O}$, the total quantity of blood behaves exactly like a solution of hæmoglobin free from $O$ (Preyer.) The amount of iron in the blood $(0.55$ in 1,000 parts) stands in direct relation to the amount of $\mathrm{Hb}$; this to the quantity of blood-corpuscles; and this, in turn, to the specific gravity of the blood. The amount of $\mathrm{O}$ in the blood, therefore, is nearly proportional to the specific gravity of the blood, and it is also in proportion to the amount of iron in the blood. Picard affirms that $2 \cdot 36$ grammes of iron in the blood can fix chemically $1 \mathrm{grm}$. $\mathrm{O}$; while, according to Hoppe-Seyler, the proportion is 1 atom iron to 2 atoms $O$.

When blood is kept long outside of the blood-vessels, the quantity of 0 gradually diminishes, and if it be kept for a length of time at a high temperature it may disappear altogether. This depends upon decomposition occurring within the blood. By this decomposition in the blood (cadaveric phenomenon), reducing substances are formed which consume the 0 . All kinds of blood, however, do not act with equal energy in consuming 0 , e.g., venous blood from active muscles acts most energetically, while that from the hepatic vein has very little effect. $\mathrm{CO}_{2}$ appears in the blood in place of the $\mathrm{O}$, and the colour darkens. The amount of $\mathrm{CO}_{2}$ produced is sometimes greater than that of the $\mathrm{O}$ consumed. 
If blood (or a solution of oxyhremoglobin) he acted upon by acids (e.\%., tartaric acid) until it is strongly acid, $\mathrm{O}$ may be pumped out in considerably less amount, while the formation of $\mathrm{CO}_{2}$ is not increased. We must, therefore, assume that, during the decomposition of the $\mathrm{Hb}$ caused by the acids (p. 33), a decomposition product becomes more highly oxidised by the intense chemical union of the $\mathrm{O}$ at the moment of its origin (Lothar Meyer, Zuntz, Strassburg). The same phenomenon occurs when oxyhrmoglobin is decomposed by boiling.

\section{Is Ozone $\left(0_{3}\right)$ Present in Blood?}

On account of the numerous and energetic oxidations whicl occur in connection with the blood, the question has often been raised as to whether the $\mathrm{O}$ of the bloor exists in the form of active $\mathrm{O}\left(\mathrm{O}_{3}\right)$, or ozone. Ozone, however, is contained neither in the blood itself (Schönbein) nor in the blood-gases obtained from it. Nevertheless, the red corpuscles (and $\mathrm{Hb}$ ) have a distinct relation to ozone.

(1.) Tests for 0zone.-Hæmoglobin acts as a conveyer of ozone, i.e., it is able to rernove the active $O$ of other bodies and to convey or transfer it at once to other easily oxidisable substances. (a.) Turpentine which has been exposed to the air for a long time always contains ozone. The tests for the latter are starch and potassium iodide, the ozone decomposing the iodide when the iodine strikes a blue with the starch. (b.) Freshly-prepared tincture of guaiacum is also rendered blue by ozone. If some tincture of guaiacum be added to turpentine there is no reaction, but on adding a drop of blood a deep blue colour is immediately produced, i.e., blood takes the ozone from the turpentine and conveys it at once to the dissolved guaiacum, which becomes blue (Schönbein, His). It is immaterial whether the $\mathrm{Hb}$ contains $\mathrm{O}$ or not.

(2.) It has been asserted also that hamoglobin acts as an o:oneproducer, i.e., that it can convert the ordinary $\mathrm{O}$ of the air into ozone. Hence the reason why red blood-corpuscles alone render guaiacum blue. This reaction succeels best when the guaiacum solution is allowed to dry on blotting-paper, and a few drops of blood (diluted 5 to 10 times) are poured on it. That the $\mathrm{Hb}$ forms ozone from the surrounding $O$, is shown by the experiment in which even red bloodcorpuscles containing carbonic oxide were found to cause the blue colour (Külıne and Scholz).

According to Pflïger, however, these reactions only occur from decomposition of the $\mathrm{Hb}$, and as a result of this view the bloodcorpuscles cannot be regarded as producers of ozone.

-Snlphuretted hydrogen is decomposed by blood (as by ozone itself) into sulphur and water. Hydric peroxide is decomposed by blood into $O$ and water [but this reaction is prevented by the addition of a small amount of hydrocyanic acid (Schönbein)]. Crystallised $\mathrm{Hb}$ does not do this, and $\mathrm{H}_{2} \mathrm{O}_{2}$ may be cautiously injected into the blood-vessels of animals. This would show that unchanged $\mathrm{Hb}$ does not produce ozone.

Various Forms of Oxygen.-There are three forms of oxygen: (1.) The 
ordinary oxygen $\left(\mathrm{O}_{2}\right)$ in the air. (2.) Active or nascent oxygen $(0)$, which never can occur in the free state, but the moment it is formed acts as a powerful oxidising agent and produces chemical compounds. It converts water into hydric peroxide-the $\mathrm{N}$ of the air into nitrous and nitric acids, and even $\mathrm{CO}$ into $\mathrm{CO}_{2}$, which ozone does not. It certainly plays an important part in the organism. (3.) Ozone $\left(\mathrm{O}_{3}\right)$, which is formed by the decomposition of several molecules of ordinary oxygen $\left(\mathrm{O}_{2}\right)$ into two atoms of $\mathrm{O}$, and the appropriation of each of these atoms by a molecule of undecomposed oxygen. It is oxygen condensed to $\frac{2}{3}$ of its rolume.

\section{Carbonic Acid and Nitrogen in Blood.}

II. Carbonic Acid.-In arterial blood there are about 30 volumes per cent. of $\mathrm{CO}_{2}$ (at $0^{\circ} \mathrm{C}$. and 1 metre pressure-Setschenow); but in venous blood the amount is very variable; e.g., in the venous blood of passive muscles there are 35 volumes per cent. (Sczelkow), while in the blood of asphyxia there may be $52 \cdot 6$ volumes per cent. The amount of $\mathrm{CO}_{2}$ in the lymph of asphyxia is less than that in the blood (Buchner, Gaule).

The $\mathrm{CO}_{2}$ in the entire mass of the blood may be extracted from it or completely pumped out, but during the process of evacuation, or removal of the gas, a new property of the red blood-corpuscles is produced, whereby they assume the function of an acid and thus aid in the chemical expulsion of the $\mathrm{CO}_{2}$. This acid-like property of the red corpuscles occurs especially in the presence of $\mathrm{O}$ and heat.

(A.) The $\mathrm{CO}_{2}$ in the Plasma.-The largest portion of the $\mathrm{CO}_{2}$ belongs to the plasma (or serum) and it appears all to be in a state of chemical combination. Serum takes up $\mathrm{CO}_{2}$ quite independently of pressure, hence it cannot be merely absorbed. A certain part of the $\mathrm{CO}_{2}$ can be removed from the serum (plasma) by the torricellian vacuum, while another part is obtained only after the addition of an acid. [This is called the "fixed" $\mathrm{CO}_{2}$, while the former is known as the "loose" $\mathrm{CO}_{2}$.]

The union of $\mathrm{CO}_{2}$ in the serum may take place in the following ways :-

(1.) $\mathrm{CO}_{2}$ is united to the soda of the plasma in the form of "sodic carbonate." This portion of the $\mathrm{CO}_{2}$ can only be displaced from its combination by the addition of an acid. (In depriving blood of its gases the red corpuscles play the rôle of an acid.)

(2.) A portion of the $\mathrm{CO}_{2}$ is loosely united to sodic carbonate in the form of sodic bicarbonate; the carbonate takes up 1 equivalent of $\mathrm{CO}_{2} ; \mathrm{Na}_{2} \mathrm{CO}_{3}+\mathrm{CO}_{2}+\mathrm{H}_{2} \mathrm{O}=2 \mathrm{NaHCO}_{3}$. This $\mathrm{CO}_{2}$ may be pumped out, as in the process the bicarbonate splits up again into the neutral carbonate and $\mathrm{CO}_{2}$. 
Preyer has objected to this view on the ground that blood is alkaline in reaction, whilst all solutions that contain $\mathrm{CO}_{2}$ in a state of absorption, or loose chemical combination, are always acid. Pfliger and Zuntz showed that blood, after being completely saturated with $\mathrm{CO}_{2}$, still remains alkaline.

As the bicarbonate only gives up its $\mathrm{CO}_{2}$ very slowly in vacuo, while blood gives off its $\mathrm{CO}_{2}$ very energetically, perhaps the soda, united with an albuminous body, combines with the $\mathrm{CO}_{2}$ and forms a complex compound, from which the $\mathrm{CO}_{2}$ is rapidly given off in vacuo.

(3.) A minimal portion of the $\mathrm{CO}_{2}$ may be chemically united in the plasma with neutral sodic phosphate (Fernet). One equivalent of this salt can fix 1 equivalent of $\mathrm{CO}_{2}$, so acid sodium phosphate and acid sodium carbonate are formed, $\mathrm{Na}_{2} \mathrm{HPO}_{4}+\mathrm{CO}_{2}+\mathrm{H}_{2} \mathrm{O}=\mathrm{NaH}_{2} \mathrm{PO}_{4}$ $+\mathrm{NaH}, \mathrm{CO}_{3}$ (Hermann). When the gases are removed the $\mathrm{CO}_{2}$ escapes, and neutral sodic phosphate remains.

It is probable, however, that almost all the sodic plosphate found in the blootash arises from the burning of lecithin; we have, therefore, to cousider only the very small amount of this salt which occurs in the plisma (Hoppe-Seyler and Siertoli).

(B.) The $\mathrm{CO}_{2}$ in the Blood-Corpuscles.-The rerl corpuscles contain $\mathrm{CO}_{2}$ in a loose chemical combination; for (1.) a rolume of blood can fix nearly as much $\mathrm{CO}_{2}$ as an equal volume of serum (Ludwig, $\mathrm{Al}$. Schmidt); and (2.) with increasing pressure the absorption of $\mathrm{CO}_{2}$ by blood takes place in a lifferent ratio from what occurs with serum (Pflüger, Zuntz). The red corpuscles may fix more $\mathrm{CO}_{2}$ than their own volume, and the union of the $\mathrm{CO}_{2}$ seems to depend upon the $\mathrm{Hb}$, for Setschenow found that, when $\mathrm{Hb}$ was acted on by $\mathrm{CO}_{2}$, its power of fixing the latter was increased, which is perhaps due to the formation of some substance (paraglobulin) more suited for fixing $\mathrm{CO}_{2}$. The colourless corpuscles also fix $\mathrm{CO}_{2}$ after the manner of the serumconstituents, and to the extent of to $\frac{1}{1:}$ of the absorbing power of serum (Setschenow).

III. Nitrogen exists in the blood to the extent of 1.4 to $1.6 \mathrm{vol}$. per cent., and it appears to be simply absorbed.

It is still donbtful whether a small part of the $\mathrm{N}$ exists chemically united in the red corpuscles. Outside the body when blood is heated, and when there is a free supply of $O$ and warmth, it gives off very minute quantities of ammonia, which are perhaps derived from the decomposition of some salt of ammonia as yet unknown (Kühne and Strauch).

\section{Arterial and Venous Blood.}

Arterial blood contains in solution all those substances which are necessary for the nutrition of the tissues, those which are employed in secretion; it also contains a rich supply of $O$. Venous bloorl must 
contain less of all these, but in addition it holds the used-up or effete substances derived from the tissues, and the products of their retrogressive metabolism being more numerous, there is in venous blood a larger amount of $\mathrm{CO}_{2}$. It is evident also that the blood of certain veins must have special characters, e.g., that of the portal and hepatic veins.

The following are the most important points of difference between arterial and venous blood :-

\section{Arterial Blood contains-m}

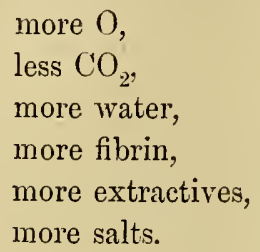

more sugar,

fewer blood-corpuscles, less urea.

It is bright red and not dichroic.

As a rule it is $1^{\circ} \mathrm{C}$. warmer.

The bright red colour of arterial blood depends on the presence of oxyhæmoglobin, whilst the dark colour of venous blood is due to its smaller proportion of oxyhæmoglobin, and the quantity of reduced hæmoglobin which it contains. The dark change of colour is not to be attributed to the larger quantity of $\mathrm{CO}_{2}$ in venous blood (Marchand); for if equal qualities of $\mathrm{O}$ be added to two portions of blood, and if $\mathrm{CO}_{2}$ be arlded to one of them, the colour is not changed (Pflüger).

\section{Quantity of Blood.}

In the adult the quantity of blood is equal to $\frac{1}{13}$ part of the bodyweight (Bischoff), in newly-born children $\frac{1}{10}$ (Welcker).

According to Schiicking, the amount of blood in a newly-born child depends to some extent upon the time at which the umbilical cord is ligatured. The amount $=\frac{1}{15}$ of the body-weight when the cord is tied at once, while if it is tied somewhat later it may be $\frac{1}{9}$. Immediate ligature of the cord may, therefore, deprive a newly-born child of 100 grammes of blood. Further, the number of corpuscles is less in a child after immediate ligature of the umbilical cord, than when it is tied somewhat later (H.elot).

Various methods are adopted to ascertain the amount of blood, but perhaps that of Welcker is the best.

The methods of Valentin (1838), and Ed. Weber (1850), are not now used, as the results obtained are not sufficiently accurate.

Method of Welcker (1854). - Begin by taking the weight of the animal to be experimented on; place a cannula in the carotid, and allow the blood to run into a flask previously weighed, and in which small pebbles (or $\mathrm{Hg}$ ) have been placed in order to defibrinate the blood by shaking. Take a part of this detibrinated blood, and make it cherry-red in colour by passing through it a stream of $\mathrm{CO}$ 
(because ordinary blood varies in colour according to the amount of $\mathrm{O}$ contained in it-Gscheidlen, Heidenhain). Tie a - -shaped cannula in the two cut ends of the carotid, and allow a 0.6 per cent. solution of common salt to flow into the vessel from a pressure bottle; collect the coloured fluid issuing from the jugular veins and inferior vena cava until the fluid is quite clear. The entire body is then chopped up (with the exception of the contents of the stomach and intestines, which are weighed, and their weight deducted from the body-weight), and extracted with water, and after twenty-four hours the fluir is expressed. This water, as well as the washings with salt solution, are collected and weighed, and part of the mixture is saturated with $\mathrm{CO}$. A sample of this dilute blood is placed in a vessel with parallel sides (l c.m. thick), opposite the light (the so-called hæmatinometer), and in a second ressel of the same dimensions, a sample of the undiluted $\mathrm{CO}$-blood is diluted with water from a burette until both fluids give the same intensity of colour. From the quantity of water required to dilute the blood to the tint of the washings of the blood-vessels, the quantity of blood in the washings is calculated. (On chopping up the muscles alone, we obtain the amount of $\mathrm{Hb}$ present in them, which is not taken into calculation-Kühne).

Quantity of Blood in Various Animals.-The quantity of blood in the mouse $=\frac{1}{12}$ to $\frac{1}{13}$; guinea-pig $\frac{1}{197} \cdot\left(\frac{1}{17}\right.$ to $\left.\frac{1}{22}\right)$; rabbit $=\frac{1}{20 \cdot 1}$ $\left(\frac{1}{15}\right.$ to $\left.\frac{1}{25}\right) ; \operatorname{dog}=\frac{1}{13}\left(\frac{1}{11}\right.$ to $\left.\frac{1}{15}\right) ;$ cat $=\frac{1}{21 \cdot 5} ;$ birds $=\frac{1}{10}$ to $\frac{1}{13} ;$ frog $=\frac{1}{15}$ to $\frac{1}{20}$; fishes $=\frac{1}{14}$ to $\frac{1}{19}$ of the body-weight (without the contents of the stomach and intestines).

The specific gravity of the blood ought always to be taken when estimating the amount of blood. The amount of blood is diminished during inanition; fat persous have relatively less blood; after hæmorrhage the loss is at first replaced by a watery fluid, while the bloodcorpuscles are gradually regenerated (p. 63).

The estimation of the quantity of blood in different organs is done by suddenly ligaturing their blood-vessels intra vitam. A watery extract of the chopped up organ is prepared, and the quantity of blood estimated as described above. Roughly, it may be said that the lungs, heart, large arteries, and veins contain $\frac{1}{4}$; the muscles of the skeleton, $\frac{1}{4}$; the liver, $\frac{1}{4}$; and other organs, $\frac{1}{4}$ (Ranke).

\section{Variations from the Normal Condition of the Blood.}

(A.) Increase of the Blood, or of its Individual Constituents.-(1.) An increase in the entire mass of the blood, uniformly in all organs, constitutes polycmia (or plethora), and in over-nourished individuals it may approach a pathological condition. A bluish-red colour of the skin, swollen reins, large arteries, hard full pulse, injection of the capillaries and smaller vessels of the visible mucous membranes are signs of this state, accompanied by congestion of the brain, giving rise to vertigo, and congestion of the lungs, as shown by breathlessness. After major amputations with little loss of blood a relative increase of blood has been found (?) (plethora apocoptica).

Transfusion.-Polyæmia may be produced artificially by the injection of blood of the same species. If the normal quantity of blood be increased 83 per cent. 
no abnormal condition occurs, because the blood-pressure is not permanently raised. The excess of blood is accommodated in the greatly distended capillaries, which may be stretched beyond their normal elasticity (Worm Müller). If it be increased to 150 per cent. there are variations in the blood-pressure, life is endangered, and there may be sudden rupture of blood-vessels (Worm Müller).

Fate of Transfused Blood.-After the transfusion of blood the formation of lymph is greatly increased; but in one to two days the serum is used up, the water is excreted chiefly by the urine, and the albumin is partly changed into urea (Landois). Hence, the blood at this time appears to be relatively richer in blood-corpuscles (Panum, Lesser, IVorm Müller). The red corpuscles break up much more slowly, and the products thereof are partly excreted as urea and partly (but not constantly) as bile pigments. Even after a month an increase of coloured blood-corpuscles has been observed (Tschirjew). That the blood-corpuscles are broken up slowly in the economy is proved by the fact that the amount of urea is much larger when the same quantity of blood is swallowed by the animal, than when an equal amount is transfused (Tschirjew, Landois). In the latter case there is a moderate increase of the urea lasting for days, a proof of the slow decomposition of the red corpuscles. Pronounced over-filling of the vessels causes loss of appetite, and a tendency to hæmorrhage of the mucous inembranes.

(2.) Polyæmia serosa is that condition in which the amount of serum-i.e., the amount of water in the blood, is increased. This may be produced artificially by the transfusion of blood-serum from the same species. The water is soon given off in the urine, and the albumin is decomposed into urea, without however, passing into the urine. An animal forms more urea in a short time from a quantity of transfused serum than from the same quantity of blood, a proof that the bloodcorpuscles remain longer undecomposed than the serum (Forster, Landois). If serum from another species of animal be used (e.g., dog's serum transfused into a rabbit), the blood-corpuscles of the recipient are dissolved; hæmoglobinuria is produced (Ponfick); and if there be general dissolution of the corpuscles, death may occur (Landois).

Polyæmia aquosa is a simple increase of the water of the blood, and occurs temporarily after copious drinking, but increased diuresis soon restores the normal condition. Diseases of the kidneys, which destroy their secreting parenchyma, produce this condition, and often general dropsy, owing to the passage of water into the tissues. Ligature of the ureter produces a watery condition of the blood.

(3.) Plethora poiycythæmica, Hyperglobulie.-An increase of thc red corpuscles has been assumed to occur when customary regular hæmorrhages are interrupted-e.g., menstruation, bleeding from the nose, \&c.; but the increase of corpuscles has not been definitely proved. There is a proved case of temporary polycythrmiaviz, when similar blood is transfused, a part of the fluid is used up, while the corpuscles remain unchanged for a considerable time. There is a remarkable increase in the number of blood-corpuscles (to 8.82 millions per cubic millimetre, p. 4) in certain severe cardiac affections where there is great congestion, and much water transudes through the vessels. In cases of hemiplegia, for the same reason, the number of corpuscles is greater on the paralysed congested side (Penzoldt). After diarrhœa, which diminishes the water of the blood, there is also an increase (Brouardel). There is a temporary increase in the hamatoblasts as a reparative process after severe hæmorrhage (p. 15), or after acute diseases. In cachectic conditions this increase continues, owing to the diminished non-conversion of these corpuscles into red corpuscles. In the last stages of cachexia the number diminishes more and more until the formation of hæmatoblasts ceases (Hayem).

(4.) Plethora hyperalbuminosa is a term applied to the increase of albumins in the plasma, such as occurs after taking a large amount of food. A similar con- 
dition is produced by transfusing the serum of the same species, whereby, at the same time, the urea is increased. Injection of egg-albumin produces albuminuria (Stokes, Lehmann).

(B.) Diminution of the Quantity of Blood, or its Individual Constituents.-(1.) Oligæmia vera, or diminution of the quantity of blood as a whole, occurs whenever there is hæmorrhage. Life is endangered in newly-born ehildren when they lose a few ounces of bloor; in children a year old, on losing half-a-pound; and in adults, when one-half of the total blood is lost. Women bear loss of blood much better than men. The periodical formation of blood after each menstruation seems to enable blood to he renewed more rapidly in their case. Stout persons, old people, and children do not bear the loss of blood well. The more rapidly blood is lost, the more dangerous it is.

Symptoms of Loss of Blood.-Great loss of blood is accompanied by general paleness and coldness of the cutaneous surface, increased oppression, twitching of the eyeballs, noises in the ears and vertigo, loss of voice, great breathlessness, stoppage of secretions, coma ; dilatation of the pupils, involuntary evacuations of urine and fæces, and lastly, general convulsions, are sure signs of death by homorrhage. In the gravest cases restitution is only possible by means of trans. fusion. Animals can bear the loss of one-fourth of their entire blood without the blood-pressure in the arteries permanently falling, because the blood-vessels contract and accommodate themselves to the smaller quantity of blood (in consequence of the stimulation of the vasomotor centre in the medulla). The loss of one-third of the total blood diminishes the blood-pressure considerably (one-fourth in the carotid of the dog). If the hæmorrhage is not such as to cause death, the fluid part of the blood and the dissolved salts are restored by absorption from the tissues, the blood-pressure gradually rises, and then the albumin is restored, though a longer time is required for the formation of red corpuscles. At first, therefore, the blood is abnormally rich in water (hydromia), and at last abnormally poor in corpuscles (oligocythcemia, hypoglobulie). With the increased lymphstream which pours into the blood, the colourless corpuscles are considerably increased above normal, and during the period of restitution fewer red corpuscles seem to be used up (e.g., for bile).

After moderate bleeding from an artery in animals, Buntzen observer that the volume of the blood was restored in several hours; after more severe hæmorrhage in 24 to 48 hours. The red blood-corpuscles after a loss of bloor equal to $1 \cdot 1$ to $4 \cdot 4$ per cent. of the body-weight, are restored only after 7 to 34 days. The generation begins after 24 hours. During the period of regeneration the number of the smallest bloor-corpuscles (hæmato-blasts) is increased. Even in man the duration of the period of regeneration depends upon the amount of blood lost (Lyon). The amount of hæmoglobin is diminished nearly in proportion to the amount of the hemorrhage (Bizzozero and Salvioli).

Metabolism in Anæmia. - The condition of the metabolism within the bodies of anæmic persons is important. The decomposition of proteids is increased (the same is the case in hunger), hence the excretion of urea is increased (Baner, Jirgensen). The decomposition of fats, on the contrary, is diminished, which stands in relation with the diminution of $\mathrm{CO}_{2}$ given off. Anæmic and chlorotic persons put on fat easily. The fattening of cattle is aided by occasional bleedings and by intercurrent periods of hunger (Aristotle).

(2.) An excessive thickening of the blood through loss of water is called Oligæmia sicca. This occurs in man after copious watery evacuations, as in cholera, so that the thick tarry blood stagnates in the vessels. Perhaps a similar condition-though to a less degree-may exist after very copious perspiration.

(3.) If the proteids in blood be abnormally diminished the conclition is called Oligæmia hypalbuminosa; they may be diminished about one-half. They are usually replaced by an excess of water in the blood. Loss of albumin from 
the blood is caused directly by albuminuria ( 25 grammes of albumin may be given off by the urine daily), persistent suppuration, great loss of milk, extensive cutaneous ulceration, albuminous diarrhœa (dysentery). Frequent and copious hæmorrhages, however, by increasing the absorption of water into the vessels, at first produce oligæmia hypalbuminosa.

Mellitæmia.-The sugar in the blood is partly given off by the urine, and in "diabetes mellitns" one kilo. $(2 \cdot 2$ lbs. $)$ may be given off daily, when the quantity of urine may rise to 25 kilos. To replace this loss a large amount of food and drink is required, whereby the urea may be increased threefold. The increased production of sugar causes an increased decomposition of albuminous tissues; hence the urea is always increased, even though the supply of albumin be insufficient. The patient loses flesh; all the glands, and even the testicles, atrophy or degenerate (pulmonary phthisis is common); the skin and bones become thinner; the nervous system holds out longest. The teeth become carious on account of the acid saliva, the crystalline lens becomes turbid from the amount of sugar in the fluid of the eye which extracts water from the lens (Kunde, Heubel), and wounds heal badly because of the abnormal condition of the blood. Absence of all carbohydrates in the food causes a diminution of the sugar in the blood, but does not cause it to disappear entirely. An excessive amount of inosite has been found in the blood and urine, constituting melliturice inosita (Vohl).

Lipæmia, or an Increase of the Fat in the Blood, occurs after every meal rich in fat, so that the serum may become turbid like milk. Pathologically, this occurs in a high degree in drunkards and in corpulent individuals. When there is great decomposition of albumin in the body (and therefore in very severe diseases), the fat in the blood increases, and this also takes place after a liberal supply of easily decomposable carbo-hydrates and much fat.

The Salts remain very persistently in the blood. The withdrawal of common salt produces albuminuria, and, if all salts be withheld, paralytic phenomena occur (Forster). Over-feeding with salted food, such as salt meat, has caused death through fatty degeneration of the tissues, especially of the glands. Withdrawal of lime and phosphoric acid produces atrophy and softening of the bones. In infectious diseases and dropsies the salts of the blood are often increased, and diminished in inflammation and cholera. $[\mathrm{NaCl}$ is absent from the urine in certain stages of pneumonia, and it is a good sign when the chlorides begin to return to the urine].

The amount of fibrin is increased in inflammations of the lung and pleura; hence, such blood forms a crusta plilogistica (p. 39). In other diseases, where decomposition of the blood-corpuscles occurs, the fibrin is increased, perhaps because the dissolved red corpuscles yield material for the formation of fibrin. After repeated hæmorrhages, Sigm. Mayer found an increase of fibrin. Blood rich in fibrin is said to coagulate more slowly than when less fibrin is present-still there are many exceptions.

For the abnormal changes of the red and white blood-corpuscles see p. 23. 


\section{Physiology of the Circulation.}

\section{General View of the Circulation.}

THE blood within the ressels is in a state of continual motion, being carried from the ventricles by the large arteries (aorta and pulmonary) and their branches to the system of capillary vessels, from which again, it passes into the reins that end in the atria of the auricles (II. Harvey).

The cause of the circulation is the difference of pressure which exists between the blood in the aorta and pulmonary artery on the one hand, and the two venæ cavæ and the four pulmonary veins on the other. The blood, of course, moves continually in its closed tubular system in the direction of least resistance. The greater the difference of pressure, the more rapid the movement will be. The cessation of the difference of pressure (as after death) naturally brings the movement to a standstill. The circulation is usually dirided into-

(1.) The greater, or systemic circulation, which includes the course of the blood from the left auricle and left ventricle, through the aorta and all its branches, the capillaries of the body and the reins, until the two venæ caræ terminate in the right auricle.

(2.) The lesser, or pulmonic circulation, which includes the course from the right anricle and right ventricle, the pulmonary artery, the pulmonary capillaries, and the four pulmonary veins springing from them, until these open into the right auricle.

(3.) The portal circulation, which is sometimes spoken of as a special circulatory system, although it represents only a second set of

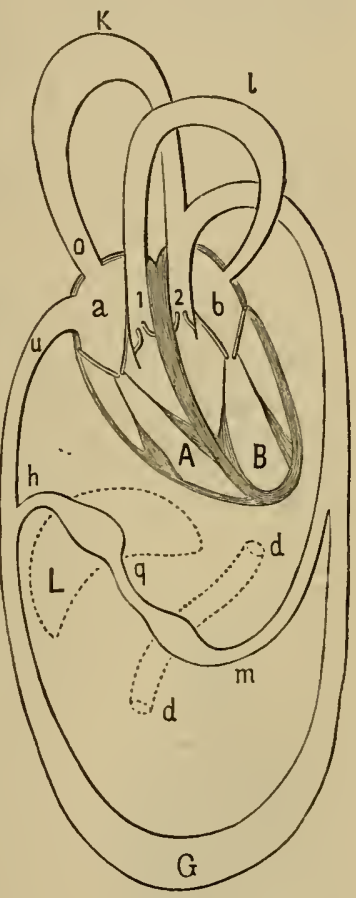

Fig. 16.

Scheme of the circulation-a, right auricle; $A$, right ventricle; $b$, left auricle; $\mathrm{B}$, left ventricle; 1 , pulmonary artery ; 2 , aorta with semilunar valves; $l$, area of pulmonary circulation; $K$, area of systemic circulation in region supplying the superior vena cava, $o ; \mathrm{G}$, area supplying the inferior vena cava, $u ; d, d$, intestine; $m$, mesenteric artery; $q$, portal vein ; L, liver; $h$, hepatic vein.

the comrse of a venous 
trunk. It consists of the vena portarum-formed by the union of the intestinal or mesenteric and splenic veins, and it passes into the liver, where it divides into capillaries, from which the hepatic veins arise. These last veins join the inferior vena cava.

Strictly speaking, however, there is no special portal circulation. Similar arrangements occur in other animals in different places - e.g., snakes have such a system in their supra-renal capsules, and the frog in its kidneys.

When an artery splits up into fine branches during its course, and these branches do not form capillaries, but reunite into an arterial trunk, a rete mirabile is formed, such as occurs in apes and the edentata. Similar arrangements may exist on veins, giving rise to venous retic mirabilia.

\section{The Heart.}

Muscular Fibres of the Heart.-The musculature of the mammalian heart consists of short ( 50 to $70 \mu$, man), very fine, transversely striated muscular fibres, which are actual uni-cellular elements (Eberth), devoid of a sarcolemma (15 to $25 \mu$ broad), and usually divided at their blunt ends, by which means they anastomose and form a network. (Fig. 17, A, B.) The individual muscle-cells contain in their

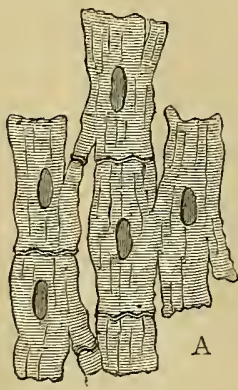

A

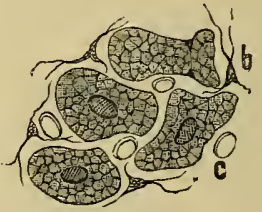

$\mathrm{B}$

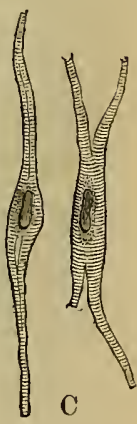

Fig. 17.

A, branched muscular fibres from the heart of a mammal ; B, transverse section of the cardiac fibres ; $b$, connective tissue corpuscles ; $c$, capillaries ; $C$, muscular fibres from the heart of a frog.

centre an oval mucleus, and are held together by a cement which is blackened by silver nitrate, and dissolved by a 33 per cent. solution of caustic potash. This cement is also dissolved by a 40 per cent. solution of nitric acid. The transverse striæ are not very distinct, and not unfrequently there is an appearance of longitudinal striation, produced by a number of very small granules arranged in rows within 
the fibres. The fibres are gathered lengthwise in bundles, or fasciculi, surrounded and separated from each other by delicate processes of the perimysium. When the connective tissue is dissolved by prolonged boiling, these bundles can be isolatea, and constitute the so-called "fibres" of the heart. The transverse sections of the bundles in the auricles are polygonal or rounded, while in the ventricles they are somewhat flattened. [The muscular mass of the heart is called the myocardium, and is invested by fibrous tissue. It is important to notice that the connective tissue of the visceral pericardium (epicardium) is continuous with that of the endocardium by means of the perimysium surrounding the bundles of muscular fibres.] The fine spaces which exist between these bundles form narrow lacunæ, lined with epithelium, and constituting part of the lymphatic system of the heart.

[The cardiac nuscular fibres occupy an intermediate position between striped and plain muscular fibres. Although they are striped they are involuntary, not being directly under the influence of the will, while they contract more slowly than a voluntary muscle of the skeleton.]

[In the frog's heart the muscular fibres are in shape elongated spindles, or fusiform, in this respect resembling the plain inuscle-cells, but they are transversely striped (Fig. 17, C). They are easily isolated by means of a 33 per cent. solution of potash or dilute alcoliol (Weissmann, Ranvier).]

\section{Arrangement of the Cardiac Muscular Fibres, and their Physiological Importance.}

The study of the embryonic heart is the key to a proper understanding of the complicated arrangement of the fibres in the adult heart. The simple tubular heart of the embryo has an outer circular and an inner longitudinal layer of fibres. The septum is formed later; hence, it is clear that a part, at least, of the fibres must be common to the two auricles, and a part also to the two ventricles, since there is, originally, but one chamber in the heart. The muscular fibres of the auricles are, however, completely separated from those of the ventricles by the fibro-cartilaginous rings. In the auricles the fundamental arrangement of the embryonic fibres partly remains, while in the ventricles it becomes obscured as these cavities undergo a sac-like dilatation, and also become twisted in a spiral manner.

(1.) The Muscular Fibres of the Auricles are completely separated from the fibres of the ventricles by the fibrous rings which surround the auriculo-ventricular orifices, and which serve as an attachment for the auriculo-ventricular valves (Fig. 18, I). The auricles are much thinner than the ventricles, and their fibres are generally arranged in two layers; the outer transverse layer is continuous over both auricles, 
whilst the inner one is directed longitudinally. The outer transverse fibres may be traced from the openings of the venous trunks anteriorly and posteriorly over the auricular walls. The longitudinal fibres are specially well marked where they are inserted into the fibro-cartilaginous rings, while in some parts of the anterior auricular wall they are not continuous. In the auricular septum, some fibres, circularly disposed around the fossa ovalis (formerly the embryonic opening of the foramen ovale) are well marked. Circular bands of striped muscle exist around the veins where they open into the heart; these are least marked on the inferior vena cava, and are stronger and reach higher $(2.5 \mathrm{~cm}$.) on the superior vena cava (Fig. 18, II). Similar fibres exist around the four pulmonary veins, where they join the left auricle, and these fibres (which are arranged as an inner circular and an outer longitudinal layer) can be traced to the hilus of the ling in man and some mammals ; in the ape and rat they extend on the pulmonary veins right into the lung. In the mouse and bat, again, the striped muscular fibres pass so far into the lungs that the walls of the smaller veins are largely composed of striped muscle (Stieda).

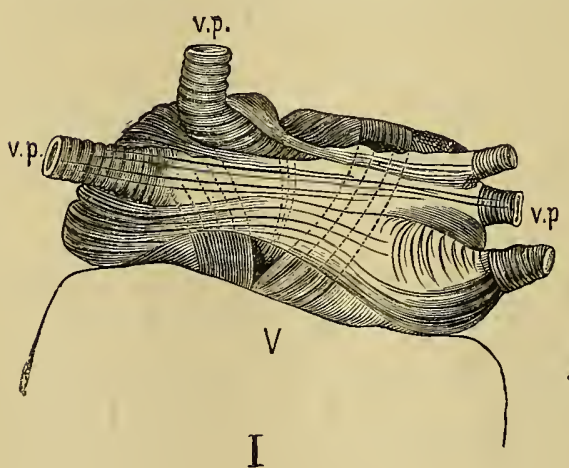

Fig. 18.

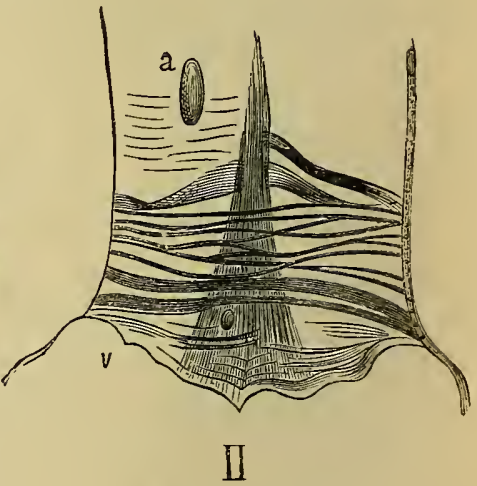

II

I. Course of the muscular fibres on the left auricle-Observe the outer transverse and inner longitudinal fibres, the circular fibres on the pulmonary veins $(v, p)$; $\mathrm{V}$, the left ventricle (John Reid). II. Arrangement of the striped muscular fibres on the superior vewa cava (Elischer)-a, opening of vena azygos; $v$, auricle.

Circular muscular fibres are found where the vena magna cordis enters the heart, and in the valvula thebesii which guards it.

From a physiological point of view the following facts are to be noted as a result of the anatomical arrangement:-

(1.) The auricles contract independently of the ventricles. This is seen when the heart is about to die; then there may be several auricular contractions for one ventricular, and at last only the auricles 
pulsate. The auricular portion of the right auricle beats longest; hence, it is called the "ultimum moriens." Independent rhythmical contractions of the renæ caræe and pulmonary voins are often noticed after the heart has ccased to beat (Haller, Nysten). [This beating can also be observed in those veins in a rabbit after the heart is cut out of the body.]

(2.) The double arrangement of the fibres (transverse and longitudinal) produces a simultaneous and uniform diminution of the auricular carity (such as occurs in most of the hollow viscera).

(3.) The contraction of the circular muscular fibres around the venous orifices, and the subsequent contraction of the auricle, cause these veins to empty themselves into the auricle; and by their presence and action they prevent any large quantity of blood from passing backward into the veins when the auricle contracts. [No valves are present in the superior and inferior vena cava in the adult heart, or in the pulmonary veins, hence the contraction of these. Circular muscular fibres play an important part in preventing any reflux of blood during the contraction of the auricles.]

\section{Arrangement of the Ventricular Fibres.}

(2.) The Muscular Fibres of the Ventricles.-The fibres in the thick wall of the ventricles are arranged in several layers (Fig. 19,A) under the pericardium. First, there is an outer longitudinal layer (A) which is in the form of single bundles on the right ventricle, but forms a complete layer on the left ventricle, where it measures about one-eighth of the thickness of the ventricular wall. A second longitudinal layer of fibres lies on the inner surface of the ventricles, distinctly visible at the orifices, and within the vertically placed papillary muscles, whilst elsewhere it is replaced by the irregularly arranged trabeculæ carneæ. Between these two layers there lies the thickest layer, consisting of more or less transversely-arranged bundles which may be broken up into single layers more or less circularly disposed. The deep lymphatic ressels run between the layers, whilst the blood-vessels lie within the substance of the layers and are surrounded by the primitive bundles of muscular fibres (Henle). All three layers are not completely independent of each other; on the contrary, the fibres which run obliquely form a gradual transition betreen the transverse layers and the inner and outer longitudinal layers. It is not, however, quite correct to assume that the outer longitudinal layer gradually passes into the transverse, and this again into the inner longitudinal layer (as is shown schematically in C); because, as Henle pointed out, the transverse fibres are relatively far greater in amount. In general, the 

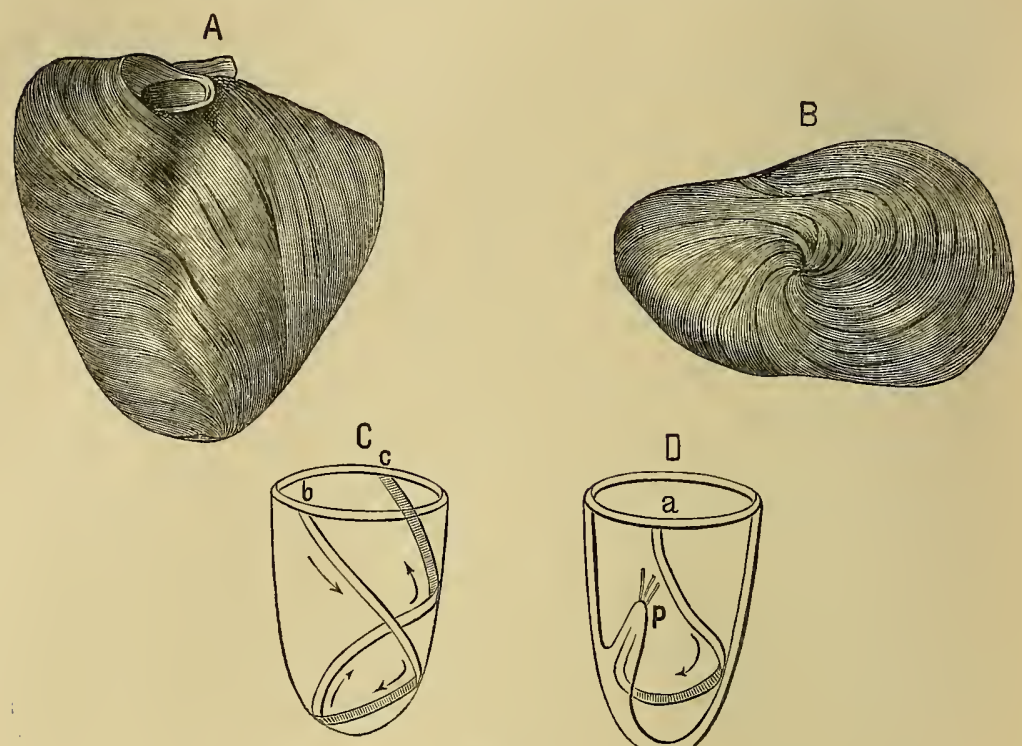

Fig. 19.

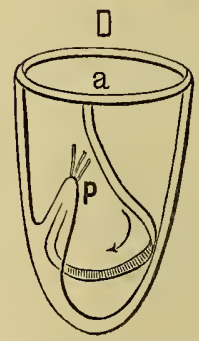

Course of the ventricular muscular fibres-A, On the anterior surface; B, View of the apex with the vortex (Henle); C, Scheme of the course of the fibres within the ventricular wall; D, Fibres passing into a papillary muscle (C. Ludwig).

outer longitudinal fibres are so arranged as to cross the inner longitudinal layer at an acute angle. The tranverse layers lying between these two form gradual transitions between these directions. At the apex of the left ventricle, the onter longitudinal fibres bend or curve so as to meet at the so-called vortex (Wirbel) B, where they enter the muscular substance, and, taking an upward and inward direction, reach the papillary muscles, $D$ (Lower); although it is a mistake to say that all the bundles which ascend to the papillary muscles arise from the vertical fibres of the outer surface: many seem to arise independently within the ventricular wall. According to Henle, all the external longitudinal fibres do not arise from the fibrous rings or the roots of the arteries.

[The assumption that the muscles of the ventricle are arrauged so as to form a figure of 8 , or in loops, seems to be incorrect; thus, fibres are said to arise at the base of the ventricle, to pass over it, and to reach the vortex, where they pass into the interior of the muscular substance, to end either in the papillary muscles, or high up on the inner surface of the heart at its base. Figs. C and D give a schematic representation of this view.]

A special layer of circular muscular fibres, which acts like a true sphincter, surrounds the arterial opening of the left ventricle, and seems to have a certain independence of action (Henle). 
Only the general arrangement of the ventricular muscular fibres has been indicated here (Lower, Casp. Wolff, 1780-92). C. Ludwig (1849), and more recently Pettigrew (1864) have made the subject a special study, and followed out its complications. According to the last observer, there are seven layers in the rentricle, viz., three external, a fourth or central layer, and three internal. These internal layers are continuous with the corresponding extcrnal layers at the apex, thus-one and seven, two and six.

\section{Pericardium, Endocardium, Valves.}

The PERICARDIUIr encloses within its two layers [visceral and parietal] a lymph space-the pericardial space-which contains a small quantity of lymph-the pericardial fluid. It has the structure of a serous membrane, i.e., it consists of connective tissue mixed with fine elastic fibres arranged in the form of a thin delicate membrane, and covered on its free surfaces with a single layer of epithelium or endothelium, composed of irregular, polygonal, flat cells.

A rich lymphatic network lies under the pericardium (fig. 20) and endocardium and also in the deeper layers of the visceral pericardium next the heart, but stomata have not been found leading from the pericardial cavity into these lymphatics, nor do these open. ings exist on the parietal layer. [Salvioli has shown that lymphatic spaces also lie between the muscular bundles.] Around the coronary arteries of the heart exist deposits of fat and lymphvessels (WedI), which lie in the furrows and grooves in the $s u b$. serose of the epicardium (visceral layer).

TheENDocARdu un (according to Luschka) does not represent the intima alone, but the entire wall of a blood-vessel. Next the cavity of the heart, it consists of a single layer of polygonal, flat,

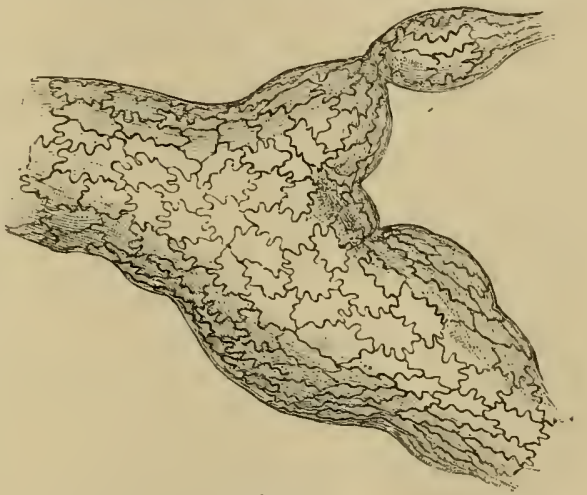

Fig. 20.

Lymphatic of the pericardium epithelium stained with nitrate of silver. nucleated endothelial cells. [Under this there is a nearly homogeneous hyaline layer (fig. 21, a), slightly thicker on the left side, which gives the endocardium its polished appearance.] Then follows, as the basis of the membrane, a layer of fine elastic fibres-stronger in the auricles, and in some places thereof assuming the characters of a fenestrated nembrane. Between these fibres a small quantity of connective tissue exists, which is in larger amount and more areolar in its characters next the myocardium. Bundles of non-striped muscular fibres (few in the auricles) are scattered and arranged for the most part longitudinally between the

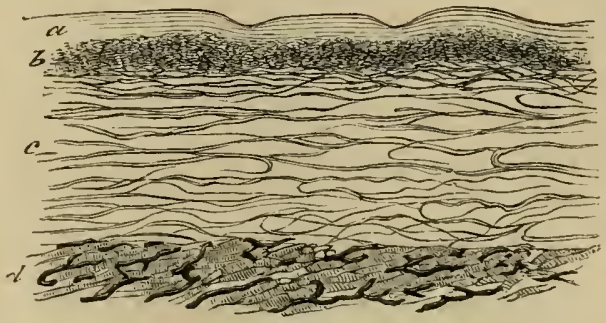

Fig. 21.

Siection of the endocardium- $a$, hyaline layer: $b$, network of fine elastic fibres; c, network of stronger elastic fibres; $d$, myocardium with blood-ressels, which do not pass into the endocardium. 
elastic fibres. These seem evidently meant to resist the distension which is apt to occur when the heart contracts and great pressure is put upon the endocardium. In all cases where high pressure is put upon walls composed of soft parts, we always find muscular fibres present, and never elastic fibres alone. No blood-vessels occur in the endocardium (Langer).

The valves also belong to the endocardium-both the semi-lunar valves of the aorta and pulmonary artery, which prevent the blood from passing back into the ventricles, and the tricuspid (right auriculo-ventricular) and mitral (left auriculo-ventricular), which protect the auricles from the same result. The lower vertebrata have valves in the orifices of the venæ cavæ which prevent regurgitation into them; while in bircls and some mammals these valves exist in a rudimentary condition.

The VALVES are fixed by means of their base to resistant fibrous rings, consisting of elastic and fibrous tissue. They are formed of two layers-(1.) the fibrous, which is a direct continuation of the fibrous rings, and (2.) a layer of elastic elements. The elastic layer of the auriculo-ventricular valves is an immediate prolongation of the endocardium of the auricles, and is directed towards the auricles. The semi-lunar valves have a thin elastic layer directed towards the arteries, which is thickest at their base. The connective-tissue layer directed towards the ventricle is about half the thickness of the valve itself.

Muscular Fibres in the Valves. - The auriculo-ventricular valves also contain striped muscular fibres (Reid, Gussenbauer). Radiating fibres proceed from the auricles and pass into the valves, which, whell the atria contract, retract the valves towards their base, and thus make a larger opening for the passage of the blood into the ventricles; according to Paladino, they raise the valves after they have been pressed down by the blood-current. This observer also described some longitudinal fibres which proceed from the ventricles to enter these valves. There is also a concentric layer of fibres arranged near their point of attachment, and directed more towards their ventricular surface. These fibres seem to contract sphincter-like when the ventricle contracts, and thus approximate the base of the valves, and so prevent too great tension being put upon them. The larger chordæ tendiniæ also contain striped muscle (Oehl), while a delicate muscular network exists in the valvula thebesii and valvula eustachii.

Purkinje's Fibres.-This name is applied to an anastomosing system of grayish fibres which exist in the sub-endocardial tissue of the ventricles, especially in the heart of the sheep and ox. The fibres are made np of polyhedral, clear cells, containing some granular protoplasm, and usually two nuclei (Fig. 22). The margins of the cells are striated. Transition forms are found between these cells and the ordinary cardiac fibres; in fact these cells become continuous with the true fully developed cardiac fibres. They represent cells which have been arrested 
in their development. They are absent in man and the lower vertebrates, but in birds and some mammals they are well marked (Schweigger-Seidel, Ranvicr).

Blood-Vessels occur in the auriculo-ventricular valves only where muscular fibres are present, while the semi-lunar valves are usually devoid of vessels except at their base. The best figures of the blood-ressels of the valves are given by Langer. The network of lymphatics in the endocardium reaches towards the middle of the valves (Eberth and Belajeff).

Weight of the Heart. -According to W. Miiller the proportion between

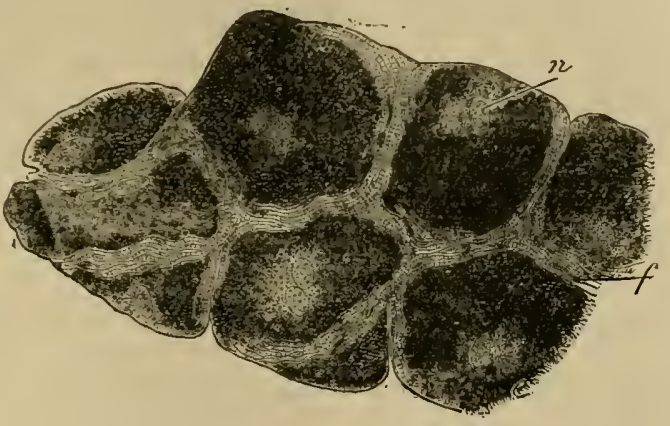

Fig. 2.2.

Purkinje's fibres isolated with dilute alcohol $-c$, cell ; $f$, striated substance ; $n$, nucleus $-\times 300$.

the weight of the body and the heart in the child, and until the body reaches 40 kilos., is 5 grams. of heart-substance to 1 kilo. of body-weight; when the body-weight is from 50 to 90 kilos., the ratio is 1 kilo. to 4 grams. of heartsubstance; at 100 kilos. 3.5 grams. As age advances, the auricles become stronger. The right ventricle is half the weight of the left. The weight of the heart of an adult man is about $9 \mathrm{oz} .(1 \mathrm{oz} .=29.2 \mathrm{grms}$. $)$; female $=8 \frac{1}{2} \mathrm{oz}$. (Clendinning as a mean of 400 observations). [According to Laennec the heart is about the size of the closed fist of the individual]. Blosfield and Dieberg give 346 grms. for the male, and 310 to 340 grms. for the female heart. The specific gravity of the heart-muscle is 1.069 (Kapff).

\section{Self-Steering Action of the Heart.}

Coronary Vessels._Many observations have been made to ascertain whether the orifices of the coronary arteries are covered by the semilunar valves during contraction of the left ventricle (Thebesius, 1739; Brücke, 1854), or whether they are permanently open (Morgagni, 1723; Hyrtl, 1855)-Fig. 23.

Anatomical Investigations. - The two coronary arteries whose branches do not anastomose (Hyrtl, Henle; but this is denied by Krause and L. Langer), arise from the beginning of the aorta in the region of the sinus of Valsalva. The position of origin varies-(1.) either the origins lie within the sinus, or $(2$.$) their openings are only$ partially reached by the margins of the semi-lunar valves (which is usually the case in the left coronary artery of man and the ox), or (3.) their orifices lie clear above the margins of the valves. Post-mortem observations seem to show that during contraction of the ventricle, it is very improbable that the semi-lunar valves constantly cover the origin of the coronary arteries. 
The Self-Steering Action of the Heart.-Brücke attempted to show that during the systole, or contraction of the ventricle, the semi-lunar valves covered the openings of the coronary arteries, so that these vessels could be filled with blood only during the diastole or relaxation of the ventricle. To him it seemed that $\left(a_{\text {. }}\right)$ the diastolic filling of the coronary arteries would help to dilate the ventricles; $(b$.$) on the con-$ trary, a systolic filling of these arteries would oppose the contraction, because the systolic filling and expulsion of the blood from the coronary arteries would diminish the force of the ventricular contraction. To this arrangement, Brïcke gave the above name.

Arguments against Brücke's View.-The following considerations militate against this theory:-(1.) Filling the coronary vessels under a high pressure in a dead heart canses a diminution of the ventricular cavity (v. Wittich). (2.) The chief trunks of the coronary arteries lie in loose sub-pericardial fatty tissue, in the cardiac sulci, hence a dilatation of the ventricle through this agency is most unlikely (Landois). (3.) Experiments on animals have shown that a coronary artery sponts, like all arteries, during the systole of the ventricle. Von Ziemssen found that in the case of a woman (Serafin), who had a large part of the anterior wall of the thorax removed by an operation, the heart being covered only by a thin membrane, the pulse in the coronary arteries was synchronous with the pulse in the pulmonary artery. H. N. Martin and Sedgwick placed a manometer in connection with the coronary artery, and another with the carotid in a large dog, and they found that the pulsations occurred simultaneously. When a coronary artery is divided, the blood flows out continuously, but undergoes acceleration during the systole of the rentricles (Endemann, Perls). (4.) If a strong intermittenj current of water be allowed to flow through a sufficiently wide tube into the left auricle of a fresh pig's heart, so that the water passes into the aorta, and if the aorta be provided with a rertical tube, the water flows continuously from the coronary arteries, and is accelerated during the systole. (5.) It is exceedingly improbable that the coronary arteries should be filled during the cliastole while all the other arteries are filled during systole of the ventricle. (6.) There is always a sufficient quantity of blood in the sinus of Valsalva to fill the arteries during the first part of the systole. (7.) The valves, when raised, are not applied directly to the aortic wall (Hamberger, Rüdinger) even by the most energetic pressure from the ventricie (Sandborg and Worm Müller). (8.) Observations on voluntary muscles have shown that the small arteries dilate during contraction of the muscle, and the blood stream is accelerated. (9.) By the systolic filling of the aorta the arterial path is elongated-this elastic distension is compensated before the diastole occurs. By the recoil of the aortic walls the layer of blood in them is driven backwards and closes the valves (Ceradini). According to Sandborg and Worm Müller, the seniInnar valves close just after the ventricles have begun to relax, which agrees with the curve obtained from the cardiac impulse (Fig. 25a, A).

During the systole, the small arterial trunks lying next the ventricular cavities have to bear a higher pressure than that borne by the aorta, and their lumen must be compressed during the systole so that their contents are propelled towards the reins.

Peculiarities of the Cardiac Blood-Vessels.-The capillary ressels of the myocardium are very numerous, corresponding to the energetic activity of she 
heart. Where they pass into veins, several nnite at once to form a thick venous trunk whereby an easy passage is offered to the blood. The reins are provided with valves so that (1.) during systole of the right auricle the venous stream is interrupted; (2.) during contraction of the ventricles the blood in the coronary reins is similarly accelerated as in the veins of muscles.

The coronary arteries are characterised by their very thick connective tissue and elastic intima, which perhaps accounts for the frequent occurrence of atheroma of these vessels (Henle). Some observers (Hyrtl and Henle) maintain that the coronary arteries do not anastomose, but this is denied by Langer and Krause. Many of the small lower vertebrates have no blood-vessels in their heart-muscle, e.g., frog (Hyrtl).

Coronary Circulation.-The phenomena produced by partial obliteration or ligature of the coronary arteries are most important. In man analogous conditions occur, as in atheroma or calcification of these arteries.

Ligature of the Coronary Arteries.-Sée and others ligatured the coronary arteries in a dog, and found that after 2 minutes the cardiac contractions gave place to twitchings of the muscular fibres, and ultimately the heart ceased to beat. Ligature of the anterior coronary artery alone, or of both its branclies, is sufficient to produce this result.

If the coronary arteries be compressed or tied in a rabbit in the angle between the bulbus aorte and the ventricle, the heart's action is soon weakened, owing to the sudden anæmia and to the retention of the decomposition products of the metabolism in the heart-muscle ( $\mathrm{v}$. Bezold, Erichsen). Ligature of one artery first affects the corresponding ventricle, then the other ventricle, and, last of all, the auricles. Hence, compression of the left coronary artery (with simultaneous artificial respiration in a curarised animal) causes slowing of the contractions, especially of the left ventricle, whilst the right one at first contracts more quickly and then, gradually, its rhythm is slowed. The contractions of the left ventricle are not only slowed but also weakened, whilst the right pulsates with undiminished force. Hence it follows that as the left half of the heart cannot expel the blood in sufficient quantity, the left auricle becomes filled, whilst the right ventricle, not being affected, pumps blood into the lungs. Edema of the lungs is produced by the high pressure in the pulmonary circulation, which is propagated from the right heart through the pulmonary vessels into the left auricle (Samuelson and Grünhagen).

According to Sig. Mayer, protracted dyspnœa causes the left rentricle to beat more feebly sooner than the right, so that the left sicle of the heart becomes congested. Perhaps this may explain the occurrence of pulmonary odema during the death agony.

Cohnheim and v. Schulthess-Rechberg found after ligature of one of the large branches of a coronary artery in a large dog, that at the end of a minute the 
pulsations become discontinuous; several, as it were, do not occur. This intermittence becomes more pronounced, the two sides of the heart do not contract simultaneously (arhythmia), the heart beats more slowly, and the blood-pressure falls. Suddenly, about 105 secs. after the ligature is applied, both rentricles cease to beat, and there is the greatest fall of the blood-pressure. After 10 to 20 secs., twitching movements occur in the ventricles, while the auricles pulsate regularly, and may continue to do so for many minutes, while the ventricles cease to beat altogether after 50 secs. According to Lukjanow, there is a peristaltic condition which operates upwards and downwards, and occurs in the period between the regular contraction and the twitching vibratory movement.

\section{The Movements of the Heart.}

Cardiac Revolution.-The movement of the heart is characterised by an alternate contraction and relaxation of the cardiac walls. The total cardiac movement is called a "CARDIAC REVolution," or a "cardiac cycle," and consists of three acts-the contraction or systole of the auricles, the contraction or systole of the rentricles, and the pause. During the pause the auricles and ventricles are relaxed; during the contraction of the auricles the ventricles are at rest; whilst during the contraction of the ventricles, the auricles are relaxed. The rest during the phase of relaxation is called the dicstole. The following is the sequence of events in the heart during a cardiac revolution:-

\section{Events During a Cardiac Revolution.}

(A.) The Blood Flows into the Auricles, and thus distends them and the auricular appendages. This is caused by-

(1.) The pressure of the blood in the renæ cavæ (right side) and the pulmonary veins (left side) being greater than the pressure in the auricles.

(2.) The elastic traction of the lungs $(\$ 60)$ which, after complete systole of the auricles, pulls asunder the now relaxed and yielding auricular walls. The auricular appendages are also filled at the same time, and they act to a certain extent as accessory reservoirs for the large supply of blood streaming into the auricles.

(B.) The Auricles Contract, and we observe in rapid succession-

(1.) The contraction and emptying of the auricular appendix towards the atrium. Simultaneously the mouths of the reins become narrowed (Haller, Nysten) owing to the contraction of their circular muscular fibres (more especially the superior vena cava and the pulmonary veins).

(2.) The auricular walls contract simultaneously towards the auriculoventricular valves and the venous orifices, whereby 
(3.) The blood is driven into the relaxed ventricles, which are considerably distended thereby.

The contraction of the auricles is followed by

(a.) A slight stagnation of the blood in the large venous trunks, as can be easily observed in a rabbit after division of the pectoral muscles so as to expose the junction of the jugular with the subclavian vein. There is no proper regurgitation of the blood, but only a partial interruption of the inflow into the auricles, because, as already mentioned, the mouths of the veins are contracted, and because the pressure in the superior vena cava and in the pulmonary veins soon holds in equilibrium any reflux of blood; and lastly, because any reflux

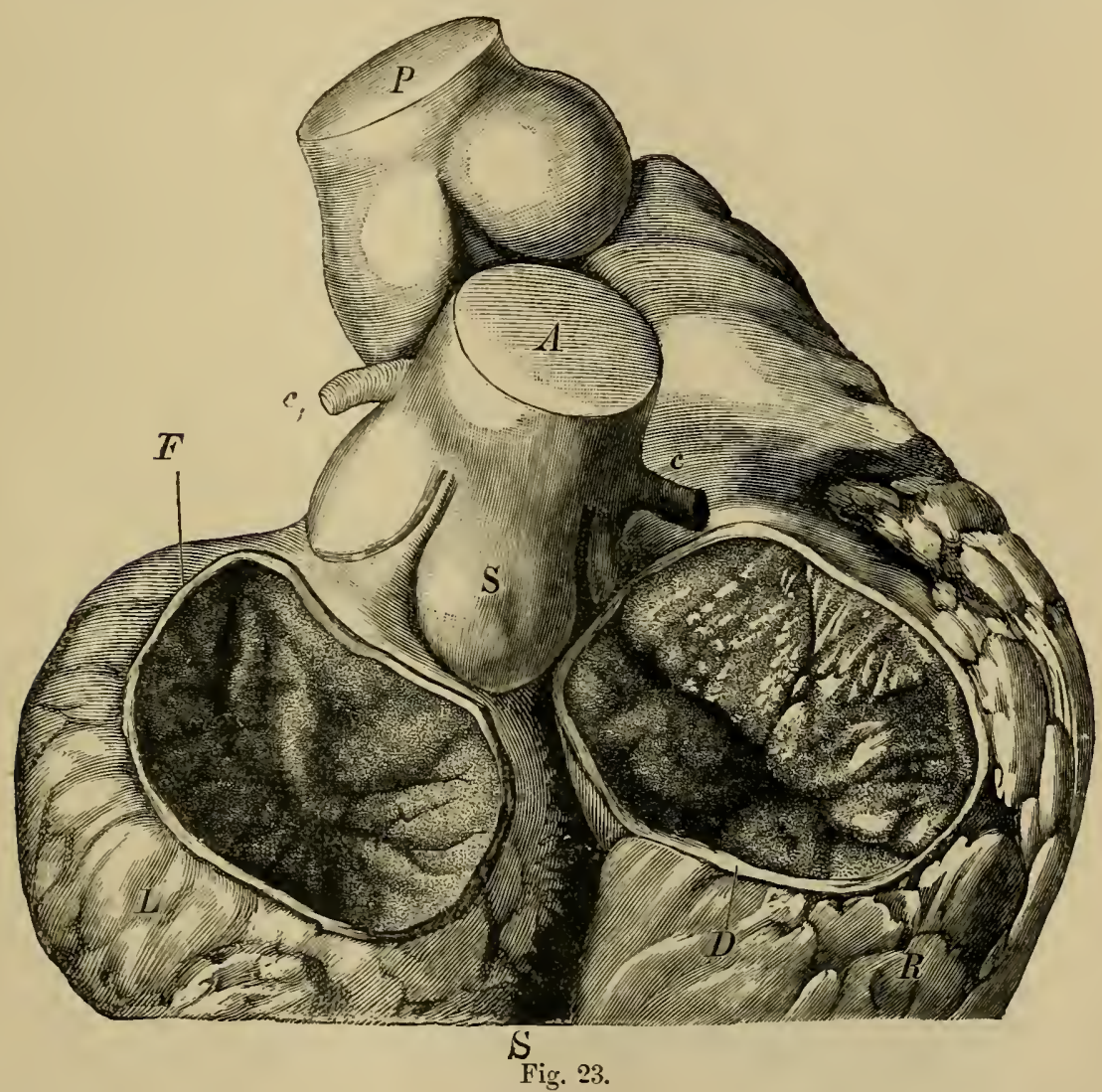

Gypsum cast of the ventricles of the human heart-viewed from behind and above; the walls have been removed, and only the fibrous rings and the auriculoventricular valves are retained-L, left, $R$, right ventricle; $S$, position of septum ; F, left fibrous ring, with mitral valve closed; D, right fibrous ring, with tricuspid closed; $A$, aorta, with the left $\left(C_{1}\right)$ and right $(C)$ coronary arteries; $S$, sinus of valsalva; $P$, pulmonary artery. 
into the cardiac veins is prevented by valves. The movement of the heart causes a regular pulsatile phenomenon in the blood of the venæ cavæ, which under abormal circumstances may produce a venous pulse (see Venous pulse).

(b.) The chief motor effect of the contraction of the auricles is the dilatation of the relaxed ventricle, which has already been dilated to a slight extent by the elastic force of the lungs.

The dilatation of the ventricles has been ascribed to the elasticity of the muscular walls - the strongly contracted ventricular walls (like a compressed indiarubber bag), in virtue of their elasticity, are supposed to return to their normal resting form, and thereby to suck in or aspirate the blood under a negative pressure. Such suction power on the part of the ventricle is, however, only effective to a very slight extent.

(c.) When the ventricles are distended by the inflowing blood, the auriculo-ventricular valves are floated up, partly by the recoil or reflexion of the blood from the ventricular wall, and partly owing to their lighter specific gravity, whereby they easily float into a more or less horizontal position. The valves are also raised to a slight extent by the longitudinal muscular fibres, which pass from the auricles into the cusps of the valve (Paladino).

(C.) The Ventricles now Contract, and simultaneously the auricles relax, whereby

(1.) The muscular walls contract forcibly from all sides, and thus diminish the ventricular cavity.

(2.) The blood is at once pressed against the under-surface of the auriculo-ventricular valves, whose curved margins are opposed to each other like teeth, and are pressed hermetically agaiust each other (Sandborg and Worm Müller). It is impossible for the blood to push the cusps backwards into the auricle, as the chordce tendinice hold fast their margins and surfaces like a taut sail. The margins of the neighbouring

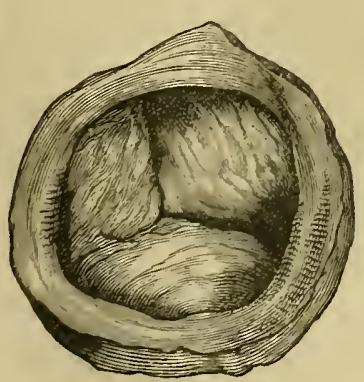

Fig. 24.

The closed semi-lunar valves of the pulmonary artery seen from below. cusps are also kept in apposition by the chordæ tendiniæ from one papillary muscle always passing to the adjoining edges of two cusps (John Reid). The extent to which the ventricular wall is shortened is compensated by the contraction of the papillary muscle, and also of the large muscular chordæ, so that the cusps cannot be pushed into the auricle.

When the valves are closed their surfaces are horizontal, so that even when the ventricles are contracted to their greatest extent, a small amount of blood remains, which is not expelled (Sandborg and Worm Müller). 
(3.) Opening of the Semi-lunar Valves.-When the pressure within the ventricle exceeds that in the arteries, the semi-lunar valves are forced open and stretched like a sail across the pocket-like sinus, without, however, being firmly or directly applied to the wall of the arteries (pulmonary and aorta), and thus the blood enters the arteries.

Negative Pressure in the Ventricle.-Goltz and Gaule found that there was a negative pressure of $23.5 \mathrm{~mm}$. $\mathrm{Hg}$. (dog) in the interior of the ventricle during a certain phase of the heart's action. They surmised that that phase coincided with the diastolic dilataiion, for which they assumed a considerable power of aspiration. Marey observed a similar condition and called it "vacuite postsystolique," but thought that it coincided with the end of the systole; while Moens is of opinion that this negative pressure within the ventricle obtaius shortly before the systole has reached its height, i.e., just before the inner surface of the veutricles and the valves, after the blood is expelled, are nearly in apposition. He explains this aspiration as being due to the formation of an empty space in the veutricle caused by the energetic expulsion of the blood through the aorta and pulmonary artery.

(D.) Pause.-As soon as the ventricular contraction ends, and the ventricles begin to relax, the semi-lunar valves close. The diastole of the ventricles is followed by the PAUSE. Under normal circumstances the right and left halves of the heart always contract or relax uniformly and simultancously.

\section{Pathological Disturbances of Cardiac Action.}

Cardiac Hypertrophy.-All resistances to the movement of the blood through the various compartments of the heart, and through the vessels communicating with it, cause a greater amount of work to be thrown upon the portion of the heart specially related to this part of the circulatory system; consequently, there is produeed an increase in the thickness of the muscular walls and dilatation of the heart. If the resistance or obstacle does not act upon one part of the heart alone, but on parts lying in the onward direction of the blood-stream, these parts also subsequently undergo hypertrophy. If in additiou to the muscular thickening of a part of the heart the cavity is simultaneously dilated, it is spoken of as eccentric hypertrophy or hypertrophy with clilatation.

The obstacles most likely to occur in the blood-vessels are narrowing of the lumen or want of elasticity in their walls ; in the heart, narrowing of the arterial or venous orifices or insufficiency or incompetency of the valves. Incompetency of the valves forms an obstruction to the movement of the blood, by allowing part of the blood to flow back or regurgitate, thus throwing extra work upon the heart.

Thus arise-(1.) Hypertrophy of the left ventricle, owing to resistance in the area of the systemic circulation, especially in the arteries and capillaries-not in the veins. Amongst the causes are, constriction of the orifice or other parts of the aorta, calcification, atheroma, and waut of elasticity of the large arteries aul irregular dilatations in their course (Aneurisms); insufficiency of the aortic valves, in which case the same pressure always obtain within the ventricle and in the aorta ; and lastly, contraction of the kidneys, so that the excretion of water by these organs is diminished. Even in mitral insufficiency compensatory hypertrophy of the left ventricle must occur, owing to the hypertrophy of the left atrium in consequence of the increased blood-pressure in the pulmonary circuit. 
(2.) Hypertrophy of the left auricle occurs in stenosis of the left auriculo-ventricular orifice, or in insufficiency of the mitral valve, and it occurs also as a result of aortic insufficiency, because the auricle has to overcome the continual aortic pressure within the rentricle.

(3.) Hypertrophy of the right ventricle, occurs $(a$.$) when there is resistance to$ the blood-stream through the pulmonary circuit. The resistance may be due to (x.) obliteration of large vascular areas in consequence of destruction, shrinking or compression of the lungs, and the disappearance of numerous capillaries in emphyeematous lungs. ( $\beta_{.}$) Overfilling of the pulmonary circuit with blood in consequence of stenosis of the left auriculo-ventricular orifice or mitral insufficiencyconsequent upon hypertrophy of the left auricle resulting from aortic insufficiency. (b.) Hypertrophy of the right ventricle will also occur when the valves of the pulmonary artery are insufficient, thus permitting the blood to flow back into the rentricle, so that the pressure within the pulmonary artery prevails within the right ventricle (very rare).

(4.) Hypertrophy of the right ausicle occurs in consequence of the last-named condition, and also from stenosis of the tricuspid orifice, or insufficiency of the tricuspid valve (rare). If several lesions occur simultaneously, the result is complex.

Artificial Injury to the Valves.-0. Rosenbach has made experiments on the action of the heart when its valves are injured artificially. If the aortic valves are perforated, with or without simultaneous injury to the mitral or tricuspid valves, the heart does more work; thus the physical defect is overcome for a time, so that the blood-pressure does not fall. The heart seems to have a store of reserve energy, which is called into play. Soon, however, dilatation takes place, on account of the regurgitation of the blood into the heart. Hypertrophy then occurs, but the compensation meanwhile must be obtained through the reserve energy of the heart.

Impeded Diastole.-Among causes which hinder the diastole of the heart arecopious effusions into the pericardium, or pressure of tumours upon the heart. The systole is greatly interfered with when the heart is united to the pericardium and to the connective tissue in the mediastinum. As a consequence the connective tissue, and eren the thoracic wall, are drawn in during contraction of the heart, so that there is a retraction of the region of the apex-beat cluring systole, and $a$ protrusion of this part during the diastole.

\section{The Apex-Beat-The Cardiogram.}

Cardiac Impulse.-By the term "apex-beat", or cardiac impulse, is understood under normal circumstances an elevation (perceptible to touch and sight) in a circumscribed area of the fifth left intercostal space, caused by the movement of the heart. [The apex-beat is felt in the fifth left intercostal space, two inches below the nipple, and one inch to its sternal side.] The impulse is more rarely felt in the fourth intercostal space, and it is much less distinct when the heart beats against the fifth rib itself. The position and force of the cardiac impulse vary with changes in the position of the body.

[Methods.-To obtain a curve of the apex-beat or a cardiogram, we may use one or other of the following cardiographs (Fig. 25). Fig. 25, A, is the first form used by Marey, and it consists of an oval wooden capsule applied in an 
air-tight manner over the apex-beat. The disc, $p$, capable of being regulated by the screw, $s$, presses upon the region of the apex-beat, while $t$ is a tube which may be connected with a registering tambour (Fig. 2S). $B$ is an improved form of the instrument, consisting essentially of a tambour, while attached to the membrane is a button, $p$, to be applied over the apex-beat. The movements of the air within the capsule are communicated by the tube, $t$, to a registering tambour. Fig. 25, C, is the pansphygmograph of Brondgeest, which consists of a Marey's tambour, in an iron horse-shoe frame, and adjustable by means of a screw, s. BurdonSanclerson's cardiograph is shown in D. The button, $p$, carried by the spring, $e$, does not rest upon the caoutchouc nembrane, but on an aluminium plate attached to it. The apparatus is adjusted to the chest by three supports. Fig. 25, E, shows a modified instrument on the same principle by Grummach and $\mathrm{v}$. Knoll. In all these figures the $t$ indicates the cxit-tube communicating with a registering tambour (Fig. 2S). D and E may be used for other purposes, e.g., for the pulse, so that they are polygraphs. See also Fig. 52.]

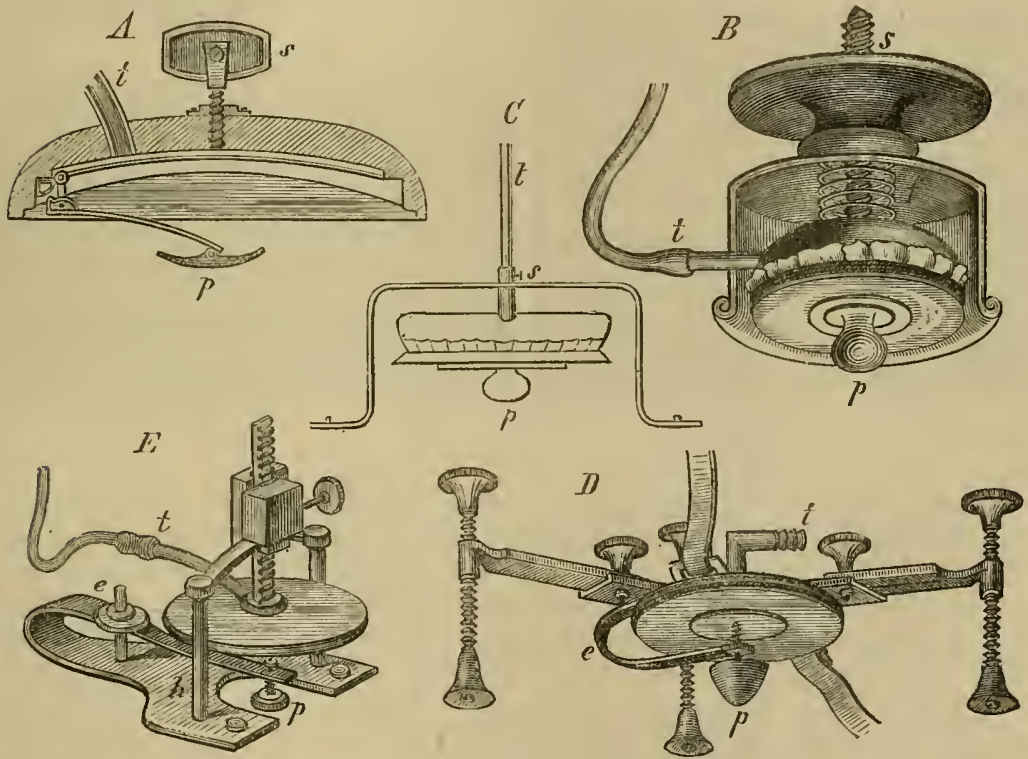

Fig. 25.

Various cardiographs-A, original form as used by Marey; $\mathrm{B}$, improved form by Marey; C, Pansphygmograph of Brondgeest; D, Cardiograph of BurdonSandersoll ; E, that of Grummach and v. Knoll.

Fig. $25 a, A$, shows the cardiogram or the impulse-curve of the heart of a healthy man; B, that of a dog, obtained by means of a sphygmograph. In both the following points are to be noticed- $a, b$, corresponds to the time of the pause and the contraction of the auricles. As the atria contract in the direction of the axis of the heart from the right and above towards the left and below, the apex of the heart moves towards the intercostal space. The two or three smaller 
A

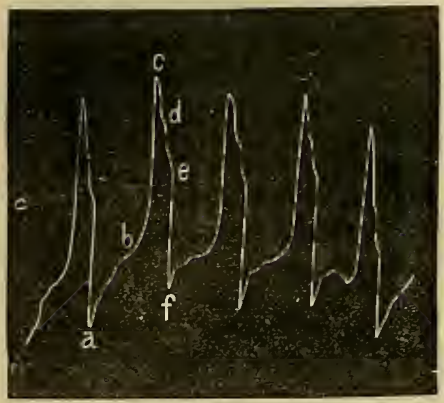

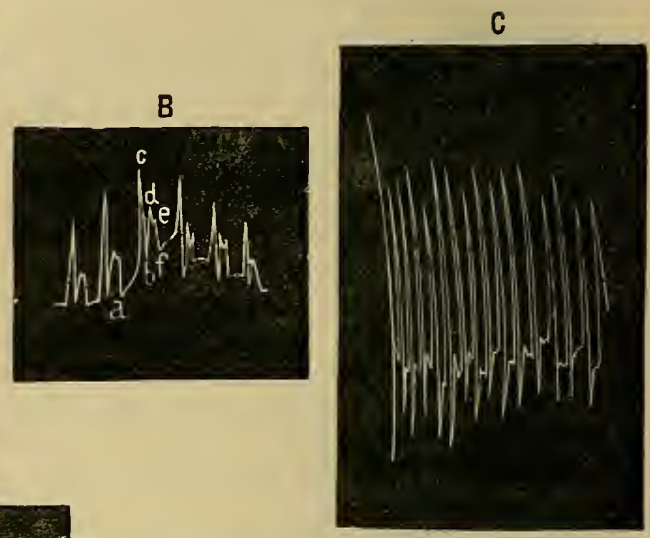

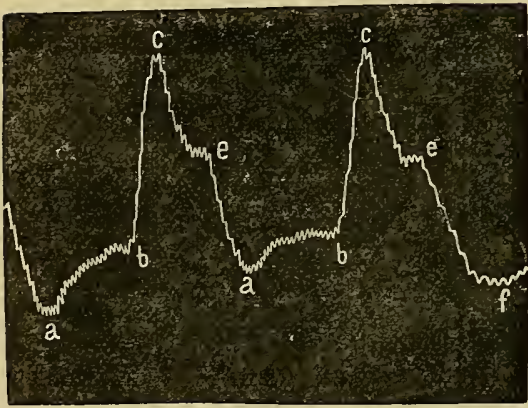

D

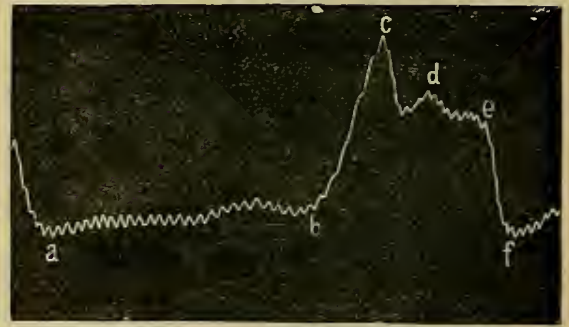

$E$

Fig. 2こ๘.

Curves taken from the apex-beat-A, normal curve from mau; $B$, from a dog; $\mathrm{C}$, very rapid curve from a dog; $\mathrm{D}$ and $\mathrm{E}$, normal curves from a man, registered on a vibrating glass-plate where each indentation $=0.01613$ secs. In all the curves, $a, b$, means contraction of the auricles; $b, c$, ventricular systole; $d$, closure of the aortic valves; $e$, closure of the pulmonary artery valves; $e, f$, relaxation or cliastole of the ventricle.

elevations are perhaps caused by the contractions of the ends of the veins, the auricular appendices, and the atria themselves.

Some observers ascribe the small elevations occurring before $b$ to the filling of the ventricle during the diastole, whereby it is pressed against the intercostal space (Manrer, Griitzner').

The portion, $b, c$, which communicates the greatest impulse to the instrument, and also to one's hand when it is placed on the apexbeat, is caused by the contraction of the ventricle, and during it the first sound of the heart occurs. Frequently, but erroneously, the cardiac impulse has been ascribed to this contraction of the ventricle. It however, is due to all those conditions which cause an elevation in the region of the apex-beat. 
The cause of the rentricular impulse has been much discussed. It depends upon the following :-

(1.) The base of the heart (auriculo-ventricular groove) represents during diastole a transversely-placed ellipse, while during contraction it has a more circular figure. Thus, the long diameter of the ellipse is diminished in the cat from 28 to $22.5 \mathrm{~mm}$. (C. Ludwig); the small diameter is increased ( $\left(\frac{1}{10}\right.$ to $\left.\frac{1}{4}\right)$, while the base is brought nearer to the chest-wall (Arnold, Ludwig)-Fig. 26, I. This alone does not cause the impulse, but the basis of the heart, being hardened during the systole and brought nearer to the chest-wall, allows the apex to execute the morement which causes the impulse.

(2.) During relaxation, the ventricle lies with its apex obliquely downwards, and with its long axis in an oblique direction-so that the angles formed by the axis of the ventricles with the diameter of the base are unequal-represents a regular cone, with its axis at right angles to its base. Hence, the apex must be erected from below and behind, forwards and upwards (Harvey-"cor sese erigere"), and when hardened during systole presses itself into the intercostal space (Ludwig)-Fig. 26, II.
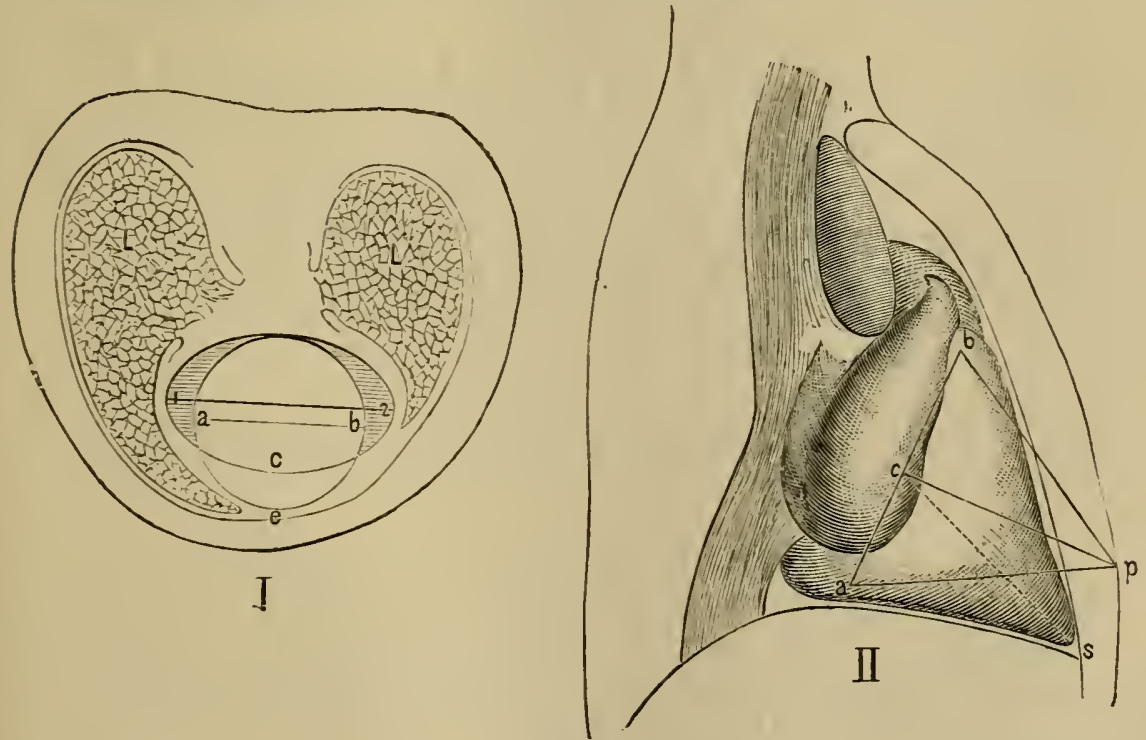

Fig. 26.

I, Schematic horizontal section through the heart and lungs, and the thoracic walls, to show the change of shape which the base of the heart undergoes during contraction of the ventricle-1, 2, transverse diameter of the ventricle during diastole; $c$, position of the thoracic wall during diastole; $a, b$, transverse diameter of the heart during systole, with $e$, the position of the anterior thoracic wall during systole. II, Side-view of the heart-s, apex during diastole; $p$, the same during systole (C. Ludwig). 
(3.) The ventricle undergoes during systole a slight spiral twisting on its long axis ("lateralem inclinationem"-Harvey), so that the apex is brought from behind more forward, and thus a greater portion of the left ventricle is turned to the front. This rotation is caused by the muscular fibres of the ventricles, which proceed from that part of the fibrous rings between the auricles and ventricles which lies next the anterior thoracic wall. The fibres pass from above obliquely downwards, and to the left, and also run in part upon the pasterior surface of the ventricle. When they contract in the axis of their direction, they tend to raise the apex, and also to bring more of the posterior surface of the heart in relation with the anterior thoracic wall (Harvey, Kuirschner, Wilckens). This rotation is favoured by the slightly spiral arrangement of the aorta and pulmonary artery (Kornitzer).

These are the most important causes, but minor causes are as follows :-

(4.) The "reaction impulse" is that movement which the ventricles are said to undergo (like an exploded gun or rocket) at the moment when the blood is discharged into the aorta and pulmonary artery, whereby the apex goes in the opposite direction-i.e., downwards and slightly outwards (Alderson 1825, Gutbrod, Skoda, Hiffelsheim). Landois, however, has shown that the mass of blood is discharged into the vessels 0.08 of a second after the beginning of the systole, while the cardiac impulse occurs with the first sound.

(5.) When the blood is discharged into the aorta and pulmonary artery, these vessels are slightly elongated, owing to the increased bloodpressure (Senac). As the heart is suspended from above by these vessels, the apex is pressed slightly downwards and forwards towards the intercostal space (?)

Guttmann and Jahn observed that the cardiac impulse disappeared after sudden ligature of the aorta and pulmonary artery, while Chauveau and Rosensteiu maintain that it persists.

As the cardiac impulse is observed in the empty hearts of dead animals, (4) and (5) are certainly of only second-rate importance. Filehne and Pentzoldt maintain that the apex during systole does not move to the left and downwards, as must be the case in (4) and (5), but that it moves upwards and to the right-a result corroborated by v. Ziemssen, which, however, is disputed by Lösch.

It is to be remembered that as the apex is always applied to the chest-wall, separated from it merely by the thin margin of the lung, it only presses against the intercostal space during systole (Kiwisch).

After the apex of the curve, $c$, has been reached at the end of the 
systole, the curve falls rapidly, as the ventricle rapidly becomes relaxed. In the descending part of the curve, at $d$ and $e$, are two elevations, which occur simultaneously with the second sound. These are caused by the sudden closure of the semi-lunar valves, which, occurring suddenly, is propagated through the axis of the ventricle to its apex, and thus causes a vibration of the intercostal space; $d$ corresponds to the closure of the aortic valves, and $e$ to the closure of the pulmonary valves. The closure of the valves in these two vessels is not simultaneous, but is separated by an interval of 0.05 to $0.09 \mathrm{sec}$. The aortic valves close sooner on account of the greater blood-pressure there (Landois, 1876, Ott and Haas, Malbranc, Maurer, Grïtzner, Langendorff, v. Ziemssen, and Ter Gregorianz).

Complete diastolic relaxation of the ventricle occurs from $c$ to $f$ in the curve. It is clear, then, that the cardiac impulse is caused chiefly by the contraction of the ventricles, while the auricular systole and the vibration caused by the closure of the semi-lunar valves are also concerned in its production.

\section{The Time 0ccupied by the Cardiac Movements.}

Methods.-The time occupied by the various phases of the movements of the heart may be cletermined by studying the apex-beat curve.

(1.) If we know at what rate the plate on which the curve was obtained moved during the experiment, of course all that is necessary is to measure the distance, and so calculate the time occupied by any event (see Pulse).

(2.) It is preferable, however, to cause a tuning-fork, whose rate of vibration is known, to write its vibrations under the curve of the apex-beat, or the curve may be written upon a plate attached to a vibrating tuning-fork (Fig. $25 a, \mathrm{D}, \mathrm{E})$. Such a curve contains fine teeth, caused by the vibrations of the tuning-fork. D and $\mathrm{E}$ are curves obtained from the carcliac impulse in this way from healthy students. In $\mathrm{D}$ the notch $d$, is not indicated. Each complete vibration of the tuning-fork, reckoned from apex to apex of the teeth $=0.01613$ sec., so that it is simply necessary to count the number of teeth and multiply to obtain the time. The values obtained vary within certain limits even in health.

Pause and Contraction of Auricles. - The value of $a b=$ pause + contraction of the auricles-is subject to the greatest variation, and depends chiefly upon the number of heart-beats per minute. The more quickly the heart beats, the smaller is the pause, and conversely. In some curves, even when the heart beats slowly, it is scarcely possible to distinguish the auricular contraction (indicated by a rise) from the part of the curve corresponding to the pause (indicated by a horizontal line). In one case (heart-beats 55 per minute) the pause $=0.4$ sec., the auricular contraction $=0.177 \mathrm{sec}$. In Fig. $25 a, \mathrm{~A}$, the time occupied by the pause the auricular contraction ( 74 beats per minute) $=0.5$ sec. 
In $\mathrm{D}$ the $a b=19$ to 20 vibrations $=0.32$ sec.; in $\mathrm{E}=26$ vibrations $=0.42 \mathrm{sec}$.

Ventricular Systole.-The ventricular systole is calculated from the beginning of the contraction, $b$ to $e$, when the semi-lunar valves are closed; it lasts from the first to the second sound. It also varies somewhat, but is more constant. When the heart beats rapidly, it is somewhat less-during slow action, greater. In $\mathrm{E}=0.32$ sec.; in $\mathrm{D}$ $=0.29$ sec. ; with 55 beats per minute Landois found it $=0.34$, with a very high rate of beating $=0.199 \mathrm{sec}$.

When the ventricle beats feebly; it contracts more slowly, as can be shown by applying the registering apparatus to the heart of an animal just killed. In Fig. 27 , from the ventricle of a rabbit just killed, the slow heart-beats, B, are seen to last longest.

A

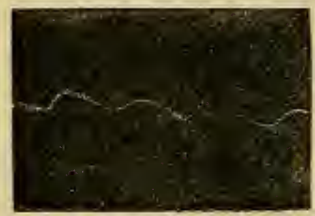

B

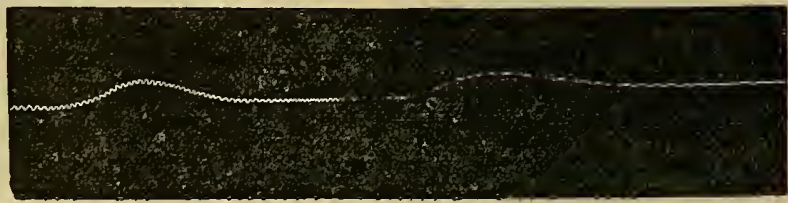

Fig. 27.

Curves obtained from the ventricle of a rabbit, and written upon a vibrating plate attached to a tuning-fork (vibration $=0.01613$ sec.) $-\mathrm{A}$, tolerably soon after death; $B$, from the dying ventricle.

In calculating the time occupied by the veutricular systole we must remember(1.) The time between the two sounds of the heart, i.e., from the beginning of the first to the end of the second sound $(b$ to $e$ ). (2.) The time the blood flows into the corta, which comes to an end at the depression between $c$ and $d$ (in Fig. 25a, E). Its commencement, however, does not coincide with $b$, as the aortic valves open 0.085 (Landois) to 0.073 (Rive) sec. after the beginning of the ventricular systole. Hence the aortic current lasts 0.08 to $0.09 \mathrm{sec}$.

This is calculated in the following way:-The time between the first sound of the heart and the pulse in the axillary artery is $0.137 \mathrm{sec}$, and of this time 0.052 sec. are occupied in the propagation of the pulse-wave along the $30 \mathrm{~cm}$. of artery lying between the root of the aorta and the axilla. Thus the pulse-wave in the aorta occurs 0.137 minus $0.052=0.085$ sec. after the beginning of the first sound.

The current in the pulmonary artery is interrupted in the depression between $d$ and $e$. (3.) Lastly, the time occupied by the muscular contraction of the ventricle, which begins at $b$, reaches its greatest extent at $c$, and is completely relaxed at $f$. The apex of the curve, $c$, may be higher or lower according to the flexibility of the intercostal space, hence the position of $c$ varies. In hypertrophy with dilatation of the left ventricle, the duration of the ventricular contraction does not greatly exceed the normal.

The time which elapses between $d$ and $e$, i.e., between the complete closure of the arortic and pulmonary valves, is greater the more the pressure in the aorta exceeds that in the pulmonary artery, as the 
valves are closed by the pressure from above, and the difference in time may be 0.05 see., or even double that time, in which ease the seeond sound appears double (compare p. 94). If the aortic pressure diminishes while that in the pulmonary artery rises, $d$ and $e$ may be so near each other that they are no longer marked as distinct elements in the curve.

The time, $e, f$, during which the ventricle relaxes varies somewhat: 0.1 sec. may be taken as a mean.

Accelerated Cardiac Action.-When the action of the heart is greatly accelerated, the panse is considerably shortened in the first instanee (Donders), and to a less extent the time of contraction of the auricles and ventricles. When the pulse-rate is very rapid, the systole of the atria coincides with the closure of the arterial valves of the preceding eontraction, as is shown in Fig. 25a, C ( $\log )$.

In registering the cardiac impulse, the apparatus is separated by a greater or less extent of soft parts from the heart itself, so that in all cases the intercostal tissues do not follow exactly the movements of the heart, and thus the eurve obtained may not coincide mathematically with the movements of the heart. It is desirable that curves be obtained from persons whose hearts are exposed, i.e., in cases of ectopia cordis.

Gibson inscribed cardiograms from the heart of a man with cleft sternum. The following were the results obtained:-Auricular contraction $=0 \cdot 115$; ventricular contraction $(b, d)=0.28$; difference between closure of valves $(d, e)=0.09$ ventricular diastole $(e, f)=0.11$; panse $=0.45 \mathrm{sec}$.

Endocardial Pressure.-In large mammals, such as the horse, Chauvean and Marey determined the duration of the events that occur within the heart, and also the endocardial pressure, by means of a cardiac sound. Small elastie bags attached to tubes were introduced through the jugular vein into the right auricle and ventricle. Each of these tubes was connected with a registering tambour (Fig. 28), and simultaneous tracings of the variations of pressure within the eavities of the heart were obtained by causing the writing-points of the levers of the tambours to write upon a revolving cylinder.

Fig. 29, A, gives the result obtained when the elastic bag was placed in the right auricle, introduced through

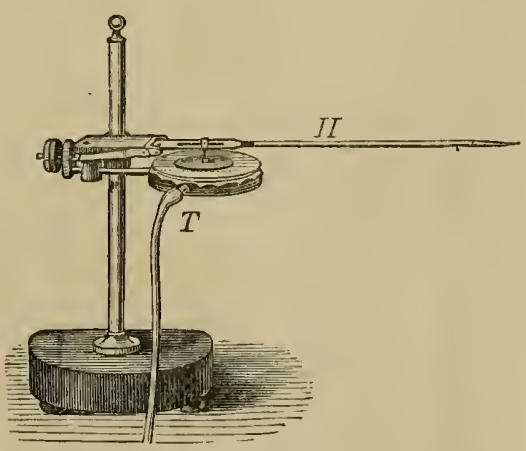

Fig. 2S.

Marey's registering tambour, consisting of a metallic capsule, $T$, with thin indiarubber stretched over it, and bearing an aluminium disc, which acts upon the writing lever, $H$. By means of a thick-walled caoutchouc tube, it may be connected with any system containing air, so as to record variations of pressure.

the jugular vein and superior vena cava; $B$, when it was pushed through the tricuspid valve into the right ventricle; $\mathrm{D}$, in the root of the aorta, pushed in 
through the carotid; $\mathrm{C}$, pushed past the semi-lunar valves into the left ventricle; while at $\mathrm{E}$ a similar bag has been placed externally between the heart's apex and the inner wall of the chest. In all cases $v=$ auricular contraction; $\mathrm{V}$, that of the ventricle; $s$, closure of semi-lunar valves, sooner in $\mathrm{C}$ than $\mathrm{B} ; \mathrm{P}=$ pause.

Method.-The carcliac sound consists of a tube containing two separate airpassages, and in connection with each of these there is a small elastic bag or ampulla. One of the bags is fixed to the free end of the sound, and communicates with one of the air-passages. The other bag is placed in connection with the second air-passage in the sound, and at such a distance, that, when the former bag lies within the ventricle, the latter is in the auricle. Each bag and air-tube in connection with it, is connected with a Marey's tambour, Fig. 28, provided with a lever which inscribes its movements upon a revolving cylinder. Any variation of pressure within the auricle or ventricle will affect the elastic ampullæ, and thus raise or depress the lever. Care must be taken that the writing-points of the levers, are placed exactly above each other. A tracing of the cardiac impulse is taken simultaneously by means of a cardiograph attached to a separate tambour.

It has still to be determined whether the auricles and ventricles act alternately, so that at the moment of the beginning of the ventricular

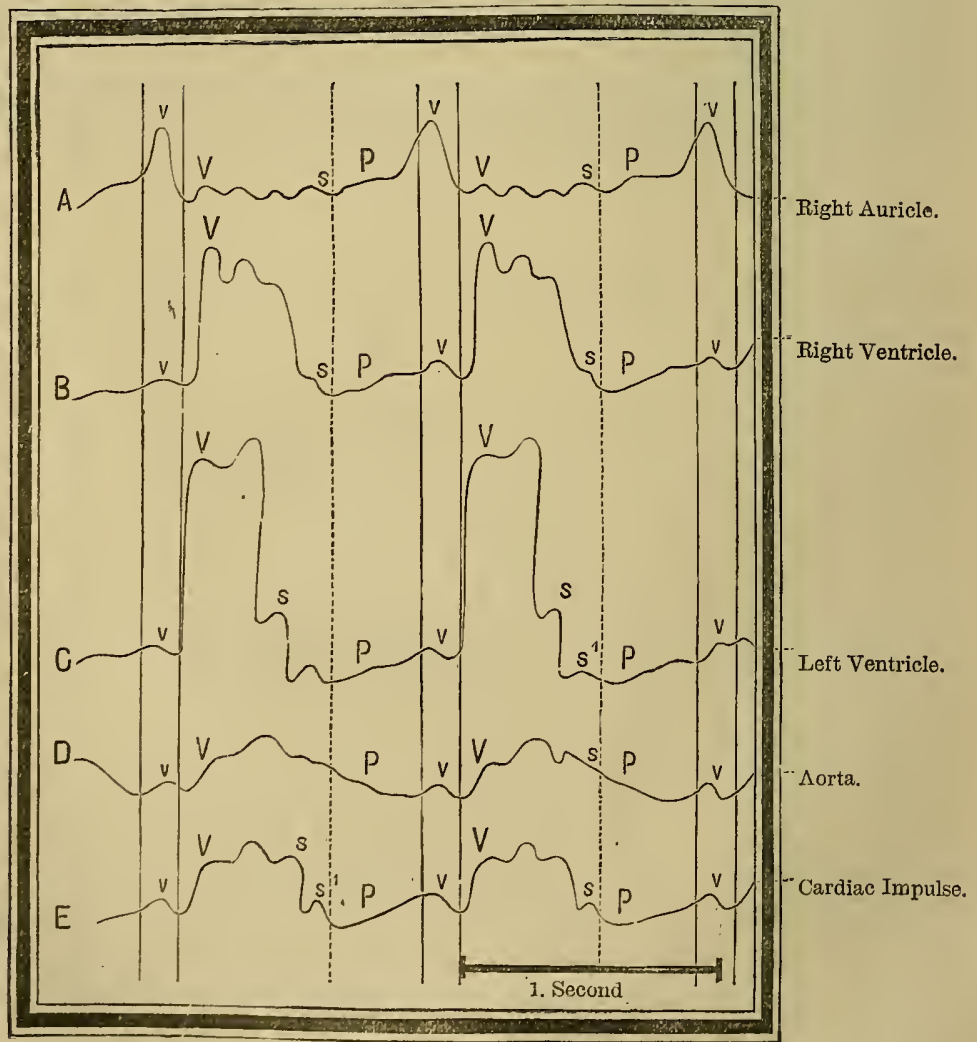

Fig. 29.

Curves obtained from the heart by the cardiac sound (Chauveau and Marey). 
contraction the auricles relax, or whether the ventrieles are contracted while the auricles still remain slightly contracted, so that the whole heart is contracted for a short time at least. The latter view was supported by Harvey, Donders, Schiff, and others, while Haller and many of the more recent observers support the view that the action of the auricles and ventricles alternates. In the case of Frau Serafin, whose heart was exposed, v. Ziemssen and Ter Gregorianz obtained curves from the auricles, which showed that the contraction of the auricles continued even after the commencement of the ventricular systole. In Marey's curve (Fig. 29) the contraction of the ventricle is represented as following that of the auricle.

\section{Pathological Disturbances of the Cardiac Impulse.}

Change in the Position of the Apex-beat.-The position of the cardiac impulse is changed-(1) by the accumulation of fluids (serum, pus, blood) or gas in one pleural cavity. A copious effusion into the left pleural cavity compresses the lung, and may displace the heart towards the right side, while effusion on the right side may push the heart more to the left. As the right heart must make a greater effort to propel the blood through the compressed lung, the cardiac impulse is usually increased. Advanced emphysema of the lung, causing the diaphragm to be pressed dowuwards, displaces the heart downwards and inwards, while conversely the pushing or pulling up of the diaphragm (by contraction of the lung, or through pressure from below) causes the apex-beat to be displaced upwards (even to the third intercostal space), and also slightly to the left. Thickening of the muscular walls and dilatation of the cavities (hypertrophy with dilatation) of the left ventricle make that ventricle longer and broader, while the increased cardiac impulse may be felt to the left of the mammary line, and in the axillary line in the sixth, seventh, or even eighth intercostal space. Hypertrophy, with dilatation of the right side, increases the breadth of the heart, while the cardiac impulse is felt more to the right, even to the right of the sternum, and at the same time it may be slightly beyond the left mammary line. In the rare cases where the heart is transposed, the apex-beat is felt on the right side. When the cardiac impulse goes to the left of the left mammary line, or to the right of the parasternal line, the heart is increased in breadth, and there is hypertrophy of the heart. A greatly increased cardiac impulse may extend to several intercostal spaces.

The cardiac impulse is abnormally vecukened during atrophy and degeneration of the cardiac muscle, or by weakening of the innervation of the cardiac ganglia. It is also weakened when the heart is separated from the chest-wall owing to the collection of fluids or air in the pericarditum, or by a greatly distended left lung; and, indeed, when the left side of the chest is filled with fluid, the cardiac impulse may be extinguished. The same occurs when the left ventricle is very imperfectly filled during its contraction (in consequence of marked narrowing of the mitral orifice), or when it can only empty itself very slowly and gradually, as during marked narrowing of the aortic orifice.

An increase of the cardiac impulse occurs during hypertrophy of the walls, as well as after the influence of various stimuli (psychical, inflammatory, febrile, toxic) which affect the cardiac ganglia. Great hypertrophy of the left ventricle 
causes the heart to heave, so that a part of the left chest-wall may be raised and also vibrate during systole.

A falling in of the anterior wall of the chest during cardiac systole occurs in the third and fourth interspaces, not unfrequently under normal circumstances, sometimes during increased cardiac action, and in eccentric hypertrophy of the ventricles. As the heart's apex is slightly displaced, and the ventricle becomes slightly smaller during its systole, the empty space is filled by the yielding soft parts of the intercostal space. When the heart is united with the pericardium and the surrounding connective tissue, which renders systolic locomotion of the heart impossible, a falling in of the chest-wall during systole takes the place of the cardiac impulse (Skoda). During the diastole a diastolic cardiac impulse of the corresponding part of the chest-wall may be said to occur.

Changes in the cardiac impulse are best ascertained by taking graphic representations of the cardiac impulse, and studying the curves so obtained. This method has been largely followed by many clinicians.

In all the following curves, $a, b$, means auricular contraction; $b$, $c$, ventricular
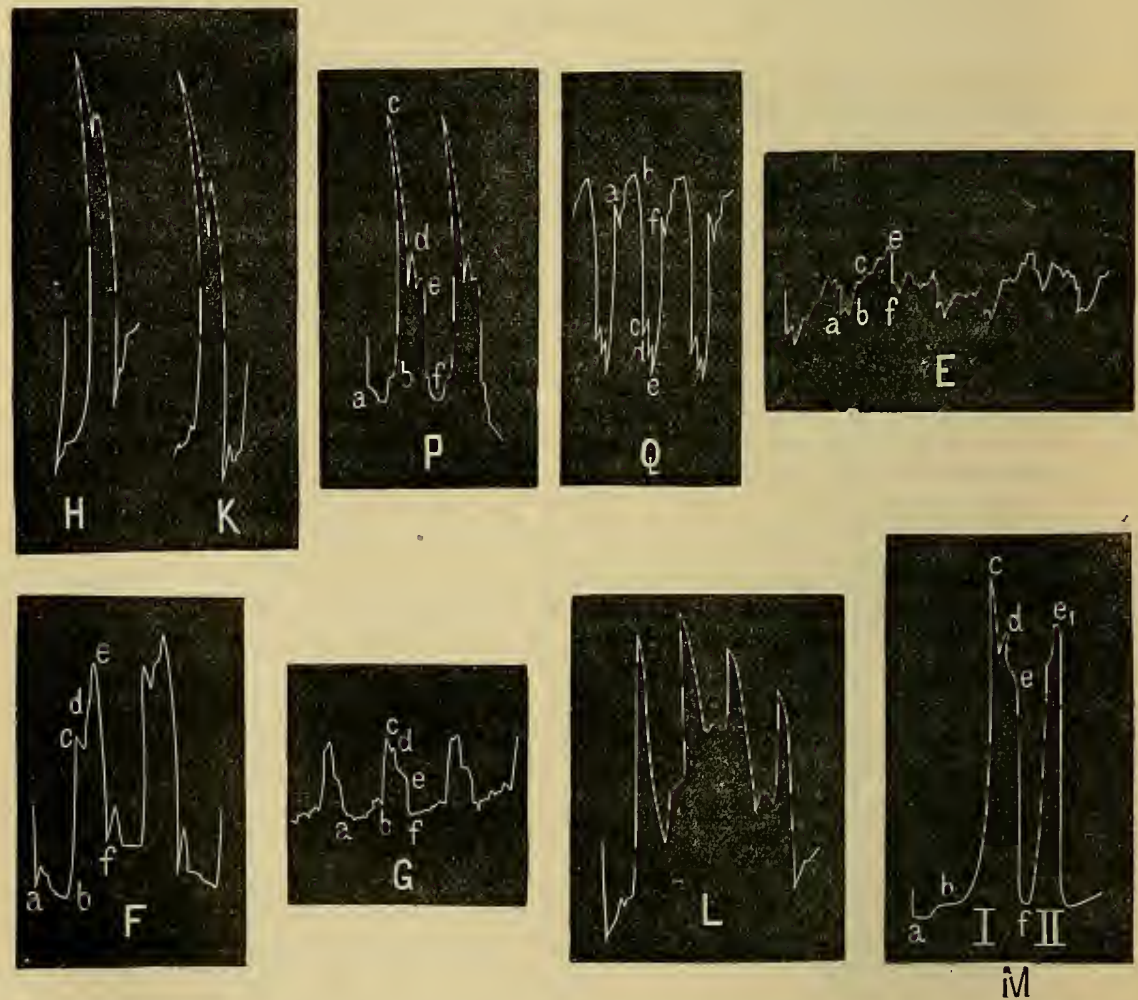

Fig. 30.

Various forms of eurves obtained from the cardiac impulse- $a, b$, Contraction of auricles ; $b, c$, ventricular systole ; $d$, closure of aortic, and ' $e$ of pulmonary valves; $e, f$, diastole of ventricle; $\mathrm{P}, \mathrm{Q}$, hypertrophy and dilatation of the left ventricle; $\mathrm{E}$, stenosis of the aortic orifice; $\mathrm{F}$, mitral insufficiency; $\mathrm{G}$, mitral stenosis; $L$, nervous palpitation in Basedow's disease; $M$, case of so-called hemisystole. 
contraction; $d$, closure of the aortic valves, and $e$ of the pulmonary; $e, f$, the time the ventricle is relaxed (Fig. 30 .)

In curve $\mathrm{P}$ (much reduced), taken from a case of marlied hypertrophy with dilatation, the ventricular contraction, $b c$, is usually very great, while the time occupied by the contraction is not much increased. $\mathrm{P}$ and $\mathrm{Q}$ were obtained from a man suffering from marked eccentric hypertrophy of the left ventricle, in consequence of insufficiency of the aortic valves. Curve $Q$ was taken intentionally over the auriculo-ventricular groove, where a falling in of the chest-wall occurred during systole; nevertheless, the individual events occurring in the heart are indicated.

Fig. $\mathrm{E}$ is from a case of aortic stenosis. The auricular contraction $(a, b)$ lasts only a short time; the ventricular systole is obviously lengthened, and after a short elevation $(b, c)$ shows a series of fine indentations $(c, c)$ caused by the blood being pressed through the narrowed and roughened aorta.

Fig. $\mathbf{F}$, from a case of insufficiency of the mitral valve, shows $(a, b)$ well marked on account of the increased activity of the left auricle, while the shock $(d)$ from the closure of the aortic valves is small on account of the diminished tension in the arterial system. On the other hand, the shock from the accentuated pulmonary sound (e) is very great, and is in the apex of the curve. On account of the great tension in the pulmonary artery, the second pulmonary tone may be so strong, and succeed the second aortic sound $(d)$ so rapidly, that both almost merge completely into each other $(\mathrm{H}$ and $\mathrm{K})$.

The curve of stenosis of the mitral orifice $(G)$ shows a long irregular notched auricular contraction $(a, b)$ caused by the blood being forced through an irregular narrow orifice. The ventricular contraction $(b, c)$ is feeble on account of its being imperfectly filled. The closures of the two valves, $d$ and $e$, are relatively far apart, and one can hear distinctly a reduplicated second sound. The aortic valves close rapidly because the aorta is imperfectly supplied with blood, while the more copious inflow of blood into the pulmonary artery causes a later contraction of its valves (Geigel).

If the heart beats rapidly and feebly-if the blood-pressure in the aorta and pulmonary artery be low, the signs of closure of the pulmonary valves may be absent-as in curve $\mathrm{L}$-taken from a girl snffering from nervous palpitation and morbus Basedowii.

In very rare cases of insufficiency of the mitral valve, it has been observed that at certain times both ventricles contract simultaneously, as in a normal heart, but that this alternates with a condition where the right ventricle alone seems to contract. Curve M is such a curve obtained by Malbranc, who called this condition intermittent hemisystole. The first curve $(\mathrm{I})$ is like a normal curve, during which the whole heart acted as usual. The curve II, however, is caused by the right side of the heart alone; it wants the closure of the aortic valves, $d$, and there was no pulse in the arteries. Owing to insufficiency of the tricuspid valve, the same person had a venous pulse with every cardiac impulse, so that the arterial and venous pulses first occurred together, and then the venous pulse alone occurred.

In these cases (Skoda, v. Bamberger, Leyden) the mitral insufficiency leads to overflowing of the right ventricle, while the left is nearly empty, so that the right side requires to contract more energetically than the left. It does not seem that the right ventricle alone contracts in these cases, but rather that the action of the left side is very feeble.

\section{The Heart-Sounds.}

On listening over the region of the heart in a healthy man, either with the ear applied directly to the chest-wall, or by means of a 
stethoscope (Laennec, 1819), we hear two characteristic sounds, the so-called "heart-sounds." Harvey was acquainted with these sounds, but they have been more carefully studied by clinicians since the time of Laennec.

The first sound [long or systolic] is somewhat duller, longer, and one-third or one-fourth deeper, than the second sound; it is less sharply defined at first, and is isochronous with the systole of the ventricles (Turner). The second sound [short or diastolic] is clearer, sharper, shorter, more sudden, and is one-third to one-fourth higher; it is sharply defined and isochronous with the closure of the semi-lunar valves. There is a very short interval between the first and second sounds, and between the second and the next following first sound a distinctly longer interval. This is the pause.

[The sounds emitted during each cardiac cycle have been compared to the pronunciation of the syllables $l \bar{u} b b$, durp. We may express the course of events with reference to the sounds, thus:- $l \bar{u} b b$, duup, pause.] Or the result may be expressed thus-

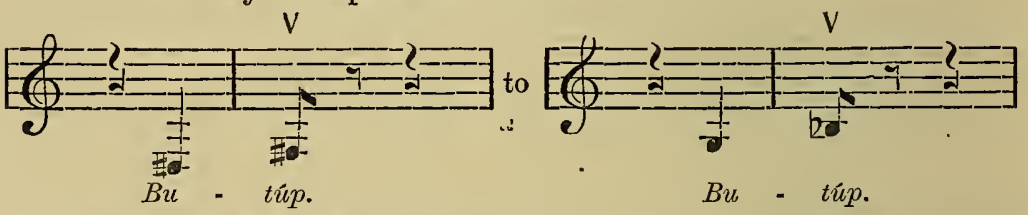

The causes of the first sound are due to two conditions. As the sound is heard in an excised heart in which the movements of the valves are arrested, and also when the finger is introduced into the auriculo-ventricular orifices so as to prevent the closure of the valves (C. Ludwig and Dogiel), one of the chief factors lies in the "musclesound" produced by the contracting muscular fibres of the ventricles (Williams, 1835).

This sound is supported and increased by the sound produced by the tension and vibration of the auriculo-ventricular valves and their chordæ tendinix, at the moment of the ventricular systole (Rouanet, Kiwisch, Bayer, Giese).

Wintrich, by means of proper resonators, has been able so to analyse the first sound as to distinguish the clear, short, valvular part from the deep, long, muscular sound.

The muscle-sound prodnced by transversely-striped muscle does not occur with a simple contraction, but only when several contractions are superposed to produce tetanus (see Muscle). The ventricular contraction is only a simple contraction, but it lasts considerably longer than the contraction of other muscles, and herein lies the cause of the occurrence of the muscle-sound during the ventricular contraction.

Defective Heart-Sounds.-In certain conditions (typhus, fatty degeneration of the heart) where the muscular substance of the heart is much weakened, the 


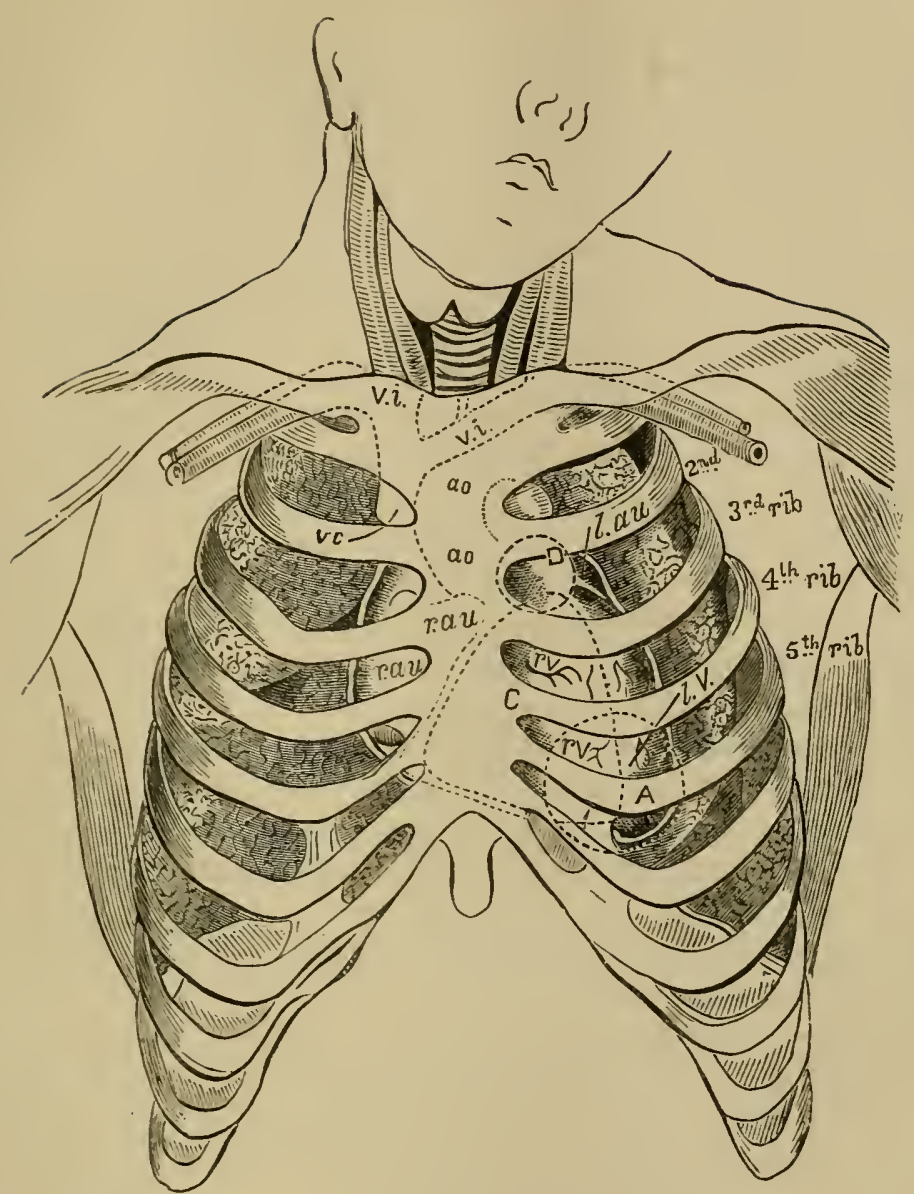

Fig. 31 .

The heart-its sereral parts and great vessels in relation to the front of the thorax. The lungs are collapsed to their normal extent, as after death, exposing the heart. The outlines of the several parts of the heart are indicated by very fine dotted lines. The area of propagation of valvular murmurs is marked out by more visible dotted lines. A, the circle of mitral murmur, corresponds to the left apex. The broad and somewhat diffused area, roughly triangular, is the region of tricuspid murmurs, and corresponds generally with the right ventricle, where it is least covered by lung. The letter $\mathrm{C}$ is in its centre. The circumscribed circular area, $\mathrm{D}$, is the part over which the pulmonic arterial murmurs are commonly heard loudest. In many cases it is an inch, or even more, lower down, corresponding to the conus arteriosus of the right ventricle, where it touches the walls of the thorax. The internal organs and parts of organs are indicated by letters as follows $-r$. au, right auricle, traced in fine dotting; $a 0$, arch of aorta, seen in the first intercostal space, and traced in fine dotting on the sternum; vi, the two innominate veins; $r v$, right ventricle; $l v$, left rentricle. 
first sound may be completely inaudible. In aortic insufficiency, in which, in consequence of the reflux of blood from the aorta into the ventricle, the mitral valve is gradually stretched, and sometimes even before the beginning of the ventricular systole, the first sound may be absent. Both pathological cases show that for the production of the first sound, muscle-sound and valve-sound must eventually work together, and that the tone is altered, or may even disappear, when one of these canses is absent.

The Cause of the Second Sound is undoubtedly due to the prompt closure, and therefore sudden stretching or tension, of the semi-lunar valves of the aorta and pulmonary artery, so that it is purely a valvular sound (Carswell and Rouanet, 1830). Perhaps it is augmented by the sudden vibration of the fluid-particles in the large arterial trunks. As already pointed out (p. 85), the aortic and pulmonary valves do not close simultaneously. Usually, however, the difference in time is so small that both valves make one sound, but the second sound may be double or divided when, through increase of the difference of pressure in the aorta and pulmonary artery, the interval becomes longer. Even in health this may be the case, as occurs at the end of inspiration or the beginning of expiration (v. Dusch).

[The second sound has all the characters of a valvular sound. That the aortic valves are concerned in its production, is proved by introducing a curved wire through the left carotid artery and hooking up one or more segments of the valve, when the sound is modified, and it may be replaced by an abnormal sound or "murmur." Again, when these valves are diseased, the sound is altered, and it may be accompanied or even displaced by murmurs.]

Where the Sounds are Heard Loudest.-The sound produced by the tricuspid valve is heard loudest at the insertion of the fifth right rib into the sternum, and from here somewhat inwards and obliquely upwards along the sternum; as the mitral valve lies more to the left and deeper in the chest, and is covered in front by the arterial orifice, the mitral sound is best heard at the apex-beat, or immediately above it, where a strip of the left ventricle lies next the chest-wall. [The sound is conducted to the part nearest the ear of the listener by the muscular substance of the heart.] The aortic and pulmonary orifices lie so close together that it is convenient to listen for the second (aortic) sound in the direction of the aorta and where it comes nearest to the surface, i.e., over the first right costal cartilage close to its junction with the sternum. The sound, although produced at the semi-lunar valves, is carried upwards by the column of blood and by the walls of the aorta.

The sound produced by the pulnonary artery is heard most distinctly in the second left intercostal space, somewhat to the left and external to the margin of the sternum (Fig. 31). 


\section{Variations of the Heart-Sounds.}

An increase of the first sound of both ventricles indicates a more energetic contraction of the ventricular muscle and a simultaneously greater and more sudden tension of the auriculo-ventricular valves. An increase of the second sound is a sign of increased tension in the interior of the corresponding large arteries. Hence, increase of the second (pulmonary) sound indicates overfilling and excessive tension in the pulmonary circuit.

Feeble weali action of the heart, as well as abnormal want of blood in the heart, causes weak heart-sounds, which is the case in degenerations of the heart-muscle.

Irregularities in structure of the individnal valves may cause the heart-sounds to become "impure." If a pathological cavity, filled with air, be so placed, and of such a form as to act as a resonator to the heart-sounds, they may assume a "metallic" character. The first and second sounds may be "reduplicated" or "divided." The reduplication of the first sound is explained by the tension of the tricuspid and that of the mitral valves not occurring simultaneously. Sometimes a sound is produced by a hypertrophied auricle producing an andible presystolic sound, i.e., a sound or "murmur," preceding the first sound. As the aortic and pulmonary valves do not close quite simnltaneously, a reduplicuted second sound is only an increase of a physiological condition (Landois). All conditions which cause the aortic valves to close rapidly (diminished amount of blood in the left ventricle) and the pulmonary valves to close later (congestion of the right ventricle-both conditions together in mitral stenosis), favour the production of a reduplicated second sound.

Cardiac Murmurs.--If irregularities occur in the valves, either in cases of stenosis or in insufficiency, so that the blood is subjected to vibratory oscillations and friction, then, instead of the heart-sounds, other sounds arise or accompany these-murmurs or bruits, which, when combined, are always accompanied by disturbances of the circulation. It is rare that tumours or other deposits projecting into the ventricles cause murmurs, unless there be present at the same time lesions of the valves and disturbances of the circulation. The cardiac murmurs or bruits are always related to the systole or diastole, and usually the systolic are more accentuated and louder. Sometimes they are so loud that the thorax trembles under their irregular. oscillations (fremitus, frémissement cataire).

In cases where diastolic mu'mur's are heard, there are always anatomical changes in the cardiac mechanism. These are insufficiency of the arterial valves, or stenosis of the auriculo-ventricular orifices (usually the left). Systolir: murnur's do not always necessitate a disturbance in the cardiac mechanism. They may occur in the left side, owing to insufficiency of the mitral valve, stenosis of the aorta, and in calcification and dilatation of the ascending part of the aorta. These murmurs occur very much less frequently on the right side, and are due to insufficiency of the tricuspid and stenosis of the pulmonary orifice.

Systolic murmurs often occur without any valvular lesion, although they are always less loud, and are caused by abnormal vibrations of the valves or arterial walls. They occur most frequently at the orifice of the pulmonary artery, less frequently at the mitral, and still less frequently at the aorta or the tricuspid orifice. Anæmia, general mal-nutrition, acute febrile affections, are the causes of these murmurs.

Murmurs also occur during a certain stage of inflammation of the pericardium (pericarditis) from the roughened surfaces of this membrane rubbing upon each other. Audible friction-sounds are thus produced, and the vibration may even be perceptible to touch. [These are "friction-sounds," and quite distinct from sounds produced within the heart itself.] 


\section{Duration of the Movements of the Heart.}

That the heart continues to beat for some time after it is cut out of the body, was known to Cleanthes, a contemporary of Herophilus, 300 B.c. The movement lasts longer in cold-blooded animals (frog, turtle, fish)-extending even to days - than in mammals. A rabbit's heart beats from 3 minutes up to 36 minutes after it is cut out of the body. The average of many experiments is about 11 minutes. Panum found the last trace of contraction to occur in the right auricle (rabbit) 15 hours after death; in a mouse's heart, 46 hours; in a dog's, 96 hours. An excised frog's heart beats, at the longest, $2 \frac{1}{2}$ days (Valentin). In a human embryo (third month) the heart was found beating after 4 hours. In this condition stimulation causes an increase and acceleration of the action. Afterwards, the ventricular contraction first becomes weaker, and soon each auricular contraction is not followed by a ventricular contraction, two or more of the former being sncceeded by only one of the latter. At the same time the ventricles contract more slowly (Fig. 27), and soon stop altogether, while the auricles still continue to beat. If the ventricles be stimulated directly, as by pricking them with a pin, they may execute a contraction. The left auricle soon ceases to beat, while the right auricle still continues to contract. The right auricular appendage continues to beat longest, as was observed by Galen and Cardanus (1550). The term "ultimum moriens" is applied to it. Similar observations have been made upon the hearts of persons who have been executed.

If the heart has ceased to beat, it may be excited to contract for a short time by direct stimulation (Harvey), more especially by heat; even under these circumstances, the auricles and their appendages are the last parts to cease contracting. As a general rule, direct stimulation, although it may callse the heart to act more vigorously for a short time, brings it to rest sooner. In such cases, therefore, the regular sequence of events ceases, and there is usually a twitching movement of the muscular fibres of the heart. C. Ludwig found that even after the excitability is extinguished in the mammalian heart, it may be restored by injecting arterial blood into the coronary arteries: lesion of these vessels is followed by enfeebled action of the heart (p. 75). Hammer found that in a man, whose left coronary artery was plugged, the pulse fell from 80 to 8 beats per minute.

Action of Gases on the Heart.-During its activity the heart uses $\mathrm{O}$, and produces $\mathrm{CO}_{2}$, so that it beats longest in pure $\mathrm{O}$ (12 hours) (Castell), and not so long in $\mathrm{N},-\mathrm{H}$ (1 hour) $-\mathrm{CO}_{2}$ (10 minutes), $\mathrm{CO}$ (42 minutes) $-\mathrm{Cl}$ (2 minutes), or in a vacuum (20 to 30 minutes) (Boyle, 1670; Fontana, Tiedemann, 1847), even when there is watery 
vapour present to prevent evaporation. If the heart be re-introduced into $O$ it begins to beat again. [An excised heart suspended in ordinary air beats three to four times as long as a heart which is placed upon a glass-plate.] A heart which has ceased to contract spontaneously may contract when an electrical stimulus is applied to it, but it does not do so for a longer time than other muscles (Budge).

\section{Innervation of the Heart.}

[When the heart is removed from the body, or when all the nerves which pass to it are divided, it still beats for some time, so that its movements must depend upon some mechanism situated within itself. The ordinary rhythmical movements of the heart are undoubtedly associated with the presence of nerve ganglia, which exist in the substance of the lieart-the intracardiac ganglia. But the morements of the heart are influenced by nervous impulses which reach it from without, so that there falls to be studied an intracardiac and an extracardiac nervous mechanism.]

\section{The Cardiac Nerves.}

The cardiac plexus is composed of the following nerves-(1.) The cardiac branches of the vagus, the branch of the same name from the external branch of the superior laryngeal, a branch from the inferior laryngeal, and sometimes branches from the pulmonary plexus of the vagus (more numerous on the right side). (2.) The superior, middle, inferior, and lowest cardiac branches of the three cervical ganglia and the first thoracic ganglia of the sympathetic. (3.) The inconstant twig of the descending branch of the hypoglossal nerve, which, according to Luschka, arises from the upper cervical ganglia. From the plexus there proceed-the deep and the superficial nerves (the latter usually at the division of the pulmonary artery under the arch of the aorta, and containing a ganglion). The following nerves may be separately traced from the plexus-

(a.) The plexus coronarius dexter and sinister (Scarpa), which contains the raso-motor nerves for these vessels (physiological proof still wanting) as well as the nerves (sensory?) proceeding from them (to the pericardium ?)

(b.) Intra-cardiac Nerves and Ganglia. - The nerves lying in the grooves of the heart and in its substance, containing numerous ganglia (Remak), which are regarded as the automatic motor centres of the heart. A nervous ring containing numerous ganglia corresponds to the margin of the septum atriorum; there is another in the auriculoventricular groove. Where the two meet, they exchange fibres. The ganglia usually lie near the pericardium. In mammals the two largest 
ganglia lie near the orifice of the superior vena cava-in birds the largest ganglion (containing thousands of ganglionic cells) lies posteriorly where the longitudinal and transverse sulci cross each other. Fine branches, also provided with small ganglia, proceed from these ganglia, and penetrate the muscular walls of the auricles and ventricles.

Nerves of the Frogss Heart.-In the frog there is a large ganglion (Remak's) near the fibres of the vagus within the wall of the sinus venosus. Branches of the vagus proceed from this ganglion along the anterior and posterior walls of the auricular septum, and each of these contains a ganglion in the auriculo-ventricular groove, these aggregations of ganglion cells constituting Bidder's ganglion. Fine branches proceed from this ganglion, but they can be traced only for a short distance, so that the greater part of the ventricle appears to be devoid of nerves.

According to Openchowsky, every part of the heart (frog, triton, tortoise) contains nerve-fibres which are connected with every muscular fibre. In the auricles, at the end of the non-medullated fibre, a tri-radiate nucleus exists which gives off fibrils to the muscular bundles.

There is a network of fine nerve-fibres distributed immediately under the endocardium-these fibres act partly in a centripetal direction on the cardiac ganglia, and are partly motor for the endocardial muscles. The parietal layer of the pericardium contains (sensory) nerve-fibres. The following kinds of nerve-cells are found - unipolar cells, the single processes of which afterwards divide; bipolar cells (Fig. $31 a)$, which in the frog possess a straight $(n)$ and usually also a spiral process $(0)$.

\section{The Automatic Motor Centres of the Heart.}

(1.) We must assume that the nervous centres which excite the cardiac movements, and maintain the rhythm of these movements, lie

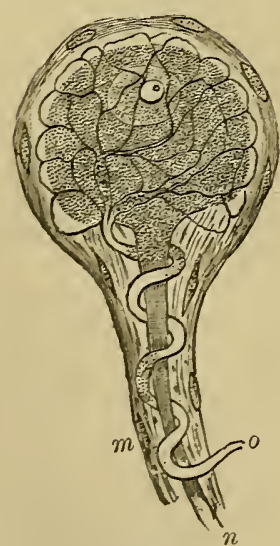

Fig. $31 a$.

Pyriform ganglionic bipolar nerve-cell from the heart of a frog (Arnold)- $m$, sheath; $n$, straight process; 0 , spiral process. within the heart, and that they are probably represented by the gangtia.

(2.) There are-not one, but several, of these centres in the heart, which are connected with each other by conducting paths. As long as the heart is intact, all its parts are made to move in rhythmical sequence from a principal central point, an impulse being conducted from this centre through the conducting paths (Donders). What the "discharging forces" of these regular progressive movements are, is unknown. If, however, the heart be subjected to the action of diffuse stimuli (e.g., strong electrical currents), all the centres are thrown into action, and a spasm-like action of the heart occurs. The dominating centre lies in the auricles, hence the regular progressive movement usually starts from them. If the excit- 
ability is diminished (e.g., by touching the septum with opiumLudwig, Hoffa), other centres seem to undertake this function, in which case the movement may extend from the ventricles to the auricles. If a heart be cut into pieces, so that the individual pieces still remain connected with each other, the regular peristaltic or wave-like movements proceeding from the auricles to the ventricle, may continue for a long time (Donders, Engelmann). If the heart, however, be completely divided into two distinct pieces (auricle and ventricle), the movements of both parts continue, but not in the same sequence- they beat at different rates.

(3.) All stimuli of moderate strength applied directly to the heart cause at first an increase of the rhythmical heart-beats; stronger stimuli cause a diminution, and it may be paralysis, which is often preceded by a convulsive movement. Increased activity exhausts the energy of the heart sooner.

(4.) The auricular centres seem to be more excitable tlan those of the ventricle; hence, in a heart left to itself the auricles pulsate longest.

(5.) The heart may be excited (reflexly) from its inner surface. Weak stimuli applied to the inner surface of the heart greatly accelerate the heart's action, the stimulus required being much feebler than that applied to the external surface of the heart. Strong stimuli, which bring the heart to rest, also act more easily when applied to the inner surface than when they are applied to its outer surface (Henry, 1832). The ventricle is always the part first to be paralysed.

(6.) In order that the heart may continue to contract, it is necessary that it be supplied with a fluid which in addition to $O$ (Ludwig, Volkmann, Goltz) must contain the necessary nutritive materials. The most perfect fluid, of course, is blood. Hence the heart ceases to beat in an indifferent fluid $(0.6$ p.c. sodium chloride), but its activity may be revived by supplying it with a proper nutritive fluid.

Cardiac Nutritive Fluids. - These nutritive fluids are such as contain serumalbumin-e.g., blood, serum, or lymph. Serum retains its nutritive properties even after it has been subjected to diffusion (Martins and Kronecker). Milk and whey (v. Ott), normal saline solution $(0.6$ per cent. $\mathrm{NaCl})$ mixed with blood, albumin, or peptone, and 0.3 per cent. sodium carbonate (Kronecker, Merunowicz, and Stiénon), or a trace of caustic soda (Gaule), or a solution of the salts of serum, are suitable.

(7.) The independent pulsations of parts of the heart which are devoid of ganglia, show that the presence of ganglia is not absolutely necessary in order to have rhythmical pulsation. Direct stimulation of the heart may cause these movements. But the ganglia are more excitable than the heart-muscle itself, and they conduct the impulses 
which lead to the regular alternating action of the various parts of the heart, so that under normal circumstances, we must assume that the action of the heart is governed by the ganglia.

The chief experiments upon which the above statements are based consist of two classes:-(1.) Where the heart is INCISED or DIVIDED; and (2.) where it is STIMULATED DIRECTLY.

(I.) Experiments by CUTTING and LIGATURING the heart. These experiments have been made chiefly upon the frog's heart. The LIGATURE experiments are performed by tightening and then relaxing a ligature placed around the heart, so that the physiological connection is destroyed, while the anatomical or mechanical connections (continuity of the cardiac wall, intact condition of its cavities) still exist. The most important of these experiments are-

(1.) Stannius' Experiment.-If the sinus venosus of a frog's heart be separated from the auricles, either by an incision or by a ligature, the auricles and ventricle stand still in diastole, whilst the veins and the remainder of the sinus continue to beat. If a second incision be made at the auriculo-ventricular groove, as a rule the ventricle begins at once to beat again, whilst the auricles remain in the condition of diastolic rest. According to the position of the second ligature or incision, the auricles may also beat along with the ventricles, or the auricles alone may beat, while the ventricles remain at rest (1852).

Explanations.-Various explanations of these experiments have been given :(a.) Remak's ganglion in the sinus vinosus is distinguished by its great excitability, while Bidder's ganglion in the auriculo-ventricular groove is less excitable; in the normal condition of the heart the motor impulse is carried from the former to the latter. If the sinus venosus be separated from the heart, Remak's ganglion has no action on the heart. The heart stops for two reasons-first, because Bidder's ganglion alone has not sufficient energy to excite it to action, and because the inhibitory fibres of the vagus going to the heart have been stimulated by being divided at this point (Heidenhain). [That stimulation of the inhibitory fibres of the vagus is not the cause of the standstill, is proved by the fact that the standstill occurs even after the administration of atropine, which paralyses the cardiac inhibitory mechanism.] The passive heart, however, may be made to contract by mechanically stimulating Bidder's ganglion-e.g., by a slight prick with a needle in the auriculo-ventricular groove (H. Munk), or by the action of a constant current of moderate strength (Eckhard), the ventricular pulsation at the same time preceding the auricular (v. Bezold, Bernstein). If the auriculo. ventricular groove be divided, the ventricle pulsates again, because Bidder's ganglion has been stimulated by the act of dividing it; while, at the same time, the ventricle is withdrawn from the inhibitory infiuence of the vagus produced by the first division at the sinus venosus. If the line of separation is so made that Bidder's ganglion remains attached to the auricles, these pulsate, and the ventricle; rests; if it be divided into halves, the auricles and ventricles pulsate, each half being excited by the portion of the ganglion in relation with it. (b.) According to another view, both Remak's ( $a$.) and Bidder's ganglia $(b$.$) are motor centres, but$ in the auricles there is in addition an inhibitory ganglionic system (c.) (Bezold, Traube). Under normal circumstances $a+b$ is strouger than $c$, while $c$ is stronger 
than $a$ or $b$ separately. If the sinus venosus be separated it beats in virtue of $a$; on the other hand, the heart rests because $c$ is stronger than $b$. If the section be made at the level of the auriculo-ventricular, the auricles stand still owing to $c$, while the ventricle beats owing to $b$.

(2.) If the ventricle of a frog's heart be separated from the rest of the heart by means of a LIGATURE, or by an INCISION carried through it at the level of the auriculo-ventricular groove, the sinus and atria pulsate undisturbed as before (Descartes, 1644), but the ventricle stands still in diastole. Local stimulation of the ventricle causes a single contraction. If the incision be so made that the lower margin of the auricular septum remains attached to the ventricle, the latter pulsates (Rosenberger, 1850).

(3.) Section of the Heart.-Engelmann's recent experiments show that if the ventricle of a frog's heart be cut up into two or more strips in a zig-zag way, so that the individual parts still remain connecterl with each other by muscular tissue, the strips still beat in a regularly progressive, rhythmical manner, provided one strip is caused to contract. The rapidity of the transmission is about 10 to $30 \mathrm{~mm}$. per sec. (Engelmann). Hence, it appears that the conducting paths for the impulse causing the contraction are not nervous, but must be the contractile mass itself. It has not been proved that nerve-fibres proceed from the ganglia to all the muscles.

[According to Marchand's experiments, it takes a very long time for the excitement to pass from the auricles to the ventricle-a much longer time, in fact, than it would require to conduct the excitement through muscle-so that it is probable that the propagation of the impulse from the auricles to the ventricle is conducted by nervous channels to the auriculo-ventricular nervous apparatus. In fact, in the mammalian heart the muscular fibres of the auricles are quite distinct from those of the ventricle.]

(4.) It is usually stated that when the apex of a frog's heart is severed from the rest of the heart, it no longer pulsates (Heidenhain, Goltz), but such an apex, if stimulated mechanically, responds with a single contraction.

Action of Fluids on the Heart.-Haller was of opinion, that the venous blood was the natural stimulus which caused the heart to contract. That this is not so, is proved at once by the fact that the heart beats rhythmically when it contains no blood.

Blood and other fluids which are supplied to an excised heart are not the cause of its rhythmical movements, but only the conditions on which these movements depend. Thus, a heart which is too feeble to contract may be made to do so by supplying it with a fluid containing proteids, when a latent intra-cardiac mechanism is brought into action, the albuminous or other fluid merely supplying the pabulum for the excitable elements. 
[Methods.-The action of fluids upon the excised frog's heart has been rendered possible by the invention of the "frog-manometer" of Ludwig. The apparatus has been improved by Ludwig's pupils, and already numerous important results have been obtained. The apparatus, Fig. 32 consists of-(1.) a double-way cannula, $c$, which is tied into the heart, $h$; (2.) a manometer, $m$, connected with $c$, and registering the movements of its mercury on a revolving cylinder, cyl; (3.) two Mariotte's flasks, $a$ and $b$, which are connected with the other limb of the cannula. Fither $a$ or $b$ can be placed in communication with the interior of the heart by means of the stop-cock, $s$. The fluid in one graduated tube may be poisoned, and the other not; $d$ is a glass vessel for fluid, in which the heart pulsates, $e$ and $e^{\prime}$ are electrodes, $e$ is inserted into the fluid in $d, e^{\prime}$ is attached to the german silver cannula which is shown in Fig. $32 a$.

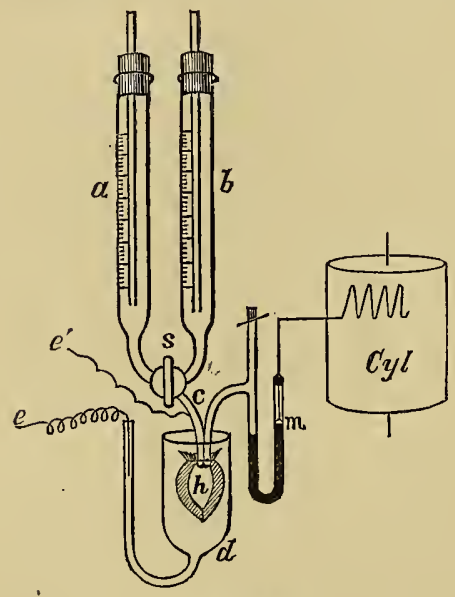

Fig. 32.

Scheme of a frog-manometer- $a, b$, Mariotte's flasks for the nutrient fluids ; $s$, stop-cock ; $c$, cannula ; $m$, manometer ; $h$, heart ; $d$, glass cup for $h ; e, e^{\prime}$, electrodes; cyl, revolving cylinder.

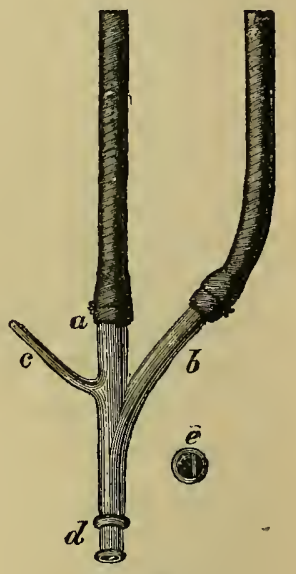

Fig. 32a.

Double-way or perfusion cannula (nat. size) for a frog's heart-c, for fixing an electrode; $d$, the heart is tied over the flanges, preventing it from slipping out; $e$, section of $d$.

In the tonometer of Roy (Fig. 33) the ventricle, $h$, or the whole heart, is placed in an air-tight chamber; $o$, filled with oil, or with oil and normal saline solution. As before, a "perfusion" cannula is tied into the heart. A piston, $p$, works up and down in a cylinder, and is adjusted by means of a thin flexible animal membrane, such as is used by perfumers. Attached to the piston by means of a thread is a writing lever, $l$, which records the variations of pressure within the chamber, 0 . When the ventricle contracts, it becomes smaller, diminishes the pressure within 0 , and hence the piston and lever rise; conversely, when the heart dilates, the lever and piston descend. Variations in the volume of the ventricle may be registered, without in any way interfering with the flow of fluids through it.

Two preparations of the frog's heart have been used-(1.) The "heart," in which case the cannula is introduced into the heart through the sinus venosus, and a 
ligature is tied over it around the auricle, or it may be the sinus venosus. Thus the auriculo-ventricular ganglia and other nervous structures remain in the preparation. This was the heart preparation employed by Luciani and Rossbach. (2.) In the "heart-apex" preparation the cannula is introduced as before, but the ligature is tied on it on the ventricle, several millimetres below the auriculo-

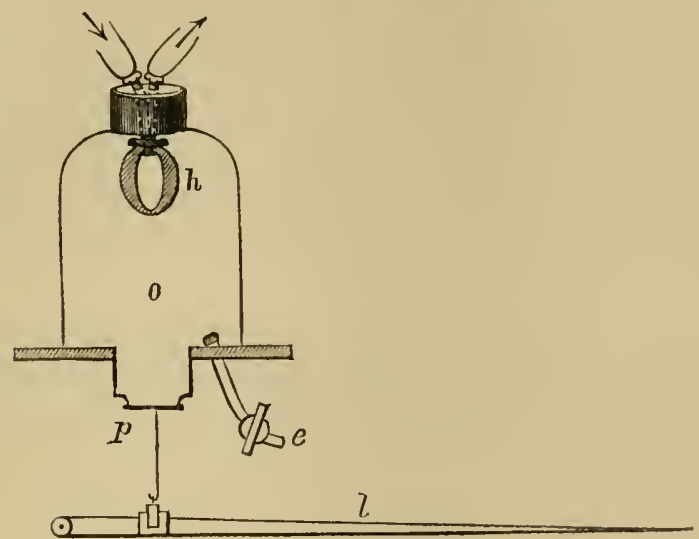

Fig. 33.

Roy's apparatus or tonometer for the heart- $h$, heart; 0 , air-tight chamber; $p$, piston; $l$, writing lever.

ventricular groove, so that this preparation contains none of the auriculo-ventricular ganglia, and, according to the usual statement, this part of the heart is devoid of nerve ganglia. This is the preparation which was used by Bowditch, Kronecker and Stirling, Merunowicz, and others. The first effect of the application of the ligature in both cases is, that both preparations cease to beat, but the "heart" nsually resumes its rhythmical contractions within several minutes, while the "heart-apex" does not contract spontaneously until after a much longer time (10 to 90 mins.).

If the "Heart-Apex" be filled with a 0.6 per cent. solution of common salt, the contractions are at first of greater extent, but they afterwards cease, and the preparation passes into a condition of "apparent death;" while if the action of the fluid be prolonged, the heart may not contract at all, even when it is stimulated electrically or mechauically. It may be made, however, to pulsate again, if it be supplied with saline solution containing blood ( 1 to 10 per cent). The "stille" or state of quiescence may last 90 mins. (Kronecker and Merunowicz). If the ventricle be nipped with wire forceps at the junction of the upper with its middle third, so as to separate the lower two-thirds of the ventrical physiologically but not anatomically from the rest of the heart, then the apex will cease to contract, althongh it is still supplied with the frog's own blood (Bernstein, Bowditch). The physiologically isolated apex may be made to beat by clamping the aortic branches to prevent blood passing out of the heart, and thus raising the intracardial pressure. The rate of the beat of the apex is independent of and slower than that of the rest of the heart. This experiment proves that the amount of pressure within the apex cavity is an important factor in the causation of the spontaneous apex beats (Gaskell). If blood-serum, to which a trace of delphinin is added, be transfused or "perfused" through the heart, it begins to beat within a minute, contiuues to beat for several seconds, and then stands still in diastole (Bowditch). Quinine (Schtschepotjew) and a mixture 
of atropine and muscarin have a similar action (v. Basch). These experiments show that, provided no nervous apparatus exists within the heart-apex, the cause of the varying contraction is to be sought for in the musculature of the heart (Kronecker), and that the stimulus necessary for the systole of the heart'sapex may arise within itself (Aubert). If there is no nervous apparatus of any kind present, then we must assume that the heart-muscle may execute rhythmical movements independently of the presence of any nervous mechanism, although it is usually assumed that the ganglia excite the heart-muscle to pulsate rhythmically. It is by no means definitely proved that the heart-apex is devoid of all nervous structures, which may act as originators of these rhythmical impulses.

The "Heart" preparation in many respects behaves like the foregoing, i.e., it is exhausted after a time by the continued application of normal saline solution $\left(0^{\circ} 6\right.$ per cent. $\left.\mathrm{NaCl}\right)$, while its activity may be restored by supplying it with albuminous and other fluids (p. 99).]

[(5.) Luciani found that such a heart, when filled with pure serum, produced groups of pulsations with a long diastolic pause between every two groups (Fig. 34). The successive beats in each group assume a "staircase" character (p. 107). These periodic groups undergo many

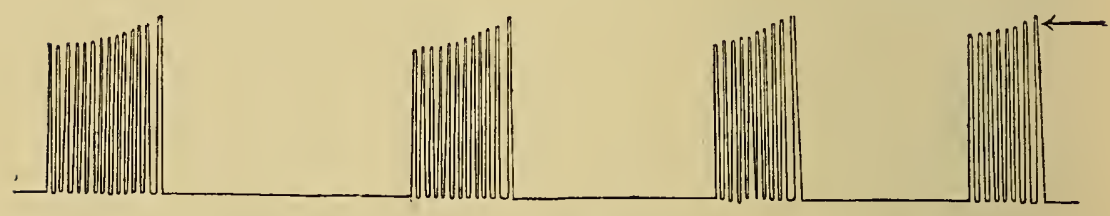

Fig. $3 t$.

Four groups of pulsations with intervening pauses, as obtained by Luciani, with their "staircase" character. The points on the abscissa were marked every ten seconds.

changes; they occur when the heart is filled with pure serum free from blood-corpuscles, and they disappear and give place to regular pulsations when defibrinated blood or serum containing hæmoglobin or normal saline solution (Rossbach) is used. They also occur when the blood within the heart has become dark-coloured-i.e., when it has been deprived of certain of its constituents-and if a trace of veratrin be added to bright-red blood they occur.]

(6.) The same apparatus permits of the application of electrical stimuli to either of the above-named preparations. An apex-preparation, when stimulated with even a weak induction shock, always gives its maximal contraction, and when a tetanising current is applied tetanus does not occur (Kronecker and Stirling). When the opening and closing shocks of a sufficiently strong constant current are applied to the heart-apex, it contracts with each closing or opening shock. [When a constant current is applied to the lower two-thirds of the ventricle (heart-apex), under certain conditions the apex contracts rhythmically. 
This is an important fact in connection with any theory of the cardiac beat.]

(7.) If the bulbus aortæ (frog) be ligatured, it still pulsates, provided the internal pressure be moderate. Should it cease to beat, a single stimulus makes it respond by a series of contractions. Increase of temperature to $35^{\circ} \mathrm{C}$., and increase of the pressure within it increase the number of pulsations (Engelmann).

(II.) Direct Stimulation of the Heart.-All direct cardiac stimuli act more energetically on the inner than on the outer surface of the heart. If strong stimuli are applied for too long a time, the ventricle is the part first paralysed.

(a.) Thermal Stimuli.-[Heat affects the number or fiequency and the amplitude of the pulsations, as well as the duration of the systole and diastole and the excitability of the heart.] Descartes (1614) observed that heat increased the number of pulsations of an eel's heart. A. v. Humboldt found that when a frog's heart was placed in lukewarm water, the number of beats increased from 12 to 40 per minute. As the temperature increases, the number of beats is at first con. siderably increased, but afterwards the beats again become fewer, and if the temperature is raisel above a certain limit the heart stands still, the myosin of which its fibres consist is coagulated, and "heat-rigor" occurs. Even before this stage is reached, howerer, the heart may stand still, the muscular fibres

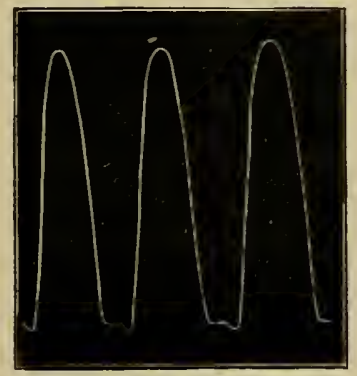

$a$

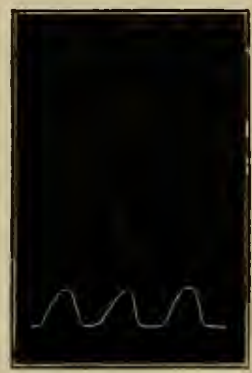

$b$

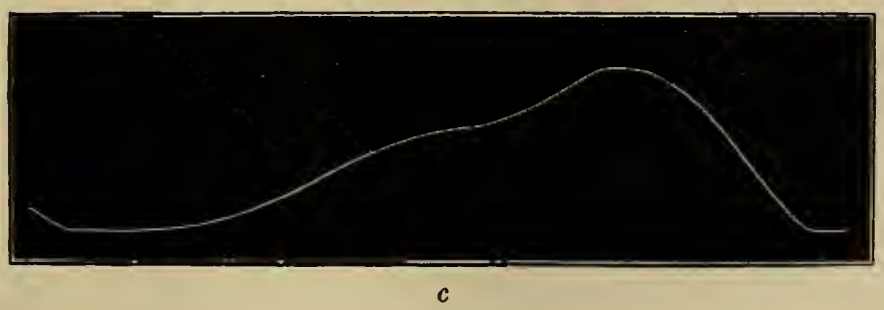

Fig. 35.

Fig. $a$, Contractions of a frog's heart at $19^{\circ} \mathrm{C} ; b$, at $34^{\circ} \mathrm{C}$; $c$, at $3^{\circ} \mathrm{C}$. 
appearing to remain contracted. The ventricles usually cease to beat before the auricles (Schelske). The size and extent of the contractions increase up to about $20^{\circ} \mathrm{C}$., but above this point they diminish (Fig. 35). The time occupied by any single contraction at $20^{\circ} \mathrm{C}$. is only about $\frac{1}{10}$ of the time occupied by a contraction occurring at $5^{\circ} \mathrm{C}$. "

A heart which has been warmed is capable of reacting pretty rapidly to intermittent stimuli, while a heart at a low temperature reacts only to stimuli occurring at a considerable interval. If a frog be kept in a cold place its heart beats slowly and does little work, but if the heart be supplied with the extract of a frog which has been kept warm, it is rendered more capable of doing work (Gaule).

Cold. - When the temperature of the blood is diminished, the heart beats slower (Kielmeyer, 1793). A frog's heart, placed between two watch-glasses and laid on ice, beats very much slower (Ludwig, 1861). The pulsations of a frog's heart stop when the heart is exposed to a temperature of $4^{\circ} \mathrm{C}$. to $0^{\circ}$ (E. Cyon). If a frog's heart be taken out of warm water, and suddenly placed upon ice, it beats more rapidly, and conversely, if it be taken from ice and placed in warm water, it beats more slowly at first and more rapidly afterwards (Aristow).

[Methods,-The effect of heat on a heart may be studied by the aid of the frogmanometer, the fluid in which the heart is placed being raised to any temperature required. For demonstration purposes, the heart of a pithed frog is excised and placed on a glass slide under a light lever, such as a straw. The slide is warmed by means of a spirit-lamp. In this way the frequency and amplitude of the contractions are readily made visible at a distance.]

[Gaskell fixes the heart by means of a clamp placed round the auriculo-ventricular groove, while levers are placed horizontally above and below the heart. These levers are fixed to part of the auricles and to the apex by means of threads. Each part of the heart attached to a lever, as it contracts, pulls upon its own lever, so that the extent and duration of each contraction may be registered. This method is applicable for studying the effect of the vagus and other nerves upon the heart (Roy).]

(b.) Mechanical Stimuli.-Pressure applied externally to the heart accelerates its action. In the case of Frau Serafin, v. Ziemssen found, that slight pressure on the auriculo-ventricular groove caused a second short contraction of both ventricles after the heart-beat. Strong pressure causes a very irregular action of the cardiac muscle. This may readily be produced by compressing the freshly excised heart of a dog between the fingers.

The intra-cardiac pressure also affects the heart-beat. If the pressure within the heart be increased, the heart-beats are gradually increased, if it be diminished the number of beats diminishes (Ludwig and Thiry). If the intra-cardiac pressure be very greatly increased, the heart's action becomes very irregular and slower (Heidenhain). A heart which has ceased to beat may, under certain circumstances, be caused to execute a single contraction if it be stimulated mechanically.

(c.) Electrical Stimuli.-A constant electrical current of moderate strength increases the number of heart-beats. v. Ziemssen found in the case of Frau Serafin (p. 74, 3), that the number of beats was doubled, when a constant uninterrupted strong current was passed through the ventricles. If the constant current be very strong, or if tetanising induction currents be used, the cardiac muscle assumes a condition resembling, but not identical with, tetanus (Ludwig and Hoffa), and of course this results in a fall of the blood-pressure (Sigm. Mayer).

When a single induction shock is applied to the ventricle of a frog's heart during systole, it has no apparent effect; but if it is applied during diastole, the succeeding contraction takes place sooner. The auricles behave in a similar manner. Whilst they are contracted, an induction shock has no effect; if, however, the stimulus is applied during diastole, it causes a contraction, which is followed by systole of the 
ventricle (Hildebrand). Even when strong tetanising induction-shocks are applied to the heart, they do not produce tetanus of the entire cardiac musculature, or as it is said, "the heart knows no tetanus" (Kronecker and Stirling). Small white local weal-like elevations-such as occur when the intestinal musculature is stimulated - appear between the electrodes. They may last several minutes. A frog's heart, which yields weak and irregular contractions, may be made to execute regular rhythmical contractions isochronous with the stimuli, if electrical stimuli are used (Bowditch). In this case the weakest stimuli (which are still active) behave like the stronger stimuli-even with the weak stimulus the heart always gives the strongest contraction possible. Hence this minimal electrical stimulus is as effective as a "maximal" stimulus (Kronecker and Stirling).

V. Ziemssen found that he could not alter the heart-beats of the human heart (Frau Serafin, p.74,3), even with strong induction currents. The ventricular diastole seemed to be less complete, and there were irregularities in its contraction. By opening and closing, or by reversing a strong constant current applied to the heart, the number of beats was increased, and the increase corresponded with the number of electrical stimuli; thus, when the electrical stimuli were $120,140,180$, the number of heartbeats was the same, the pulse beforehand being $S 0$. When 150 shocks per minute were applied the action of the heart assumed the characters of the pulsus alternans (p. 143). Minimal stimuli were also found to act like maximal stimuli. The normal pulse-rate of $S 0$ was reduced to 60 and 50 , when the number of shocks was reduced in the same ratio. The rhythm became at the same time somewhat irregular. In these experiments a strong current is required, and v. Basch found that the same was true for the frog's heart. Even in healthy persons, v. Ziemssen ascertained that the energy and rhythm of the heart could be modified by passing an electrical current through the uninjured chest-wall.

[Method.-The apparatus (Fig. 32.) is also well adapted for studying the effect of electrical currents upon the heart. Bowditch, Kronecker and Stirling, and other observers, used the "heart-apex," as it does not contract spontaneously for some time after the ligature is applied. One electrode is attached to the cannula, and the other is placed in the fluid in which the heart is bathed.]

[Opening induction shocks, if of sufficient strength, cause the heart to contract, while weak stimuli have no effect; on the other hand, moderate stimuli, when they do cause the heart to contract, always cause a maximal contraction, so that a minimal stimulus acts at the same time like a maximal stimulus. The heart either contracts or it does not contract, and when it contracts the result is always a "maximal" contraction. Bowditch found, that the excitability of the heart was increased by its own movements, so that after a heart had once contracted, the strength of the stimulus required to excite the next contraction may be greatly diminished, and yet the stimulus be effectual. Usually the amplitude of the first beat so produced is not so great as the second beat, and the second is less than the third, so that a "staircase" ("Treppe") of beats of successively greater extent are produced (Fig. 34.) This staircase arrangement occurs even when the strength of the stimulus is kept constant, so that the production of one contraction facilitates the occurrence of the succeeding one. A staircase arrangement of the pulsations is also seen in Luciani's groups (p. 104). The question, whether a stimulus will cause a contraction, denends upon what particular phase the heart is in, when the shock is applied. Even comparatively weak stimuli will cause a heart to contract, provided the stimuli are applied at the proper moment and in the proper tempo-i.e. to say, they become what are called "infallible." If stimuli are applied to the heart, at intervals which are longer than the time the heart takes to execute its contraction, they are effectual or "adequate," but if they are applied before the period of pulsation comes to an end, then they are ineffectual (Kronecker). It is quite clear, there- 
fore, that the relation of the strength of the stimulus, to the extent of the contrac. tion of the cardiac muscle, is quite different from what occurs in a muscle of the skeleton, where within certain limits the amplitude of the contraction bears a relation to the stimulus, while in the heart the contraction is always maximal.]

(d.) Chemical Stimuli-Many chemical substances, when applied in a dilute solution, to the inner surface of the heart, increase the heart-beats, while if they are concentrated or allowed to act too long, they diminish the heart-beats, and paralyse it. Bile (Budge), bile salts (Röhrig) diminish the heart-beats (also when they are absorbed into the blood as in jaundice); in very dilute solutions both increase the heart-beats (Landois). A similar result is produced by acetic, tartaric, citric (Bobrik), and phosphoric acids (Leyden). Chloroform and ether, applied to the inner surface, rapidly diminish the heart-beats, and then paralyse it; but very small quantities of ether ( 1 per cent.) accelerate the heart-beat of the frog (Kronecker and $M^{\prime}$ Gregor-Robertson), while a solution of $1 \frac{1}{2}$ to 2 per cent. passed through the heart arrests it temporarily or completely. A dilute solution of opium, strychnia, or alcohol applied to the endocardium, increases the heart-beats (C. Ludwig); if concentrated they rapidly arrest its action. Chloral-hydrate paralyses the leart (P. v. Rokitansky).

Action of Gases.--When blood containing different gases was passed through a frog's heart, Klug found that blood containing sulphurous acid rapidly and completely killed the heart ; chlorine stimulated the heart at first, and ultimately killed it; and laughing-gas rapidly killed it also. Blood containing sulphuretted hydrogen paralysed the heart without stimulating it. Carbonic oxide also paralysed it, but if fresh blood was transfused, the heart recovered. [Blood containing $\mathrm{O}$ excites the heart (Castell), while the presence of much $\mathrm{CO}_{2}$ paralyses it, and the presence of $\mathrm{CO}_{2}$ is more injurious than the want of $\mathrm{O} . \mathrm{H}$ and $\mathrm{N}$ have no effect.]

Rossbach found on stimulating the ventricle of a frog's heart at a circumscribed area, either mechanically, chemically, or electrically, during systole, that the part so stimulated relaxes in partial diastole. The immediate direct after-effect of this stimulation is, that the muscular fibres in the part irritated remain somewhat shrivelled. This part ceases to act, and has lost its vital functions. If the stimulus is applied during diastole, the part irritated always relaxes sooner, and its diastole lasts longer than does that of the parts which were not stimulated. If weak stimuli are allowed to act for a long time upon any part of the ventricle of a frog's heart, the part so stimulated always relaxes sooner than the non-stimulated parts, and its diastole is also prolonged.

Cardiac Poisons are those substances whose action is characterised by special effects upon the movements of the heart. Amongst these are the neutral salts of potash. [Until 1863 it was believed that these salts were just as slightly active on the heart as the soda salts, but Bernard and Grandeau showed that very small doses of these salts produced death, the heart standing still in diastole. An excised frog's heart ceases to beat after one-half to one minute when it is placed in a 2 per cent. solution of potassic chloride.] Even a very dilute solution of yellow prussiate of potash injected into the heart of a frog causes the ventricle to stand still in systole.

As early as 1691, Clayton and Moulin showed the poisonous acticn of potassium sulphate, and alum, as compared with the non-poisonous action of sodiun chloride, which was demonstrated by Courten in 1679. Antiar (Java arrow-poison) causes the ventricle to stand still in systole and the auricles in diastole. Some heartpoisons in small doses, diminish the heart's action, and in large doses not unfrequently accelerate it-e.g., digitalis, morphia, nicotin. Others, when given in small doses, accelerate its action, and in large doses slow it-veratria, aconitin, camphor. 
Special Actions of Cardiac Poisons.-The complicated actions of various poisons upon the heart, have led observers to suppose that there are various intracardiac mechanisms on which these substances may act. Besides the muscular fibres of the heart and its automatic ganglia, some toxicologists assume that there are inhibitory ganglia into which the inhibitory fibres of the vagus pass, and accelerator ganglia, which are connected with the accelerating nerve-fibres of the heart. Both the inhibitory and accelerator ganglia are connected with the automatic ganglia by conducting channels.

Iuscarin stimulates permanently the inhibitory ganglia, so that the heart stands still (Schmiedeberg and Koppe). As atropin aud daturin paralysc these ganglia, the stand-still of the heart brought about by muscarin may be set aside by atropin. [If a frog's heart be excised and placed in a watch-glass, and a few drops of a very dilute solution of muscarin be placed on it with a pipette, it ceases to beat within a few minutes, and will not beat again. If, however, the muscarin be remored, and a solution of atropine applied to the heart, it will resume its contrac. tions after a short time.] Physostigmin [Calabar bean] excites the energy of the cardiac muscle to such an extent, that stimulation of the vagus no longer causes the heart to stand still. Iodine-aldehyd, chloroform, and chloral-hydrate paralyse the automatic ganglia. The heart stands still, and it cannot be made to contract again by atropine. The cardiac muscle itself remains excitable after the action of muscarin and iodine-aldehyd, so that if it be stimulated it contracts. [According to Gaskell, antiarin and digitalin solutions produce an alteration in the condition of the muscular tissue of the apex of the heart of the same nature as that produced by the action of very dilute alkali solution, while the action of a blood solution containing muscarin closely resembles that of a dilute acid solution (\$ 65).]

[Nature of a Cardiac Contraction.-The question as to whether this is a simple contraction or a compound tetanic contraction, has been much discussed. This much is certain, that the systolic contraction of the heart is of very much longer duration ( 8 to 10 times) than the contraction of a skeletal muscle produced by stimulation of its motor nerve. When the sciatic nerve of a nerve-muscle preparation ("rheoscopic limb") is adjusted upon a contracting heart, a simple secondary twitch of the limb, and not a tetanic spasm, is produced when the heart (auricle or ventricle) contracts. This of itself is not sufficient proof that the systole is a simple spasm, for tetanus of a muscle does not in all cases give rise to secondary tetanus in the leg of a rheoscopic limb. Thus, a simple "initial" contraction occurs, when the nerve is applied to a muscle tetanised by the action of strychnia, and the contracted diaphragm gives a similar result. The question as to whether the heart can be tetanised, has been answered in the negative, and as yet it has not been shown that the heart can be tetanised in the same way that a skeletal muscle is tetanised.]

The peripheral or extra-cardiac nerres will be discussed in connection with the Nerrous System.

\section{The Cardio-Pneumatic Movement.}

As the heart within the thorax occupies a smaller space during the systole than during the diastole, it follows that when the glottis is open, 
air must be drawn into the chest when the heart contracts; whenever the heart relaxes, i.e., during diastole, air must be expelled through the open glottis. But we must also take into account the degree to which the larger intrathoracic vessels are filled with blood. These movements of the air within the lungs, although slight, seem to be of importance in hybernating animals. In animals in this condition, the agitation of the gases in the lungs favours the exchange of $\mathrm{CO}_{2}$ and $\mathrm{O}$ in the lungs, and this slow current of air is sufficient to aerate the blood passing through the lungs. [Ceradini called the diminution of the volume of the entire heart which occurs during systole meiocardie, and the subsequent increase of volume when the heart is distended to its maximum, auxocardie.]

Method.-The cardo-pneumatic movements-i.e., the movement of the respiratory gases dependent on the movements of the heart and great vessels-may be demonstrated in animals and man. A manometric flame may be used. Insert one limb of a Y-tube into the opened trachea of an animal, while the other limb passes to a small gas-jet, and connect the other tube with a gas-jet. It is clear that the movements of the heart will affect the column of gas, and thus affect the flame. Large animals previously curarised are best. It may also be done in man by inserting the tube into one nostril, while the other nostril and the mouth are closed. [A simpler and less irritating plan is to till a wide curved glass-tube with tobacco smoke, and insert one end of the tube into one nostril while the other nostril and the mouth are closed. If the glottis be kept open, and respiration be stopped, then the movements of the column of smoke within the tube are obvious.]

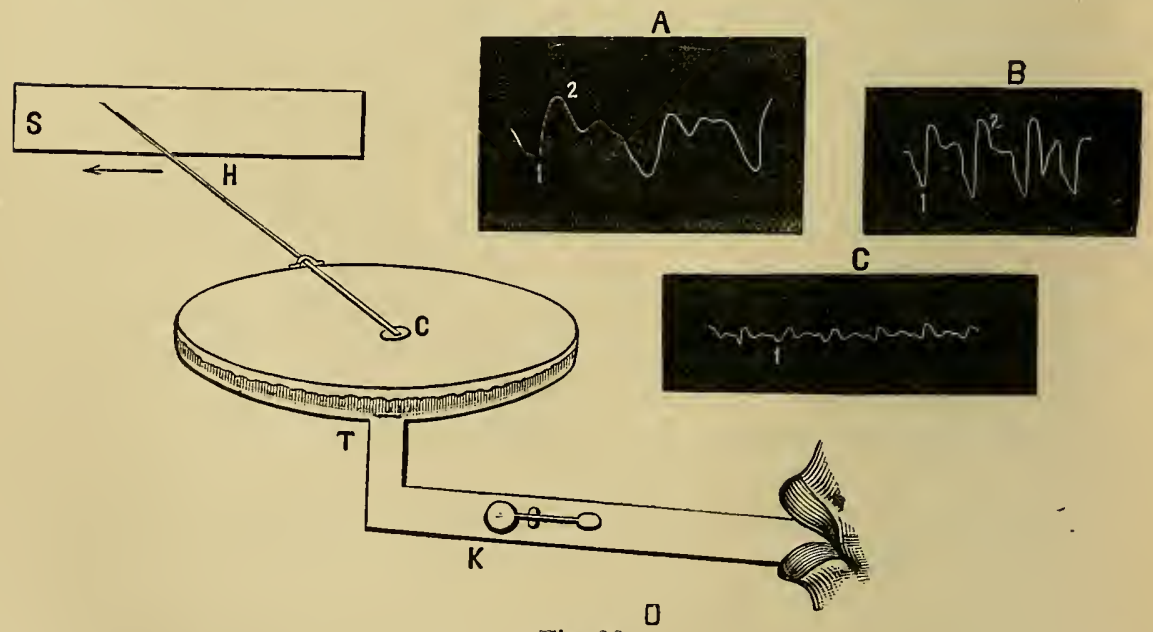

Fig. 36.

Landois' cardio-pneumograph, and the curves obtained therewith-A and B, from man, 1 and 2, correspond to the periods of the first and second heart-sounds; $\mathrm{C}$, from dog; D, method of using the apparatus.

Cardio-Pneumograph.-Ceradini employed a special instrument, while Landois uses his cardio-pneumograph which ccnsists of a tube (D), about one inch 
in diameter and six to eight inches in length; the tube is bent at a right angle, and communicates with a small metal capsule about the size of a saucer ( $T$ ), over which a membrane composed of collodion and castor oil is loosely stretched. To this membrane is attached a glass-rod $(\mathrm{H})$ used as a writing-style, which records its movements on a glass-plate (S) moved by clock-work. A small valve $(K)$ is placed on the side of the tube (D), which enables the experimenter to breathe when necessary. The tube (D) is held in an air-tight manner between the lips, the nostrils being closed, the glottis open, and respiration stopped. Fig. 36, $A, B, C$, are curves obtained in this way. In them we observe-

(a) At the moment of the first sound (1.), the respiratory gases undergo a sharp expiratory movement, because at the moment of the first part of the ventricular systole the blood of the ventricle has not left the thorax, while venous blood is streaming into the right auricle through the venæ cavæ, and because the dilating branches of the pulmonary artery compress the accompanying bronchi. The blood of the right veutricle has not yet left the thorax, it passes merely into the pulmonary circuit. The expiratory movement is diminished somewhat by $(\alpha)$ the muscular mass of the ventricle occupying slightly less bulk during the contraction, and $(\beta)$ owing to the thoracic cavity being slightly increased by the fiftl intercostal space being pushed forward by the cardiac impulse.

(b) Immediately after (1.), there follows a strong inspiratory current of the respiratory gases. As soon as the blood from the root of the aorta reaches that part of the aorta lying outside the thorax, more blood leaves the chest than passes into it simultancously through the venæe cava.

(c) After the second sound (at 2.), indicated sometimes by a slight depression in the apex of the curve, the arterial blood accumulates, and hence there is another expiratory movement in the curve.

(d) The peripheral wave-movements of the blood from the thorax cause another inspiratory movement of the gases.

(e) More blood flows into the chest through the veins, and the next heart-beat occurs.

\section{Influence of the Respiratory Pressure on the Dilatation and Contraction of the Heart.}

The variation in pressure to which all the intra-thoracic organs are subjected, owing to the increase and decrease in the size of the chest caused by the respiratory movements, exerts an influence on the movements of the heart, as was proved by Carson in 1820, and by Donders in 1854. Examine first the relations in different passive conditions of the thorax, when the glottis is open.

The diastolic dilatation of the cavities of the heart (excluding the pressure of the venous blood and the elastic stretching of the relaxed muscle-wall) is fundamentally due to the elastic traction of the lungs. This is stronger the more the lungs are distended (inspiration), and is less active the more the lungs are contracted (expiration). Hence it follows :-

(1.) When the greatest possible expiratory effort is made (of course, with the glottis open) only a small amount of blood flows into the cavities of the heart; the heart in diastole is small and contains a small 
amount of blood. Hence the systole must also be small, which further gives rise to a small pulse-beat.

(2.) On taking the greatest possible inspiration, and therefore causing the greatest stretching of the elastic tissue of the lungs, the elastic traction of the lungs is, of course, greatest-30 mm. Hg. (Donders). This force may act so energetically as to interfere with the contraction of the thin-walled atria and appendices, in consequence of which these cavities do not completely empty themselves into the ventricles. The heart is in a state of great distension in diastole, and is filled with blood ; nevertheless, in consequence of the limited action of the auricles, only small pulse-beats are observed. In several individuals Donders found the pulse to be smaller and slower; afterwards it became larger and faster.

(3.) When the chest is in a position of moderate rest, whereby the elastic traction is moderate $(7.5 \mathrm{~mm}$. Hg.-Donders), we have the condition most favourable to the action of the heart-sufficient diastolic dilatation of the cavities of the heart, as well as unhindered emptying of them during systole.

A very important factor, is the influence exerted upon the action of the heart, by the voluntary increase or diminution of the intra-thoracic pressure.

(1.) Valsalva's Experiment.-If the thorax is fixed in the position of deepest inspiration, and the glottis be then closed, and if a powerful expiratory effort be made by bringing into action all the expiratory muscles, so as to contract the chest, the cavities of the heart are so compressed that the circulation of the blood is temporarily interrupted. In this expiratory phase the elastic traction is very limited, and the air in the lungs being under a high pressure also acts upon the heart and the intra-thoracic great vessels. No blood can pass into the thorax from without; hence the visible veins swell up and become congested, the blood in the lungs is rapidly forced into the left ventricle by the compressed air in the lungs, and the blood soon passes out of the chest. Hence the lungs and the heart contain little blood. Hence also there is a greater supply of blood in the systemic than in the pulmonary circulation and the heart. The heart-sounds disappear, and the pulse is absent (E. H. Weber, Donders).

(2.) J. Müller's Experiment.-Conversely, if after the deepest possible expiration the glottis be closed, and the chest be now dilated with a great inspiratory effort, the heart is powerfully dilated, the elastic traction of the lungs, and the very attenuated air in these organs act so as to dilate the cavities of the heart in the direction of the lungs. More blood flows into the right heart, and in proportion as the right auricle and rentricle can orercome the traction outwards, the blood- 
vessels of the lungs become filled with blood, and thus partly occupy the lung-space. Much less blood is driven out of the left heart, so that the pulse may disappear. Hence, the heart is distended with blood, and the lungs are congested, while the aortic system contains a small amount of blood-i.e., the systemic circulation is comparatively empty, while the heart and the pulmonary vessels are engorged with blood.

In normal respirction, the air in the lungs during inspiration is under slight pressure, while during expiration the pressure is higher, so that these conditions favour the circulation; inspiration favours the supply of blood (and lymph) through the venæ cavæ, and favours the occurrence of diastole. In operations where the axillary or jugular vein is cut, air may be sucked into the circulation during inspiration, and cause death. Expiration favours the flow of blood in the aorta and its branches, and aids the systolic emptying of the heart. The arrangement of the valves of the heart causes the blood to move in a definite direction through it.
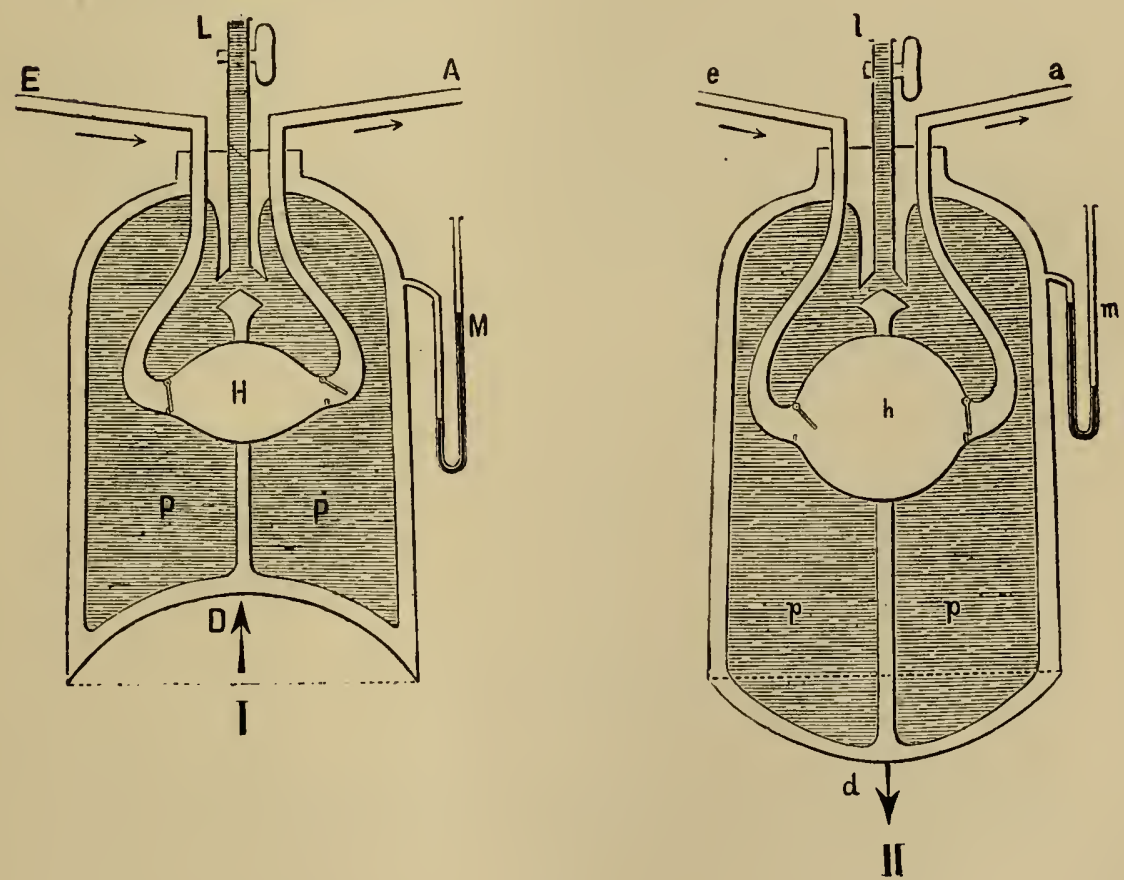

Fig .37.

Apparatus for demonstrating the action of inspiration, II, and expiration, I, on the heart and on the blood-stream-P, $p$, lungs ; $\mathrm{H}, h$, heart ; $\mathrm{L}, l$, closed glottis ; $\mathrm{M}, m$, manometers; $\mathrm{E}$, $e$, ingoing blood-stream, vein ; $\mathrm{A}$, a, outgoing blood-stream, artery; $\mathrm{D}$, diaphragm during expiration; $d$, during inspiration. 
The elastic traction of the lungs aids the lesser circulation through the lungs within the chest; the blood of the pulmonary capillaries is exposed to the pressure of the air in the lungs, while the blood in the pulmonary veins is exposed to a less pressure, as the elastic traction of the lungs, by dilating the left auricle favours the outflow from the capillaries into the left auricle. The elastic traction of the lungs acts slightly as a disturbing agent on the right ventricle, and, therefore, on the movement of blood through the pulmonary artery, owing to the overpowering force of the blood-stream through the pulmonary artery, as against the elastic traction of the lungs (Donders).

The above apparatus (Fig. 37) shows the effect of the inspiratory and expiratory movements on the dilatation of the heart, and on the blood-stream in the large blood-vessels. The large glass-vessel represents the thorax; the elastic membrane, $\mathrm{D}$, the diaphragm ; $\mathrm{P}, p$, the lungs ; $\mathrm{L}$, the trachea supplied with a stop-cock to represent the glottis; $\mathrm{H}$, the heart; $\mathrm{E}$, the venæ cavæ; $\mathrm{A}$, the aorta. If the glottis be closed, and the expiratory phase imitated by pushing up $\mathrm{D}$ as in $\mathrm{I}$, the air in $\mathrm{P}, \mathrm{P}$ is compressed, the heart, $\mathrm{H}$, is compressed, the venous valve closes, the arterial is opened, and the fluid is driven out through A. The manometer, $\mathrm{M}$, indicates the intrathoracic pressure. If the glottis be closed, and the inspiratory phase imitated, as in II, $p, p$ and $h$ are dilated, the venous valve opens, the arterial valve closes; hence, venous blood flows from $e$ into the beart. Thus, inspiration always favours the venous stream, and hinders the arterial; while expiration hinders the venous, and favours the arterial stream. If the glottis $\mathrm{L}$ and $l$, be open, the air in $\mathrm{P}, \mathrm{P}, p, p$ will be changed during the respiratory movements $\mathrm{D}$ and $d$, so that the action on the heart and blood-vessels will be diminished, but it will still persist, although to a much less extent. 


\section{The Circulation.}

\section{The Flow of Fluids through Tubes.}

Toricelli's Theorem (1643) states that the velocity of eflux $(v)$ of a fluidthrough an opening at the bottom of a cylinclrical vessel-is exactly the same as the velocity which a borly falling freely would acquire, were it to fall from the surface of the fluid to the base of the orifice of the outflow. If $h$ be the heiglat of the propelling force, the velocity of efflux is given by the formula-

$$
v=\sqrt{2} y h \text { (where } y=9 \cdot S \text { metre). }
$$

The rapidity of outflow increases (as shown experimentally) with increase in the height of the propelling force, $h$. The former occurs in the ratio, 1, 2, 3, when $h$ increases in the ratio, $1,4,9-i$. e., the velocity of efflux is as the square root of the height of the propelling force. Hence, it follows that the velocity of efflux depends upon the height of the liquid above the orifice of outflow, and not upon the nature of the fluid.

Resistance.-Toricelli's theorem, however, is only valid when all resistance to the outflow is absent; but, in fact, in every physical experiment such resistance exists. Hence, the propelling force, $h$, has not only to cause the efflux of the fluid, but has also to overcome resistance. 'These two forces may be expressed by the heights of two columns of water placed over each otherviz., by the height of the column of water causing the outflow, F, and the height of the column, D, which

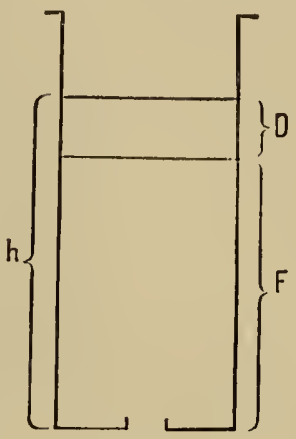

Fig. 38.

Cylindrical vessel filled with water-h, height of the column of fluid; $\mathrm{D}$, height of column of fluid required to overcome the resistance; and $F$, height of column of fluid causing the efflux. overcomes the resistance opposed to the outflow of the fluid. So that

$$
h=\mathrm{F}+\mathrm{D} \text {. }
$$

\section{Propelling Force-Velocity of the Current, and Lateral Pressure.}

In the case of a fluid flowing through a tube, which it fills completely, we have to consider the propelling force, $h$, eausing the fluid to flow through the various sections of the tube. The amount of the propelling force depends upon two factors :-

(1.) On the velocity of the current, $v$;

(2.) On the pressure (amount of resistance) to which the fluid is subjected at the various parts of the tube, $D$.

(1.) The velocity of the current, $v$, is estimated-(a.) from the lumen, $l$, of the 
tube; and (b.) from the quantity of fluid, $q$, which flows through the tube in the unit of time. So that $v=q: l$. Both values, $q$ as well as $l$, can be accurately measured. (The circumference of a round tube, whose diameter $=d$ is $3 \cdot 14 . d$. The sectional area (lumen of the tube) is $l=\frac{3 \cdot 14}{4}, d^{2}$.) Having in this way determined $v$, from it we may calculate the height of the column of fluid, $\mathrm{F}$, which will give this velocity-i.e., the height from which a body must fall in vacuo, in order to attain the velocity, $v$, In this case $\mathrm{F}=\frac{v^{2}}{4 g}$ (where $g=$ the distance traversed by a falling body in 1 sec. $=4.9$ metre).

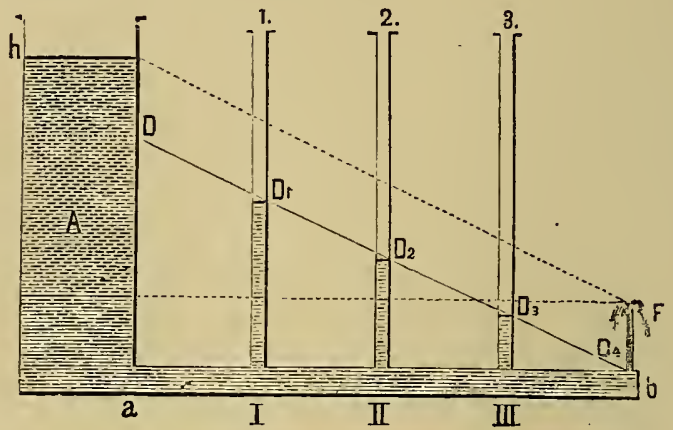

Fig. 39.

A cylindrical vessel filled with water $-a, b$, outflow tube, along which are placed at intervals vertical tubes, $1,2,3$, to estimate the pressure.

(2.) The pressure, D (amount of resistance), is measured directly by placing manometers at different parts of the tube (Fig. 39).

The propelling force at any part of the tube is

$$
\text { or, } \quad \begin{aligned}
h & =\mathrm{F}+\mathrm{D} ; \\
& h=\frac{v^{2}}{4 g}+\mathrm{D} . \quad \text { (Donders). }
\end{aligned}
$$

This is proved experimentally by taking a tall cylindrical vessel, $\mathrm{A}$, of sufficient size, which is kept filled with water at a constant level, $h$. The ontflow rigid tube, $a, b$, has in connection with it a number of tubes placed vertically $1,2,3$, constituting a piezometer. At the end of the tube, $b$, there is an opening with a short tube fixed in it, from which the water issues to a constant height, provided the level of $h$ is kept constant. The height to which it rises depends on the height of the column of fluid causing the velocity, F. As the pressure in the manometric tubes, $\mathrm{D}^{1}, \mathrm{D}^{2}, \mathrm{D}^{3}$, can be read off directly, the propclling force of the water at the sections of the tubes, I, II, III, is-

$$
h=\mathrm{F}+\mathrm{D}^{1} ;-\mathrm{F}+\mathrm{D}^{2} ;-\mathrm{F}+\mathrm{D}^{3} .
$$

At the end of the tube, $b$, where $\mathrm{D}^{4}=0, h=\mathrm{F}+0$, i.e., $h=\mathrm{F}$. In the cylinder itself it is the constant pressure, $h$, which causes the movement of the fluid.

It is clear, that the propelling force of the water gradually diminishes as we pass from the part where the fluid passes out of the cylinder into the tube towards the end of the tube, $b$. The water in the pressure-cylinder, falling from the height, $h$, only rises as high as $F$ at $b$. This diminution of the propelling power is due to the 
presence of REsISTANCES, which oppose the current in the tube, i.e., part of the energy is transformed into heat. As the propelling power at $b$ is represented only by $\mathrm{F}$, while in the vessel it is $h$, the difference must be due to the sum of the resistances, $\mathrm{D}=h-\mathrm{F}$; hence it follows that $h=\mathrm{F}+\mathrm{D}$ (Donders).

\section{Estimation of Resistance.}

Estimation of the Resistance.-When a fluid flows through a tube of uniform calibre the propelling force, $h$, diminishes from point to point on account of the uniformly acting resistance, hence the.sum of the resistances in the whole tube is directly proportional to its length. In a uniformly wide tube, Huid flows through each sectional area with equal velocity, hence $v$ and also $\mathrm{F}$ are equal in all parts of the tube. The diminution which $h$ (propelling force) undergoes can only occur from a diminution of pressure $D$, as $F$ remains the same throughout (and $h=\mathrm{F}+\mathrm{D}$ ). Experiment with the pressure-cylinder shows, that as a matter of fact, the pressure towards the outtlow end of the tube becomes gradually diminished.

In a uniformly wide tube, the height of the pressure in the manometers expresses the resistances opposed to the current of fluid, which it has to overcome in its course from the point investigated to the free orifice of effux.

Nature of the Resistance.-The resistance opposed to the flow of a fluid, depends upon the colesion of the particles of the fluid amongst themselves. During the current, the outer layer of fluid which is next the wall of the tube, and which moistens it, is at rest (Girard, Poiseuille). All the other layers of fluid, which may be represented as so many cylindrical layers, one inside the other, move more rapidly as we proceed towards the axis of the tube, the axial thread or stream being the most rapidly moving part of the liquid. On account of the movement of the cylindrical layers, one within the other, a part of the propelling energy must be used up. The amount of the resistance greatly depends upon the amount of the cohesive force which the particles of the fluid have for each other; the more firmly the particles cohere with each other, the greater will be the resistance, and vice versâ. Hence, the sticky blood-current experiences greater resistance than water or ether.

Heat diminishes the cohesion of the particles, hence it also diminishes the resistance to the flow of a current. These resistances are first develojed by, and result from, the movement of the particles of the fluid, they being, as it were, torn from each other. The more rapid the current, therefore, i.e., the larger the number of particles of fluid which are pulled asunder in the unit of time, the greater will be the sum of the resistance. As already mentioned, the laycr of fluid lying next the tube, and moistening it, is at rest, hence the material which composes the tube exerts no influence on the resistance.

\section{Effect of Tubes of Unequal Calibre.}

Unequal Diameter.-When the velocity of the current is uniform, the resistance depends upon the diamcter of the tube-the smaller the diameter, the greater the resistance; the greater the diameter, the less the resistance. The resistance in narrow tubes, however, increases more rapidly than the diameter of the tube decreases, as has been proved experimentally.

In tubes of unequal calibre, at different parts of their course, the relocity of the current varies-it is slower in the wide part of the tube and more rapid in the 
narrow parts. As a general rule, in tubes of unequal diameter the velocity of the current is inversely proportional to the diameter of the corresponding section of the tube; i.e., if the tube be cylindrical, it is inversely proportional to the square of the diameter of the circular transverse section. In tubes of uniform diameter, the propelling force of the moving fluid diminishes uniformly from point to point, but in tubes of unequal calibre it does not diminish uniformiy. As the resistance is greater in narrow tubes, of course the propelling force must diminish more rapidly in them than in wide tubes. Hence, within the wide parts of the tube the pressure is greater than the sum of the resistances still to be overcome, while in the narrow portions it is less than these.

Tortuosities and Bending of the Vessels add new resistance, and the fluid presses more strongly on the convex side than on the concave side of the bend, and there the resistance to the flow is greater than on the concave side.

Division of a tube into two or more branches is a source of resistance, and diminishes the propelling power. When a tube divides into two smaller tubes, of course some of the particles of the fluid are retarded, while others are accelerated on account of the unequal velocities of the different layers of the fluid. Many particles which had the greatest velocity in the axial layer come to lie more towards the side of the tube where they move more slowly; and conversely many of those lying in the outer layers reach the centre, where they move more rapidly. Hence, some of the propelling force is used up in this process, and the pulling asunder of the particles where the tube divides acts in a similar manner. If two tubes join to form one tube, new resistance is thereby cansed which must diminish the propelling force. The sum of the mean velocities in both branches is independent of the angle at which the division takes place (Jacobson). If a branch be opened from a tube, the principal current is accelerated to a considerable extent, no matter at what angle the branch may be given off.

\section{Currents through Capillary Tubes.}

Poiseuille proved experimentally, that the flow in the capillaries is subject to special conditions-

(1.) The quantity of fluid which flows out of the same capillary tube is proportional to the pressure.

(2.) The time necessary for a given quantity of fluid to flow out (with the like pressure, diameter of tube and temperature), is proportional to the length of the tubes.

(3.) The product of the outflow (other things being equal) is as the fourth power of the diameter.

(4.) The velocity of the current is proportional to the pressure and to the square of the diameter, and inversely proportional to the length of the tube.

(5.) The resistance in the capillaries is proportional to the velocity of the current.

\section{Movement of Fluids and Wave-Motion in Elastic Tubes.}

(1.) When an uninterrupted uniform current flows through an elastic tube, it follows exactly the same laws as if the tube had rigid walls. If the propelling power increases or diminishes, the elastic tubes become wider or narrower, and they behave, as far as the movement of the fluid is concerned, as wider or narrower rigid tubes. 
(2.) Wave-Motion.-If, however, more fluid be forced in jerks into an elastic tube, i.e., interruptedly-the first part of the tubc dilates suddenly, corresponding to the quantity of fluid propelled into it. The jerk communicates an oscillatory movement to the particles of the fluid, which is communicated to all the fluid particles from the beginning to the end of the tube; a positive wave is thus rapidly propagated throughout the whole length of the tube. If we imagine the elastic tube to be closed at its peripheral end, the positive wave will be reflected from the point of occlusion, and it may be propagated to and fro through the tube until it finally disappears. In such a closed tube a sudden jet of fluid produces only a wave-movement, i.e., only a vibratory movement, or an alteration in the shape of the liquid, there being no actual translation of the particles along the tube.

(3.) If, however, fluid be pumped interrnptedly or by jerks into an elastic tube filled with fluid, in which there is already a continuous current, the movement of the current is combined with the wave-movement. We must carefully distinguish the movement of the current of the fluid, i.e., the translation of a mass of fluid through the tube, from the wave-movement, the oscillatory novement, or movement of change of form in the column of fluid. In the former, the particles are actually translated, while in the latter they merely vibrate. The current in elastic tubes is slower than the wave-movement, which is propagated with great rapidity.

This last case obtains in the arterial system. The blood in the arteries is already in a state of continual movement, directed from the aorta to the capillarics (movement of the current of blood); by means of the systole of the left ventricle a quantity of fluid is suddenly pumped into the aorta, and causes a positive wave (pulse-wave) which is propagated with great rapidity to the terminations of the arteries, while the current of the blood itself moves much more slowly.

Rigid and Elastic Tubes.-It is of importance to contrast the movement of fluids in rigid and in elastic tubes. If a certain quantity of fluid be forced into a rigid tube under a certain pressure, the same quantity of fluid will flow out at once at the other end of the tube, provicled there be no special resistance. In an elastic tube, immediately after the forcing in of a certain quantity of fluid, at first only a small quantity flows ont, and the remainder flows out only after the propelling force has ceased to act.

If an equal quantity of fluid be periodically injected into a rigid tube, with each jerk an equal quantity is forced out at the other end of the tube, and the outflow lasts exactly as long as the jerk or the contraction, and the panse between two periods of outflow is exactly the same as between the two jerks or contractions. In an elastic tube it is different, as the outflow continues for a time aftei the jerk; hence, it follows that a continuons outflow current will be produced in elastic tubes, when the time between two jerks is made shorter than the duration of the outflow after the jerk has been completed. When fluid is pumped periodically into rigid tubes, it causes a sharp abrupt ontflow isochronous with the inflow, and the outflow becomes continuous only when the inflow is continuous and uninterrupted. In elastic tubes, an intermittent current under the above conditions causes a continuous outflow which is increased with the systole or contraction.

\section{Structure and Properties of the Blood-Vessels.}

In the body the large vessels carry the blood to and from the various tissues and organs, while the thin-walled capillaries bring the blood into 
intimate relation with the tissues. Through the excessively thin walls of the capillaries the fluid part of the blood transudes, to nourish the tissues outside the capillaries. [At the same time fluids pass from the tissues into the blood. Thus, there is an exchange between the blood and the fluids of the tissues. The fluid after it passes into the tissues constitutes the lymph, and acts like a stream irrigating the tissue elements.]

I. The Arteries are distinguished from veins by their thicker walls, due to the greater development of smooth muscular and elastic tissues-the middle coat (tunica media) of the arteries is specially thick, while the outer coat (t. adventitia) is relatively thin.

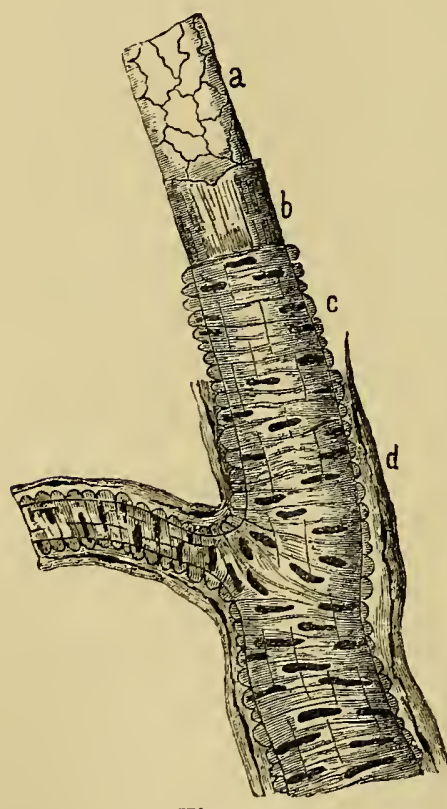

Fig. 40.

Small artery to show the various layers which compose its walls$a$, endothelium; $b$, internal elastic lamina; $c$, circular muscular fibres of the middle coat; $d$, the connective tissue outer coat (T. adventitia).
[The absence of valves is by no means a characteristic feature.]

The arteries consist of three coats (Fig. 40). (1.) The Tunica intima, or inner coat, consists of a layer of (a) irregular, long, fusiform nucleated squamous cells forming the excessively thin transparent endothelium (His, 1866), immediately in contact with the blood-stream. [Like other endothelial cells, these cells are held together by a cement substance which is blackened by the action of silver nitrate.]

Outside this lies a very thin, more or less fibrous, layer - sub-epithelial layer-in which numcrous spindle or branched protoplasmic cells lie embedded within a corresponding system of plasma canals. Outside this is an elastic lamina (b), which in the smallest arteries is a structureless or fibrous elasticmembrane-in arteries of medium size it is a fenestrated membrane(Henle), while in the largest arteries there nay be several layers of elastic laminæ or fenestrated elastic membrane mixed with connective tissue. [In some arteries the elastic membrane is distinctly fibrous, the fibres being chiefly arranged longitudinally. It may be stripped off, when it forms a brittle elastic membrane, which has a great tendency to curl up at its margins. In a transverse section of a middle-sized artery it appears as a bright wavy line, but the curves are probably produced 
by the partial collapse of the vessel. It forms an important guide to the pathologist in enabling him to determine which coat of the artery is diseased.]

In middle-sized and large arteries a few non-striped muscular fibres are disposed longitudinally between two elastic plates or laminæ (K. Bardeleben). Along with the circular muscular fibres of the middle coat, they may act so as to narrow the artery, and they may also aid in keeping the lumen of the vessel open and of uniform calibre. It is not probable that when they act by themselves they dilate the vessel.

(2.) The Tunica media, or midule coat, contains much non-striped nuscle $(c)$, which in the smallest arteries consist of transversely disposed non-striped muscular fibres lying between the endothelium and the T. adventitia, while a finely granular tissue with few elastic fibres forms the bond of union between them. As we proceed from the very smallest to the small arteries, the number of muscular fibres becomes so great as to form a well-marked fibrous ring of non-striped muscle, in which there is comparatively little connective tissue. In the large arteries the amount of connective tissue is considerably increased, and between the layers of fine connective tissue numerous (as many as 50) thick, elastic fibrous or fenestrated laminx are concentrically arranged.

A few non-striped fibres lie scattered amongst these, and some of them are arranged transversely, while a few have an oblique or longitudinal direction.

The first part of the aorta and pulmonary artery, and the retinal arteries are devoid of muscle. The descending aorta, common iliac, and popliteal have longitudinal fibres between the transverse ones. Longitudinal bundles lying inside the media occur in the renal, splenic, and internal spermatic arteries. Longitudinal bundles occur both on the outer and inner surfaces of the umbilical arteries, which are very muscular.

(3.) The Tunica adventitia, or outer coat, in the smallest arteries consists of a structureless membrane with a few connective tissue corpuscles attached to it; in someuthat lerger arteries there is a layer of fine fibrous elastic tissue mixed with bundles of fibrillar connective tissue $(d)$. In arteries of middle size, and in the largest arteries the chief mass consists of bundles of fibrillar connective tissue containing connective tissue corpuscles. The bundles cross each other in a variety of directions, and fat cells often lie between them. Next the media there are numerous fibrous or fenestrated elastic lamellæ. In medium sized and small arteries the elastic tissue next the media takes the form of an independent elastic membrane (Henle's external elastic 
membrane). Bundles of non-striped muscle, arranged longitudinally,

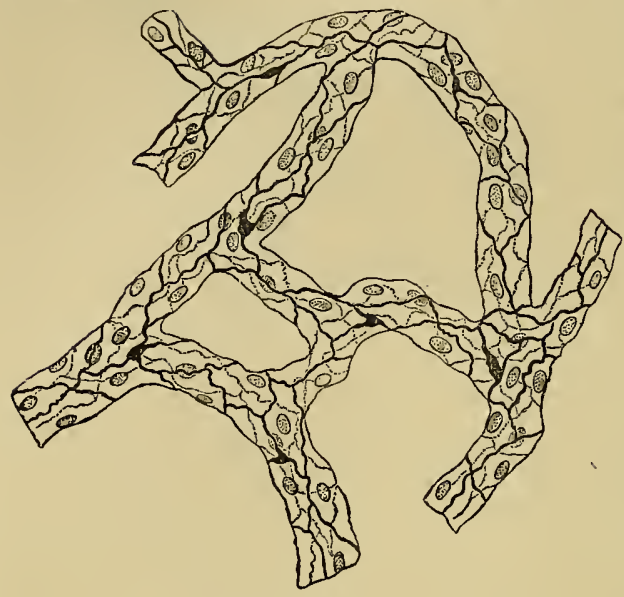

Fig. 41.

Capillaries-The outlines of the endothelial cells marked off from each other by the cement which is blackened by the action of silver nitrate. The nuclei of the cells are obvious. occur in the adventitia of the arteries of the penis, and in the renal, splenic, spermatic, iliac, hypogastric, and superior mesenteric arteries.

II. The Capillaries, while retaining their diameter, divide and reunite so as to form net-works, whose shape and arrangement differ considerably in different tissues. The diameter of the capillaries varies considerably, but as a general rule, it is such as to admit freely a single row of blood-corpuscles. In the retina and muscle the diameter is $5-6 \mu$, and in bone-marrow, liver, and choroid $10-20 \mu$. The tubes consist of a single layer of transparent, excessively thin nucleated endothelial cells joined to each other by their margins (Hoyer, Auerbach, Eberth, Aeby, 1865).

[The nuclei contain a well-marked intra-nuclear plexus of fibrils, like other nuclei.] The cells are more fusiform in the smaller capillaries and more polygonal in the larger. The body of the cells presents the characters of very faintly refractive protoplasm, but it is doubtful whether the body of the cell is endowed with the property of contractility.

Action of Silver Nitrate.-If a dilute solution ( $\frac{1}{4}$ per cent.) of silver nitrate be injected into the blood-vessels, the cement substance of the epithelium [and of the muscular fibres as well] is revealed by the presence of the black "silver-lines." The blackened cement substance shows little specks and large black slits at different points. It is not certain whether these are actual holes ( $\mathrm{J}$. Arnold) through which colourless corpuscles may pass out of the vessels, or are merely larger accumulations of the cement substance.

[Arnold called these small areas in the black silver lines when they were large stomata, and when small stigmata. They are most numerous after venous congestion, and after the disturbances which follow inflammation of a part (Cohnheim, Winiwarter). They are not always present. The existence of cement substance between the cells may also be inferred from the fact that indigo- 
sulphate of soda is deposited in it (Thoma), and particles of cinnabar and China ink are fixed in it, when these substances are injected into the blood (Foa).]

Fine anastomosing fibrils derived from non-medullated NERVES terminate in small end-buds in relation with the capillary wall; ganglia in connection with capillary nerves occur only in the region of the sympathetic (Bremer and Waldeyer).

[If a capillary is examined in a perfectly fresh condition (while living) and without the addition of any reagent, it is impossible to make out any line of demarcation between adjacent cells owing to the uniform refractive index of the entire wall of the tube.]

The small ressels next in size to the capillaries and continuous with them have a completely structureless covering in addition to the endothelium.

III. The Veins are generally distinguished from the arteries by their lumen being wider than the lumen of the corresponding arteries; their walls are thinner on account of the smaller amount of non-striped muscle and elastic tissue (the non-striped muscle is not unfrequently arranged longitudinally in reins). They are also more extensile (with the same strain). The adventitia is usually the thickest coat.

The occurrence of valves is limited to the veins of certain areas. (1.) The intima consists of a layer of shorter and broader endothelial cells, under which in the smallest veins there is a structureless elastic membrane, sub-epithelial layer, which is fibrous in veins somewliat larger in size, but in all cases is thinner than in the arteries. In large veins it may assume the characters of a fenestrated membrane, which is double in some parts of the

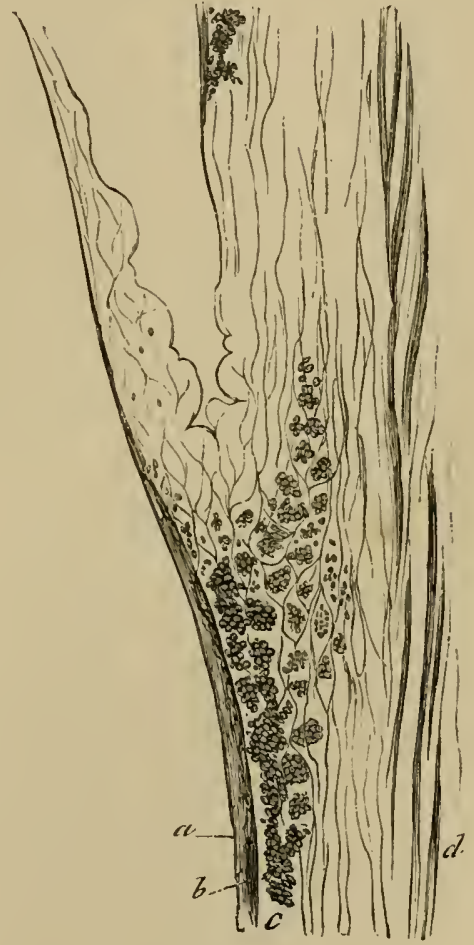

Fig. 42.

Longitudinal section of a vein at the level of a valve-- $a$, hyaline layer of the internal coat; $b$, elastic lamina; $c$, groups of smooth muscular fibres divided transversely; $d$, longitudinal muscular fibres in the adventitia. crural and iliac veins. Isolated muscular fibres exist in the intima of the femoral and popliteal veins. 
(2.) The media of the larger veins consists of alternate layers of elastic and muscular tissue united to each by a considerable amount of connective tissue, but this coat is always thinner than in the corresponding arteries. This coat diminishes in the following order in the following vessels-popliteal, veins of the lower extremity, veins of the upper extremity, superior mesenteric, other abdominal veins, hepatic, pulmonary, and coronary veins. The following veins contain no muscle-veins of bone, central nervous system, and its membranes, retina, the superior cava, with the large trunks that open into it, the upper part of the inferior cava. Of course, in these cases, the media is very thin. In the smallest veins the media is formed of fine connective tissue, with very few muscular fibres scattered in the inner part.

(3.) The adventitia is thicker than that of the corresponding arteries; it contains much connective tissue usually arranged longitudinally, and not much elastic tissue. Longitudinally arranged muscular fibres occur in some veins (renal, portal, inferior cava near the liver, veins of the lower extremities). The valves consist of fine fibrillar connective tissue with branched cells. An elastic network exists on their convex surface, and both surfaces are covered by endothelium. The valves contain many muscular fibres (Fig. 42). [Ranvier has shown that the shape of the epithelial cells covering the two surfaces of the valves differs. On the side over which the blood passes, they are more elongated than on the cardiac side of the valve, where the long axes of the cell are placed transversely.]

The sinuses of the dura mater are spaces covered with endothelium. The spaces are either duplicatures of the membrane, or channels in the substance of the tissue itself.

Cavernous spaces we may imagine to arise by numerous clivisions and anastomoses of tolerably large veins of unequal calibre. The vascular wall appears to be much perforated and like a sponge, the internal space being traversed by threads and strands of tissue, which are covered with endothclium on their surfaces, that are in contact with the blood. The surrounding wall consists of connective tissue which is often very tongh, as in the corpus cavernosum, and it not unfrequently contains non-striped muscle.

Cavernous Formations of an analogous nature on arterics, are the carotid-gland of the frog, and a similar structure on the pulmonary arteries and aorta of the turtle, and the coccygeal-gland of man (Luschka). This structure is richly supplied with sympathetic nerve-fibres, and is a convoluted mass of ampullated or fusiform dilatations of the middle sacral artery (Arnold), surrounded and permeated by non-striped muscle (Eberth).

Vasa Vasorum.-[These are small vessels which nourish the coats of the arteries and veins. They arise from one part of a vessel and enter the walls of 
the same vessel, or another at a lower level. They break up chiefly in the outer coat, and none enter the inner coat.] In structure they resemble other small blood-vessels, and the blood circulating in the arterial or venous wall is returned by small veins.

Intercellular blood-channels.-Intercellular blood-channels of narrow calibre and without walls occur in the granulation tissue of healing wounds. At first blood-plasma alone is found between the formative cells, but afterwards the bloodcurrent forces blood-corpuscles through the channels. The first blood-vessels in the developing chick are formed in a similar way from the formative cells of the mesoblast.

Properties of the Blood-Vessels.-The larger blood-vessels are cylindrical tubes composed of several layers of various tissues, more especially elastic tissue and plain muscular fibres, and the whole is lined by a smooth layer of epithelium. One of the most important properties is the CONTRACTILITY of the vascular wall, in virtne of which the blood-vessel becomes contracted, so that the calibre of the vessel and, therefore, the supply of blood to a part are altered. The contractility is due to the plain muscular fibres which are, for the most part, arranged circularly. It is most marked in the small arteries, and of course is absent where no muscular tissue occurs. The amount and intensity of the contraction depend upon the development of the muscular tissue; in fact, the two go hand-in-hand. [If an artery be exposed in the living body it soon contracts under the stimulus of the atmosphere (J. Hunter) acting upon the muscular fibres.]

[Action of Alkalies and Acids on the Vascnlar System.-Gaskell finds that very dilute alkalies and acids have a remarkable effect on the blood-vessels and also upon the heart. A very dilute solution of lactic acid ( 1 part to 10,000 parts of saline solution), passed through the blood-vessels of a frog, always enlarges the calibre of the blood-ressels, while an alkaline solution (1 part sodium hydrate to 10,000 or 20,000 parts saline solution) always diminishes their size, usually to absolute closure, and incleed the artificial constriction of the blood-vessels may be almost complete. These fluids are antagonistic to each other as far as regards their action on the calibre of the arteries. Microscopic observations which confirmed these results, were also macle on the blood-vessels of the mylohyoid muscle of the frog. Dilute alkaline solutions act on the heart in the same way. After a series of beats, the ventricle stops beating, the stand-still being in a state of contraction. Very dilute lactic acid causes the ventricle to stand still in the position of complete relaxation. The action of the acid and alkali solutions are antagonistic in their action on the ventricle. Gaskell attaches considerable importance to the "tonic" and "atonic" conditions of the whole vascular system produced by very dilute solutions of alkalies and acids respectively.]

That the capillaries undergo dilatation and contraction, owing to variations in size of the protoplasmic elements of their walls, must be admitted.

Stricker has described capillaries as "protoplasm in tubes," and observed that they exhibited movements when stimulated in living animals. Golubew described an active state of contraction of the capillary wall, but he regarded the nuclei as 
the parts which underwent change. Tarchanoff found that mechanical or electrical stimulation caused a change in the shape and size of the nuclei, so that he regards these as the actively contractile parts. [Severini also attaches great importance to the contractility of the capillaries.] Stricker's observations were made on the capillaries of tadpoles. These phenomena became less marked as the animal became older. Rouget observed the same result in the capillaries of new-born mammals. As the capillaries are excessively thin and delicate, and as they are soft structures, it is obvious that the form of the individual cells must depend to a considerable extent upon the degree to which the vessels are filled with blood. In vessels which are distended with blood the endothelial cells are flattened, but when the capillaries are collapsed, they project more or less into the lumen of the vessel (Renaut).

[It is a well-kuown fact that the capillaries present great variations in their diameter at different times. As these variations are usually accompanied by a corresponding contraction or dilatation of the arterioles, it is usually assumed that the variations in the diameter of the capillaries are due to differences of the pressure within the capillaries themselves-viz., to the elasticity of their walls. Every one is agreed that the capillaries are very elastic, but the experiments of Roy and Graham Brown show that they are contractile as well as elastic, and these observers conclude that under normal conditions, it is by the contractility of the capillary wall as a whole that the diameter of these vessels is changed, and to all appearance their contractility is constantly in action. "The individual capillaries (in all probability) contract or expand in accordance with the requirements of the tissues through which they pass. The regulation of the,vascular blood-flow is thus more complete than is usually imagined "(Roy and Graham Brown).]

Physical Properties.-Amongst the physical properties of the bloodvessels, ELASTICITY is the most important; their elasticity is small in amount, i.e., they offer little resistance to any force applied to them so as to distend or elongate them, but it is perfect in quality, i.e., the blood-vessels rapidly regain their original size and form after the force distending them is removed.

[The elasticity of the arteries is of the utmost importance in aiding the conversion of the interrupted flow of the blood in the large arteries into a uniform flow in the capillaries. E. H. Weber compared the elastic wall of the arteries with the air in the air-chamber of a fire-engine. In both cases an elastic medium is acted upon-the air in the one case and the elastic tissue in the other-which in turn presses upon the fluid, propelling it onwards continually, while the action of the pump or the heart, as the case may be, is intermittent.]

According to E. H. Weber, Volkmann, and Wertheim, the elongation of a bloodvessel (and most moist tissues) is not proportional to the weight used to extend it, the elongation being relatively less with a large weight than with a small one, so that the curve of extension is nearly [or, at least, bears a certain relation to] a hyperbola.

According to Wundt, we have not only to consider the extension produced at first by the weight, but also the subsequent "elastic after-effect," which occurs gradually. The elongation which occurs during the last few momeuts occurs so slowly and so gradually that it is well to observe the effect by means of a magnifying lens. Variations from the general law occur to this extent, that if a certain weight is exceeded, less extension, and, it may be, permanent elongation of the artery not unfrequently occur. K. Bardeleben found, especially in veins elongated to 40 or 50 per cent. of their original length, that when the weight employed increased by an equal amount each time, the elongation was proportional 
to the square-root of the weight. This is apart from any elastic after-effect. Veins may be extended to at least 50 per cent. of their length without passing the limit of their elasticity.

[Roy has made careful experiments upon the elastic properties of the arterial wall. A portion of an artery, so that it could be distended by any desired internal pressure, was inclosed in a small vessel containing olive oil. The small vessel with oil was arranged in the same way as in Fig. 33 for the heart. The variations of the contents were recorded by means of a lever writing on a revolving cylinder. The aorta and other large arteries were found to be most elastic and most distensible at pressures corresponding more or less exactly to their normal blood-pressure, while in veins the relation between internal pressure and the cubic capacity is very different. In them the maximum of distensibility occurs with pressures immediately above zero. Speaking generally, the cubic capacity of an artery is greatly increased by raising the intra-arterial tension, say from zero to about the normal internal pressure which the artery sustains during life. Thus in the rabbit the capacity of the aorta was quadrupled by raising the intra-arterial pressure from zero to $200 \mathrm{~mm}$. Hg., while that of the carotid was more than six times as great at that pressure as it was in the undistended condition. The pulmonary artery is distinguished by its excessive elastic distensibility. Its capacity (rabbit) was increased more than twelve times on raising the internal pressure from zero to about $36 \mathrm{~mm}$. Hg. Veins, on the other hand, are distinguished by the relatively small increase in their cubic capacity produced by greatly raising the internal pressure, so that the enormous changes in the capacity of the veins during life, are due less to differences in the pressure than to the great differences in the quantity of blood which they contain (Roy).]

Pathological.-Interference with the nutrition of an artery alters its elasticity [and that in cases where no structural changes can be found]. Marasmus preceding death causes the arteries to become wicler than normal (Roy). Age also influences their elasticity-in some old people they become atheromatous and even calcified. [The ratio of expansion of strips of the aortic wall to the weights employed to stretch them, remains much the same from childhood up to a certain age (Roy).]

Cohesion.-Blood-vessels are endowed with a very large amount of cohesion, in virtue of which they are able to resist even considerable internal pressure without giving way. The carotid of a sheep is ruptured only when fourteen times the usual pressure it is called upon to bear is put upon it (Volkmann). A greater pressure is required to rupture a vein than an artery with the same thickness of its wall.

\section{The Pulse-Historical.}

Although the movement of the pulse in the superficially placed arteries was known to the ancients, still the pulse, as it was affected by disease, was more studied by the older physicians than the normal pulse. Hippocrates (460 to 377 B.C.) speaks of the former as $\sigma \phi u \gamma u o$ s, while Herophilus ( 300 B.C.) contrasted the normal pulse $(\pi \alpha \lambda \mu o ́ s)$ with the pulse of disease ( $\sigma \phi v \gamma \mu o ́ s)$. He lays special stress upon the relative time occupied by the dilatation aud contraction of the arterial tube, and compares these phenomena with the notes of music. He established the fact that the rhythm of the pulse varies in the newly-born, in the adult, and in the aged. Further, he distinguished the size, fulness, quickness, and frequency of the pulse. Erasistratus ( +280 B.c.), a contemporary of Herophilus, made correct 
observations on the pulse-wave. He points out that the pulse occurs sooner in arteries near the heart than in those placed further away from it, because the pulse proceeding from the heart passes towards the periphery. Erasistratus placed a cannula in the course of an artery, and he found that the pulse could still be felt on the distal side of this point. Archigenes gave the name dicrotic pulse to a condition which he had observed in febrile conditions. Galen (131 to 202 A.D.) gave more exact details as to the relations of the dilatation and contraction of the arteries during the movement of the pulse, and supplied much information on the pulse-rhythm, and the influence of temperament, age, sex, period of the year, climate, sleep and waking, cold and warm baths, on its rate and other qualities. Cusanus (1565) was the first person to count the pulse-beats with the aid of a watch.

\section{Instruments for Investigating the Pulse.}

The individual phases of the movement of the pulse could only be accurately investigated by the application of instruments to the arteries.

(1.) Poiseuille's Box Pulse-Measurer (1829).-An artery (Fig. 43, a, a) is exposed and placed in an oblong box $(K, K)$ filled with an indifferent fluid. A vertical tube with a scale attached communicates with the interior of the box. The column of fluid undergoes a variation with every pulse-beat.

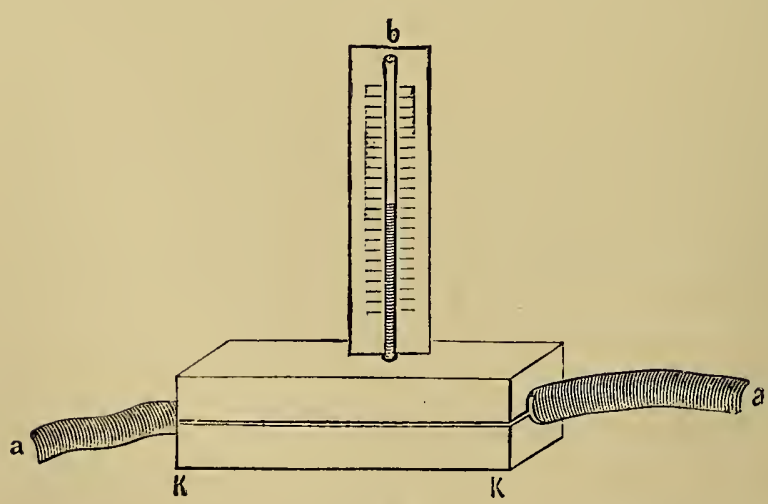

Fig. 43.

Poiseuille's pulse-measurer- $a, a$, exposed artery ; $\mathbf{K}, \mathbf{K}$, the box consisting of two pieces; $b$, vertical tube, with scale attached.

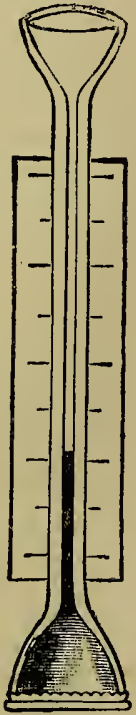

Fig. 44.

Sphygmometer of Hérisson and Chelius.

(2.) Hérisson's Tubular Sphygmometer consisted of a glass-tube whose lower end was covered with an elastic membrane (Fig. 44). The tube was partly filled with $\mathrm{Hg}$. The membrane was placed over the position of a pulsating artery, so that its beat caused a movement in the $\mathrm{Hg}$. Chelius used a similar instrument, 
and he succeeded with this instrument in showing the existence of the doublebeat (dicrotism) in the normal pulse (1S50).

(3.) Vierordt's Sphygmograph (1855).-In this, one of the earliest sphygmographs, Vierordt departed from the principle of a fluctuating fluid column, and adopted the principle of the lever. Upon the artery rested a small pad, which moved a complicated system of levers. At first he used a straw six inches long, which rested on the artery. The point of one of the levers inscribed its movements upon a revolving cylinder. This iustrument was soon discarded.

(4.) Marey's Sphygmograph consists of a combination of a lever with an elastic spring. It consists of an elastic spring (Fig. 45, A) fixed at one end, $\approx$, free at the other end, and provided with an ivory pad, $y$, which is pressed by the spring upon the radial artery. On the upper surface of the pad there is a vertically-placed fine toothed rod, $k$, which is pressed upon by a weak spring, $e$, so that its teeth dove-tail with similar teeth in the small wheel, $t$, from whose axis there projects a long, light, wooden lever, $v$, running nearly parallel with the elastic spring. This lever has a fine style at its free-end, $s$, which writes upon a smoked plate, $\mathrm{P}$, moved by clock-work, $\mathrm{U}$, in front of the style. Marey's instrument, as improved by Mahomed and others, has been very largely used.

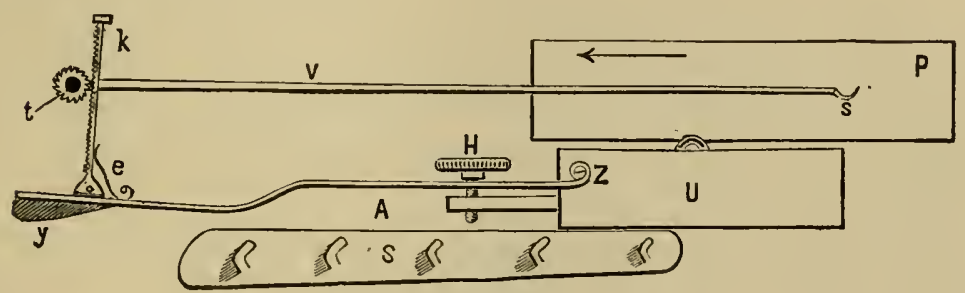

Fig. 45.

Scheme of Marey's sphygmograph-A, spring with ivory pad, $y$, which rests on the artery; $e$, weak spring pressing $k$ into $t ; v$, writing lever; $\mathrm{P}$, piece of smoked glass or paper moved by clock-work, $U ; H$, screw to limit excursion of $\mathrm{A} ; s$, arrangement for fixing the instrument to the arm of the patient.

[Its more complete form, as in Fig. 46, where it is shown applied to the arm, consists of--(1.) a steel spring, A, which is provided with a pad resting on the artery, and moves with each movement of the artery; (2.) the lever, C, which records the movement of the artery and spring in a magnified form on the smoked paper, $G$; (3.) an arrangement, $L$, whereby the exact pressure exerted upon the artery is indicated on the dial, M (Mahomed); (4.) the clock-work, H, which moves the smoked paper, $G$, at a uniform rate; (5.) a frame-work to which the various parts of the instrument are attached, and by means of which the instrument is fastened to the arm by the straps, $K, K$ (Byrom Bramwell).

[Application.- In applying the sphygmograph, cause the patient to seat himself beside a low table, and place his arm on the double-inclined plane (Fig. 46). In the newer form of instrument, the lid of the box is so arranged as to unfold to make this support. The fingers ought to be semi-flexed. Mark the position of the radial artery with ink. See that the clock-work is wound ap, and apply the 
ivory pad exactly over the radial artery where it lies upon the radius, fixing it to the arm by the non-elastic straps, K, K (Fig. 46). Fix the slide holding the smoked paper in position. The best paper to use is that with a very smooth surface (albuminised or enamelled card) smoked over the flame of a turpentine lamp, or over a piece of burning camphor. The writing-style is so arranged as to write upon the smoked paper with the least possible friction. The most important part

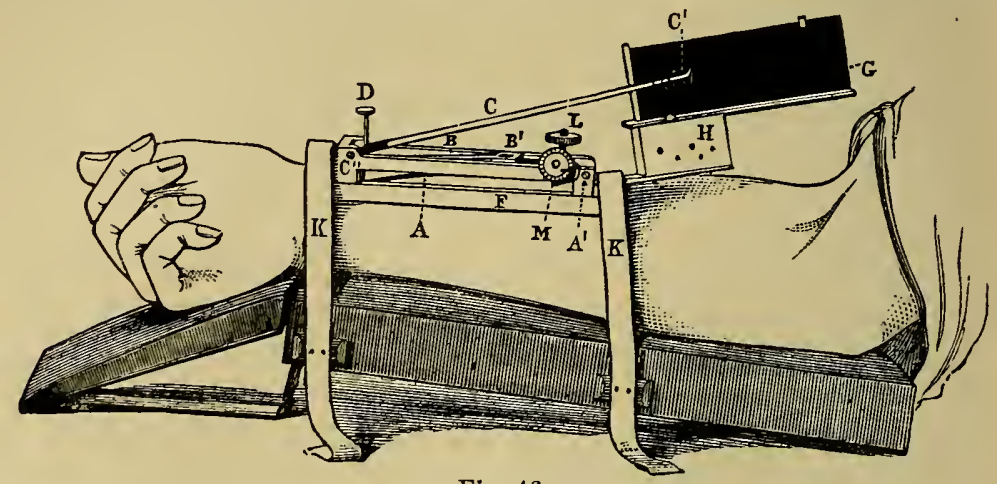

Fig. 46.

Marey's improved sphygmograph as used when a tracing is taken-A, steel spring ; B, first lever; C, writing lever; $\mathrm{C}^{\prime}$, its free writing end; $D$, screw for bringing $\mathrm{B}$ in contact with $\mathrm{C}$; $\mathrm{G}$, slide with smoked paper; $\mathrm{H}$, clockwork; L, screw for increasing the pressure; M, dial indicating the amount of pressure; $\mathrm{K} . \mathrm{K}$, straps for fixing the instrument to the arm, and the arm to the double-inclined plane or support (Byrom Bramwell).

of the process is to regulate the pressure exerted upon the artery by means of the milled head, L. This must be determined for each pulse, but the rule is to graduate the pressure until the greatest amplitude of movement of the lever is obtained. Set the clock-work going, and a tracing is obtained, which must be "fixed " by dipping it in a rapidly drying varnish-e.g., photographic. In every case scratch on the tracing with a needle the name, date, and amount of pressure employed.]

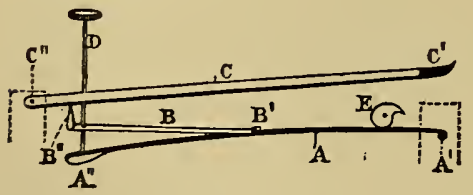

Fig. 47.

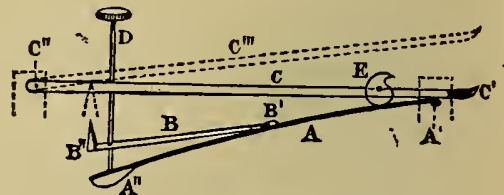

Fig. 48.

[Fig. 47.-Scheme showing the essential part of the instrument when in working order-i.e., the turned up knife-edge, $\mathrm{B}^{\prime \prime}$, of the short lever in contact with the writing lever, C. Every movement of the steel spring at $A^{\prime \prime}-i . e$. , the artery - will in this position be communicated to the writing lever.

[Fig. 48. -Scheme showing the essential parts of the instrument after increase of the pressure. The knife-edge, $\mathrm{B}^{\prime \prime}$, is no longer in contact with the writing lever, and the movements of the steel spring, $\mathrm{A}^{\prime \prime}-i . e$., the artery-are no longer communicated to it. In order to put the instrument into working order, the knife-edge, B", must be raised to the position indicated by the dotted lines. This is effected by means of the screw, D (Byrom Bramwell).] 
[(5.) Dudgeon's Sphygmograph.-This is a most convenient form of sphygmograph. Fig. 49 shows its actual size.

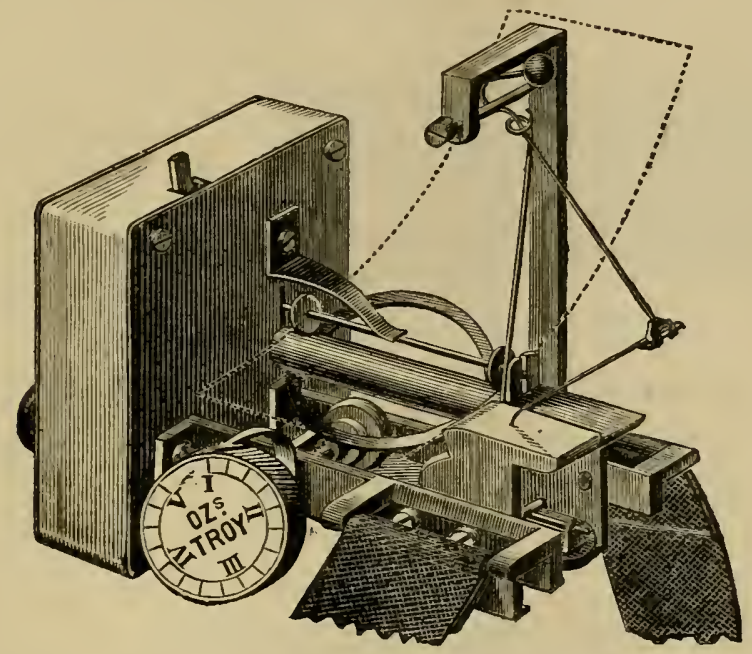

Fig. 49.-Dudgeon's sphygmograph.

The instrument after being carefully adjusted upon the radial artery is kept in position by an inelastic strap. The pressure of the spring is regulated by the eccentric wheel to any amount from 1 to 5 ounces.

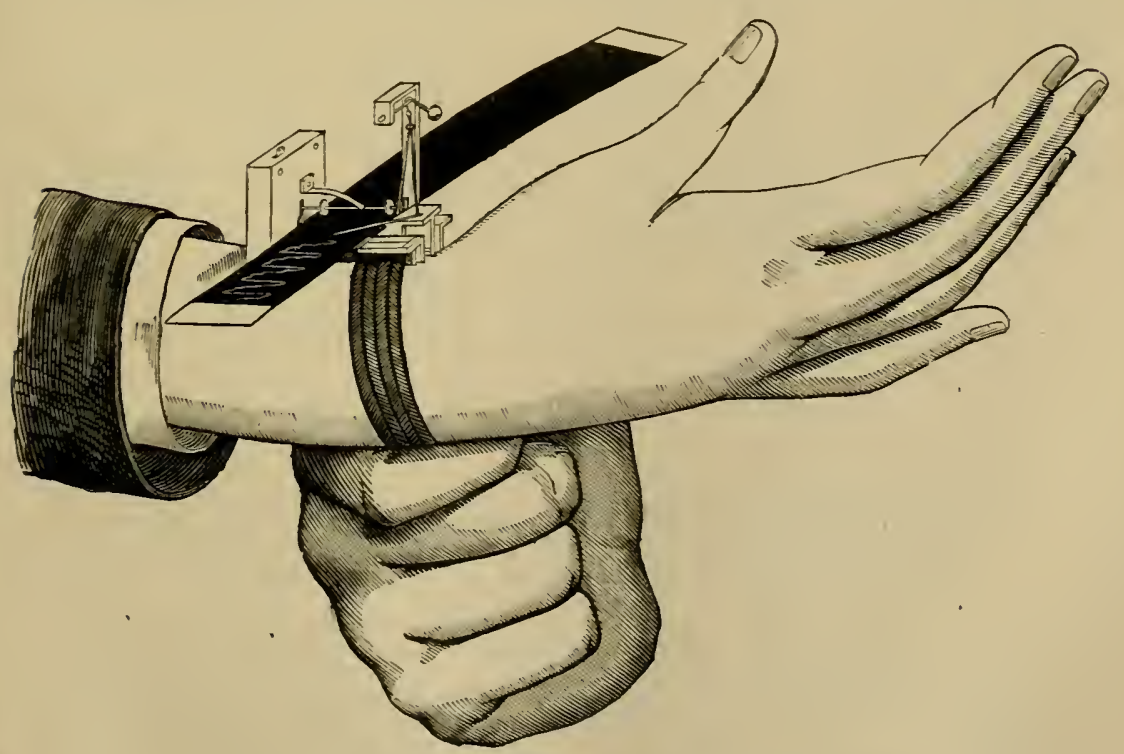

Pig. 50.-Mode of applying Dudgeon's sphygmograph. 
As in other instruments, the tracing paper is moved in front of the writing-needle by means of clock-work. The writing-levers are so adjusted that the movements of the artery are magnified fifty times.]

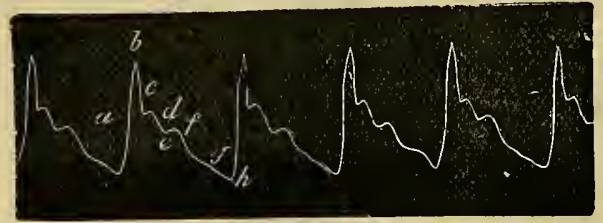

Fig. 51.-Sphygmogram-pressure $2 \mathrm{oz}$.

[Fig. 51 is a sphygmogram taken with this instrument from a healthy individual. Itrepresents a perfect tracing $-a$, the vertical upward, systolic or percussion wave; $b$, apex ; $c$, on the descent; $d$, first tidal or predicrotic wave; $e$, aortic notch ; $f$, dicrotic wave (Dudgeon).]

(6.) Marey's Tambours are also employed for registering the movements of the pulse. They are used in the same way as the pansphygmograph of Brondgeest. Fig. 52 shows their arrangement. Two pairs of metallic cups (S, S and $\mathrm{S}^{\prime}, \mathrm{S}^{\prime}$, Upham's capsules) are pierced in the middle by

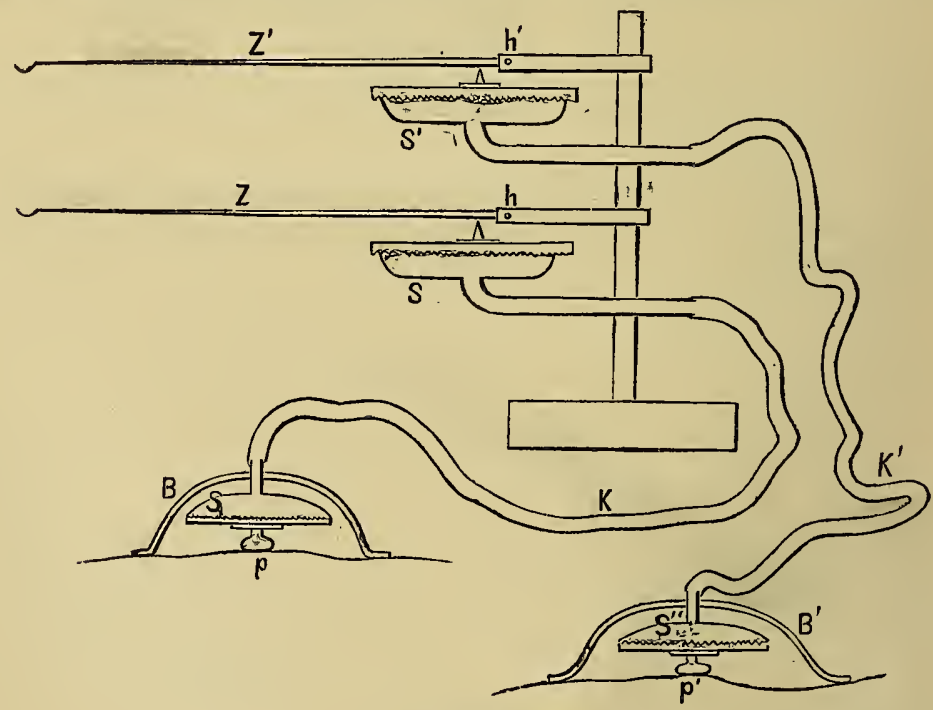

Fig. 52.

Scheme of Brondgeest's sphygmograph, on the principle of Upham and Marey's tambours-S, $S^{\prime}$, recciving and recording $\left(S, S^{\prime}\right)$ tambours with writing-levers, $\mathrm{Z}$ and $\mathrm{Z}^{\prime} ; \mathrm{K}, \mathrm{K}^{\prime}$, conducting tubes : $p$ over heart, $p^{\prime}$ over a distant artery. This illustration also shows the principle of Marey's cardiograph.

thin metal tubes, whose free-ends are connected with caoutchouc tubes, $\mathrm{K}$ and $\mathrm{K}^{\prime}$. All the four metallic vessels are covered with an elastic membrane. On $\mathrm{S}$ and $\mathrm{S}^{\prime}$ are fixed two knob-like pads, $p$ and $p^{\prime}$, which are applied to the pulsating arteries, and the metal arcs, $B$ and 
$\mathrm{B}^{\prime}$, retain them in position. On the other tambours are arranged the writing levers, $Z$ and $Z^{\prime}$. Pressure on the one tambour necessarily compresses the air and makes the other, with which it is connected, expand, so as to move the writing-lever. This arrangement does not give absolutely exact results; still, it is very easily used and is convenient. In Fig. 52 a double arrangement is shown, whereby one instrument, $B$, may be placed over the heart, and the other, $\mathrm{B}^{\prime}$, on a distant artery.

Landois' Angiograph.-To a basal plate, G, G, are fixed two upright supports, $p$, which carry between them at their upper part the movable lever, $d, r$, carrying a rod bearing a pad, $e$, directed downwards, which rests on the pulse. The short arm carries a counterpoise, $d$, so as exactly to balance the long arm. The long arm has fixed to it at $r$ a vertical rod provided with teeth, $h$, which is presserl against a toothed wheel firmly fixed on the axis of the very light writing-lever, $e f$, which is supported between two uprights, $q$, fixed to the opposite end of the basal plate, G, G. The writing-lever is equilibriated by means of a light weight. The writing-needle, $k$, is fixed by a joint to $e$, and it writes on the plate, $t$. The first-mentioned lever, $d, r$, carries a shallow plate, $Q$, just above the pad, into which weights may be put to weight the pulse. In this instrument the weight can be measured

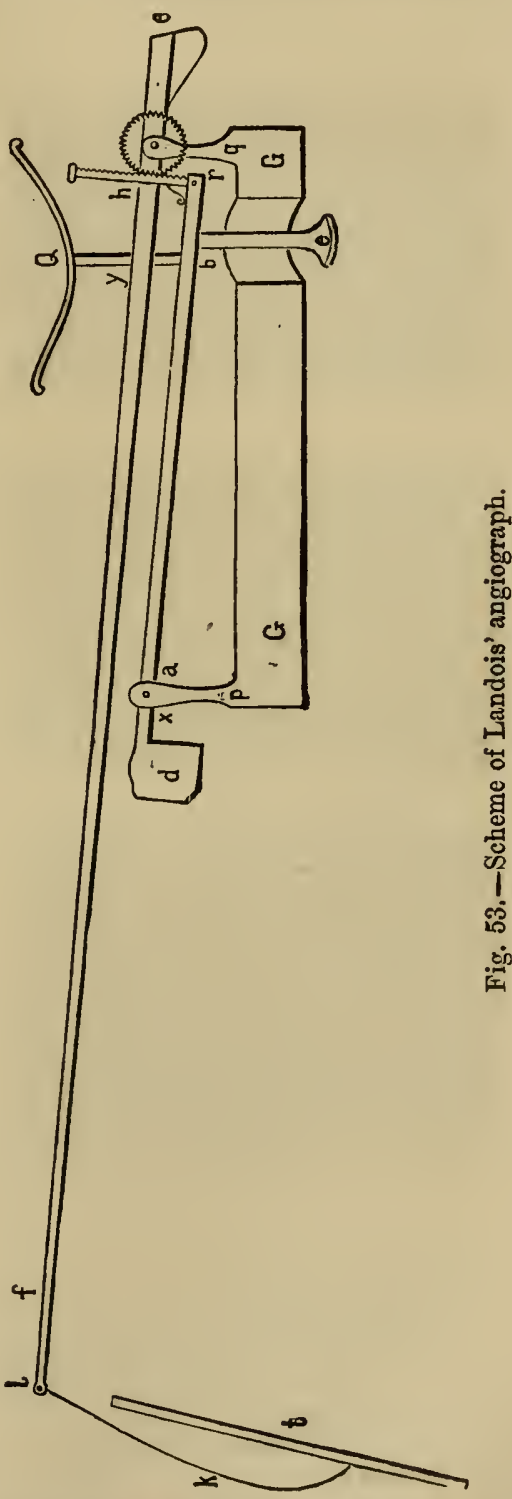
and varied; the writing-lever moves vertically and not in a curve, 
as in Marey's apparatus, which greatly facilitates the measuring of the curves. (Fig. 53.)

Other sphygmographs are used, both in this country and abroad, including that of Sommerbrodt, which is a complicated form of Marey's sphygmograph, and those of Pond and Mach. In choosing a sphygnograph, that instrument is to be preferred which yields a curve corresponding most closely with the variations of the pressure within the artery, in which the resistance of the instrument

A

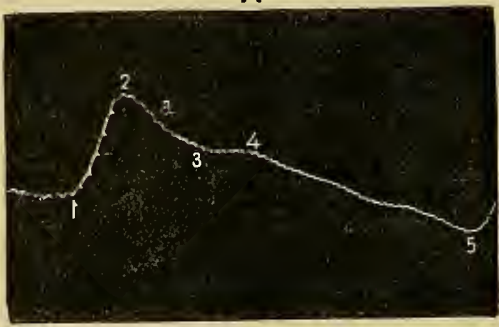

B

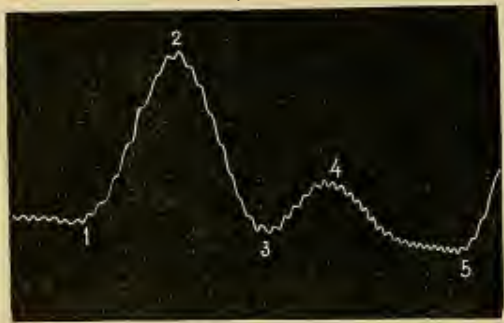

C

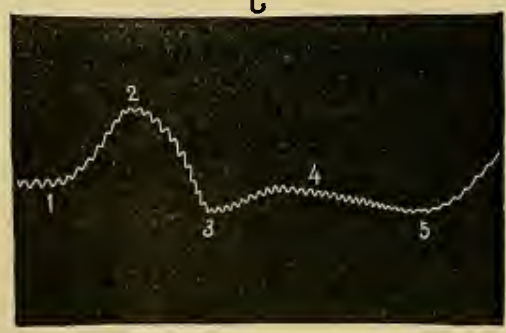

Fig. 54.

Pulse-curves of the carotid, radial, and posterial tibial arteries of a healthy student, obtained by Landois' angiograph writing upon a plate attached to a vibrating tuning.fork. Each double vibration corresponds to $0.01613 \mathrm{sec}$. is small, which gives the largest curve, and in which the part in contact with the artery is not greatly displaced from its position of equilibrium (Mach).

\section{Characters of a Pulse-Curve.-} In every pulse-curve--SPHYGMOGRAM or ARTERIOGRAM-we can distinguish the ascending part (ascent) of the curve, the apex, and the descending part (descent). Secondary elevations scarcely ever occur in the ascent, which is usually represented by a straight line, while they occur constantly in the descent. Such elevations occurring in the descent are called catacrotic, and those in the ascent, anacrotic (Landois). When the recoil elevation or dicrotic wave occurs in a well-marked form in the descent, the pulse is said to be dicrotic, and when it occurs twice, tricrotic.

Measuring Pulse-Curves.-If the smoked surface on which the tracing is inscribed is moved at a uniform rate by means of the clock-work, then the height and length of the curve are measured by means of an ordinary rule. If we know the rate at which the paper was moved, then it is easy to calculate the duration of any event in the curve. For exact observation a low-power microscope with a micrometer in the eye-piece should be used.

For the method of smoking the paper and fixing the tracings see p. 130. 
It is very convenient to write the curve upon a plate of glass fixed to a tuning-fork kept in vibration. Every part of the curve shows little elevations (whose rate of vibration is known beforehand). All that is required is to count the number of vibrations in order to ascertain the duration of any part of the curve.

Fig. 54 was taken in this way from (A) the carotid, (B) the radial, and (C) the posterior tibial arteries of a healthy student. The results are :-

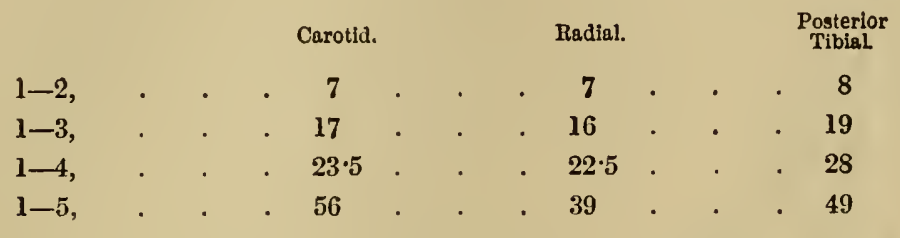

This method has also been used for the registration of other physiological processes-e.g., contraction of muscle.

Landois' Gas.Sphygmoscope.-A superficially placed artery communicates its movements to the overlying skin, and also to any freely movable body in contact with the skin. In this instrument (Fig. 55) a thin layer of air over the pulsating artery, $a$, is enclosed by means of a thin piece of metal, which is so adjusted that its concave side forms a tunnel of air over the artery. The narrow space between the metallic wall, $b$, and the skin, $a$, is filled with ordinary gas, one end of the metal shield being connected by means of a tube, $g$, with the gas-supply, while to the other end there is attached by means of a short piece of eaoutchouc, $x, q$, a bent glass-tube, $t$, with a very small aperture which acts as a gas-burner. The gas is allowed to flow through the apparatus at a low pressure, and is so regulated that the flame, $v$, is only a few millimetres in height. The flame rises isochronously with every pulse-beat, and the dicrotic beat in the normal pulse is quite observable.

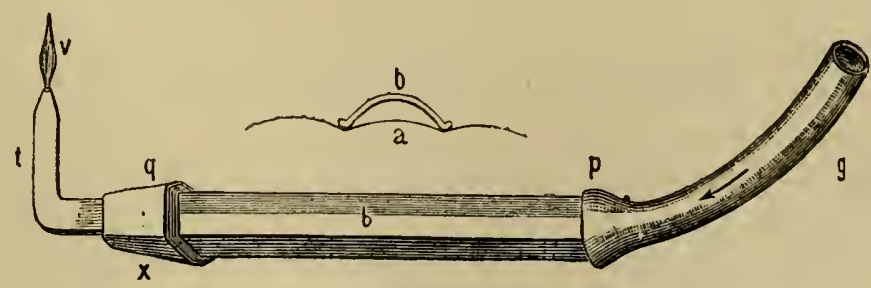

Fig. 55.

Landois' gas-sphygmoscope- $a$, skin over artery; $b$, metal plate; $p, g$, gas ; $x, q$, caoutchouc tube attaching glass gas-burner, $t$ to $b$.

Czermak photographed a beam of light set in motion by the movements of the pulse.

Hæmautography. - Expose a large artery of an animal, and divide it so that the stream of blood issuing from it strikes against a piece of paper drawn in front 
of the blood-stream. A curve (Fig. 56) is obtained which corresponds very closely with the pulse-tracing obtained from a normal

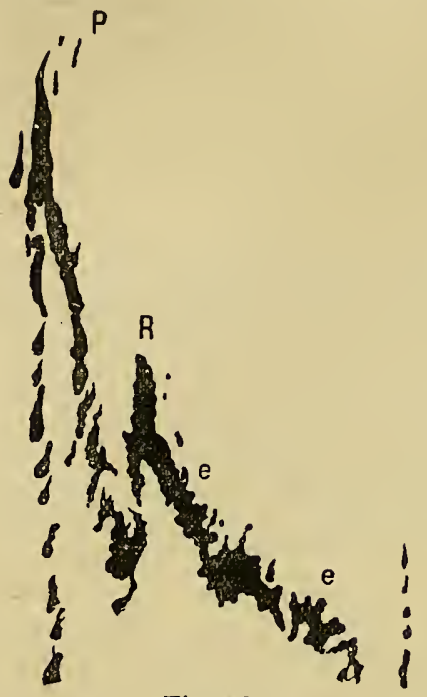

Fig. 56.

Hæmautographic curve of the posterior tibial artery of a large dog $-\mathrm{P}$, primary pulse wave; $\mathrm{R}$, dicrotic or recoil wave; $e, e$, elevations due to elasticity. artery. In addition to the primary wave, $P$, there is a distinct "recoil-elevation," or dicrotic wave, $\mathrm{R}$, and slight vibrations, $e, e$, due to variations in the elasticity of the arterial wall. The interest which attaches to a curve obtained in this way is, that it shows the movements to occur in the blood itself, and these movements to be communicated as waves to the arterial wall. By estimating the amount of blood in the various parts of the curve we obtain a knowledge of the amount of blood discharged by the divided artery during the systole and diastole (i.e., the narrowing and dilatation) of the arterythe ratio is $7: 10$. Thus in the unit of time, during arterial dilatation rather more than twice as much blood flows out as happens during arterial contraction.

Microphone.-Fix a small piece of wax over the radial artery, and to it attach a very fine vertical wire which is brought into contact with the charcoal of a microphone held over the artery. The primary pulse wave and dicrotic wave are distinctly heard in a telephone brought into connection with the microphone (Landois). All these methods are well suited for demonstrating the pulse, but for accuracy resort must be had to some form of recording instrument.

\section{The Pulse-Curve or Sphygmogram.}

A sphygmogram consists of several curves, each one of which corresponds with a beat of the heart. Each pulse-curve consists of (1.) the ascending part which occurs during the dilatation (diastole) of the artery; (2.) the apex, ( $\mathrm{P}$ in Fig. 58 and $b$ in Fig. 57); (3.) the descending part, corresponding to the contraction (systole) of the artery. The most noticeable peculiarity of the pulse-curve is the existence of turo completely distinct elevations occurring in the descent. The more distinct of the two occurs as a well-marked elevation about the middle of the descent ( $\mathrm{R}$ in Fig. 58 and $f$ in Fig. 57 ); it is called the DICROTIC WAVE, or with reference to its mode of origin, the "recoil wave." The ascent, also called up-stroke or percussion stroke (Mahomed), in a normal sphygmogram, is nearly vertical, while the apex of the percussion stroke is usually pointed.

[In Fig. 57, each part of the curve between the base of one up- 
stroke and the base of the next up-stroke corresponds to a beat of the heart, so that this figure shows five heart-beats and part of a sixtl. The part, $a, b=$ the ascent, $b$, the apex of the up-stroke, and $b$ to $h$, the descent, with a curve, $d$, called the first tidal or predicrotic wave, $e$, an angle or notch, the

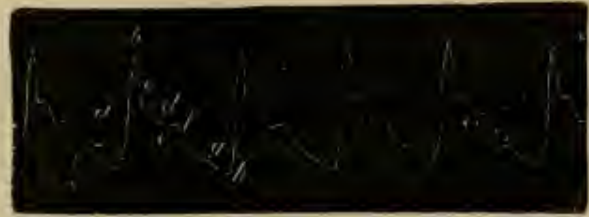

Fig. 57.

Sphygmogram of radial artery-pressure $2 \mathrm{oz}$. aortic notch, $f$, a second elevation, called the dicrotic wave, $g$, a slight curve, sometimes called the second tidal wave. The descent is continued to $h$, where the ascent of the next heart-beat begins.]

\section{Origin and Characters of the Dicrotic Wave.}

The dicrotic or recoil wave, which is always present in a normal pulse, is caused thus :-During the ventricular systole a mass of blood is propelled into the already full aorta, whereby a positive wave is rapidly transmitted from the aorta throughout the arterial system, even to the smallest arterioles, in which this primary wavo is extinguished. As soon as the semi-lunar valves are closed, and no more blood flows into the arterial system, the arteries which were previously distended by the mass of blood suddenly thrown into them, recoil or contract, so that in virtue of the elasticity (and contractility) of their walls, they exert a counter-pressure upon the column of blood, and thus the blood is forced onwards. There is a free passage for it towards the periphery, but towards the centre (heart) it impinges upon the already closed semi-lunar valves. This developes a new positive wave, which is propagated peripherally through the arteries, where it disappears in their finest branches. In those cases where there is sufficient time for the complete development of the pulse-curve (as in the short course of the carotids, and in the arteries of the upper arm, but not in those of the lower extremity, on account of their length), a second wave of reflection may be cansed in exactly the same way as the first.

Just as the pulse occurs later in the more peripherally placed arteries than in those near the heart, so the secondary wave reflected from the closed aortic valves must appear later in the peripheral arteries. Both kinds of waves, the primary pulse wave, the secondary, and eventually even the tertiary reflected wave arise in the same place, and take the same course, and the longer the course they have to travel to any part of the arterial system, the later they arrive at their destination. 
The following points regarding the dicrotic wave have been ascertained experimentally :-

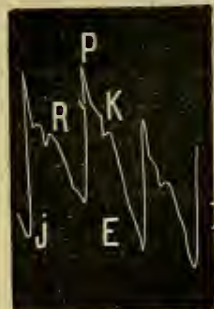

I

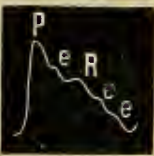

VI

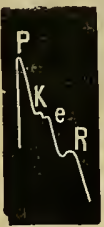

II

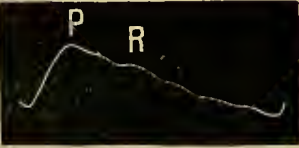

VII

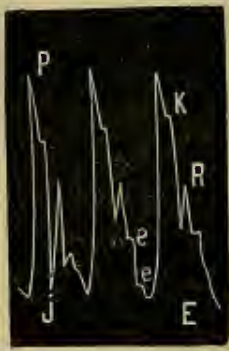

IV

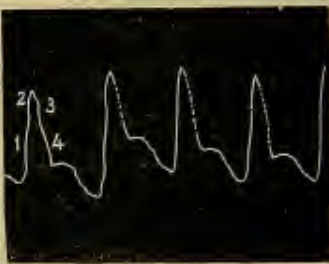

VIII

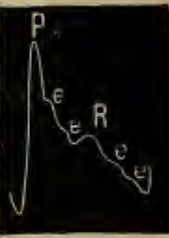

V

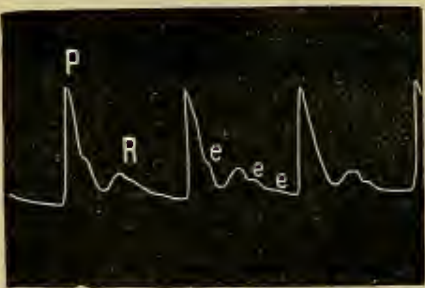

IX

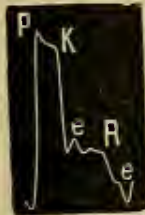

$\mathrm{X} 1$

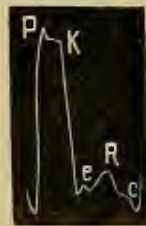

XII

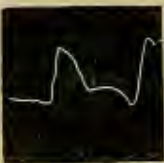

XIII

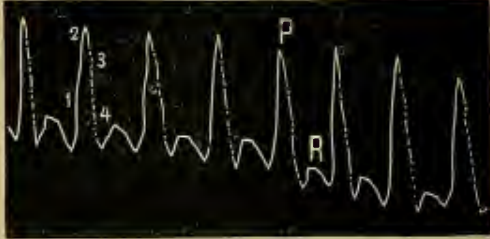

$\mathbf{X}$

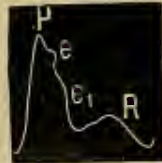

XIT

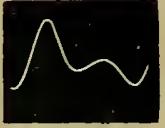

$\mathrm{XV}$

Fig. 58.

I, II, III, Suhygmogram of carotid artery; IV, axillary; $V$ to IX, radial ; $\mathrm{X}$, dicrotic radial pulse; XI, XII, crural; XIII, posterior tibial; XIV, $\mathrm{XV}$, pedal. In all the curves $-\mathrm{P}$, indicates apex; $\mathrm{R}$, dicrotic wave; $e, e$, elevations due to elasticity; $K$, elevation cansed by closure of the semi-lunar valves of the aorta.

(1.) The dicrotic wave occurs later in the descending part of the 
curve, the further the artery experimented upon is distant from the heart (Landois, 1863). Compare the curves, Fig. 54, p. 134.

The shortest accessible course is that of the carotid: where the dicrotic wave reaches its maximum 0.35 to $0.37 \mathrm{sec}$. after the beginning of the pulse. In the upper extremity the apex of the dicrotic wave is 0.36 to 0.38 to $0.40 \mathrm{sec}$. after the beginning of the pulse-beat. The longest course is that of the arteries of the lower extremity. The apex of the dicrotic wave occurs 0.45 to 0.52 to $0.59 \mathrm{sec}$. after the base of the curve. It varies with the height of the individual.

(2.) The dicrotic elevation in the descent is lower (Naumann), and is less distinct (Landois), the further the artery is situated from the heart. This is just what one would expect-viz., the longer the distance which the wave has to travel the less distinct it must become.

(3). It is more pronounced in a pulse where the primary pulse-wave is short and energetic (Marey, Landois). It is greatest relatively when the systole of the heart is short and energetic.

(4.) It is greater the lower the tension or pressure of the blood within the arteries (Marey, Landois), [and is best developed in a soft pulse]. In Fig. 58, IX and $\mathrm{X}$ were obtained when the tension of the arterial wall was low; V and VI, medium; and VII with high tension.

Conditions Influencing Arterial Tension.--It is diminished at the beginning of inspiration, by hæmorrhage, stoppage of the heart, heat, an elevated position of parts of the body; it is increased at the beginning of expiration by accelerated action of the heart, stimulation of vaso-motor nerves, diminished outflow of blood at the periphery, and by inflammatory congestion (Knecht); further, by certain poisons, as lead, amyl nitrite; compression of other large arterial trunks, action of cold and electricity on the small cutaneous vessels, and by impeded outflow of venous blood. When a large arterial trunk is exposed the stimulation of the air causes it to contract, resulting in an increased tension within the vessel. In many diseased conditions the arterial tension is greatly increased-[e.g., in Bright's disease, where the kidney is contracted ("granular"), and where the left ventricle is hypertrophied].

In all these conditions increased arterial tension is indicated by the dicrotic wave being less high and less distinct, while with diminished arterial tension it is a larger and apparently more independent elevation. Moens has shown that the time between the primary elevation and the dicrotic wave increases with increase in the diameter of the tube, with diminution of its thickness, and when its coefficient of elasticity diminishes.

\section{Origin and Characteristics of the Elastic Elevations.}

Besides the dicrotic wave, a number of small less-marked elevations occur in the course of the descent in a sphygmogram (Fig. 58,e,e). These elevations are caused by the elastic tube being thrown into vibrations by the rapid energetic pulse-wave, just as an elastic membrane vibrates when it is suddenly stretched. The artery also executes 
vibratory movements when it passes suddenly from the distended to the relaxed condition. These small elevations in the pulse-curve, caused by the elastic vibrations of the arterial wall, are called "elastic elevations" by Landois.

(1.) The elastic vibrations increase in number in one and the same artery with the degree of tension of the elastic arterial wall. A very high tension occurs in the cold stage of intermittent fever, in which case these elevations are well marked. (2.) If the tension of the arterial wall be greatly diminished these elevations may disappear, so that while diminished tension favours the production of the dicrotic wave, it acts in the opposite way with reference to the "elastic elevations." (3.) In diseases of the arterial walls affecting their elasticity, these elevations are either greatly diminished or entirely abolished. (4.) The farther the arteries are distant from the heart, the higher are their elevations. (5.) When the mean pressure within the arteries is increased by preventing the outflow of blood from them, the elastic vibrations are higher and nearer the apex of the curve. (6.) They vary in number and length in the pulse-curves obtained from different arteries of the body.

When the arm is held in an upright position, after five minutes the blood-vessels empty themselves, and collapse, while the elasticity of the arteries is diminished.

\section{Dicrotic Pulse.}

Sometimes during fever,'especially when the temperature is high, a dicrotic pulse may be felt, each pulse-beat, as it were, being composed of two beats (Fig. 58, X),

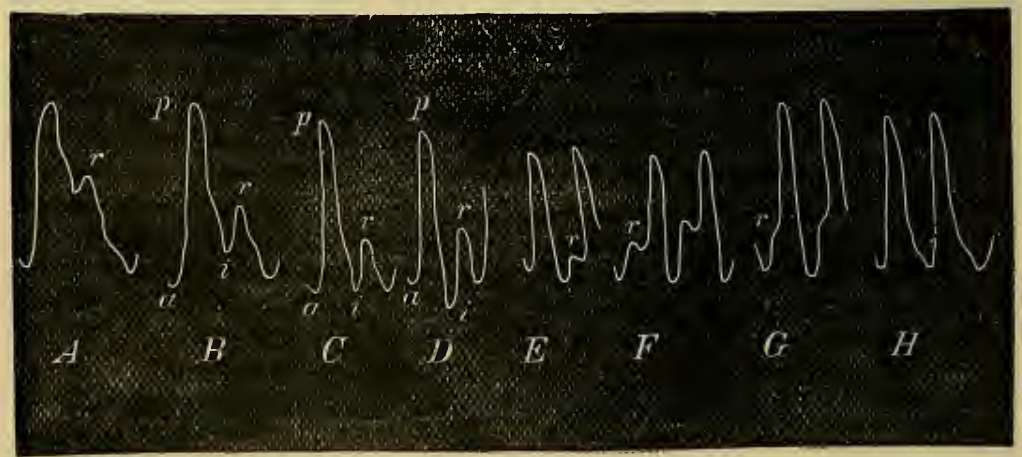

Fig. 59.

Pulsus dicrotus $-P$. caprizans; $P$. monocrotus. '

one beat being large and the other small, and more like an after-beat. Both beats correspond to one beat of the heart. The two beats are quite distinguishable by the touch. The phenomenon is only an exaggerated condition of what occurs 
in a normal pulse. The sensible second beat is nothing more than the greatly increased dicrotic elevation, which, under ordinary conditions, is not felt by the finger.

Conditions.-The occurrence of a dicrotic pulse is favoured (1) by a short primary pulse-wave, as in fevers, where the heart beats rapidly; (2) by a diminished tension within the arterial system. A short systole and diminished arterial blood-pressure are the most favourable conditions for causing a dicrotic pulse. The double-beat may be felt only at certain parts of the arterial system, whilst at other parts only a single beat is felt. A favourite site is the radial artery of one or the other side, where conditions favourable to its occurrence appear to exist. This seems to be due to a local diminution of the blood-pressure in this area, owing to the paralysis of its vaso-motor nerves (Landois). If the tension be increased by compressing other large arterial trunks or the veins of the part, the double-beat becomes a simple pulse-beat. The dicrotic pulse in fever seems to be due to the increased temperature $\left(39^{\circ}\right.$ to $40^{\circ} \mathrm{C}$.), whereby the artery is more distended, and the heart-beat is shorter and more prompt (Riegel).

(3.) It is absolutely necessary that the elasticity of the arterial wall be normal. The dicrotic pulse does not occur in old persons with atheromatous arteries (Landois). In Fig. 59, A, B, C, we observe the gradual passage of the normal radial curve, $\mathrm{A}$, into the dicrotic beat, $\mathrm{B}, \mathrm{C}$, where the dicrotic wave, $r$, appears as an independent elevation. If the frequency of the pulse increases more and more in fever, the next following pulse-beat may occur in the ascending part of the dicrotic wave, D, E, F, and it may even occur close to the apex, G (P. caprizans). If the next following beat occurs in the depression, $i$, between the primary elevation, $p$, and the dicrotic elevation, $r$, the latter entirely disappears, and the curves, $\mathrm{H}$, assume what Landois calls the "monocrotic " type.

\section{Characters of the Pulse.}

\section{Pulsus Frequens and Rarus.}

Frequency.-According as a greater or less number of beats occurs in a given time, e.g., per minute, the pulse is said to be frequent or rare. The normal rate, in man $=71$ per minute, and somewhat more in the female; in fever it may exceed 120 ( 250 have boen counted by Bowles), while in other diseases it may fall to 40 , and even 10 to 15 (de Haen), 17 (Hartog), and 14 (Cornil); but such cases are rare, and are probably due to an affection of the cardiac nerves. The frequency of the pulse is usually increased when the respirations are deeper, but not more numerous, i.e., rapid shallow respirations do not affect the frequency of the pulse, but deep respirations do (Knoll).

\section{Pulsus Celer and Tardus.}

Celerity or Rapidity.-If the pulse-wave is developed so that the distension of the artery slowly reaches its height and the relaxation also takes place gradually, we have the p. tardus or slow pulse, the opposite condition gives rise to the $\mathrm{p}$. celer or quick pulse. The rapidity of the pulse is increased by quick action of the heart, power of expansion of the arterial walls, easy efflux of blood owing to the dilatation of the small arteries, and by nearness to the heart. [The quickness has reference to a single pulse-beat, the frequency to a number of beats.] In a quick pulse, the curve is high and the angle at the apex is acute, while in a slow pulse the ascent is low and the angle at the apex is large. 


\section{Conditions affecting the Pulse-Rate.}

Frequency in Health.-In man the normal pulse-rate $=71$ to 72 beats per minute, in the female about 80 . In some individuals the pulse-rate may be higher (90 to 100), in others lower (50), and such a fact must be borne in mind. The following conditions influence it-

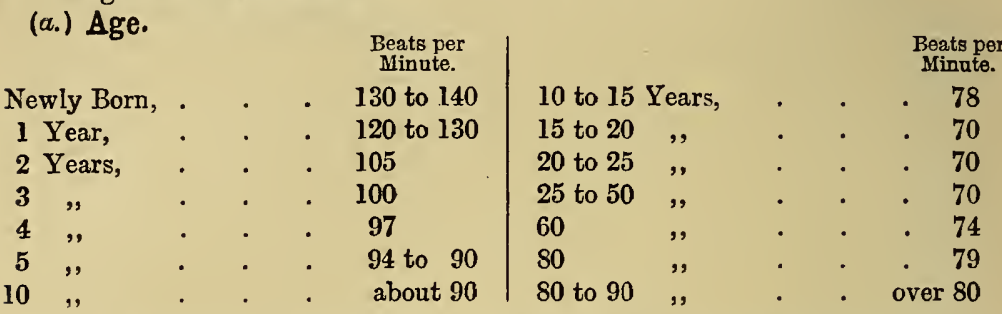

(b.) The length of the body has a certain relation to the frequency of the pulse. The following results have been obtained by Czarnecki from the formulæ of Volkmann and Rameaux-

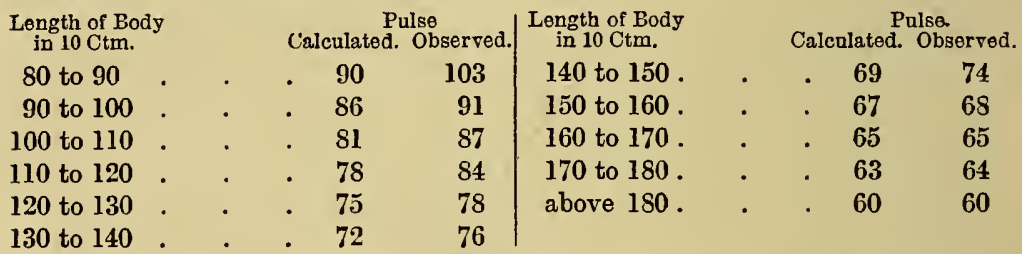

(c.) The pulse-rate is increased by muscular activity, by every increase of the arterial blood-pressure, by taking of food, increased temperature, painful sensations, and by psychical disturbances. [Increased heat or fever (Pyrexia) increases the frequency, and as a rule the increase varies with the height of the temperature. Dr. Aitken states that an increase of the temperature of $1^{\circ} \mathrm{F}$. above $98^{\circ} \mathrm{F}$. corresponds with an increase of ten pulse-beats per minute; thus,

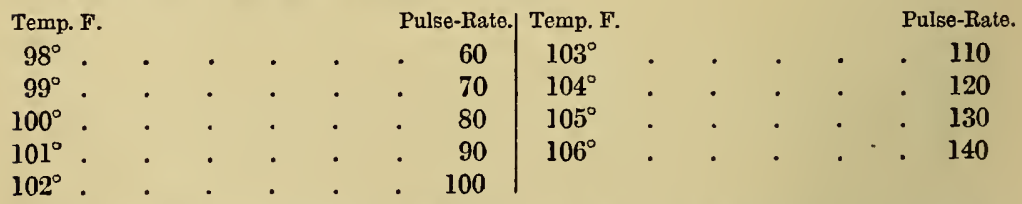

This is merely an approximate estimate.] It is more frequent when a person is standing than when he lies down. Music accelerates the pulse and increases the blood-pressure in dogs and men (Dogiel). Exposure to increased barometric pressure diminishes the frequency.

The variation of the pulse-rate during the day -3 to 6 a.m. $=61$ beats; 8 to $11 \frac{1}{2}$ a.m. $=74$. It then falls towards 2 p.m.; towards 3 (at dinner-time) another increase takes place and goes on until 6 to 8 p.m., $=70$; and it falls until midnight $=54$. It then rises again towards 2 a.m., when it soon falls again, and afterwards rises as before towards 3 to $6 \mathrm{a} . \mathrm{m}$.

\section{Variations in the Pulse-Rhythm.}

On applying the fingers to the normal pulse we feel beat after beat occurring at apparently equal intervals. Sometimes in a normal series a beat is omitted $=$ pulsus intermittens, or intermittent pulse; at other times the beats become 
smaller and smaller, and after a certain time begin as large as before $=P$. myurus. When an extra beat is intercalated in a normal serics $=P$. intercurrens. The regular alternation of a high and a low beat $=P$. culternans (Traube). In the $P$. bigeminus of Traube the beats occur in pairs, so that there is a longer pause

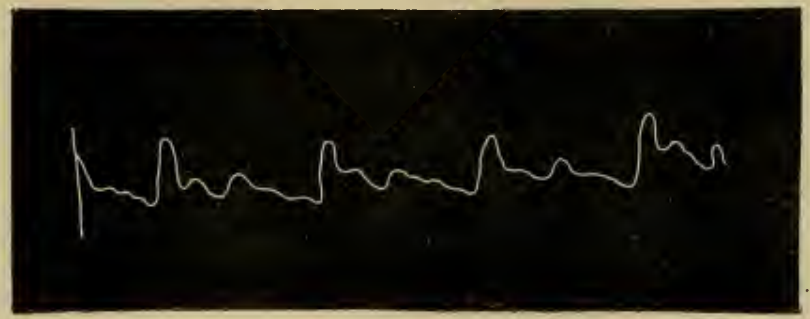

Fig. 60 .

Pulsus alternans.

after cvery two beats. Traulue found that he conld produce this form of pulse in curarised dogs by stopping the artificial respiration for a long time. The $P$. trigeminus and quadrigeminus occur in the same way, but the irregularities occur after every third and fourth beat. Knoll found that in animals such irregularities of the pulse were apt to occur, as well as great irregularity in the rhythm generally, when there is great resistance to the circulation, and consequently the heart has great deniands upon its energy. The same occurs in man, when an improper relation exists between the force of the cardiac muscle and the work it has to do (Riegel). Complete irregularity of the heart's action is called arhythmia cordis.

\section{Variations in the Strength, Tension, and Volume of the Pulse.}

The relative strength of the pulse (p. fortis and debilis), i.e., whether the pulse is strong or weak, is estimated by the weight which the pulse is able to raise. A sphygmograph, provided with an index indicating the amount of pressure exerted upon the spring pressing upon the artery, may be used (Fig. 46). In this case, as soon as the pressure exerted upon the artery overcomes the pulse-beat, the lever ceases to move. The weight employed indicates the strength of the pulse. [The finger may be, and generally is, used. The finger is pressed upon the artery until the pulse-beat in the artery beyond the point of pressure is obliterated. In health it requires a pressure of several ounces to do this. Handfield Jones uses a SPHYGMOMETER for this purpose. It is constructed like a cylindrical letterweight, and the pressure is exerted by means of a spiral spring which has been carefully graduated.] The pulse is hard or soft when the artery, according to the mean blood pressure, gives a feeling of greater or less resistance to the finger, and this quite independent of the energy of the individual pulse-beats $(P$. durus and mollis).

In estimating the tension of the artery and the pulse, i.e., whether it is hard or soft, it is important to observe whether the artery has this quality only during the pulse-wave, i.e., if it is hard during diastole, or whether it is hard or soft during the period of rest of the arterial wall. All arteries are harder and less compressible during the pulse-beat than during the period of rest, but an artery which is very hard during the pulse-beat may be hard also during the pause 
between the pulse-beats, or it may be very soft, as in insufficiency of the aortic valves. In this case, after the systole of the left ventricle, owing to the incompetency of the semi-lunar valves, a large amount of blood flows back into the ventricle, so that the arteries are thereby suddenly rendered partially empty. [The sudden collapse of the artery gives rise to the characteristic "pulse of unfilled arteries."]

Under similar conditions, the volume of the pulse is obvious from the size of the sphygmogram, so that we speak of a large and a small pulse (P. magnus and parvus). Sometimes the pulse is so thready and of such diminished volume that it can scarcely be felt. A large pulse occurs in disease when, owing to hypertrophy of the left ventricle, a large amount of blood is forced into the aorta. A small pulse occurs under the opposite condition, when a small amount of blood is forced into the aorta, either from a diminution of the total amount of the blood, or from the aortic orifice being narrowed, or from disease of the mitral valve; again, where the ventricle contracts feebly, the pulse becomes small and thready. Sometimes the pulse differs on the two sides, or it may be absent on one side.

Waldenburg constructed a "pulse-clock" to register the tension, the diameter of the artery, and the volume of the pulse upon a dial. It does not give a graphic tracing, the results being marked by the position of an indicator.

\section{The Pulse-Curves of Various Arteries.}

1. Carotid (Fig. 54, A.; Fig. 58, I, II, III ; Fig. 64, C and $\mathrm{C}_{3}$ ).

The ascending part is very steep-the apex of the curve (Fig. 58, P) is sharp and high. Below the apex there is a small notch-the "AORTIC NOTCH" (Fig. 58, K)-which depends on a positive wave formed in the root of the aorta, owing to the closure of the aortic valves, and propagated with almost wholly undiminished energy into the carotid artery. Quite close to this notch, if the curve be obtained with minimal friction, the first elastic vibration occurs (Fig. $58, \mathrm{II}, e)$. Above the middle of the descending part of the curve is the dicrotic elevation, $R$, produced by the reflection of a positive wave from the already closed semi-lunar valves. The dicrotic wave is relatively small on account of the high tension in the carotid artery. After this the curve falls rapidly, but in its lowest third two small elevations may be seen. Of these the former is due to elastic vibration. The latter represents a second dicrotic wave-(Fig. 58, III, R), (Landois, Moens). Here there is a true tricrotism, which is more easily obtained from the carotid on account of the shortness of the arterial channel.

Moens describes the "aortlc elevation" as occurring at the moment of the closure of the acrtic valves.

\section{Axillary Artery (Fig. 58, IV).}

In this curve the ascent is very steep, while in the descent near the apex there is a small (aortic) elevation, $\mathrm{K}$, caused by a positive wave, produced by the closure of the aortic valves. Below the middle there 
is a tolerably high dicrotic clevation, $R$, higher than in the carotid curve; because in the axillary artery the arterial tension is less, and permits a greater development of the dicrotic wave. Further on, two or three small elastic vibrations occur, $e, e$.

3. Radial Artery (Fig. 54, B ; Fig. 58, V-X; Fig. 64, $R$ and $R_{1}$ ).

The line of ascent (Fig. 58) is tolerably high and sudden-somewhat in the form of a long $f$. The apex, $\mathrm{P}$, is well marked. Below this, if the tension be high, two elastic vibrations may occur $(\mathrm{V}, e, e)$, but if it be low, only one (VI to IX, e). About the middle of the curve is the well-marked dicrotic elevation, $R$.

This wave is least pronounced in a small liard pulse, and when the artery is much distended (Fig. 58, VII, $\mathrm{R}_{1}$ ); it is larger when the tension is low (Fig. 56, IX, R), and is greatest of all when the pulse is dicrotic $(\mathrm{X}, \mathrm{R})$. Two or three small elastic elevations occur in the lowest part of the curve.

\section{Femoral Artery (Fig. 58, XI, XII).}

The ascent is steep and high-the apex of the curve is not unfrequently broad, and in it the closure of the aortic valves $(K)$ is indicated. The curve falls rapidly towards its lower third. The dicrotic elevation, $\mathrm{R}$, occurs late after the beginning of the curve, and there are also small elastic elevations $(e, e)$.

\section{Pedal Artery (Fig. 58, XIV, XV), and Posterior Tibial}

(Fig. 54, C, and Fig. 58, XIII).

In pulse-curves obtained from these arteries, there are well-marked irdications that the apparatus (heart) producing the waves is placed at
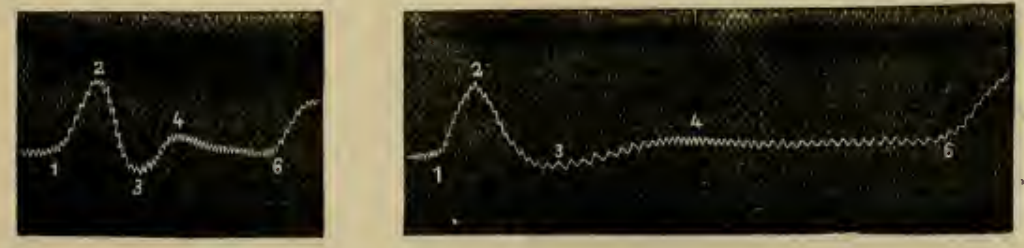

Fig. 61.

A, curve of posterior tibial, and $\mathrm{B}$, pedal artery of a man. Curves written by the angiograph upon a vibrating plate attached to a tuning-fork.

a considerable distance. The ascent is oblique and low-the dicotric elevation occurs late. Two elastic vibrations (Fig. 58, XIV, e,e) occur 
in the descent, but they occur very close to the apex, while the elastic vibrations at the lower part of the curve are feebly marked. In Fig. $61, \mathrm{~A}$ is from the posterior tibial, and $\mathrm{B}$ from the pedal artery of the same individual. When measured, they give the following result :-

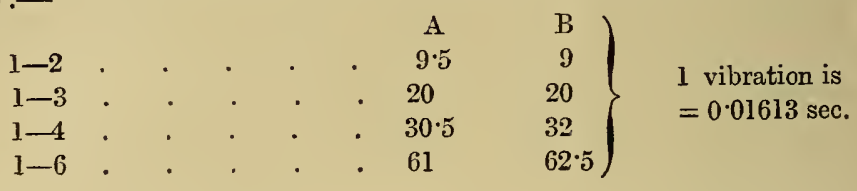

\section{Anacrotism.}

As a general rule, the line of ascent of a pulse-curve has the form of an $f$, and is nearly vertical. The arterial walls are thrown into elastic vibration by the pulsebeat, and the number of vibrations depends greatly upon the tension of the arterial walls.

The distension of the artery, or what is the same thing, the ascent of the sphygmogram, usually occurs so rapidly that it is equal to one elastic vibration. The elongated $f$-shape of the ascent is fundamentally just a prolonged elastic vibration. When the number of vibrations causing the elastic variation is small, and when the line of ascent is prolonged, two elevations occasionally occur in the line of ascent. Such a condition may occur normally (Fig. 56, VIII at 1 and $2 ; X$ at 1 and 2). When a series of closely-placed elastic vibrations occur in the upper part of the line of ascent, so that the apex appears dentate and forms an angle with the line of ascent, then the condition becomes one of Anacrotism (Fig. 62,a,a), which, when it becomes so marked, may be characterised as pathological (Landois). Anacrotism of the pulse occurs when the time of the influx of the blood is longer than the time occupied by an elastic vibration. Hence it takes place :-

(1.) In dilatation and hypertrophy of the left ventricle, e.g., Fig. 62, A, a tracing from the radial artery of a man suffering from contracted kidney. The large volume of blood expelled with each systole requires a long time to dilate the tense arteries.

(2.) When the extensibility of the arterial wall is diminished even the normal amount of blood expelled from the heart at every systole requires a long time to dilate the artery. This occurs in old people where the arteries tend to become rigid, e.g., in atheroma. Cold also stimulates the arteries so that they become less extensile. Within one hour after a tepid bath, the pulse assumes the anacrotic form (Fig. 62, D)-(G. v. Liebig.)

(3.) When the blood stagnates in consequence of great diminution in the velocity of the blood-stream, as occurs in paralysed limbs, the volume of blood propelled into the artery at every systole no longer produces the normal distension of the arterial coats, and anacrotic notches occur (Fig. 62, B).

(4.) After ligature of an artery, when blood slowly reaches the peripheral part of the vessel through a relatively small collateral circulation, it also occurs. If the brachial artery be compressed so that blood slowly reaches the radial, the radial pulse may become anacrotic. It often occurs in stenosis of the aorta, as the blood has difficulty in getting into the aorta (Fig. 62, C).

Recurrent Pulse.-If the radial artery be compressed at the wrist, the pulse-beat reappears on the distal side of the point of pressure through the arteries of the palm of the hand (Janaud, Neidert). The 
curve is anacrotic, and the dicrotic wave is diminished, while the elastic clevations are increased.
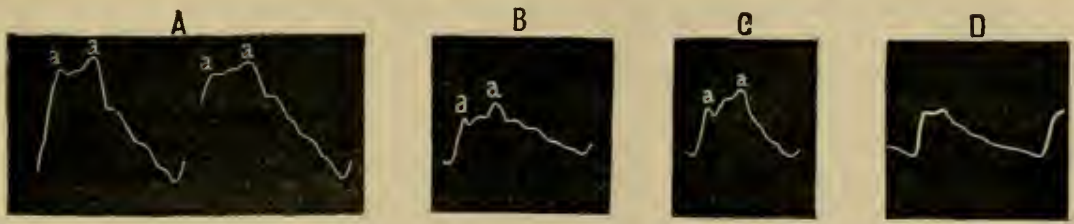

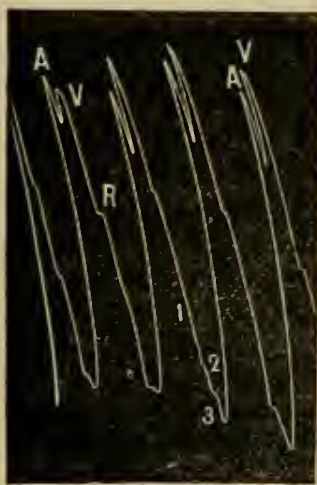

I.

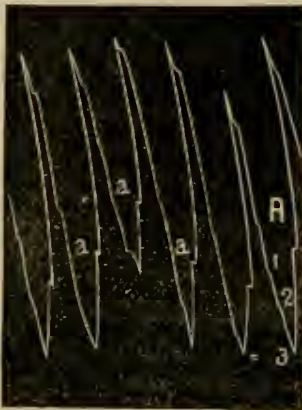

II.
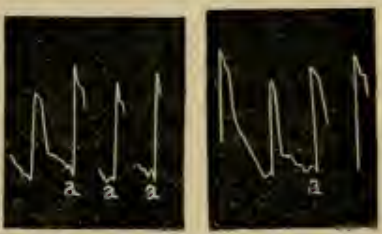

III.

Fig. 62.

Anacrotic pulse-curves-A, B, C, D, from the radial artery ; $a$, $a$, the anacrotic notches; I., II., III., curves with anacrotic elevations- $a$ in insufficiency of the aortic valves.

(5.) A special form of anacrotism occurs in cases of vell-marked insufficiency of the aortic valves. Practically, in these cases, the aorta remains permanently open. The contraction of the left auricle causes in the blood a wave-motion, which is at once propagated through the open mouth of the aorta into the large blood-vessels. This wave is followed by the wave caused by the contraction of the hypertrophied left ventricle, but of course the former wave is not so large as the latter. In insufficiency of the aortic valves, the auricular wave occurs before the ventricular wave in the ascending part of the curve. The auricular is well marked only in the large vessels, for it soon becomes lost in the peripheral vessels. Fig. 62, I., was obtained from the carotid of a man suffering from well-marked insufficiency of the aortic valves, with considerable hypertrophy of the left ventricle and left auricle. The ascent is steep, caused by the force of the contracted heart. In the apex of the curve are two projections, $A$ is the anacrotic auricular wave, and $V$ is the ventricular ware. Fig. 62, II., is a curve obtained from the subclavian artery of the same individual. In the femoral artery the auricular projection is only obtained when the friction of the writing-style is reduced to the minimum, and when it occurs it immediately precedes the beginning of the ascent (Fig. 62 III., a). The pulse-curve, in cases of aortic insufficiency, is also characterised by-(1) its considerable height; (2) the rapid fall of the lever from the apex of the curve, because a large part of the blood which is forced into the aorta regurgitates 
into the left ventricle when the ventricle relaxes; (3) not unfrequently a projeciion occurs at the apex, due to the elastic vibration of the tense arterial wall; (4) the dicrotic wave $(R)$ is small compared with the size of the curve itself, because the pulse-wave, owing to the lesion of the aortic valves, has not a sufficiently large surface to be reflected from. The great height of the curve is explained by the large amount of blood projected into the aortic system by the greatly hypertrophied and dilated ventricle.

\section{Influence of the Respiratory Movements on the Pulse-Curve.}

The respiratory movements influence the pulse in two ways:-(1) in a purely physical way, by diminishing the arterial pressure during each inspiration and increasing it during expiration; (2) the respiratory movements are accompanied by stimulation of the vasomotor centre, which produces variation of the blood-pressure.

(a.) Normal Respiration.-During inspiration, owing to the dilatation of the thorax, more arterial blood is retained within the chest, while at the same time, venous blood is sucked into the right auricle by aspiration; as a consequence of this, the tension in the arteries during inspiration must be less. The diminution of the chest during expiration favours the flow in the arteries, while it retards the flow of the venous blood in the venæ cavæ-two factors which raise the tension in the arterial system. The difference of pressure explains the difference in the form of the pulse-curve obtained during inspiration and expiration, as in Fig. 63 and Fig. 58, I., III., IV., in which J indicates the part of the curve which occurred during inspiration, and $E$ the expiratory portion. The following are the points of difference:-(1.) The greater distension of the arteries during expiration causes all the parts of the

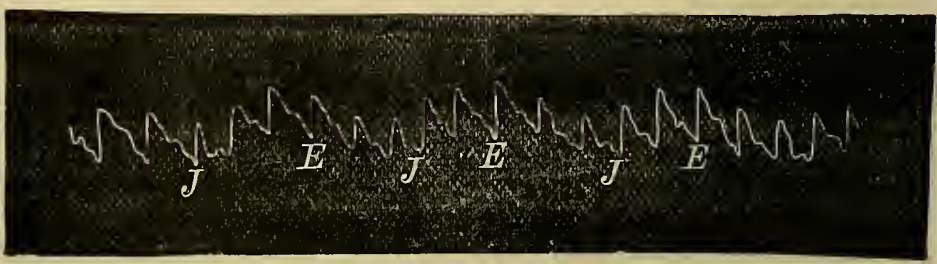

Fig. 63.

Influence of the respiration upon the sphygmogram, after Riegel-J, during inspiration; E, during expiration.

curve occurring during this phase to be higher; (2.) the line of ascent is lengthened during expiration, because the expiratory thoracic movement helps to increase the force of the expiratory wave; (3.) 
owing to the increase of the pressure the dicrotic wave must be less during expiration; (4.) for the same reason the elastic elevations are more distinct and occur higher in the curve near its apex. The frequency of the pulse is slightly greater during expiration than during inspiration.

(b.) This purely mechanical effect of the respiratory movements is modified by the simultaneous stimulation of the vasomotor centre which accompanies these movements. At the beginning of inspiration the blood-pressure in the arteries is lowest, but it begins to rise during inspiration, and increases until the end of the inspiratory act, reaching its maximum at the beginning of expiration. During the remainder of the expiration the blood-pressure falls until it reaches its lowest level again at the beginning of inspiration (compare $\S 85, f$ ), the pulsecurves are similarly modified, and exhibit the signs of greater or less tension of the arteries corresponding to the phases of the respiratory movements (Klemensiewicz, Knoll, Schreiber, Löwit). [There is, as it were, a displacement of the blood-pressure curve relative to the respiratory curve.]

Forced Respiration. - With regard to the effect produced on the pulse-curve by a powerful expiration and a forced inspiration, observers are by no means agreed.

Valsalva's Experiment.-Strong expiratory pressure is best produced by closing the mouth and nose, and then making a great expiratory effort; at first there is increase of the blood-pressure and the formation of pulse-waves resembling those which occur in ordinary expiration, the dicrotic wave being less developed; but, when the forced pressure is long continued, the pulse-curves have all the signs of diminished tension (Riegel, Frank, and Sommerbrodt). This effect is due to the action of the vasomotor centre, which is affected reflexly from the pulmonary nerves. We must assume that forced expiration, such as occurs in Valsalva's experiment, acts by depressing the activity of the vasomotor centre (compare Vol. $i i$.) Coughing, singing, and declaiming, act like Valsalva's experiment, while the frequency of the pulse is increased at the same time (Sommerbrodt). After the cessation of Valsalva's experiment, the blood-pressure rises above the normal state (Sommerbrodt), almost as much as it fell below it; the normal condition being restored within a few minutes (Lenzmann).

Muiller's Experiment.-When the thorax is in the expiratory phase, close the mouth and nose, and take a deep inspiration so as forcibly to expand the chest $(\$ 60)$. At first the pulse-curves have the characteristic signs of diminished tension, viz., a higher and more distinct dicrotic wave; then the tension can, by nervous influences, be increased, just as in Fig. 64, where $\mathrm{C}$ and $\mathrm{R}$ are tracings taken from the 
carotid and radial arteries respectively, during Müller's experiment, in which the dicrotic waves, $r, r$, indicate the diminished tension in the vessels. In $C_{1}$ and $R_{1}$, taken from the same person during Valsalva's experiment, the opposite condition occurs.

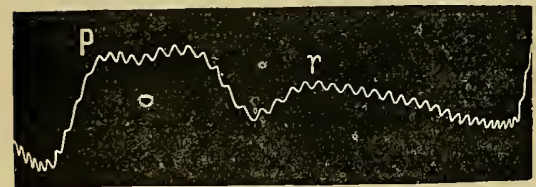

C

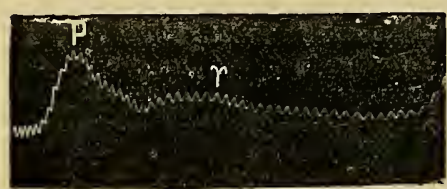

$\mathrm{C}_{1}$

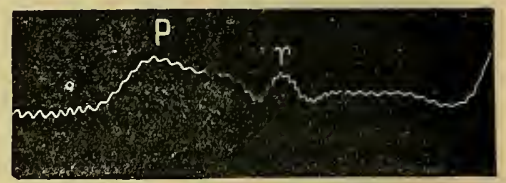

$\mathbf{R}$

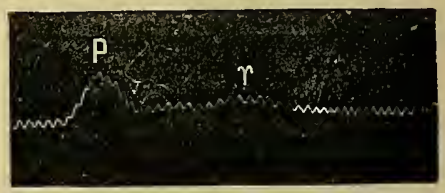

$\mathrm{R}_{1}$

Fig. 64.

$C$, curve from the carotid, and $R$, radial, during Müller's experiment; $C_{1}$ and $R_{1}$, from the same vessels during Valsalva's experiment. Curves written on a vibrating surface.

On expiring into a vessel resembling a spirometer (see Respiration), (Waldenburg's respiration apparatus), and filled with compressed air, the same result is obtained as in Valsalva's experiment-the blood-pressure falls and the pulse-beats increase; conversely, the inspiration from this apparatus of air under less pressure acts like Müller's experiment, i.e., it increases the effect of the inspiration, and afterwards increases the blood-pressure, which may either remain increased on continuing the experiment, or may fall (Lenzmann).

The inspiration of compressed air diminishes the mean blood-pressure (Zuntz), and the after-effect continues for some time. The pulse is more frequent both during and after the experiment. Expiration in rarified air increases the bloodpressure (Zuntz, Lenzmann). The effects which depend upon the action of the nervous system do not occur to the same extent in all cases. Exposure to com. pressed air in a pneumatic cabinet lowers the pulse-curve, the elastic vibrations become indistinct, and the dicrotic wave diminishes and may disappear ( $v$. Vivenot). The heart's beat is slowed and the blood-pressure raised (Bert,

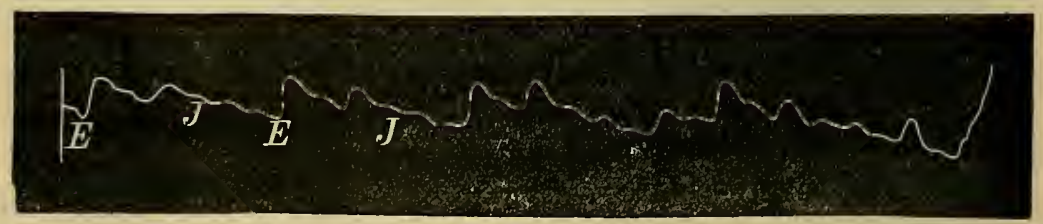

Fig. 65.

Pulsus paradoxus, after Kussmaul-E, expiration; J, inspiration.

Jacobsohn, Lazarus). Exposure to rarified air causes the opposite result, which is a sign of diminished arterial tension. 
Pulsus Paradoxus.-Under pathological conditions, especially when there is union of the heart or its large vessels with the surrounding parts, the pulse during inspiration may be extremely small and changed, or may even be absent. This condition has been called pulsus paradoxus (Griesinger, Kussmaul).

It depends upon a diminution of the arterial lumen during the inspiratory movement. Even in health, it is possible by a change of the inspiratory movement to produce the p. paradoxus (Riegel, Sommerbrodt).

\section{Influence of Pressure upon the Form of the Pulse-Curve.}

It is most important to know the actual pressure which is applied to an artery while a sphygmogram is being taken. The changes affect the form of the curve as well as the relation of the individual parts thereof. In Fig. 66, $a, b, c, d, e$, are radial curves; $a$ was taken with minimal pressure, $b$ with 100, $c 200, d 250$, and $e$ 450 grammes pressure, while $A, B, C, D$, show the relations as to the time of occurrence of the individual phenomena where the weight was successively increased. The study of these curves yields the following results:-(1) When the weight is small, the dicrotic wave is relatively less; the whole curve is high. (2) With a moderate weight (100-200 grammes) the dicrotic wave is best marked, the whole curve is somewhat lower. (3) On increasing the weight, the size of the
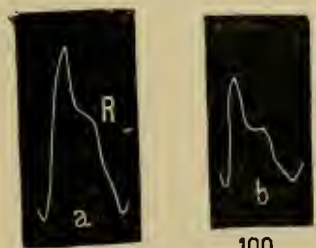

100

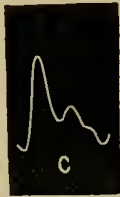

200

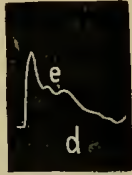

250

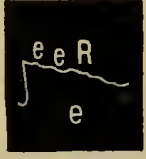

450

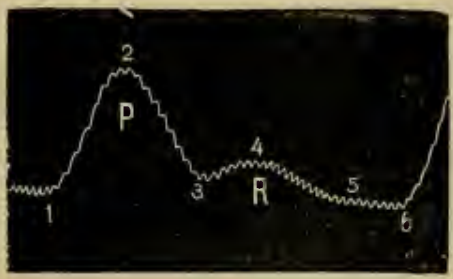

A 100

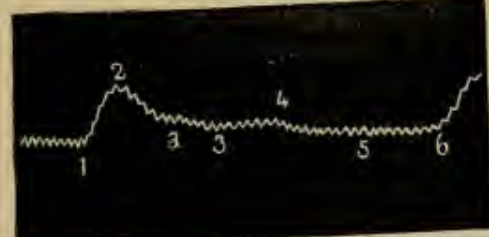

C 220

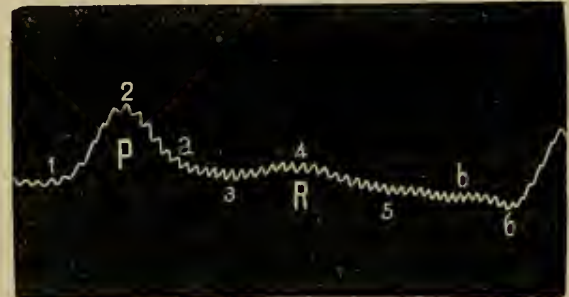

B 170

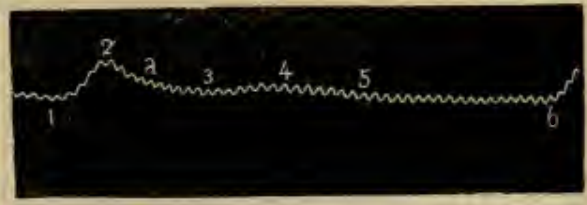

240

Fig. 66.

Various forms of curves (radial) obtained by gradually increasing the pressure. 
dicrotic wave again diminishes. (4) The fine elastic vibrations preceding the dicrotic wave appear first when a weight of 220 to 300 grammes is used. (5) The rapidity of the pulse changes with increasing weight, the time occupied by the ascent becoming shorter, the descent becoming longer. (6) The height of the entire curve decreases as the weight increases. In every sphygmogram the pressure under which it was obtained ought always to be stated.

In Fig. 66, A, B, C, D, are curves obtained from the radial artery of a healthy student. The pressure exerted upon the artery for A was $100 ; \mathrm{B}, 170 ; \mathrm{C}, 220$; and D, 240 grms. The time occupied by the various events was:-

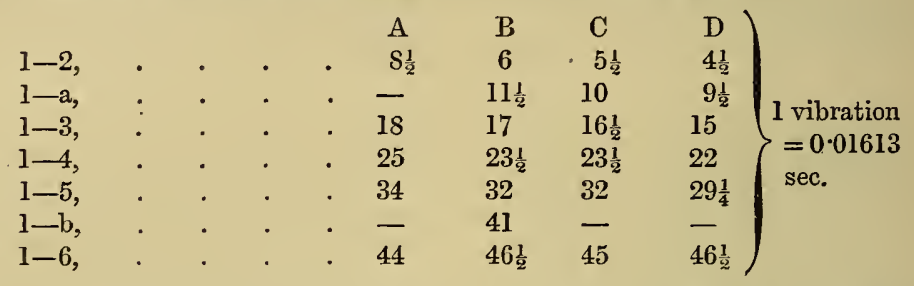

If pressure be excerted upon an artery for a long time the strength of the pulse is gradually increased. If, after subjecting an artery to considerable pressure, a lighter weight be used, not unfrequently the pulse-curve assumes the form of a dicrotic pulse, owing to the greater development of the dicrotic elevation. When strong pressure is applied, the blood is forced to find its way through collateral channels. When the chief artery ceases to be compressed, the total area is, of course, considerably and suddenly enlarged, which results in the production of a dicrotic elevation. Fig. 58, X, is such a dicrotic curve obtained after considerable lressure had been applied to the artery.

\section{Rapidity of Transmission of Pulse-Waves.}

The pulse-wave proceeds throughout the arterial system from the root of the aorta, so that the pulse is felt sooner in parts lying near the heart than in the peripheral arteries. E. H. Weber calculated the rate of the pulse-wave as 9.240 metres [ $28 \frac{1}{2}$ feet] per sec. from the difference in time between the pulse in the external maxillary artery and the dorsal artery of the foot. Czermak showed that the elasticity was not equal in all the arteries, so that the velocity of the pulse-wave cannot be the same in all. The pulse-wave is propagated more slowly in the arteries with soft extensile walls than in arteries with resistant and thick walls, so that it is transmitted more rapidly in the arteries of the lower extremities than in those of the upper. It is still slower in children.

\section{Propagation of the Pulse-Wave in Elastic Tubes.}

Waves similar to the pulse may be produced in elastic tubes. (1) According to E. H. Weber the velocity of propagation of the waves is 11.295 metres per sec.; 
according to Donders, 11 - 14 metres (34-43 feet). (2) According to E. H. Weber increased internal tension causes only an inconsiderable decrease; Rive found a great decrease; Donders found no obvious difference; while Marey found an increased velocity. (3) Donders found the velocity to be the same in tubes, $2 \mathrm{~mm}$. in diameter, as in wider tubes, but Marey believes that the velocity varies when the diameter of the tube changes. (4) The velocity is less the smaller the elastic coefficient. (5) The velocity increases with increased thickness of the wall, while it diminishes when the specific gravity of the fluid increases.

Moens has recently formulated the following laws as to the velocity of propagation of waves in elastic tubes:-(1) It is inversely proportional to the square root of the specific gravity of the fluid. (2) It is as the square root of the thickness of the wall, the lateral pressure being the same. (3) It is inversely as the square root of the diameter of the tube, the lateral pressure being the same. (4) It is as the square root of the elastic coefficient of the wall of the tube, the lateral pressure being the same (Valentin).

Experiments with Caoutchouc Tubes.-For this purpose Iandois employs the following apparatus (Fig. 67):-A large tuning-fork, A (35 cmtr. long), carries on one of its arms a glass-plate, $P$ ( $25 \mathrm{cmtr}$. long, and $5 \mathrm{cmtr}$. broad), while the other arm is weighted, G. The tuning-fork is fixed by an iron holder, $T$, to a movable piece of wood which can be pushed along with the hand in a groove on a support $\mathrm{H}, \mathrm{H}$. When the glass-plate is smoked, the curved needle of the angiograph writes its movements upon it. The fork, when it vibrates, makes little teeth in the curve, and the value of each vibration is estimated beforehand. Every complete vibration in this instrument is equal to $0.01613 \mathrm{sec}$.

Velocity of the Waves in Elastic Tubes filled with Water or Mercury. -Take a soft extensible elastic tube, A, 8. 80 metres long, $1 \mathrm{~mm}$. thick, and

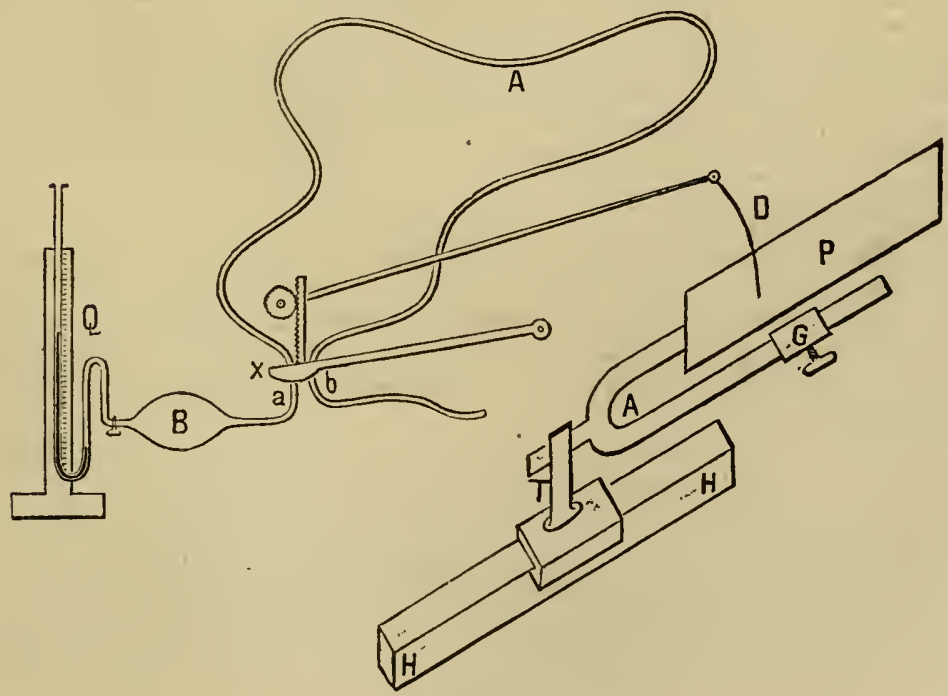

Fig. 67.

Instrument for measuring the velocity of the pulse-wave in an elastic tube containing water or mercury-A, tuning.fork; $B$, ampulla; $A$, elastic tube; $P$, glass-plate smoked; $Q$, manometer; $x$, pad of lever of angiograph; writingstyle, $D$. 
$7 \mathrm{~mm}$. diameter. If 1 metre of the tube is weighted with 1 kilo, it elongates 68 cmtr. An ampulla, B, capable of containing $50 \mathrm{cmtr}$., is fixed to one end of the tube, while to the other end of the ampulla is fixed a mercurial manometer, $Q$.

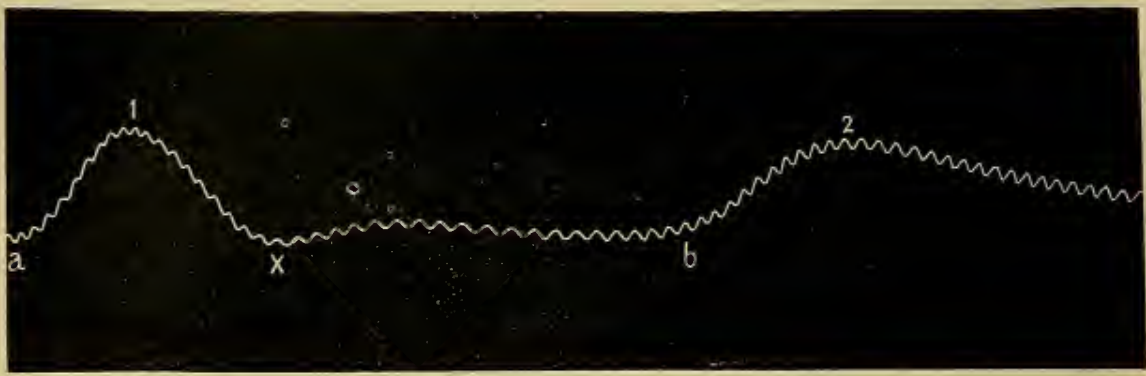

Fig. $67 a$.

Pulse-curve from an elastic tube registered upon a plate attached to a vibrating tuning-fork.

The tube, $A$, is shut close to the ampulla every time the pressure is measured, in order to obviate the occurrence of oscillation in the mercury. A certain portion of the tube, say 8 metres, is measured. The beginning, $a$, and end, $b$, of this stretch of tubing are placed under the pad, $x$, of the angiograph. When a positive wave is produced by compressing the ampulla, the writing-lever is raised twice, the first time when the wave passes the first part of the tube, $a$, under the pad, and the second time when the end part of the tube, $b$, is distended by the wave. The curve obtained is shown in Fig. $67 a$, in which the two elevations, 1 and 2 , are obvious. The time between the two may be ascertained by counting the number of vibrations of the tuning-fork. The experiments gave the following results:-

(A.) The velocity of the wave is 11.809 metres per sec.

(B.) The intra-vascular pressure has a decided influence on the velocity: thus, in the tube, $\mathrm{A}$, with $18 \mathrm{cmtr}$. (Hg.) pressure, the velocity per metre $=0.093 \mathrm{sec}$, while with 21 cmtr. pressure $(\mathrm{Hg})=$.0.095 sec. per metre.

(C.) The specific gravity of the liquid influences the velocity of the pulse-wave. In mercury the wave is propagated four times more slowly than in water (Marey and Landois).

(D.) The velocity in a tube which is more rigid and not so extensile is greater than in a tube which is easily distended.

\section{Velocity of the Pulse-Wave in Man.}

Landois obtained the following results in a student whose height was 174 centimetres:-Difference between carotid and radial $=0.074 \mathrm{sec}$. (the distance being taken as 62 centimetres); carotid and femoral $=0.068$ sec.; femoral (inguinal region) and posterior tibial $=0.097 \mathrm{sec}$. (distance estimated at 91 centimetres).

The velocity of the pulse-wave in the arteries of the upper extremities $=9.43$ metres per sec, and in those of the lower extremity 9.40 metres per second. The velocity is greater in the less extensible arteries 
of the lower extremities than in those of the upper limb. For the same reason it is less in the peripheral arteries and in the yielding arteries of children (Czermak).

E. H. Weber estimated the velocity at 9.24 metres per sec.; Garrod; 9-10.8 metres; Grashey, $s \cdot 5$ metres; Moens, $S \cdot 3$ metres, and with diminished pressure during Valsalva's experiment (p. 112) $7 \cdot 3$ metres.

In animals, hremorrhage (Haller), slowing of the heart produced by stimulation of the vagus (Moens), section of the spinal cord, deep morphia-narcosis, and dilata. tion of blood-vessels by heat, produce slowing of the velocity, while stimulation of the spinal cord accelerates it (Grunmach).

The ware-length of the pulse-wave is obtained by multiplying the duration of the inflow of blood into the aorta $=0.08$ to 0.09 secs. (p. S6) by the velocity of the pulse-wave.

Method.-Place the knobs of two tambours (Fig. 52) upon the two arteries to be investigated, or place one over the apex-beat and the other upon an artery. These receiving tambours are connected with two registering tambours, as in Brondgeest's pansphygmograph ( $\$ 67$, Fig. 52) so that their writing-levers are directly over each other, and so arranged as to write simultaneously on one vibrating. plate attached to a tuning-fork. [Or they may be made to write upon a revolving cylinder, whose rate of movement is ascertained by causing a tuning-fork of a known rate of vibration to write under them.] In Fig. $68, \mathrm{H}$ is the curve obtained from the heart, and $\mathrm{C}$ from the radial artery. The apparatus is improved by using rigid tubes and filling them with water, in which all impulses are rapidly communi. cated. In arteries which are distant from each other, or in the case of the heart and an artery, the two knobs of the receiving tambours may be connected by means of a $\mathrm{Y}$-tube with one writing-lever. In Fig. $68, \mathrm{~B}$ is a curve from the radial artery taken in this way. In it $v \mathrm{HP}$ indicates contraction of the ventricle; $\mathrm{H}$, the apex of the ventricular contraction; $\mathrm{P}$, the primary apex of the radial curve; $v$, the

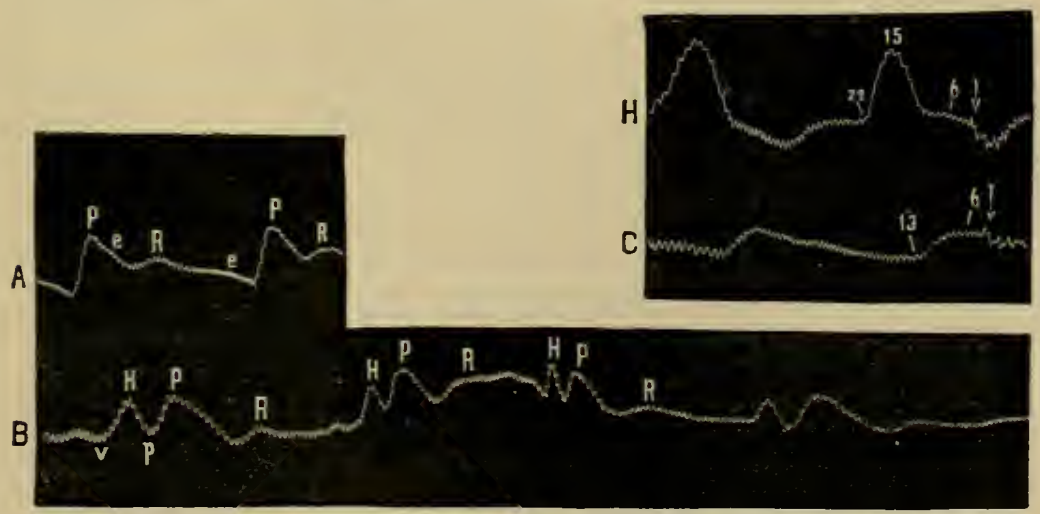

Fig. 68 .

$A$, curre of radial artery on a vibrating surface ( 1 vib. $=0.01613$ sec.); $P$, apex of curve ; $e, e$, elastic vibrations; $\mathrm{R}$, dicrotic wave; $\mathrm{B}$, curve of same radial taken along with the heart-beat; $v, \mathrm{H}, \mathrm{P}$, contraction of the ventricle; $\mathrm{H}$, curve of the heart-beat; $\mathrm{C}$, of the radial artery, taken simultaneously. The arrows indicate the identical points in both curves. In $\mathrm{B}, v$ to $p=9$ vibrations. 
beginning of the ventricular contraction; $p$, of the radial pulse. $\mathrm{A}$ is the curve of the radial artery alone. From these curves, as well as from $H$ and $C$, it is evident that in this instance 9 vibrations occur between the beginning of the ventricular contraction (in $\mathrm{H}$ at 22) until the beginning of the pulse in the radial artery (in $\mathrm{C}$ at 13), so that $0.15 \mathrm{sec}$. elapses between these two events ( 1 vibration $=0.01613 \mathrm{sec}$.).

In Fig. 69 the difference between the carotid and the posterior tibial pulse $=$ $0 \cdot 137$ sec.

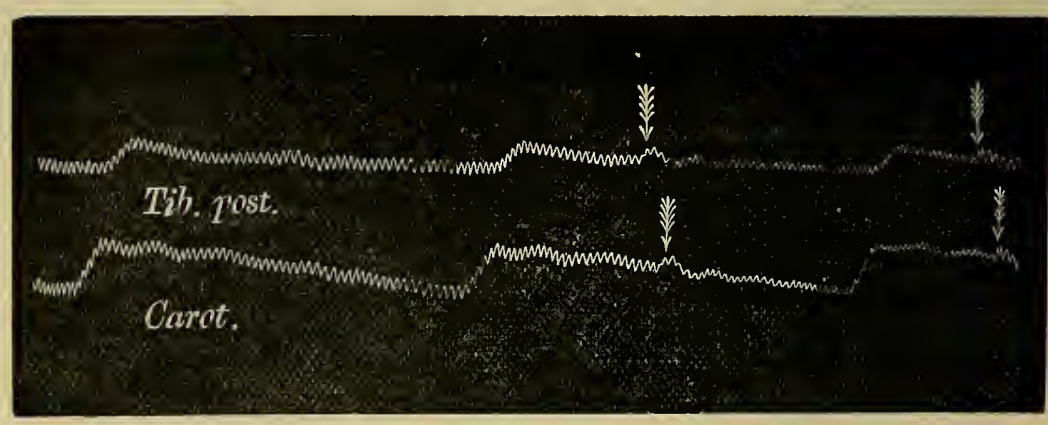

Fig. 69.

Curves of the carotid and posterior tibial taken simultaneously with Brondgeest's pansphygmograph writing upon a vibrating plate, attached to a tuning-fork. The arrows indicate the identical moment of time in each curve.

Pathological.-In cases of diminished extensibility of the arteries, e.g., in atheroma (p. 127), the pulse-wave is propagated more rapidly. Local dilatations of the arteries, as in aneurisms, cause a retardation of the wave, and a similar result arises from local constrictions. Relaxation of the walls of the vessels in high fever retards the movement (Hamernjk).

\section{Further Pulsatile Phenomena.}

1. In the mouth and nose, when they are filled with air, and the glottis closed, pulsatile phenomena (due to the arteries in their soft parts), may be found communicating a movement to the contained air. The curves obtained are relatively small, and closely resemble the curve of the carotid. A similar pulse is obtained in the tympanum with intact membrana tympani, and when the soft parts of the tympanum are congested (Schwartze, Tröltsch).

2. Entoptical Pulse,-After violent exercise, an illumination corresponding to each pulse-beat, occurs on a dark optical field. When the optical field is bright, an analogous darkening occurs (Landois). The ophthalmoscope occasionally reveals pulsation of the retinal arteries (Jäger), which becomes marked in insufficiency of the aortic valves (Quincke, $\mathrm{O}$. Becker, Helfreich).

3. Pulsatile Muscular Contraction.-The orbicularis palpebrarum muscle contracts under similar conditions synchronously with the pulse; and it is perhaps due to the pulse-beat exciting the sensory nerves reflexly. 'The brothers Weber found that not unfrequently while walking, the step and pulse gradually and in. voluntarily coincide.

4. When the legs are crossed as one sits in a chair, the leg which is supported is raised with each pulse-beat, and it gives also a second or dicrotic elevation.

5. If, while a person is quite quiet, the incisor teeth of the lower jaw be made just to touch the upper incisors very lightly, we detect a double beat of the lower 
against the upper teeth, owing to the pulse-beat in the extcrnal maxillary artery raising the lower jaw. The second elevation is due to the closure of the semi-lunar valves, and not to a dicrotic wave.

6. Brain and Fontanelles.-The large arteries at the base of the brain communicate a movement to it, while similar movements occur with respiration-rising during expiration and falling during inspiration. These movements are visible in the fontanelles of infants. The respiratory movements depend upon variations in the amount of blood in the veins of the cranial cavity, and also upon the respiratory variations of the blood-pressure.

7. Amongst pathological phenomena, are the beating in the epigastrium, as in hypertrophy of the right or left ventricle, caused, it may be, by deep insertion of the diaphragm, and it may be partly by the beating of a dilated abdominal aorta or coeliac axis.

Abnormal dilatations (aneurisms) of the arteries cause an abnormal pulsation, while they produce slowing in the velocity of the pulse-wave in the corresponding artery. Hence the pulse appears later in such an artery than in the artery on the healthy side. Hypertrophy and dilatation of the left ventricle cause the arteries near the heart to pulsate strongly. In the analogous condition of the right ventricle, the beat of the pulmonary artery may be seen and felt in the second left intercostal space.

\section{Vibrations communicated to the Body by the action of the Heart.}

The beating of the heart and large arteries communicates vibrations to the body as a whole, but the vibration is not simple but compound.

Gordon was the first to represent this pulsatory vibration graphically. If a

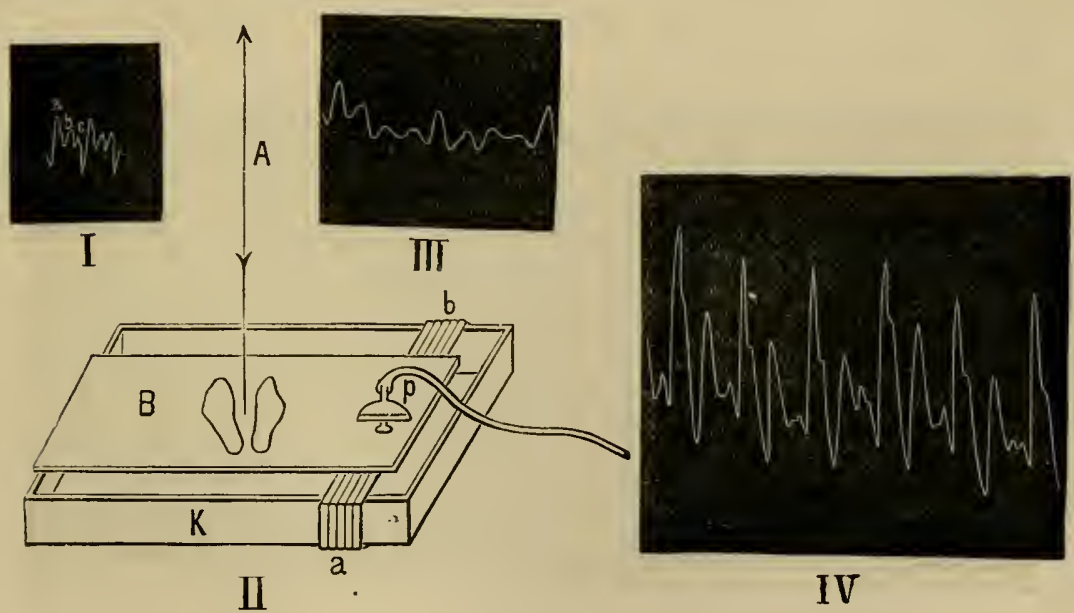

Fig. 70 .

II, Elastic support for registering the molar motions of the body-K, a wooden box; $\mathbf{B}$, feet of patient ; $p$, cardiograph ; $a, b$, elastic tubing. I, III-Vibration curves of a healthy person. IV-Similar curve obtained from a patient suffering from insufficiency of the aortic valves and great hypertrophy of the heart. 
person be placed in an erect attitude in the scale of a large balance, the index oscillates, and its movements coincide with the heart's movements.

Fig. 70, I, shows a curve obtained by Gordon, written directly by the index of the spring balance. The lowest part of the curve corresponds to the systole of the ventricle.

Landois employed the following arrangement:-Take a long four-sided box, $\mathrm{K}$, open at the top, and arrange several coils, $a, b$, of stout caoutchonc tubing round one end. A wooden board, B, smaller than the opening in the box, is so placed that it rests with one end on the caoutchouc tubing, and with the other on the narrow end of the box. The person to be experimented upon, A, stands vertically and firmly on this board. A receiving tambour, $p$, is placed against the surface of the board next the elastic tube, which registers the vibrations of the foot support. Fig. III is a curve showing such vibrations, each heart-beat being followed in this case by four oscillations. It corresponds to $I$. To ascertain the relations and causes of these vibrations, it is necessary to obtain, simnltaneously, a tracing of the heart and the vibratory curve. For this purpose use the two tambours of Brondgeest's pansphygmograph (p. 71), placing one nob or pad over the heart, and the other on the foot-support, and allow the writing-tambours to inscribe their vibrations on a glass-plate attached to a tuning-fork.

In the lower or cardiac impulse curve, Fig. 71, the rapidly-rising part is due to the ventricular systole. It contains 8 vibrations ( 1 vib. $=0.01613 \mathrm{sec}$.). The beginning of the ventricular systole is indicated in the fig. by $-36-3-17$.

If the corresponding numbers in the upper or vibratory curve are studied, it is obvious that at the moment of ventricular systole the body makes a downward vibration, i.e., it exercises greater pressure upon the foot-support. Gordon interprets his curve as giving exactly the opposite result. This downward motion, however, lasted only during 5 vibrations of the tuning-fork : during the last 3 vibrations, corresponding to the systole, there is an ascent of the body corresponding to a less pressure upon the foot-plate. When the ventricle empties itself, it undergoes a movement in a downward and outward direction-Gutbrodt's "reaction impulse."

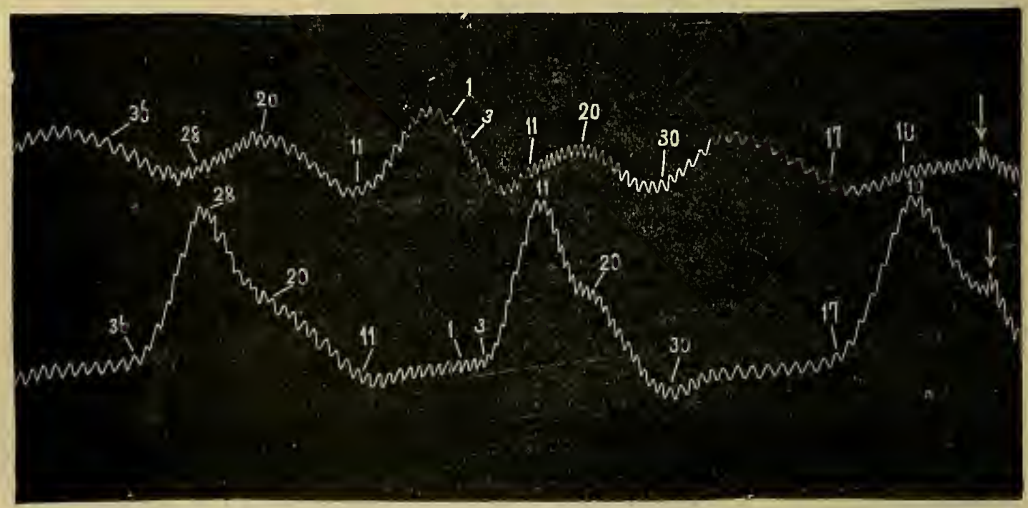

Fig. 71.

The upper curve is the vibration-curve of a healthy person, and the lower one a tracing of the apex beat.

In the upper curve analogous numbers are employed to indicate the vibrations occurring simultaneously, viz,,$-28-11-10$. The closure of the semi-lunar 
valves is well marked in the three heart-beats at 20-20. This closure is indicated in analogous points in both curves, after which there is a descent of the foot-support, and this corresponds to the downward propagation of the pulse-wave through the aorta to the vessels of the feet.

In insufficiency of the aortic valves, as shown in Fig. 70, IV, the vibration com. municated to the body is very considerable.

\section{The Blood-Current.}

The closed and much-branched vascular system, whose walls are endowed with elasticity and contractility, is not only completely filled with blood but it is over-filled. The total volume of the blood is somewhat greater than the capacity of the entire vascular system. Hence it follows that the mass of blood must exert pressure on the walls of the entire system, thus causing a corresponding dilatation of the elastic vascular walls (Brunner). This occurs only during life; after death the muscles of the vessels relax, and fluid passes into the tissues, so that the blood-vessels come to contain less fluid and some of the vessels may be emptied.

If the blood were uniformly distributed throughout the vascular system and under the same pressure, it would remain in a position of equilibrium (as after death). If, however, the pressure be raised in one section of the tube the blood will move from the part where the pressure is higher to where it is lower; so that the blood-current is a result of the difference of pressure within the vascular system. If either the aorta or the venæ cavæ be suddenly ligatured in a living animal, the blood continues to flow, gradually more slowly, until the difference of pressure is equalised throughout the entire vascular system.

The velocity of the current will be greater the greater the difference of pressure, and the less the resistance opposed to the blood-stream.

The difference of pressure which causes the current is produced by the heart (E. H. Weber). Both in the systemic and pulmonary circulations the point of highest pressure is in the root or beginning of the arterial system, while the poirt of lowest pressure is in the terminal portion of the venous orifices at the heart. Hence, the blood flows continually from the arteries through the capillaries into the venous trunks.

The heart keeps up the difference of pressure required to produce this result; with each systole of the ventricles a certain quantity of blood is forced into the begimning of the arteries, while at the same time an equal amount flows from the venous orifices into the auricles during their diastole (E. H. Weber).

Donders added another important fact-viz., that the action of the heart not only causes the difference of pressure necessary to establish a blood-current, but that it also raises the mean pressure within the vascular 
system. The terminations of the veins at the heart are wider and more extensible than the arteries where they arise from the heart. As the heart propels a volume of blood into the arteries equal to that which it receives from the veins, it follows that the arterial pressure must rise more rapidly than the venous pressure diminishes, since the arteries are not so wide nor so extensible as the veins. Thus the total pressure must also increase.

The volume of blood expelled from the ventricles at every systole would give rise to a jerky or intermittent movement of the blood stream-1. if the tubes had rigid walls, as in such tubes any pressure exerted upon their contents is propagated momentarily throughout the length of the tube, and the motion of the fluid ceases when the propelling force ceases. 2. The flow would also be intermittent in character in elastic tubes if the time between two successive systoles were longer than the duration of the current necessary for the compensation of the difference of pressure caused by the systole. If the time between two successive systoles be shorter than the time necessary to equilibrate the pressure, the current will become continuous, provided the resistance at the periphery of the tube be sufficiently great to bring the elasticity of the tube into action. The more rapidly systole follows systole, the greater becomes the difference of pressure, and the more distended the elastic walls. Although the current thus produced is continuous, a sudden rise of pressure is caused by the forcing in of a mass of blood at every systole, so that with every systole there is a sudden jerk and acceleration of the blood-stream corresponding to the pulse (compare $\S 64$ ).

This sudden jerk-like acceleration of the blood-current is propagated throughout the arterial system with the velocity of the pulse-wave: both phenomena are due to the same fundamental cause. Every pulse-beat causes a temporary rapid progressive acceleration of the particles of the fluid. But just as the form-movement of the pulse is not a simple movement, neither is the pulsatile acceleration a simple acceleration. It follows the course of the development of the pulsewave. The pulse-curve is the graphic representation of the pulsatory acceleration of the blood-stream. Every rise in the curve corresponds to an acceleration, every depression to a retardation of the current.

Method.-These facts are capable of demonstration by means of very simple physical experiments. [Tie a Higginson's syringe to a piece of an ordinary gaspipe. On forcing water through the tube by compressing the elastic pump, the water will flow out at the other end of the tube in jets, while during the intervals of pulsation no water will flow out. As the walls of the tube are rigid, just as much fluid flows out as is forced into the tube. If a similar arrangement be made, and a long elastic tube be used, a continuous outflow is obtained, provided the pulsations occur with sufficient rapidity and the length of the tube, or the resist- 
ance at its periphery, be sufficient to bring the elasticity of the tube into action. This can be done by putting a narrow cannula in the outflow end of the tube, or by placing a clamp on it so as to diminish the exit aperture. This apparatus converts the intermittent flow into a continuous current.] The fire-engine is a good example of the conversion of an intermittent inflow into a uniform outflow. The air in the reservoir is in a state of elastic tension, and it represents the elasticity of the vascular walls. When the pump is worked slowly, the outflow of the water occurs in jets, and is interrupted. If the pumping movement be sufficiently rapid, the compressed air in the reservoir causes a continutous outflow, which is distinctly accelerated at crery movement of the pump.

Current in the Capillaries.--In the capillary vessels the pulsatile acceleration of the current ceases with the extinction of the pulsewave. The great resistance which is offered to the current towards the capillary area canses both to disappear. It is only when the capillaries are greatly dilated, and when the arterial blood-pressure is high, that the pulse is propagated through the capillaries into the beginning of the veins. A pulse is observed in the veins of the submaxillary gland after stimulation of the chorda tympani nerve, which contains the vascular or vaso-dilator nerves for the blood-vessels of this gland. If the finger be constricted with an elastic band so as to hinder the return of the venous blood, and to increase the arterial blood-pressure, while at the same time dilating the capillaries, an intermittent increased redness occurs, which corresponds with the wellknown throbbing sensation in the swollen finger. This is due to the capillary pulse. [Roy and Graham Brown found that pulsatile phenomena were produced in the capillaries, by increasing the extra-vascular pressure (p. 173). Quincke called attention to the capillary pulse which can often be seen under the finger nails. Extend the fingers completely, when a whitish area appears under the nails. A red area near the free margin of the nail advances and retires with each pulse-beat. It is well-marked in some diseased conditions of the heart, and is probably produced by increased extra-vascular pressure.]

\section{Schemata of the Circulation.}

E. H. Weber constructed a scheme of the circulation. It consisted of a forcepump with properly arranged valves to represent the heart, portions of gut for the arteries and veins, and a piece of glass tubing containing a piece of sponge to represent the capillaries. Various schemes have been invented, including the very complicated one of Marey [and the thoroughly practical one of Rutherford].

\section{Capacity of the Ventricles.}

Since the right and left ventricles contract simultaneously, and just the same volume of blood passes through the pulmonary as 
through the systemic circulation, it follows that the right ventricle must be just as capacious as the left. The capacity of the ventricles has been estimated in the following ways:-

(1.) Directly, by filling the dead ventricle with blood (Santorini, 1724; Legallois and Collin). This method is unsatisfactory and inaccurate. (2.) All the vessels of the relaxed heart are ligatured, the heart excised, and the contents of the cavities estimated (Abegg, 1848). (3.) Volkmann estimated the capacity to be $\frac{1}{400}$ of the body-weight-i.e., for a man of 75 kilos. $=197 \cdot 5 \mathrm{grms}$.

\section{Estimation of the Blood-Pressure.}

(A.) In Animals: Method of Hales.-The Rev. Stephen Hales (1727) was the first to introduce a long glass tube into a blood-vessel in order to estimate the blood-pressure by measuring the height of the column of blood, i.e., how high the blood rose in the tube. The tube was provided at its lower end with a copper tube bent at a right angle (Pitot's tube). [The tube he used was one-sixth of an inch bore and about nine feet long; and was inserted into the femoral artery of a horse. The height to which the blood rose in the tube was noted, as well as the oscillations that occurred with every pulsation. From the height of the column of fluid he calculated the force of the heart.]

(2.) The Hæmadynamometer of Poiseuille.-This observer (1828) used a $\mathrm{U}$-shaped tube partially filled with mercury-a manometer-which was brought into connection with a blood-vessel by means of a rigid tube. [The mercury oscillated with every pulsation, and the extent of the oscillations was read off by means of a scale attached to the bent tube. He called the instrument a homadynamometer].

[(3.) Vierordt used a tube five or six feet long, and filled it with a solution of sodium carbonate, thus preventing much blood from entering the tube, while at the same time the soda solution prevented the coagulation of the blood.]

(4.) C. Ludwig's Kymograph.-C. Ludwig employed a U-shaped manometer of the same kind, but he placed a light float (Fig. 72, $d, s$ ) upon the surface of the mercury in the open limb of the tube. A writing-style, $f$, placed transversely on the free-end of the float, inscribed the movements of the float-and, therefore, of the mercury - upon a cylinder, $c$, caused to revolve at a uniform rate. This apparatus registered the height of the blood-pressure, as well as the pulsatile and other oscillations occurring in the mercury. Volkmann called this instrument a kymograph or "wave-writer." The difference of the height of the column of mercury, $c, d$, in both limbs of the tube indicates the pressure within the vessel. If the height of the column of mercury be multiplied by 13.5 , this gives the height of the corresponding column of blood. Setschenow placed a stop-cock in the lower bend, $h$, of the tube. If this be closed so as just to permit a small aperture of communication to remain, the pulsatile vibrations no longer appear, and the apparatus indicates the mean pressure. By the term mean pressure is meant the limit of pressure, above and below which the oscillations occurring in an ordinary blood- 
pressure tracing range. [Briefly, it is the average elevation of the mercurial column.]
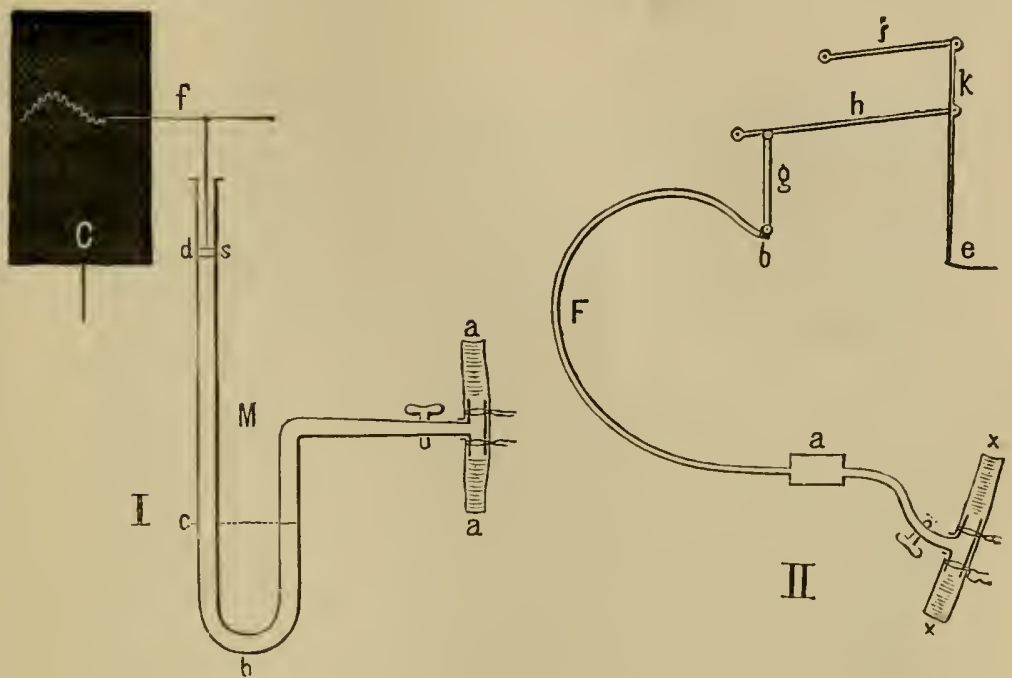

Fig. 72.

I, Scheme of C. Ludwig's kymograph ; II, Fick's spring-kymograph.

In a blood-pressure tracing, such as Fig. 74, each of the smaller waves corresponds to a heart-beat, the ascent corresponding to the systole and the descent to the diastole. The large undulations are clne to the respiratory movements. It is clear that the heart-beat is expressed as a simple rise and fall (Fig. 74), so that the curve of the heart-beat obtained with a mercurial kymograph differs from a sphygmographic curve. A perfect recording instrument ought to indicate the height of the blood-pressure and also the size, form, and duration of any wave-motion communicated to it. The mercurial manometer does not give the true form of the pulse-wave, as the mercury, when once set in motion, executes vibrations of its own, owing to its great inertia, and thus the finer movements of the pulse-wave are lost. Hence a mercurial kymograph is nsed for registering the blood-pressure, and not for obtaining the exact form of the pulse-wave. Instruments with less inertia and with no vibrations peculiar to themselves, are required for this purpose. [The theory of the mercurial manometer has been carefully worked out by Mach and also by v. Kries.]

[Method.-Expose the carotid of a chloralised rabbit, and isolate a portion of the vessel between two ligatures, or two spring clamps. With a pair of scissors make an oblique slit into the artery, and into it insert a straight glass cannula, directing the open end of the cannula towards the heart. Fill the cannula with a saturated solution of sodinm carbonate, taking care that no air-bubbles enter, and connect it with the lead tube which goes to the descending limb of the manometer. The tube which connects the artery with the manometer must be flexible and yet inelastic, and a lead tube is best. It is usual to connect a pressure-bottle, containing a saturated solution of sodium carbonate, by means of an elastic tube, with the tube attached to the manometer. This bottle can be raised or lowered. Before beginning the experiment, raise the pressure-bottle until there is a positive pressure of several inches of mercury in the manometer, or until the pressure is about equal to the estimated blood-pressure, and then clamp the tube 
of the pressure-bottle where it joins the lead tube. By having this positive pressure, the escape of blood from the artery into the solution of sodium carbonate is to a large extent avoided. When all is ready, the ligature on the carcliac side

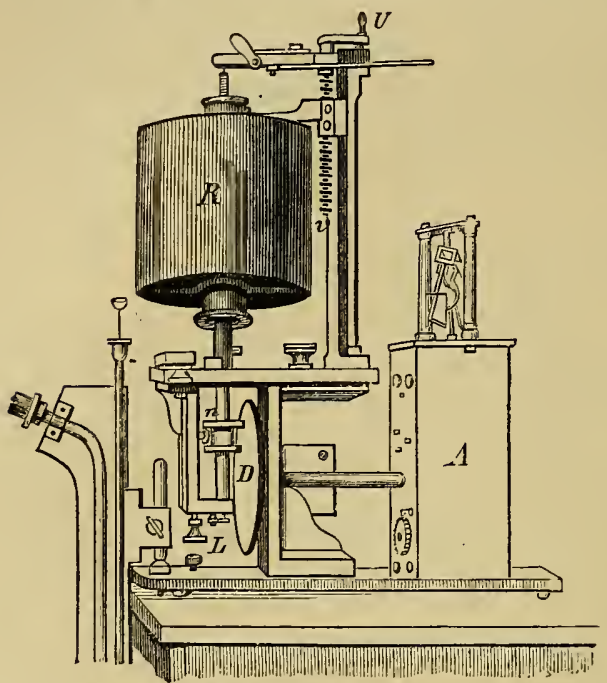

Fig. 73.

Ludwig's improved form of revolving cylinder, $R$, which is moved by the clock-work in the box, $A$, and regulated by a Foucault's regulator placed on the top of the box. The disc, $D$, moved by the clock-work, presses upon the two wheels, $n$, which can be raised or lowered by the screw, $L$, thus altering the position of $n$ on $D$, so as to cause the cylinder to rotate at different rates. The cylinder itself can be raised by the handle, $v$. On the left side of the figure is a mercurial manometer. When the cylinder is used, it is covered with smoked smooth paper. of the cannula is removed, and immediately the float begins to oscillate and inscribe its movements upon the recording surface. The fluid within the artery exerts pressure laterally upon the sodium carbonate solution, and this in turn trans. mits it to the mercury.]

[When we have occasion to take a tracing for any length of time, it must be written upon a strip of paper which is moved at a uniform rate in front of the writing. style on the float (Fig. 73). Various arrangements are em. ployed for this purpose, but it is usual to cause a cylinder to revolve so as to unfold a roll or riband of paper placed on a movable bobbin. As the cylinder revolves, it gradually winds off the strip of paper, which is kept applied to the revolving surface by ivory friction wheels. In Fick's complicated kymo. graph a long strip of smoked paper is used. The writingstyle may consist of a sable brush, or a fine glass pen filled with aniline blue dissolved in water, to which a little alcohol and glycerine are added.]

[In orcler to measure the height of the pressure, we must know the position of the

abscissa or line of no pressure, and it may be recorded at the same time as the blood-pressure or afterwards.]

[In Fig. 74, $O-x$ is the zero-line or the abscissa, and the height of the vertical lines or ordinates may be measured by the millimetre scale on the left of the figure. The height of the blood-pressure is obtained by drawing ordinates from the curve to the abscissa, measuring their length, and multiplying by two.]

(5.) Spring-Kymograph. -A. Fick (1864) constructed a "spring-kymograph" on the principle of Bourdon's manometer (Fig. 72, II).

A hollow C-shaped metallic spring, F, is filled with alcohol. One end of the hollow spring is closed, and the other end, covered by a membrane, is brought into connection with a blood-vessel by a junction-piece filled with a solution of sodium carbonate. As soon as the communication with the artery is opened, the pressure rises, and the spring, of course, tends to straighten itself. To the closed end, $b$, 
there is fixed a vertical rod attached to a series of levers, $h, i, k, e$, one of which writes its movements upon a surface moving at a uniform rate. The blood-pressure and the periodic variations of the pulse are both recorded, although the latter is not done with absolute accuracy.

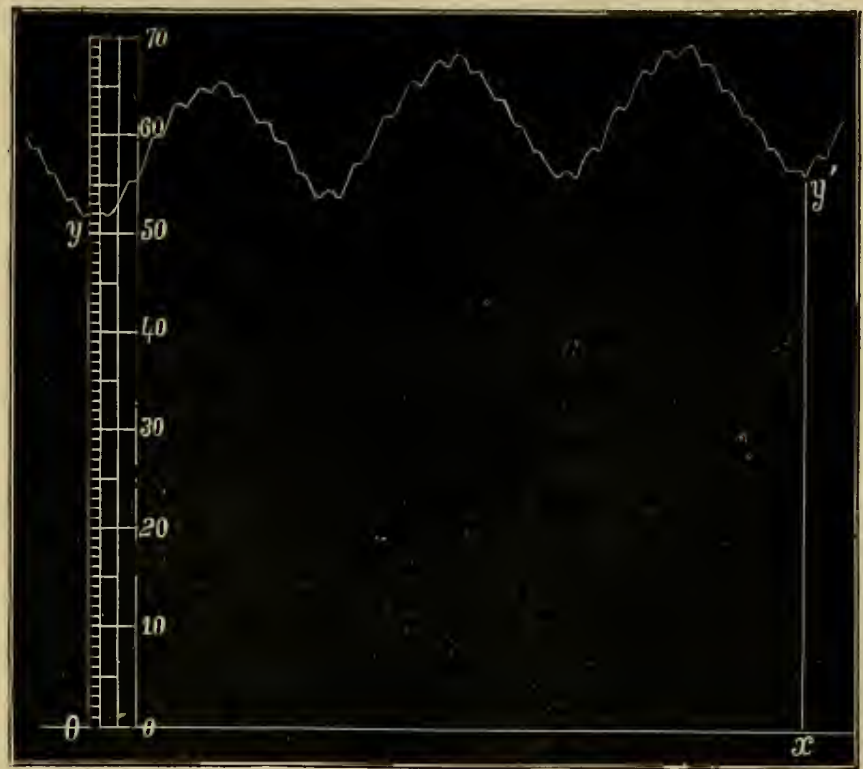

Fig. 74 .

Blood-pressure curve of the carotid of a dog obtained with a mercurial manometer. $O-x=$ line of no pressure, zero line, or abscissa; $y-y^{\prime}$ is the blood-pressure tracing with small waves, each one caused by a heart-beat, and the large waves due to the respiration. A millimetre scale shows the height of the pressure in millimetres of mercury.

[Hering improved Fick's instrument (Fig. 75). $\quad a, b, c$, is the hollow spring filled with alcohol, and communicating at $a$ with the lead tube, $d$, passing to the cannula in the artery. To $c$ is attached a series of light wooden levers with a writingstyle, $s$. The lower part of 4 dips into a vessel, $e$, filled with oil or glycerine which serves to damp the vibrations of the levers. At $f$ is a syringe communicating with the tube, $d$, filled with solution of sodic carbonate, and used for regulating the amount of fluid in the tube connecting the manometer with the blood-vessel. The whole apparatus can be raised or lowered on the toothed rod, $h$, by means of the millhead opposite, $g$, to which all the parts of the apparatus are attached.]

(B.) In Man the blood-pressure may be estimated by means of a properly graduated sphygmograph (p. 130). The pressure required to abolish the movement of the lever indicates approximately the vascular tension. Landois (Schöbel) investigated the radial pulse in a healthy student, and obtained a mean blood-pressure equal to 550 grammes.

(2.) By a manometric method v. Basch estimated the blood-pres- 
sure. He placed a capsule containing fluid upon a pulsating artery, and the capsule communicated with a mercurial manometer. As soon as the pressure within the manometer slightly exceeded that within the

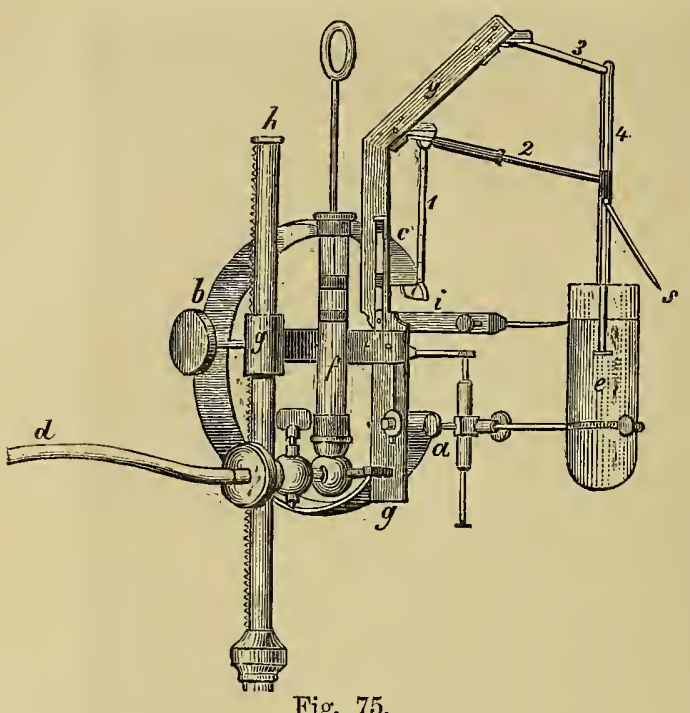

Fig. 75.

Fick's Spring-manometer, as improved by Hering. artery, the artery was compressed so that a sphygmograph placed on a peripheral portion of the vessel ceased to beat. Both arrangements, however, do not give the exact pressure within the artery, they only indicate the pressure which is required to compress the artery and the overlying soft parts. The pressure required to compress thearterial walls, however, is very small compared with the blood-pressure. It is only $4 \mathrm{~mm}$. Hg. v. Basch estimated the pressure in the radial artery of a healthy man to be $135-165$ millimetres of mercury.

In children the blood-pressure increases with age, height, and weight. In the superficial temporal artery from $2-3$ years, it is $=97 \mathrm{~mm}$; from $12-13$ years, 113 mm. Hg. (A. Eckert, c. $\S 100$ ). The blood-pressure is raised immediately after bodily movements; it is higher when a person is in the horizontal position than when sitting, and in sitting than in standing (Friedmann). After a cold as well as after a warm bath (L. Lehmann), the first effect is an increase of bloodpressure and of the quantity of urine (Grefberg).

\section{Blood-Pressure in the Arteries.}

The following results have been obtained by experiment on systemic arteries:-

(a.) Mean Blood-Pressure.--The blood-pressure is very considerable, varying within pretty wide limits; in the large arteries of large mammals, and perhaps in man it is $=140-160$ millimetres $(5 \cdot 4$ to $6 \cdot 4$ inches) of a mercurial colunn.

The following results have been olstained, those marked thus * by Poiseuille, and those + by Volkmann :- 
* Carotid, Horse, $161 \mathrm{~mm}$.

$+\quad, \quad$, 122-214 mm.

* " Dog, $151 \mathrm{~mm}$.

", $\quad$, $130-190 \mathrm{~mm}$. (Ludwig).

$+\quad$, (roat, 118-135 mm.

$+\quad$ Rabbit, $90 \mathrm{~mm}$.

+ , Fowl, S8-171 mm.
+ Aorta of frog, $22-29 \mathrm{~mm}$.

+ Gill artery of Pike, 35̃-S $4 \mathrm{~mm}$.

Brachial artery of man during an operation, 110-120 mm. (Faivre). Perhaps too low owing to the injury.

The pressure in the aorta of mammals varies from 200 to $250 \mathrm{~mm}$. $\mathrm{Hg}$. .

As a general rule, the blood-pressure in large animals is higher than in small animals, because in the former the blood-channel is considerably longer, and there is greater resistance to be overcome. In very young and in very old animals the pressure is lower than in individuals in the prime of life.

(b.) Branching of the Blood-Vessels.-Within the large arteries the blood-pressure diminishes relatively little as we pass towards the periphery, because the difference of the resistance in the different sections of large tubes is very small. As soon, however, as the arteries begin to divide frequently, and undergo a considerable diminution in their lumen, the blood-pressure in them rapidly diminishes, because the propelling energy of the blood is much weakened owing to the resistance which it has to overcome (p. 118).

(c.) Amount of Blood.-The blood-pressure is increased with greater filling of the arteries, and vice versâ; it

\section{Increases}

1. With increased and accelerated action of the heart;

2. In plethoric persons;

3. After increase of the quantity of blood by direct transfusion, or after a copious meal.

\section{Decreases}

1. During diminished and enfeebled action of the heart;

2. In anæmic persons;

3. After hæmorrhage or considerable excretions from the blood by sweating, the urine, severe diarrhœa.

The blood-pressure does not vary in the same proportion as the variations in the amount of blood. The vascular system, in virtue of its muscular tissue, has the property, within liberally wide limits, of accommodating itself to larger or smaller quantities of blood (C. Ludwig and Worm Müller, $§ 102, d)$. Small and moderate hæmorrhages (in the dog to $2 \cdot S$ per cent of the body-weight) have no obvious effect on the blood-pressure. After a slight loss of blood the pressure may even rise (Worm Müller). If a large amount of blood be withdrawn, it causes a great fall of the blood-pressure (Hales, Magendie), and when hæmorrhage oscurs to 4-6 per cent. of the body-weight, the blood-pressure $=0$. The transfusion of a moderate amount of blood does not raise the mean arterial blood-pressure. [There are important practical deductions from these experiments, viz., that the blood-pressure cannot be diminished directly by moderate blood-letting, and that the blood-pressure is not necessarily high in plethoric persons.]

(d.) Capacity of the Vessels.-The arterial pressure rises when the capacity of the arterial system is diminished, and conversely. The plain circularly-disposed muscular fibres of the arteries are the chief 
agents concerned in this process. When they relax, the arterial bloodpressure falls, and when they contract, it rises. These actions of muscular fibres are controlled and regulated by the action of the vasomotor nerves (vol. ii.)

(e.) Collateral Vessels.-The arterial pressure within a given area of the vascular system must rise or fall according as the neighbouring areas are diminished, whether by the application of pressure, or a ligature, or are rendered impervious, or as these areas dilate. The application of cold or warmth to limited areas of the bodyincreasing or diminishing the atmospheric pressure on a part-the paralysis or stimulation of certain vaso-motor areas (vol. ii.), all produce remarkable variations in the blood-pressure. [The effect of dilatation of a large vascular area on the arterial pressure is well shown by what happens when the blood-vessels of the abdomen are dilated. If the central end of the superior cardiac nerve of a rabbit be stimulated, after a few seconds the blood-vessels of the abdomen dilatc, and gradually there is a steady fall of the blood-pressure in the systemic arteries.' Fig. 76 is a blood-pressure tracing showing the height of the blood-pressure before stimulation, $a$. The stimulation was continued from $a$ to $b$, and after a certain latent period, there is a steady fall of the blood-pressure. The nerve which causes this reflex

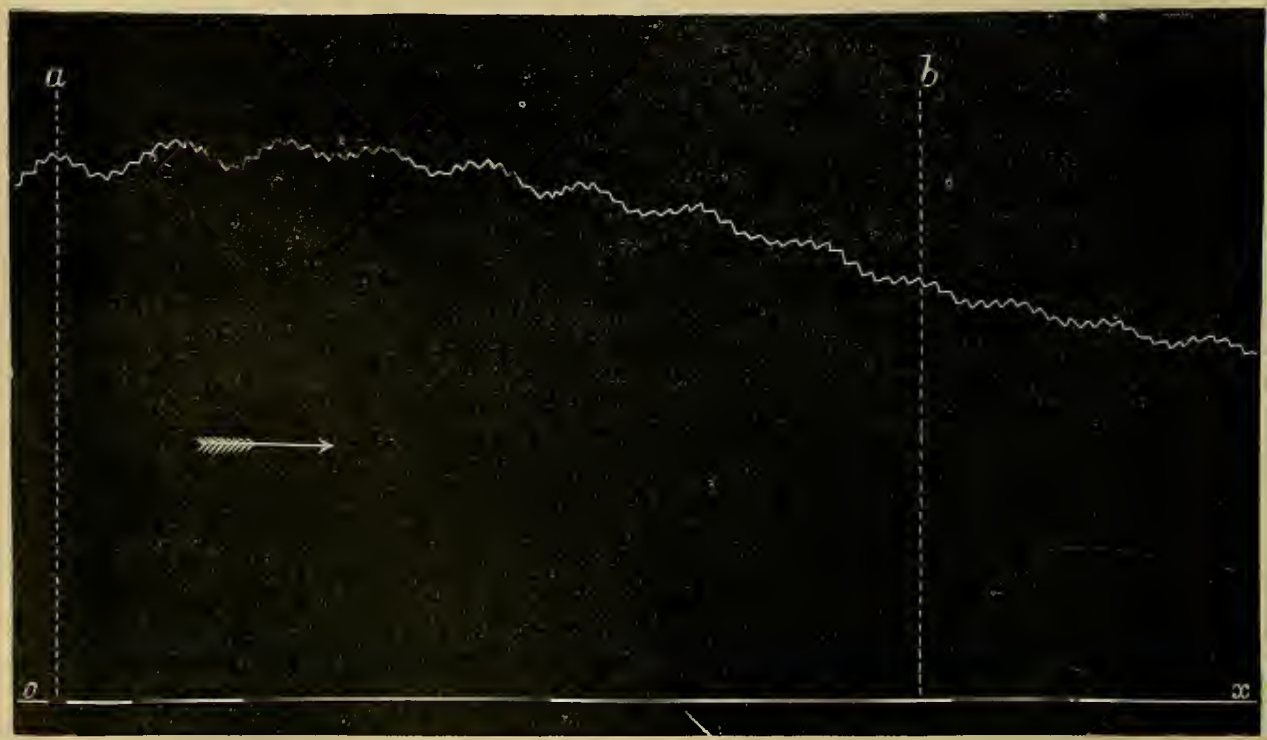

Fig. 76.

Kymographic tracing showing the effect on the blood-pressure of stimulation of the central end of the depressor nerve in the rabbit. Stimulation began at $a$, and ended at $b ; 0-x$, the abscissa. 
dilatation of the abdominal blood-vessels, and consequent lowering of the blood-pressure, is also called the depressor nerve.

(f.) Respiratory Undulations.-The arterial pressure also undergoes regular variations or undulations owing to the respiratory movernents. These undulations are called respiratory undulations (Figs. 74 and 77). Stated broadly, during every strong inspiration the pressure rises, and during expiration it falls $(\$ 74)$. This is not quite correct(see below). These undulations may be explained by the fact, that with every expiration, the blood in the aorta is subjected to an increase of pressure through the compressed air in the chest; with every inspiration, on the other land, it is diminished owing to the diminution of the air in the lungs acting upon the aorta. Besides, the inspiratory movements of the chest aspirate blood from the venæ cavæ towards the heart, while expiration retards it, and thas influences the blood-pressure. The undulations are most marked in the arteries lying nearest to the heart. The respiratory undulations are due in part to a stimulation or condition of excitement of the vaso-motor centre, which runs parallel with the respiratory movements. This stimulation of the vaso-motor centre causes the arteries to contract, and thus the blood-pressure is raised. The variations in the pressure which depend upon a varying activity of the vaso-motor centre are known as the curves of Truube and Hering (p. 171). In Fig. 77 are represented a blood-pressure tracing and a curve of the movements of respiration (thick line) taken simultaneously in a dog by C. Ludwig and Einbrodt. The blood-pressure tracing was obtained from the carotid artery, while

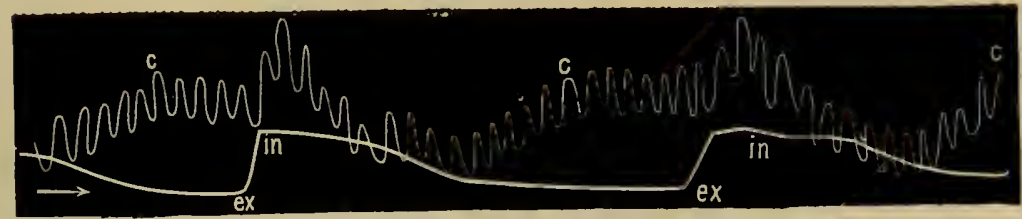

Fig. 77.

Kymographic blood-pressure tracing (upper, thin line), and respiration curve (lower, thick line), taken simultaneously-ex, expiration ; in, inspiration ; $c, c$, heart-beats. The large curves in the blood-pressure tracing are due to respiration (Ludwig and Einbrodt).

the pressure within the thorax was measured by means of a manometer placed in connection with one pleural cavity. In this curve, when expiration begins (at $e x$ ), and as the expiratory-pressure rises, the bloodpressure rises, while when inspiration begins (at in) both fall. The blood-curve, however, begins to rise (at $c$ ) before expiration com- 
mences-i.e., during the last part of the act of inspiration. This is due to the contraction of the arteries, caused by impulses sent from the vaso-motor centre. It is also aided by the circumstance that during inspiration there is an increased inflow of venous blood to the heart, so that when it contracts, more blood is forced into the arteries. [The maxima and minima of the two curves do not coincide exactly, but in addition the number of pulse-beats is greater in the ascent than in the descent. This is well-marked in a blood-pressure tracing from a dog's carotid, while in a rabbit this difference of the pulse-rate is but slightly marked. The smaller number of pulse-beats during the descent-i.e., during the greater part of expiration-is due to the activity of the cardioinhibitory centre in the medulla oblongata. This is proved by the fact, that section of both vagi in the dog causes the difference of pulse-rate to disappear, while other conditions remain the same as before, except that the heart beats more rapidly. It would seem that during the ascent, the cardio-inhibitory centre is comparatively inactive. It is clear, therefore, that the respiratory and cardio-inhibitory centres in the medulla oblongata act to a certain extent in unison, so that it is reasonable to suppose that other centres situated in close proximity to these may also act in unison with them, or, as it were, "in sympathy." As already stated, the vaso-motor centre is also in action during a particular part of the time.]

[If a $\operatorname{dog}$ be curarised and artificial respiration established, the respiratory undulations still occur, although in a modified form. In artificial respiration, the mechanical conditions, as regards the intrathoracic pressure, are exactly the reverse of those which obtain during ordinary respiration. Air is forced into the chest during artificial respiration, so that the pressure within the chest is increased during inspiration, while in ordinary inspiration the pressure is diminished. Thus, the same mechanical explanation will not suffice for both cases.]

If the artificial respiration be suddenly interrupted in a curarised animal, the blood-pressure rises steadily and rapidly. This rise is due to the stimulation of the vaso-motor centre in the medulla oblongata by the impure blood. This causes contraction of the small arteries throughout the body, which retards the out-flow from the large arteries, and thus the pressure within them is raised. [Stated broadly, the arterial pressure depends on the central organ-the heart, and on the condition of the peripheral organs-the small arteries. Both are influenced by the nervous system. If the action of the vaso-motor centre be eliminated by dividing the spinal cord in the cervical region, arrest of the respiration causes a very slight rise of the blood-pressure; hence, it is evident that venous blood acts but slightly 
on the heart, or on any local peripheral nervous mechanism, or on the muscular fibres of the arteries. This experiment shows that it is the vaso-motor centre which is specially acted upon by the venous blood.]

[Traube-Hering Curves.-The following experiment proves that the varying activity of the vaso-motor centre suffices to produce undulations in the blood-pressure tracing. Take a dog, curarise it, expose both vagi and establish artificial respiration; then estimate the bloodpressure in the carotid. After section of the vagi, the heart will continue to beat more rapidly, but it will be undisturbed by the cardio-inhibitory centre. Thus the central factor in the causation of the blood-pressure remains constant. Suddenly interrupt the respiration and, as already stated, the blood-pressure will rise steadily and uniformly, owing to the stimulation of the vaso-motor centre by the venous blood. In this case the peripheral factor or state of tension of the small arteries throughout the body is influenced by the condition of the nerve-centre which controls their action. After a time, the bloodpressure tracing shows a series of bold curves higher than the original tracing. These can only be due to an alteration in the state of the small arteries, brought about by a condition of rhythmical activity of the vaso-motor centre. These curves were described and figured by Traube, and are called the Traube or Traube-Hering curves. As in other conditions, stimulation gives place to exhaustion, and soon the venous blood paralyses the vaso-motor centre and the small arteries relax, blood flows freely out of the larger arteries, and the blood-pressure rapidly sinks. Variations in the blood-pressure have been observed after a mechanical pump has been substituted for the heart, i.e., after all respiratory movements have been set aside, so that the only factor which would account for the phenomena of the Traube-Hering curves is the variation in the peripheral resistance in the small arteries, determined by the condition of the vaso-motor centre.]

The respiratory undulations of the blood-pressure become more pronounced the greater the force of the respirations, which produce greater variations of the intrathoracic pressure. In man, the diminution of the pressure within the trachea is $1 \mathrm{~mm}$. Hg. luring tranquil inspiration, while during forced respiration, when the respiratory passage is closed, it may be $57 \mathrm{~mm}$. Conversely, during ordinary expiration, the pressure is increased within the trachea $2-3 \mathrm{~mm}$. $\mathrm{Hg}$, while during forced expiration, owing to the compression of the abdominal muscles, it may reach $87 \mathrm{~mm}$. $\mathrm{Hg}$.

The increase of the blood-pressure during inspiration, as well as the fall during expiration, must in part depend upon the pressure within the abdomen. As the diaphragm descends during inspiration, it presses upon the abdominal contents, including the abdominal vessels, 
whereby the blood-pressure must be increased. The reverse effect occurs during expiration (Schweinburg). [Section of both phrenic nerves and opening of the abdominal cavity cause the respiratory undulations almost entirely to disappear. The respiratory undulations, therefore, depend in great part upon the changes of the abdominal pressure and the effect of these changes on the amount of blood in the abdominal vessels. When making a blood-pressure experiment, pressure upon the abdomen of the animal with the hand, causes the blood-pressure to rise rapidly.]

(g.) Variations with each Pulse-beat.-The mean arterial pressure undergoes a variation with each heart-beat or pulse-beat, causing the socalled pulsatory undulations (Fig. 77,c). The mass of blood forced into the arteries with each ventricular systole causes a positive wave and an increase of the pressure corresponding with it, which of course corresponds in its development and in its form with the pulse-curve.

In the large arteries Volkmann found the increase during the heart-beat to be $=\frac{1}{16}$ (horse) and ${ }_{17}^{1}(\mathrm{dog})$ of the total pressure.

None of the apparatus described in $\$ 84$ gives an exact representation of the pulsecurve. They all show simply a rise and fall, a simple curve. The sphygmograph alone gives a true expression of the undulations in the blood-pressure which are due to the heart-beat.

(h.) If the heart's action be arrested or interrupted by continued stimulation of the vagus (Brunner, 1855), or by a high positive respiratory-pressure (Einbrodt), the arterial blood-pressure falls enormously, while it rises in the veins as the blood flows into them from the arteries to equilibrate the difference of pressure in the two sets of vessels. This experiment shows, that even when the difference of pressure is almost entirely set aside, the passive blood presses upon the arterial walls-i.e, on account of the overfilling of the bloodvessels, a slight pressure is exerted upon the walls, even when there is no circulation (Brunner). [As already stated, the arterial pressure depends on the condition of the central organ-the heart-and on the peripheral organs-the small arteries. If the action of the heart be arrested, then the blood-pressure rapidly falls. Fig. 78 shows the effect on the blood-pressure, of arresting the action of the heart, by stimulation of the peripheral end of the vagus. There is a sudden fall of the arterial pressure, as shown by the rapid fall of the curve from $a$.

For the effects of the nervous system upon the blood-pressure, see "Vaso-motor Centre" (vol. ii.)

Pathological.-In persons suffering from granular or contracted kidney and sclerosis of the arteries, in lead poisoning, and after the injection of ergotin, which causes contraction of the small arteries, it is found, on employing the method of 
v. Basch, that the blood-pressure is raised. It is also increased in cases of cardiac bypertrophy with dilatation, and by digitalis in cardiac affections, while it falls

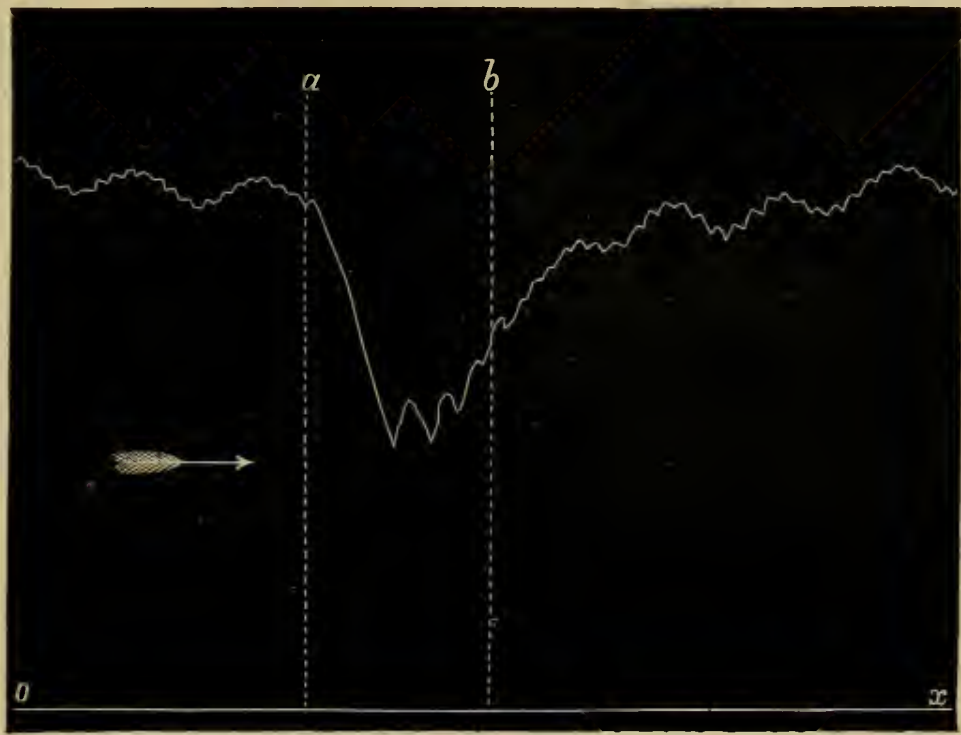

Fig. 78.

Blood-pressure tracing taken with a mercurial kymograph from the carotid of a rabbit; $o-x$, abscissa; vagus nerve stimulated between the vertical lines, $a$ and $b$.

after the injection of morphia (Kristeller). The blood-pressure falls in fever (Wetzel), a fact also indicated in the sphygmogram (\$ 69). In chlorosis and phthisis the blood-pressure is low (Waldenburg).

\section{Blood-Pressure in the Capillaries.}

Methods.-Direct estimation of the capillary pressure is not possible on account of the smallness of the capillary tubes. If a glass plate of known dimensions be placed on a portion of the skin rich in blood-vessels, and if it be weighted until the capillaries become pale, we obtain approximately the pressure necessary to overcome the capillary pressure. N. v. Kries placed a small glass plate (Figs. 79, 80) $2 \cdot 5-5 \mathrm{sq} . \mathrm{mm}$., on a suitable part of the skin, e.g., the skin at the root of the nail on the terminal phalanx, or on the ear in man, and on the gum in rabbits. Into a scalepan attached to this, weights were placed until the skin became pale. The pressure in the capillaries of the hand, when the hand is raised, Kries found to be $24 \mathrm{~mm}$. $\mathrm{Hg}$.; when the hand hangs down, $54 \mathrm{~mm}$. Hg.: in the ear, $20 \mathrm{~mm}$., and in the gum of a rabbit, $32 \mathrm{~mm}$.

[Roy and Graham Brown ascertained the hydrostatic pressure necessary to occlude the vessels in transparent parts placed under the microscope, e.g., the web of a frog's foot, tongue or mesentery of a frog, the tails of newts and small fishes. The upper surface of the part to be inrestigated, e.g., the web of a frog's foot, is made just to touch a thin glass plate. The under surface is in contact with a delicate trans. 
parent membrane covering the upper end of a small brass cylinder, whose lower end contains a piece of glass fitted air-tight into it. The interior of the brass cylinder communicates by means of a tube with an arrangement for obtaining any desired pressure, and the amount of the pressure is indicated by a manometer. Air pressure is used, and this is obtained by compressing a caoutchouc bag between two brass plates. The membrane to be investigated lies between two transparent

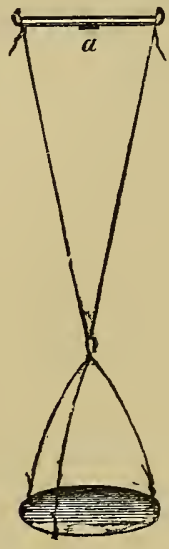

Fig. 79.

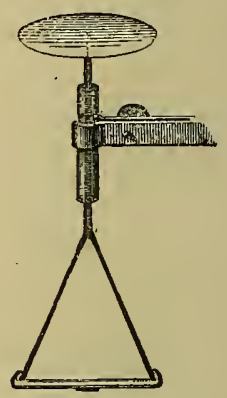

Fig. 80.

Apparatus used by $v$. Kries for estimating the capillary pressure- $\alpha$, the small square of glass. In Fig. 79 the scale-pan for the weights is below, and in Fig. 80, above.

media, an upper one of glass and a lower one of transparent membrane, on which the pressure acts. Any change in the vessels is observable by means of the microscope. These observers conclude from their experiments that the capillaries are contractile, and that their contractility is, to all appearance, in constant action. The regulation of the peripheral blood-stream is due not only to the cerebro-spinal vaso-motor centres, but also to independent peripheral vaso-motor mechanisms, which may be nervous in their nature, or are due to some direct action on the walls of the vessels (p. 126).]

Conditions influencing Capillary Pressure.-The intra-capillary bloodpressure in a given area increases-(1.) When the afferent small arteries dilate. When they are dilated the blood-pressure within the large arteries is propagated more easily into them. (2.) By increasing the pressure in the small afferent arteries. (3.) By narrowing the diameter of the veins leading from the capillary area. Closure of the veins may quadruple the pressure (v. Kries). (4.) By increasing the pressure in the veins (e.g., by altering the position of a limb). A diminution of the capillary pressure is caused by the opposite conditions.

Changes in the diameter of the capillaries influence the internal pressure. We have to consider the movements of the capillary wall itself (protoplasma-movements, Stricker-p. 125), as well as the pressure, swelling, and consistence of the surround- 
ing tissues. The resistance to the blood-stream is greatest in the capillary area, and it is evident that the blood in a long capillary must exert more pressure at the commencement than at the end of the capillary; in the middle of the capillary area the blood-pressure is just about one-half of the pressure within the large arteries (Donders). The capillary pressure must also vary in different regions of the body. Thus, the pressure within the intestinal capillaries, in those constituting the glomeruli of the kidney, and in those of lower limbs when the person is in the erect posture, must be greater than in other regions, depending in the former cases partly upon the double resistance caused by two sets of capillaries, and in the latter case partly on purely hydrostatic causes.

\section{Blood-Pressure in the Veins.}

In the large venous trunks near the heart (innominate, sub-clavian, jugular) a mean negative pressure of about $-0.1 \mathrm{~mm} . \mathrm{Hg}$. prevails $(\mathrm{H}$. Jacobson). Hence, the lymph-stream can flow unhindered. As the distance of the veins from the heart increases, there is a gradual increase of the lateral pressure; in the external facial vein (sheep) $=+3 \mathrm{~mm}$. brachial, $4.1 \mathrm{~mm}$., and in its branches $9 \mathrm{~mm}$.; crural, $11.4 \mathrm{~mm}$. (Jacobson). [The pressure is said to be negative when it is less than that of the atmosphere.]

Conditions Influencing the Venous Pressure.-(1.) All conditions which diminish the difference of pressure between the arterial and venous systems increase the venous pressure and vice versâ.

(2.) General plethora of blood increases it; anæmia diminishes it.

(3). Respiration, or the aspiration of the thorax, affects specially the pressure in the veins near the heart; during inspiration, owing to the diminished tension, blood flows towards the chest, while during expiration it is retarded. The effects are greater the deeper the respiratory movements, and these may be very great when the respiratory passages are closed $(\$ 60)$.

[When a vein is exposed at the root of the neck, it collapses during inspiration, and fills during expiration - a fact which was known to Valsalva. The respiratory movements do not affect the venous stream in the peripheral veins. The veins of the neck and face become distended with blood during crying, and on making violent expiratory efforts, as in blowing upon a wind-instrument; while every surgeon is well acquainted with the fact that air is particularly apt to be sucked into the veins, especially in operations near the root of the neck. This is due to the negative intra-thoracic pressure occurring during inspiration.]

(4.) Aspiration of the Heart.-Blood is sucked or aspirated into the auricles when they dilate, so that there is a double aspirationone synchronous with inspiration, and the other, which is but slight, synchronous with the heart-beat. There is a corresponding retardation of the blood-stream in the venæ cavæ, caused by the contraction of the auricle (see p. 77,a). The respiratory and cardiac 
undulations are occasionally observable in the jugular vein of a healthy person ( $\$ 99)$.

[Braune showed that the femoral vein under Poupart's ligament collapsed when the lower limb was rotated outwards' and backwards, but filled again when the limb was restored to its former position. All the veins which open into the femoral vein have valves, which permit blood to pass into the femoral vein, but prevent its reflux. This mechanism acts to a slight degree as a kind of suction and pressure apparatus when a person walks, and thus favours the onward movement of the blood.]

(5.) Changes in the position of the limbs or of the body, for hydrostatic reasons, greatly alter the venous pressure. The veins of the lower extremity bear the greatest pressure, while at the same time they contain most muscle (K. Bardeleben, $\S 65$ ). Hence, when these muscles from any cause become insufficient, dilatations occur in the veins, giving rise to the production of varicose veins.

[(6.) Movements of the Voluntary Muscles.-Veins which lie between muscles are compressed when these muscles contract, and as valves exist in the veins the flow of the blood is accelerated towards the heart; if the outflow of the blood be obstructed in any way, then the venous pressure on the distal side of the obstruction may be greatly increased. When a fillet is tied on the upper-arm, and the person moves the muscles of the fore-arm, the course of the superficial veins can be distinctly traced on the surface of the limb.]

[(7.) Gravity exercises a greater effect upon the blood-stream in the extensile veins than upon the stream in the arteries. It acts on the distribution of the blood, and thus indirectly on the motion of the bloodstream. It favours the emptying of descending veins, and retards the emptying of ascending veins, so that the pressure becomes less in the former and greater in the latter. If the position of the limb be changed, the conditions of pressure are also altered (Paschutin). If a person be suspended with the head hanging downwards, the face soon becomes turgid, the position of the body favouring the inflow of blood through the arteries, and retarding the outflow through the veins. If the hand hangs down it contains more blood in the veins than if it is held for a short time over the head, when it becomes pale and bloodless. As Lister has shown, the condition of the vessels in the limb are influenced not only by the position of the limb, but also by the fact that a nervous mechanism is called into play.]

[Ligature of the Portal Vein.-The pressure and other conditions vary in particular veins. Thus, if the portal vein be ligatured, there is congestion of the capillaries and rootlets of the portal vein, and dilatation of all the blood-vessels in the abdomen, and gradually nearly all the blood of the animal accumulates within its belly, so that, paradoxical as it may seem, an animal may be bled into its own belly. As a consequence of sudden and complete ligature of this vein, the arterial 
blood-pressure gradually and rapidly falls, and the animal dies very quickly. If the ligature be removed before the blood-pressure falls too much, the auimal may recover.

Ligature of the Veins of a Limb.-The effect of ligaturing or compressing all the veins of a limb is well seen in cases where a bandage has been applied too tightly. It leads to congestion and increase of pressure within the veins and capillaries, incrcased transudation of fluid throngh the capillaries, and consequent redema of the parts beyond the obstruction. Ligature of one vein does not always produce œdema, but if several veins of a limb be ligatured, and the vaso-motor nerves be divided at the same time, the rapid production of cedema is ensured. In pathological cases the pressure of a tumour upon a large vein may produce similar results.]

\section{Blood-Pressure in the Pulmonary Artery.}

Methods.-(1.) Direct estimation of the blood-pressure in the pulmonary artery by opening the chest was made by C. Ludwig and Beutner (1850). Artificial respiration was kept up, and the manometer was placed in connection with the left branch of the pulmonary artery.

The circulation through the left lung of cats and rabbits was thereby completely cut off, and in dogs to a great extent interrupted. There was an additional disturbing element, viz., the removal of the elastic force of the lungs owing to the opening of the chest, whereby the venous blood no longer flows normally into the right heart, while the right heart itself is under the full pressure of the atmosphere. The estimated pressure in the $\operatorname{dog}=29.6$; in the cat $=17.6$; in the rabbit, $12 \mathrm{~mm}$. Hg. - -i.e., in the dog 3 times, the rabbit 4 times, and the cat 5 times less than the carotid pressure.

(2.) Hering (1850) experimented upon a calf with ectopia cordis. He introduced glass tubes directly into the heart, by pushing them through the muscular walls of the ventricles. The blood rose to the height of 21 inches in the right tube, and $33 \cdot 4$ inches in the left.

(3.) Chauveau and Faivre (1S56) introduced a catheter through the jugular vein into the right ventricle, and placed it in connection with a manometer (p. 87).

Indirect measurements have been made by comparing the relative thickness of the walls of the right and left ventricles, or the walls of the pulmonary artery and aorta, for tlere must be a relation between the pressure and the thickness of the muscle in the two cases.

Beutner and Marey estimated the relation of the pulmonary artery to the aortic presssure as 1 to 3 ; Goltz and Gaule as 2 to 5; Fick and Badoud found a pressure of $60 \mathrm{~mm}$. in the pulmonary artery of the dog, and in the carotid $111 \mathrm{~mm}$. Hg. The blood-pressure within the pulmonary artery of a child is relatively higher than in the adult (Beneke).

The lungs within the chest are kept in a state of distension, owing to the fact that a negative pressure exists on their outer pleural surface. When the glottis is open, the inner surface of the lung and the walls of the capillaries in the pulmonary air-vesicles are exposed to the full pressure of the air. The heart and the large blood-vessels within the chest are not exposed to the full pressure of the atmosphere, but only 
to the pressure which corresponds to the atmospheric pressure minus the pressure exerted by the elastic traction of the lungs $(\S 60)$. The trunks of the pulmonary artery and veins are subjected to the same conditions of pressure. The elastic traction of the lungs is greater, the more they are distended. The blood of the pulmonary capillaries will, therefore, tend to flow towards the large blood-vessels. As the elastic traction of the lungs acts chiefly on the thin-walled pulmonary veins, while the semi-lunar valves of the pulmonary artery, as well as the systole of the right ventricle, prevent the blood from flowing backwards, it follows that the blood in the capillaries of the lesser circulation must flow towards the pulmonary veins.

If tubes with thin walls be placed in the walls of an elastic distensible bag, the lumen of these tubes changes according to the manner in which the bag enclosing them is distended. If the bag be directly inflated so as to increase the pressure within it, the lumen of the tubes is diminished (Funke and Latschenberger). If the bag be placed within a closed space, and the tension within this space be diminished so that the bag thereby becomes distended, the tubes in its wall dilate. In the latter case-viz., by negative aspiration-the lungs are kept distended within the thorax, hence the blood-vessels of the lungs containing air are wider than those of collapsed lungs (Quincke and Pfeiffer, Bowditch and Garland, De Jager). Hence also, more blood flows through the lungs distended within the thorax than through collapsed lungs. The dilatation which takes place during inspiration acts in a similar manner. The negative pressure that obtains within the lungs during inspiration causes a considerable dilatation of the pulmonary veins into which the blood of the lungs flows readily, whilst the blood under high pressure in the thick-walled pulmonary artery scarcely undergoes any alteration. The velocity of the blood-stream in the pulmonary vessels is accelerated during inspiration (De Jager, Lalesque).

The blood-pressure in the pulmonary circuit is raised when the lungs are inflated. Contraction of small arteries, which causes an increase of the blood-pressure in the systemic circulation, also raises the pressure in the pulmonary circuit, because more blood flows to the right side of the heart (v. Openchowski).

The vessels of the pulmonary circulation are very distensible and their tonus is slight. [Occlusion of one branch of the pulmonary artery does not raise the pressure within the aorta (Beutner). Even when one pulmonary artery is plugged with an embolon of paraffin, the pressure within the aortic system is not raised (Lichtheim). Thus; when a large branch of the pulmonary artery becomes impervious, the obstruction is rapidly compensated, and this is not due to the action of 
the nervous system. The vaso-motor system has much less effect upon the pulmonary blood-vessels than upon those of the systemic circulation (Badoud, Hofmokl, Lichtheim). The compensation seems to be due chiefly to the great distensibility and dilatation of the pulmonary vessels (Lichtheim)].

We know little of the effect of physiological conditions upon the pulmonary artery. According to Lichtheim suspension of the respiration causes an increase of the pressure. [In one experiment he found that pressure within the pulmonary artery was increased, while it was not increased in the carotid, and he regards this experiment as proving the existence of vaso-motor nerves in the lung.] Morel found that electrical and mechanical stimulation of the abdominal organs caused a considerable rise of pressure in the pulmonary artery $(\mathrm{dog})$.

During the act of great straining, the blood at first flows rapidly out of the pulmonary veins and afterwards ceases to flow, because the inflow of blood into the pulmonary vessels is interfered with. As soon as the straining ceases, blood flows rapidly into the pulmonary vessels (Lalesque).

Severini found that the blood-stream through the lungs is greater and more rapid when the lungs are filled with air rich in $\mathrm{CO}_{2}$ than when the air within them is rich in $O$. He supposes that these gases act upon the vascular ganglia within the lung, and thus affect the diameter of the vessels.

Pathological.-Increase of the pressure within the area of the pulmonary artery occurs frequently in man, in certain cases of heart disease. In these cases the second pulmonary sound is always accentuated, while the elevation caused thereby in the cardiogram is always more marked and occurs earlier ( $\$ 52)$.

[Influence of the Nervous System.-The pulmonary circulation is much less dependent on the nervous system than the systemic circulation. Very considerable variations of the blood-pressure within the other parts of the body may occur, while the pressure within the right heart and pulmonary artery is but slightly affected thereby. The pressure is increased by electrical stimulation of the medulla oblongata, and it falls when the medulla is destroyed. Section and stimulation of the central or peripheral ends of the vagi, stimulation of the splanchnics, and of the central end of the sciatic, have but a minimal influence on the pressure of the pulmonary artery (Aubert).]

\section{Measurement of the Velocity of the Blood-Stream.}

Methods. (1.) A. W. Volkmann's Hæmadromometer.-A glass tube of the shape of a hair-pin, $60-130 \mathrm{ctm}$. long and 2 or $3 \mathrm{~mm}$. broad, with a scale etched on it, or attached to it, is fixed to a metallic basal plate, B, so that each limb passes to a stop-cock with three channels. The basal plate is perforated along its length, and carries at each end short cannulæ, $c, c$, which are tied into the ends of a divided artery. The whole apparatus is first filled with water [or, better, 
with salt solution]. The stop-cocks are moved simultaneously, as they are attached to a toothed wheel and have at first the position given in Fig. 81, I, so that the blood simply flows through the hole in the basal piece, i.e., directly from one end of the artery to the other. If at a given moment the stop-cock is turned in the direction indicated in Fig. 81, II, the blood has to pass through the glass tube, and the time it takes to make the circuit is noted, and as the length of the tube is known, we can easily calculate the velonity of the blood.

Volkmann found the velocity to be in the carotid $(\operatorname{dog})=205-357 \mathrm{~mm}$. carotid (horse) $=$ $306 ;$ maxillary (horse) $=232 ;$ metatarsal $=56$ $\mathrm{mm}$. per second. The method has very obvious

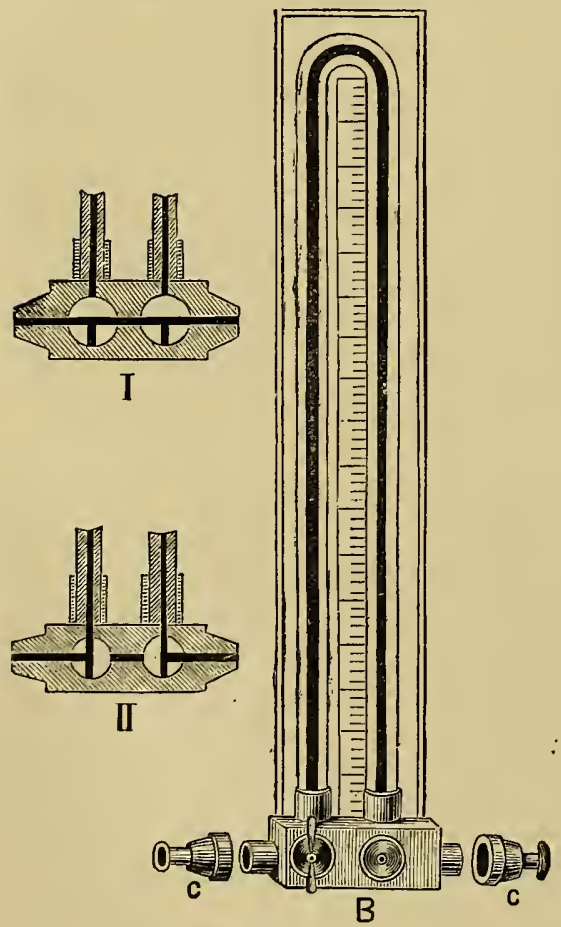

Fig. 81 .

Volkmann's Hæmadromometer (B)-I, blood flows from artery to artery; II, blood must pass through the glass tube of $\mathrm{B}$; $c, c$, cannulæ for the divided artery.

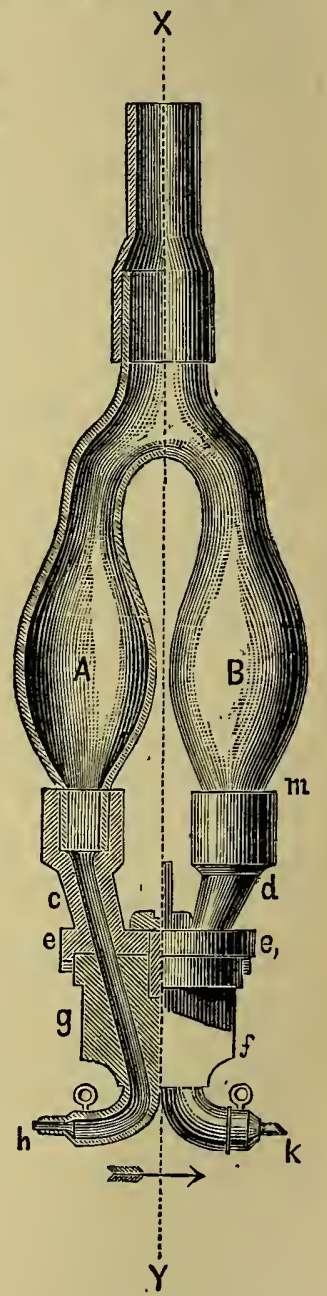

Fig. 82.

Ludwig \& Dogiel's Stromuhr or Rheometer$\mathrm{X}, \mathrm{Y}$, axis of rotation ; $\mathrm{A}, \mathrm{B}$, glass bulbs; $h, k$, cannulæ inserted in the divided artery; $e, e_{1}$, rotates on $g, f ; c, d$, tubes.

defects arising from the narrowness of the tube; the introduction of such a tube offers new resistance, while there are no respiratory or pulse variations observable in the stream in the glass tube. 
(2.) C. Ludwig and Dogiel (1867) devised a STROMUHR or RHEOMETER for measuring the amount of blood which passed through an artery in a given time (Fig. 82). It consists of two glass bulbs, $\mathrm{A}$ and $\mathrm{B}$, of exactly the same capacity. These bulbs communicate with each other, above, their lower ends being fixed by means of the tubes, $c$ and $d$, to the metal disc, $e e_{1}$. This disc rotates round the axis, $X Y$, so that, after a complete revolution the tube, $c$, communicates with $f$, and $d$ with $g ; f$ and $g$ are provided with horizontally placed cannulæ, $h$ and $k$, which are tied into the ends of the divided artery. The cannula, $h$, is fixed in the central end, and $k$ in the peripheral end of the artery (e.g., carotid); the bulb, A, is filled with oil and $B$ with defibrinated blood; at a certain moment the communication through $h$ is opened, the blood flows in, driving the oil before it, and passes into $B$, while the defibrinated blood flows through $k$ into the peripheral part of the artery. As soon as the oil reaches $m$-a moment which is instantly noted, or, what is better, inscribed upon a revolving cylinder-the bulbs, A, B, are rotated upon the axis, $X \mathrm{Y}$, so that $\mathrm{B}$ comes to occupy the position of A. The same experiment is repeated, and can be continued for a long time. The quantity of blood which passes in the unit of time $(1 \mathrm{sec}$.) is calculated from the time necessary to fill the bulb with blood. Important results are obtained by means of this instrument.

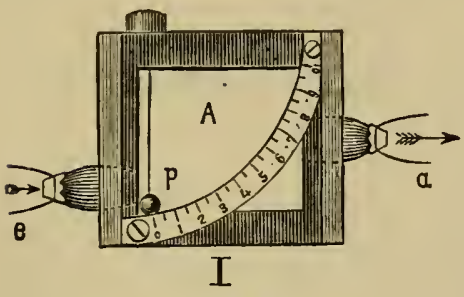

Fig. 83.

Vierordt'sHæmatachometer-A, glass; $e$, entrance; $a$, exit cannula; $p$, pendulum.

(3.) Vierordt's Hæmatachometer (1858) consists of a small metal box (Fig. 83) with parallel glass sides. To the narrow sides of the box are fitted an entrance, $e$, and an exit cannula, $a$. In its interior is suspended, against the entrance opening, a pendulum, $p$, whose vibrations may be read off on a curved scale. [This instrument, as well as Volkmann's apparatus, has only a historical interest. ]

(4.) Chauveau and Lortet's (Dromograph) (1860) is constructed on the same principle. A tube, A, B (Fig. S4) of sufficient diameter, with a side-tube fixed to it, $\mathrm{C}$, which can be placed in connection with a manometer, is introduced into the carotid artery of a horse. At $a$ a small piece is cut out and provided with a covering of gutta-percha which has a small hole in it; through this a light pendulum, $a, b$, with a long index, $b$, projects into the tube, i.e., into the bloodcurrent, which causes the pendulum to vibrate, and the extent of the vibrations can be read off on a scale, $S, S$. G is an arrangement to permit the instrument to be held. Both this and the former instrument are tested beforehand with a stream of water sent through them with varying velocities.

The curve of the velocity may be written off on a smoked glass, 
moving parallel with the index $b$. The dromograph curve, III, shows the primary elevation, $\mathrm{P}$, and the dicrotic elevation, $\mathrm{R}$.
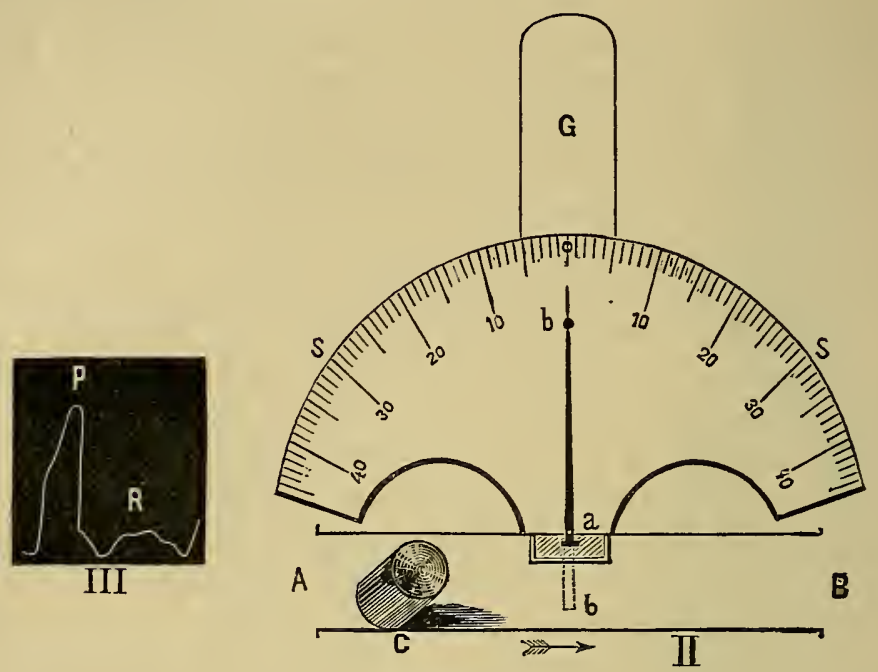

Fig. 84 .

Dromograph-A, B, tube inserted in artery; C, lateral tube connected with a manometer; $b$, index moving in a caoutchouc membrane, $a$; $\mathrm{G}$, handle. III, curve obtained by dromograph.

\section{Velocity of the Blood in Arteries, Capillaries, and Veins.}

(1.) Division of Vessels.-In estimating the velocity of the blood, it is important to remember that the sectional area of all the branches of the aorta becomes greater as we proceed from the aorta towards the capillaries, so that the capillary area is 700 times greater than the sectional area of the aorta (Vierordt). As the veins join and form larger trunks, the venous area gradually becomes smaller, but the sectional area of the venous orifices at the heart is greater than that of the corresponding arterial orifices.

The eommon iliacs are an exception; the sum of their sectional areas is less than that of the aorta; the sections of the four pulmonary veins are together less than that of the pulmonary artery.

(2.) Sectional Area.-An equal quantity of blood must pass through every section of the circulatory system, through the pulmonic as well as through the systemic circulation, so that the same amount of blood must pass through the pulmonary artery and aorta, notwithstanding the very unequal blood-pressure in these two vessels. 
(3.) Lumen.-The velocity of the current, therefore, in various sections of the vessels must be inversely as their lumen.

(4.) Capillaries.-Hence, the velocity must diminish very considerably as we pass from the root of the aorta and the pulmonary artery towards the capillaries, so that the velocity in the capillaries of mammals $=0.8$ millimetre per sec.; frog $=0.53 \mathrm{~mm}$. (E. H. Weber); $\operatorname{man}=0.6$ to 0.9 (C. Vierordt). According to A. W. Volkmann the blood in mammalian capillaries flows 500 times slower than the blood in the aorta. Hence the total sectional area of all the capillaries must be 500 times greater than that of the aorta. Donders found the velocity of the stream in the small afferent arteries, to be 10 times faster than in the capillaries. A pulsatory acceleration, more rapid during its first phase, is observable in the small arteries, althongh these are not themselves distended thereby.

Veins.-The current becomes accelerated in the veins, but in the larger trunks it is 0.5 to 0.75 times less than in the corresponding arteries.

(5.) Mean Blood-Pressure.-The velocity of the blood does not depend upon the mean blood-pressure, so that it may be the same in congested and in anæmic parts (Volkmann, Hering).

(6.) Difference of Pressure.-On the other hand, the velocity in any section of a vessel is dependent on the difference of the pressure which exists at the commencement and at the end of that particular section of a blood-vessel; it depends, therefore, on (1) the vis a tergo (i.e., the action of the heart), and (2) on the amount of the resistance at the periphery (dilatation or contraction of the small vessels-C. Ludwig and Dogiel).

(7.) Pulsatory Acceleration.-With every pulse-beat a corresponding acceleration of the blood-current (as well as of the blood-pressure) takes place in the arteries, so that every ascent of the sphygmogram corresponds to an acceleration, and every descent to a diminished velocity of the blood-stream. The variations in the velocity caused by the heartbeat are recorded in Fig. 84, obtained by Chauveau's dromograph from the carotid of a horse. The velocity-curve corresponds with a sphygmogram-P represents the primary elevation and $\mathrm{R}$ the dicrotic wave. This acceleration, as well as the pulse, disappears in the capillaries. In large vessels, Vierordt found the increase of the velocity during the systole to be greater by $\frac{1}{4}$ to $\frac{1}{2}$ than the velocity during the diastole.

(8.) Respiratory Effect.-Every inspiration retards the velocity in the arteries, every expiration aids it somewhat; but the value of these agencies is very small.

If we compare what has already been said regarding the effect of the respiration 
on the contraction and dilatation of the heart and on the blood-stream $(\S 60)$, it is clear that respiration favours the blood-stream, so does artificial respiration. When artificial respiration is interrupted, the blood-stream becomes slower (Dogiel). If the suspension of respiration lasts somewhat longer, the current is again accelerated on account of the dyspnœic stimulation of the vaso-motor centre (Heidenhain) (see Vaso-motor centre, vol. ii.)

(9.) Conditions Affecting Velocity in the Veins.-Many circumstances affect the velocity of the blood in the veins. (1) There are regular variations in the large veins near the heart (Valsalva) due to the respiration and the movements of the heart ( $\$ 50$, and 60$)$. (2) Irregular variations due to pressure-e.g., from contracting muscles (\$ 87), friction on the skin in the direction or against the direction of the venous current, the position of a limb or of the body. The pump-like action of the veins of the groin during walking has been referred to $(\S 87)$. When the lower limb is extended and rotated outwards, the femoral vein in the iliac fossa collapses, owing to an internal negative pressure; when the thigh is flexed and raised, it fills under a positive pressure (Braune). A similar condition obtains in walking.

\section{Estimation of the Capacity of the Ventricles.}

Vierordt calculated the capacity of the left ventricle from the velocity of the blood-stream, and the amount of blood discharged per second by the right carotid, right subclavian, the two coronary arteries, and the aorta below the origin of the innominate artery. He estimated that with every systole of the heart, 172 cubic centimetres (equal to 182 grammes) of blood were discharged into the aorta; this, therefore, must be the capacity of the left ventricle (compare $\S 83$ ).

\section{The Duration of the Circulation.}

The question as to how long the blood takes to make a complete circuit through the course of the circulation was first answered by Hering (1829) in the case of the horse. He injected a 2 per cent. solution of potassium ferrocyanide into a special vein, and ascertained (by means of ferric chloride) when this substance appeared in the blood taken from the corresponding vein on the opposite side of the body. The ferrocyanide may also be injected into the central or cardiac end of the jugular vein, and the time noted at which its presence is detected in the blood of the peripheral end of the same vein]. Vierordt (1858) improved this method by placing under the corresponding vein of the opposite side a rotating disc, in which was fixed a number of cups at regular intervals. The first appearance of 
the potassium ferrocyanide is detected by adding ferric chloride to the serum, which separates from the samples of blood after they have stood for a time. The duration of the circulation is as follows :-

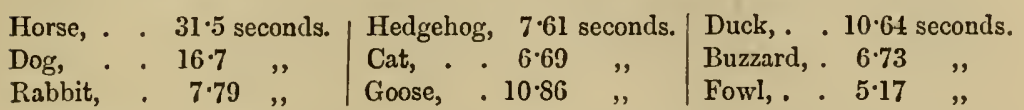

Results. - When these numbers are compared with the frequency of the normal pulse-beat in the corresponding animals, the following deductions are obtained :-

(1.) The mean time required for the circulation is accomplished during 27 heart-beats-i.e., for $\operatorname{man}=23.2$ seconds, supposing the heart to beat 72 times per minute.

(2.) Generally, the mean time for the circulation in two warm-blooded animals is inversely as the frequency of the pulse-beats.

Conditions Influencing the Time.-The time is influenced by the following factors :-

1. Long vascular channels (e.g., from the metatarsal vein of one foot to the other foot) require a longer time than short channels (as between the jugulars). The difference may be equal to 10 per cent. of the time required to complete the entire circuit.

2. In young animals (with shorter vascular channels and higher pulse-rate) the time is shorter than in old animals.

3. Rapid and energetic cardiac contractions (as during muscular exercise) diminish the time. Hence rapid and at the same time less energetic contractions (as after section of both vagi), and slow but vigorous systoles (e.g., after slight stimulation of the vagus) have no effect.

C. Vierordt estimated the quantity of blood in a man, in the following manner. In all warm-blooded animals, 27 systoles correspond to the time for completing the circulation. Hence, the total mass of the blood must be equal to 27 times the capacity of the ventricle, i.e., in man, 187.5 grams. $\times 27=5062.5$ grams. This is equal to $\frac{1}{13}$ of the body-weight, in a person weighing 65.8 kilos. (compare § 49).

It is not to be forgotten that the salt used is to some extent poisonous (p. 108).

\section{Work of the Heart.}

Johann Alfons Bernoulli (1679) and Julius Robert Mayer estimated the work done by the heart. The work of a motor is expressed in kilogramme-metres-i.e., the number of kilos. which the motor can raise in the unit of time to the height of 1 metre.

The left ventricle expels 0.188 kilo. of blood (Volkmann) with each systole, and in doing so it overcomes the pressure in the aorta, which is equal to a column of blood 3.21 metres in height (Donders). [The amount of blood expelled from each ventricle during the systole is about 180 grms. (6 ozs.) It is forced out against a pressure of 
$250 \mathrm{~mm} . \mathrm{Hg} .=3.21$ metres of blood.] The work of the heart at each systole is $0.188 \times 3.21=0.604$ kilogramme-metres. If the number of beats $=75$ per minute, then the work of the left ventricle in 24 hours $=(0.604 \times 75 \times 60 \times 24)=65,230$ kilogramme-metres. While the "work" done by the right ventricle is about one-third that of the left, and therefore $=21,740$ kilogramme-metres. Both ventricles do work equal to 86,970 kilogramme-metres. A workman during eight hours produces 300,000 kilogramme-metres-i.e., about four times as much as the heart. As the whole of the work of the heart is consumed in overcoming the resistance within the circulation, or rather is converted into heat, the body must be partly warmed thereby $(425.5$ gramme-meters are equal to 1 heat-unit-i.e., the force required to raise 425.5 grammes to the height of 1 metre may be made to raise the temperature of 1 cubic centimetre of water $1^{\circ} \mathrm{C}$.) So that 204,000 " heat-units" are obtained from the transformation of the kinetic energy of the heart.

One gramme of coal when burned yields 8,080 heat-units, so that the heart yields as much energy for heating the body as if about 25 grammes of coal were burned within it to produce heat.

\section{Blood-Current in the Smallest Vessels.}

Methods.-The most important observations for this purpose are made by means of the microscope on transparent parts of living animals. Malpighi was the first to observe the circulation in this way in the lung of a frog (1661).

The following parts have been employed :-the tails of tadpoles and small fishes; the web, tongue, mesentery, and lungs of curarised frogs (Cowper, 1704); the wing of the bat, the third eyelid of the pigeon or fowl, the mesentery; the vessels of the liver of frogs and newts (Gruithuisen), of the pia mater of rabbits, of the skin on the belly of the frog, of the mucous membrane of the inner surface of the human lip (Hüter's Cheiloangioscope, 1879); of the conjunctiva of the eyeball and eyelids. All these may be examined by reflected light.

[Entoptical appearances of the circulation (Purkinje, 1825). Under certain conditions a person may detect the movement of the blood-corpuscles within the blood-vessels of his own eye. The best method is that of Rood, viz., to look at the sky through a dark blue glass, or through several pieces of cobalt glass placed over each other (Helmholtz)].

Form and Arrangement of Capillaries.-Regarding the form and arrangement of the capillaries, we find that-

1. The diameter, which in the finest, permits only the passage of single corpuscles in a row-one behind the other-may vary from $5 \mu-2 \mu$, so that two or more corpuscles may move abreast when the capillary is at its widest.

2. The length is about $0.5 \mathrm{~mm}$. They terminate in small veins.

3. The number is very variable, and the capillaries are most numerous in those tissues, where the metabolism is most active, as in lungs, liver, muscles-less numerous in the sclerotic and in the nerve-trunks. 
4. They form numerons anastomoses, and give rise to net-works, whose form and arrangement are largely determined by the arrangement of the tissue elements them. selves. They form simple loops in the skin, and polygonal net-works in the serous membranes, and on the surface of many gland tubes; they occur in the form of elongated net-works, with short connecting branches in muscle and nerve, as well as between the straight tubules of the kidney; they converge radially towards a central point in the lobules of the liver, and form arches in the free margins of the iris, and on the limit of the sclerotic and cornea.

[A good contrast as to the vascularity of two adjacent parts is seen in the gray and white matter of the brain, the former being very vascular, the latter but slightly so.]

[Direct termination of Arteries in Veins.-Arteries sometimes terminate directly in veins, without the intervention of capillaries, e.g., in the ear of the rabbit, in the terminal phalanges of the fingers and toes in man and some animals, in the cavernous tissue of the penis (Hoyer). They may be regarded as secondary channels which protect the circulation of adjacent parts, and they may also be related to the heat-regulating mechanisms of peripheral parts (Hoyer).]

End-Arteries,-In connection with the termination of arteries in capillaries, it is important to determine if the arterioles are "end or terminal arteries," i.e., if they do not form any further anastomoses with other similar arterioles, but terminate directly in capillaries, and thus only communicate by capillaries with neighbonring arterioles-or the arteries may anastomose with other arteries just before they break up into capillaries. This distinction is important in connection with the nutrition of parts supplied by such arteries (Cohnheim).

Capillary Circulation.-On observing the capillary circulation, we notice that the red corpuscles move only in the axis of the current (axial current), while the lateral transparent plasma-current flowing on each side of this central thread is free from these corpuscles. [The axial current is the more rapid.] This plasma layer or "Poiseuille's space" is seen in the smallest arteries and veins, where $\frac{3}{5}$ is taken up with the axial current, and the plasma layer occupies $\frac{1}{5}$ on each side of it (Fig. 85). A great many, but not all, of the colourless corpuscles run in this layer. It is much less distinct in the capillaries. Rud. Wagner stated that it is absent in the finest vessels of the lung and gills [although Gunning was unable to confirm this statement.] The coloured corpuscles move in the smallest capillaries in single file one after the other; in the larger vessels, several corpuscles may move abreast, with a gliding motion, and in their course they may turn over and even be twisted if any obstruction is offered to the blood-stream. As a general rule, in these vessels the movement is uniform, but at a sharp bend of the vessel it may partly be retarded and partly accelerated. Where a vessel divides, not unfrequently a corpuscle remains upon the projecting angle of the division, and is doubled over it so that its ends project into the two branches of the tube. There it may remain for a time, until it is dislodged, when it soon regains its original form on account of its elasticity. Not unfrequently we see a red corpuscle becoming bent where two vessels meet, but on all occasions it rapidly regains 
its original form. This is a good proof of the elasticity of the coloured corpuscles.

Colourless Corpuscles.-The motion of the colourless corpuscles is quite different in character; they roll directly on the vascular wall, moistened on their peripheral zone by the plasma in Poiseuille's space, their other surface being in contact with the thread of coloured corpuscles in the centre of the stream. Schklarewsky has shown by physical experiments, that the particles of least specific gravity in all capillaries (e.g., of glass) are pressed toward the wall, while those of greater specific gravity remain in the middle of the stream. [Graphite and particles of carmine were suspended in water, and caused to circulate through capillary tubes placed under a microscope, when the graphite kept the centre of the stream, and the carmine moved in the layer next the wall of the tube.]

When the colourless corpuscles reach the wall of the vessel, they must roll along, partly on account of their surface being sticky, whereby they readily adhere to the vessel, and partly because one surface is directed towards the axis of the vessel where the movement is most rapid, and where they receive impulses directly from the rapidly moving coloured blood-corpuscles (Donders). The rolling motion is not always uniform, not unfrequently it is retrograde in direction, which seems to be due to an irregular adhesion to the vascular wall. Their slower movement (10 to 12 times slower than the red corpuscles) is partly due to their stickiness, and partly to the fact that as they are placed near the wall, a large part of their surface lies in the peripheral threads of the fluid, which of course move more slowly (in fact the layer of fluid next the wall is passivep. 117).

[D. J. Hamilton finds that, when a frog's web is examined in a vertical position, by far the greater proportion of leucocytes float on the upper surface, and only a few on the lower surface, of a small bloodvessel. In experiments to determine why the coloured corpuscles float or glide exclusively in the axial stream, while a great many, but not all, of the leucocytes roll in the peripheral layers, Hamilton ascertained that the nearer the suspended body approaches to the specific gravity of the liquid in which it is immersed, the more it tends to occupy the centre of the stream. He is of opinion that the phenomenon of the separation of the blood-corpuscles in the circulating fluid is due to the colourless corpuscles being specifically lighter, and the coloured either of the same or of very slightly greater specific gravity than the bloodplasma. Hamilton controverts the statement of Schklarewsky, and he finds that it is the relative specific gravity of a body which ultimately determines its position in a tube. These experiments point to the 
immense importance of a due relation subsisting between the specific gravity of the blood-plasma and that of the corpuscles.]

In the vessels first formed in the incubated egg, as well as in those of young tadpoles, the movement of the blood from the heart occurs in jerks (Spallanzani, 1768). The velocity of the blood-stream is influenced by the diameter of the vessels, which undergo periodic changes of calibre. This change occurs not only in vessels provided with muscular fibres, but also in the capillaries, which vary in diameter, owing to the contraction of the cells composing their walls (p. 125).

The velocity of the blood is greater in the pulmonary than in the systemic capillaries (Hales, 1727); hence, we must conclude that the total sectional area of the pulmonary capillaries is less than that of all the systemic capillaries.

\section{Passage of the Blood-Corpuscles out of the Vessels-Diapedesis.}

Diapedesis.-If the circulation be studied in the vessels of the mesentery, we may observe colourless corpuscles passing out of the vessels in greater or less numbers (Fig. S5). The mere contact with the air suffices to excite slight inflammation. At first, the colourless corpuscles in the plasma-space move more slowly; several accumulate near each other, and adhere to the walls-soon they bore into the wall, ultimately they pass quite through it, and may wander for a distance into the peri-vascular tissues. It is doubtful whether they pass through the so-called "stomata" which exist between the endothelial cells, or whether they simply pass through the cement-substance between the endothelial cells (p. 122). This process is called Diapedesis, and consists of several acts :- $(\alpha$.$) The adhesion of lymph-cells or$ colourless corpuscles to the inner surface of the vessel (after moving more slowly along the wall up to this point). (b.) They send processes into and through the vascular wall. (c.) The body of the cell is drawn after or follows the process, whereby the corpuscle appears constricted in the centre (Fig. 85, c). (d.) The com. plete passage of the corpuscle through the wall, and its farther motion in virtue of its own amoboid movements. Hering observed that in large vessels with perivascular lymph spaces, the corpuscles passed into these latter, hence cells are found in lymph before it has passed through lymphatic glands. The cause of the diapedesis is partly due to the independent locomotion of the corpuscles, and it is partly a physical act, viz., a filtration of the colloid mass of the cell under the force of the blood-pressure (Hering)-in the latter respect depending upon the intra-vascular pressure and the velocity of the blood-stream. Hering regards this process, and even the passage of the coloured corpuscles through the vascular wall as a normal process. The RED corpuscles pass out of the vessels when the venous outflow is obstructed, which also causes the transudation of plasma through the vascular wall. The plasma carries the coloured corpuscles along with it, and at the moment of their passage through the wall they assume extraordinary shapes, owing to the tension put upon them, regaining their shape as soon as they pass out (Colnnheim). 
This remarkable phenomenon was described by Waller in 1846. Cohnheim has

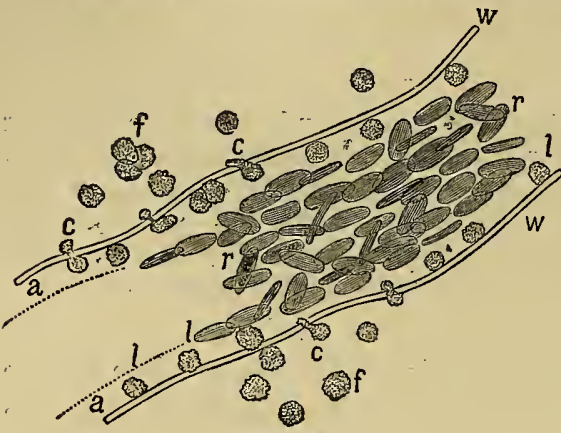

Fig. 85.

Small vessel of themesentery of a frog, showing the diapedesis of the colourless corpuscles $-w, w$, vascular walls ; $a, a$, Poiseuille's space; $r, r$, red corpuscles ; $l, l$, colourless corpuscles adhering to the wall, and $c, c$, in various stages of extrusion ; $f, f$, extruded corpuscles. recently re-described it, and according to him the out-wandering is a sign of inflammation, and the colourless corpuscles which accumulate in the tissues are to be regarded as true pus corpuscles, which may undergo further increase by division.

Stasis. - When as strong stim ulus acts on a vascular part, hyperæmic redness and swelling occur. Microscopic observation shows, that the capillaries and the small vessels are dilated and overfilled with blood-corpuscles; in some cases a temporary narrowing precedes the dilatation; simultaneously the velocity of the stream changes: rarely there is a temporary acceleration, mose frequently it becomes slower. If the action of the stimulus or irritant be continued, the retardation becomes considerable, the stream moves in jerks, then follows a to and fro movement of the blood-column-a sign that stagnation has taken place in other vascular areas. At last, the blood-stream comes completely to a standstill-STASIS-and the blood-vessels are plugged with blood-corpuscles. Numerous colourless blood-corpuscles are found in the stationary blood. Whilst these various processes are taking place, the colourless corpuscles-more rarely the red-pass out of the vessels. Under favourable circumstances the stasis may disappear. The swelling which occurs in the neighbourhood of inflamed parts is chiefly due to the exudation of plasma into the surrounding tissues. [The vapour of chloroform causes hyperæmia of the web (Lister).]

\section{Movement of the Blood in the Veins.}

As already mentioned, in the smallest veins coming from the capillaries, the blood-stream is more rapid than in the capillaries themselves, but less so than in the corresponding arteries. The stream is uniform, and if no other conditions interfered with it, the venousstream towards the heart ought to be uniform, but many circumstances affect the stream in different parts of its course. Amongst these are:(1) The relative laxness, great distensibility, and the ready compressibility of the walls, even of the thickest veins. (2) The incomplete filling 'of the veins, which does not amount to any considerable distension of their walls. (3) The numerous and free anastomoses between adjoining veins, not only between veins lying in the same plane, but also between superficial and deep veins. Hence, if the course of the blood be obstructed in one direction, it readily finds another outlet. 
(4) The presence of numerous valves which permit the blood-stream to move only in a centripetal direction (Fabricius ab Aquapendente). They are absent from the smallest veins, and are most numerous in those of middle size.

Law of the Position of Valves.-The venous valves always have two pouches, and are placed at definite intervals, which correspond to the $1,2,3$, or $\mathrm{n}^{\text {th }}$ power of a certain "fundamental distance," which is $=7 \mathrm{~mm}$. for the lower extremity and $5.5 \mathrm{~mm}$. for the upper. Many of the original valves disappear. On the proximal side of every valve a lateral branch opens into the vein, while on the distal side of each branch lies a valve. The same is true for the lymphatics (K. Bardeleben).

Effect of Pressure.-As soon as pressure is applied to the veins, the next lowest valves close, and those immediately above the seat of pressure open and allow the blood to move freely toward the heart. The pressure may be exerted from without, as by anything placed against the body; the thickened contracted muscles, especially the muscles of the limbs, compress the veins. That the blood flows out of a divided vein more rapidly when the muscles contract, is shown during venesection. If the muscles are kept contracted, the venous blood passing out of the muscles collects in the passive parts-e.g., in the cutaneous veins. The pulsatile pressure of the arteries accompanying the veins favours the venous current (Ozanam).

From a hydrostatic point of view, the valves are of considerable importance, as they serve to divide the column of blood into segments (e.g., in the crural vein in the erect attitude), so that the fine bloodvessels in the foot are not subjected to the whole amount of the hydrostatic pressure in the veins.

The velocity of the venous blood has been measured directly (with the hæmadromometer and the stromuhr-\$ \$9). Volkmann found it to be $225 \mathrm{~mm}$. per sec. in the jungular vein. Reil observed that $2 \frac{1}{2}$ times more blood flowed from an arterial orifice than from a veuous orifice of the same size. The velocity of the venous current obviously depends upon the sectional area of the vessel. Borelli estimated the capacity of the venous system to be 4 times greater than that of the arterial ; while, according to Haller, the ratio is 9 to 4 .

As we proceed from the small veins towards the venæ cavæ, the sectional area of the veins, taken as a whole, becomes less, so that the velocity of the current increases in the same ratio. The velocity of the current in the venæ cavæ may be about half of that in the aorta (Haller).

As the pulmonary veins are narrower than the pulmonary artery, the blood moves more rapidly in the former. The velocity of the blood-current in the veins is accelerated during inspiration-compare $\S 88$ (De Jager).

[Active pulsation occurs in the veins of the wing of the bat (Schiff).] 


\section{Sounds or Bruits within Arteries.}

These murmurs, sounds, or bruits occur either spontaneously, or are produced by the application of external pressure, whereby the lumen of the vessel is diminished. In four-fifths of all healthy men two sounds-corresponding in duration and other characters to the two heart-sounds-are heard in the carotid (Conrad, Weil). Sometimes only the second heart-sound is distinguishable, as its place of origin is near to the carotid. They are not true arterial sounds, but are simply "propagated heart-sounds."

Arterial Sounds or murmurs are readily produced by pressing upon a strong artery-e.g., the crural in the inguinal region, so as to leave only a narrow passage for the blood ("Stenosal murmur"). A fine blood-stream passes with great rapidity and force through this narrow part, into a wider portion of the artery lying behind the point of compression. Thus arises the "pressure-stream" (P. Niemeyer), or the "fluid vein" ("Veine fluide" of Chauveau.) The particles of the fluid are thrown into rapid oscillation, and undergo vibratory movements, and by their movement produce the sound within the peripheral dilated portion of the tube. A sound is produced in the fluid by pressure (Corrigan, Heynsius). The sounds are not caused by vibrations of the vascular wall, as supposed by Bouillaud.

A murmur of this sort is the "sub-clavicular murmur" (Röser), occasionally heard during systole in the subclavian artery; it occurs when the two layers of the pleura adhere to the apex of the lung (especially in tubercular diseases of the lungs), whereby the subclavian artery undergoes a local constriction due to its being made tense and slightly curved (Friedreich). This result is indicated in a diminution or absence of the pulse-wave in the radial artery (Weil).

Arterial murmurs are favoured by-(1) Sufficient delicacy and elasticity of the arterial walls (Th. Weber). (2) Diminished peripheral resistance-e.g., an casy outflow of the fluid at the end of the stream (Kiwisch). (3) Accelerated current in the vascular system generally. (4) A considerable difference of the pressure in the narrow and wide portions of the tube (Marey). (5) Large calibre of the arteries.

It is obvious that arterial murmurs will occur in the human body:-(a.) When, owing to pathological conditions, the arterial tube is dilated at one part, into which the blood-current is forcibly poured from the normal narrow tube. Dilatations of this sort are called aneurisms, within which murmurs are generally audible. (b.) When pressure is exerted upon an artery-e.g., by the pressure of the greatly enlarged arteries during pregnancy, or by a large tumour pressing upon a large artery. (c.) A murmur corresponding to each pulse-beat is heard., especially where two or more large arteries lie together; hence, during pregnancy, we hear the uterine murmur, or placental bruit, or souffle in the greatly dilated uterine arteries. It is much less distinct in the umbilical arteries of the cord (umbilical murmurs). Similar sounds are heard throngh the thin walls of the head of infants (Fisher, 1833). A unurmur due to the systole of the heart is often heard in the carotid (Jurasz). In such 
cases where no source of external pressure is discoverable, and when no aneurism is present, the spontaneously occurring sounds are favoured, when at the moment of arterial rest (cardiac systole) the arterial walls are distended to the slightest extent, and when dnring the movement of the pulse (cardiac diastole) the tension is most rapid (Traube, Weil)-i.e., when the low systolic minimum tension of the arterial wall passes rapidly into the high maximum tension. This is especially the case in insufficiency of the aortic valves, in which case the sounds in the arteries are audible over a wide area. If the minimum tension of the arterial wall is relatively great, even during diastole, the sounds in the arteries are greatly diminished.

In insufficiency of the aortic valves, characteristic sounds may be heard in the crural artery. If pressure be exerted upon the artery, a double blowing murmur is heard; the first one is due to a large mass of blood being propelled into the artery synchronously with the heart-beat, the second to the fact that a large quantity of blood flows back into the heart during diastole (Duroziez, 1S61). If no pressure be exercised two sounds are heard, and these seem to be due to a wave propagated into the arteries by the auricles and ventricles respectively (Landois)-compare $\S 73$, Fig. 62 , III. In atheroma a double sound may sometimes be heard $(\$ 73,2)$.

\section{Venous Murmurs.}

I. Bruit de Diable.-This sound is heard above the clavicles in the furrow between the two heads of the sterno-mastoid, most frequently on the right side, and in 40 per cent. of all persons examined. It is either a continuous or a rhythmical murmur, occurring during the diastole of the heart or during inspiration; it has a whistling or rushing character, or even a musical quality, and arises within the bulb of the common jugular vein. When this sound is heard without pressure being exerted by the stethoseope, it is a pathological phenomenon. If, however, pressure be exerted, and if, at the same time, the person examined turns his head to the opposite side a similar sound is heard in nearly all cases (Weil). The pathological bruit de diable occurs especially in anæmic persons, in lead-poisoning, syphilitic and scrofulous persons, sometimes in young persons, and less frequently in elderly people. Sometimes a thrill of the vascular wall may be felt.

Causes.-It is due to the vibration of the blood flowing in from the relatively narrow part of the common jugular vein into the wide bulbous portion of the vessel, and seems to occur chiefly when the walls of a thin part of the rein lie close to each other, so that the current must purl through it. It is clear that pressure from without, or lateral pressure, as by turning the head to the opposite side, must favour its occurrence. Its intensity will be increased when the velocity of the stream is increased, hence inspiration and the diastolic action of the heart (both of which assist the venous current) increase it. The erect attitude acts in a similar manner. A similar bruit is sometimes, though rarely, heard in the subclavian, axillary, thyroid (scrofula), facial, innominate and crural veins and superior cava. 
II. Regurgitant Murmurs.-On making a sudden effort, a murmur may be heard in the crural vein during expiration, which is caused by a centrifugal current of blood, owing to the incompetence or absence of the valves in this region. If the valves at the jugular bulb are not tight, there may be a bruit with expiration (expiratory jugular vein bruit-Hamernjk), or during the cardiac systole (systolic jugular vein bruit-v. Bamberger).

III. Valvular Sounds in Veins.-When the tricuspid valve is incompetent, during the ventricular systole, a large volume of blood is propelled backwards into the venæ cavæ. The venous valves are closed suddenly thereby and a sound produced. This occurs at the bulb or dilatation on the jugular vein (v. Bamberger), and in the crural vein at the groin (N. Friedreich), i.e., only as long as the valves are competent. Forced expiration may cause a valvular sound in the crural vein. No sound is heard in the veins under perfectly normal circumstances.

\section{The Venous Pulse-Phlebogram.}

Methods.-A tracing of the movements of a vein, taken with a lightly weighted sphygmograph, has a characteristic form and is called a phlebogram (Fig. 86). In order to interpret the various events of the phlebogram it is most important to record simultaneously the events that take place in the heart. The auricular contraction (compare Fig. 29, p. 88), is synchronous with $a b$; be, with the ventricular systole, during which time the first sound occurs, whilst $a, b$ is a presystolic movement. The carotid pulse coincides nearly with the apex of the cardiogram, i.e., almost simultaneously with the descending limb of the phlebogram (Riegel).

Occasionally in healthy individuals a pulsatile movement, synchronous with the action of the heart, may be observed in the common jugular vein. It is either confined to the lower part of the vein, the socalled bulb, or extends farther up along the trunk of the vein. In the latter case, the valves above the bulb are insufficient, which is by no means rare, even in health. The wave-motion passes from below upwards, and is most obvious when the person is in the passive horizontal position, and it is more frequent on the right side, becanse the right vein lies nearer the heart than the left.

The venous pulse resembles very closely the tracing of the cardiac impulse (Landois). Compare Fig. 86, 1, with Fig. 25a, A, p. 82 .

It is obvious that, as the jugular vein is in direct communication with the right auricle, and as the pressure within it is low, the systole of the right auricle must cause a positive wave to be propagated towards the peripheral end of the jugular vein. Fig. 86, 9 and 10, are venous pulses of a healthy person with insufficiency of the valves of the jugular vein. In these curves, the part $a, b$, corresponds to the contraction of the auricle. Occasionally this part consists of two elevations, corresponding to the contraction of the atrium and auricle respectively. As the blood in the right auricle receives an impulse from the sudden tension of the tricuspid valve, isochronous with the systole of the right ventricle, there is a positive wave in the jugular vein in Fig. 86, 9 and 
10, indicated by $b, c$. Lastly, the sudden closure of the pulmonary valves may even be indicated (e). As the aorta lies in direct relation with the pulmonary artery, the sudden closure of its valves may also be indicated (Fig. 86, 9, at $d$ ). During the diastole of the auricle and ventricle, blood flows into the heart, so that the vein partly collapses and the lever of the recording instrument descends (Riegel, FrançoisFranck).

The blood in the sinuses of the brain also undergoes a pulsatile movement, owing to the fact that during cardiac diastole much blood flows into the veins (Mosso). Under favourable circumstances, this movement may be propagated into the veins of the retina, coustituting the venous retinal pulse of the older observers (Helfrich).

Jugular Vein Pulse.-The venous pulse in the jugular vein is far better marked in insufficiency of the tricuspid valve, and the vein may pulsate violently, but if its valves be perfect the pulse is not propagated along the vein, so that $a$ pulse in the jugular vein is not necessarily a sign of insufficiency of the tricuspid valve, but only of insufficiency of the valve of the jugular vein (Friedreich).

Liver Pulse.-The ventricular systole is propagated into the valve-less
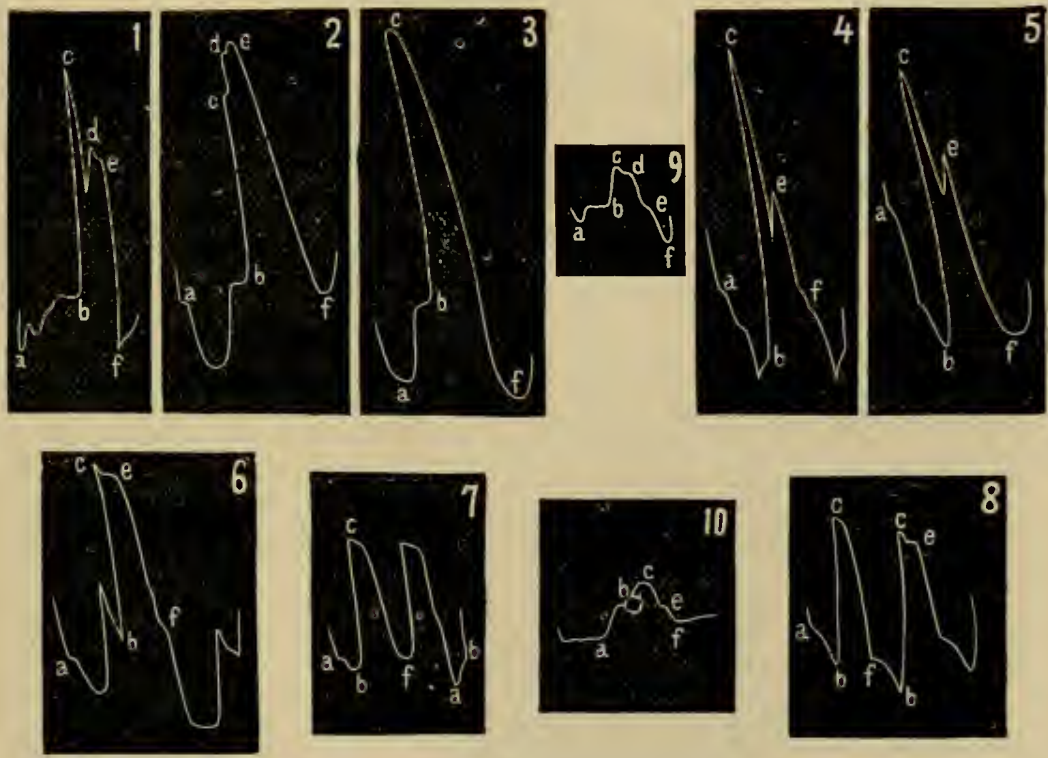

Fig. S6.

Various forms of venous pulses, chiefly after Friedreich-1-8 from insufficiency of the tricuspid; 9 and 10 , pulse of the jugular vein of a healthy person. In all the curves, $a, b=$ contraction of the right auricle; $b, c$, of the right ventricle ; $d$, closure of the aortic valves; $e$, closure of the pulmonary valves; $e, f$, diastole of the right ventricle.

inferior vena cava, and causes the liver pulse. With each systole blood passes into the hepatic veins, so that the liver undergoes a systolic swelling and injection. 
Fig. 86, 2-8, are curves of the pulse in the common jugular vein (after Friedreich). Although at first sight the curves appear to be very different, they all agree in this, that the various events occurring in the heart during a cardiac revolution are indicated more or less completely. In all the curves, $a, b=$ auricular contraction. The auricle, when it contracts, excites a positive wave in the veins (Gendrin, 1843, Marey, Friedreich). The elevation, $b, c$, is caused by the large blood-wave produced in the veins, owing to the emptying of the ventricle. It is always greater, of course, in insufficiency of the tricuspid valves than under normal circumstances (Fig. 86, 9 and 10). In the latter case, the closure of the tricuspid valve causes only a slight wave-motion in the auricle. The apex, $c$, of this wave may be higher or lower, according to the tension in the vein and the pressure exerted by the sphygmograph. As a general rule, at least one notch $(4,5,6, e)$ follows the apex, due to the prompt closure of the valves of the pulmonary artery. The closure of the closely adjacent aortic valves may cause a small secondary wave near to $e$ (as in 1 and $2, d)$. The curve falls towards $f$, corresponding to the diastole of the heart.

A well-marked venous pulse occurs when the right auricle is greatly congested, as in cases of insufficiency of the mitral valve or stenosis of the same orifice. In rare cases, in addition to the pulse in the common jugular vein, the external jugular, the facial, thyroid, external thoracic veins, or even the veins of the upper and lower extremities may pulsate.

A similar pulsation must occur in the pulmonary veins in mitral insufficiency, but of course the result is not visible.

On rare occasions, a pulse occurs in the veins on the back of the hand and foot, owing to the arterial pulse being propagated througl the capillaries into the veins. This may occur under normal circumstances, when the peripheral ends of the arteries become dilated and relaxed (Quincke), or when the blood-pressure within these vessels rises rapidly and falls as suddenly, as in insufficiency of the aortic valves.

In progressive effusion into the pericardium, at first the carotid pulse becomes smaller and the venous pulse larger; beyond a certain pressure, the latter ceases (Riegel).

\section{Distribution of the Blood.}

Methods. - The methods adopted do not give exact results. J. Ranke ligatured the parts during life, removed them, and investigated the amount of blood while the tissues were still warm.

In a rabbit, one-fourth of the total amount of the blood is found in each of the following: $-a$, in the passive muscles; $b$, in the liver; $c$, in the organs of the circulation (heart and great vessels); $d$, in all other parts together (J. Ranke).

The amount of blood is influenced by-(1) The anatomical distribution (vascularity or the reverse) of the vessels as a whole; (2) the diameter of the vessels, which depends upon physiological causes- $(a)$ on the blood-pressure within the vessels; (b) on the condition of the vaso-motor or vaso-dilatator nerves; (c) on the condition of the tissues themselves, e.g., the vessels of the intestine during absorption; by the vessels of muscle during muscular contraction; of vessels in inflamed parts. 
Activity of an Organ.-The most important factor, however, is the state of activity of the organ itself; hence, the saying, "ubi irritatio, ibi affluxus." We may instance the congestion of the salivary glands and the gastric mucous membrane during digestion, and the increased vascularity of muscles during contraction. As the activity of organs varies at different times, the amount of blood in the part or organ goes hand-in-hand with the variations in its states of activity (J. Ranke). When some organs are congested others are at rest; during digestion, there is muscular relaxation and less mental activity : violent muscular exertion retards digestion-during great congestion of the cutaneous vessels the activity of the kidneys diminishes. Many organs (heart, muscles of respiration, certain nerve-centres) seem always to be in a uniform state of activity and vascularity.

During the activity of an organ, the amount of blood in it may be increased 30 per cent., nay even 47 per cent. The motor organs of young muscular persons are relatively more vascular than those of old and feeble persons ( $J$. Ranke).

During a condition of mental activity, the carotid is dilated, the dicrotic wave in the carotid curve is increased (the radial shows the opposite condition), and the pulse is increased in frequency (Gley).

In the condition of increased activity, a more rapid renevval of the blood seems to occur; after muscular exertion the duration of the circulation diminishes (Vierordt).

Age.-The development of the heart and large vessels determines a different distribution of the blood in the child from that which obtains in the adult. The heart is relatively small from infancy up to puberty, the vessels are relativcly large; while after puberty the heart is large, and the vessels are relatively smaller. Hence, it follows that the blood-pressure in the arteries of the systemic circulation must be lower in the child than in the adult. The pulmonary artery is relatively wide in the child, while the aorta is relatively small ; after puberty both vessels have nearly the same size. Hence, it follows that the blood-pressure in the pulmonary vessels of the child is relatively higher than that in the adult (Beneke).

\section{Plethysmography.}

Plethysmograph.--In order to estimate and register the amount of blood in a limb Mosso devised an instrument (Fig. 87), which he termed a Plethysmograph. It is constructed on the same principle as the less perfect apparatus of Chelius and Fick.

It consists of a long cylindrical glass-vessel, $G$, suited to accommodate a limb. The opening through which the limb is introduced is closed with caoutchouc, and the vessel is filled with water. There is an opening in the side of the vessel in which a manometer tube, filled to a certain height with water, is fixed. As the arm is enlarged with the increased supply of arterial blood passing into it at each 
pulse-beat, of course the water column in the manometer is raised. Fick placed a foat upon the surface of the water, and thus enabled the variations in the volume of the fluid to be inscribed on a revolving cylinder. The curve obtained resem.

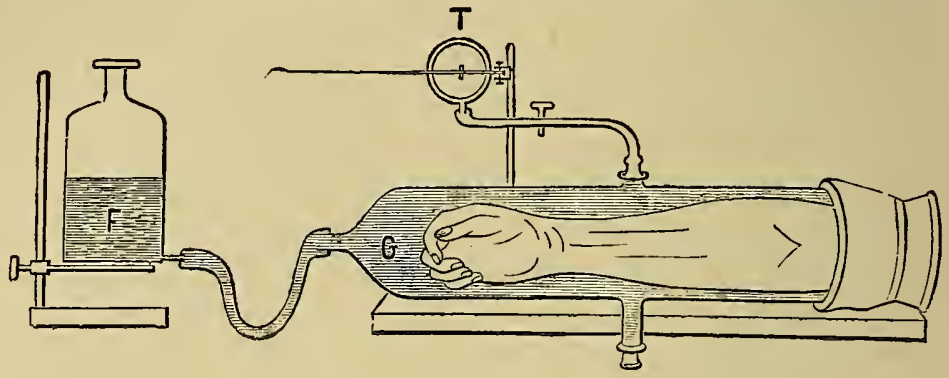

Fig. 87.

Mosso's Plethysmograph-G, glass-vessel for holding a limb; F, flask for varying the water-pressure in $\mathrm{G}$; $\mathrm{T}$, recording apparatus.

bled the pulse-curve; it was even dicrotic. In Fig. 87 the movement of the fluid is represented as conveyed to a Marey's tambour, $T$, similar to the recording apparatus employed in Brondgeest's Pansphygmograph (p. 87).

From the curve obtained we learn that-(1.) The pulsatile variations in the volume are similar to the pulse-curve. As the venous current is regarded as uniform in the passive limb, every increase of the volumecurve indicates a greater velocity of the arterial current towards the periphery, and vice versî (Fick). (2.) The respirctory undulations correspond to similar variations in the blood-pressure tracing $(\S 85, f)$. Vigorous respiration and cessation of the respiration cause a diminution of the volume. The limb swells during straining (v. Basch) and coughing, and diminishes during sighing. (3.) Certain periodic undulations occur, due to the regular periodic contractions of the small arteries. (4.) Other undulations, due to various accidental causes, affect the blood-pressure: changes of the position of a limb acting hydrostatically, and dilatation or contraction of the vessels in other vascular regions. (5.) Movement of the muscles of the limb under observation causes diminution of volume (experiment of Fr. Glisson, 1677); as the venous current is accelerated, the musculature is also very slightly diminished in volume, even when the intra-muscular vessels are dilated. (6.) Mental exercise causes a diminution in the volume of the limb, and so does sleep (Mosso). Music influences the blood-pressure in dogs, the pressure rising or falling under different conditions. The stimulation of the auditory nerve is transmitted to the medulla oblongata, where it acts so as to cause acceleration of the action of the heart (Dogiel). Compression of the afferent artery causes a decrease, and compression of the vein an increase in the volume of the limb (Mosso). 


\section{Transfusion of Blood.}

Transfusion is the introduction of blood from one animal into the vascular system of another animal.

Historical.-The first indication of direct transfusion from blood-vessel to blood-vessel dates from the time of Cardanus in 1556. After the discovery of the circulation in England, J. Potter (1638) evolved the idea of transfusion of blood. Numerous experiments were made on animals. New blood was transfused in order to restore life in animals that had been bled. Boyle and Lower conducted these and other experiments. The blood of the same species, as well as the blood of other species, was employed. The first case of transfusion on man was performed by Jean Denis in Paris (1667), lamb's blood being used. At the present time, when transfusion is practised on man, only human blood is used.

(a.) The RED CORPUSCLES are the most important elements in connection with the restorative powers of the blood. They seem to preserve their functions even in blood which has been defibrinated outside the body (Prevost and Dumas, 1821). The effect of various reagents upon them has already been noticed $(\S 4, \mathrm{~A})$.

(b.) With regard to the GASES of the blood-corpuscles, oxygenated (arterial) blood never acts injuriously; but venous blood overcharged with carbonic acid ought only to be transfused when the respiration is sufficient to oxygenate the blood as it passes through the pulmonary capillaries, whereby venous is transformed into arterial blood. If the respiratory movements have ceased, or are inperfectly performed, the blood becomes rapidly richer in carbonic acid and in this condition reaches the heart; thence it is propelled into the blood-vessels of the medulla oblongata, where it acts as a powerful stimulus of the respiratory centre, causing dyspnoea, convulsions, and death.

(c.) The FIBRIN, or the substances from which it is formed ( $\$ 29$ ), do not seem to play any part in connection with the restorative powers of the blood; hence, defibrinated blood performs all the functions of non-defibrinated blood within the body (Panum, Landois).

(d.) The investigations of Worm Mïller showed that an excess of 83 per cent. of blood might be transfused into the vascular system of an animal without producing any injurious effects. Hence it follows that the vascular system has the power of accommodating large quantities of blood within it. That the vascular system can accommodate itself to a diminished amount of blood has been known for a long time.

When Employed.-The transfusion of blood is used-(1.) in acute ancemia (§ 41, I), e.g., after copious hæmorrhage. New blood from the same species of animal is introduced directly into the vessels, to supply the place of the blood lost by the hæmorrhage. 
(2.) In cases of poisoning, where the blood has been rendered useless by being mixed with a poisonous substance, and hence is unable to support life. In such cases, remove a considerable quantity of the blood, and replace it by fresh blood. Carbonic oxide is a poison of this kind (Kühne), and its effects on the body have already been described (compare p. 32). The indication is also obtained for a similar practice in poisoning with ether, chloral, chloroform, opium, morphia, strychnine, cobra poison.

(3.) Under certain pathological conditions, the blood may become so altered in quality as to be unable to support life. The morphological elements of the blood may be altered, and so may the relative proportion of its other constituents. Amongst these conditions, may be cited the pathological condition of uræmia, due, it may be, to the accumulation of urea or the products of its decomposition within the blood [or to the retention of the potash and other urinary salts-Feltz and Ritter]; accumulation of the biliary constituents in the blood (Cholæmia), and great increase of the carbonic acid. All these three conditions, when very pronounced, may cause death. In these cases part of the impure blood may be replaced by normal human blood (Landois).

Amongst conditions where the morphological constituents of the blood are altered qualitatively or quantitatively are: hydræmia (excessive amount of water in the blood $\$ 41,1$ ); oligocythæmia (abnormal diminution of red blood-corpuscles). When these conditions are highly developed, more especially in pernicious anæmia $(\S 10,2)$, healthy blood may be substituted. Transfusion is not suited for persons suffering from leukæmia (compare p. 23).

After-Effects.-A quarter or half an hour after normal blood has been injected into the blood-vessels of a man, there is a greater or less febrile reaction, according to the amount of blood transfused (compare Fever).

Operation.-The operative procedure to be adopted in the process of transfusion varies according as defibrinated or non-defibrinated blood is used. In order to defibrinate blood, some blood is withdrawn from a vein of a healthy man in the ordinary way, it is collected in an open vessel and whipped or beaten with a glass rod until all the fibrin is completely removed from it. It is then filtered through an atlas filter, heated to the temperature of the body (by placing it in warm water) and injected by means of a syringe into an artery opened for the purpose. A vein (e.g., basilic or great saphenous) may be selected for the transfusion, in which case the blood is driven in, in the direction of the heart; if an artery is selected (radial or posterior tibial) the blood is injected towards the periphery (Hüter), or towards the heart (Landois, Unger, Schäfer).

Dangers. - It is most important not to permit the entrance of air into the circulation, for if it be introduced in sufficient quantity, it may cause death. When air enters the circulation it reaches the right side of the heart where, owing to the movement of the blood, it forms air-bubbles and makes a froth. The air-bubbles are pumped into the branches of the pulmonary artery, in which they become impacted, arrest the pulmonary circulation, and rapidly cause death. 
If non-defibrinated human blood is used, the blood may be passed directly from the arm of the giver to the arm of the receiver by means of a flexible tube. The tube used must be filled with normal saline solution to prevent the entrance of air.

Peritoneal Transfusion.-Recently, the injection of defibrinated blood into the peritoneal cavity has been recommended. The blood so injected is absorbed (Ponfick). Even after twenty minutes the number of blood-corpuscles in the blood of the recipient (rabbit) is increased, and the number is greatest on the first or second day (Bizzozero and Golgi). The operation, however, may cause death, and one fatal case, owing to peritonitis, is recorded (Mosler). It is evident that this method of transfusion is not applicable in cases where blood must be introduced into the circulation as rapidly as possible $(e . q .$, after severe hæmorrhage or in certain cases of poisoning). [Blood has been injected into the subcutaneous cellular tissue of the abdomen in cases of great debility.]

Heterogeneous Blood.-The blood of animals ought never to be transfused into the blood-vessels of man. Some surgeons have transfused blood directly from the carotid of a lamb into the human subject. It is to be remembered, however, that the blood-corpuscles of the sheep are rapidly dissolved by human blood, so that the active constituents of the blood are rendered useless (Landois). As a general rule, the blood-serum of many mammals dissolves the blood-corpuscles of other mammals $(\$ 5,5)$.

Solution of the Blood-Corpascles.-The serum of dug's J)lood is a powerful solvent, while that of the blood of the horse and rabbit dissolves corpuscles relatively slowly. The blood-corpuscles of mammals vary very greatly with reference to their power to resist the solvent action of the serum of other animals. The red blood-corpuscles of rabbit's blood are rapidly dissolved by the blood-serum of other animals, whilst those of the cat and dog resist the solvent action much longer. Solution of the corpuscles occurs in defibrinated as well as in ordinary blood. When the blood of a rabbit or lamb is injected into the blood-vessels of a dog they are dissolved in a few minutes. If blood be withdrawn by pricking the skin with a needle, the partially dissolved corpuscles may be detected.

Liberation of Hæmoglobin and Hæmoglobinuria.-As a consequence of the solution of the coloured corpuscles, the blood-plasma is reddened by the liberated hæmoglobin. Part of the dissolved material may be used up in the body of the recipient, some of it for the formation of bile, but if the solution of the corpuscles has been extensive, the hæmoglobin is excreted in the urine (hæmoglobinuria) in less amount in the intestine, the bronchi, and serous cavities (Panum). Bloody urine has been observed in man after the injection of 100 grammes of lamb's blood. Even some of the recipient's own corpuscles may be dissolved, as in the case where the recipient's blood-corpuscles are dissolved by the serum of the transfused blood-e.g., transfusing dog's blood into man. In the rabbit, whose corpuscles are readily dissolved, the transfusion of the blood-serum of the dog, man, pig, sheep, or cat produces serious symptoms, and even death. The dog, whose corpuscles are more resistant, bears transfusion of other kinds of blood well.

Dangers.-When foreign or heteroyeneous blood (i.e., blood from a different species) is transfused, two phenomena, which may be dangerous to life, occur:-

(1.) Before the corpuscles are dissolved they usually run together and form sticky masses consisting of 10 or 12 corpuscles, which are apt to occlude capillaries. After a time they give up their hæmoglobin, leaving the stroma, which yields a sticky fibrin-like mass that may occlude fine vessels (p. 48).

(2.) The presence of a large quantity of dissolved hæmoglobin may cause extensive coagulation within the blood-vessels. The injection of dissolved hæmoglobin causes extensive coagulations (Naunyn and Francken).

The coagulation occurs usually in the venous system and in the large vessels, and may cause death either suddenly or after a considerable time. 
Dissolved hrmoglobin seems greatly to increase the activity of the fibrin-ferment (s 30), perhaps by accelerating the decomposition of the colourless corpuscles. Hæmoglobin exposed to the air gradually loses this property; and the fibrinferment, when in contact with hæmoglobin, is either destroyed or rendered less active (Sachssendahl).

Vascular Symptoms.-As a result of the above-named causes of occlusion of the vessels, there are often signs of the circulation being impeded in various organs, In man, after transfusion of lamb's blood, the skin is bluish-red, in consequence of the stagnation of blood in the cutaneous vessels. Difficulty of breathing occurs from obstruction in the capillaries of the lung ; while there may be rupture of small bronchial vessels, causing sanguineous expectoration. The dyspnœa may increase, especially when the circulation through the medulla oblongata-the seat of the respiratory centre-is interfered with. In the digestive tract, for the same reason, increased peristalsis, evacuation of the contents of the rectum, vomiting, and abdominal pain may occur. These phenomena are explained by the fact that disturbances of the circulation in the intestinal vessels cause increased peristaltic movements. Degeneration of the parenchyma of the kidney occurs as a result of the occlusion of some of the renal vessels. The uriniferous tubules become plugged with cylinders of coagulated albumin (Ponfick). Owing to the occlusion of numerous small muscular branches the muscles may become stiff, or coagulation of their myosin may occur. Other symptoms, referable to the nervous system, the sense-organs and heart, are all due to the interference with the circulation through them. An important symptom is the occurrence of a considerable amount of fever half an hour or so after the transfusion of heterogeneous blood. When many vessels are occluded, rupture of some small blood-vessels may take place. This explains. the occurrence of slight yet persistent hæmorrhages, which occur on the free surfaces of the mucous and serous membranes, and in the parenchyma of organs, as well as in wounds. The blood coagulates with difficulty, and imperfectly.

Transfusion of other Fluids.-Other substances have been transfused. Normal Saline Solution $(0.6$ p.c. $\mathrm{NaCl})$ aids the circulation in a purely mechanical way (Goltz), and it even excites the circulation (Kronecker, Sander, Ott). In severe anæmia this fluid cannot maintain life (Eulenburg and Landois). The injection of PEPToNe, even in moderate amount, is dangerous to life, as it causes paralysis of the vessels. The injection of MiLK is accompanied with danger; fever occurs after the injection, and the milk globules cause the occlusion of many vessels, producing subsequent degenerations. Fat may appear in the urine, and there may be fatty infiltration of the urinary tubules. The urine contains sugar and albumin, the liver cells often contain fatty granules, and the weight of the body diminishes. If too large a quantity of milk be transfused, death occurs. When unboiled milk is injected, numerous bacteria are developed in the blood (Schäfer). 


\section{The Blood-Glands.}

\section{The Spleen.}

Structure.-The spleen is covered by the peritoneum, except at the hilus. Under this skrous covering there is a tough thick elastic fibrous CAPSULE, which closely invests the organ and gives a covering to the vessels which enter or leave it at the hilus, so that fibrous tissue is carried into the organ along the course of the vessels. [The capsule cannot be separated without tearing the splenic pulp.] Numerous TRABECULE pass into the spleen from the deep surface of the capsule. These trabeculæ branch and anastomose so as to produce a net-work or sustentacular tissue, which is continuous with the connective tissue, prolonged inwards and surrounding the blood-vessels (Fig. 88). Thus, the connective tissue in the spleen, as in other viscera, is continuous

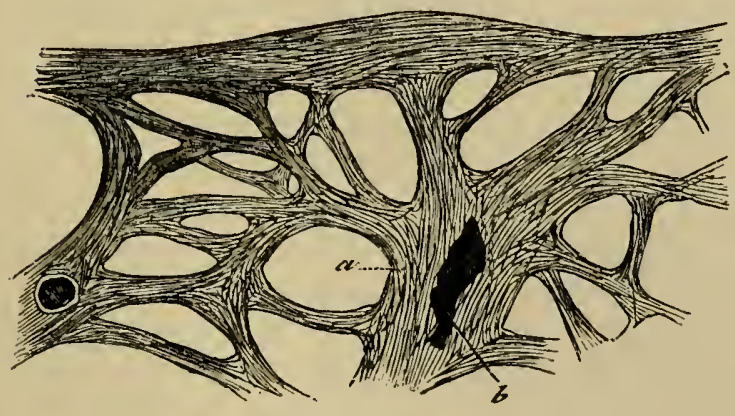

Fig. 88 .

Trabecula of the spleen of a cat with the splenic pulp washed out- $a$, trabecula; $b$, vein.

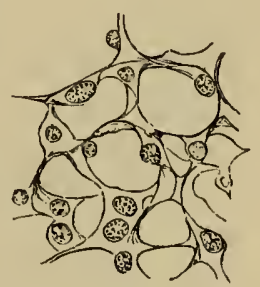

Fig. 89.

Spleen of a cat injected with gelatine, show ing the adenoid reticulum.

throughout the organ. In this way an irregular dense net-work is formed, comparable to the meshes of a bath-sponge. [This net-work is easily demonstrated by washing out the pulp lying in its meshes by means of a stream of water, when a beautiful soft semi-elastic net-work of rounded and flattened threads is obtained.]

The Capsule is composed of interlacing bundles of connective tissue 
mixed with numerous fine fibres of elastic tissue and some non-striped muscular fibres.

Reticulum.-Within the meshes of the trabecular framework there is dispersed a very delicate net-work (reticulum) of adenoid tissue (Billroth), which, with the other coloured elements that fill up the meshes, constitute the splenic pulp (Fig. 89). The reticulum is continuous with the fibres of the trabeculæ. [If a fine section of the spleen be "pencilled" in water, so as to remove the cellular elements, the preparation presents much the same characters as a section of a lymph-gland similarly treated, viz., a very fine net-work of adenoid tissue, continuous with, and surrounding the walls of, the blood-vessels. The spaces of this tissue (His) are filled with lymph and blood-corpuscles.]

The Pulp is a dark reddish-coloured semi-fluid material, which may be squeezed or washed out of the meshes in which it lies. It contains a large number of coloured blood-corpuscles, and becomes brighter when it is exposed to the action of the oxygen of the air.

Blood-Vessels and Malpighian Corpuscles.-The large splenic artery splits up into several branches before it enters the spleen, and it is accompanied in its course by the vein. Both vessels and their branches are enclosed in a fibrous sheath, which becomes continuous with the trabeculæ. The smaller branches of the artery gradually lose this fibrous investment, and each one ultimately divides into a group or pencil of arterioles (PENICILLI) which do not anastomose with each other. [Thus each branch is terminal-a condition which is of great importance in connection with the pathology of embolism or infarction of the vessels of the spleen.] At the points of division of the branches of the artery, or scattered along their course, are small oval or globular masses of adenoid tissue about the size of a small millet seed ( $\frac{1}{80}$ to $\frac{1}{20}$ inch in diameter) - the MLALPIGHIAN CORPUSCLES. [These bodies are visible to the naked eye as small, round, or oval white structures, about the size of millet seed, in a section of a fresh spleen. They are very numerous- $[7,000$ in man (Sappey)]-and are readily detected in the dark reddish pulp. We must be careful not to mistake sections of the trabeculæ for them. These corpuscles consist of adenoid tissue, whose meshes are loaded with lymph-corpuscles, and they present exactly the same structure as the solitary follicles of the intestine (compare Lymphatic Glands).

[They are just small lymphatic accumulations around the arteriesperiarterial masses of adenoid tissue similar to those masses that occur in a slightly different form in other organs, e.g., the lungs. In a section of the spleen the artery may pass through the centre of the mass or through one side of it, and in some cases the tissue is collected unequally on opposite sides of the vessel, so that it is 
lob-sided. They are not surrounded by any special envelope. In some animals the lymphatic tissue is continued for some distance along the small arteries, so that to some extent it resembles a perivascular sheath of adenoid tissue (WV. Müller, Schweigger-Seidel). In a well injected spleen, a few fine capillaries may be found within these corpuscles (Sanders). The capillaries distributed in the substance of the Malpighian corpuscle (Fig. 90) form a net-work, and ultimately pour their blood into the spaces in the pulp. According to Robin and Legros, these vessels are comparable to the vasa vasorum of other blood-vessels. According to Cadiat, the

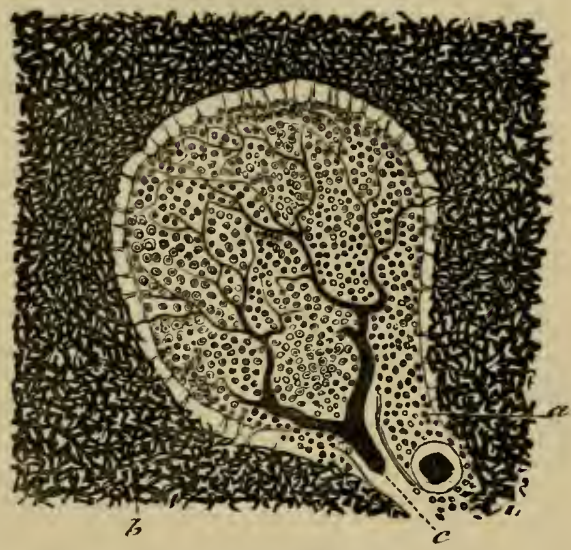

Fig. 90.

Malpighian corpuscle of the spleen of a cat injected $-a$, artery around which the corpuscle is placed; $b$, meshes of the pulp injected; $c$, the artery of the corpuscle ramifying in the lymphatic tissue composing it. The clear space around the corpuscle is the lymphatic sinus. corpuscles are separated from the splenic pulp by a lymplatic sinus, which is traversed by efferent capillaries passing to the pulp (Fig. 90).]

Connection of Arteries and Veins.-It is very difficult to determine what is the exact mode of termination of the arteries within the spleen, more especially as it is extremely difficult to inject the blood-vessels of the spleen. According to Stieda, W. Müller, Peremeschko, and Klein, the fine "capillary arteries" formed by the division of the small arteries do not open directly into the capillary veins, but the connection between the arteries and veins is by means of the "intermediary intercellular spaces" of the reticulum of the spleen, so that according to this view, there is no continuous channel lined throughout by epithelium connecting these vessels one with another. Thus the blood of the spleen flows into the spaces of the adenoid reticulum just as the lymplstream flows through the spaces in a lymph-gland. According to Billroth and Kölliker, a closed blood-channel actually does exist between the capillary arteries and the veins, consisting of dilated spaces (similar to those of erectile tissue). These intermediary spaces are said to be completely lined by spindle-shaped epithelium, which abuts externally on the reticulum of the pulp. [According to Frey, owing to the walls of the terminal vessels being incomplete, there being clefts or spaces between the cells composing them, the blood 
passes freely into spaces of the adenoid tissue of the pulp "in the same way as the water of a river finds its way amongst the pebbles of its bed," these "intermediary passages" being bounded directly by the cells and fibres of the net-work of the pulp. From these passages the venous radicles arise. At first, their walls are imperfect and cribriform, and they often present peculiar transverse markings due to the circular disposition of the elastic fibres of the reticulum. The small veins have at first a different course from the arteries. They anastomose freely, but they soon become ensheathed, and accompany the arteries in their course.]

Elements of the Pulp.-The morphological elements are very various-(1.) Lympl corpuscles of various sizes, sometimes partly swollen, and at other times with granular contents. (2.) Red bloodcorpuscles. (3.) Transition forms between 1 and 2 [although this is denied by some observers ( $\$ 7 \mathrm{C})]$. (4.) Cells containing red bloodcorpuscles and pigment granules. [These cells exhibit amoboid movements.] (Compare § 8.)

[The Lymphatics undoubtedly arise within the spleen. The lymphatics which leave the spleen are not numerous (Kölliker). There are two systems-a superficial, capsular, and trabecular system; and a peri-vascular set. The superficial lymphatics in the capsule are rather more numerous. Some of them seem to communicate with the lymphatics within the organ (Tomsa, Kölliker). In the horse's spleen, they communicate with the lymphatics in the trabeculæ, and with the peri-vascular lymphatics. The exact mode of origin of the peri-vascular system is unknown, but in part at least it begins in the spaces of the adenoid tissue of the Malpighian corpuscles and peri-vascular adenoid tissue, and runs along the arteries towards the hilus. There seem to be no afferent lymphatics in the spleen such as exist in a lymphatic gland.]

The Nerves of the spleen are composed for the most part of nonmedullated nerve-fibres, and run along with the artery. Their exact mode of termination is unknown, but they probably go to the bloodvessels and to the muscular tissue in the capsule and trabeculæ. [They are well seen in the spleen of the ox, and in their course very small ganglia placed wide apart, have been found by Remak and W. Stirling.]

Chemical Composition.-Several of the more highly oxidised stages of albuminous bodies exist in the spleen. Besides the ordinary constituents of the blood, there exist:-leucin, tyrosin, xanthin, hypoxanthin; also lactic, butyric acetic, formic, succinic, and uric acids, and perhaps glycero-phosphoric acid (Salkowski); Cholesterin, a glutin-like body, inosite, a pigment containing iron, and even free iron oxide (Nasse). The ash is rich in phosphoric acid and iron-poor in chlorine compounds. The splenic juice is alkaline in reaction; the specific gravity of the 
spleen $=1059-1066$. [The watcry extract of the spleen contains a proteid combined with iron.]

The Functions of the spleen are obscure, but we know some facts on which to form a theory. [The spleen differs from other organs in that no very apparent effect is produced by it, so that we must determine its uses in the economy from a consideration of such facts as the following-(1.) The effects of its removal or extirpation. (2.) The changes which the blood undergoes as it passes through it. (3.) Its chemical composition. (4.) The results of experiments upon it. (5.) The effects of diseases.]

(1.) Extirpation.-The spleen may be removed from an animal without the organism suffering any very obvious change (Galen). The human spleen has been successfully removed by Köberle, Péan, Zacaralla (1849), and others. As a result (compensatory ?) the lymphatic glands enlarge, but not constantly, while the blood-forming activity of the red marrow of bone is increased. Small brownish-red patches were observed in the intestines of frogs after extirpation of the spleen. These new formations are regarded by some observers as compensatory organs. Tizzoni asserts that new splenic structures are formed in the omentum (horse, dog) after the destruction of the parenchyma and blood-vessels of the spleen. The spleen is absent extremely seldom (Meinhard, Koch, and Waclismuth). [Schindeler found that animals after extirpation of the spleen became very ravenous, but there was no other marked symptom.]

Schiff stated that after extirpation of the spleen, the pancreatic juice failed to digest proteids. The evidence in support of this statement is unsatisfactory, and Mosler affirms that this operation has no effect either on gastric or pancreatic digestion. Heidenhain also found a similar negative result. The operation ought to be performed on young animals, as old animals often succumb to it.

(2.) According to Gerlach and Funke the spleen is a BLOOD-Forming GLAND. As already mentioned (p. 20) the blood of the splenic vein contains far more colourless corpuscles than the blood of the splenic artery. Many of these corpuscles undergo fatty degeneration, and disappear in the blood-stream (Virchow). That colourless blood-corpuscles are formed within the spleen seems to be proved by the enormous number of these corpuscles which are found in the blood in cases of hyperplasia of the spleen or leukæmia (Bennett, 1852, Virchow). Bizzozero and Salvioli found that several days after severe hæmorrhage, the spleen became enlarged, and its parenchyma contained numerous red nucleated hæmato-blasts.

Accorling to Schiff, extirpation of the spleen has no effect, either upon the absolute or relative number of coloured or colourless corpuscles. [According to the more accurate observations of Picard and Malassez, there is a temporary 
diminution of the coloured blood-corpuscles and their hæmoglobin, after extirpation of the spleen.]

(3.) Other observers (Kölliker and Ecker) regard the spleen as an organ in which COLOURED BLOOD-CORPUSCLES ARE DESTROYED, and they consider the large protoplasmic cells containing pigment granules (p. 16) as a proof of this. According to the observations of von Kusnetzow, these structures are merely lymph-corpuscles, which, in virtue of their amoboid movements, have entangled coloured blood-corpuscles. [Such corpuscles exhibit similar properties when placed upon a warm stage.] Similar cells occur in extravasations of blood (Virchow). The coloured blood-corpuscles within the lymph-cells gradually become disintegrated, and give rise to the production of granules of hæmatin and other derivatives of hæmoglobin. Hence, the spleen contains more iron than corresponds to the amount of blood present in it. When we consider that the spleen contains a large number of extractives derived from the decomposition of proteids, it is very probable that coloured blood-corpuscles are destroyed in the spleen. Further, the juice of the spleen contains salts similar to those that occur in the red bloodcorpuscles.

The blood of the spleen is said to undergo other changes, but the following statements must be accepted with caution :-The blood of the splenic vein contains more water and fibrin ; its red blood-corpuscles are smaller, brighter, less flattened, more resistant, and do not form rouleaux; its hæmoglobin crystallises more easily, and there is a larger proportion of $\mathrm{O}$ during digestion.

[It would thus appear that the spleen has a very direct relation to the blood; that coloured blood-corpuscles undergo disintegration, and that colourless corpuscles are manufactured within it.]

(4.) Contraction.-In virtue of the plain muscular fibres in its capsule and trabeculæ, the spleen undergoes variations in its volume (Kölliker). Stimulation of the spleen (Rud. Wagner, 1849) or its nerves, by cold, electricity, quinine, eucalyptus, ergot of rye, and other "splenic reagents" (Mosler) causes it to contract, whereby it becomes paler, and its surface may even appear granular. After a meal, the spleen increases in size, and it is usually largest about five hours after digestion has begun-i.e., at a time when the digestive organs have almost finished their work, and have again become less vascular. After a time it regains its original volume. For this reason the spleen was formerly regarded as an apparatus for regulating the amount of blood in the digestive organs.

[The congestion of the spleen after a meal is more probably related to the formation of new colourless corpuscles than to the destruction of red corpuscles. It may be, however, that some of the products of 
digestion are partially acted upon in the spleen, and undergo further change in the liver.]

There is a relation between the size of the spleen and that of the liver, for it is found that when the spleen contracts-e.g., by stimulation of its nerves - the liver becomes enlarged as if it were injected with more blood than usual (Drosdow and Botschetschkarow).

[Oncograph.-Botkin, and more recently Roy, have studied various conditions which affect the size of the spleen. Roy's observations are most important. He enclosed the spleen of a living animal (dog) in a box with rigid walls, and filled with oil after the manner of the plethysmograph ( $(101)$. Any variations in the size of the organ caused a variation in the amount of oil within the box, and these variations were recorded. This instrument Roy termed an "ONCOGRAPH" (o $\chi^{\circ}$ os, volume). The blood-pressure was recorded at the same time.

Roy finds that the circulation through the spleen is peculiar, and that it is not due to the blood-pressure within the arteries, but is carried on chiefly by a rhythmical contraction of the muscular fibres of the capsule and trabeculæ. The spleen undergoes very regular rhythmical contractions (systole) and dilatations (diastole). This alternation

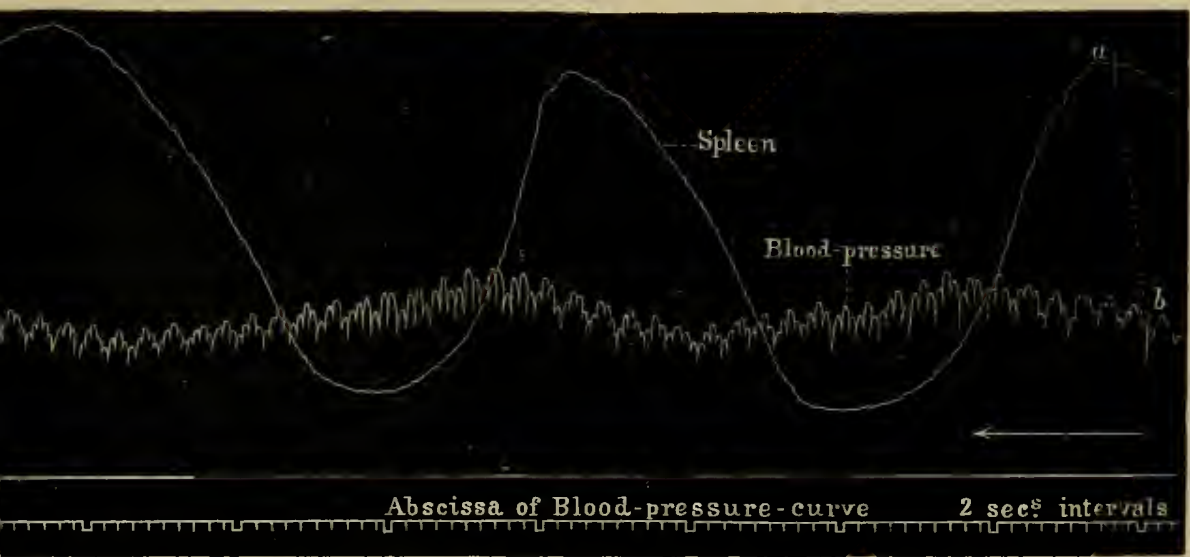

Fig. 91 .

Tracing of a splenic curve, reduced one-half, taken with the oncograph. The upper line with large waves is the splenic curve, each ascent corresponds to an increase, and each descent to a diminution in the volume of the spleen. The curve beneath is a blood-pressure tracing from the carotid artery. The lowest line indicates the time, the interruptions of the marker occurring every two seconds. The vertical lines, $a$ and $b$, give the relative positions of the lever point of the oncograph, and of the point of the recording style of the kymograph respectively (Roy). 
of systole and diastole may last for hours, and the two events together occupy about one minute (Fig. 91). Changes in the arterial bloodpressure have comparatively little influence on the volume of the spleen. The rhythmical contractions, although modified, still go on after section of the splenic nerves. This would seem to indicate that the spleen has an independent (nervous) mechanism within itself causing its movements.]

Influence of Nerves.-Section of the splenic nerves causes an increase in the size of the spleen; and when the nerves at the hilum are extirpated it swells and assumes a deep purple colour. The nerves have their centre in the medulla oblongata, and so far they are comparable to vaso-motor nerves. Stimulation of the medulla oblongata, either directly or by means of asphyxiated blood, causes contraction of the spleen [hence, the spleen is "small and contracted" in death from asphyxia.] The fibres proceed down the cord, and are probably joined by other fibres derived from ganglion cells lying opposite the first to the fourth cervical vertebræ, which cells also act on the spleen. The fibres leave the cord in the dorsal region, enter the left splanchnic, pass through the semi-lunar ganglion, and thus reach the splenic plexus (Jaschkowitz.) Stimulation of the peripheral ends of these nerves causes contraction of the spleen, and so does cold applied to the spleen directly or over the region of the organ. In this last case the result is brought about reflexly. Section or paralysis of these nerves causes dilatation, and so does curara or continued nareosis (Bulgak). [Botkin found that the application of the induced current to the skin over the spleen, in a case of leukæmia, caused well-marked contraction of the spleen in all its dimensions; the spleen becoming firmer, and its surface more irregular. The result lasted much longer than the duration of the stimulus. The same occurred in a case of enlarged lymphatic glands. After a time the organ began to enlarge. After every stimulation the number of colourless corpuscles in the blood increased, and the condition of the patient improved.]

[There is a popular notion that the spleen is influenced by the condition of the nervous system. Botkin found that depressing emotions increased its size, while exhilarating ideas diminished it. The causes of these changes are referable not only to changes in the amount of blood in the spleen, but also to the greater or less degree of contraction of its muscular tissue. And it would appear that, like the small arteries, the muscular tissue of the spleen is in a state of tonic contraction. The size of the spleen may be influenced reflexly. Thus, Tarchanoff found that stimulation of the central end of the vagus, when the splanchnics were intact, caused contraction of the spleen, while stimulation of the central end of the sciatic also caused contraction, but to a less degree. 
It is quite certain that all the phenomena are not due to the action of vaso-motor nerves on the splenic blood-vessels. There is a certain amount of independent action of the muscular fibres of the organ, and it is not improbable that the innervation of the spleen is similar to the innervation of arteries, and that it has a motor centre in the cord capable of being influenced by afferent nerves, and sending out efferent impulses.]

[Roy confirmed most of these results, and found that stimulation of (1) the central end of a sensory nerve, (2) of the peripheral ends of both splanchnics, (3) of the peripheral ends of both vagi, caused contraction of the spleen. But even after section of the splanchnics and vagi, stimulation of a sensory nerve still caused contraction, so that there must be some other channel as yet unknown. Bochefontaine found that electrical stimulation of certain parts of the cortex cerebri produced contraction of the spleen.] Sensory nerves seem to occur only in the peritoneum covering the spleen.

Pressure on the splenic vein causes enlargement of the spleen (Mosler); hence, increased pressure in this vein (congestion of the portal vein, cessation of hæmorrhoidal and menstrual discharges) also causes its enlargement. With regard to the action of "splenic reagents," such as Quinine, on the contraction of the spleen, $\operatorname{Binz}$ is of opinion that this drug retards the formation of the colourless blood-corpuscles, so that its chief function is interfered with and the organ becomes less vascular. It is not definitely decided, however, whether it is contraction or dilatation of the spleen that alters the proportion of red and white corpuscles in the blood.

Splenic Tumours. - The increase in size of the spleen in various diseases early attracted the attention of physicians. The healthy spleen undergoes several variations in volume during the course of a day, corresponding with the varying activity of the digestive organs. In this respect the spleen resembles the arteries. In many fevers the spleen becomes greatly enlarged, probably due to paralysis of its uerves. It is greatly increased in intermittent fever or ague, and often during the course of typhus. When it becomes abnormally enlarged, and remains so after repeated attacks of ague, it is greatly hypertrophied and constitutes "ague cake." In cases of splenic leukæmia it is greatly enlarged, and at the same time there is a great increase in the number of colourless corpuscles in the blood, and also a decrease of the coloured ones (p. 23).

\section{The Thymus.}

During foetal life this gland is largely developed, and it increases during the first two or three years of life, remaining stationary until the tenth or fourteenth year, when it begins to atrophy and undergo fatty degeneration. [The degeneration begins at the outer part of each lobule and progress inwards (His).]

Structure.-["It consists of an aggregation of lymph-follicles (resembling the glands of Peyer) or masses of adenoid tissue held together by a framework of connective tissue which contains blood-vessels, lymphatics, and a few nerves (Fig. 92). The framework of connective tissue gives off septa which divide the gland into lobes, these being further subdivided by finer septa in to lobules, the lobules being separated 
by fine intra-lobular lamellæ of connective tissue into follicles $(0.5-1.5 \mathrm{~mm}$.). These follicles make up the gland substance, and they are usually polygonal when seen in a section. Each follicle consists of a cortical and a medullary part, and the matrix or framework of both consists of a fine adenoid reticulum whose meshes are filled with lymph-corpuscles" (Fig. 93, a).] Many of these corpuscles exhibit various stages of disintegration. In the medulla are found the concentric corpuscles of Hassall. ["They consist of a central granular part, around which are disposed layers of flattened nucleated endothelial cells arranged concentrically. When seen in a section they resemble the 'cell-nests' of epithelioma (Fig. 93, b).

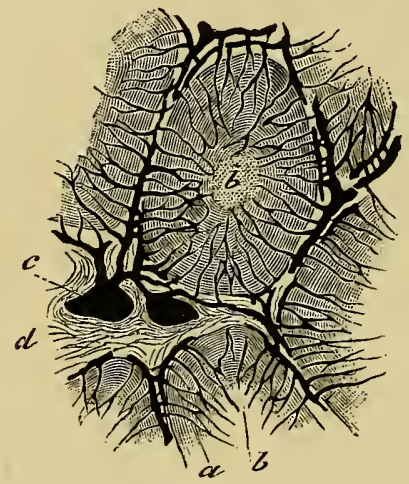

Fig. 92.

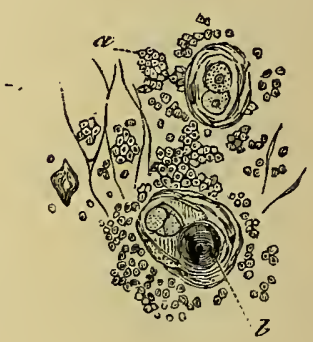

Fig. 93.

Section of the thymus gland of a cat, showing one complete lobule with an outer cortical part, a centre, $b$, and parts of adjoining lobules $-a$, lymphoid tissue; $c$, bloodvessels injected; $d$, connective tissue.

Elements of the thymus $(\times 300)-$ $a$, lymph-corpuscles; $b$, con. centric corpuscle of Hassall.

They have also been compared to similar bodies which occur in the prostate. They are most numerous when the gland undergoes its retrograde metamorphosis."]

Simon, His, and others described a convoluted blind canal, the "central canal," as occurring within the gland, and on it the follicles were said to be placed. Other observers, Jendrassik and Klein, either deny its existence or regard it merely as a lymphatic or an artificial product. Numerous fine lymphatics penetrate into the interior of the organ, and many are distributed over its surface, but their mode of origin is unknown. [They seem to be channels through which the lymph-corpuscles are conveyed away from the gland.] Numerous blood-vessels are also distributed to the septa and follicles (Fig 92, c).

Chemical Composition.-Besides gelatin, albumin, soda-albumin, there are sugar and fat, leucin, xanthin, hypoxanthin, formic, acetic, butyric, and succinic acids. Potash and phosphoric acid are more abundant in the ash than soda, calcium, magnesium (? ammoninm), chlorine, and sulphuric acid (v. GorupBesanez).

[Function.-As long as it exists, it seems to perform the functions of a true lymph-gland. This view is supported by the fact that in reptiles and amphibians, which do not possess lymph-glands, the thymus remains as a permanently active organ. That the thymus forms colourless corpuscles was first maintained by Hewson, and confirmed by His and Jendrassik. Extirpation (Friedleben) gave few 
positive results, but chemical investigation shows that the parenchyma contains a large number of products indicating considerable metabolic activity. The volume of the gland undergoes variations both in health and disease.]

\section{The Thyroid.}

Structure.-In a connective tissue net-work rich in cells there lie numerous completely closed sacs $(0.04-0.1 \mathrm{~mm}$. in diameter), which in the embryo and the newly-born animal are composed of a membrana propria lined by a single layer of nucleated cubical cells (Fig. 94). The sacs contain a transparent, viscid, albuminous fluid. [Not unfrequently the sacs contain many coloured blood-corpuscles (Baber). As in other glands, there are lobes and lobules.] Each sac is surrounded by a plexus of capillaries which do not penetrate the membrana propria. There are also numerous lymphatics. At an early jeriod the sacs dilate, their cellular lining atrophies, and their contents undergo colloid degeneration. When the gland vesicles are greatly enlarged, "goitre" is produced.

The Chemical Composition of this gland has not been much investigated. In addition to the ordinary constituents, leucin, xanthin, sarkin, lactic, succinic, and volatile fatty acids have been found.

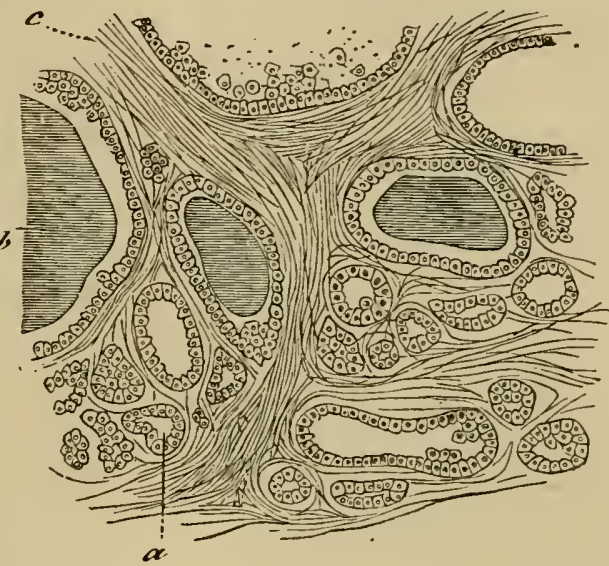

Fig. 94.

Section of the thyroid gland $(\times 250)-a$, small closed vesicles lined by low columnar epithelium; $b$, colloid masses distending the vesicles; $c$, connective tissue between the vesicles.

Functions.-Its functions are quite unknown. Perhaps it nay be an apparatus for regulating the blood supply to the head (?). It becomes enlarged in Basedow's disease, in which there is great palpitation, as well as protrusion of the eyeball [Exophthalmos], which seem to depend upon a simultaneous stimulation of the accelerating nerve of the heart, and the sympathetic fibres for the smooth muscles in the orbital cavity and the eyelids, as well as of the inhibitory fibres of the vessels of the thyroid. In many localities it is common to find swelling of the thyroid constituting goitre, which is sometimes, but far from invariably, associated with idiocy and cretinism.

\section{The Supra-Renal Capsules.}

Structure,-These organs are invested by a thin capsule which sends processes into the interior of the organ. They consist of an outer (broad) or cortical layer and an inncr (narrow) or medullary layer. The former is yellowish in colour, firm and striated, while the latter is softer and deeper in tint. In the outermost zone of the cortex (Fig. 95, b), the trabeculæ form polygonal meshes which contain the 
cells of the gland substance; in the broader middle zone, the meshes are elongated and the cells filling them are arranged in columns radiating outwards. Here the cells are transparent and nucleated, often containing oil globules; in the innermost

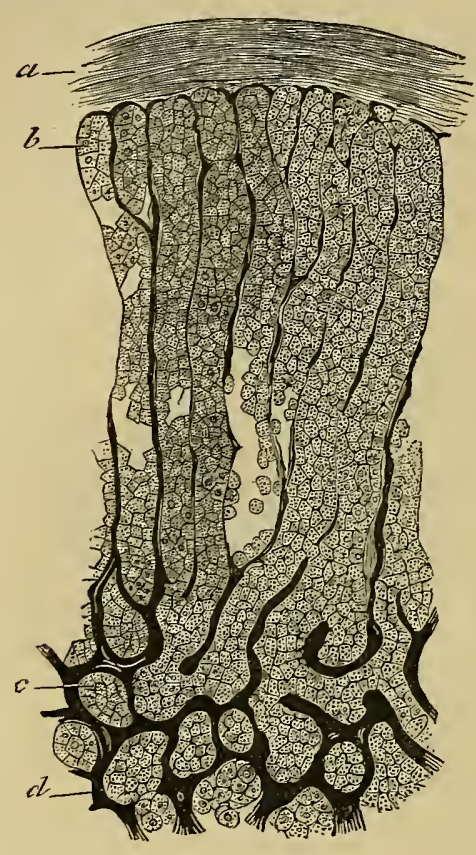

Fig. 95.

Section of a human supra-renal capsule $-a$, capsule; $b$, gland cells of the cortex arranged in columns; $c$, glandular net-work of the medulla; $d$, blood-vessels. narrow zone the polygonal arrangement prevails, and the cells often contain yellowish-brown pigment. In the medulla (c) the stroma forms a reticulum containing groups of cells of very irregular shape.

Numerous blood-vessels occur in the gland, especially in the cortex. [The nerves are extremely numerous, and are derived from the renal and solar plexuses. Many of the fibres are medullated. After they enter the gland, numerous ganglionic cells occur in the plexuses which they form. Indeed, some observers regard the cells of the medulla as nervous. Undoubtedly, numerous multipolar nerve-cells exist within the gland.]-(Eberth, Creighton, v. Brunn).

Chemical Composition.-The suprarenals contain the constituents of connective-tissue and nerve-tissue; also lencin, hypoxanthin, benzoic, hippuric, and taurocholic acids, taurin, inosit, fats, and a body which becomes pigmented by oxidation. Amongst inorganic substances potash and phosphoric acid are most abundant.

The function of the supra-renal bodies is quite unknown. It is noticeable, however, that in Addison's disease (" bronzed skin") which is perhaps primarily a nervous affection, these glands have frequently, but not invariably, been found to be diseased. Owing to the injury to adjacent abdominal organs extirpation of these organs is often, although not always, fatal. Brown-Séquard thinks they may be concerned in preventing the over-production of pigment in the blood.

\section{Hypophysis Cerebri-Coccygeal and Carotid Glands.}

The hypophysis cerebri, or pituitary body, consists of an anterior lower or larger lobe partly embracing the posterior lower or smaller lobe. These two lobes are distinct in their structure and development. The posterior lobe is a part of the brain, and belongs to the infundibulum. The nervous elements are displaced by the ingrowth of connective-tissue and blood-vessels. The anterior portion represents an inflected and much-altered portion of ectoderm, from which it is developed. It contains gland-like structures, with connective-tissue, lymphatics and blood-vessels, the whole being surrounded by a capsule. According to Ecker and Mihalkowicz, it resembles the supra-renal capsule in its structure, while, 
according to other observers, in some animals it is more like the thyroid. Its functions are entirely unknown.

Coccygeal and Carotid Glands.-The former, which lies on the tip of the coccyx, is composed, to a large extent, of plexuses of small more or less cavernous arteries, supported and enclosed by septa and a capsule of connective-tissuc (Luschka). Between these lie polyhedral granular cells arranged in net-works. The carotid gland has a similar structure (p. 124). Their functions are quite unknown. Perhaps both organs may be regarded as the remains of embryonal hlood-vessels (Arnold).

\section{Comparative.}

The heart in fishes as well as in the larræ of amphibians with gills, is a simple venous heart consisting of an auricle and a ventricle. The rentricle propels the blood to the gills where it is oxygenated (arterialised); thence it passes into the aorta to be distributed to all parts of the body, and returns through the capillaries of the body and the veins to the heart. The amphibians (frogs) have two anricles and one ventricle. From the latter there proceeds one ressel which gives off the pulmonary arteries, and as the aorta supplies the rest of the body with blood, the veins of the systemic circulation carry their blood to the right auricle, those of the lung into the left auricle. In fishes and amphibians there is a dilatation at the commencement of the aorta, the bulbus arteriosus, which is partly provided with strong muscles. The reptiles posscss two separate auricles, and two imperfectly separated ventricles. The aorta and pulmonary artery arise separately from the two latter chambers. The venous blood of the systemic and pulmonary circulations flows separately into the right and left auricles, and the two streams are mixed in the ventricle. In some reptiles the opening in the ventricular septum seems capable of being closed. The crocodile has two quite separate ventricles. The lower vertebrates have valves at the orifices of the venæ cavæ, which are rudimentary in birds and some maminals. All birds and mammals have two completely separate auricles and two separate ventricles. In the halicore the apex of the ventricles is deeply cleft. Some animals have accessory hearts, e.g., the eel in its caudal vein. They are very probably lymph-hearts (Robin). The veins of the wing of the bat pulsate (Schiff). The lowest vertebrate, amphioxus, has no heart, but only a rhythmically-contracting vessel.

Amongst blood-glands the thymus and spleen occur throughout the vertebrata, the latter being absent only in amphioxus and a few fishes.

Amongst invertebrata a closed vascular system, with pulsatile movement, occurs here and there, e.g., amongst echinodermata (star-fishes, sea-urchins, holothurians) and the higher worms. The insects have a pulsating "dorsal vessel" as the central organ of the circulation, which is a contractile tube provided with valves and dilated by muscular action; the blood being propelled rhythmically in one direction into the spaces which lie amongst the tissues and organs, so that these animals do not possess a closed vascular system. The mollusca have a heart with a lacunar vascular system. The cephalopods (cuttle-fish) hare three hearts-a simple arterial heart, and two venous simple gill-hearts, each placed at the base of the gills. The vessels form a completely closed circnit. The lovest animals have either a pulsatile vesicle, which propels the colourless juice into the tissues (infusoria), or the vascular apparatus may be entirely absent.

\section{Historical Retrospect.}

The ancients held various theories regarding the movement of the blood, but they knew nothing of its circulation. According to Aristotle (384 B.c.), the heart, the 
acropolis of the body, prepared in its cavities the blood, which streamed through tine arteries as a nutrient fluid to all parts of the body, but never returned to the heart.

With Herophilus and Erasistratus (300 B.c.), the celebrated physicians of the Alexandrian school, originated the erroneous view that the arteries contain air, which was supplied to them by the respiration (hence the name artery). They were led to adopt this view from the empty condition of the arteries after death. By experiments upon animals, Galen disproved this view (131-201 A.D.) - "Whenever I injured an artery," he says, "blood always flowed from the wounded vessel. On tying part of an artery between two ligatures, the part of the artery so included is always filled with blood."

Still, the idea of a single centrifugal movement of the blood was retained, and it was assumed that the right and left sides of the heart communicated directly by means of openings in the septum of the heart until Vesalius showed that there are no openings in the septum. Michael Servetus (the Spanish monk, burned at Geneva, at Calvin's instigation, in 1553) discovered the pulmonary circulation. Cesalpinus confirmed this observation, and named it "Circulatio." Fabricius ab Aquapendente (Padua, 1574) investigated the valves in the veins more carefully (although they were known in the fifth century to Theodoretus, Bishop in Syria), and he was acquainted with the centripetal movement of the blood in the veins. Up to this time it was imagined that the veins carried blood from the centre to the periphery, although Vesalius was acquainted with the centripetal direction of the blood-stream in the large venous trunks. At length, William Harvey, who was a pupil of Fabricius (1604) demonstrated the complete circulation (1616-1619), and published his great discovery in 1628. [For the history of the discovery of the circulation of the blood, see the works of Willis on "W. Harvey," "Servetus and Calvin," those of Kirchner, and the various Harveian orations.]

According to Hippocrates, the heart is the origin of all the vessels; he was acquainted with the large vessels arising from the heart, the valves, the chordæ tendiniæ, the auricles, the closure of the semi-lunar valves. Aristotle was the first to apply the terms aorta and venæ cavæ; the school of Erasistratus used the term carotid, and indicated the functions of the venous valves. In Cicero a distinction is drawn between arteries and veins. Celsus mentions that if a vein be struck below the spot where a ligature has been applied to a limb, it bleeds, while Aretaeus (50 A.D.) knew that arterial blood was bright and venous dark. Pliny ( 79 A.D.) described the pulsating fontanelle in the child. Galen (13I-203 A.D.) was acquainted with the existence of a bone in the septum of the heart of large animals (ox, deer, elephant). He also surmised that the veins communicated with the arteries by fine tubes. The demonstration of the capillaries, however, was only possible by the use of the microscope, and employing this instrument, Malpighi (1661) was the first to demonstrate the capillary circulation. Leuwenhoek (1674) described the capillary circulation more carefully, as it may be seen in the web of the frog's foot and other transparent membranes. Blancard (1676) proved the existence of capillary passages by means of injections. William Cooper (1697) proved that the same condition exists in warm-blooded animals, and Ruysch made similar injections. Stenson (born 1638) established the muscular nature of the heart, although the Hippocratic and Alexandrian schools had already surmised the fact. Cole proved that the sectional area of the blood-stream became wider towards the capillaries (1681). Joh. Alfons Borelli (1608-1679) was the first to estimate the amount of work done by the heart. 


\section{Physiology of Respiration.}

THE Object of respiration is to supply the oxygen necessary for the oxidation processes that go on in the body, as well as to remove the carbonic acid formed within the body. The most important organs for this purpose are the lungs. There is an outer and an inner respiration-the former embraces the exchange of gases between the external air and the blood-gases of the respiratory organs (lungs and skin)-the latter, the exchange of gases between the blood in the capillaries of the systemic circulation and the tissues of the body.

[The pulmonary apparatus consists of (1) an immense number of small sacs-the air-vesicles-filled with air, and covered externally by a very dense plexus of capillaries; (2) air-passages-the nose, pharynx, larynx, trachea, and bronchi communicating with (1); (3) the thorax with its muscles, acting like a pair of bellows, and moving the air within the lungs.]

\section{Structure of the Air-Passages and Lungs.}

The lungs are compound tubular (racemose?) glands which separate $\mathrm{CO}_{2}$ from the blood. Each lung is provided with an excretory duct (bronchus) which joins the common respiratory passage of both lungs-the trachea.

Trachea.-The trachea and extra-pulmonary bronchi are similar in structure. The basis of the trachea consists of a number (16-20) of C-shaped incomplete cartilaginous hoops placed over each other. These rings consist of hyaline cartilage, and are united to each other by means of tough fibrous tissue containing much elastic tissue, the latter being arranged chiefly in a longitudinal direction. The function of the cartilages is to keep the tube open under varying conditions of pressure. Pieces of cartilage having a similar function occur in the bronchi and their branches, but they are absent from the bronchioles, which are less than $1 \mathrm{~mm}$. in diameter. In the smaller bronchi the cartilages are fewer and scattered more irregularly. [In a transverse section of a large intra-pulmonary bronchus, two, three, or more pieces of cartilage, each invested by its perichondrium, may be found.] At the points of bifurcation of the bronchi, the cartilages assume the form of irregular plates embedded in the bronchial wall.

An external fibrous layer of connective-tissue and elastic fibres covers the trachea and the extra-pulmonary bronchi externally. Towards the œsophagus, the elastic elements are more numerous, and there are also a few bundles of plain muscular fibres arranged longitudinally. Within this layer there are bundles of non-striped muscular fibres which pass transversely between the cartilages behind, and also in the intervals between the cartilages, [These pale reddish fibres con. 
stitute the trachealis muscle, and are attached to the inner surfaces of the cartilages by means of elastic tendons at a little distance from their free-ends (Munniks, 1697). The arrangement varies in different animals-thus, in the cat, dog, rabbit, and rat the muscular fibres are attached to the external surfaces of the cartilages, while in the pig, sheep, and ox they are attached to their internal surfaces (Stirling).] Some muscular fibres are arranged longitudinally external to the transverse fibres (Kramer). The function of these muscular fibres is to prevent too great distension when there is great pressure within the air-passages.

The mucous membrane consists of a basis of very fine connective-tissue containing much adenoid-tissue with numerous lymph-corpuscles. It also contains numerous elastic fibres, arranged chiefly in a longitudinal direction under the basement membrane. They are also abundant in the deep layers of the posterior part of the membrane opposite the intervals between the cartilages. A small quantity of loose sub-mucous connective-tissue containing the large blood-vessels, glands, and lymphatics unites the mucous membrane to the perichondrium of the cartilages. The epithelium consists of a layer of columnar ciliated cells with several layers of immature cells under them. [The superticial layer of cells is columnar and ciliated (Fig. 97, $b$ ), while those lying under them present a variety of forms, and below all is a layer of somewhat flattened squames, $c$, resting on the basement membrane, $d$. These squames constitute a layer quite distinct from the basement membrane, and they form the layer described by Débove. They are active germinating cells, and play a most important part in connection with the regeneration of the epithelium, after the superficial layers have been shed, in such conditions as bronchitis (v. Drasch, Hamilton). Not unfrequently a little viscid mucus $(a)$ lies on the free-ends of the cilia. In the intermediate layer, the cells are more or less pyriform or battledore-shaped (Hamilton), with their long tapering process inserted amongst the deepest layer of squames. According to Drasch, this long process is attached to one of these cells and is an outgrowth from it, the whole constituting a "foot-cell."]

Underneath the epithelial is the homogeneous basement membrane, through which fine canals pass connecting the cement of the epithelium with spaces in the mucosa. [This membrane is well marked in the human trachea, where it plays an important part in many pathological conditions, e.g., bronchitis. It is stained bright red with picrocarmine.] The cilia act so as to carry any secretion towards the larynx. Goblet cells exist between the ciliated columnar cells. Numerous small compound tubular mucous glands occur in the mucous membrane, chiefly between the cartilages. Their ducts open on the surface by means of a slightly funnel-shaped aperture into which the ciliated epithelium is prolonged for a short distance. [The acini of some of these glands lie outside the trachealis muscle. The acini are lined by cubical or columnar secretory epithelium. In some animals (dog) these cells are clear, and present the usual characters of a mucous-secreting gland; in man, some of the cells may be clear, and others "granular," but the appearance of the cells depends upon the physiological state of activity.] These glands secrete the mucus, which entangles particles inspired with the air, and is carried towards the larynx by ciliary action. [Numerous lymphatics exist in the mucous and sub-mucous coat, and not unfrequently small aggregations of adenoid tissue occur (especially in the cat) in the mucous coat, usually around the ducts of the glands. They are comparable to the solitary follicles of the alimentary tract. The blood-vessels are not so numerous as in some other mucous membranes. [A plexus of nerves containing numerous ganglionic cells at the nodes exists on the posterior surface of the trachealis muscle. The fibres are derived from the vagus, recurrent laryngeal, and sympathetic (C. Frankenhauser, W. Stirling, Kandarazi).]

[The mucous membrane of the trachea and extra-pulmonary bronchi, therefore, consists of the following layers from within outwards :- 
(1.) Stratified columnar ciliated epithelium.

(2.) A layer of flattened cells (Débove's membrane).

(3.) A clear homogeneous basement membrane.

(4.) A basis of areolar tissue, with adenoid tissue and blood-vessels, and outside this a layer of longitudinal elastic fibres.

Outside this, again, is the sub-mucous coat, consisting of loose areolar tissue, with the larger vessels, lymphatics, nerves, and mucous glands.]

[The Bronchi.-In structure, the extra-pulmonary bronchi resemble the trachea. As they pass into the lung they divide dichotomously very frequently, and the branches do not anastomose. The subdivisions become finer and finer, the finest

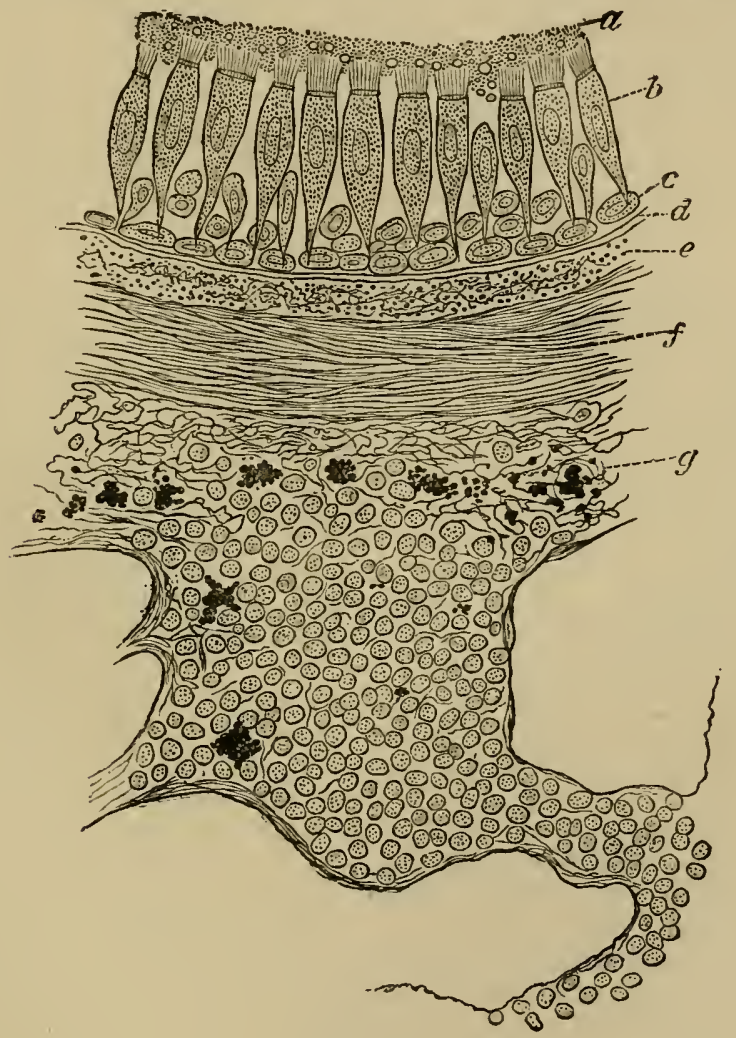

Fig. 97.

Transverse section of part of a normal human bronchus $(\times 450)-\alpha$, precipitated mucus on the surface of the ciliated epithelium, $b ; b$, ciliated columnar epithelium; $c$, deep germinal layer of cells (Débove's membrane); $d$, elastic basement membrane; $e$, elastic fibres divided transversely (inner fibrous layer); $f$, bronchial muscle (non-striped); $g$, outer fibrous layer with leucocytes and pigment granules (black) deposited in it. The lower part of the figure shows a mass of a denoid tissue.

branches being called terminal bronchi, or bronchioles, which open separately into clusters of air-vesicles.] 
[In the niddle-sized intra-pulmonary bronchi, the usual characters of the mucous membrane are retained, only it is thinner; the cartilages assume the form of irregular plates situated in the outer wall of the bronchus; while the muscular fibres are disposed in a complete circle constituting the bronchial muscle (Fig. $97, f)$. When this muscle is contracted, 'or when the bronchus as a whole is contracted, the mucous membrane is thrown into longitudinal folds, and opposite these folds the elastic fibres form large elevations. This muscle is particularly well-developed in the smaller microscopic bronchi. Numerous elastic fibres, $e$, disposed longitudinally, exist under the basement membrane, $d$. They are continuous with those of the trachea, and are continued onwards into the lung. The mucous membrane of the larger intra-pulmonary bronchi consists of the following layers from within outwards:-

(1.) Stratified columnar ciliated epithelium (Fig. 97, b).

(2.) Débove's membrane (Fig. 97, c).

(3.) Transparent homogeneous basement membrane (Fig. 97, d).

(4.) Areolar tissue with longitudinal elastic fibres (Fig. 97, e).

(5.) A continuous layer of non-striped muscular fibres disposed circularly (bronchial muscle-Fig. 97, $f$ ).

Outside this is the sub-mucous coat, consisting of areolar tissue mixed with much adenoid tissue (Fig. 97, g), sometimes arranged in the form of cords, the lymphfollicular cords of Klein. It also contains the acini of the numerous mucous glands, blood-vessels, and lymphatics. The ducts of the glands perforate the muscular layer, and open on the free surface of the mucous membrane. The sub-mucous coat is connected by areolar tissue with the perichondrium of the cartilages. Outside the cartilages are the nerves and nerve ganglia accompanying the bronchial vessels. A branch of the pulmonary artery and pulmonary vein usually lie on opposite sides of the bronchus, while there are several branches of the bronchial arteries and veins. Fat cells also occur in the peri-bronchial tissue.]

In the small bronchi the cartilages and glands disappear, but the circular muscular fibres are well developed. They are lined by lower columnar ciliated epithelium, containing goblet cells.

Bronchioles.-After repeated subdivision, the bronchi form the "smallest bronchi" (about 05-1 mm.) or lobular bronchial tubes. Each tube is lined by a layer of ciliated epithelium, but the glands and cartilages have disappeared. These tubes have a few lateral alveoli or air-cells communicating with them. Each smallest bronchus ends in a "respiratory bronchiole" (Kölliker), which gradually becomes beset with more air-cells, and in which squamous epithelium begins to appear between the ciliated epithelial cells. [Each bronchiole opens into several wider alveolar or lobular passages. Each passage is completely surrounded with air-cells, and from it are given off several similar but wider blind branches, the infundibula, which, in their turn, are beset on all sides with alveoli or air-cells. Several infundibula are connected with each bronchiole, and the former are wider than the latter. Each bronchiole, with its alveolar passages, infundibula, and airvesicles, is termed a lobule, whose base is directed outwards, and whose apex may be regarded as a terminal bronchus. The lung is made up of an immense number of these lobules, separated from each other by septa of connective-tissue, the interlobular septa (Fig. 100, e) which are continuous on the one hand with the sub-pleural connective-tissue, and on the other with the peri-bronchial connective-tissue.]

[It is evident that there is an alteration in the structure of the bronchi, as we proceed from the larger to the smaller tubes. The cartilages and glands are the first structures to disappear. The circular bronchial muscle is well developed in the smaller bronchi, and bronchioles, and exists as a continuous thin layer over the alveolar passages, but it is not continued over and between the air-cells. Elastic fibres, continuous, on the one hand, with those in the smaller bronchi, and 
on the other, with those in the walls of the air-cells, lie outside the muscular fibres in the bronchioles and infunclibula. In the respiratory bronchioles, the ciliated epithelium is reduced to a single layer, and is mixed with the stratified form of epithelium, while, where the alveolar passages open into the air-cells or alveoli, the epithelium is non-ciliated, low, and polyhedral.]

Alveoli or Air-Cells.-The form of the air-cells, which are $250 \mu\left({ }_{1}^{\frac{1}{0} \sigma}\right.$ inch) in diameter, may be more or less spherical, polygonal, or cup-shaped. They are disposed around and in communication with the alveolar passages. 'Their form is determined by the existence of a nearly structureless membrane, composed of slightly fibrillated comnective-tissue containing a few corpuscles. This is surrounded by numerous fine elastic fibres which give to the pulnonary parenchyma its well-marked elastic characters (Fig. 99, e,e). These fibres often bifurcate, and are arranged with reference to the alveolar wall. They are very resistant, and in some cases of lung-disease may be recognised in the sputum. A few non-striped muscular fibres exist in the delicate connective - tissue between adjoining air-ves. icles (Moleschott). These muscular fibres sometimes become greatly developed in certain diseases (IW. Stirling). The air-cells are linerl by two kinds of cells-(1) large, transparent, clear polygonal (nucleated?) squames or placoids $(22-45 \mu)$ lying over and between the capil. laries in the alveolar wall (Fig. 98, a); (2) small irregular "granular" nucleated cells $(7-15 \mu)$ arranged singly or in groups (two or three) in the interstices betwcen the capillaries. They are well seen in a cat's lung (Fig. 98, d). [When acted on with nitrate of silver the cement-substance bounding the clear cells is stained, but the small cells become of a uniform brown granular appearance, so that they are readily recognised. Small holes or "pseudo-stomata"

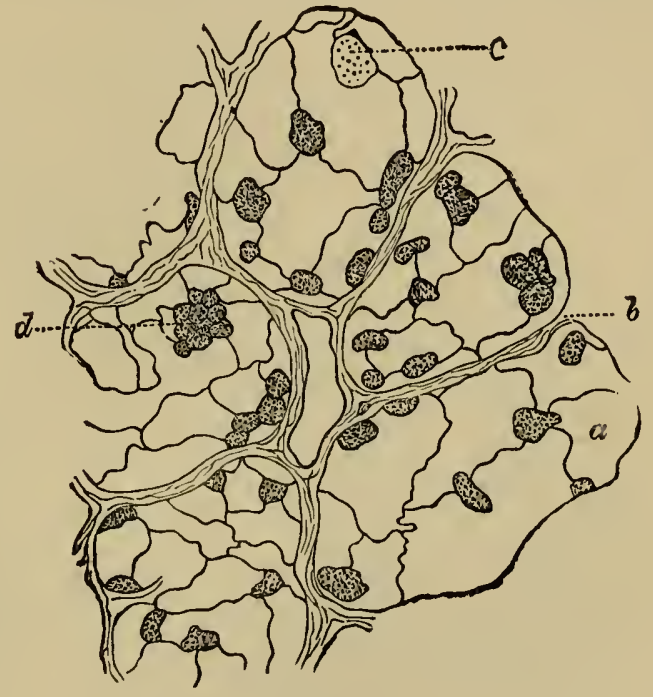

Fig. 9s.

Air-vesicles from a kitten whose lungs were injected with silver nitrate $(\times 450)-a$, outlines of fullydeveloped squamous epithelinm; $b$, alveolar wall; $c$, young epithelial cell losing its granular appearance; $d$, aggregation of young epithelial cells germinating. seem to exist in the cement-substance, and are most obvious in distended alveoli (Klein). They open into the lymph-canalicular system of the alveolar wall (Klein), and through them the lymph-corpuscles, which are always to be found on the surface of the air-vesicles, migrate, and carry with them into the lymphatics particles of carbon derived from the air.] In the alveolar walls is a very dense plexus of fine capillaries (Fig. 99, c), which lie more towards the cavity of the air-vesicle (Rainey), being covered only by the epithelial lining of the air-cells. Between two adjacent alveoli there is only a single layer of capillaries (man), and on the boundary line between two air-cells the course of the capillaries is twisted, thus projecting sometimes into the one alveolus, sometimes into the other. 
The Blood-vessels of the lung belong to two different systems:-(A) PoLMONARY VESSELS (lesser circulation). The branches of the pulmonary artery accompany the bronchi and are closely applied to them. [As they proceed they branch, but the branches do not anastomose, and ultimately they terminate in small arterioles which supply several adjacent alveoli, each arteriole splitting up into capillaries for several air-cells (Fig. 99, v,c). An efferent vein usually arises at the opposite side of the air-cells and carries away the purified blood from the capillaries. In their course these veins unite to form the pulmonary veins which are joined in their course by a few small bronchial veins (Zuckerkandl). The veins usually anastomose in the earlier part of their course, whilst the corresponding arteries do not.] Although the capillary plexus is very fine and dense, its sectional area is less than the sectional area of the systemic capillaries, so that the blood-stream in the pulmonary capillaries must be more rapid than that in the capillaries of the body generally. The pulmonary veins, unlike veins generally, are collectively

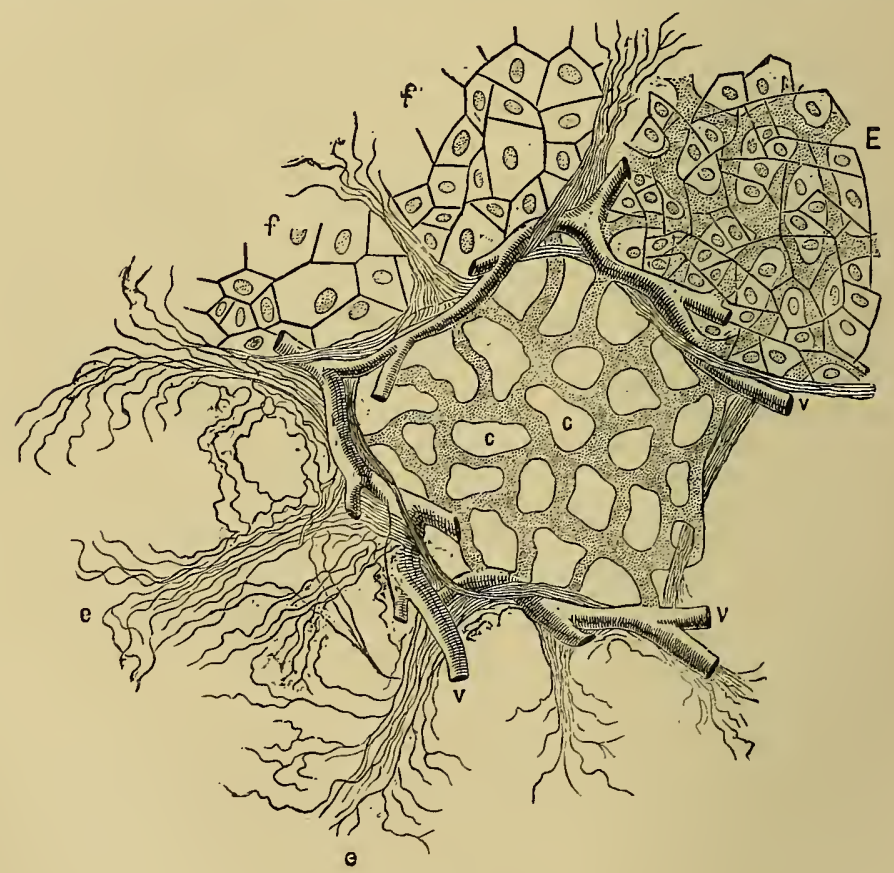

Fig. 99.

Semi-schematic representation of the air vesicles of the lung $-v, v$, blood-vessels at the margins of an alveolus; $c, c$, its blood capillaries; $\mathrm{E}$, relation of the squamous epithelium of an alveolus to the capillaries in its wall; $f$, alveolar epithelium shown alone; $e, e$, elastic tissue of the lung.

narrower than the pulmonary artery (water is given off in the lung), and they have no valves. [The pulmonary artery contains venous blood, and the pulmonary veins pure or arterial blood].

(B) The Bronchial vessels represent the nutrient system of the lungs. They (1-3) arise from the aorta (or intercostal arteries) and accompany the bronchi without anastomosing with the branches of the pulmonary artery. In their course they give branches to the lymphatic glands at the hilum of the lung, 
to the walls of the large blood-vessels (vasa vasorum), the pulmonary pleura, the bronchial walls, and the interlobular septa. The blood which issues from their capillaries is returned-partly by the pulmonary veins-hence, any considerable interference with the pulmonary circulation causes congestion of the bronchial mucous membrane, resulting in a catarrhal condition of that membrane. The greater part of the blood is returned by the bronchial veins which open into the rena azygos, intercostal vein, or superior vena cava. The veins of the smaller bronchi (fourth order onwards) open into the pulmonary veins, and the anterior bronchial also communicate with the pulmonary veins (Zuckerkandl).

[The Pleura.-Each pleural cavity is distinct, and is a large serous sac, which really belongs to the lymphatic system of the lung. The pleura consists of two layers, visceral and parietal. The visceral pleura covers the lung; the parietal portion lines the wall of the chest, and the two layers of the corresponding pleura are continuous with one another at the root of the lung. The visceral pleura is the thicker, and may readily be separated from the inner surface of the chest. Structurally, the pleura resembles a serons membrane, and consists of a thin layer of fibrous tissue covered by a layer of endothelium. Under this layer, or the pleura proper, is a deep or sub-serous layer of looser areolar tissue, containing many elastic fibres. This layer of the pleura pulmonalis of some animals, as the guinea-pig, contains a net-work of noll-striped muscular fibres (Klein). Over the lung it is also continuous with the interlobular septa. The interlobular septa (Fig. $100, e)$ consist of bands of fibrous tissue separating adjoining lobules, ancl

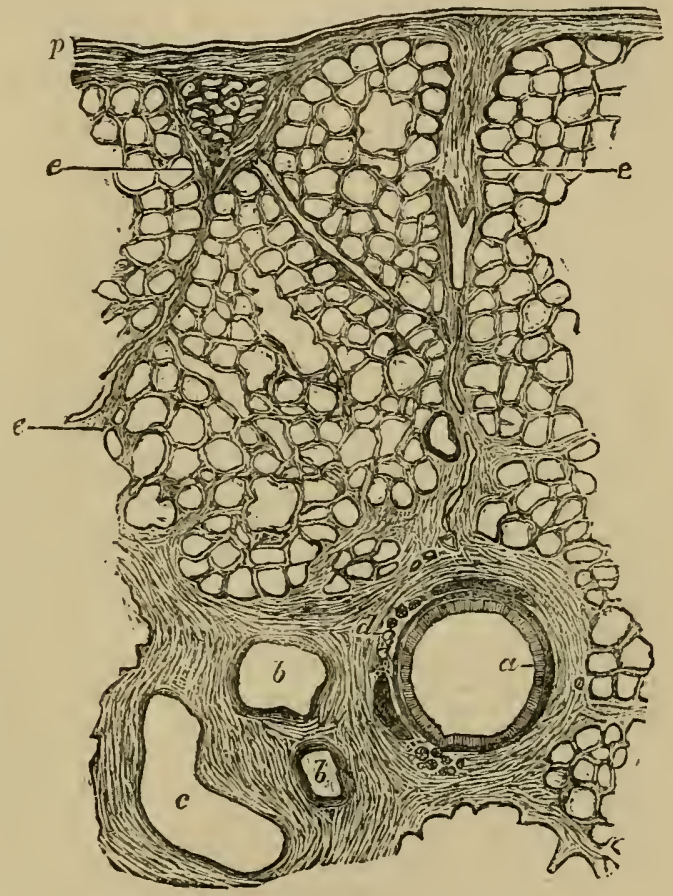

Fig. 100.

Normal human lung ( $\times 50$ and reduced $\$)-a$, small bronchus; $b b$, branches of the pulmonary artery; $c$, branch of the pulmonary vein; $e$, interlobular septa, continuous with tlie deep layer of the pleura, $p$.

they become continuous with the peri-bronchial connective-tissue entering the lung at its hilum. Thus the tibrous framework of the lung is continuous throughout the lung, just as in other organs. The connection of the sub-pleural fibrous tissue with the connective-tissue within the substance of the lung, has most important pathological bearings. The interlobular septa contain lymphatics and blood-vessels. The endothelium covering the parietal layer is of the ordinary squamous type, but on the pleura pulmonalis the cells are less flattened, more polyhedral, and granular. They must necessarily vary in shape with changes in 
the volume of the lung, so that they are more flattened when the lung is distended, as rluring inspiration (Klein). The pleura contains many lymphatics, which communicate by means of stomata with the pleural cavity.]

[The Lymphatics of the lung are numerous and are arranged in several systems. The various air-cells are connected with each other by very delicate connective-tissue, and according to J. Arnold in some parts this interstitial tissue presents characters like those of adenoid tissue; so that the lung is traversed by a system of juice-canals or Saft-canälchen.] [In the deep layer of the pleura, there is a (a) sub-pleural plexus of lymphatics partly derived from the pleura, but chiefly from the lymph-canalicular system of the pleural alveoli. Some of these branches proceed to the bronchial glands, but others pass into the interlobular septa, where they join $(b)$ the peri-vascular lymphatics which arise in the lymph-canalicular system of the alveoli. These trunks, provided with valves, run alongside the pulmonary artery and vein, and in their course they form frequent anastomoses. Special vessels arise within the walls of the bronchi and occur chiefly in the outer coat of the latter, constituting $(c)$ the peri-bronchial lymphatics, which anastomose with $b$. The branches of these two sets run towards the bronchial glands. Not unfrequently (cat) masses of adenoid tissue are found in the course of these lymphatics (Klein)]. The lymph-canalicular system and the lymphatics become injected when fine coloured particles are inspired, or are introduced into the air-cells artificially. The pigment particles pass through the semi-fluid cement substance into the lymph-canalicular system and thence into the lymphatics (v. Wittich); or, according to Klein, they pass through actual holes or pores in the cement (p. 221). [This pigmentation is well seen in coal-miners' lung or anthracosis, where the particles of carbon pass into and are found in the lymphatics. Sikorski and Kuttner showed that pigment reached the lymphatics in this way during life. If pigment, China ink, or indigo carmine be introduced into a frog's lung, it is found in the lymphatic system of the lung. Ruppert, and also Schottelius, showed that the same result occurred in dogs after the inhalation of charcoal, cinnabar, or precipitated Berlin blue, and von Ins after the inhalation of silica. A. Schestopal used China ink and cinnabar suspended in $\frac{3}{4}$ p.c. salt solution.]

Excessively fine lymph-canals lie in the wall of the alveoli in the interspaces of the capillaries, and there are slight dilatations at the points of erossing (Wydwozoff). According to Pierret and Renaut every air-cell of the lung of the ox is surrounded by a large lymph-space, such as occurs in the salivary glands. When a large quantity of fluid is injected into the lung it is absorbed with great rapidity, even blood-corpuscles rapidly pass into the lymphatics. [Nothnagel found that, if blood was sucked into the lung of a rabbit, the blood-corpuscles were found within the interstitial connective-tissue of the lung after $3 \frac{1}{2}-5$ minutes, from which he concludes that the communications between the cavity of the air. cells and the lymphatics must be very numerous.]

The superficial lymphatics of the pulmonary pleura communicate with the pleural cavity by means of free openings or stomata (Klein), and the same is true of the lymphatics of the parietal pleura, but these stomata are confined to limited areas over the diaphragmatic pleura. [The lymphatics in the costal pleura occur over the intercostal spaces and not over the ribs (Dybkowski).] The large arteries of the lung are provided with lymphatics which lie between the middle and outer coats (Grancher).

[The movements of the lung during respiration are most important factors in moving the lymph onwards in the pulmonary lymphatics. The return of the lymph is prevented by the presence of valves.]

[The Nerves of the lung are derived from the anterior and posterior pulmonary plexuses, and consist of branches from the vagus and sympathetic. They enter the lungs and follow the distribution of the bronchi, several sections of nerve. 
trunks being usually found in a section of a large bronchial tube. These nerves lie outside the cartilages, and are in close relation with the branches of the bronchial arteries. Medullated and non-medullated nerve-fibres occnr in the nerves, which also contain numerous small ganglia (Remak, Klein, Stirling). In the ling of the calf these ganglia are so large as to be macroscopic. The exact mode of termination of the nerve-fibres within the lung has yet to be ascertained in mammals, but some fibres pass to the bronchial muscle, others to the large blood-vessels of the lung, and it is highly probable that the mucous glands are also supplied with nerve filaments. In the comparatively simple lungs of the frog, nerves with numerous nerve-cells in their course are found (Arnold, Stirling), and in the very simple lung of the newt, there are also numerous nerve-cells disposed along the course of the intra-pulmonary nerves. Some of these fibres terminate in the uniform layer of non-striped muscle which forms part of the pulmonary wall in the frog and newt, and others end in the muscular coat of the pulmonary blood-vessels (Stirling). The functions of these ganglia are unknown, but they may be compared to the nerve-plexuses existing in the walls of the digestive tract.]

The Function of the Non-striped Muscle of the entire bronchial system seems to be to offer a sufficient amount of resistance to increased pressure within the air-passages; as in forced expiration, speaking, singing, blowing, etc. The vagus is the motor-nerve for these fibres, and according to Longet (1842), the "lung-tonus" during increased tension depends upon these muscles. Stimulation of the lower end of the vagus causes a slight contraction of the bronchial muscles, but the movement is neither sudden nor considerable. It is highly doubtful if bronchial (spasmodic) asthma depends upon contraction of these muscular fibres due to stimulation of the vagus.

Chemistry.--In addition to connective, elastic, and muscular tissue, the lungs contain lecithin, inosit, uric acid (taurin and leucin in the ox), guanin, xanthin (?), hypoxanthin $(\operatorname{dog})$-soda, potash, magnesium, oxide of iron, much phosphcric acid, also chlorine, sulphuric and silicic acids-in diabetes sugar occurs-in purulent infiltration glycogen and sugar-in renal degeneration urea, oxalic acid, and ammonia salts; and in diseases where decomposition takes place, leucin and tyrosin.

[Physical Properties of the Lungs.-The lungs, in virtue of the large amount of elastic tissue which they contain, are endowed with great elasticity, so that when the chest is opened, they collapse. If a cannula with a small lateral opening be tied into the trachea of a rabbit's or sheep's lungs, the lungs may be inflated with a pair of bellows, or elastic pump. After the artificial inflation, the lungs, owing to their elasticity, collapse and expel the greater part of the air. As much air remains within the light spongy tissue of the lungs, even after they are removed from the body, a healthy lung floats in water. If the air-cells are filled with pathological fluids or blood, as in certain diseased conditions of the lung (pneumonia), then the lungs or parts thereof may sink in water. The lungs of the fotus, before respiration has taken place, sink in water, but after respiration has been thoroughly established in 
the child, the lungs float. Hence, this hydrostatic test is largely used in medico-legal cases, as a test of the child having breathed. If a healthy lung be squeezed between the fingers, it emits a peculiar and characteristic fine crackling sound, owing to the air within the air-cells. A similar sound is heard on cutting the vesicular tissue of the lung. The colour of the lungs varies much; in a young child it is rose-pink, but afterwards it becomes darker, especially in persons living in towns or a smoky atmosphere, owing to the deposition of granules of carbon. In coal-miners the lungs may become quite black.]

\section{Mechanism of Respiration.}

The mechanism of respiration consists in an alternate dilatation and contraction of the chest. The dilatation is called inspiration, the contraction expiration. As the whole external surfaces of both elastic lungs are applied directly, and in an air-tight manner by their smooth moist pleural investment to the inner wall of the chest, which is covered by the parietal pleura, it is clear, that the lungs must be distended with every dilatation of the chest, and diminished by every contraction thereof. These movements of the lungs, therefore, are entirely passive, and are dependent on the thoracic movements (Galen).

On account of their complete elasticity and their great extensibility, the lungs are able to accommodate themselves to any variation in the size of the thoracic carity, without the two layers of the pleura becoming separated from each other. As the capacity of the non-distended chest is greater than the volume of the collapsed lungs after their removal from the body, it is clear that the lungs even in their natural position within the chest are distended, i.e., they are in a certain state of ELASTIC TENSION ( $(60)$. The tension is greater the more disterided the thoracic cavity, and vice versit. As soon as the pleural cavity is opened by perforation from without, the lungs, in virtue of their elasticity, collapse, and a space filled with air is formed between the surface of the lungs and the inner surface of the thoracic wall (pneumothorax). The lungs so affected are rendered useless for respiration; hence a double pneumo-thorax causes death.

It is also clear that, if the pulmonary pleura be perforated from within the lung, air will pase from the respiratory passages into the pleural sac, and also give rise to pneumo-thorax.

[Not unfrequently the surgeon is called on to open the chest, say by removing a portion of a rib to allow of the free exit of pus from the pleural cavity. If this be done with proper precautions, and if the external wound be allowed to heal, after a time the air in the pleural cavity becomes absorbed, the collapsed lung tends to regain its original form, and again becomes functionally active.] 
Estimation of Elastic Tension.-If a manometer be introduced through an intercostal space into the pleural cavity, in a dead subject, we can measure, by means of a column of mercury, the amount of the elastic tension required to keep the lung in its position. This is equal to $6 \mathrm{~mm}$. in the dead subject, as well as in the condition of expiration. If, however, the thorax be brought into the position of inspiration by the application of traction from without, the elastic tension may be increascd to $30 \mathrm{~mm}$. $\mathrm{Hg}$. (Donders).

If the glottis be closed and a deep inspiration taken, the air within the lungs must become rarified, because it has to fill a greater space. If the glottis be suddenly opened, the atmospheric air passes into the lungs until the air within the lungs has the same density as the atmosphere. Conversely, if the glottis be closed, and if an expiratory effort be made, the air within the chest must be compressed. If the glottis be suddenly opened, air passes out of the lungs until the pressure outside and inside the lung is equal. As the glottis remains open during ordinary respiration, the equilibration of the pressure within and without the lungs will take place gradually. During tranquil inspiration there is a slight negative pressure; during expiration a slight positive pressure in the lungs; the former $=1 \mathrm{~mm}$., the latter, $2-3 \mathrm{~mm}$. Hg. in the human trachea (measured in cases of wounds of the trachea).

\section{Quantity of Gases Respired.}

As the lungs within the chest never give out all the air they contain, it is clear, that only a part of the air of the lungs is changed during inspiration and expiration. The volume of this air will depend upon the depth of the respirations.

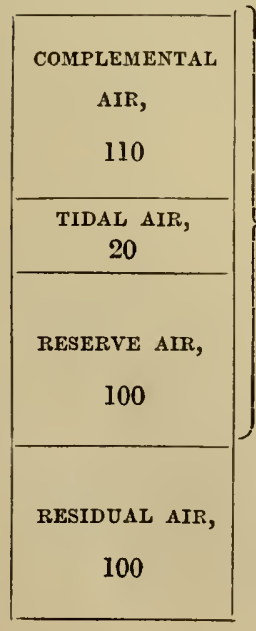

Hutchinson (1846) distinguishes the following points :-

(1.) Residual Air is the volume of air which remains in the chest after the most complete expiration. It is equal to $1,230-1,640$ c.c. $[100-130$ cubic inches.]

(2.) Reserve or Supplemental Air is the volume of air which can be expelled from the chest after a normal quiet expiration. It is equal to $1,240-1,800$ c.c. [100 cubic inches.]

(3.) Tidal Air is the volume of air which is taken in and given out at each respiration. It is equal to 500 cubic centimetres [ 20 cubic inches.]

(4.) Complemental Air is the volume of air that can be forcibly inspired over and above what is taken in at a normal respiration. It amounts to about 1,500 c. c. [100-130 cubic inches.] 
(5.) Vital Capacity is the term applied to the volume of air which can be forcibly expelled from the chest after the deepest possible inspiration. It is equal to 3,772 c.c. (or 230 cubic inches) for an Englishman (Hutchinson), and 3,222 for a German (Haeser).

Hence, after every quiet inspiration, both lungs contain $(1+2+3)$ $=3,000-3,900$ c.ctmr. [220 cubic inches]; after a quiet expiration $(1+2)=2,500-3,400$ c.ctmr. [200 cubic inches.] So that about $\frac{1}{6}$ to $\frac{1}{7}$ of the air in the lungs is subject to renewal at each respiration.

Estimation of Vital Capacity.-The estimation of the vital capacity was formerly thought to be of great consequence, but at the present time not much importance is attached to it, nor is it frequently measured in cases of disease. It is estimated by means of the SPIROMETER of

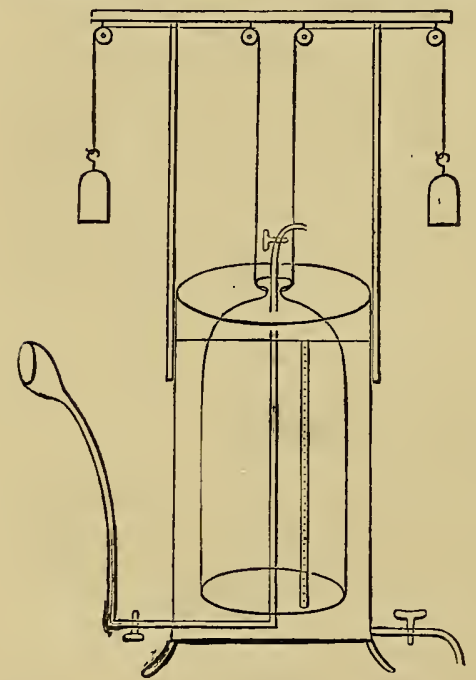

Fig. 101.

Hutchinson. This instrument (Fig. 101), consists of a graduated cylinder filled with water and inverted like a gasometer over water, and balanced by means of a counterpoise. Into this cylinder a tube projects, and this tube is connected with a mouth-piece. The person to be experimented upon takes the deepest possible inspiration, closes his nostrils, and breathes forcibly into the mouth-piece of the tube. After doing so the tube is closed. The cylinder is raised by the air forced into it, and after the water inside and outside the cylinder is equalised, the height to which the cylinder is raised indicates the Scheme of Hutchinson's Spirometer. amount of air expired, or the vital or respiratory capacity. In a man of average height, 5 feet 8 inches, it is equal to 230 cubic inches.

The following circumstances affect the vital capacity :-

(1.) The height. - Every inch added to the height of persons between 5 and 6 feet, gives an increase of the vital capacity $=130$ c.c. [ 8 cubic inches.]

(2.) The body-weight. - When the body-weight exceeds the normal by 7 per cent., there is a diminution of $\mathbf{3 7}$ c.c. of the vital capacity for every kilo. of increase.

(3.) Age. - The vital capacity is at its maximum at 35 ; there is an annual decrease of 23.4 c.c. from this age onwards to 65 , and backwards to 15 years of age.

(4.) Sex-It is less in women than men, and even where there is the same circumference of chest, and the same height in a man and a woman, the ratio is $10: 7$.

(5.) Position.-More air is respired in the erect than in the recumbent position.

(6.) Disease.-Abdominal and thoracic diseases diminish it. 


\section{Number of Respirations.}

In the adult, the number of respirations varies from 16 to 24 per minute, so that about 4 pulse-beats occur during each respiration. The number of respirations is influenced by many conditions :-

(1.) The position of the body.-In the adult, in the horizontal position, Guy counted 13, while sitting 19 , while standing 22 , respirations per minute.

(2.) The age.-Quetelet found the mean number of respirations in 300 individuals to be :-

$\left.\begin{array}{cccc}\text { Year. } & & & \text { Respirations. } \\ 0-1, & . & . & 44 \\ 5, & . & . & 26 \\ 15-20, & . & . & 20 \\ 20-25, & . & . & 15 \cdot 7 \\ 25-30, & . & . & 16 \\ 30-50, & . & . & 15 \cdot 1\end{array}\right\}$ Average Number

(3.) The state of activity.-Gorham counted in children of 2 to 4 years of age, during standing 32 , in sleep 24, respirations per minute. During bodily exertion the number of respirations increases before the heart-beats. [Tery slight muscular exertion suffices to increase the frequency of the respirations.]

[(4.) The temperature of the surrounding medium. - The respirations become more numerous the higher the surrounding temperature, but this result only occurs when the actual temperature of the blood is increased, as in fever.

(5.) Digestion.-There is a slight variation during the course of the day, the increase being most marked after mid-day dinner (Vierordt).

(6.) The will can to a certain extent modify the number and also the depth of the respirations, but after a short time the impulse to respire overcomes the voluntary impulse.

(7.) The gases of the blood have a marked effect, and so has the heat of the blood in fever.]

\section{Time occupied by the Respiratory Movements.}

The time occupied in the various phases of a respiration can only be accurately ascertained by obtaining a curve or pneumatogram of the respiratory movements.

Methods.-Vierordt and C. Ludwig transferred the movements of a part of the chest-wall to a lever which inscribed its movements upon a revolving cylinder. Riegel (1S73) constructed a "double stethograph" on the same principle. This instrument is so arranged that one arm of the lever may be applied in connection with the healthy side of a person's chest, and the other on the unsound side.

(2.) An air-tambour, such as is used in Brondgeest's pansphygmograph (Fig. 103, A) may be used. It consists of a brass vessel, a, shaped like a small sancer. The mouth of the brass vessel is corered with a double layer of caoutchouc membrane, $b, c$, and air is forced in between the two layers until the external membrane bulges outwards. This is placed on the chest, and the apparatus is fixed in position by means of the bands, $d, d$. The cavity of the tambour communicates by means of a canutchouc tube, $s$, with a recording tambour which inscribes its 


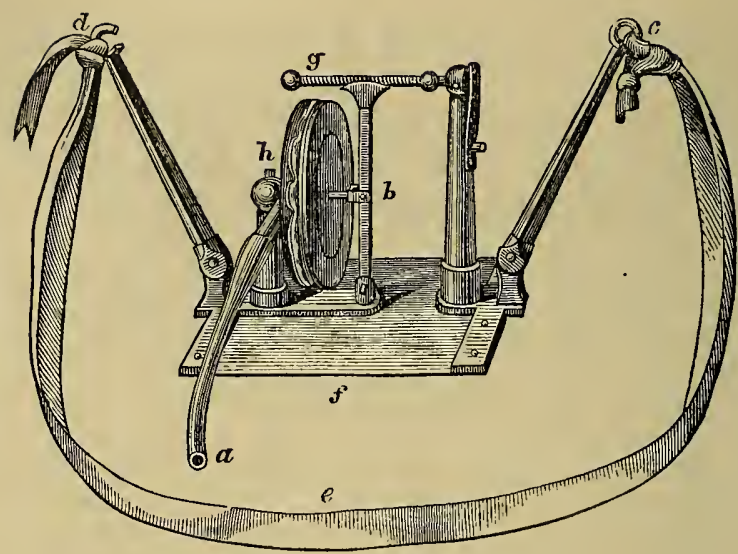

Fig. 102.

Marey's Stethograph.

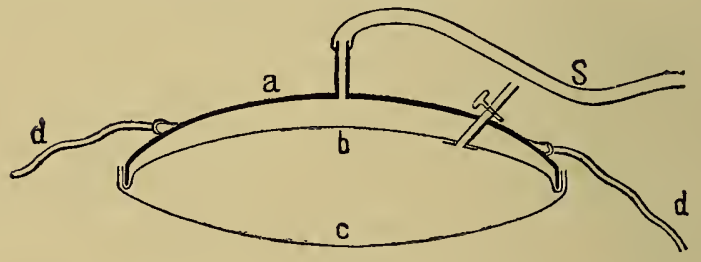

A

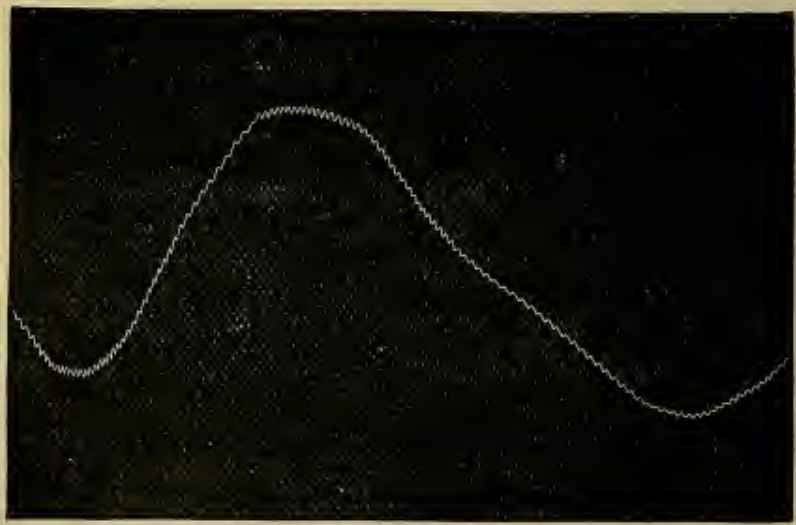

B

Fig. 103.

A, Brondgeest's tambour for registering the respiratory movements $-b$, $c$, inner and outer caoutchouc membranes; $a$, the capsule; $d, d$, cords for fastening the instrument to the chest; $\mathrm{S}$, tube to the recording tambour; $\mathrm{B}$, normal respiratory curve obtained on a vibrating plate (each vibration $=0.01613 \mathrm{sec}$.). 
movements upon a revolving cylinder. Every dilatation of the chest compresses the membrane, and thus the air within the tambour is also compressed.

(3.) A caunula or œsophageal sound may be introduced into that portion of the œsophagus which lies in the chest, and a connection established with an Upham's capsule - p. 132 (Rosenthal).

Marey's Stethograph or Pncumograph. - [There are two forms of this instrument, one modified by P. Bert and the more modern form (Fig. 102). A tambour $(h)$ is fixed at right angles to a thin elastic plate of steel $(f)$. The aluminium disc on the caoutchouc of the tambour is attached to an upright $(b)$, whose end lies in contact with a horizontal screw $(g)$. Two arms $(d, c)$ are attached to opposite sides of the steel plate, and to them the belt (e) which fastens the instrument to the chest is attached. When the chest expands these two arms are pulled asunder, the steel plate is bent and the tambour is affected, and any movement of the tambour is transmitted to a registering tambour by the air in the tube $(a)$.]

In the case of animals placed on their backs, Snellen introduced a long needlc vertically through the abdominal walls into the liver. Rosenthal opened the abdomen and applied a lever to the under surface of the diaphragm, and thus registered its movements (PHRENOGRAPH).

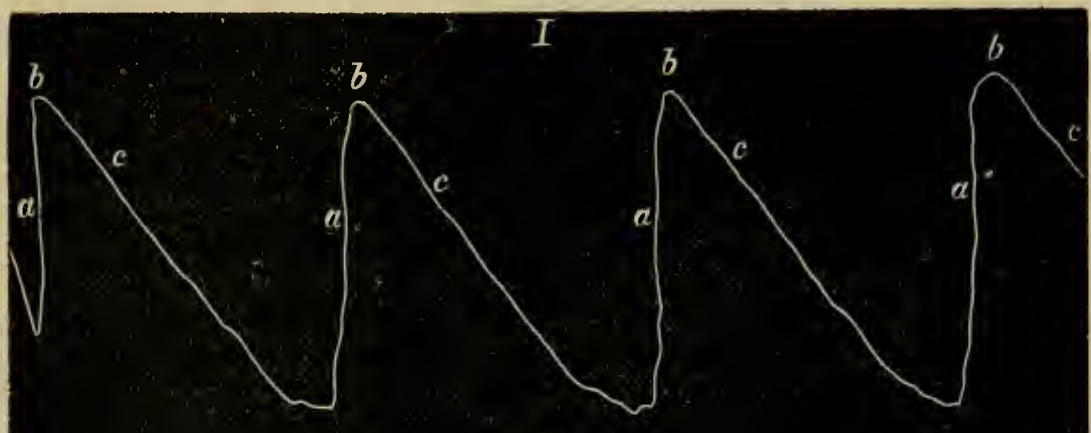

II

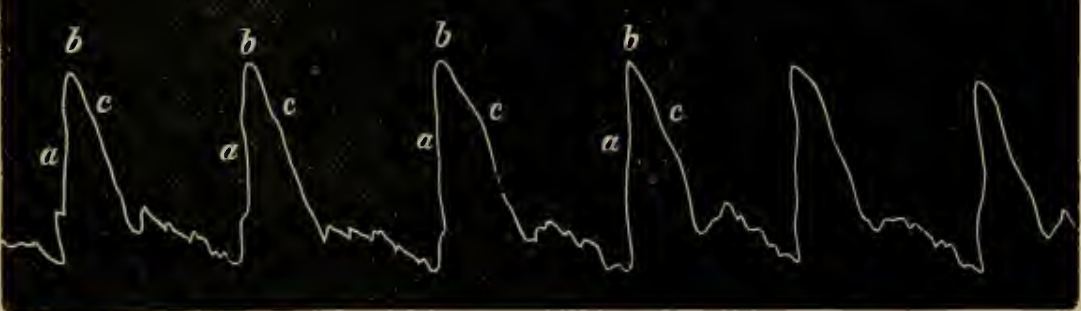

Fig. 104.

Pueumatogram obtained by means of Riegel's Stethograph-I, normal curves; II, curve from a case of emphysema; $a$, ascending limb; $b$, apex; $c$, descending limb of the curve. The small elevations are due to the cardiac impulse.

The curve (Fig. 103, B) was obtained by placing the tambour of a Brondgeest's pansphygmograph upon the xiphoid process, and recording 
the movement upon a plate attached to a vibrating tuning-fork. The inspiration (ascending limb) begins with moderate rapidity, is accelerated in the middle, and towards the end again becomes slower. The expiration also begins with moderate rapidity, is then accelerated, and becomes much slower at the latter part, so that the curve falls very gradually.

Inspiration is slightly shorter than expiration.-According to Sibson, the ratio for an adult is as 6 to 7 ; in women, children, and old people, 6 to 8 or 6 to 9 . Vierordt found the ratio to be 10 to $14 \cdot 1$ (to $24 \cdot 1$ ); J. R. Ewald, 11 to 12 . It is only occasionally that cases occur where inspiration and expiration are equally long, or where expiration is shorter than inspiration. When respiration proceeds quietly and regularly, there is usually no pause (complete rest of the chest-walls) between the inspiration and expiration (Riegel). The very flat part of the expiratory curve has been wrongly regarded as due to a pause. Of course, we may make a voluntary pause between two respirations, or at any part of a respiratory act.

Some observers, however, have described a pause as occurring between the end of expiration and the beginning of the next inspiration (expiration pause), and also another pause at the end of inspiration (inspiration pause). The latter is always of very short duration, and considerably shorter than the former.

During very deep and slow respiration, there is usually an expiration pause, while it is almost invariably absent during rapid breathing. An inspiration pause is always absent under normal circumstances, but it may occur under pathological conditions.

In certain parts of the respiratory curve slight irregularities may appear, which are sometimes due to vibrations communicated to the thoracic walls by vigorous heart-beats (Fig. 104).

The "type" of respiration may be ascertained by taking curves from various parts of the respiratory movements. Hutchinson showed that in the female, the thorax is dilated chiefly by raising the sternum and the ribs (Respiratio costalis), while in man it is caused chiefly by a descent of the diaphragm (Respiratio diaphragmatica or abdominalis). In the former, there is the so-called "costal type," in the latter the "abdominal or diaphragmatic type."

Forced Respiration.-This difference in the type of respiration in the sexes occurs only during normal quiet respiration. During deep and forced respiration, in both sexes the dilatation of the chest is caused chiefly by raising the chest and the ribs. In man, the epigastrium may be pulled in sooner than it is protruded. During sleep, the type of respiration in both sexes is thoracic, while at the same time the inspiratory dilatation of the chest precedes the elevation of the abdominal wall (Mosso).

It is not determined whether the costal type of respiration in the female depends upon the constriction of the chest by corsets or other causes (Sibson), or whether it is a natural adaptation to the child-bearing function in women (Hutchinson). Some observers maintain that the difference of type is quite distinct, even in sleep, when all constrictions are removed, and that similar differences are noticeable in young children. This is denied by others, while a third class of observers hold 
that the costal type occurs in children of both sexes, and they ascribe as a cause the greater flexibility of the ribs of children and women, which permits the muscles of the chest to act more efficiently upon the ribs. [When a child sucks, it breathes exclusively through the nose, hence catarrhal conditions of the nasal mucous membrane are franght with danger to the child.]

\section{Pathological Variations of the Respiratory Movements.}

I. Changes in the mode of movement.-In persons suffering from disease of the respiratory organs, the dilatation of the chest may be diminished (to the extent of 6 or $5 \mathrm{cmtr}$.) on both sides or only on one side. In affections of the apex of the lung (in phthisis), the sub-normal expansion of the upper part of the wall of the chest may be considerable. Retraction of the soft parts of the thoracic wall, the xiphoid process and the parts where the lower ribs are inserted, occurs in cases where air cannot freely enter the chest during inspiration, e.g., in narrowing of the larynx; when this retraction is contined to the upper part of the thoracic wall, it indicates that the portion of the lung lying under the part so affected is less extensile and diseased.

Harrison's Groove.-In persons suffering from chronic difficulty of breathing, and in whom, at the same time, the diaphragm acts energetically, there is a slight groove which passes horizontally outwards from the xiphoid cartilage, caused by the pulling in of the soft parts and corresponding to the insertion of the diaphragm.

The duration of inspiration is lengthened in persons suffering from narrowing of the trachea or larynx; expiration is lengthened in cases of dilatation of the lung, as in emphysema, where all the expiratory muscles must be brought into action (Fig. 104).

II. Variations in the Rhythm. - When the respiratory apparatus is much affected, there is either an increase or a deepening of the respirations, or both. When there is great difficulty of breathing, this is called Dyspnœa.

Canses of Dyspnœa.-(1) Limitation of the exchange of the respiratory gases in the blood due to-(a) Diminution of the respiratory surface (as in some diseases of the Iungs); (b) narrowing of the respiratory passages; $(c)$ diminution of the red blood-corpuscles; $(d)$ disturbances of the respiratory mechanism (e.g., due to affections of the respiratory muscles or uerves, or painful affections of the chest-wall); (e) impeded circulation through the lungs due to various forms of heart-disease. (2) Heat-dyspnca. - The frequency of the respirations is increased in febrile conditions. The warm blood acts as a direct irritant of the respiratory centre in the mednlla oblongata, and raises the number of respirations to $30-60$ per minute ("Heat-dyspncea"). If the carotids be placed in warm tubes, so as to heat the blood going to the medulla oblongata, the same phenomena are produced ( $A$. Fick.) See also "Respiratory centre" (vol. ii.).

Cheyne-Stokes' Phenomenon. - This remarkable phenomenon occurs in certain diseases, where the normal supply of blood to the brain is altered, or where the quality of the blood itself is altered, e.g., in certain affections of the brain and heart, and in uræmic poisoning. Respiratory pauses of one-half to three-quarters of a minute alternate with a short period $\left(\frac{1}{2}-\frac{3}{4} \mathrm{~min}\right.$.) of increased respiratory activity, and during this time $20-30$ respirations occur. The respirations constituting this "series" are shallow at first; gradually they become deeper and more dyspnoeic, and finally become shallow or superficial again. Then follows the pause, and thus there is an alternation of pauses and series (or groups) of modified respirations. During the pause, the pupils are contracted and inactive; and when the respirations begin, they dilate and become sensible to light; the eyeball is moved as a whole at the same time (Lenbe). Hein observed that consciousness was abolished during the panse, 
and that it returued when respiration commenced. A few muscular contractions may occur towards the end of the pause (rare).

With regard to the eauses of this phenomenon there is some doubt. According to Rosenbach, the anomalous nutrition of the brain causes certain intracranial centres, especially the respiratory centre, to be less excitable and to be sooner exhausted, and this condition reaches its maximum during the respiratory pause. During the pause these centres recover, and they again become more active. As soon as they are again exhausted, their activity ceases. Luciani also regards variations in the excitability of the respiratory centre as the cause of the phenomenon, which he compares with the periodic contraction of the heart (p. 104). He observed this phenomenon after injury to the medulla oblongata above the respiratory centre, and after apnœa produced in animals deeply narcotised with opium. It also occurs in the last stages of asphyxia, during respiration in a closed space. Mosso found a similar phenomenon normally in the hybernating dormouse (Myoxus.)

Periodic Respiration of Frogs.-If frogs be kept under water, or if the aorta be clamped, after several hours, they become passive. If they be taken out of the water, or if the clamp be removed from the aorta, they gradually recover and always exhibit the Cheyne-Stokes' phenomenon. In such frogs the blood. current may be arrested temporarily, while the phenomenon itself remains (Sokolow and Luchsinger). If the blood-current be arrested by ligature of the aorta, or if the frogs be bled, the respirations occur in groups. This is followed by a few single respirations, and then the respiration ceases completely. During the pause between the periods, mechanical stimulation of the skin causes the discharge of a group of respirations (Siebert and Langenclorff). Muscarin and digitalin cause periodic respiration in frogs [which is not due to the action of these drugs on the heart.]

\section{General View of the Respiratory Muscles.}

\section{(A.) Inspiration.}

\section{During Ordinary Inspiration are Active.}

1. The diaphragm (Nervus phrenicus.)

2. The Mm. levatores costarum longi et breves (Rami posteriores Nn. dorsalium).

3. The $\mathrm{Mm}$. intercostales externi et intercartilaginei $(\mathrm{Nn}$. intercostales).

\section{During Forced Respiration are Active.}

(a.) Muscles of the Trunk.

1. The three Mm. scaleni (Rami musculares of the plexus cervicalis et irachialis).

2. M. sternocleidomastoideus (Ram. externus $N$. accessorii).

3. M. trapezius (R. externus N. accessorii et Ram. musculares plexus cervicalis).

4. M. pectoralis minor ( $N n$. thoracici anteriores).

5. M. serratus posticus superior ( $N$. dorsalis scupulae).

6. Mm. rhomboidei ( $N$. dorsalis scapulae). 
7. Mm. extensores columnae vertebralis (Ram. posteriores neriorum dorsalium).

[8. Mm. serratus anticus major (N. thoracicus longus). ? ?]

(b.) Muscles of the Larynx.

1. M. sternoliyoideus (Ram. descendens hypoglossi).

2. M. sternothyreoideus (Ram. descendens hypoglossi).

3. M. crico-arytaenoideus posticus ( $N$. laryngeus inferior vayi).

4. M. thyreo-arytaenoideus ( $N$. laryngeus inferior vagi).

(c.) NIuscles of the Face.

1. M. dilatator narium anterior et posterior ( $N$. facialis).

2. M. levator alae nasi ( $N$. facialis).

3. The dilators of the mouth and nares, during forced respiration, ["gasping for breath"] (N. facialis).

(d.) Muscles of the Pharynx.

1. M. levator veli palatini ( $N$. facialis).

2. M. azygos uvulae ( $N$. facialis).

3. According to Garland, the pharynx is always narrowed.

\section{(B.) Expiration.}

\section{During Ordinary Respiration.}

The thoracic cavity is diminished by the weight of the chest, the elasticity of the lungs, costal cartilages, and abdominal muscles.

\section{During Forced Expiration.}

The Abdominal Muscles.

1. The abdominal muscles [including the obliquus externus and internus, and transversalis abdominis] ( $N n$. abdominis internis anteriores e nervis intercostalibus, 8-12).

2. Mm. intercostales interni, so far as they lie between the osseous ribs, and the $\mathrm{Mm}$. infracostales ( $\mathrm{N} n$. intercostales).

3. M. triangularis sterni ( $N n$. intercostales).

4. M. serratus posticus inferior (Ram. externi nerv. dorsalium).

5. M. quadratus lumborum (Ram. muscular e plexu lumbali).

\section{Action of the Individual Respiratory Muscles.}

(A.) Inspiration.-(1.) The Diaphragm arises from the cartilages and the adjoining osseous parts of the lower six ribs (costal portion), by two thick processes or crura from the upper three or four lumbar vertebrae, and a sternal portion from the back of the ensiform process. 
It represents an arched double cupola or dome-shaped partition, directed towards the chest; in the larger concavity on the right side lies the liver, while the smaller arch on the left side is occupied by the spleen and stomach. During the passive condition, these viscera are pressed against the under surface of the diaphragm, by the elasticity of the abdominal walls and by the intra-abdominal pressure, so that the arch of the diaphragm is pressed upwards into the chest. The elastic traction of the lungs also aids in producing this result. The greater part of the upper surface of the central tendon of the diaphragm is united to the pericardium. The part on which the heart rests, and which is perforated by the inferior vena cava (foramen quadrilaterum) is the deepest part of the middle portion of the diaphragm during the passive condition.

Action of the Diaphragm.-When the diaphragm contracts, both arched portions become flatter, and the chest is thereby elongated from above downwards. In this act, the lateral muscular parts of the diaphragm pass from an arched condition into a flatter form (Fig. 105),

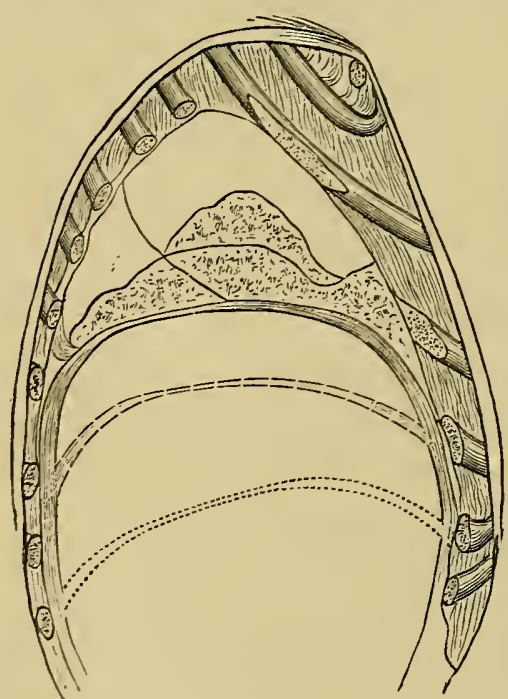

Fig. 105.

Sagittal section through the second rib on the right side. This figure shows that when the arched muscular part of the diaphragm contracts, a wedgeshaped space, with its apex downwards, is formed around the circum. ference of the lower part of the chest, so that the chest is enlarged from above downwards. and during a forced inspiration, the lowest lateral portions, which during rest are in contact with the chest-wall, become separated from it. The middle of the central tendon where the heart rests (fixed by means of the pericardium and inferior vena cava) takes no share in this movement; hence, this part is highest in the thorax during a forced inspiration.

Undoubtedly, the diaphragm is the most powerful agent in increasing the cavity of the chest. Brücke, in fact, believes that in addition to increasing the length of the thoracic cavity from above downwards, it also increases the transverse diameter of the lower part of the chest. It presses upon the abdominal viscera from above, and strives to press these outwards, thus tending to push out the adjoining thoracic wall.

If the contents of the abdomen are removed from a living animal, every time the diaphragm contracts, the ribs are drawn inwards (Haller). This, of course, hinders the chest from becoming wider below, hence the presence of the abdominal viscera seems to be necessary for the normal activity of the diaphragm.

The immense importance of the diaphragm as the great inspiratory muscle is proved by the fact that, after both phrenic nerves (third and fourth cervica nerves) 
are divided, death occurs (Budge, Eulenkamp). The phrenic nerve contains some sensory fibres for the pleura, pericardium, and a portion of the diaphragm (Schreiber, Henle, Schwalbe).

The contraction of the diaphragm is not to be regarded as a "simple muscular contraction," since it lasts 4 to 8 times longer than a simple contraction; it is rather a short tetanic contraction, which we may arrest at any stage of its activity without bringing into action any antagonistic muscles (Kronecker and Marckwald).

(2.) The Elevators of the Ribs.-The ribs at their vertebral ends (which lie much higher than their sternal ends) are united by means of joints by their heads and tubercles to the bodies and transverse processes of the vertebre. A horizontal axis can be drawn through both joints, around which the ribs can rotate upwards and downwards. If the axes of rotation of each pair of ribs be prolonged on both sides until they meet in the middle line, the angles so formed are greatest above $\left(125^{\circ}\right.$ ), and smaller below $\left(\mathrm{SS}^{\circ}\right)$ (A. W. Volkmann). Owing to the ribs being curved, we can imagine a plane which, in the passive (expiratory) condition of the chest, has a slope from behind and inwards to the front and outwards. If the ribs move on their axis of rotation this plane becomes more horizontal, and the thoracic cavity is increased in its transverse diameter. As the axis of rotation of the upper ribs runs in a more frontal, and that of the lower ribs in a more sagittal, direction, the elevation of the upper ribs causes a greater increase from before backwards, and the lower ribs from within outwards (as the movements of ribs which are directed downwards are vertical to the axis). The costal cartilages undergo a slight tension at the same time, which brings their elasticity into play.
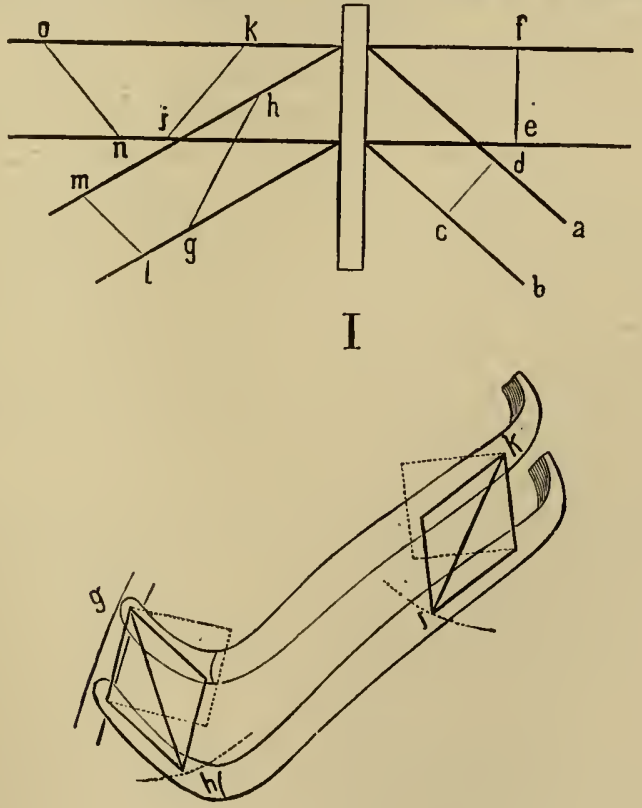

Fig. 106.

Scheme of the action of the intercostal muscles.

Changes in the Chest.-All "inspiratory muscles" which act directly upon the chest-wall, do so by raising the ribs:-(a.) When the ribs are 
raised, the intercostal spaces are widened. (b.) When the upper ribs are raised, all the lower ribs and the sternum must be elevated at the same time, because all the ribs are connected with each other by means of the soft parts of the intercostal spaces. (c.) During inspiration, there is an elevation of the ribs and a dilatation of the intercostal spaces. (The lowest rib is an exception; during forced respiration, at least, it is drawn downwards). (d.) If, on a preparation of the chest, the ribs be raised as in inspiration, we may regard all those muscles as elevators of the ribs, whose origin and insertion become approximated. Everyone is agreed that the scaleni and levatores costarum longi et breves, the serratus posticus superior, are inspiratory muscles. These are the most important inspiratory muscles which act upon the ribs.

Intercostal Muscles.-With regard to the action of the intercostal muscles, there is a great difference of opinion. According to the above experiment, the external intercostals and the intercartilaginous parts of the internal intercostals act as inspiratory muscles, whilst the remaining portions of the internal intercostals (as far as they are covered by the external) are elongated when the ribs are raised, while they shorten when the chest-wall descends. A muscle shortens only during its activity. The internal intercostals were regarded by Hamberger (1727) as depressors of the ribs or expiratory muscles.

In Fig. 106, I, when the rods, $a$ and $b$ (which represent the ribs) are raised, the intercostal space must be widened $(e f>c d)$. On the opposite side of the figure, it is evident that when the rods are raised, the line, $g h$, is shortened ( $i k<g h$, direction of the external intercostals) $-l m$ is lengthened ( $l m<0 n$, direction of internal intercostals). Fig. 106, II, shows, that when: the ribs are raised, the intercartilaginei, indicated by $g h$, and the external intercostals, indicated by $l k$, are shortened. When the ribs are raised, the position of the muscular fibres is indicated by the diagonal of the rhomb becoming shorter.

The mode of action of the intercostal muscles is an old story. Galen (131-203 A.D.) regarded the externals as inspiratory, the internals as expiratory. Hamberger (1727) accepted this proposition and considered the intercartilaginei also as inspiratory. Haller took both the external and internal intercostals as inspiratory, while Vesalius (1540) regarded both as expiratory. Landerer observing that the upper two or three intercostal spaces became narrower during inspiration, regarded both as active during inspiration and expiration. They keep one rib attached to the other, so that their action is to transmit any strain put upon them to the wall of the chest. On this view they will be in action, even when the distance between their points of attachment becomes greater. Landois regards the external intercostals and intercartilaginei as active only during inspiration, the internal intercostals only during expiration. [Martin and Hartwell exposed the internal intercostals and observed whether they contracted along with the diaphragm, or whether the contractions of these two muscles alternate. As the result of their experiments, they conclude that "the internal intercostal muscles are expiratory throughout their whole extent, at least in the dog and cat; and that in the former animal they are almost 'ordinary' muscles of respiration, while in the latter they are 'extraordinary' respiratory muscles."] Landois is of opinion that the chief 
action of these muscles is not to raise or depress the ribs, but rather that the external intercostals and the intercartilaginei offer resistance to the inspiratory dilatation of the intercostal spaces and to the simultaneously increased elastic tension of the lungs. Internal intercostals act during powcrful expiratory efforts, (e.g., coughing), and oppose the distension of the lungs and chest cansed by this act. Unless muscles were present to resist the uninterrupted tension and pressure, the intercostal substance would become so distended that respiration would be impossible. [According to Rutherford, the internal intercostals are probably muscles of inspiration.]

The Pectoralis Minor and (? Serratus Anticus Major) can only act as elevators of the ribs, when the shoulders are fixed, partly by the rhomboidei, and partly by fixing the shoulder-joint and supporting the arms, as is done instinctively by persons suffering from breathlessness.

(3.) Muscles acting upon the Sternum, Clavicle and Vertebral Column. -When the head is fixed by the muscles of the neck, the sternocleidomastoid can raise the manubrium sterni, and the sternal end of the clavicle, so that the thorax is raised and thereby dilated. The scaleni also aid in this act. The clavicular portion of the trapezius may act in a similar, although less energetic, manner. When the vertebral column is straightened, it causes an elevation of the upper ribs, and a dilatation of the intercostal spaces which aid inspiration. During deep respiration, this straightening of the vertebral column takes place involuntarily.

(4.) Laryngeal Movements.-During laboured respiration, with every inspiration, the larynx descends and the glottis is opened. At the same time the palate is raised, so as to permit a free passage to the air entering through the mouth.

(5.) Facial Movements.-During laboured respiration, the facial muscles are involved; there is an inspiratory dilatation of the nostrils (well marked in the horse and rabbit.) When the need for respiration is very great, the mouth is gradually widened, and the person as it were gasps for breath. During expiration, the muscles that are active during 4 and 5 relax, so that a position of equilibrium is established without there being any active expiratory movement to counteract the inspiratory movement. During inspiration the pharynx becomes narrower (Garland.)

(B.) Expiration.-Ordinary expiration occurs without the aid of muscles, owing to the weight of the chest, which tends to fall into its normal position from the position to which it was raised during inspiration. This is aided by the elasticity of the various parts of the chest. When the costal cartilages are raised, which is accompanied by a slight rotation of their lower margins from below forwards and upwards their elasticity is called into play. As soon, therefore, as the inspiratory forces cease, the costal cartilages return to their normal position 
-i.e., the position of expiration-and tend to untwist themselves; at the same time, the elasticity of the distended lungs draws upon the thoracic walls and the diaphragm. Lastly, the tense and elastic abdominal walls, which, in man chiefly, are stretched and pushed forward, tend to return to their non-distended passive condition when the abdominal viscera are relieved from the pressure of the contracted diaphragm. (When the position of the body is reversed, the action of the weight of the chest is removed, but in place of it, there is the weight of the viscera, which press upon the diaphragm.)

The abdominal muscles [obliquus internus and externus, transversalis abdominis and levator ani] are always active during laboured respiration. They act by diminishing the abdominal cavity, and they press the abdominal contents upwards against the diaphragm. When they act simultaneously, the abdominal cavity is diminished throughout its whole extent. The Triangularis sterni depresses the sternal ends of the united cartilages and bones, from the third to sixth ribs downwards; and the Serratus posticus inferior depresses the four lowest ribs, causing the others to follow. It is aided by the Quadratus lumborum, which depresses the last rib. According to Henle, the serratus posticus inferior fixes the lower ribs for the action of the slips of the diaphragm inserted into them, so that it acts during inspiration. According to Landerer, the downward movement of the ribs in the lower part of the thorax dilates the chest.

In the erect position, when the vertebral column is fixed, deep inspiration and expiration naturally alter the position of the centre of gravity, so that during inspiration, owing to the protrusion of the thoracic and abdominal walls, the centre of gravity lies somewhat more to the front. Hence, with each respiration there is an involuntary balancing of the body. During very deep inspiration, the accompanying straightening of the vertebral column and the throwing backwards of the head compensate for the protrusion of the anterior walls of the trunk.

\section{Relative Dimensions of the Chest.}

It is important, from a physician's point of view, to know the dimensions of the thorax, and also the variations it undergoes at different parts. The diameter of the chest is ascertained by means of callipers; the circumference with a flexible centimetre or other measure.

In strong men, the circumference of the upper part of the chest (immediately under the arms) is 88 centimetres ( 34.3 inches), in females 82 centimetres ( 32 inches); on the level of the ensiform process 82 centimetres ( 32 inches) and 78 centimetres ( $30 \cdot 4$ inches) respectively. When the arms are placed horizontally, during the phase of moderate expiration, the circumference immediately under the nipple and the angles of the scapulæ is equal to half the length of the body; in man 
82 , and during deep inspiration 89 centimetres. The circumference at the level of the ensiform cartilage is 6 centimetres less. In old people, the circumference of the upper part of the chest is diminished, so that the lower part becomes the wider of the two. The right half of the chest is usually slightly larger than the left half, owing to the greater development of the muscles on that side. The long diameter of the chest-from the clavicle to the margin of the lowest rib-varies very much.

The transverse diameter in man above and below is $25-26$ centimetres $(9 \cdot 7-10 \cdot 1$ inches), in females $23-24$ centimetres $(8 \cdot 9-9 \cdot 2$ inches); above the nipple it is 1 centimetre more. The antero-

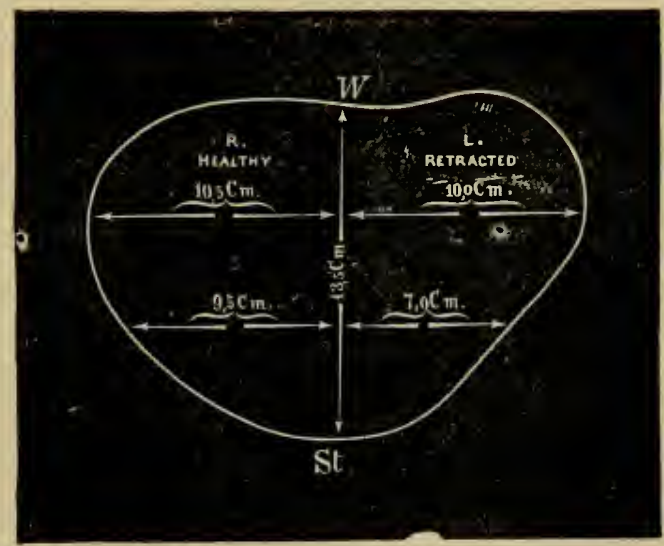

Fig. 107.

Curve taken with the cyrtometer - Left side of the chest retractel in a girl twelve years of age (Eichhorst).

posterior diameter (distance of anterior chest-wall from the tip of a spinous process) in the upper part of the chest is $=17$ ( 6.6 inches), in the lower 19 centimetres $(7 \cdot 4$ inches). Valentin found, that in man during the deepest inspiration the chest on a level with the groove in the heart was increased about $\frac{1}{12}$ to $\frac{1}{7}$; while Sibson estimates the increase at the level of the nipple to be $\frac{1}{10}$.

Thoraco-meter.-In order to obtain a knowledge of the degree of movementrising or falling-of the chest-wall during respiration, various instruments have been invented. The thoraco-meter of Sibson (Fig. 108) measures the elevation in different parts of the sternum. It consists of two metallic bars placed at right angles to each other; one of them, A, is placed on the vertebral column. On B there is placed a movable transverse bar, $\mathrm{C}$, which carries on its free-end a toothed rod, $\mathrm{Z}$, directed downwards. The lower end of this rod is provided with a pad which rests on the sternum, while its toothed edge drives a small wheel which 
moves an index, whose excursions are indicated on a circle with a scale attached to it.

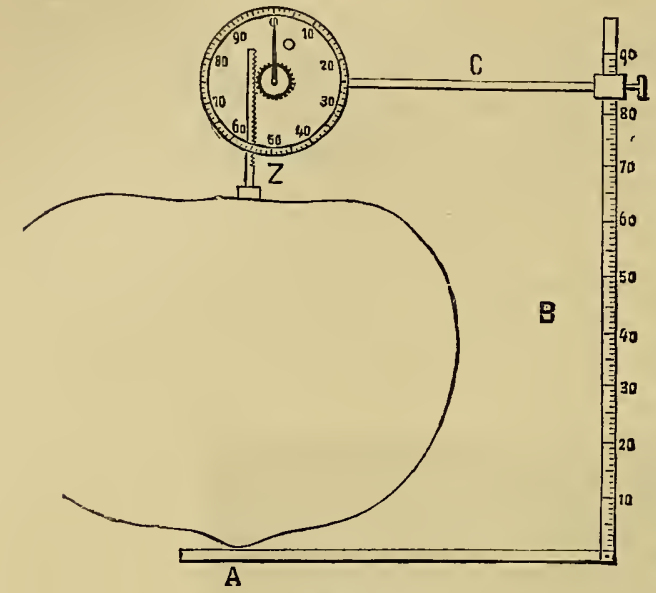

Fig. 108.

Sibson's Thoraco-meter.
The Cyrtometer of Woillez is very useful. A brass chain, composed of movable links, is applied in a definite direction to part of the chest-wall, e.g., trans. versely on a level with the nipple, or vertically upon the mammillary or axillary lines anteriorly. There are freely movable links at two parts which permit the chain to be easily removed, so that as a whole it still retains its form. The chain is laid upon a sheet of paper, and a line drawn with a peucil around its inner margin gives the form of the thorax (Fig. 107).

Limits of the Lungs.-The extent and boundaries of the lungs are ascertained in the living subject by means of Percussion, which consists in lightly tapping the chest-wall by means of a hammer (percussionhammer). A small ivory or bony plate (pleximeter), held in the left-hand, is laid on the chest, and the hammer is made to strike this plate, whereby a sound is emitted, which sound varies with the condition of the subjacent lung-tissue. Wherever the lung substance in contact with the chest-wall contains air, a clear resonant tone or sound - such as is obtained by striking a vessel containing air, a clear percussion sound-is obtained. Where the lung does not contain air, a dull sound-like striking a limb-is obtained. If the parts containing air be very thin, or are only partially filled with air, the sound is "muffled."

Fig. 109, along with Fig. 31, indicate the relations of the lungs to the anterior surface of the chest. The apices of the lungs reach 3-7 centimetres $(1 \cdot 1-2 \cdot 7$ inches) above the clavicles anteriorly, while posteriorly they extend from the spines of the scapulæ as high as the seventh spinous process. The lower margin of the right lung in the passive position (moderate expiration) of the chest, commences at the right margin of the sternum at the insertion of the sixth rib, runs under the right nipple, nearly parallel to the upper border of the sixth rib, and descends a little in the axillary line, to the upper margin of the seventh rib. On the left side (apart from the position of the heart), the lower limit reaches as far down anteriorly as the right. In Fig. 109 the line, $a, t, b$, shows the lowest limit of the passive lungs. Posteriorly, 
both lungs reach as far down as the tenth rib. During the deepest inspiration, the lungs descend anteriorly as far as between the sixth and seventh ribs, and posteriorly to the eleventh rib-whereby the diaphragm is separated from the thoracic (wall (Fig. 105). During the deepest expiration, the lower margins of the lungs are elevated almost as much as they descend during inspiration. In Fig. 109, $m, n$, indicates the margin of the right lung during deep inspiration; $h, l$, during deep expiration.

It is important to observe the relation of the margin of the left lung

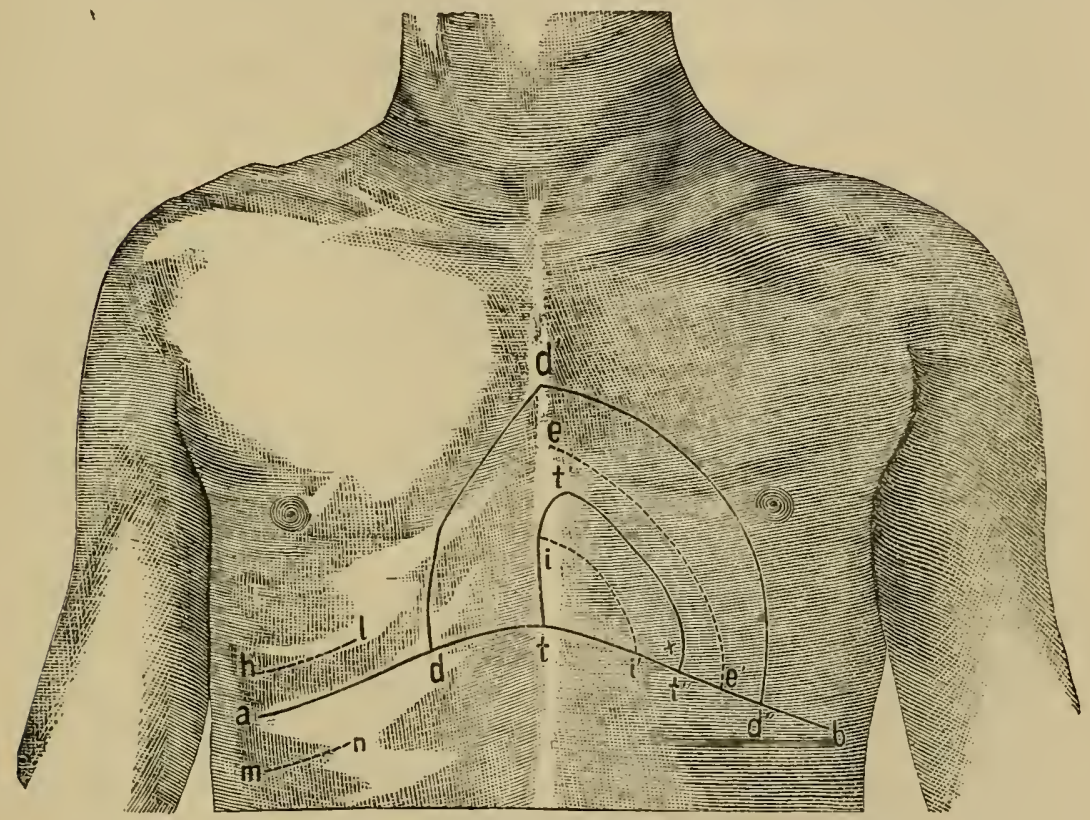

Fig. 109.

Topography of the lungs and heart during inspiration and expiration (v. Dusch)$h, l$, upward limit of margin of lung during deepest expiration; $m, n$, lower limit during deepest inspiration; $t, t^{\prime}, t^{\prime \prime}$, triangular area where the heart is uncovered by lung, dull pereussion sound; $d, d^{\prime}, d^{\prime \prime}$, muffled percussion sound; $i, i^{\prime}$, anterior margin of left lung reaches this line during deep inspiration, and during deep cxpiration it recedes as far as $e, e^{\prime}$.

to the heart. In Fig. 109, a somewhat triangular space, reaching from the middle of the point of insertion of the fourth rib to the sixth rib on the left side of the sternum, is indicated. In the passive chest, the heart lies in contact with the thoracic wall in this triangular area. This area is represented by the triangle, $t, t^{\prime}, t^{\prime \prime}$, and percussion over it gives a dull sound (superficial dulness).

In the area of the larger triangle, $d, d^{\prime}, d^{\prime \prime}$, where the heart is 
separated from the chest-wall by the thin anterior margins of the lung, peruussion gives a muffled sound, while further outwards a clear lung percussion sound is obtained. During deep inspiration, the inner margin of the left lung reaches over the heart as far as the insertion of the mediastinum, whereby the dull sound is limited to the smallest triangle, $t, i, i^{\prime}$. Conversely, during very complete expiration, the margin of the lung recedes so far that the cardiac dulness embraces the space, $t, e, e^{\prime}$.

\section{Pathological Variations of the Percussion Sounds.}

The normal clear resonant percussion sound of the lungs becomes muffled when infiltration takes place into the lungs, so as to diminish the normal amount of air within them, or when the lungs are compressed from without, e.g., by effusion of fluid into the pleura. The percussion sound becomes clearer when the chest-wall is very thin, as in spare individuals during very deep inspiration, and especially in emphysema, where the air-vesicles of certain parts of the lung (apices and margins) become greatly dilated.

The pitch of the percussion sound ought also to be noted. It depends upon the greater or less tension of the elastic pulmonary tissue, and on the elasticity of the thoracic wall. The tcnsion of the elastic tissue is increased during inspiration and diminished during expiration, so that even under physiological conditions, the pitch of the sound varies.

The sound is said to be tympanitic (Skoda) when it has a musical quality resembling the timbre of a sound produced on a drum, and when it has a slight variation in pitch. If a caoutchouc ball be placed near the ear, on tapping it gently, a well-marked tympanitic sound is heard, and the sound is of higher pitch the smaller the diameter of the ball. A tympanitic sound is always produced on tapping the trachea in the neck. A tympanitic sound produced over the chest is always indicative of a diseased condition. It occurs in cases of cavities or vomicæ within the substance of the lung (the sound becomes deeper when the mouth, or, better, the mouth and nose, are closed), when air is present in one pleural cavity, as well as in conditions where the tension of the pulmonary tissues is diminished. The tympanitic sound resembles the metallic tinkling which is heard in large pathological cavities in the lungs, or which occurs when the pleural cavity contains air, and when the conditions which permit a more uniform reflection of the sound-waves within the cavity are present.

When percussing a chest, we may determine whether the substance lying under the portion of the chest under examination presents great or small resistance to the blow, either of the percussion-hammer or of the tips of the fingers, as the case may be.

Phonometry.-If the stem of a vibrating tuning-fork be placed on the chestwall over a part containing air, its sound is intensified; but if it be placed over a portion of the lung which contains little or no air its sound is enfeebled (von Baas).

Historical. -The actual discoverer of the art of percussion was Auenbrugger $(+1809)$. Piorry and Skoda developed the art and theory of percussion, while Skoda originated and developed the physical theory (1839). 


\section{The Normal Respiratory Sounds.}

Normal Vesicular Sound.-If the ear directly, or through the medium of a stethoscope, be placed in connection with the chest-wall, we hear over the entire area, where the lung is in contact with the chest, the so-called "vesicular" sound, which is audible only during inspiration. It is a fine sighing or rustling sound. It is said to be caused by the sudden dilatation of the air-vesicles (hence "vesicular") during inspiration, and it is also ascribed to the friction of the current of air entering the alveoli.

The sound has, at one time, a soft, at another, a sharper character; the latter occurs constantly in children up to 12 years of age. In their case, the sound is sharper, because the air, in entering resicles onethird narrower, is subjected to greater friction. As the air passes out of the air-vesicles during expiration, it gives rise to a feeble sighing sound of an indistinct soft character.

Bronchial Respiration. - Within the larger air-passages-larynx, trachea, bronchi-during inspiration and expiration, there are loud sounds like a sharp h or ch-the "bronchial"- the laryngeal, tracheal, or "tubular" sound, or breathing. This sound is also heard between the scapulæ, at the level of the fourth dorsal vertebra (bifurcation of trachea), and it occurs also during expiration, being slightly louder on the right side, owing to the slightly greater calibre of the right bronchus.

At all other parts of the chest, the vesicular sound obscures the tubular or bronchial sound. If the air-vesicles are deprived of their air, the tubular breathing becomes distinct. It is asserted that, when lungs containing air are placed orer the trachea, the tubular sound there produced becomes vesicular. In this case, we must suppose that the resicular sound arises from the tubular breathing becoming weakened, and being acoustically altered, by being conducted through the lung alveoli (Baas, Penzoldt). A sighing sound is often produced at the apertures of the nose and mouth during forced respiration.

\section{Pathological Respiratory Sounds.}

Historical.-Although sereral abnormal sounds in connection with diseases of the respiratory organs were known to Hippocrates (succussion-sound, friction, and several catarrhal sounds), still, Laënnec was the discoverer of the method of auscultation (1S16), while Skoda greatly extended our knowledge of its facts.

(1.) Bronchial breathing occurs over the entire area of the lung, either when the air-vesicles are devoid of air, which may be caused by the exudation of fluid or solid constituents, or when the lungs are compressed from without. In both 
cases vesicular sounds disappear, and the condensed or solidified lung-tissue conducts the urubular sound of the large bronchi to the surface of the chest. It also occurs in large cavities, with resistant walls near the surface of the lung, provided these cavities communicate with a large bronchus.

(2.) The amphoric sound is compared to that produced by blowing over the mouth of an empty bottle. It occurs either when a cavity-at least the size of the fist-exists in the lung, which is so blown into during respiration that a peculiar amphoric-like sound with a metallic timbre is produced; or when the lung still contains air, and is capable of expansion; as there is still air in the pleural cavity, it acts as a resonator, and causes an amphoric sound, simultaneous with the change of air in the lungs.

(3.) If obstruction occurs in the course of the air-passages of the lungs, various results may accrue, according to the nature of the resistance:- $\left(a_{0}\right)$ owing to various causes, e.g., in the apices of the lungs there may be partial swelling of the walls of the air-tubes, or infiltration into the air-cells which hinders the regular supply of air. In these cases, parts of the lung are not supplied with air continuously; it only reaches them periodically. In these cases a cog-wheel sound occurs. A similar sound may be heard occasionally in a normal lung, when the muscles of the chest contract in a periodic spasmodic manner. (b.) When the air entering large bronchi causes the formation of bubbles in the mucus which may have accumulated there, "mucous rales" are produced. They also occur in small spaces when the walls are separated from their fluid contents by the air entering during inspiration, or when the walls, being adherent to each other, are suddenly pulled asunder. The rales are distinguished as moist (when the contents are fluid), or as $d r y$ (when the contents are sticky); they may be inspiratory, expiratory, or continuous, or they may be coarse or fine; further, there is the very fine crepitation or crackling sound, and lastly, the metallic tinkling caused in large cavities through resonance. (c.) When the mucous membrane of the bronchi is greatly swollen, or is so covered with mucus that the air must force its way through, deep sonorous ronchi (ronchi sonori) may occur in the large airpassages, and clear shrill sibillant sounds (ronchi sibilantes) in the smaller ones. When there is extensive bronchial catarrh, not unfrequently we feel the chestwall vibrating with the rale sounds (Bronchial fremitus).

(4.) If fluid and air occur together in one pleural cavity in which the lung is collapsed, on moving the person's thorax vigorously, we hear a sound such as is produced when air and water are shaken together in a bottle. This is the soccussion sound of Hippocrates. Much more rarely, this sound is heard under similar conditions in large pulmonary cavities.

(5.) When the two apposed surfaces of the pleura are inflamed, have become soft, and are covered with exudation, they move over each other during respiration, and in doing so, give rise to FRICTION sounds, which can be felt (often by the patient himself), and can also be heard. The sound is comparable to the sound produced by bending new leather.

(6.) When we speak or sing in a loud tone, the walls of the chest vibrate (PECTORAL FREMITUS), because the vibration of the vocal cords is propagated throughout the entire bronchial ramifications. The vibration is, of course, greatest near the trachea and large bronchi. If there be much exudation or air in the pleura, or great accumulation of mucus in the bronchi, the pectoral fremitus is diminished or altogether absent.

All conditions which cause bronchial breathing increase the pectoral fremitus. Under normal circumstances, therefore, it is louder where bronchial breathing is heard normally. The ear hears an intensified sound, which is called BRoNCHOPHONY. If through effusion into the pleura or inflammatory processes in the lungtissue the bronchi are pressed flat, a peculiar bleating sound (AGOPHONY) may be heard. 


\section{Pressure in the Air-Passages During Respiration.}

Normal Respiration.-If a manometer be tied into the trachea of an animal, so that the respiration goes on completely undisturbed, during every inspiration there is a negative pressure $(-3 \mathrm{~mm} . \mathrm{Hg}$.) and during expiration a positive pressure (Donders). Donders placed the $U$-shaped manometer tube in one nostril, closed his mouth, leaving the other nostril open, and respired quietly. During every quiet inspiration, the mercury showed a negative pressure of $1 \mathrm{~mm}$., and during expiration a positive pressure of $2-3 \mathrm{~mm}$. (Hg.)

Forced Respiration.-As soon as the air was inspired or expired with greater force, the variations in pressure became very much greater, e.g., during speaking, singing, and coughing. The inspiratory pressure was $=-57 \mathrm{~mm}$. (36-74), the greatest expiratory pressure $+87(82-100)$ $\mathrm{mm}$. Hg. (Donders). The pressure of forced expiration therefore, is 30 $\mathrm{mm}$. greater than the inspiratory pressure.

Resistance to Inspiration.-Notwithstanding this, we must not conclude that the expiratory muscles act more powerfully than the inspiratory; for during inspiration, a variety of resistances has to be overcome, so that after these have been met, there is only a residue of the force for the aspiration of the mercury. The resistances to be overcome by the inspiratory muscles are:-(1.) The elastic tension of the lungs, which during the deepest expirations $=6 \mathrm{~mm}$.; during the deepest inspirations $=30 \mathrm{~mm}$. Hg. (\$107). (2.) The raising of the weight of the chest. (3.) The elastic torsion of the costal cartilages. (4.) The depression of the abdominal contents, and the elastic distension of the abdominal walls. All these not inconsiderable resistances, which the inspiratory muscles have to overcome, act during expiration, and aid the expiratory muscles. The forces concerned in inspiration are decidedly much greater than those of expiration.

As the lungs within the chest, in virtue of their elasticity, continually strive to collapse, necessarily they must cause a negative pressure within the chest. This amounts in dogs during inspiration, to $7 \cdot 1$ to $7.5 \mathrm{~mm}$. Hg., and during expiration to $4 \mathrm{~mm}$. Hg. (Heynsius). The analogous values for man have been estimated at $4.5 \mathrm{~mm}$. Hg. and $3 \mathrm{~mm}$. Hg., by Hutchinson.

Even the greatest inspiratory or expiratory pressure is always much less than the blood-pressure in the large arteries; but if the pressure be calculated upon the entire respiratory surface of the thorax, very considerable results are obtained.

Effects of the first Respiration on the Thorax.-Until birth, the airless lungs are completely collapsed (atelectic) within the chest, and fill it, so that on opening the chest in a dead foetus, pneumo-thorax does not occur (Bernstein). 
Supposing, however, respiration to have been fully established after birth, and air to have freely entered the lungs, if a manometer be placed in connection with the trachea and the chest be opened, the manometer will register a pressure of $6 \mathrm{~mm} . \mathrm{Hg}$., due to the collapse of the elastic lungs. Bernstein supposes that the thorax assumes a new permanent form, due to the first respiratory distension; it is as if, owing to the respiratory elevation of the ribs, the thorax had become permanently too large for the lungs, which are, therefore, kept permanently distended, but collapse as soon as air passes into the pleura. When a lung has once been filled with air, it cannot be emptied by pressure from without, as the small bronchi are compressed before the air can pass out of the alveoli. The expiratory muscles cannot possibly expel all the air from the lungs, while the inspiratory muscular force is sufficient to distend the lungs beyond their elastic equilibrium. Inspiration distends the lungs, increasing their elastic tension, while expiration diminishes the tension without abolishing it.

\section{Appendix to Respiration.}

Nasal Breathing.-During quiet respiration, we usually breatheor ought to breathe-through the nostrils, the mouth being closed. The current of air passes through the pharyngo-nasal cavity-so that in its course during inspiration, it is (1) warmed and rendered moist, and thus irritation of the mucous membrane of the air-passages by the cold air is prevented; (2) small particles of soot, or other foreign substances in the air, adhere to, and become embedded in the mucus covering the somewhat tortuous walls of the respiratory passages, and are carried outwards by the agency of the ciliated epithelium of the respiratory passages; (3) disagreeable odours and certain impurities are detected by the sense of smell.

If a lung be inflated, air constantly passes through the walls of the alveoli and trachea. This also occurs during violent expiratory efforts (cutaneous emphysema in whooping-cough), so that pueumo-thorax may occur (J. R. Ewald and Koberts).

Pulmonary Edema, or the exudation of lymph or serum into the pulmonary alveoli, occurs:-(1) When therc is very great resistance to the blood-stream in the aorta or its branches, e.g., by ligaturing all the arteries going to the head (Sig. Mayer), or the arch of the aorta, so that only one carotid remains pervious (Welch). (2) When the pulmonary veins are occluded. (3) When the left ventricle, owing to mechanical injury, ceases to beat, while the right ventricle goes on contracting (p. 75). These conditions produce at the same time anæmia of the vaso-motor centre, which results in stimulation of that centre, and consequent contraction of all the small arteries. Thus, the blood-stream through the veins to the right heart is favoured, and this in its turn favours the production of cdema of the lungs.

\section{Peculiarly Modified Respiratory Movements.}

(1.) Coughing.-Consists in a sudden violent expiratory explosion after a previous deep inspiration and closure of the glottis, whereby the glottis is forced open and any substance, fluid, gaseous or solid, in contact with the respiratory mucous membrane is violently ejected through the open mouth. It is produced 
voluntarily or reflexly; in the latter case, it can be controlled by the will only to a limited extent.

[Causes.-A congh may be discharged reflexly from a large number of surfaces. -(1) A draught of cold air striking the skin, especially of the upper part of the body. (2) More frequently it is discharged from the respiratory mucous mem. brane, especially of the larynx, the sensory branches of the vagus and the superior laryngeal nerve being the afferent nerves. (3) Sometimes an offending body, such as a pea in the external anditory meatus gives rise to coughing, the afferent nerve heing the auricular branch of the vagus. (4) There seems to be no doubt that there may be a "gastric congh," especially in cases of indigestion, produced by stimulation of the gastric branches of the vagus.]

(2.) Hawking, or clearing the throat.-An expiratory current is forced in a continuous stream through the narrow space between the root of the tongue and the depressed soft palate, in order to assist in the removal of foreign bodies. When the act is carried out periodically the closed glottis is suddenly forced open, and it is comparable to a voluntary gentle cough. This act can only be produced voluntarily.

(3.) Sneezing consists in a sudden violent expiratory blast through the nose, for the removal of muciss or foreign bodies (the mouth being rarely open) after a simple or repeated spasm-like inspiration-the glottis remaining open. It is usually caused reflexly by stimulation of sensory nerve-fibres of the nose [nasal branch of the fiftl nerve], or by sudden exposure to a bright light (Cassius Felix, A.D. 97) [the afferent nerve is the optic]. This reflex act may be interfered with to a certain extent, or even prevented, by stimulation of sensory nerves, firmly compressing the nose where the nasal nerve issues. The continued use of sternutatories, as in persons who take snuff, dulls the sensory nerves, so that they no longer act when stimulated reflexly.

(4.) Snoring occurs during respiration through the open mouth, whereby the inspiratory and expiratory stream of air throws the uvula and soft palate into vibration. It is involuntary, and usually occurs during sleep, but it may be produced voluntarily.

(5.) Gargling consists in the slow passage of the expiratory air-current in the form of bubbles through a fluid lying between the tongue and the soft palate, when the head is held backwards. It is a voluntary act.

(6.) Crying, caused by emotional conditions, consists in short, deep inspirations, long expirations with the glottis narrowed, rclaxed facial and jaw muscles, secretion of tears, often combined with plaintive inarticulate expressions. When crying is long continued, sudden and spasmodic involuntary contractions of the diaphragm occur, which cause the inspiratory sounds in the pharynx and larynx known as sobbing. This is an involuntary act.

(7.) Sighing is a prolonged inspiration, usually combined with a plaintive sound often caused involuntarily, owing to painful or unpleasant recollections.

(8.) Laughing is due to short, rapid expiratory blasts through the tense vocal cords which cause a clear tone, and there are characteristic inarticulate sounds in the larynx, with vibrations of the soft palate. The mouth is usually open, and the countenance has a characteristic expression, owing to the action of the $M$. zygomaticus major. It is usually involuntary, and can only be suppressed, to a certain degree, by the will (by forcibly closing the mouth and stopping respiration).

(9.) Yawning is a prolonged, deep inspiration occurring after successive attempts at numerous inspirations-the mouth, fauces, and glottis being wide open; expiration shorter-both acts often accompanied by prolonged characteristic sounds. It is quite involuntary, and is usually excited by drowsiness or ennui.

[(10.) Hiccough is due to a spasmodic involuntary contraction of the diaphragm, causing an inspiration, which is arrested by the sudden closure of the glottis, so 
that a characteristic sound is emitted. Not unfrequently it is due to irritation of the gastric mucous membrane, and sometimes it is a very troublesome symptom in uræmic poisoning.]

\section{Chemistry of Respiration.}

\section{Quantitative Estimation of Carbonic Acid, Oxygen, and Watery Vapour.}

I. Estimation of $\mathrm{CO}_{2} \cdot-1$. The volume of $\mathrm{CO}_{2}$ is estimated by means of the anthracometer (Fig. 110, II) of Vierordt. The volume of gas is collected in a graduated tube, $r r$, provided with a bulb at one end (previously filled with water and carefully calibrated, i.e., the exact amount which each part of the tube contains is accurately measured), and the tube is closed. The lower end has a stop-cock, $h$, and to this is screwed a flask, $n$, completely filled with a solution of caustic potash; the stop-cock is then opened, the potash solution is allowed to ascend into the tube, which is moved about until all the $\mathrm{CO}_{2}$ unites with the potash to form potassium carbonate. Hold the tube vertically and allow the potash to run back into the flask, close the stop-cock, and remove the bottle with the potash. Place the stop-cock under water, open it and allow the water to ascend in the tube, when the space in the tube occupied by the fluid indicates the volume of $\mathrm{CO}_{2}$ which is combined with the potash.

2. By weight.-A large quantity of the mixture of gases which has to be investigated is made to pass through a Liebig's bulb filled with caustic potash. The potash apparatus having been carefully weighed beforehand, the increase of weight indicates the amount of $\mathrm{CO}_{2}$ which has been taken up by the potash from the air passed through it.

3. By Titration.-A large volume of the air to be investigated is conducted through a known volume of a solution of barium hydrate. The $\mathrm{CO}_{2}$ unites with the barium and forms barium carbonate. The fluid is neutralised with a standard solution of oxalic acid, and the more barium that has united with the $\mathrm{CO}_{2}$ the smaller will be the amount of oxalic acid used, and vice versâ.

II. Estimation of Oxygen.-According to volume-(a) By the union of the $\mathrm{O}$ with potassium pyrogallate. The same procedure is adopted as for the estimation of $\mathrm{CO}_{2}$, only the flask, $n$, is filled with the pyrogallate solution instead of potash. (b) By exposure in an eudiometer (see Blood gases, p. 55).

III. Estimation of Watery Vapour.-The air to be investigated is passed through a bulb containing concentrated sulphuric acid or through a tube filled with pieces of calcium chloride. The amount of water is directly indicated by the increase of weight.

\section{Methods of Investigation.}

I. Collecting the Expired Air.-1. The air expired may be collected in the cylinder of the spirometer ( $(108)$ which is suspended in concentrated salt solution to avoid the absorption of $\mathrm{CO}_{2}$. 
The apparatus of Andral and Gavarret is thus used:-The operator breathed several times into a capacious cylinder (Fig. 110). A mouth-piece (M) was placed air-tight over the mouth while the nostrils were closed. The direction of the respiratory current was regulated by two so-called "Müller's valves" (mercurial), $(a$ and $b$ ). With every inspiration the bottle or valve, $a$ (filled below with $\mathrm{Hg}$. and hermetically closed above) permits the air inspired to pass to the lungs-during every expiration, the expired air can pass only through $b$ to the collecting cylinder $\mathrm{C}$.

2. If the gases given off by the skin are to be collected, a limb, or whatever part

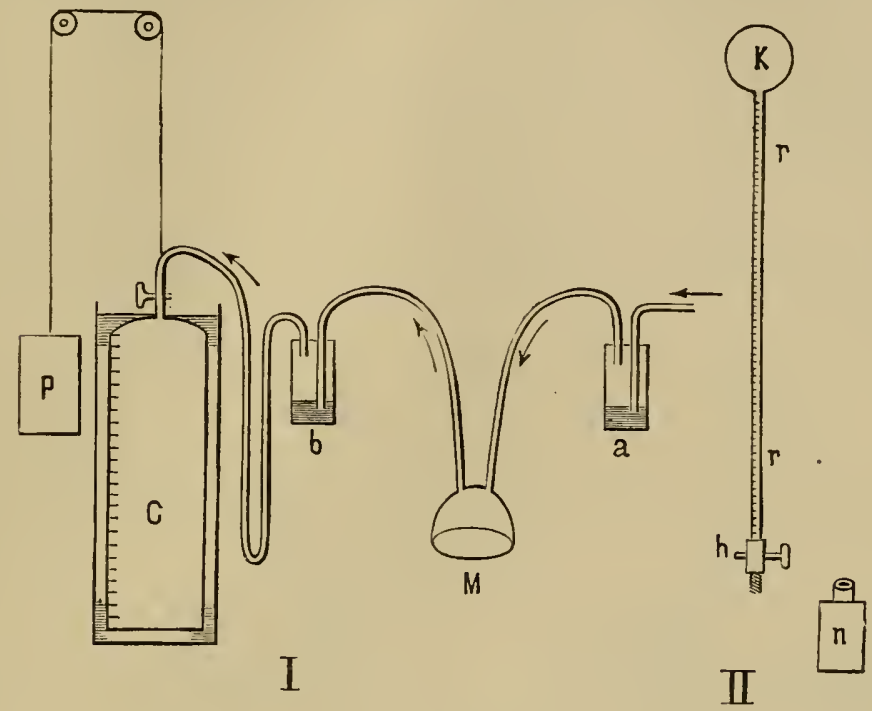

Fig. 110.

I. Apparatus of Andral and Gavarret for collecting the expired air- $C$, large cylinder to collect the air expired; $\mathrm{P}$, weight to balance cylinder; $a, b$, two Müller's valves; M, mouth-piece. II. Anthracometer of Vierordt.

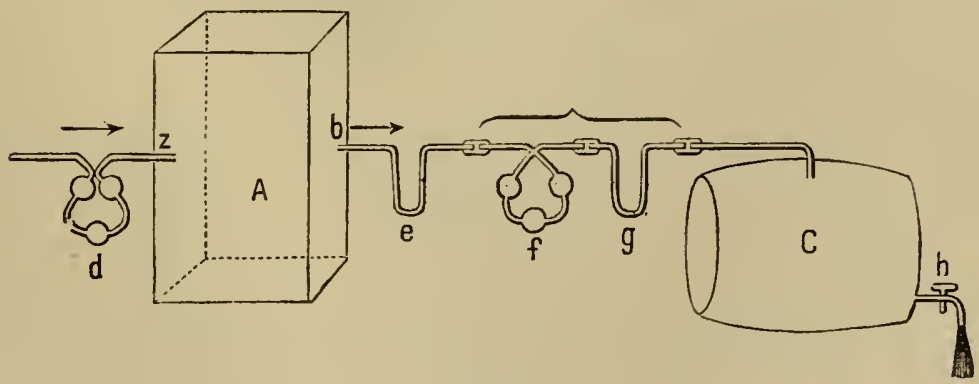

Fig. 111.

Respiratory Apparatus of Scharling- $d$, bulb containing caustic potash to absorb $\mathrm{CO}_{2}$ from in-going air; $\mathrm{A}$, box for man or animal experimented on; $e$ and $g$, tubes containing sulphuric acid to absorb watery vapour; $f$, potash bulb to absorb $\mathrm{CO}_{2}$ given off; $\mathrm{C}$, vessel filled with water to aspirate air through the foregoing system; $h$, stop-cock. 
is to be investigated, is secured in a closed vessel, and the gases so obtained are analysed.

II. The most important apparatus for this purpose are those of-(a.) Scharling (Fig. 111), which consists of a closed box, A, of sufficient size to contain a man. It has two openings-an entrance opening, $z$, and an exit, $b$. The latter is connected with an aspirator, C, a large barrel filled with water. When the stop-cock, $h$, is opened and the water flows out of the barrel, fresh air will rush in continuously into the box, A, and the air mixed with the expired gases will be drawn towards C. A Liebig's bulb, $d$, filled with caustic potash, is connected with the entrance tube, $z$, through which the in-going air must pass, whereby it is completely dejprived of $\mathrm{CO}_{2}$, so that the person experimented on is supplied with air free from $\mathrm{CO}_{2}$. The air passing out by the exit tube, $b$, has to pass first through $e$, where it gives up its watery vapour to sulphuric acid, whereby the amount of watery vapour is estimated by the increase of the weight of the apparatus, $e$. Afterwards the air passes through a bulb, $f$, containing caustic potash, which absorbs all the $\mathrm{CO}_{2}$, while the tube, $g$, filled with sulphuric acid, absorbs any watery vapour that may have come from $f$. The increase of weight of $f$ and $g$ indicate the amount of $\mathrm{CO}_{2}$. The total volume of air used is known from the capacity of $\mathbf{C}$.

(b.) Regnault and Reiset's Apparatus is more complicated, and is used when it is necessary to keep animals for some time under observation in a bell-jar. It consists (Fig. 112) of a globe, $\mathrm{R}$, in which is placed the dog to be experimented on. Around this is placed a cylinder, $g g$ (provided with a thermometer, $t$ ) which may be used for calorimetric experiments. A tube, $c$, leads into the globe, $R$; through this tube passes a known quantity of pure oxygen (Fig. 112, 0 ). To absorb

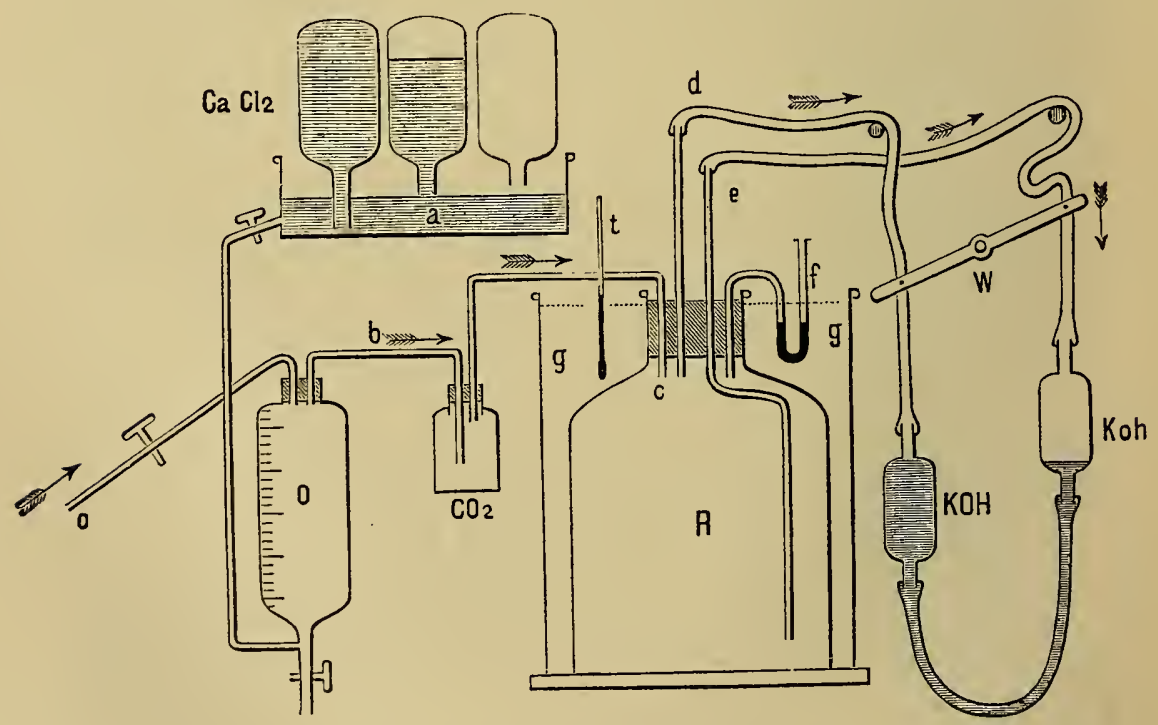

Fig. 112.

Scheme of the Respiration Apparatus of Regnault and Reiset-R, globe for animal; $g g$, outer casing for $\mathrm{R}$, provided with a thermometer, $t ; d$ and $e$, exit tubes to movable potash bulbs, $\mathrm{KOH}$ and $\mathrm{Koh}$; $\mathrm{O}$, in-going oxygen; $\mathrm{CO}_{2}$, vessel to absorb any carbonic acid; $\mathrm{Ca} \mathrm{Cl}_{2}$, apparatus for estimating the amount of $O$ supplied; $f$, manometer. 
any trace of $\mathrm{CO}_{2}$, a vessel containing potash (Fig. 112, $\mathrm{CO}_{2}$ ) is placed in the course of the tube. The vessel for measuring the 0 is emptied towards $R$, through a solution of calcium chloride from a large pan $\left(\mathrm{Ca} \mathrm{Cl}_{2}\right)$ provided with large flasks. Two tubes, $d$ and $e$, lead from $\mathrm{R}$, and are united by caoutclouc tubes with the potash buibs ( $\mathrm{KOH}, \mathrm{Koh}$ ), which can be raised or depressed alternately by means of the beam, W. In this way they aspirate alternately the air from $R$, and the caustic potash absorbs the $\mathrm{CO}_{2}$. The increase of weight of these flasks after the expcriment indicates the amount of $\mathrm{CO}_{2}$ expired. The manometer, $f$, shows whether there is a diffcrence of the pressure outside and inside the globe, $R$.

(c.) V. Pettenkofer has invented the most complete apparatus (Fig. 113). It consists of a chamber, $\mathrm{Z}$, with metallic walls, and provided with a door and a window. At $a$ is an opening for the admission of air, while a large double suctionpump, $\mathrm{P} \mathrm{P}_{1}$ (driven by means of a steam-engine) continually renews the air within the chamber. The air passes into a vessel, $b$, filled with pumice-stone saturated with sulphuric acid, in which it is dried; it then passes through a large gas-meter, $c$, which measures the total amount of the air passing through it.

After the air is measured, it is emptied outwards by means of the pump, $P P_{1}$. From the chief exit tube, $x$, of the chamber, provided with a small manometer, $q$, a narrow laterally placed tubc, $n$, passes, conducting a small secondary stream,

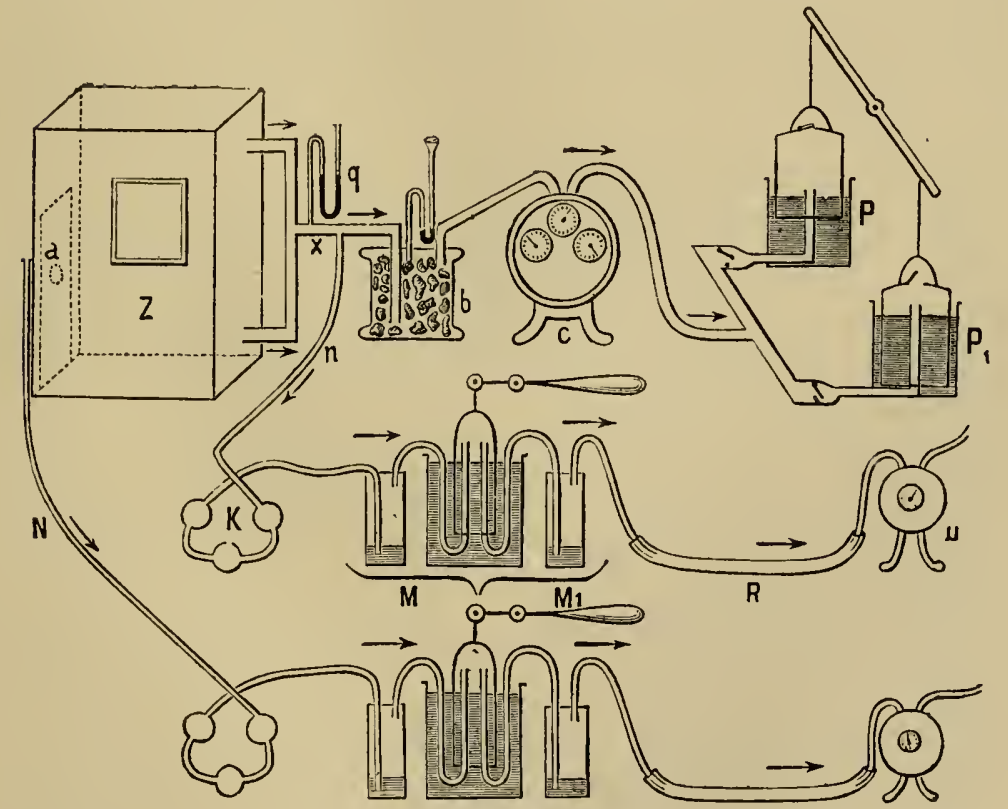

Fig. 113.

Respiration Apparatus of $\mathrm{v}$. Pettenkofer-Z, chamber for person experimented on; $x$, exit tube with manometer, $q$; $b$, vessel with sulphuric acid; C, gas-meter; $\mathrm{PP}_{1}$, pump; $n$, secondary current, with, $k$, bulb; $\mathrm{MM}_{1}$, suction apparatus; $u$, gas-meter; $\mathrm{N}$, stream for investigating air before it enters $\mathrm{Z}$.

which is chemically investigated. This current passes through the suction. apparatus, $\mathrm{M} \mathrm{M}_{1}$ (constructed on the principle of Müller's mercurial valve, and driven by a steam-engine). Before reaching this apparatus, the air passes through the bulb, K, filled with sulphuric acid, whose increase in weight indicates the amount of watery vapour. After passing through $\mathrm{MM}_{1}$, it goes through the 
tube, $\mathrm{R}$, filled with baryta solution, which takes up the $\mathrm{CO}_{2}$. The quantity of air which passes through the accessory current, $n$, is measured by the small gasmeter, $u$, from which it passes outwards. The second accessory stream, $\mathrm{N}$, enables us to investigate the air before it enters the chamber, and it is arranged in exactly the same way as $n$.

The increase of $\mathrm{CO}_{2}$ and $\mathrm{H}_{2} \mathrm{O}$ in the accessory stream, $n$ (i.e., more than in $\mathrm{N}$ ), indicates the amount of $\mathrm{CO}_{2}$ given off by the pressure in the chamber, $\mathrm{Z}$.

\section{Composition and Properties of Atmospheric Air.}

\section{DRY AIR contains :-}

$\begin{array}{llll}\text { Gas. } & & \\ \mathrm{O}, & \cdot & \cdot & \\ \mathrm{N}, & \cdot & \cdot & . \\ \mathrm{CO}_{2}, & \cdot & . & .\end{array}$

By Weight.

$23 \cdot 015$

$76 \cdot 985$
By Volume.

20.96

$79 \cdot 02$

$0.03-0.034$

2. Aqueous VAPOUR is always present in the air, but it varies greatly in amount, and generally increases with the increase of the temperature of the air. In connection with the moisture of the air we distinguish (a), the absolute moisture, i.e., the quantity of watery vapour which a volume of air contains in the form of vapour; and $(b)$, the relative moisture, i.e., the amount of watery vapour which a volume of air contains with respect to its temperature.

Experience shows that people generally can breathe most comfortably in an atmosphere which is not completely saturated with aqueous vapour according to its temperature, but is only saturated to the extent of 70 per cent. If the air be too dry it irritates the respiratory mucous membrane; if too moist, there is a disagreeable sensation, and if it be too warm a feeling of closeness. Hence, it is important to see that the proper amount of watery vapour is present in the air of our sittingrooms, bedrooms, and hospital wards.

The absolute amount of moisture varies:-In towns during the day it increases with increase of temperature, and diminishes when the temperature falls; it also varies with the direction of the wind, season of the year, height above sea-level.

The relative amount of moisture is greatest at sumrise, least at midday; small on high mountains; greater in winter than in summer; larger with a south or a west wind than with a north or an east wind.

The air in midsummer contains absolutely three times as much watery vapour as in midwinter, nevertheless the air in summer is relatively drier than the air in winter.

3. The air Expands BY Heat. Rudberg found that 1,000 volumes of air, at $0^{\circ}$, expanded to 1,365 when heated to $100^{\circ} \mathrm{C}$.

4. The DEnsity of the air diminishes with increase of the height above the sea-level.

\section{Composition of Expired Air.}

1. The expired air contains $\mathrm{MORE} \mathrm{CO}_{2}$-in normal respiration $=4 \cdot 38$ vols. per cent. (3.3 to 5.5 per cent.), so that it contains nearly 100 times more $\mathrm{CO}_{2}$ than the atmospheric air.

2. It contains LESS $O(4 \cdot 782$ vols. per cent. less $)$ than the atmospheric air, i.e., it contains only 16.033 vols. per cent. of $O$. 
3. Respiratory Quotient.-Hence, during respiration, more 0 is taken into the body from the air than $\mathrm{CO}_{2}$ is given off (Lavoisier); so that the volume of the expired air is $\left(\frac{1}{40}-\frac{1}{50}\right)$ smaller than the volume of the air inspired, both being calculated as dry, at the same temperature, and at the same barometric pressure. The relation of the $\mathrm{O}$ absorbed to the $\mathrm{CO}_{2}$ given off, is $4 \cdot 38: 4 \cdot 782$. This is expressed by the "respiratory quotient"-

$$
\frac{\mathrm{CO}_{2}}{\mathrm{O}}\left(=\frac{4 \cdot 38}{4 \cdot 782}\right)=0.906
$$

4. An excessively small quantity of $\mathrm{N}$ is added to the expired air (Regnault and Reiset). Seegen found that all the $\mathrm{N}$ taken in with the food did not reappear in the excreta (urine and fæces), and he assumed that a small part of it was given off by the lungs.

5. During ordinary respiration, the expired air is saturated with watery vapour. It is evident, therefore, that when the watery vapour in the air varies, the lungs give off different quantities of water from the body. The percentage of watery vapour falls during rapid respiration (Moleschott).

6. The expired air is WARMIER $\left(36.3^{\circ} \mathrm{C}\right)$, that is, very near the temperature of the body, and even although the temperature of the surrounding atmosphere be very variable, the temperature of the expired air still remains nearly the same.

The Instrument (Fig. 114) was used by Valentin and Brunner to determine the temperature of the expired air. It consists of a glass tube, A, A, with a mouth-piece, B, and in it is a fine thermometer, C. The operator breathes through the nose and expires slowly through the month-piece into the tube.

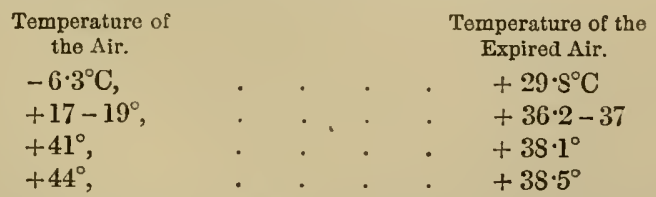

7. The diminution of the volume of the expired air mentioned under (3) is far more than compensated by the warming which the inspired air undergoes in the respiratory passages, so that the volume of the expired air is one-ninth greater than the air inspired.

S. A very small quantity of AmLONIA is found in the expired air (Regnault and Reiset) $=0.0204$ grammes in 24 hours (Lossen); it is probably derived from the blood, for blood exposed to the air evolves ammonia (Brücke).

9. Small quantities of $\mathrm{H}$ and $\mathrm{CH}_{4}$ are expired, both being absorbed from the intestine. In herbivora, Reiset found that 30 litres of $\mathrm{CH}_{*}$ were expired in 24 hours.

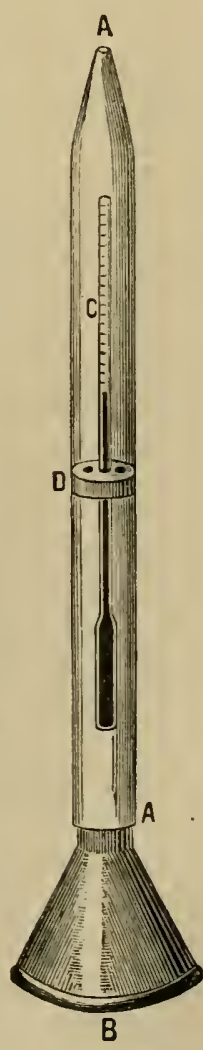

Fig. 114. 


\section{Daily Quantity of Gases Exchanged.}

As under normal circumstances more $O$ is absorbed than there is $\mathrm{CO}_{2}$ given off (equal volumes of $\mathrm{O}$ and $\mathrm{CO}_{2}$ contain equal quantities of 0 ), a part of the $\mathrm{O}$ must be used for other oxidation-processes in the body. According to the extent of these latter processes, the ratio of the $\mathrm{O}$ taken in to the $\mathrm{CO}_{2}$ given out-

$$
\left(\frac{\mathrm{CO}_{2}}{\mathrm{O}}=0.906 \text { normally }\right) \text { must vary. }
$$

The amount of $\mathrm{CO}_{2}$ given off may be less than the "mean" above stated. The quantity of $\mathrm{CO}_{2}$ alone is not a reliable indication of the entire exchange of gases during respiration; we must estimate simultaneously the amount of $\mathrm{O}$ absorbed, and the $\mathrm{CO}_{2}$ given off.

\section{Review of Daily Gaseous Income and Expenditure.}

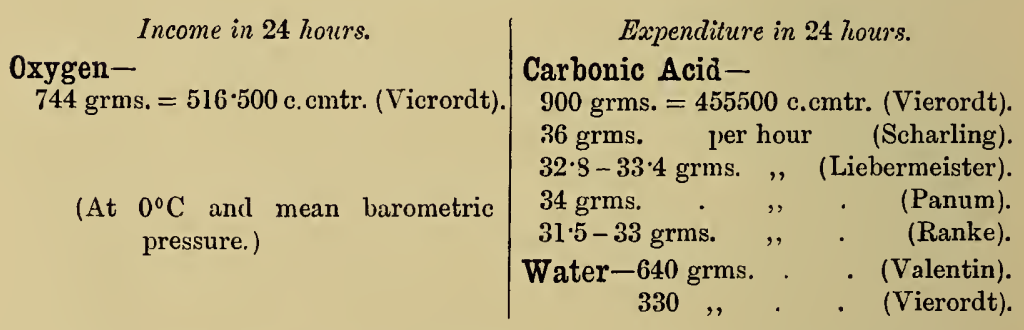

\section{Conditions Influencing the Gaseous Exchanges.}

The formation of $\mathrm{CO}_{2}$, in all probability, consists of two distinct processes. First, compounds containing $\mathrm{CO}_{2}$ seem to be formed in the tissues which are oxidation products of substances containing carbon. The second process consists in the separation of this $\mathrm{CO}_{2}$, which, however, takes place without the absorption of $O$. Both processes do not always occur simultaneously, and the one process may exceed the other in extent (L. Hermann, Plüger).

According to Schmiedeberg, the oxidation in the tissues depends upon a synthesis with the liberation of $\mathrm{H}_{2} \mathrm{O}$, the blood supplying the necessary $\mathrm{O}$.

The following affect these processes:-

1. Age.-Until the body is fully developed, the $\mathrm{CO}_{2}$ given off increases, but it diminishes as the bodily energies decay. Hence, in young persons the $\mathrm{O}$ absorbed is relatively greater than the $\mathrm{CO}_{2}$ given off; at other periods both values are pretty constant. Example :- 


\begin{tabular}{|c|c|c|c|c|c|c|}
\hline \multirow[b]{2}{*}{ Age-years. } & \multicolumn{6}{|c|}{ In 24 hours. } \\
\hline & \multicolumn{4}{|c|}{$\begin{array}{c}\mathrm{CO}_{2} \text { Gram. excreted. } \\
\text { = Carbon. }\end{array}$} & \multicolumn{2}{|c|}{ Absorbed Gram. } \\
\hline 8 & 443 & Gram. & $=121$ & rbon. & 375 & mmes. \\
\hline 15 & 766 & ” & $=209$ & , & 652 & $"$ \\
\hline 16 & 950 & , & $=259$ & $"$ & 809 & , \\
\hline $18-20$ & 1003 & 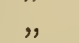 & $=274$ & $"$ & 854 & " \\
\hline $20-24$ & 1074 & 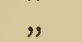 & $=293$ & $\eta$ & 914 & " \\
\hline $40-60$ & 889 & $"$ & $=242$ & $"$ & 757 & " \\
\hline $60-80$ & 810 & , & $=221$ & " & 689 & , \\
\hline
\end{tabular}

The absolute amount of $\mathrm{CO}_{2}$ given off is less in children than in adults; but if the $\mathrm{CO}_{2}$ given off be calculated with reference to body-weight, then, weight for weight, a child gives off twice as much $\mathrm{CO}_{2}$ as an adult.

2. Sex.-Males, from the eighth year onwards to old age, give off about one-third more $\mathrm{CO}_{2}$ than females (Andral and Gavarret). This difference is more marked at puberty, when the difference may rise to one-half. After cessation of the menses, there is an increase, and in old age the amount of $\mathrm{CO}_{2}$ given off diminishes. Pregnancy increases the amount, owing to causes which are easily understood.

3. The Constitution.-As a general rule, muscular, energetic persons use more $\mathrm{O}$ and excrete more $\mathrm{CO}_{2}$ than less active persons of the same weight.

4. Alternation of Day and Night.-The $\mathrm{CO}_{2}$ given off is diminished during sleep about one-fourth (Scharling). This diminution is caused by the constant heat of the surroundings (bed), darkness, absence of muscular activity, and the non-taking of food (see 5, 6, 7, 9). It does not seem that any $\mathrm{O}$ is stored up during sleep ( $\mathrm{S}$. Lewin). After awaking in the morning, the respirations are more rapid and deeper, and thus the amount of $\mathrm{CO}_{2}$ given off is increased. It decreases during the forenoon, until dinner at mid-day causes another increase. It falls during the afternoon, and increases again after supper.

During hybernation, when no food is taken, and when the respirations cease, or are enormously diminished, the respiratory exchange of gases is carried out by diffusion and by the cardio-pneumatic movements (p. 109). The $\mathrm{CO}_{2}$ given off falls to $\frac{1}{75}$, the $\mathrm{O}$ taken in to $\frac{1}{41}$ of what they are in the waking condition (Valentin). Much less $\mathrm{CO}_{2}$ is given off than $\mathrm{O}$ taken in, so that the body-weight may increase through the excess of $O$.

5. Temperature of the Surroundings.-Cold-blooded animals become warmer when the temperature of their environment is raised, and they give off. more $\mathrm{CO}_{2}$ in this condition than when they are cooler (Spallanzani)-e.g., a frog with the temperature of the surroundings at 
$39{ }^{\circ} \mathrm{C}$. excreted three times as much $\mathrm{CO}_{2}$ as when the temperature was $6^{\circ} \mathrm{C}$. (Moleschott).

Warm-blooded animals behave somewhat differently when the temperature of the surrounding medium is changed. When the temperature of the animal is lowered thereby, there is a considerable decrease in the amount of $\mathrm{CO}_{2}$ given off, as in cold-blooded animals ; but if the temperature of the animal be increased (also in fever), the $\mathrm{CO}_{2}$ is increased (C. Ludwig and Sanders-Ezn). Exactly the reverse obtains when the temperature of the surroundings varies, and the bodily temperature remains constant. As the cold of the surrounding medium increases, the processes of oxidation within the body are increased through some as yet unknown reflex mechanism; the number and depth of the respirations increase, whereby more $\mathrm{O}$ is taken in and more $\mathrm{CO}_{2}$ is given out (Lavoisier). A man in January uses $32 \cdot 2$ grammes $O$ per hour ; in July only 31.7 grammes. In animals, with the temperature of the surroundings at $8^{\circ} \mathrm{C}$., the $\mathrm{CO}_{2}$ given off was one-third greater than with a temperature of $38^{\circ} \mathrm{C}$. When the temperature of the air increasesthe body temperature remaining the same-the respiratory activity and the $\mathrm{CO}_{2}$ given off diminish, while the pulse remains nearly constant (Vierordt). On passing suddenly from a cold to a warm medium the amount of $\mathrm{CO}_{2}$ is considerably diminished; and conversely, on passing from a warm to a cold medium, the amount is considerably increased (compare Regulation of Temperature).

6. Muscular exercise causes a considerable increase in the $\mathrm{CO}_{2}$ given out (Scharling), which may be three times greater during walking than during rest (Ed. Smith). Ludwig and Sczelkow estimated the $\mathrm{O}$ taken in and the $\mathrm{CO}_{2}$ given off by a rabbit during rest, and when the muscles of the hind limbs were tetanised. During tetanus the $\mathrm{O}$ and $\mathrm{CO}_{2}$ were increased considerably, but in tetanised animals more $\mathrm{O}$ was given off in the $\mathrm{CO}_{2}$ expired than was taken up simultaneously during respiration. The passive animal absorbed nearly twice as much $O$ as the amount of $\mathrm{CO}_{2}$ given off (compare Metabolism in Muscle).

7. Taking of food causes constantly a not inconsiderable increase in the $\mathrm{CO}_{2}$ given off, which depends upon the quantity taken, and the increase generally occurs about an hour after the chief meal-dinner (Vierordt). During inanition, the exchange of gases diminishes considerably until death occurs (Letellier). At first the $\mathrm{CO}_{2}$ given off diminishes more quickly than the $\mathrm{O}$ is taken up. The quality of the food influences the $\mathrm{CO}_{2}$ given off to this extent, that substances rich in carbon (carbohydrates and fats) cause a greater excretion of $\mathrm{CO}_{2}$ than substances which contain less $\mathrm{C}$ (albumins). Regnault and Reiset found that a dog gave off 79 per cent. of the $\mathrm{O}$ inspired after a flesh diet, and 91 per cent. after a diet of starch. If easily oxidisable substances (glycerine or 
lactate of soda), are injected into the blood, the $\mathrm{O}$ taken in, and the $\mathrm{CO}_{2}$ given off, undergo a considerable increase (Ludwig and Scheremetjewsky). Alcohols, tea, and ethereal oils, diminish the $\mathrm{CO}_{2}$ (Prout, Vierordt). [Ed. Smith found that the effects produced by alcoholic drinks varied with the nature of the spirituous liquor. Thus brandy, whisky, and gin diminish the amount, while pure alcohol, rum, ale, and porter tend to increase it.]

8. The Number and Depth of the Respirations have practically no influence on the formation of $\mathrm{CO}_{2}$, or the oxidation-processes within the body, these being regulated by the tissues themselves, by some mechanism as yet unknown (Pflüger). They have a marked effect, however, upon the removal of the already formed $\mathrm{CO}_{2}$ from the body. An increase in the number of respirations (their depth remaining the same), as well as an increase of their depth, the number remaining the same, cause an absolute increase in the amount of $\mathrm{CO}_{2}$ given off, which with reference to the total amount of gases exchanged, is relatively diminished. The following example from Vierordt illustrates this :-

\begin{tabular}{|c|c|c|c|c|c|}
\hline $\begin{array}{l}\text { No. of Resps. } \\
\text { per min. }\end{array}$ & Vol. of Air. & $\underset{\mathrm{CO}_{2} .}{\text { Amount of }}=\underset{\mathrm{CO}_{2}}{\mathrm{CO}_{2}}$ & $\begin{array}{l}\text { Depth of } \\
\text { Resps. }\end{array}$ & $\begin{array}{c}\text { Amount of } \\
\mathrm{CO}_{2}\end{array}$ & $=\underset{\mathrm{CO}_{2}}{\text { per cent. }}$ \\
\hline $\begin{array}{l}12 \\
24 \\
48 \\
96\end{array}$ & $\begin{array}{r}6000 \\
12000 \\
24000 \\
48000\end{array}$ & $\begin{aligned} 258 \text { c.cmtr. } & =4,3 \% \\
420 \quad, & =3,5, \\
744 \quad " & =3,1, \\
1392 \quad ", & =2,9,\end{aligned}$ & $\begin{array}{r}500 \\
1000 \\
1500 \\
2000 \\
3000\end{array}$ & $\begin{array}{ll}21 & \text { c.cmtr. } \\
36 & ", \\
51 & ", \\
64 & ", \\
72 & , "\end{array}$ & $\begin{array}{l}=4.3 \% \\
=3.6, \\
=3.4, \\
=3.2 \% \\
=2.4, \%\end{array}$ \\
\hline
\end{tabular}

9. Exposure to a bright light causes an increase in the $\mathrm{CO}_{2}$ given off in frogs (Moleschott, 1855); in maminals and birds (Selmi and Piacentini); even in frogs deprived of their lungs (Fubini); or in those whose spinal cord has been divided high up (Chasanowitz). The consumption of $O$ is increased at the same time (Pflüger and v. Platen). The same results occur in blind persons, although to a less degree. Bluish-violet light is almost as active as white light, while red light is less active (Moleschott and Fubini).

10. The experiments of Grehant on dogs, seem to show that intense inflammation of the bronchial mucous membrane influences the $\mathrm{CO}_{2}$ given off.

11. Amongst poisons, thebaia increases the $\mathrm{CO}_{2}$ given off, while morphia, codeia, narcein, narcotin, papaverin, diminish it (Fubini).

\section{Diffusion of Gases within the Lungs.}

The air within the air-vesicles contains most $\mathrm{CO}_{2}$ and least $\mathrm{O}$, and as we pass from the small to the large bronchi and onwards to the trachea, the composition of the air gradually approaches more closely to that of the atmosphere (Allan and Pepys). Hence, if the air expired be collected in two portions, the first half (i.e., the air from the larger airpassages), contains less $\mathrm{CO}_{2}(3 \cdot 7 \mathrm{rols}$. per cent.) than the second half $(5 \cdot 4$ vols. per cent.). This difference in the percentage of gases gives 
rise to a diffusion of the gases within the air-passages ; the $\mathrm{CO}_{2}$ must diffuse from the air-vesicles outwards, and the $\mathrm{O}$ from the atmosphere and nostrils inwards (compare p. 52). This movement is aided by the cardio-pneumatic movement (Landois, p. 109). In hybernating animals and in persons apparently but not actually dead, the exchange of gases within the lungs can only occur in the above-mentioned ways.

For ordinary purposes this mechanism is insufficient, and there are added the respiratory movements whereby atmospheric air is introduced into the larger air-passages, from which and into which the diffusion currents of $\mathrm{O}$ and $\mathrm{CO}_{2}$ pass, on account of the difference of tension of the gases.

\section{Exchange of Gases between the Blood of the Pulmonary Capillaries and the Air in the Air-Vesicles.}

This exchange of gases occurs almost exclusively through the agency of chemical processes (independent of the diffusion of gases).

Method. - It is important to ascertain the tension of the $\mathrm{O}$ and $\mathrm{CO}_{2}$ in the venous blood of the pulmonary capillaries. Pfluger and Wolfberg estimated the tension by "catheterising the lungs." An elastic catheter was introduced through an opening in the trachea of a dog into the bronchus leading to the lowest lobe of the left lung. An elastic sac was placed round the catheter, and when the latter was introduced into the bronchus, the sac around the catheter was distended so as to plug the bronchus. No air can escape between the catheter and the wall of the bronchus. The outer end of the catheter was closed at first, and the dog was allowed to respire quietly. After four minutes the air in the air-vesicles was completely in equilibrium with the blood-gases. The air of the lung was sucked out of the catheter by means of an air-pump, and afterwards analysed.

Thus we may measure indirectly the tension of the $\mathrm{O}$ and $\mathrm{CO}_{2}$ in the venous blood of the pulmonary capillaries. The direct estimation of the gases in different kinds of blood is made by shaking up the blood with another gas. The gases so removed indicate directly the proportion of blood-gases.

The following tabular arrangement indicates the tension and percentage of $\mathrm{O}$ and $\mathrm{CO}_{2}$ in arterial and venous blood, in the atmosphere, and in the air of the alveoli :-

I.

O-Tension in arterial blood $=29.6 \mathrm{~mm}$.

$\mathrm{Hg}$. (corresponding to a mixture containing 3.9 vol. per cent. of 0 ).

II.

$\mathrm{CO}_{2} \cdot$ Tension in arterial blood $=21 \mathrm{~mm}$. $\mathrm{Hg}$. (corresponding to $2 \cdot 8$ vol, per cent.)
III.

O-Tension in venous blood $=22 \mathrm{~mm}$. Hg. (corresponding to $\$ \cdot 9$ vol. per cent.)

IV.

$\mathrm{CO}_{2}$-Tension in venous blood $=41 \mathrm{~mm}$. $\mathrm{Hg}$. (corresponding to 5.4 rol. per cent.) 
V.

0 -Tension in the air of the alveoli of the catheterised lung $=27.44 \mathrm{~mm}$. $\mathrm{Hg}$. (corresponding to $3.6 \mathrm{vol}$. per cent.)

VI.

$\mathrm{CO}_{2}$-Tension in the air of the alveoli of the catheterised lung $=27 \mathrm{~mm}$. $\mathrm{Hg}$. (corresponding to 3.56 vol. per cent.)
VII.

O-Tension in the atmosphere $=15 \mathrm{sm}$. $\mathrm{Hg}$. (corresponding to $20 . \mathrm{S}$ vol. per cent.)

VIII.

$\mathrm{CO}_{2}$-Tension in the atmosphere $=0.3 \mathrm{~S}$ $\mathrm{mm}$. $\mathrm{Hg}$. (corresponding to $0.03-0.0 \mathrm{~J}$ rol. per cent.)

When we compare the tension of the $\mathrm{O}$ in the air (VII. $=158 \mathrm{~mm}$. $\mathrm{Hg}$.) with the tension of the $\mathrm{O}$ in venous blood (III. $=22 \mathrm{~mm}$. Hg., or $\mathrm{V} .=27.44 \mathrm{~mm}$. Hg.), we might be inclined to assume that the passage of the $\mathrm{O}$ from the air of the air-vesicles into the blood was due solely to diffusion of the gases; and similarly, we might assume that the $\mathrm{CO}_{2}$ of the venous blood (IV. or VI.) diffused into the air-vesicles, because the tension of the $\mathrm{CO}_{2}$ in the air is much less (VIII.) There are a number of facts, however, which prove that the exchange of the gases in the lungs is chiefly due to chemical forces.

Absorption of 0.-With regard to the absorption of $\mathrm{O}$ from the air in the alveoli into the venous blood of the lung capillaries, whereby the blood is arterialised, it is proved that this is a chemical process. The gas-free (reduced) hæmoglobin takes up $\mathrm{O}$ to form oxyhæmoglobin $(\S 15$, 1). That this absorption has nothing to do directly with the diffusion of gases, but is due to a chemical combination of the atomic compounds, is shown by the fact, that, when pure $O$ is respired, the blood does not take up more $\mathrm{O}$ than when atmospheric air is respired; further, that animals made to breathe in a limited closed space can absorb almost all the $\mathrm{O}$-even to traces-into their blood before suffocation occurs. Of course if the absorption of $\mathrm{O}$ were due to diffusion, in the former case more $\mathrm{O}$ would be absorbed, while in the latter case the absorption of $\mathrm{O}$ could not possibly occur to such an extent as it does.

The law of diffusion comes into play in connection with the absorption of $\mathrm{O}$ to this extent, viz., that the $\mathrm{O}$ diffuses from the air-cells of the lung into the blood-plasma, where it reaches the blood-corpuscles suspended in the plasma. The hæmoglobin of the blood-corpuscles forms at once a chemical compound (oxyhæmoglobin) with the $\mathrm{O}$.

Even in very rarified air, such as is met with in the upper regions of the atmosphere during a balloon ascent, the absorption of $\mathrm{O}$ still remains independent of the partial pressure (Loth. Mayer, Fernet). But a much longer time is required for this process at the ordinary temperature of the body, so that in rarified air, the absorption of $O$ is greatly delayed, but it is not diminished. This is the cause of death in æronauts who have ascended so high that the atmospheric pressure is diminished to one-third (Setschenow).

Excretion of $\mathrm{CO}_{2}$. - With regard to the excretion of $\mathrm{CO}_{2}$ from the blood, we must remember that the $\mathrm{CO}_{2}$ in the blood exists in two con- 
ditions. Part of the $\mathrm{CO}_{2}$ forms a loose or feeble chemical compound, while another portion is more firmly combined. The former is obtained by those means which remove gases from fluids containing them in a state of absorption, so that in removing the $\mathrm{CO}_{2}$ from the blood it is difficult to determine whether the $\mathrm{CO}_{2}$, so removed, obeyed the law of diffusion, or if it was expelled by chemical means.

Although it is convenient to represent the excretion of $\mathrm{CO}_{2}$ from the blood into the air-vesicles of the lung, as due to equilibration of the tension of the $\mathrm{CO}_{2}$ on opposite sides of the alveolar membrane, i.e., to diffusion-nevertheless, chemical processes play an important part in this act. The absorption of $\mathrm{O}$ by the coloured corpuscles acts, at the same time, in expelling $\mathrm{CO}_{2}$. This is proved by the fact that the expulsion of $\mathrm{CO}_{2}$ from the blood takes place more readily when $\mathrm{O}$ is simultaneously admitted (Ludwig and Holmgren).

The free supply of $\mathrm{O}$ not only favours the removal of the $\mathrm{CO}_{2}$, which is loosely combined, but it also favours the expulsion of that portion of the $\mathrm{CO}_{2}$ which is more firmly combined, and which can only be expelled by the addition of acids to the blood (Ludwig, Schöffer and Sczelkow). That the oxygenated blood-corpuscles (i.e., their oxyhæmoglobin) are concerned in the removal of $\mathrm{CO}_{2}$, is proved by the fact that $\mathrm{CO}_{2}$ is more easily removed from serum which contains oxygenated blood-corpuscles than from serum charged with $\mathrm{O}$.

[The following scheme may serve to illustrate the extent to which diffusion comes into play. The $\mathrm{O}$ must pass through the alveolar membrane, $\mathrm{AB}$-including the alveolar epithelium and the wall of the capillaries-as well as the blood-plasma, to reach the hæmoglobin of the blood-corpuscles. Similarly, the $\mathrm{CO}_{2}$ must leave the salts of the plasma with which it is in combination, and diffuse in the opposite direction, through the wall of the capillaries, the alveolar membrane and epithelium, to reach the air-vesicles. Let $\mathrm{AB}$ represent the

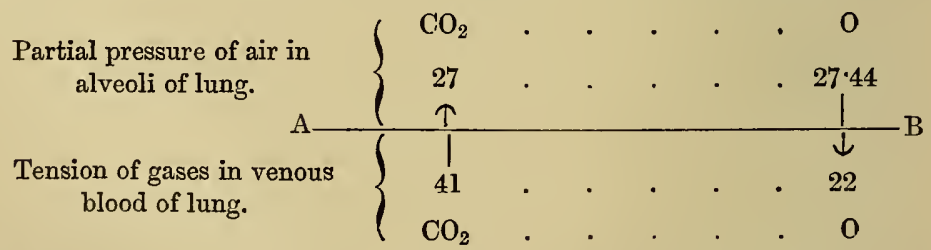

alveolar membrane; on the one side of it is represented the partial pressure of the $\mathrm{CO}_{2}$ and $\mathrm{O}$ in the air-vesicles; and on the other, the partial pressure of the $\mathrm{CO}_{2}$ and $\mathrm{O}$ in the venous blood entering the lung. The arrows indicate the direction of diffusion.]

Theories.- Various theories have been proposed to account for the expulsion of the $\mathrm{CO}_{2}$ from its state of chemical combination in the blood due to the action of the oxygenated blood-corpuscles. (a.) It is possible that the $\mathrm{CO}_{2}$ in the blood- 
corpuscles (perhaps united with paraglobulin ?-Setschenow) is expelled by the 0 taken up; (b.) the acid reaction of the hæmoglobin (Preyer) may act so as to expel the $\mathrm{CO}_{2}$ out of the corpuscles and the plasma; $(c$.$) by the absorption of \mathrm{O}$ volatile fatty acids may be formed from the hæmoglobin (Hoppe-Seyler). These acids may act so as to expel the $\mathrm{CO}_{2}$.

Nature of the Process.-The exchange of gases between the blood and the air in the lungs has been represented by Donders as due to a process of dissociation.

\section{Dissociation of Gases.}

Many gases form true chemical compounds with other bodies (i.e., they combine according to their equivalents), when the contact of these bodies is effected under conditions such that the partial pressure of the gases is high. The chemical compound formed under these conditions is broken up, whenever the partial pressure is diminished, or when it reaches a certain minimum level, which varies with the nature of the bodies forming the compound. Thus, by increasing and diminishing the partial pressure alternately, a chemical compound of the gas may be formed and again broken up. This process is called Dissociation of the gases. The minimal partial pressure is constant for each of the different substances and gases, but tempercuture, as in the case of the absorption of gases, has a great effect on the partial pressure; with increase of temperature the partial pressure, under which dissociation occurs, diminishes.

As an example of the dissociation of a gas, take the case of calcinm carbonate. When it is heated in the air to $440^{\circ} \mathrm{C}, \mathrm{CO}_{2}$ is given off from its state of chemical combination, but is taken up again and a chemical compound formed, which is changed into chalk when it cools.

Dissociation in the Blood.-The chemical combinations containing $\mathrm{CO}_{2}$ and those containing $\mathrm{O}$ within the blood-stream behave in a similar manner-viz., the salts of the plasma, which are combined with $\mathrm{CO}_{2}$, and the oxyhæmoglobin. If these compounds of $\mathrm{O}$ and $\mathrm{CO}_{2}$ are placed under conditions where the partial pressure of these gases is very low-i.e., in a medium containing a very small amount of these gases, the compounds are dissociated-i.e., they give off $\mathrm{CO}_{2}$ or $\mathrm{O}$. If after being dissociated, they are placed under conditions where, owing to the large amount of these gases, the partial pressure of $\mathrm{O}$ or of $\mathrm{CO}_{2}$ is high, these gases are taken up again, and enter into a condition of chemical combination.

The hæmoglobin of the blood in the pulmonary capillaries finds plenty of $\mathrm{O}$ in the alveoli; hence, it unites with the $\mathrm{O}$ owing to the high partial pressure of the $O$ in the lung, and so forms the compound oxyhæmoglobin. On its course through the capillaries of the systemic 
circulation, the oxyhæmoglobin of the blood comes into relation with tissues poor in $\mathrm{O}$; the oxyhæmoglobin is dissociated, the $\mathrm{O}$ is supplied to the tissues, and the blood freed from this $O$, returns to the right heart, and passes to the lungs, where it takes up new $O$.

The blood whilst circulating meets with most $\mathrm{CO}_{2}$ in the tissues; the high partial pressure of the $\mathrm{CO}_{2}$ in the tissues causes the $\mathrm{CO}_{2}$ to unite with certain constituents in the blood so as to form chemical compounds, which carry the $\mathrm{CO}_{2}$ from the tissues to the lungs. In the air of the lungs, however, the partial pressure of the $\mathrm{CO}_{2}$ is very low, dissociation of these chemical compounds occurs under the low partial pressure, and the $\mathrm{CO}_{2}$ passes into the air-cells of the lung, from which it is expelled during expiration. It is evident that the giving up of $\mathrm{O}$ from the blood to the tissues, and the absorption of $\mathrm{CO}_{2}$ from the tissues, go on side by side and take place simultaneously, while in the lungs the reverse processes occur also simultaneously.

\section{Cutaneous Respiration.}

Methods.-If a man or an animal be placed in the chamber of a respiratory apparatus (Scharling's, or v. Pettenkofer's), and if tubes be so arranged that the respiratory gases do not enter the chamber, of course we obtain only the "perspiration" of the skin in the chamber. It is less satisfactory to leave the head of the person outside the chamber, while the neck is fixed air-tight in the wall of the chamber. The extent of the cutaneous respiration of a limb may be ascertained by enclosing it in an air-tight vessel (Röhrig) similar to that used for the arm in the plethysmograph (p. 198).

Loss by Skin.-A healthy man loses by the skin, in 24 hours, $\frac{1}{67}$ of his body-weight (Séguin), which is greater than the loss by the lungs, in the ratio of $3: 2$ (Valentin, 1843). Only 10 grammes-150 grains (Scharling), or it may be 3.9 grammes - 60 grains (Aubert), of the entire loss is due to the $\mathrm{CO}_{2}$ given off by the skin. The remainder of the excretion from the skin is due to water, containing a few salts in solution. When the surrounding temperature is raised, the $\mathrm{CO}_{2}$ is increased (Gerlach), in fact it may be doubled (Aubert); violent muscular exercise has the same effect.

0 Absorbed.-The $\mathrm{O}$ taken up by the skin is either equal to (Regnault and Reiset), or slightly less than, the $\mathrm{CO}_{2}$ given off. As the $\mathrm{CO}_{2}$ excreted by the skin is only $\frac{1}{2} \frac{1}{2} \sigma$ of that excreted by the lungs, while the $\mathrm{O}$ taken in $=\frac{1}{180}$ of that taken in by the lungs, it is evident that the respiratory activity of the skin is very slight. Animals whose skin has been covered by an impermeable varnish die not from suffocation, but from other causes.-(See Artificial Diminution of Temperature.) 
In animals with a thin moist epidermis (frog) the exchange of gases is much greater, and in them the skin so far supports the lungs in their function, and may even partly replace them functionally. In mammals with thick dry cutaneous appendages, the exchange of gases is, again, much less than in man.

\section{Internal Respiration.}

Where $\mathrm{CO}_{2}$ is Formed.-By the term "internal respiration" is understood the exchange of gases between the capillaries of the systemic circulation and the tissues of the various organs of the body. As the organic constituents of the tissues, during their activity, undergo gradual oxidation, and form, amongst other products, $\mathrm{CO}_{2}$ : we may assume--(1.) That the chief focus for the absorption of $\mathrm{O}$ and the formation of $\mathrm{CO}_{2}$ is to be sought for within the TISsuEs themselves. That the $O$ from the blood in the capillaries rapidly penetrates or diffuses into the tissues is shown by the fact, that the blood in the capillaries rapidly loses $\mathrm{O}$ and gains $\mathrm{CO}_{2}$, while blood containing $\mathrm{O}$, and kept warm outside the body, changes very slowly and incompletely. If portions of fresh tissues be placed in defibrinated blood containing $\mathrm{O}$, then the $\mathrm{O}$ rapidly disappears (Hoppe-Seyler). Frogs deprived of their blood exhibit an exchange of gases almost as great as normal. This shows that the exchange of gases must take place in the tissues themselves (Pflüger and Oertmann). If the chief oxidations took place in the blood and not in the tissues, then, during suffocation, when $O$ is excluded, the substances which use up 0 , i.e., those substances which act as reducing agents, ought to accumulate in the blood. But this is not the case, for the blood of asplyyxiated animals contains mere traces of reducing materials (Pflüger). It is difficult to say how the $\mathrm{O}$ is absorbed by the tissues, and what becomes of it immediately it comes in contact with the living elements of the tissues. Perhaps it is temporarily stored up, or it may form certain intermediate less oxidised compounds. This may be followed by a period of rapid formation and excretion of $\mathrm{CO}_{2}$. On this supposition, it is evident that the absorption of $\mathrm{O}$ and the excretion of $\mathrm{CO}_{2}$ need not occur to the same extent, so that the amount of $\mathrm{CO}_{2}$ given off at any period is not necessarily an index of the amount of $\mathrm{O}$ absorbed during the same period.

[There are two views as to where the $\mathrm{CO}_{2}$ is formed as the blood passes through the tissues. One view is that the seat of oxidation is in the blood itself, and the other is that it is formed in the tissues. If we knew the tension of the gases in the tissues the problem would be easily solved, but we can only arrive at a knowledge of this subject indirectly, in the following ways] :-

$\mathrm{CO}_{2}$ in Cavities. - That the $\mathrm{CO}_{2}$ is formed in the tissues is supported by the fact, that the amount of $\mathrm{CO}_{2}$ in the fluids of the cavities of the body is greater than the $\mathrm{CO}_{2}$ in the blood of the capillaries. 
Pflüger and Strassburger found the tension of $\mathrm{CO}_{2}$ to be, in

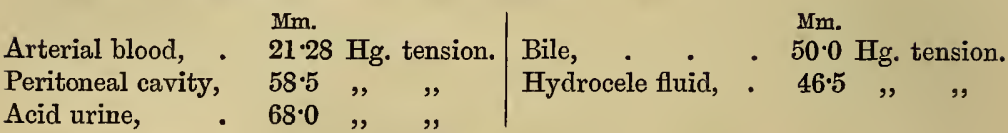

The large amount of $\mathrm{CO}_{2}$ in these fluids can only arise from the $\mathrm{CO}_{2}$ of the tissues passing into them.

Gases of Lymph.-In the lymph of the ductus thoracicus the tension of $\mathrm{CO}_{2}=33.4$ to $37 \cdot 2 \mathrm{~mm}$. $\mathrm{Hg}$., which is greater than in arterial blood, but considerably less than in venous blood $(41.0 \mathrm{~mm}$. $\mathrm{Hg})$. This does not entitle us to conclude that in the tissues from which the lymph comes, only a small quantity of $\mathrm{CO}_{2}$ is formed, but rather that in the lymph there is less attraction for the $\mathrm{CO}_{2}$ formed in the tissues than in the blood of the capillaries, where chemical forces are active in cansing it to combine, or that in the course of the Iong lymph-current, the $\mathrm{CO}_{2}$ is partly given back to the tissues, or that $\mathrm{CO}_{2}$ is formed in the blood itself. Further, the muscles, which are by far the largest producers of $\mathrm{CO}_{2}$, contain few lymphatics, nevertheless they supply much $\mathrm{CO}_{2}$ to the blood.

The amount of free "non-fixed" $\mathrm{CO}_{2}$ contained in the juices and tissues indicates that the $\mathrm{CO}_{2}$ passes from the tissues into the blood; still, Preyer believes that in venous blood $\mathrm{CO}_{2}$ undergoes chemical combination. The exchange of $\mathrm{O}$ and $\mathrm{CO}_{2}$ varies much in the different tissues. The muscles are the most important organs, for in their active condition they excrete a large amount of $\mathrm{CO}_{2}$, and use up much $O$. The $O$ is so rapidly used up by them that no free $O$ can be pumped out of muscular tissue (L. Hermann). The exchange of gases is more vigorous during the activity of the tissues. Nor are the salivary glands, kidneys, and pancreas any exception, for although when these organs are actively secreting, the blood flows out of the dilated veins in a bright red stream, still the relative diminution of $\mathrm{CO}_{2}$ is more than compensated by the increased volume of blood. which passes through these organs.

(2.) In the BLOOD itself, as in all tissues, $\mathrm{O}$ is used up and $\mathrm{CO}_{2}$ is formed. This is proved by the following facts :-That blood withdrawn from the body becomes poorer in $\mathrm{O}$ and richer in $\mathrm{CO}_{2}$; that in the blood of asphyxia, free from $\mathrm{O}$, and in the blood-corpuscles (Afanassieff), there are slight traces of reducing agents, which become oxidised on the addition of $\mathrm{O}$ (A. Schmidt). Still, this process is comparatively insignificant as against that which occurs in all the other tissues. That the walls of the vessels-more especially the muscular fibres in the walls of the small arteries-use $\mathrm{O}$ and produce $\mathrm{CO}_{2}$ is unquestionable, although it is so slight that the blood in its whole arterial course undergoes no visible change.

Ludwig and his pupils have proved that $\mathrm{CO}_{2}$ is actually formed in the blood. If the easily oxidisable lactate of soda be mixed with blood, and this blood be caused to circulate in an excised but still living organ, such as a ling or kidney, more $\mathrm{O}$ is used up and more $\mathrm{CO}_{2}$ is formed than in unmixed blood similarly transfused.

(3.) That the tissues of the living lungs use $\mathrm{O}$ and give off $\mathrm{CO}_{2}$ is probable. When C. Ludwig and Müller passed arterial blood through the blood-vessels of a lung deprived of air, the $\mathrm{O}$ was diminished and the $\mathrm{CO}_{2}$ increased. 
As the total amount of $\mathrm{CO}_{2}$ and $\mathrm{O}$ found in the entire blood, at any one time, is only 4 grammes, and as the daily excretion of $\mathrm{CO}_{2}=900$ grammes, and the $\mathrm{O}$ absorbed daily $=744$ grammes, it is clear that exchange of gases must go on with great rapidity, that the $\mathrm{O}$ absorbed must be used quickly, and the $\mathrm{CO}_{2}$ must be excreted.

Still, it is a striking fact that oxidation-processes of such magnitude as, e.g., the union of $\mathrm{C}$ to form $\mathrm{CO}_{2}$, occur at a relatively low temperature of the blood and the tissues. It has been assumed that the blood acts as an ozone-producer, and transfers this active form of $O$ to the tissues. Liebig showed that the alkaline reaction of most of the juices and tissues favours the processes of oxidation. Numerous organic substances, which are not altered by $\mathrm{O}$ alone, become rapidly oxidised in the presence of free alkalies, e.g., gallic acid, pyrogallic acid, and sugar; while many organic acids, which are unaffected by ozone alone, are changed into carbonates, when in the form of alkaline salts (Gorup-Besanez), and in the same way, when they are introduced into the body in the form of acids, they are partly or wholly excreted in the urine, but when they are administered as alkaline compounds they are changed into carbonates.

\section{Respiration in a Closed Space.}

Respiration in a closed or confined space causes :-(1) a gradual diminution of $\mathrm{O}$; (2) a simultaneous increase of $\mathrm{CO}_{2} ;(3)$ a diminution in the volume of the gases. If the space be of moderate dimensions, the animal uses up almost all the $\mathrm{O}$ contained therein (Nysten), and dies ultimately from spasms caused by the asphyxia. The $\mathrm{O}$ is absorbed, therefore-independently of the laws of absorption-by chemical means. The $\mathrm{O}$ in the blood is almost completely used up (Setschenow). In a larger closed space, the $\mathrm{CO}_{2}$ accumulates rapidly, before the diminution of $\mathrm{O}$ is such as to affect the life of the animal. $\mathrm{As} \mathrm{CO}_{2}$ can only be excreted from the blood when the tension of the $\mathrm{CO}_{2}$ in the blood is greater than the tension of $\mathrm{CO}_{2}$ in the air, as soon as the $\mathrm{CO}_{2}$ in the surrounding air in the closed space becomes the same as in the blood, the $\mathrm{CO}_{2}$ will be retained in the blood, and finally $\mathrm{CO}_{2}$ may pass back into the body. This occurs in a large closed space, when the amount of $O$ is still sufficient to support life, so that death occurs under these circumstances (in rabbits) through poisoning with $\mathrm{CO}_{2}$, causing diminished excitability, loss of consciousness, and lowering of temperature, but no spasms (Worm Müller). In pure $\mathrm{O}$, animals breathe in a normal way; the quantity of $\mathrm{O}$ absorbed and the $\mathrm{CO}_{2}$ excreted is quite independent of the percentage of $\mathrm{O}$, so that the former occurs through chemical agency independent of pressure. In closed spaces filled with $\mathrm{O}$, animals died by re-absorption of the $\mathrm{CO}_{2}$ excreted. Worm Müller found that rabbits died after absorbing $\mathrm{CO}_{2}$ equal to half the volume of their body, although the air still contained 50 per cent. $O$. Animals can breathe quite quietly a mixture of air containing 14.8 per cent. ( 20.9 per cent. normal); with 7 per cent. 
they breathe with difficulty; with 4.5 per cent. there is marked dyspnœa; with 3 per cent. O there is tolerably rapid asphyxia (W. Müller). The air expired by man normally contains $14-18$ per cent. $O$. If animals be supplied with a mixture of gases similar to the atmosphere, in which $\mathrm{N}$ is replaced by $\mathrm{H}$, they breathe quite normally (Lavoisier and Seguin); the $\mathrm{H}$ undergoes no great change.

Dyspnœa occurs when the respired air is deficient in $O$, as well as when it is overcharged with $\mathrm{CO}_{2}$, but the dyspncea in the former case is prolonged and sevele; in the latter, the respiratory activity soon ceases. The want of $O$ causes a greater and more prolonged increase of the blood-pressure than is caused by excess of $\mathrm{CO}_{2}$. Lastly, the consumption of $\mathrm{O}$ in the body is less affected when the $\mathrm{O}$ in the air is diminished than when there is excess of $\mathrm{CO}_{2}$. If air containing a diminished amount of $\mathrm{O}$ be respired, death is preceded by violent phenomena of excitement and spasms, which are absent in cases of death by breathing air overcharged with $\mathrm{CO}_{2}$. In poisoning with $\mathrm{CO}_{2}$, the excretion of $\mathrm{CO}_{2}$ is greatly diminished, while with diminution of $\mathrm{O}$, it is almost unchanged (C. Friedländer and E. Herter).

Cl. Bernard found that, when an animal breathed in a closed space, it became partially accustomed to the condition. On placing a bird under a bell-jar, it lived several hours: but if several hours before its death another bird fresh from the outer air were placed under the same bell-jar, the second bird died at once, with convulsions.

Frogs, when placed for several hours in air devoid of $O$, give off just as much $\mathrm{CO}_{2}$ as in air containing $\mathrm{O}$, and they do this without any obvious disturbance (Pflüger, Aubert). Hence, it appears that the formation of $\mathrm{CO}_{2}$ is independent of the absorption of $\mathrm{O}$, and the $\mathrm{CO}_{2}$ must be formed from the decomposition of other compounds. Ultimately, however, complete motor paralysis occurs, whilst the circulation remains undisturbed (Aubert).

\section{Dyspnoea and Asphyzia.}

[The causes of dyspncea have already been referred to ( $\$ 111$ ), and those of asphyxia are referred to in detail in vol. ii. under Nervous Mechanism of Respiration. If from any cause, an animal be not supplied with a due amount of air, normal respiration becomes greatly altered, passing through the phases of hyperpnoea, or increased respiration, dyspnœa or difficulty of breathing, to the final condition of suffocation or asphyxia. The phenomena of asphyxia may be developed in an animal by closing its trachea by means of a clamp, and in fact by any means which prevent the entrance of air or blood into the lungs.

The phenomena of asphyxia are usually divided into several stages. -1. During the first stage there is hyperpnoea, the respirations being deeper, more frequent, and laboured. The extraordinary muscles of respiration-both those of inspiration and expiration-referred to in $\S 118$, are called into action, the condition of dyspnca being rapidly produced, and the struggle for air becomes more and more severe. During this time the oxygen of the blood is being used up, the blood 
itself is becoming more and more venous. This venous blood circulating in the medulla oblongata, and spinal cord stimulates the respiratory centres, thus causing these violent respirations. This stage usually lasts about a minute and gradually gives place to-

2. The second stage, when the inspiratory muscles become less active, while those concerned in laboured expiration contract energetically, and indeed almost every muscle in the body may contract; so that this stage of violent expiratory efforts ends in general convulsions. The conrulsions are due to stimulation of the respiratory centres by the venous blood. The convulsive stage is short, and is usually reached in a little over one minute. This storm is succeeded by-

3. The third stage, or stage of exhaustion, the transition being usually somewhat sudden. This condition is brought about by the venous blood acting on and paralysing the respiratory centres. The pupils are widely dilated, consciousness is abolished, and the activity of the reflex centres is so depressed that it is impossible to discharge a reflex act, even from the cornea. The animal lies almost motionless, with flaccid muscles, and to all appearance dead, but every now and again, at long intervals, it makes a few deep inspiratory efforts, showing that the respiratory centres are not quite, but almost paralysed. Gradually, the pauses become longer and the inspirations feebler and of a gasping character. As the venous blood circulates in the spinal cord it causes a large number of muscles to contract, so that the animal extends its trunk and limbs. It makes one great inspiratory spasm, the mouth being widely open and the nostrils dilated, and ceases to breathe. After this stage, which is the longest and most variable, the heart becomes paralysed, partly from being over-distended with venous blood, and partly, perhaps, from the action of the renous blood on the cardiac tissues, so that the pulse can hardly be felt. To this pulseless condition the term "asphyxia" ought properly to be applied. In connection with the resuscitation of asphyxiated persons, it is important to note that the heart continues to beat for a few seconds after the respiratory movements have ceased.

The whole series of phenomena occupies from 3 to 5 minutes, according to the animal operated on, and depending also upon the suddenness with which the trachea was closed. If the causes of suffocation act more slowly, the phenomena are the same, only they are developed more slowly.

The Circulation.-The post-mortem appearances in man or in an animal are generally well marked. The right side of the heart, the pulmonary artery, the venæ caræ, and the veins of the neck are engorged with dark venous blood. The left side is comparatively empty, because the rigor mortis of the left side of the heart, and the elastic recoil of the systemic arteries, force the blood towards the 
systemic veins. The blood itself is almost black, and is deprived of almost all its oxygen, while its hæmoglobin is nearly all in the condition of reduced hæmoglobin, while ordinary venous blood contains a considerable amount of reduced and oxyhæmoglobin. The blood of an asphyxiated animal practically contains none of the latter, and much of the former.

It is important to study the changes in the circulation in connection with the outward phenomena exhibited by an animal during suffocation.

We may measure the blood-pressure in any artery of an animal while it is being asphyxiated, or we may open its chest, maintain artificial respiration, and place a manometer in a systemic artery, e.g., the carotid, and another in a branch of the pulmonary artery. In the latter case, we can watch the order of events in the heart itself, when the artificial respiration is interrupted. It is well to study the events in both cases.

If the blood-pressure be measured in a systemic artery, e.g., the carotid, it is found that the blood-pressure rises very rapidly and to a great extent during the first and second stages; the pulse-beats at first are quicker, but soon become slower and more vigorous. During the third stage it falls rapidly to zero. The great rise of the blood-pressure during the first and second stages is chiefly due to the action of the venous blood on the general vaso-motor centre, causing constriction of the small systemic arteries. The peripheral resistance is thus greatly increased, and it tends to cause the heart to contract more vigorously, but the slower and more vigorous beats of the heart are also partly due to the action of the venous blood on the cardio-inhibitory centre in the medulla.

If the second method be adopted, viz., to open the chest, keep up artificial respiration, and measure the blood-pressure in a branch of the pulmonary artery, as well as in a systemic artery, e.g., the carotid-we find that when the artificial respiration is stopped, in addition to the rise of the blood-pressure indicated in the carotid manometer, the cavities of the heart and the large veins near it are engorged with venous blood. There is, however, but a slight comparative rise in the blood-pressure in the pulmonary artery. This may be accounted for, either by the pulmonary artery not being influenced to the same extent as other arteries, by the vaso-motor centre, or by its greater distensibility (Lichtheim-compare $\S 88$ ). But, as the heart itself is supplied through the coronary arteries with venous blood, its action soon becomes weakened, each beat becomes feebler, so that soon the left ventricle ceases to contract, and is unable to overcome the great peripheral resistance in the systemic arteries, although the right ventricle may still be contracting. As the blood becomes more venous, the vasomotor centre becomes paralysed, the small systemic arteries relax, and the blood flows from them into the veins, while the blood-pressure in 
the carotid manometer rapidly falls. The left ventricle, now relieved from the great internal pressure, may execute a few feeble beats, but they can only be feeble, as its tissues have been subjected to the action of the very impure blood. More and more blood accumulates in the right side from the causes already mentioned.

The violent inspiratory efforts in the early stages aspirate blood from the veins towards the right side of the heart, but of course this factor is absent when the chest is opened.]

[Recovery from the condition of asphyxia.-If the trachea of a dog be closed suddenly and completely, the average duration of the respiratory movements is 4 minutes 5 seconds, while the heart continues to beat for about 7 minutes. Recovery may be obtained if proper means be adopted before the heart ceases to beat; but after this, never.

If a dog be drowned, the result is different. After complete submersion for $1 \frac{1}{2}$ minutes, recovery did not take place. In the case of drowning, air passes out of the chest, and water is inspired into and fills the air-vesicles. It is rare for recovery to take place in a person deprived of air for more than five minutes. If the statements of sponge-divers are to be trusted, a person may become accustomed to the deprival of air for a longer time than usual. In cases where recovery takes place after a much longer period of submersion, it has been suggested that, in these cases, syncope occurs, the heart beats but feebly or not at all, so that the oxygen in the blood is not used up with the same rapidity. It is a well-known fact that newly-born and young puppies can be submerged for a long time before they are suffocated.]

Artificial Respiration.-The methods of performing artificial respiration in persons apparently suffocated are fully given in vol. $i i$, under Nervous Mechanism of Respiration.

\section{Respiration of Foreign Gases.}

No gas without a sufficient admixture of $\mathrm{O}$ can support life. Even with completely innocnous and indifferent gases, if no $O$ be mixed with them, they cause suffocation in 2 to 3 minutes.

I. Completely indifferent gases are $\mathrm{N}, \mathrm{H}, \mathrm{CH}_{4}$. The living blood of an animal breathing these gases yields no $\mathrm{O}$ to them (Pflüger).

II. Poisonous gases.-(a.) Those that displace $\mathrm{O}$, and form a permanent stable compound with the hæmoglobin-(1.) CO (\$ 16 and 17). (2.) CNH (Hydrocyanic acid) displaces (?) 0 from hæmoglobin, with which it forms a more stable compound and kills exceedingly rapidly. It prevents $\mathrm{O}$ being changed into ozone in the blood. Blood-corpuscles charged with hydrocyanic acid lose the property of decomposing liydric peroxide into water and $O(\$ 17,5)$.

(b.) Narcotic gases. - (1.) $\mathrm{CO}_{2}-\mathrm{v}$. Pettenkofer characterises air containing $\mathrm{O}$ with $\cdot 1$ p.c. $\mathrm{CO}_{2}$ as "bad air ;" still, air in a room containing this amount of $\mathrm{CO}_{2}$ produces a disagreeable feeling rather from the impurities mixed with it than from the actual amount of $\mathrm{CO}_{2}$ itself. Air containing $\mathrm{I}$ p.c. $\mathrm{CO}_{2}$ produces decided discomfort, and with 10 p.c. it endangers life, while larger amounts cause death with symptoms of coma. (2.) $\mathrm{N}_{2} \mathrm{O}$ (nitrous oxide) respired, mixed with $\frac{7}{5}$ volume 0 , causes, after 1 to 2 minutes, a short temporary stage of excitement ("Laughing gas" of $\mathrm{H}$. Davy), which is succeeded by unconsciousness, and afterwards an increased excretion of $\mathrm{CO}_{2}$. (3.) Ozonised air causes similar effects (Binz). 
(c.) Reducing gases. - (1.) $\mathrm{H}_{2} \mathrm{~S}$ (sulphuretted hydrogen) rapidly robs bloodcorpuscles of $\mathrm{O}: \mathrm{S}$ and $\mathrm{H}_{2} \mathrm{O}$ being formed, and death occurs rapidly before the gas can decompose the hæmoglobin (Hoppe-Seyler).

(2.) $\mathrm{PH}_{3}$-Phosphuretted hydrogen is oxidised in the blood to form phosphoric acid and water with decomposition of the hæmoglobin (Dybkowski, Koschlakoff, and Popoff).

(3.) $\mathrm{AsH}_{3}$, arseniuretted hydrogen and $\mathrm{SbH}_{2}$, antimoniuretted hydrogen, act like $\mathrm{PH}_{3}$, but in addition, the hæmoglobin passes out of the stroma and appears in the urine.

(4.) $\mathrm{C}_{2} \mathrm{~N}_{2}$, cyanogen absorbs $\mathrm{O}$, and decomposes the blood (Rosenthal and Laschkewitsch).

III. Irrespirable gases, i.e., gases which, on entering the larynx, cause reflex spasm of the glottis. When introduced into the trachea they cause inflammation and death. Under this category come hydrochloric, hydrofluoric, sulphurous, nitrous, and nitric acids, ammonia, chlorine, fluorine, and ozone.

\section{Accidental Impurities of the Air.}

Dust Particles.-Amongst these are dust particles which occur in enormous amount suspended in the air, and thereby act injuriously upon the respiratory organs. The ciliated epithelium of the respiratory passages eliminates a large number of them. Some of them, however, reach the air-vesicles of the lung, where they penetrate the epithelium, reach the interstitial lung-tissue and lymphatics and so pass with the lymph-stream into the bronchial glands. Particles of coal or charcoal are found in the lungs of all elderly individuals, and blacken the alveoli. In moderate amount these black particles do not seem to do any harm in the tissues, but when there are large accumulations they give rise to lung affections, which lead to disintegration of these organs. [In coal-miners, for example, the luug-tissues along the track of the lymphatics and in the bronchial glands are quite black, constituting "coal-miners' lung."'] In many trades various particles occur in the air; miners, grinders, stone-masons, file-makers, weavers, spinners, tobacco manufacturers, millers, and bakers, suffer from lung affections caused by the introduction of particles of various kinds inhaled during the time they are at work.

There seems no doubt that the seeds of some contagious diseases may be inhaled. Diphtheritic bacteria become localised in the pharynx and in the larynxglanders in the nose-measles in the bronchi-hay-monads in the nose. Many seeds of disease pass into the mouth along with air, are swallowed, and undergo development in the intestinal tract, as is probably the case in cholera and typhoid fever.

\section{Ventilation of Rooms.}

Fresh air is as necessary for the healthy as for the sick. Every healthy perion ought to have a cubic space of 800 cubic feet, and every sick person 1000 cubic feet of space. [The space allowed per individual varies greatly, but 1000 cubic feet is a fair average. If the air in this space is to be kept sweet, so that the $\mathrm{CO}_{2}$ does not exceed $\cdot 06$ p.c., 2000 cubic feet of air per hour must be supplied.] In France only 42 cubic feet per head are allowed in barracks, 60 cubic feet in hospitals. In Prussia in barracks 420-500 cubic feet are allowed for every soldier, for hospital 600-720; in England 600 cubic feet per head. When there is overcrowding in a room the amount of $\mathrm{CO}_{2}$ increases. v. Pettenkofer found the normal amount of $\mathrm{CO}_{2}(\cdot 04$ to $\cdot 05$ per 1000) increased in comfortable rooms to 
$0.54-0.7$ per 1000 ; in badly ventilated sick chambers $=2.4$; in overcrowded auditoriums, $3 \cdot 2$; in pits $=4.9$; in school-rooms, $7 \cdot 2$ per 1000 . Although it is not the quantity of $\mathrm{CO}_{2}$ which makes the air of an overcrowded room injurious, but the excretions from the outer and inner surfaces of the body, which give a distinct odour to the air, quite recognisable by the sense of sinell, still, the amount of $\mathrm{CO}_{2}$ is taken as an index of the presence and amount of these other deleterious sub. stances. The question as to whether the ventilation of a room or ward occupied by persons is sufficient, is ascertained by estimating the amount of $\mathrm{CO}_{2}$. A room which does not give a disagreeable, somewhat stuffy, odour has less than 0.7 per 1000 of $\mathrm{CO}_{2}$, while the ventilation is certainly insufficient if the $\mathrm{CO}_{2}=1$ per 1000 .

As the air contains only 0.0005 cubic meter $\mathrm{CO}_{2}$ in 1 cubic meter of air, and as an adult produces hourly 0.0226 cubic meters $\mathrm{CO}_{2}$, calculation shows that every person requires 113 cubic meters of fresh air per hour, if the $\mathrm{CO}_{2}$ is not to exceed 0.7 per $1000:$ for $0.7: 1000=(0.0226+x \times 0.0005): x$, i.e., $x=113$.

In ordinary rooms, where every person is allowed the necessary space $(1000 \mathrm{cubic}$ feet) the air is sufficiently renewed by means of the pores in the walls of the room, by the opening and shutting of doors, and by the fireplace, provided the damper is kept open.

It is most important to notice that the natural ventilation be not interfered with by dampness of the walls, for this influences the pores very greatly. At the same time, damp walls are injurious to health by conducting away heat, and in them the germs of infectious diseases may develop (Lindwurm).

\section{Formation of Mucus in the Respiratory Passages-Sputum.}

The respiratory mucous membrane is covered normally with a thin layer of mucus (Fig. 97). By its presence this substance so far inhibits the formation of new mucus by protecting the mucous glands from the action of cold or other irritative agents. New mucus is secreted as that already formed is removed. An increased secretion accompanies congestion of the respiratory mucous membrane. Division of the nerves on one side of the trachea (cat) causes redness of the tracheal mucous membrane and increased secretion (Rossbach).

Effects of rearents on the mucous secretion.-If ice be placed on the belly of an animal so as to cause the animal to "take a cold," the respiratory mucous membrane first becomes pale, and afterwards there is a copious mucous secretion, the membrane becoming deeply congested. The injection of sodium carbonate and ammonium chloride limits the secretion. The local application of alum, silver nitrate, or tannic acid makes the mucous membrane dry, and the epithelium is shed. The secretion is excited by apomorphin, emetin, pilocarpin, and ipecacuanha, while it is limited by atropin and morphia (Rossbach).

Normal Sputum.-Under normal circumstances some mucus-mixed with a little saliva-may be coughed up from the back of the throat. In catarrhal conditions of the respiratory mucous membrane, the sputum is greatly increased in amount, and is often mixed with other characteristic products. Microscopically, sputum contains :-

1. Epithelial cells-chiefly squames from the mouth and pharynx 
(Fig. 115), more rarely alveolar epithelium and ciliated epithelium (7) from the respiratory passages. The epithelial cells are often altered, having undergone maceration or other changes. Thus some cells may have lost their cilia (6).

The epithelium of the alveoli (2) is squamous epithelium, the cells being 2 to 4 times the breadth of a colourless blood-corpuscle. These cells occur chietly in the morning sputum in individuals over 30 years of age. In younger persons their presence indicates a pathological condition of the pulmonary parenchyma (Guttman, H. Schmidt, and Bizzozero). They often undergo fatty degeneration, and they may contain pigment granules (3); or, they may present the appearance of what Buhl has called "myelin degenerated cells;" i.e., cells filled with clear refractive drops of various sizes, some colourless, others coloured particles, the latter having been absorbed (4). Mucin in the form of myelin drops (5)? is always present in sputum.

2. Lymphoid cells (9) are to be regarded as colourless blood-corpuscles which have wandered out of the blood-vessels; they are most numerous in yellow sputum, and less numerous in the clear, mucus-like excretion. The lymph-cells often present alterations in their characters; they may be shrivelled up, fatty, or present a granular appearance.
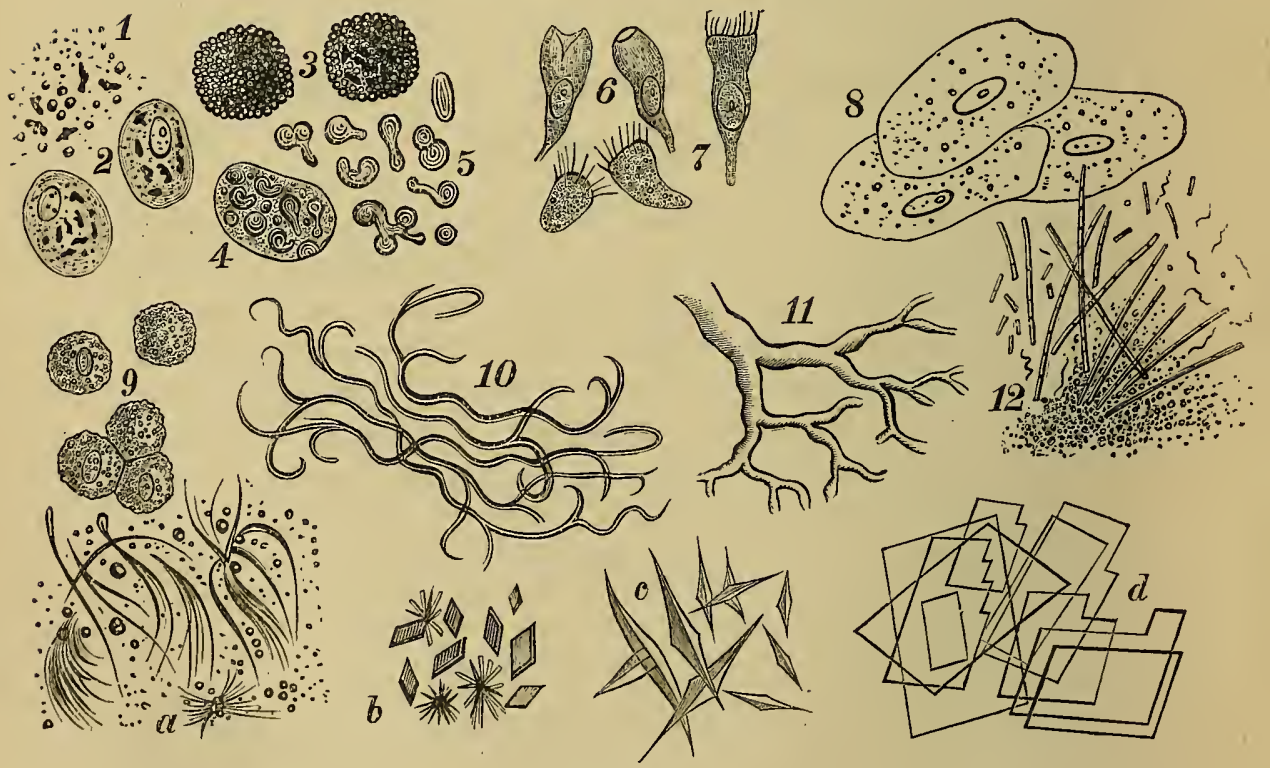

Fig. 115.

Various objects found in sputum-1, Detritus and particles of dust; 2, alveolar epithelium with pigment; 3, fatty and partly pigmented alveolar epithelium; 4 , alveolar epithelium containing myelin-forms; 5 , free myelin-forms; 6,7 , ciliated epithelium, some changed, others without cilia; 8 , squamous epithelium from the mouth; 9 , leucocytes; 10, elastic fibres; 11 , fibrin-cast of a small bronchus; 12, leptothrix buccalis with cocci, bacteria, and spirochæti; $a$, fatty acid crystals and free fatty granules; $b$, hæmatoidin; $c$, Charcot's crystals ; $d$, Cholesteriu. 
The fluid substance of the sputum contains much mucus arising from the mucous glands and goblet cells; together with nuclein, and lecithin, and the constituents of saliva according to the amount of the latter mixed with the secretion. Albumin occurs only during inflammation of the respiratory passages, and its amount increases with the degree of inflammation. Urea has been found in cases of nephritis.

Pathological.-In cases of catarrh, the sputum is at first usually sticky and clear (sputa cruda), but later it becomes more firm and yellow (sputa cocta). Under pathological conditions there may be found in the sputum-(a.) Red bloodcorpuscles from rupture of a blood-vessel. (b.) Elastic-fibres (10) from disintegration of the alveoli of the lung; usually the bundles are finc, curved, and the fibres branched. [In certain cases it is well to add a solntion of caustic potash, which dissolves the other elements and leaves the elastic fibres untouched.] Their presence always indicates destruction of the lung-tissue. (c.) Colourless plugs of fibrin (11), casts of the smaller or larger bronchi, occur in some cases of fibrinous exudation into the finer air-passages. (d.) Crystals of various kinds-Crystals of fatty acids (Fig. 115, a) in bundles of fine needles. They indicate great decomposition of the stagnant secretion-colourless, sharp-pointed, octagonal, or rhombic plates $-(c)$ (Charcot's crystals) of unknown nature (perhaps tyrosin), Hæmatoidin (b) and cholesterin crystals $(d)$ occur much more rarely. $\left(f_{0}\right)$ Fungi and other lower organisms frequently occur. The threads of leptotlrix buccalis (12); Oidium albicans in the mouth of sucklings, rol-shaped bacilli and bacteria. In phthisis, the tubercle-bacillus of Koch.

Abnormal coloration of the sputum-red from blood-wlien the blood remains long in the lung it undergoes a regular series of changes and tinges the sputum dark red, bluish brown, brownish yellow, deep yellow, yellowish green, or grass green. The sputum is sometimes yellow in jaundice. The sputum inay be tinged by what is inspired [as in the case of the "black-spit" of miners.]

The odour of the sputum is more or less unpleasant. It becomes very disagreeable when it has remained long in pathological lung cavities, and it is stinking in gangrene of the lung.

\section{Action of the Atmospheric Pressure.}

At the normal pressure of the atmosphere (height of the barometer, 760 millimetres $\mathrm{Hg}$.), pressure is exerted upon the entire surface of the body $=15,000$ to 20,000 kilos., according to the extent of the superficial area (Galileo). This pressure acts equally on all sides upon the body, and occurs also in all internal cavities containing air, both those that are constantly filled with air (the respiratory passages and the spaces in the superior maxillary, frontal, and ethmoid bones), and those that are temporarily in direct communication with the outer air (the digestive tract and tympanum ). As the fuids of the body (blood, lymph, secretions, parenchymatous juices) are practically incompressible, their volume remains practically unchanged under the pressure; but they will absorb gases from the air corresponding to the prevailing pressure (i.e., the partial pressure of the individual gases), and according to their temperature (compare $\S 33$ ). 
The solids consist of elementary parts (cells and fibres), each of which presents only a microscopic surface to the pressure, so that for each cell the prevailing pressure of the air can only be calculated at a few millimetres-a pressure under which the most delicate histological tissues undergo development. As an example of the action of the pressure of the atmospheric pressure upon large masses, take that brought about by the adhesion of the smooth, sticky, moist articular surfaces of the shoulder and hip joints. In these cases, the arm and the leg are supported without the action of muscles. The thighbone remains in its socket after section of all the muscles and its capsule (Brothers' Weber). Even when the colytoid cavity is perforated, the limb does not fall out of its socket. The ordinary barometric variations affect the respiration-a rise of the barometric pressure excites, while a fall diminishes, the respirations. The absolute amount of $\mathrm{CO}_{2}$ remains the same $(\S 127,8)$.

A Great Diminution of the Atmospheric Pressure, such as occurs in ballooning (highest ascent, 8,600 meters), or in ascending mountains, causes a series of characteristic phenomena :-(1.) In conseqnence of the diminution of the pressure upon the parts directly in contact with the air, they become greatly congested, hence, there is redness and swelling of the skin and free mucous membranes; there may be hæmorrhage from the nose, lungs, gums, turgidity of the cutaneous veins; copious secretion of sweat, great secretion of mucus. (2.) $\Lambda$ feeling of weight in the limbs, a pressing outwards of the tympanic membrane (until the tension is equilibrated by opening of the Eustachian tube), and as a consequence noises in the ears and difficulty of hearing. (3.) In consequence of the diminished tension of the $O$ in the air (\$129), there is difficulty of breathing, pain in the chest, whereby the respirations (and pulse) become more rapid, deeper, and irregular. When the atmospheric pressure is diminished $\frac{1}{2}-\frac{1}{3}$, the amount of 0 in the blood is diminished (Bert, Fränkel and Geppert), the $\mathrm{CO}_{2}$ is imperfectly removed from the blood, and in consequence there is diminished oxidation within the body. When the atmospheric pressure is diminished to one-half, the amount of $\mathrm{CO}_{2}$ in arterial blood is lessened; and the amount of $\mathrm{N}$ diminishes proportionally with the decrease of the atmospheric pressure (Fränkel and Gepert). The diminished tension of the air prevents the vibrations of the vocal cords from occurring so forcibly, and hence the voice is feeble. (5.) In consequence of the amount of blood in the skin, the internal organs are relatively anæmic; hence, there is diminished secretion of urine, muscular weakness, disturbances of digestion, dullness of the senses, and it may be unconsciousness, and all these phenomena are intensified by the conditions mentioned under (3). Some of these phenomena are modified by usage. The highest limit at which a man may still retain his senses is placed by Tissandier at an elevation of 8,000 metres $(280 \mathrm{~nm} . \mathrm{Hg})$. In dogs the blood-pressure falls, and the pulse becomes small and diminished in frequency, when the atmospheric pressure falls to $200 \mathrm{~mm}$. $\mathrm{Hg}$.

Those who live upon high mountains suffer from a disease (mal de montagne), which consists essentially in the above symptoms, although it is sometimes complicated with anæmia of the internal organs. Al. v. Humboldt found that in those who lived on the Andes, the thorax was capacious. At 6,000 to 8,000 feet above sea-level, water contains only one-third of the absorbed gases, so that fishes cannot live in it (Boussingault). Animals may be subjected to a farther diminution of the atmospheric pressure, by being placed under the receiver of an air-pump. Birds 
die when the pressure is reduced to $120 \mathrm{~mm}$. Hg.; mammals at $40 \mathrm{~mm}$. Hg.; frogs endure repeated evacnations of the receiver, whereby they are much dis. tended, owing to the escape of gases and water, but after the entrance of air they become greatly compressed. The cause of death in mammals is ascribed by Hoppe-Seyler to the evolution of bubbles of gas in the blood; these bubbles stop up the capillaries, and the circulation is arrested. Local diminution of the atmo spheric pressure causes marked congestion and swelling of the part, as occurs when a cupping-glass is used.

Great Increase of the Atmospheric Pressure.-The phenomena, which are, for the most part, the reverse of the foregoing, have been observed in pneumatic cabinets and in diving bells, where men may work even under $4 \frac{1}{2}$ atmospheres pressure. The phenomena are:-(1.) Paleness and dryness of the external surfaces, collapse of the cutaneous veins, diminution of perspiration, and mucous secretions. (2.) The tympanic membrane is pressed inwards (until the air escapes through the Eustachian tube, after causing a sharp sound), acute sounds are heard, pain in the ears, and difficulty of hearing. (3.) A feeling of lightness and freshness during respiration, the respiration becomes slower (by 2-4 per minute), inspiration easier and shorter, expiration lengthened, the pause distinct. The capacity of the lungs increases, owing to the freer movement of the diaphragm, in consequence of the diminution of the intestinal gases. Owing to the more rapid oxidations in the body, muscular movement is easier and more active. The $\mathrm{O}$ absorbed and the $\mathrm{CO}_{2}$ excreted are increased. The venous blood is reddened. (4.) Difficulty of speaking, alteration of the tone of the voice, inability to whistle. (5.) Increase of the urinary secretion, more muscular energy, more rapid metabolism, increased appetite, subjective feeling of warmth, pulse beats slower, and pulse-curve is lower (compare p. 150). In animals subjected to excessively high atmospheric pressure, P. Bert found, that the arterial blood contained 30 vols. per cent. $\mathrm{O}$ (at $760 \mathrm{~mm}$. Hg.); when the amount rose to 35 vol. per cent., death occurred with convulsions. Compressed air has been used for therapeutical purposes, but in doing so a too rapid increase of the pressure is to be avoided. Waldenburg has constructed such an apparatus, which may be used for the respiration of air under a greater or less pressure.

\section{Comparative and Historical.}

Mammals have lungs similar to those of man. The lungs of birds are spongy, united to the chest-wall, and there are openings on their surface communicating with thin-walled "air-sacs" which are placed amongst the viscera. The air-sacs communicate with cavities in the bones, which give the latter great lightness (Aristotle). The diaphragm is absent. In reptiles the lungs are divided into greater and smaller compartments ; in snakes one lung is abortive, while the other has the elongated form of the body. The amphibians (frog) possess two simple lungs, each of which represents an enormous infundibulum with its alveoli. The frog pumps air into its lungs by the contraction of its throat, the nostrils being closed and the glottis opened. When young-until their metamorphosis-frogs breathe like fishes by means of gills. The perennibranchiate amphibians (Proteus), retain their gills throughout life. Amongst fishes, which breathe by gills and use the $\mathrm{O}$ absorbed by the water, the Dipnoi have in addition to gills a swim-bladder provided with afferent and efferent vessels, which is comparable to the lung. The Cobitis respires also with its intestine (Erman, 1S0S). Insects and centipedes respire by " tracheæ," which are branched canals distributed throughout the body ; they open on the surface of the body by openings (stigmata) which can be closed. Spiders respire by means of tracheæ and tracheal sacs, crabs by gills. The molluses and cephalopods have gills, some gasteropods have gills and others lungs. 
Amongst the lower invertebrata some breathe by gills, others by means of a special "water-vascular system," and others again by no special organs.

Aristotle (384 B. C.) regarded the object of respiration to be the cooling of the body, so as to moderate the internal warmth. He observed correctly that the warmest animals breathe most actively, but in interpreting the fact he reversed the cause and effect. Galen (131-203 A.D.), thought that the "soot" was removed from the body along with the expired water. The most important experiments on the mechanics of respiration date from Galen; he observed that the lungs passively follow the movements of the chest; that the diaphragm is the most important muscle of inspiration; that the external intercostals are inspiratory; and the internal, expiratory. He divided the intercostal nerves and muscles, and observed that loss of voice occurred. On dividing the spinal cord higher and higher, he found that as he did so, the muscles of the thorax lying higher up, became paralysed. Oribasius (360 A.D.) observed that in double pneumothorax both lungs collapsed. Vesalius (1540) first described artificial respiration, as a means of restoring the beat of the heart. Malpighi (166I) described the structure of the lungs. J. A. Borelli ( +1679$)$ gave the first fundamental description of the mechanism of the respiratory movements. The chemical processes of respiration could only be known after the discovery of the individual gases therein concerned. Van Helmont $(+1644)$ detected $\mathrm{CO}_{2}$. [Joseph Black (1757) discovered, by the following experiment, that $\mathrm{CO}_{2}$ or "fixed air" is given out during expiration:-take two jars of lime water, breathe into one through a bent glass tube, and force ordinary air through the other, when a white precipitate of calcium carbonate will be found to occur in the former.] In $\mathbf{1 7 7 4}$ Priestley discovered 0 . Lavoisier detected N (1775), and ascertained the composition of atmospheric air, and he regarded the formation of $\mathrm{CO}_{2}$ and $\mathrm{H}_{2} \mathrm{O}$ of the breath as a result of a combustion within the lungs themselves. J. Ingen-Houss (1730-1790) discovered the respiration of plants. Vogel and others proved the existence of $\mathrm{CO}_{2}$ in venous blood, and Hoffmann and others that of $\mathrm{O}$ in arterial blood. The more complete conception of the exchange of gases was, howerer, only possible after Magnus had extracted and analysed the gases of arterial and venous blood (p. 55). 


\section{Physiology of Digestion.}

\section{The Niouth and its Glands.}

THE mucous membrane of the cavity of the mouth, which becomes continuous with the skin at the red margin of the lips, has a number of sebaceous glands in the region of the red part of the lip. The buccal mucous membrane consists of bundles of fine fibrous tissue mixed with elastic fibres, which traverse it in every direction. Papillæ-simple or compound-occur near the free surfaces. The sub-mucous tissue, which is directly continuous with the fibrous tissue of the mucous membrane itself, is thickest where the mucous membrane is thickest, and densest where it is firmly fixed to the periosteum of the bone and to the gum; it is thinnest where the mucous membrane is most movable, and where there are most folds. The cavity of the montl is lined by stratified squamous epithelium (Fig. 115, 8), which is thickest, as a rule, where the longest papillæ occur.

All the glands of the mouth, including the salivary glands, may be divided into different classes according to the nature of their secretions.

1. The serous or albuminous glands [true salivary], whose secretion contains a certain amount of albumin, e.g., the human parotid.

2. The mucous glands, whose secretion in addition to some albumin, contains the characteristic constituent mucin.

3. The mixed [or muco-salivary] glands, some of the acini secreting albumin and others mucin-e.g., the human maxillary gland (Heidenhain). The structure of these glands is referred to under the salivary glands.

Numerous mucous glands (labial, buccal, palatine, lingual, molar) bave the appearance of small macroscopic bodies lying in the sub-mucosa. They are branched tubular glands, and the contents of their secretory cells consist partly of mucin, which is expelled from them during secretion. The excretory ducts of these glands, which are lined by cylindrical epithelium, are constricted where they enter the mouth. Not unfrequently one duct receives the secretion of a neighbouring gland.

The glands of the tongue form two groups, which differ morphologically and physiologically. (1.) The mucous glands (Weber's glands), occurring chiefly near the root of the tongue, are branched tubular glands lined with clear transparent secretory cells whose nuclei are placed near the attached end of the cells. The acini have a distinct membrana propria. (2.) The serous glands (Ebner's) are acinous glands occurring in the region of the circumvallate papillæ (and in animals near the papillæ foliatæ). They are lined with turbid granular epithelium with a central nucleus, and they secrete saliva (Henle). (3.) The glands of Blandin and Nuhn are placed near the tip of the tongue, and consist of mucous and serous acini, so that they are mixed glands (Podwisotzky).

The blood-vessels are moderately abundant, and the larger trunks lie in the 
sub-mucosa, whilst the finer twigs penetrate into the papillæ, where they form either a capillary net-work or simple loops.

The larger lymphatics lie in the sub-mucosa, whilst the finer branches form a fine net-work placed in the mucosa. The lymph-follicles also belong to the lymphatic system. On the dorsum of the posterior part of the tongue they form an almost continuous layer. They are round or oval (1-1.5 mm. in diameter), and placed in the sub-mucosa. They consist of adenoid tissue loaded with lymphcorpuscles. The outer part of the adenoid reticulum is compressed so as to form a kind of capsule for each follicle. Similar follicles occur in the intestine as solitary follicles, in the small intestine they are collected together into Peyer's patches, and in the spleen they occur as Malpighian corpuscles. On the dorsum of the tongue several of these follicles form a slightly oval elevation, which is surrounded by connective tissue. In the centre of this elevation there is a depression into which a mucous gland opens, which fills the small crater with mucus.

The Tonsils have fundamentally the same structure. On their surface are a number of depressions into which the ducts of small mucous glands opeu. These depressions are surrounded by groups (10-20) of lymph-follicles, and the whole is environed by a capsule of connective tissue. After E. H. Weber discovered lymphatics in the neighbourhood of the tonsils, Brücke referred these structures to the lymphatic system. Large lymph-spaces, communicating with lymphatics, occur in the neighbourhood of the tonsils, but as yet a direct connection between the spaces in the follicles and the lymph-vessels has not been proved to exist. Stohr found that numerous leucocytes passed between the epithelium covering the tonsils, and reached the mouth.

Nerves.-Numerous medullated nerve-fibres occur in the sub-mucosa, pass into the mucosa and terminate partly in the individual papillæ in Krause's End-bulbs, which are most abundant in the lips and soft palate, and not so numerous in the cheeks and in the floor of the mouth. The nerves administer not only to common sensation, but they also are the organs of transmission for tactile (heat and pressure) impressions. It is highly probable, however, that some nerve-fibres end in fine terminal fibrils, between the epithelial cells, such as occur in the cornea and elsewhere.

\section{The Salivary Glands.}

Structure of the Ducts.-The three pairs of salivary glands, submaxillary, sub-lingual, and parotid, are compound tubular glands. Fig. 116, A, shows a fine duct, terminating in the more or less flaskshaped alveoli or acini. [Each gland consists of a number of lobes, and each lobe in turn of a number of lobules, which, again, are composed of acini. All these are held together by a framework of connective tissue. The larger branches of the duct lie between the lobules, and constitute the interlobular ducts, giving branches to each lobule which they enter, constituting the intralobular ducts. These intralobular ducts branch and finally terminate in connection with the alveoli, by means of an intermediary or intercalary part. The larger interiobar and interlobular ducts consist of a membrana propria, strengthened outside with fibrous and elastic tissue, and in some places also by non-striped muscle, while the ducts are lined by columnar epithelial cells. In the largest branches, there is a second row of smaller cells, lying between 
the large cells and the membrana propria. The intralobular ducts are lined by a single layer of large cylindrical epithelium. As is shown in Fig. 116, E, the nuclens occurs about the middle of the cell, while the outer half, i.e., next the basement membrana of the cell, is finely striaterl longitudinally, which is due to fibrillæ; the inner half next the lumen is granular. The intermediary part is narrow, and is lined with a single layer of flattened cells, each with an elongated oval nucleus. There is usually a narrow "neck," where the intralobular duct becomes continuous with the intermediary part, and here the cells are polyherlral (Klein).

The acini, or alveoli, are the parts where the actual process of secretion takes place. They vary somewhat in shape-some are tubular, others hranched, some are dilated and resemble a Florence flask, and scveral of them usually open into one intermediary part of a duct. Each
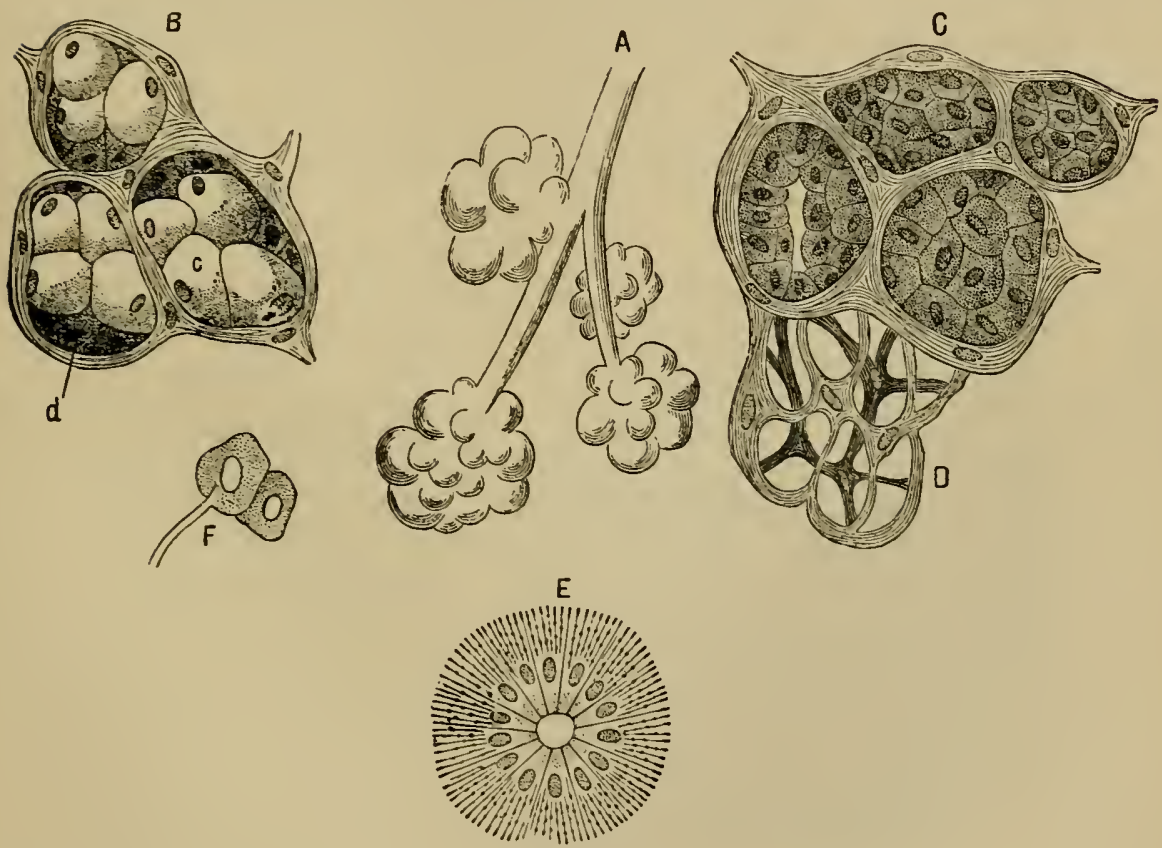

Fig. 116.

A, duct ant acini of the partid gland of a $\log ; B$, acini of the sub-maxillary gland of a dog ; $c$, refractive mucous cells; $d$, granular half-moous of Gianuzzi; $\mathrm{C}$, similar alveoli after prolonged secretion; $\mathrm{D}$, basket-shaped tissue investment of an acinus; $\mathrm{E}$, transverse section of an excretory duct lined with cylindrical "rodded" epithelium; F, entrance of a non-medullated nerve-fibre into a secretory cell.

alveolus is bounded by a basement membrane, with a reticulate structure made up of nucleated, branched and anastomosing cells, so as to resemble a basket (D). There is a homogeneous membrane bounding the 
alreoli in addition to this basket-shaped structure. Immediately outside this membrane is a lymph-space (Gianuzzi), and outside this again the net-work of capillaries is distributed. [The extent to which this lymph-space is filled with lymph determines the distance of the capillaries from the membrana propria. The interalveolar lymph-spaces communicate with large lymph-spaces between the lobules, which in turn communicate with perirascular lymphatics around the arteries and reins.] The lymphatics emerge from the gland at the hilum.

The secretory cells rary in structure, according as the salivary gland is a mucous [sub-maxillary and sub-lingual of the dog and cat], a serous [parotid of man, and mammals, and sub-maxillary of rabbit], or a mixed gland [human sub-maxillary and sub-lingual].

Mucous Acini.-The secretory cells of mucous glands, and the mucous acini of mixed glands (Fig. 11 $\tau$ ), are lined by a single layer of "mucin cells" (Heidenhain) (Fig. 116, B, c), which are large cells distended with mucin, or at least with a hypothetical substance, mucigen, which yields mucin. The mucin cells are more or less spheroidal in shape, clear, shining, highly refractire, and nearly fill the acinus. The flattened nuclens is near the wall of the

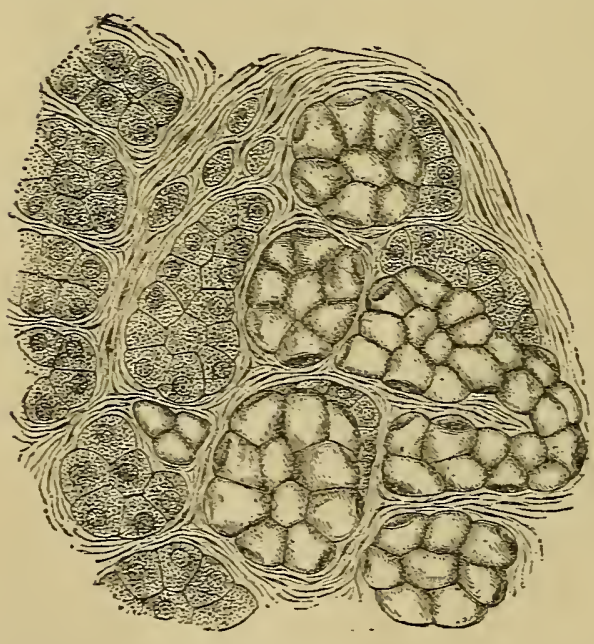

Fig. 117.

Section of part of the human sub-maxillary gland. On the left of the figure is a group of serous alveoli, and on the right a group of mucous alreoli. acinus. Each cell has a fine process which orerlaps the fixed part of the cell next to it. Owing to the fact that the body of each cell is infiltrated with mucin, these cells do not stain with carmine, although the nucleus and its immediately investing protoplasm do. Another kind of cell occurs in the sub-maxillary gland of the dog. It forms a halfmoon-shaped structure (Gianuzzi) lying in direct contact with the wall of the acinus. Each "halfmoon" or "crescent" consists of a number of small, closely packed, angular, strongly albuminous cells with small oral nuclei, which, however, are separated only with difficulty. Hence, Heidenhain has called them "composite marginal cells" $(\mathrm{B}, d$.$) They are granular, darker, devoid$ 
of mucin, and stain readily with pigments. [In the sub-maxillary gland of the eat there is a complete layer of these "marginal" carmine-staining cells lying between the mucous cells and the membrana propria.]

[Serous Acini.-In true serous glands (parotid of man and mammals) and in the serous acini of mixed glands, the acini are lined by a single layer of secretory columnar finely granular cells, which in the quiescent condition completely fill the acinus, so that scarcely any lumen is left. Just before secretion, or when these cells are quiescent, Langley has shown that they are large and filled with numerous granules, which obscure the presence of the nucleus. As secretion takes place, these granules seem to be used up or discharged into the lumen; at least, the outer part of each cell gradually becomes clear and more transparent, and this condition spreads towards the inner part of the cell.]

[In the mixed or muco-salivary glands (Klein), (e.g., human submaxillary), some of the alveoli are mucous and others serous in their characters, but the latter are always far more numerous, and the one kind of acinus is directly continuous with the others (Fig. 117)].

\section{Histological Changes during the Activity of the Salivary Glands.}

[The condition of physiological activity of the gland-cells is accompanied by changes in the histological characters of the secretory cells.]

[Serous Glands.-The changes in the secretory cells have been carefully studied in the parotid of the rabbit. The histological appearances vary somewhat, according as the glands are examined in the fresh condition or after liardening in various reagents, such as absolute alcohol. When the gland is at rest, in a preparation hardened in alcohol, and stained with carmine, the cells consist of a pale, almost uncoloured substance, with a few fine granules, and a small irregular red-stained, shrivelled nucleus, devoid of a nucleolus. The appearance of the nucleus suggests the idea of its being shrivelled by the action of the hardening reagent (Fig. 118)].

[During activity, if the gland be caused to secrete by stimulating the sympathetic, all parts of the cells undergo a change (Figs. 118, 119) (1) The cells diminish somewhat in size; (2) the nuclei are no longer irregular, but round, with a sharp contour and nucleoli; (3) the substance of the cell itself is turbid, owing to the diminution of the clear substance, and the increase of the granules, especially near the nuclei; (4) at the same time, the whole cell stains more deeply with carmine (Heidenhain). 
On studying the changes which occur in a living serous gland, Langley found that, cluring rest, the substance of the cells of the parotid is pervaded by fine granules, which are so numerous as to obscure the nucleus, while the outlines of the cells are indistinct. No lumen is visible in the acini during activity, the granules disappear from the outer zone of the cells, the cells themselves becoming more distinct and smaller. After

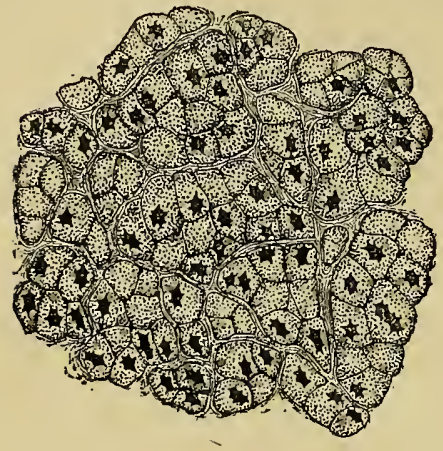

Fig. 118.

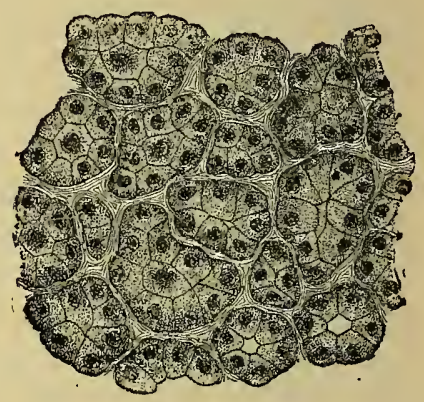

Fig. 119.

Sections of a "serous" gland-the parotid of a rabbit, Fig. 118, at rest; Fig. 119, after stimulation of the cervical sympathetic.

prolonged secretion, the granules largely disappear from the cell-substance except quite near the inner margin. The cells are smaller, their outlines more distinct, their round nuclei apparent, and the lumen of the acini is wide and distinct. Thus, it is evident that, during rest, granules are manufactured, which disappear during the activity of the cells, the disappearance taking place from without inwards. Similar changes occur in the cells of the pancreas.]

[Mucous Glands.--More complex changes occur in the mucous glands, such as the sub-maxillary or orbital glands of the dog (Lavdovsky). The appearances vary according to the intensity and duration of the secretory activity.

The mucous cells at rest are large, clear, and refractive, containing a flattened nucleus (Fig. 116, B, $c$ ), surrounded with a small amount of protoplasm, and placed near the basement membrane. The clear substance does not stain with carmine, and consists of mucigen lying in the wide spaces of an intracellular plexus of fibrils. After prolonged secretion, produced, it may be, by strong and continued stimulation of the chorda, the mucous cells of the sub-maxillary gland of the dog undergo a great change.] The distended, refractive, and "mucous-cells," which occur in the quiescent gland, and which do not stain with carmine, do not appear after the gland has been in a state of activity. Their place is taken by small dark protoplasmic cells (Fig. 116, C), devoid of mucin. These cells 
readily stain with carmine, whilst their nucleus is scarcely, if at all, coloured by the dye. The researches of $R$. Heidenhain (1868) have shed much light on the secretory activity of the salivary glands.

The change may be produced in two ways. Either it is due to the "mucons cells" during secretion becoming broken up, so that they yield their mucin directly to the saliva; in saliva rich in mucin, small microscopic pieces of mucin are found, and sometimes mucous cells themselves are present. Or, we must assume that the mucous cells simply eliminate the mucin from their bodies (Ewald, Stöhr); while, after a period of rest, new mucin is formed. According to this view, the dark granular cells of the glands, after active secretion, are simply mucous cells, which have given out their mucin. If we assume, with Heidenhain, that the mucous cells break up, then these granular non-mucous cells must be regarded as new formations produced by the proliferation and growth of the composite marginal cells, i.e., the crescents, or half-moons of Gianuzzi.

[During rest, the protoplasm seems to manufacture mucigen, which is changed into and discharged as mucin in the secretion, when the gland is actively secreting. Thus, the cells become smaller, but the protoplasm of the cell seems to increase, new mucigen is manufactured during rest, and the cycle is repeated.]

\section{The Nerves of the Salivary Glands.}

The nerves are for the most part medullated, and enter at the hilun of the gland, where they form a rich plexus provided with ganglia between the lobules. [According to Klein, there are no ganglia in the parotid gland.]

All the salivary glands are supplied by branches from two different nerves-from the sympathetic and from a cranial nerve.

1. The sympathetic nerve gives branches to (a.) the sub-maxillary and the sub-lingual glands, derived from the plexus on the external maxillary artery; $(b$.$) to the parotid gland from the carotid plexus.$

2. The facial nerve gives branches to the sub-maxillary and sub-lingual glands from the chorda tympani which accompanies the lingual branch of the fifth nerve. The branches to the parotid reach it in a roundabout way. They arise from the tympanic branch of the glosso-pharyngeal nerve (dog). The tympanic plexus sends fibres to the small superficial petrosal nerve (Eckhard, Loeb, Heidenhain), and with it these fibres run to the anterior surface of the pyramid in the temporal bone, and, after passing through the foramen lacerum anticum, reach the otic ganglion. This ganglion sends branches to the auriculo-temporal nerve (itself derived from the third branch of the trigeminus), which, as it passes upwards to the temporal region under cover of the parotid, gives branches to this gland (v. Wittich).

The sub-maxillary ganglion, which gives branches to the sub- 
maxillary and sub-lingual glands, receives fibres from the tympanicolingual nerve, as well as sympathetic fibres from the plexus on the external maxillary artery.

Termination of the nerve-fibres. - With regard to the ultimate distribution of these nerves we can distinguish (1) the vaso-motor nerves, which give branches to the walls of the blood-vessels; and (2) the secretory nerves proper. Pflüger states, with regard to the latter, that (a.) medullated nerve-fibres penetrate the acini; the sheath of Schwann (gray sheath) unites with the membrana propria of the acinus; the medullated fibre--still medullated-passes between the secretory cells, where it divides and becomes non-medullated, and its axial cylinder terminates in connection with ${ }^{7}$ the nucleus of a secretory cell. [This, however, is not proved] (Fig. 116, F).

(b) According to Pflüger, some of the nerve-fibres end in multipolar ganglion cells, which lie outside the wall of the acinus, and these cells send branches to the secretory cells of the acini. [These cells probably correspond to the branched cells of the basket-shaped structure.]

(c) Again, he describes medullated fibres which enter the attached end of the cylindrical epithelium lining the excretory ducts of the glands (E). Pflüger thinks that those fibres entering the acini directly are cerebral, while those with ganglia in their course are derived from the sympathetic system.

$[(d)$ The direct termination of nerve-fibres has been observed in the salivary glands of the cockroach by Kupffer.]

\section{Action of the Nervous System on the Secretion of Saliva.}

A. Sub-maxillary Gland.-Stimulation of the facial nerve at its origin (Ludwig and Rahn) causes a profuse secretion of a thin watery saliva, which contains a very small amount of specific constituents (Eckhard). Simultaneously with the act of secretion, the bloodvessels of the glands become dilated, and the capillaries are so distended that the pulsatile movement in the arteries is propagated into the veins. Nearly four times as much blood flows out of the veins (Cl. Bernard), the blood being of a bright red colour, and contains onethird more $O$ than the venous blood of the non-stimulated gland. Notwithstanding this relatively high percentage of $O$, the secreting gland uses more $\mathrm{O}$ than the passive gland $(\S 131,1)$.

[If a cannula be placed in Wharton's duct, e.g., in a dog, and the chorda tympani be divided, no secretion flows from the cannula. On stimulating the peripheral end of the chorda tympani with an interrupted current of electricity, the same results-copious secretion of saliva and vascular dilatation, with increased flow of blood through the gland- 
occur, as when the origin of the seventh nerve itself is stimulated. The watery saliva is called chorda saliva.]

Two functionally different kinds of nerve-fibres occur in the facial nerve-(1) True secretory fibres, (2) vaso-dilator fibres. The increased amount of secretion is not due simply to the increased blood supply.

II. Stimulation of the sympathetic nerve causes a scanty amount of a very thick, sticky, mucous secretion (Eckhard), in which the specific salivary constituents, mucin, and the salivary corpuscles are very abundant. The specific gravity of the saliva is raised from 1,007 to 1,010 . Simultaneously the blood-vessels become contracted, so that the blood flows more slowly from the veins, and has a dark bluish colour.

The sympathetic also contains two kinds of nerve-fibres-(1) True secretory fibres, and (2) vaso-constrictor fibres.

Relation to Stimu lus.- On stimulating the cerebral nerves, at first with a weak and gradually with a stronger stimulus, there is a gradual development of the secretion in which the solid constitnents--occasionally the organic-are increased (Heidenhain). If a strong stimulus be applied for a long time, the secretion diminishes, becomes watery, and is poor in specifie constitnents, especially in the organic elements, which are more affected than the inorganic (C. Ludwig and Becher). After prolonged stimulation of the sympathetic, the secretion resembles the chorda saliva. It would seem, therefore, that the chorda and sympathetic saliva are not specifically distinct, but vary only in degree. On continuing the stimulation of the nerves up to a certain maximal limit, the rapidity of secretion becomes greater, and the percentage of salts also increases to a certain maximum, and this independently of the former condition of the glands. The percentage of organic constitnents also depends on the strength of the nervous stimulation, but not on this alone, as it is essentially contingent upon the condition of the gland before the secretion took place, and it also depends upon the duration and intensity of the previous secretory activity. Very strong stimulation of the gland leaves an "after-effect" which predisposes it to give off organic constitnents into the secretion (Heidenhain).

Relation to Blood Supply.-The secretion of saliva is not simply the result of the amount of blood in the glands; that there is a factor independent of the changes in the state of the vessels is shown by the following facts :-

(1.) The secretory activity of the glands when their nerves are stimulated continues for some time after the blood-vessels of the gland have been ligatured (Ludwig, Czermack). [If the head of a rabbit be cut off, stimulation of the seventh nerve, above where the chorda leaves it, canses a flow of saliva which cannot be accounted for on the supposition that the saliva already present in the salivary glands is forced out of them. Thus we may have secretion withont a blood-stream. The saliva is really secreted from the lymph present in lymph-spaces of the gland (Ludwig)].

(2.) Atropin and Daturin extinguish the activity of the secretory fibres in the chorda tympani, but do not affect the vaso-dilator fibres (Heidenhain). The same results occur after the injection of acids and alkalies into the excretory duct (Gianuzzi). 
(3.) The pressure in the excretory duct of the salivary gland-measured by means of a manometer tied into it-may be nearly twice as great as the pressure within the arteries of the glands (Ludwig), or even in the carotid itself. The pressure in Wharton's duct may reach $200 \mathrm{~mm}$. Hg.

(4.) Just as in the case of muscles and nerves, the salivary glands become fatigned or exhausted after prolonged action. This result may also be brought about by injecting acids or alkalies into the duct, which shows that the secretory activity of the gland is independent of the circulation (Gianuzzi).

[The vascular dilatation and the increased flow of saliva due to the activity of the secretory cells, produced by stimulation of the chorda tympani, although they occur simultaneonsly, do not stand in the relation of cause and effect. We may cause vascular dilatation without an increased flow of saliva, as already stated (2). If atropin be given to an animal, stimulation of the chorda produces dilatation of the bloodvessels, but no secretion of saliva. Atropin paralyses the secretory fibres, but not the vaso-dilator fibres (Fig. 120). The increased supply of blood, while not causing, yet favours the act of secretion, by placing a larger amount of pabulum at the disposal of the secretory elements, the cells.]

[The experiment described under (3.) proves, in a definite manner, that the passage of the water from the blood-vessels, or at least from the lymph into the acini of the gland, cannot be due to the bloodpressure; that, in fact, it is not a mere process of filtration, such as occurs in the glomeruli of the kidney. In the case of the salivary gland, where the pressure within the gland may be double that of the arterial pressure, the water actually moves from the lymph against very great resistance. We can only account for this result by ascribing it to the secretory activity of the gland-cells themselves. Whether the activities of the gland-cells, as suggested by Heidenhain, are governed directly by two distinct kinds of nerve-fibres, a set of solid-secreting fibres, and a set of water-secreting fibres, remains to be proved.]

All these facts lead us to conclude that the nerves exercise a direct effect upon the secretory cells, apart from their action on the blood-vessels. This physiological consideration goes hand in hand with the anatomical fact of the direct continuation of nerve-fibres with the secretory cells. When the chorda tympani is extirpated on one side in young dogs, the sub-maxillary gland on that side does not develop so much-its weight is 50 per cent. less-while the mucous cells and the "crescents" are smaller than on the sound side (Bufalini).

During secretion, the temperature of the gland rises $1.5^{\circ} \mathrm{C}$ (Ludwig), and the blood flowing from the veins is often warmer than the arterial blood.

"Paralytic Secretion" of Saliva.-By this term is meant the continued secretion of a thin watery saliva from the sub-maxillary gland, which occurs 24 hours after the secretion of the cerebral nerves (chorda of the seventh), i.e., those branches of them that go to this gland, whether the 
sympathetic be divided or not (Cl. Bernard). It increases until the eighth day, after which it gradually diminishes, while the gland tissue degenerates. The injection of a small quantity of curara into the artery of the gland also causes it. Perhaps it arises from the secretion, which stagnates within the gland after section of the nerves, acting as a direct stimulus to secretion (Heidenhain). Perhaps it may be explained as a degeneration effect, comparable to the fibrillar contractions which occur in a muscle after secretion of its motor nerve.

B. Sub-lingual Gland.-Very probably the same relations obtain as in the sub-maxillary gland.

C. Parotid Gland.-In the dog, stimulation of the sympathetic alone, causes no secretion; it occurs when the glosso-pharyngeal branch to the parotid is simultaneously excited. This branch may be reached within the tympanum in the tympanic plexus. A thick secretion containing much organic matter is thereby obtained. Stimulation of the cerebral branch alone yields a clear thin watery secretion, containing a very small amount of organic substances, but a considerable amount of the salts of the saliva (Heidenhain).

Reflex Secretion of Saliva.-[If a cannula be placed in Wharton's duct, e.g., in a dog, during fasting, no saliva will flow out. If the mucous membrane of the mouth be stimulated by a sapid substance placed on the tongue, there is a copious flow of saliva. If the sympathetic nerve be divided, secretion still takes place when the mouth is stimulated, but if the chorda tympani be cut, secretion no longer takes place. Hence, the secretion is a reflex act; in this case, the lingual is the afferent, and the chorda the nerve-carrying impulses from a centre situated in the medulla oblongata (Fig. 120).] In the intact body, the secretion of saliva

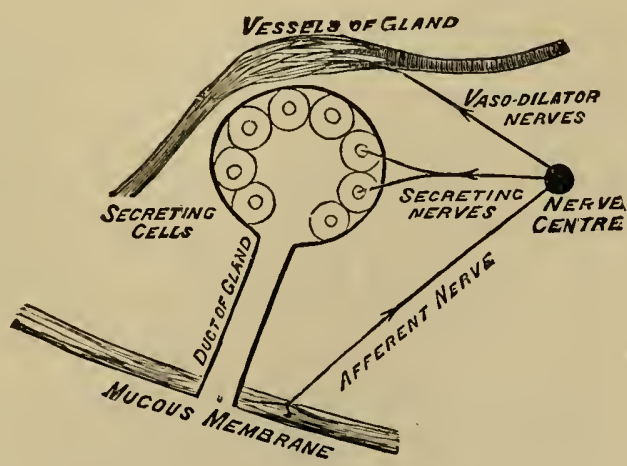

Fig. 120. occurs through a reflex stimulation of the nerves concerned, whereby under normal circumstances the secretion is always watery (chorda or facial saliva). The centripetal or afferent nerve-fibres concerned are:(1) The nerves of taste. (2) The sensory branches of the trigeminus of the entire cavity of the mouth and the glosso-pharyngeal (which appear to be capable of being stimulated by mechanical stimuli, pressure, 
tension, displacement). The movements of mastication also cause a secretion of saliva. Pflüger found that one-third more saliva was secreted on the side where mastication took place; and $\mathrm{Cl}$. Bernard observed that the secretion ceased in horses during the act of drinking. (3) The nerves of smell, excited by certain odours. (4) The gastric branches of the vagus (Frerichs, Oehl). A rush of saliva into the mouth usually precedes the act of romiting (p. 310).

(5) The stimulation of distant sensory nerves, e.g., the central end of the sciatic -certainly through a complicated reflex mechanism-causes a secretion of saliva (Owsjannikow and Tschierjew). Perhaps the secretion of saliva, which sometimes occurs during pregnancy, is caused in the same reflex manner.

Stimulation of the conjunctiva, e.g., by applying an irritating fluid to the eye of carnivorous animals, causes a reflex secretion of saliva (Aschanbrandt).

The reflex centre for the secretion of saliva lies in the medulla oblongata, at the origin of the seventh and ninth cranial nerves (Eckhard, Loeb). The centre for the sympathetic fibres is also placed here (Grützner and Chlapowski). This region is connected by nerve-fibres with the cerebrum; hence, the thought of a-savory morsel, sometimes when one is hungry, causes a rapid secretion of a thin watery. fluid-[or, as we say, "makes the mouth water"]. If the centre be stimulated directly by a mechanical stimulus (puncture), salivation occurs, while asphyxia has the same effect. The reflex secretion of saliva may be inhibited by stimulation of certain sensory nerves, e.g., by pulling out a loop of the intestine (Pawlow). Stimulation of the cortex cerebri of a dog, near the sulcus cruciatus, is often followed by secretion of saliva (Eulenberg and Landois, Bochefontaine, Bubnoff, and Heidenhain). Disease of the brain in man sometimes causes a secretion of saliva, owing to the effects produced on the intracranial centre.

So long as there is no stimulation of the nerves, there is no secretion of saliva, as in sleep (Mitscherlich). Directly after the section of all the nerves, secretion stops, for a time at least.

Pathological Conditions and Poisons.-Certain affections, as inflammatiou of the mouth, neuralgia, ulcers of the mucous membrane, affections of the gums, due to teething or the prolonged administration of mercury, often produce a copious secretion of saliva (or ptyalism). Certain poisons cause the same effect by direct stimulation of the nerves, as Calabar bean (Physostigmin), digitalin, and especially pilocarpin. Many poisons, especially the narcotics-above all, atropin-paralyse the secretory nerves, so that there is a cessation of the secretion, and the mouth becomes dry ; while the administration of muscarin in this condition causes secretion (Prevost). Pilocarpin acts on the chorda tympani, causing a profuse secretion, and, if atropin be given, the secretion is again arrested. Conversely, if the secretion be arrested by atropin, it may be restored by the action of pilocarpin or physostigmin. Nicotin, in small does, excites the secretory nerves, but in large doses paralyses them (Heidenhain). Daturin, cicutin, and iodide of æthylstrychnine, paralyse the chorda. 
Theory of Salivary Secretion.-Heidenhain has recently formulated the following theory regarding the secretion of saliva:- "During the passive or quies cent condition of the gland, the organic materials of the secretion are formed from and by the activity of the protoplasm of the secretory cells. A quiescent cell, which has been inactive for some time, therefore contains little protoplasm, and a large amount of these secretory substances. In an actively secreting gland, there are two processes occurring together, but independent of each other, and regulated by two different classes of nerve-fibres; secretory fibres cause the act of secretion, while trophic fibres cause chemical processes within the cells, partly resulting in the formation of the soluble constituents of the secretion, and partly in growth of the protoplasm. According to the number of both kinds of fibres present in a nerve passing to a gland, such nerve being stimulated, the secretion takes place more rapidly (cerebral nerve) or more slowly (sympathetic), while the secretion contains less or more solid constituents. The cerebral nerves contain many secretory fibres and few trophic fibres, while the sympathetic contains many trophic, but few secretory fibres. The rapidity and chemical composition of the secretion vary, according to the strength of the stimulus. During continued secretion, the supply of secretory materials in the gland-cells is used up more rapidly than it is replaced by the activity of the protoplasm; hence, the amount of organic constituents diminishes, and the microscopic characters of the cells are altered. The microscopic characters of the cells are altered also by the increase of the protoplasm, which takes place in an active gland. The mucous cells disappear, and seem to be dissolved after prolonged secretion, and their place is taken by other cells derived from the proliferation of the marginal cells. The energy which causes the current of fluid depends upon the protoplasm of the glaud-cells."

The saliva is diminished in amount in man in cases of paralysis of the facial or sympathetic nerves, as is observed in unilateral paralysis of these nerves.

\section{The Saliva of the Individual Glands.}

(a.) The Parotid Saliva is obtained by placing a fine caunula in Steno's duct (Eckhard) ; it has an alkaline reaction, but during fasting, the first few drops may be neutral or even acid on account of free $\mathrm{CO}_{2}$ (Oehl)-its specific gravity is 1,003 to 1,004 . When allowed to stand it becomes turbid, and deposits, in addition to albuminous matter, calcium carbonate, which is present in the fresh saliva in the form of bicarbonate.

Salivary calculi are formed in the ducts of the salivary glands, owing to the deposition of lime salts, and they contain only traces of the other salivary constituents; in the same way is formed the tartar of the teeth, which contains many threads of leptothrix, and the remains of low organisms which live in decomposing saliva in carious cavities between the teeth.

It contains small quantities (more abundant in the horse) of a globulin-like body, and never seems to be without CNKS sulphocyanide of potassium (or sodium-Treviranus, 1814), which, however, is absent in the sheep and $\operatorname{dog}$ (Brettel).

The sulphocyanide gives a clark red colour (ferric sulphocyanide) with ferric chloride. It also reduces iodic acil when added to saliva, causing a yellow colour from the liberation of iodine, which may be detected at once by starch (Solera). 
Mucin is absent, hence the parotid saliva is fluid, is not sticky, and can readily be poured from one vessel into another. It contains 1.5-1.6 per cent. of solids (Mitscherlich, van Setten) in man, of which $0.3-1.0$ per cent. is inorganic.

Amongst the organic substances the most important are Ptyalin, a small amount of urea (Gobley), and traces of a volatile acid (Caproic ?)

Of the inorganic constituents-the most abundant are potassium and sodium chlorides; then potassium, sodium, and calcium carbonates, some phosphates and a trace of an alkaline sulphate.

(b.) The Sub-maxillary Saliva is obtained by placing a cannula in Wharton's duct; it is alkaline, and may be strongly so. When allowed to stand for a long time, fine crystals of calcium carbonate are deposited, together with an amorphous albuminous body. It always contains mucin (which is precipitated by acetic acid); hence, it is usually somewhat tenacious. Farther, it contains ptyalin, but in less amount than in parotid saliva ; and, according to Oehl, only 0.0036 per cent. of potassium sulphocyanide.

Chemical Composition.-Sub-maxillary saliva (dog) :

Water, . . . 991.45 per 1,000 , Organic Matter, . . $2 \cdot 89 "$,

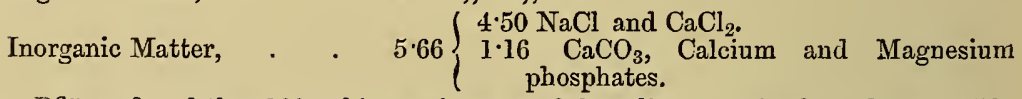

Pfluger found that 100 cubic centimetres of the saliva contained $0.60-64 \cdot 7 \mathrm{CO}_{2}$ (part could be pumped out, and part required the addition of phosphoric acid); $0.8 \mathrm{~N}$.; or, in 100 vol. gas, $0.910 ; 97.88 \mathrm{CO}_{2}, 1.21 \mathrm{~N}$.

(c.) The Sub-lingual Saliva is obtained by placing a very fine cannula in the ductus Rivinianus (OehI), is strongly alkaline in reaction, very sticky and cohesive, contains much mucin, numerous salivary corpuscles, and some potassium sulphocyanide (Louget).

\section{The Mixed Saliva in the Mouth.}

The fluid in the mouth is a mixture of the secretions from the salivary glands, and the secretions of the mucous glands of the mouth.

(1.) Physical Characters.-The mixed saliva of the mouth is a somewhat opalescent, tasteless, odourless, slightly glairy, fluid, with a specific gravity of 1,004-1,009, and an alkaline reaction. The amount secreted in 24 hours $=200$ to 1,500 grammes $(7-70$ oz.) ; according to Bidder and Schmidt, however, 1,000 to 2,000 grammes. The solid constituents $=5.8$ per 1,000 .

Composition.-The solids are:-Epithelium and mucus, $2 \cdot 2 ;$ ptyalin and albumin, 1.4 ; salts, 2.2 ; potassium sulphocyanide, 0.04 per 1,000 . The ash contains chiefly potash, phosphoric acid, and chlorine (Hammerbacher).

Decomposition products of epithelium, salivary corpuscles, or the remains of food, 
may render it acid temporarily, as after long fasting, and after much speaking (Hoppe-Seyler). Even ontside the body, saliva containing much epithelium becomes acid before it putrifies (Gorup-Besanez). The reaction is acid in some cases of dyspepsia and in fever, owing to the stagnation and insufficient secretion.

(2.) Microscopic Constituents. - (a.) The salivary corpuscles are slightly larger than the white blood-corpuscles $(S-11 \mu)$, and are nucleated protoplasmic globular cells without an envelope. During their living condition, the particles in their interior exhibit molecular or Brownian movement. The dark granules lying in the protoplasm are thrown into a trembling movement, from the motion of the fluid in which they are suspended. This dancing motion stops when the cell dies.

[The Brownian movements of these suspended granules are purely physical, and are exhibited by all fine microscopic particles suspended in a limpicl fluide.g., gamboge rubbed up in water, particles of carmine, charcoal, \&c.]

(b.) Pavement epithelial cells from the mucous membrane of the mouth and tongue; they are very abundant in catarrh of the mouth (Fig. 115, S).

(c.) Living organisms, which live and thrive in the cavities of teeth nourished by the remains of food. Amongst these are Leptothrix buccalis (Fig. 115, 12) and small bacteria-like organisms.

(3.) Chemical Properties-(a.) Organic Constituents. - Serum-albumin is precipitated by heat and by the addition of alcohol. In saliva, mixed with much water and shaken up with $\mathrm{CO}_{2}$, a globulin-like body is precipitated; mucin occurs in small amount. Amongst the extractives, the most important is ptyalin (Berzelius); fats and urea occur only in traces. In twenty-four hours 130 milligrammes of potassium or sodium sulphocyanide are secreted.

(b.) Inorganic Constituents.- Sodium and potassium chlorides, potassium sulphate, alkaline and earthy phosphates, ferric phosphate.

Abnormal Constituents.-In diabetes mellitus, lactic acid, derived from a further decomposition of grape-sugar, is found (Lehmann). It dissolves the lime in the teeth, giving rise to diabetic dental caries. Frerichs found leucin, and Vulpian increase of albumin in albuminuria. Of foreign substances taken into the body, the following appear in the saliva:-Mercury, potassium, iodine, and bromine.

Saliva of New-born Children.-In new-born children, the parotid alone contains ptyalin. The diastatic ferment seems to be developed in the sub-maxillary gland, and pancreas at the earliest after two months. Hence, it is not advisable to give starchy food to infants. No ptyalin has been found in the saliva of infants suffering from thrush (Oidium albicans-Zweifel).

The diastatic action of saliva is not absolutely necessary for the suckling, feeding as it does upon milk. The mouth during the first two months is not moist, but at a later period saliva is copiously secreted (Korowin); after the first six months, the salivary glands 
increase considerably. The eruption of the teeth-owing to the irritation of the mucous membrane-produces a copious secretion of saliva.

\section{Physiological Action of Saliva.}

I. The most important part played by saliva in digestion is its diastatic or amylolytic action (Leuchs, 1831)-i.e., the transformation of starch into dextrin and some form of sugar. This is due to the ptyalin -a hydrolytic ferment or enzym - which acts in very minute quantity, so that starch takes up water and becomes soluble, the ferment itself undergoing no essential change in the process. [Ptyalin belongs to the group of unorganised ferments. Like all other ferments it acts only within a certain range of temperature, being most active about $40^{\circ} \mathrm{C}$. Its energy is permanently destroyed by boiling. It acts best in a slightly alkaline or neutral medium.]

Action on Starch.- [Starch grains consist of granulose or starch enclosed by coats of cellulose. Cellulose does not appear to be affected. by saliva, so that saliva acts but slowly on raw, unboiled starch. If the starch be boiled so as to swell up the starch grains and rupture the cellulose envelopes, the amylolytic action takes place rapidly.]

[If starch paste or starch-mucilage, made by boiling starch in water, be acted upon by saliva, especially at the temperature of the body, the first physical change observable is the liquefaction of the paste, the mixture becoming more fluid and transparent. The change takes place in a few minutes. When the action is continued, important chemical changes occur.]

According to O'Sullivan, Musculus, and v. Mering, the diastatic ferment of saliva (and of the pancreas) by acting upon starch or glycogen forms maltose and dextrin (both soluble in water). Several closely allied varieties of dextrin, distinguishable by their colour reactions, seem to be produced (Brücke). Erythrodextrin is formed first; it gives a red colour with iodine, then a reducing dextrin-Achroodextrin, which gives no colour reaction with iodine. The sugar formed by the action of ptyaline upon starch is maltose $\left(\mathrm{C}_{12} \mathrm{H}_{22} \mathrm{O}_{11}+\mathrm{H}_{2} \mathrm{O}\right)$, which is distinguished from grape-sugar $\left(\mathrm{C}_{12} \mathrm{H}_{24} \mathrm{O}_{12}\right)$ by containing one molecule less of water, which, however, it holds as a molecule of water of hydration, as indicated in the formula given above (Ad. Mayer). [Maltose also differs from grape-sugar in its greater rotatory power on polarised light, and in its less power of reducing cupric oxide.]

[Thus, it will be seen that between the original starch and the final product, maltose, several intermediate bodies are formed. The starch 
gives a blue with iodine, but after it has been acted on for a time it gives a red or violet colour, indicating the presence of erythrodextrin, there being a simultaneous production of sugar; but ultimately no colour is obtained on adding iodine-achroodextrin, which gives no colour with iodine, and maltose being formed. The presence of the maltose is easily determined by testing with Fehling's solution.

Brown and Heron suggest that the final result of the transformation may be represented by the equation-

$$
\begin{aligned}
& 10\left(\mathrm{C}_{12} \mathrm{H}_{20} \mathrm{O}_{10}\right)+8 \mathrm{H}_{2} \mathrm{O}=8\left(\mathrm{C}_{12} \mathrm{H}_{22} \mathrm{O}_{11}\right)+2\left(\mathrm{C}_{12} \mathrm{H}_{20} \mathrm{O}_{10}\right) \\
& \text { Soluble starch. Water. Maltose. Achroodextrin.] }
\end{aligned}
$$

The ferment slowly changes maltose into grape-sugar or dextrose. This result may be brought about much more rapidly by boiling maltose with dilute sulphuric or hydrochloric acid. Achroodextrin ultimately passes into maltose, and this again into dextrose; the other form of dextrin does not seem to undergo this change (Seegen's Dystropodextrin). For the further changes that maltose undergoes in the intestine, see Intestinal Juice, ii., 2.

[The formula of starch is usually expressed as $\mathrm{C}_{6} \mathrm{H}_{10} \mathrm{O}_{5}$, but the researches already mentioned, and those of Brown and Heron, make it probable that it is more complex, which we may provisionally represent by $n\left(\mathrm{C}_{12} \mathrm{H}_{20} \mathrm{O}_{10}\right)$.]

According to Musculus and Meyer, erythrodextrin is a mixture of dextrin and soluble starch.

Preparation of Ptyalin.-(1.) Like all other hydrolytic ferments, it is carried down with any copious precipitate that is produced in the fluid which contains it. It is easily isolated from the precipitate. The saliva is acidulated with phosphoric acid, and lime-water is added until the reaction becomes alkaline, when a precipitate of basic calcium phosphate occurs, which carries the ptyaline along with it. This precipitate is collected on a filter and washed with water, which dissolves the ptyaline, and from its watery solution it is precipitated by alcohol as a white powder. It is redissolved in water and reprecipitated, and is obtained pure (Cohnheim).

(2.) V. Wittich's method.-The salivary glands [rat] are chopped up, placed in absolute alcohol for twenty-four hours, taken out and dried, and afterwards placed in glycerine for several days. The glycerine extracts the ptyalin. It is precipitated by alcohol from the glycerine extract.

(3.) William Roberts recommends the following solutions for extracting ferments from organs which contain them:-(1) A 3-4 p.c. solution of a mixture of two parts of boracic acid and 1 part borax. (2) Water, with 12-15 p.c. of alcohol. (3) One part chloroform to 200 of water.

Diastatic action of Saliva.-(a.) The diastatic or sugar-forming action is known by:-(1) The disappearance of the starch. When a small quantity of starch is boiled with several hundred times its volume of water, starch mucilage is obtained, which strikes a blue colour with iodine. If to a small quantity of this starch a sufficient amount of saliva be added, and the mixture kept for some time at the temperature of the body, the blue colour disappears. (2) The presence of sugar is proved directly by using the tests for sugar ( $\$ 149$ ).

(b.) The action takes place more slowly in the cold than at the temperature of 
the body-its action is enfeebled at $55^{\circ} \mathrm{C}$., and destroyed at $75^{\circ} \mathrm{C}$. (Paschutin). The most favourable temperature is $35^{\circ}-39^{\circ} \mathrm{C}$.

(c.) The ptyalin itself does not seem to be changed during its action, but ptyalin which has been used for one experiment, is less active when used the second time (Paschutin).

(d.) Saliva acts best in a slightly alkaline medium, but it also acts in a neutral and even in a slightly acid fluid; strong acidity prevents its action. The ptyalin is only active in the stomach when the acidity is due to organic acids (lactic or bntyric), and not when free hydrochloric acid is present (von den Velden). In both cases, however, dextrin is formed. Ptyalin is destroyed by hydrochloric acid or digestion by pepsin (Chittenden and Griswold). Even butyric and lactic acids formed from grape-sugar in the stomach may prevent its action; but if the acidity be neutralised, the action is resumed (Cl. Bernard).

(e.) The addition of common salt, ammonium chloride, or sodium sulphate (4 p.c. solution), increases the activity of the ptyalin, and so do $\mathrm{CO}_{2}$, acetate of quinine, strychnia, morphia, curara, 0.025 p.c. sulphuric acid.

(f.) Much alcohol and canstic potash destroy the ptyalin; long exposure to the air weakens its actiou. Salicylic acid and much atropin arrest the formation of sugar.

(g.) Ptyalin acts very feebly and very gradually upon raw starch, only after 2-3 hours (Schiff); while upon boiled starch it acts rapidly. [Hence the necessity for boiling thoroughly all starchy foods.]

(h.) The various kinds of starch are changed more or less rapidly according to the amount of cellulose which they contain; raw potato-starch after 2-3 hours, raw maize-starch after 2-3 minutes (Hammarsten). Starch cellulose is dissolved at $55^{\circ} \mathrm{C}$. (Nägeli). When the starches are powdered and boiled, they are changed with equal rapidity.

(i.) A mixture of the saliva from all the glands is more active than the saliva from any single gland (Jakubowitsch), while mucin is inactive. Ptyalin differs from diastase in so far that the latter first begins to act at $+66^{\circ} \mathrm{C}$. Ptyalin decomposes salicin into saligenin and grape-sugar (Frerichs and Stïdler), but it has no action on caue-sugar and amygdalin.

II. Saliva dissolves those substances which are soluble in water; while the alkaline reaction enables it to dissolve some substances which are not soluble in water alone, but require the presence of an alkali.

III. Saliva moistens dry food and aids the formation of the "bolus," while by its mucin it aids the act of swallowing, the mucin being given off unchanged in the fæces. The ultimate fate of ptyalin is unknown.

[IV. Saliva also aids articulation, while according to Liebig it carries down into the stomach small quantities of 0.$]$

[V. It is necessary to the sense of taste to dissolve sapid substances, and bring them in relation with the end-organs of the nerves of taste.]

The presence of a peptone-forming ferment has recently been detected in saliva (Hüfner, Munk, Kühne). This ferment is likewise said to occur in the saliva of the horse, which can also convert cane-sugar into invert sugar, and slightly emulsionise fats (Ellenberger and v. Hofmeister). According to Hofmeister, the saliva of the sheep has a digestive action on cellulose.

Saliva has no action on proteids, while on fats it produces a very feeble emụlsion, 


\section{Tests for Sugar.}

1. Trommer's Test depends upon the fact that in alkaline solutions sugar acts as a reducing agent; in this case a metallic oxide is changed into a suboxide. To the fluid to be investigated, add $\frac{1}{2}$ of its volume of a solution of caustic potash (soda) specific gravity $1 \cdot 25$, and a few drops of a weak solution of cupric sulphate, which causes at first a bluish precipitate consisting of hydrated cupric oxide, but it is redissolved giving a clear blue fluid, if sugar be present. Heat the upper stratum of the fluid, and a yellow or red ring of cuprous oxide is obtained, which indicates the presence of sugar; $2 \mathrm{CuO}-\mathrm{O}=\mathrm{Cu}_{2} \mathrm{O}$.

The solution of hydrated cupric oxide is caused by other organic substances; but the final stage, or the production of cuprons oxide is obtained only with certain sugars-grape, fruit and milk (but not cane) sugar. Fluids which are turbid must be previously filtered, and if they are highly coloured they must be treated with basic lead acetate; the lead acetate is afterwards removed by the addition of sodium phosphate aud subsequent filtration. If very small quantities of sugar are present along with compounds of ammonia, a yellow colour instead of a yellow precipitate may be obtained. In doing the test, care must be taken not to add too much cupric sulphate.

[2. Fehling's Solution is an alkaline solution of potassio-tartarate of copper. Boil a small quantity of the deep-blue coloured Fehling's solution in a test tube, and add to the boiling test a few drops of the fluid supposed to contain the sugar. If sugar be present the copper solution is reduced, giving a yellow or reddish precipitate. The reason for boiling the test itself is, that the solution is apt to decompose when kept for some time, when it is precipitated by heat alone. This is one of the best and most reliable tests for the presence of sugar. In Pavy's modification of this test, ammonia is nsed instead of a caustic alkali.]

3. Böttger's Test. - Alkaline bisınuth oxide solution ( 5 grammes each of basic bismuth nitrate, and tartaric acid, 30 cubic centimetres water, and caustic soda sufficient for neutralisation) is reduced to bismuth suboxicle by sugar, with the formation of a dark olive green and ultimately black precipitate.

4. Moore and Heller's Test.-Caustic potash or soda is added until the mixture is strongly alkaline; it is afterwards boiled. If sugar be present, a yellow, brown or brownish-black colouration is obtained. If nitric acid be added, the odour of burned sugar (caramel) and formic acid is obtained.

5. Mulder and Neubauer's Test.-A solution of indigo-carmine rendered alkaline with sodic carbonate, is added to the sugar-solution until a slight bluish colour is obtained. When the mixture is heated the colour passes into purple, red, and yellow. When shaken with atmospheric air, the fluid again becomes blue.

In all cases where albumin is present it must be removed-in urine by acidulating with acetic acid and boiling; in blood, by adding four times its volume of alcohol and afterwards filtering, while the alcohol is expelled by heat. 


\section{Quantitative Estimation of Sugar.}

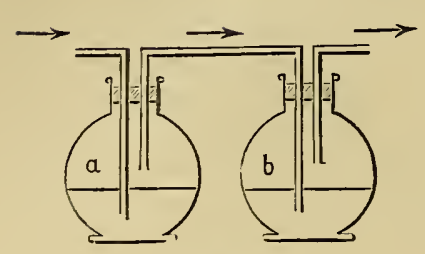

Fig. 121.

Apparatus for the quantitative estimation of sugar by fermentation.

I. By Fermentation.-Into the glass vessel (Fig. 121, a) a measured quantity (20 cmtr.) of the fluid (sugar) is placedalong with some yeast, while $b$ contains concentrated sulphuric acid. The whole apparatus is now weighed. When exposed to a sufficient temperature $\left(10-40^{\circ} \mathrm{C}\right.$.), the sugar splits into 2 molecules of alcohol and 2 of carbonic acid,

$$
\begin{aligned}
\mathrm{C}_{6} \mathrm{H}_{12} \mathrm{O}_{6} & =2\left(\mathrm{C}_{2} \mathrm{H}_{6} \mathrm{O}\right)+2\left(\mathrm{CO}_{2}\right), \\
\text { Grape-sugar } & =2 \text { alcohol }+2 \text { carbonic acid ; }
\end{aligned}
$$

and in addition there are formed traces of glycerine and succinic acid. The $\mathrm{CO}_{2}$ escapes from $b$, and as it passes through the $\mathrm{H}_{2} \mathrm{SO}_{4}, \mathrm{CO}_{2}$ yields to the latter its water. The apparatus is weighed after two days, when the reaction is ended, and the amount of sugar is calculated from the loss of weight in the $20 \mathrm{cmtr}$. of fluid. 100 parts of water-free sugar $=48.89$ parts $\mathrm{CO}_{2}$, or 100 parts $\mathrm{CO}_{2}$ correspond to 204.54 parts of sugar.

II. Titration.-By means of Fehling's solution, which consists of cupric sulphate, tartrate of potash and soda, caustic soda, and water. It is made of such a strength that all the copper in 10 cubic centimetres of the solution is reduced by 0.05 grammes of grape-sugar (see Urine, vol. ii.).

III. The amount may also be estimated by the polarisation apparatus (see Urine, vol. ii.).

\section{Mechanism of the Digestive Apparatus.}

This embraces the following acts :-

1. The introduction of the food; the movements of mastication and those of the tongue; insalivation and the formation of the bolus of food.

2. Deglutition.

3. The movements of the stomach, of the small and large intestine.

4. The excretion of fæcal matters.

\section{Introduction of the Food.}

Fluids are taken into the mouth in three ways:-(1) By suction, the lips are applied air-tight to the vessel containing the fluid, while the tongue is retracted (the lower jaw being often depressed) and acts like the piston in a suction pump, thus causing the fluid to enter the mouth. Herz found that the negative pressure caused by an infant while sucking $=3-10 \mathrm{~mm}$. Hg. (2) The fluid is lapped when it is brought into direct contact with the lips, and is raised by aspiration and mixed with air so as to produce a characteristic sound in the mouth. (3) Fluid may be poured into the mouth, and as a general rule the lips are applied closely to the vessel containing the fluid.

The solids when they consist of small particles are licked up with the lips, aided by the movements of the tongue. In the case of large. 
masses, a part is bitten off with the incisor teeth, and is afterwards brought under the action of the molar teeth by means of the lips, cheeks, and tongue.

\section{The Movements of Mastication.}

The articulation of the jaw is provided with an interarticular cartilage (Vidius, 1567)-the meniscus-which prevents direct pressure being made upon the articular surface when the jaws are energetically closed, and which also divides the joint into two cavities, one lying over the other. The capsule is so lax that, in addition to the raising and depressing of the lower jaw, it permits of the lower jaw being displaced forwards upon the articular tubercle, whereby the meniscus moves with it, and covers the articular surface.

The process of mastication consists of the following movements :-

(a.) The elevation of the jaw is accomplished by the combined action of the Temporal, Masseter, and Internal Pterygoid Muscles. If the lower jaw was previously so far depressed that its articular surface rested upon the tubercle, it now passes backwards upon the articular surface.

(b.) The depression of the lower jaw is caused by its own weight, aided by the action of the anterior bellies of the Digastrics, the Myloand Genio-hyoid and Platysma (Haller). The muscles act during forcible opening of the mouth. The necessary fixation of the hyoid bone is obtained through the action of the Omo- and Sterno-hyoid, and by the Sterno-thyroid and Thyro-hyoid.

When the articular surface of the lower jaw passes forwards on to the tubercle, the External Pterygoids actively aid in producing this (Bérard).

(c.) Displacement of both or one articular surface forwards or backwards. During rest, when the mouth is closed, the incisor teeth of the lower jaw fall within the arch of the upper incisors. When in this position, the jaw is protruded by the External Pterygoids, whereby the articular surface passes on to the tubercle (and, therefore, downwards), while the lateral teeth are thereby separated from each other. The jaw is retracted by the Internal Pterygoids without any aid from the posterior fibres of the Temporals. When one articular surface is carried forwards, the jaw is protruded and retracted by the External and Internal Pterygoid of the same side. At the same time, there is a transverse movement, whereby the back teeth of the protruded side are separated from each other.

During mastication, when the individual movements of the lower jaw are variously combined, the food to be masticated is kept from passing outwards by the action of the muscles of the lips (Orbicularis oris) and the Buccinators, while the tongue continually pushes the particles between the molar teeth. The energy of the muscles of 
mastication is regulated by the sensibility of the teeth, and the muscular sensibility of the muscles of mastication, as well as by the general sensibility of the mucous membrane of the mouth and lips. At the same time, the mass is mixed with saliva, the divided particles cohere, and are formed into a mass or bolus of a long, oval shape by the muscles of the tongue. The bolus then rests on the back of the tongue, ready to be swallowed.

Nerves of Mastication.-The muscles of mastication and the buccinator receive their motor nerves from the third branch of the trigeminus; the mylohyoid and the anterior belly of the digastric being supplied from the same source. The genio-, omo-, and sterno-hyoid, sterno-thyroid, and thyro-hyoid are supplied by the hypoglossal, while the facial supplies the posterior belly of the digastric, the stylo-hyoid, the platysma, and the museles of the lips. The general centre for the muscles of mastication lies in the medulla oblongata.

When the mouth is closed, the jaws are kept in contact by the pressure of the air, as the cavity of the mouth is rendered free from air, and the entrance of air is prevented anteriorly by the lips, and posteriorly by the soft palate. The pressure exerted by the air is from $2-4 \mathrm{~mm}$. Hg. (Metzger and Donders).

\section{Structure and Development of the Teeth.}

A tooth is just a papilla of the mucous membrane of the gum, which has undergone a characteristic development. In its simplest form, as in the teeth of the lamprey, the connective-tissue basis of the papilla is covered with many layers of cormeous epithelium. In human teeth, part of the papilla is transformed into a layer of calcified dentine, while the epithelium of the papilla produces the enamel, the fang of the tooth being covered by a thin accessory layer of bone, the crusta petrosa or cement.

The dentine or ivory which surrounds the pulp cavity and the canal of the fang (Fig. 122) is very firm, elastic, and brittle. Like the matrix of bone, dentine,

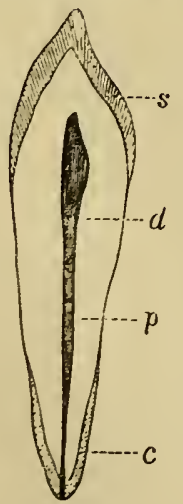

Fig. 122.

Vertical section of a tooth $-p$, pulp cavity; $d$, dentine; $c$, cement; $s$, enamel.

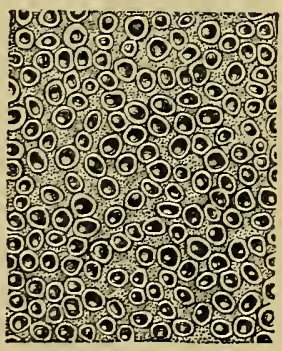

Fig. 123.

Transverse section of dentineThe light rings are the walls of the dentinal tubules; the dark centres with the light points are the fibres of Tomes lying in the tubules. when treated in a certain way, presents a fibrillar structure (v. Ebner). It is permeated by innumerable long, tortuous, wavy tubes-thedentinaltubuies (Leeuwe nhoek, 1678)each of which communicates with the pulp cavity by means of a fine opening, and passes more or less horizontally outwards as far as the outer layers of the dentine. The tubules are bouuded by an extremely resistant, thin, cuticular membrane, which strongly resists the action of chemical reagents. These tubules are tilled completely by soft fibres, the "fibres of Tomes" (1840), which are merely greatly elongated and branched processes of the odontoblasts of the pulp (Waldeyer, 1865). 
The dentinal tubules, as well as the fibres of Tomes, anastomose throughout their entire extent by means of fine processes. As the fibres approach the enamel, which they do not penetrate, some of them bend on themselves, and form a loop (Fig. 124, c), whilst others pass into the "interglobular spaces," which are so abundant in the outer part of the dentine (Czermak, 1850). These interglobular spaces are small spaces bounded by curved surfaces. Certain curved lines "Schreger's lines" (1S00), may be detected with the naked eye in the dentine (e.g., of the elephant's tusk) running parallel with the contour of the tooth. They are caused by the fact that at these parts all the chief curves in the dentinal tubules follow a similar course (Retzius, $183 \mathrm{~J}$ ).

The enamel, the hardest substance in the body (resembling apatite), covers the crown of the teeth. It consists of hexagonal flattened prisms (Malpighi, 1687) arranged side by side like a palisade (Fig. 124, $a$ and B). They are 3-5 $\mu$ ( $\frac{\sigma^{2} \sigma 0}{\text { inch) }}$ broad, not quite uniform in thickness, curved slightly in different directions, and

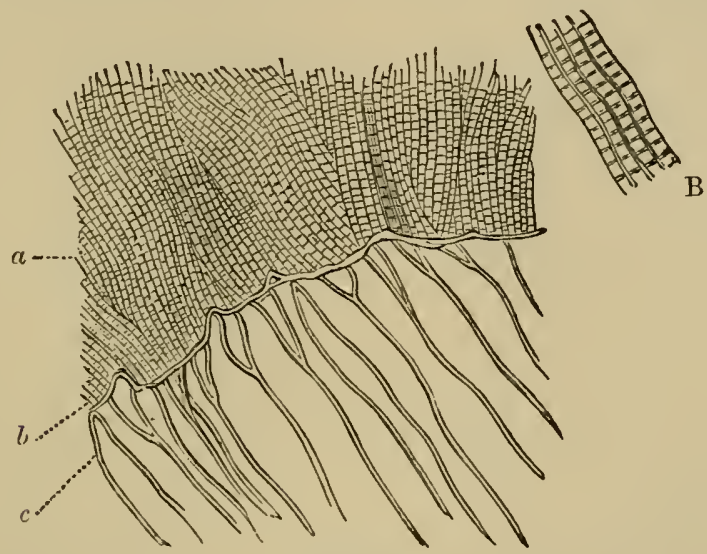

Fig. 124.

Section of a tooth between the dentine and enamel $-a$, enamel; $c$, dentinal tubules; $\mathrm{B}$, enamel prisms highly magnified.

owing to inequalities of thickness, they exhibit transverse markings. They are elongated, calcified, cylindrical, epithelial cells derived from the dental papilla.

Retzius described dark-brown lines running parallel with the outer boundary of the enamel, due to the presence of pigment. The fully-formed enamel is negatively doubly refractive and uniaxial, while the developing enamel is positively doubly refractive (Hoppe-Seyler).

The cuticala or Nasmyth's membrane (1S39) covers the free surface of the enamel as a completely structureless membrane $1-2 \mu$ thick, but in quite young teeth it exhibits an epithelial structure, and is derived from the outer epithelial layer of the enamel-organ.

The cement (John Hunter, 177S) or crusta petrosa, is a thin layer of bone covering the fang (Fig. 125, a). The bone lacunæ communicate directly with the dentinal tubules of the fang. Haversian canals and lamellæ are only found where the layer of cement is thick, and the former may communicate with the pulp-cavity (Salter). Very thin layers of cement may be deroid of bone-corpuscles. Sharpey's fibres occur in the cement of the dog's tooth (Wal. deyer); while in the horse's tooth single bone-corpuscles are enveloped by a capsule (Gerber). In the periodontal membrane, which is just the periosteum of the alveolus, coils of blood-ressels similar to the renal glomeruli occur. They anastomose with each other, and are surrounded with a delicate capsule of connective-tissue (C. Wedl). 
Chemistry of a Tooth.-The teeth consist of a gelatine-yielding matrix infiltrated with calcium phosphate and carbonate (like bone).-1. The dentine contains-organic matter, $27 \cdot 70$; calcium phosphate and carbonate, $72 \cdot 06$; magnesium phosphate, $0 \cdot 75$, with traces of iron, fluorine, and sulpburic acid (Aeby, HoppeSeyler).

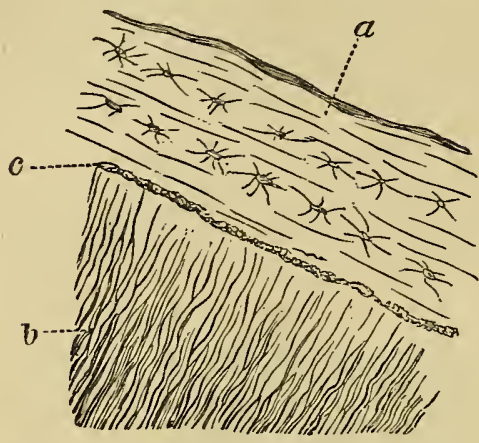

Fig. 125.

Transverse section of the fang $-a$, cement with bone-corpuscles; $b$, dentine with dentinal tubules; $c$, boundary between both.

2. The enamel contains an organic proteid matrix allied to the substance of epithelium. It contains $3 \cdot 60$ organic matter and 96.00 of calcium phosphate and carbonate, 1.05 magnesium phosphate, with traces of calcium fluoride and an insoluble chlorine compound.

3 . The cement is identical with bone.

The pulp in a fully-grown tooth represents the remainder of the dental papilla around which the dentine was deposited. It consists of a very vascular indistinctly fibrillar connectivetissue, laden with cells. The layers of cells, resembling epithelium, which lie in direct contact with the dentine are called odontoblasts (Waldeyer, 1865), i.e., those cells which build up the dentine. These cells send off long branched processes into the dentinal tubules, whilst their nucleated bodies lie on the surface of the pulp and form connections by processes with other cells of the pulp and with neighbouring odontoblasts. Numerous non-medullated nerve-fibres (sensory from the Trigeminus) whose mode of termination is unknown, occur in the pulp.

The periosteum or periodontal membrane of the fang is, at the same time, the alveolar periosteum, and consists of delicate connective-tissue with few elastic fibres and many nerves.

The gums are devoid of mucous glands, are very vascular, and often provided with long vascular papillæ which are sometimes compound.

Development of a Tooth.-It begins at the end of the second month of foetal

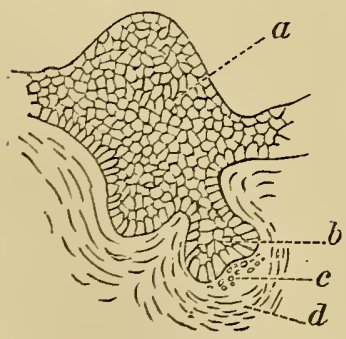

Fig. 126.

$a$, Dental ridge ; $b$, enamelorgan; $c$, beginning of the dentine-germ; $d$, first indication of the toothsac. life. Along the whole length of the fotal gum is a thick projecting ridge (Fig. 126, a) composed of many layers of epithelium. A depression, the dental groove also filled with epithelium, occurs in the gum, and runs along under the ridge. The dental groove becomes deeper throughout its entire length, and on transverse section presents the appearance of a dilated flask (b), while at the same time it is filled with elongated epithelial cells, which form the "enamel-organ." A conical papilla (the "dentinegerm") grows up from the mucous tissue, of which the gum consists, towards the enamel-organ (Fig. $127, c)$, so that the apex of the papilla comes to have the enamel-organ resting upon it like a double cap. Afterwards, owing to the development of counectivetissue, the parts of the enamel-organ lying between and uniting the individual dentine-germs disappear, and gradually the connective-tissue forms a tooth. sac enclosing the papilla and its enamel-organ $(d)$. 
Those epithelial cells (Fig. 127, 3) of the enamel-organ, which lie next the top of the papilla, are cylindrical, and become calcified to form enamel prisms. The layer of cells of the donble cap, which is directed towards the tooth-sac (1), becomes flattened, fuses, undergoes a horny transformation, and becomes the cuticula, whilst the cells which lie between both layers undergo an intermediate metamorphosis, so that they come to resemble the branched stellate cells of the mucous tissue (2), and gradually disappear altogether.

The dentine is formed in the most superficial layer of the projecting connectivetissue of the dental papilla, owing to the calcification of the continuous layer of odontoblasts which occurs there (Figs. 127 and 128, $k$ ). During the process, fibres

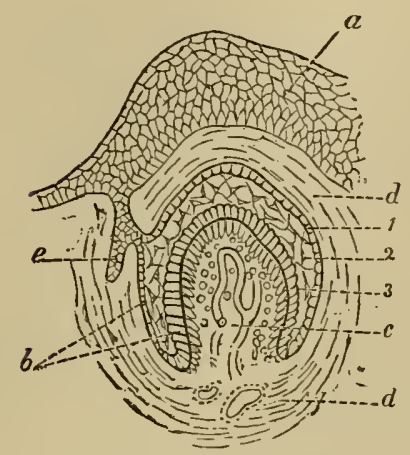

Fig. 127.

$u$, Dental ridge; $\dot{v}$, enamel-organ with 1 , onter epithelinm; 2 , middle stellate layer; 3 , enamel-prism cell layer; $c$, dentine-germ with blood-vessels and the long osteoblasts on the surface; $d$, tooth-sac; $e$, secondary enamel-germ.

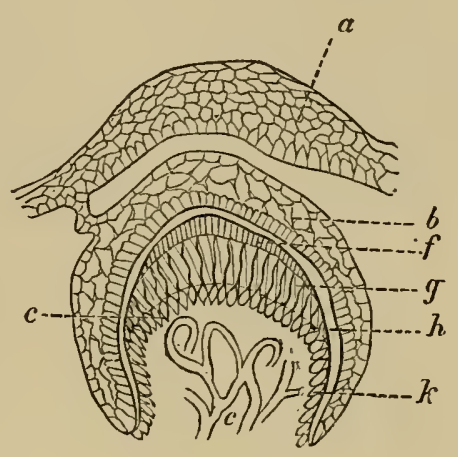

Fig. 12S.

a, Dental ridge; $b$, enamel-organ; $c$, dentine-germ ; $f$, enamel ; $/$, dentine; $h$, interval between enamel-organ and the position of the tooth; $k$, layer of odontoblasts.

or branches of these cells are left unaffected, and remain as the fibres of Tomes. Exactly the same process occurs as in the formation of bone, the odontoblasts forming around themselves a calcified matrix. The cement is formed from the soft connective-tissue of the dental alveolus.

Dentition.-During the development of the first (temporary or milk) teeth a special enamel-organ (Fig. 127, e) is formed near these, but it does not undergo development until the milk-teeth are shed; even the papilla is wanting at first. When the permanent tooth begins to develop, it opens into the alveolar wall of the milk-teeth from below.

'The tissue of this dental sac causes erosion, or eating away of the fang and even of the body of the milk-teeth, without its blood-vessels undergoing atrophy. The chief agents in the absorption are the amœboid cells of the granulation tissue. [Multinuclear giant-cells also erode the fangs of the teeth.]

Eruption of the Teeth.-The following is the order in which the twenty milkteeth cut the gum-i.e., from the seventh month to the second year:-Lower central incisors, upper central incisors, upper lateral incisors, lower lateral incisors, first molar, canine, the second molars.

[The figures indicate in months the period of eruption of each tootl..] 


\begin{tabular}{|c|c|c|c|c|}
\hline Molars. & Canines. & Incisors. & Canines. & Molars. \\
\hline 2412 & 18 & 9779 & 18 & 2412 \\
\hline
\end{tabular}

The permanent teeth succeed the milk-teeth, the process beginning about the seventh year. Ten teeth in each jaw take the place of the milk-teeth, while six teeth appear further back in each jaw. Thus the total number of permanent teeth is thirty-two. As the sacs, from which the permanent teeth arc developed, are formed before birth, they merely undergo the same process of development as the temporary teeth, only at a much later period. The last of the permanent molars--the wisdom-tooth-may not cut the jaw until the seventeenth to the twentyfifth year. At the sixth year the jaw contains the largest number of teeth, as all the temporary teeth are present, and, in addition, the crowns of all the permanent teeth, except the wisdom-tooth, making forty-eight in all.

[Eruption of Permanent Teeth.-The age at which each tooth cuts the gum is given in years in the following table :-

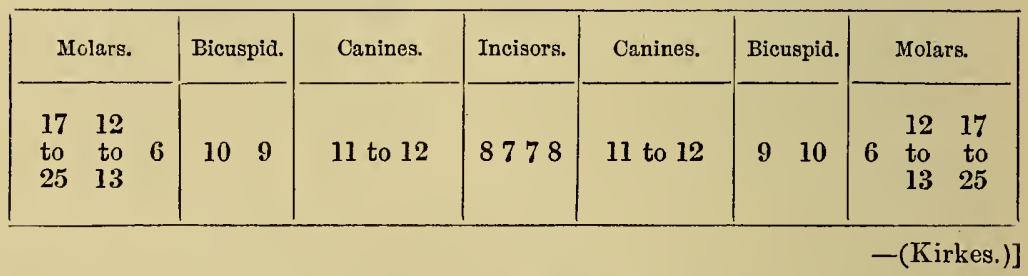

\section{Movements of the Tongue.}

The tongue being a muscular organ (Aretaeus, A.D. 81), and extremely mobile, plays an important part in the process of mastication :-(1) It keeps the food from passing from between the molar teeth. (2) It collects into a bolus the finely-divided food after it is mixed with saliva. (3) When the tongue is raised, the bolus lying on its dorsum is pushed backwards into the pharynx, whence it passes into the osophagus.

The muscular fibres of the tongue run in three directions-longitudinally, from base to tip; transversely, the fibres for the most part proceeding outwards from the vertically placed septum linguæ; vertically, from below upwards. Some of the muscles are confined to the tongue (intrinsic), while others (extrinsic), are attached beyond it to the hyoid bone, lower jaw, to the styloid process, and the palate.

Microscopically, the fibres are transversely striated, with a delicate sarcolemma, and very often they are divided where they are inserted into the mucous membrane (Leeuwenhock). The muscular bundles cross each other in various directions, and in the interspaces fat cells and glands occur.

On analysing the lingual movements, we may distinguish changes in form and changes in position :- 
(1.) Shortening and broadening by the longitudinal muscle, aided by the hyo-glossus.

(2.) Elongation and narrowing, by the transversus linguæ.

(3.) The dorsum rendered concave by the transversus and the simultaneous action of the median vertical fibres.

(4.) Arching of the dorsum :-(a) transversely by contraction of the lowest transverse bundles; $(b)$ longitudinally by the action of the lowest longitudinal muscles.

(5.) Protrusion, by the genio-glossus, while at the same time the tongue usually becomes narrower and longer (2).

(6.) Retraction, by the hyo-glossus and stylo-glossus, and (1) usually occurring at the same time.

(7.) Depression of the tongue into the floor of the mouth, by the hyoglossus. The floor of the mouth may be made deeper by simultaneously rlepressing the hyoid bone.

(8.) Elevation of the tongue towards the gums:-(a) At the tip by the anterior part of the longitudinal fibres; $(b)$ in the middle by elevating the entire hyoid bone by the mylo-hyoid ( $N$. trigeminus); $(c)$ at the root by the stylo-glossus and palato-glossus, as well as indirectly by the stylo-hyoid ( $N$. facialis).

(9.) Lateral movements, whereby the tip of the tongue passes to the right or the left; these are caused by the contraction of the longitudinal fibres of one side.

Motor Nerves.-The proper motor nerve of the tongue is the hypoglossal. When this nerve is divided or paralysed on one side, the tip of the tongue lying in the floor of the month is directed towards the sound side, because the tonus of the non-paralysed longitudinal fibres shortens the sound side slightly. If the tongue be protruded, however, the tip passes towards the paralysed side. This arises from the direction of the genio-glossus (from the middle downwards and outwards), and the tongue follows the direction of its action. The tongues of animals which have been killed exhibit fibrillar contractions of the muscles, sometimes lasting for a whole day (Cardanus, 1550).

\section{Deglutition.}

The onward movements of the contents of the digestive canal are effected by a special kind of action whereby the tube or canal contracts upon its contents, and as this contraction proceeds along the tube, the contents are thereby carried along. This is the "peristaltic movement," or peristalsis.

In the first and most complicated part of the act of deglutition, we distinguish in order the following individual movements:-

(1.) The aperture of the month is closed by the orbicularis oris $(N$. facialis). 
(2.) The jaws are pressed against each other by the muscles of mastication ( $N$. trigeminus), while at the same time the lower jaw affords a fixed point for the action of the muscles attached to it and the hyoid bone.

(3.) The tip, middle, and root of the tongue, one after the other, are pressed against the hard palate, whereby the contents of the mouth are propelled towards the pharynx.

(4.) When the bolus has passed the anterior palatine arch (the mucus of the tonsillar glands making it slippery again), it is prevented from returning to the mouth by the palato-glossi muscles which lie in the anterior pillars of the fauces, coming together like two side-screens or curtains, meeting the raised dorsum of the tongue (Stylo-glossus -Dzondi, 1831).

(5.) The morsel is now behind the anterior palatine arch and the root of the tongue, and has reached the pharynx, where it is subjected to the successive action of the three pharyngeal constrictor-muscles which propel it onwards. The action of the superior constrictor of the plarynx is always combined with a horizontal elevation (Levator veli palatini; $N$. facialis), and tension (Tensor veli palatini; $N$. trigeminus, otic ganglion) of the soft palate (Bidder, 1838). The upper constrictor presses (through the pterygo-pharyngets) the posterior and lateral walls of the pharynx tightly against the posterior margin of the horizontal tense soft palate (Passavant), whereby the margins of the posterior palatine arches (palato-pharyngeus) are approximated. The pharyngo-nasal cavity is thus completely shut off, so that the bolus cannot be pressed backwards into the nasal cavity. In cases where the soft palate is defective, part of the food usually passes into the nose.

(6.) The bolus is propelled onwards by the successive contractions of the constrictors of the pharynx until it passes into the œsophagus. At the same time the entrance to the glottis is closed, else the morsel would pass into the larynx.

Falk and Kronecker assert, that by the rapid contraction of the transversely striped muscles which diminish the aperture of the mouth, the bolus is projected into the csophagus, so that peristalsis of the pharynx and ossophagus only occurs during forced deglutition.

If we make a series of efforts to swallow, one after the other, contraction of the esophagus takes place only after the last attempt. Kronecker and Meltzer found that stimulation of the glosso-pharyngeal nerve inhibited the act of deglutition and the propagation of the movement through the osophagus. Section of both nerves causes tonic spasm of the osophagus and cardia. 
The closure of the glottis is effected in the following manner:-(a.) The whole larynx-the lower jaw being fixed-is raised upwards and forwards, while at the same time the root of the tongue hangs over it. The hyoid bone is raised forwards and upwards by the genio-hyoid, anterior belly of the digastric and mylo-hyoid; the larynx is approximated close to the hyoid bone (Berengar, 1521) by the thyro-hyoid.

(b.) When the larynx is raised so that it comes to lie below the overhanging root of the tongue, the epiglottis is pressed downwards over the entrance to the glottis, and the bolus passes over it. In addition, the epiglottis is pulled down by the special muscular fibres of the reflector epiglottidis (Thiele) and aryepiglotticus.

Injury to the Epiglottis.-Intentional injury of the epiglottis in animals, or its destruction in man may cause fluids to "go the wrong way," i.e., into the glottis, whilst solid food can be swallowed without disturbance. In dogs, at any rate, coloured fluids placed on the root of the tongue have been observed to pass directly into the pharynx without coming into contact with, so as to tinge, the upper surface of the epiglottis (Magendie, Schiff).

(c.) Lastly, the closure of the glottis by the constrictors of the larynx also prevents the entrance of substances into the larnyx (Czermak).

In order that the descending bolus may be prevented from carrying the pharynx with it, the stylo-pharyngeus, salpingo-pharyngeus, and baseo-pharyngeus contract upwards when the constrictors act.

Nerves.-Deglutition is voluntary only during the time the bolus is in the mouth. When the food passes through the palatine arch into the gullet the act becomes involuntary, and is, in fact, a well-regulated reflex action. When there is no bolus to be swallowed, voluntary movements of deglutition can be accomplished only within the mouth; the pharynx only takes up the movement provided a bolus (food or saliva) mechanically excites the reflex act. The sensory nerves which, when mechanically stimulated, excite the involuntary act of deglutition, are, according to Schrœeder van der Kolk, the palatine branches of the trigeminus (from the sphenopalatine ganglion) and the pharyngeal branches of the vagus (Waller, Prevost). The centre for the nerves concerned (for the striped muscles) lies in the superior olives of the medulla oblongata. Swallowing can be carried out when a person is unconscious, or after destruction of the cerebrum, cerebellum, and pons. [Even in the deep coma of alcoholism, the tube of a stomach-pump is readily carried into the stomach reflexly, provided the surgeon passes it back into the pharynx to bring it within the action of the constrictors of the pharynx.] The nerves of the pharynx are derived from the pharyngeal plexus, which receives branches from the vagus, glosso-pharyngeal, and sympathetic. 
Within the resophagus, whose stratified epithelium is moistened with the mucus derived from the mucous. glands in its walls, the downward movement is involuntary, and depends upon a complicated reflex movement discharged from the centre for deglutition-there is a peristaltic movement of the outer longitudinal and inner circular nonstriped muscular fibres.

In the upper part of the œsophagus which contains striped muscular fibres, the peristalsis takes place more quickly than in the lower part. The movements of the œsophagus never occur independently, but are always the continuation of a foregoing act of deglutition. If food be introduced into the cesophagus through a hole in its wall, there it lies; and it is only carried downwards when a movement to swallow is made (Volkmann). The peristalsis extends along the whole length of the œsophagus, even when it is ligatured or when a part of it is removed (Mosso). If a dog be allowed to swallow a piece of flesh tied to a string, so that the flesh goes half-way down the csophagus, and if the flesh be withdrawn, the peristalsis still passes downwards (C. Ludwig and Wild).

The motor nerve of the cesophagus is the vagus (not the accessory fibres); after it is divided, the food lodges in the lower part of the cesophagus. Very large and very small masses are swallowed with more difficulty than those of moderate size. Dogs can swallow an olive-shaped body weighted with a counterpoise of 450 grammes (Mosso). When the thorax is greatly distended, as in Miiller's experiment, or greatly diminished, as in Valsalva's experiment (p. 112), deglutition is rendered more difficult.

Goltz observed, that the œsophagus and stomach (frog) became greatly more excitable, i.e., the excitability of the ganglionic plexuses in their walls was increased, when the brain and spinal cord or both vagi were destroyed. These organs contracted energetically after slight stimulation, while frogs whose central nervous system was intact, swallowed fluids simply by peristalsis. Females, and sometimes men also, with marked weakening of the nervous system (Hysteria), not unfrequently have similar spasmodic contractions of the œsophageal region (globus hystericus). After section of both vagi, Schiff observed spasmodic contraction of the œsophagus.

[Structure of the Esophagus.-The walls of the cosophagus are composed of three coats-mucous, sub-mucous, and muscular.

The mucous coat is firm and is thrown into longitudinal folds, which disappear when the tube is distended. It is lined by several layers of stratified squamous epithelium. The membrane itself is composed, especially at its inner part, of dense fibrous tissue, which projects in the form of papillæ, into the stratified epithelium. At its outer part is a continuous layer of non-striped muscle, the muscularis mucosce.

The sub-mucous coat is thicker than the foregoing, and consists of loose connective-tissue, with the acini of small compound tubular mucous glands imbedded in it. The ducts pierce the muscularis mucosæ to open on the inner surface of the tube.

The muscular coat consists of an inner, thicker, circular, and an outer, thinner, longitudinal layer of non-striped muscle. In man, the upper third of the gullet consists of striped muscular fibres. Outside the muscular coat is a layer of fibrous tissue with elastic fibres. 
As in the intestine, there are two plexuses of nerves with ganglia; one in the sub-mucous coat and the other between the two muscular coats. Blood-vessels and numerous lymphatics lie in the mucous and sub-mucous coats.]

\section{5\%. Movements of the Stomach.}

When the stomach is empty, the great curvature is directed downwards and the lesser upwards; but when the stomach is full, it rotates on an axis running horizontally through the pylorus and cardia, so that the great curvature appears to be directed to the front and the lesser backwards.

Arrangement of the Muscular Fibres.-The non-striped muscular fibres of the stomach are arranged in three directions or layers, an outer longitudinal continuous with those of the oesophagus. This layer is best developed along the curvatures, especially the lesser. At the pylorus the fibres form a thick layer, and become continuous with the longitudinal fibres of the duodenum. The circular fibres form a complete layer, but at the pylorus they are well marked and constitute the pyloric sphincter-muscle, or valve; whilst at the cardia (inlet), such a muscular ring is absent (Gianuzzi). The innermost oblique or diagonal layer is incomplete.

The movements of the stomach are of two kinds:-(1.) The rotatory or churning movements, whereby the parts of the wall of the stomach lying in contact with the contents or ingesta glide to and fro with a slow rubbing movement. Such movements seem to occur periodically, every period lasting several minutes (Beaumont). By these movements the contents are moistened with the gastric juice, while the masses of food are partly broken down. The formation of hair-balls in the stomach of dogs and oxen indicates that such rotatory movements of the contents of the stomach take place. (2.) The other kind of movement consists in a periodically occurring peristalsis, whereby, as with a push, the portions of the contents of the stomach first dissolved are forced into the duodenum. They begin after a quarter of an hour (Busch), and recur until about five hours after a meal (Beaumont). This peristalsis is most pronounced towards the pyloric end, and the muscles of the pyloric sphincter relax to allow the contents to pass into the duodenum. According to Riudinger, the longitudinal muscular fibres, when they contract, especially when the pyloric end is filled, may act so as to dilate the pylorus.

The strongly muscular walls of the stomach of grain-eating birds effect a trituration of the food. The mechanical force thereby exerted was often experimented 
upon by the older physiologists, who found that glass balls and lead tubes, which could be compressed onily by a weight of 40 kilos., were broken or compressed in the stomach of a turkey.

Inflience of Nerves on the Movements.-[The stomach is supplied by the vagi and by the sympathetic, the right vagus being distributed to the posterior surface, and the left to the anterior surface, of the stomach.] The ganglionic plexus of nerve-fibres and nerve-cells (Averbach's), which lies between the muscular coats of the stomach, must be regarded as its proper motor centre, and to it motor impulses are conducted by the vagi. Section of both vagi does not abolish, but it diminishes the movements of the stomach. The muscular fibres of the cardia may be excited to action, or their action inhibited by fibres which run in the vagus ( $\mathrm{Nn}$. constrictores, et dilatator cardix), (v. Openchowski). [If the vagi be divided in the neck, there is a short temporary spasmodic contraction of the cardiac aperture. On stimulating the peripheral end of the vagus with electricity, after a latent period of a few seconds, the cardiac end contracts, more especially if the stomach be distended, but the movements are slight if the stomach be empty.]

Stimulation of the coliac plexus causes movements in the stomach of ruminants (Eckhard), perhaps indirectly through the effect upon the blood-vessels.

Local electrical stimulation of the surface of the stomach eauses circular constrictions of the organ, which disappear very gradually, while the movement is often propagated to other parts of the gastric wall. When heated to $25^{\circ} \mathrm{C}$, the excised empty stomach exhibits movements (Calliburces). . Injury to the pedunculi cerebri, optic thalamus, medulla oblongata, and even to the cervical part of the spinal cord, according to Schiff, causes paralysis of the vessels of certain arcas of the stomach, resulting in congestion and subsequent hæmorrhage into the mucous membrane.

[It is no uncommon occurrence to find hæmorrhage into the gastric mucous membrane of rabbits, after they have been killed by a violent blow on the head.]

\section{Vomiting.}

Mechanism.-Vomiting is caused by contraction of the walls of the stomach, whereby the pyloric sphincter is closed. It occurs most easily when the stomach is distended-(dogs usually greatly distend the stomach by swallowing air before they vomit); it readily occurs in infants, in whom the cul de sac at the cardia is not developed. It is quite certain that in children, this vomiting occurs through contraction of the walls of the stomach without the spasmodic action of the abdominal walls. When the vomiting is violent, the abdominal muscles act energetically. [The act of vomiting is generally preceded 
by a feeling of nausea, and usually there is a rush of saliva into the mouth, caused by a reflex stimulation of afferent fibres in the gastric branches of the vagus, the efferent nerve being the chorda tympani. After this a deep inspiration is taken, and the glottis closed, so that the diaphragm is firmly pressed downwards against the abdominal contents, and it is kept contracted; the lower ribs are pulled in. The diaphragm being kept contracted and the glottis closed, a violent expiratory effort is made, so that the contraction of the abdominal muscles acts upon the abdominal contents, the stomach being forcibly compressed. The cardiac orifice is opened at the same time, and the contents of the stomach are ejected. The chief agent seems to be the abdominal compression, but the walls of the stomach also help, though only to a slight extent.]

The contraction of the walls of the stomach, which causes a general diminution of the gastric cavity, is not a true antiperistalsis, as can be seen in the stomach when it is exposed (Galen). The cardia is opened by the longitudinal muscular tibres (Schiff) which pull towards the lower orifice of the cesophagus, so that when the stomach is full they must act as dilators. The act of vomiting is preceded by a ructus-like dilating movement of the intra-thoracic part of the cesophagus, which is caused thus:-The glottis is closed, inspiration occurs suddenly and violently, whereby the cesophagus is distended by gases proceeding from the stomach (Lüttich). The larynx and hyoid bone by the combined action of the geniohyoid, sternohyoid, sternothyroid, and thyrolyoid muscles are forcibly pulled forwards, so that the air passes from the pharynx downwards into the upper section of the oesophagus (Landois). If the abdominal walls contract suduenly, and if this sudden impulse be aided by the movements of the stomach itself, the contents of the stomach are forced outwards. During continued vomiting, antiperistalsis of the duodenum may occur, whereby bile passes into the stomach, and becomes mixed with its contents.

Children, in whom the fundus is absent, vomit more easily than adults. The callacity of the stomach of a new-born child is $35-43$ cubic centimetres; after 14 days, 153-160 c.c.; at 2 years, 740 c.c.

Magendie was of opinion that the abdominal muscles alone were concerned in vomiting, as he found that romiting occurred when he replaced the stomach by a bag. This was much too crude an experiment. But it only succeeds when the lowest part of the œesophagus has been removed (Fantini, Schiff). The view of Gianuzzi, that the abdominal muscles are the chief factor, because animals poisoned with curara-in whom these muscles are paralysed, but not the walls of the stomach-cannot vomit, is too wide a deduction.

Influence of Nerves. - The centre for the movements concerned in vomiting lies in the medulla oblongata, and is in relation with the respiratory centre, as is shown by the fact, that nausea may be overcome by rapid and deep respirations. In animals, vomiting may be inhibited by vigorous artificial respiration. On the other hand, the administration of certain emetics prevents the occurrence of apnoea.

The act of vomiting is most easily excited (chemically or mechanically) by stimulation of the centripetal or afferent nerves of the mucous membrane of the soft palate, pharynx, root of the tongue (glosso- 
pharyngeal nerve), stomach, and further, by stimulation of the uterus (pregnancy), intestine (inflammation of the abdomen), urinary apparatus (passing a renal calculus), and also by direct stimulation of the vomiting centre.

Vomiting produced by the thought of something disagreeable appears to be caused by the conduction of the excitement from the cerebrum to the vomiting centre. Vomiting is very common in cliseases of the brain. Section of both vagi prevents vomiting.

Emetics act (1) partly by mechanically or chemically stimulating the ends of the centripetal (afferent) nerves of the mucous membrane. Tickling the fauces, touching the surface of the exposed stomach $(\operatorname{dog})$; and many chemical emeticse.g., cupric and zinc sulphate and other metallic salts-act in this way. (2) Other substances cause vomiting when they are introduced into the blood (without being first introduced into the stomach), and act directly upon the vomiting centre, e.g., apomorphin. (3) Lastly, there are some substances which act in both ways, e.g., tartar emetic. Emetics may also remove mucus from the lungs, and in this case it is probable that the emetic acts upon the respiratory centre, and so favours the respirations. [According to Lauder Brunton, cupric sulphate acts even when injected into the blood.]

Vomiting is analogous to the process of rumination in animals that chew the cud. Some persons can empty their stomach in this way.

\section{Movements of the Intestine.}

Peristalsis. - The best example of peristaltic movements is afforded by the small intestine; the progressive narrowing of the tube proceeds from above downwards, thus propelling the contents before it. Frequently after death, or when air acts freely upon the gut, we may observe that the peristalsis develops at various parts of the intestine simultaneously, whereby the loops of intestine present the appearance of a heap of worms creeping amongst each other. The advance of new intestinal contents again increases the movement. In the large intestine, the movements are more sluggish and less extensive. The peristaltic movements may be seen and felt when the abdominal walls are very thin, and also in hernial sacs. They are more lively in vegetable feeders than in carnivora. The peristalsis is perhaps conducted directly through the muscular substance itself (as in the heart and ureterEngelmann).

Method of Observation. - Open the abdomen of an animal under a. 6 p.e. saline solution to prevent the exposure of the gut to air (Sanders, and Braam-Houckgeest).

The ileo-colic valve (Bauhin's valve; 1579, known to Rondelet in 1554), as a rule, prevents the contents of the large intestine from passing backwards into the small intestine. The movements of the stomach and intestine cease during sleep (Busch).

However, when fluid is slowly introduced into the rectum through a tube, it may pass upwards into the intestine, and even go through the ileo-colic valve into the small intestine. 
Muscarin excites very lively peristalsis of the intestines, which may be set aside by atropin (Schmiedeberg and Koppe).

Pathological. - When any condition excites an acute inflammation of the intestinal mucous membrane, catarrh is rapidly produced, and very strong contractions of the inflamed parts filled with food, take place. When these parts of the gut become empty, the movements are not stronger than normal. If new material passes into the inflamed part, the peristalsis recurs, and is more lively than normal, and the result is diarrhoea (Nothnagel). Sometimes, a greatly contracted part of the small intestine is pushed into the piece of gut directly continuous with it, giving rise to invagination or intussusception.

Antiperistalsis-i.e., a movement which sets in and travels in an upward direction towards the stomach, does not occur uormally. That such a condition takes place has been inferred from the fact, that in cases where the intestine is occluded (Ileus) fiecal matter is vomited. The most recent experiments of Nothnagel throw doubts upon this view, as he failed to observe antiperistalsis in cases where the intestine was occluded artificially. The fæcal odour of the ejecta may result from the prolonged retention of the material within the small intestine.

\section{Excretion of Fæcal Matter.}

The contents of the small intestine remain in it about three hours, and about twelve hours in the large intestine, where they become less watery. The contents assume the characters of fres, and become "formed" in the lower part of the great intestine. The freces are gradually carried along by the peristaltic movement, until they reach a point a little abore that part of the rectum which is surrounded by both sphincters; the internal sphincter consisting of non-striped, and the external of striped muscle.

Immediately after the fæces have been expelled, the external sphincter (Fig. 129, S, and Fig. 130) usually contracts vigorously, and remains in this condition for some time. Afterwards it relaxes, when the elasticity of the parts surrounding the anal opening, particularly of the two sphincters, suffices to keep the anus closed. In the interval between two evacuations, there does not seem to be a continued tonic contraction of the sphincters. As long as the fæces lie above the rectum, they do not excite any conscious sensations, but the sensation of requiring to go to stool occurs, when the frees pass into the rectum. At the same time, the stimulation of the sensory nerves of the rectum causes a reflex excitement of the sphincters. The centre for these movements (Budge's centrum anospinale) lies in the lumbar region of the spinal cord; in the rabbit, between the sixth and seventh, and in the dog, at the fifth lumbar vertebra (Masius).

In animals, whose spinal cord is divided above the ceutre, a slight touch in the region of the auus causes this orifice to contract, but after this lively reflex contraction, the sphincters relax again, and the anus may remain open for a time. This occurs, because the voluntary impulses which proceed from the brain to cause the contraction of the external sphincter are absent. Landois observed, that in 


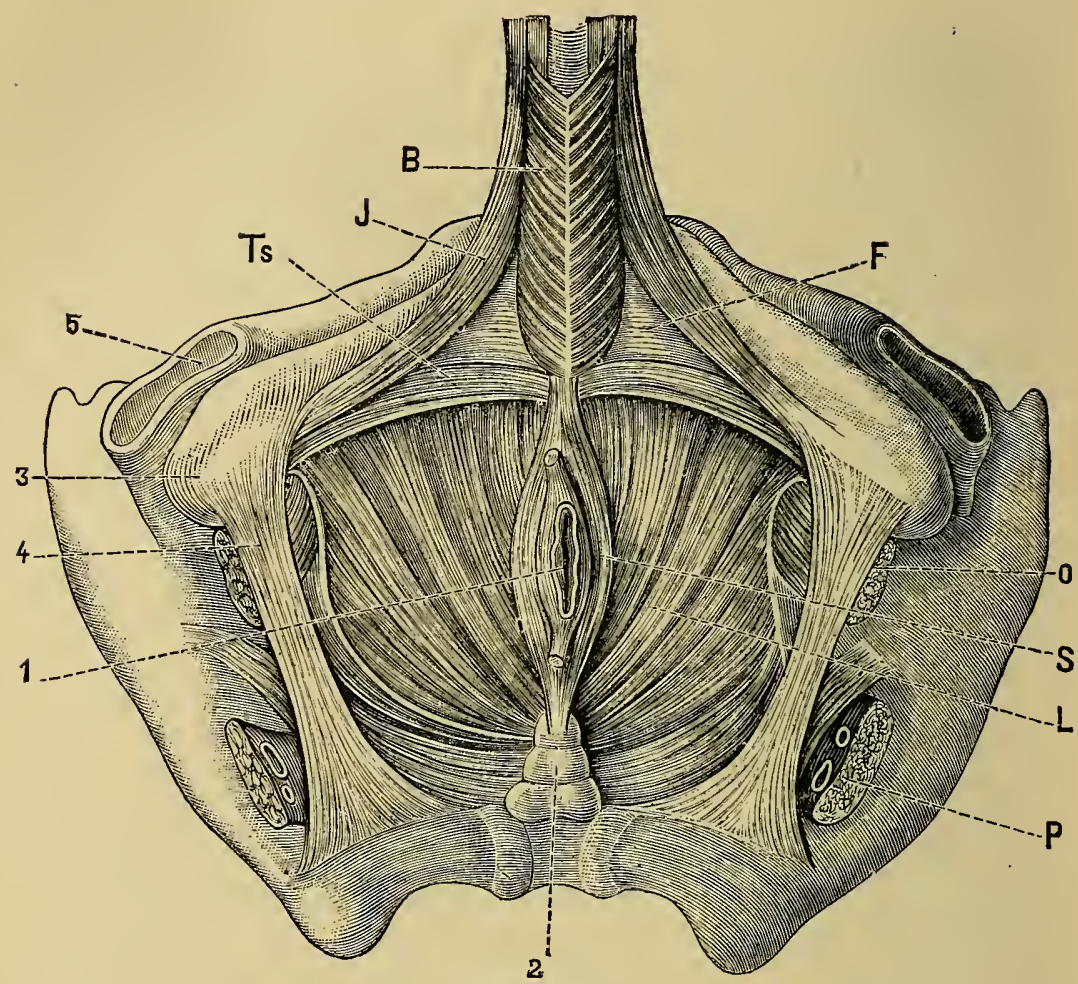

Fig. 129.

The Perinæum and its Muscles-1, Anus; 2, coccyx; 3, tuberosity; 4, sciatic ligament; 5 , cotyloid cavity ; $\mathrm{B}$, bulbo-cavernosus muscle; $\mathrm{Ts}$, superficial transverse perineal muscle; $\mathrm{F}$, fascia of the deep transverse perineal muscle; $\mathrm{J}$, ischio-cavernosus muscle; $\mathrm{M}$, obturator internus; $\mathrm{S}$, external anal sphincter; L, levator ani ; $\mathrm{P}$, pyriformis (Henle).

dogs with the posterior roots of their lower lumbar and sacral nerves divided, the anus remained open, and not unfrequently a mass of fæces remained half ejected. As the sensibility of the rectum and anus was abolished in these animals, the sphincters could not contract reflexly, nor could there be any voluntary contraction of the sphincters.

The external sphincter can be contracted voluntarily from the cerebrum, like any voluntary muscle, but the closule can only be effected up to a certain degree. When the pressure from above is very great, the energetic peristalsis at last overcomes the strongest voluntary impulses. Stimulation of the peduncles of the cerebrum and of the spinal cord below this point, causes contraction of the external sphincter.

Defæcation.-The evacuation of the fæces, which in man usually occur's at certain times, begins with a lively peristalsis of the large intestine, which passes downwards to the rectum. In order that the mass of 
fæces may not excite reflexly the sphincter-muscles, in consequence of mechanical stimulation of the sensory nerves of the rectum, there seems to be an inhibitory centre for the reflex action of the sphincters, which is set in action, owing, as it appears, to voluntary impulses. Its seat is in the brain; Masius thinks it is in the optic thalami, from whence fibres pass through the peduncles of the cerebrum to the lumbar part of the spinal cord. When this inhibitory apparatus is in action, the frecal mass passes through the anus, without causing it to close reflexly.

The strong peristalsis which precedes defæcation can be aided, and to a certain degree, excited by voluntary, short, movements of the external sphincter and levator ani, whereby the plexus myentericus of the large intestine is stimulated mechanically, thus causing lively peristaltic

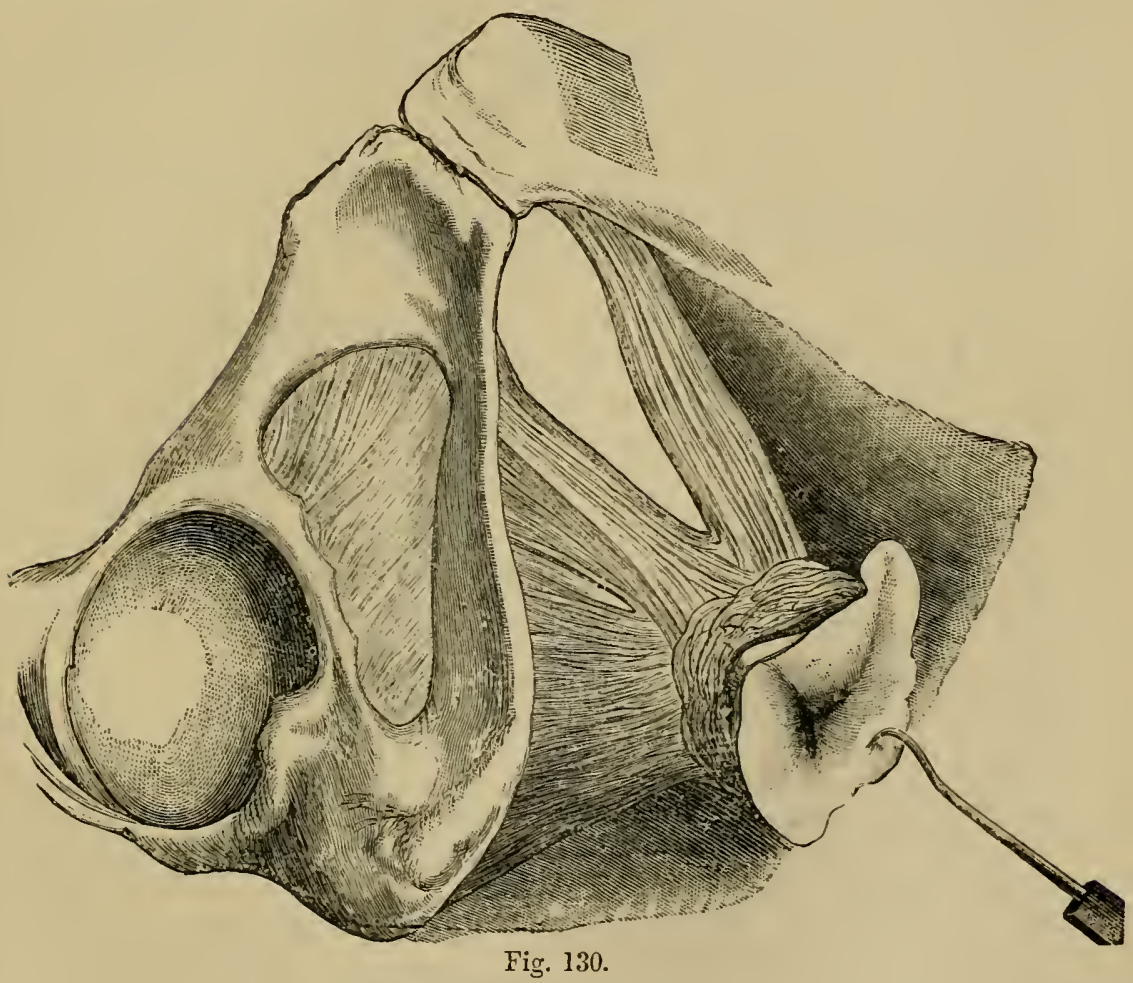

Levator ani and Sphincter ani externus.

movements in the large intestine. The expulsion of the fæces is also aided by the pressure of the abdominal muscles, and most efficiently when a deep inspiration is taken, so as to fix the diaphragm, whereby the abdominal cavity is diminished to the greatest extent. The soft 
parts of the floor of the pelvis during a strong effort at stool, are driven downwards in the form of a cone, causing the mucous membrane of the anus, which contains much venous blood, to be everted. The function of the levator ani (Figs. 129, 130) is, to raise voluntarily the soft parts of the floor of the pelvis, and to pull the anus to a certain extent upwards over the descending fæcal mass. At the same time, it prevents the distension of the pelvic fascia. As the fibres of both levatores converge below and become united with the fibres of the external sphincter, they aid the latter, during energetic contraction of the sphincter; or, as Hyrtl puts it, the levatores are related to the anus, like the two cords of a tobacco pouch. During the periods between the evacuation of the gut, the fæces appear only to reach the lower end of the sigmoid flexure. As a rule, from thence downwards, the rectum is normally devoid of fæces. It seems that the strong circular fibres of the muscular coat, which Nélaton has called sphincter ani tertius, when they are well developed, contract and prevent the entrance of the fæces. When the tendency to the evacuation of the rectum is very pressing, the anus may be closed more firmly from without, by energetically rotating the thigh outwards, and contracting the muscles of the gluteal region.

\section{Influence of Nerves on the Intestinal Movements.}

Auerbach's Plexus.-The intestinal canal contains an automatic motor centre within its walls-the plexus myentericus of Auerbach-which lies between the longitudinal and circular muscular fibres of the gut. It is this plexus which enables the intestine when cut out of the body to execute, apparently spontaneously, movements for some time.

[Structure.-The plexus of Auerbach consists of non-medullated nerve-fibres which form a dense plexus, groups of ganglion cells occurring at the nodes (Fig. 131). A similar plexus extends throughout the whole intestine between the longitudinal and circular muscular coats from the œsophagus to the rectum. Branches are given off to the muscular bundles. A similar, but not so rich a plexus lies in the sub-mucous coat, Meissner's plexus, which gives branches to supply the muscularis mucosæ, the smooth muscular fibres of the villi, and the glands of the intestine (Fig. 132).]

1. If this centre is not affected by any stimulus, the movements of the intestine cease-comparable to the condition of the medulla oblongata in apnoea (Sig. Mayer and v. Basch). The same is truejust as in the case of the respiration-during intra-uterine life, in consequence of the foetal blood being well supplied with $O$. This condition may be termed aperistalsis. It also occurs during sleep, perhaps on account of the greater amount of $\mathrm{O}$ in the blood during that state. 


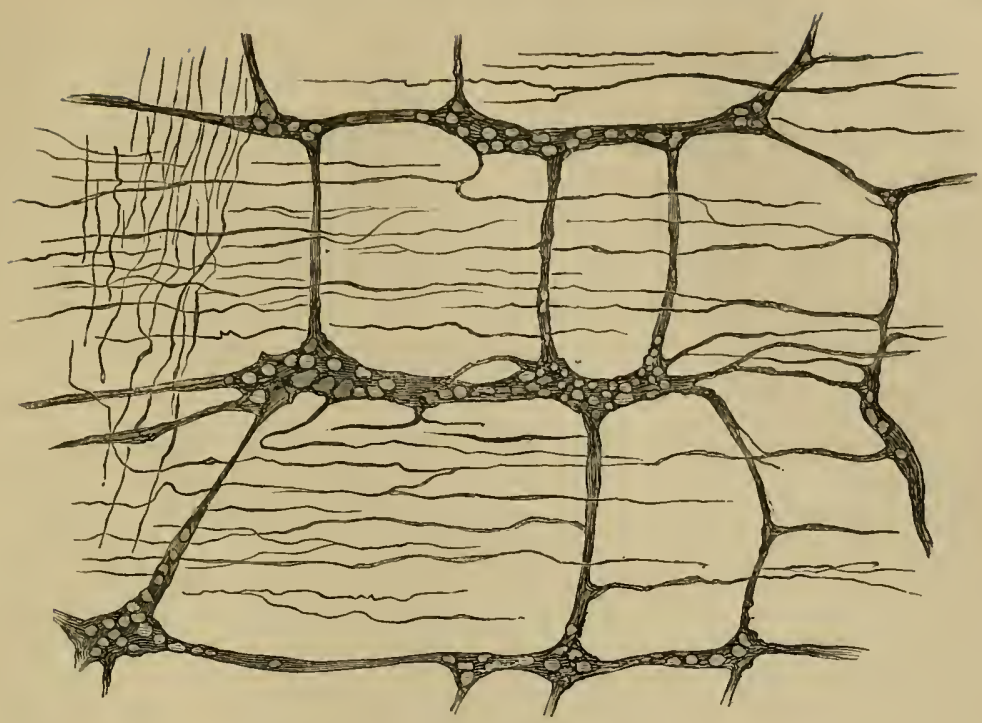

Fig. 131.

Plexus of Auerbach, prepared from the small intestine of a dog, by the action of gold chloride. The nerve-cells are shown at the nodes, while the fibrils proceeding from the ganglia, and the anastomosing fibres, lie between the muscular bundles.

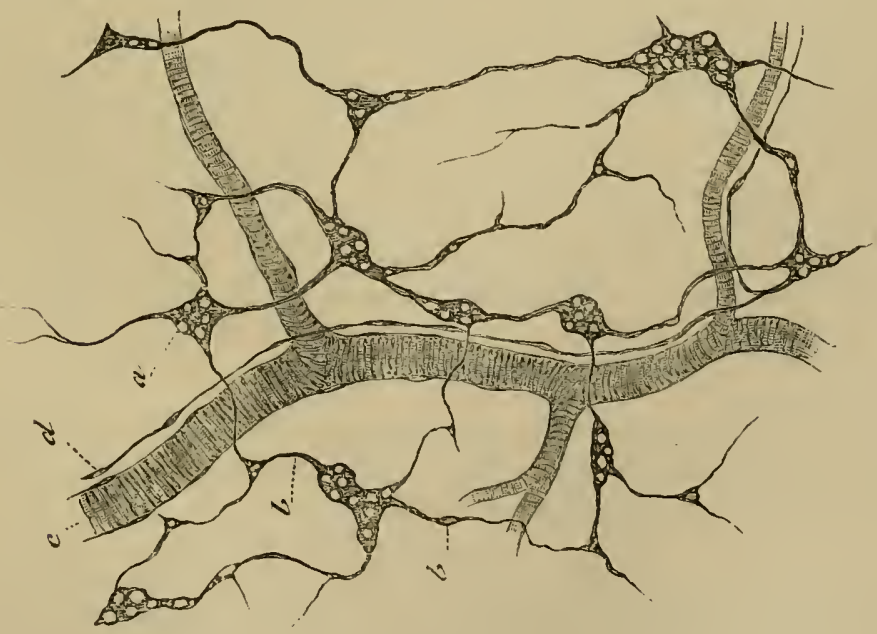

Fig. 132.

Plexus of Meissner- $a$, ganglia; $b$, anastomosing fibres; $c$, artery; $d$, vaso-motor nerve-fibres accompanying $c$.

2. When blood containing the normal amount of blood-gases passes through the intestinal blood-vessels, the quiet peristaltic movements of 
health occur (euperistalsis) provided no other stimulus be applied to the intestine.

3. All stimuli applied to the plexus myentericus increase the peristalsis, which may become so very violent as to cause evacuation of the contents of the large gut, and may even produce spasmodic contraction of the musculature, of the intestine. This condition may be termed dysperistalsis, corresponding to dyspnœa. The condition of the blood flowing through the intestinal vessels has a most important effect on the peristaltic movements.

Condition of the Blood.-Dysperistalsis may be produced by $(a)$ interrupting the circulation of blood in the intestines, no matter whether anæmia (as after compressing the aorta-Schiff,) or venous hyperæmia be produced. The stimnlating condition is the want of $\mathrm{O}$, i.e., the increase of $\mathrm{CO}_{2}$. Very slight disturbance in the intestinal blood-vessels, e.g., venous congestion after copious transfusion into the veins, whereby the abdominal and portal veins become congested, canses increased peristalsis. The intestines become nodulated at one part and narrow at another, and involuntary evacuation of the fæces takes place when there is congestion, owing to the plugging of the intestinal blood-vessels when blood from another species of animal is used for transfusion (p. 202-Landois). The marked peristalsis which occurs on the approach of death is undoubtedly due to the derangements of the circulation, and the consequent alteration of the amount of gases in the blood of the intestine. The same is true of the increased movements of the intestines which occur as a result of psychical excitement, e.g., grief. The stimulus, in this case, passes from the cerebrum through the medulla oblongata (vaso-motor centre) to the intestinal nerves and causes anæmia of the intestine, (corresponding to the palor occurring elsewhere). When the normal condition of the circulation is restored, the peristalsis diminishes. (b) Direct stimulation of the intestine, conducted to the plexus myentericus, causes dysperistalsis; direct exposure of the intestines to the air (stronger when $\mathrm{CO}_{2}$ or $\mathrm{Cl}$ is present)--the introduction of various irritating substances into the intestine-increased filling of the intestine when there is any difficulty in emptying the gut (often in man)-direct stimulation of various kinds (also inflammation), all act upon the intestine, either from without or from within. Induction shocks applied to a loop of intestine in a hernial sac cause lively peristalsis in the hernia. The intestinal movements are favoured by heat, and cease below $19^{\circ} \mathrm{C}$. (Horwath).

4. The continued application of strong stimuli causes the dysperistalsis to give place to rest, owing to over-stimulation, which may be called "intestinal paresis," or exhaustion.

This condition is absolutely different from the passive condition of the intestine in aperistalsis. Continued congestion of the intestinal blood-vessels ultimately causes intestinal paralysis, e.g., when transfusion of foreign blood causes coagulation within these vessels (Landois). Filling the blood-vessels with "indifferent" fluids, after the peristalsis has been previously caused by compressing the aorta, also causes cessation of the movements $(0$. Nasse). The movements cease when the intestines are cooled to $19^{\circ} \mathrm{C}$. (Horwath), while severe inflammation of the intestine has a similar effect. Under favourable circumstances, the intestine may recover from this condition. Arterial blood admitted into the vessels of the exhausted intestine causes peristalsis, which at first is more vigorous than normal. 
5. The continued application of strong stimuli causes complete paralysis of the intestine, such as occurs after violent peritonitis, or inflammation of the musculature or mucous coat in man. In this condition, the intestine is greatly distended, as the paralysed musculature does not offer sufficient resistance to the intestinal gases which are expanded by the heat. This constitutes the condition of meteorism.

Influence of Nerves.-With regard to the nerves of the intestine, stimulation of the vagus increases the movements fof the small intestine), either by conducting impressions to the plexus myentericus, or by causing contraction of the stomach, which stimulates the intestine in a purely mechanical manner (Braam-Houckgeest). The splanchnic is (1) the inlibitory nerve of the small intestine (Pfluiger), but only as long as the circulation in the intestinal blood-vessels is undisturbed, and the blood in the capillaries does not become venous (Sigm. Mayer, and von Basch); when the latter condition occurs, stimulation of the splanchnic increases the peristalsis. If arterial blood be frecly supplied, the inhibitory action continues for some time (O. Nasse). Stimulation of the origin of the splanchnics, of the spinal cord in the dorsal region (under the same conditions), and even when general tetanus has been produced by the administration of strychuia, causes an inhilitory effect. O. Nasse concludes from these experiments that the splanchnic contains-(2) inhibitory fibres which are easily exhausted by a venous condition of the blood, and also motor fibres which remain excitable for a longer time, because after death, stimulation of the splanchnics always causes peristalsis, just like stimulation of the vagus. (3) The splanchnic is also the vaso-motor nerve of all the intestiual blood-vessels, so that it governs the largest vascular area in the body. When it is stimulated, all the vessels of the intestine, which contain muscular fibres in their walls, contract; when it is divided, they dilate. In the latter case, a large amount of blood accumulates within the blood-vessels of the abdomen, so that there is anæmia of the other parts of the body, which may be so great as to cause death-owing to the deficient supply of blood to the medulla oblongata. (4) The splanchnic is the sensory nerve of the intestine, and as such, under certain circumstances, it may give rise to extremely painful sensations.

As stimulation of the splanchnic contracts the blood-vessels, von Basch has raised the question, whether the intestine does not come to rest, owing to the want of the blood, which acts as a stimulns. But, when a weak stimulus is applied to the splanchnic, the intestine ceases to move before the blood-vessels contract (van Braam-Houckgeest); it wonld therefore seem that the stimulation diminishes the excitability of the plexus myentericus.

According to Engelmann and v. Brakel, the peristaltic movement is chiefly propagated by direct muscular conduction, as in the heart and ureter, without the intervention of any nerve-fibres. 
Effect of Drugs. - Amongst the reagents which act upon the intestinal movements are:-(1) Such as diminish the excitability of the plexus myentericus, i.e., which lessen or even abolish intestinal peristalsis, e.g., belladonna. (2) Such as stimulate the inhibitory fibres of the splanchnic, and in large doses paralyse themopium, morphia (Nothnagel); 1 and 2 produce constipation. (3) Other agents excite the motor apparatus-nicotin (even causing spasm of the intestine), muscarin, caffein, and many laxatives, which act as purgatives. The movements produced by muscarin are abolished by atropin (Schmiedeberg and Koppe). These substances accelerate the evacuation of the intestine, and, owing to the rapid movement of the intestinal contents, only a small amount of water is absorbed; so that the evacuations are frequently fluid. (4) Amongst purgatives, colocynth and croton oil act as direct irritants. With regard to drugs of this sort, they seem to cause a watery transudation into the intestine (C. Schmidt, Moreau), just as croton oil causes vesicles when applied to the skin. (5) Calomel is said to limit the absorptive activity of the intestinal wall, and to control the decompositions in the intestine. The stools are thin and greenish from the admixture of bili-verdin. (6) Certain saline purgatives-sodium sulphate, magnesium sulphate, cause fluid evacuations by retaining the water in the intestine (Buchheim); and it is said, that if they be injected into the blood-vessels of animals, they cause constipation (Aubert).

If a crystal of a potash salt be applied to the intestine, it causes a local constriction, accompanied by lively movement extending about 10 centimetres above where the crystal was applied. Soda salts are not so powerful, and they seem to act upon the nerves and not upon the musculature (Nothnagel, K. Bardeleben).

[Action of Saline Cathartics.-From an extended investigation recently made by Matthew Hay on the action of saline cathartics, it would appear certain that a salt exerts a genuine excito-secretory action on the glands of the intestines, whilst at the same time, in virtue of its low diffusibility, it impedes absorption. Thus, between stimulated secretion and impeded absorption there is an accumulation of fluid within the canal, which, partly from ordinary dynamical laws, partly from a gentle stimulation of the peristaltic movements excited by distension, reaches the rectum and results in purgation. Purgation does not ensue when water is withheld from the diet for one or two days previous to the administration of the salt in a concentrated form. This absence of effect is due to a deficiency of water in the blood, so that the blood cannot, through the intestinal glands, yield enough fluid to the salt in order to produce purgation. When a concentrated solution of a salt is administered to an animal whose alimentary canal is known, from a few hours' preliminary fasting, to be empty, but whose blood is in a natural state of dilution, the blood becomes rapidly very concentrated, and reaches the maximum of its concentration in from half an hour to an hour and a half; within four hours the blood has gradually returned to its normal state of concentration without having reabsorbed fluid from the intestine. It apparently recoups itself from the tissue-fluids. After a few days' abstention from water, the tissuefluids are so much diminished as not to be able any longer to recoup the blood, and the blood itself gradually becomes concentrated; hence a concentrated saline solution fails to excite any secretion when administered.

It is also interesting in connection with saline catliartics that the salt-sulphate of magnesia or sulphate of soda-becomes split up in the small intestine, and the acid is more rapidly absorbed than the base. A portion of the absorbed acid shortly afterwards returns to the intestines, evidently through the intestinal glands. After the maximum of excretion of the acid has been reached, the salt begins very slowly and gradually to disappear by absorption, which is checked only by the occurrence of purgation. The salt does not purge when injected into the blood, and excites no intestinal secretion; nor does it purge when injected subcutaneously, unless on account of its causing local irritation of the abdominal subcutaneous tissue, which acts reflexly on the intestines, dilating their bloodvessels, and perhaps stimulating their muscular movements.] 


\section{Structure of the Stomach.}

Structure.-[The walls of the stomach consist of four coats, which are from without inwarls-

(1) The serous layer, from the peritoneum.

(2) The muscular layer, composed of three layers of non-striped muscular fibres- $(a)$, longitudinal; $(b)$, circular; $(c)$, oblique (sce p. 309).

(3) The sub-mucous layer, of loose connective-tissue, with the larger blood-vessels, lymphatics, and nerves.

(4) The mucous layer.]

The well-developed mucous membrane of the stomach is thrown into a series of folds or ruge, in the contracted condition of the organ. With the aid of a hand-lens, it is seen to be beset with small irregular depressions or pits (Vidius, 1567-Fig. 133). Throughout its entire extent it is covered by a single layer of moderately tall, narrow cylindrical epithelium, which seems to consist of mucus-secreting goblet cells (Fig. 135, $)$ ). The epithelium is sharply defined at the cardia from the stratified epithelium of the cesophagus, and also at the pylorus, from the true cylindrical epithelium with the striated disc in the duodenum. [The cells in the passive condition seem to consist of two zones, an outer clear part, next the lumen of the organ, consisting of a substance (mucigen) which yields mucus, the attached end of the cell being granular.] The oval nucleus lies about the centre of the cells. Spindleshaped, nucleated cells, probably for replacing the others, are said by Ebstein to occur at their bases. All the cells are open at their free-ends, so that the mucus is readily discharged, leaving the cells empty (F. E. Schultze). Numerous tubular glands of two distinct kinds are placed vertically, like rows of test-tubes, in the mucous membrane.

Fundus-glands.-On making a vertical section of the cardiac portion of the gastric mucous membrane, and submitting it to

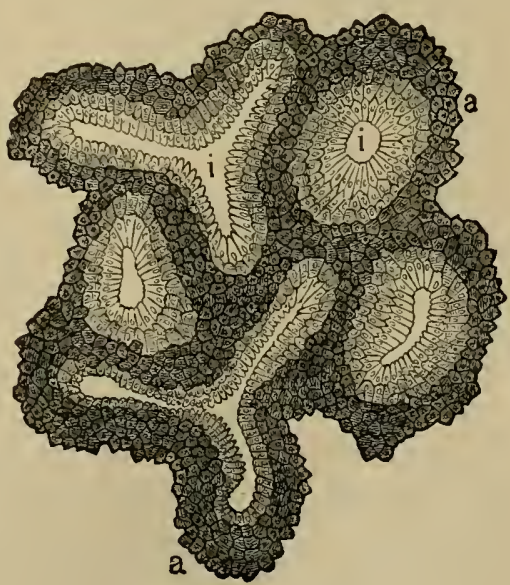

Fig. 133.

Surface section of the dog's gastric mucous membrane, showing the crater-like depressions or pits, $i i$; $a$, the elevations round $i$.

microscopic examination, it is seen to consist of a number of tubular glands 
placed side by side. These are the fundus-glands (Heidenhain), otherwise called peptic, or cardiac. Several gland-tubes, which are wider below, usually open into the short duct (Fig. 136). Each gland consists of a structureless membrana propria with anastomosing branched cells in relation with it. The duct is lined by a layer of cells like those lining the stomach, while the secretory part of the tubes is lined throughout by a layer of granular, short, small, polyhedral, or columnar nucleated cells. These cells border the very narrow lumen, and were called chief or principal cells by Heidenhain; they are also known as central cells (Fig. 134, II, a), or adelomorphous (áon $\lambda$ os, hidden). At various places, between these cells and the membrana propria are large oval, or angular, well-defined granular, densely reticulated, nucleated cells, the parietal cells of Heidenhair, or the delomorphous cells of Rollett

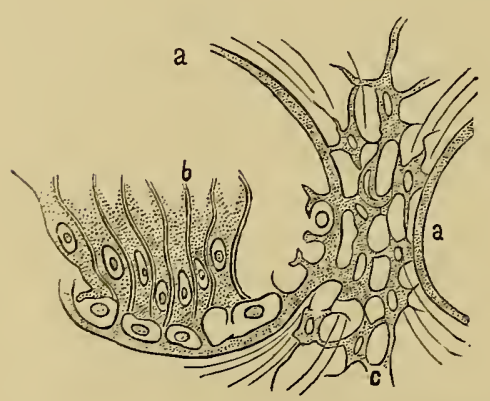

$\mathrm{J}$

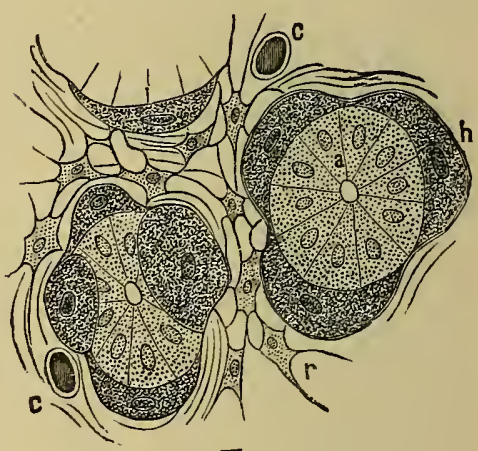

II

Fig. 134.

I, Transverse section of a duct of a fundus-gland- $a$, membrana propria; $b$, mucus secreting goblet cells; $c$, adenoid interstitial substance. II, Transverse section of a fundus-gland $-\alpha$, chief cells; $h$, parietal cells; $r$, adenoid-tissue between the gland-tubes; $c$, divided capillaries.

(Fig. 134, II, h). They are most numerous in the neck of the glands, and least so in the deep blind end of the tubes. These cells are stained deeply by osmic acid and aniline blue, so that they are readily distinguished from the other cells. They bulge out the membrana propria of the gland opposite where they are placed. The parietal cells in man are said to reach to the lumen of the gland-tubes (Stöhr). Isolated cells are sometimes found under the epithelium of the surface of the stomach (Heidenhain), and occasionally in individual pyloric glands (Stöhr). The fundus-glands are most numerous (about 5 millions, according to Sappey), and are of considerable size in the fundus.

2. The Pyloric Glands occur only in the region of the pylorus, 
where the mucous membrane is more yellowish-white in colour (Fig. 135, A). These glands are generally branched at their lower ends, so that several tubes open into a single duct [which, in contradistinction to the duct of the other glands, is wide and long, extending often to half the depth of the mucous membrane. The duct is lined by epithelium like that lining the stomach, while the secretory part is lined by a single layer of short, finely granular, columnar cells, whose secretion is quite different from that of the cells lining the duct. The lumen is well-defined. Nussbaum has occasionally found other cells, which stain deeply with osmic acid, between the bases of these. Ebstein regards these cells as forming pepsin. It is to be remembered that the appearance of the cells differs according to their state of physiological activity (Figs. 137 and 138). When they are exhausted they are smaller and more granular, owing to the denser
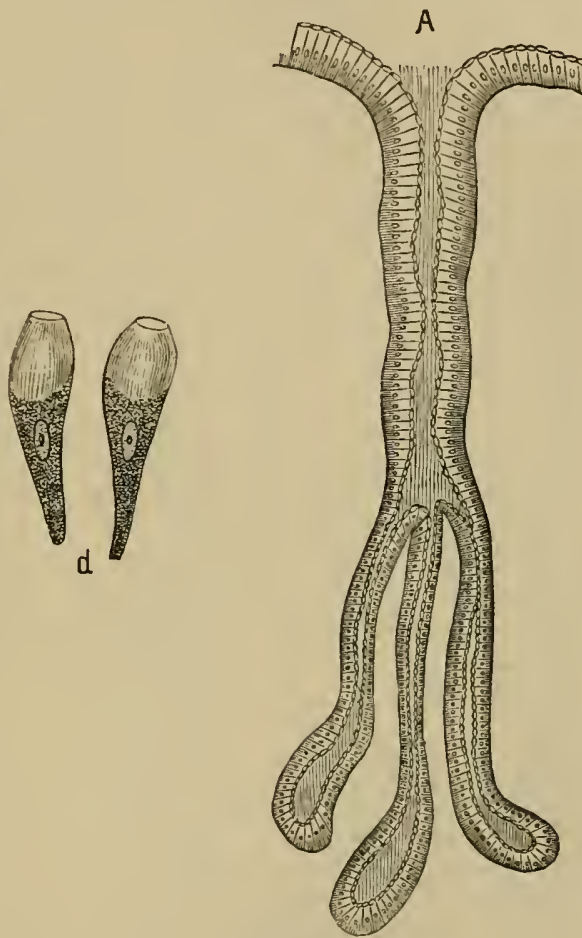

Fig. 135.

A, Isolated pyloric gland; $d$, isolated goblet cells.

reticulation of their net-work; at any rate, they are granular in preparations hardened in alcohol (Fig. 138).]

Muscularis Mucosæ.-The glands are supported by very delicate connectivetissue mixed with adenoid-tissue (Fig. 134). Below this are two layers, circular and longitudinal, of nou-striped muscle, the muscularis mucosa, and from it fine processes of smooth muscular fibres pass up between groups of the glands towards the free epithelial surface of the gastric mucous membrane. These muscular processes are said to be concerned in emptying the glands. [In the gastric mucous membrane of the cat, there is a clear homogeneous layer which is stained red by picrocarmine, and placed immediately internal to the muscularis mucosæ. It is pierced by the processes passing from the muscularis mucosæ.]

Masses of adenoid-tissue oceur in the mucous membrane, especially near the pylorus, constituting lymph-follicles, which are comparable to the solitary glands of the small intestine. 
The Lymphatics are numerous, and begin close under the epithelium by dilated extremities or loops (Fig. 136, d); they run vertically, and anastomose in the mucosa between the gland-tubes, which they envelope in sinus-like spaces. They join large trunks in the mucosa; another plexus of large vessels exists in the submucosa (Lovèn).

[The Nerves.-A plexus of non-medullated nerve-fibres and a few ganglion cells

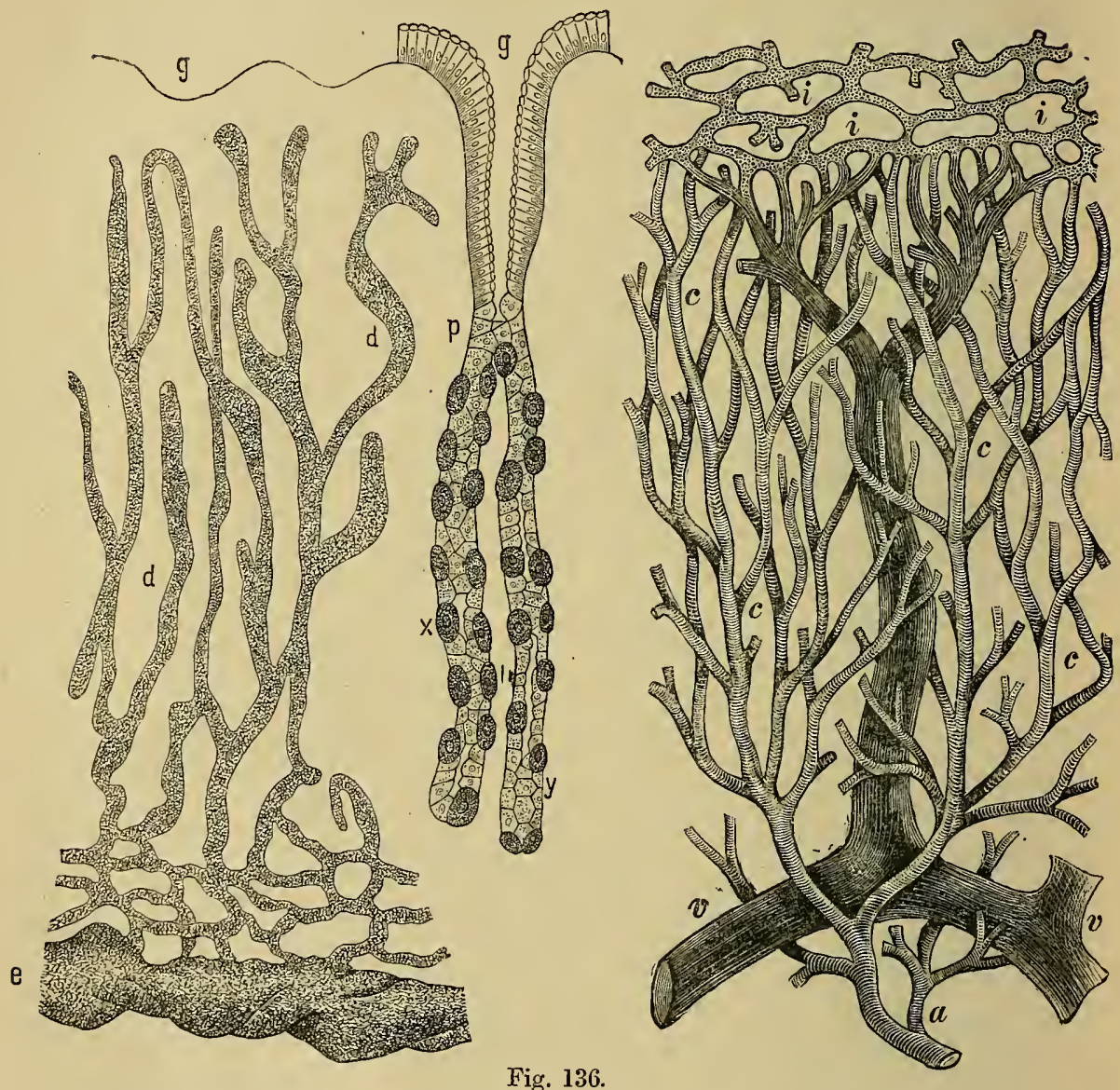

Vertical section of the gastric mucous membrane- $g g$, pits on the surface; $p$, neck of fundus-glands opening into a duct, $g ; x$, parietal, and $y$, chief cells ; $a, v, c$, artery, vein, capillaries; $d, d$, lymphatics, emptying into a large trunk, $e$. (Partly schematic).

exist in the muscular coat (Auerbach's), and another (Meissner's) in the submucosa.]

The Blood-vessels are very numerous. Small arterial branches, $a$, run in the sub-mucosa and ascend between the glands to form a longitudinal capillary net-work, $c c$, which forms a narrow net-work under the epithelium, and between its meshes the gland-ducts open $(g)$. The veins gradually collect from this horizontal capillary net-work arrl run towards the large veins of the sub-mucosa, $v$. 


\section{The Gastric Juice.}

Properties.-The gastric juice is a tolerably clear colourless fluid, with a strong acid reaction, sour taste, and peculiar characteristic odour; it rotates the plane of polarised light to the left (Hoppe-Seyler). It is not rendered turbid by boiling, and resists putrefaction for a long time. Its specific gravity $=1002.5(\log , 1005)$, and it contains only $\frac{1}{2}$ p.c. of solid constituents. The quantity of gastric juice secreted in 24 hours was estimated by Beaumont, from observations upon Alexis St. Martin, who had a gastric fistula (1834) -at only 180 grms. daily (!); by Grünewald (1853), in a similar case, as equal to 26.4 p.c. of the bodyweight; while Bidder and Schmidt (from corresponding observations on (logs) estimated it as equal to $6 \frac{1}{2}$ kilos. daily, corresponding to $\frac{1}{10}$ of the body-weight. It contains :-

(1.) Pepsin (Tli. Schwann, 1836), the characteristic nitrogenous hydrolytic ferment or enzym, which dissolves proteids-3 per 1000 .

(2.) Hydrochloric Acid (Prout, 1824), 0.2-0.3 (according to Richet, $0 \cdot 8-2 \cdot 1)$ per 1000 ; (in the dog, 15 times more). This occurs free in the gastric juice, as the latter always contains more free chlorine than bases, to which it can be united (C. Schmidt). Lactic acir is usually met with, but it arises from the fermentation of the carbohydrates of the food.

[It has been for a long time disputed, whether the acidity of the gastric juice is due to hydrochloric acid or to free lactic acid. The most reliable of recent methods for determining this, point conclusively to hydrochloric acid as the cause of the acidity (Richet and others).]

Tests.-Free hydrochloric acid is detected by the following reactions:-0.025 p.c. solution of methylviolet becomes blue; or, alkaline solution of tropæolin becomes lilac; or, red Bordeaux wine is treated with amylic alcohol until its colour almost disappears-when, if dilute hydrochloric acid be added, a rose colour is obtained.

(3.) The large amount of mucus which covers the surface of the mucous membrane is to be regarded as the secretion from the goblet cells of the mucous membrane (p. 321). [The reaction of the mucus covering the walls of the empty stomach is in many cases alkaline (M. Hay).]

\section{(4.) Mineral Salts (2 per 1000).}

They are chiefly sodium and potassium chloricles, less calcic chloride (ammonium chloride, also in animals), and the compounds of phosphoric acid with lime, magnesium, and iron.

Amongst foreign substances, which may be introduced into the body, the following appear in the gastric juice, HI, after the use of potassium iodide-potassium sulpho-cyanide, ferric lactate, and sugar, and ammonium carbonate in uræmia. 


\section{Secretion of Gastric Juice.}

After the discovery of the two kinds of glands in the stomach, and after it was found that the fundus-glands contained two different forms of cells, the question as to whether the different constituents of gastric juice were formed by different histological elements came to be investigated.

Changes of the Cells during Digestion.-During the course of digestion, the cells of the fundus (and pyloric glands, dog) undergo

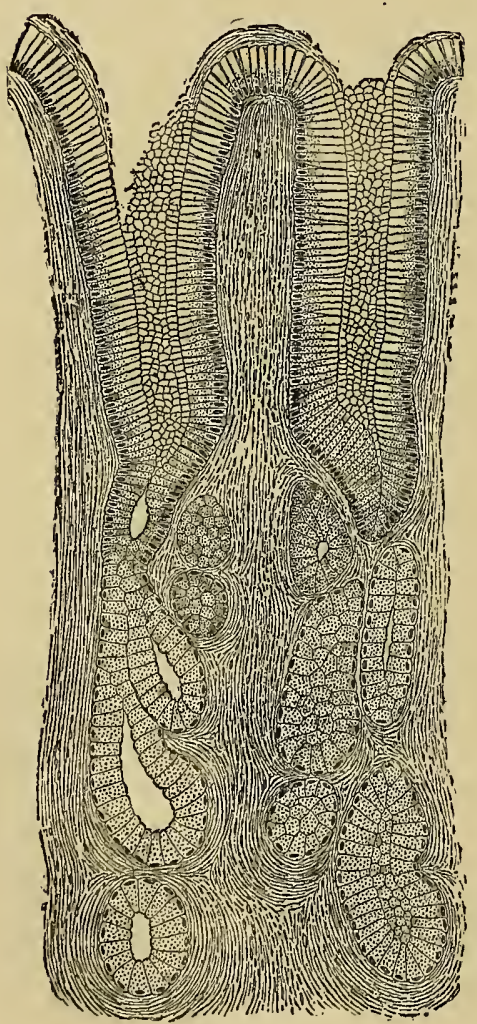

Fig. 137.

Section of the pyloric mucous membrane (Ebstein).

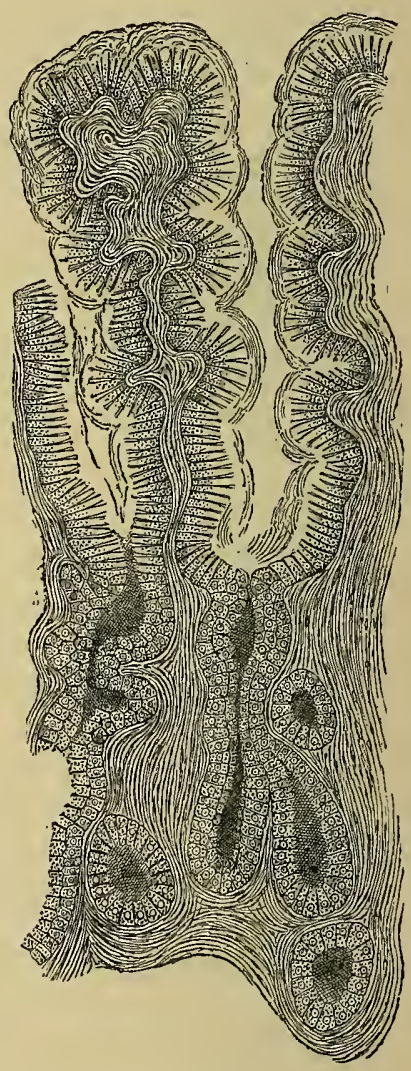

Fig. 138.

Pyloric glands, showing changes of the cells during digestion (Ebstein).

important changes (Heidenhain, Ebstein). During hunger, the chief cells are clear and large, the parietal investing cells are small, the pyloric cells clear and of moderate size. During the first six hours of 
digestion, the chief cells become cnlarged and moderately turbid or granular, the parietal cells also enlarge, while the pyloric cells remain unchanged. The ehief cells become diminished and more turbid or granular until the 9th hour, the parietal cells are still swollen, and the pyloric eells enlarge. During the last hours of digestion, the chief cells again beeome larger and elearer, the parietal cells diminish, the pyloric cells decrease in size and become turbid (Figs. 137 and 138).

[Langley gives a different description of the appearances presented by these cclls, cluring different phases of secretory activity. The results may be reconciled by remembering that the gland-cells were examined under different conditions. The secretory cells consist of a cell-substance composed of $(a)$ a framework of living protoplasm, either in the form of an intra-cellular fibrillar net-work (Klein), or in flattened bands. The meshes of this framework enclose at least two chemical substances, viz., (b) a hyaline substance in contact with the framework, and (c) spherical granules which are embedded in the hyaline substance (Langley). Speaking generally, during active secretion, the granules decrease in number and size, the hyaline substance increases in amount, the net-work grows. This is the reverse of what is stated above as the observation of Heidenhain, but the granular appearance described by Heidenhain after secretion is very probably due to the action of the hardening agent, alcohol. Langley found that in the living condition, or after the use of osmic acid, in some animals at least, the chief cells are granular during rest, but during a state of activity two zones are differentiated, an onter one, which is clear, owing to the disappearance of the granules, and an inner more or less granular one. Granules reappear in the outer part after rest. During digestion, the parietal cells increase in size, but do not become grannlar. In all cells oontaining much pepsinogen, distinct granules are present, and the quantity of pepsinogen varies directly with the number and size of the granules. In the glands of some animals there is little difference between the resting and activc phases (Langley). Compare Serous Glands, p. 284, and Pancreas, § 168.]

The Pepsin is formed in the chief cells (Heidenhain). When these are clear and large they contain much pepsin, when they are contracted and turbid the amount is small (Griitzner). The pyloric glands are also said to secrete pepsin, but only to a small extent (Ebstein, Griitzner, Klemensiewicz). Pepsin accumulates during the first stage of hunger, and it is eliminated cluring digestion and also during prolonged hunger. According to Ebstein, Griitzner, and Langley, pepsin as such, is not present within the cells, but only a "mother-substance," a pepsinogen. substance (zymogen), which oecurs in the granules of the chief cells (Langley). This zymogen or mother-substance by itself, has no effect upon proteids; but if it be treater with hydrochloric acid or sodium chloride, it is changed into pepsin. Pepsin and pepsinogen may be extracted from the gastric mucous membrane by means of water free from acids.

The pyloric glands secrete pepsin, but no acid.-Klemensiewicz excised in a living dog the pyloric portion of the stomach, and afterwards stitched together the duodenum and the remaining part of the stomach. The excised pyloric part with its vessels intact, he stitched to the abdominal wall, after sewing its lower end. 
The animals experimented on died, at the latest, after six days. The secretion of this part was thin, alkaline, and contained 2 p.c. of solids, including pepsin.

In the frog, the alkaline glands of the œesophagus contain only chief cells which produce pepsin; while the stomach has glands which secrete acid (and perhaps some pepsin), and are lined by parietal cells (Partsch, v. Swiecicki). Amongst fishes, the carps have no fundusglands in the stomach (Luchau).

[The secreting portions of glands of the cardiac sac (crop) of the herring, are lined by a single layer of polygonal cells (W. Stirling).]

The hydrochloric acid is formed, according to Heidenhain, by the parietal cells. It occurs on the free surface of the gastric mucous membrane as well as in the ducts of the gastric glands. The deep parts of the glands are usually alkaline. Free $\mathrm{HCl}$ is detected in human gastric juice, within 45 minutes to 1-2 hours after a moderate meal (von den Velden, and others), and 3-4 hours after a full meal (Edinger); the amount gradually increases during the process of digestion (Kretschy and Uffelmann).

CI. Bernard injected potassium ferrocyanide and afterwards lactate of iron into the veins of a dog. After death, blue colouration occurred only in the upper, acid layers of the mucous membrane. Nevertheless we must assume, that the hydrochloric acid is secreted in the parietal cells of the fundus of the glands, and that it is rapidly carried to the surface along with the pepsin.

Brücke neutralised the surface of the gastric mucous membrane with magnesia usta, chopped up the mucous membrane with water and left it for some time, when the fluid had again an acid reaction.

With regard to the formation of a free acid, the following statements may be noted:-The parietal cells form the hydrochloric acid from the chlorides which the mucous membrane takes up from the blood. According to Voit, the formation of acid ceases if chlorides be withheld from the food. The active agent is lactic acid, which splits up sodium chloride and forms free $\mathrm{HCl}$ (Maly). The base set free is excreted by the urine, rendering it at the same time less acid (Jones, Maly). The formation of acid is arrested during hunger. According to H. Schulz, watery solutions of alkaline and earthy chlorides are decomposed, even at a low temperature, by $\mathrm{CO}_{2}$, free hydrochloric acid being formed.

[A solution of sulphate of soda, not sufficiently strong to cause inflammatory redness of the gastric mucous membrane, yet concentrated enough to excite secretion, canses, when injected into the empty stomach, the pouring ont of an alkaline, not an acid secretion (Matthew Hay).]

Secretion.-When the stomach is empty, there is no secretion of gastric juice; this occurs only after appropriate (mechanical, thermal, or chemical) stimulation. In the normal condition, it takes place immediately on the introduction of food, but also of indigestible substances, 
such as stones. The mucous membrane becomes red, and the circulation more active, so that the venous blood becomes brighter. [That the vagi are concerned in this vascular dilatation, is proved by the fact, that if both nerves be divided during digestion, the gastric mucous membrane becomes pale (Rutherford).] The secretion is probably caused reflexly, and the centre is perhaps in the wall of the stomach itself, (Meissner's plexus in the sub-mucous coat). It is asserted that the idea of food, especially during hunger, excites secretion. As yet we do not know the effect produced upon the secretion by stimulation or destruction of other nerves-e.g., vagus, sympathetic. [There is no nerve passing to the stomach, whose stimulation causes a secretion of gastric juice, as the chorda tympani does in the submaxillary gland. If the ragi be divided sufficiently low down not to interfere with respiration, the introduction of food still canses a secretion of gastric juice; even if the sympathetic branches be divided at the same time, secretion still goes on (Heidenhain). This experiment points to the existence of local secretory centres in the stomach. But there is evidence to show that there is some connection, perhaps indirect, between the central nervous system and the gastric glands. Richet observed a case of complete occlusion of the osophagus in man, produced by swallowing a caustic alkali. A gastric fistula was made, through which the person could be nourished. On placing sugar or lemon-juice in the person's mouth, Richet observed secretion of gastric juice. In this case, no saliva could be swallowed to excite secretion, so that it must have taken place through some nervous channels. Even the sight or smell of food caused secretion. Emotional states also are known to interfere with gastric digestion.]

Heidenhain isolated a part of the mucous membrane of the fundus so as to form a blind-sac of it, and he found that mechanical stimulation caused merely local secretion. If, however, at the same time, absorption of digested matter also occurred, secretion took place over larger surfaces.

The statement of Schiff, that active gastric juice is secreted only after absorption of the so-called peptogenic substances (especially dextrin), is denied.

Action of Alcohol.-Small doses of alcohol, introduced into the stomach, increase the secretion of gastric juice; large doses arrest it. Artificial digestion is not affected by 10 p.c. of alcohol, is retarded by 20 p.c., and is arrested by stronger doses. Beer and wine hinder digestion, and in an undiluted form they interfere with artificial digestion (Bucliner).

The gastric juice, which passes into the duodenum after gastric digestion is completed, is neutralised by the alkali of the intestinal mucous membrane and the pancreatic juice. Part of the pepsin is re-absorbed as such, and is found in traces in the urine and musclejuice (Brücke). 
If the gastric juice be completely discharged externally through a gastric fistula, the alkalinity of the intestine is so strong, that the urine becomes alkaline (Maly).

The acid gastric juice of the new-born child is already fairly active; casein is most easily digested by it, then fibrin and the other proteids (Zweifel). When the amount of acid is too great in the stomach of sucklings, large firm indigestible masses of casein are apt to be formed (Simon, Biedert-see Millk). This occurs more especially after the use of cow's milk.

\section{Methods of obtaining Gastric Juice.}

Historical.-Spallanzani caused starviug animals to swallow small pieces of sponge, enclosed in perforated lead capsules, and after a time, when the sponges had become saturated with gastric juice, he removed them from the stomach. To avoid the admixture with saliva, the sponges are best introduced throngh an opening in the cesophagus (Manassein). Starving animals were forced to swallow small stones, which excited the secretion of gastric juice. After a time, the animals were killed, and the juice collected.

Dr. Beaumont (1S25), an American physician, was the first to obtain human gastric juice, from a Canadian named Alexis St. Martin, who was injured by a gunshot wound, whereby a permanent gastric fistula was established. Various substances were introduced through the external opening, which was partially covered with a fold of skin, and the time required for their solution was noted. Bassow (1842,) Blondlot (1843), and Bardeleben (1849) were thereby led to make artificial gastric fistulæ.

Gastric Fistula.-The anterior abdominal wall is opened by a medium incision just below the ensiform cartilage, the stomach is exposed, and its anterior wall opened and afterwards stitched to the margins of the abdominal walls. A strong cannula is placed in the fistula thus formed. A silver cannula about an inch wide, and with a flange, is introduced into the stomach, so that the flange lies in contact with the gastric mucous membrane. The inner surface of the tube of the cannula is provided with a screw into which a similar cannula is screwed, and its flange comes in contact with the abdominal wall. When the two are placed together they have the form of $T_{\bar{a}}^{b}$, where $a$ passes into $b$. [When the two parts of the cannula are screwed together, the flanges keep the abdominal walls and gastric walls in contact, until they become united organically.] As a rule, the tube is kept corked. If the ducts of the salivary glands be tied, a perfectly uncomplicated object for investigation is obtained.

According to Leube, dilute human gastric juice may be obtained by means of a syphon-like tube introduced into the stomach. Water is introduced first, and after a time it is withdrawn.

Artificial Gastric Juice.-An important advance was made when Eberle (1834) prepared " artificial gastric juice," by extracting the pepsin from the gastric nucous membrane with dilute hydrochloric acid. A certain degree of concentration, however, is required (Schwann). Four litres of a watery solution of $0 \cdot 5-1 \cdot 0-1 \cdot 7$ of pure hydrochloric acid per 1000 (Briicke) are sufficient to extract the chopped-up mucous membrane of the pig's stomach. Half a litre is infused with the stomach and renewed every six hours. The collected fluid is afterwards filtered (Hoppe-Seyler.) The substance to be digested is placed in this fluid, and the whole is kept at the temperature of the body, but it is necessary to add a little $\mathrm{HCl}$ from time to time (Schwann). The $\mathrm{HCl}$ may be replaced by ten times its volume of lactic acid (Lehmann) and also by nitric acid; while oxalic, sulphuric, phosphoric, acetic, formic, succinic, tartaric, and citric acid are much less active; butyric and salicylic acids are inactive. 
Von Wittich's Glycerine Method.-(a) Glycerine extracts pepsin in a very pure form. The mucous membrane is rubbed up with glass until it forms a pulp, mixed with glycerine, and allowed to stand for eight days. The fluid is presser through clotl, and the filtrate mixed with alcohol, thus precipitating the pepsin, which is washed with alcohol and afterwards dissolved in the dilute $\mathrm{HCl}$, to form an artificial digestive fluid. [The addition of a few drops of the glycerine extract to dilute $\mathrm{HCl}$, is sufficient for experiments on artificial digestion.]

(b.) Or the mucous membrane may be placed for 24 hours in alcohol, and afterwards dried and extracted for $\mathrm{S}$ days with glycerine.

(c.) Wm. Roberts has userl other agents for extracting enzyms (p. 295).

Preparation of pure pepsin.-Bricke pours on the pounded mucous membrane of the pig's stomach a 5 per cent. solution of phosphoric acid, and afterwards adds lime water until the acid reaction is scarcely distinguishable. A copious precipitate, which carries the pepsin with it, is produced. This precipitate is collected on cloth, repeatedly washed with water, and afterwards dissolved in very dilute $\mathrm{HCl}$. A copious precipitation is caused in this fluid, by gradually adding to it a mixture of cholesterin in four parts of alcohol and one of ether. The cholesterin-pulp is collected on a filter, washed witl water containing acetic acid, and afterwards with pure water. The cholesterin-pulp is placed in ether to dissolve the cholesterin, and the ether is then removed. The small watery deposit contains the pepsin in solution.

Properties.-Pepsin so prepared is a colloid substance; it does not react like albumin with the following tests, viz.:-it does not give the xanthoprotein reaction (p. 333), is not precipitated by acetic acid and potassium ferrocyanide, nor by tannic acid, mercuric chloride, silver nitrate, or indine. In other respects it belongs to the group of albumins. It is rendered inactive in an acid fluid by heating it to $55^{\circ}-60^{\circ} \mathrm{C}$. (Ad. Mayer).

\section{Process of Gastric Digestion.}

Chyme. - The finely divided mixture of food and gastric juice is called chyme. The gastric juice acts upon certain constituents of this chyme.

\section{I.-Action on Proteids.}

Pepsin and the dilute hydrochloric acid, at the temperature of the body, transform proteids into a solulle form, to which Lehmann (1850) gave the name of "Peptone." During this change, they are first transformed into a substance which has the characters of syntonin (Mulder). Syntonin is an acid-albumin or albuminate; when neutralised by an alkali [e.g., sodium carbonate], the albuminate is again precipitated. An intermediate product is formed, a body which, as it were, stands midway between albumin and peptone. This is called propeptone (Schmidt-Mülheim), and is identical with Kühne's hemialbuminose and Meissner's parapeptone. It is not coagulated by heat, but is precipitated. 
by concentrated solution of common salt. It is soluble in water in the presence of weak acids and alkalies. It is precipitated by nitric acid and adheres firmly to the walls of the reagent glass; it dissolves in nitric acid with the aid of heat, giving an intense yellow colour, and is again precipitated in the cold (E. Salkowski). The compound of nitric acid with propeptone is of the nature of a salt, and it is deposited in the form of sphæroids.

By the continued action of the gastric juice, the propeptone passes into a true soluble peptone. The unchanged albumin behaves like an anhydride with respect to the peptone. The formation of peptone is due to the taking up of a molecule of water, under the influence of the hydrolytic ferment pepsin, and the action takes place most readily at the temperature of the body. Gelatin is changed into a gelatin-peptone. The greater the amount of pepsin (within certain limits), the more rapidly does the solution take place. The pepsin suffers scarcely any change, and if care be taken to renew the hydrochloric acid so as to keep it at a uniform amount, the pepsin can dissolve new quantities of albumin. Still, it seems that some pepsin is used up in the process of digestion (Grützner). Proteids are introduced into the stomach either in a solid (coagulated) or fluid condition. Casein alone of the fluid forms is precipitated or coagulated, and afterwards dissolved. The non-coagulated proteids are transformed into syntonin, without being previously coagulated, and are then changed into propeptone and directly peptonised, i.e., actually dissolved.

When albumin is digested by pepsin at the temperature of the body, a not inconsiderable amount of heat disappears, as can be proved by calorimetric experiment (Maly). Hence, the temperature of the chyme in the stomach falls $0.2^{\circ}-0.6^{\circ} \mathrm{C}$ in $2-3$ hours (v. Vintschgau and Dietl).

Coagulated albumin may be regarded as the anhydride of the fluid form, and the latter again as the anhydride of peptone. The peptones, therefore, represent the highest degree of hydration of the proteids.

Hence, peptones may be formed fiom proteids by those reagents which usually cause hydration, viz., treatment with strong acids, (from fibrin, with $0.2 \mathrm{HCl}-$ v. Wittich), caustic alkalies, putrefactive, and various other ferments and ozone (Gorup-Besanez).

The anhydride proteid has been prepared from the hydrated form. Henniger and Hofmeister, by boiling pure peptone with dehydrating substances (anhydrous acetic acid at $80^{\circ} \mathrm{C}$.), have succeeded in decomposing it into a body resembling syntonin.

Properties of Peptones:-(1) They are completely soluble in water. (2) They diffuse very easily through membranes (Funke), and are twelve times as diffusible as fluid albumin; the fibrin-peptone is said 
to crystallise (Drechsel). (3) They filter quite easily through the pores of animal membranes (Acker). ( 4 ) They are not precipitated by boiling nitric acid, acetic acid and potassium ferrocyanide, weak alcohol, or metaphosphoric acid. (5) They are precipitated from neutral or feebly acid solutions by mercuric chloride, mercuric nitrate [Millon's reagent], silver nitrate, basic lead acctate, potassio-mercuric iodide, tannic acid, picric acid, bile acids, strong alcohol, phosphoro-wolframic acid, and phosphoro-molybdic acid (Brücke).

(6) With Millon's reagent they react like proteids, and give a red colour, and with nitric acid give the yellow xantho-protein reaction. (7) With caustic potash or soda and a small quantity of cupric sulphate, they give a beautiful purplish-red colour (Biuret-reaction). (8) They rotate the plane of polarised light to the left.

The biuret-reaction is obtained with propeptone, as well as with a form of albumin, which is formed during artificial digestion and is soluble in alcohol. It is called Alkophyr by Briicke.

[Darby's fluid meat gives all the above reactions, and is very useful for studying the tests for peptones.]

Preparation.-Pure peptones are prepared by taking fluid which contains them and neutralising it with barium carbonate, evaporating upon a water-bath, and filtering. The barium is removed from the filtrate by the careful addition of sulphuric acid, and subsequent filtration (Hoppe-Seyler). Brieger extracted from gastric peptones by anylic alcohol a peptone-free poison, with actions like those of curara. It belongs to the group of ptomaines-i.e., alkaloids obtained from dead bodies.

Peptones are undoubtedly those modifications of albumin or proteids which, after their absorption from the intestinal canal into the blood, are destined to be used to make good the proteids used up in the human organism. By giving peptones (instead of albumin) as food, life can not only be maintained, but there may eren be an increase of the body-weight (Plósz and Maly, Adamkiewicz).

The N-equilibrium in the metabolism of the body may be kept up by administering 1.11 grammes of peptones, artificially prepared from flesh, per kilo. of the body-weight (Catillon). After being absorbed into the blood-stream, peptones are retransformed, first into propeptone, and then into serum-albumin.

Conditions Affecting Gastric Digestion.-The presence of already-formed peptones interferes with the action of the gastric juice, in so far as the greater. concentration of the fluid interferes with and limits the mobility of the fluid particles (Hoppe-Seyler). Boiling concentrated acids, alum, and tannic acid, alkalinity of the gastric juice (e.g., by the admixture of much saliva) abolish the action. The salts of the heavy metals, which cause precipitates with pepsin, peptone, and mucin, interfere with gastric digestion, and so do concentrated solutions of alkaliue salts, common salt, magnesium and sodium sulphates. Alcohol precipitates the pepsin, but by the subsequent addition of water it is rodissolved, so that 
digestion goes on as before. Any means that prevent the proteid bodies from sweilling up, as by binding them firmly, impede digestion. Slightly over half a pint of cold water does not seem to disturb healthy digestion, but it does so in cases of disease of the stomach. Copious draughts of water and violent muscular exercise, disturb digestion; while warm clothing, especially over the pit of the stomach, aids it. Menstruation retards gastric digestion.

[The action of gastric juice on proteids may be observed outside the body, and we can prove, as is shown in the following table, after Rutherford, that pepsin and an acid-e.g., hydrochloric, along. with water-are essential to the formation of gastric peptones:-

\begin{tabular}{|l|l|l|}
\hline \multicolumn{1}{|c|}{ Beaker A. } & \multicolumn{1}{|c|}{ Beaker B. } & \multicolumn{1}{c|}{ Beaker C. } \\
\hline $\begin{array}{l}\text { Water. } \\
\text { Pepsin, 0.3 per cent. }\end{array}$ & $\begin{array}{l}\text { Water. } \\
\text { HCl, 0.2 per cent. } \\
\text { Fibrin. }\end{array}$ & $\begin{array}{l}\text { Water. } \\
\text { Pepsin, 0.3 per cent. } \\
\text { HCl, } 0 \cdot 2,, \\
\text { Fibrin. }\end{array}$ \\
\hline Unchanged. & $\begin{array}{r}\text { Keep all in water-bath } \\
\text { at } 38^{\circ} \mathrm{C} .\end{array}$ & \\
\hline $\begin{array}{c}\text { Fibrin swells up, be- } \\
\text { comes clear, and is } \\
\text { changed into acid- } \\
\text { albumin or syntonin. }\end{array}$ & $\begin{array}{l}\text { Fibrin ultimately } \\
\text { changed into } \\
\text { peptone. }\end{array}$ \\
\hline
\end{tabular}

The fibrin is obtained by beating blood, and afterwards washing and boiling it to destroy any traces of pepsin. The fibrin may be coloured with carmine, and from the rapidity with which the fibrin is dissolved -i.e., the depth of the colour of the fluid-we may estimate the digestive power of the gastric juice. Similar experiments may be made with unboiled white of egg, mixed with nine volumes of water, and filtered through muslin.]

[In all animals gastric digestion is essentially an acid digestion, and between the native proteid, fibrin, albumin, or any other form of proteid, and the end-product peptone, there are many intermediate substances and bye-products, whose properties and characters have still to be investigated. If the peptones be decomposed, small quantities of leucin and tyrosin are produced. W. Roberts obtained a bitter substance during gastric digestion.]

\section{Action on other Constituents of Food.}

Milk coagulates when it enters the stomach, owing to the precipitation of the casein, and in doing so, it entangles some of the milk globules. During the process of coagulation, lheat is given off (Mosso, Ad. 
Mayer). The free hydrochloric acid of the gastric juice is itself sufficient to precipitate it; the acid removes from the alkali-albuminate or casein the alkali which keeps it in solution. Hammarsten separated a special ferment from the gastric juice-quite distinct from pepsin-the mill-curdling ferment which, quite iudependently of the acid, precipitates the casein either in neutral or alkaline solutions. It is this ferment or rennet which is used to coagulate casein in the making of cheese. [Rennet is an infusion of the fourth stomach of the calf in brine.]

One part of the renuet-ferment can precipitate 800,000 parts of casein. When casein coagulates, two new proteids seem to be formed-the coagulated proteid which constitutes cheese, and a body resembling peptone dissolved in the whey. The addition of calcium chloride accelerated, while water retarded the coagulation (Hammarsten). - See Milk.

Casein is first precipitated in the stomach, then a body like syntonin is formed, and finally peptone. During the process, a substance containing phosphorus and resembling nuclein appears (Lubavin).

There is a "lactic acid ferment" (Hammarsten) also present, which changes milk-sugar into lactic acid. Part of the milk-sugar is changed in the stomach and intestine into grape-sugar.

Action on Carbohydrates.-Gastric juice does not act as a solvent of sturch, inulin, or gums. Cane-sugar is slowly changed into grape-sugar (Bouchardat and Sandras, 1845, Lehmann). According to Uffelmann, the gastric mucus, and according to Leube, the gastric acids are the chief agents in this process. [Matthew Hay has failed to find any organic ferment in the stomach capable of digesting sugar.] During the digestion of true cartilage, there is formed a chondrin-peptone, and a body which gives the sugar reaction with Trommer's test. Perfectly pure elastin yields an elastin-peptone, similar to albumin-peptone, and hemi-elastin similar to hemi-albuminose (Horbaczervski).

Fats formerly were stated not to be acted on, but the recent researches of Cash and Ogata show that a small part of the fats is broken up into glycerine and fatty acids.

[We still require further observations on the gastric digestion of fats. Richet observed in his case of fistula (p. 329), that fatty matters remained a long time in the stomach, and Ludwig found the same result in the dog. In some dyspeptics, rancid eructations often take place towards the end of gastric digestion. W. Roberts suggests that there may be some slight decomposition of neutral fats and liberation of fatty acids. In this connection, it is important to remember that fatty acids are liberated from nentral fats by bacteroid ferments (zyniophytes).]

\section{Action of Gastric Juice on the various Tissues,}

(1.) The gelatin-yielding substance (collagen) of all the connective-tissues (connective-tissue, white fibro-cartilage, and the matrix of bone), as well as glutin, 
are dissolved and peptonised by the gastric juice (Uffelmann). (2.) The structureless membranes (membranæ propriæ) of glands, sarcolemma, Schwann's sheath of nerve-fibres, capsule of the lens, the elastic laminæ of the cornea, the membranes of fat cells are dissolved, but the true elastic (fenestrated) membranes and fibres are not affected. (3.) The striped-muscular substance, after solution of the sarcolemma, breaks up transversely into discs, and, like non-striped muscle, is dissolved and forms a true soluble peptone, but parts of the muscle always pass into the intestine. (4.) The albuminous constituents of the soft cellular elements of glands, stratified epithelium, endothelium, lymph-cells, form peptones, but the nuclein of the nuclei does not seem to be dissolved. (5.) The horny parts of the epidermis, nails, hair, as well as chitin, silk, conchiolin, and spongin of the lower animals are indigestible, and so are amyloid-substance and wax. (6.) The red blood-corpuscles are dissolved, the hæmoglobin decomposed into hæmatin and a globulin-like substance; the latter is peptonised, while the former remains unchanged, and is partly absorbed and transformed into bile-pigment. Fibrin is easily dissolved to form propeptone and fibrin-peptone. (7.) Mucin, which is also secreted by the goblet cells of the stomach: passes through the intestines unchanged. (8.) Vegetable fats are not affected by the gastric juice; these cells yield their protoplasmic contents to form peptones, while the cellulose of the cellwall, in the case of man at least, remains undigested. During putrefaction in the intestine, some cellulose seems to be transformed into sugar.

Why the Stomach does not digest itself.-That the stomach can digest living things is shown by the following facts:-The limb of a living frog was introduced through a gastric fistula into the stomach of a dog (Cl. Bernard)-the ear of a rabbit (Pavy) was also introduced--aud both were partly digested. The margins of a gastric ulcer and of gastric fistulæ in man are attacked by the gastric juice. John Hunter (1772) discussed the question as to why the stomach does not digest itself. Not unfrequently after death the posterior wall of the stomach is found digested, [more especially if the person die after a full meal and the body be kept in a warm place, whereby the contents of the stomach may escape into the peritoneum. Cl. Bernard showed, that if a rabbit be killed and placed in an oven at the temperature of the body, the walls of the stomach are attacked by its own gastric juice. Fishes also are frequently found with their stomach partially digested after death]. It would seem, therefore, that so long as the circulation continues, the tissues are protected from the action of the acid by the alkaline blood; this action cannot take place if the reaction be alkaline (Pavy). Ligature of the arteries to the stomach, according to Pavy, causes digestive softening of the gastric mucous membrane. The thick layer of mucus may also aid in protecting the stomach from the action of its own gastric juice (Cl. Bernard).

\section{Gases in the Stomach.}

The stomach always contains a certain quantity of gases, which are derived partly from the gases swallowed with the saliva, partly from gases which pass backwards from the duodenum, and partly from air swallowed directly.

If the larynx and hyoid bone (p. 311) are suddenly and forcibly raised upwards and forwards, there passes into the space behind the larynx a considerable amount of air, which, on the latter regaining its position, is swallowed, owing to the peristalsis of the cesophagus. We can feel the passage of such a mass of air as it passes along the esophagus. In this way a considerable volume of air may be swallowed. 
The air in the stomach is constantly undergoing changes, whereby its $\mathrm{O}$ is absorbed by the blood, and for 1 vol. of $\mathrm{O}$ absorbed 2 vols. of $\mathrm{CO}_{2}$ are returned to the stomach from the blood. Hence, the amount of $\mathrm{O}$ in the stomach is very small, the $\mathrm{CO}_{2}$ very considerable (Planer).

Gases in the Stomach-Vol. per cent. (Planer).

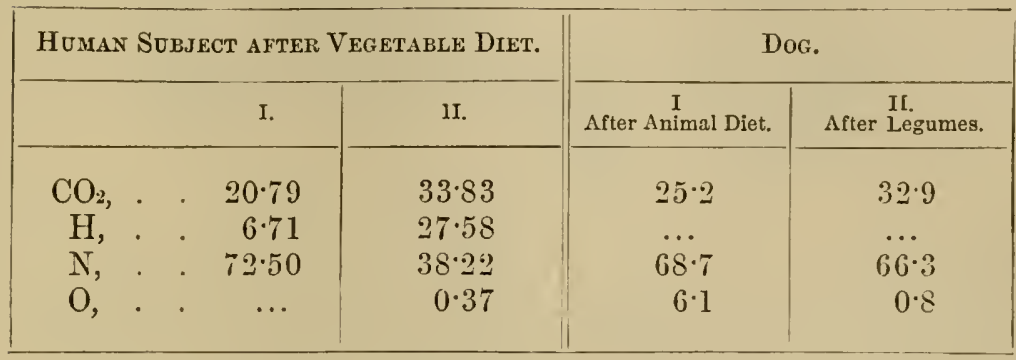

A part of the $\mathrm{CO}_{2}$ is set free by the acid of the stomach from the saliva, which contains much $\mathrm{CO}_{2}$ (p. 292). The $\mathrm{N}$ acts as an indifferent substance.

Abnormal development of gases in persons suffering from gastric catarrh, only occurs when the gastric contents are neutral in reaction; during the butyric acid fermentation $\mathrm{H}$ and $\mathrm{CO}_{2}$ are formed, while the acetic-acid and lactic-acid fermentations do not cause the formation of gases. Narsh gas $\left(\mathrm{CH}_{4}\right)$ has also been found, but it must come from the intestine, as it can only be formed when no 0 is present (Intestinal Guses).

\section{Structure of the Pancreas.}

The pancreas is built on the type of compound tubular or acinotubular glands, and in its general arrangement into lobes, lobules and system of ducts and acini, it corresponds exactly to the true salivary glands. The epitheliun lining the ducts is not at all, or only faintly, striated. The acini are tubular or flaskshaped, and often convoluted. They consist of a membrana propria, resembling that of the salivary-glands, lined by a single layer of somewhat cylindrical cells, with a more or less conical apex towards the very narrow lumen of the acini. [As in the salivary glands, there is a narrow intermediary part of the ducts opening into the acini, and lined by flattened epithelium]. The cells lining the acini consist of two zones (Fig. 139) :-

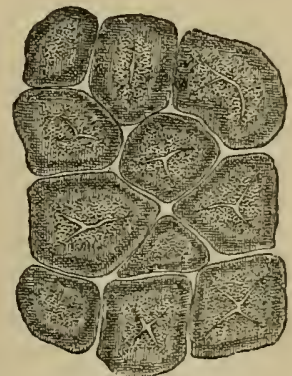

Fig. 139.

Section of the tubes of the pancreas in the fresh condition.

(1.) The smaller purietal layer (outer) is transparent, homogeneous, sometimes faintly striated, and readily stained with carmine and $\log$ - 
wood; and (2.) the inner layer (Bernard's granular layer) is strongly granular, and stains but slightly with carmine. It undoubtedly contributes to the secretion by giving off material, the granules being dissolved, and this zone becoming smaller(Heidenhain). The spherical
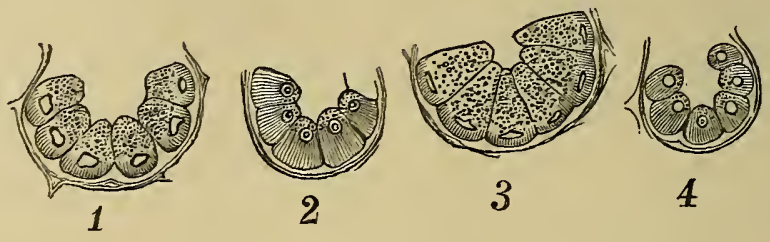

Fig. 140.

Changes of the pancreatic cells in various stages of activity-i, During hunger ; 2 , in the first stage of digestion; 3 , in the second stage; 4 , during paralytic secretion.

nucleus lies between the two zones. [The lumen of the acini is very small, and, according to Langerhans, spindle-shaped or branched cells (centro-acinar cells) lie in it, and send their processes between the secretory cells, thus acting as supporting cells for the elements of the wall of the acini].

During secretion, there is a continuous change in the appearance of the cell-substance; the granules of the inner zone dissolve in the secretion; the homogeneous substance of the outer zone is reversed and transformed into granules, which pass towards the inner zone (Heidenhain, Kühne, and Lea).

Changes in the Cells during Digestion.-During the first stage (6-10 hours) the granular inner zone diminishes in size, the granules disappear, while the striated outer zone increases in size (Fig. 140, 2). In the second stage (10-20 hours) the inner zone is greatly enlarged and granular, while the outer zone is small (Fig. 140, 3). During hunger the outer zone again enlarges (Fig. 140, 1). In a gland where paralytic secretion takes place, the gland is much diminished in size, the cells are shrivelled (Fig. 140,4) and greatly changed (Heidenhain). According to Ogata, some cells actually disappear during secretion. When a coloured injection is forced into the duct under a high pressure, fine intercellular passages between the secreting cells are formed (Saviotti's canals), but they are artificial products.

Duct.-The axially-placed excretory duct consists of an inner thick and an outer loose wall of connective and elastic tissues, lined by a single layer of nonstriated columnar epithelium. Small mucous glands lie in the largest trunks. The connective-tissue separates the gland into lobes and lobules. Non-medullated nerves, with ganglia in their course, pass to the acini, but their mode of termina. tion is unknown. The blood-vessels form a rich capillary plexus round some acini, while round others there are very few. Kühne and Lea found peculiar small cells in groups between the alveoli, and supplied with convoluted capillaries like glomeruli. Their significance is entirely unknown. [They are probably lymphatic in their nature.] The lymphatics resemble those of the salivary glands. 'The pancreas contains water, proteids, ferments, fats, and salts. 
[In making experiments upon the pancreatic secretion, it is important to remember, that the number of pancreatic ducts varies in different animals. In man there is just one duct opening along with the common bile-duct at Vater's ampulla, at the junction of the middle and lower thirds of the duodenum. The rabbit has two ducts, the larger opening separately about 16 inches below the entrance of the bile-duct. The dog and cat have each two ducts opening separately.]

In a gland which has been exposed for some time, leucin, butalanin, tyrosin, often xanthin and guanin are found; lactic and fatty acids seem to be formed from chemical decompositions taking place.

\section{The Pancreatic Juice.}

Method of obtaining the pancreatic juice. Regner de Graaf (1664) tied a cannula in the pancreatic duct of a dog, and collected the juice in a small bag placed in the abdomen. Other experimenters brought the tube through the abdominal wall, and made a temporary tistula, which after some days became inflamed so that the cannula fell out. To make a permanent fistula, a duodenal fistula (like a gastric fistula) is made, and Wirsung's duct is catheretised with a fine tube; or the abdomen is opened ( $\mathrm{dog})$, and the pancreatic duct is pulled forward and stitched to the abdominal wall, with which in certain cases it unites.

The secretion obtained from a permanent fistula is a copious, slightly active, watery secretion containing much sodium carbonate; while the thicl: fluid obtained from the fistula before inflammation sets in acts far more energetically. This thick secretion, which is small in amount, is the normal secretion. The copious watery secretion is perhaps caused by the increased transudation from the dilated bloodvessels (possibly in consequence of the paralysis of the vaso-motor nerves). It is, therefore, in a certain sense, a "paralytic secretion" (p. 2S8). The quantity varies much, according as the fluid is thick or thin.

During digestion, a large dog secretes $1-1.5$ grammes of a thick secretion (Cl. Bernard). Bidder and Schmidt obtained in 24 hours 35-117 grammes of a watery secretion per kilo. of a dog.

When the gland is not secreting, and is at rest, it is soft, and of a pale yellowish-red colour, but during secretion it is red and turgid with blood, owing to the dilatation of the blood-ressels.

The normal secretion is transparent, colourless, odourless, saltish to the taste, and has a strong allaline reaction, owing to the presence of sodium carbonate, so that when an acid is added, $\mathrm{CO}_{2}$ is given off. It contains albumin and alkali-albuminate; like thin white of egg it is sticky, somewhat viscid, flows with difficulty, and is coagulated by heat into a white mass. In the cold, there separates a jelly-like albuminous coagulum. Nitric, hydrochloric, and sulphuric acids cause a precipitate; while the precipitate caused by alcohol is redissolved by water. Cl. Bernard found in the pancreatic juice of a $\operatorname{dog} 8 \cdot 2$ p.c. of 
organic substances, and 0.8 p.c. of ash. The juice (dog) analysed by Carl Schmidt contained in 1000 parts:-

$$
\begin{aligned}
& \text { (Organic, . . } 81 \cdot 84 \text { (Common Salt, . . . 7.36 } \\
& \text { Solids, 90.38 in Inorganic, . . 8.54 Sodic Phosphate, . . 0.45 } \\
& 1,000 \text { parts, . (like those of "Sulphate, . 0 010 } \\
& \text { blood-serum). Soda, . . . . . 0:32 } \\
& \text { Lime, . . . . . 0.22 } \\
& \text { Magnesia, . . . 0.05 } \\
& \text { Potassic Sulphate, . } 0.02 \\
& \text { Ferric Oxide, . . } 0.02
\end{aligned}
$$

The more rapid and more profuse the secretion, the poorer it is in organic substances (Weinmann, Bernstein), while the inorganic remain almost the same; nevertheless, the total quantity of solids is greater than when the quantity secreted is small (Bernstein). Traces of leucin (Radziejewski) and soaps are contained in the fresh juice. [It usually contains few or no structural elements. Any structural elements present in the fresh juice, as well as its proteids, are digested by the peptone-forming ferment of the juice, especially if the juice be kept for some time. If the fresh juice is allowed to stand for some time and then mixed with chlorine water, a red colour is obtained.]

Concretions are rarely formed in the pancreatic ducts; they usually consist of calcic carbonate. Dextrose has been! found in the juice in diabetes, and urea in jaundice.

The statement made by Schiff that the pancreas secretes only after the absorption of dextrin has not been confirmed. The secretory activity of the pancreas is not dependent on the presence of the spleen.

\section{Digestive Action of the Pancreatic Juice.}

The presence of at least four hydrolytic ferments or enzymes makes the pancreatic juice one of the most important digestive fluids in the body.

I. The Diastatic Action (Valentin, 1844) is caused by a diastatic ferment, amylopsin, a substance which seems to be identical with the saliva ferment; but it acts much more energetically than the ptyalin of saliva, on raw starch as well as upon boiled starch; at the temperature of the body the change is effected almost at once, while it takes place more slowly at a low temperature. Glycogen is changed into dextrin and grape-sugar, and achroodextrin (Brücke's) into sugar. Even cellulose is said to be dissolved (Schmulewitsch), and gum changed into sugar by it (v. Voit).

According to v. Mering and Musculus, the starch (as in the case of the saliva p. 294) is changed into maltose, a reducing-dextrin and grape-sugar; so also is glycogen.

Amylopsin slranges achroodextrin into maltose; at $40^{\circ} \mathrm{C}$. maltose is slowly 
changed into dextrose (Brown and Heron), but canc-sugar is not changed into invertin.

The ferment is precipitated by alcohol, while it is extracted by glycerine without undergoing any essential change. All conditions which destroy the diastatic action of saliva (p. 296) similarly affect its action, but the admixture with acid gastric juice (its acid bcing neutralised) or bile does not seem to have any injurious influence. This ferment is absent from the pancreas of new-born children (Korowin). The ferment is isolated by the same methods as obtain for the salivaptyalin (p. 295); but the tryptic ferment is precipitated at the same time. The addition of neutral salts (4 p.c. solution) e.g., potassium nitrate, common salt, ammonium chloride, increases the diastatic action.

II. The Tryptic Action (Cl. Bernard, 1855), or the action on proteids, depends upon the presence of a liydrolytic ferment which Corvisart (1858) called pancreatin, and W. Kühne (1876) termed trypsin. Trypsin acts upon proteids at the temperature of the body, when the reaction is alkaline, and changes them first into a globulin-like body, propeptone (p. 331), and then into a tine peptone, sometimes called tryptone. The proteids do not swell up before they are changed into peptone. When the proteid has been previously swollen up by the action of an acid, or when the reaction of the medium is acid, the transformation is interfered with. Gelatin is peptonised by it; but nuclein (Bokay) and hæmoglobin withstand solution (Hoppe-Seyler). Trypsin acts upon the connective-tissues just like pepsin ( $\$ 166$, III.).

When the trypsin is allowed to act upon the peptone formed by its own action, the peptone is partly changed into the amido-acid, leucin, or amido-caproic acid $\left(\mathrm{C}_{6} \mathrm{H}_{13} \mathrm{NO}_{2}\right)$, and tyrosin $\left(\mathrm{C}_{9} \mathrm{H}_{11} \mathrm{NO}_{3}\right)$, which belongs to the aromatic scries (Kühne). Hypoxanthin, xanthin (Salomon) and asparaginic acid $\left(\mathrm{C}_{4} \mathrm{H}_{7} \mathrm{NO}_{4}\right)$, are also formed during the digestion of fibrin and gluten, and so are glutaminic acid $\left(\mathrm{C}_{5} \mathrm{H}_{9} \mathrm{NO}_{4}\right)$, amido-valerianic acid $\left(\mathrm{C}_{5} \mathrm{H}_{11} \mathrm{NO}_{2}\right)$. Gelatin is first changed into a gelatin-peptone, and afterwards is decomposed into glycin and ammonia.

If the action of the pancreatic juice be still further prolonged, especially if the reaction be alkaline, a body with a strong, stinking, disagreeable fæcal odour, indol $\left(\mathrm{C}_{8} \mathrm{H}_{7} \mathrm{~N}\right)$, volatile fatty acids, skatol $\left(\mathrm{C}_{9} \mathrm{H}_{9} \mathrm{~N}\right)$, and phenol $\left(\mathrm{C}_{6} \mathrm{H}_{6} \mathrm{O}\right)$ are formed, while, at the same time, $\mathrm{HCO}_{2} \mathrm{H}_{2} \mathrm{SCH}_{4}$ and $\mathrm{N}$ are given off. The formation of indol and the other substances just mentioned depends upon putrefaction ( $\$ 184$, III.) Their formation is prevented by the addition of salicylic acid or thymol, which kills the organisms upon which putrefaction depends (Hüfner, Kühne).

[If some fibrin be placed in pancreatic juice, or in a 1 per cent. solution of sodium carbonate containing the ferment trypsin, peptones are rapidly formed. When we compare gastric with pancreatic digestion, we find that there are marked differences. The fibrin in pancreatic digestion is eroded, or eaten away, and never swells up. The process 
takes place in an alkaline medium, and never in an acid one. In fact, a 1 per cent. solution of sodium carbonate seems to play the same part in assisting trypsin as a $\cdot 2$ per cent. solution of $\mathrm{HCl}$ does for pepsin in gastric digestion. In gastric digestion there is a by-product, syntonin, formed in addition to the true peptones. In pancreatic digestion a body resembling alkali-albumin, which passes into a globulin-like body, and ultimately into a tryptic peptone, is formed. Of the peptones so formed, one is called anti-peptone, and it is not further changed, but part of the proteid is changed in a by-product, hemi-peptone. This body, when acted upon, yields leucin and tyrosin. When putrefaction takes place, the bodies above-mentioned are also formed. We might represent the action of trypsin thus :-

Proteid + trypsin +1 per cent. sodium carbonate, kept at $38^{\circ} \mathrm{C}=$ formation of a globulin-like body, and then anti-peptone and hemipeptone are formed.

\begin{tabular}{|c|c|c|}
\hline \multirow[t]{3}{*}{ ANTI-PEPTONE } & \multicolumn{2}{|c|}{ HEMI-PEPTONE } \\
\hline & yields & yields \\
\hline & $\begin{array}{l}\text { Normal Digestive } \\
\text { Products. }\end{array}$ & $\begin{array}{l}\text { Putrefactive } \\
\text { Products. }\end{array}$ \\
\hline $\begin{array}{l}\text { Undergoes } \\
\text { no further } \\
\text { change. }\end{array}$ & $\begin{array}{l}\text { Leucin, } \\
\text { Tyrosin, } \\
\text { Hypoxanthin, } \\
\text { Asparaginic Acid. }\end{array}$ & $\begin{array}{l}\text { Indol, } \\
\text { Skatol, } \\
\text { Phenol, } \\
\text { Volatile Fatty Acids, } \\
\mathrm{HCO}_{2} \mathrm{H}_{2} \mathrm{~S} \text {, } \\
\mathrm{CH}_{4} \mathrm{~N} \text {. }\end{array}$ \\
\hline
\end{tabular}

It seems that trypsin in pure water can act slowly upon fibrin to produce peptone. Pepsin cannot do this without the aid of an acid.]

When proteids are boiled for a long time with dilute $\mathrm{H}_{2} \mathrm{SO}_{4}$, we obtain peptone, then leucin and tyrosin (Kühne); gelatin yields glycin. Hypoxanthin and xanthin are obtained in the same way by similarly boiling fibrin, and the former may even be obtained by boiling fibrin with water (Chittenden).

It is very remarkable that the juice of the green fruit of the papaya tree (Carica papaya) possesses digestive properties (Roy, Wittmack), and the action is due to an albuminous peptonising ferment, closely related to trypsin, and called caricin or papain. The milky juice of the fig-tree has a similar action.

According to Gorup-Besanez, sprouting malt, vetch, hop, hemp during sprouting, and the receptacle of the artichoke contain a peptonising ferment.

Leucin, tyrosin, glutaminic and asparaginic acids, and xanthin are formed in the seeds of some plants; hence we may assume that the processes of decomposition in some seeds are closely allied to the fermentative actions that occur in the intestine (Salomon).

Origin of Trypsin.-It is formed within the pancreas from a "mothersubstance" or zymogen (Heidenhain), which takes up oxygen. The 
zymogen is found in small amount, 6 to 10 hours after a meal, in the inner zone of the secretory cells, but, after $\mathbf{1 6}$ hours, it is very abundant in the inner zone of the cells. It is soluble in water and glycerine. Trypsin is formed in the watery solution from the zymogen, and the same result occurs when the pancreas is chopped up and treated with strong alcohol (W. Kühne). The addition of sodium chloride, carbonate, and glycocholate, favours the activity of the tryptic ferment (Heidenhain).

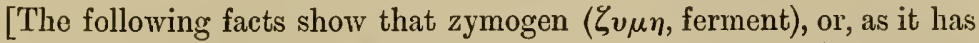
been called, trypsinogen, is the precursor of trypsin, that it exists in the gland-cells, and requires to be acted upon before trypsin is formed. If a glycerine extract be made of a pancreas taken from an animal just killed, and if another extract be made from a pancreas which has been kept for twenty-four hours, it will be found that an alkaline solution of the former has practically no effect on fibrin, while the latter is powerfully proteolytic. If a fresh and still warm pancreas be rubbed up with an equal volume of a 1 per cent. solution of acetic acid, and then extracted with glycerine, a powerfully proteolytic extract is at once obtained. Trypsin is formed from zymogen by the action of acetic acid (Hcidenhain). There is reason to believe that trypsin is formed from zymogen by oxidation, and that the former loses its proteolytic power after removal of its oxygen. The amount of zymogen present in the gland-cells seems to depend upon the number and size of the granules present in the inner granular zone of the secretory cells.]

Trypsin is never absent from the pancreas of new-born children (Zweifel), and it may be extracted by water, which, however, also dissolves the albumin. Kühne has carefully separated the albumin and obtained the ferment in a pure state. It is soluble in water, insoluble in alcohol. Pepsin and hydrochloric acid together act upon trypsin and destroy it; hence it is not advisable to administer trypsin by the mouth, as it would be destroyed in the stomach (Ewald, Mays).

III. The action on neutral fats is twofold:-(1) It acts upon fats so as to form a fine permanent emulsion (Eberle). (2) It causes fats to take up a molecule of water and split into glycerine and fatty acids:-

$$
\begin{aligned}
& \text { Tristearin. Water. Glycerine. Stearic Acid. } \\
& \left(\mathrm{C}_{57} \mathrm{H}_{110} \mathrm{O}_{6}\right)+3\left(\mathrm{H}_{2} \mathrm{O}\right)=\left(\mathrm{C}_{3} \mathrm{H}_{8} \mathrm{O}_{3}\right)+3\left(\mathrm{C}_{18} \mathrm{H}_{36} \mathrm{O}_{2}\right) \text {. }
\end{aligned}
$$

The latter result is due to the action of an easily decomposable fatsplitting ferment (Cl. Bernard), also called steapsin. Lecithin is decomposed by it into glycero-phosphoric acid, neurin and fatty acids (Bokay). After the decomposition is completed, the fatty acids are saponified by the alkali of the pancreatic and intestinal juices.

Emulsification.-The most important change effected on fats in the small intestine, is the production of an emulsion, or their sub-division into exceedingly minute particles. This is necessary in order that the fats may be taken up by 
the lacteals. If the fat to be emulsified contains a free fatty acid, i.e., if it be slightly rancid, and if the fluid with which it is mixed be alkaline, emulsification takes place extremely rapidly (Brücke). A drop of cod-liver oil, which in its unpurified condition always contains fatty acids, on being placed in a drop of 0.3 p.c. solution of soda, instantly gives rise to an emulsion (Gad). The excessively minute oil globules that compose the emulsion are first covered with a layer of soap, which soon dissolves, and in the process small globules are detached from the original oil globules. The fresh surface is again covered by a soap film, and the process is repeated over and over again until an excessively fine emulsion is obtained (G. Quincke). If the fat contain much fatty acid and the solution of soda be more concentrated, "myelin-forms" are obtained similar to those which are formed when fresh nerve-fibres are teased in water (Brücke). Animal oils emulsionise more readily than vegetable oils; castor oil does not emulsionise (Gad).

[Pancreatic Extracts.-The action of the pancreas may be tested by making a watery extract of a perfectly fresh gland. Such an extract always acts upon starch and generally upon fats, but this extract and also the glycerine extract vary in their action upon proteids at different times. If the extract-watery or glycerine -be made from the pancreas of a fasting animal, the tryptic action is slight or absent, but is active if it be prepared from a gland 4 to 10 hours after a meal.]

The pancreas of new-born children contains trypsin and the fat-decomposing ferment, but not the diastatic one (Zweifel). A slight diastatic action is obtained after two months, but the full effect is not obtained until after the first year (Korowin).

IV. According to Kühne and W. Roberts, the pancreas contains a milk-curdling ferment, which may be extracted by means of concentrated solution of common salt.

\section{The Secretion of the Pancreatic Juice.}

As in other glands, we distinguish a quiescent state, during which the gland is soft and pale, and a state of secretory activity, during which the organ swells up and appears pale red. The latter condition only occurs after a meal, and is caused probably in a reflex way owing to stimulation of the nerves of the stomach and duodenum. Kühne and Lea found that all the lobules of the gland were not active at the same time. The pancreas of the herbivora secretes uninterruptedly [but in the dog, secretion is not constant].

Time of Secretion.-According to Bernstein and Heidenhain, the secretion begins to flow when food is introduced into the stomach, and reaches its maximum 2-3 hours thereafter. The amount falls towards the 5 th or 7 th hour, and rises again (owing to the entrance of the chyme into the duodenum) towards the 9 th and 11 th hour, gradually falling towards the 17th-24th hour, until it ceases completely. When more food is taken the same process is repeated. As a general rule, when the secretion occurs rapidly it contains less solids than when it takes place slowly.

Condition of Blood-vessels.-During secretion, the blood-vessels behave like the blood-vessels of the salivary glands after stimulation of 
the chorda-they dilate, and the venous blood is bright red-thus, it is probable that a similar nervous mechanism exists, [but as yet no such mechanism las been discovered.] The secretion is excreted at a pressure of more than $17 \mathrm{~mm}$. Hg. (rabbit).

Effect of nerves upon the secretion. The nerves arise from the hepatic, splenic, and superior mesenteric plexuses, together with branches from the vagus and sympathetic. The secretion is excited by stimulation of the medulla oblongata (Heidenhain and Landan), as well as by direct stimulation of the gland itself by induction shocks (Kuihne and Lea). The secretion is suppressed by atropin, by producing vomiting (Cl. Bernard), by stimulation of the central end of the vagus (C. Ludwig and Bernstein), as well as by stimulation of other sensory nerves-e.g., the crural and sciatic (Afanassiew and Pawlow). Extirpation of the nerves accompanying the blood-vessels prevents the above-named stimuli from acting. Under these circumstances a thin "paralytic secretion" with feeble digestive powers is formed, but its amount is not influenced by the taking of food (Bernstein).

Extirpation of the gland may be performed (Schiff), or the duct ligatured in animals (Frerichs), withont causing any very great change in their nutrition; the absorption of fat from the intestine does not cease. After the duct is ligatured it may be again restored. Ligature of the duct may cause the formation of cysts in the duct and atrophy of the gland-substance. Pigeons soon die after this operation (Langendorff).

\section{Preparation of Peptonised Food.}

[Peptonised food may be given to patients whose digestion is feeble. Dr. Wm. Roberts, of Manchester, uses various forms of this food. Food may be peptonised either by peptic or tryptic digestion, but the former is not so suitable as the latter, because in peptic digestion the grateful odour and taste of the food are destroyed, while bitter by-products are formed. Hence, Dr. Roberts employs pancreatic digestion, which yields a more palatable and agreeable product. As trypsin is destroyed by gastric digestion, obviously it is useless to give extract of the pancreas to a patient along with his food.

Peptonised Milk.-"A pint of milk is diluted with a quarter of a pint of water and heated to $60^{\circ} \mathrm{C}$. Two or three tea-spoonfuls of Benger's liquor pancreaticus, together with 10 or 20 grains of bicarbonate of soda, are then mixed therewith." Keep the mixture at $38^{\circ} \mathrm{C}$. for about two hours, and then boil it for two or three minutes, which arrests the ferment action.

Peptonised Gruel, prepared from oatmeal, or any farinaceous food, is more agreeable than peptonised milk, as the bitter flavour does not appear to be developed in the pancreatic digestion of vegetable proteids. 
Peptonised Milk-Gruel yielded Roberts the most satisfactory results, as a complete and highly nutritious food for weak digestions. Make a thick gruel from any farinaceous food, e.g., oatmeal, and while still hot add to it an equal volume of cold milk, when the mixture will have a temperature of $52^{\circ} \mathrm{C}$. $\left(125^{\circ} \mathrm{F}\right.$.). To each pint of this mixture, add two or three tea-spoonfuls of liquor pancreaticus and 20 grains of bicarbonate of soda. It is kept warm for two hours under a "cosey." It is then boiled for a few minutes and strained. The bitterness of the digested milk is almost completely covered by the sugar produced during the process (Roberts).

Peptonised soups and beef-tea have also been made and used with success.]

\section{Structure of the Liver.}

The liver, the largest gland in the body, consists of innumerable small lobules or acini, 1-2 millimetres $\left(\frac{1}{24}-\frac{1}{12}\right.$ inch) in diameter. These lobules are visible to the naked eye. All the lobules have the same structure.

1. The Connective-tissue and Capsule.-The liver is covered by a thin fibrous firmly adherent capsule, which has on its free surface a layer of endothelium derived from the peritoneum. The capsule sends fine septa into the organ between the lobules, but it is also continued into the interior at the transverse fissure, where it surrounds the portal vein, hepatic artery, and bile duct, and accompanies these structures as the Capsule of Glisson or interlobular connective-tissue. The spaces in which these three structures lie are known as portal canals. In some animals (pig, camel, polar bear), the lobules are separated from each other by the somewhat lamellated connective-tissue of Glisson's eapsule, but in man this is but slightly developed, so that adjoining lobules are more or less fused. Very delicate connective-tissue, but small in amount, is also found within the lobules (Fleischl, Kupffer). Leucocytes are sometimes found in the tissue of Glisson's capsule.

2. Blood-vessels, - (a.) Branches of the Venous System.-If the vena porta be traced from its entrance into the liver at the portal fissure, it will be found to give off numerous branches lying between the lobules, and ultimately forming small trunks which reach the periphery of the lobules, where they form a rich plexus. These are the interlobular veins (Fig. 141,V.i). From these veins numerous capillaries $(c, c)$ are given off to the entire periphery of the lobule. The capillaries converge towards the centre of the lobule. As they proceed inwards, they form elongated meshes, and between the capillaries lie rows or columns of liver-cells $(d, d)$. The capillaries are relatively wide, and are so disposed as to lie between the edges of the columns of cells, and never between the surfaces of two neighbouring cells. The capillaries converge towards the centre of each lobule, where they join to form one large vein, the intralobular or central vein $(V . c)$, which traverses each lobule, reaches its surface at one point, passes out, and joins similar veins from other lobules to form the sublobular veins $(V . s)$. These in turn unite to form wide veins, the origins of the hepatic veins, which open into the vena cava inferior.

(b.) Branches of the Hepatic Artery. - The branches of the hepatic artery accompany the branches of the portal vein and bile duct in the portal canals between the lobules, and in their course they give off capillaries to supply the walls of the 
portal vein and larger bile ducts. The branches of the hepatic artery anastomose frequently where they lie between the lobules. On reaching the periphery of the lobules, a certain number of capillaries are given off, which penetrate the lobule

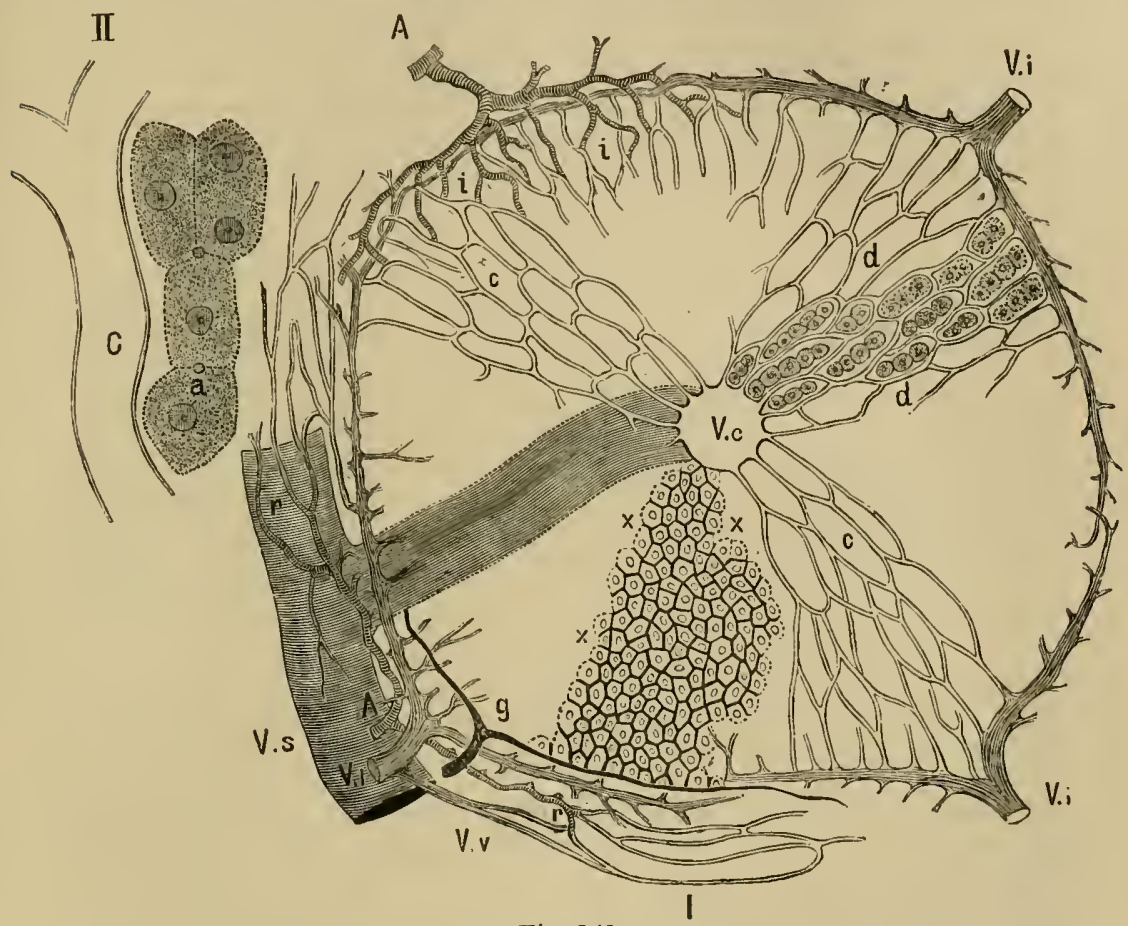

Fig. 141.

I, Scheme of a liver lobule-V.i, V. $i$, interlobular veins (portal); V. $c$, central or intra-lobular vein (hepatic); $c$, $c$, capillaries between both; V. $s$, sublobular vein; V. $v$, vena vascularis; A, A, branches of the hepatic artery, giving branches, $r, r$, to Glisson's capsule and the larger vessels, and ultimately forming the venæ vasculares at $i, i$, opening into the intralobular capillaries; $g$, branches of the bile ducts; $x, x$, intralobular bile capillaries between the liver-cells; $d, d$, position of the liver-cells between the meshes of the blood capillaries. II, Isolated liver-cells $-c$, a blood capillary; $a$, fine bile capillary channel.

and terminate in the capillaries of the portal vein $(i, i)$. Those capillaries, however, which supply the walls of the portal vein and large bile ducts $(r, r)$, terminate in veins which end in the portal vein (V. $v$-Ferrein).

Several branches-capsular-pass to the surface of the liver, where they form a wide-meshed plexus under the peritoneum. The blood is returned by veins which open into branches of the portal vein.

[Pathologists draw a sharp distinction between different zones within a hepatic lobule. Thus, the central area, capillaries, and cells are the zone of the hepatic vein, which is specially liable to cyanotic changes; the area next the periphery of the lobule is the portal vein zone, whose cells under certain circumstances are particularly apt to undergo fatty degeneration; while there is an area lying midway between the two foregoing-the hepatic artery zone-which is specially liable to amyloid or waxy degeneration.] 
3. The Hepatic Cells (Fig. 14I, II, a) are irregular polygonal cells of about $\frac{1}{1000}$ th of an inch $(34-45 \mu)$ in diameter (Fig. 142). The arrangement of the capillaries within a lobule determines the arrangement of the liver-cells. The livercells form anastomosing columns which radiate from the centre to the periphery of each lobule (Fig. 143). [The liver-cells are usually stated to be devoid of an envelope, although Haycraft states that they possess one. They usually contain a single nucleus with one or more nucleoli, but sometimes two nuclei occur. The protoplasm and nucleus of each cell contains a plexus of fibrils just like other epithelial cells. In some animals, globules of oil and pigment grannles are found

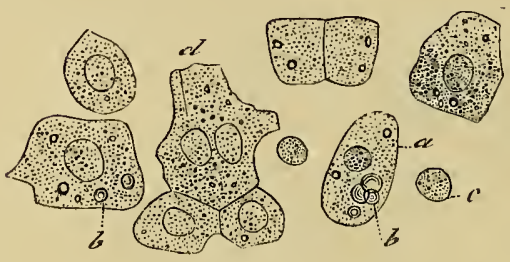

Fig. 142.

Human liver-cells-the cell protoplasm contains biliary colouring matter and oilglobules $b ; d$, has two nuclei.

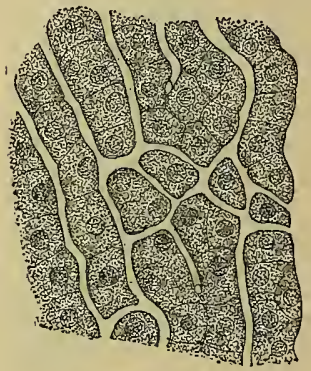

Fig. 143.

Appearance of the livercells after withholding food for 36 honrs.

in the cell protoplasm (Fig. 142).] Each cell is in relation with the wide-meshed blood-capillaries $(d, d)$, and also with the much narrower mesh-work of bile $\operatorname{ducts}\left(\mathrm{I}, x_{\mathbf{s}}\right)$

It is important to observe that the appearance of the cells varies with the period of digestion. During hunger, the liver cells are finely granular and very cloudy (Fig. 143). About 13 hours after a full meal, especially of starchy food, they contain coarse glancing masses of glycogen (Fig. 144, 2). The protoplasm near the surface of the cell is condensed, and a fine net-work stretches towards the centre of the cell, and in it is suspended the nucleus (Kupffer, Heidenhain). [The net-work within the cells is best seen after solution of the glycogen.]

4. The bile ducts. - The finest bile capillaries or canaliculi arise from the centre of the lobule, and indeed throughout the whole lobule, they form a regular anastomosing net-work of very fine tubes or channels. Each cell is surrounded by a polygonal-usually hexagonal-mesh $(x, x)$. The bile capillaries always lie in the middle of the surfaces between two adjoining cells (II, a), where they form actual intercellular passages (Hering). [According to some observers, they are merely excessively narrow channels (1-2 mm. wide) in the cement-substance between the cells, while according to others, they have a distinct delicate wall (Fritsch). The bile capillary net-work is much closer and finer than the blood capillary net-work.

[Thus, there are three net-works within each lobule-(1) a net-work of capillaries; (2) a net-work of hepatic cells; (3) a net-work of bile capillaries.]

Excessively minute intracellular passages are said to pass from the bile capillaries into the interior of the liver-cells, where they communicate with certain small cavities or vacuoles (Asp), Kupffer, Pflüger)-(Fig. 144, 3). As the blood capillaries run along the edges of the liver-cells, and the bile capillaries between the opposed surfaces of adjacent cells, the two systems of canals within the 
lobule are kept separate. Some bile capillaries run along the edges of the liver-cells in the human liver, especially during embryonic life (Zuckerkandl, Toldt).

Towards the peripheral part of the lobule, the bile capillaries are larger, while adjoining channels anastomose, and leave the lobule, when they become interlobular ducts $(g)$, which join with other similar ducts to form larger interlobular bile ducts. These accompany the hepatic artery and portal vein, and leave the liver at the transverse fissure. The finer interlobular ducts frequently anastomose in Glisson's capsule (Asp), possess a structureless basement membrane, and are lined by a single layer of low polyhedral epithelial cells. The larger interlobular ducts have a distinct wall consisting of connective and elastic tissue, mixed with circularly disposed smooth muscular fibres. Capillaries are supplied to the wall, which is lined by a single layer of columnar epithelium. A sub-mucosa occurs only in the largest bile ducts, and in the gallbladder. Smooth muscular fibres, arranged in single bundles, occur in the largest dncts, and as longitudinal and circular layers in the gall-bladder, whose nucous membrane is provided with numer. ous folds and depressions. The epithelium lining the gall-bladder is cylindrical, with a distinct clear disc, and between these cells are goblet cells. Small branched tubular mucous glands occur in the large bile ducts and in the gall-bladder.

Vasc Aberrantia are isolated bile ducts which occur on the surface of the liver, but have no relation to any system of liver lobules. They occur at the sharp margin of the liver, in the region of the inferior vena cava, of the gall-bladder, and of the parts near the portal fissure. It seems that the

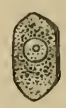

1
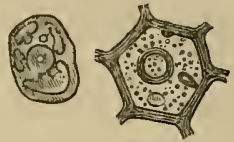

23

Fig. 144.

1, Liver-cell during fasting; 2 , containing masses of gly cogen; 3 , a liver-cell surrounded with bile-channels from which fine twigs proceed into the cell-substance where they end in vacuolelike enlargements. From a rabbit's liver injected with Berlin blue from the bile duct.

liver lobules to which they originally belonged have atrophicd and disappeared (Zuckerkandl and Toldt).

5. The Lymphatics begin as pericapillary tubes around the capillaries within the lobules (MacGillavry). They emerge from the lobule, and run within the walls of the branches of the hepatic and portal veins, and afterwards surround the venous trunks (Fleischl, A. Budge), thus forming the interlobular lymphatics. These unite to form larger trunks, which leave the liver partly at the portal fissure, partly along with the hepatic veins, and partly at different points on the surface of the organ. There is a narrow superficial mesh-work of lymphatics under the peritoneum-sub-peritoneal-which communicates with the thoracic lymphatics through the triangular ligament and suspensorium, while on the under surface, they communicate with the lymphatics of the interlobular connective tissue.

6. The Nerves consist partly of medullated and partly of non-medullated fibres from branches of the sympathetic and left vagus to the hepatic plexus. They accompany the branches of the hepatic artery, and ganglia occur on their branches within the liver. Some of the nerve-fibres are vaso-motor in function, and, according to Pflüger, other nerve-fibres terminate directly in connection with liver-cells, although this observation has still to be confirmed.

Pathological.- The connective tissue between the lobules may undergo great increase in anount, especially in alcohol- and gin-drinkers, and thus the substance of the lobules may be greatly compressed, owing to the cicatricial contraction of the newly-formed connective tissue (Liver Cirrhosis). In such interlobular con. nective tissue, newly-formed bile ducts are found (Cornil, Charcot, and others).

Ligature of the cluctus choleclochus, after a time, causes interstitial inflammation of the liver. In rabbits and guinea-pigs, the liver parenchyma disappears, and its 
place is taken by newly-formed connective tissue and bile ducts (Charcot and Gombault). In all these cases of interstitial inflammation, there is proliferation of the epithelium of the bile ducts (Foà, Salvioli).

\section{Chemical Composition of the Liver-Cells.}

(1.) Proteids.-The fresh soft parenchyma of the liver is alkaline in reaction; after death, coagulation occurs, the cell contents appear turbid, the tissue becomes friable, and gradually an acid reaction is developed. This process closely resembles what occurs in muscle, and is due to the coagulation of a myosin-like body, which is soluble during life, but after death undergoes spontaneous coagulation (Plósz). The liver contains other albuminous bodies ; one coagulating at $45^{\circ} \mathrm{C}$, another at $70^{\circ} \mathrm{C}$, and one which is slightly soluble in dilute acids and alkalies. The cell nuclei contain nuclein (Plósz). The connective tissue yields gelatin.

(2.) Glycogen or Animal Starch-1 $12-2 \cdot 6$ p.c.-is most closely related to inulin, is soluble in water, but diffuses with difficulty, is a true carbohydrate (Cl. Bernard and v. Hensen, 1857), and has the formula $6\left(\mathrm{C}_{6} \mathrm{H}_{10} \mathrm{O}_{5}\right)+\mathrm{H}_{2} \mathrm{O}$ (Külz and Bornträger). It is stored up in the liver-cells (Bock and Hoffmann), in amorphous granules around the nuclei, but it is not uniformly distributed in all parts of the liver (v. Wittich). Like inulin, it gives a deep red colour with solution of iodine in iodide of potassium. It is changed into dextrin and sugar (p. 294) by diastatic ferments, and when boiled with dilute mineral acids, it yields grape-sugar.

Preparation of Glycogen.-Let a rabbit have a hearty meal, and kill it three or four hours thereafter. The liver is removed immediately after death; it is cut into fine pieces, plunged in boiling water and boiled for some time in order to obtain a watery extract of the liver-cells. [It is placed in boiling water to destroy the ferment present in the liver, which would transform the glycogen into grape-sugar.] To the cold filtrate are added alternately dilute hydrochloric acid and potassio-mercuric iodide as long as a precipitate occurs. The albuminates or proteids are precipitated by the iodine compound in the presence of free HCl. It is then filtered, when a clear opalescent fluid, containing the glycogen in solution, is obtained. The glycogen is precipitated from the filtrate, as a white amorphous powder, on adding an excess of 70-80 p.c. alcohol. The precipitate is washed with 60 p.c., and afterwards with 95 p.c. alcohol, then with ether, and lastly, with absolute alcohol; it is dried over sulphuric acid and weighed (Brücke).

Conditions which influence its amount.-If large quantities of starch, milk-, fruit-, or cane-sugar, or glycerine, but not mannite or glycol (Luchsinger), or inosite (Külz), be added to the proteids of the food, the amount of glycogen in the liver is very greatly increased (to 12 p.c. in the fowl), while a purely albuminous or purely fatty diet diminishes it enormously. During hunger, it almost disappears (Pavy and Tscherinoff). The injection of dissolved carbohydrates into a 
mesenteric vein of a starving rabbit causes the liver, previously free from glycogen, to contain glycogen (Naunyn).

During life, under normal conditions, the glycogen in the liver is either not transformed into grape-sugar (Pavy, Ritter, Eulenberg), or, what is more probable, only a very small amount of it is so changed. The normal amount of sugar in blood is $0.5-1$ per 1000 , although the blood of the hepatic vein contains somewhat more. A considerable amount is transformed into sugar only when there is a decided derangement of the hepatic circulation, and in these circumstances, the blood of the hepatic vein contains more sugar. The glycogen undergoes this change very rapidly after death, so that a liver which has been dead for some time always contains more sugar and less glycogen.

The ferment which effects this change can be obtained from the extract of the liver-cells by the same means as are applicable for obtaining other similar ferments, such as ptyalin; but it does not seem to be formed within the liver-cells, but only passes very rapidly from the blood into them. The ferment seems to be rapidly formed when the blood-stream undergoes considerable derangement (Ritter, Schiff). A similar ferment is formed when red blood-corpuscles are dissolved (Tiegel), and, as there is a destruction of red blood-corpuscles taking place continually within the liver, this is one source from which the ferment may be formed, whereby minute quantities of sugar would be continually formed in the liver.

If glycogeu is injected into the blood, achroodextrin appears in the urine, and also hremoglobin, as glycogen dissolves red blood-corpuscles (Böhm, Hoffmanu).

Ligature of the bile duct canses decrease of the glycogen in the liver (v. Wittich); it appears as if, after this operation, the liver loses the property of forming glycogen from the materials supplied to it.

(3.) The following substances have also been found in the liver-cells:Fats in the form of highly refractive granules in the liver-cells, as well as in the bile ducts; sometimes, when the food contains much fat (more abundant in drunkards and the phthisical), olein, palmitin, stearin, volatile fatty acids, and sarcolactic acid are found.

[Fatty granules are of common occurrence within the cells of the liver, and when they do not occur in too great amount, do not seem to interfere very greatly with the functions of the liver-cells. These fatty granules are common in disease, constituting fatty infiltration and degeneration, and in such cases the cells within a lobule of the liver, next the portal vein, are usually most highly charged with the fatty particles. Fatty particles occur if too much fatty food be taken, and they are commonly found in the livers of stall-fed animals, and the well-known pâte-de. foie gras is largely composed of the livers of geese, which have been fed on large amounts of farinaceous food, and which have been subjected to other unfavourable hygienic conditions. Fatty granules are recognised by their highly refractive appearance, by their solubility in ether, and by being blackened by osmic acid.]

There are also found traces of cholesterin, minute quantities of urea, uric acid. 
[Leucin (?guanin), sarkin, xanthin, cystin, and tyrosin occur pathologically in certain diseases where marked chemical decompositions occur.]

4. The inorganic substances found in the human liver are-potassium, sodium, calcium, magnesium, iron, manganese, chlorine, and phosphoric, sulphuric, carbonic, and silicic acids ; while copper, zinc, lead, mercury, and arsenic, are accidentally deposited in the hepatic tissue.

\section{Diabetes Mellitus, or Glycosuria.}

The formation of large quantities of grape-sugar by the liver, and its passage into the blood (p. 62), and from the blood into the urine, are related to the above-mentioned normal conditions. Extirpation of the liver in frogs (Moleschott), or destruction of the hepatic cells as by fatty degeneration from poisoning with phosphorus or arsenic (Salkowski) do not cause this condition. It occurs for several hours, however, after the injury of a certain part-the centre for the hepatic vaso-motor nerves-of the floor of the lower part of the fourth ventricle (Cl. Bernard's piqûre); also after section of the vaso-motor channels in the spinal cord, from above down to the exit of the nerves for the liver, viz., to the lumbar region, and in the frog to the fourth vertebra (Schiff). When the vaso-motor nerves, which proceed from this centre to the liver, are cut or paralysed in any part of their course, mellituria or glycosuria is produced. All the nerve-channels do not run through the spinal cord alone. A number of vaso-motor nerves leave the spinal cord higher up, pass into the sympathetic, and thus reach the liver; so that destruction of the superior (Pavy), as well as of the inferior cervical sympathetic ganglion, and the first thoracic ganglion (Eckhard), of the abdominal ganglia (Klebs, Munk), and often of the splanchnic itself (Hensen, v. Graefe), produces diabetes. The paralysis of the blood-vessels causes the liver to contain much blood, and the intrahepatic blood-stream is slowed. This disturbance of the circulation causes a great accumulation of sugar in the liver, as the blood-ferment has time to act upon the glycogen and transform it into sugar. By stimulation of the sympathetic at the lowest cervical and first thoracic ganglion, the hepatic vessels at the periphery of the liver lobules become contracted and pale (Cyon, Aladoff). It is remarkable that glycosuria when present may be set aside by section of the splanchnic nerves. This is explained by supposing that the enormous dilatation and congestion, or the hyperæmia, of the abdominal blood-vessels thereby produced, renders the liver anæmic.

A number of poisons which paralyse the hepatic vaso-motor nerves produce diabetes in a similar way-curara (when artificial respiration is not maintained), chloroform, ether, chloral, amyl nitrite, carbon disulphide, morphia, mercuric 
chloride, and (?) CO. But congestion of the liver produced in other ways appears to cause diabetes-e.g., after mechanical stimulation of the liver. To this class belongs the injection of dilute saline solutions into the blood (Bock, Hoffmann), whereby either the change in form or the solution of the coloured blood-corpuscles causes the congestion. The circumstance that repeated blood-letting makes the blood richer in sugar may, perhaps, be explained by the slowing of the circulation. [Injection of a solution of a neutral salt into a ligatured loop of the small intestine sometimes causes mellituria (M. Hay).]

Continued stimulation of peripheral nerves may act reflexly upon the centre for the vaso-motor nerves of the lirer. Diabetes has been observed to occur after stimulation of the central end of the vagus $(\mathrm{Cl}$. Bernard, Eckhard, Külz, Lobeck), and also after stimulation of the central end of the depressor nerve (Filehne). Even section and subsequent stimulation of the central end of the sciatic nerve causes diabetes (Schiff, Külz, Böhm and Hoffmann, Froning), and thus is explained the occurrence of diabetes in people who suffer from sciatica.

According to Schiff, the stagnation of blood in other vascular regions of the body may cause the ferment to accumulate in the blood to such an exent that liabetes occurs. The glycosuria that occurs after compression of the aorta or portal vein may perhaps be ascribed to this cause, but perhaps the pressure produced by these procedures may paralyse certain nerves. According to Eckhard, injury to the vermiform process of the cerebellum of the rabbit causes diabetes. In man, affections of the above-named nervous regions cause diabetes.

Theoretical. - In order to explain the more immediate cause of these phenomena several hypotheses have been advanced :-

(a.) The liver glycogen may be transformed unhindered into sugar, as the blood in its passage through the liver deposits or gives up the ferment to the livercells (see above). So that the normal function of the raso-motor system of the liver, and its centre in the floor of the fourth ventricle, may be regarded as, in a certain sense, an "inhibitory system" for the formation of sugar.

(b.) If we assume that under normal conditions, there is continually a small quantity of sugar passing from the liver into the hepatic vein, we might explain the diabetes as due to the disappearance of those decompositions-diminished burning-up of the sugar in the blood-which are coustantly removing the sugar from the blood. In fact, diabetic persons have been found to consume less $O$ ( . Pettenkofer and Voit), and to have an increased formation of urea.

Sources of Glycogen.-The "mothcr-substance" of the glycogen of the liver has been variously stated to be the carbo-hydrates of the food (Pavy), fats (olive oil, Salomon), glycerine (van Deen, Weiss), taurin and glycin (the latter splitting into glycogen and urea-Heynsius and Küthe), the proteids (Cl. Bernard), and gelatin (Salomon). If it is derived from the albumins, it must be formed from a non-nitrogenous derivative thereof.

Effects of Food.-Rabbits whose livers have been rendered free from glycogen by starvation, yield new glycogen from their livers when they are fed with cane-sugar, grape-sugar, maltose, or starch. Forced muscular movements soon make the liver of dogs free from glycogen, and exposure to cold diminishes its amount. Dextrin and 
grape-sugar occur in the dead liver (Limpricht, Külz), but in addition, some glycogen is found for a considerable time after death, in the liver and in the muscles.

Other Situations.-Glycogen is by no means confined to the liver-cells; it occurs during foetal life in all the tissues of the body of the embryo, also in young animals (Kühne), and in the placenta (Bernard). In the adult it occurs in the testicle (Kühne), in the muscles (MacDonnel, O. Nasse), in numerous pathological products, in inflamed lungs (Kühne), and also in the corresponding tissues of the lower animals. [It also occurs in the chorionic villi (Cl. Bernard), in colourless blood-corpuscles, in fresh pus cells which still exhibit amoboid movements, and in fact in all developing animal cells, with amoboid movement; it is a never-failing constituent in cartilage, and in the muscles and liver of invertebrata, such as the oyster (Hoppe-Seyler).]

Persons suffering from diabetes require a large amount of food; they suffer greatly from thirst, and drink much fluid. They exhibit signs of marked emaciation, when the loss of the body is greater than the supply. In severe cases towards death, not unfrequently a peculiar comatose condition-diabetic coma-occurs, when the breath often has the odour of acetone, which is also found in the urine (Petters). But neither acetone nor its precursor, aceto-acetic acid, nor æethyl-diacetic acid, nor the unknown substance, in diabetic urine, which gives the red colour with ferric chloride, is the cause of the coma (Frerichs and Brieger). The urinary tubules often show the signs of coagulationnecrosis, which is recognised by a clear swollen-up condition of the dead cells (Ebstein). As yet there is no satisfactory explanation of those rarer cases of "acetonæmia" without diabetes (Kanlecti, Cantini, v. Jacksch).

\section{The Functions of the Liver.}

[We have still much to learn regarding the functions of the liver, but it has two distinct functions-one obvious, the other not. (1) The liver secretes bile, which is formed by the hepatic cells, and leaves the organ by the bile-ducts, to be poured by them into the duodenum. (2) But the liver-cells also form glycogen, which does not pass into the ducts, but in some, altered and diffusible form passes into the bloodstream, aud leaves the liver by the hepatic veins. Hence, the study of the liver materially influences our conception of a secreting organ. In this case, we have the products of its secretory activity leaving it by two different channels-the one by the ducts, and the other by the bloodstream. The relation of the liver to the blood-corpuscles has already been mentioned (pp. 13-17).]

\section{Constituents of the Bile.}

Bile is a yellowish brown or dark green-coloured transparent fluid, with a sweetish strongly bitter taste, feeble musk-like odour and 
neutral reaction. The specific gravity of human bile from the gallbladder $=1026-1032$, while that from a fistula $=1010-1011$ (Jacobsen). It contains :-

(1.) Mucus, which gives bile its sticky character, and not unfrequently makes it alkaline, is the product of the mucous glands and the goblet-cells of the mucous membrane of the larger bile-ducts. When bile is exposed to the air, the mucus causes it to putrefy rapidly. It is precipitated by acetic acid, or alcohol. [Bile from the gall-bladder, when poured from one vessel into another, shows the presence of mucin in the form of thin threads connecting the fluids in the two vessels. When such bile is treated with alcohol, it no longer exhibits this property, but flows like a non-viscid watery fluid. The bile formed in the ultimate bile-ducts does not seem to contain mucin or mucus, but bile from the gall-bladder always does.]

(2.) The Bile Acids.-Glycocholic and taurocholic acids, so-called conjugate acids, are united with soda (in traces with potash) to form glycocholate and taurocholate of soda, which have a bitter taste. In human bile (as well as in that of birds, many mammals, and amphibians), taurocholic acid is most abundant; in other animals (pig, ox) glycocholic acid is most abundant. These acids rotate the plane of polarised light to the right.

(a.) Glycocholic acid, $\mathrm{C}_{26} \mathrm{H}_{43} \mathrm{NO}_{6}$ (first discovered and described as cholic acid by Gmelin, and called, by Lehmann, glycocholic acid). When boiled with caustic potash, or baryta water, or with dilute mineral acids, it takes up $\mathrm{H}_{2} \mathrm{O}$ (Strecker, 1848), and splits into-

Glycin $(=$ Glycocoll $=$ Gelatin Sugar $=$ Amidoacetic acid $)=\mathrm{C}_{2} \mathrm{H}_{5} \mathrm{NO}_{2}$.

+Cholalic acid (also called Cholic acid). . . $\quad=\mathrm{C}_{24} \mathrm{H}_{40} \mathrm{O}_{5}$. $=$ Glycocholic acid + Water . . $\quad=\mathrm{C}_{26} \mathrm{H}_{43} \mathrm{NO}_{6}+\mathrm{H}_{2} \mathrm{O}$.

(b.) Taurocholic acid, $\mathrm{C}_{26} \mathrm{H}_{45} \mathrm{NSO}_{7}$, when similarly treated, takes up water and splits into-

Taurin (= Amidoæthyl-sulphuric acid) $=\mathrm{C}_{2} \mathrm{H}_{7} \mathrm{NSO}_{3}$.

+ Cholalic acid . . . $\quad=\mathrm{C}_{24} \mathrm{H}_{40} \mathrm{O}_{5}$.

$=$ Taurocholic acid + Water . . $\quad=\mathrm{C}_{26} \mathrm{H}_{45} \mathrm{NSO}_{7}+\mathrm{H}_{2} \mathrm{O}$ (Strecker).

Preparation of the Bile acids.-Bile is evaporated to $\frac{1}{4}$ of its volume, rubbed up into a paste with excess of animal charcoal, and dried at $100^{\circ} \mathrm{C}$. The black mass is extracted with absolute alcohol, which is filtered until it is clear. After a part of the alcohol has been removed by distillation, the bile salts are precipitated in a resinous form, and on the addition of excess of ether, there is formed immediately a crystalline mass of glancing needles (Platner's "crystallised bile"). The alkaline salts of the bile acils are freely soluble in water or alcohol, and insoluble in ether. Neutral lead acetate precipitates the glycocholic acid-as lead glycocholate -from the solution of botll salts; the precipitate is collected on a filter, dissolved in hot alcohol, and the lead is precipitated as lead sulphide by $\mathrm{H}_{2} \mathrm{~S}$; after removal of the lead sulphide, the addition of water precipitates the isolated glycocholic acid. If, after precipitating the lead glycocholate, the filtrate be treated with 
basic leacl acetate, a precipitate of lead taurocholate is formed, from which the acid may be obtained in the same way as described above (Strecker).

When human bile is similarly treated, instead of the "crystallised bile," a resinous non-crystalline precipitate is obtained. Boiling with baryta water isolates the cholalic acid from it, which is obtained from its barium salt by adding hydrochloric acid. When dissolved in ether, it occurs in the form of prismatic crystals if petroleum-ether is added. The anthropocholic acid $\left(\mathrm{C}_{18} \mathrm{H}_{28} \mathrm{O}_{4}-\mathrm{H}\right.$. Bayer), so obtained is not soluble in water, but readily so in alcohol, and rotates the ray of polarised light to the left.

With regard to the decomposition products of the bile acids, glycin, as such, does not occur in the body, but only in the bile in combination with cholalic acid, in urine in combination with benzoic acid, as hippuric acid, and lastly, in gelatin in complex combination.

Cholalic acid rotates the ray of polarised light to the right, and its chemical composition is unknown (perhaps it is to be regarded as benzoic acid, in which a complex of atoms similar to oleic acid is introduced-Hoppe-Seyler). It occurs free only in the intestine, where it is derived from the splitting up of taurocholic acid, and it passes in part into the fæces. It is insoluble in water, soluble in alcohol, but soluble with difficulty in ether, from which it separates in prisms. Its crystalline alkaline salts are readily soluble in water.

Cholalic acid is replaced in the bile of many animals by a nearly related acid, e.g., in pig's bile, by hyo-cholalic acid (Strecker, Gundlach); in the bile of the goose, cheno-cholalic acid is present (Marsson, Otto).

When cholalic acid is boiled with concentrated $\mathrm{HCl}$, or dried at $200^{\circ} \mathrm{C}$, it becomes an anhydride, thus:-

$$
\begin{aligned}
& \text { Cholalic acid }=\mathrm{C}_{24} \mathrm{H}_{40} \mathrm{O}_{5}, \text { produces } \\
& \text { Choloidinic acid }=\mathrm{C}_{24} \mathrm{H}_{38} \mathrm{O}_{4}+\mathrm{H}_{2} \mathrm{O} \text {, and this again yields } \\
& \text { Dyslysin. } \\
& =\mathrm{C}_{24} \mathrm{H}_{36} \mathrm{O}_{3}=\mathrm{H}_{2} \mathrm{O} .
\end{aligned}
$$

(Choloidinic acid is, however, not improbably a mixture of cholalic acid and dyslysin; dyslysin, when fused with caustic potash, is changed into cholalate of potash-Hoppe-Seyler). If anthropocholic acid be heated to $185^{\circ} \mathrm{C}$, it gives up 1 molecule of water, and yields anthropochol-dyslysin (Bayer).

By oxidation cholalic acid yields a tribasic acid, as yet uninvestigated, and a fair amount of oxalic acid, but no fatty acids (Clève).

Pettenkofer's Test._The bile acids, cholalic acids, and their anhydrides, when dissolved in water, yield on the addition of $\frac{2}{3}$ concentrated sulphuric acid (added in drops so as not to heat the fluid above $70^{\circ} \mathrm{C}$ ), and several drops of a 10 p.c. solution of cane-sugar, a reddish purple transparent fluid, which shows two absorption-bands at E \& F (Schenk).

[A very good method is to mix a few drops of the cane-sugar solution with the bile, and to shake the mixture until a copious froth is obtained. Pour the sulphuric acid down the side of the test-tube, and then the characteristic colour is seen in the froth.]

According to Drechsel, it is better to add phosphoric acicl, instoad of sulphuric 
acid, until the fluid is syrupy, then add the cane-sugar, and afterwards place the whole in boiling water. When investigating the amount of bile acids in a liquid, the albumin must be removed beforehand, as it gives a reaction similar to the bile acids, but in that case the red fluid has only one absorption-band. If only small quantitics of bile acids are present, the fluid must in the first place bo concentrated by evaporation.

The origin of the bile acids takes place within the liver. After its extirpation, there is no accumulation of biliary matters in the blood (Joh. Müller, Kunde, Moleschott).

How the formation of the nitrogenous bile acids is effected is quite unknown. They must be obtained from the decomposition of albuminous materials, and it is important to note that the amount of bile acids is increased by albuminous food.

Taurin contains the sulphur of albumin ; bile salts contains 4-4.6 p.c. of sulphur (Voit), which may perhaps be derived from the stroma of the dissolved red bloorl. corpuscles.

(3.) The Bile Pigments.-The freshly secreted bile of man and many animals has a yellowish-brown colour, due to the presence of bilirubin (Städler). When it remains for a considerable time in the gall-bladder, or when alkaline bile is exposed to the air, the bilirubin absorbs $\mathrm{O}$ and becomes changed into a green pigment, biliverdin. This substance is present naturally, and is the chief pigment in the bile of herbivora and cold-blooded animals.

(a.) Bilirubin $\left(\mathrm{C}_{32} \mathrm{H}_{36} \mathrm{~N}_{4} \mathrm{O}_{6}\right)$ is, according to Städler and Maly, perhaps united with an alkali; it crystallises in transparent fox-red clinorhombic prisms. It is insoluble in water, soluble in chloroform, by which substance it nay be separated from biliverdin, which is insoluble in chloroform. It unites as a monobasic acid with alkalies, and as such is soluble. It is identical with Virchow's hæmatoidin (p. 35).

Preparation.-It is most easily prepared from the red (bilirubin-chalk) gall. stones of man or the ox. The stones are pounded, and their chalk dissolved by hydrochloric acid ; the pigment is then extracted with chloroform.

That bilirubin is derived from hæmoglobin is very probable, considering its identity with hæmatoidin. Very probably red blood-corpuscles are dissolved in the liver, and their hæmoglobin changed into bilirubin.

(b.) Biliverdin (Heintz), $\mathrm{C}_{32} \mathrm{H}_{36} \mathrm{~N}_{4} \mathrm{O}_{8}$, is simply an oxidised derivative of the former, from which it can be obtained by various oxidation processes. It is readily soluble in alcohol, very slightly so in ether, and not at all soluble in chloroform. It occurs in considerable amount in the placenta of the bitch. As yet it has not been retransformed by reducing agents into bilirubin.

Tests for Bile Pigments.-Bilirubin and biliverdin may occur in other fluids-e.g., the urine, and are detected by the Gmelin-Heintz' reaction. When nitric acid containing some nitrous acid is added to the liquid containing these pigments, a play of colours is obtained, beginning with green (biliverdin), blue, violet, red, ending with yellow. 
[This reaction is best done by placing a drop of the liquid on a white porcelain plate, and adding a drop of the impure nitric acid.]

(c.) If when the blue colour is reached, the oxidation process is arrested, bilicyanin (Heynsius, Campbell), in acid solution blue, (in alkaline violet) is obtained, which shows two ill-defined absorption-bands near D (Jaffe). Capranica advises that the acid fluids be shaken with a mixture of chloroform and alcohol $(1: 1)$. This mixture absorbs the pigment; pour off the fluid and add bromine in alcohol ( $\frac{1}{2}$ p.c.), and the play of colour is obtained.

(d.) Bilifuscin occurs in small amount in decomposing bile and in gall-stones $=$ bilirubin $+\mathrm{H}_{2} \mathrm{O}$.

(e.) Biliprasin (Städler) also occurs $=$ bilirubin $+\mathrm{H}_{2} \mathrm{O}+\mathrm{O}$.

(f.) The yellow pigment, which results from the prolonged action of the oxidising reagent, is the choletelin $\left(\mathrm{C}_{16} \mathrm{H}_{18} \mathrm{~N}_{2} \mathrm{O}_{6}\right)$ of Maly; it is amorphous, and soluble in water, alcohol, acids, and alkalies.

(g.) Hydro-bilirubin.-Bilirubin absorbs $\mathrm{H}+\mathrm{H}_{2} \mathrm{O}$ (by putrefaction, or by the treatment of alkaline watery solutions with the powerfully reducing sodium amalgam), and becomes converted into Maly's hydrobilirubin $\left(\mathrm{C}_{32} \mathrm{H}_{14} \mathrm{~N}_{4} \mathrm{O}_{7}\right)$, which is slightly soluble in water, and more easily soluble in solutions of salts, or alkalies, alcohol, ether, chloroform, and shows an absorption-band at $b, \mathbf{F}$. This substance, which, according to Hammarsten, occurs in normal bile, is a constant colouringmatter of fæces, and was called stercobilin by Vaulair and Masius, but is identical with hydro-bilirubin (Maly). It is, however, probably identical with the urinary pigment urobilin of Jaffé (Stokvis, p. 35).

(4.) Cholesterin, $\mathrm{C}_{26} \mathrm{H}_{44} \mathrm{O}\left(\mathrm{H}_{2} \mathrm{O}\right)$ is an alcohol which rotates the
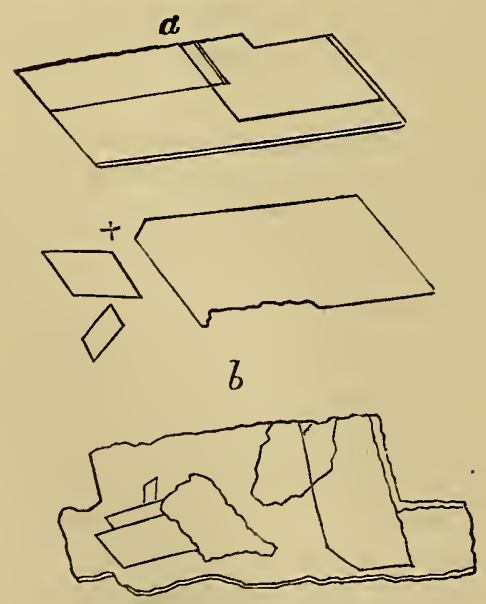

Fig. 145.

Crystals of Cholesterin $-a$, regularly laminated; $b$, irregularly laminated, partially injured forms; $\times 300$ (Aitken after Wedl). ray of polarised light to the left, and whose constitution is unknown; it occurs also in blood, yelk, nervous matter, and [gall-stones]. It forms transparent rhombic plates, which usually have a small oblong piece cut out of one corner (Fig. 145, a). It is insoluble in water, soluble in hot alcohol, in ether, and chloroform. It is kept in solution in the bile by the bile salts.

Preparation.-It is most easily prepared from so-called white gallstones, which not unfrequently consist almost entirely of cholesterin, by extracting them with hot alcohol after they are pulverised. Crystals are excreted after evaporation of the alcohol, and they give a red colour 
with sulphuric acid ( 5 vol. to 1 vol. $\mathrm{H}_{2} \mathrm{O}-$ Moleschott), while they give a blue as cellulose does-with sulphuric acid and iodine. When dissolved in chloroform, 1 drop of concentrated sulphuric acid causes a deep red colour (H. Schiff).

(5.) Amongst the other organic constituents of bile are:-Lecithin (p. 36), or its decomposition product, neurin (cholin), and glycerophosphoric acid (into which lecithin may be artificially transformed by boiling with baryta); Palmitin, Stearin, Olein, as well as their soda soaps; Diastatic Ferment (Jacobson, v. Wittich); traces of Urea (Picard); (in ox bile, acetic acid and propionic acid, united with glycerine and metals, Dogiel).

[The proportion of diastatic ferment is not greater than in the tissues of the body generally (M. Hay).]

\section{(6.) Inorganic constituents of bile (0.6 to 1 p.c.):-}

They are-sodium chloride, potassium chloride, calcic and magnesic phosphate, and much iron, which in fresh bile gives the ordinary reactions for iron, so that iron must occur in one of its oxidised compounds in bile (Kunkel); manganese and silica. Freshly secreted bile contains in the dog more than 50 vol., and in the rabbit 109 vol. per cent. $\mathrm{CO}_{2}$ (Pflüger, Boguljubow, Charles), partly united to alkalies, partly absorbed, the latter, however, being almost completely absorbed within the gall-bladder.

\section{The mean composition of human bile is :-}

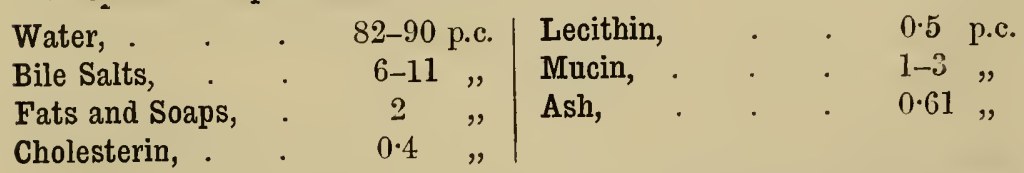

Farther, unchanged fat probably always passes into the bile, but is again absorbed therefrom (Virchow). The amount of $\mathrm{S}$ in dry dog's bile $=2 \cdot 8-3 \cdot 1$ p.c., the $\mathrm{N}=7-10$ p.c. (Spiro); the sulphur of the bile is not oxidised into sulphuric acid, but it appears as a sulphur-compound in the urine (Kunkel, v. Voit).

\section{Secretion of Bile.}

The secretion of bile is not a mere filtration of substances already existing in the blood of the liver, but it is a chemical production of the characteristic biliary constituents, accompanied by oxidation, within the hepatic cells, to which the blood of the gland only supplies the raw material. The liver-cells themselves undergo histological changes during the process of digestion (Heidenhain, Kayser). It is secreted continually; it is partly stored up in the gall-bladder, and is poured out copiously during digestion.

The higher temperature of the blood of the hepatic vein, as well as the large amount of $\mathrm{CO}_{2}$ in the bile (Pflïger), indicate that oxidations occur within the liver. The water of the bile is not merely 
filtered through the blood-capillaries, as the pressure within the bileducts may exceed that in the portal vein.

(2.) The quantity of bile was estimated by $\mathrm{v}$. Wittich from a biliary fistula, at 533 cubic centimetres in 24 hours (some bile passed into the intestine); by Westphalen, at 453-566 grms.; [by Murchison, at 40 oz.]; Joh. Ranke, on a biliary-pulmonary fistula, at 652 cubic centimetres. The last observation gives 14 grms. (with 0.44 grms. solids) per kilo. of man in 24 hours.

Analogous values for animals are-1 kilo. dog, $32 \mathrm{grm} .(1 \cdot 2$ solids $)-$ Kölliker, H. Müller; 1 kilo. rabbit, $137 \mathrm{grm}$. (2.5 solids)-Bidder and Schmidt; 1 kilo. guinea-pig, 176 grms. (5.2 solids)-Bidder and Schmidt.

(3.) The excretion of bile into the intestine shows two maxima during one period of digestion; the first, from 3 to 5 hours, and the second, from 13 to 15 hours after food. The cause is due to simultaneous reflex excitement of the hepatic blood-vessels, which become greatly dilated.

(4.) The influence of food is very marked. The largest amount is secreted after a flesh diet, with some fat added; less after vegetable food; a very small amount with a pure fat diet; it stops during hunger. Draughts of water increase the amount, with a corresponding relative diminution of the solid constituents.

(5.) The influence of blood supply is variable :-

(a.) Secretion is greatly favoured by a copious and rapid blood supply. The blood-pressure is not the prime factor, as ligature of the cava above the diaphragm, whereby the greatest blood-pressure occurs in the liver, arrests the secretion (Heidenhain).

(b.) Simultaneous ligature of the hepatic artery (diameter $5 \frac{1}{2} \mathrm{~mm}$.) and the portal vein (diameter $6 \mathrm{~mm}$.) abolishes the secretion (Röhrig). These two vessels supply the raw material for the secretion of bile.

(c.) If the hepatic artery be ligatured, the portal vein alone supports the secretion (Simon, Schiff, Schmulewitsch, Asp). According to Kottmeier, Betz, Cohnheim and Litten, ligature of the artery or one of its branches ultimately causes necrosis of the parts supplied by that branch, and eventually of the entire liver, as this artery is the nutrient vessel of the liver.

$(d$.$) If the branch of the portal vein to one lobe be ligatured, there is only a$ slight secretion in that lobe, so that the bile must be formed from the arterial blood (Schmulewitsch and Asp). Complete ligature of the portal vein rapidly causes death. [The blood-pressure falls rapidly and the blood accumulates in the blood-vessels of the abdomen. In fact, the accumulation of the blood within the abdomen takes place to so great an extent, that practically the animal is bled into its own abdomen (p. 176).]

Neither the ligature of the hepatic artery by itself (Schiff, Betz), nor the gradual obliteration of the portal vein by itself, causes the cessation of the secretion, but it is diminished. That sudden ligature of the portal vein causes cessation is explained by the fact, that in addition to diminution of the secretion, the enormous stagnation of blood in the rootlets of the portal vein in the abdominal organs makes the liver very anæmic, and thus prevents it from secreting.

(e.) If the blood of the hepatic artery is allowed to pass into the portal vein (which has been ligatured on the peripheral side), secretion continues (Schiff). 
(f.) Profusc loss of blood arrests the secretion of bile, before the muscular and nervous apparatus become paralysed. A more copious supply of blood to other organs-e.g., to the muscles of the trunk-during vigorous exercise, diminishes the secretion, while the transfusion of large quantities of blood increases it (Landois); but if too high a pressure is caused in the portal vein, by introducing blood from the carotid of another animal, it is diminished (Heidenhain).

(g.) The influence of nerves.-All conditions which cause contraction of the abdominal blood-vessels-e.g., stimulation of the ansa Vieussenii, of the inferior cervical ganglion, of the hepatic nerves (Afanassiew) of the splanchnics, of the spinal cord (either directly by strychnia, or reflexly through stimulation of sensory nerves) affect the secretion; and so do all conditions which cause stagnation or congestion of the blood in the hepatic vessels (section of the splanchnic nerves, diabetic puncture, $\$ 175$ ), section of the cervical spinal cord (Heidenhain). Paralysis (ligature) of the hepatic nerves causes at first an increase of the biliary secretion (Afanassieff).

(h.) With regard to the raw material supplied to the liver by its blood-vessels, it is important to note the difference in the composition of the blood of the hepatic and portal veins. The blood of the hepatic vein contains more sugar (?), lecithin, cholesterin (Drosdoff), and blood-corpuscles, but less albumin, fibrin, hæmoglobin, fat, water, and salts.

(6.) The formation of bile is largely dependent upon the decomposition of coloured blood-corpuscles, as they supply the material necessary for the formation of some of its constituents.

Hence, all conditions which cause solution of the coloured blood-corpuscles are accompanied by an increased formation of bile ( $\$ 180$ ).

(7.) Of course a normal condition of the hepatic cells is required for a normal secretion of bile.

Biliary Fistulæ. - The mechanism of the biliary secretion is studied in animals by means of biliary fistulæ. Schwann opened the belly by a vertical incision a little to the right of the ensiform process, cut into the fundus of the gall-bladder, and sewed its margins to the edges of the wound in the abdomen, and afterwards introduced a cannula into the wound. As a rule all the bile is discharged externally; but to be quite certain that this is so, the common bile-duct ought to be tied between two ligatures, and divided. After a fistula is freshly made the secretion falls. This depends upon the removal of the bile from the body. If bile be supplied the secretion is increased. Regeneration of the divided bile-duct may occur in dogs. v. Wittich observed a biliary fistula in man. [A temporary biliary fistula may also be made. The abdomen is opened in the same way as described above. A long bent glass cannula is introduced and tied into the common bile-duct, and the cystic duct is ligatured or clamped. The tube is brought ont through the wound in the abdomen. Necessarily all the bile must be discharged by the tube].

\section{Excretion of Bile.}

[In connection with the excretion of bile, we must keep in view two distinct mechanisms. (1) The bile-secreting mechanism dependent upon the liver-cells, which are always in a greater or less degree of activity; (2) the bile-expelling mechanism, which is specially active at certain periods of digestion (p. 360).] 
This occurs-(1.) Owing to the continual pressure of the newlyformed bile within the interlobular bile-ducts forcing onward the bile in the excretory ducts.

(2.) Owing to the interrupted periodic compression of the liver from above, by the diaphragm, at every inspiration. Farther, every inspiration assists the flow of blood in the hepatic veins, and every respiratory increase of pressure within the abdomen favours the current in the portal vein.

It is probable that the diminution of the secretion of bile, which occurs after bilateral division of the vagi, is to be explained in this way; still it is to be remembered, that the vagus sends branches to the hepatic plexus. It is not decided whether the biliary excretion is diminished after section of the phrenic nerves and paralysis of the abdominal muscles.

(3.) Owing to the contraction of the smooth muscles of the larger bileducts and the gall-bladder. Stimulation of the spinal cord, from which the motor nerves for these structures pass, causes acceleration of the outflow, which is afterwards followed by a diminished outflow (Heidenhain, J. Munk). Under normal conditions, this stimulation seems to occur reflexly, and is caused by the passage of the ingesta into the duodenum, which, at the same time, excites movement of this part of the intestine.

(4.) Direct stimulation of the liver (Pflüger), and reflex stimulation of the spinal cord (Röhrig), diminish the excretion; while extirpation of the hepatic plexus (Pflüger), and injury to the floor of the fourth ventricle (Heidenhain) do not exert any disturbing influence.

(5.) A relatively small amount of resistance causes bile to stagnate in the bile-ducts.

A manometer, tied into the gall-bladder of a guinea-pig, supports a column of 200 millimetres of water; and secretion can take place under this pressure (Heidenhain, Friedländer, Barisch). If this pressure be increased, or too long sustained, the watery bile passes from the liver into the blood, even to the amount of four times the weight of the liver, thus causing solution of the red bloodcorpuscles by the absorbed bile; and very soon thereafter, hæmoglobin appears in the urine.

\section{Reabsorption of Bile.}

\section{Phenomena of Jaundice (Icterus; Cholæmia).}

Absorption Jaundice.-When an impediment or resistance is offered to the outflow of bile into the intestine-e.g., by a plug of mucus, or a gall-stone which occludes the bile-duct, or where a tumour or pressure from without, makes it impervious - the bile-ducts become filled with bile and cause an enlargement of the liver. The pressure within the bile-ducts is increased. As soon as the pressure has reached a certain amount, which it soon does when the bile-duct is occluded (in the dog $275 \mathrm{~mm}$. of a column of bile-Afanassiew)-reabsorption of bile from the distended larger bile-ducts takes place into the lymphatics (not the blood: 
vessels) of the liver (Saunders, 1795); the bile acids pass into the lymphatics of the liver. [The lymphatics can be seen at the portal fissure filled with a deep yellowcoloured lymph]. The lymph passes into the thoracic duct, and so into the blood (Fleischl, Kunkel, Kufferath). Even when the pressure is very low within the portal vein, bile may pass into the blood, without any obstruction to the bile-duct being present. This is the case in Icterus neonatorum, as after ligature of the umbilical cord, no more blood passes through the umbilical vein; farther, in the icterus of hunger, as the portal vein is relatively empty, owing to the feeble absorption from the intestinal canal (Cl. Bernard, Voit, Naunyn).

II. Cholremia may also occur, owing to the excessive production of bile (hypercholia), the bile not being all excreted into the intestine, so that part of it is reabsorbed. This takes place when there is solution of a great number of bloodcorpuscles $(\$ 178,6)$, which yields material for the formation of bile. Thick inspissated bile accumulates in the bile-ducts, so that stagnation, with subsequent reabsorption of the bile, takes place (Afanassiew). The transfusion of heterogeneous blood by dissolving coloured blood-corpuscles acts in this direction (p. 201). Icterus is a common phenomenon after too copions transfusion of the same blood. The blood-corpuscles are dissolved by the injection into the bloor of heterogeneous blood-serum (Landois), by the injection of bile acids into the vessels (Frerichs), and by other salts, by phosphoric acid, water (Herrmann), chloral, inhalation of chloroform, and ether (Nothnagel, Bernstein); the injection of dissolved hæmoglobin into the arteries (Kühne), or into a loop of small intestine, acts in the same way (Naunyn).

Icterus Neonatorum.-When, owing to compression of the placenta within the uterus, too much blood is forced into the blood-vessels of the newly-born infant, a part of the surplus blood during the first few days becomes dissolved, whereby the hæmoglobin passes into bilirubin, thus cansing jaundice (Virchow, Violet).

When the jaundice is caused by the absortion of bile already formed in the liver, it is called hepatogenic or absorption-jaundice. The following are the symptoms :-

Phenomena.-(1.) Bile pigments and bile acids pass into the tissues of the body; hence, the most pronounced external symptom is the yellowish tint or jaundice. The skin and the sclerotic become deeply coloured yellow. In pregnancy the fertus is also tinged.

(2.) Bile pigments and bile acids pass into the urine (not into the saliva, tears, or mucus), and their presence is ascertained by the nsual tests ( $\$ 177)$. When there is much bile pigment, the urine is coloured a deep yellowish brown, and its froth is citron yellow; while strips of gelatin or paper dipped into it also become coloured. Occasionally bilirubin (= hæmatoidin) erystals occur in the urine.

(3.) The freces are "clay coloured" (because the hydrobilirubin of the bile is absent from the fæcal matter)-very hard (because the fluid of the bile does not pass into the intestine); contain much fat (in globules and crystals), because the fat is not sufficiently digested in the intestine without bile, so that more than 60 p.c. of the fat taken with the food reappears in the fæces (v. Voit); they have a very disagreeable odour, because bile normally greatly limits the putrefaction in the intestine. The evacuation of the faces occurs slowly, partly owing to the hardness of the frees, partly because of the absence of the peristaltic movements of the intestine, owing to the want of the stimulating action of the bile.

(4.) The heart-beats are greatly diminished, e.g., to 40 per minute. This is due to the action of the bile salts, which at first stimulate the cardiac ganglia, and then weaken them. The injection of bile salts into the heart, produces at first a temporary acceleration of the pulse (Landois), and afterwards slowing (Röhrig). The 
same occurs when they are injected into the blood, but in this case, the stage of excitement is very short. The phenomenon is not affected by section of the vagi. It is probable, that when the action of the bile salts is long continued they act upon the heart-muscle (Traube). In addition to the action on the heart, there is sloving of the respiration and diminution of temperature.

(5). That the nervous system, and perhaps also the muscles, are affected, either by the bile salts or by the accumulation of cholesterin in the blood (Flint, K. Muiller), is shown by the rery general relaxation, sensation of fatigue, weakness and drowsiness, lastly deep coma-sometimes there is sleeplessness, itchiness of the skin, eren mania, aud spasms. Löwit, after injecting bile into animals, observed phenomena referable to stimulation of the respiratory, cardio-inhibitory, and rasomotor nerre-centres.

(6.) In rery pronounced jaundice, there may be "yellow vision" (Lucretius Carus), owing to impregnation of the retina and macnla lutea with the bile pigment.

(7.) The bile acids in the blood dissolve the red blood-corpuscles. The hæmoglobin is changed into new bile pigment, and the globulin-like body of the hæmoglobin may form urinary cylinders or casts in the urinary tubules (Nothnagel), which are ultimately washed out of the tubules by the urine.

Passage of substances into the Bile.-Various substances pass into the bile, such substances being in the blood, riz, the metals (v. Sartoris, Mohnheim, Orfila)-copper, lead, zinc, nickel, silver, bismuth (Wichert), arsenic, antimony, iron; these substances are also deposited in the hepatic tissues. Potassium iodide, bromide, and sulphocyanide (Peiper), and turpentine also pass into the bile, and, to a less degree, cane-sugar and grape-sugar (Mosler); sodium salicylate, and carbolic acid (Peiper). If a large amount of water be injected into the blood, the bile becomes albuminous (Mosler); mercuric and mercurous chlorides canse an increase of the water of the bile (G. Scott). Sugar has been found in the bile in diabetes; leucin and tyrosin in typhos, lactic acid and albumin in other pathological conditions of this fluid.

[Influence of Drugs on the Secretion of Bile.-Two methods are adopted, one by means of permanent fistula, and the other by establishing temporary fistulae. The latter is the more satisfactory method, and the experiments are usually made on fasting curarised dogs. A suitable cannula is introduced into the common bile-dnct, as described at p. 361, the animal is curarised, artificial respiration being kept up, while the drug is injected into the stomach or intestine. Pührig used this method, which was improved by Rutherford and Vignal. Röhrig found that some purgatives, croton oil, colocynth, jalap, aloes, rhubarb, senna, and other substances, increased the secretion of bile. Rutherford and Vignal investigated the action of a large number of drugs on the bile-secreting mechanism. They found that croton oil is a feeble hepatic stimulant, wlile podophyllin, aloes, colchicum, enonymin, iridin, sanguinarin, ipecacuan, colocynth, sodium phosphate, phytolaccin, sodium benzoate, sodium salicylate, dilute nitrohydrochloric acid, ammonium phosphate, mercuric chloride (corrosive sublimate), are all powerful, or very considerable, hepatic stimulants. They found that some substances stimulate the intestinal glands, but not the liver, e.g., magnesium sulphate, castor oil, gamboge, ammonium chloride, manganese sulphate, calomel. Other substances stimnlate the liver as well as the intestinal glands, althongh not to the same extent, e.g., scammony (powerful intestinal, feeble hepatic stimulant); colocynth excites both powerfully; jalap, sodium sulphate, baptisin, act with considerable power both on the liver and the intestinal glands. Calabar bean stimulates the liver, and the increased secretion cansed thereby may be reduced by sulphate of atropin, although the latter drug, when given alone, does not notably affect the secretion of bile. The injection of water or bile slightly increases the secretion. In all cases where purgation was produced by purely intestinal stimulants, such as 
magnesium sulphate, gamboge, and castor oil, the secretion of bile was diminished. In all such experiments it is most important that the temperature of the animal be lept up by covering it with cotton wool, else the secretion of bile diminishes.

As yet, we cannot say definitely whether these substances stimulate the secretion of bile, by exciting the mucous membrane of the duodenum or other part of the small intestine, and thereby inducing reflex excitement of the liver. Their action does not seem to be due to increase of the blood-stream through the liver. More probably, as Rutherford suggests, these drugs act directly on the hepatic cells or their nerves. Acetate of lead directly depresses the biliary secretion, while some substances affect it indirectly.]

Cholesteræmia.-Flint ascribes great importance to the excretion of cholesterin by the bile, with reference to the metabolism of the nervous system. Cholesterin, which is a normal ingredient of nervous-tissue, is excreted by the bile; and if it be retained in the blood, "cholesteræmia," with grave nervous symptoms, is said to occur. This, however, is problematical, and the phenomena described arc probably referable to the retention of the bile acids in the blood.

\section{Functions of the Bile.}

[(1) Bile is concerned in the digestion of certain food-stuffs;

(2) part of it is absorbed;

(3) part is excreted.]

(A.) Bile plays an important part in the absorption of fats :-

(1.) It cmulsionises neutral fats ( $\$ 170, \mathrm{III}$.), whereby the fatty granules pass more readily through or between the cylindrical epithelium of the small intestine into the lacteals. It does not decompose neutral fats into glycerine and a fatty acid, as the pancreas does.

When, however, fatty acids are dissolved in the bile (Lenz) the bile salts are decomposed, the bile acids being set free, while the soda of the decomposed bile salts readily forms a soluble soap with the fatty acids. These soaps are soluble in the bile, and increase considerably the emulsifying power of this fluid. Bile can dissolve directly fatty acids to form an acid fluid, which has high enulsionising properties (Steiner).

(2.) As fluid fat flows more rapidly through capillary tubes when they are moistened with bile, it is concluded that when the pores of the absorbing wall of the small intestine are moistened with bile, the fatty particles pass more easily through them.

(3.) Filtration of fat takes place through a membrane moistened with bile or bile salts under less pressure than when it is moistened witl water or salt solutions ( $r$. Wistinghausen).

(4.) As bile, like a solution of soap, has a certain relation to watery solutions, as well as to fats, it permits diffusion to take place between these two fluids, as the membrane is moistened by both fluids ( $v$. Tristinghausen).

It is clear, therefore, that the bile is of great importance in the preparation and in the absorption of fats. This is forcibly illustrated by experiments on animals, in which the bile is entirely discharged exterually through a fistula. Dogs, under these conditions, absorbed at most 40 p.c. of the fat taken with the food (r. Yoit). 
The chyle of such animals is very poor in fat, is not white but transparent; the fæces, however, contain much fat, and are oily. Such animals are voracious (Nasse); the tissues of the body contain little fat, even when the nutrition of the animals has not been much interfered with. Persons suffering from disturbances of the biliary secretion, or from liver affections, ought, therefore, to abstain from fatty food.

(B.) Fresh bile contains a diastatic ferment which transforms starch into sugar (Nasse, Jacobson, v. Wittich), and also glycogen into sugar (Bufalini).

(C.) Bile excites contractions of the muscular coats of the intestine, and contributes thereby to absorption.

(1.) The bile acids act as a stimulus to the muscles of the villi, which contract from time to time, so that the contents of the lymph-spaces [origins of the lacteals] are emptied towards the larger lymphatics, and the villi are thus in a position to absorb more (Schiff). [The villi act like numerous small pumps, and expel their contents, which are prevented from returning by the presence of valves in the larger lymphatics.]

(2.) The musculature of the intestine itself seems to be excited, perhaps through the agency of the plexus myentericus. In animals with a biliary fistula, and in which the bile-duct is obstructed, the intestinal peristalsis is greatly diminished, while the salts of the bile aciads administered by the mouth cause diarrhœa and vomiting (Leyden, Schülein). As contraction of the intestine aids absorption, bile is also necessary in this way for the absorption of the dissolved food stuffs.

(D.) The bile moistens the walls of the intestine, as it is copiously excreted. It gives to the fæces their normal amount of water, so that they can be readily evacuated. Animals with biliary fistula, or persons with obstruction of the bile-ducts, are very costive. The mucus of the bile aids the forward movement of the ingesta through the intestinal canal. [Thus, in a certain sense, bile is a natural purgative.]

(E.) The bile diminishes putrefactive decomposition of the intestinal contents (Valentin). [Thus, it is an antiseptic.]

(F.) When the strongly acid contents of the stomach pass into the duodenum, the glycocholic acid is precipitated by the gastric acid, and carries the pepsin with it (Burkart). Some of the albumin, which has been simply dissolved, but as yet not peptonised, is also precipitated, but it does not seem that peptone or propeptone are precipitated by the mixture of the bile acids (Maly and Emich). The bile salts are decomposed by the acid of the gastric juice. When the mixture is rendered alkaline by the pancreatic juice and the alkali derived from the decomposition of the bile salts, the pancreatic juice acts energetically in this alkaline medium (Moleschott).

When bile passes into the stomach, as in vomiting, the acid of the gastric juice unites with the bases of the bile salts; so that sodium chloride and free bile acids are formed, and the acid reaction is thereby somewhat diminished. The bile 
acids are not effective for carrying on gastric digestion; the peptone is precipitated by them; neutralisation also causes a precipitate of pepsin and mucin. As soon, however, as the walls of the stomach secrete new acid, the pepsin is redissolved. The bile which passes into the stomach deranges gastric digestion, by shrivelling the proteids, which can only be peptonised when they are swollen up.

\section{Fate of the Bile in the Intestine.}

Some of the biliary constituents are completely evacuated with the freces, while others are reabsorbed by the intestinal walls.

(1.) Mucin passes unchanged into the fæces.

(2.) The bile pigments are reduced, and are partly excreted with the freces as hydrobilivubin $(\$ 177,3 \mathrm{~g})$, and partly as the identical end-product urobilin by the urine.

Hydrobilirubin is absent from Meconium, while bilirubin and biliverdin and an unknown red oxidation product of it are present (Zweifel). Hence, no reduction - but rather oxidation-processes occur in the fotal intestine (Hoppe-Seyler).

(3.) Cholesterin is given off with the freces.

(4.) The bile salts are for the most part reabsorbed by the walls of the jejunum and ileum, to be re-employed in the animal's economy. Tappeiner fouml them in the chyle of the thoracic duct-minute quantities pass from the blood into the urine. Only a very small amount of glycocholic acid appears unchanged in the fæces. The taurocholic acid, as far as it is not absorbed, is easily decomposed in the intestine, by the putrefactive processes, into cholalic acid and taurin; the former of these is found in the freces, but the taurin at least seems not to be constantly present.

As putrefactive decomposition does not occur in the fotal intestine, unchanged tanrocholic acid is found in meconium (Zweifel). The anhydride stage of cholalic acid (the artificially prepared choloidinic acid ?), dyslysin, is an artificial product, and does not occur in the frees (Hoppe-Seyler).

(5.) The freces contain mere traces of Lecithin (Wegscheider, Bokay).

The greatest part of the most important biliary constituents, the bile acids, re-enter the blood, and thus is explained why animals with a biliary fistula, where all the bile is removed (without the animal being allowed to lick the bile), rapidly lose weight. This depends partly upon the digestion of the fats being interfered with, and also upon the direct loss of the bile salts. If such dogs are to maintain their weight, they must eat twice as much food. In such cases, carbohydrates most beneficially replace the fats. If the digestive apparatus is otherwise intact, the animals, on account of their voracity, may eren increase in weight, but the flesh and not the fat is increased.

The fact that bile is secreted during the fotal period, whilst none of the other digestive fluids is, proves that it is an excretion.

The cholalic acid which is reabsorbed by the intestinal walls passes into the body, and seems ultimately to be burned to form $\mathrm{CO}_{2}$ and $\mathrm{H}_{2} \mathrm{O}$. The glycin (with 
hippuric acid) forms urea, as the urea is increased after the injection of glycin (Horsford, Schultzen, Nencki). The fate of taurin is unknown. When large quantities are introduced into the human stomach, it reappears in the urine, as tauro-carbonic acid, along with a small quantity of unchanged taurin. When injected subcutaneously into a rabbit, nearly all of it reappears in the urine.

\section{The Intestinal Juice.}

The human intestine is ten times longer than the length of the body, as measured from the vertex to the anus. It is longer comparatively than that of the omnivora (Heming). Its minimum length is 507, its maximum 1,149 centimetres; its capacity is relatively greater in children (Beneke). In childhood the absorptive elements, in adults the secreto-chemical processes, appear to be most active (Baginsky).

The succus entericus is the digestive fluid secreted by the numerous glands of the intestinal mucous membrane. The largest amount is produced by Lieberkühn's glands, while in the duodenum, there is added the scanty secretion of the small compound tubular Brunner's glands.

Brunner's glands are small convoluted, branched, tubular glands, lying in the sub-mucosa of the duodenum. Their fine ducts run inwards, pierce the mucous membrane, and open at the bases of the villi, The acini are lined by cylindrical cells, like those lining the pyloric glands. In fact, Brunner's glands are structurally and anatomically identical with the pyloric glands of the stomach. During hunger, the cells are turbid and small, while during digestion they are large and clear. The glands receive nerve-fibres from Meissner's plexus (Drasch).

I. The Secretion of Brunner's Glands. - The granular contents of the secretory cells of these glands, which occur singly in man, but form a continuous layer in the duodenum of the sheep, besides albuminous substances, consist of mucin and a ferment-substance of unknown constitution. The watery extract of the glands causes:-(1) Solution of proteids at the temperature of the body (Krolow). (2) It also has a diastatic (?) action. It does not appear to act upon fats. [Brown and Heron have shown that the secretion of Brunner's glands, more actively than any other glands of the intestines, converts maltose into glucose.]

On account of the smallness of the objects, such experiments are only made with great difficulty, and, therefore, there is a certain amount of uncertainty with regard to the action of the secretion.

Lieberkühn's glands are simple tubular glands resembling the finger of a glove [or a test tube], which lie closely packed, vertically near each other, in the mucous membrane; they are most numerous in the large intestine, owing to the absence of villi in this region. They consist of a structureless membrana propria lined by a single layer of low cylindrical epithelium, between which numerous goblet-cells occur, the goblet-cells being fewer in the small intestine and much more numerous in the large (Fig. 146). The glands of the small intestine yield a thin secretion, while those of the large intestine yield a large amount of sticky mucus from their goblet-cells (Klose and Heidenhain). 
II. The Secretion of Lieberkühn's glands is, from the duodenum onwards, the chief constituent of the intestinal juice.

Intestinal Fistula.-The intestinal juice is obtained by making a Thiry's fistula (1561). A loop of the intestine of a $\operatorname{dog}$ is pulled forward, and a piece about four inches in length is cut out, so that the continuity of the intestinal tube is broken, but the mesentery and its blood-vessels are not divided. One end of this tube is closed, and the other end is left open and stitched to the abdominal wall. After the two ends of the intestine, from which this piece was taken, have been carefully brought together with sutures, so as to establish the continuity of the intestinal canal, animals still continue to live. The excised piece of intestine yields a secretion which is uncontaminated with any other digestive secretion.

[Thiry's method is very unsatisfactory, as judged from the action of the separated loop in relation to medicaments, probably owing to its mucous membrane becoming atrophied from disuse, or injured by inflammation. Meade Smith has lately used a better method, in which he makes a small opening in the intestine, throngh which he introduces two small hollow and collapsed india-rubber balls, one above and the other below the opening, which are then distended by inflation until they completely block a certain length of the intestine. The loop thus blocked off having been previously well washed out, is allowed to become filled with succus, which is secreted on the application of various stimuli. By means of Bernard's gastric cannula (p. 330) inserted into the fistula in the loop, the secretion can be removed when desired.]

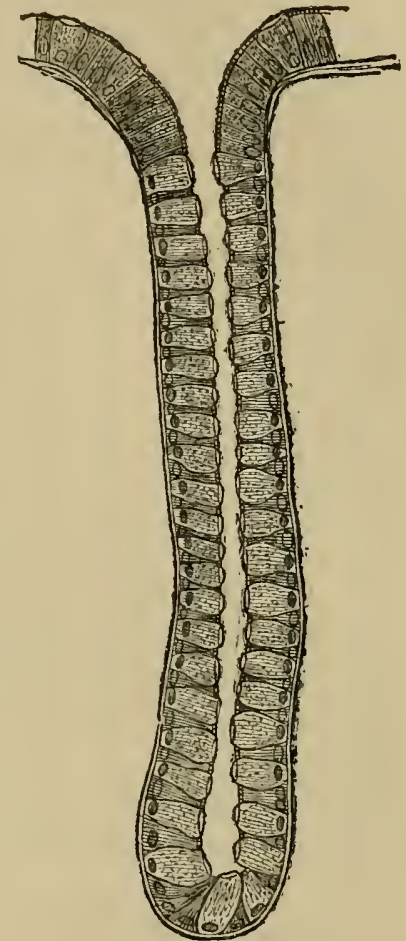

Fig. 146.

Lieberkuihn's Gland, from the large intestine of a dog.

The intestinal juice of such fistulæ flows spontaneously in very small amount, and is increased during digestion; it is increasedespecially its mucus-by mechanical, chemical, and electrical stimuli; at the same time, the mucous membrane becomes red, so that $100 \square$ centimetres yields 13 to 18 grammes of this juice in an hour (Thiry, Masloff).

Characters.-The juice is light yellow, opalescent, thin, strongly alkaline, specific gravity 1011, evolves $\mathrm{CO}_{2}$ when an acid is added; it contains albumin and ferments; mucin occurs in the juice of the large intestine. Its composition is-proteids $=0.80$ p.c.; other organic substances $=0.73$ p.c.; salts, 0.88 p.c.; amongst these-sodium carbonate, $0.32-0.34$ p.c.; water, 97.59 p.c. 
[The intestinal juice obtained by Meade Smith's method contained only 0.39 per cent. of organic matter, and in this respect agreed closely with the juice which $A$. Morean procured by dividing the mesenteric nerves of a ligatured loop of intestine.]

[The secretion of the large intestine is much more viscid than that of the small intestine.]

Actions of succus entericus. - The digestive functions of the fluid of the small intestine are:-

(1.) It has less diastatic action than either the saliva or the pancreatic juice (Schiff, Busch, Quincke, Garland), but it does not form maltose; while the juice of the large intestine is said to possess this property (Eichhorst). V. Wittich extracted the ferment with a mixture of glycerine and water.

[The diastatic action of the small intestine is incomparably weaker than that of the saliva, or pancreatic juice, and barely exceeds that of the tissues and fluids of the bodies generally. A similarly weak diastatic action is possessed by the secretion of the colon.]

(2.) It converts maltose into grape-sugar. It seems, therefore, to continue the diastatic action of saliva $(\S 148)$ and pancreatic juice (\$170) which usually form only maltose. Thus maltose seems to be transformed into grape-sugar by the intestinal juice.

(3.) Fibrin is slowly (by the trypsin and pepsin-Kühne) peptonised (Thiry, Leube); less easily albumin (Masloff), fresh casein, flesh raw or cooked, vegetable albumin (Kölliker, Schiff); probably gelatin also is changed by a special ferment into a solution which does not gelatinise (Eichhorst).

[The ferment for this purpose is mainly contained in Brunner's glands, and in Peyer's patches (Brown and Heron).]

(4.) Fats are only partly emulsionised (Schiff), and afterwards decomposed (Vella).

[M. Hay has never observed any emulsifying action. The apparent emulsification in certain instances is due to shaking the alkaline juice with a rancid oil, containing free fatty acids, when a certain quantity of a soap is at once formed.]

(5.) According to $\mathrm{Cl}$. Bernard, invertin occurs in intestinal juice (this ferment can also be extracted from yeast), whereby cane-sugar $\left(\mathrm{C}_{12} \mathrm{H}_{22} \mathrm{O}_{11}\right)$ takes up water $\left(+\mathrm{H}_{2} \mathrm{O}\right)$ and becomes converted into invert sugar, which is a mixture of left rotating sugar (lævulose, $\mathrm{C}_{6} \mathrm{H}_{12} \mathrm{O}_{6}$ ) and of grape-sugar (dextrose, $\mathrm{C}_{6} \mathrm{H}_{12} \mathrm{O}_{6}$ ). Heat seems to be absorbed during the process (Leube). (See Carbohydrates for the various kinds of sugar).

[Hoppe-Seyler has suggested that this ferment is not a natural product of the body, but is introduced from without with the food. Matthew Hay has recently disproved this theory by, amongst other reasons, finding it to be invariably present in the intestine of the foetus. It is found in every portion of the small intestine, but not in the large intestine, nor in any other part of the body, and is much less diffusible than diastase.] 
Fate of the Ferments. - With regard to the digestive ferments, Langley is of opinion that they are destroyed in the intestinal canal; the diastatic ferment of saliva is destroyed by the $\mathrm{HCl}$ of the gastric juice; pepsin and rennet are acted upon by the alkaline salts of the pancreatic and intestinal juices, and by trypsin; while the diastatic and peptic ferments of the pancreas disappear under the influence of the acid fermentation in the large intestine.

The action of the Nervous System on the secretion of the intestinal juice is not well determined. Section or stimulation of the vagi has no apparent effect; while extirpation of the large sympathetic abdominal ganglia causes the intestinal canal to be filled with a watery fluid, and gives rise to diarrhœa (Budge). This may be explained by the paralysis of the vaso-motor nerves, and also by the section of large lymphatic vessels during the operation, whereby absorption is interfered with and transudation is favoured.

Moreau's Experiment.--A similar result is caused by extirpation of the nerves which accompany the blood-vessels going to a loop of intestine (Moreau). [Moreau placed four ligatures on a loop of intestine at equal distances from each other. The ligatures were tied so that three loops of intestine were shut off. The nerves to the middle loop were divided, and the intestine was replaced in the abdominal cavity. After a time, a very small amount of secretion, or none at all, was found in two of the ligatured compartments of the gut-i.e., in those with the nerves and blood-ressels intact-but the compartment whose nerves had been divided contained a watery secretion.]

The secretion of the intestiual and gastric juices is diminished in man in certain nervous affections (hysteria, hypochondriasis, and various cerebral riseases); while in other conditions, these secretions are increased.

If an isolated intestinal fistula be made, and various drugs administered, experiment shows that the mucous membrane excretes iodine, bromine, lithium, sulphocyanides, but not potassium ferrocyanide, arsenious or boracic acid (Quincke), or iron salts (Glaevecke).

In sucklings, not unfrequently a large amount of acid is formed when the fungi in the intestine (Leube) split up milk-sugar or grape-sugar into lactic acid. Starch changed into grape-sugar may undergo the same abnormal process; hence, infants ought not to be fed with starchy food.

\section{Fermentation Processes in the Intestine.}

Those processes, which are to be regarded as fermentations or putrefactire processes, are quite different from those caused by the action of distinct ferments (Frerichs, Hoppe-Seyler). The putrefactive changes are connected with the presence of lower organisms, so-called fermentation or putrefaction producers (Nencki); and they may develop in suitable media outside the body. The lower organisms which cause the intestinal fermentation are swallowed with the food and the drink, and also with the saliva. When they are introduced, fermentation and putrefaction begin, and gases are evolved.

Intestinal Gases._-During the whole of the foetal period until birth, this fermentation cannot occur; hence, gases are never present in the intestine of the newly-born (Breslau). The first air-bubbles pass into the intestine with the saliva which is swallowed, even before food has been taken. The germs of organisms are thus introduced into the 
intestinal tract, and give rise to the formation of gases. The evolution of intestinal gases goes hand-in-hand with the fermentations. Atmospheric air is also swallowed, and an exchange of gases takes place in the intestine, so that the composition of the intestinal gases depends upon various conditions.

Kolbe and Ruge collected the gases from the anus of a man, and found in 100 vols. :-

\begin{tabular}{|c|c|c|c|c|c|}
\hline Food. & $\mathrm{CO}_{2}$ & H. & $\mathrm{CH}_{4}$. & N. & $\mathrm{H}_{2} \mathrm{~S}$. \\
\hline $\begin{array}{l}\text { Milk, : } \\
\text { Flesh, : } \\
\text { Peas, : }\end{array}$ & $\begin{array}{l}16 \cdot 8 \\
12 \cdot 4 \\
21 \cdot 0\end{array}$ & $\begin{array}{r}43 \cdot 3 \\
2 \cdot 1 \\
4 \cdot 0\end{array}$ & $\begin{array}{r}0.9 \\
27 \cdot 5 \\
55 \cdot 9\end{array}$ & $\begin{array}{l}35 \cdot 3 \\
57 \cdot 8 \\
18 \cdot 9\end{array}$ & $\begin{array}{l}\text { Quantity not } \\
\text { estimated. }\end{array}$ \\
\hline
\end{tabular}

With regard to the formation of gas and the processes of fermentation we note-

1. Air bubbles are swallowed when food is taken. The 0 thereof is rapidly absorbed by the walls of the intestinal tract, so that in the lower part of the large intestine, even traces of $\mathrm{O}$ are absent. In exchange, the blood-vessels in the intestinal wall give off $\mathrm{CO}_{2}$ into the intestine, so that a part of the $\mathrm{CO}_{2}$ in the intestine is derived by diffusion from the blood.

2. $\mathrm{H}$ and $\mathrm{CO}_{2} \mathrm{NH}_{3}$ and $\mathrm{CH}_{4}$ are also formed from the intestinal contents by fermentation, which takes place even in the small intestine (Planer).

Fungi as Exciters of Fermentation.-The chief agents in the production of fermentations, putrefaction and other similar decompositions are undoubtedly the group of the fungi called Schizomycetes. They are small unicellular organisms of various forms, globular (Micrococcus), short rods (Bacterium), long rods (Bacillus), or spiral threads (Vibrio, Spirillum, Spirochata). The mode of reproduction is by division, and they may either remain single or unite to form colonies. Each organism is usually capable of some degree of motion. They produce profound chemical changes in the fluids or media in which they grow and multiply, and these changes depend upon the vital activity of their protoplasm. These minute microscopic organisms take certain constituents from the "putrient fluids" in which they live, and use them partly for building up their own tissues and partly for their own metabolism. In these processes, some of the substances so absorbed and assimilated undergo chemical changes, some ferments seem thereby to be produced, which in their turn may act upon material present in the nutritive fluid.

These fungi consist of a capsule or envelope enclosing protoplasmic contents. Many of them are provided with excessively delicate cilia, by means of which they move about. The new organisms produced by the division of pre-existing ones, sometimes form large colonies visible to the naked eye, the individual fungi being united by a jelly-like mass, the whole constituting zoogloea. In some fungi, reproduction takes place by spores; more especially when the nutrient fluids are poor in nutritive materials. The bacteria form longer rods or threads which are jointed, and in each joint or segment small (1-2 $\mu$ ) highly refractive globules or spores are developed (Fig. 148, 7). In some cases, as in the butyric acid fermentation, the rods 
become fusiform before spores are formed. When the envelope of the mother-cell is ruptured or destroyed, the spores are liberated, and if they fall upon or into a suitable medium, they germinate and reproduce organisms similar to those from which they sprung. The process of spore-production is illustrated in Fig. 147, 7, 8, 9 , and in $1,2,3,4$ is shown the process of germination in the butyric acid fungus. The spores are very tenacious of life; they may be dried when they resist death for a very long time; some of them are not killed by being boiled. Some fungi exhibit their vital activities only in the presence of $\mathrm{O}$ (Aërobes), while others require the exclusion of $\mathrm{O}$ (Anaërobes, Pasteur). According to the products of their action, they are classified as follows:-Those that produce fermentations (zymogenic schizomycetes); those that produce pigments (chromogenic): those that produce disagreeable odlours, as during putrefaction (bromogenic); and those that when introduced into the living tissues of other organisms produce pathological conditions, and even death (patlogenic). All these different kinds occur in the human body.
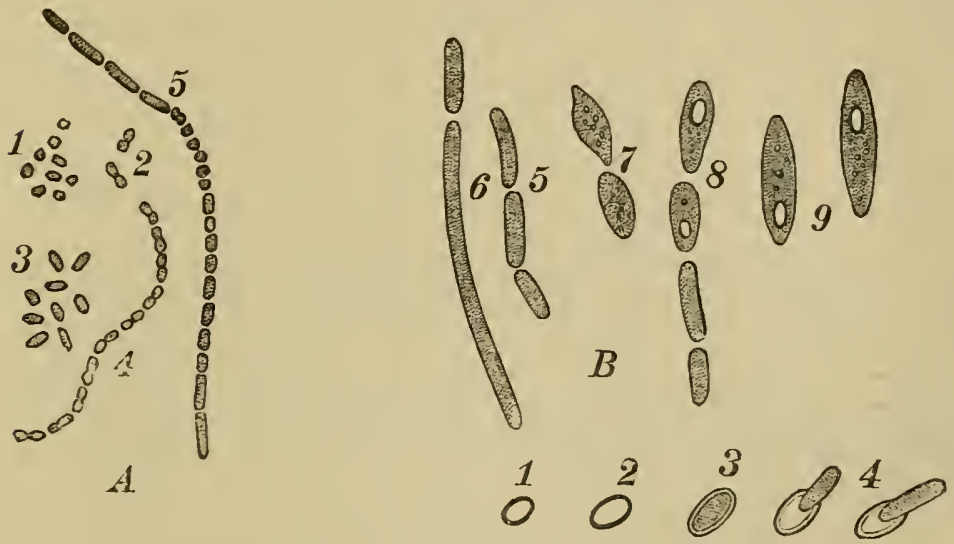

Fig. 147 .

A, Bacterium accti in the form of-cocei (1); diplococci (2); short rods (3), and jointed threads $(4,5)$. B, Bacillus butyricus-(1) isolated spores; $(2,3,4)$ germinating condition of the spores; $(5,6)$ short and long rods; $(7,8,9)$ formation of spores within a cellular fungus.

When we consider that numerous fungi are introduced into the intestinal canal with the food and drink-that the temperature and other conditions within this tube are specially farourable for their development; that there also they meet with sufficient pabulum for their development and reproduction-we cannot wonder that a rich crop of these organisms is met with in the intestine, and that they produce these numerous decompositions.

I. Fermentation of the Carbo-hydrates.-(1.) Bacterium lacticum (Cohn), (Ferment lactique, Pasteur) are biscuit-shaped cells, 1.5-3 $\mu$ in length, arranged in groups or isolated. They split up sugar into lactic acid;

$$
1 \text { grape-sugar }=\mathrm{C}_{6} \mathrm{H}_{12} \mathrm{O}_{6}=2\left(\mathrm{C}_{3} \mathrm{H}_{6} \mathrm{O}_{3}\right)=2 \text { milk-sugar. }
$$

Milk-sugar $\left(\mathrm{C}_{12} \mathrm{H}_{22} \mathrm{O}_{4}\right)$ may be split up by the same ferment causing it to take up $\mathrm{H}_{2} \mathrm{O}$, and forming 2 molecules of grape-sugar, $2\left(\mathrm{C}_{6} \mathrm{H}_{12} \mathrm{O}_{6}\right)$, which are again split into 4 molecules of lactic acid, $4\left(\mathrm{C}_{3} \mathrm{H}_{6} \mathrm{O}_{3}\right)$. 
The fungi which occur everywhere in the atmosphere are the cause of the spontaneous acidification, and subsequent coagulation of milk.-See Milk.

(2.) Bacillus butyricus (B. 'amylobacter, Van Tieghem; Clostridium butyricum, Vibrion butyrique, Pasteur), which in the presence of starch is often coloured blue by iodine, changes lactic acid into butyric acid, together with $\mathrm{CO}_{2}$ and $\mathrm{H}$ (Prazmowski).

$$
2\left(\mathrm{C}_{3} \mathrm{H}_{6} \mathrm{O}_{3}\right) \text { lactic acid }=\left\{\begin{array}{c}
\mathrm{C}_{4} \mathrm{H}_{8} \mathrm{O}_{3}=1 \text { butyric acid. } \\
2\left(\mathrm{CO}_{2}\right)=2 \text { carbonic acid. } \\
4 \mathrm{H}=4 \text { hydrogen. }
\end{array}\right.
$$

This fungus (Fig. 147, B) is a true anaërobe, and grows only in the absence of $O$. The lactic acid fungus uses $O$ very largely, and is, therefore, its natural precursor. The butyric acid fermentation is the last change undergone by many carbohydrates, especially of starch and inulin. It takes place constantly in the fæces.

(3.) A fungus, whose nature is not yet determined, causes alcohol to be formed from carbohydrates (Fitz). The presence of yeast may cause the formation of alcohol in the intestine, and in both cases also from milk-sugar, which first becomes changed into dextrose (p. 298, I).

(4.) Bacterium aceti (Fig. 147, A) converts alcohol into acetic acid outside the body. Alcohol $\left(\mathrm{C}_{2} \mathrm{H}_{6} \mathrm{O}\right)+\mathrm{O}=\mathrm{C}_{2} \mathrm{H}_{4} \mathrm{O}$ (Aldehyd) $+\mathrm{H}_{2} \mathrm{O}$. Acetic acid $\left(\mathrm{C}_{2} \mathrm{H}_{4} \mathrm{O}_{2}\right)$ is formed from aldehyd by oxidation. According to Nägeli, the same fungus causes the formation of a small amount of $\mathrm{CO}_{2}$ and $\mathrm{H}_{2} \mathrm{O}$. As the acetic fermentation is arrested at $35^{\circ} \mathrm{C}$., this fermentation cannot occur in the intestine, and the acetic acid, which is constantly found in the fæces, must be derived from another source. During putrefaction of the proteids with exclusion of air, acetic acid is produced (Nencki).

(5.) Sturch and cellulose are partly dissolved by the schizomycetes of the intestine. If cellulose be mixed with cloacal-mucus (HoppeSeyler), or with the contents of the intestine (Tappeiner), $n$ molecules, $\left[n\left(\mathrm{C}_{6} \mathrm{H}_{10} \mathrm{O}_{5}\right)\right.$, take up $n$ molecules of water, $+n\left(\mathrm{H}_{2} \mathrm{O}\right)$, and produce three times $n$ molecules $\mathrm{CO}_{2}$, and three times $n$ molecules of marshgas $3 n\left(\mathrm{CH}_{4}\right)$.

(6.) Fungi, whose nature is unknown, can partly transform starch (? and cellulose) into sugar, others excrete invertin-e.g., the Leukonostoc mesenteriodes, which develops in the juice of turnips. Invertin changes cane-sugar into invert-sugar ( $(183, \mathrm{II}, 5)$.

II. Fermentation of the Fats.-In certain putrefactive conditions, organisms of an unknown nature can callse neutral fats to take up water and split into glycerine and fatty acids. Glycerine- $-\mathrm{C}_{3} \mathrm{H}_{5}$ $(\mathrm{HO})_{3}$-is a triatomic alcohol, and is capable of undergoing several fermentations, according to the fungus which acts upon it. With a. neutral reaction, in addition to succinic acid, a number of fatty acids, $\mathrm{H}$ and $\mathrm{CO}_{2}$, are formed.

Fitz found under the influence of the hay-fungus (Bacillus subtilis, Fig. 148) alcohol with caproic, butyric, and acetic acids; in other cases butylic alcohol is the chief product. 
The fatty acids, especially as chalk soaps, form an excellent material for fermentation. Calcium formiate mixed with cloacal-mucus ferments and yields calcium carbonate, $\mathrm{CO}_{2}$ and $\mathrm{H}$; calcium acetate, under the same conditions, produces calcium carbonate, $\mathrm{CO}_{2}$ and $\mathrm{CH}_{4}$. Amongst the oxy-acids, we are acquainted with the fermentations of lactic, glycerinic, malic, tartaric, and citric acids.

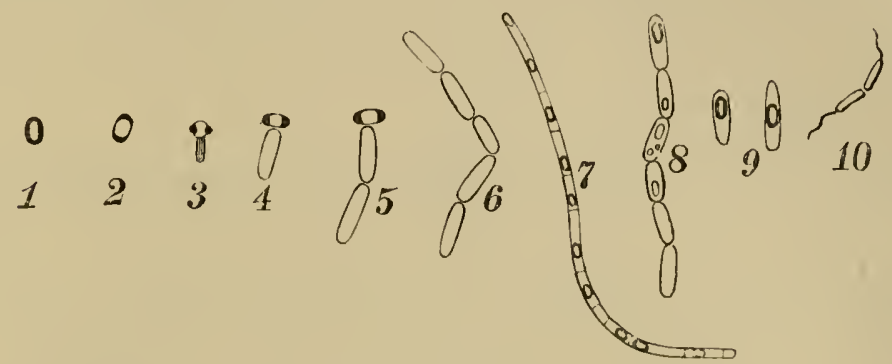

Fig. 14S.

Bacillus subtilis-1, spore ; 2, 3, 4, germination of the spores ; 5,6 , short rods ; 7 , jointed thread, with the formation of spores in each segment or cell; 8 , short rods, some of them containing spores; 9 , spores in single short rods; 10 , fungus with a cilium.

According to Fitz, lactic acid (in combination with chalk), produces propionic and acetic acids, $\mathrm{CO}_{2} \mathrm{H}_{2} \mathrm{O}$. Other ferments cause the formation of valerianic acid. Glycerinic acil, in addition to alcohol and succinic, yields chiefly acetic acid; malic acid forms succinic and acetic acid. The other acids above cnumerated yield somewhat similar products.

III. Fermentation of the Proteids.-There do not seem to be fungi of sufficient activity in the intestine to act upon undigested proteids and their derivatives. Many schizomycetes, however, can produce a peptonising ferment.

We have already seen that pancreatic digestion (p. 341), acts upon the proteids, forming, among other products, amido-acids, leucin, tyrosin, and other bodies. Under normal conditions, this is the greatest decomposition produced by the pancreatic juice. The putrefactive fermentation of the large intestine (Hüffner, Nencki) causes further and more profound decompositions. Leucin $\left(\mathrm{C}_{6} \mathrm{H}_{13} \mathrm{NO}_{2}\right)$ takes up two molecules of water and yields valerianic acid $\left(\mathrm{C}_{5} \mathrm{H}_{10} \mathrm{O}_{2}\right)$, ammonia, $\mathrm{CO}_{2}$ and $2\left(\mathrm{H}_{2}\right)$; glycin behaves in a similar manner. Tyrosin $\left(\mathrm{C}_{9} \mathrm{H}_{11} \mathrm{NO}_{3}\right)$ is decomposed into indol $\left(\mathrm{C}_{8} \mathrm{H}_{7} \mathrm{~N}\right)$, which is constantly present in the intestine (Kühne), $\mathrm{CO}_{2} \mathrm{H}_{2} \mathrm{ON}$ (Nencki). If $\mathrm{O}$ be present, other decompositions take place. These putrefactive products are absent from the intestinal canal of the fotus and the newly-born (Senator). During the putrefactive decomposition of proteids, $\mathrm{CO}_{2} \mathrm{H}_{2} \mathrm{~S}$, also $\mathrm{H}$ and $\mathrm{CH}_{4}$, are formed; the same result is obtained by boiling them with 
alkalies. Gelatin, under the same conditions, yields much leucin and ammonia, $\mathrm{CO}_{2}$, acetic, butyric, and valerianic acids, and glycin (Nencki). Mucin and nuclein undergo no change. Artificial pancreatic digestion experiments rapidly tend to undergo putrefaction.

The substance which causes the peculiar fæcal odour is produced by putrefaction, but its nature is not known. It clings so firmly to indol and skatol that these substances were formerly regarded as the odorous bodies, but when they are prepared pure they are odourless (Bayer). The above-mentioned putrefactive processes, which also occur in pancreas undergoing decomposition, may be interrupted by antiseptics (salicylic acid). The putrefactive products of the pancreas give a red colour or precipitate with chlorine water.

Indol.-Amongst the solid substances in the large intestine formed only by putrefaction is indol $\left(\mathrm{C}_{8} \mathrm{H}_{7} \mathrm{~N}\right)$, a substance which is also formed when proteids are heated with alkalies, or by overheating them with water to $200^{\circ} \mathrm{C}$. It is the stage preceding the indican in the urine. If the products of the digestion of the proteids-the peptones-are rapidly absorbed, there is only a slight formation of indol; but when absorption is slight, and putrefaction of the products of pancreatic digestion occurs, much indol is formed, and indican appears in the urine.

Jaffé found much indican in the urine in strangulated hernia, and when the small intestine was obstructed. Landois observed the same after the transfusion of heterogeneous blood.

A. Bayer prepared indigo-blue artificially from ortho-phenyl-propionic acid, by boiling it with dilute caustic soda, after the addition of a little grape-sugar. He obtained indol and skatol from indigo-blue. Hoppe-Seyler found that on feeding rabbits with ortho-nitrophenyl-proprionic acid, much indican was present in the urine.

Phenol $\left(\mathrm{C}_{6} \mathrm{H}_{6} \mathrm{O}\right)$ is formed by putrefaction in the intestine, and it is also formed when fibrin and pancreatic juice putrefy outside the body (Baumann), while Brieger found it constantly in the fæces. It seems to be increased by the same circumstances that increase indol (Salkowski), as an excess of indican in the urine is accompanied by an increase of phenylsulphuric acid in that fluid.

Hydrocinnamic acid (phenylpropionic acid) may also be obtained from putrefying flesh and fibrin. It is completely oxidised in the body into benzoic acid, and appears as hippuric acid in the urine. Thus is explained the formation of hippuric acid from a purely albuminous $\operatorname{diet}$ (E. and H. Salkowski).

Skatol $\left(\mathrm{C}_{9} \mathrm{H}_{9} \mathrm{~N}=\right.$ methylindol)-(Brieger), is a constant human fæcal substance, and has been prepared artificially by Nencki and Secretan from egg-albumin, by allowing it to putrefy for a long time under water. It also appears in the urine as a sulphuric acid compound. The excretin of human fæces, described by Marcet, is related to cholesterin, but its history and constitution are unknown. 
It is of the utmost importance, in connection with the processes of putrefaction, to determine whether they take place when oxygen is excluded or not (Pasteur). When $\mathrm{O}$ is absent, reductions take place; oxy-acids are reduced to fatty acids, and $\mathrm{HCH}_{4}$ and $\mathrm{H}_{2} \mathrm{~S}$ are formed; while the $\mathrm{H}$ may produce further reductions. If $\mathrm{O}$ be present, the nascent $\mathrm{H}$ separates the molecule of free ordinary oxygen $\left(=\mathrm{O}_{2}\right)$ into two atoms of active oxygen $(=0)$. Water is formed on the one hand, while the second atom of $\mathrm{O}$ is a powerful oxidizing agent (HoppeSeyler).

[It is not improbable that some substances, as sulphur, are in part renderer] soluble and absorbed by the action of the nascent hydrogen evolved by the schizomycetes, forming a soluble hydrogen compound with the substance (Matthew Hay).]

It is remarkable that the putrefactive processes, after the development of phenol, indol, skatol, cresol, phenylpropionic and phenylacetic acids, are afterwards limited, and after a certain concentration is reached they cease altogether. The putrefactive process produces antiseptic substances which kill the microorganisms (Wernich), so that we may assume, that these substances limit to a certain extent the putrefactive processes in the intestine.

The reaction of the intestine immediately below the stomach is acid, but the pancreatic and intestinal juices cause a neutral and afterwards an alkaline reaction, which obtains along the whole small intestine. In the large intestine, the reaction is generally acid, on account of the acid fermentation and the decomposition of the ingesta and the freces.

\section{Processes in the Large Intestine.}

Within the large intestine, the fermentative and putrefactive processes are certainly more prominent than the digestive processes proper, as only a very small amount of the intestinal juice is found in it (Kühne). The absorptive function of the large intestine is greater than its secretory function, as at the beginning of the colon, its contents are thin and watery, but in the further course of the intestine they become more solid. Water and the products of digestion in solution are not the only substances absorbed, but under certain circumstances, unchanged fluid egg-albumin (Voit and Bauer, Czerny and Latschenberger), milk and its proteids (Eichhorst), flesh-juice, solution of gelatin, myosin with common salt, may also be absorbed. Experiments with acid-albumin, syntonin, or blood-serum gave no result. Toxic substances are absorbed more rapidly than from the stomach (Savory). The frcal matters are formed or rather shaped in the lower part of the gut. The cæcum of many animals, e.g., rabbit, is of considerable size, and in it fermentation seems to occur with considerable energy, giving rise to an acid reaction. In man, the chief function of 
the cæcum is absorption, as is shown by the great number of lymphatics in its walls. From the lower part of the small intestine and the cæcum onwards, the ingesta assume the fæcal odour.

The amount of fæces is about 170 grms. (60-250 grms.) in 24 hours; but if much indigestible food be taken, it may be as much as 500 grms. The amount is less, and the absolute amount of solids is less, after a diet of flesh and albumin, than after a vegetable diet. The fæces are rendered lighter by the evolution of gases, and hence they float on water.

The consistence of the frces depends on the amount of water present-it is usually about 75 per cent. The amount of water depends partly on the food-pure flesh diet causes relatively dry fæces, while substances rich in sugar yield frees with a relatively large amount of water. The quantity of water taken has no effect upon the amount of water in the fæces. But the energy of the peristalsis has this effect, that the more energetic it is, the more watery the frces are, because sufficient time is not allowed for absorption of the fluid from the ingesta. Paralysis of the blood- and lymph-vessels, after section of the nerves, leads to a watery condition of the fæces (p. 371).

The reaction is often acid in consequence of lactic acid being developed from the carbo-hydrates of the food. Numerous other acids produced by putrefaction are also present $(\S 184)$. If much ammonia be formed in the lower part of the intestine, a neutral or even alkaline, reaction may obtain. A copious secretion of mucus favours the occurrence of a neutral reaction.

The odour, which is stronger after a flesh diet than after a vegetable diet, is caused by some fæcal products of putrefaction, which have not yet been isolated; also by volatile fatty acids and by sulphuretted hydrogen, when it is present.

The colour of the fæces depends upon the amount of altered bilepigments mixed with them, whereby a bright yellow to a dark-brown colour is obtained.

The colour of the food is also of importance. If much blood be present in the food, the fæces are almost brownish-black from hæmatin; green vegetables = brownish green from chlorophyll; bones $(\mathrm{dog})=$ white from the amount of lime; preparations of iron = black from the formation of sulphide of iron. [The pigment of claret tinges the fæces.]

The fæces contain-

(1.) The unchanged residue of animal or vegetable tissues used as food; hairs, horny and elastic tissues; most of the cellulose, woody fibres, spiral vessels of vegetable cells, gum.

(2.) Portions of digestible substances, especially when these have been taken in too large amount, or when they have not been sufficiently 
broken up by chewing. Portions of muscular fibres, ham, tendon, cartilage, particles of fat, coagulated albumin-vegetable cells from potatoes and vegetables, raw starch, dc.

All food yields a certain amount of residue-white bread, $3 \cdot 7$ p.c.; rice, $4 \cdot 1$ p.c.; flesh, 4.7 p.c.; potatoes, $9 \cdot 4$ p.c.; cabbage, 14.9 p.c.; black bread, 15 p.c.; yellow turnip, 20.7 p.c. (Rubner).

(3.) The decomposition products of the bile-pigments, which do not now give the Gmelin-Heintz reaction; as well as the altered bile-acids $(\$ 177,2)$. This reaction, however, may be obtained in pathological stools, especially in those of a green colour; unaltered bilirubin, biliverdin, glycocholic, and taurocholic acids occur in meconium (Zweifel, Hoppe-Seyler).

(4.) Unchanged mucin and nuclein-the latter occasionally after a diet of bread, together with cylindrical epithelium in a state of partial solution, from the intestinal canal, and occasional drops of oil. Cholesterin is very rare. The less the mucus is mixed with the frces, the lower the part of the intestine from which it was derived (Nothnagel).

(5.) After a milk diet and also after a fatty diet, crystalline needles of lime, combined with fatty acids, chalk-soaps, constantly occur, even in sucklings (Wegscheider). Even unchanged masses of casein and fat occur during the milk-cure. Compounds of ammonia, with the acids mentioned at p. 375 , the result of putrefaction, belong to the frecal matters (Brieger).

(6.) Amongst inorganic residuces, soluble salts rarely occur in the freces because they diffuse readily-e.g., common salt, and the other alkaline chlorides, the compounds of phosphoric acid, and some of those of sulphuric acid. The insoluble compounds, of which ammoniacomagnesic or triple phosphate, neutral calcic phosphate, yellow coloured lime salts, calcium carbonate, and magnesium phosphate are the chief, form 70 p.c. of the ash. Some of these insoluble substances are derived from the food, as lime from bones, and in part they are excreted after the food has been digested, as ashes are eliminated from food which lias been burned.

The excretion of inorganic substances is sometimes so great, that they form incrustations around other fæcal matters. Usually ammoniaco-magnesic phosphate occurs in large crystals by itself, or it may be mixed with magnesium phosphate.

(7.) A considerable portion of normal fæcal matter consists of micrococci and microbacteria (Bacterium termo-Woodward, Nothnagel). Bacillus subtilis is not very plentiful, while yeast is seldom absent (Frerichs, Nothnagel). In stools that contain much starch, the bacillus amylobacter, which is tinged blue with iodine, occurs (p. 374), 
and other small globular or rod-like fungi, which give a similar reaction (Nothnagel, Uffelmann). Bienstock, who has devoted attention to the microbes of the fæces, finds two kinds of bacteria in all fæces; both resemble B. subtilis (Fig. 148) very closely, but they are distinguished from it by their mode of development. They do not cause any fermentative action. There are several other forms found in the frecal evacuations, under different circumstances.

The changes of the intestinal contents have been studied on persons with an accidental intestinal fistula, or an artificial anus.

\section{Pathological Variations.}

A. The taking of food may be interfered with by spasm of the muscles of mastication (usually accompanied by general spasms), stricture of the œsophagus, by cicatrices after swallowing caustic fluids (e.g., caustic potash, mineral acids), or by the presence of a tumour, such as cancer. Inflammation of all kinds in the mouth or pharynx interferes with the taking of food. Impossibility of swallowing occurs as part of the general phenomena in disease of the medulla oblongata, in consequence of paralysis of the motor centre (superior olives) for the facial, vagus, and hypoglossal nerves, and also for the afferent or sensory fibres of the gloss-pharyngeal, vagus, and trigeminus. Stimulation or abnormal excitation of these parts causes spasmodic swallowing, and the disagreeable feeling of a constriction in the neck (globus hystericus).

B. The secretion of saliva is diminished during inflammation of the salivary glands; occlusion of their ducts by concretions (salivary calculi); also by the use of atropin, daturin, and during fever, whereby the secretory (not the vaso-motor) fibres of the chorda appear to be paralysed (p. 287). When the fever is very high, no saliva is secreted. The saliva secreted during moderate ferer is turbid and thick, and usually acid. As the fever increases, the diastatic action of the saliva diminishes (Uffelmann). The secretion is increased, by stimulation of the buccal nerves (inflammation, ulceration, trigeminal neuralgia), so that the saliva is secreted in great quantity. Mercury and jaborandi cause secretion of saliva, the former causing stomatitis, which excites the secretion of saliva reflexly. Even diseases of the stomach accompanied by vomiting, cause secretion of saliva. A very thick tenacious sympathetic saliva occurs when there is violent stimulation of the vascular system during sexual excitement, and also during certain psychical conditions. The reaction of the saliva is acid in catarrh of the mouth, in fever in consequence of decomposition of the buccal epithelium, and in diabetes mellitus in consequence of acid fermentation of the saliva which contains sugar. Hence, diabetic persons often suffer from carious teeth. Unless the mouth of an infant be kept scrupulously clean, the saliva is apt to become acid.

C. Disturbances in the activity of the musculature of the stomach may be due to paralysis of the muscular layers, whereby the stomach becomes distended, and the ingesta remain a long time in it. A special form of paralysis of the stomach is due to non-closure of the pylorus (Ebstein). This may be due to disturbances of innervation of a central or peripheral nature, or there may be actual paralysis of the pyloric sphincter, or anæsthesia of the pyloric mucous membrawe, which acts reflexly upon the sphincter muscle; and lastly, it may be due to the reflex impulse not being transferred to the efferent fibre within the nerve centre. Abnormal activity of the gastric musculature hastens the passage of the ingesta into the intestine; vomiting often occurs. 
Gastric digestion is delayed by violent bodily or mental exercise, and sometimes it is arrested altogether. Sudden mental excitement may have the same effect. These effects are very probably caused through the vaso-motor nerves of the stomach. Feeble and imperfect digestion may be of a purely nervous nature (Dyspepsia nerrosa-Leube; Neurasthenia gastrica-Burkart). [According to J. W. Fraser, all infused beverages, tea, coffee, cocoa, retard the peptic digestion of proteids, with few exceptions. The retarding action is less with coffee than with tea. The tannic acid and volatile oil seem to be the retarding ingredients in teas.]

Inflammatory or catarrhal affections of the stomach, as well as ulceration and new formations, interfere with digestion, and the same result is caused by eating too much food which is difficult of digestion, or taking too much highly spiced sauces or alcohol. In the case of a dog suffering from chronic gastric catarrh, Gritzner observed that the secretion took place continuously, and that the gastric juice contained little pepsin, was turbid, sticky, feebly acid, and even alkaline. The introduction of food did not alter the secretion, so that in this condition the stomach really obtains no rest. The chief cells of the gastric glands were turbid. Hence, in gastric catarrb, we ought to eat frequently, but take little at a time, while at the same time dilute $(0.4$ p.c.) hydrochloric acid ought to be administered. Small doses of common salt seem to aid digestion. [In cases of carcinoma of the stomach, the acid reaction of the gastric juice is almost invariably absent.]

Feeble digestion may be caused either by imperfect formation of acid or pepsin, so that both substances may be administered in such a condition. [It may also be due to deficient muscular power in the wall of the stomach.] In other cases, lactic, butyric, and acetic acids are formed, owing to the presence of lowly organisms. In such cases, small doses of salicylic acid are useful (HoppeSeyler), together with some hydrochloric acid. Pepsin need not be given often, as it is rarely absent, even from the diseased gastric mucous membrane. Albumin has been found in the gastric juice in cases of gastric catarrh and cholera.

D. Digestion during Fever and Anæmia.-Beaumout found that in the case of Alexis St. Martin, when fever occurred, a small amount of gastric juice was secreted; the mucous membrane was dry, red, and irritable. Dogs suffering from septicæmic fever, or renderel anæmic by great loss of blood, secrete gastric juice of feeble digestive power and containing little acid (Manassein). Hoppe-Seyler investigated the gastric juice of a typhus patient, in which Von der Velden found no free acid, and he found the same in gastric catarrh, fever, and in cancer of the stomach. The gastric juice of the typhus patient did not digest artificially, even after the addition of hydrochloric acid. The diminution of acid, under these circumstances, favours the occurrence of a neutral reaction, so that, on the one hand, digestion cannot proceed, and, on the other, fermentative processes (lactic and butyric acid fermentations with the evolution of gases) occur. These results are associated with the presence of micro-organisms and Sarcina ventriculi (Goodsir). He advises the administration of hydrochloric acid and pepsin, and when there are symptoms of fermentation, small doses of salicylic acid. Uffelinann found the secretion of a peptone-forming gastric juice ceased in fever, when the fever is severe at the outset, when a feeble condition occurs, or when the temperature is very high. The amount of juice secreted is certainly diminished during fever. The excitability of the mucous membrane is increased, so that romiting readily occurs. The increased excitability of the vaso-motor nerves during fever (Heidenhain) is disadvantageous for the secretion of the digestive fluids. Beaumont observed that fluids are rapidly absorbed from the stomach during fever, but the absorption of peptones is diminished on account of the accompanying catarrhal condition of the stomach, and the altered functional activity of the muscularis mucosæ (Leube).

Many salts when given in large amount disturb gastric digestion-e.g., the 
sulphates. While the alkaloids, morphia, strychnia, digitalin, narcotin, veratria have a similar action; quinine favours it (Wolberg). In some nervous individuals a "peristaltic un-rest of the stomach," conjoined with a dyspeptic condition, occurs (Kussmaul).

E. In acute diseases, the secretion of bile is affected ; it becomes less in amount and more watery, i.e., it contains less specific constituents. If the liver undergoes great structural change, the secretion may be arrested.

F. Gallstones.-When decomposition of the bile occurs, gallstones are formed in the gall-bladder or in the bile-ducts. Some are white, and consist almost entirely of stratified layers of crystals of cholesterin. The brown forms consist of bilirubinlime and calcium carbonate, often mixed with iron, copper, and manganese. The gallstones in the gall-bladder become facetted by rubbing against each other. The nucleus of the white stones often consists of chalk and bile colouring matters, together with nitrogenous residues, derived from shed epithelium, mucin, bile salts and fats. Gallstones may occlude the bile-duct and cause cholæmia. When a small stone becomes impacted in a duct, it gives rise to excessive pain constituting hepatic colic, and may even cause rupture of the bile-duct with its sharp edges.

G. Nothing certain has been determined regarding the pancreatic secretion in disease, but in fever, it appears to be diminished in amount and digestive activity. The suppression of the pancreatic secretion [as by a cancerous tumour of the head of the pancreas] is often accompanied by the appearance of fat in the form of globules or groups of crystals in the fæces.

$H$. Constipation is a most important derangement of the digestive tract. It may be cuused by-1. Conditions which obstruct the normal channel, e.g., constriction of the gut from stricture-in the large gut after dysentery, tumours, rotation on its axis of a loop of intestine (volvulus), or invagination, occlusion of a coil of gut in a hernial sac, or by the pressure of tumours or exudations from without, or congenital absence of the anus. 2. Too great dryness of the contents, caused by too little water in the articles of diet, diminution of the amount of the digestive secretions, e.g., of bile in icterus; or in consequence of much fluid being given off by other organs, as after copious secretion of saliva, milk, or in fever. 3. Variations in the functional activity of the muscles and motor-nervous apparatus of the gut may cause constipation, owing to imperfect peristalsis. This condition occurs in inflammations, degenerations, chronic catarrh, diaphragmatic inflammation. Affections of the spinal cord, and sometimes also of the brain, are usually accompanied by slow evacuation of the intestine. Whether diminished mental activity and hypochoudrias are the cause of or are caused by constipation is not proved. Spasmodic contraction of a part of the intestine may cause temporary retention of the intestinal contents, and, at the same time, give rise to great pain or colic; the same is true of spasm of the anal sphincter, which may be excited reflexly from the lower part of the gut. The fæcal masses in constipation are usually hard and dry, owing to the water being absorbed; hence they form large masses or scybala within the large intestine, and these again give rise to new resistance.

Amongst the reagents which prevent evacuation of the bowels, some paralyse the motor apparatus temporarily, e.g., opium, morphia; some diminish the secretion of the intestinal mucons membrane, and cause constriction of the blood-vessels, as tannic acid, vegetables containing tannin, alum, chalk, lead acetate, silver nitrate, bismuth nitrate.

I. Increased evacuation of the intestinal contents is usually accompanied by a watery condition of the frecs, constituting diarrhœa.

The causes are:-

1. A too rapid movement of the contents through the intestine, chiefly through the large intestine, so that there is not time for the normal amount of absorption to take place. The increased peristalsis depends upon stimulation of the motor- 
nervous apparatus of the intestine, usually of a reflex nature. Rapid transit of the contents through the intestine causes the evacuation of certain substances, which cannot be digested in so short a time.

2. The stools become thinner from the presence of much water, mucus, and the admixture with fat, ancl by eating fruit and vegetables. In rare cases, when the evacnations contain much mucin, Charcot's crystals (Fig. 115, c) occur. In ulceration of the intestine, lencocytes (pus) are present (Nothnagel).

3. Diarrhea may occur as a consequence of disturbance of the diffusion-processes through the intestinal walls, as in affections of the epithelium, when it becomes swollen in inflammatory or catarrhal conditions of the intestinal mucons membranc. [Irritation over the abclomen, as from the subcutaneons injection of small quantities of saline solutions, canses cliarrhœa (M. Hay).]

4. It may also be due to increased secretion into the intestine, as in capillary diffusion, when magnesium sulphate in the intestine attracts water from the blood.

The same occurs in clolcra, when the stools are copious and of a rice-water character, and are loaded with epithelial cells from the villi. The transudation into the intestine is so great that the blood in the arteries becomes very thick, and may even on this account cease to circulate:

Transuclation into the intestine also takes place as a consequence of paralysis of the vaso-motor nerves of the intestine. This is perliaps the case in diarrhoea followin: upon a cold. Certain substances seem directly to excite the secretory organs of the intestines or their nerves, such as the drastic purgatives ( $p .3(64)$. Pilocarpin injected into the blood causes great secretion (Masloff).

During febrilc conditions, the secretion of the intestinal glands seems to be altered quantitatively and qualitatively, with simultaneons alteration of the functional activity of the musculature and the organs of absorption, while the excitability of the mucous membrane is increased (Uffelmann). It is important to note that in many acute febrile diseases, the amount of common salt in the urine climinishes, and increases again as the fever subsides.

\section{8\%. Comparative.}

Salivary Glands.-Amongst Mammals the herbivora have larger salivary glands than the carnivora; while midway between both are the omnivora. The whale has no salivary glands. The pinnipedia have a small parotid, which is absent in the echidna. The dog and many carnivora have a special gland lying in the orbit, the orbital or zygomatic gland. In Birds the salivary glands open at the angle of the mouth, in them the parotid is absent. Amongst Reptiles the parotid of some species is so changed as to form poison glands; the tortoise has sublingual glands; reptiles have labial glands. The Amphibia and Fishes have merely small glands scattered over the mouth. The salivary glands are large in Insects; some of them secrete formic acid. The salivary glands are well cleveloped in molluses, and the saliva of dolium galea contains more than 3 p.c. of free sulphuric acid (?) The cephalopods have double glands.

A Crop is not present in any mammal ; the stomach is either simple, as in man, or, as in many rodents, it is divided into two halves, into a cardiac and a pyloric portion. The stomach of ruminants is compound, and consists of four cavities. 'The intestine is short in flesh-eating animals and long in herbivora. The cæeum is a very large and important digestive organ in herbirora, and in most rodents; it is small in man, and absent in carnirora. The osophagus in grain-eating Birds not unfrequently has a blind diverticulum or crop for softening the food. In the crop of pigeons during the breeding season, there is formed a peculiar secretion - "pigeon's milk," which is used to feed the young (J. Hunter). The 
stomach consists of a glandular proventriculus and a strong muscular stomach which is covered with horny epithelium and triturates the food. There are usually two fluid diverticula on the small intestine near where it joins the large gut. In Fishes the intestinal canal is usually simple; the stomach is merely a dilatation of the tube; and at the pylorus there may be one, but usually many, blind glandular appendages (the appendices pyloricæ). There are usually longitudinal folds in the intestinal mucous membrane, but in some fishes, e.g., the shark, there is a spiral valve. [It is curious to find that the inversive (cane-sugar) ferment is wanting in the herbivora, as the cow, horse, and sheep, but is present in the carnivora, as the dog and cat. It is also met with in birds and reptiles, and in many of the invertebrates, as the ordinary earth-worm (Matthew Hay).]

In Amphibia and Reptiles the stomach is a simple dilatation; the gut is larger in vegetable feeders than in flesh feeders. The liver is never absent in vertebrates, although the gall-bladder frequently is. The pancreas is absent in some fishes.

Digestion in Plants.-The observations on the albumin-digesting power of some plants (Canby, 1869; Ch. Darwin, 1875) are extremely interesting. The sundew or drosera has a series of tentacles on the surface of its leaves, and the tentacles are provided with glands. As soon as an insect alights upon a leaf it is suddenly seized by the tentacles, the glands pour out an acid juice over the prey, which is gradually digested; all except the chitinous structures. The secretion, as well as the subsequent absorption of the products of digestion, are accomplished by the activity of the protoplasm of the cells of the leaves. The digestive juice contains a pepsin-like ferment and formic acid. Similar phenomena are manifested by the Venus flytrap (Dionæa), by pinguicula, as well as by the cavity of the altered leaves of nepenthes. About fifteen species of these "insectivorous" or carnivorous plants are known.

\section{Historical.}

Digestion in the Mouth. - The Hippocratic school was acquainted with the vessels of the teeth; Aristotle ascribed an uninterrupted growth to these organs, and he farther noticed that animals that were provided with horns, and had cloven hoofs, had an imperfect set of teeth-the upper incisors were absent. It is curious to note that in some cases where men have had an excessive formation of hairy appendages, the incisor teeth have been found to be badly developed. The muscles of mastication were known at an early period; Vidius ( +1567$)$ described the tempero. maxillary articulation with its meniscus. The older observers regarded the saliva as a solvent, and in addition, many bad qualities, especially in starving animals, were ascribed to it. This arose from the knowledge of the saliva of mad animals, and the parotid saliva of poisonous snakes. Human saliva, without organisms, is poisonous to birds (Gautier). The salivary glands have been known for a long time. Galen (131-203 A.D.) was acquainted with Wharton's duct, and Aëtius (270 A.D.) with tlie sub-maxillary and sub-lingual glands. Hapel de la Chenaye (1780) obtained large quantities of saliva from a horse, in which he was the first to make a salivary fistula. Spallanzani (1786) asserted that food mixed with saliva was more easily digested than food moistened with water. Hamberger and Siebold investigated the reaction, consistence, and specific gravity of saliva, and found in it mucus, albumin, common salt, calcium, and sodium phosphates. Berzelius gave the name ptyalin to the characteristic organic constituent of saliva, but Leuchs (1S31) was the first to detect its diastatic action.

Gastric Digestion.-Digestion was formerly compared to boiling, whereby solution was effected. According to Galen, only substances that have been dissolved passed through the pylorus into the intestine. He described the movements of the stomach and the peristalsis of the intestines. Aelian gave names to 
the four stomachs of the ruminants. Vidius $(+1567)$ noticed the numerous small apertures of the gastric glands. Van Helmont ( $\dagger 1644)$ expressly notices the acidity of the stomach. Reaumur (1752) knew that a juice was secreted by the stomach, which effected solution, and with which he and Spallanzani performed experiments on digestion outside the body. Carminati (1785) found that the stomachs of carnivora during digestion secreted a very acid juice. Prout (1824) discovered the hydrochloric acid of the gastric juice, Sprott and Boyd (1836) the glands of the gastric mucous membrane, while Wasmann and Bischoff noted the two kinds of gastric glands. After Beaumont (1834) had made his observations upon Alexis St. Martin, who had a gastric fistula, caused by a gunshot wound, Bassow (1842) and Blondlot (1843) made the first artificial gastric fistulæ upon animals. Eberle (1834) prepared artificial gastric juice. Mialhe called albumin, when altered by gastric digestion, albuminose; Lehmann, who investigated this substance more carefully, gave it the name peptone. Schwann isolated pepsin (1836), and established the fact of its activity in the presence of hydrochloric acid.

Pancreas, Bile, Intestinal Digestion.-The pancreas was known to the Hippocratic School; Maur. Hoffinann (1642) demonstrated its duct (fowl), and Wirsung described it in man. Regner de Graaf (1664) collected the pancreatic juice from a fistula, and Tiedmann and Gmelin found it to be alkaline, while Leuret and Lassaigne found that it resembled saliva. Valentin discovered its diastatic action, Eberle its emulsionising power, and Cl. Bernard (1846) its tryptic and fat-splitting properties. The last-mentioned function was referred to by Purkinje and Pappenheim (1836).

Aristotle characterised the bile as a useless excretion; according to Erasistratus (304 B.c.), fine invisible channels conduct the bile from the liver into the gallbladder. Aretaeus ascribed icterus to obstruction of the bile-duct. Benedetti (1493) described gall-stones. According to Jasolinus (1573), the gall-bladder is emptied by its own contractions. Sylvius de la Boë noticed the lymphatics of the liver (1640); Walaeus, the connective-tissue of the so-called capsule of Glisson (1641). Haller indicated the uses of bile in the digestion of fats.

The liver-cells were described by Henle, Purkinje, and Dutrochet (1838). Heynsius discovered the urea, and $\mathrm{Cl}$. Bernard (1853) the sugar in the liver, and he and Hensen (1857) found glycogen in the liver. Kiernan gave a more exact description of the hepatic blond-vessels (1S34). Beale injected the lymphatics, and Gerlach the finest bile-ducts. Schwann (1844) made the first biliary fistula; Demarcay particularly referred to the combination of the bile acids with soda (1S38); Strecker discovered the soda compounds of both acids, and isolated them.

Corn. Celsus mentions nutrient enemata (3-5 A.D.) Fallopius (1561) described the valvulæ eonniventes and villi of the intestinal mucous membrane, and the nervous plexus of the mesentery. The agminated glands or patches of Peyer were known to Severinus (1645). 


\section{Physiology of Absorption.}

\section{The Organs of Absorption.}

THE mucous membrane of the whole intestinal tract, as far as it is covered by a single layer of columnar epithelium-i.e., from the cardiac orifice of the stomach to the anus-is adapted for absorption. The mouth and osophagus, lined as they are by stratified squamous epithelium, are much less adapted for this purpose. Still, poisoning is caused by placing potassium cyanide in the mouth.

The channels of absorption in the intestinal tract are-(1) the capillary blood-vessels; and (2) the lacteals of the mucous membrane. Almost the whole of the substances absorbed by the former pass into the rootlets of the portal vein, and traverse the liver, while those that enter the lacteals really pass into lymphatics, so that the chyle passes through the thoracic duct, and is poured by it into the blood, where the thoracic duct joins the subclavian vein.

Watery solutions of salts-e.g., potassium iodide (in $\frac{1}{10}-1 \frac{1}{2}$ hours), grape-sugar, poisons, peptones, and in a still higher degree, alcoholic solutions of poisons are absorbed from the stomach.

The greatest area of absorption is undoubtedly the small intestine, especially its upper half (Landois and Lépine).

\section{Structure of the Small and Large Intestines.}

[The wall of the small intestine consists of four coats; which from without inwards are named serous, muscular, sub-mucous, and mucous.

The serous coat has the same structure as the peritoneum-i.e., a thin basis of fibrous tissue covered on its outer surface by endothelium.

The muscular coat consists of a thin outer longitudinal and an inner thicker circular layer of non-striped muscular fibres.

The sub-mucous coat consists of loose connective-tissue containing large bloodvessels and nerves, and it connects the muscular with the mucous coat.]

The mucous coat is the most internal coat, and its absorbing surface is largely increased by the presence of the valvulæ conniventes and villi. [The valvulce conniventes are permanent folds of the mucous membrane of the small intestine, arranged across the long axis of the gut. They pass round a half or more of the inner surface of the gut. They begin a little below the 
commencement of the duodenum, and are large and well marked in the duodenum, and remain so as far as the upper half of the jejunum, where they begin to become smaller, and finally disappear about the lower part of the ileum.] The villi are characteristic of the small intestine, and are confined to it; they occur everywhere as closely-set projections over and between the ralvulæ conniventes (Fig. 149). When the inner surface of the mucous membrane is examined in water, it has a velvety appearance owing to their presence. [They vary in length from $\frac{1}{50}$ to $\frac{1}{30}$ of an inch, are most numerous and largest in the upper part of the intestine, duodenum, and jejunum, where absorption is most active, but they are less abundant in the ileum. Their total number has been calculated at four millions by Krause.] Each villus is a projection of the entire mucous membrane, so that it contains within itself representatives of all the tissue elements of the mucosa. The orifices of

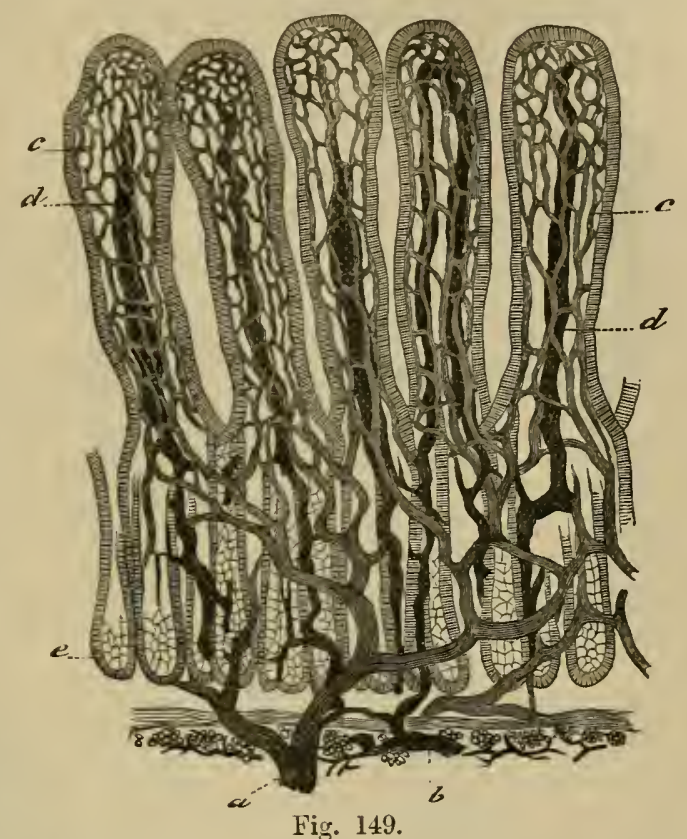

Mucous membrane of the small intestine of the dog; the lacteals are black and the blood-vessels lighter$a$, artery; $b$, lymphatic; $c$, plexus of capillaries in the villi: $d$, lacteal; $e$, Lieberkühn's glands. the glands of Lieberkïhn open between the bases of villi (Fig, 151).

Each villus, be it cylindrical or conical in shape, is covered by a single layer of columnar epithelium, whose protoplasm is reticulated, and contains a welldefined nucleus with an intranuclear plexus of fibrils. The ends of the epithelial cells directed towards the gut are polygonal, and present the appearance of a mosaic (Fig. 150, D). When looked at from the side, their free surface is seen to be covered with a clear, highly refractive disc or "cuticula," which is marked with vertical striæ. These striæ were supposed by Kölliker to represent pores for the absorption of fatty particles, but this has not been confirmed, while Brettauer and Steinach regarded them as produced by prisms placed side by side.

According to some observers ( $v$. Thanhoffer), however, this clear disc is the optical expression of a thinning of the cell membrane, comparable to the thickened flange around the bottom of a vessel, such as is used for collecting gases. On this supposition, the upper end of each cell is open, and from it there projects pseudopodia-like bundles of protoplasmic processes (Fig. 150, B). These processes are supposed to be extended beyond the margin of the cell and again rapidly retracted, and in so acting they are said to carry the fatty particles into the interior of the cells, much as the pseudopodia of an amoeba entangles its food. [This view has not been contirmed by a sufficient number of observers.] Between the epithelial cells are the so-called goblet-cells (Fig. 150, C). [Each goblet-cell is more or less 
like a chalice, narrower above and below, and broad in the middle, with a tapering fixed extremity. The outer part of each cell is filled with a clear substance or mucigen, which, on the addition of water, yields mucus. The mucigen lies in the intervals of a fine net-work of fibrils, which pervades the cell protoplasm. The protoplasm, containing a globular or triangular nucleus, is pushed into the lower part

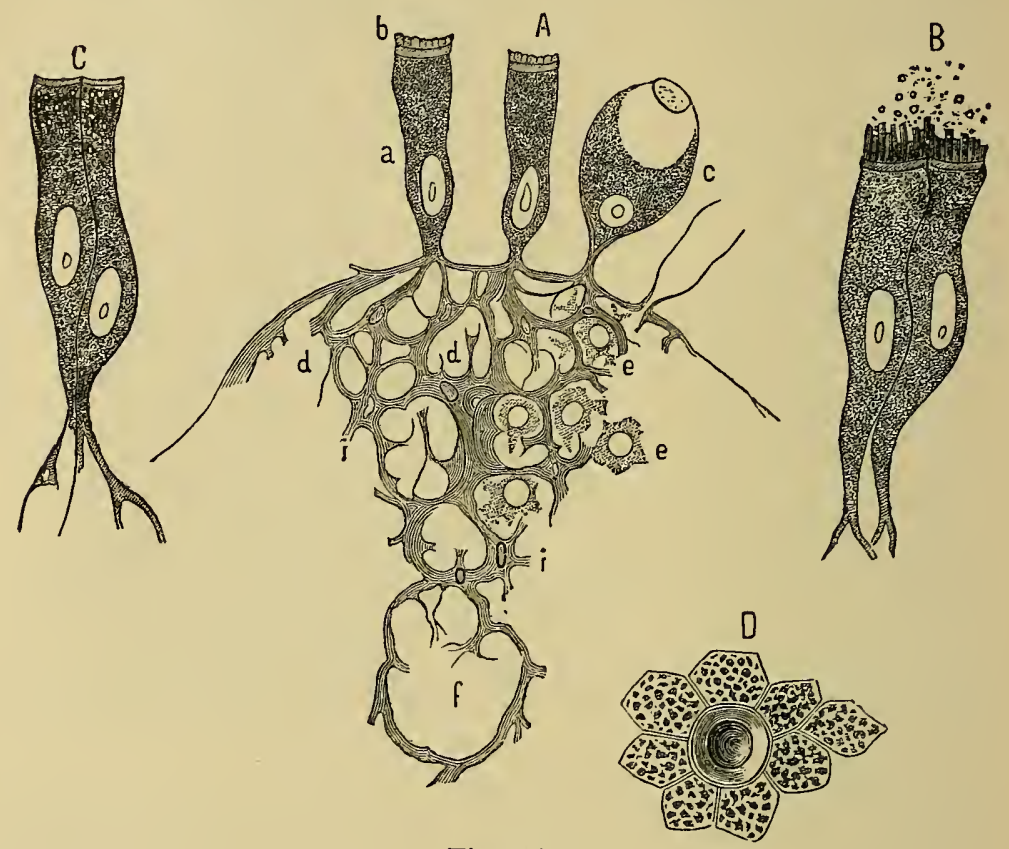

Fig. 150.

Scheme of an intestinal villus-A, Transverse section of part of a villus; $a$, columnar epithelium with, $b$, clear disc; $c$, goblet-cell ; $i, i$, adenoid reticulum; $d, d$, spaces within the same and containing leucocytes, $e, e ; f$, section of the central lacteal; $B$, scheme of a cell with processes supposed to be projected from its interior; C, columnar epithelium after the absorption of fatty granules; D, the columnar epithelium of a villus seen from above with a goblet-cell in the centre.

of the cell. These goblet-cells are simply altered columnar epithelial cells, which secrete mucus in their interior. They are more numerous under certain conditions. Not unfrequently in sections of the mucous membrane of the gut, after it is stained with logwood, we may see a deep blue plug of mucus partly exuded from these cells. When looked at from above they give the appearance seen in Fig. 150, D.] The epithelial cells are shed in enormous numbers in cholera, and in poisoning with arsenic and muscarin (Böhm).

[The epithelial cells covering the villus are placed upon a layer of squamous epithelium (basement membrane)-the sub-epithelial membrane of Débove. This basement membrane is said to be connected by processes with the so-called branched cells of the adenoid tissue of the villus, while it also sends up processes between the epithelial covering.]

The villus itself consists of a basis of adenoid tissue, containing in its centre one 
or more lacteals, closely invested with a few longitudinal smooth muscular fibres, derived from the muscularis mucosæ, and a plexus of blood-vessels.

The adenoid tissue of the villus consists of a reticulum of fibrils with endothelial plates at its nodes. The spaces of the adenoid tissue form a spongy net-work of inter-communicating channels containing stroma-cells or leucocytes (Fig. 150,A,e,e). These leucocytes or lymph-corpuscles have been seen to contain fatty granules, and they may, perhaps, play an important part in the absorption of fatty particles.

The lymphatic or lacteal lies in the axis of the villus (Fig. 149, d). By some observers, the lacteal is regarded merely as a space in the centre of the villus, but more probably it has a distinct wall composed of endothelial cells, with apertures or stomata here and there between the cell-plates. These stomata place the interior of the lacteal in direct communication with the spaces of the adenoid tissue. It is very probable that white blood-corpuscles wander out of the blood-vessels of the villi into the spaces of the adenoid tissue, where they become loaded with fatty granules, and pass into the central lacteal. Znwarykin and Wiedersheim suppose that the leucocytes pass from the parenchyma of the villus towards the epithelial layer, and even between the epithelial cells, from which they return towards the axis of the villus, laden with substances which they have taken into their interior (p. 399).

A small artery placed eccentrically passes into each villus. In man it begins to divide about the middle of the villus, but in animals it usually runs to the apex before it divides. The capillaries resulting from the division of the artery form a fine dense net-work placed superficially, immediately under the epithelium of the surface. The blood is carried out of a villus by one or two veins (Fig. 149, $a, c$ ).

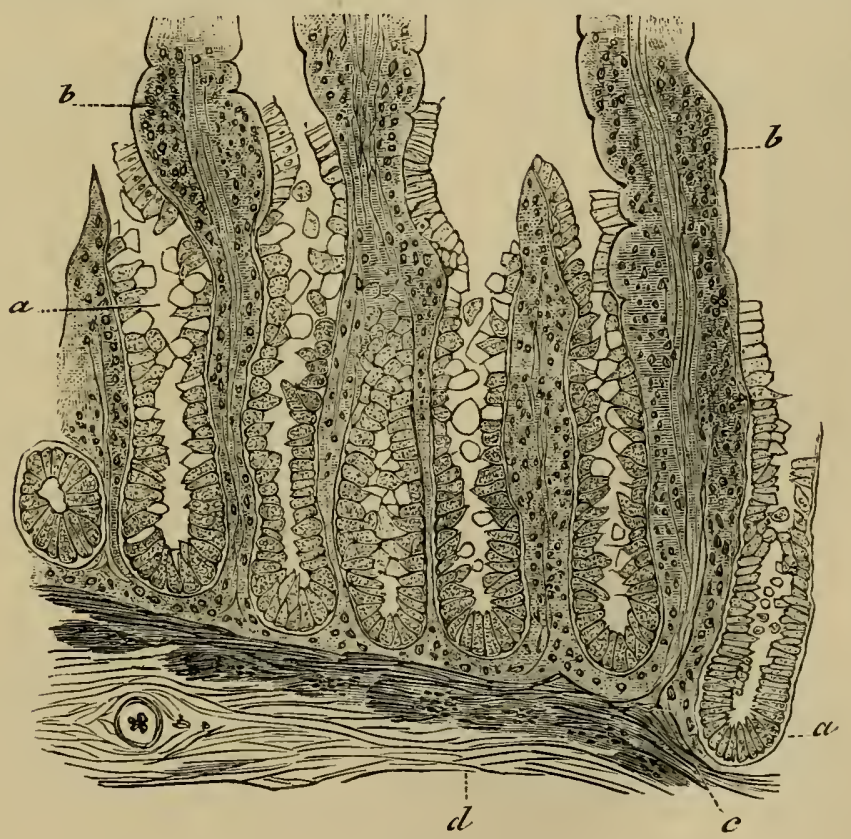

Fig. 151.

Section of the mucous membrane of the small intestine, showing Lieberkuhn's glands- $a$, with irregular epithelium; $b$, villi, cut short; $c$, muscularis mucosæ; $d$, sub-mucous tissue. 
iNon-striped muscular fibres are present in villi (Henle). Some are arranged longitudinally from base to apex, immediately outside the central lacteal. When they contract they tend to empty the lacteal (Brïcke). A few muscular fibres are placed more superficially, and run in a more transverse direction. [The muscular fibres of the villi are direct prolongations of the muscularis mucosæ].

Nerves pass into the villi from Meissner's plexus lying in the sub-mucous coat. The nerves to the villi are said to have small granular ganglionic cells in their course, and they terminate partly in the muscular fibres and partly in the arteries of the villi.

[On making a vertical section of the mucous membrane of the small intestine, it is seen to consist of a net-work of adenoid tissue loaded with leucocytes. This tissue forms its basis, and in it are placed vertically side by sicle, like test-tubes in a stand, immense numbers of simple tubular glands-the Crypts of Lieberkühn (Fig. 151). They open above at the bases of the villi, while their lower extremity reaches almost to the muscularis mucosæ. Each tube consists of a basement membrane lined by a single layer of columnar epithelium, leaving a wide lumen, the cells lining them being continuous with those that cover the mucous membrane. Some goblet-cells are often found between the columnar epithelium. Immediately below the bases of the follicles of Lieberkïhn is the muscularis mucosa, consisting of two or three narrow layers of non-striped muscular fibres arranged circularly and longitudinally. It is continuous with the muscularis mucosæ of the stomach, and extends throughout the whole intestine. It sends fibres upwards into the villi.]

[Brunner's glands are compound tubular glands lying in and confined to the sub-mucous coat of the duodenum. Their ducts perforate the muscularis mucosæ to open on the surface. They seem to be the homologues of the pyloric glands of the stomach (p. 368).]

[Solitary follicles are small round or oval white masses of adenoid tissue, with their deeper parts embedded in the sub-mucosa, and their apices projecting into the mucosa of the intestine. They begin at the pyloric end of the

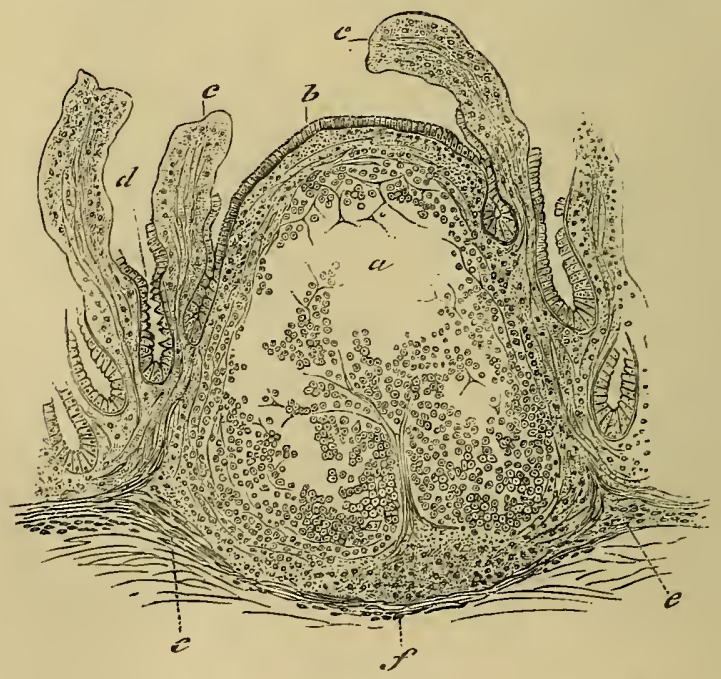

Fig. 152.

Section of a solitary follicle of the small intestine (human), showing-a, lymphfollicle covered with epithelium $(b)$ which has fallen from the villi, $c ; d$, Lieberkühn's follicle ; $e$, muscularis mucosæ; $f$, sub-mucous tissue. 
stomach and are found throughout the whole intestine. They consist of small masses of adenoid tissue loaded with leucocytes (Fig. 152). They are well supplied with blood-vessels (p. 406), although no lymphatic vessels enter them. They are surrounded by lymphatics, and, in fact, they may be said to hang into a lymphstream.]

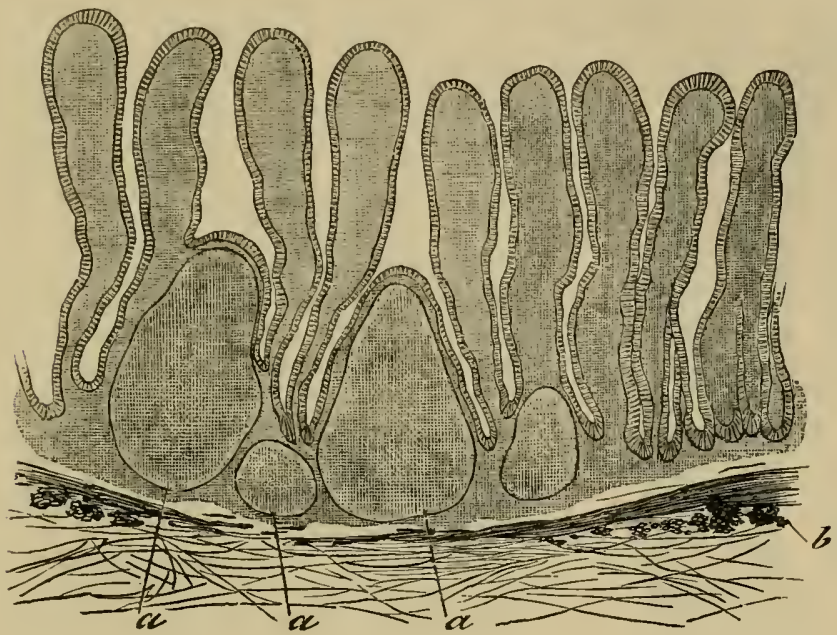

Fig. 153.

Diagram of a vertical section of the mucous membrane of the small intestine of a dog, showing the closed follicles, $a a ; b$, muscnlaris mucosæ.

[Peyer's glands, or agminated glands, consist of groups of lymph-follicles like the foregoing (Fig. 153). The masses are often more or less fused together, their bases lie in the sub-mucosa, while their summits project into the mucosa, where they are covered merely by the columnar epithelium of the intestine. The lymph-corpuscles often project between the epithelium. The patches so formed have their long axis in the axis of the intestine, and they are always placed opposite the attachment of the mesentery. Like the solitary glands, they are well supplied with blood-vessels, while around them is a dense plexus of lymphatics or lacteals. They are most abundant in the lower part of the ileum. These glands are specially affected in typhoid fever.]

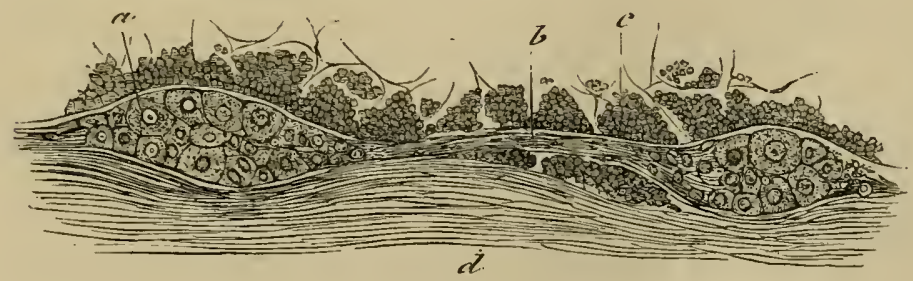

Fig. 154.

Auerbach's plexus shown in section (human)- $a$, ganglionic cells; $b$, nerve fibres; $c$, section of the circular muscular fibres; $d$, longitudinal muscular fibres. 
Nerves of the Intestine.-Thoughout the whole intestinal tract, there exists the plexus myentericus of Auerbach (Fig. 154), lying between the longitudinal and circular muscnlar coats. This plexus consists of non-medullated nerves with groups of ganglionic cells at the nodes. Fibres

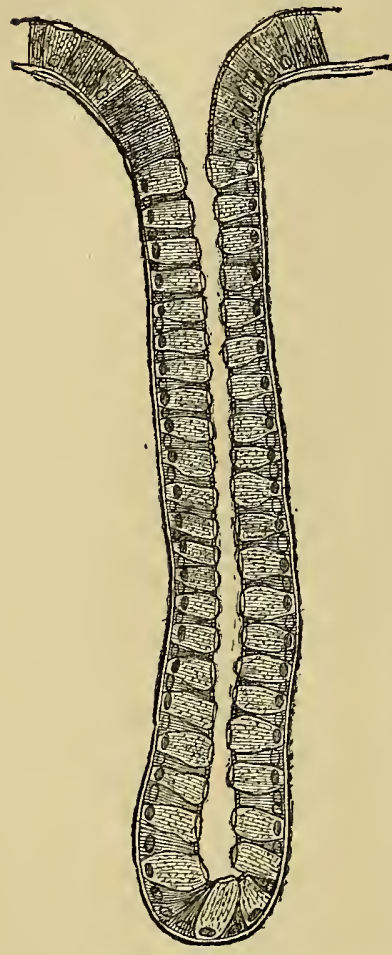

Fig. 155. are given off by it to the muscular coats.

Connected by branches with the foregoing and lying in the sub-mucosa, is the plexus of Meissner, which is much finer, the meshes being .wider, the nodes smaller, but also provided with ganglionic cells. It supplies the muscular fibres and arteries of the mucosa, including those of the villi. It also supplies branches to Lieberkühn's glands (Drasch).-Compare Figs. 131 and 132.

[Structure of the Large Intestine.-It has four coats like those of the small intestine. The serous coat has the same structure as that of the small intestine. The muscular coat has external longitudinal fibres occurring all round the gut, but they form three flat ribband-like longitudinal bands in the cæcum and colon. Inside this coat are the circular fibres. The submucosa is practically the same as that of the small intestine. The mucosa is characterised by negative characters. It has no villi and no Peyer's patches, but otherwise it resembles structurally the small intestine, consisting of a basis of adenoid with the simple tubular glands of Lieberkülın (Fig. 155). These glands are very numerous and somewhat longer than those of the small intestine, and they always contain far more goblet-cells. The cells lining them are devoid of a clear disc. Solitary glands occur throughout the entire length of the large intestine. At the bases of Lieberkühn's glands is the muscularis mucosce. The blood-vessels and

Lieberkühu's gland from the nerves have a similar arrangement to those in large intestine (dog). the stomach.]

\section{Absorption of the Digested Food.}

\section{The physical forces concerned are endosmosis, diffusion, and filtration.}

All the constituents of the food, with the exception of the fats, which in part are changed into a fine emulsion, are brought into a state of solution by the digestive processes. These substances pass through the walls of the intestinal tract, either into the blood-vessels of the mucous membrane or into the beginning of the lymphatics. In this passage of the fluids two physical processes come into playendosmosis and diffusion as well as filtration.

I. Endosmosis and diffusion occur between two fluids which are capable of forming an intimate mixture with each other, e.g., hydrochloric acid and water, but never between two fluids which do not form a perfect mixture, such as oil and water. If two fluids capable of mixing with each other, but of different compositions, be separated from each other by means of a septum with physical pores, (which occur even in a homogeneous membrane), an exchange of the constituents in 
the fluids occurs until both fluids have the same composition. This exchange of fluids is termed endosmosis or diosmosis.

If we remember that within the intestinal tract, there are relatively concentrated solutions of those substances which have been brought into solution by the digestive juices-peptone, sugar, soaps, and solutions of the salts-while separated from these by the porous mucous membrane and the walls of the bloodand lymph-capillaries is the blood, which contains relatively less of these substances, it is clear that an endosmotic current must set in towards the blood and lymph-vessels.

Diffusion.-If the two mixible fluids are placed in a vessel, the one fluid over the other, but without being separated by a porous septum, an exchange of the particles of the fluids also occurs, until the whole mixture is of uniform composition. This process is called Diffusion.

Conditions Influencing Diffusion.-Graham's investigatious showed that the rapidity of diffusion is influenced by a variety of conditions:-(I) The nature of the fluids themselves is of importance; acids diffuse most rapidly; the alkaline salts more slowly; and most slowly, fluid albumin, gelatin, gum, dextrin. These last do not crystallise, and perhaps do not form true solntions. (2) The more concentrated the solutions, the greater the diffusion. (3) Heat accelerates, while cold retards, the process. (4) If a solution of a body which diffuses with difficulty be mixed with an easily diffusible one, the former diffuses with still greater difficulty. (5) Dilute solutions of several substances diffuse into each other without any difficulty, but if concentrated solutions are employed, the process is retarded. (6) Double salts, one constituent of which diffuses more readily than the other, may be chemically separated by diffusion.

The exchange of the fluid particles takes place independently of the hydrostatic pressure. Fig. 156 represents an endosmometer. A glass cylinder is filled with distilled water, and into this is placed a flask, $J$, without a bottom, instead of which a membrane, $m$, is tied on. A glass tube, $\mathrm{R}$, is fixed firmly by means of a cork into the neck of the flask. The flask is filled up to the lower end of the tube with a concentrated salt solution, and is then placed in the cylindrical vessel until both fluids are on the same level, $x$. The fluid in the tube, $\mathrm{R}$, soon begins to rise, because water passes through the membrane into the concentrated solution in the flask, and this independently of the hydrostatic pressure. Particles of the concentrated salt solution pass into the cylinder and mix with the water, F. These outgoing and ingoing currents continue until the fluids without and within $J$ are of uniform composition, whereby the fluid in $R$ always stands higher (e.g., at $y)$, while it is lowered in the cylinder. The circumstance of the level of the fluid within the trube being so high and remaining so, is due to the fact that the pores in the membrane are ton fine to allow the hydrostatic pressure to act throngh them.

Endosmotic Equivalent.-Experiment has shown, that equal weights of different soluble substances attract different amounts of distilled water through the membrane-i.e., a known weight of a soluble substance (in the flask) can be

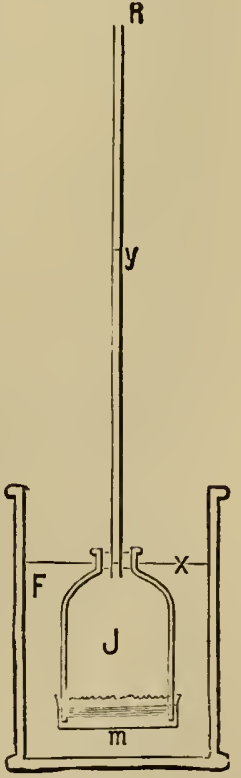

Fig. 156.

Endosmometer for Diffusion. exchanged by endosmosis for a definite weight of water. The term endosmotic equivalent indicates the weight of distilled water that passes into the flask of the endosmometer, in exchange for a known weight of the soluble substance (Jolly). For 1 gram. alcohol $4 \cdot 2$ grams. water were exchanged; while for 1 gram. $\mathrm{NaCl}, 4 \cdot 3$ 
grains. water passed into the endosmometer. The following numbers give the endosmotic equivalent of

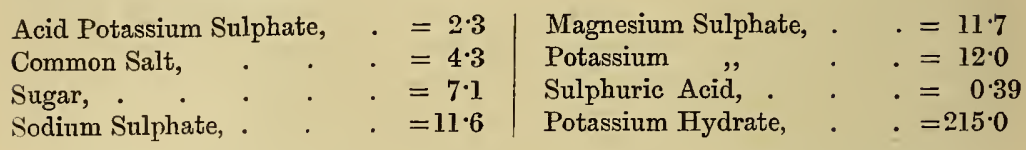

The amount of the substance which passes through the membrane into the water of the cylinder is proportional to the concentration of the solution (Vierordt). If the water in the cylinder, therefore, be repeatedly renewed, the endosmosis takes place more rapidly, and the process of equilibration is accelerated. The larger the pores of the membrane, and the smaller the molecules of the substance in solution, the more rapid is the endosmosis. Hence, the rapidity of endosmosis of different substances varies-thus, the rapidity of sugar, sodium sulphate, common salt, and urea is in the ratio of $1: 1 \cdot 1: 5: 9 \cdot 5$ (Eckhard, Hoffmann).

The endosmotic equivalent is not constant for each substance. It is influenced by-(1) The temperature, which as it increases, generally increases the endosmotic equivalent. (2) It also varies with the degree of concentration of the osmatic solutions, being greater for dilute solutions of the substances (C. Ludwig and Cloëtta).

If a substance other than water be placed in the cylinder, an endosmotic current occurs on both sides until complete equality is obtained. In this case, the currents in opposite directions disturb each other. If two substances be dissolved in the water in the flask at the same time, they diffuse into water without affecting each other. (3) It also varies with membranes of varying porosity. Common salt, which gives an endosmotic equivalent with a pig's bladder $=4 \cdot 3$, gives 6.4 when an ox bladder is used; 2.9 with a swimming bladder; and 20.2 with a collodion membrane (Harzer).

Colloids.-There is a number of fluid substances which, on account of the great size of their molecules, do not pass, or pass only with difficulty, through the pores of a membrane impregnated with gelatinous bodies, which diffuse slowly. These substances are not actually in a true state of solution, but exist in a very dilute condition of imbibition. Such substances are the fluid proteids, starches, dextrin, gum, and gelatin. These diffuse when no septum is present, but diffuse with difficulty or not at all through a porous septum. Graham called these substances Colloids, because when concentrated, they present a glue-like or gelatinous appearance; farther, they do not crystallise, while those substances which diffuse readily are crystalline, and are called Crystalloids. Crystallisable substances may be separated from non-crystallisable by this process, which Graham called Dialysis. Mineral salts favour the passage of colloids through membranes (Baranetzky).

That Endosmosis takes place in the intestinal canal tract, through the mucous membrane and the delicate membranes of the blood- and lymph-capillaries, cannot be denied. On the one side of the membrane, within the intestine, are the highly diffusible peptones, sugar, and soaps, and within the blood-vessels are the colloids which are scarcely diffusible, e.g., the proteids of blood and lymph.

II. Filtration is the passage of fluids through the coarse intermolecular pores of a membrane owing to pressure. 'The greater the pressure, and the larger and more numerous the pores, the more rapidly does the fluid pass through the membrane; increase of temperature also accelerates it. Those substances which are imbibed by the membrane filter most rapidly, so that the same substance filters through 
different membranes with varying rapidity. The filtration is usually slower, the greater the concentration of the fluid. The filter has the property of retaining some of the substances from the solution passing through it, e.g., colloid substances-or water (in dilute solutions of nitre). In the former case, the filtrate is more dilute, in the latter, more concentrated than before filtration. Other substances filter without undergoing any change of concentration. Many membranes behave differently, according to which surface is placed next the fluid; thus the shell-membrane of an egg permits filtration only from without inwards; [and the same is true to a much less extent with an ordinary filter paper-the smooth side of the filter paper ought always to be placed next the fluid to be filtered]. There is a similar difference with the gastric and intestinal mucous membrane.

Filtration of the soluble substance may take place from the canal of the digestive tract when:-(1) The intestine contracts and thus exerts pressure upon its contents. This is possible when the tube is narrowed at two points, and the musculature between these two points contracts upon the fluid contents. (2) Filtration, under negative pressure, may be caused by the villi (Brücke). When the villi contract energetically, they empty their contents towards the blood- and lymph-vessels. The lymph-vessels remain empty, as the chyle is prevented from passing backwards into the origin of the lacteal within the villi, owing to the presence of numerous valves in the lymphatics. When the villi pass again into the relaxed condition, they again become filled with the fluids of the irtestinal contents.

\section{Absorptive Activity of the Wall of the Intestine.}

The process of digestion produces from the food, partly solutions and partly finely divided emulsions, whose fine particles are surrounded by an albuminous envelope, the haptogen membrane [of Ascherson], whereby these particles become more stable. Unchanged colloid substances may also be present in the intestinal tract.

I. Absorption of Solutions.-True solutions undoubtedly pass by endosmosis into the blood-vessels and lymphatics of the intestinal walls, but numerous facts indicate, that the protoplasm of the cells of the tube take an active part in the process of absorption. The forces concerned have not as yet been referred simply to physical and chemical processes.

(1.) The Inorganic Substances.-Water and the soluble salts necessary for nutrition are easily absorbed. When saline solutions pass by endosmosis into the vessels, water must pass from the intestinal vessels into the intestine. The amount of water, however, is small, owing to the small endosmotic equivalent of the salts to be absorbed. More salts are absorbed from concentrated than from dilute solutions (Funke). 
If large quantities of salts, with a high endosmotic equivalent, are introduced into the intestine, e.g., magnesium or sodium sulphate, these salts retain the water necessary for their solution, and thus diarrhœa is caused (Poiseuille, Buchheim). Conversely, when these substances are injected into the blood a large quantity of water passes from the intestine into the blood, so that constipation occurs, owing to dryness of the intestinal contents (Aubert). [M. Hay concludes from his experiments (p. 320), that salts, when placed in the intestines, do not abstract water from the blood, or are themselves absorbed, in virtue of an endosmotic relation being established between the blood and the saline solution in the intestines. Absorption is probably due to filtration and diffusion, or processes of imbibition other than endosmosis, as yet little understood. The result obtained by Aubert, which is not constant, is mostly caused by the great diuresis which the injected salt excites.]

Numerous inorganic substances, which do not occur in the body, are absorbed by endosmosis from the intestine, e.g., dilute sulphuric acid, potassium iodide, chlorate, and bromide and many other salts.

(2.) The soluble carbohydrates, such as the sugars of which the chief representative is grape-sugar, with a relatively high endosmotic equivalent. Cane-sugar is changed by a special ferment into invert sugar, which is a mixture of grape-sugar and lævulose (p. 370). Perhaps a very small proportion of the cellulose is changed into grape-sugar. The absorption appears to take place somewhat slowly, as only very small quantities of grape-sugar are found in the chylevessels or the portal vein at any time. According to v. Mering, the sugar passes from the intestine into the rootlets of the portal vein; dextrin also occurs in the portal vein. When the blood of the portal vein is boiled with dilute sulphuric acid, the amount of sugar is increased (Naunyn). The amount of sugar absorbed depends upon the concentration of its solution in the intestine; hence, the amount of sugar in the blood is increased, after a diet containing much of this substance (C. Schmidt and v. Becker), so that it may appear in the urine, in which case, the blood must contain at least 0.6 per cent. of sugar (Lehmann and Uhle). A small amount of cane-sugar has also been found in the blood (Cl. Bernard, Hoppe-Seyler). The sugar is used up in the bodily metabolism; some of it is perhaps oxidised in the muscles (Zimmer).

(3.) The peptones have a small endosmotic equivalent (Funke), a 2-9 per cent. solution $=7-10$. Owing to their great diffusibility, they are readily absorbed, and they are the chief representatives of the proteids which are absorbed. The amount absorbed depends upon the concen- 
tration of their solution in the intestine. They pass into the bloodvessels (Schmidt-Mülheim). When animals are fed on peptones (with the necessary fat or sugar), they serve to maintain the body-weight (Maly, Plósz, and Györgyai). Only minute quantities of peptone have as yet been found in the blood (Drosdorff); hence, it is assumed, either that they are rapidly converted into true albuminous bodies, or that in part at least, they undergo further decompositions, with which we are as yet unacquainted. As, however, they can compensate for the total metabolism of the proteids within the body, we must assume that they are converted into proteids.

Schmidt-Mülheim has recently found that, four hours after feeding a pig with fibrin, a large quantity of crystalline propeptone (p. 331) can be obtained from the blood. When 5 c.c. of a 20 per cent. solution of peptone in 0.6 per cent. $\mathrm{NaCl}$ solution, for every kilo. of a dog, are injected into the blood, death is produced owing to paralysis of the blood-vessels (compare 28, II, $f$ ). Fano is of opinion that the red blood-corpuscles take np the peptone, and subject it to further changes.

(4.) Unchanged true proteids filter with great difficulty, and much albumin remains upon the filter. On account of their high endosmotic equivalent they pass with extreme difficulty, and only in traces through membranes. Nevertheless, it has been conclusively proved that unchanged proteids can be absorbed (Brücke), e.g., casein, soluble myosin, alkali-albuminate, albumin mixed with common salt, gelatin (Voit, Baner, Eichhorst). They are absorbed even from the large intestine (Czerny and Latschenberger), although the human large intestine cannot absorb more than 6 grms. daily. But the amount of unchanged proteids absorbed is always very much less than the amount of peptone.

Egg-albumin without common salt, syntonin, serum-albumin, and fibrin are not absorbed (Eichhorst). Landois observed in the case of a young man who took the whites of 14-20 eggs along with $\mathrm{NaCl}$, that albumin was given off by the urine for 4-10 hours thereafter. The amount of albumin given off rose until the third day and ceased on the fifth day. The more albumin that was taken the sooner the albuminuria appeared and the longer it lasted. The unchanged egg-albumin reappeared in the urine. If egg-albumin be injected into the blood, part of it reappears in the urine ( $\$ 41,2)$ (Stokvis, Lehmann).

(5.) The soluble fat-soaps represent only a fraction of the fats of the food which are absorbed; the greater part of the neutral fats being absorbed in the form of very fine particles-as an emulsion. The absorbed soaps have been found in the chyle, and as the blood of the portal vein contains more soaps during digestion than during hunger, it has been assumed that the soaps pass into the intestinal bloodcapillaries. The investigations of Lenz, Bidder, and Schmidt render it probable that the organism can absorb only a limited amount of fat within a given period; the amount perhaps bears a relation to the 
amnunt of bile and pancreatic juice. The maximum per 1 kilo. (cat) was $0 \cdot 6$ grms. of fat per hour.

It appears as if the soaps reunite with glycerine in the parenchyma of the villi, to form neutral fats, as Perewoznikoff and Will found, after injecting these two ingredients into the intestinal canal. C. A. Ewald found that fat was formed when soaps and glycerine were brought into contact with the fresh intestinal mucous membrane. Perhaps this is the explanation of the observation of Bruch, who found fatty particles within the blood-vessels of the villi.

Absorption of other Substances.-Of soluble substances which are introduced into the intestinal canal, some are absorbed and others are not. The following are absorbed-alcohol, part of which appears in the urine (not in the expired air), viz., that part which is not changed into $\mathrm{CO}_{2}$ and $\mathrm{H}_{2} \mathrm{O}$, within the body; tartaric, citric, malic, and lactic acids; glycerine, inulin (Komanos); gum and vegetable mucin, which give rise to the formation of glycogen in the liver.

Amongst colouring matters alizarin (from madder), alkannet, indigo-sulphuric acid, and its soda salt are absorbed; hæmatin is partly absorbed, while chlorophyll is not. Metallic salts seem to be kept in solution by proteids, are perhaps absorbed along with them, and are partly carried by the blood of the portal vein to the liver (ferric sulphate has been found in chyle). Numerous poisons are very rapidly absorbed, e.g., hydrocyanic acid after a few seconds; potassium cyanide has been found in the chyle.

II. Absorption of the smallest particles.-The largest amount of the fats is absorbed in the form of a milk-like emulsion formed by the action of the bile and the pancreatic juice, and consisting of excessively small granules of uniform size (v. Frey). The fats themselves are not chemically changed, but remain as undecomposed neutral fats. The particles seem to be surrounded by a delicate albuminous envelope, or haptogen membrane, partly derived from the pancreatic juice [probably from its alkali-albuminate]. The villi of the small intestine are the chief organs concerned in the absorption of the fatty emulsion, but the epithelium of the stomach and that of the large intestine also take a part. The fatty granules are recognised in the villi-(1) Within the delicate canals? (p. 387) in the clear band of the epithelium (Kölliker). [It is highly doubtful if the vertical lines seen in the clear disc of the epithelium of the intestine are due to pores.] (2) The protoplasm of the epithelial cells is loaded with fatty granules of various sizes during the time of absorption, while the nuclei of the cells remain free, although, from the amount of fat within the cells, it is often difficult to distinguish them. (3) The granules pass into the spaces of the parenchyma of the villi; these spaces communicate freely with each other. (4) The origin of the lacteal in the axis of the villus is found to be filled with fatty granules.

The amount of fat in the chyle of a dog, after a fatty meal, is 8-10 per cent., while the fat disappears from the blood within thirty hours. 
With regard to the forces concerned in the absorption of fats, v. Wistinghausen proved, that when a porous membrane is moistened with bile, the passage of fatty particles through it is thereby facilitated, but this fact alone does not explain the copious and rapid absorption of fats. It appears probable, that the protoplasm of the epithelial cells is actively concerned in the process, and that it takes the particles into its interior. Perhaps a fine protoplasmic process is thrown out by these cells, just as pseudopodia are thrown out and retracted by lower organisms. It is possible that absorption may take place through the open mouths of the goblet-cells. The protoplasm of the epithelial cells is in direct communication with the numerous protoplasmic lymph-cells within the reticulum of the villi, so that the particles may pass into these, and from them through the stomata (?) between the endothelial cells into the central lacteal of the villus. According to this view, the absorption of fatty particles, and perhaps also the absorption of true proteids, is due to an active vital process, as indicated by the observations of Brücke and v. Thanhoffer. This view is supported by the observation of Grünhagen, that the absorption of fatty particles in the frog is most active at the temperature at which the motor phenomena of protoplasm are most lively. That it is due to simple filtration alone is not a satisfactory explanation, for the amount of fatty particles in the chyle is independent of the amount of water in it. If absorption was chiefly due to filtration, we would expect that there would most probably be a direct relation between the amount of water and the fat (Ludwig and Zawilsky). [The observations of Watney have led him to suppose that the fatty particles do not pass through the cell protoplasm to reach the lacteal, but that they pass through the cementsubstance between the epithelial cells covering a villus. If this view be correct, the absorbing surface is thereby greatly diminished.]

[Schäfer suggests that the leucocytes, which have been observed between the columnar cells of the villi of the smill intestine, are carriers of at least part of the fat from the lumen of the gut to the lacteal ; they also, perhaps, alter it for further use in the economy (p. 389)].

The activity of the cells of the intestine with pseudopodial processes may be studied in the intestinal canal of Distomum hepaticum. Sommer has figured these pseudopodial processes actively engaged in the absorption of particles from the intestine.

Spina observed that the intestinal epithelium of the larve of flies shortened when they were stimulated with electricity, and absorbed fluid from the intestinal canal. The cells of the villi of the frog also react to electrical stimulation.

The increase in the size of the cells occurs simultaneously with the contraction of the intestine. Spina also supports the view that the cells, in virtue of their activity, possess the property of absorbing fluid from the intestinal contents and again giving it up. An exchange of fluids in the opposite direction never takes place.

The statements of former observers that particles of charcoal, pigments, and 
even mammalian blood-corpuscles (in the frog) were absorbed by the epithelial cells of the intestine, and passed into the blood, are erroneous. Even for the absorption of completely fluid substances, endosmosis and filtration seem to be scarcely sufficient. An active participation of the protoplasm of the cells seems here also-in part at least-to be necessary, else it is difficult to explain how very slight disturbances in the activity of these cells-e.g., from intestinal catarrhcause sudden variations of absorption, and even the passage of fluids into the intestine.

If absorption was due to diffusion alone, when alcohol is injected into the intestine, water ought to pass into the intestine, but this does not occur. Brieger found that the injection of a $0.5-1$ per cent. solution of salts into a ligatured loop of intestine did not cause water to pass into the intestine; but it appeared when a 20 per cent. solution was injected.

\section{Influence of the Nervous System.}

With regard to the influence of the nervous system upon intestinal absorption we know very little. After extirpation of the semi-lunar ganglion (Budge), as well as after section of the mesenteric nerves (Moreau), the intestinal contents become more fluid, and are increased in amount. This may be partly due to diminished absorption. v. Thanhoffer states, that he observed the protrusion of threads from the epithelial cells of the small intestine only after the spinal cord, or the dorsal nerves, had been divided for some time.

[Matthew Hay injected saline solutions directly into the exposed intestine. He found that a 20 per cent. solution of sulphate of soda always excites a profuse secretion, but that a 10 per cent. solution only does so, or rather, that it only increases in bulk, when injected in sufficient quantity-a certain weight of salt failing to increase the bulk of the fluid secretion when dissolved as a 10 per cent. solution, but exciting a profuse secretion when forming a 20 per cent. solution. Secretion, he has reason to believe, is active in both-perhaps, almost equally activebut absorption is greatly impeded in the case of the concentrated salt, by its injurious action on the absorptive mechanism of the mucous membrane. Moreau has recently maintained that, under such circumstances, there is actually no absorption, but Hay has disproved this, by observing that strychnia injected into a loop of intestine, containing the concentrated salt, still causes death, although after an interval three times longer than when the loop contains a 10 per cent. solution of the salt.

Hay has also observed that the local effect of a ligature applied to the intestine is to excite secretion from the mucous membrane in its immediate vicinity, and therefore add to the bulk of the saline solution; whereas the reflex effect of a ligature, as exercised through the nervous system, is to diminish the quantity of the secreted fluid in a remote portion of the intestine, probably by stimulating and accelerating absorption. Division of the vagi does not affect the nature or the quantity of the secretion].

\section{Feeding with "Nutrient Enemata."}

In cases where food cannot be taken by the mouth-e.g., in stricture of the œsophagus, continued vomiting, \&c., food is given per rectum (Celsus, 3-5 A.D.). As the digestive activity of the large intestine is very slight, fluid food ought to be given in a condition ready to be absorbed, and this is best done by introducing 
it into the rectum throngh a tube with a funnel attached, and allowing the food to pass in slowly by its own weight. The patient must endeavour to retain the enema as long as possible. When the fluid is slowly and gradually introduced, it may pass above the ileo-cæcal valve.

Solutions of grape-sugar, and perhaps a small amount of soap solution, are useful; and amongst nitrogenons substances the commercial flesh, bread, or milk peptones of Sanders-Ezn, Adamkiewicz, in Germany, and Darby's fluid meat in this country, are to be recommended. The amount of peptone required is 1.11 grms. per kilo. of body-weight (Catillon); less useful are butter-milk, egg-albumin with common salt. Leube uses a mixture of $150 \mathrm{grms}$. flesh, with 50 grms. pancreas and 100 grms. water, which he injects into the rectum where the proteids are peptonised and absorbed. The method of nutrient enemati only permits imperfect nutrition, and at most only $\frac{1}{4}$ of the proteids necessary for maintaining the metabolism of the body is absorbed (v. Voit, Bauer).

\section{Chyle-Vessels and Lymphatics.}

Within the tissues of the body, and even in those tissues which do not contain blood-vessels-e.g., the cornea, or in those which contain few blood-vessels, there exists a system of vessels or channels which contain the juices of the tissues, and within these vessels the fluid always moves in a centripetal direction. These canals arise within the tissues in a variety of ways, and unite in their course to form delicate and afterwards thicker tubes, which ultimately terminate in two large trunks which open at the junction of the jugular and subclavian veins; that on the left side is the thoracic duct, and that on the right, the right lymphatic trunk.

Lymphatics.-With regard to the lymph and its movements in different organs, it is to be noticed that this occurs in different ways in different places. (1) In many tissues, the lymphatics represent the nutrient channels, by which the fluid which transudes through the neighbouring vessels is distributed, as in the cornea and in many connective tissues. (2) In many tissues, as in glands-e.g., the salivary glands (Gianuzzi) and the testis, the lymph-spaces are the first reservoirs for fuid, from which the cells during the act of secretion derive the fluid necessary for that process. (3) The lymphatics have the general function of collecting the fluid which saturates the tissues, and carrying it back again to the blood. The capillary blood-system may be regarded as an irrigation system, which supplies the tissues with nutrient fluids, while the lymphatic system may be regarded as a drainage apparatus, which conducts away the fluids that lave transuded through the capillary walls. Some of the decomposition products of the tissues, proofs of their retrogressive metabolism, become mixed with the lymph-stream, so that the lymphatics are at the same time absorbing vessels. Substances introduced into the parenchyma of the tissues in other ways, e.g., by subcutaneous injection, are partly 
absorbed by the lymphatics. A study of these conditions shows, that the lymphatic system represents an appendix to the blood-vascular system, and further, that there can be no lymph system when the blood-stream is completely arrested; it acts only as a part of the whole, and with the whole.

Lacteals.-When we speak of the lymphatics proper as against the chyle-vessels or lacteals, we do so from anatomical reasons, because the important and considerable lymphatic channels coming from the whole of the intestinal tract are, in a certain sense, a fairly independent province of the lymphatic vascular area, and are endowed with a high absorptive activity, which, from ancient times, has attracted the notice of observers. The contents of the chyle-vessels or lacteals are mixed with a large amount of fatty granules, giving the chyle a white colour, which distinguishes them at once from the clear watery contents of the true lymphatics. From a physiological point of view, however, the lacteals must be classified with the lymphatics, for, as regards their structure and function, they are true lymphatics, and their contents consist of true lymph mixed with a large amount of absorbed substances, chiefly fatty granules. [The contents of the lacteals are white only during digestion, at other times they are clear like lymph].

\section{Origin of the Lymphatics.}

The mode of origin of the lymphatics varies within the different tissues. The following modes are known :-

1. Origin in Spaces.-Within the connective-tissues (connective-tissue proper, bone), are numerous stellate, irregular, or branched, spaces which communicate with each other by numerous tubular processes (Fig. 157, s); in these communicating spaces lie the cellular elements of these tissues. These spaces, however, are not completely filled by the cells, but an interval exists between the body of the cell and the wall of the space, which is greater or less according to the condition of movement of the protoplasmic cell. These spaces are the so-called "juice-canals" or "saftcanälchen," and they represent the origin of the lymphatic vessels (v. Recklinghausen). As they communicate with neighbouring spaces, the movement of the lymph is provided for. The cells which lie in the spaces, and which were formerly but erroneously regarded by Virchow as the origins of the lymphatics, exhibit amceboid movements. Some of these cells remain permanently, each in its own space, within which, however, it may change its form-these are the so-called "fixed" connective-tissue corpuscles, and bone-corpuscles-while others merely wander or pass into these spaces, and are called "wandering cells," or "leucocytes;" but the latter are merely lymph-corpuscles, or colourless blood-corpuscles which have passed out of the blood-vessels into the origin of the lymphatics. These cells exhibit amoboid movements. These spaces communicate with the small tubular lymphatics-the so-called lymph-capillaries (L). The spaces lie close together where they pass into a lymph-capillary $(a)$. The lymph-capillary, which is usually of greater diameter than the blood-capillary, generally lies in the middle 


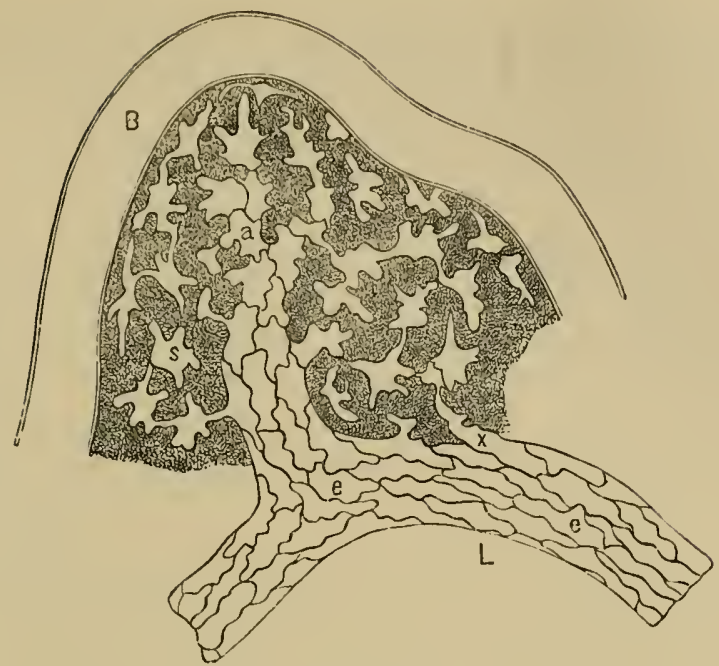

Fig. 157 .

Origin of lymphatics-From the central tendon of the diaphragm of a rabbit (semi-diagrammatic); $s$, the juice-canals, comununicating at $x$ with the lymphatics; $a$, origin of the lymphatics by the confluence of several juicecanals. The tissue has been stained with nitrate of silver.

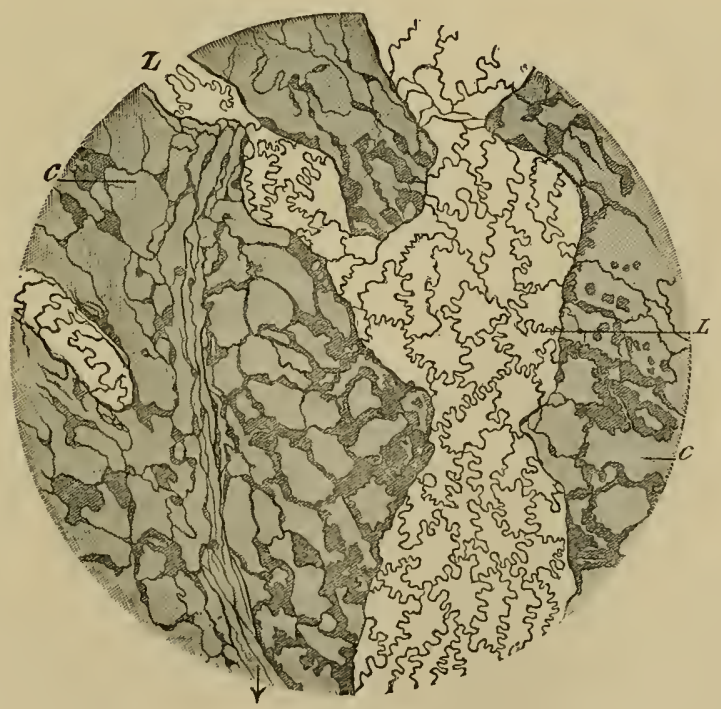

Fig. 15s.

Central tendon of the diaphragm of the rabbit stained with silver nitrate and viewed from the pleural side-L, lymphatic with its sinuous endothelium; c, cells of the connective-tissue brought into view by the silver nitrate. 
of the space within the capillary arch (B). The finest lymphatics are lined by a layer of delicate, nucleated endothelial cells $(e, e)$, with characteristic sinuous margins, whose characters are easily revealed by the action of silver nitrate (Fig. 158, L). This substance blackens the cement substance which holds the endothelial cells together. Between the endothelial cells are small holes, or stomata, by means of which the lymph-capillaries communicate (at $x$ ) with the juice canals.

It is assumed that the blood-vessels communicate with the juice canals (J. Arnold, Thoma, Uskoff), and that fluid passes out of the thin-walled capillaries through their stomata (p. 122) into these spaces. This fluid nourishes the tissues, the tissues take up the substances appropriate to each, while the effete materials pass back into the spaces, and from these reach the lymphatics, which ultimately discharge them into the venous blood.

Whether the cells within these spaces are actively concerned in the pouring out of the blood-plasma, or take part in its movement, is matter for conjecture. We can imagine that by contracting their body, after it has been impregnated with fluid, this fluid may be propelled from space to space towards the lymphatics. The leucocytes wander through these spaces until they pass into the lymphatics. Fine particles which are contained in these spaces-e.g., after tattooing the skin and even fatty particles after inunction-are absorbed by the leucocytes, and carried by them to other parts of the body. [The pigment particles used to tattoo the finger are asually found within the first lyinphatic gland at the elbow.]

After what has been said regarding the passage of colourless bloodcorpuscles through the stomata of the blood-capillaries, or through the walls of the smaller blood-vessels ( $(95)$, the passage of cellular elements from the blood-vessels into the origin of the lymphatics is to be considered as a normal process (E. Hering). Granular colouring matter passes from the blood into the protoplasmic body of the cells within the lymph-spaces; and only when the granular pigment is in large amount, does it appear as a granular injection in the branches of the juice-spaces (Uskoff).

(2.) The origin of lymphatics within villi-i.e., of the chyle vessel or lactealhas been described at p. 389. The central lacteal communicates with the lacunar interstitial spaces in the adenoid tissue of the villus, and this again with the protoplasmic body of the epithelial cells. It is assumed that the lymph-corpuscles, which lie in the meshes of the adenoid tissue, pass into the central lacteal (His), while new cells are continually passing out of the blood-capillaries of the villi into the tissue, where they perhaps undergo increase through division.

(3.) Origin of lymphatics in perivascular spaces (Fig. 159).-The smallest bloodvessels of bone, the central nervous system, retina, and the liver, are completely surrounded by wide lymphatic tubes, so that the blood-vessels are completely bathed by a lymph-stream. In the brain, these lymphatics are partly composed of delicate connective-tissue fibres, which traverse the lymph-space and become attached to the wall of the included blood-vessel (Roth). Fig. 159, B, represents a transverse section of a small blood-vessel, B, from the brain; $p$ is the divided perivascular space. This space is called the perivascular space of His, but in 
addition to it, the blood-vessels of the brain have a lymph-space within the adventitia of the blood-vessels (Virchov-Rolin's space). It is partly lined by welldefined endothelium. Where the blood-vessels begin to increase considerably in

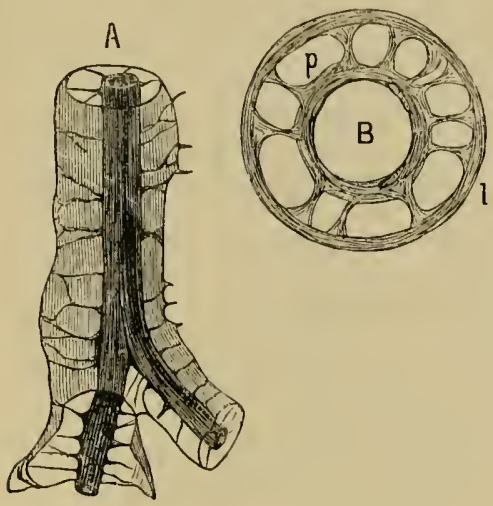

Fig, 159.

Perivascular lymphatics-A, aorta of tortoise; $\mathrm{B}$, artery from the brain.

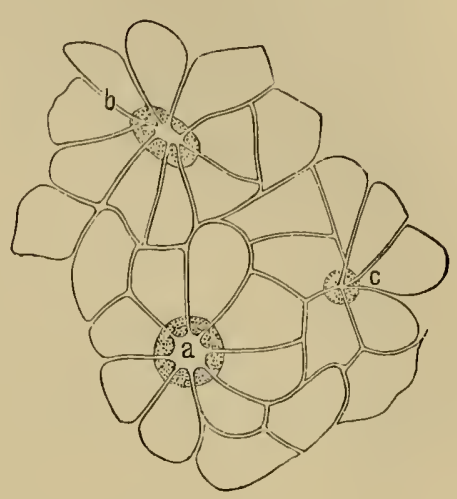

Fig. 160.

Stomata from the great lymph-sac of a frog- $a$, stoma open; $b$, half-closed; $c$, closed.

diameter, they pass through the wall of the lymphatics, and the two vessels afterwards take separate courses. In all cases, where there is a perivascular space, the passage of lymph-and blood-corpuscles into the lymphatics is greatly facilitated. In the tortoise, the large blood-vessels are often surrounded with perivascular lymphatics. Fig. 159, A, gives a representation of the aorta surrounded by a perivascular space (Gegenbaur) which is visible to the unaided eye. In mammals, the perivascular spaces are microscopic.

(4.) Origin in the form of interstitial slits within organs.-Within the testis, the lymphatics begin simply in the form of numerous slits, which occur between the coils and twists of the seminal tubules. They take the form of elongated spaces bounded by the curved cylindrical surfaces of the tubules. The surfaces, however, are covered with endothelium. The lymphatics of the testis get independent walls after they leave the parenchyma of the organ. In many other glands, the gland-substance is similarly surrounded by a lymph-space. The blood-vessels pour the lymph into these spaces and from them the secreting cells obtain the materials necessary for the formation of their secretion.

(5.) Origin by means of free stomata on the walls of the larger serous cavitics (Fig. 160, a).-The investigations of v. Recklinghausen, Ludwig, Dybkowsky, Schweigger-Seidel, Dogiel, and others have shown, that the old view of Mascagni, that the serous cavities freely communicate with the lymphatics, is correct. The investigation of the serous surfaces, most easily accomplished on the septum of the great abdominal lympli-sac of the frog, by means of silver nitrate, reveals the presence of relatively large free openings or stomata lying between the endothelium. Each stoma is bounded by several cells, which have a granular appearance, and are capable of undergoing a change of shape, so that the size of the stoma depends upon the degree of contraction of these cells; thus the stoma may be open $(a)$, lialf open $(b)$, or completely closed $(c)$. These stomata are the origin of the lymphatics. The serous cavities belong therefore to the lymphatic system, and fluids placed in the serous cavitics readily pass into the 
lymphatics. The cavities of th peritoneum, plenra, pericardium, tunica vaginalis testis, arachnoid space, aqueous chambers of the eye (Schwalbe), and the labyrinth of the ear, are true lymph-cavities, and the fluid they contain is to be regarded as lymph.

(6.) Free open pores have been observed on some mucous menbranes, which are regarded as the origin of lymphatics, e.g., in the bronchi (Klein)-the nasal mucous membrane (Hjalmar-Heiberg), in the trachea and larynx.

The larger lymphatics resemble in structure the veins of corresponding size. The valves are particularly numerous in the lymphatics, so that a distended lymphatic resembles a chain of pearls. [Lymphatics have dilations here and there in their course (Fig. 158).]

\section{The Lymph-Glands.}

The so-called lymphatic glands belong to the lymph apparatus. They are incorrectly termed glands, as they are much branched lacunar labyrinthine spaces merely composed of adenoid tissue, and intercalated in the course of the lymphatic vessels.

There are simple and compound lymph-glands.

(1.) The simple lymph-glands, or, more correctly, lymph-follicles, are small rounded bodies, about the size of a pin-head. They consist of a mass of adenoid tissue (Fig. 161, A), i.e., of a very delicate net-work of fine reticular fibres with nuclei at their points of intersection, and in the spaces of the mesh-work lie the lymph and the lymph-corpuscles. Near the surface, the tissue is somewhat denser, where it forms a capsule, which is not however a true capsule, as it is permeated with numerous small sponge-like spaces. Small lymphatics come directly into contact with these lymph-follicles, and often cover their surface in the form of a

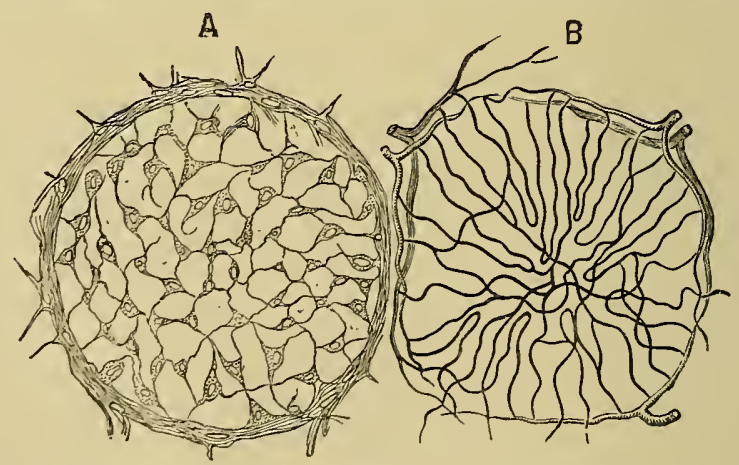

Fig. 161.

Two lymph-follicles-A, a small follicle highly magnified, showing the adenoid reticulum; B, a follicle less highly magnified, showing injected blood-vessels.

close net-work. The surface of the lymph-follicles is not unfrequently placed in the wall of a lymph-vessel, so that it is directly bathed by the lymph-stream. Although no direct canal-like opening leads from the follicle into the lymphatic stream, in relation with it a communication must exist, and this is obtained by the numerous spaces in the follicle itself, so that a lymph-follicle is a true lymphatic apparatus (Brïcke) whose juices and lymph-corpuscles can pass into the nearest lymphatic. The follicles are surrounded by a net-work of blood-vessels which 
sends loops of capillaries into their interior (Fig. 161, B). We may assume that lymph-corpuscles pass from these rapillaries into the follicle.

In connection with these follicles, including those of the back of the tongue, the solitary glands of the intestine and the adenoid tissue in the bronchial tract, the tonsils, Peyer's patches, it is important to remember that enormous numbers of lencocytes pass out between the epithelial cells covering these follicles. The extruded lencocytes undergo disintegration subsequently (Ph. Stöhr).

(2.) The compound lymph-glands-the so-called lymphatic glands-represent a collection of lymph-follicles, whose form is somewhat altered. Every lymphgland is covered externally with a connective-tissue capsule (Fig. 162, c), which contains numerous non-striped muscular fibres (O. Heyfelder). From its inner surface, numerous septa and trabeculæ $(t r)$ pass into the interior of the gland, so that the gland-substance is divided into a large number of compartments. These compartments in the cortical portion of the gland have a somewhat rounded form, and constitute the alveoli, while in the medullary portion they have a more elongated and irregular form. [On making a section of a lymph-gland we can readily distinguish the cortical from the medullary portion of the gland.] All the compartments are of equal dignity, and they all communicate with each other by means of openings, so that the septa bound a rich net-work of spaces within the gland, which communicate on all sides with eacl other.

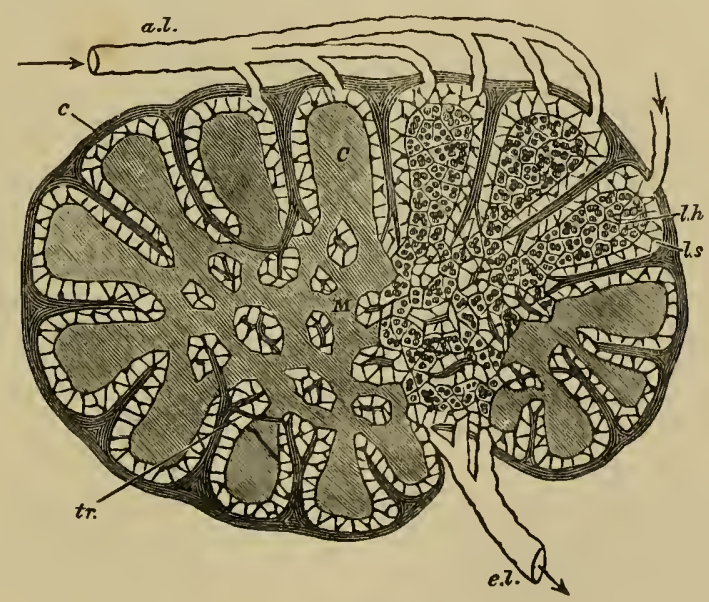

Fig. 162.

Diagrammatic section of a lymphatic gland $-a, l$, afferent; $e, l$, efferent lymphatics ; C, cortical substance; $\mathbf{M}$, reticular cords of medulla; $l, s$, lymph-sinus; $c$, capsule, with trabeculæ, $t r$.

These spaces are traversed by the follicular threads (Fig. 163, $f, f$ ). These represent the contents of the spaces, but they are smaller than the spaces in which they lie, and do not come into contact anywhere with the walls of the spaces. If we imagine the spaces to be injected with a mass, which ultimately shrinks to one-half of its original volume, we obtain a conception of the relation of these follicular threads to the spaces of the gland. The blood-vessels of the gland $(b)$ lie within these follicular threads. They are surrounded by a tolerably thick crust of adenoid tissue, with very fine meshes $(x, x)$ filled with lymph-corpuscles, and with its surface $(0,0)$ covered by the cells of the adenoid reticulum, in such a way as to leave free communications through the narrow meshes. 
Between the surface of the follicular threads and the inner wall of all the spaces of the gland, lies the lymph-channel or lymph-path $(\mathrm{B}, \mathrm{B})$, which is traversed by a reticulum of adenoid tissue, containing rclatively few lymph-corpuscles. It is very probable that these lymph-paths are lined by endothelium (v. Reckliughausen).

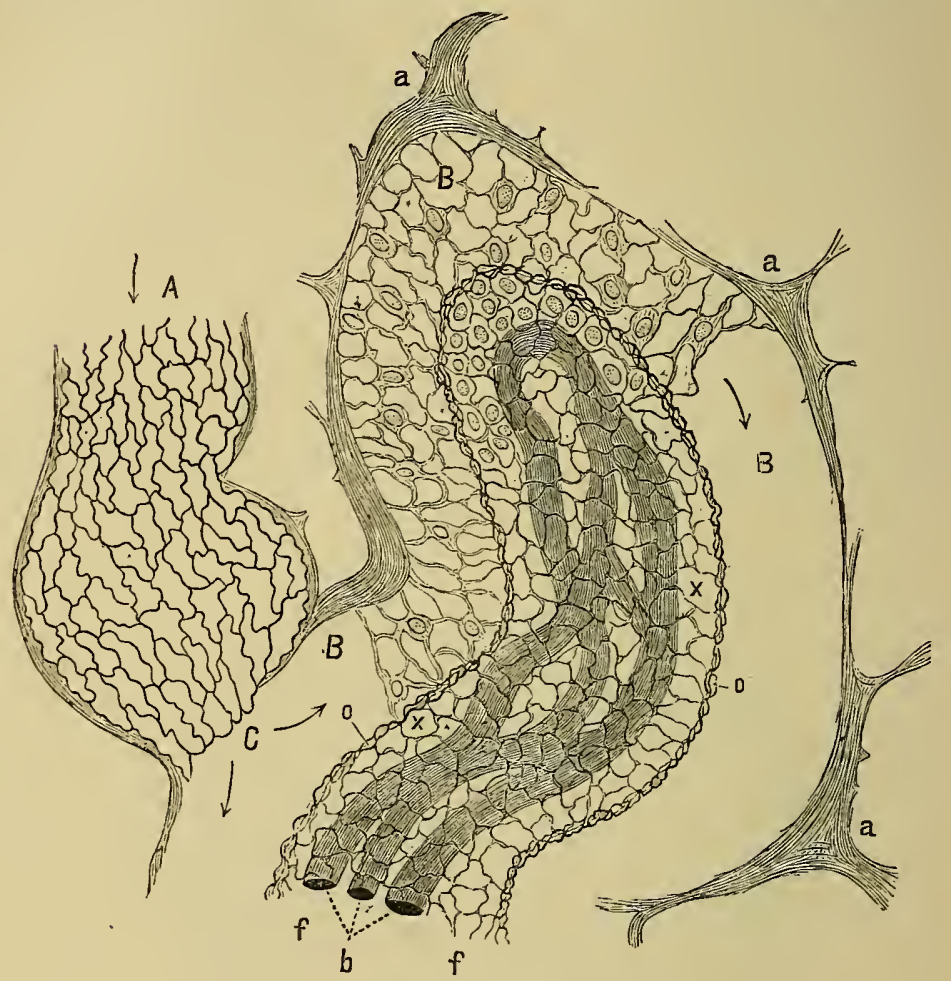

Fig. 163.

Part of a lymphatic gland-A, Vas afferens ; B, B, lymph-spaces within the gland; $a$, $a$, septa or trabeculæ seen on edge ; $f, f$, follicular strand from the medulla; $x, x$, its adenoid reticulum; $b$, its blood-vessels; $o, o$, narrow meshed part limiting the follicular strands from the lymph-space.

The vasa afferentia (Fig. 162, $a, l$ ), of which there are usually several, expand upon the surface of the gland, perforate the onter capsule, and pour their contents into the lymph-paths (C) of the gland. The vasa efferentia, which are less numerous than the afferentia, and come out at the hilum, form large, wide, almost cavernous dilatations, and they anastomose near the gland $(e, l)$. Throngh them the lymph passes out at the opposite surface of the gland. The lymph percolates through the fland, and passes along the lymph-paths, which represent a kind of rete mirabile interposed between the afferent and efferent lymph-vessels.

During its passage throngh this conplicated branched system of spaces, the movement of the Iymph through the gland is retarded, and, owing to the numerous resistances which occur in its path, it has very little propulsive energy. The lymph-corpuseles which lie in the meshes of the adenoid reticulum are washed out of the gland by the lymph-stream (Briicke). The lymph-corpuscles lying within the follicular threads, pass through the narrow meshes (0) 
into the lymph-paths. The formation of lymph-corpuscles occurs either locally, from division of the pre-existing cells, or new leucocytes wander ont into the follicular threads. The movcment of the lymph through the gland is favoured by the muscular action of the capsule. When the capsule contracts energetically, it must compress the gland like a sponge, and the direction in which the fluid moves is regulated by the position and arrangement of the valves.

The researches of Teichmann, His, Frey, Brücke, and v. Recklinghausen have chiefly contributed to the elucidation of the morphological and physiological relations of the lymph-glands.

In addition to the constituents of lymph, the following clemical substances have been found in lymphatic glands:-Lencin (Frerichs and Stideler) and Xanthin.

\section{Properties of Chyle and Lymph.}

(1.) Both fluids are albuminous, colonrless, clear juices, containing lymph-corpuscles (§9), which are identical with the colourless bloodcorpuscles. In some places, e.g., in the lymphatics of the spleen, especially in starving animals (Nasse), and in the thoracic duct, a few coloured blood-corpuscles have been found. The lymph-corpuscles are supplied to the lymph and chyle, from the lymphatic glands and the adenoid tissue. They also pass out of the blood-vessels and wander into the lymphatics, and as coloured blood-corpuscles have also been seen to pass out of the blood-vessels (Stricker, J. Arnold), this explains the occasional presence of these corpuscles in some lymphatics; but when the pressure within the veins is high near the orifice of the thoracic duct, red blood-corpuscles may pass into the thoracic duct. In addition, the chyle contains numerous fatty granules each surrounded with an albuminous envelope. [Thus the chyle, in addition to the constituents of the lymph, contains, especially during digestion, a very large amount of fat in the form of the finely emulsionised fat of the food, which gives it its characteristic white or milky appearance. During hunger, the fluid in the lacteals resembles ordinary lymph. The fine fat granules constitute the so-called "molecular basis" of the chyle.]

Composition of Lymph. - The lymph consists of a plesma with lymphcorpuscles suspended in it. The corpuscles-for the most part investigated in the form of pus-cells-consist of swollen-up protcid and soluble paraglobulin, together with lecithin, cerebrin, cholesterin, and fat, while their nuclei yield nuclein. Nuclein contains $P$, and is prepared by the artificial digestion of pus, as it alone remains undigested; it is soluble in alkalies, and is precipitated from this solution by acids. It gives a feeble xanthoproteic reaction. When subjected to the prolonged action of alkalies and acids, it yields substances allied to albumin and syntonin. Miescher found glycogen in the lymph-corpuscles of serous fluids. The lymph-plasma contains the three fibrin-factors (\$ 29), 
derived very probably from the breaking up of lymph-corpuscles. When lymph is withdrawn from the body, these substances cause it to coagulate. Coagulation occurs slowly, owing to the formation of a soft jellylike, small "lymph-clot," which contains most of the lymph-corpuscles. The exuded fluid or lymph-serum contains alkuli-albuminate (precipitated by acids), serum-albumin (coagulated by heat), and paraglobulin-the two latter occurring in the same proportion as in blood-serum; 37 per cent. of the coagulable proteids is paraglobulin (Salvioli). Peptone has been found in chyle (? and perhaps also in lymph); also urea (Wurtz), leucin and sugar.

(2.) Chyle, which occurs within the lacteals of the intestinal tract can only be obtained in very small amount before it is mixed with lymph, and hence the difficulty of investigating it. A few lymphcorpuscles occur even in the origin of lacteals within the villi, but their number increases in the vessels beyond the intestine, more especially after the chyle has passed through the mesenteric glands. The amount of solids, which undergoes a great increase during digestion, on the contrary, diminishes when chyle mixes with lymph. After a diet rich in fatty matters, the chyle contains innumerable fatty granules $(2-4 \mu$ in size). [This is the so-called "molecular basis" of the chyle.] The amount of fibrin-factors increases with the increase of lymph-corpuscles, as they are formed from the breaking-up of the lymph-corpuscles. Grohé found a diastatic ferment in chyle, which was probably absorbed from the intestine, occasionally sugar, to 2 per cent. (Colin), and after much starchy food, lactates have been found (Lehmann).

The Chyle of a person who was executed contained:-

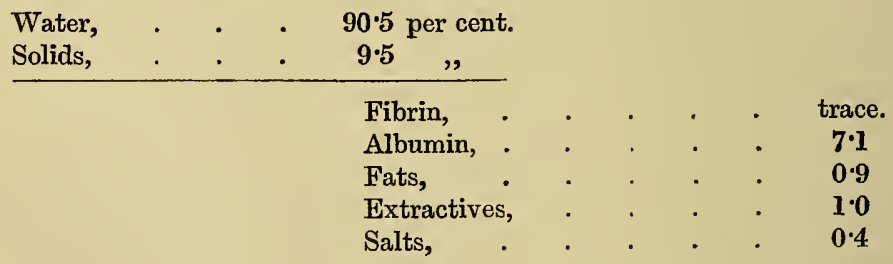

Cl. Schmidt found the following inorganic substances in 1000 parts of chyle (horse):-

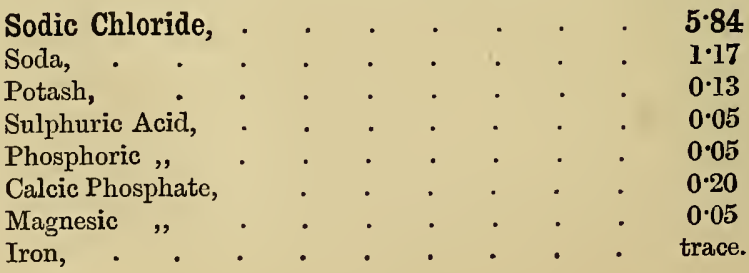


(3.) The Lymph obtained from the beginning of the lymphatic system also contains very few lymph-corpuscles; it is clear, transparent, and colourless, and closely resembles the fluids of serous cavities. That the lymph coming from different tissues varies somewhat, is highly probable, but this has not been proved. After lymph has passed through lymphatic glands, it contains more corpuscles, and also more solids, especially albumin and fat. Ritter counted 8,200 lymphcorpuscles in 1 cubic centimetre of the lymph of a dog.

Hensen and Dähnhardt obtained pure lymph in considerable quantity from a lymphatic fistula, in the leg of a man. It had an alkaline reaction, and a saline taste. It had the following composition, which may be compared with the composition of serous transudations:-

\begin{tabular}{|c|c|c|}
\hline $\begin{array}{l}\text { Pure Lymph } \\
\text { (Hensen \& Dïhnhardit). }\end{array}$ & $\begin{array}{l}\text { Cerebrospinal Fluid } \\
\text { (Hoppe-Seyler). }\end{array}$ & $\begin{array}{l}\text { Pericardial Flnid } \\
\text { (v. Gorup-Besanez). }\end{array}$ \\
\hline $\begin{array}{lcc}\text { Water, . } & . & 98 \cdot 63 \\
\text { Solids, . } & . & 1.37 \\
\text { Fibrin, . } & . & 0 \cdot 11 \\
\text { Albumin, } & . & 0.14 \\
\text { Alkali-albuminate, . } & 0.09 \\
\text { Extractives, . } & . & \ldots \\
\text { Urea, Leucin, } & . & 1.05 \\
\text { Salts, } & . & 0.85 \\
70 \text { vol. per cent. of ab- } \\
\text { sorbed } \mathrm{CO}_{2}, 50 \% \text { of which } \\
\text { could be pumped out, and } \\
20 \% \text { by the addition of an } \\
\text { acid. }\end{array}$ & $\begin{array}{l}\qquad \begin{array}{c}98 \cdot 74 \\
1 \cdot 25 \\
\ldots \\
0 \cdot 16 \\
\ldots \\
\ldots \\
\ldots\end{array} \\
\text { The cerebrospinal fluid, } \\
\text { and abdominal - lymph } \\
\text { contain a kind of sugar } \\
\text { (without the property of } \\
\text { rotating polarised light- } \\
\text { Hoppe-Seyler). }\end{array}$ & $\begin{array}{c}95 \cdot 51 \\
4 \cdot 4 S \\
0 \cdot 08 \\
2 \cdot 46 \\
\ldots \\
1 \cdot 26 \\
\ldots\end{array}$ \\
\hline
\end{tabular}

100 parts of the Ash of lymph contained the following substances:-

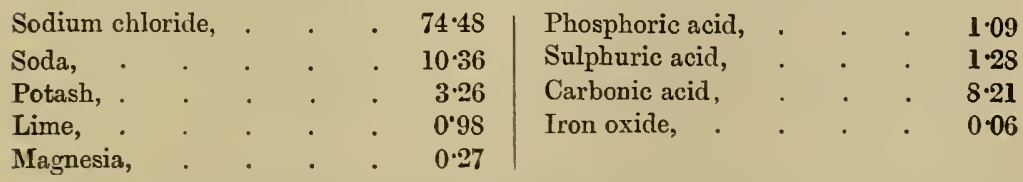

Just as in blood, potash and phosphoric acid are most abundant in the corpuscles, while soda (chiefly sodium chloride) is most abundant in the lymph-serum. The potash and phosphoric acid compounds are most abundant in cerebro-spinal fluid, according to C. Schmidt. The amount of water in the lymph rises and falls with that of the blood. Dog's lymph contains much $\mathrm{CO}_{2}-$ more than 40 vols. per cent., of which 17 per cent. can be pumped out, and 23 per cent. expelled by acids, while there are only traces of $\mathrm{O}$ and 1.2 vols. per cent. $\mathrm{N}$ (Ludwig, Hammersten). 
The observation that when lymph is collected from large vessels and exposed to the air it becomes red (Funke) is as yet unexplained; but it is certainly not due to the formation of coloured corpuscles from colourless ones, owing to contact with the $\mathrm{O}$ of the air.

\section{Quantity of Lymph and Chyle.}

When it is stated that the total amount of the lymph and chyle passing through the large vessels in 24 hours is equal to the amount of the blood (Bidder and C. Schmidt), it must be remembered that this is merely a conjecture. Of this amount one-half may be lymph and the other half chyle. The formation of lymph in the tissues takes place continually, and without interruption. Nearly 6 kilos. of lymph were collected in 24 hours from a lymphatic fistula in the arm of a woman, by Gubler and Quevenne; 70 to 100 grms. were collected in $1 \frac{1}{2}$ to 2 hours from the large lymph-trunk in the neck of a young horse.

The following conditions affect the amount of chyle and lymph:-

(1.) The amount of chyle undergoes very considerable increase during digestion, more especially after a full meal, so that the lacteals of the mesentery and intestine are distended with white or milky chyle. During hunger, the lymph-vessels are collapsed, so that it is difficult to see the large trunks.

(2.) The amount of lymph increases with the activity of the organ from which it proceeds. Active or passive muscular movements greatly increase its amount. Lesser obtained in this way 300 cubic centimetres lymph from a fasting dog, whereby its blood became so inspissated as to cause death.

(3.) All conditions which increase the pressure upon the juices of the tissues increase the amount of lymph, and vice versî. These conditions are :-

(a.) An increase of the blood-pressure, not only in the whole vascular system, but also in the vessels of the corresponding organ, augments the amount of lymph and vice versî (Ludwig, Tomsa). This however is doubtful, as has been shown by Paschutin and Emminghaus. [In order to increase the amount of lymph depending upon pressure within the vessels, what must happen is increased pressure within the capillaries and veins].

(b.) Ligature or obstruction of the efferent veins greatly increases the amount of lymph which flows from the corresponding parts (Bidder, Emminghaus). It may be doubled in amount (Weiss). Tight bandages cause a swelling of the parts on the peripheral side of the bandage, owing to a copious effusion of lymph into the tissue (congestive cedema).

(c.) An increased supply of arterial blood acts in the same way, but to a less degree. Paralysis of the vaso-motor nerves (Ludwig), or stimulation of vasodilatur fibres (Gianuzzi), by increasing the supply of blood increases the amount of lymph; while diminution of the blood supply, owing to stimulation of vaso-motor fibres or other causes, diminishes the amount. Even after ligature of both carotids, as the head is still supplied with blood by the vertebrals, the lymph-stream in the large cervical lymphatic, does not cease (WW. Krause). 
(4.) When the total amount of the blood is increased, by the injection of blood, serum, or milk into the arteries, much fluid passes into the tissues and increases the formation of lymph.

(5.) The formation of lymph still goes on for a short time after death, and after complete cessation of the action of the heart, but only to a slight extent. If fresh blood be caused to circulate in the body of an animal, while it is still warm, more lymph flows from the lymphatics (Genersich). It appears as if the tissues obtained plasma from the blood for a time after the stoppage of the circulation. This perhaps explains the circumstance that some tissues, c.g., connective-tissues, contain more fluid after death than during life, whilst the blood-vessels have given out a considerable amount of their plasma after deatl.

(6.) The amount of lymph is increased under the influence of curara (Lesser, Paschutin), and so is the amount of solids in the lymph. A large amount of lymph collects in the lymph-sacs [especially the sublingual] of frogs poisoned with curara, which is partly explained by the fact that the lymph-hearts are paralysed by curara (Bidder). The amount of lymph is also increased in inflamed parts (Lassar).

\section{Origin of Lymph.}

(1.) Origin of the Lymph-Plasma.-The lymph-plasma may be regarded as fluid which has been pressed through the walls of the bloodvessels by the blood-pressure, i.e., by filtration, into the tissues. The salts which pass most readily through membranes, go through nearly in the same proportion as they exist in blood-plasma-the fibrin-factors to about two-thirds, and albumin to about one-half of that in the blood. As in the case of other filtration processes, the amount of lymph must increase with increasing pressure. This was proved by Ludwig and Tomsa, who found that when they passed blood-serum under varying pressures through the blood-vessels of an excised testis, the amount of transuded fluid which flowed from the lymphatics varied with the pressure. This "artificial lymph" had a composition similar to that of the natural lymph. Even the amount of albumin increased with increasing pressure. The lymph-plasma is mixed in the different tissues with the decomposition products, the results of the metabolism of the tissues.

When the muscles are in action, not only is the lymph poured out more rapidly, but more lymph is formed. The tendons and fascix of the muscles of the skeleton which are provided with numerous small stomata, absorb the lymph from the muscles. By the alternate contraction and relaxation of these fibrous structures, they act like suctionpumps, whereby the lymphatics are alternately filled, and emptied, while 
the lymph is propelled onwards. Even passive movements act in the same way. If solutions be injected under the fascia lata, they may be propelled onwards to the thoracic duct by passive movements of the limb (Ludwig, Schweigger-Seidel, and Genersich).

(2.) The Origin of the Lymph-Corpuscles varies-1. A very considerable number of the lymph-corpuscles are derived from the lymphatic glands; they are washed out of these glands into the vas efferens by the lymph-stream, hence, the lymph always contains more corpuscles after it has passed through a lymph-gland. Small isolated lymph-follicles permit corpuscles to pass through their limiting layer into the lymph-stream. 2. A second source is those organs whose basis consists of adenoid tissue, and in whose meshes numerous lymphcorpuscles occur-e.g., the mucous membrane of the entire intestinal tract, red marrow of bone, the spleen. In these cases, the cells reach the origin of the lymph-stream by their own amoeboid movements. 3. As lymph-corpuscles are returned to the blood-stream, where they appear as colourless blood-corpuscles, so they again pass out of the blood-capillaries into the tissues, partly owing to their amoboid movements (Cohnheim), and they are partly expelled by the blood-pressure (Hering). In rare cases, lymph-corpuscles wander from lymphatic spaces back again into the blood-vessels (v. Recklinghausen).

Fine particles of cinnabar or milk-globules introduced into the blood soon pass into the lymphatics, and the vaso-motor nerves do not affect the process. The extrusion of particles is greater during venous congestion, just as with diapedesis (p. 189), than when the circulation is undisturbed; inflammatory affections of the vascular wall also favour their passage. The vessels of the portal system are especially pervious (Riitimeyer).

(4.) By increase of the lymph-corpuscles by division, and also by proliferation of the fixed connective-tissue corpuscles (His). This process certainly occurs during inflammation of many organs. This has been proved for the excised cornea kept in a moist chamber (v. Recklinghausen, Hoffmann); the nuclei of the cornea-corpuscles proliferate also (Stricker, Norris). That the connective-tissue corpuscles proliferate is shown by the enormous production of lymph-corpuscles in acute inflammations (with the formation of pus)-e.g., in extensive erysipelas, and inflammatory purulent effusions into serous cavities, where the number of corpuscles is too great to be explained, by the wandering of blood-corpuscles out of the blood-vessels.

Decay of Lymph-Corpuscles.-The lymph-corpuscles disappear partly where the lymphatics arise. The occurrence of the fibrin-factors in the lymph-formed as they are from the breaking-up of lymphcorpuscles-would seem to indicate this. In inflammation of connective-tissue, in addition to the formation of numerous new lymph- 
corpuscles, a considerable number seems to be dissolved; hence the lymph, and also the blood, in this case contains more fibrin.

Lymph-corpuscles are also dissolved within the blood-stream, and help to form the fibrin-factors.

\section{Movement of Chyle and Lymph.}

The ultimate cause of the movement of the chyle and lymph depends upon the difference of the pressure at the origin of the lymphatics, and the pressure where the thoracic duct opens into the venous system.

(1.) The forces which are active at the origin of the lymphatics are concerned in moving the lymph, but these must vary according to the place of origin-(a) The lacteals receive the first impulse towards the movements of their contents - the chyle-from the contraction of the muscular fibres of the villi (p. 390). When these contract and shorten, the axial lacteal is compressed, and its contents forced in a centripetal direction towards the large lymphatic trunks. When the villi relax the numerous valves prevent the return of the chyle into the villi. (b) Within those lymphatics which take the form of peri-vascular spaces, every time the contained blood-vessel is dilated the surrounding lymph will be pressed onwards. (c) In the case of the pleural lymphatics with open mouths, every inspiratory movement acts like a suction-pump upon the lymph (Dybkowsky), and the same is the case with the openings (stomata) of the lymphatics on the abdominal side of the diaphragm (Ludwig, Schweigger-Seidel). (d) In the case of those vessels which begin by means of fine juice-canals, the movement of the lymph must largely depend upon the tension of the juices of the parenchyma, and this again must depend upon the tension or pressure in the blood-capillaries, so that the blood-pressure acts like a vis a tergo in the rootlets of the lymphatics.

[In some organs peculiar pumping arrangements are brought into action. As already mentioned, the abdominal surface of the central tendon of the diaphragm is provided with stomata, or open communications between the peritoneal cavity and the lymphatics in the substance of the tendon. v. Recklinghausen found that milk put upon the peritoneal surface of the central tendon showed little eddies caused by the milk-globules passing through the stomata and entering the lymphatics. The central tendon consists of two layers of fibrous tissue arranged in different directions. When the diaphragm moves during respiration, these layers are alternately pressed together and pulled apart. Thus the spaces are alternately dilated and contracted, lymph being drawn into the lymphatics (Fig. 164, $h$ ) through the stomata]. 
[Ludwig's Experiment.-Tie a respiration cannula in the trachea of a dead rabbit; cut across the body of the animal immediately below the diaphragm; remove the viscera, and ligature the vessels passing between the thorax and abdomen; tie the thorax to a ring, and hang it up with the head downwards;

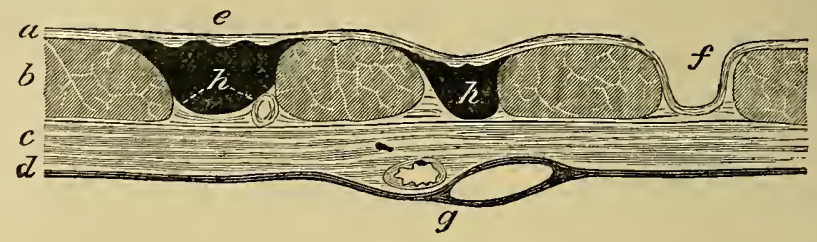

Fig. 164.

Section of central tendon of diaphragm-The injected lymph spaces, $h$ and $h$, are

black. At $f$ the walls of the space are collapsed (Brunton, after Ludwig and

Schweigger-Seidel).

pour a solution of Berlin blue upon the peritoneal surface of the diaphragm; connect the respiration cannula either with a pair of bellows or an apparatus for artificial respiration, and imitate the respiratory movements. After a few minutes, the lymphatics are filled with a blue injection showing a beautiful plexus.]

[The same kind of pumping mechanism exists over the costal pleura (p. 224).]

[The fascia covering the muscles is another similar mechanism. The fascia consists of two layers of fibrous tissue, with intervening lymphatics (Fig. 165). When a muscle contracts, lymph is forced out

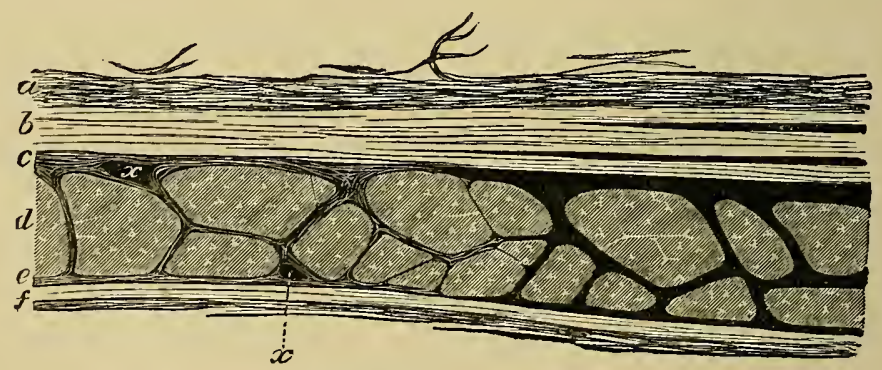

Fig. 165.

Injected lymph spaces from the fascia lata of the dog-The injected spaces are black in the figure (Brunton, after Ludwig and Schweigger-Seidel).

from between the layers of the fascia, while when it relaxes, the lymph from the muscle, carrying with it some of the waste products of muscular action, passes out of the muscle into the fascia, between the now partially separated layers.]

(2.) Within the lymph-trunks themselves, the independent contraction of their muscular fibres partly aids the lymph-stream. Heller observed in the mesentery of the guinea-pig, that the peristaltic movement of 
the lymphatic wall passed in a centripetal direction. The numerous ralves prevent any reflux. The contraction of the surrounding muscles, and every pressure upon the ressels and the tissues aid the current (Ludwig, Noll). If the outflow of blood from the veins is interfered with, lymph flows copiously from the corresponding tissues (Nasse, Tomsa). [If a cannula be tied in a lymphatic of a dog, a few drops of lymph flow out at long intervals. But if even passive movements of the limb be made, e.g., simply flexing and extending the limb, the outflow becomes very considerable and continuous.]

(3.) The lymph-glands, which occur in the course of the lymphatics, offer very considerable resistance to the lympl-strean, which must pass through the lympli-paths, whose spaces are traversed by adenoid tissue, and contain a few lymph-corpuscles. But this is, to a certain extent, compensated by the non-striped muscle which exists in the capsule and trabecula of the glands. When they contract, they force on the lymph, while the valves prevent its reflux. Enlarged lymphatic glands have been seen to contract when stimulated electrically. [Botkin has stimulated enlarged lymphatic glands with electricity in cases of lenkæmia.]

(4.) As the lymph-vessels gradually join and form larger vessels, and finally form one trunk, the transverse section, or sectional area, diminishes, so that the velocity of the current and the pressure are increased. Nevertheless, the velocity is always small; it varied from 230-300 millimetres per minute in the large lymphatic in the neck of a horse (Weiss), a fact which enables us to conclude that the movement must be very slow in the small vessels. The lateral pressure at the same place, was 10-20 mm., and in the dog 5-10 mm. of a weak solution of soda (Weiss, Noll), although it was found to be $12 \mathrm{~mm}$. $\mathrm{Hg}$. in the thoracic duct of a horse (Weiss).

(5.) The respiratory movements exercise a considerable influence upon the lymph-stream in the thoracic duct, and in the right lymphatic duct; every inspiration favours the passage of the venous blood, and also of the lymph towards the heart, whereby the tension in the thoracic duct may even become negative (Bidder). [The diastolic suction of the heart by diminishing the pressure in the veins, also favours the inflow of lymph into the thorax.]

(6.) Lymph-hearts exist in certain cold-blooded animals (Panizza, Joh. Miiller). The frog has two axillary hearts (above the shoulder near the vertebral column), and two sacral hearts, one on each side of the coccyx near the anus. They beat, but not synchronously, about 60 times per minute, and contain 10 cubic centimetres of lymph. They have transversely striped muscular fibres in their walls, and are also provided with nerve ganglia (Waldeyer). The posterior pair pump the lymph into the branch of the Vena iliaca communicans, and the anterior pair into the Vena subscapularis. Their pulsation depends partly, but not 
exclusively, upon the spinal cord, for if the cord be rapidly destroyed, they may cease to pulsate (Volkmann), but not unfrequently they continue to pulsate after removal of the cord (Valentin, Luchsinger). A second source of their pulsatile movements is to be sought for in Waldeyer's ganglia. Stimulation of the skin, intestine, or blood-heart influences them reflexly-partly accelerating and partly retarding them. If the coccygeal nerve, which connects the sacral hearts to the spinal cord, be clivided, these effects do not occur (v. Wittich). Strychnia accelerates their movements (Scherhej). Antiar paralyses the lymph-heart and the blood-heart at the same time (Vintschgau), while curara paralyses the former alone (Bidder).

In other amphibians, there are two lymph-hearts, in the ostrich and cassowary and some swimming birds (Panizza), and in the embryo chick (A. Budge). They occur in some fishes-e.g., near the caudal vein of the eel.

(7.) The nervous system has a direct effect upon the lymph-stream, on account of its connection with the muscles of the lymphatics and lymph-glands, and with the lymph-hearts where these exist. Farther, Kühne observed that the cornea-corpuscles contracted when the corneal nerves were stimulated. Goltz also observed that when a dilute solution of common salt was injected under the skin of a frog, it was rapidly absorbed, but if the central nervous system was destroyed it was not absorbed.

If inflammation be produced in the posterior extremities of a dog, and if the sciatic nerve be divided on one side, celema and a simultaneous increase of the lymph-stream occur on that side (Jankowski).

Ligatnre the leg of a frog, except the nerves, so as to arrest the circulation, and place the leg in water; it swells up very rapidly, but a dead limb does not swell up. So that absorption is independent of the continuance of the circulation. Section of the sciatic nerve, or destruction of the spinal cord (but not section of the brain), arrests absorption (Lautenbach).

\section{Absorption of Parenchymatous Effusions.}

Fluids which pass from the blood-vessels into the spaces in the tissues, or those injected subcutaneously, are absorbed chiefly by the blood-vessels, but also by the lymphatics. Small particles, as after tattooing with cinnabar or China ink, may pass from the tissue-spaces into the lymphatics-and so do blood-corpuscles from extravasations of blood, and fat granules from the marrow of a broken bone. If all the lymphatics of a part are ligatured, absorption takes place quite as rapidly as before (Magendie); hence, absorbed fluid must pass through the thin membranes of the blood-vessels. The corresponding experiment of ligaturing all the bloodvcssels, when no absorption of the parenchymatous juices takes place (Emmert, Henle, v. Dusch), does not prove that the lymphatics are not concerned in absorption, for, after ligaturing the blood-vessels of a part, of course the formation of lymph, and also the lymph-stream, must cease.

When fluids are injected under the skin, absorption takes place very rapidlymore rapidly than when the substance is given by the mouth. The subcutaneous injection of many drugs is now extensively used, but of course the substances used must not corrode, irritate, or coagulate the tissues. Some substances do not act when given by the mouth, as snake poison, poisons from dead bodies or putrid things, although they act rapidly when introduced subcutaneously. If emulsin be given by the mouth, and amygdalin be injected into the veins of an animal, hydro. 
cyanic acid is not formed, as the emulsin seems to be destroyed in the alimentary canal. If the emulsin, lowever, be injected into the blood, and the amygdalin be given by the mouth, the animal is rapidly poisoned, owing to the formation of hydrocyanic acid, as the amygdalin is rapidly absorbed from the intestinal canal. The amygdalin, a glucoside $\left(\mathrm{C}_{20} \mathrm{H}_{27} \mathrm{NO}_{11}\right)$, is acted upon by fresh emulsin like a ferment; it takes up $2\left(\mathrm{H}_{2} \mathrm{O}\right)$ and yields hydrocyanic acid $(\mathrm{C} \mathrm{H} \mathrm{N})$, + oil of bitter almonds $\left(\mathrm{C}_{7} \mathrm{H}_{6} \mathrm{O}\right)$, + sugar $2\left(\mathrm{C}_{6} \mathrm{H}_{12} \mathrm{O}_{6}\right)-(\mathrm{Cl}$. Bernard).

When serum is injected subcutaneously, it is rapidly absorbed; it is decomposed within the blood-stream, and increases the amount of urea (p. 62,2). Albuminous solutions, oil, peptones and sugars are also absorbed (Eichhorst).

\section{Congestion of Lymph and Serous Effusions.}

\section{Edema and Dropsy.}

[As aptly illustrated by Lauder Brunton, the lymph-spaces may be represented by cisterns, each of which is provided with supply pipes-the arteries and capillaries; while there are two exit pipes-the veins and lymphatics. In health, the balance between the inflow and outflow is such, that the spaces are merely moistence with fluid. When a cannula is placed in a lymphatic vessel in a dog, only a few drops of lymph flow out at long intervals. Emminghaus found that, if the veins of the limb be ligatured, the lymph flows much more quickly. This is in part due to the increased transudation of fluid from the small blood-vessels, but as Brunton suggests, it may also be due to fluid passing away by the lymphatics when it can no longer be carried away by the veins. We cannot say what is the relative share of the veins and lymphaties, nor in the above experiment do we know how much is due to increased transudation or diminished absorption. When there is an undue accumulation of fluid in the lymph-spaces, we have the condition termed dropsy.]

If the efferent veins and lymphatics of an organ be ligatured, or if resistance be offered to the outflow of their contents, congestion and a copious transudation of lymph into the tissues take place. These are most marked in the skin and subcutaneous cellular tissue. The soft parts swell up, without pain or redness, and a doughy swelling, which pits on pressure with the finger, results. These are the signs of lymph-congestion, which is called œdema when the fluid is watery.

Under similar circumstances, lymph is effused into the serous cavities. If at the same time, a large number of colourless blood-corpuscles pass out of the bloodvessels into the cavity, the fluid becomes more and more like pus. In order that these corpuscles may proliferate, a considerable percentage of albumin is necessary. When the pressure within the serous cavity rises above that in the small blood-vessels, water may pass into the blood. These sero-purulent effusions not unfrequently undergo changes, and yield decompasition products, such as leucin, tyrosin, xanthin, kreatin, kreatinin (?), uric acid (?), urea. Endothelium from the serous cavity (Quincke), sugar in pleuritic effusions (Eichlrorst), and in œdemas with little albumin (Rosenbach), cholesterin frequently in hydrocele fluid, and succinic acid in the fluid of echinococci have all been found in these effusions.

The effusion of lymph may arise not only from pressure upon the lymphatics, but also from inflammation and thrombosis of the lymphatics themselves, in which cases not unfrequently new Iymphatics are formed, so that the communication is re-established. Sometimes the ductus thoracicus bursts and lymph is poured directly into the abdomen or thorax. [Ligature of the thoracic duct results in rupture of the receptaculum chyli and escape of chyle and lymph into the large serous cavities (Ludwig).]

When dropsy or effusion of fluids occurs into serous cavities, there is always a 
greater transudation of fluid through the blood-vessels. The abdominal bloodvessels, and those which yield a watery effusion under normal circumstances, are those most liable to be affected.

Transudation is favoured by-(1) Venous congestion, in which case the effusion usually contains little albumin, and few lymph-corpuscles, while the coloured-corpuscles on the contrary are more numerous the greater the venous obstruction. Ranvier produced œdema artificially by ligaturing the vena cava in a dog, and at the same time dividing the sciatic nerve. The paralytic dilatation of the blood-vessels thereby produced caused an increased amount of blood to pass to the limb, while the blood-pressure was raised, and both factors favoured the transudation of fluid. [Ranvier's experiment proves that mere ligature of the venous trunk of a limb by itself is not sufficient to cause edema. The œdema is due to the concomitant paralysis of the vaso-motor nerves. If the motor roots of the sciatic nerve alone be divided along with ligature of the vena cava, no cedema occurs, but if the vaso-motor fibres are divided at the same time, the limb rapidly becomes œedematous. There is such an increased transudation through the vascular walls that the veins and lymphatics cannot remove it with sufficient rapidity, and cedema occurs. If there be weakness of the vaso-motor nerves, slight obstruction is sufficient to produce cedema (Lauder Brunton).] When the leg veins are occluded with an injection of gypsum, œdema occurs (Sotnischewsky). (2) Some unknown physical changes occur in the protoplasm of the endothelium of the capillaries and blood-vessels, which favour the transudation of albumin, hæmoglobin, and even blood-corpuscles. This occurs when abnormal substances accumulate in the blood-e.g., dissolved hæmoglobin-and when the blood contains little $O$ or albumin. The same has been observed after exposure to too high temperatures, and the swelling of soft parts in the neighbourhood of an inflammatory focus seems due to the transudation of fluid through the altered vascular wall. It is probable that a nervous influence may affect particular areas, through its action on the bloodvessels of the part (it may be upon the protoplasm of the blood-capillaries). The transudations of this nature usually contain much albumin and many lymphcorpuscles. (3) When the blood contains a very large amount of water the tendency to transudation of fluid is increased. After a time it may produce the changes indicated in (2), and when long continued may increase the permeability of the vascular wall (Cohnheim). Watery lymphatic effusions from wateryblood-"cachectic oedema"-occur in feeble and badly nourished individuals. [One of the commonest forms of dropsy is the slight cedema of the legs in anæmic persons, in whom the heart and lungs are healthy. Many factors are involved-the watery condition of the blood, the condition of nutrition of the capillaries, and probably a tendency to vaso-motor paresis (Brunton).]

[(4) Ostroumoff found that stimulation of the lingual nerve not only causes the blood-vessels of the tongue to dilate, but the corresponding side of the tongue becomes œdematous. If a solution of dilute hydrochloric acid or quinine (p. 287) be injected into the duct of the submaxillary gland, and the chorda tympani stimulated, there is no secretion of saliva, but the gland becomes œdematous. In an animal poisoned with atropin, stimulation of the chorda causes dilatation of the blood-vessels, although there is no secretion of saliva, nevertheless the gland does not become œdematous (Heidenhain). As Brunton suggests, this experiment points to some action of atropin on the blood-vessels which has hitherto been entirely overlooked.]

\section{Comparative Physiology.}

In the frog, large lymph-sacs, lined with endothelium, exist under the skin, while large lymph-sacs lie in relation with the vertebral column-one on each side 
-separated by a thin membrane, perforated with stomata, from the abdominal cavity. This is the cysterna lymphatica magna of Panizza. Some amphibians and many reptiles have large lymph-spaces under the skin, which occupy the whole of the dorsal region of the body. All reptiles and the tailed amphibians have large elongated reservoirs for lymph along the course of the aorta. The lymph apparatus of the tortoise (Fig. 159) is very extensive.

The osseous fishes have in the lateral parts of their backs an elongated lymphtrunk, which reaches from the tail to the antecior fins, and is connected with the dilated lymphatic rootlets in the base of the tail and in the fins. The largest internal lymph-sinus is in the region of the cesophagus. Many birds possess a sinus-like dilatation or lymph-space in the region of the tail. The lymph-spaces communicate with the venous system-with valves properly arranged-usually in connection with the upper vena cava. Lymph-hearts have already been referred to $(\mathrm{p}, 417)$.

In carnivora, the lymph-glands of the mesentery are united into one large com. pact mass, the so-called " pancreas Asellii."

\section{Historical.}

Although the Hippocratic School was acquainted with the lymph-glands from their becoming swollen from time to time, and although Herophilus and Erasistratus had seen the mesenteric glands, yet Aselli (1662) was the first who accur. ately described the lacteals of the mesentery with their valves. Pecquet (1648) discovered the receptaculum chyli; Rudbeck and Thom. Bartholinus the lymphatic vessels (1650-52); Eustachius (1563) was acquainted with the thoracic duct, which Gassendus (1654) maintained that he was the first to see; Lister noticed that the chyle became blue when indigo was injected into the intestine (1671); Sömmcring observed the separation of fibrin when lymph coagulated; Reuss and Emmert discovered the lymph-corpuscles. The chemical investigations date from the first quarter of this century; they were carried out by Lassaigne, Tiedemann, Gmelin, and others. The two last observers noticed that the white colour of chyle was due to the presence of small fatty granules. 


\section{Physiology of Animal Heat.}

\section{Sources of Heat.}

Sources. - The heat of the body is an uninterrupted evolution of kinetic energy, which we must represent to ourselves as due to vibrations of the corporeal atoms. The ultimate source of the heat is contained in the potential energy taken into the body with the food, and with the $\mathrm{O}$ of the air absorbed during respiration. The amount of heat formed depends upon the amount of energy liberated (see Introduction).

The energy of the food-stuffs may be called "latent heat," if we assume that when they are used up in the body, chiefly by a process

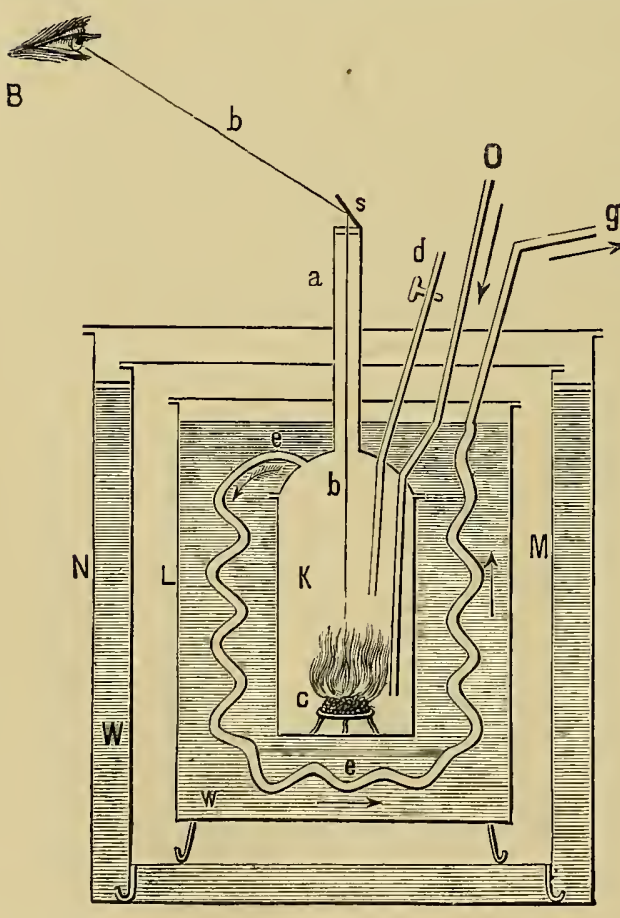

Fig. 166.

Water calorimeter of Favre and Silbermann.

of combustion, kinetic energy is liberated only in the form of heat. As a matter of fact, however, mechanical energy and electrical energy are developed from the potential energy. In order to obtain a unit measure for the energy liberated, it is advisable to express all the potential energy as heat-units.

The Calorimeter.-This instrument enables us to transform the potential energy of the food into heat, and, at the same time, to measure the number of heat-units produced.

Favre and Silbermann used a water-calorimeter (Fig. 166). The substance to be burned is placed in a large cylindrical combustion chamber (K), suspended in a large cylindrical vessel $(\mathrm{L})$ filled with water $(w)$, so that the combustion chamber is completely surrounded by the water. Three tubes open into the upper 
part of the chamber; one of them $(O)$ supplies the air which is necessary for combustion, it reaches almost to the bottom of the chamber; the second tube $(a)$ is fixed in the middle of the lid, and is closed above with a thick glass plate, and on this is placed, at an angle, a small mirror $(s)$ which enables an observer to see into the interior of the chamber, and to observe the process of combustion at $c$. The third tube $(d)$ is used only when combustible gases are to be burned in the chamber. It can be closed by means of a stop-cock. A lead tube $(e, e)$ with many twists on it, passes from the upper part of the chamber through the water, and finally opens at g. The gaseous products of combustion pass out through this tube, and in doing so help to heat the water. The cylindrical vessel with the water is closed with a lid which transmits the four tubes. The water cylinder stands on four feet within a large cylinder (M), which is filled with some good non-conductor of heat, and this again is placed in a large vessel filled with water (W). This is to prevent any heat reaching the inner cylinder from without. A weighed quantity of the substance $(c)$ to be investigated, is placed in the combustion chamber. When combustion is ended, during which the inner water must be repeatedly stirred, the temperature of the water is ascertained by means of a delicate thermometer. If the increase of the temperature and the amount of water are known, then it is easy to calculate the number of heat-units produced by the combustion of a kuown weight of the substance (see Intraduction).

The ice-calorimeter may also be used. The inner cylinder is filled with ice and not with water, and ice is also placed in the outer cylinder to prevent any heat from without from acting upon the inner ice. The heat given off from the combustion chamber causes a certain amount of the ice to melt, and the water thereby produced is collected and measured. It requires 79 heat-units to melt $1 \mathrm{grm}$. of ice to $1 \mathrm{grm}$. of water at $0^{\circ} \mathrm{C}$.

Just as in a calorimeter, although much more slowly, the food-stuffs within our body are burned up, oxygen being supplied, and thus potential energy is transformed into kinetic energy, which, in the case of a person at rest-i.e., when the muscles are inactive, almost completely appears in the form of heat (see Introduction).

Favre, Silbermann, Frankland, Rechenberg, B. Danilewsky, and others have made calorimetric experiments on the heat produced by food. Thus, therc are produced by

1 grm. Albumin 4,998 heat-units

1 ,, Ox-flesh 5,103 ,,

$1 \mathrm{grm}$. Albumin 4,263 ,

1 ,, Ox-flesh 4,368 ,"

\section{f Completely dried and completely}

$\{$ burned.

(When burned to urea (i.e., the heat-units corresponding to the urea ( 1 grm. $=2,206$ calories) is deducted from those of the albumin and flesh.

1 gramme of the following dry substances yields heat-units :-

\begin{tabular}{|c|c|c|c|c|c|c|c|c|c|}
\hline Casein, & & & 5,785 & Palmitin, . & & S,SS3 & Glutin, & & \\
\hline Potatoes, & & & 3,752 & Olein, & . & 8 , & Legumin, & & 5,573 \\
\hline Milk, . & & & 5,093 & Glycerin, & - & 4,179 & Blood tibrin, & & 5,709 \\
\hline Bread, & & & 3,984 & Leucin, . & & 6 , & Peptone, . & & 4,914 \\
\hline ice, . & & & 3,813 & Creatin, & & 4 & Glutin, . & & 5,493 \\
\hline tarch, & & & 4,479 & Grape-sugar, & & 3,939 & Chondrin, & & . 4,909 \\
\hline$f$ & & & 6,460 & Car & & 4,173 & Flesh extract & & \\
\hline 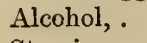 & & & $8,95 \mathrm{~S}$ & Mill & & 4,162 & (Liebig), & & - 3,206 \\
\hline tearin, . & & & 9,036 & Veget & rin, & 31 & & & \\
\hline
\end{tabular}


When we know the weight of any of the above-named substances consumed by a man in twenty-four hours, a simple calculation enables us to determine how many heat-units are formed in the body by oxidation-i.e., provided the substance is completely oxidised.

Sources of Heat.-The individual sources of heat are to be found in the following :-

(1.) In the transformation of the chemical constituents of the food, endowed with a large amount of potential energy, into such substances as have little or no energy.

The organic substances used as food consist of $\mathrm{C}, \mathrm{H}, \mathrm{O}, \mathrm{N}$, so that there takes place- (a) Combustion of $\mathrm{C}$ into $\mathrm{CO}_{2}$, of $\mathrm{H}$ into $\mathrm{H}_{2} \mathrm{O}$, whereby heat is produced; $1 \mathrm{grm}$. $\mathrm{C}$ burned to produce $\mathrm{CO}_{2}$ yields 8,080 heat-units, while $1 \mathrm{grm} . \mathrm{H}$ oxidised to $\mathrm{H}_{2} \mathrm{O}$ yields 34,460 heat-units. The $\mathrm{O}$ necessary for these processes is absorbed during respiration, so that, to a certain extent at least, the amount of heat produced may be estimated from the amount of $\mathrm{O}$ consumed. The same consumption of $\mathrm{O}$ gives rise to the same amount of heat whether it is used to oxidise $\mathrm{H}$ or $\mathrm{C}$ (Pflüger). There is a relation amounting to cause and effect, between the amount of heat produced in the body and the $\mathrm{O}$ consumed. The cold-blooded animals, which consume little $O$ have a low temperature; amongst warm-blooded animals, 1 kilo. of a living rabbit takes up within an hour $0.914 \mathrm{grm} . \mathrm{O}$, and its body is heated to a mean of $38^{\circ} \mathrm{C}$. 1 kilo. of a living fowl uses $1 \cdot 186$ grms. $\mathrm{O}$, and gives a mean temperature of $43.9^{\circ} \mathrm{C}$. (Regnault and Reiset). The amount of heat produced is the same whether the combustion occurs slowly or quickly; the rapidity of the metabolism, therefore, affects the rapidity, but not the absolute amount of heat production. The combustion of inorganic substances in the body, such as the sulphur into sulphuric acid, the phosphorus into phosphoric acid, is another, although very small, source of heat.

(b.) In addition to the processes of combustion or oxidation, all those chemical processes in our body, by which the amount of the available potential energy which is present is diminished, in consequence of a greater satisfaction of atomic affinities, lead to the production of heat. In all cases where the atoms assume more stable positions with their affinities satisfied, chemical energy passes into kinetic thermal energy, as in the alcoholic fermentation of grape-sugar, and other similar processes.

Heat is also developed during the following chemical processes :-

(a) During the union of bases with acids (Andrews). The nature of the base determines the amount of heat produced, while the nature of the acid is without effect. Only in those cases where the acid, e.g., $\mathrm{CO}_{2}$, is unable to set aside the alkaline reaction, the amount of heat produced is less. The formation of compounds of chlorine (e.g., in the stomach) produces heat. 
( $\beta$ ) When a neutral salt is changed into a basic one (Andrews). In the blood, the sulphuric and phosphoric acids derived from the combustion of $S$ and $P$ are united with the alkalies of the blood to form basic salts. The decomposition of the carbonates of the blood by lactic and phosphoric acids forms a double source of heat, on the one hand, by the formation of a new salt, as well as by the liberation of $\mathrm{CO}_{2}$, which is partly absorbed by the blood.

$(\gamma)$ The combination of hæmoglobin with 0 (\$36).

In connection with those chemical processes, whereby the heat of the body is produced, heat-absorbing intermediate compounds are not unfrequently formed. Thus, in order that the final stage of more complete saturation of the affinities be reached, intermediary atomic groups are formed, whereby heat is absorbed. Heat is also absorbed when the solid aggregate condition is dissolved during retrogressive processes. But these intermediary processes whereby heat is lost, are very small, compared with the amount of heat liberated when the end-products are formed.

(2.) Certain physical processes are a second source of heat.

(a) The transformation of the kinetic mechanical energy of internal organs, when the work done is not transferred outside the body, produces heat. Thus the whole of the kinetic energy of the heart is changed into heat, owing to the obstructions which are opposed to the blood-stream. The same is true of the mechanical energy evolved by many muscular viscera. The torsion of the costal cartilages, the friction of the current of air in the respiratory organs, and the ingesta in the digestive tract, all yield heat.

An excessively minute amount of the mechanical energy of the heart is transferred to surrounding bodies by the cardiac impulse and the superficial pulse-beats, but this is infinitesimally small. During respiration, when the respiratory gases and other substances are expired, i very small amount of energy disappears externally, which does not become changed into heat. If we assume that the daily work of the circulation exceeds $\$ 6,000$ kilogram-metres, the heat evolved is equal to $204,000$ calories, in 24 hours ( $\$ 93)$, which is sufficient to raise the temperature of a person of medium size, $2^{\circ} \mathrm{C}$. In former times, Boerhave and others thought that the heat of the body was chiefly due to the friction of the blood within the vessels.

(b) When, owing to muscular activity, the body produces work which is transferred to external objects, e.g., when a man ascends a tower or mountain, or throws a heavy weight, a portion of the kinetic energy passes into heat, owing to friction of the muscles, tendons, and the articular surfaces, as well as to the shock and pressure of the ends of the bones against each other.

(c) The electrical currents which occur in muscles, nerves, and glands, very probably are changed into heat. The chemical processes which produce heat evolve electricity, which is also changed into heat. This source of heat, however, is very small. 
(d) Other processes are the formation of heat from the absorption of $\mathrm{CO}_{2}$ (Henry), by the concentration of water as it passes through membranes (Regnault and Pouillet), in imbibition (Matteucci, 1834), formation of solids-e.g., of chalk in the bones. After death, and in some pathological processes during life, the coagulation of blood (Valentin, Schiffer), and the production of rigor mortis, are sources of heat.

\section{Homoiothermal and Poikilothermal Animals.}

In place of the old classification of animals into "cold-blooded" and "warm-blooded," another basis of classification seems desirable, viz., the relation of the temperature of the body to the temperature of the surrounding medium.

Bergmann introduced the word homoiothermal animals for the warm-blooded animals (mammals and birds), because these animals can maintain a very uniform temperature, even although the surrounding temperature be subject to considerable variations. The so-called coldblooded animals are called poikilothermal, because the temperature of their bodies rises or falls, within wide limits, with the heat of the surrounding medium.

When homoiothermal animals are kept for a long time in a cold medium, their heat production is increased, and when they are kept for a long time in a warm medium it is diminished.

Fordyce gave a proof of the nearly uniform temperature in man. A man remained ten minutes in an oven containing very dry hot air, and yet the temperature of the palm of his hand, mouth, and urine was increased only a few tenths of a degree.

Becquerel and Brechet investigated the temperature of the human biceps (by means of thermo-electric needles), when the arm had been one hour in ice, and yet the temperature of the muscular tissue was cooled only $0.2^{\circ} \mathrm{C}$. The same muscle did not undergo any increase in temperature, or at most $0 \cdot 2^{\circ} \mathrm{C}$, when the man's arm was placed for a quarter of an hour in water at $42^{\circ} \mathrm{C}$.

If heat be rapidly abstracted or rapidly supplied to the body, so as to produce rapid variation of the temperature, life is endangered.

Poikilothermal animals behave very differently; the temperature of their bodies generally follows, although with considerable variations, the temperature of the surroundings. When the temperature of the surroundings is increased, the amount of heat produced is increased, and when the surrounding temperature falls, the amount of heat evolved within the body also falls.

The following table shows very clearly the characters of poikilothermal animals, e.g., frogs (Rana Esculenta), which were placed in air and water of varying temperatures. The frogs were fixed to an iron support, and immersed up to the mouth. The temperature was measured by means of a thermometer introduced through the mouth into the stomach. 


\begin{tabular}{|c|c|c|c|}
\hline \multicolumn{2}{|c|}{ In Water. } & \multicolumn{2}{|c|}{ In Air. } \\
\hline $\begin{array}{l}\text { Temperature of the } \\
\text { Water. }\end{array}$ & $\begin{array}{l}\text { Temperature of Frog's } \\
\text { Stomach. }\end{array}$ & $\begin{array}{l}\text { Temperature of the } \\
\text { Air. }\end{array}$ & $\begin{array}{l}\text { Temperature of Frog's } \\
\text { Stomach. }\end{array}$ \\
\hline $\begin{array}{l}41.0^{\circ} \mathrm{C} . \\
35 \cdot 2 \\
30.0 \\
23.0 \\
20.6 \\
11.5 \\
5.9 \\
2.8\end{array}$ & $\begin{array}{l}38 \cdot 0^{\circ} \mathrm{C} \text {. } \\
34 \cdot 3 \\
29 \cdot 6 \\
22 \cdot 6 \\
20 \cdot 7 \\
12 \cdot 9 \\
5 \cdot 0 \\
5 \cdot 3\end{array}$ & $\begin{array}{l}40 \cdot 4^{\circ} \mathrm{C} \text {. } \\
35 \cdot 8 \\
27 \cdot 4 \\
19 \cdot 8 \\
16 \cdot 4 \\
14 \cdot 7 \\
6 \cdot 2 \\
5.9\end{array}$ & $\begin{array}{l}31 \cdot 7^{\circ} \mathrm{C} \text {. } \\
24 \cdot 2 \\
19 \cdot 7 \\
15 \cdot 6 \\
14 \cdot 6 \\
10 \cdot 2 \\
7 \cdot 6 \\
S \cdot 6\end{array}$ \\
\hline
\end{tabular}

Temperature of different Animals,-Birds-Gull, $37 \cdot \mathrm{S}^{\circ}$; swallow, $44 \cdot 03^{\circ}$. Mammals-Dolphin, $35.5^{\circ}$; mouse, $41 \cdot 1^{\circ}$. Reptiles-Snakes, $10^{\circ}-12^{\circ}$, but higher when incubating. Amphibians and fishes $-0.5^{\circ}-3^{\circ}$ above the temperature of the surroundings. Arthropoda $-0 \cdot 1^{\circ}-5 \cdot 8^{\circ}$ above the surroundings. Bees in a hive, $30^{\circ}-32^{\circ}$, and when swarming, $40^{\circ}$. The following animals have a temperature higher than the surrounding temperature:-Cephalopods, $0.57^{\circ}$; molluses, $0.46^{\circ}$; echinoderms, $0.40^{\circ}$; medusæ, $0.27^{\circ}$; polyps, $0.21^{\circ} \mathrm{C}$.

\section{Methods of Estimating Temperature- Thermometry.}

Thermometry.-By using thermometric apparatus, we are enabled to obtain information regarding the degree of heat of the body to be investigated. For this purpose, the following methods are employed:-

A. The Thermometer (Galileo, 1603).--Sanctorius made the first thermometric observations on man (1626). Celsius (1701-1744) divided his thermometer into 100 parts, and each part was again divided into 10 parts, so that $\frac{1}{10}{ }^{\circ} \mathrm{C}$. could be easily read off. All thermometers which have been used for a long time give too high readings (Bellani), hence they should be compared, from time to time, with a normal thermometer. When taking the temperature, the bulbs ought to be surrounded for 15 minutes, and during the last 5 minutes the mercury column ought not to vary. A very sensitive thermometer will indicate the temperature after 7 seconds if the urine-stream be directed upon its bulb (Oertmann). Minimal and maximal thermometers are often of use to the physician.

Walferdin's metastatic thermometer (Fig. 167) is specially useful for comparative observation. The tube is very narrow in comparison with the bulb, and in order that the stem be not too long, it is constructed so that the amount of mercury can be varied. A quantity of mercury is taken, so that with the temperature expected the thread of mercury will stand about the middle of the stem. A small bulb at the upper part of the stem receives the excess of $\mathrm{Hg}$. Suppose a temperature between $37^{\circ}-40^{\circ} \mathrm{C}$. is to be measured, the bulb is first heated a little over $40^{\circ} \mathrm{C}$., it is then suddenly cooled, and shaken at the same time, so that the thread of mercury is thereby suddenly broken above $40^{\circ}$. The tube is so narrow that $1^{\circ} \mathrm{C}$. is equal to about 10 centimetres of the length of the tube, so that $\frac{1}{100}{ }^{\circ} \mathrm{C}$. is still 1 millimetre in length. The scale is divided empirically, but the value of the divisions must be compared with a normal thermometer.

Kronecker and Meyer used very small maximal "outflow thermometers" (Dulong and Petit), and caused them to pass through the intestinal canal, or through large blood-vessels. The mercury flows out of the short open tube, and of course more Hows out the higher the temperature. After these small bulbs have passed through the animal, a comparison is instituted with a normal thermometer to determine at what temperature the mercury reaches the free margin of the tube.

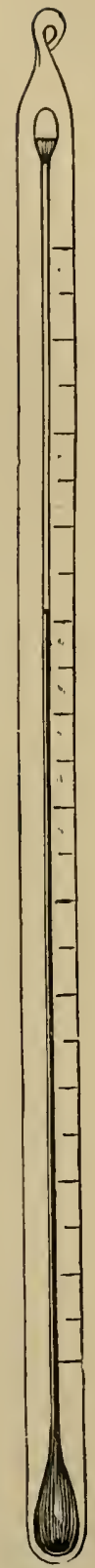

Fig. 167.

Walferdin's

Metastatic

Thermo-

meter. 

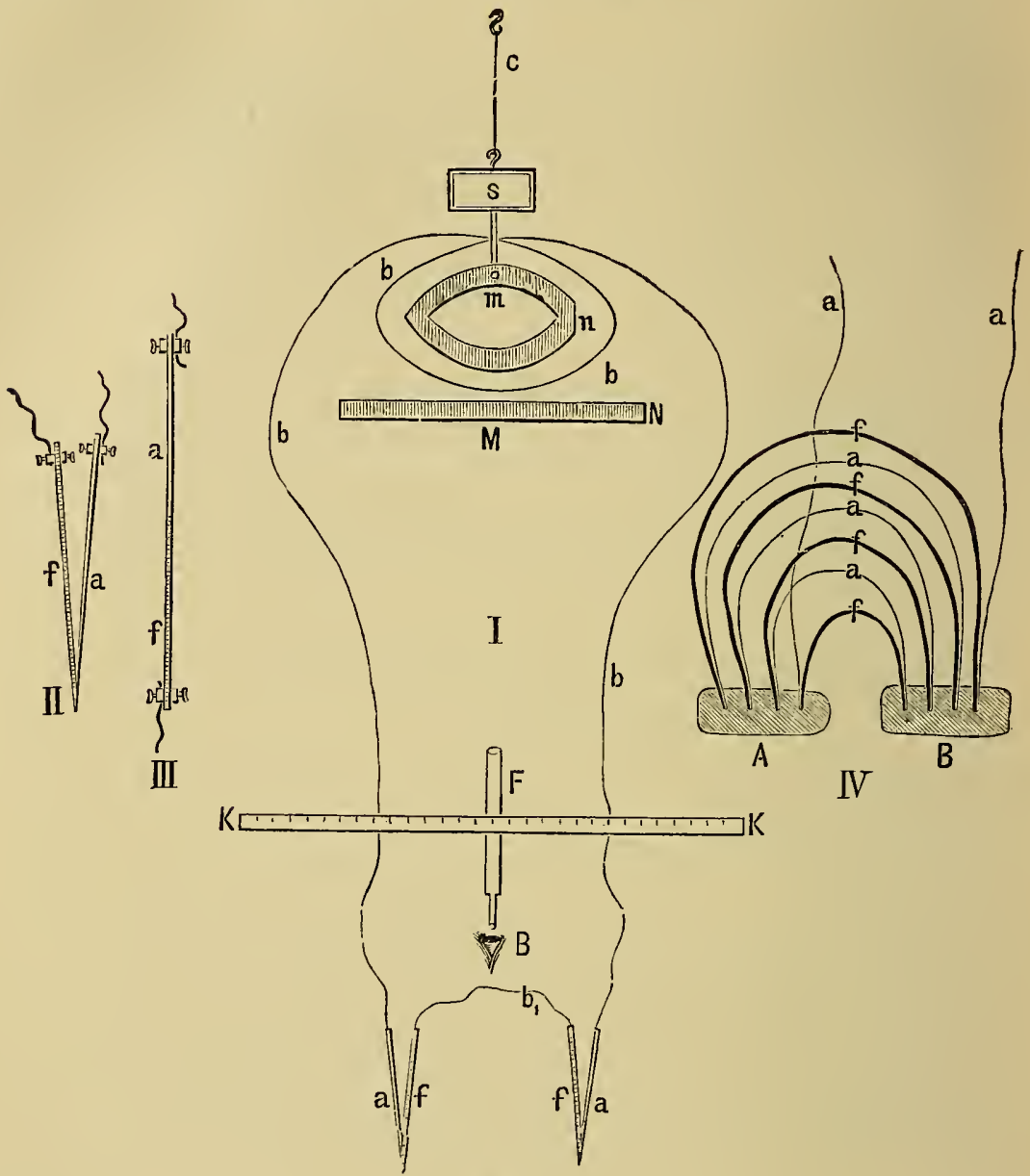

Fig. 168.

Scheme of thermo-electric arrangements for estimating the temperature.

B. Thermo-electric Method,-This method enables us to determine the temperature accurately and rapidly (Fig. 168, I). The thermo-electric galvanometer of Meissner? and |Meyerstein consists of a circular magnet $(\mathrm{m})$, suspended by a thread of silk (c), to which a small mirror (S) is attached. A large stationary bar magnet (M) is placed near the magnet $(m)$, so that the north poles ( $n$ and $N$ ) of both magnets point in the same direction, and it is so arranged that the suspended magnet is caused to point to the north by a minimal action of $\mathbf{M}$.

A thick copper wire $(b, b)$ is coiled several times round $m$ (although in the Fig. it is represented as a single coil), and the ends of the wire are soldered to two thermo-elements,"each composed of two different metals-iron and German silver, the two similar free elements being united by a wire $\left(b_{1}\right)$, so that the two thermoelements form part of a closed circuit. A horizontal scale $(K, K)$ is placed at a distance of 3 metres from the mirror, so that the divisions of the scale are seen in the mirror. The scale itself rests upon a telescope (F) directed towards the mirror. The observer (B) who looks through the telescope can see the divisions of the scale 
in the mirror. When the magnet, and with it the mirror, swing out of the magnetic meridian, the observer notices other divisions of the scale in the mirror. When one of the thermo-elements is heated, an electrical current is produced, which passes from the iron to the German silver in the heated couple, and canses a deviation of the suspended magnet. Suppose a person were swimming in the direction of the current in the conducting wire, then the north pole of the magnet goes to the north (Ampère). The tangent of the angle $\phi$, through which the freely moveable magnet is diverted by a galvanic current, from its position of rest or zero, in the magnetic meridian, is the same as the galvanic stream; $G$ is proportional to the magnetic energy $D$, i.e., tang. $\phi=\frac{\mathrm{C}^{*}}{\mathrm{D}^{*}}$. If $\mathrm{G}$ is to remain the same, and the tang. $\phi$ to be as large as possible, the magnetic energy must be diminished as much as possible. If the magnetism of the suspended magnet be indicated by $m$, and that of the earth by $\mathrm{T}$, the magnetic directing energy $\mathrm{D}=\mathrm{T} m$, so that $\mathrm{D}$ can be diminished in two ways: (1) by diminishing the magnetic moment of the suspended magnet, as may be clone by using a pair of astatic needles, such as are used in Nobili's galvanometer; (2) and also by weakening the magnetism of the earth, by placing an accessory stationary magnet (Hauy's rod) in the same direction, and near the sus. pended magnet. An important arrangement for rajidly getting the magnet to zero is the dead-beat arrangement of Gauss (not figured in the scheme).

It consists of a thick copper cylinder, on which the wire of the coil is wound. This mass of copper may be regarded as a closed multiplicator with a very large transverse section. The vibrating magnet induces a current of electricity in this closed circuit, whose intensity is greatest when the velocity of the excursion of the magnet is greatest, and which takes the opposite direction as soon as the magnet returns towards zero. These induced currents cause a diminution of the vibrations of the magnet in this way, that the arc of vibration of the magnet diminishes very rapidly, almost in a geometrical progression. The induced damping-current is stronger, the less the resistance in the closed circuit, and in the damper or dead-beat arrangement itself, the greater the section of the copper ring. This damping arrangement limits the oscillations of the magnet, and it comes to rest rapidly and promptly after 3 or 4 small vibrations, so that much time is saved. The angle of deviation is so small that the angle itself may be taken instead of the tangent.

The thermo-electric needles of Dutrochet (II) may be placed in the circuit. They consist of iron and German silver soldered at their points; or the needles of Becquerel (III) may be used. They consist of the same metals soldered in a straight line, one behind the other. The needles must always be covered by a varnish, which will prevent the parenchymatous juices from acting upon them, and so causing a current. Before the experiment we must deternine what extent of excursion on the scale is obtained with a certain temperature. In order to determine this, a delicate thermometer is fixed to each of the thermo-couples, and both are placed in oil baths, which differ in temperature-say by $1^{\circ} \mathrm{C}$. - as can be determined by the thermometers. When the current is closed, the excursion on the scale will indicate $1^{\circ} \mathrm{C}$. Suppose that the excursion was $150 \mathrm{~mm}$., then each $\mathrm{mm}$. of the scale would be equal to ${ }_{1}^{\frac{1}{50}}{ }^{\circ} \mathrm{C}$. When this is determined, the two thermo-needles may be placed in the different tissues or organs of animals, and, of course, we obtain the difference of temperatnre in these places. Or one thermo-couple may be placed in a bath of constant temperature (nearly that of the body), in which is placed a delicate thermometer, while the other needle is introduced into the organ to be investigated. In this case, we obtain the difference of temperature between the tissue and the source of the constant heat. The electric current passes in the warmer needle from the iron to the German silver, and thus through the wires of the apparatus. For small differences of temperature, such as occur in the body, the thermo-electric energy is always proportional to the 
difference of temperature of the two needles or couples. In place of a single pair of needles several may be used, whereby the sensitiveness of the apparatus is greatly increased. Helmholtz found that by using 16 antimony-bismuth couples, he could detect an increase of $\frac{1}{4000}{ }^{\circ} \mathrm{C}$. Schiffer prepared a simple thermopile (IV) by soldering together alternately four pairs of wires of iron $(f)$ and German silver $(a)$. These are placed in the two organs (A and B), which are to be investigated, whereby a very high degree of exactness is obtained.

\section{Temperature Topography.}

Although the blood, in virtue of its continual motion, completing, as it does, the circulation in $\mathbf{2 3}$ seconds, must exercise a very considerable influence on the equilibration of the temperature in different organs, nevertheless a completely uniform temperature does not exist, and the temperature varies in different parts:-

\section{Temperature of the Skin.-}

Middle of the sole of the foot, $\quad$. $32 \cdot 26^{\circ} \mathrm{C}$.

Near tendo achillis, . . . 33.85

Anterior surface of leg, : : 33.05

Middle of calf, $\quad . \quad: \quad 33.85$

Bend of knee, $\quad: \quad: \quad: \quad: 35.00$

Middle of upper arm, . . . $34 \cdot 40$

Inguinal fold, $: \quad: \quad$. $35 \cdot 80$

Near cardiac impulse, : : : $\quad 34 \cdot 40$

J. Davy made these observations directly after standing, while naked, with the temperature of the room at $21^{\circ} \mathrm{C}$. Only the under surface of the thermometer touched the skin.

In the closed axilla, 36.49 (mean of 505 individuals); $-36 \cdot 5$ to $37 \cdot 25$ (Wunderlich); $-36.89^{\circ} \mathrm{C}$ (Liebermeister).

The temperature of the skin of the head is higher in the region of the forehead and parietal region than in the occipital region; the left side is warmer than the right (Maragliano). Dyspncea increases the temperature of the skin (Heidenhain, Fränkel).

Method.-Liebermeister determines the temperature of free cutaneous surfaces thus:-The bulb of the thermometer is heated slightly above the temperature expected; after the mercury begins to fall, the bulb is placed on the skin, and if the bulb has the same temperature as the skin, the mercury remains stationary. This experiment must be repeated several times.

\section{Temperature of the Cavities.-}

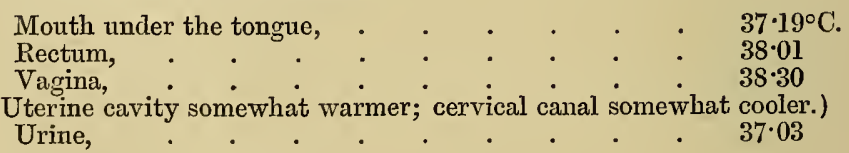

The temperature falls in the stomach during digestion (p. 332). Cold injections $\left(11^{\circ} \mathrm{C}\right.$.) into the rectum rapidly lowe rthe temperature in the stomach $1^{\circ} \mathrm{C}$. (Winternitz).

3. The Temperature of the Blood is, as a mean, $39^{\circ} \mathrm{C}$. The venous blood in internal viscera is warmer than the arterial, but it is cooler in peripheral parts:- 


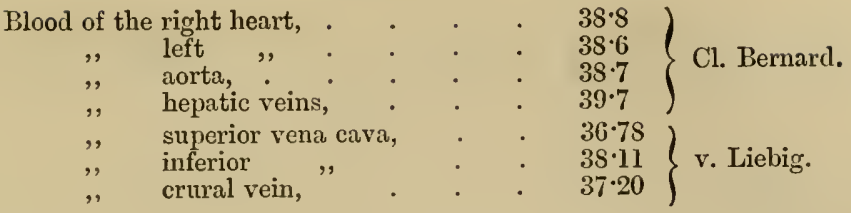

The lower temperature of the blood in the left heart may be explained by the blood becoming cooled in its passage through the lungs during respiration. According to Heidenhain and Körner, the right heart is slightly warmer because it lies in relation with the warm liver, whilst the left heart is surrounded by the lung which contains air. This observation of Malgaigne (1832), Berger, and G. v. Liebig is disputed by others, who say that the left heart is slightly warmer (Jacobson and Bernhardt) because the combustion processes are more active in arterial blood, and heat is evolved during the formation of oxyhæmoglobin (Gamgee). The blood in the veins is usually cooler than in the corresponding arteries (Haller), owing to the superficial position of the former, whereby they give off heat during their long course; thus the blood of the jugular vein is $\frac{1}{2}$ to $2^{\circ} \mathrm{C}$. lower than the blood in the carotid (Colin); the crural vein $\frac{3}{4}-1^{\circ}$ cooler than in the crural artery (Becquerel and Brechet). Superficial veins, more especially those of the skin, give off much heat, and their blood is, therefore, somewhat cooler. The warmest blood is that of the hepatic vein, $397^{\circ} \mathrm{C}$. (Cl. Bernard), partly owing to the great chemical changes which occur within the liver (p. 432), and partly to its protected situation. By means of small ontflow thermometers introduced into the circulation, Kronecker and Meyer found the following temperatures in three starving dogs:-Vena azygos, $37 \cdot 7(38 \cdot 0)(39 \cdot 0)$; right ventricle, $38.3(39.2)$ (39.2); branch of the pulmonary artery, $38 \cdot 4(38 \cdot 6)(40 \cdot 2)$. At the same time, the temperature in the stomach was $38 \cdot 6(37 \cdot 3)(40 \cdot 0)$, and in the rectum, $39.5(39 \cdot 5)$ $(39 \cdot 4)$; the maximum temperature in the last two dogs was $40 \cdot 1$ and $41 \cdot 2$, hence in the starving condition, the temperature of the stomach was less than the temperature of the blood in the pulmonary circulation.

4. Temperature of the Tissues.-The individual tissues are warmer: (1) the greater the transformation of kinetic energy into heat, i.e., the greater the tissue metabolism; (2) the more blood they contain; (3) and the more protected their situation. According to Heidenhain and Körner, the cerebrum is the warmest organ in the body.

Berger measured the temperature of the tissues of a sheep, and found the following:-

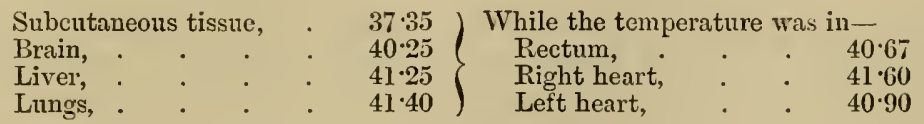

Becquerel and Brechet found the temperature of the human subcutaneous tissue to be $2 \cdot 1^{\circ} \mathrm{C}$. lower than that of the neighbouring muscles. The horny tissues do not produce heat, and their low temperature is due to the conduction of heat from the parts on which they grow. The temperature of the cornea partly depends on that of the iris, and the more contracted the pupil is, it receives more heat from the blood-vessels of the iris. 


\section{Conditions influencing the Temperature of Organs.}

The temperature of the individual organs is by no means constant; it is influenced by many conditions; amongst these are the following:-

(1.) The more heat that is produced independently within a part, the higher is its temperature. As the amount of heat produced within a part depends upon its metabolism, therefore, when the metabolism is increased, the amount of heat produced is similarly increased.

(a) Glands produce more heat during the act of secretion, as is proved by the higher temperature of their secretion, or by the higher temperature of the venous blood flowing out of their veins. Ludwig found that when he stimulated the chorda tympani, the secretion of the submaxillary gland was $1.5^{\circ} \mathrm{C}$. warmer than the blood in the carotid, which supplied the gland with blood. The blood in the renal vein in a kidney which is secreting is warmer than the blood in the renal artery. The secreting liver produces much heat. Cl. Bernard investigated the temperature of the blood of the portal and hepatic veins during hunger, at the beginning of digestion, and when digestion was most active, and he found:-

Temperature of portal vein, . $37 \cdot 8^{\circ} \mathrm{C}$. $\}$ After 4 days $\{$ Blood of right heart, " hepatic ,". 38.4$\}$ starvation. \{

(Hunger period.)

$\left.\begin{array}{cc}\left.\text { Temperature of portal vein, }: \begin{array}{l}39 \cdot 9 \\ \text {, }\end{array}\right\} \text { Bepatic, } & 39.5\end{array}\right\}$ Beginning of digestion.

Temperature of portal vein, . $39 \cdot 7$ Digestion most $\{$ Blood of right heart

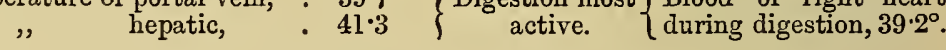

When a dog receives a moderate diet, the mean temperature in the stomach is $39^{\circ} \mathrm{C}$., in the rectum, $39.5^{\circ} \mathrm{C}$; at the end of the first day of hunger, in the stomach, $38.7^{\circ}$, in the rectum, $39.3^{\circ}$; while after food, in both situations it is $40^{\circ}$. Chemical or mechanical stimulation of the gastric mucous membrane, or even the sight of food, has a similar action (Kronecker and Meyer).

(b) When the muscles contract they evolve heat (Bunsen, 1805). Davy found that an active muscle became $0.7^{\circ} \mathrm{C}$. warmer; while Becquerel (1835), by means of a thermo-galvanometer, found that human muscles, when kept contracted for five minutes, became $1^{\circ} \mathrm{C}$. warmer (see Physiology of Muscle).

This is one of the reasons why the temperature may rise above $40^{\circ}$ during rapid running. A temperature obtained by energetic muscular action usually does not fall to the normal until after resting for $1 \frac{1}{2}$ hours (Billroth). The low temperature of paralysed limbs depends partly upon the absence of the muscular contractions.

(c) With regard to the effect of sensory nerves upon the tempera- 
ture, one of the first points to ascertain is whether the circulation is accelerated or retarded by their stimulation, or whether the respiration is increased or diminished $(\$ 214, \mathrm{II} ., 3)$, and whether the muscles of the skeleton are relaxed or contracted reflexly $(\$ 214, \mathrm{I}, 3)$. In the former case, the temperature of the interior and rectum is increased; in the latter, diminished.

That there are heat-regulating nerve-centres has not been definitely proved; with regard to the influence of vaso-motor nerves see vol. ii.

(d) The temperature of the body rises during mental exertion. Davy observed an increase of $0.3^{\circ} \mathrm{C}$. after vigorous mental exertion.

Lombard observed that the temperature of the forehead rose $0.5^{\circ} \mathrm{C}$. during mental activity and emotional disturbances. The part of the forehead corresponded to the posterior region of both upper frontal eonvolutions, to the anterior central convolution, and (?) to the anterior part of the posterior central convolution. The temperature was higher on the left side.

(e) The parenchymatous fluids, serous fluids, and lymph produce little heat owing to their feeble metabolism, hence they have the same temperature as their surroundings; the epidermal and horny tissues do not produce heat, they merely conduct it from subjacent structures.

(2.) The temperature depends, to a large extent, upon the amount of blood in an organ, and also upon the rapidity with which the blood is renewed by the circulation. This is best observed in the difference of the temperature between a cold pale bloodless hand and a warm red congested one.

Becquerel and Brechet found, that the temperature of the human biceps fell several tenths of a degree, when the axillary artery was compressed. Ligature of the iliac artery in a $\log$ cansed a fall of $\frac{1}{2}^{\circ} \mathrm{C}$. within 18 minutes; while the removal of the ligature caused the temperature to rise rapidly to normal. Ligature of the crural artery and vein in a dog causes a fall of several degrees (Landois). If the extremities be kept suspended in the air, they become bloodless and cold.

Liebermeister has pointed out a difference with regard to the external and internal parts of the body. The external parts give off more heat than they produce, so that they become cooler the more slowly new blood flows into them, and warmer the greater the rapidity of the blood-stream through them. Acceleration of the blood-stream, therefore, causes the temperature of peripheral parts to approximate more and more to the temperature of internal organs, while retardation of the blood-stream causes them to approach the temperature of the surrounding medium. Exactly the reverse is the case with internal parts, where a large amount of heat is produced, and heat is given up almost alone to the blood which flows throngh them. Their temperature must fall when the bloodstream through them is accelerated, and it is raised when the blood-stream is retarded (Heidenhain). Hence it follows, that the greater the difference of the temperature between peripheral and internal parts, the slower must be the velocity of the circulation.

(3.) If the position of an organ be such, or if other conditions 
cause it to give off heat by conduction or radiation, then its temperature falls.

A good example of this is the skin, which varies greatly in temperature according to the temperature of the surrounding medium, whether it is covered or uncovered, whether it is dry or moist with sweat (which abstracts heat when it evaporates). When much cold food or drink is taken the stomach is cooled, and when ice-cold air is breathed the respiratory passages as far as the bronchi are cooled.

\section{Estimation of the Amount of Heat- Calorimetry.}

Calorimetry is the method of determining the amount of heat possessed by any body, or what amount of heat it is capable of producing. The unit of measurement is the "heat-unit," i.e., the amount of heat (or potential energy) required to raise the temperature of 1 gramme of water, $1^{\circ} \mathrm{C}$. (see Introduction).

Experiment has shown that equal quantities of different substances require very unequal amounts of heat to raise them to the same temperature, e.g., 1 kilo. water requires nine times as much heat as 1 kilo. iron to raise it to the same temperature. In the human body, therefore, which is composed of very different substances, unequal amounts of heat will be required to raise them all to the same temperature. The same amount of heat transferred to two different substances will raise them to different temperatures. Hence, bodies of different temperatures may contain equal amounts of heat. The amount of heat required to raise a definite quantity (e.g., 1 gramme) of a substance to a certain higher degree (e.g., $1^{\circ} \mathrm{C}$.) is called "specific heat" (Wilkie, 1780). The specific heat of water (which of all bodies has the highest specific heat) is taken as $=1$. By "heat-capacity" is meant, that property of bodies in virtue of which they must absorb a given amount of heat in order to have a certain temperature (Crawford).

Calorimetry is employed:-I. To determine the specific heat of the different organs of the body.-Only a few observations have been made. The mean specific heat of the following animal parts (water $=1$ ) is:-

$\begin{array}{lll}\text { Human Blood } & =1.02 \\ \text { Arterial ," } & =1.031 \\ \text { Venous } & =0.892 \\ \text { Cow's Milk } & =0.992 \\ \text { Human Muscle } & =0.741 \\ \text { Ox , } & =0.787\end{array}$

$\left.\begin{array}{ll}\text { Compact Bone } & =0.3 \\ \text { Spongy ", } & =0.71 \\ \text { Fat-tissue } & =0.712 \\ \text { Striped Muscle } & =0.825 \\ \text { Defibrinated Blood } & =0.927\end{array}\right\}$ 蹗

The specific heat of the human body, as a whole, is about that of an equal volume of water.

Kopp has estimated the specific heat of solids and fluids by the following method (Fig. 169):-The solid to be investigated is broken in pieces about the size of a pea, and placed in a test-tube, A, with thin walls, which is closed above with a cork, from which a copper-wire with a hook on it projects. The test-tube contains a certain quantity of fluid which does not dissolve the substance, but which lies between its pieces and covers it. It is weighed three times to ascertain the 
weight (1) of the empty glass, (2) after it is filled with the solid substance, (3) after the fluid is added, so that we obtain the wcight of the solid substance, $m$, and that of the fluid, $f$. The test-tube and its contents are placed in a mercury bailh, BB, and this again in an oil bath, C C, and the whole is raised to a high temperature. Into $\mathrm{BB}$ there is introduced a fine thermometer, $\mathrm{T}$. When the tube, $A$, has reached the necessary temperature (say $40^{\circ}$ ) it is rapidly placed in the water of the accompanying calorimeter-box, D D. The water in this box, which also contains a thermometer, D, is kept in motion until it has completely
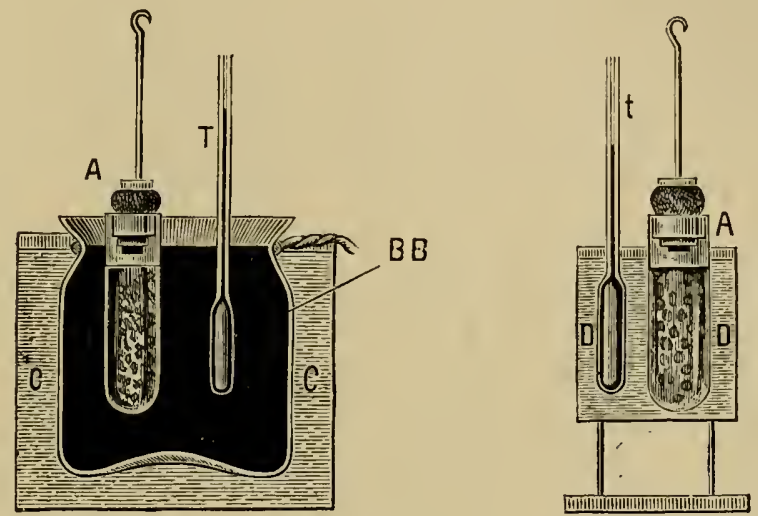

Fig. 169.

Kopp's apparatus for the estimation of specific heat.

absorbed all the heat given off by $A$. Let $T$ represent the temperature to which $A$ and its contents were raised in the mercury bath, and $T_{1}$ the temperature to which it fell in the calorimeter; let $s$ be the specific heat, and $m$ the weight of the solid substance in the test-tube, while $\sigma$ and $\mu$ represent the specific heat of the weight of the interstitial fluid in the test-tube; and lastly, let $w$ equal the amount of water in contact with $\mathrm{A}$, which absorbs and gives off heat; then $\mathrm{W}$ represents the amount of heat which the test-tube and its contents give off during cooling.

$$
\mathrm{W}=(s . m+w+\sigma \cdot \mu)\left(\mathrm{T}-\mathrm{T}_{1}\right) .
$$

The amount of heat, $W_{1}$, absorbed by the calorimeter is

$$
\mathrm{W}_{1}=\mathrm{M}\left(t_{1}-t\right),
$$

where MI represents the amount of water in the calorimeter, and $t$ the original temperature of the water in the calorimeter, and $t_{1}$ the temperature to which it is raised by placing $A$ in it. If $W$ and $W_{1}$ are equal, then

$$
\text { The specific heat, } s=\frac{\mathbf{M}\left(t_{1}-t\right)-(w+\sigma . \mu)\left(\mathrm{T}-\mathrm{T}_{1}\right)}{m\left(\mathrm{~T}-\mathrm{T}_{1}\right) .}
$$

If a fluid substance is placed in the test-tube, and its weight $=m$, and its specific heat $=s$, the formula for the specific heat of the fluid to be investigated is

$$
s=\frac{M\left(t_{1}-t\right)-w\left(\mathrm{~T}-\mathrm{T}_{1}\right)}{m\left(\mathrm{~T}-\mathrm{T}_{1}\right)}
$$

This is a subject which has been very slightly cultivated. J. Rosenthal in his investigations used an ice-oalorimeter (\$ 206). 
II Calorimetry is more important for determining the amount of heat produced in a giren time by the bodr as a whole, or by its inciriäual parts.

Laroisier and Laplace made the first calorimetric obserrations on animals in 17SS, br means of an ice-calorimeter; a guinea-pig melted 13 ozs. of ice in 10 hours Crawford, and afterwards Dulong and Despretz (1824), used Rumford"s water-calorimeter, which is similar to the one alreadr described-riz., of Farre and Sibarmant. Small animals are placed in the inner thin-walled copper chamber $(K)$, which is placed in a water-batb surrounded on all sides by some non-conducting material. We require to know the amount of water, and its original temperature. The number of calories is obtained from the increase of the temperature at the end of the experiment, which lasts sereral hours. The air is supplied to the animal through a special apparatus resembling a gasometer. The amount of $\mathrm{CO}_{2}$ in the gases erolred is estimated chemicalls.

According to Despretz, a bitch forms 14,610 heat-units per houri.e., 393,000 in 24 hours. Other things being equal, a man seven times hearier than this mould produce in 24 hours about $2,750,000$ calories. Senator found that a dog weighing 6,330 grms. produced 15,370 calories, and excreted at the same time 3,67 grms. $\mathrm{CO}_{2}$ The first calorimetric experiments on man were made by Scharling (1849). Liebermeister estimated the amount of heat given off by a man placed in a cold bath, which was surrounded with a woollen corering. Lejden placed a lower limb in the calorimeter, whereby 6,000 grms. water were raised $1^{\circ} \mathrm{C}$. in an hour. If we assume that the total superficial area of the body is fffteen times greater than that of the leg, the human body rould produce $2,376,000$ calories in 24 hours.

\section{Thermal Conductivity of Animal Tissues.}

The thermal conductirity of animal tissues is of special interest in connection with the skin and subcutaneous fatty tissne. The fatty lajer under the skin, more especially in the whale, walrus, and seal, forms a protective corering, whereby the conduction of heat from internal organs is rendered almost impos. sible. Inrestigations apon this subject, howerer, are fer. Griess (1870) attempted to estimate the thermal conductirity by beating one part of the tissue, and determining when and in what direction pieces of wax placed on the tissue to be inrestirated began to melt. He inrestigated the stomach of the sheep, the blander, skin, hoof, horn, and bones of an ox, deer's horn, irory, mother-of-pearl, Ehell of haliotis. He found that fibrous tissues conducted heat more readily in the direction of their fibres than at right angles to the course of the fibres. Hence, the fircures obtained from the melted wax mere usually elliptical. Landois has made similar obserrations, and he finds that tissues conduct better in the direction of their fibres. After bones, blood-clot vous the best conductor, then

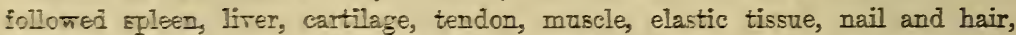
bloniless skin, sastric mucous membrane, washed fibrin. It is specially interesting to zote how much better skin containing blood in its blood-ressels conducts compared with bloodless ekin. Hence little heat is giren off from a bloodless Ekin, while congested skin conducts and gires off much more heat. 
Like all other substances, the human body is enlarged by heat. A man weighing 60 kilos., and whose temperature is raised from $37^{\circ} \mathrm{C}$. to $40^{\circ} \mathrm{C}$., is enlarged about 62 cubic centimetres. Connective-tissue (tendon) is extended by heat, while elastic tissue, the skin, like caoutchouc, are contracted (Lombard and Walton).

\section{Variations of the Mean Temperature.}

(1.) General Climatic and Somatic Influences.-In the tropics, the mean temperature of the body is about $\frac{1}{2}^{\circ} \mathrm{C}$. higher than in temperate climates, where again it is several tenths of a degree warmer than in cold climates (J. Davy); but this has recently been denied by Boileau and Pinkerton. This difference is comparatively trivial, when we remember that a man is subjected to a variation of over $40^{\circ} \mathrm{C}$. in passing from the equator to the poles. Observations on more than 4000 persons show that when a person goes from a warm to a cold climate, his temperature is but slightly diminished, but when he goes from a cold to a warm climate his temperature rises relatively considerably more. In the temperate zone, the temperature of the body during a cold winter is usually $0.1-0.3^{\circ} \mathrm{C}$. lower than it is on a warm summer day. The elevation of a place above sea-level has no obvious effect on the temperature of the body. There seems to be no difference in different races, nor in the sexes, other conditions being the same. Persons of powerful physique and constitution are said to have generally a slightly higher temperature, than feeble, weak, anæmic persons.

(2.) Influence of the General Metabolism.-As the formation of heat depends upon the transformation of chemical compounds, whose chief final products, in addition to $\mathrm{H}_{2} \mathrm{O}$, are $\mathrm{CO}_{2}$ and urea, the amount of heat formed must go pari pasu with the amount of these excreta. The more rapid metabolism which sets in after a full meal causes a rise of temperature to several tenths of a degree ("Digestion-fever"). As the metabolism is much diminished during hunger, this explains why the mean temperature in a fasting man is $36.6^{\circ}$, while it is $37.17^{\circ}$ on ordinary days (Lichtenfels and Fröhlich).

Jürgensen also found that the temperature fell on the first day of inanition, (although there was a temporary rise on the second day). In experiments made upon starving animals, the temperature at first fell rapidly, then remained constant for a considerable time, while during the last days it fell considerably. Schmidt starved a cat-on the 15 th day the temperature was $38 \cdot 6^{\circ}$; on the 16 th, $38.3^{\circ} ; 17$ th, $37.64^{\circ} ; 18$ th, $35.8^{\circ} ; 19$ th $($ death $)=33 \cdot 0^{\circ}$. Chossat found that starring mammals and birds had a temperature $16^{\circ} \mathrm{C}$. below normal on the day of their deatl.

(3.) Influence of Age.-Age has a decided effect upon the temperature of the body. The extent of the general metabolism is in part an 
index of the heat of the body at different ages, but it is possible that other unknown influences also are active.

\begin{tabular}{|c|c|c|c|}
\hline Age. & $\begin{array}{l}\text { Mean Temperature at } \\
\text { the Ordinary Temp. }\end{array}$ & Normal Limits. & Where Measurcd. \\
\hline $\begin{array}{c}\text { Newly-born, } \\
5-9 \text { year, } \\
15-20 \text {,, } \\
21-30 \text {,, } \\
25-30 \text {,, } \\
31-40 \quad, \\
41-50 \quad, \\
51-60 \quad, \\
80 \quad,\end{array}$ & $\begin{array}{l}37 \cdot 45^{\circ} \mathrm{C} . \\
37 \cdot 72 \\
37 \cdot 37 \\
37 \cdot 22 \\
36 \cdot 91 \\
37 \cdot 1 \\
36 \cdot 87 \\
36 \cdot 83 \\
37 \cdot 46\end{array}$ & $\begin{array}{c}37 \cdot 35-37 \cdot 55^{\circ} \mathrm{C} . \\
37 \cdot 87-37 \cdot 62 \\
36 \cdot 12-38 \cdot 1 \\
\ldots \\
\ldots \\
36 \cdot 25-37 \cdot 5 \\
\ldots \\
\ldots \\
\ldots\end{array}$ & $\begin{array}{c}\text { Rectum. } \\
\text { Mouth and Rectum. } \\
\text { Axilla. } \\
,, \\
, \\
, \\
\text { Mouth. }\end{array}$ \\
\hline
\end{tabular}

Newly-born Animals exhibit peculiarities owing to the sudden change in their conditions of existence. Immediately after birth, the infant is $0.3^{\circ}$ warmer than the vagina of the mother, viz., $37.86^{\circ}$. A short time after birth, the temperature falls $0.9^{\circ}$, while 12-24 hours afterwards, it has risen to the normal temperature of an infant, which is $37.45^{\circ}$. Several irregular variations occur during the first weeks of life. During sleep, the temperature of an infant falls $0.34^{\circ}$ to $0.56^{\circ}$, while continued crying may raise it several tenths of a degree. old people, on account of their feeble metabolism, produce little heat;

\begin{tabular}{|c|c|c|c|c|c|c|c|}
\hline Time. & $\begin{array}{l}\text { Bären- } \\
\text { spruny. }\end{array}$ & J. Davy. & Hallmann. & Gierse. & Jürge & isen. & Jäger. \\
\hline $\begin{array}{rr}\text { Morning, } 5 \\
6 \\
7 \\
8 \\
9 \\
10 \\
11 \\
11 \\
\text { Mid-day, } 12 \\
1 \\
2 \\
3 \\
4 \\
5 \\
6 \\
7 \\
8 \\
9 \\
10 \\
11 \\
12 \\
1 \\
\text { Night, } \\
2 \\
3 \\
4\end{array}$ & 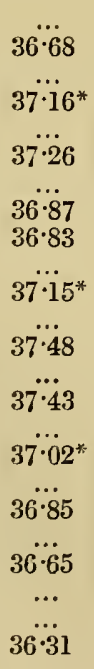 & $\begin{array}{c}\ldots \\
36 \cdot 94^{*} \\
\ldots \ddot{89} \\
36.8 \\
36 \cdot 89 \\
\ldots \\
\ldots \\
37 \cdot 05 \\
\ldots \\
37 \cdot 17 \\
37 \cdot 05^{*} \\
6 \frac{1}{2}=36 \cdot 83 \\
7 \frac{1}{2}=36 \cdot 50^{*} \\
\ldots \\
\ldots \\
36.72 \\
\ldots \\
36 \cdot 44 \\
\ldots \\
\ldots \\
\ldots\end{array}$ & $\begin{array}{c}\ldots \\
36 \cdot 63 \\
36 \cdot 80^{*} \\
\ldots \\
10 \frac{1}{2}=37 \cdot 36 \\
\ldots \\
\ldots \\
37 \cdot 21 \\
\ldots \\
\ldots \\
\ldots \\
5 \frac{1}{2}=37 \cdot 31 \\
\ldots \\
37 \cdot 31^{*} \\
\ldots \\
\ldots \\
36 \cdot 70 \\
\ldots \\
\ldots \\
\ldots \\
\ldots \\
\ldots\end{array}$ & $\begin{array}{c}\ldots \\
\ldots \cdot \\
36 \cdot 98 \\
37 \cdot 08^{*} \\
\ldots \cdot \\
37 \cdot 23 \\
\ldots \\
\dddot{*} \\
37 \cdot 13 \\
37 \cdot 50^{*} \\
37 \cdot 43 \\
\ldots \cdot \\
37 \cdot 43 \\
37 \cdot 29 \\
\ldots \\
\ldots \\
\ldots \\
37 \cdot 29 \\
36 \cdot 81 \\
\ldots \\
\ldots \\
\ldots \\
\ldots \\
\ldots\end{array}$ & $\begin{array}{l}36 \cdot 7 \\
36 \cdot 7 \\
36 \cdot 7^{*} \\
36 \cdot 8 \\
36 \cdot 9 \\
37 \cdot 0 \\
37 \cdot 2 \\
37 \cdot 3^{*} \\
37 \cdot 3 \\
37 \cdot 4 \\
37 \cdot 4^{*} \\
37 \cdot 4 \\
37 \cdot 5 \\
37 \cdot 5 \\
37 \cdot 5 * \\
37 \cdot 4 \\
37 \cdot 4 \\
37 \cdot 3 \\
37 \cdot 2 \\
37 \cdot 1 \\
37 \cdot 0 \\
36 \cdot 9 \\
36 \cdot 8 \\
36 \cdot 7\end{array}$ & $\begin{array}{l}36 \cdot 6 \\
36 \cdot 4 \\
36 \cdot 5 \\
36 \cdot 7 \\
36 \cdot 8 \\
37 \cdot 0 \\
37 \cdot 2 \\
37 \cdot 3^{*} \\
37 \cdot 3 \\
37 \cdot 4 \\
37 \cdot 3^{*} \\
37 \cdot 3 \\
37 \cdot 5 \\
37 \cdot 6 \\
37 \cdot 6^{*} \\
37 \cdot 7 \\
37 \cdot 5 \\
37 \cdot 4 \\
37 \cdot 1 \\
36 \cdot 9 \\
36 \cdot 9 \\
36 \cdot 7 \\
36 \cdot 7 \\
36 \cdot 7\end{array}$ & $\begin{array}{l}36 \cdot 9 \\
37 \cdot 1 \\
37 \cdot 5^{*} \\
37 \cdot 4 \\
37 \cdot 5 \\
37 \cdot 5 \\
37 \cdot 3 \\
37 \cdot 5 * \\
37 \cdot 4 \\
37 \cdot 5 \\
37 \cdot 5 \\
37 \cdot 5 * \\
37 \cdot 5 \\
37 \cdot 4 \\
37 \cdot 3 \\
37 \cdot 1 * \\
36 \cdot 9 \\
36 \cdot 8 \\
36 \cdot 8 \\
36 \cdot 9 \\
36 \cdot 9 \\
36 \cdot 8 \\
36 \cdot 7 \\
36 \cdot 7\end{array}$ \\
\hline
\end{tabular}

[* Indicates taking of food.] 
they become cold sooner, and hence ought to wear warm clothing to keep up their temperature.

(4.) Periodical Daily Variations.-In the course of 24 hours there are regular periodic variations in the mean temperature, and these occur at all ages. As a general rule, the temperature continues to rise during the day (maximum at 5-S p.m.), while it continues to fall during the night (minimum 2-6 a.m.). The mean temperature occurs at the third hour after breakfast (Lichtenfels and Fröhlich).

According to Lichtenfels and Fröhlich, the morning temperature rises 4-6 hour's after breakfast until its first maximum, then it falls until dinner time; and it rises again within two hours, to a second maximum, falls again towards evening, while supper does not appear to cause any obvious increase. The daily variation of the temperature is given in Fig. 170, according to Liebermeister and Jürgensen. According to Bonnal, the minimum occurs between 12-3 a.n. (in winter 36.05 , in suminer $36 \cdot 45^{\circ} \mathrm{C}$ ), the maximum hetween 2-4 p.m.

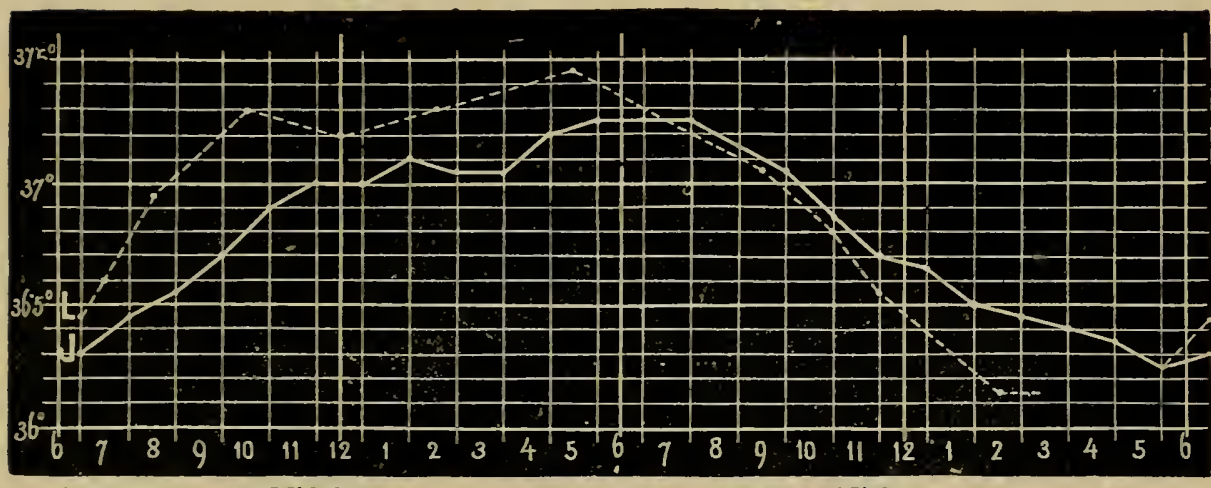

Morning. Mid-day. Evening. Night. Morning.

Fig. 170.

Variations of the daily temperature in health during 24 , hours - L. . . . . . . . ., after Liebermeister: J_-, after Jürgensen.

As the variations occur when a person is starved-although those that occur at the periods at which food ought to have been taken are less-it is obrious that the variations are not due entirely to the taking of food.

The daily variation in the frequency of the pulse (p. 142) often coincides with variation of the temperature. Bärensprung found that the mid-day temperaturemaximum slightly preceded the pulse-maximum.

If we sleep during the day, and do all our daily duties during the night, the above described typical course of the temperature is inverted (Krieger). With regard to the effect of activity or rest, it appears that the activity of the muscles during the day, tends to increase the mean temperature slightly, while at night, the mean temperature is less than in the case of a person at rest (Liebermeister). 
The peripheral parts of the body exhibit more or less regular variations of their temperature. In the palm of the hand, the progress of events is the following :-After a relatively high night-temperature, there is a rapid fall at 6 a.m., which reaches its minimum at 9-10 a.m. This is followed by a slow rise, which reaches a high maximum after dinner ; it falls between 1-3 p.m., and after two to three hours reaches a minimum. It rises from 6-8 p.m., and falls again towards morning. A rapid fall of the temperature in a peripheral part correspends to a rise of temperature in internal parts (Römer).

(5.) Many operations upon the body affect the temperature. After hcemorrhage, the temperature falls at first, but it rises again several tenths of a degree, and is usually accompanied by a shiver or slight rigor; several days thereafter, it falls to normal, and may even fall somewhat below it. The sudden loss of a large amount of blood causes a fall of the temperature of $\frac{1}{2}-2^{\circ} \mathrm{C}$. Very long continued hæmorrhage (dog) causes it to fall to $31^{\circ}$ or $29^{\circ} \mathrm{C}$. (Marshall Hall).

This is obviously due to the diminution of the processes of oxidation in the anæmic body, and to the enfeebled circulation. Similar conditions causing diminished metabolism effect the same result. Continued stimulation of the peripheral end of the vagus, so that the heart's action is enormously slowed, diminishes the temperature several degrees in rabbits (Landois and Ammon).

The transfusion of a considerable quantity of blood raises the temperature about half an hour after the operation. This gradually passes into a febrile attack, which disappears within several hours. When blood is transfused from an artery to a vein of the same animal a similar result occurs (Albert and Stricker) ( $\$ 102)$.

(6.) Many poisons diminish the temperature-e.g., chloroform (Scheinesson), and the anæsthetics, as also alcohol, digitalis, quinin, aconitin, muscarin. These may act upon the blood so as to limit its oxidising power, or they may render the tissues less liable to undergo molecular transformations for the production of heat. In the case of the anæsthetics, the latter effect perhaps occurs, and is due possibly to a semi-coagulation of the nervous substance (?).

The temperature is increased by strychnin, nicotin, picrotoxin, veratrin (Hogyes), laudanin (F. A. Falck). Curara (muscarin-Hogyes), laudanosin (F. A. Falck), give an uncertain effect.

(7.) Various diseases have a decided effect upon the temperature. Loewenhardt found that in insane persons, several weeks before their death, the rectal temperature was $30^{\circ}-31^{\circ} \mathrm{C}$; Bechterew found in dementica paralytica, before death $27.5^{\circ} \mathrm{C}$. (rectum); the lowest temperature observed, and life retained, in a drunk person was $24^{\circ} \mathrm{C}$. (Reinke, Nicolaysen). The temperature is increased in fever, and the highest point reached just before death, and recorded by Wunderlich, was $44 \cdot 65^{\circ} \mathrm{C}$. (compare $\S 220$ ). 
The mean height of all the temperatures taken during a day in a patient is called the "daily mean," and according to Jaeger it is $37.13^{\circ}$ in the rectum in health. A daily mean of more than $37.8^{\circ}$ is a "fever temperature," while a mean under $37 \cdot 0^{\circ} \mathrm{C}$. is regarded as a "collapse temperature."

\section{Regulation of the Temperature.}

As the bodily temperature of man and similar animals is nearly constant, notwithstanding great variations in the temperature of their surroundings, it is clear that some mechanism must exist in the body, whereby the leat-economy is constantly regulated. This may be brought about in two ways; either by controlling the transformation of potential energy into heat, or by affecting the amount of heat given off according to the amount produced, or to the action of external agencies.

\section{Regulatory arrangements governing the production of heat.-} Liebermeister estimates the amount of heat produced by a healthy man at 1.8 calories per minute. It is highly probable that, within the body, there exist mechanisms which determine the molecular transformations, upon which the evolution of heat depends (Hoppe-Seyler, Liebermeister). This is accomplished chiefly in a reflex manner. The peripheral ends of cutaneous nerves (by thermal stimulation), or the nerves of the intestine and the digestive glands (by mechanical or chemical stimulation during digestion or inanition) may be stimulated, whereby impressions are conveyed to the heat-centre which sends out impulses through efferent fibres to the depots of potential energy, either to increase or diminish the extent of the transformations occurring in them. The nerve channels herein concerned are entirely unknown. Many considerations, however, go to support such an hypothesis. The following phenomena indicate the existence of mechanisms regulating the production of heat:-

(1.) The temporary application of moderate cold raises the bodily temperature, while heat, similarly applied to the external surface, lowers it ( $\$ 222$ and 224).

(2.) Cooling of the surroundings increases the amount of $\mathrm{CO}_{2}$ excreted, by increasing the production of heat (Liebermeister, Gildermeister), while the $\mathrm{O}$ consumed is also increased simultaneously; heating the surrounding medium diminishes the $\mathrm{CO}_{2}$ (compare Respiration, p. 257).

D. Finkler found, from experiments upon guinea-pigs, that the production of heat was more than donbled when the surrounding temperature was diminished $24^{\circ} \mathrm{C}$. The metabolism of the guinea-pig is increased in winter 23 per cent. as compared with summer, so that the same relation obtains as in the case of a diminution of the surrounding temperature of short duration. 
C. Ludwig and Sanders-Em found, that in a rabbit there was a rapid increase in the amount of $\mathrm{CO}_{2}$ given off, when the surroundings were cooled from $38^{\circ}$ to $6^{\circ}-7^{\circ} \mathrm{C}$, , while the excretion was diminished when the surrounding temperature was raised from $4^{\circ}-9^{\circ}$ to $35^{\circ}-37^{\circ}$, so that the thermal stimulation, due to the temperature of the surrounding medium, acted upon the combustion within the body. Pfliger found that a rabbit which was dipped in cold water used more $\mathrm{O}$ and excreted more $\mathrm{CO}_{2}$.

If the cooling action was so great as to reduce the bodily temperature to $30^{\circ}$, the exchange of gases diminished, and where the temperature fell to $20^{\circ}$, the exchange of gases was diminished one-half. It is to be remembered, however, that the excretion of $\mathrm{CO}_{2}$ does not go hand in hand with the formation of $\mathrm{CO}_{2}$, so that the increased excretion of $\mathrm{CO}_{2}$ in a cold bath is perhaps due to more complete expiration, and Berthelot has proved that the formation of $\mathrm{CO}_{2}$ is not a certain test of the amount of heat produced. If mammals be placed in a warm bath, which is $2^{\circ}-3^{\circ}$ higher than their own temperature, the excretion of $\mathrm{CO}_{2}$ and the consumption of $\mathrm{O}$ are increased, owing to the stimulation of their metabolism (Pflüger), while the excretion of urea is also increased in animals (Naunyn) and in man (Schleich).

(3.) Cold acting upon the skin causes involuntary muscular movements (shivering, rigors), and also voluntary movements, both of which produce heat.

The cold excites the action of the muscles, which is connected with processes of oxidation (Pflüger). After poisoning with curara, which paralyses voluntary motion, this regulation of the heat falls to a minimum (Röhrig and Zuntz).

(4.) Variations in the temperature of the surroundings affect the appetite for food; in winter, and in cold regions, the sensation of hunger and the appetite for the fats, or such substances as yield much heat when they are oxidised, are increased; in summer, and in hot climates, they are diminished. Thus the mean temperature of the surroundings, to a certain extent, determines the amount of the heatproducing substances to be taken in the food. In winter the amount of ozone in the air is greater, and thus the oxidising power of the inspired air is increased.

II. Regulatory mechanisms governing the excretion of heat.The mean amount of heat given off by the human skin in 24 hours, by a man weighing 82 kilos. is $2,092-2,592$ calories-i.e., $1 \cdot 36-1 \cdot 60$ per minute.

(1.) Increased temperature causes dilatation of the cutaneous vessels; the skin becomes red and congested, soft, and with more fluids, so that it becomes a better conductor of heat; the epithelium is moistened, and sweat appears upon the surface. Thus increased excretion of heat is provided for, while the evaporation of the sweat also abstracts heat.

Cold causes contraction of the cutaneous vessels; the skin becomes pale, less soft, poorer in juices, and collapsed; the epithelium becomes dry, and does not permit fluids to pass through it to be evaporated, so that the excretion of heat is diminished. The excretion of heat from 
the periphery, and the transverse thermal conduction through the skin, are diminished by the contraction of the vessels and muscles of the skin, and by the expulsion of the well conducting blood from the cutaneous and sub-cutaneous vessels. The cooling of the body is very much affected, owing to the diminution of the cutaneous blood-stream, just as occurs when the current through a coil or worm of a distillation apparatus is greatly diminished (Winternitz). If the blood-vessels dilate, the temperature of the surface of the body rises, the difference of temperature between it and the surrounding cooler medium is increased, and thus the excretion of heat is increased. Tomsa has shown that the fibres of the skin are so arranged anatomically, that the tension of the fibres produced by the erector pili muscles causes a diminution in the thickness of the skin, this result being brought about at the expense of the easily expelled blood.

Landois and Hauschild ligatured the arteries alone, or the arteries and veins (dog)-e.g., the axillary artery and vein, the crurals, the carotids and the jugular veins, and found that in a short time the temperature rose several tenths of a degree.

By the systematic application of stimuli-e.g., cold baths, and washing with cold water, the muscles of the skin and its blood-vessels may be caused to contract, and become so vigorous and excitable, that when cold is suddenly applied to the body or to a part of it the excretion of heat is energetically prevented, so that cold baths and washing with cold water are, to a certain extent, "gymnastics of the cutaneous muscles," which, under the above circumstances, protect the body from cold (Rosenthal, du Bois-Reymond).

(2.) Increased temperature causes increased heart-beats, while diminished temperature diminishes the number of contractions of the heart (p. 105). The relatively warm blood is pumped by the action of the heart from the internal organs of the body to the surface of the skin, where it readily gives off heat. The more frequently the same volume of blood passes through the skin-27 heart-beats being necessary for the complete circuit of the blood-the greater will be the amount of heat given off and conversely. Hence, the frequency of the heart-beat is in direct relation to the rapidity of cooling (Walther). In very hot air (over $100^{\circ} \mathrm{C}$.) the pulse rose to over 160 per minute. The same is true in fever (p. 142). Liebermeister gives the following numbers with reference to the temperature in an adult:-

Pulse-beats, per min., 78.6-91:2-99.8-105.5 - 110-137.5.

Temperature in $\mathrm{C}^{\circ}$., $37^{\circ}-38^{\circ}-39^{\circ}-40^{\circ}-41^{\circ}-42^{\circ}$.

(3.) Increased temperature increases the number of respirations.Under ordinary circumstances, a much larger volume of air passes through the lungs when it is warmed almost to the temperature of the 
body. Farther, a certain amount of watery vapour is given off with each expiration, which must be evaporated, whereby heat is abstracted. Energetic respiration aids the circulation, so that respiration acts indirectly in the same way as (2). According to other observers, the increased consumption of $\mathrm{O}$ favours the combustion in the body (p. 259, 8), whereby the increased respiration must act in producing an amount of heat greater than normal. This excess is more than compensated by the cooling factors above-mentioned. Forced respiration produces cooling, even when the air breathed is heated to $54^{\circ} \mathrm{C}$., and saturated with watery vapour (Lombard).

(4.) Covering of the body.-Animals become clothed in winter with a winter fur or covering, while in summer their covering is lighter, so that the excretion of heat in surroundings of different temperatures is thereby rendered more constant. Many animals which live in very cold air or water are protected from too rapid excretion of heat by a thick layer of fat under the skin. Man provides for a similar result by adopting summer and winter clothing.

The position of the body is also important; pulling the parts of the body together, approximation of the head and limbs, keep in the heat; spreading out the limbs, erection of the hairs, pluming the feathers, allow more heat to be evolved. If a rabbit be kept exposed to the air with its legs extended for three hours, the rectal temperature will fall from $39^{\circ} \mathrm{C}$. to $37^{\circ} \mathrm{C}$. Man may influence his temperature by remaining in a warm or a cold room-by taking hot or cold drinks, hot or cold baths-remaining in air at rest or air in motion, e.g., by using a fan.

Stimulation of the central end of a sensory nerve (sciatic) increases the surface temperature and diminishes the internal temperature (Ostroumow, Mitropolsky).

\section{Clothing.}

Warm clothing is the equivalent of food.-As clothes are intended to keep in the heat of the body, and heat is produced by the combustion and oxidation of the food, we may say, the body takes in heat directly in the food, while clothing prevents it from giving off too mnch heat. Summer clothes weigh 3-4 kilo, and winter ones, 6-7 kilo.

In connection with clothes, the following considerations are of importance :-

(1.) Their capacity for conduction.-Those substances which conduct heat badly keep us warmest. Hare-skin, down, beaver-skin, raw silk, taffeta, sheeps' wool, cotton wool, flax, spun-silk, are given in order, from the worst to the best conductors. (2.) The capacity for radiation.-Coarse materials radiate more heat than smooth, but colour has no effect. (3.) Relation to the sun's rays.-Dark materials absorb more heat than light-coloured ones. (4.) Their hygroscopic properties are important, whether they can absorb much moisture from the skin and gradually give it off by evaporation or the reverse. The same weight of wool takes up twice as much water as linen; hence, the latter gives it off in evaporation more rapidly. Flannel next the skin, therefore, is not so easily moistened, nor does it so rapidly become cold by evaporation; hence, it protects against the 
action of cold. (5.) The permeability for air is of importance, but does not stand in relation with the heat-conducting capacity. The following substances are arranged in order from the most to the least permeable-flannel, buckskin, linen, silk, leather, waxcloth.

\section{Income and Expenditure of Heat- Balance of Heat.}

As the temperature of the body is maintained within narrow limits, the amount of heat taken in must balance the heat given off, i.e., exactly the same amount of potential energy must be transformed in a given time into heat, as heat is given off from the body.

An adult produces as much heat in half an hour as will raise the temperature of his body $1^{\circ} \mathrm{C}$. If no heat was given off, the body would become very hot in a short time; it would reach the boilingpoint in 36 hours, supposing the production of heat continued uninterruptedly.

The following are the most important calculations on this subject :-

\section{A. According to Helmholtz.}

Helmholtz was the first to estimate numerically the amount of heat produced by a man.

(1.) Heat-income--(a) A healthy adult, weighing 82 kilos., expires in 24 hours, 878.4 grms. $\mathrm{CO}_{2}$ (Scharling). The combustion of the $\mathrm{C}$ therein into $\mathrm{CO}_{2}$ produces . . 1,730,760 Cal.

(b) But he takes in more $\mathrm{O}$ than reappears in the $\mathrm{CO}_{2}$; the excess is used in oxidation-processes, $e . g$., for the formation of $\mathrm{H}_{2} \mathrm{O}$, by union with $\mathrm{H}$, so that 13,615 grms. $\mathrm{H}$ will be oxidised by the excess of $\mathrm{O}$, which gives

$$
\frac{318,600,,}{2,049,360 \text { Cal. }}
$$

(c) About 25 per cent. of the heat must be referred to sources other than combustion (Dulong), so that the total $=2,732,000 \mathrm{Cal}$.

$2,732,000$ calories are actually sufficient to raise the temperature of an adult weighing $80-90$ kilos., from $10^{\circ}$ to $38-39^{\circ} \mathrm{C}$, i.e., to a normal temperature.

(2.) Heat-expenditure.-(a) Heating the food and drink, which have a mean temperature of $12^{\circ} \mathrm{C}$. . . . . . . 70,157 Cal. $=2.6$ per cent.

(b) Heating the air respired $=16,400$ grm., with an initial temperature of $20^{\circ} \mathrm{C}$. - . 70,032, $=2 \cdot 6$, (When the temperature of the air is $0^{\circ}, 140,064 \mathrm{Cal} .=5.2$ per cent.)

(c) Evaporation of $656 \mathrm{grm}$. water by the lungs, $397,536 \mathrm{Cal} .=14 \cdot 7$ per cent.

(d) The remainder given off by radiation and evaporation of water by the skin, $\quad(77.5$ per cent. $t o)=80.1$ per cent. 


\section{B. According to Dulong.}

(1.) Heat-income--Dulong, and after him Boussingault, Liebig and Dumas, sought to estimate the amount of heat from the $\mathrm{C}$ and $\mathrm{H}$ contained in the food. As we know that the combustion of 1 grm. $C=8,040$ heat-units, and $1 \mathrm{grm} . \mathrm{H}=$ 34,460 heat-units, it would be easy to determine the amount of heat were the $\mathrm{C}$ simply converted into $\mathrm{CO}_{2}$ and the $\mathrm{H}$ into $\mathrm{H}_{2} \mathrm{O}$. But Dulong omitted the $\mathrm{H}$ in the carbohydrates (e.g., grape-sugar $=\mathrm{C}_{6} \mathrm{H}_{12} \mathrm{O}_{6}$ ) as producing heat, because the $\mathrm{H}$ is already combined with $\mathrm{O}$, or at least is the proportion in which it exists in water.

This assumption is hypothetical, for the atoms of $\mathrm{C}$ in a carbohydrate may be so firmly united to the other atoms, that before oxidation can take place, their relations must be altered, so that potential energy is used up, i.e., heat must be rendered latent; so that these considerations rendered the following example of Dulong's method given by Vierordt very problematical.

An adult eats in 24 hours, 120 grm. proteids, 90 grm. fat, and 340 grm. starch (carbohydrates). These contain:--

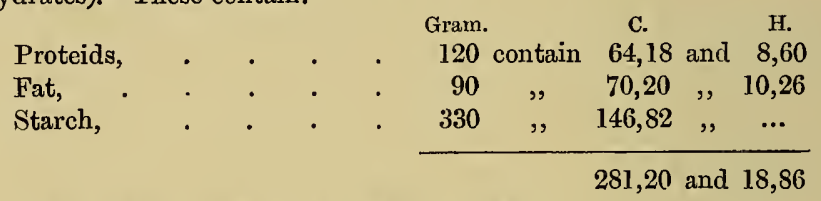

The urine and fæces contain still unconsumed, $29,8, \quad 6,3$

Remainder to be burned, . . . . 251,4 and 12,56

As $1 \mathrm{grm}$. $\mathrm{C}=8,040$ heat-units and $1 \mathrm{grm} . \mathrm{H}=34,460$ heat-units, we have the following calculation:-

$$
\begin{aligned}
& 251,4 \times 8,040=2,031,312 \text { (from combustion of } \mathrm{C} \text { ). } \\
& 12,56 \times 34,460=432,818 \text { ( , " , H). } \\
& \text { 2,464,130 heat-ınits. }
\end{aligned}
$$

(2.) Heat-expenditure:-

Heat-units. Percent. of the excreta,

1. 1,900 grm. are excreted daily by the urine and fæces, and they are $25^{\circ}$ warmer than the food, $47,500 \quad 1 \cdot 8$

2. $13,000 \mathrm{grm}$. air are heated (from $12^{\circ}$ to $37^{\circ} \mathrm{C}$.) (heat-capacity of the air $=0.26$ ), . . . 84,500 3.5

3. $330 \mathrm{grm}$. water are evaporated by the respiration

( 1 grm. = 582 heat-units), . . . . 192,060 7·2

4. $660 \mathrm{grm}$. water are evaporated from the skin, $384,120 \quad 14 \cdot 5$

Total, . . . . . 708,180

Remainder radiated and conducted from the skin, $1,791,810 \quad 72$

Total amount of heat-units given off, $\quad . \quad 2,500,000 \quad 100$

\section{Heat-income, according to Frankland.}

Frankland burned the food directly in a calorimeter, and found that 1 grm, of the following substances yielded:-

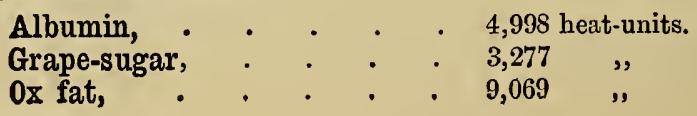


The albumin, however, is only oxidised to the stage of urea, hence the heatunits of urea must be deducted from 4,998, which gives 4,263 heat-units obtainable from $1 \mathrm{grm}$. albumin. When we know the number of grammes consumed, a simple multiplication gives the number of heat-units.

The heat-units will vary, of course, with the nature of the food. J. Ranke gives the following:-

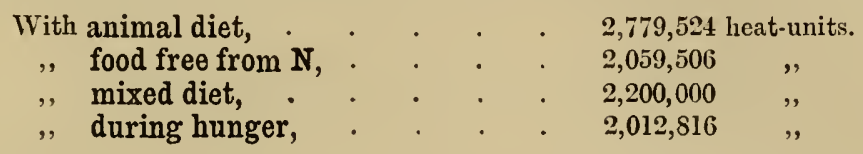

\section{Variations in Heat-production.}

According to Helmholtz, an adult weighing 82 kilos. produces 2,732,000 calories in 24 hours.

(1.) Influence of the body-weight. - Accepting the above number, Immermann has given the following formula for the heat-production in living tissues:-

$$
w: W=\sqrt[3]{p^{2}}: \sqrt[3]{\mathrm{P}^{2}}
$$

(where $\mathrm{WV}=2,732,000 ; \mathrm{P}=S 2$ kilos. $\left[\mathrm{W}: \sqrt[3]{\mathrm{p}^{2}}=144,75\right] ; \mathrm{p}=$ body-weight of the person to be investigated, and $w$ represents the heat-production which is required.)

It is highly desirable that IV $: \sqrt[3]{p^{2}}(=m)$ was ascertained as a mean from a large number of observations, then the heat production for any body-weight $p$ would be

$$
\mathrm{w}=\mathrm{m} \sqrt[3]{\mathrm{j}^{2}} .
$$

(2.) Age and Sex.-The heat-production is less in infancy and in old age, and it is less in proportion in the female than in the male.

(3.) Daily Variation. - The heat-production shows variations in 24 hours corresponding with the temperature of the body $(\$ 213,4)$.

(4.) The heat-production is greater in the waking condition, during physical and mental exertion, and during digestion, than in the opposite conditions.

\section{Relation of Heat-production to the Work of the Body.}

The potential energy supplied to the body may be transformed into heat and potential energy (see Introduction). In the passive condition, almost all the potential energy is changed into heat; the workman, however, transforms potential energy into work-mechanical work-in addition to heat. These two may be compared by using an equivalent measurement, thus, 1 heat-unit (energy required to raise 1 gramme of water $1^{\circ} \mathrm{C}$.) $=425 \cdot 5$ gramme-metres.

The following example may serve to illustrate the relation between heatproduction and the production of work:-Suppose a small steam-engine to be placed within a capacious calorimeter, and a certain quantity of coal to be burned, then as long as the engine does not perform any mechanical work, heat alone is produced by the burning of the coal. Let this amount of heat be estimated, and a second experiment made by burning the same amount of coal, but allow the engine to do 
a certain amount of work-say, raise a weight-by a suitable arrangement. This work must, of course, be accomplished by the potential energy of the heating material. At the end of this experiment, the temperature of the water will be much less than in the first experiment, i.e., fewer heat-units have been transferred to the calorimeter when the engine was heated than when it did no work.

Comparative experiments of this nature have shown, that in the second experiment the useful work is very nearly proportional to the decrease of the heat (Hirn).

In good steam-engines only $\frac{1}{20}$, and in the very best $\frac{1}{8}$, of the potential energy is changed into mechanical energy, while $\frac{19}{20}-\frac{7}{8}$ passes into heat.

Compare this with what happens within the body:-A man in a passive condition forms from the potential energy of the food between $2 \frac{1}{2}-2 \frac{3}{4}$ million calories. The work done by a workman is reckoned at 200,000 kilogramme-metres.

If the organism were entirely similar to a machine, a smaller amount of heat, corresponding to the work done, would be formed in the body. As a matter of fact, the organism produces less heat from the same amount of potential energy when mechanical work is done. There is one point of difference between a workman and a working machine. The workman consumes much more potential energy in the same time than a passive person; much more is burned in his body, and hence, the increased consumption is not only covered, but even over-compensated. Hence, the workman is warmer than the passive person, owing to the increased muscular activity $(\$ 210,1, b)$. Take the following example :-Hirn (1858) remained passive, and absorbed $30 \mathrm{grm} .0$ per hour in a calorimeter, and produced 155 calories. When in the calorimeter he did work equal to 27,450 kilogramme-metres, which was transferred beyond it; he absorbed $132 \mathrm{grm}$. O, and produced only 251 calories.

In estimating the work done, we must include only the heat equivalent of the work transferred beyond the body; lifting weights, pushing anything, throwing a weight, and lifting the body, are examples. In ordinary walking, there is no loss of heat (apart from overcoming the resistance of the air); when descending from a height there may be increased warmth of the body.

The organism is superior to a machine in as far as it can, from the same amount of potential energy, produce more work in proportion to heat. Whilst the very best steam-engine gives $\frac{1}{8}$ of the potential energy in the form of work, and $\frac{7}{8}$ as heat, the body produces $\frac{7}{5}$ as work and $\frac{4}{5}$ as heat. Chemical energy can never do work alone, in a living or dead motor, without heat being formed at the same time.

\section{Accommodation for Varying Degrees of Temperature.}

All substances which possess high conductivity for heat, when brought into contact with the skin, appear to be very much colder or 
hotter than bad conductors of heat. The reason of this is that these bodies abstract far more heat, or conduct more heat than other bodies. Thus the water of a cool bath, being a better conductor of heat, is always thought to be colder than air at the same temperature. In our climate it appears to us that

Air,

at $18^{\circ} \mathrm{C}$, is molerately warm; "25 $25^{\circ}-25^{\circ} \mathrm{C}$., hot; above $28^{\circ}$, very hot.

\section{Water,}

at $18^{\circ} \mathrm{C}$, is cold; from $18-29^{\circ} \mathrm{C}$, cool; ,2 $29-35 \cdot 5^{\circ} \mathrm{C}$., warm;

,, 37.5 and above, hot.

As long as the temperature of the body is higher than that of the surrounding medium, heat is given off, and that the more rapidly the better the conducting power of the surrounding medium. As soon as the temperature of the surrounding medium rises higher than the temperature of the body, the latter absorbs heat, and it does so the more rapidly the better the conducting power of the medium. Hence, hot water appears to be warmer than air at the same temperature. A person may remain eight minutes in a bath at $45.5^{\circ} \mathrm{C}$. (dangerous to life!); the hands may be plunged into water at $50.5^{\circ} \mathrm{C}$, but not at $51.65^{\circ} \mathrm{C}$., while at $60^{\circ}$ violent pain is produced.

A person may remain for eight minutes in air heated to $99.95-$ $127^{\circ} \mathrm{C}$, and a temperature of $132^{\circ} \mathrm{C}$. has been borne for ten minutes (Tillett, 1763). The body-temperature rises only to $38 \cdot 6-38 \cdot 9^{\circ}$ (Fordyce, Blagden, 1774). This depends upon the air being a bad conductor, and thus it gives less heat to the body than water would do. Farther, and what is more important, the skin becomes covered with sweat, which evaporates and abstracts heat, while the lungs also give off more watery vapour. The enormously increased heart-beats-over 160 -and the dilated blood-vessels, enable the skin to obtain an ample supply of blood for the formation and evaporation of sweat. In proportion as the secretion of sweat diminishes, the body becomes unable to endure a hot atmosphere; hence it is that in air containing much watery vapour a person cannot endure nearly so high a temperature as in dry air, so that heat must accumulate in the body. In a Turkish vapour bath of $53^{\circ}$ to $60^{\circ} \mathrm{C}$, the rectal temperature rises to $40 \cdot 7-41 \cdot 6^{\circ} \mathrm{C}$. (Barthels, Jürgensen, Krishaber). A person may work continuously in air at $31^{\circ} \mathrm{C}$. which is almost saturated with moisture (Stapff).

If a person be placed in water at the temperature of the body, the normal temperature rises $1^{\circ} \mathrm{C}$. in 1 hour, and in $1 \frac{1}{2}$ hours about $2^{\circ} \mathrm{C}$. (Liebermeister). A gradual increase of the temperature from 38.6 to $40 \cdot 2^{\circ} \mathrm{C}$. causes the axillary temperature to rise to $39 \cdot 0^{\circ} \mathrm{C}$. within fifteen minutes. 
Rabbits placed in a warm box at $36^{\circ} \mathrm{C}$. acquire a constant temperature of $42^{\circ} \mathrm{C}$, and lose weight; but if the temperature of the box be raised to $40^{\circ}$, death occurs, the body-temperature rising to $45^{\circ} \mathrm{C}$. (J. Rosenthal).

\section{Storage of Heat in the Body.}

As the uniform temperature of the body, under normal circumstances, is due to the reciprocal relation between the amount of heat produced and the amount given off, it is clear that heat must be stored up in the body when the evolution of heat is diminished. The skin is the chief organ regulating the evolution of heat; when it and its blood-vessels contract, the heat evolved is diminished, when they dilate it is increased. Heat may be stored up when-

(a) The skin is extensively stimulated, whereby the cutaneous vessels are temporarily.contracted (Röhrig). (b) Any other circumstances prevent heat from being given off by the skin (Winternitz). (c) When the vaso-motor centre is excited, causing all the blood-vessels of the body-those of the skin included-to contract. This seems to be the cause of the rise of temperature after transfusion of blood (Landois), and the rise of temperature after the sudden removal of water from the body seems to admit of a similar explanation; as the inspissated blood occupies less space, and the contracted vessels of the skin admit less blood. (d) When the circulation in the cutaneous vessels of a large area is mechanically slowed, or when the smaller vessels are plugged by the injection of some sticky substance, or by the transfusion of foreign blood, the temperature rises (§ 102). Landois found that ligature of both carotids, and the axillary and crural arteries, caused a rise of $1^{\circ} \mathrm{C}$. within two hours.

It is also obvious that when a normal amount of heat is given off, an increased production of heat must raise the temperature. The rise of the temperature after muscular or mental exertion, and during digestion seems to be caused in this way. The rise which occurs several hours after a cold bath is probably due to the reflex excitement of the skin causing an increased production (Jürgensen).

When the temperature of the body, as a whole, is raised $6^{\circ} \mathrm{C}$., death takes place, as in sunstroke. It seems as if there was a molecular decomposition of the tissues at this temperature; while, if a slightly lower temperature be kept up continuously, fatty degeneration of many tissues occurs (Litten). If animals, which have been exposed artificially to a temperature of over $42^{\circ}-44^{\circ} \mathrm{C}$., be transferred to a cooler. atmosphere, their temperature becomes sub-normal $\left(36^{\circ} \mathrm{C}\right.$.) and may remain so for several days.

\section{Fever.}

Fever consists in a greatly increased tissue metabolism (especially in the musclesFinkler, Zuntz), with simultaneous increase of the temperature. Of course the mechanism regulating the balanoe of formation and expenditure of heat is disturbed. 
During fever, the body is greatly incapacitated for performing mechanical work. It is cvident, therefore, that the large amount of potential energy transformed is almost all converted into heat, so that the non-transformation of the energy into mechanical work is another important factor.

We may take intermittent fever or ague as a type of fever, in which violent attacks of fever of several hours duratiou alternate with periods free from fever. This enables us to analyse the symptoms. The symptoms of fever are:-

(1.) The increased temperature of the body.- $\left(38^{\circ}-39^{\circ} \mathrm{C}\right.$., slight; from $39^{\circ}-41^{\circ} \mathrm{C}$. and upwards, severe). The ligh temperature occurs not only in cases where the skin is red, and has a hot burning feeling (calor mordax), but even during the rigor or the shivering stage, the temperature is raised (Ant. de Haen, 1760). The congested red skin is a good conductor of heat, while the pale bloodless skin conducts badly; hence, the former feels hot to the touch (v. Bärensprungcompare $\$ 212)$.

(2.) The increased production of heat (assumed by Lavoisier and Crawford) is proved by calorimetric observations. This is, in small part, due to the increased activity of the circulation being changed into heat $(\$ 206,2, a)$, but for the most part it is due to increased combustion within the body.

(3.) The increased metabolism gives rise to the "consuming" or "wasting" character" of fever, which was known to Hippocrates and Galen, and in 1852 v. Bärensprung asserted that "all the so-called febrile symptoms show that the metabolism is increased." The increase of the metabolism is shown in the increased excretion of $\mathrm{CO}_{2}=70^{\circ}-80^{\circ}$ per cent. (Leyden and Fränkel), while more $\mathrm{O}$ is consumed, although the respiratory quotient remains the same (Zuntz and Lilienfeld). According to $\mathrm{D}$. Finkler, the $\mathrm{CO}_{2}$ excreted shows greater variations than the $\mathrm{O}$ consumed. The excretion of urea is increased $\frac{1}{3}$ to $\frac{2}{3}$. In dogs suffering from septic fever, Naunyn observed that the urea began to increase before the temperature rose, "prefelvile rise." Part of the urea, however, is sometimes retained during the fever, and appears after the fever is over, "epicritical excretion of urea" (Naunyn). The uric acid is also increased; the urine pigment (\$ 19) derived from the hæunoglobin, may be increased 20 times, while the excretion of potash may be seven-fold.

It is important to observe, that the oxidation or combustion processes within the body of the fever patient, are greatly increased, when he is placed in a warmer atmosphere. The oxidation processes in fever, however, are also increased under the influence of cooler surrouudings $(\$ 214, I, 2)$, but the increase of the oxidation in a warm medium is very much greater than in the cold (D. Finkler). The amount of $\mathrm{CO}_{2}$ in the blood is diminished, but not at once after the onset even of a very severe fever (Goppert).

(4.) The diminished excretion of heat varies in different stages of a fever. We distinguish several stages in a ferer-(a) The cold stage, when the loss of heat is greatly diminished, owing to the pale bloodless skin, but at the same time the heat-production is increased $1 \frac{1}{2}-2 \frac{1}{2}$ times. The sudden and considerable rise of the temperature during this stage shows that the diminished excretion of heat is not the only cause of the rise of the temperature. (b) During the hot stage, the heat given off from the congested red skin is greatly increased, but at the same time more heat is produced. Liebermeister assumes that a rise of $1,2,3,4^{\circ} \mathrm{C}$. corresponds to an increased production of heat of $6,12,18,24$ per cent. (c) In the sweating stage, the excretion of heat through the red moist skin and evaporation are greatest, more than two to three times the normal (Leyden). The heat-production is either increased, normal, or sub-normal, so that under these conditions the temperature may also be sub-normal $\left(36^{\circ} \mathrm{C}\right.$.).

(5.) The heat-regulating mechanism is injured.-A warm temperature of the surroundings raises the temperature of the fever patient more than it does that of a non-febrile person. The depression of the heat-production, which 
enables normal animals to maintain their normal temperature in a warm medium (\$214), is much less in fever (D. Finkler).

The accessory phenomena of fever are very important:-Increase in the intensity and number of the heart-beats $(\$ 214, \mathrm{II}, 2)$ and respirations (in adults 40 , and children 60 per min.), both being compensatory phenomena of the increased temperature; further, diminished digestive activity $(\S 186, D)$ and intestinal movements; disturbances of the cerebral activities; of secretion; of muscular activity; slower excretion-e.g., of potassium iodide through the urine (Bachrach, Scholze). In severe fever, molecular degenerations of the tissues are very common.

For the condition of the blood-corpuscles in fever see p. 23, the vascular tension, $\S 69$; the saliva, $\S 146$.

Quinine, the most important febrifuge, causes a decrease of the temperature by limiting the production of heat (Lewizky, Binz, Naunyn, Quincke, Arntz). Toxic doses of the metallic salts act in the same way, while there is at the same time diminished formation of $\mathrm{CO}_{2}$ (Luchsinger).

\section{Artificial Increase of the Bodily Temperature.}

If mammals are kept consiantly in air at $40^{\circ} \mathrm{C}$., the excretion of heat from the body ceases, so that the heat produced is stored up. At first, the temperature falls somewhat for a very short time (Obernier), but soon a decided increase occurs. The respirations and pulse are increased, while the latter becomes irregular and weaker. The $\mathrm{O}$ absorbed and $\mathrm{CO}_{2}$ given off are diminished after 6-8 hours (Litten), and death occurs after great fatigue, feebleness, spasms, secretion of saliva, and loss of consciousness, when the bodily temperature has been increased $4^{\circ}$ or at most $6^{\circ} \mathrm{C}$. Death does not take place, owing to rigidity of the muscles, for the coagulation of the myosin of mammals' muscles occurs at $49-50^{\circ} \mathrm{C}$, in birds at $53^{\circ} \mathrm{C}$, and in frogs at $40^{\circ} \mathrm{C}$. If mammals are suddenly placed in air at $100^{\circ} \mathrm{C}$., death occurs (in 15-20 min.) very rapidly, and with the same phenomena, while the bodily temperature rises $4-5^{\circ} \mathrm{C}$. In rabbits, the body-weight diminishes 1 grm. per min. Birds bear a high temperature somewhat longer; they die when their blood reaches $48-50^{\circ} \mathrm{C}$.

Even man may remain for some time in air at $100-110-132^{\circ} \mathrm{C}$, but in 10-15 minutes there is danger to life. The skin is burning to the touch, is red, a copious secretion of sweat bursts forth, and the cutaneous veins are fuller and redder (Crawford). The pulse and respirations are greatly accelerated. Violent headache, vertigo, feeblcness, stupefaction indicate great danger to life. The rectal temperature is only $1-2^{\circ} \mathrm{C}$. higher. The high temperature of fever may even be dangerous to human life. If the temperature remains for any length of time at $42.5^{\circ} \mathrm{C}$, death is almost certain to occur. Coagulation of the blood in the arteries is said to occur at $42 \cdot 6^{\circ} \mathrm{C}$. (Weikart). If the artificial heating does not produce death, fatty infiltration and degenera- 
tion of the liver, heart, kidneys, and muscles begin after $36-48$ hours (Litten).

Cold-blooded animals, if placed in hot air or warm water, soon have their temperature raised $6-10^{\circ} \mathrm{C}$. The highest temperature compatible with life in a frog must be below $40^{\circ} \mathrm{C}$., as the frog's heart and muscles hegin to coagulate at this temperature. Death is preceded by a stage resembling death, during which lifo may be saved.

Most of the juicy plants die in half an hour in air at $52^{\circ} \mathrm{C}$, , or in water at $46^{\circ} \mathrm{C}$. (Sachs). Dried seeds of corn may still germinate after long exposure to air at $120^{\circ} \mathrm{C}$. Lowly organised plants, such as alga, may live in water at $60^{\circ} \mathrm{C}$. (Hoppe-Seyler). Several bacteria withstand a boiling temperature (Tyndall, Chamberland).

\section{Employment of Heat.}

Action of Heat.-The short, but not intense, action of heat on the surface causes, in the first place, a transient slight decrease of the bodily temperature, partly because it retards reflexly the production of heat (Kernig), and partly becanse, owing to the dilatation of the cutaneous vessels and the stretching of the skin, more heat is given off (Senator). A warm bath above the temperature of the blood at once increases the bodily temperature.

Therapeutic Uses.-The application of heat to the entire body is used where the bodily temperature has fallen, or is likely to fall, very low, as in algid stage of cholera, and in infants born prematurely. The general application of heat is obtained by the use of warm baths, packing, vapour, insolation, and the copious use of hot crinks. The local application of heat is obtained by the use of warm wrappings, partial baths, plunging the parts in warm earth or sand, or placing wounded parts in chambers filled with heated air. After removal of the heating agent, care must be taken to prevent the great escape of heat due to the dilatation of the blood-vessels.

\section{Increase of Temperature-Post-mortem Phenomena.}

Heidenhain found that in a dead dog, before the body cooled, there was a constant temporary rise of the temperature, which slightly exceeded the normal. The same observation had been occasionally made on human bodies immediately after death, especially when death was preceded by muscular spasms. Thus, Wunderlich measured the temperature 57 minutes after death in a case of tetanus, and found it to be $45 \cdot 375^{\circ} \mathrm{C}$.

The causes are :-

(1.) A temporary increased production of heat after death, due chiefly to the change of the semi-solid myosin of the muscles into a solid form (rigor mortis). As the muscle coagulates, heat is produced (v. Walther, Fick). All conditions which cause rapid and intense coagulation of the muscles-e.g., spasms, favour a post-mortem rise of temperature (see Physiology of $M$ uscle); a rapid coagulation of the blood has a similar result (p. 42).

(2.) Immediately after death, a series of chemical processes occur within the body, whereby heat is produced. Valentin placed dead rabbits in a chamber, so that no heat could be given off from the body, and he found that the internal temperature of the animal's body was increased. The processes which cause a rise of 
temperature post mortem are more active during the first than the second hour; and the higher the temperature at the moment of death, the greater is the amount of heat evolved post mortem (Quincke and Brieger).

(3.) Another cause is the diminished excretion of heat post mortem. After the circulation is abolished, within a few minutes little heat is given off from the surface of the body, as rapid excretion implies that the cutaneous vessels must be continually filled with warm blood.

\section{Action of Cold on the Body.}

A short temporary slight cooling of the skin (removing one's clothes in a cool room, a cool bath for a short time, or a cool douche) causes either no change or a slight rise in the bodily temperature (Liebermeister). The slight rise, when it occurs, is due to the stimulation of the skin causing reflexly a more rapid molecular transformation, and therefore a greater production of heat (Liebermeister), while the amount of heat given off is diminished owing to contraction of the small cutaneous vessels and the skin (Jürgensen, Senator, Speck). The continuous and intense application of cold causes a decrease of the temperature (Currie), chiefly by conduction, notwithstanding that at the same time there is a greater production of heat. After a cold bath, the temperature may be $34^{\circ}, 32^{\circ}$, and even $30^{\circ} \mathrm{C}$.

As an after-effect of the great abstraction of heat, the temperature of the body after a time remains lower than it was before ("primary aftereffect"-Liebermeister); thus after an hour it was- $-0.22^{\circ} \mathrm{C}$. in the rectum. There is a "secondary after-effect" which occurs after the first after-effect is over, when the temperature rises (Jürgensen). This effect begins 5-8 hours after a cold bath, and is equal to $+0.2^{\circ} \mathrm{C}$. in the rectum. Hoppe-Seyler found that some time after the application of heat, there was a corresponding lowering of the temperature.

Taking Cold,-If a rabbit be taken from a surrounding temperature of $35^{\circ} \mathrm{C}$, and suddenly cooled, it shivers, and there may be temporary diarrhœa. After two days, the temperature rises $1.5^{\circ} \mathrm{C}$., and albuminuria occurs. There are microscopic traces of interstitial inflammation in the kidneys, liver, lungs, heart, and nerve-sheaths, the dilated arteries of the liver and lnng contain thrombi, and in the neighbourhood of the veins are accumulations of leucocytes. In pregnant animals, the fœtus shows the same conditions (Lassar). Perhaps the greatly cooled blood acts as an irritant causing inflammation (Rosenthal).

Action of Frost.-The continued application of a high degree of cold causes at first contraction of the blood-vessels of the skin and its muscles, so that it becomes pale. If continued paralysis of the cutaneous vessels occurs, the skin becomes red, owing to congestion of its vessels. As the passage of fluids through the capillaries is rendered more difficult by the cold, the blood stagnates, and the skin assumes a livid appearance, as the $O$ is almost completely used up. Thus the peripheral circulation is slowed. If the action of the cold be still more intense, the peripheral circulation stops completely, especially in the thinnest and most exposed organs- 
ears, nose, toes, and fingers. The sensory nerves are paralysed, so that there is numbness and loss of sensibility, and the parts may even be frozen through and through. As the slowing of the circulation in the superficial vessels gradually affects other areas of the circulation, the pulmonary circulation is enfeebled, and diminished oxidation of the blood occurs, notwithstanding the greater amount of $\mathrm{O}$ in the cold air, so that the nerve centres are affected. Hence, arise great dislike to making movements or any muscular effort, a painful sensation of fatigue, a peculiar and almost irresistible desire to sleep, cerebral inactivity, blunting of the sense-organs, and lastly, coma. The blood freezes at $-3.9^{\circ} \mathrm{C}$, , while the juices of the superficial parts freeze sooner. Too rapid movements of the frost-bitten parts ought to be avoided. Rubbing with snow, and the very gradual application of heat, produce the best results. Partial death of a part is not unfrequently produced by the prolonged action of cold.

\section{Artificial Lowering of the Temperature in Animals.}

Phenomena.-The artificial cooling of warm-blooded animals, by placing them in cold air or in a freezing mixture gives rise to a series of characteristic phenomena (A. Walther). If the animals (rabbits) are cooled so that the temperature (rectum) falls to $18^{\circ}$, they suffer great depression without, however, the voluntary or reflex movements being abolisher. The pulse falls from 100 or 150 to 20 beats per minute, and the blood-pressure falls to several millimetres of $\mathrm{Hg}$. The respirations are few and shallow. Suffocation does not cause spasms (Howarth), the secretion of urine stops, and the liver is congested. The animal may remain for 12 hours in this condition, and when the muscles and nerves show signs of paralysis, coagulation of the blood occurs after numerous blood-corpuscles have been destroyed. The retina becomes pale, and death occurs with spasms and the signs of asphyxia.

If the bodily temperature be reduced to $17^{\circ}$ and under, the voluntary movements cease before the reflex acts (Richet and Rondeau).

An animal cooled to $18^{\circ} \mathrm{C}$, and left to itself, at the same temperature of the surroundings, does not recover of itself, but if artificial respiration be employed, the temperature rises $10^{\circ} \mathrm{C}$. If this be combined with the application of external warmth, the animals may recover completely, even when they have been apparently dead for forty minutes. Walther cooled adult animals to $9^{\circ} \mathrm{C}$., and recovered them by artificial respiration and external warmth; while Howarth cooled young animals to $5^{\circ} \mathrm{C}$. Mammals, which are born blind, and birds, which come out of the egg devoid of feathers, cool more rapidly than others. Morphia, and more so, alcohol, accelerate the cooling of mammals; hence, drunk men are more liable to die when exposed to cold. 
Artificial Cold-Blooded Condition.-Cl. Bernard made the important observation, that the muscles of animals that had been cooled remained irritable for a long time, both to direct stimuli as well as to stimuli applied to their nerves; and the same is the case when the animals are asphyxiated for want of $\mathrm{O}$. An "artificial cold-blooded condition," i.e., a condition in which warm-blooded animals have a lower temperature, and retain muscular and nervous excitability (Cl. Bernard), may also be caused in warm-blooded animals, by dividing the cervical spinalcord and keeping up artificial respiration; further, by moistening the peritoneum with a cool solution of common salt (Wegner).

Hybernation presents a series of similar phenomena. Valentin found that hybernating animals become half-awake when their bodily temperature is $28^{\circ} \mathrm{C}$.; at $18^{\circ} \mathrm{C}$. they are in a somnolent condition, at $6^{\circ}$ they are in a gentle sleep, and at $1 \cdot 6^{\circ} \mathrm{C}$. in a deep sleep. The heart-beats and the blood-pressure fall, the former to S-10 per minute. The respiratory, urinary, and intestinal movements cease completely, and the cardio-pneumatic movement alone (p. 110) sustains the slight exchange of gases in the lungs. They cannot endure cooling to $0^{\circ} \mathrm{C}$.; they awake before the temperature falls so low. Hybernating animals may be cooled to a greater degree than other mammals; they give off heat rapidly, and they become warm again rapidly, and even spontaneously. New-born mammals resemble hybernating animals more closely in this respect than do adults.

Cold-blooded Animals may be cooled to $0^{\circ}$. Even when the blood has been frozen and ice formed in the lymph of the peritoneal cavity, frogs may recover. In this condition they appear to be dead, but when placed in a warm medium they soon recover. A frog's muscle so cooled will contract again (Kühne). The germs and ova of lower animals, e.g., insects' eggs, survive continued frost; and if the cold be moderate, it merely retards development. Bacteria survive a temperature of $-87^{\circ} \mathrm{C}$; ; yeast, even- $100^{\circ} \mathrm{C}$. (Frisch).

Varnishing the Skin causes a series of similar phenomena. The varnished skin gives off a large amount of heat by radiation (Krieger), and sometimes the cutaneous vessels are greatly dilated (Laschkewitsch). Hence the animals cool rapidly and die, although the consumption of $\mathbf{O}$ is not diminished. If cooling be prevented (Valentin, Schiff, Brunton) by warming them and keeping them in warm wool, the animals live for a longer time. The blood post mortem does not contain any poisonous substances, nor even are any materials retained in the blood which can cause death, for if the blood be injected into other animals, these remain healthy. Varnishing the human skin does not seem to be dangerous(Senator).

\section{Employment of Cold.}

Cold may be applied to the whole or part of the surface of the body in the following conditions :-

(a) By placing the body for a time in a cold bath to abstract as much heat as possible, when the bodily temperature in fever rises so high as to be dangerous to life. This result is best accomplished and lasts longest when the bath is gradually cooled from a moderate temperature. If the body be placed at once in cold water, the cutaneous vessels contract, the skin becomes bloodless, and thus obstacles are placed in the way of the excretion of heat. A bath gradually cooled in this way is borne longer (v. Ziemssen). The addition of stimulating substances, 
e.g., salts, which cause dilatation of the cutaneous vessels, faeilitates the excretion of heat; even salt-water conduets heat better. If alcohol be given internally at the same time, it lowers the temperature.

(b) Cold may be applied locally by means of ice in a bag, which causes contraction of the cutaneous vessels and contraction of the tissues (as in inflammation), while at the same time heat is abstracted locally.

(c) Heat may be abstracted locally by the rapid evaporation of volatile substanees (ether, carbon disulphide), which causes numbness of the sensory nerves. The introduction of media of low temperature into the body, respiring cool air, taking cold drinks, or the injection of cold fluids into the intestine acts locally, and also produces a more general action. In applying cold it is important to notice that the initial contraction of the vessels and the contraction of the tissues are followed by a stronger dilatation and turgescence.

\section{2\%. Heat of Inflamed Parts.}

"Calor" or heat is reckoned one of the fundamental phenomena of inflammation, in addition to rubor (redness), tumor (swelling), and dolor (pain). But the apparent increase in the heat of the inflamed parts is not above the temperature of the blood. Simon, in 1S60, asserted that the arterial blood flowing to an inflamed part was cooler than the part itself; but $v$. Bürensprung denies this, as J. Hunter did, and so does Jacobson, Bernhardt, and Laudien. The outer parts of the skin in an inflamed part are warmer than usual, owing to the dilatation of the vessels (rubor) and the consequent acceleration of the blood-stream in the inflamed part, and owing to the swelling (tumor) from the presence of good heatconducting fluids; but the heat is not greater than the heat of the blood. It is not proved that an increased amount of heat is produced, owiug to inereased molecular decompositions within an inflamed part.

\section{Historical and Comparative.}

Accorling to Aristotle, the heart prepares the heat within itself, and sends it along with the blood to all parts of the body. This doctrine prevailed in the time of Hippocrates and Galen, and oceurs even in Cartesius and Bartholinus (1667, "flamula cordis"). The iatro-mechanical school (Boerhave, van Swieten) ascribed the heat to the friction of the blood on the walls of the ressels. The iatro-chemical school, on the other hand, songht the source of heat in the fermentations that arose from the passage of the absorbed substances into the blood (van Helmont, Sylvius, Ettmüller). Lavoisier (1777) was the first to aseribe the heat to the combustion of carbon in the lungs.

After the construction of the thermometer by Galileo, Sanctorius (1626) made the first thermometric observations on sick persons, while the first calorimetric observations were made by Lavoisier and Laplace.

Comparative observations are given at $\S 207$, and also undcr Hybernation (p. 456). 


\section{Physiology of the Metabolic Phenomena of the Body.}

By the term metabolism are meant all those phenomena, whereby all -even the most lowly-living organisms are capable of incorporating the substances obtained from their food into their tissues, and making them an integral part of their own bodies. This part of the process is known as assimilation. Further, the organism in virtue of its metabolism forms a store of potential energy, which it can transform into kinetic energy, and which, in the higher animals at least, appears most obvious in the form of muscular work and heat. The changes of the constituents of the tissues, by which these transformations of the potential energy are accompanied, result in the formation of excretory products, which is another part of the process of metabolism. The normal metabolism requires the supply of food quantitatively and qualitatively of the proper kind, the laying up of this food within the body, a regular chemical transformation of the tissues, and the preparation of the effete products which have to be given out through the excretory organs.

\section{General View of the most Important Substances used as Food.}

Water.

When we remember that 58.5 per cent. of the body consists of water, that water is being continually given off by the urine and fæces, as well as through the skin and lungs, that the processes of digestion and absorption require water for the solution of most of the substances used as food, and that numerous substances excreted from the body require water for their solution-e.g., in the urine, the great importance of water and its continual renewal within the organism are at once apparent. As put by Hoppe-Seyler, all organisms live in water, and even in running-water, a saying which ranks with the old saying"Corpora non agunt nisi fluida."

Water-as far as it is not a constituent of all fluid foods-occurs in 
different forms as drink:-(1) Rain-water, which most closely resembles distilled or chemically pure water, always contains minute quantitics of $\mathrm{CO}_{2} \mathrm{NH}_{3}$, nitrous and nitric acids.

(2) Spring-water usually contains much mineral substance. It is formed from the deposition of watery vapour or rain from the air, which permeates the soil containing much $\mathrm{CO}_{2}$; the $\mathrm{CO}_{2}$ is dissolved by the water, and aids in dissolving the alkalies, alkaline earths, and metals, which appear in solution as bicarbonates-e.g., of lime or iron oxide. The water is removed from the spring by proper mechanical appliances, or it bubbles up on the surface in the form of a "spring." (3) The running-water of rivers usually contains much less mineral matter than spring-water. Springwater floating on the surface rapidly gives off its $\mathrm{CO}_{2}$, whereby many substances-e.g., lime-are thrown out of solution, and deposited as insoluble precipitates.

Gases._Spring-water contains little $\mathrm{O}$, but much $\mathrm{CO}_{2}$, which latter gives to it its fresh taste. Hence, vegetable organisms flourish in spring-water, while animals requiring, as they do, much $\mathrm{O}$, are but poorly represented in such water. Water flowing freely gives up $\mathrm{CO}_{2}$, and absorbs $\mathrm{O}$ from the air, and thus affords the necessary conditions for the existence of fishes and other marine animals. Riverwater contains $\frac{1}{30}-\frac{1}{20}$ of its volume of absorbed gases, which may be expelled by boiling or freezing.

Drinking-water is chiefly obtained from springs. River-water, if used for this purpose, must be filtered to get rid of mechanically suspended impurities. For household purposes a charcoal filter may be used, as the charcoal acts as a disinfectant. Alum has a remarkable action; if 0.0001 per cent. be added, it makes turbid water clear.

Investigation of Drinking-water.-Drinking-water, even in a thick layer, ought to be completely colourless, not turbid, and without odour. Any odour is best recognised by heating it to $50^{\circ} \mathrm{C}$, and adding a little caustic soda. It ought not to be too hard-i.e., it ought not to contain too much lime (and magnesia) salts.

By the term "degree of hardness" of a water is meant the unit amount of lime (and magnesia) in 100,000 parts of water; a water of $20^{\circ}$ of hardness contains 20 parts of lime (calcium oxide) combined with $\mathrm{CO}_{2}$, sulphuric, or hydrochloric acids (the small amount of magnesia may be neglected). A good drinking water ought not to exceed $20^{\circ}$ of hardness. The hardness is determined by titrating the water with a standard soap solution, the result being the formation of a scum on the surface.

The hardness of unboilec $\vec{l}$ water is called its total hardness, while that of boiled water is called permanent hardhess. Boiling drives off the $\mathrm{CO}_{2}$, and precipitates the calcium carbonate, so that the water at the same time becomes softer.

The presence of sulphuric acid, or sulphates, is determined by the water becoming turbid on adding a solntion of barium chloride and hydroclloric acid.

Chlorine occurs in small amount in pure spring water, but when it occurs there 
ir large amount-apart from its being derived from saline springs, near the sea or manufactories-we may conclude that the water is contaminated from waterclosets or dunghills, so that the estimation of chlorine is of importance. For this purpose use a solution, A, of 17 grms. of crystallised silver nitrate in 1 litre of distilled water; 1 cubic centimetre of this solution precipitates 3.55 milligrammes of chlorine as silver chloride. Use also B, a cold saturated solution of neutral potassium chromate. Take 50 cubic centimetres of the water to be investigated, and place it in a beaker, add to it 2-3 drops of $B$, and allow the fluid $A$ to run into it from a burette until the white precipitate first formed remains red, even after the fluid has been stirred. Multiply the number of cubic centimetres of A used by $7 \cdot 1$, and this will give the amount of chlorine in 100,000 parts of the water. Example-50 c.cmtr. requires $2 \cdot 9$ c.cmtr. of the silver solution, so that 100,000 parts of the water contain $2 \cdot 9 \times 7 \cdot 1=20.59$ parts chlorine (Kubel, Tiemann). Good water ought not to contain more than 15 milligrammes of chlorine per litre.

The presence of lime may be ascertained by acidulating 50 cubic centimetres of the water with $\mathrm{HCl}$ and adding ammonia in excess, and afterwards adding ammonia oxalate; the white precipitate is lime oxalate. According to the degree of turbidity we judge whether the water is "soft" (poor in lime), or "hard" (rich in lime).

Magnesia is determined by taking the clear fluid of the above operation, after removing the precipitate of lime and adding to it a solution of sodium phosphate and some ammonia ; the crystalline precipitate which occurs is magnesia.

The more feeble all these reactions are which indicate the presence of sulphuric acid, chlorine, lime, and magnesia, the better is the water. In addition, good water ought not to contain more than traces of nitrates, nitrites, or compounds of ammonia, as their presence indicates the decomposition of nitrogenous organic substances.

For nitric acid, take 100 cubic centimetres of water acidulated with 2 to 3 drops of concentrated sulphuric acid, add several pieces of zinc together with a solution of potassium iodide, and starch solution-a blue colour indicates nitric acid. The following test is very delicate:-Add to half a drop of water in a capsule 2 drops of a watery solution of Brucinum sulphuricum, and afterwards several drops of concentrated sulphuric acid; a rose-red colouration indicates the presence of nitric acid.

The presence of nitrous acid is ascertained by the blue colouration which results from the addition of a solution of potassium iodide, and solution of starch after the water has been acidulated with sulphuric acid.

Compounds of ammonia are detected by Nessler's reagent, which gives a yellow or reddish colouration when a trace of ammonia is present in water; while a large amount of these compounds gives a brown precipitate of the iodide of mercury and ammonia.

The contamination of water by decomposing animal substance is determined by the amount of $\mathrm{N}$ it contains. In most cases it is sufficient to determine the amount of nitric acid present. For this purpose we require (A) a solution of 1.871 grms. potassium nitrate in 1 litre distilled water-1 cubic centimetre contains 1 milligramme nitric acid; (B) a dilute solution of indigo, which is prepared by rubbing together 1 part of pulverised indigotin with 6 parts $\mathrm{H}_{2} \mathrm{SO}_{4}$, and allowing the deposit to subside, when the blue fluid is poured into 10 times its volume of distilled water and filtered. This fluid is diluted with distilled water until a layer, 12-15 mm. in thickness begins to be transparent.

To test the activity of $\mathrm{B}$, place 1 cubic centimetre of $\mathrm{A}$ in 24 cubic centimetres water, add some common salt and 50 cubic centimetres concentrated sulphuric acid, and allow $B$ to flow from a burette into this mixture until a faint green colour is obtained. The number of cubic centimetres of $B$ used correspond to 1 milligramme of nitric acid. 
25 cubic centimetres of the water to be investigated are mixed with 50 cubic centimetres of concentrated $\mathrm{H}_{2} \mathrm{SO}_{4}$, and titrated with $\mathrm{B}$ until a green colour is obtained. This process must be repeated, and on the second occasion the solution $\mathrm{B}$ must be allowed to flow in at once, when usually somewhat more indigo solution is required to obtain the green solution. The number of cubic centimetres of $B$ (corresponding to the strength of $\mathrm{B}$, as determined above), indicates the amount of nitric acid present in $\mathbf{2 5} \mathrm{c.cmtr}$. of the water investigated. As much as $\mathbf{1 0}$ milligrammes nitric acid have been found in spring-water (Marx, Trommsdorff).

Sulphuretted Hydrogen is recognised by its odour ; also by a piece of blotting. paper moistened with alkaline solution of lead becoming brown, when it is held over the boiling water. If it occurs as a compound in the water, sodium nitroprusside gives a reddish violet colour.

It is of the greatest importance that drinking water should be free from the presence of organic matter in a state of decomposition. Organic matter in a state of decomposition, and the organisms therewith associated, when introduced into the body, may give rise to fatal maladies, e.g., cholera and typhoid fever. This is the case when the water-supply has been contaminated from water which has percolated from water-closets, privies, and dung-pits. The presence of organic matter may be detected thus-(1) A considerable amount of the water is evaporated to dryness in a porcelain vessel, if the residue be heated again a brown or black colour indicates the presence of a considerable amount of organic matter; and if it contain $\mathrm{N}$, there is an odour of ammonia. Good water treated in this way gives only a light-brown. The presence of micro-organisms may be determined microscopically after eraporating a small quantity of the water on a glass-slide. (2) The addition of potassio-gold chloride added to the water gives a black frothy precipitate after long standing. (3) A solution of potassium permanganate, added to the water in a covered jar, gradually becomes decolourised, and a brownisl precipitate is formed.

Water containing much organic matter should never be used as drinking water, and this is especially the case when there is an epidemic of typhoid fever, cholera, or diarrhœa. In all such circumstances, the water ought to be boiled for a long time, whereby the organic germs are killed. The insipid taste of the water after boiling may be corrected by adding a little sugar or lime juice.

\section{Structure and Secretion of the Mammary Glands.}

About 20 galactoferous ducts open singly upon the surface of the nipple. Each of these, just before it opens on the surface, is provided with an oval dilatationthe sinus lacteus. When traced into the gland, the galactoferous ducts divide like the branches of a tree, and a large branch of the duct passes to each lobe of the gland, all the lobes being held together by loose connective-tissue. Only during lactation do all the fine terminations of the ducts communicate with the globular glandular acini. Every gland acinus consists of a membrana propria, surrounded externally with a net-work of branched connective-tissue corpuscles, and lined internally with a somewhat flattened polyhedral layer of nucleated secretory cells (Fig. 171.) The size of the lumen of the acini depends upon the secretory activity of the glands; when it is large it is filled with milk containing numerous refractive fatty granules. The milk-ducts consist of fibrillar connective-tissue. Some fibres are arranged longitudinally, but the chief mass are disposed circularly, and are permeated externally with elastic fibres, while in the finer ducts, there is a membrana propria continuous with that of the gland acini. The ducts are lined by cylindrical epithelium. 
During the first few days after delivery, the breasts secrete a small amount of milk of greater consistence, and of a yellow colour-the colostrum-in which

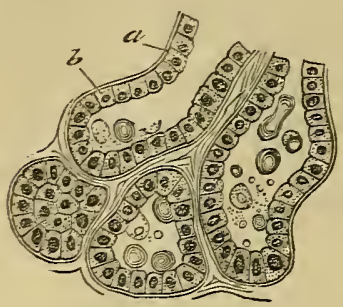

Fig. 171.

Acini of the mammary gland of a sheep during lactation$a$, membrana propria; $b$, secretory epithelium. large cells filled with fatty granules occurthe colostrum-corpuscles. Sometimes a nucleus is observable within them, and rarely they exhibit amœboid movements (Fig. 172, $c, d, e$ ). The regular secretion of milk begins after 3-4 days. It was formerly supposed that the cells of the acini underwent a fatty degeneration, and thus produced the fatty granules of the milk. It is more probable, from the observations of Stricker, Schwarz, Partsch and Heidenhain, that the cells of the acini manufacture the fatty granules, and their protoplasm eliminates them, at the same time forming the clear fluid part of the milk.

Changes during Secretion.-Partsch and Heidenhain found that the secretory cells in the passive non-secreting gland (Fig. 172, I) were flat, polyhedral, and uni-nucleated, whilst the secreting cells (Fig. II) often con-

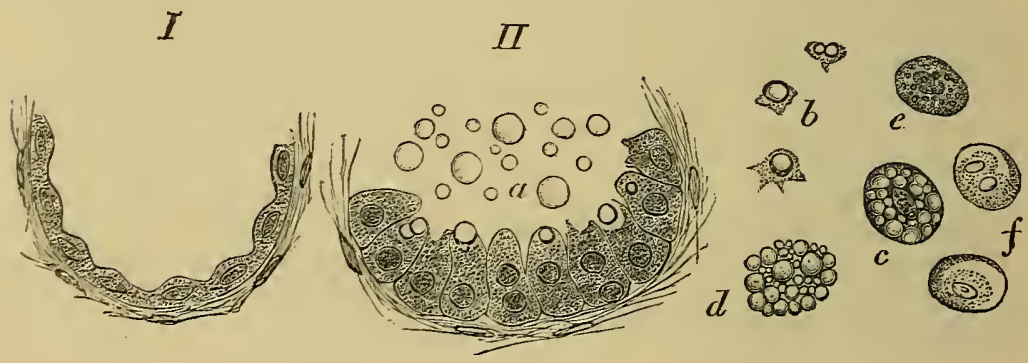

Fig. 172.

I-Inactive acinus of the mamma. II-During the secretion of milk; $a, b$, milkglobules ; $c, d, e$, colostrum-corpuscles ; $f$, pale cells (bitch).

tained several nuclei, were more albuminous, higher, and cylindrical in form. The edge of the cell directed towards the lumen of the acinus undergoes characteristic changes during secretion. Fatty granules are formed in this part of the cell, and are afterwards extruded. The decomposed portion of the cell is dissolved in the milk, and the fatty granules become free as milk-globules. (Fig. II, a). If nuclei are present in that part of the cell which is broken up, they also pass into the milk and give rise to the presence of nuclein in the secretion.

Besides the milk-globules and colostrum-corpuscles, Rauber has found leucocytes undergoing fatty degeneration and single pale cells $(f)$. Occasionally milk-globules are found with traces of the cell-substance adhering to their surface (b).

Formation of Milk.-Concerning the formation of the individual constituents 
of milk, H. Thierfelder, who digested fresh mammary glands directly after death, found that during the digestion of the glands at the temperature of the body, a reducing substance, probably lactose, was formed by a process of fermentation. The mother substance (saccharogen) is soluble in water, but not in alcohol or ether, is not destroyed by boiling, and is not identical with glycogen. The ferment which forms the lactose is connected with the glaud-cells-it does not pass into the milk, nor into a watery extract of the gland. During the digestion of the mammary glands, at the temperature of the body, casein is formed, probably from serumalbumin, by a process of fermentation. This ferment occurs in the milk.

The nipple and its areola are characterised by the presence of pigment-more abundant during preguancy -in the rete Malpighii of the skin, and by large papillæ in the cutis vera. Some of the papillæ contain touch-corpuscles. Numerous nonstriped muscular fibres surround the milk-ducts in the deep layers of the skin and in the subcutaneous tissue, which contains no fat. These muscular fibres can be traced following a longitudinal course to the termination of the ducts on the surface. The small glands of Mfontgomery, which occur on the areola during lactation, are small milk-glands, each with a special duct opening on the surface of the elevation.

Arteries proceed from several sources to supply the mamma, but their branches do not accompany the milk-ducts; the gland acini are each surrounded by a network of capillaries, which communicate with those of adjoining acini by small arteries and veins. The veins of the areola are arranged in a circle (circulus Halleri). The nerves are derived from the supraclavicular, and the II-IV-VI intercostals; they proceed to the skin over the gland, to the very sensitive nipple, to the blood-vessels and non-striped muscle of the nipple, and to the gland acini, where their mode of termination is still unknown. Lymphatics surround the alveoli, and they are often full. The milk appears to be prepared from the lymph contained in the lymphatics surrounding the acini.

The comparative anatomy of the mamma.-The rodents, insectivora, and carnivora, have 10 to 12 teats, while some of them have only 4. The pachydermata and ruminantia have $2-4$ abdominal teats, the whale has 2 near the vulva. The apes, bats, vegetable-feeding whales, elephants, and sloths have 2, like man. In the marsupials the tubes are arranged in groups, which open on a patch of skin devoid of hair without any nipple. The young animals remain within the mother's pouch, and the milk is expelled into their mouths by the action of a muscle-the compressor mammæ.

The development of the human mamma begins in both sexes during the third month; at the fourth and fifth months, a few simple tubular gland-ducts are arranged radially around the position of the future nipple, which is devoid of hair. In the new-born child the ducts are branched twice or thrice, and are provided with dilated extremities, the future acini. Up to the 12th year in both sexes, the ducts continue to divide dendritically, but without any proper acini being formed.

In the girl at puberty the ducts branch rapidly; but the acini are formed only at the periphery of the gland, while during pregnancy, acini are also formed in the centre of the glaud, while the connective-tissue at the same time becomes somewhat more opened out. At the climacteric period, or menopause, all the acini and numerous fine milk-ducts degenerate. In the adult male, the gland remains in the non-developed infantile condition. Accessory or supernumerary glands upon the breast and abdomen are not uncommon, sometimes the mamma occurs in the axilla, on the back, over the acromion process, or on the leg. A slight secre. tion of milk in a newly-born infant is normal.

During the evacuation of the milk (500-1500 cubic centimetres daily), there is not only the mechanical action of sucking, but also the activity of the gland itself. This consists in the erection of the nipple, whereby its non-striped muscular fibres compress the siuuses on the milk-ducts, and empty them, so that the milk may flow out in streams. The gland acini are also excited to secretion reflexly by the stim- 
ulation of the sensory nerves of the nipple. The vessels of the gland are dilated, and there is a copious transudation into the gland, the transuded fluid being manufactured into milk under the influence of the secretory protoplasm. The amount of secretion depends upon the blood-pressure (Röhrig). During sucking, not only is the milk in the gland extracted, but new milk is formed, owing to the accelerated secretion. Emotional disturbances-anger, fear, \&c.-arrest the secretion. Laffont found that stimulation of the mammary nerve (bitch) caused erection of the teat, dilatation of the vessels, and secretion of milk. After section of the cerebro-spinal nerves going to the mamma, Eckhard observed that erection of the teat ceased, although the secretion of milk in a goat was not interrupted. The rarely observed galactorrhœa is perhaps to be regarded as a paralytic secretion analogous to the paralytic secretion of saliva. Heidenhain and Partsch found that the secretion (bitch) was increased by injecting strychnine or curara after section of the nerves of the gland. The "milk-fever," which accompanies the first secretion of milk, probably depends on stimulation of the vaso-motor nerves, but this condition must be studied in relation with the other changes which occur within the pelvic cavity after birth. [Some substances, such as atropin, arrest the secretion of milk.]

\section{Milk and its Preparations.}

Milk represents a complete or typical food in which all the constituents necessary for maintaining the life and growth of the body are present. To every 10 parts of proteids there are 10 parts fat and 20 parts sugar. Relatively more fat than albumin is absorbed from the milk (Rubner); while a part of both is excreted in the fæces,

Characters.-Milk is an opaque, bluish-white fluid with a sweetish taste and a characteristic odour, probably due to the peculiar volatile substances derived from the cutaneous secretions of the glands, and it has a specific gravity of 1026-1035 (Radenhausen). When it stands for a time, numerous milk-globules, butter-globules, or cream, collect on its surface, under which there is a watery bluish fluid. Human milk is always alkaline, cow's milk may be alkaline, acid or amphoteric; while the milk of carnivora is always acid.

Milk-Globules.-When milk is examined microscopically, it is seen to contain numerous small highly refractive oil-globules, floating in a clear fluid-the mill-plasma (Fig. 172,a, $b$,); while colostrum-corpuscles and epithelium from the milk-ducts are not so numerous. The white colour and opacity of the milk are due to the presence of the milkglobules which reflect the light; the globules consist of a fat, or butter, surrounded with a very thin envelope of casein. If acetic acid be added to a microscopic preparation of milk, this caseous envelope is dissolved, the fatty granules are liberated, and they run together to form irregular masses. If cow's milk be shaken with caustic potash, the casein envelopes are dissolved, and if ether be added, the milk becomes clear and transparent, as the ether dissolves 
out all the fatty particles in the solution. Ether cannot extract the fat from cow's milk until acetic acid or caustic potash is added to liberate the fats from their envelopes; but shaking with ether is sufficient to extract the fats from human milk (Radenhausen). Some observers deny that an envelope of casein exists, and according to them milk is a simple emulsion, kept emulsionised owing to the colloid swollen up casein in the milk-plasma. The treatment of milk with potash and ether makes the casein unable any longer to preserve the emulsion (Soxhlet).

The fats of the milk-globules are the triglycerides of stearic, palmitic, myristic, oleic, arachinic (butinic), capric, caprylic, caproic, and butyric acids, with traces of acetic and formic acids (Heintz), and cholesterin (Schmidt-Mülheim). When milk is beaten or stirred for a long time (i.e., churned), the fat of the milk-globules is ultimately obtained in the form of butter, owing to the rupture of the envelopes of casein.

Butter is soluble in alcohol and ether, and it is clarified by heat $\left(60^{\circ} \mathrm{C}\right.$.), or by washing in water at $40^{\circ} \mathrm{C}$. When allowed to stand exposed to the air it becomes rancid, owing to the glycerine of the neutral fats being decomposed by fungi into acrolein and formic acid, while the volatile fatty acids give it its rancid odour.

The milk-plasma, obtained by filtration through clay filters or membranes, is a clear, slightly opalescent fluid, and contains casein ( $\$ 249$, III, 3), serum-albumin (p. 49), and to a less extent a body resembling albumin (lactoproteïn-Millon, Liebermann); galactin, albuminose, and globulin; peptone $(0.13$ per cent.); nuclein, diastatic ferment (in human milk-Béchamp). Milk-sugar ( 252$)$, a carbohydrate resembling dextrin (Ritthausen), (? lactic acid), lecithin, urea, extractives;sodic and potassic chlorides, alkaline phosphates, calcium and magnesium sulphates, alkaline carbonates, traces of iron, fluorine, and silica; $\mathrm{CO}_{2} \mathrm{~N}, \mathrm{O}$.

When milk is boiled the albumin coagulates, while the surface also becomes covered with a thin scum or layer of casein, which has become insoluble.

When milk is filtered through fresh animal membranes (Hoppe-Seyler), or through a clay filter, the casein does not pass through (Helmholtz, Zahn, Kehrer), while burned pulverised clay and animal charcoal also attract the casein (Dupre and Hermann).

The coagulation of milk depends upon the coagulation of its casein. In milk, casein is combined with calcium phosphate, which keeps it in solution; acids which act on the calcium phosphate cause coagulation of the casein (acetic and tartaric acids in excess redissolve it). All acids do not coagulate human milk (Biedert). It is coagulated by two or more drops of hydrochlcric acid $(0.1$ per cent.) or acetic acid $(0.2$ per cent.). The spontaneous coagulation of milk after it has stood for a time, especially in a warm place, is due to the formation of lactic acid, which is 
formed from the milk-sugar in the milk by the action of bacterium lacticum [which is introduced from without (Pasteur, Cohn, Lister)]. It changes the neutral alkaline phosphate into the acid phosphate, takes the casein from the calcium phosphate, and precipitates the casein (p. 373). The ferment may be isolated by means of alcohol.

Rennet, which contains a special ferment, coagulates milk with an alkaline reaction (sweet whey). This ferment decomposes the casein into the precipitated cheese, and also into the slightly soluble whey-albumin (Hammarsten, Köster), so that the coagulation by rennet is a process quite distinct from the coagulation of milk by the gastric and pancreatic juices. When the milk is coagulated we obtain the curd, consisting of casein with some milk-globules entangled in it; the whey contains some soluble albumin and fat, and the great proportion of the salts and milk-sugar, together with lactic acid.

Boiling (by killing all the lower organisms), sodium bicarbonate ( $106 \mathrm{v})$, ammonia, salicylic acid $\left(\frac{1}{5000}\right)$, glycerine, and ethereal oil of mustard prevent the spontaneous coagulation. Fresh milk makes tincture of guaiacum blue, but boiled milk does not do so (Schacht, C. Arnold). When milk is exposed to the air for a long time, it gives off $\mathrm{CO}_{2}$, and absorbs $\mathrm{O}$; the fats are increased (? owing to the development of fungi in the milk), and so are the alcoholic and ethereal extracts, from the decomposition of the casein (Hoppe-Seyler, Kemmerich). According to Schmidt-Mülheim, some of the casein becomes converted into peptone, but this occurs only in unboiled milk.

Composition, -100 parts of milk contain-

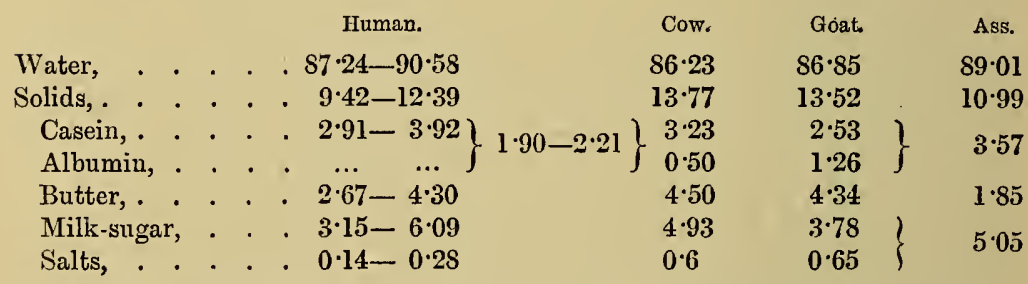

Human milk contains less albumin, which is more soluble than the albumin in the milk of animals.

Colostrum contains much serum-albumin, and very little casein, while all the other substances, and especially the fats, are more abundant.

Gases.-Pfliuger and Setschenow found in 100 vols. of milk $5 \cdot 01-7 \cdot 60 \mathrm{CO}_{2}$; $0.09-0.32 \mathrm{O} ; 0.70-1.41 \mathrm{~N}$, according to volume. Only part of the $\mathrm{CO}_{2}$ is expelled by phosphoric acid.

Salts.-The potash salts (as in blood and muscle) are more abundant than the soda compounds, while there is a considerable amount of calcium phosphate, which is necessary for forming the bones of the infant. Wildenstein found in 100 parts of the ash of human milk-sodium chloride, $10 \cdot 73$; potassium chloride, 26.33 ; potash, 21.44 ; lime, $18 \cdot 78$; magnesia, 0.87 ; phosphoric acid, 19; ferric phosphate, 0.21 ; sulphuric acid, 2.64 ; silica traces. The amount of salts present is affected by the salts of the food.

Conditions Influencing the Composition.-The more frequently the breasts are emptied, the richer the milk becomes in casein. The last milk obtained at any time is always richer in butter, as it comes from the most distant part of the gland-viz., the acini (Reiset, Heynsius, Forster, de Leon). Some substances are 
diminished and others increased in amount, according to the time after delivery. The following are increased:-Until the 2nd month after delivery, casein and fat; until the 5th month, the salts (which diminish progressively from this time onwards); from S-10th months, the sugar. The following aro diminished:-From 10-24th months, casein; from 5-6th and 10-11th months, fat; during lst month, the sugar; from the 5th month, the salts.

[That cow's milk is influenced by the pasture and food is well-known. 'Turnip as food give a peculiar odour, taste, and flavour to milk, and so do the fragrant grasses. The mental state of the nurse influences the quantity and quality of the milk, while many substances given as medicines reappear in the milk, such as dill, copaiba, conium, aniseed, garlic, potassium iodide, arsenic, mercury, opium, rhubarb, or its active principle, and the cathartic principle of senna. Jaborandi is the nearest approach to a galactagogue, but its action is temporary. Atropin is a true anti-galactagogue. The composition of the milk may be affected by using fatty food, by the use of salts, and above all by the diet (Dolan).]

[Milk may be a vehicle for communicating disease-by direct contamination from the water used for adulteration or cleansing; by the milk absorbing deleterous gases; by the secretion being altered in diseased animals.]

The greater the amount of milk that is secreted (woman), the more casein and sugar, and the less butter it contains. The milk of a primipara is less watery. Rich feeding, especially proteids (small amount of vegetable food), increase the amount of milk and the casein, sugar, and fat in it; a large amount of carbohydrates (not fats) increases the amount of sugar.

If other than human inilk has to be used, ass's milk most closely resembles human milk. Cow's milk is best when it contains plenty fatty matters-it must be diluted with its own volume of water at first, and a little milk-sugar added. The casein of cow's milk differs qualitatively from that of human milk (Biedert); its coagulated flocculi or curd are much coarser than the fine curd of human milk, and they are only $\frac{3}{4}$ dissolved by the digestive juices, while human milk is completely dissolved. Cow's milk when boiled is less digestible than unboiled (E. Jessen).

Milk ought not to be kept in zinc vessels owing to the formation of zinc lactate.

Tests for Milk.-The amount of cream is estimated by placing the milk for 24 hours in a tall cylindrical glass graduated into a humdred parts; the cream collects on the surface, and ought to form from 10-24 vols. per cent. The specific gravity (fresh cow's milk, 1,029-1,034; when creamed, 1,032-1,040) is estimated with an araeometer or lactometer at $15^{\circ} \mathrm{C}$. The sugar is estimated by titration with Fehling's solution (p. 298), but in this case 1 cubic centimetre of this solution corresponds to $0.0067 \mathrm{grm}$. of milk-sugar; or its amount may be estimated with the polariscopic apparatus (vol. ii). The proteids are precipitated and the fats extracted with ether. The fats in fresh milk form about 3 per cent., and in skimmed milk 11 per cent. The amount of water in relation to the milkglobules is estimated by the lactoscope (the diaphänometer of Donné, modified by Vogel and Hoppe-Seyler), which consists of a glass-vessel with plane parallel sides placed 1 centimetre apart. A measured quantity of milk is taken, and water is added to it from a burette until the outline of a candle flame placed at a distance of 1 metre can be distinctly seen through the diluted milk. This is done in a dark room. For 1 cubic centimetre of good cow's milk, 70-\$5 centimetres water are required.

$V$ arious substances pass into the milk when they are administered to the mothermany odoriferous vegetable bodies, e.g., anise, vermuth garlic, \&c.; opium, indigo, salicylic acid, iodine, iron, zinc, mercury, lead, bismuth, antimony. In osteomalacia the amount of lime in the milk is increased (Gusserow). Potassium iodide 
dirninishes the secretion of milk by affecting the secretory function (Stumpf). Amongst abnormal constituents are-hæmoglobin, bile-pigments, mucin, blood-corpuscles, pus, fibrin. Numerous fungi and other low organisms develop in evacuated milk, and the rare blue milk is due to the development of Bacterium cyanogenëum (Fuchs, Neelsen). The blue colour is due to aniline blue derived from casein (Erdmann). The milk-serum is blue, not the fungus. Blue milk is unhealthy, and causes diarrhoea (Mosler). Red and yellow milk are produced by a similar action of chromogenic fungi (p. 373). The former is produced by Micrococcus prodigiosus, which is colourless. The colour seems to be due to fuchsin. The yellow colour is produced by Bacterium synxanthum (Ehrenberg), and the colour is also due to an aniline substance (Schröter).

Preparations of Milk.-(1.) Condensed milk-80 grms. cane-sugar are added to 1 litre of milk; the whole is evaporated to $\frac{1}{5}$; and while hot sealed up in tin cans (Lignac). For children one teaspoonful is dissolved in a pint of cold water, and then boiled.

(2.) Koumiss is prepared by the Tartars from mare's milk. Koumiss and sour milk are added to milk, the whole is violently stirred, and it undergoes the alcoholic fermentation, whereby the milk-sugar is first changed into galactose, and then into alcohol; so that koumiss contains 2-3 per cent. of alcohol; while the casein is at first precipitated, but is afterwards partly redissolved and changed into acid-albumin and peptone (Dochmann).

(3.) Cheese is prepared by coagulating milk with rennet, allowing the whey to separate, and adding salt to the curd. When kept for a long time cheese "ripens," the casein again becomes soluble in water, probably from the formation of soda albuminate; in many cases it becomes semi-fluid when it takes the characters of peptones. When further decomposition occurs, leucin and tyrosin are formed. The fats increase at the expense of the casein, and they again undergo further change, the volatile fatty acids giving the characteristic odour. The formation of peptone, leucin, tyrosin, and the decomposition of fat recalls the digestive processes.

\section{Eggs.}

Eggs must also be regarded as a complete food, as the organism of the young chick is developed from them. The yelk contains a characteristic proteid body, Vitellin ( $(249)$, and an albuminate in the envelopes of the yellow yelk spheres-Nuclein, from the white yelk; fats in the yellow yelk (palmitin, olein), cholesterin, much lecithin; and as its decomposition product, glycerin-phosphoric acid-grapesugar, pigments (luteïn), and a body containing iron and related to hæmoglobin; lastly, salts qualitatively the same as in blood-quantitatively as in the blood-corpuscles-gases.

The chief constituent of the white of egg is egg-albumin ( $\$ 249)$, together with a small amount of palmitin and olein partly saponified with soda; grape-sugar, extractives; lastly salts, qualitatively resembling those of blood, but quantitatively like those of serum, and a trace of fluorine.

Relatively more of the nitrogenous constituents than the fatty constituents of eggs are absorbed (Rubner). 


\section{Flesh and its Preparations.}

Flesh, in the form in which it is eaten, contains in addition to the muscle-substance proper, more or less of the elements of fat, connective- and elastic-tissue mixed with it. The following results refer to flesh freed as much as possible from these constituents. The chief proteid constituent of the contractile muscular substance is myosin (Kühne); serum-albumin occurs in the fluid of the fibres, in the lymph and blood of muscle. The fats are for the most part derived from the interfibrillar fat cells, and so are lecithin and cholesterin from the nerves of the muscles; the gelatin is derived from the connective-tissue of the perimysium, perineurium, and the walls of blood-vessels and tendons. The red colour of the flesh is due to the hæmoglobin present in the sarcous substance (Kühne, Gscheidlen). Elastin occurs in the sarcolemma, neurilemma, and in the elastic fibres of the perimysium and walls of the vessels; the small amount of keratin is derived from the endothelium of the vessels. The chief muscular substance, the result of the retrogressive metabolism of the sarcous substance, is kreatin (Chevreul-0.25 per cent., Perls) ; kreatinin, the inconstant inosinic acid, then lactic, or rather sarcolactic acid (see Muscle). Farther, taurin, sarkin, xanthin, uric acid, carnin, inosit (most abundant in the muscles of drunkards), dextrin (in horse and rabbit, not constant-Sanson, Limpricht); grape-sugar (Meissner), but it is very probably derived post mortem from glycogen $(0.43$ per cent.), which occurs in considerable amount in fotal muscles $(O$. Nasse); lastly, fatty acids. Amongst the salts, potash and phosphoric acid compounds (Braconnot) are most abundant; magnesium phosphate exceeds calcium phosphate in amount.

In 100 parts FLESH there is, according to Schlossberger and v. Bibra-

\begin{tabular}{|c|c|c|c|c|c|c|c|c|}
\hline & Ox. & Calf. & Deer. & Pig. & Man. & Fowl. & Carp. & Frog. \\
\hline Water, & $77 \cdot 50$ & $78 \cdot 20$ & $74 \cdot 63$ & $78 \cdot 30$ & $74 \cdot 45$ & $77 \cdot 30$ & $79 \cdot 78$ & $80 \cdot 43$ \\
\hline Solids, & $22 \cdot 50$ & $21 \cdot 50$ & $25 \cdot 37$ & $21 \cdot 70$ & $25 \cdot 55$ & $22 \cdot 7$ & $20 \cdot 22$ & $19 \cdot 57$ \\
\hline Soluble Albumin, & $2 \cdot 20$ & $2 \cdot 60$ & $1 \cdot 94$ & $2 \cdot 40$ & 1.93 & $3.0\}$ & $2 \cdot 35$ & $1 \cdot 86$ \\
\hline Colouring Matter, & & & & & & & $\cdots$ & $\cdots$ \\
\hline Glutin, . & $1 \cdot 30$ & $1 \cdot 60$ & 0.50 & 0.80 & $2 \cdot 07$ & $1 \cdot 2$ & 1.98 & $2 \cdot 4 S$ \\
\hline Alcoholic Extract, . & $1 \cdot 50$ & $1 \cdot 40$ & $4 \cdot 75$ & $1 \cdot 70$ & $3 \cdot 71$ & $1 \cdot 4$ & $3 \cdot 47$ & $3 \cdot 46$ \\
\hline $\begin{array}{l}\text { Fats, } \\
\text { Insoluble Albumin, }\end{array}$ & $\ldots$ & ... & $1 \cdot 30$ & .. & $2 \cdot 30$ & $\ldots$ & $1 \cdot 11$ & $0 \cdot 10$ \\
\hline Blood-vessels, \&c., & $17 \cdot 50$ & $16 \cdot 2$ & $16 \cdot 81$ & $16 \cdot 81$ & $15 \cdot 54$ & $16 \cdot 5$ & $111 \cdot 31$ & $11 \cdot 67$ \\
\hline
\end{tabular}


Iit 100 parts ASH there is-

\begin{tabular}{|c|c|c|c|c|c|c|c|c|}
\hline & & & & & Horse. & Ox. & Calf. & Pig. \\
\hline Potash, & - & . & . & • & $39^{\circ} 40$ & $35 ' 94$ & $34: 40$ & $37 \cdot 79$ \\
\hline Soda, & . & $\cdot$ & - & . & $4 \cdot 86$ & ... & $2 \cdot 35$ & $4 \cdot 02$ \\
\hline Magnesia, . & . & • & - & - & $3 \cdot 88$ & $3 \cdot 31$ & $1 \cdot 45$ & $4 \cdot 81$ \\
\hline Chalk, & . & - & - & - & $1 \cdot 80$ & $1 \cdot 73$ & 1.99 & $7 \cdot 54$ \\
\hline Potassium, & - & 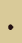 & . & . & $\ldots$ & $5 \cdot 36$ & $\cdots$ & $\ldots$ \\
\hline Sodium, . & - & - & - & • & $1 \cdot 47\}$ & ... & $\{10.50]$ & 0.40 \\
\hline Chlorine, . & - & $\cdot$ & $\cdot$ & & & $4 \cdot 86$ & $\{10.59\}$ & 0.62 \\
\hline Iron oxide, & . & • & • & . & $1 \cdot 0$ & 0.98 & $0 \cdot 27$ & 0.35 \\
\hline Phosphoric & Acid, & . & $\cdot$ & $\cdot$ & $46^{\prime} 74$ & $34: 36$ & $48 \cdot 13$ & $44: 47$ \\
\hline Sulphuric &, & . & . & - & $0 \cdot 30$ & $3 \cdot 37$ & $\ldots$ & $\ldots$ \\
\hline Silicic & , & • & . & - & ... & $2 \cdot 07$ & 0.81 & ... \\
\hline Carbonic & $"$ & . & . & • & $\ldots$ & $8 \cdot 02$ & ... & ... \\
\hline Ammonia, & - & . & - & . & $\cdots$ & $0 \cdot 15$ & $\cdots$ & ... \\
\hline
\end{tabular}

The amount of fat in flesh varies very much according to the condition of the animal. After removal of the visible fat, human flesh contains $7 \cdot 15$, ox $11 \cdot 12$, calf 10.4 , sheep 3.9 , wild goose 8.8 , fowl 2.5 per cent.

The amount of extractives is most abundant in those animals which exhibit energetic muscular action; hence it is largest in wild animals. The extract is increased after vigorous muscular action, when sarcolactic acid is developed, and the flesh becomes more tender and is more palatable. Some of the extractives excite the nervous system, e.g., kreatin and kreatinin; and others give to flesh its characteristic agreeable taste ("osmasome "), but this is also partly due to the different fats of the flesh, and is best developed when the flesh is cooked. The extractives in 100 parts of flesh-in man and pigeon, 3 ; deer and duck, 4 ; swallow, 7 per cent.

Preparation, or Cooking of Flesh.-As a general rule, the flesh of young animals, owing to the sarcolemma, connective-tissue, and elastic constituents being less tough, is more tender and more easily digested than the flesh of old animals; after flesh has been kept for a time it is more friable and tender, as the inosit becomes changed into sarcolactic acid and the glycogen into sugar, and this again into lactic acid, whereby the elements of the flesh undergo a kind of maceration. Finely-divided flesh is more digestible than when it is eaten in large pieces. In cooking meat, the heat ought not to be too intense, and ought not to be continued too long, as the muscular fibres thereby become hard and shrink very much. Those parts are most digestible which are obtained from the centre of a roast where they have been heated to $60-70^{\circ} \mathrm{C}$., as this temperature is sufficient, with the aid of the acids of the flesh, to change the connective-tissue into gelatin, whereby the fibres are loosened, so that the gastric juice readily attacks them. In roasting beef, apply heat suddenly at first, to coagulate a layer on the surface, which prevents the exit of the juice.

Meat Soup is best prepared by cutting the flesh into pieces and placing them for several hours in cold water, and afterwards boiling. Liebig found that 6 parts per 100 of ox flesh were dissolved by cold water. When this cold extract was boiled, 2.95 parts were precipitated as coagulated albumin, which is chiefly removed by "skimming," so that only 3.05 parts remain in solution. From 100 parts of flesh of fowl, 8 parts were extracted, and of these $4 \cdot 7$ coagulated and 3.3 
remained dissolved in the soup. By boiling for a very long time, part of the albumin may be redissolved (Mulder). The dissolved substances are:1. Inorganic salts of the meat, of which $82 \cdot 27$ per cent. pass into the soup; the earthy phosphates chiefly remain in the cooked meat. 2. Kreatin, kreatinin, the inosinates and lactates which give to broth or beef-tea their stimulating qualities, and a small amount of aromatic extractives. 3. Gelatin, more abundantly extracted from the flesh of young aninals. According to these facts, therefore, flesh-broth or beef-tea is a powerful stimulant, supplying muscle with restoratives, but is not a food in the ordinary sense of the term. The flesh after the extraction of the broth is still available as a food.

Liebig's Extract of Meat is an extract of flesh evaporated to a thick syrupy consistence. It contains no fat or gelatin, and is chiefly a solution of the extractives and salts of flesh.

[Mastermann has shown that the chemical analysis of beef-tea is analogous to that of urine, except that it contains less urea and uric acid.]

\section{Vegetable Foods.}

The nitrogenous constituents of plants are not so easily absorbed as animal food (Rubner). Carbohydrates, starch, and sugar are very completely absorbed, and even a not inconsiderable proportion of cellulose nay be digested (Weiske, König). The more fats that are contained in the vegetable food, the less are the carbohydrates digested and absorbed (Rubner).

1. The cereals are most important vegetable foods; they contain proteids, starch, salts, and water to 14 per cent. The nitrogenous glutin is most abundant under the husk

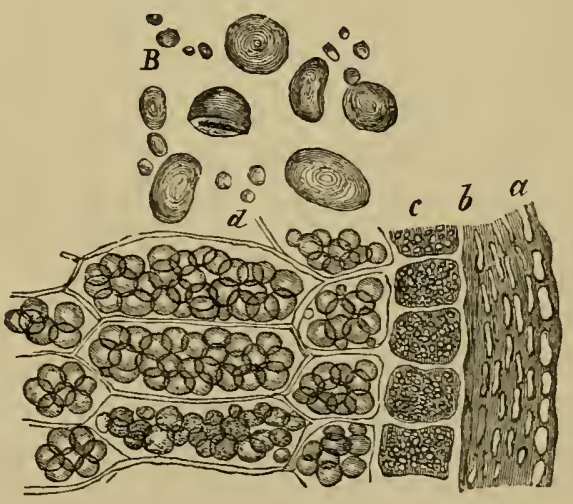

Fig. 173.

Microscopic characters of wheat $-\times 200 ; a$, cells of the bran; $b$, cells of thin cuticle; $c$, glutin cells; $d$, starch cells; $B$, wheat starch $\times 350$.

(Payen). The use of whole

meal containing the outer layers of the grain is highly nutritive, but bread containing much bran is somewhat indigestible (Rubner). [A section of a wheat grain with its layers of glutin is shown in Fig. 173.] Their composition is the following:- 


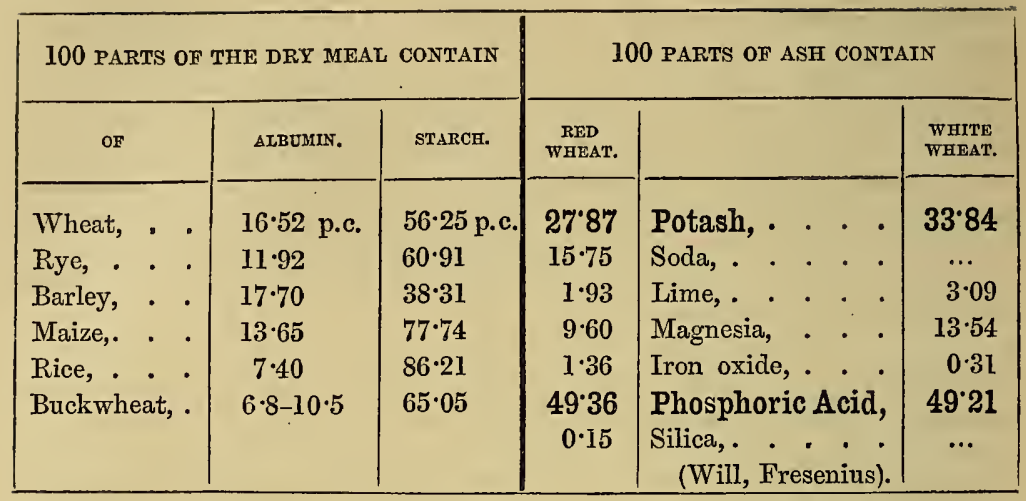

It is curious to observe that soda is absent from white wheat, its place being taken by other alkalies. Rye contains more cellulose and dextrin than wheat, but less sugar; rye bread is usually less porous.

In the preparation of bread, the meal is kneaded with water until dough is formed, and to it is added salt and yeast (saccharomycetes cerevisiæ). When placed in a warm oven, the proteids of the meal begin to decompose and act as a ferment upon the swollen up starch, which becomes in part changed into sugar. The sugar is farther decomposed into $\mathrm{CO}_{2}$ and alcohol, the $\mathrm{CO}_{2}$ forms bubbles, which make the bread spongy and porous. The alcolnol is driven off by the baking $\left(200^{\circ}\right)$, while much soluble dextrin is formed in the crust of the bread.

2. The Pulses contain much albumin, especially vegetable casein or legumin; together with starch, lecithin, cholesterin, and 9-19 per cent. water. Peas contain 28.02 proteids, and 38.81 starch: beans 28.54 , and 37.50 : lentils, $29 \cdot 31$, and 40 , and more cellulose. Owing to the absence of glutin they do not form dough, and bread cannot be prepared from them. On account of the large amount of proteids which they contain they are admirably adapted as food for the poorer classes.

3. Potatoes contain 70-81 per cent. water. In the fresh juicy cellular tissue, which has an acid reaction, from the presence of phosphoric, malic, and hydrochloric acids, there is 16-23 per cent. of starch, 2.5 soluble albumin, globulin (Zöller), and a trace of asparagin. The envelopes of the cells swell up by boiling, and are changed into sugar and gums by dilute acids. The poisonous solanin occurs in the sprouts. In 100 parts of potato ash, May found 46.96 potash, $2 \cdot 41$ sodium chloride, $8 \cdot 11$ potassium chloride, 6.50 sulphuric acid derived from burned proteids, $7 \cdot 17$ silica.

4. In Fruits the chief nutrient ingredients are sugar and salts; the organic acids give them their characteristic taste, the gelatinising substance is the soluble so-called pectin $\left(\mathrm{C}_{32} \mathrm{H}_{48} \mathrm{O}_{32}\right)$, which can be prepared artificially by boiling the very insoluble pectose of unripe fruits and mulberries. 
5. The Green Vegetables are specially rich in salts, which resemble the salts of the blood; thus, dry salad contains 23 per cent. of salts, which closely resemble the salts of the blood. Of much less importance are the starch, cell-substance, dextrin, sugar and the small amount of albumin which they contain.

\section{Condiments-Coffee-Tea-Chocolate- Alcoholic Drinks.}

Some substances are used along with food, not so much on account of their nutritive properties as on account of their stimulating effects and agreeable qualities, which are exerted partly upon the organ of taste, and partly upon the nervous system. These are called condiments.

Coffee, tea, and chocolate are prepared as infusions of these substances. Their chief active ingredients are respectively caffein, thein $\left(\mathrm{C}_{\mathrm{S}} \mathrm{H}_{10} \mathrm{~N}_{4} \mathrm{O}_{2}+\mathrm{H}_{2} \mathrm{O}\right)$, and theobromin $\left(\mathrm{C}_{7} \mathrm{H}_{8} \mathrm{~N}_{4} \mathrm{O}_{2}\right)$, which are regarded as alkaloids of the vegetable bases, and which have recently been prepared artificially from xanthin (E. Fischer).

These "alkaloids" occur as such in the plants containing them; they behave like ammonia; they have an alkaline reaction, and form crystalline salts with acids. All these vegetable bases act upon the nervous system; some more feebly (as the above), others more powerfully (quinine); some stimulate powerfully, or completely paralyse (morphia, atropin, strychnin, curarin, nicotin, muscarin).

All these substances act on the nerrous system; they quicken thought, accelerate movement, and stir one to greater activity. In these respects they resemble the stimulating extractives--kreatin and kreatinin-of beef-tea. Coffee contains about $\frac{1}{3}$ per cent of caffein, part of which is only liberated by the act of roasting. Tea has 6 per cent. of thein, whilst green tea contains 1 per cent. ethereal oil, and black tea $\frac{1}{2}$ per cent.; in green tea there is 18 per cent., in black 15 per cent. tannin; green tea yields about 46 per cent., and the black scarcely 30 per cent. of extract.

The inorganic salts present are also of importance; tea contains 3.03 per cent. of salts, and amongst these are soluble compounds of iron, manganese, and soda salts. In coffee, which yields 3.41 per cent. of ash, potash salts are most abundant; in all three substances the other salts which occur in the blood are also present.

Alcoholic Drinks owe their action chiefly to the alcohol which they contain. The alcohol, when taken into the body, undergoes certain changes and produces certain effects:- -1 . It is oxidised chiefly into $\mathrm{CO}_{2}$ and $\mathrm{H}_{2} \mathrm{O}$, so that it is so far a source of heat. As it undergoes this change very readily, when taken to a certain extent it may act as a substitute for the consumption of the tissues of the body, especially when the amount of food is insufficient. Small doses diminish the 
decomposition of the proteids to the extent of 6-7 per cent. Only a very small part of the alcohol is excreted in the urine; the odour of the breath is not due to alcohol, but to other volatile substances mixed with it, e.g., fusel oil, \&c. 2. In small doses it excites, while in large doses it paralyses, the nervous system. By its stimulating qualities it excites to greater action, which, however, is followed by depression. 3. It diminishes the sensation of hunger. 4. It excites the vascular system, accelerates the circulation, so that the muscles and nerves are more active owing to the greater supply of blood. It also gives rise to a subjective feeling of warmth. In large doses, however, it paralyses the vessels, so that they dilate, and thus much heat is given off $(\S 213,7, \S 227)$. The action of the heart also becomes affected, the pulse becomes smaller, feebler, and more rapid. In high altitudes, the action of alcohol is greatly diminished, owing to the diminished atmospheric pressure whereby it is rapidly given off from the blood.

Alcohol in small doses is of great use in conditions of temporary want, and where the food taken is insufficient in quantity. When alcohol is taken regularly, more especially in large doses, it affects the nervous system, and undermines the psychical and corporeal faculties, partly from the action of the impurities which it may contain, such as fusel oil, which has a poisonous effect upon the nervous system, partly by the direct effects, such as catarrh and inflammation of the digestive organs, which it produces, and lastly, by its effect upon the normal metabolism.

Preparation.-Alcoholic drinks are prepared by the fermentation of various carbohydrates, such as sugar derived from starch. The alcoholic fermentation, such as occurs in the manufacture of beer, is caused by the development of the yeast plant, Saccharomycetes cerevisiæ; while in the fermentation of the grape (wine), S. ellipsoideus is the species present. The yeast takes the substances necessary for the maintenance of its organic processes directly from the mixture of the sugar-viz., carbohydrates, proteids, and salts, especially calcium and potassium phosphates and magnesium sulphate. These substances undergo decomposition

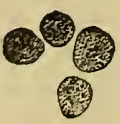

1.

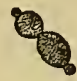

2.

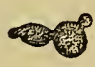

3.

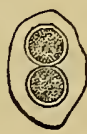

4.

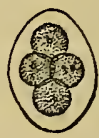

5.

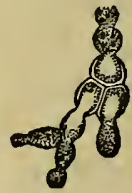

6.

Fig. 174.

1, Isolated yeast cells; 2, 3, yeast cells budding; 4, 5, so-called endogenous formation of cells; 6 , sprouting and formation of buds.

within the cells of the yeast plant, which multiply during the process, and there are produced alcohol and $\mathrm{CO}_{2}$ (p. 298), together with glycerine $(3 \cdot 2-3 \cdot 6$ per cent.) 
and succinic acid $(0 \cdot 6-0.7$ per cent.). Yeast is either added intentionally or it reaches the mixture from the air, which always contains its spores. When yeast is completely excluded, or if it be killed by boiling, [or if its action be prevented by the presence of some germicide], the fermentation does not occur. The alcoholic fermentation is due to the vital activity of a low organism (Schwann, Mitscherlich, Pasteur).

In the preparation of brandy, the starch of the grain or potatoes is first changed into sugar by the action of diastase or maltin. Yeast is added, and fermentation thereby produced; the mixture is distilled at $78 \cdot 3^{\circ} \mathrm{C}$. The fusel oil is prevented from mixing with the alcohol by passing the vapour through heated charcoal. The distillate contains 50-55 per cent. of alcohol.

In the preparation of wine, the saccharine juice of the grape-the must-after being expressed from the grapes is exposed to the air at $10-15^{\circ} \mathrm{C}$., and the yeast cells, which are floating about, drop into it and excite fermentation, which lasts 10-14 days, when the yeast sinks to the bottom. The clear wine is drawn off into casks, where it becomes turbid by undergoing an after.fermentation, until the sugar is converted into alcohol and $\mathrm{CO}_{2}$, which is accompanied by the deposition of some yeast and tartar. If all the sugar is not decomposed-which occurs when there is not sufficient nitrogenous matter present to nourish the yeast-a sweet wine is obtained. Wine contains $89-90$ per cent. water, 7-8 per cent. alcohol, together with æthylic, propylic, and butylic alcohol. The red colour of some wines is due to the colouring matter of the skin of the grapes, but if the skins be removed before fermentation, red grapes yield white wine.

When wine is stored it develops a fine flavour or bouquet. The characteristic vinous odour is due to ananthic ether. The salts of wine closely resemble the salts of the blood.

In the preparation of beer the grain is moisten, and allowed to germinate when the temperature rises, and the starch (68 per cent. in barley) is changed into sugar. Thus " malt" is formed, which is dried, and afterwards pulverised, and extracted with water at $70-75^{\circ}$, the watery extract being the "wort." Hops are added to the wort, and the whole is evaporated, when the proteids are coagulated. Hops give beer its bitter taste, make it keep, while their tannic acid precipitates any starch that may be present, and clarifies the wort. After being boiled, it is cooled rapidly $\left(12^{\circ} \mathrm{C}\right.$.); yeast is added, and fermentation goes on rapidly and with considerable efferrescence at $10^{\circ}-14^{\circ}$. Beer contains $75-95$ per cent. water; alcohol, 2-5 per cent. (porter and ale, to $S$ per cent.); $\mathrm{CO}_{2}, 0.1-0.8$ per cent.; sugar, 2-8 per cent.; gum, dextrin, 2-10 per cent.; the hops yield traces of protein, fat, lactic acid, ammonia compounds, the salts of the grain and of the hops.

In the ash, there is a great preponderance of phosphoric acid and potash, both of which are of great importance for the formation of blood. In 100 parts of ash there are 40.8 potash, 20.0 phosphorus, magnesium phosphate 20 , calcium phosphate $2 \cdot 6$, salica $16^{\circ} 6$ per cent. The formation of blood, muscle, and other tissues from the consumption of beer is due to the phosphoric acid and potash, while if too much be taken, the potash produces fatigue.

Condiments are taken with food, partly on account of their taste, and partly because they excite secretion. Common salt, in a certain sense, is a condiment. We may also include many substances of unknown constitution which act upon the gustatory organs, e.g., substances in the crust of bread and in meat which has been roasted. 


\section{Phenomena and Laws of Metabolism.}

\section{Equilibrium of the Metabolism.}

By this term is meant that, under normal physiological conditions, just as much material is absorbed and assimilated from the food, as is removed from the body by the excretory organs in the form of effete or end-products, the result of the retrogressive tissue changes. The income must always balance the expenditure; wherever a tissue is used up, it must be replaced by the formation of new tissue. As long as the body continues to grow, the increase of the body corresponds to a certain increase of formation, whereby the metabolism of the growing parts of the body is 2.5 to 6.3 times greater than that of the parts already formed (Crusius). Conversely, during senile decay, there is an excess of expenditure from the body.

Methods.-The normal equilibrium of the metabolism of the body is investigated-(1) By determining chemically that the sum of all the substances passing into the body is equal to the sum of all the substances given off from it. Thus the $\mathrm{C}, \mathrm{N}, \mathrm{H}, \mathrm{O}$, salts and water of the food, and the $\mathrm{O}$ inspired, must be equal to the $\mathrm{C}, \mathrm{H}, \mathrm{N}, \mathrm{O}$, salts and water given off in the excreta (urine, fæces, air expired, water excreted). (2) The physiological equilibrium is determined empirically by observing that the body retains its normal weight with a given diet; so that by simply weighing a person, a physician is enabled to determine exactly the state of convalescence of his patient.

The tedious process of making an elementary analysis of the metabolic substances was first undertaken in the Munich School by v. Bischoff, v. Voit, v. Pettenkofer, and others. Their observations showed, that in the circulation of materials the $\mathbf{C}$ and $\mathrm{N}$ were the most important. The total amount of $\mathrm{C}$ taken in the food, if the metabolism be in a condition of physiological equilibrium, must be equalled by the $\mathrm{C}$ in the $\mathrm{CO}_{2}$ given off by the lungs and skin ( 90 per cent.), together with the relatively small amount of $\mathrm{C}$ in the organic excreta of the urine and fæces (10 per cent.). With regard to the $\mathrm{N}$, nearly all the $\mathrm{N}$ taken in with the food is excreted within 24 hours in the form of urea. A very small amount of nitrogenous matter is excreted in the fæces, while the other nitrogenous urinary constituents (uric acid, kreatin, \&c.) represent about 2 per cent. of $\mathrm{N}$. A trace of the $\mathrm{N}$ is given off by the breath (p. 255), and a minute proportion in combination, in the epidermal scales (50 milligrammes daily in the hair and nails) and in the sweat.

That nearly all the $\mathrm{N}$ taken in the food reappears in the urine and fæces, as v. Voit showed for carnivora, and Henneberg, Stohman and Grouven for herbivora, and v. Ranke for man, is contradicted partly by old and partly by new observations (Barral, Boussingault, Bischoff, Regnault and Reiset, Seegen and Nowak), which go to show that the 
whole of the $\mathrm{N}$ cannot be recovered from these excretions, but on the contrary there is a considerable deficit.

According to Seegen and Nowak, 1 kilo. weight of a living animal excretes of gaseous $N$ per hour, thus-rabbit, 4-5 milligrammes (according to Leo, only ${ }_{12}^{1}$ of this value); $\operatorname{dog}, 8$ milligranmes; fowls, pigeons, 7-9 milligrammes. According to Leo, only 0.55 per cent. of the albumin transformed within the body (assuming 15 per cent. $N$ in albumin) is given off in the form of gaseous $N$.

The $\mathrm{H}$ leaves the body chiefly in the form of water-a part, however, is in combination in other excreta; the 0 is chiefly excreted as $\mathrm{CO}_{2}$ and water; a little is given off in combination in other excreta; water is given off by evaporation from the lungs and skin. As $\mathrm{H}$ is oxidised to form $\mathrm{H}_{2} \mathrm{O}$, more water is excreted than is taken in. With regard to the salts, most of the readily soluble salts are given off by the urine; less, especially potash salts and rather insoluble salts, in the fæces, while others, e.g., common salt, are given off in the sweat. Of the sulphur of albumin, about one-half is excreted in the sulphur compounds in the urine, and the other half in the fæces (taurin) and in the epidermal tissues.

Every body has a minimum and a maximum limit with reference to its metabolism, according to the amount of work done by the body, and its weight. If less food be given than is necessary to maintain the former, the body loses weight; while, if more be given after the maximum limit is reached, the food so given is not absorbed, but remains as a floating balance and is given off with the fæces. When food is liberally supplied and the weight increases, of course the minimum limit rises; hence, during the process of "feeding" or "fattening," the income necessary is very much greater than in poorlyfed animals, for the same increase of the body-weight. By continuing the process, a condition is at last reached, in which the digestive organs are just sufficient to maintain the existing condition, but cannot act so as to admit of new additions being made to the body-weight (v. Bischoff, v. Voit, v. Pettenkofer).

By the term "luxus consumption" is meant the direct combustion or oxidation of the superfiuous food stuffs absorbed into the blood. This, however, does not exist; on the contrary, the material in the juices is always being used for building up the tissues. The albumin found in the fluids, which everywhere permeate the tissues, has been called "circulating albumin," and, according to v. Voit, it may undergo decomposition sooner than the organised "organ albumin," which forms an integral part of the tissues.

According to v. Voit only 1 per cent. of the organ albumin present in the body, while 70 per cent. of the circulating albumin, is transformed in 24 lours.

The excretion of $\mathrm{N}$ after taking food is not equal from hour to hour ; it rises rapidly at first, reaches a maximum in 5-6 hours, and then gradually falls. The same is the case with the excretion of $S$ and $P$, only in these cases, after a flesh diet, the maximum is reached at the fourth hour. After the addition of fat to a 
flesh diet, the excretion of $\mathrm{N}$ and $\mathrm{S}$ is uniform from hour to hour (Feder and $\mathrm{v}$. Voit).

\section{Quality and Quantity of the Income in a Healthy Adult.}

As far as his organisation is concerned, man belongs to the omnivorous animals, i.e., those that can live upon a mixed diet.

Requisites.-Man requires for his existence and to maintain health the following four groups of foods; none of them must be absent from the food for any length of time. They are :-

1. Water-for an adult in his food and drink, 2,700-2,800 grms. daily ( $\$ 229$ and $\S 247,1$ ).

2. Inorganic Substances are an integral part of all tissues, and without them the tissues cannot be formed. They occur in ordinary food. The addition of too much salt increases the consumption of water, and this in turn increases the transformation of $\mathrm{N}$ in the body (Weiske). If an animal be deprived of salts, nutrition is interfered with; food deprived of its lime affects the formation of the bones; deprival of common salt causes albuminuria (247, A, III).

The alkaline salts serve to neutralise the sulphuric acid formed by the oxidation of the sulphur of the proteids (E. Salkowski, Bunge, Lunin).

Sodium acetate in large doses causes diuresis, and diminishes the transformation of nitrogenous substances in the body, and the same diminution is caused by sodium sulphate and phosphate; sodium carbonate (? Ott) increases the transformation of nitrogenous substances (J. Mayer), diminishes the uric acid, and increases the urea in the urine.

Only in times of famine is man driven to eat large quantities of inorganic substances, to extract the organic matter mixed therewith. A. v. Humboldt states, in regard to the inhabitants of the Orinocco, that they eat a kind of earth which contains innumerable infusoria.

3. At least one animal or vegetable albuminous body or proteid $(\S 248, \S 250)$. The proteids are required to replace the used-up nitrogenous tissues, e.g., for muscles. They contain 15.4 to 16.5 per cent. $\mathrm{N}$.

Asparagin, in combination with gelatin, can replace albumin in the food (Weiske), while asparagin alone limits the decomposition of albumin in herbivora (Weiske, Zuntz, Bahlmann, Lehmann), but not in carnivora (J. Munk). Ammoniacal salts, glycocoll, sarkosin, and benzamid, increase the amount of albu$\min$ in the body.

4. At least one fat ( $(251)$, or a digestible carbohydrate ( $\$ 252)$. These chiefly serve to replace the transformed fats and non-nitrogenous constituents. Owing to the large amount of $\mathrm{C}$ which they contain, when they undergo oxidation, they form the chief source of the 
heat of the body ( $\$ 206)$. Fats and carbohydrates may replace each other in the food, and in inverse proportion too, corresponding to the amount of $\mathrm{C}$ which each contains. According to v. Voit, for this purpose 175 parts of starch by weight are equal to 100 parts of fat.

\section{Animal Foods.}

Explanation of the sigus.

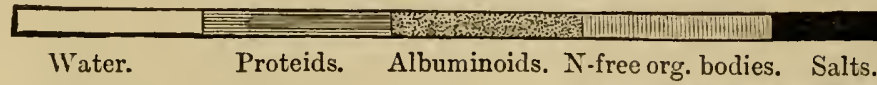

Beef.

\begin{tabular}{|c|c|c|c|c|c|c|}
\hline 62 & 205 & 2.5 \\
\hline 55 & 1
\end{tabular}

Fowl.

73

Fish.

76

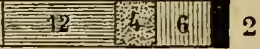

Egg.

73,5

$\sqrt{17}=$

Cow's milk.

86

Human milk.

\section{Vegetable Foods.}

Explanation of the signs.

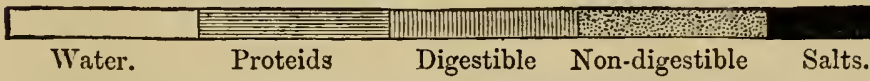

$\mathrm{N}$-free organ bodies.

Wheaten-bread.

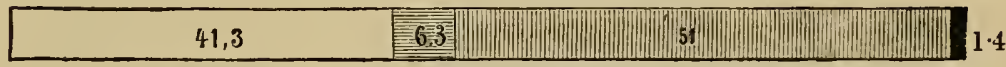

Peas.

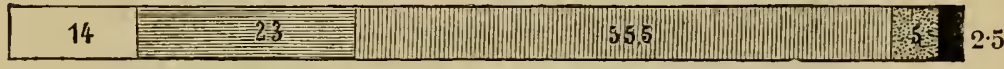

Rice.

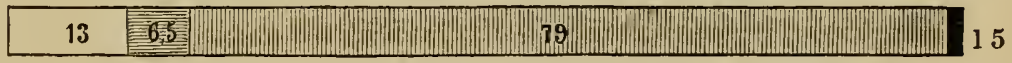

Potatoes.

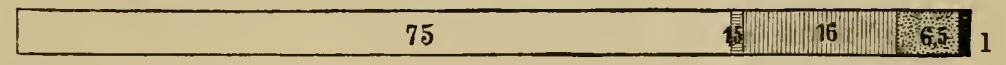

White Turnip.

Cauliflower.

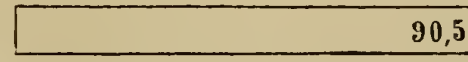

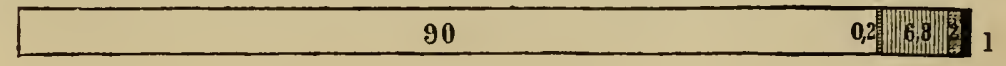


Proportion.-With regard to the relative proportions of the various kinds of food which ought to be taken, experience has shown that the diet best suited for the body must contain 1 part of nitrogenous foods to $3 \frac{1}{2}$ or, at most, $4 \frac{1}{2}$ of the non-nitrogenous. Looking at ordinary foods from this point of view, we see how far they correspond to this requirement, and how several substances may be combined to produce a satisfactory diet.

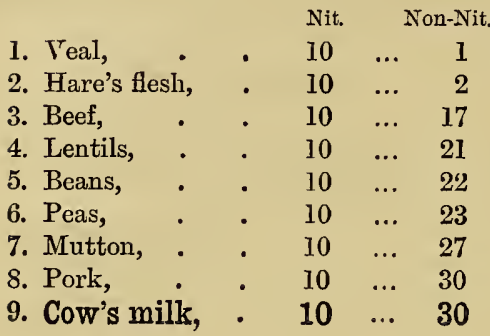

\begin{tabular}{lrrr} 
& Nit. & \multicolumn{2}{c}{ Non-Nit } \\
10. Human milk, & 10 & $\ldots$ & 37 \\
11. Wheaten-flour, 10 & $\ldots$ & 46 \\
12. Oat-meal, . & 10 & $\ldots$ & 50 \\
13. Rye-meal, . & 10 & $\ldots$ & 57 \\
14. Barley-meal, & 10 & $\ldots$ & 57 \\
15. White potatoes, & 10 & $\ldots$ & 86 \\
16. Blne, & 10 & $\ldots$ & 115 \\
17. Rice, . . & 10 & $\ldots$ & 123 \\
18. Buckwheat meal, 10 & $\ldots$ & 130
\end{tabular}

An examination of this table shows that, in addition to human milk, wheatflour has the right proportion of nitrogenous to non-nitrogenous substances. A man who tries to nourish himself on beef alone, commits as great a mistake as one who would feed himself with potatoes alone. Experience has taught people that man may live upon milk and eggs, but that in addition to flesh we must eat bread or potatoes, while pulses require fat or bacon.

The diet varies with the climate and with the season of the year. As the organism must produce more heat in cold latitudes, the inhabitants of northern climes must eat more non-nitrogenous foods, such as fats and sugars or starches, which, on account of the large amount of $\mathbf{C}$ they contain, are admirably adapted for producing heat (p. 442).

The graphic representation of the composition of Foods (Fig. 175), taken from Fick, shows at once the relative proportions of the food constituents and how they vary from the standard of 1 nitrogenous to $3 \frac{1}{2}-4 \frac{1}{2}$ non-nitrogenous.

The absolute amount of food stuffs required by an adult in 24 hours depends upon a variety of conditions. As the food represents the chemical reservoir of potential energy, from which the kinetic energy (in its various forms) and the heat of the body are obtained, the absolute amount of food must be increased when the body loses more heat, as in winter, and when more muscular activity (work) is accomplished. As a general rule an adult requires daily 130 grammes proteids, 84 grammes fats, 404 grammes carbohydrates. 
A Healthy Adult Requires in 24 Hodrs-

\begin{tabular}{|c|c|c|c|c|}
\hline \multirow{2}{*}{ Food in Grammes. } & \multirow{2}{*}{$\begin{array}{c}\text { At Rest } \\
\text { (Piayfair). }\end{array}$} & \multirow{2}{*}{$\begin{array}{c}\text { Moderate } \\
\text { Work } \\
\text { (Moleschott). }\end{array}$} & \multicolumn{2}{|c|}{ Laborious Work- } \\
\hline & & & (Playfair). & $\begin{array}{l}\text { (v. Pettenkofer } \\
\text { and ₹. Voit) }\end{array}$ \\
\hline Proteids, & $70 \cdot 87$ & 130 & $155 \cdot 92$ & 137 \\
\hline Fats, . & $25 \cdot 35$ & 84 & $70 \cdot 87$ & 117 \\
\hline Carboliydrates (Sugar, & & & . & \\
\hline Starch, etc.), . & $310 \cdot 20$ & 404 & $567 \cdot 50$ & 352 \\
\hline
\end{tabular}

In an analogous example from Vierordt, the elementary substances in the food are given (p. 446), and compared with the income and expenditure.

As Adult Doing a Moderate Amount of Work Takes in:-

\begin{tabular}{|c|c|c|c|c|c|c|c|c|}
\hline & & & & & c. & II. & N. & o. \\
\hline \multirow{4}{*}{$\begin{array}{r}120 \\
90 \\
330\end{array}$} & Grammes & Albumin, & containing, & & $64 \cdot 18$ & $8 \cdot 60$ & $18 \cdot 88$ & $28 \cdot 34$ \\
\hline &, & Fats & " & $\cdot$ & $70 \cdot 20$ & $10 \cdot 26$ & $\cdots$ & $9 \cdot 54$ \\
\hline & " & Starch & , & . & $146 \cdot 82$ & $20 \cdot 33$ & ... & $162 \cdot 85$ \\
\hline & & & & & $281 \cdot 20$ & $39 \cdot 19$ & $18 \cdot 88$ & $200 \cdot 73$ \\
\hline
\end{tabular}

Add $744 \cdot 11 \mathrm{grm}$. $O$ from the air by respiration.

, 2,818, $\quad \mathrm{H}_{2} \mathrm{O}$.

" 32, Inorganic compounds (Salts).

The whole is equal to $3 \frac{1}{6}$ kilo., i.e., about $2_{2}^{1} \sigma$ of the body-weight; so that about 6 per cent. of the water, about 6 per cent. of the fat, about 1 per cent. albumin, and about 0.4 per cent. of the salts of the body are daily transformed within the organism.

An Adolt Dolng Moderate Work Gives off :-

\begin{tabular}{|c|c|c|c|c|c|c|c|}
\hline \multicolumn{3}{|c|}{. } & Water. & c. & Н. & N. & 0. \\
\hline \multirow{2}{*}{\multicolumn{2}{|c|}{$\begin{array}{l}\text { By respiration, } \\
\text { Transpiration, } \\
\text { Urine, . . } \\
\text { Fæces, }\end{array}$}} & \multirow[t]{2}{*}{$\dot{.}$} & $\begin{array}{r}330 \\
660 \\
1,700 \\
123\end{array}$ & $\begin{array}{r}248 \cdot 8 \\
2 \cdot 0 \\
9 \cdot 8 \\
20 \cdot 0\end{array}$ & $\begin{array}{c}\ldots \\
\ldots \\
3 \cdot 3 \\
3 \cdot 0\end{array}$ & $\begin{array}{c}? \\
\ldots \\
15 \cdot 8 \\
3 \cdot 0\end{array}$ & $\begin{array}{c}651 \cdot 15 \\
7 \cdot 2 \\
11 \cdot 1 \\
12 \cdot 0\end{array}$ \\
\hline & & & 2,818 & $281 \cdot 2$ & $6 \cdot 3$ & $18 \cdot 8$ & $681 \cdot 45$ \\
\hline
\end{tabular}

Add to this (besides 2,818 grammes water as drink), 296 grammes water formed in the body by the oxidation of $H$. These 296 grammes of water contain 32,89 grammes $H$, and 263,41 grammes $0 ; 26$ grammes of salts are given off in the urine, and 6 by the fæces.

Effect of Age.-The investigations of the Munich School have shown, that the following numbers represent the smallest amount of food necessary for different ages :- 


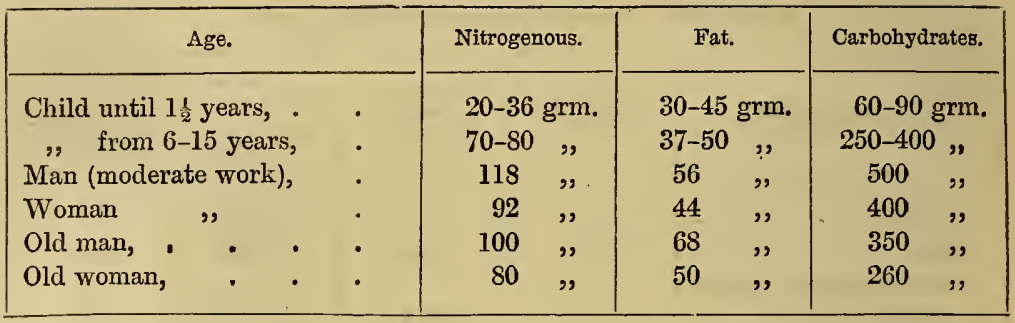

In most of the ordinary articles of diet, nitrogenous and nonnitrogenous substances are present, but in very varying proportion in the different foods. Man requires that these shall be in the proportion of $1: 3 \frac{1}{2}$ to $1: 4 \frac{1}{2}$.

If food be taken in which this proportion is not observed, in order to obtain the necessary amount of that substance which is contained in too small proportion in his food, he must consume far too much food. Moleschott finds that a person, in order to obtain the 130 grammes of proteids necessary, must use

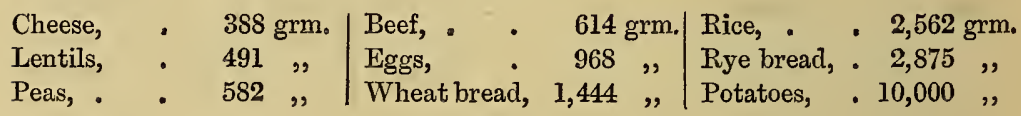

provided he were to take only one of these substances as food; so that it is perfectly obvious that if a workman were to live on potatoes alone, in order to get the necessary amount of $\mathrm{N}$, he would have to consume an altogether preposterous amount of this kind of food.

To obtain the 448 grammes of carbohydrates, or the equivalent amount of fat $(100: 175)$, necessary to support him, a man must eat

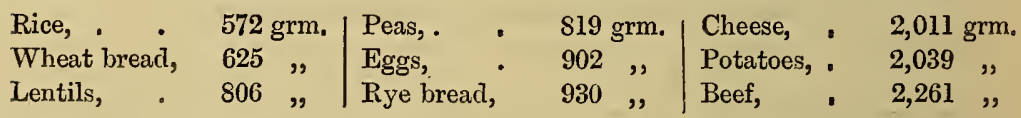

So that if he were to live upon cheese or flesh alone, he would require to eat an enormous amount of these substances.

In the case of the herbivora, the proportion of nitrogenous to non-nitrogenous food necessary is 1 of the former to 8 or 9 parts of the latter.

\section{Metabolism during Hunger and Starvation.}

If a warm-blooded animal be deprived of all food, it must, in order to maintain the temperature of its body and to produce the necessary amount of mechanical work, transform and utilise the potential energy of the constituents of its own body. The result is that its bodyweight diminishes from day to day, until death occurs from starvation. 
In order to investigate the condition of inanition it is necessary-(1) to weigh the animal daily; (2) to estimate daily all the $\mathrm{C}$ and $\mathrm{N}$ given off from the body in the fæces, urine, and expired air. The $\mathrm{N}$ and $\mathrm{C}$, of course, can only be obtained from the decomposition of tissues containing them.

The following table from Bidder and Schmidt shows the amounts of the different excreta in the ease of a starved cat:-

\begin{tabular}{|c|c|c|c|c|c|c|c|c|}
\hline Day. & $\begin{array}{c}\text { Body- } \\
\text { weight. }\end{array}$ & $\begin{array}{l}\text { Water } \\
\text { taken. }\end{array}$ & Urine. & Urea. & $\begin{array}{l}\text { Inorganic } \\
\text { substances } \\
\text { in Urine. }\end{array}$ & $\begin{array}{l}\text { Dry } \\
\text { Fæces. }\end{array}$ & $\begin{array}{c}\text { Expired } \\
\text { C. }\end{array}$ & $\begin{array}{c}\text { Water in } \\
\text { Urine } \\
\text { and Fæces. }\end{array}$ \\
\hline 1. & 2,464 & $\ldots$ & $9 \mathrm{~S}$ & $7 \cdot 9$ & $1 \cdot 3$ & $1 \cdot 2$ & $13 \cdot 9$ & $91 \cdot 4$ \\
\hline 2. & $\mathbf{2 , 2 9 7}$ & $11 \cdot 5$ & 54 & $5 \cdot 3$ & 0.8 & $1 \cdot 2$ & $12 \cdot 9$ & 50.5 \\
\hline 3. & 2,210 & $\ldots$ & 45 & $4 \cdot 2$ & 0.7 & $1 \cdot 1$ & 13 & $42 \cdot 9$ \\
\hline 4. & 2,172 & $68 \cdot 2$ & 45 & $3 \cdot 8$ & 0.7 & $1 \cdot I$ & $12 \cdot 3$ & 43 \\
\hline 5. & 2,129 & $\ldots$ & 55 & $4 \cdot 7$ & 0.7 & $1 \cdot 7$ & $11 \cdot 9$ & $54 \cdot I$ \\
\hline 6. & 2,024 & ... & 44 & $4: 3$ & $0 \cdot 6$ & 0.6 & $1 I \cdot 6$ & $4 I \cdot 1$ \\
\hline 7. & 1,946 & $\ldots$ & 40 & $3 \cdot 8$ & 0.5 & 0.7 & 11 & $37 \cdot 5$ \\
\hline 8. & 1,873 & ... & 42 & $3 \cdot 9$ & 0.6 & $1 \cdot 1$ & $10 \cdot 6$ & 40 \\
\hline 9. & 1,782 & $15 \cdot 2$ & 42 & 4 & 0.5 & $1 \cdot 7$ & $10 \cdot 6$ & $4 I \cdot 4$ \\
\hline 10. & 1,717 & ... & 35 & $3 \cdot 3$ & 0.4 & $1 \cdot 3$ & $10 \cdot 5$ & 34 \\
\hline $1 \mathrm{I}$. & 1,695 & 4 & 32 & $2 \cdot 9$ & 0.5 & $1 \cdot 1$ & $10 \cdot 2$ & 30.9 \\
\hline 12. & 1,634 & $22 \cdot 5$ & 30 & $2 \cdot 7$ & 0.4 & $1 \cdot 1$ & $10 \cdot 3$ & $29 \cdot 6$ \\
\hline 13. & 1,570 & $7 \cdot I$ & 40 & $3 \cdot 4$ & 0.5 & 0.4 & $10 \cdot 1$ & $36 \cdot 6$ \\
\hline 14. & $1,5 \mathrm{IS}$ & 3 & 41 & $3 \cdot 4$ & 0.5 & 0.3 & $9 \cdot 7$ & 38 \\
\hline 15. & 1,434 & ... & $4 \mathrm{I}$ & $2 \cdot 9$ & 0.4 & 0.3 & $9 \cdot 4$ & $38 \cdot 4$ \\
\hline 16. & 1,389 & ... & $4 S$ & 3 & 0.4 & 0.2 & s.8 & $45 \cdot 5$ \\
\hline 17. & 1,335 & $\ldots$ & 28 & $1 \cdot 6$ & 0.2 & 0.3 & $7 \cdot 8$ & $26 \cdot G$ \\
\hline \multirow[t]{2}{*}{$18 . \dagger$} & 1,267 & $\cdots$ & 13 & 0.7 & $0 \cdot 1$ & 0.3 & $6 \cdot 1$ & $12 \cdot 9$ \\
\hline & $-\mathrm{I}, 197$ & $13 I \cdot 5$ & 775 & $65 \cdot 9$ & $9 \cdot 8$ & $15 \cdot 8$ & $190 \cdot \mathrm{S}$ & $734 \cdot 4$ \\
\hline
\end{tabular}

The cat lost 1,197 grm. in weight before it died, and this amount is apportioned in the following way: $204.43 \mathrm{grm}$. $(=17.01$ per cent. $)$; loss of albumin, $132.75 \mathrm{grm}$. (=11.05 per cent.); loss of fat, 863.82 grm., loss of water ( $=71.91$ per cent. of the total body-weight).

Amongst the general phenomena of inanition, it is found that strong, well-nourished dogs die after 4 weeks, man after 21-24 days (Moleschott)-( 6 melancholics who took water died after 41 days); small mammals and birds, 9 days, and frogs 9 months. Vigorous adults die when they lose $\frac{4}{10}$ of their body-weight, but young individuals die much sooner than adults.

The symptoms of inanition are obvious:-The mouth is dry, the walls of the alimentary canal become thin, and the digestive secretions cease to be formed, pulse-beats and respirations are fewer; urine very acid from the presence of an increased amount of sulphuric and phosphoric acids, whilst the chlorine compounds rapidly diminish 
and almost disappear. The blood contains less water and the plasma less albumin, the gall-bladder is distended, which indicates a continuous decomposition of blood-corpuscles within the liver. The liver is small and very dark-coloured, the muscles are very brittle and dry, so that there is great muscular weakness, and death occurs with the signs of great depression and coma.

The relations of the metabolism are given in the foregoing table, the diminished excretion of urea is much greater than that of $\mathrm{CO}_{2}$, which is due to a larger amount of fats than proteids being decomposed.

According to the calculation, there is daily a tolerably constant amount of fat used up, while, as starvation continues, the proteids are decomposed in much smaller amounts from day to day, although the drinking of water accelerates their decomposition. The excretion of $\mathrm{CO}_{2}$ therefore falls more slowly than the total body-weight, so that the unit-weight of the living animal from day to day may even show an increased production of $\mathrm{CO}_{2}$. The amount of $\mathrm{O}$ consumed, depends of course upon the oxidation of proteids (which require less $\mathrm{O}$ ), and of fats (which require more $\mathrm{O}$ ).

According to D. Finkler, starving animals consume nearly as much $\mathrm{O}$ as wellnourished animals, so that the energy of oxidation is scarcely altered during inanition. Corresponding to this, the temperature of a starving animal is the same as normal. The respiratory quotient (p. 255) falls from 0.9 to $0 \cdot 7$, and the excretion of $\mathrm{CO}_{2}$ diminishes more rapidly than the consumption of $\mathrm{O}$. It would be wrong, however, to conclude, from the diminished condition of $\mathrm{CO}_{2}$, that the oxidation also was diminished, as the simultaneous consumption of $\mathrm{O}$ is the only guide to the energy of the metabolism. As starving animals use up their own flesh and fat, they form less $\mathrm{CO}_{2}$ than well-nourished animals which oxidise carbohydrates.

Loss of weight of Organs.-It is of importance to determine to what extent the individual organs and tissues lose weight; some undergo simple loss of weight, e.g., the bones, the fat undergoes very considerable and rapid decomposition, whilo other organs, as the heart, undergo little change, because they seem to be able to nourish themselves from the transformation products of other tissues.

A starving cat, according to v. Voit, lost-

\begin{tabular}{|c|c|c|c|c|c|c|}
\hline & present. & body-weight & & present. & & $y$-weight \\
\hline 1. Fat, & 97 & $26 \cdot 2$ & 10. Lungs, & $17 \cdot 7$ & $\ldots$ & 0.3 \\
\hline 2. Spleen, & $66 \cdot 7$ & 0.6 & 11. Pancreas, & $17 \cdot 0$ & $\ldots$ & $0 \cdot 1$ \\
\hline 3. Liver, & 53.7 & $4 \cdot 8$ & 12. Bones, & $13 \cdot 9$ & $\ldots$ & $5 \cdot 4$ \\
\hline 4. Testicles, & $40 \cdot 0$ & $0 \cdot 1$ & 13. CentralNervo & & & \\
\hline 5. Muscles, & $30 \cdot 5$ & $42 \cdot 2$ & System, & $3 \cdot 2$ & $\ldots$ & $0 \cdot 1$ \\
\hline 6. Blood, & $27 \cdot 0$ & $3 \cdot 7$ & 14. Heart, & $2 \cdot 6$ & $\ldots$ & 0.02 \\
\hline 7. Kidneys, & $25 \cdot 9$ & 0.6 & 15. Total loss of & & & \\
\hline 8. Skin, & $20 \cdot 6$ & $8 \cdot 8$ & the rest of the & & & \\
\hline 9. Intestine, & $18 \cdot 0$ & $2 \cdot 0$ & body, & $36 \cdot 8$ & $\ldots$ & $5 \cdot 0$ \\
\hline
\end{tabular}


There is a very important difference according as the animals before inanition have been fed freely on flesh and fat, or as they have merely had a subsistence diet. Well-fed animals lose weight much more rapidly during the first few days than on the later days. v. Voit thinks that the albumin derived from the excess of food occurs in a state of loose combination in the body as "circulating" or "storage-albumin," so that during hunger, it must decompose-more readily and to a greater extent than the "organ-albumin," which forms an integral part of the tissues (p. 477). Further, in fat individuals, the decomposition of fat is much greater than in slender persons.

\section{Metabolism during a purely Flesh Diet- Albumin or Gelatin.}

A man is not able to maintain his metabolism in equilibrium on a purely flesh diet; if he were compelled to live on such a diet, he would succumb. The reason is obvious. In beef, the proportion of nitrogenous to non-nitrogenous elementary constituents of food is $1: 1 \cdot 7$ (p. 480). A healthy person excretes 280 grammes of carbon, in the form of $\mathrm{CO}_{2}$, in the expired air and in the urine and fæces. If a man is to obtain 280 grammes $\mathrm{C}$ from a flesh diet he must consumedigest and assimilate-more than 2 kilos. of beef in 24 hours. But our digestive organs are unequal to this task for any length of time. The person is soon obliged to take less beef, which would necessitate the using of his own tissues, at first the fatty parts and afterwards the proteid substances.

A carnivorous animal (dog), whose digestive apparatus, being specially adapted for the digestion of flesh, has a short intestine, and powerfully active digestive fluids, can only maintain its metabolism in a state of equilibrium when fed on a flesh diet free from fat, provided its body is already well supplied with fat, and is muscular. It consumes $\frac{1}{25}$ to $\frac{1}{20}$ part of the weight of its body in flesh, so that the excretion of urea increases enormously. If it eats a larger amount, it may "put on flesh," when, of course, it requires to eat more to maintain itself in this condition, until the limit of its digestive activity is reached. If a well-nourished dog is fed on less than $\frac{1}{25}$ to $\frac{1}{2}$ of its body-weight of flesh, it uses part of its own fat and muscle, gradually diminishes in weight, and ultimately succumbs. Poorly fed non-muscular dogs are unable from the very beginning to maintain their metabolism in equilibrium for any length of time on a purely flesh diet, as they must eat so large a quantity of flesh, that their digestive organs cannot digest it. The herbivora cannot live upon flesh food, as their digestive apparatus is adapted solely for the digestion of vegetable food.

Exactly the same result occurs with other forms of proteids, as with flesh. It has been proved that gelatin may to a certain extent replace proteids in the food, in the proportion of 2 of gelatin to 1 of 
albumin. The carnivora which can maintain their metabolism in equilibrium by eating a large amount of flesh, can do so with less flesh when gelatin is added to their food. A diet of gelatin alone, which produces much urea, is not sufficient for this purpose, and animals soon lose their appetite for this kind of food (v. Bischoff, v. Voit, v. Pettenkofer, Oerum).

Owing to the great solubility of gelatin, the value of gelatin as a food used to be greatly discussed, and now again the addition of gelatin in the form of calf's-foot jelly is recommended to invalids. When chondrin is given along with flesh for a time, grape-sugar is found in the urine (Bödeker).

\section{A Diet of Fat or of Carbohydrates.}

If fat alone be given as a food, the animal lives but a short time. The animal so fed secretes even less urea than when it is starving; so that the consumption of fat limits the decomposition of the animal's own proteids. This depends upon the fact that, fat being an easily oxidised body, yields heat chiefly, and becomes sooner oxidised than the nitrogenous proteids which are oxidised with more difficulty. If the amount of fat taken be very large, all the $\mathrm{C}$ of the fat does not reappear, e.g., in the $\mathrm{CO}_{2}$ of the expired air; so that the body must acquire fat, whilst at the same time it decomposes proteids. The animal thus becomes poorer in proteids and richer in fats at the same time.

When carbohydrates alone are given, they must first be converted by the act of digestion into sugar. The result of such feeding coincides pretty nearly with the results of feeding with fat alone. But the sugar is more easily burned or oxidised within the body than the fat, and 17 parts of a carbohydrate are equal to 10 parts of fat. Thus the diet of carbohydrates limits the excretion of urea more readily than a purely fat diet. The animals lose flesh and appear even to use up part of their own fat.

The direct introduction of grape-sugar and cane-sugar into the blood does not increase the amount of oxygen used, although the amount of $\mathrm{CO}_{2}$ formed is increased (Wolfers).

\section{Mixture of Flesh and Fat,}

\section{or of Flesh and Carbohydrates.}

Since an amount of flesh equal to $\frac{1}{25}-\frac{1}{20}$ of the weight of the body is required to nourish a dog, which is fed on a purely flesh diet, if the necessary amount of fat or carbohydrates be added to the diet, a quantity of flesh three or four times less is required. A carbohydrate has a greater effect in diminishing the amount of urea 
excreted, than a quantity of fat, which requires the same amount of 0 to oxidise it, as is required by the amount of carbohydrates consumed. When the amount of flesh is insufficient, the addition of fat or carbohydrates to the food always limits the decomposition of the animal's own substance. Lastly, when too much flesh is given along with these substances, the weight of the body increases more with them than without them. Under these circumstances, the animal's body puts on more fat than flesh.

The consumption of $\mathrm{O}$ in the body is regulated by the mixture of flesh and non-nitrogenous substances, rising and falling with the amount of flesh consumed. It is remarkable that more $O$ is consumed when a given amount of flesh is taken, than when the same amount of flesh is taken with the addition of fat (v. Pettenkofer and v. Voit).

It scems that instead of fat, the corresponding amount of fatty acids has the same effect on the metabolism. They are absorbed as an emulsion just like the fats. When so absorbed, they seem to be reconverted into fats in their passage from the intestine to the thoracic duct (J. Munk, Will). Glycerin does not diminish the decomposition of albumin within the body (Lewin, Tschirwinsky, J. Munk). According to Lebedeff and v. Voit, it diminishes the decomposition of the fats, and is tberefore a food.

\section{Origin of Fat in the Body.}

I. Part of the fat of the body is derived directly from the food, i.e., it is absorbed and deposited in the tissues. This is shown by the fact that, with a diet containing a small amount of albumin, the addition of more fat causes the deposition of a larger amount of fat in the body (v. Voit, Hofmann).

Lebedeff found that dogs, which were starved for a month, so as to get rid of all their own fat, on being fed with linseed oil or mutton suet and flesh, had these fats restored to their tissues. These fats, therefore, must have been absorbed and deposited.

II. A second source of the fats is their formation from albuminous bodies (Liebig and others).

In the case of the formation of fat from proteids, which may yield 11 per cent. of fat, these proteids split up into a non-nitrogenous and a nitrogenous atomic compound. The former, during a diet containing much albumin, when it is not completely oxidised into $\mathrm{CO}_{2}$, and $\mathrm{H}_{2} \mathrm{O}$, is the substance from which the fat is formed-the latter leaves the body oxidised chiefly to the stage of urea (Hoppe-Seyler, Fürstenberg, v. Voit, v. Pettenkofer).

Examples.-That fats are formed from proteids is shown by the following :-1. A cow which produces $\mathbf{I} \mathrm{lb}$. of butter daily, does not take nearly this aniount of 
fatty matter in its food, so that the fat would appear to be formed from vegetable proteids. 2. Carnivora giving suck, when fed on plenty of flesh and some fat, yield milk rich in fat. 3. Dogs fed with plenty of flesh and some fat, add more fat to their bodies than the fat contained in the food. 4. Fatty degeneration, e.g., of nerve and muscle, is due to a decomposition of proteids. 5. The transformation of entire bodies, e.g., such as have lain for a long time surrounded with water, into a mass consisting almost entirely of palmitic acid (the adipocere of Fourcroy), is also a proof of the transformation of part of the proteids into fats. 6. Fungi are also able to form fat from albumin during their growth (v. Naegeli, and O. Löw).

Fats not merely absorbed.-Experiments which go to show that the fat of animals, during the fattening process, is not absorbed as such, from the food:-1. Fattening occurs with flesh and soaps; it is most improbable that the soaps are retransformed into neutral fats by taking up glycerin and giving up alkali (Kühne and Radziejewski). 2. If a lean dog be fed with flesh and palmitin-of stearin-soda-soap, the fat of its body contains in addition to palmitin and stearin, olein fat; so that the last must be formed by the organism from the proteids of the flesh. Further, Ssubotin found that, when a lean dog was fed on lean meat and spermaceti-fat, a very small amount of the latter was found in the fat of the animal. Although these experiments show, that the fat of the body must be formed from the decomposition of proteids, they do not prove that all the fat arises in this way, and that none of it is absorbed and redeposited.

III. According to v. Voit, no fat is formed in the body directly, e.g., by reduction from carbohydrates. As fattening occurs on a diet of pure flesh with the addition of carbohydrates, we must assume that the carbohydrates are consumed or oxidised in the body, and that, thereby, a non-nitrogenous body derived from the proteids is prevented from being burned up, and that it is changed into fat and stored up as such.

From experiments upon animals, however, Lawes, Lehmann, Heiden, v. Wolff, think they are entitled to conclude that the carbohydrates absorbed are directly concerned in the formation of fats, a view which is supported by Henneberg, B. Schulze, Gilbert and Soxhlet. According to Pasteur, glycerine (the basis of neutral fats) may be formed from carbohydrates.

Formerly it was believed that bees could prepare wax from honey alone; this is a mistake-an equivalent of albumin is required in addition-the necessary amount is found in the raw honey itself.

\section{Corpulence.}

The addition of too much fat to the body is a pathological phenomenon which is attended with disagreeable consequences. With regard to the causes of obesity, without doubt there is an inherited tendency (in 33-56 per cent. of the cases-Bouchard, Chalmers) in many families-and in some breeds of cattle-to lay up fat in the body, while other families may be richly supplied with fat, and yet remain lean. The chief cause, however, is taking too much food, i.e., more than the amount required for the normal metabolism; corpulent people, in order to maintain their bodies, must eat absolutely and relatively more than persons of spare habit, under analogous conditions of nutrition (p. 477). 
Conditions favouring Corpulence.-The following conditions favour the occurrence of corpulence:-(1) A diet rich in proteids, with a corresponding addition of fat or carbohydrates. As flesh or muscle is formed from proteids, and part of the fat of the body is also formed from albumin (1. 487), the assumption that fats and carbohydrates fatten, or when taken alone, act as fattening agents, is completely without foundation. No one ever becomes fat without taking plenty of albumin. (2) Diminished disintegration of materials within the bodye.g. (a) diminished muscular activity (much sleep and little exercise); (b) abrogation of the sexual furctions (as is shown by the rapid fattening of castrated animals, as well as by the fact that some women, after cessation of the menses, readily become corpulent) ; (c) diminished mental activity (the obesity of dementia), phlegmatic temperament. On the contrary, vigorous mental work, excitable temperament, care and sorrow, counteract the deposit of fat; $(d)$ diminished extent of the respiratory activity, as occurs when there is a great deposition of fat in the abdomen, limiting the action of the diaphragm (breathlessness of corpulent people), whereby the combustion of the fatty matters, which become deposited in the body, is limited; (e) a corpulent person requires to use relatively less heatgiving substances in his body, partly because he gives off relatively less heat from his compact body, than is done by a slender long-bodied individual, and partly because the thick layer of fat retards the conduction of heat (p. 444). Thus corresponding to the relatively diminished production of heat, more fat may be stored up; $(f)$ a diminution of the red blood-corpuscles, which are the great exciters of oxidation in the body, is generally followed by an increase of fat-fat people, as a rule, are fat because they have relatively less blood (p. 63)-women with fewer red blood-corpuscles are usually fatter than men; $(g)$ the consumption of alcohol favours the conservation of fat in the body, the alcohol is easily oxidised, and thus prevents the fat from being burned up (\$235).

Well-nourished individuals are usually at first both muscular and endowed with a fair amount of fatty tissue. When they begin to put on fat, the development of the muscular system lags behind, partly because the increasing corpulence leads to diminished activity of the muscular system, so that this system is involved secondarily. Some lively corpulent people, nevertheless, retain their muscular energy. When those conditions which favour corpulence are specially active, corpulence may ultimately pass into a condition of great obesity.

Besides the iuconvenience of the great size and weight of the body, corpulent people suffer from breathlessness-they are easily fatigued, are liable to intertrigo between the folds of the skin, the heart becomes loaded with fat, and they not unfrequently are subject to apoplexy.

In order to counteract corpulence we ought to-(1) Reduce uniformly all articles of cliet. The diet and body ought to be weighed from week to week, and as long as there is no diminution in the body-weight the amount of food onght to be gradually and uniformly reduced (notwithstanding the appetite). This must be done very gradually and not suddenly. It is not advisable to limit the amount of fat and carbohydrates alone, as is done in the Banting-cure or Bantingism. Apart altogether from the fact that fat is formed from proteids, if too little non. nitrogenous food be taken, severe disturbance of the bodily metabolism is apt to occur. (2) The muscular activity ought to be greatly developed by doing plenty of muscular work, or taking plenty of exercise, both physical and mental. (3) Favour the evolution of heat by taking cold baths of considerable duration, and afterwards rubbing the skin strongly so as to cause it to become red; farther, dress lightly; and at night use light bed-clothing; tea and coffee are useful, as they excite the circulation. (4) Use gentle laxatives; acid fruits, cider, alkaline carbonates (Marienbad, Carlsbad, Vichy, Neuenahr, Ems, \&c.). The copious drinking of water is also serviceable, as it favours the metabolism.

Fatty Degeneration.-The process of fattening consists in the deposition of 
drops of fat within the fat-cells of the panniculus and around the viscera, as well as in the marrow of bone (but they are never deposited in the subcutaneous tissue of the eyelids, of the penis, of the red part of the lips, in the ears and nose). This is quite different from the fatty atrophy or fatty degeneration which occurs in the form of fatty globules or granules in albuminous tissues-e.g., in muscular fibres (heart), gland-cells (liver, kidney), cartilage-cells, lymph-and pus-corpuscles, as well as in nerve-fibres separated from their nerve-centres The fat in these cases is derived from albumin, much in the same way as fat is formed in the gland-cells of the mammary and sebaceous glands. Marked fatty degeneration not unfrequently occurs after severe fevers, and after artificial heating of the tissues; when a too small amount of $O$ is supplied to the tissues, as occurs in cases of phosphorus poisoning (Bauer); in drunkards ; after poisoning with arsenic and other substances; and after some disturbances of the circulation and innervation. Some organs are especially prone to undergo fatty degeneration during the course of certain diseases.

\section{The Metabolism of the Tissues.}

The blood-stream is the chief medium whereby new material is supplied to the tissues and the effete products removed from them. The lymph which passes through the thin capillaries, comes into actual contact with the tissue elements. Those tissues which are devoid of blood-vessels in their own substance, such as the cornca and cartilage, receive nutrient fluid or lymph from the adjacent capillaries, by means of their cellular elements which act as juice-conducting media. Hence, when the normal circulation is interfered with, as by atheroma or calcification of the walls of the blood-vessels, these tissues are secondarily affected [this, for example, is the case in arcus senilis of the cornea, due to a fatty degeneration of the corneal tissue, owing to some affection of the blood-vessels on which the cornea depends for its nutrition]. Total compression or ligature of all the blood-vessels, results in necrosis of the parts supplied by the ligatured blood-vessels.

Hence, there must be a double current of the tissue juices; the afferent or supply current, which supplies the new material, and the efferent stream which removes the effete products. The former brings to the tissues the proteids, fats, carbohydrates and salts from which the tissues are formed. That such a current exists is proved by injecting an indifferent, easily recognisable substance into the blood, e.g., potassium ferrocyanide, when its presence may be detected in the tissues, to which it has been carried by the out-going current. The efferent stream carries away the decomposition products from the various tissues, more especially urea, $\mathrm{CO}_{2}, \mathrm{H}_{2} \mathrm{O}$ and salts, and these are transferred as quickly as possible to the organs through which they are excreted. That such a current exists is proved by injecting such a substance as potassium ferrocyanide into the tissues, 
e.g., subcutaneously, when its presence may be detected in the urine within 2 to 5 minutes. If the current from the tissues to the blood is so active that the excretory organs cannot eliminate all tho effete products from the blood, then these products are found in the tissues. This occurs when certain poisons are injected subcutaneously, when they pass rapidly into the blood and are carricd in great quantity to other tissues, e.g., to the nervous system, on which they act with fatal effect, before they are eliminated to any great extent from the blood, by the action of the excretory organs.

The effete materials are carried away from the tissucs by two channels, viz., by the veins and by the lymphatics, so that if these be interfered with, the metabolism of the tissues must also suffer. When a limb is ligatured so as to compress the veins and the lymphatics, the efferent stream stagnates to such an extent that considerable swelling of the tissues may occur (œdema, p. 419.)

H. Nasse found, that the blood of the jugular vein is 0.225 per 1000 specifically heavier than the blood of the carotid, and contains 0.9 parts per 1000 more solids; 1000 cubic centimetres of blood circulating through the head yield about 5 cubio centimetres of transudation into the tissues.

The extent and intensity of the metabolism of the tissues depend upon a variety of factors.

1. Upon their activity.-The increased activity of an organ is indicated by the increased amount of blood going to it, and by the more active circulation through it $(\S 100)$. When an organ is completely inactive, such as a paralysed muscle, or the peripheral end of a divided nerve, the amount of blood and the nutritive exchange of fluids diminish within these parts. The parts thus thrown out of activity become pale, relaxed, and ultimately undergo fatty degeneration. The increased metabolism of an organ during its activity has been proved experimentally in the case of muscle, and also in the brain (Speck).

Langley and Sewell have recently observed directly the metabolic changes within sufficiently thin lobules of glands during life. The cells of serous glands (p. 283), and those of mucous and pepsinforming glands (p. 327), during quiescence, become filled with coarse granules which are dark in transmitted light, and white in reflected light, which granules are consumed or disappear during glandular activity. During sleep, when most organs are at rest, the metabolism is limited; darkness also diminishes it, while light excites it, obviously owing to nervous influence. The variations in the total metabolism of the body are reflected in the excretion of $\mathrm{CO}_{2}$ and urea, which may be expressed graphically in the form of a curve corresponding 
with the activity of the organism; this curve corresponds very closely with the daily variations in the respirations, pulse, and temperature.

2. The composition or quality of the blood has a marked effect upon the current on which the metabolism of the tissues depends. Very concentrated blood, which contains a small amount of water, as after profuse sweating, severe diarrhœea, e.g., in cholera, makes the tissues dry, while if much water be absorbed into the blood, the tissues become more succulent and even cedema may occur. When much common salt is present in the blood and when the red bloodcorpuscles contain a diminished amount of $O$, and especially if the latter condition be accompanied by muscular exertion causing dyspnœa, a large amount of albumin is decomposed, and there is a great formation of urea. Hence, exposure to a rarified atmosphere is accompanied by increased excretion of urea (Fränkel, Penzoldt, and R. Fleischer). Certain abnormal conditions of the blood produce remarkable results; blood charged with carbonic oxide cannot absorb $\mathrm{O}$ from the air, and does not remove $\mathrm{CO}_{2}$ from the tissues (compare p. 31). The presence of hydrocyanic acid in the blood (p. 33), is said to interrupt at once the chemical oxidation processes in the blood (Mialhe), so that rapid asphyxia, owing to cessation of the internal respiration, occurs (Ed. Wagner). Fermentation is interrupted by the same substance in a similar way. A diminution of the total amount of the blood causes more fluid to pass from the tissues into the blood (p. 63), but the absorption of substances-such as poisons or pathological effusions (Kaup), from the tissues or intestines is delayed. If the substances which pass from the tissues into the blood be rapidly eliminated from it, absorption takes place more rapidly.

3. The blood-pressure is of importance in so far that, when it is greatly increased, the tissues contain more fluid, while the blood itself becomes more concentrated, to the extent of 3-5 per 1000 (Nasse). We may convince ourselves that blood-plasma easily passes through the capillary wall, by pressing upon the efferent vessel coming from the chorium deprived of its epidermis, e.g., by a burn or a blister, when the surface of the wound becomes rapidly suffused with plasma. Diminution of the blood-pressure produces the opposite result.

4. Increased temperature of the tissues favours the metabolism, so that the excretion of $\mathrm{CO}_{2}$ and the production of urea are increased ( $\S 220,221$ ); while diminution of the temperature has the opposite result (§225).

5. The influence of the Nerrous system on the metabolism is twofold. On the one hand, it acts indirectly through its effect upon the blood-vessels, by causing them to contract or dilate through the 
agency of vaso-motor nerves, whereby it influences the amount of blood supplied, and also affects the blood-pressure. But in addition to this, and quite independently of the blood-vessels, it is probable that certain special nerves-the so-called trophic nerves, influence the metabolism or nutrition of the tissues (see Trophic Nerves). That nerves do influence directly the transformation of matter within the tissues is shown by the secretion of saliva resulting from the stimulation of certain nerves, after cessation of the circulation (p. 287), and by the metabolism during the contraction of bloodless muscles. Increased respiration and apnoea are not followed by increased oxidation (Pflüger) (compare p. 259).

\section{Regeneration of Organs and Tissues.}

The extent to which lost parts are replaced varies greatly in different organs. Amongst the lower animals, the parts of organs are replaced to a far greater extent than amongst warm-blooded animals. When a hydra is divided into two parts, each part forms a new individual-nay, if the body of the animal be divided into several parts in a particular way, then each part gives rise to a new individual (Spallanzani). The Planarians also show a great capability of reproducing lost parts (Dugès). Spiders and crabs can reproduce lost feelers, limbs, and claws; snails, part of the head, feelers, and eyes, provided the central nervous system is not injured. Many fishes reproduce fins, even the tailfin. Salamanders and lizards can produce an entire tail, including bones, muscles, and even the posterior part of the spinal cord; while the triton reproduces an amputated limb, the lower jaw, and the eye. This reproduction necessitates that a small stump be left, while total extirpation of the parts prevents reproduction (Philippeaux).

In amphibians and reptiles, the regeneration of organs and tissues as a whole, takes place after the type of the embryonic development (Fraisse, Götte), and the same is true as regards the histological processes which occur in the regenerated tail and other parts of the body of the earth-worm (Bülow).

The extent to which regeneration can take place in mammals and in man is very slight, and even in these cases, it is chiefly confined to young individuals. A true regeneration occurs in-

1. The blood (compare $\S 7$ and $\S 41$ ), including the plasma, the colourless and coloured corpuscles.

2. The epidermal appendages (see Skin, vol. ii.), and the epithelium of the mucous membranes are reproduced by a proliferation of the cells of the deeper layers of the epithelium, with simultaneous division of their nuclei. Epithelial cells are reproduced as long as the matrix on which they rest and the lowest layer of cells are intact. When these are destroyed cell-regeneration from below ceases, and the cells at the margins are concerned in filling up the deficiency. Regeneration, therefore, either takes place from below or from the margins of the wound in the epithelial covering; leucocytes also wander into the part, 
while the deepest layer of cells forms large multi-nucleated cells which reproduce by division polygonal, flat nucleated cells (Klebs, Heller). The nails grow from the root forwards; those of the fingers in 4-5 months, and that of the great toe in about 12 months, although growth is slower in the case of fracture of the bones. The matrix is co-extensive with the lunule, and if it be destroyed the nail is not reproduced (see rol. ii.). The eyelashes are changed in 100-150 days (Donders), the other hairs of the body somewhat more slowly. If the papilla of the hair follicle be destroyed, the hair is not reproduced. Cutting the hair favours its growth, but hair which has been cut does not grow longer than uncut hair. After hair has grown to a certain length it falls out. The hair never grows at its apex (Aristotle). The epithelial cells of mucous membranes and secretory glands seem to undergo a regular series of changes and renewal. The presence of secretory cells in the milk $(\$ 231)$ and in the sebaceous secretion (vol. ii.) proves this; the spermatozoa are replaced by the action of spermatoblasts. In catarrhal conditions of mucous membranes, there is a great increase in the formation and excretion of new epithelium, while many cells are but indifferently formed and constitute mucous corpuscles. The crystalline lens, which is just modified epithelium, is reorganised just like epithelium; its matrix is the anterior wall of its capsule, with the single layer of cells covering it. If the lens be removed and this layer of cells retained, these cells proliferate and elongate to form lens fibres, so that the whole cavity of the empty lens capsule is refilled. If much water be withdrawn from the body, the lens fibres become turbid (Kunde, Koehnhorn). [A turbid or opaque condition of the lens may occur in diabetes, or after the transfusion of strong common salt or sugar solution into a frog.]

3. The blood-vessels undergo extensive regeneration, and they are regenerated in the same way as they are formed (p. 13). Capillaries are always the first stage, and around them the characteristic coats are added to form an artery or a vein. When an artery is injured and permanently occluded, as a general rule the part of the vessel up to the nearest collateral branch becomes obliterated, whereby the derivatives of the endothelial lining, the connective tissue-corpuscles of the wall and the leucocytes change into spindle-shaped cells and form a kind of cicatricial tissue. Blind and solid outshoots are always found on the blood-vessels of young and adult animals, and are a sign of the continual degeneration and regeneration of these vessels (Sigm. Mayer).

4. The contractile substance of muscle may undergo regeneration after it has become partially degenerated. This takes place after amyloid or wax-like degeneration, such as occurs not unfrequently after typhus and other severe fevers. This is chiefly accomplished by an 
increase of the muscle corpuscles. After being compressed, the muscular nuclei disappear and at the same time the contractile contents degenerate (Heidelberg). After several days, the sarcolemma contains numerous nuclei which reproduce new muscular nuclei and the contractile substance (Kraske, Erbkam). In fibres injured by a subcutaneous wound, Neumann found that, after 5-7 days, there was a bud-like elongation of the cut ends of the fibres, at first without transverse striation, but with striation ultimately. If a large extent of a muscle be removed, it is replaced by cicatricial connectivetissue.

Non-Striped muscular fibres are also reproduced; the nuclei of the injured fibres divide after becoming enlarged, and exhibit a wellmarked intra-nuclear plexus of fibrils. The nuclei divide into two, and from each of these a new fibre is formed, probably by the differentiation of the peri-nuclear protoplasm.

5. After a nerve is divided, the two ends do not join at once so as to permit the function of the nerve to be established. On the contrary, marked changes occur which are described in vol. $i i$. If a piece be cut out of a nerve-trunk, the peripheral end of the divided nerve degenerates, the axial cylinder and the white substance of Schwann disappear. The interval is filled up at first with juicy cellular tissue. The subsequent changes are fully described in vol. $i i$. There seems to be in peripheral nerves a continual disappearance of fibres by fatty degeneration, accompanied by a consecutive formation of new fibres (Sigm. Mayer). The regeneration of peripheral ganglionic cells is unknown. v. Voit, however, observed that a pigeon, part of whose brain was removed, had within five months reproduced a nervous mass within the skull consisting of medullated nerve-fibres and nerve-cells. Eichhorst and Naunyn found that in young dogs, whose spinal cord was divided between the dorsal and lumbar regions, there was an anatomical and physiological regeneration to such an extent that voluntary movements could be executed. Vaulair, in the case of frogs, and Masius in dogs, found that mobility or motion was first restored and afterwards sensibility. Regeneration of the spinal ganglia does not occur.

6. If a portion of a secretory gland be removed, as a general rule, it is not reproduced. But the bile-ducts (p. 350), and the pancreatic duct may be reproduced (p. 345). According to Philippeaux and Griffini, if part of the spleen be removed, it is reproduced (compare p. 207). Tizzoni and Collucci observed the formation of new livercells and bile-ducts after injury to the liver.

7. Amongst connective-tissues, cartilage, provided its perichondrium be not injured, reproduces itself by division of its cartilage cells 
(Legrand, Ewetzky, Schklarewsky); but usually when a part of a cartilage is removed, it is replaced by connective-tissue.

8. When a tendon is divided, proliferation of the tendon cells occurs, and the cut ends are united by connective-tissue.

9. The reproduction of bone takes place to a great extent under certain conditions. If the articular end be removed by excision, it may be reproduced, although there is a considerable degree of shortening. Pieces of bone which have been broken off or sawn off heal again, and become united with the original bone (Jakimowitsch). If a piece of periosteum be transplanted to another region of the body, it eventually gives rise to the formation of new bone in that locality. If part of a bone be removed, provided the periosteum be left, new bone is rapidly reproduced; hence, the surgeon takes great care to preserve the periosteum intact in all operations where he wishes new bone to be reproduced. Even the marrow of bone, when it is transplanted, gives rise to the formation of bone. This is due to the osteoblasts adhering to the osseous tissue (P. Bruns, MacEwen).

In fracture of a long bone, the periosteum deposits on the surface of the ends of the broken bones, a ring of substance which forms a temporary support, the external callus. At first this callus is jelly-like, soft, and contains many corpuscles, but afterwards, it becomes more solid and somewhat like cartilage. A similar condition occurs within the bone, where an internal callus is formed. The formation of this temporary callus is due to an inflammatory proliferation of the connective-tissue corpuscles, and partly to the osteoblasts of the periosteum and marrow. According to Rigal and Vignal, the internal callus is always osseous, and is derived from the marrow of the bone.

The outer and inner callus becomes calcified and ultimately ossified, whereby the broken ends are reunited. Towards the fortieth day, a thin layer of bone is formed (intermediary callus) between the ends of the bone. When this begins to be definitely ossified, the outer and inner callus begins to be absorbed, and ultimately the intermediary callus has the same structure as the rest of the bone.

There are many interesting observations connected with the growth and metabolism of bones. 1. The addition of a very small amount of phosphorus (Wagner) or arsenious acid (Maas) to the food causes considerable thickening of the bones. This seems to be due to the non-absorption of those parts of the bones which are usually absorbed, while new growth is continually taking place. 2. When food devoid of lime salts is given to an animal, the growth of the bones is not arrested (v. Voit), but the bones become thinner, whereby all parts, even the organic basis of the bone, undergo a uniform diminution (Chossat, A. Milne-Edwards). 3. Feeding with madder makes the bones red, as the colouring matter is deposited with the bone salts in the bone, especially in the growing and last formed parts. In birds, the shell of the egg becomes coloured. 4. The continued use of lactic acid dissolves the bones (Siedamgrotzky and Hofmeister). The ash of bone is thereby diminished. If lime salts be withheld at the same time, the effect is greatly increased, so that the bones come to resemble rachitic bones. The normal development of bone is described in vol. $i$.

When a lost tissue is not replaced by the same kind of tissue, its place is always taken by cicatricial connective-tissue. 
When this is the case, the part becomes inflamed and swollen, owing to an exudation of plasma. The blood-vessels become dilated and congested, and, notwithstanding the slower circulation, the amount of blood is greater. The blood-vessels are increased, owing to the formation of new ones. Colourless bloodcorpuscles pass out of the vessels and reproduce themselves, and many of them undergo fatty degeneration, whilst others take up nutriment and become converted into large uninucleated protoplasma-cells, from which giant-cells are developed (Ziegler, Cohnheim). The newly-formed blood-vessels supply all these elements with blood.

\section{Transplantation of Tissues.}

The nose, ear, and even a finger, after having been severed from the body by a clean cut, have, under certain circumstances, become united to the part from which they were removed.

The skin is frequently transplanted by surgeons, as, for example, to form a new nose. The piece of skin is cut from the forehead or arm, to which it is left attached by a bridge of skin. The skin is then stitched to the part which it is desired to cover in, and when it has become attached in its new situation, the bridge of skin is severed.

Reverdin cut a piece of skin into pieces about the size of a pea and fixed them on an ulcerated surface, where they, as it were, took root, grew, and sent off from their margins epithelial out-growths, so that ultimately the whole surface was covered with epithelium.

The excised spur of a cock was transplanted and fixed in the comb of the same animal where it grew (John Hunter).

P. Bert cut off the tail and legs of rats and transplanted them under the skin of the back of other rats, where they united with the adjoining parts.

Ollier found that, when periosteum was transplanted it grew and reproduced bone in its new situation. Even blood and lymph may be transfused (Trans. ficsion-p. 199).

All these results seem only to be possible between individuals of the same species, although Helferich has recently found that a piece of a dog's muscle, when substituted for human muscle, united to the adjoining muscle and became functionally active. [While $J$. $\mathrm{K}$. Wolfe has transplanted the conjunctiva of the rabbit to the human eye]. Most tissues, however, do not admit of transplantation, e.g., glands and the sense-organs. They may be removed to other parts of the body, or into the peritoneal cavity, without exciting any inflammatory reaction; they, in fact, behave like inert foreign matter.

\section{Increase in Size and Weight during Growth.}

The length of the body, which at birth is usually $\frac{1}{3 \cdot 5}$ of the adult body, undergoes the greatest elongation at an early period:-in the first year, 20 ; in the second, 10 ; in the third, about 7 centimetres; whilst from $5-16$ years the annual increase is about $5 \frac{1}{2}$ centimetres. In the twentieth year the increase is very slight. From 50 onwards the size of the body diminishes, owing to the intervertebral discs becoming thinner, and the loss may be 6-7 centimetres about the eightieth year. The weight of the body ( $\frac{1}{20}$ of an adult) sinks during the first 5-7 days, owing to the evacuation of the meconium and the small amount of food which is taken at first.

The increase of weight is greater in the same time than the increase in length. Within the first year a child trebles its weight. The greatest weight is usually 
reached about 40, while towards 60 a decrease begins, which at 80 may amount even to 6 kilo. The results of measurements, chiefly by Quetelet, are given in the following table:-

\begin{tabular}{|c|c|c|c|c|c|c|c|c|c|}
\hline \multirow{2}{*}{ Age. } & \multicolumn{2}{|c|}{ Length (Cmtr.) } & \multicolumn{2}{|c|}{ Weight (Kilo.) } & \multirow{2}{*}{ Age. } & \multicolumn{2}{|c|}{ Length (Cmtr.) } & \multicolumn{2}{|c|}{ Weight (Kilo.) } \\
\hline & Man. & Woman. & Man. & Woman. & & Man. & Woman. & Man. & Woman. \\
\hline 0 & $49 \cdot 6$ & $48 \cdot 3$ & $3 \cdot 20$ & $2 \cdot 91$ & 15 & $155 \cdot 9$ & $147 \cdot 5$ & $46 \cdot 41$ & $41 \cdot 30$ \\
\hline 1 & $69 \cdot 6$ & 69.0 & $10 \cdot 00$ & $9 \cdot 30$ & 16 & $161 \cdot 0$ & $150 \cdot 0$ & $53 \cdot 39$ & $44 \cdot 44$ \\
\hline 2 & $79 \cdot 6$ & $78 \cdot 0$ & $12 \cdot 00$ & $11 \cdot 40$ & 17 & $167 \cdot 0$ & $154 \cdot 4$ & $57 \cdot 40$ & $49 \cdot 08$ \\
\hline 3 & 86.0 & $85 \% \cdot 0$ & $13 \cdot 21$ & $12 \cdot 45$ & 18 & $170 \cdot 0$ & $156 \cdot 2$ & $61 \cdot 26$ & $53 \cdot 10$ \\
\hline 4 & $93 \cdot 2$ & $91 \cdot 0$ & $15 \cdot 07$ & $14 \cdot 18$ & 19 & 170.6 & . & $63 \cdot 32$ & $\ldots$ \\
\hline 5 & $99 \cdot 0$ & $97 \cdot 0$ & $16 \cdot 70$ & $15 \cdot 50$ & 20 & $171 \cdot 1$ & $157 \cdot 0$ & $65 \cdot 00$ & $54 \cdot 46$ \\
\hline 6 & $104 \cdot 6$ & $103 \cdot 2$ & $18 \cdot 04$ & $16 \cdot 74$ & 25 & $172 \cdot 2$ & $157 \cdot 7$ & $68 \cdot 29$ & $55 \cdot 08$ \\
\hline 7 & $111 \cdot 2$ & $109 \cdot 6$ & $20 \cdot 16$ & $18 \cdot 45$ & 30 & $172 \cdot 2$ & $157 \cdot 9$ & $68 \cdot 90$ & $55 \cdot 14$ \\
\hline 8 & $117 \cdot 0$ & $113 \cdot 9$ & $22 \cdot 26$ & $19 \cdot 82$ & 40 & $171 \cdot 3$ & $155 \cdot 5$ & $68 \cdot 81$ & $56 \cdot 65$ \\
\hline 9 & $122 \cdot 7$ & 120.0 & $24 \cdot 09$ & $22 \cdot 44$ & 50 & $167 \cdot 4$ & $153 \cdot 6$ & $67 \cdot 45$ & $58 \cdot 45$ \\
\hline 10 & $128 \cdot 2$ & $124 \cdot 8$ & $26^{\circ} 12$ & $24 \cdot 24$ & 60 & $163 \cdot 9$ & $151 \cdot 6$ & $65 \cdot 50$ & $56 \cdot 73$ \\
\hline 11 & $132 \cdot 7$ & $127 \cdot 5$ & $27 \cdot 85$ & $26 \cdot 25$ & 70 & $162 \cdot 3$ & $151 \cdot 4$ & 63.03 & $53 \cdot 72$ \\
\hline 12 & 135.9 & $132 \cdot 7$ & $3 I \cdot 00$ & 30.54 & so & $161 \cdot 3$ & $150 \cdot 6$ & $61 \cdot 22$ & $5 \mathrm{I} \cdot 52$ \\
\hline 13 & 140.3 & 138.6 & $35 \cdot 32$ & $34 \cdot 65$ & 90 & $\ldots$ & $\ldots$ & $57 \cdot 83$ & $49 \cdot 34$ \\
\hline 14 & 148.7 & $144 \cdot 7$ & $40 \cdot 50$ & $38 \cdot 10$ & & (Ch & $y$ from & uetele & \\
\hline
\end{tabular}

Between the 12th and 15th years, the weight and size of the female are greater than of the male. Growth is most active in the last months of fotal life, and afterwards from the 6 th to 9 th year, until the 13 th to 16 th. The full stature is reached about 30 , but not the greatest weight (Thoma). 


\section{General View of the Chemical Constituents of the 0rganism.}

\section{7. (A.) Inorganic Constituents.}

I. Water forms 58.5 per cent. of the whole body, but it occurs in different quantity in the different tissues; the kidneys contain the most water, $82 \cdot 7$ per cent.; bones, 22 per cent.; teeth, 10 per cent.; while enamel contains the least, 0.2 per cent.

[ Water is of the utmost importance in the ecoumy, and it is no paradox to say that all organisms live in water, for though the entire animal may not live in water, all its tissues are bathed by watery fluids, and the essential vital processes occur in water (p. 458). A constant stream of water may be said to be passing through organisms, a certain quantity of water is taken in with the food and drink, which ultimately reaches the blood, while from the blood a constant loss is taking place by the urine, the sweat and breath. The greater quantity of the water in our bodies is derived from without, but it is probable that a small amount is formed within our bodies by the action of free oxygen on certain organic substances. According to some observers, peroxide of hydrogen $\left(\mathrm{H}_{2} \mathrm{O}_{2}\right)$ is also present in the body.]

II. Gases.-[Oxygen is absorbed from the air, and enters the blood, where it forms a loose chemical compound, with the colouring matter or hæmoglobin, while a small amount exists in a free state, or is simply absorbed.] Hydrogen is found in the alimentary canal. Nitrogen [like oxygen, is absorbed from the atmosphere by the blood, in which it is dissolved, and from which it passes into other fluids of the body. It is probable that a very small quantity is formed within the body.]

The presence of Marsh gas $\left(\mathrm{CH}_{4}\right)$ (p. 255), ammonia $\left(\mathrm{NH}_{3}\right)$, and sulphuretted hydrogen $\left(\mathrm{H}_{2} \mathrm{~S}\right)$ (p. 372) has been referred to already.

III. Salts._Sodium chloride [is one of the most important inorganic substances present in the body. It occurs in all the tissues and fluids of the body, and it plays a most prominent part in connection with the diffusion of fluids through membranes, and its presence is necessary for the solution of the globulins (p. 502). In some cases it exists in a state of combination with albuminous bodies, as in the blood-plasma. Common salt is absolutely necessary for one's existence; if it be withdrawn entirely, life soon comes to an end. About 15 grammes are given off in twenty-four hours, the great part being excreted by the urine. Boussingault showed that, the addition of a certain amount of common salt to the daily food of cattle greatly improved their condition.]

Calcium phosphate $\left[\left(\mathrm{Ca}_{3} \mathrm{P}_{2} \mathrm{O}_{8}\right)\right.$ is the most abundant salt in the body, as it forms more than one-half of our bones, but it also occurs in dentine, enamel, and to a 
much less extent in the other solids and fluids of the body. Amongst secretions, milk contains relatively the largest amount $(2 \cdot 72)$ per cent. In milk it is necessary for forming the calcareous matter of the bones of the infant. It gives bones their hardness, solidity, and rigidity. It is chiefly derived from the food, and as only a small quantity is given off in the excretions, it seems not to undergo rapid removal from the body.]

Sodium phosphate $\left(\mathrm{PNa}_{3} \mathrm{O}_{4}\right)$, acid sodium phosphate $\left(\mathrm{PNa}_{2} \mathrm{HO}_{4}\right)$, acid potassium phosphate $\left(\mathrm{PK}_{2} \mathrm{HO}_{4}\right)$. [The sodium phosphate and the corresponding potash salt give most of the fluids of the body their alkaline reaction. The alkaline reaction of the blood-plasma is partly due to alkaline phosphates which are chiefly derived from the food. The acid sodium phosphate is the chief cause of the acid reaction of the urine. A small quantity of phosphoric acid is formed in the body owing to the oxidation of "lecithin" which contains phosphorus, and also forms an important constituent of nerve-tissue.]

Sodium carbonate $\left(\mathrm{Na}_{2} \mathrm{CO}_{3}\right)$ and sodium bicarbonate $\left(\mathrm{NaHCO}_{3}\right)$ [exist in small quantities in the food, and are chiefly formed in the body from the decomposition of the salts of the vegetable acids. They occur in the blood-plasma, where they play an important part in carrying the $\mathrm{CO}_{2}$ from the tissues to the lungs.]

Sodium and potassium sulphates $\left(\mathrm{NaSO}_{4}\right.$, and $\left.\mathrm{K}_{2} \mathrm{SO}_{4}\right)$ [exist in very small quantity in the body, and are introduced with the food, but part is formed in the body from the oxidation of organic bodies containing sulphur.]

[Potassium chloride $\left(\mathrm{KCl}_{2}\right)$ is pretty widely distributed, and it occurs specially in muscle, coloured blood-corpuscles, and milk. Calcium fluoride $\left(\mathrm{CaFl}_{2}\right)$ occurs in small quantity in bones and teeth. Calcium carbonate $\left(\mathrm{CaCO}_{3}\right)$ is associated with calcium phosphate in bone, tooth, and in some fluids, but it occurs in relatively much smaller amount. It is kept in solution by alkaline chlorides, or by the presence of free carbonic acid.]

Ammonium chloride $\left(\mathrm{NH}_{4} \mathrm{Cl}\right)$, - [Minute traces occur in the gastric juice and the urine.]

Magnesium phosphate $\left(\mathrm{Mg}_{3} \mathrm{PO}_{4}\right)$ [occurs in the tissues and fluids of the body along with calcium phosphate, but in very much smaller quantity.]

IV. FreeAcids.-Hydrochloric acid $(\mathrm{HCl})$ [occurs free in the gastric juice, but in combination with the alkalies it is widely distributed as chlorides.] Sulphuric acid $\left(\mathrm{H}_{2} \mathrm{SO}_{4}\right)$ [is said to occur free' in] the saliva of certain gasteropods, as Dolium galea. In the body it forms sulphates, being chiefly in combination with soda and potash.]

V. Bases.-Silicon as silicic acid $\left(\mathrm{SiO}_{2}\right)$; manganese, iron, the last forms an integral constituent of the blood pigment ; copper (?), p. 352.

\section{8. (B.) Organic Compounds.}

\section{The Albuminous or Proteid Substances.}

\section{Proteids and their Allies,}

Proteids and their allies are composed of $\mathrm{C}, \mathrm{H}, \mathrm{O}, \mathrm{N}$, and $\mathrm{S}$, and are derived from plants (see Introduction).

[According to Hoppe-Seyler their general percentage composition is

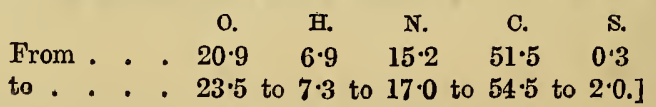


They exist in all animal fluids, and in nearly all the tissues. They occur partly in the fluid form, although Brücke maintains that the molecule of albumin exists in a condition midway between a state of imbibition and a true solution-and partly in a more concentrated condition.

Besides forming the chief part of muscle, nerve, and gland, they occur in nearly all the fluids of the body, including the blood, lymph, and serous fluids, but in health mere traces occur in the sweat, while they are absent from the bile and the urine. White of egg is the type. In the alimentary canal they are changed into peptones. The chief products derived from their oxidation within the body are $\mathrm{CO}_{2} \mathrm{H}_{2} \mathrm{O}$, and especially urea, which contains nearly all the $\mathrm{N}$ of the proteids.

Constitution.-Their chemical constitution is quite unknown. The $\mathrm{N}$ seems to exist in two distinct conditions, partly loosely combined, so as to yield am. monia readily when they are decomposed, and partly in a more fixed condition. According to Pfliiger, part of the $\mathrm{N}$ in living proteid bodies exists in the form of cyanogen. The proteids form a large group of closely related substances, all of which are perhaps modifications of the same body. When we remember that the infant manufactures most of the proteids of its ever-growing body from the casein of milk, this last view seems not improbable.

Characters.-Proteids, the anhydrides of peptones are colloids (p. 394), and therefore do not diffuse easily through animal membranes; they are amorphous and do not crystallise, and hence are isolated with difficulty; some are soluble and others are insoluble in water; they are insoluble in alcohol; they rotate the ray of polarised light to the left; in a flame, they give the odour of burned horn. Various metallic salts and alcohol precipitate them from their solution, and they are coagulated by heat, mineral acids and the prolonged action of alcohol. Caustic alkalies dissolve them (yellow), and from this solution they are precipitated by acids.

Decompositions. - When acted upon in a suitable manner by acids and alkalies, they give rise to the decomposition products-leucin (10-18 per cent.), tyrosin (0.25-2 per cent.), asparaginic acid, glutamic acid, and also volatile fatty acids, benzoic and hydrocyanic acids, and aldehydes of benzoic and fatty acids; also, indol (Hlasiwetz, Habermann). Similar products are formed during pancreatic digestion (p. 342), and during putrefaction (p. 376).

Reactions.-They are coagulated by (1) nitric acid, and when boiled therewith give a yellow, the xanthoproteic reaction; the addition of ammonia gives a deep orange colour.

(2) Millon's reagent (nitrate of mercury with nitrous acid); when heated with this reagent above $60^{\circ} \mathrm{C}$., they give a red, probably owing to the formation of tyrosin. [If the proteids are present in large amount, a red precipitate occurs, but if mere traces are present only the fluid becomes red.]

(3) The addition of a few drops of solution of cupric sulphate, and the subsequent addition of caustic potash or soda give a violet colour, which deepens on boiling, [or the same colour may be obtained by adding a few drops of Fehling's solution.]

(4) They are precipitated by acetic acid and potassium ferrocyanide.

(5) When boiled with concentrated hydrochloric acid they give a violet-red colour.

(6) Sulphuric acid containing molybdic acid gives a blue colour (Fröhde).

(7) Their solution in acetic acid is coloured violet with concentrated sulphuric acid, and shows the absorption-band of hydrobilirubin (Adamkiewicz).

(8) Iodine is a good microscopic reagent, which strikes a brownish-yellow, while sulphuric acid and cane-sugar give a purplish-violet (E. Schultze).

[ (9) When boiled with acetic acid and an equal volume of a concentrated solution of sodium sulphate, they are precipitated. This method is frequently used for removing proteids from other liquids, as it does not interfere with the presence of other substances.] 


\section{The Animal Proteids and their Characters.}

They have been divided into classes:-

\section{Class I.-Native Albumins.}

Native Albumins occur in a natural condition in the solids and fluids of the body. They are soluble in water, and are not precipitated by alkaline carbonates, $\mathrm{NaCl}$, or by very dilute acids. Their solutions are coagulated by heat at $65^{\circ}-73^{\circ} \mathrm{C}$. Dried at $40^{\circ} \mathrm{C}$., they yield a clear yellow amber-coloured friable mass, "soluble albumin," which is soluble in water.

(1.) Serum-albumin, whose chemico-physical characters are given at p. 49, and its physiological properties at $\S 41$. Almost all its salts may be removed from it by dialysis, when it no longer coagulates with heat (Schmidt). It is coagulated by strong alcohol, and is easily dissolved in strong hydrochloric acid. When precipitated, it is readily soluble in strong nitric acid. It is not coagulated when shaken up with ether. The addition of water to the hydrochloric solution precipitates acid-albumin.

(2.) Egg-albumin.-When injected into the blood-vessels or under the skin, or even when introduced in large quantity into the intestine, part of it appears unchanged in the urine (p. 397). When shaken with ether, it is precipitated. These two reactions serve to distinguish it from (1). The specific rotation is$37 \cdot 8^{\circ}$.

(Metalbumin and Paralbumin have been found by Scherer in ropy solutions in ovarian cysts; they are only partially precipitated by heat. The precipitate thrown down by the action of strong alcohol is soluble in water. They are not precipitated by acetic acid, by acetic acid and potassium ferrocyanide, by mercuric chloride, or by saturation with magnesium sulphate. Concentrated sulphuric acid and acetic acid give a violet colour (Adamkiewicz). According to Hammarsten, metalbumin is a mixture of paralbumin and other proteid substances. On being boiled with dilute sulphuric acid they yield a reducing substance (? sugar)).

\section{Class II._Globulins.}

They are native proteids, which are insoluble in distilled water, but are soluble in dilute saline solutions, sodium chloride of 1 per cent., and in magnesium sulphate, These solutions are coagulated by heat, and are precipitated by the addition of a large quantity of water. Most of them are precipitated from their sodium chloride solution by the addition of crystals of sodium chloride, and also by saturating their neutral solution at $30^{\circ}$ with crystals of magnesium sulphate. When acted upon by dilute acids, they yield acid-albumin, and by dilute alkalies, alkalialbumin.

(1.) Globulin (Crystallin) is obtained by passing a stream of $\mathrm{CO}_{2}$ through a watery extract of the crystalline lens.

(2.) Vitellin is the chief proteid in the yolk of egg. It is also said to occur in the chyle (?) and in the amniotic fluid (Weyl). Both of the foregoing are not precipitated from their neutral solutions by saturation with sodium chloride.

(3.) Para-globulin or Serum-globulin (p. 44).

(4.) Fibrinogen (p. 45).

(5.) Myosin is the chief proteid in dead muscle. Its coagulation in muscle post mortem constitutes rigor mortis. If muscle be repeatedly washed and afterwards treated with a 10 per cent. solution of sodium chloride, it yields a viscid fluid which, when dropped into a large quantity of distilled water, gives a white flocculent precipitate of myosin. It is also precipitated from its $\mathrm{NaCl}$ solution by crystals of $\mathrm{NaCl}$. For Kühne's method of preparation, see Muscle.

(6.) Globin (Preyer), the proteid residue of hæmoglobin. 


\section{Class III.-Derived Albumins (Albuminates).}

(1.) Acid-Albumin or Syntonin.-When proteids are dissolver in the stronger acids, e.g., hydrochloric, they become changed into acid-albumins. They are precipitated from solution by the addition of many salts $\left(\mathrm{NaCl}, \mathrm{Na}_{2} \mathrm{SO}_{4}\right)$ or by neutralisation with an alkali, e.g., sodic carbonate, but they are not precipitated by heat. The concentrated solution gelatinises in the cold, and is redissolved by heat. Syntonin, which is obtained by the prolonged action of dilute hydrochloric acid (2 per 1000) upon minced muscle, is also an acid-albumin. It is formed also in the stomach during digestion. According to Soyka, the alkali- and acidalbumins differ from each other only in so far as the proteid in the one case is united with the base (metal) and in the other with the acid.

(2.) Alkali-Albumin.-If egg- or serum-albumin be acted upon by dilute alkalies, a solution of alkali-albumin is obtained. Strong caustic potash acts upon white of egg and yields a thick jelly (Lieberkühn). The solution is not precipitated by heat, but is precipitated by the addition of an acid.

(3.) Casein is the chief proteid in milk (p. 466). It is precipitated by acids and by rennet at $40^{\circ} \mathrm{C}$. In its characters it is closely related to alkali-albuminate, but, according to $\mathrm{O}$. Nasse, it contains more $\mathrm{N}$. It contains a large amount of phosphorus (0.S3 per cent.). It may be precipitated from milk by diluting it with several times its volume of water and adding dilute acetic acid, or by adding magnesium sulphate crystals to milk and shaking vigorously. Owing to the large amount of phosphorus which it contains, it is sometimes referred to the nucleoalbumins. When it is digested with dilute $\mathrm{HCl}(0 \cdot 1$ per cent.) and pepsin at the temperature of the body, it gradually yields nuclein.

\section{Class IV.-Fibrin.}

For fibrin, see p. 39, and for the fibrin-factors, p. 43.

\section{Class V.-Peptones.}

For peptones and propeptones, see p. 331 .

\section{Class VI.-Lardacein and Other Bodies.}

There fall to be mentioned the "yelk-plates," which occur in the yelk:Ichthin (cartilaginous fishes, frog); Ichthidin (osseous fishes); Ichthulin (salmon); Emydin (tortoise-Valenciennes and Fremy); also the indigestible Amyloid substance (Virchow) or lardacein, which occurs chiefly as a pathological infiltration into various organs, as the liver, spleen, kidneys, and blood-vessels. It gives a blue with iodine and sulphuric acid (like cellulose), and a mahogany-brown with iodine. It is difficult to change it into an albuminate by the action of acids and alkalies.

\section{Class VII.-Coagulated Proteids.}

When any native albumins or globulins are coagulated, e.g., at $70^{\circ} \mathrm{C}$., they yield bodies with altered characters, insoluble in water and saline solutions, but soluble in boiling strong acids and alkalies, when they are apt to split up. They are dissolved during gastric and pancreatic digestion to produce peptones.

\section{Appendix: Vegetable Proteid Bodies.}

Plants, like animals, contain proteid bodies, although in less amount. They occur either in solution in the juices of living plants or in the solid form. In composition and reaction they resemble animal proteids. Vegetable proteids have frequently been obtained in a crystalline form (Radlkofer), e.g., from the seeds of the gourd (Grübler) and various oleaginous seeds (Ritthausen). 
1. Vegetable albumin is found dissolred in most juices of plants and closely resembles animal albumin. If the dough of wheat be washed with water, and the starch be allowed to subside, on boiling the supernatant fluid the regetable albumin is coagulated.

2. Glutin (vegetable fibrin) occurs in cereal grains, and its peculiar glutinous or sticky characters, when mixed with water, enable it to form dough. From wheat, which may contain as much as 17 per cent., it is prepared by washing away all the starch from the dough with a stream of water. This is best effected by washing the dough in a muslin bag or orer a fine siere. It is elastic, gray, insoluble in water and alcohol, and soluble in dilute acids ( $1 \mathrm{HCl}$ per 1000), and in alkalies. Glutin is a complex substance. If it be boiled with water a sticky rarnish-like mass is obtained, gliadin (animal gelatin). If this substance is treated with strong alcohol it dissolres, bat a slimy body remains undissolred, mucedin. If glutin be digested with alcohol, a brownish-yellow substance, giutinfilorin (Ritthausen) is extracted from it.

3. Vegetable casein occurs specially in the leguminosæ. It is slightly soluble in water, but readily soluble in weak alkalies, and in solutions of basic calcic phosphate. These solutions, like animal casein, are precipitated by acids or rennet. The rarieties of it are-(a) Legumin in peas, beans, lentils (Eirhof, 1805); it has an acid reaction, is insoluble in water, easily soluble in dilute alkalies, and in rery dilute $\mathrm{HCl}$ or acetic acid; $(b)$ the casein-like body occurring in hops and almonds which closely resembles $(a)$, and is called conglutin (Ritthansen). Vegetable casein, like animal casein, is an alkali-albuminate, and is precipitated by the same substances; it is not precipitated by boiling. When long exposed to the air, its solution coagulates with the formation of lactic acid.

\section{0. (2.) The Albuminoids.}

These substances closely resemble true proteids in their composition and origin, and are amorphous non-crystalline colloids; some of them do not contain S, but the most of them hare not been prepared free from ash. Their reactions and decomposition products closely resemble those of the proteids; some of them produce, in addition to leucin and tyrosin, glycin and alanin (amido-propionic acid). They occur as organised constituents of the tissues and also in a fluid form. It is unknown whether they are formed by oxudation from proteid bodies or by synthesis.

1. Macin is the characteristic substance present in mucus. It contains no $\mathrm{S}$. That obtained from the sub-maxillary gland contains C. 52.31, H. 7•22, N. 11·84, 0. 28.63 (Obolensky). It dissolres in water, making it sticky or slimy, and can be filtered. It is precipitated by acetic acid and alcohol; and the alcohol precipitate is again soluble in water. It is not precipitated by acetic acid and ferro-cyanide of potassium, but $\mathrm{H}_{\mathrm{N}} \mathrm{OO}_{3}$ and other mineral acids precipitate it (Scherer). It occurs in salira (p. 292), in bile, in mucous glands, secretions of mucons membranes, in mucous tissue, in synoria, and in tendons (A. Rollet). Pathologically it occurs not unfrequently in eysts; in the animal kingdom, especially in snails and in the skin of holothurians (Eichwald). It yields leucin and 7 per cent. of tyrosin when it is decomposed by prolonged boiling with sulphuric acid.

2. Nuclein (Miescher-p. 409) C. 29, H. 49, N. 9, P. 3, 0. 22, is slightly soluble in rater, easily in ammonia, alkaline carbonates, strong $\mathrm{HNO}_{3}$; it gires the biuret-reaction; no reaction with Millon's reagent; when decomposed it yields phosphorus. It occurs in the nuclei of pus and blood-corpuscles (p. 36), in spermatozoids, yelk-spheres, liver, brain, and milk, yeast, fungi, and many seeds. Its most remarkable characteristic is the large quantity of phosphorus it contains, nearly 10 per cent. Hypoxanthin and guanin have been obtained as decomposition products from it (Kossel). 
3. Keratin occurs in all horny and epidermic tissues (epidermic scales, hairs, nails, feathers)-C. $50 \cdot 3-52.5$; H. 6.4-7 ; N. $16 \cdot 2-17 \cdot 7$; O. 20.8-25 ; S. 0.7-5 per cent., is soluble only in boiling caustic alkalies, but swells up in cold concentrated acetic acid. When decomposed by $\mathrm{H}_{2} \mathrm{SO}_{4}$ it yields 10 per cent. leucin and 3.6 per cent. tyrosin.

4. Fibroin is soluble in strong alkalies and mineral acids, in ammoniosulphate of copper ; when boiled with $\mathrm{H}_{2} \mathrm{SO}_{4}$ it yields 5 per cent. tyrosin, leucin, and glycin. It is the chief constituent of the cocoons of insects and threads of spiders.

5. Spongin, allied to fibroin, occurs in the bath-sponge, and yields as decomposition products, leucin and glycin (Städeler).

6. Elastin, the fundamental substance in elastic tissue, is soluble only when boiled in concentrated caustic potash (C. 55-55.6; H. 7.1 $7 \cdot 7$; N. 16.1-17.7; $0.19 \cdot 2-21 \cdot 1$ per cent.) It yields $36-45$ per cent. of leucin and $\frac{1}{2}$ per cent. of tyrosin.

7. Gelatin, obtained from connective-tissues by prolonged boiling with water; it gelatinises in the cold (C. 52.2-50.7; H. 6.6-7.2; N. 17.9-1s.S ; S. $+0,23.5-25$; (S. 0.6 per cent.). [The ordinary connective-tissues are supposed to contain the hypothetical anhydride collagen, while the organic basis of bone is called ossein.] It rotates the ray of polarised light strongly to the left. By prolonged boiling and digestion it is converted into a peptone-like body (gelatinpeptone), which does not gelatinise (p. 332). [It swells up, but does not dissolve in cold water; when dissolved in warm water and tinged with Berlin blue or carmine it forms the usual coloured mass which is employed by histologists for making fine transparent injections of blood-ressels.] A body resembling gelatin is found in leukæmic blood and in the juice of the spleen (p. 206). When decomposed with sulphuric acid it yields glycin, ammonia, leucin, but no tyrosin. It gives insoluble precipitates with mercuric chloride and tannin.

8. Chondrin (Joh. Muiller) occurs in the matrix of hyaline cartilage and between the fibres in fibro-cartilage. It is obtained from hyaline cartilage and the cornea by boiling. It occurs also in the mantie of molluses (C. 49.5-50.9; H. 6.6-7 $\cdot 1$; N. $14 \cdot 4-14 \cdot 9 ; S+0.27 \cdot 2-29$; S. 0.4 per cent.). When boiled with sulphuric acid it yields leucin; with hydrochloric acid, and when digested chondro-glucose (Meissner); it belongs to the glucosides, which contain $\mathrm{N}$. When acted upon by oxidising reagents it is converted into gelatin (Brame). The substance which yields chondrin is called chondrogen, which is perhaps an anhydride of chondrin. The following properties of gelatin and chondrin are to be noted:-

\begin{tabular}{|c|c|c|}
\hline Reagent. & Gelatin. & Cliondrin. \\
\hline $\begin{array}{l}\text { Acids, } \\
\text { Tannic acid, mercuric } \\
\text { chloride, } \\
\text { Chlorine water, platinic } \\
\text { chloride, . } \\
\text { Alum, silver, iron, col- } \\
\text { per, lead salts, } \\
\text { Potassic ferrocyanide and } \\
\text { acetic acid, . } \\
\text { Alcolol, }\end{array}$ & $\begin{array}{l}\text { Not precipitated, } \\
\text { Precipitated, . } \\
\text { Precipitated. } \\
\text { Precipitated, . } \\
\text { Not precipitated. } \\
\text { Precipitated, precipitate } \\
\text { soluble in water. }\end{array}$ & $\begin{array}{l}\text { Precipitated by acetic } \\
\text { acid, dilute } \mathrm{HCl} \text { and } \\
\mathrm{H}_{2} \mathrm{SO}_{4} \text {. } \\
\text { Give slight opalescence. } \\
\text { Precipitated copiously. }\end{array}$ \\
\hline Specific Rotation, . & $-130^{\circ}$ & $-213^{\circ}$ \\
\hline
\end{tabular}


9. The hydrolytic ferments have recently been called Enzymes by $W$. Kühne, in order to distinguish them from organised ferments, such as yeast. The enzymes, hydrolytic or organic ferments, act only in the presence of water. They act upon certain bodies causing them to take up a molecule of water. They all decompose hydric peroxide into water and 0 . They are most active between 30 $35^{\circ} \mathrm{C}$, and are destroyed by boiling, but when dry they may be subjected to a temperature of $100^{\circ}$ without being destroyed. Their solutions, if kept for a long time, gradually lose their properties and undergo more or less decomposition.

[Table showing the unorganised ferments present in the body, and their actions :-

\begin{tabular}{|c|c|c|}
\hline Fluid or Tissues. & Ferment. & Actions. \\
\hline Saliva, . $\cdot\{$ & $\begin{array}{l}\text { 1. Ptyalin, - . } \\
\text { (See also p. 296.) }\end{array}$ & Converts Starch chiefly into Maltose. \\
\hline Gastric Juice, \{ & $\begin{array}{l}\text { 1. Pepsin, . . } \\
\text { 2. Milk-curdling, } \\
\text { 3. Lactic AcidFerment, } \\
\text { 4. Fat-splitting, . }\{\end{array}$ & $\begin{array}{l}\text { Converts Proteids into Peptones in an } \\
\text { Acid Medium, certain by-products } \\
\text { being formed (p. 331). } \\
\text { Curdles Casein of Milk. } \\
\text { Splits up Milk-sugar into Lactic Acid. } \\
\text { Splits up Fats into Glycerin and } \\
\text { Fatty Acids. }\end{array}$ \\
\hline $\begin{array}{c}\text { Pancreatic } \\
\text { Juice, . }\end{array}$ & 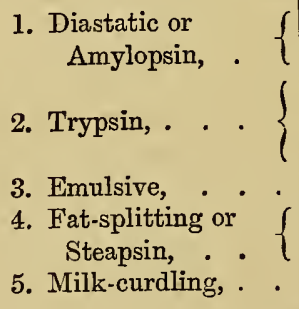 & $\begin{array}{l}\text { Converts Starch chiefly into Maltose. } \\
\text { Changes Proteids into Peptones in } \\
\text { an Alkaline Medium, certain by- } \\
\text { products being formed (p. 341). } \\
\text { Emulsifies Fats. } \\
\text { Splits Fats into Glycerin and Fatty } \\
\text { Acids. } \\
\text { Curdles Casein of Milk. }\end{array}$ \\
\hline $\begin{array}{l}\text { Intestinal } \\
\text { Juice, }\end{array}$ & $\begin{array}{l}\text { 1. Diastatic, . . } \\
\text { 2. Proteolytic, . . . } \\
\text { 3. Invertin, . . . } \\
\text { 4. Milk-curdling, . }\end{array}$ & $\begin{array}{l}\text { Does not form Maltose, but Maltose is } \\
\text { changed into Glucose (p. 370). } \\
\text { Fibrin into Peptone (?). } \\
\text { Changes Cane- into Grape-Sugar. } \\
\text { (? in Small Intestine.) }\end{array}$ \\
\hline $\begin{array}{l}\text { Blood, } \cdot \cdot \cdot \\
\text { Chyle, } \cdot \cdot \\
\text { Liver, } \quad \cdot \quad \cdot \\
\text { Milk, . } \cdot \text {. } \\
\text { Most Tissues, }\end{array}$ & Diastatic Ferments. & \\
\hline $\begin{array}{l}\text { Muscle, } \cdot \text {. } \\
\text { Urine, } \cdot \text {. }\end{array}$ & Pepsin. & \\
\hline Blood, $\cdot\{$ & $\begin{array}{l}\text { Fibrin-forming } \\
\text { Ferment. }\end{array}$ & (Modified from W. Roberts).] \\
\hline
\end{tabular}


(a.) Sugar-forming or diastatic ferment occurs in saliva (p. 294), pancreatic juice (p. 340), intestinal juice (p. 335), bile (p. 366), blood (p. 36), chyle (p. 410), liver (p. 351), in human milk (p. 465). Invertin in intestinal juice (p. 370).(Cl. Bernard.)

Almost all dead tissues, organic fluids, and even proteids, although only to a slight degrec, may act diastatically. Diastatic ferments are very generally distributed in the vegetable kingdom.

(b.) Proteolytic or Ferments which act upon proteids.-Pepsin in gastric juice and in muscle (p. 332), in vetches, myxomycetes (Krukenberg), trypsin in the pancreatic juice (p. 341), and a similar ferment in the intestinal juice (p. 370).

(c.) Fat-decomposing in pancreatic juice (p. 343) in the stomach (p. 335).

(d.) Milk-coagulating in the stomach (p. 335), pancreatic juice (p. 344), and perhaps also in the intestinal juice (?)-W. Roberts.

[The importance of fermentative processes has alrcady been referred to in detail under "Digestion." Ferments are bodies which excite chemical changes in other matter with which they are brought into contact. They are divided into two classes :-

(1.) Unorganised, soluble or non-living.

(2.) Organised, or living.

(1.) The Unorganised ferments are those mentioned in the above table. They seem to be nitrogenous bodies, although their exact composition is unknown, and it is doubtful if they have ever been obtained perfectly pure. They are produced within the body, in many secretions, by the vital activity of the protoplasm of cells. They are termed soluble because they are soluble in water, glycerine, and some other substances (p. 295), while they can be precipitated by alcohol and some other reagents. They do not multiply during their activity, nor is their activity prevented by a certain proportion of salicylic acid. They are not affected by oxygen subjected to the compression of many atmospheres (P. Bert). They are non-living. Their other properties are referred to above].

[(2.) The Organised or living ferments are represented by yeast (p. 474). Other living ferments belonging to the schizomycetes, occurring in the intestinal canal, are referred to in $\$ 184$. Yeast causes fermentation by splitting up sugar into $\mathrm{CO}_{2}$ and alcohol (p. 298), but this result only occurs so long as the yeast is living. Hence, its activity is coupled with the vitality of the cells of the yeast. If yeast be boiled, or if it be mixed with carbolic or salicylic acid, or chloroform, all of which destroy its activity, it cannot produce the alcoholic fermentation. As yet no one has succeeded in extracting from yeast a substance which will excite the alcoholic fermentation. All the organised ferments grow and multiply during their activity at the expense of the substances in which they occur. Thus the alcoholic fermentation depends upon the "life" of the yeast. They are said to be killed by oxygen subjected to the compression of many atmospheres (P. Bert). But it is important to note that Hoppe-Seyler has extracted from dead yeast (killed by ether), an unorganised ferment which can change cane-sugar into grapesugar.

All purely physiological processes in the body, except some in the intestinal canal, depend upon unorganised ferments].

10. Hæmoglobin, the colouring matter of blood, which, in addition to $\mathrm{C}, \mathrm{H}, \mathrm{O}, \mathrm{N}$, and $\mathrm{S}$, contains iron, may be taken with the albuminoids (p. 23). 


\section{(3.) Glucosides containing Nitrogen.}

In addition to chondrin, the following glucosides containing nitrogen, when subjected to hydrolytic processes, may combine with water, and form sugar and other substances:-

Cerebrin (see Nervous System) $=\mathrm{C}_{57} \mathrm{H}_{110} \mathrm{~N}_{2} \mathrm{O}_{25}$ (Geoghegan).

Protagon occurs in nerves, and contains phosphorus.

Chitin, $2\left(\mathrm{C}_{15} \mathrm{H}_{26} \mathrm{~N}_{2} \mathrm{O}_{10}\right)$, is a glucoside containing nitrogen, and occurs in the cutaneous coverings of arthropoda, and also in their intestine and tracheæ; it is soluble in concentrated acids, e.g., hydrochloric or nitric acid, but insoluble in other reagents. According to Sandwick, chitin is an amin-derivative of a carbohydrate with the general formula $\mathrm{n}\left(\mathrm{C}_{12} \mathrm{H}_{20} \mathrm{O}_{10}\right)$. The hyalin of worms is closely related to chitin. (Solanin, amygdalin, p. 49, and salicin, \&c., are glucosides of the vegetable kingdom.)

\section{(4.) Colouring Matters containing Nitrogen.}

Their constitution is unknown, and they occur only in animals. They are in all probability derivatives of hæmoglobin. They are-(1) homatin (p. 33) and hoematoidin (p. 33). (2) Bile-pigments (p. 357). (3) Urine-pigments (except Indican). (4) Melanin, C. 44.2, H3, N.9.9, O.42*6, or the black pigment, which occurs partly in epithelium (choroid, retina, iris, and in the deep layers of epidermis in coloured races) and partly in connective-tissue corpuscles (Lamina fusca of the choroid).

\section{II.-Organic Acids free from Nitrogen.}

(1.) The fatty acids with the formula $\mathrm{C}_{n} \mathrm{H}_{2 \mathrm{n}-1} \mathrm{O}(\mathrm{OH})$ occur in the body partly free and partly in combination. Free volatile fatty acids occur in decomposing cutaneous secretions (sweat). In combination, acetic acid and caproic acid occur as amido-compounds in glycin (= amido-acetic acid), and leucin (= amidocaproic acid). More especially do they occur united with glycerine to form neutral fats, from which the fatty acid is again set free by pancreatic digestion (p. 343).

(2.) The acids of the acrylic acid series, with the formula $\mathrm{C}_{n} \mathrm{H}_{2 \mathrm{n}-3} \mathrm{O}(\mathrm{HO})$, are represented in the body by one acid: oleic acid, which in combination with glycerine yields the neutral fat olein.

\section{Fats.}

Fats occur very abundantly in animals, but they also occur in all plants; in the latter more especially in the seeds (nuts, almonds, cocoa nut, poppy), more rarely in the pericarp (olive) or in the root. They are obtained by pressure, melting, or by extracting them with ether or boiling alcohol. They contain much less 0 than the carbohydrates, such as sugar and starch; they give a greasy spot on paper, and when shaken with colloid substances, such as albumin, they yield an emulsion. When treated with superheated steam, or with certain ferments (p. 507, c), they take up water and yield glycerine and fatty acids, and if the latter be volatile they have a rancid odour. Treated with caustic alkalies they also take up water, and are decomposed into glycerine and fatty acids; the fatty acid unites with the alkali and forms a soap, while glycerine is set free. The soapsolution dissolves fats. 
Glycerine is a tri-atomic alcohol, $\mathrm{C}_{3} \mathrm{H}_{5}(\mathrm{OH})_{3}$, and unites with (1) the following mono-basic fatty acids (those occurring in the body are printed in italics) :-

\begin{tabular}{|c|c|c|c|c|c|}
\hline 1. Formic & acid, & $\mathrm{C} \mathrm{H}_{2} \mathrm{O}_{2}$ & 10. Capric & acid, & $\mathrm{C}_{10} \mathrm{H}_{20} \mathrm{O}_{2}$ \\
\hline 2. Acetic & " & $\mathrm{C}_{2} \mathrm{H}_{2} \mathrm{O}_{2}$ & 11. Laurostearic & " & $\mathrm{C}_{12} \mathrm{H}_{24} \mathrm{O}_{2}$ \\
\hline 3. Propionic & ", & $\mathrm{C}_{3} \mathrm{H}_{6} \mathrm{O}_{2}$ & 12. Myristic & ", & $\mathrm{C}_{14} \mathrm{H}_{28} \mathrm{O}_{2}$ \\
\hline 4. Butyric & ", & $\mathrm{C}_{4} \mathrm{H}_{3} \mathrm{O}_{2}$ & 13. Palmitic & " & $\mathrm{C}_{16} \mathrm{H}_{32} \mathrm{O}_{2}$ \\
\hline [Isobutyric & , & $\left.\begin{array}{lll}\mathrm{C}_{4} & \mathrm{H}_{8} & \mathrm{O}_{2}\end{array}\right]$ & [Margaric & & $\mathrm{C}_{17} \mathrm{H}_{34} \mathrm{O}_{2}$ \\
\hline 5. Valerianic & " & $\mathrm{C}_{5} \mathrm{H}_{10} \mathrm{O}_{2}$ & \multicolumn{3}{|c|}{ is a mixture of 13 and 14.] } \\
\hline 6. Caproic & 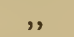 & $\mathrm{C}_{6} \mathrm{H}_{12} \mathrm{O}_{2}$ & 14. Stearic & acid, & $\mathrm{C}_{18} \mathrm{H}_{36} \mathrm{O}_{2}$ \\
\hline 7. Enanthylic & ," & $\mathrm{C}_{7} \mathrm{H}_{14} \mathrm{O}_{2}$ & 15. Arachinic & 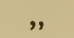 & $\mathrm{C}_{20} \mathrm{H}_{40} \mathrm{O}_{2}$ \\
\hline 8. Caprylic & $"$ & $\mathrm{C}_{8} \mathrm{H}_{16} \mathrm{O}_{2}$ & 16. Hyänic & " & $\mathrm{C}_{25} \mathrm{H}_{50} \mathrm{O}_{2}$ \\
\hline 9. Pelargonic & ", & $\mathrm{C}_{9} \mathrm{H}_{18} \mathrm{O}_{2}$ & 17. Cerotinic & ", & $\mathrm{C}_{27} \mathrm{H}_{54} \mathrm{O}_{2}$ \\
\hline
\end{tabular}

The acids form a homologous series with the formula $\mathrm{C}_{n} \mathrm{H}_{2 \mathrm{n}-1} \mathrm{O}(\mathrm{OH})$. With every $\mathrm{CH}_{2}$ added their boiling point rises $19^{\circ}$. Those containing most carbon are solid, and non-volatile; those containing less $\mathrm{C}$ (up to and including capric acid) are fluid like oil, have a burning acid taste, and a rancid odour.

The earlier members of the series may be obtained by oxidation from the later, by $\mathrm{CH}_{2}$ being removed, while $\mathrm{CO}_{2}$ and $\mathrm{H}_{2} \mathrm{O}$ are formed; thus, butyric acid is obtained from propionic acid.

Nos. 13 and 14 are found in human and animal fat, less abundant and more inconstant are $12,11,6,8,10,4$. Some occur in sweat, and in milk (p. 465). Many of them are developed during the decomposition of albumin and gelatin. Most of the above (except 15-17) occur in the contents of the large intestine (p. 376).

(2.) Glycerine also unites with the mono-basic oleic acid, which also forms a series, whose general formula is $\mathrm{C}_{\mathrm{n}} \mathrm{H}_{2 \mathrm{n}}-{ }_{3} \mathrm{O}(\mathrm{OH})$; and they all contain $2 \mathrm{H}$ less than the corresponding members of the fatty acid series. The corresponding fatty acids can be obtained from the oleic acid series and vice versa. Oleic acid (olein-elainic acid), $\mathrm{C}_{1 \mathrm{~s}} \mathrm{H}_{34} \mathrm{O}_{2}$, is the only one found in the organism; united with glycerine, it forms the fluid fat, olein (Gottlieb, 1846). The fat of new-born children contains more glyceride of palmitic and stearic acid than that of adults, which contains more glyceride of oleic acid (L. Langer). Oleic acid also occurs united with alkalies (in soaps), and (like some fatty acids) in the lecithins (p. 36). If lecithin be acted on with barium hydrate, we obtain insoluble stearic, or oleic, or palmitic acids and barium oleate, together with dissolved neurin and baric glycerinphosphate. It appears as if there were several lecithins, of which the most abundant are the one with stearic acid and that with palmitin +oleic acid radicle (Diakonow).

The neutral fats, the glycerides of fatty acids, and of oleic acid, are triple ethers of the tri-atomic alcohol glycerine.

With the neutral fats may be associated glycerin-phosphoric acid, an acid glycerin-ether, formed by the union of glycerine and phosphoric acid, with the giving off of a molecule of water $\left(\mathrm{C}_{3} \mathrm{H}_{9} \mathrm{PO}_{6}\right)$; it is a decomposition product of lecithin (p. 36).

(3.) The glycolic acids-(acids of the lactic acid series) have the formula $\mathrm{C}_{n} \mathrm{H}_{2 \mathrm{n}-2} \mathrm{O}(\mathrm{OH})_{2}$. They are formed by oxidation from the fatty acid series by substituting $\mathrm{OH}$ (hydroxyl) for 1 atom of $\mathrm{H}$ of the fatty acids. Conversely, fatty acids may be obtained from the glycolic acids. The following acids of this series occur in the body :-

(a.) Carbonic Acid (oxy-formic acid) $\mathrm{CO}(\mathrm{OH})_{2}$; in this form, however, it only makes salts. Free carbonic acid or carbon dioxide is an anhydride of the same $=\mathrm{CO}_{2}$.

(b.) Glycolic Acid (oxy-acetic acid), $\mathrm{C}_{2} \mathrm{H}_{2} \mathrm{O}(\mathrm{OH})_{2}$, does not occur free in the body. 
One of its compounds, glycin (glycocoll, amidoacetic acid, or gelatin-sugar), occurs as a conjugate acid, viz., as glycocholic acid in the bile (p. 355), and as hippuric acid in the urine. Glycin exists in complex combination in gelatin.

(c.) Lactic Acid (oxy-propionic acid), $\mathrm{C}_{3} \mathrm{H}_{4} \mathrm{O}(\mathrm{OH})_{2}$, occurs in the body in two isomeric forms-1. The ethylidene-lactic acid, which occurs in two modificationsas the right rotatory sarcolactic acid (paralactic), a metabolic product of muscle; and as the ordinary optically inactive product of "lactic fermentation," which occurs in gastric juice, in sour milk (sauerkraut, acid cucumber), and can be obtained by fermentation from sugar (p. 373). 2. The isomer, ethylene-lactic acid, occurs in the watery extract of muscles.

(d.) Leucic acid (oxy-caproic acid), $\mathrm{C}_{6} \mathrm{H}_{12} \mathrm{O}_{3}$, does not occur as such, but only in the form of one of its derivatives, leucin (amido-caproic acid), as a product of the metabolism in many tissues, and is formed during pancreatic digestion (p. 342). Leucic acid may be prepared from leucin, and glycolic acid from glycin by the action of nitrous acid.

(4.) Acids of the 0xalic Acid or Succinic Acid Series having the formula, $\mathrm{C}_{n} \mathrm{H}_{2 n-4} \mathrm{O}_{2}(\mathrm{OH})_{2}$, are bi-basic acids, which are formed as completely oxidised products by the oxidation of fatty acids and glycolic acid $\left(\mathrm{H}_{2} \mathrm{O}\right.$ being removed); and it is important to note their origin from substances rich in carbon, e.g., fats, carbohydrates, and proteids.

(a.) Oxalic Acid, $\mathrm{C}_{2} \mathrm{O}_{2}(\mathrm{OH})_{2}$, arises from the oxidation of glycol, glycin, cellulose, sugar, starch, glycerine, and many vegetable acids-it occurs in the urine as calcium oxalate.

(b.) Succinic Acid, $\mathrm{C}_{4} \mathrm{H}_{4} \mathrm{O}_{2}(\mathrm{OH})_{2}$, has been found in small amount in animal solids and fluids; spleen, liver, thymus, thyroid; in the fluids of echinococcus, of hydrocephalus, and of hydrocele, and more abundantly in dog's urine after fatty and flesh food; in rabbit's urine after feeding with yellow turnips. It is also formed in small amount during alcoholic fermentation (p. 298).

(5.) Cholalic Acids in the bile (p. 356) and in the intestine (p. 367).

(6.) Aromatic Acids-Benzoic acid (= phenyl-formic acid) occurs in urine united with glycin, as hippuric acid (see Urine).

\section{III.-Alcohols.}

Alcohols are those bodies which originate from carbohydrates, in which the radicle hydroxyl $(\mathrm{HO})$ is substituted for one or more atoms of $\mathrm{H}$. They may be regarded as water, $\underset{\mathrm{H}}{\mathrm{H}}\} \mathrm{O}$, in which the half of the $\mathrm{H}$ is replaced by a $\mathrm{CH}$ compound. Thus, $\mathrm{C}_{2} \mathrm{H}_{6}$ (ethyl-hydrogen) passes into $\underset{\mathrm{H}}{\mathrm{C}} \mathrm{C}_{5}$ O (ethylic alcohol).

(a.) Cholesterin, $\left.\mathrm{C}_{26} \mathrm{H}_{43} \mathrm{H}\right\}$, is a true mon-atomic alcohol, and occurs in blood, yelk, brain, bile (p. 358), and generally in vegetable cells.

(b.) Glycerine, $\mathrm{C}_{2} \mathrm{H}_{5}\left\{\begin{array}{l}\mathrm{OH} \\ \mathrm{OH} \\ \mathrm{OH}\end{array}\right.$ is a tri-atomic alcohol. It occurs in neutral fats united with fatty acids and oleic acid; it is formed by the splitting-up of neutral fats during pancreatic digestion (p. 343), and during the alcoholic fermentation (p. 298).

(c.) Phenol (= phenylic acid, carbolic acid, oxybenzol, p. 376).

(d.) Brenzkatechin (= dioxybenzol).

(e.) The Sugars are closely related to the alcohols, and they may be regarded as polyatomic alcohols. Their constitution is unknown. Together with a series of closely-related bodies they form the great group of the Carbohydrates, some of which occur in the animal body, while others are widely distributed in the végetable kingdom. 


\section{The Carbohydrates.}

These substances, which occur in plants and animals, have received their name, because in addition to $\mathrm{C}$ (at least 6 atoms), they contain $H$ and $O$, in the proportion in which these occur in water. They are all solid, chemically indifferent, and without odour. They have either a sweet taste (sugars), or can be readily changed into sugars by the action of dilute acids; they rotate the ray of polarised light either to the right or left; as far as their coustitution is concerned, they may be regarded as fatty bodies, as hexatomic alcohols, in which $2 \mathrm{H}$ are wanting.

They are divided into the following group:-

I. Division. Glucoses $\left(\mathrm{C}_{6} \mathrm{H}_{12} \mathrm{O}_{6}\right)$-(I) Grape-sugar (glucose, dextrose, or diabetic sugar) occurs in minute quantities in the blood, chyle, muscle (? liver), urine, and in large amount in the urine in diabetes mellitus (p. 352). It is formed by the action of diastatic ferments upon other carbohydrates, during digestion. In the vegetable kingdom, it is extensively distributed in the sweet juices of many fruits and flowers (and thus it gets into honey). It is formed from cane-sugar, maltose, dextrin, glycogen, and starch, by boiling with dilute acids. It crystallises in warty masses with one molecule of water of crystallisation; unites with bases, salts, acids, and alcohols, but is easily decomposed by bases; it reduces many metallic oxides (p. 297). Fresh solutions have a rotatory power of $+106^{\circ}$. By fermentation with yeast, it splits up into alcohol and $\mathrm{CO}_{2}$ (p. 298); with decomposing proteids, it splits into two molecules of lactic acid (p. 373); the lactic acid splits up under the same conditions in alkaline solutions, into butyric acid, $\mathrm{CO}_{2}$ and $\mathrm{H}$. For the qualitative and quantitative estimation of glucose, see $\$ 149$ and $\$ 150$. In alcoholic solution, it forms very insoluble compounds with chalk, barium, or potassium, and it also forms a crystalline compound with common salt.

(2.) Galactose, obtained by boiling milk-sugar (lactose) with dilute mineral acids; it crystallises readily, is very fermentable, and gives all the reactions of glucose. When oxidised with nitric acid it becomes transformed into mucic acid. Its specific rotatory power $=+88 \cdot 08^{\circ}$.

(3.) Laevulose (left-fruit-, invert- or mucin-sugar) occurs as a colourless syrup in the acid-juices of some fruits and in honey; is non-crystallisable, and insoluble in alcohol; specific rotatory power $=-106^{\circ}$. It is formed normally in the intestine (p. 370), and occurs rarely as a pathological product in urine.

II. Division contains carbohydrates with the formula $\mathrm{C}_{12} \mathrm{H}_{22} \mathrm{O}_{11}$, and which may be regarded as anhydrides of the first division-(1) Milk-sugar or lactose occurs only in milk, crystallises in cakes (with one molecule of water) from the syrupy concentrated whey; it rotates polarised light to the right $=+59 \cdot 3$, and is much less soluble in water and alcohol than grape-sugar. When boiled with dilute mineral acids it passes into galactose, and can be directly transformed into lactic acid only by fermentation; the galactose, however, is capable of undergoing the alcoholic fermentation with yeast (Koumis preparation, p. 468). For its quantitative estimation, see Milk.

(2.) Maltose $\left(\mathrm{C}_{12} \mathrm{H}_{22} \mathrm{O}_{11}\right)+\mathrm{H}_{2} \mathrm{O}$ (O'Sullivan) has one molecule of water less than grape-sugar $\left(\mathrm{C}_{12} \mathrm{H}_{24} \mathrm{O}_{12}\right)$, is formed during the action of a diastatic ferment, such as saliva upon starch (p. 294); is soluble in alcohol, right rotatory power= $150^{\circ}$, it is crystalline, while its reducing power is only two-thirds that of dextrose.

(3. Saccharose (cane-sugar) occurs in sugar-cane and some plants, it does not reduce solutions of copper, is insoluble in alcohol, is right rotatory, and not capable of fermentation. When boiled with dilute acids, it becomes changed into a mixture of easily fermentable glucose (right-rotatory) and laevulose (invert-sugar) which ferments with difficulty and is left-rotatory (p. 370). When oxidised with uitric acid, it passes into glucic acid and oxalic acid.) 
(4. Melitose, from Eucalyptus-manna; Melezitose, from Larch-manna; Trehalose (Mycose), from Ergot; all right-rotatory, and do not reduce alkaline cupric solutions.)

III. Division, contains carbohydrates with the formula, $\mathrm{C}_{6} \mathrm{H}_{10} \mathrm{O}_{5}$, which may be regarded as anhydrides of the second division.

1. Glycogen, with a rotatory power of $211^{\circ}$ (Böhm and Hoffmann, Külz), does not reduce cupric oxide. It occurs in the liver (p. 350), muscles, many embryonic tissues, the embryonic area of the chick (Külz), in normal and.pathological epithelium (Schiele), and according to Pavy, in the spleen, pancreas, kidney, ovum, brain and blood, together with a small amount of glucose. It also occurs in the oyster and some of the molluscs (Bizio).

2. Dextrin was discovered by Limpricht in the muscles of the horse. It is right-rotatory $=+138^{\circ}$, soluble in water and forms a very sticky solution, from which it is precipitated by alcohol or acetic acid; it is tinged slightly red with iodine. It is formed in roasted starch, (hence it occurs in large quantity in the crust of bread-see Bread, p. 472), by dilute acids, and in the body by the action of ferments (p. 294). It is formed from cellulose by the action of dilute sulphuric acid. It occurs in beer, and is found in the juices of most plants.

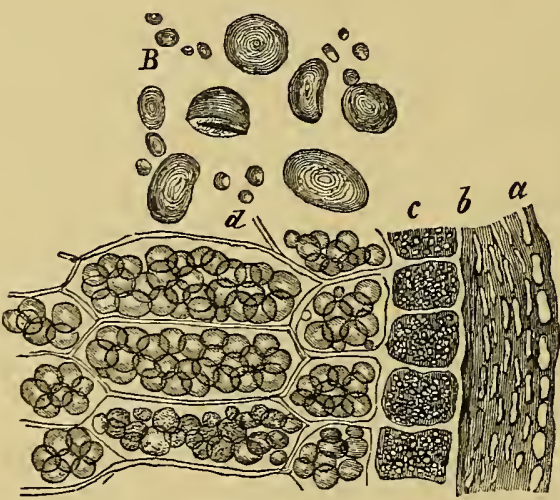

Fig. 176.

Section of a wheat grain- $d$, starch-corpuscles within regetable cells; $B$, starch-corpuscles with concentric markings (See also Fig. 173).

(3. Amylum or Starch occurs in the "mealy" parts of many plants, is formed within vegetable cells, and consists of concentric layers with an excentric nucleus (Fig. 176, B) The diameter of starch grains varies greatly with the plant from which they are derived. At $72^{\circ} \mathrm{C}$. it swells up in water and forms mucilage; in the cold, iodine colours it blue. Starch grains always contain more or less cellulose and a substance which is coloured red with iodine (erythrogranulose) (see p. 294). It and glycogen are transformed into dextrose by certain digestive ferments in the saliva, pancreatic and intestinal juices, and artificially by boiling with dilute sulphuric acid.)

(4. Gum occurs in vegetable juices (specially in acaciæ and mimosæ), is partly soluble in water (arabin), partly swells up like mucin (bassorin). Alcohol precipitates it.)

(5. Inulin, a crystalline powder occurring in the root of chicory, dandelion, and specially in the bulbs of the dahlia; it is not coloured blue by iodine.)

(6. Lichenin occurs in the intercellular substance of Iceland moss (Cetraria islandica) and algæ; is transformed into glucose by dilute sulphuric acid.)

(7. Paramylum occurs in the form of granules resembling starch, in the infusorian, Euglena viridis.)

(8. Cellulose occurs in the cell-walls of all plants (in the exo-skeleton of arthropoda, and the skin of snakes); soluble only in ammonio-cupric oxide; rendered blue by sulphuric acid and iodine. Boiled with dilute sulphuric acid, it yields dextrin and glucose. Concentrated nitric acid mixed with sulphuric acid changes it (cotton) into nitro-cellulose (gun cotton) $\mathrm{C}_{6} \mathrm{H}_{7}\left(\mathrm{NO}_{2}\right)_{3} \mathrm{O}_{5}$, which dissolves in a mixture of ether and alcohol and forms collodion.) 
(9. Tunicin is a substance rescmbling cellulose, and occurs in the integument of the tunicata or ascidians.)

IV. Division contains the carbohydrates which do not ferment.

1. Inosit (phasco-mannit, musçle-sugar) occurs in muscle (Scherer), lung, liver, spleen, kidney, brain of ox, human kidney; pathologically in urine and the fluid of echinococcus. In the regetable kingdom, in beans (leguminosa), and the juice of the grape. It is an isomer of grape-sugar; optically it is inactive, crystallises in warts with two molecules of water, in long monoclinic crystals; it has a sweet taste, is insoluble in water, does not give Trommer's reaction, is capable of undergoing only the sarcolactic acid fermentation. (Nearly allied are Sorbin from sorbic acid-Scyllit from the intestines of the hag-fish and skate-and Eukalyn arising from the fermentation of melitose.)

\section{IV.-Derivatives of Ammonia and their Compounds.}

The anımonia derivatives are obtained from the proteids, and are decomposition products of their netabolism.

(1.) Amines, i.e., compound ammonias which can be obtained from anmonia $\left(\mathrm{NH}_{3}\right)$, or from ammonium-hydroxide $\left(\mathrm{NH}_{4}-\mathrm{OH}\right)$, by replacing one or all the atolus of $\mathrm{H}$ by groups of carbohydrates (alcohol raclicals). The amine derived from one molecule of ammonia is called moncmine. We are only acquainted with
$\left.\begin{array}{r}\mathrm{H} \\ \mathrm{H} \\ \mathrm{CH}_{3}\end{array}\right\} \mathrm{N}$
Methylamine and Tri-Methylamine
$\mathrm{CH}_{3}$ )
$\mathrm{CH}_{3}, \mathrm{~N}$,
$\mathrm{CH}_{3}$ )

as decomposition products of cholin (neurin) and of kreatin. Neurin occurs in lecithin in a very complex combination (see Lecithin under Fats, and also p. 36).

(2.) Amides, i.e., derivatives of acids, which have exchanged the hydroxyl (HO) of the acids for $\mathrm{NH}_{2}$. Urea, $\mathrm{CO}\left(\mathrm{NH}_{2}\right)_{2}$, the biamid of $\mathrm{CO}_{2}$, is the chief endproduct of the metabolism of the nitrogenous constitnents of our bodies (see Urine). Carbonic acid containing water $=\mathrm{CO}(\mathrm{OH})_{2}$; in it both $\mathrm{OH}$ are replaced by $\mathrm{NH}_{2}$-thus we get $\mathrm{CO}\left(\mathrm{NH}_{2}\right)_{2}$, urea.

(3.) Amido-acids, i.e., nitrogenous compounds (p. 341), which show partly the character of an acid and partly that of a weak base, in which the atoms of $\mathrm{H}$ of the acil radicle are replaced by $\mathrm{NH}_{2}$, or by the substituted ammonia groups.

(a.) Glycin-(p. 355), (or anido-acetic acid, glycocoll, gelatin-sugar is formed by boiling gelatin with dilute sulphuric acid. It has a sweet taste (gelatin-sugar), behaves as a weak acid, but also unites with acids as an amine-base. It occurs as glycin + benzoic acid = hippuric acid in urine ; and also as glycin + cholalic acid $=$ glyco-cholic acid in bile (p. 355). (b.) Leucin-(p. 341) = amido-caproic acid. (c.) Serin-(=? amido-lactic acid) obtained from silk-gelatin. (ll.) Asparaginic acid-(amido-succinic acid); and (e.) Glutaminic acid obtained by the splitting up of proteids (p. 342). Other amido-acids are $-(f$.$) Cystin =amido-lactic acid in$ which $O$ is replaced by $S$ (see Urine). (g.) Taurin-(p. 355), amido-ethyl-sulphuric acid occurs (except in certain glands) chiefly in combination with cholalic acid, as taurocholic acid in bile. Tyrosin (parahydro-oxyphenyl-amido-propionic acid), an amido-acid of unknown constitution, occurs along with lencin during pancreatic digestion (p. 341), is a decomposition product of proteids, and occurs plentifully in the urine in acute yellow atrophy of the liver.

To the amido-acids are related-(a.) Kreatin in muscle, brain, blood, urine, regarded as methyl-uramirlo-acetic acid $\left(\mathrm{C}_{4} \mathrm{H}_{9} \mathrm{~N}_{3} \mathrm{O}_{2}\right)$. It has been prepared artificially. When boiled with baryta water, it takes up $\mathrm{H}_{2} \mathrm{O}$, and splits into urea; and (b.) Sarkosin $\left(\mathrm{C}_{3} \mathrm{H}_{7} \mathrm{NO}_{2}\right)$, methyl-amido-acetic acid. When boiled with water, heated with strong acids, in the presence of putrefying substances, kreatin gives off water, and is changed into kreatinin $\left(\mathrm{C}_{4} \mathrm{H}_{4} \mathrm{~N}_{3} \mathrm{O}\right)$. This strong base can be rechanged by alkalies into kreatin. 
(4.) Ammonia Derivatives of Unknown Constitution.-Uric acid, allantoin (see Urine) is formed by the oxidation of uric acid by means of potassium permanganate; cyanuric acid in dog's urine; inosinic acid in muscle; gurnin in traces in the liver and pancreas, in guano, the excrements of spiders, in the skin of amphibia and reptiles, in the silver sheen of many fishes (A. Ewald and Krukenberg); by oxidation it yields urea; hypoxanthin or sarkin occurs along with xanthin in many organs and in urine. Kossel prepared hypoxanthin from nuclein by prolonged boiling of the latter. It may be obtained from fibrin by putrefaction, by gastric and pancreatic digestion, and by dilute acids (Salomon, H. Krause, Chittenden); xanthin is prepared by oxidation from hypoxanthin. It occurs very rarely in the form of a urinary calculus. Paraxanthin in urine, and a similar body carnin in flesh (\$233).

\section{Aromatic Substances.}

1. Monatomic phenols-(a) Phenol (hydroxyl of benzol) in the intestine (p. 376). Phenylsulphuric acid in urine. (b) Kresol in the form of ortholkresol and parokresol, united with sulphuric acid, occur in urine. 2. Diatomic phenols -(a) Benzkatechin united with sulphuric acid in urine. 3. Aromatic oxyacids -(a) Hydroparacumaric acid; (b) Paraoxyphenylacetic acid in urine. 4. Indol and skatol in the intestine (p. 376), conjoined with sulphuric acid in urine.

\section{Historical.}

According to Aristotle, the organism requires food for three purposes-for growth, for the production of heat, and to compensate for the loss of the bodily excreta. The formation of heat takes place in the heart by a process of concoction, the heat so formed being distributed to all parts of the body by means of the blood, while the respiration is regarded as an act whereby the body is cooled. Galen accepted this view in a sonewhat modified form; according to him, the metabolic processes may be compared to the processes going on in a lamp; the blood represents the oil; the heart, the wick; the luugs, the fanning apparatus. According to the view of the iatrochemical school (van Helmont), the metabolic processes of the body are fermentations, whereby the food is mixed with the juices of the body. Since the middle of the seventeenth century (Boyle), the knowledge of the metabolic processes has followed the development of chemistry. A. v. Haller regarded heat as due to chemical processes-the food continually supplying the waste which is excreted from the body. After the discovery of oxygen (1774, by Priestley and Scheele), Lavoisier formulated the theory of combustion in the lungs, whereby carbonic acid and water were formed. Mitscherlich compared the decomposition-processes in the living body with putrefactive processes. Magendie was the first to emphasise the difference between nitrogenous and non-nitrogenous foods, and he showed that the latter alone were not able to support life. Even gelatin alone is not sufficient for this purpose.

The greatest advance in the theory of nutrition was made by J. v. Liebig, who laid the foundation of our present knowledge of this subject. According to Liebig, foods may be divided into two classes, viz., the "plastic," suitable for the construction of the organism, and the "respiratory" for the maintenance of the temperature; to the former class he referred the albuminates or proteids, to the latter, the non-nitrogenous carbohydrates and fats.

Amongst recent observers, the Munich School, as represented by v. Bischoff, v. Pettenkofer and v. Voit, has done most to give us an exact knowledge of this department of physiology. 





\section{COLUMBIA UNIVERSITY}

This book is due on the date indicated below, or at the expiration of a definite period after the date of borrowing, as provided by the rules of the Library or by special arrangement with the Librarian in charge.

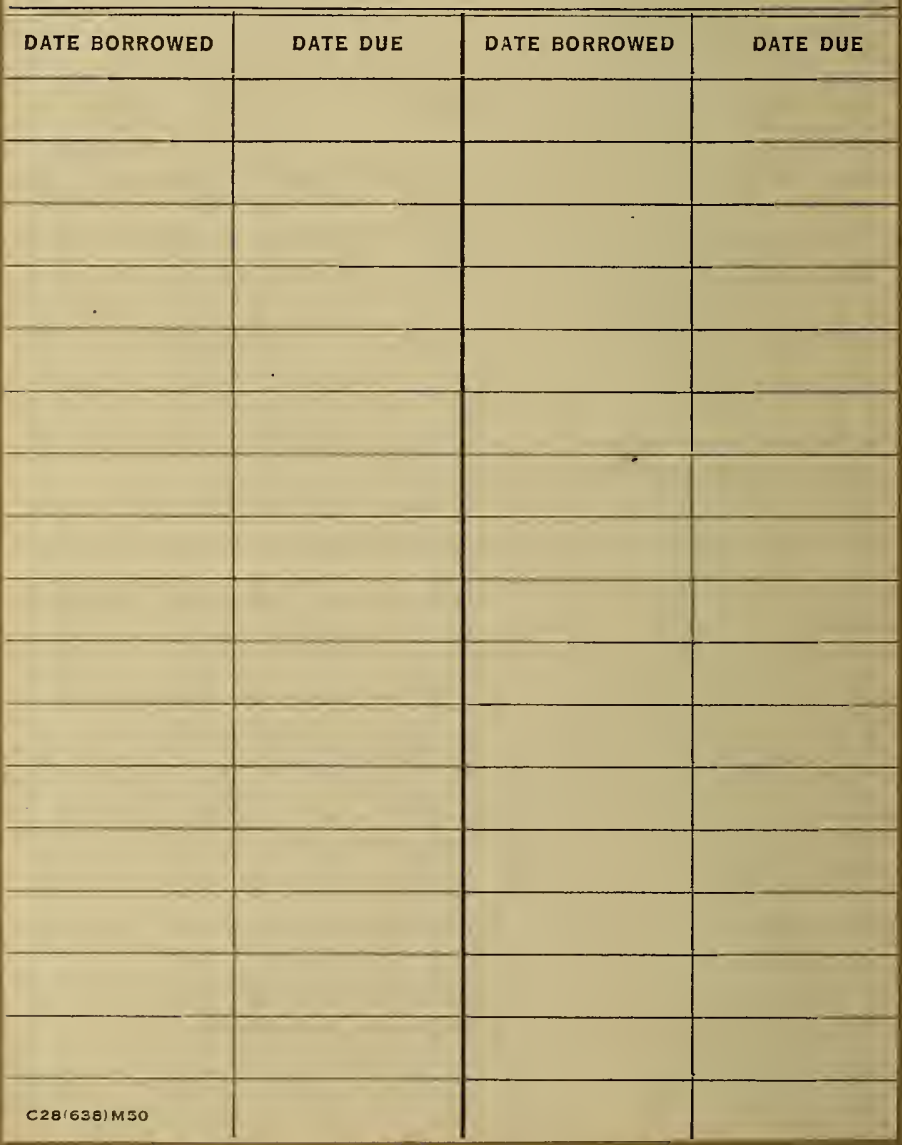




\section{QP34}

L232

Lando is จ.1 
